Taxonomy and systematics

\title{
Checklist of the native vascular plants of Mexico
}

\author{
Catálogo de las plantas vasculares nativas de México \\ José Luis Villaseñor \\ Departamento de Botánica, Instituto de Biología, Universidad Nacional Autónoma de México, Apartado postal 70-233, 04510 Ciudad de México, Mexico \\ Received 7 March 2016; accepted 6 May 2016 \\ Available online 4 August 2016
}

\begin{abstract}
An updated inventory of the native vascular plants of Mexico records 23,314 species, distributed in 2,854 genera, 297 families, and 73 orders. The flora includes 1,039 species of ferns and lycophytes, 149 gymnosperms, and 22,126 angiosperms. On average, the number of synonyms per species is 1.3 (mode $=1)$. The number of species places Mexico as the country with the fourth largest floristic richness in the world, although among the non-insular countries, by its number of endemic species (about 50\%) is second only surpassed by South Africa. The species distribution among higher taxonomic categories, and the richness and endemism values in the 32 states of Mexico are discussed. This compilation allows us to assess the flora's contribution to the overall Mexican biodiversity.

All Rights Reserved (C) 2016 Universidad Nacional Autónoma de México, Instituto de Biología. This is an open access item distributed under the Creative Commons CC License BY-NC-ND 4.0.
\end{abstract}

Keywords: Biodiversity; Biomes; Endemism; Flora of Mexico; Floristic studies; Inventories

\section{Resumen}

Un inventario actualizado de plantas vasculares nativas de México registra 23,314 especies, distribuidas en 2,854 géneros, 297 familias y 73 órdenes. La flora incluye 1,039 especies de helechos y licofitas, 149 gimnospermas y 22,126 angiospermas. En promedio se registran 1.3 sinónimos por cada nombre aceptado (moda =1). Por su número de especies, México ocupa el cuarto lugar a nivel mundial; entre los países continentales ocupa el segundo por el número de especies endémicas (alrededor del 50\%), sólo por debajo de Sudáfrica. Se discute la distribución taxonómica de las especies entre las distintas categorías taxonómicas superiores, así como los valores de riqueza y endemicidad entre los 32 estados del país. Esta recopilación permite evaluar la contribución de la flora a la biodiversidad de México.

Derechos Reservados (C) 2016 Universidad Nacional Autónoma de México, Instituto de Biología. Este es un artículo de acceso abierto distribuido bajo los términos de la Licencia Creative Commons CC BY-NC-ND 4.0.

Palabras clave: Biodiversidad; Biomas; Endemismo; Flora de México; Estudios florísticos; Inventarios

\section{Introduction}

The concept of biodiversity, applied to floristic richness, considers the number of taxa (categories of the taxonomic hierarchy) present in any geographical or administrative unit, such as county, state or country. With this number, it is possible to quantitatively evaluate diversity and compare it among areas. There are international agreements that prioritize the quantification

\footnotetext{
E-mail address: vrios@ib.unam.mx

Peer Review under the responsibility of Universidad Nacional Autónoma de México.
}

of biodiversity of the signatory countries, especially those with poor or insufficient knowledge of biodiversity at the national and/or regional levels, as is the case of Mexico (Conabio, 2012).

Mexico has a long and growing tradition of studying its vascular flora, reflected in the significant increase in recent decades of specimens housed in national scientific collections and abroad, backed by an immense bibliography. However, the knowledge of national floristic richness is still unsatisfactory mainly due to the difficulty of synthesizing scattered information in such publications along with the lack of well-curated databases of specimens documenting this richness. It is also clear that most genera require additional taxonomic study (revisions 
or monographs), and large areas of land remain unexplored to date.

The first estimates of the vascular flora of Mexico, proposed more than 2 decades ago, quoted between 17,000 and 30,000 species (reviewed in Villaseñor, 2003). A decade ago, an extensive literature review led to an estimate of about 22,351 species of vascular plants (Villaseñor, 2003, 2004). Later, Llorente-Bousquets and Ocegueda (2008), collaborating with many specialists, published the first list of species of vascular plants of Mexico, which included 22,332 species, a figure remarkably similar to that reported by Villaseñor (2003). Their list was the first publication that documents in detail aspects of Mexican plant biodiversity (Conabio, 2008). Unfortunately, the exercise carried out by the Conabio (the Mexican Biodiversity Commission) has been little used, probably because the general public has limited access to such information, and the databases still have little impact on the presentation and management of biological information. Moreover, due to the dynamism of taxonomy, published scientific names are constantly changed due to updates and corrections, or added in publication of numerous new species. Therefore, the documented information on Mexico's floristic richness should be regularly updated through the publication of floristic lists or catalogs that synthesize information on the species reported.

A catalog (floristic list or checklist) represents a more or less critical summary of the information gathered or known about the plant species (or other taxonomic designation) of a region (Nimis, 1996), and it may vary in content or approach. Sometimes, as in this work, they only list the scientific names collected for the country; on other occasions they provide additional information, such as representative specimens, literature for particular taxonomic groups, synonymy, or specific comments aimed to clarify doubts or taxonomic conflicts (see, for example, Dávila et al., 2006; García-Mendoza \& Meave, 2011; Guzmán, Arias, \& Dávila, 2003; Ibarra-Manríquez, Villaseñor, \& Durán, 1995; Villaseñor, Ortiz, Beutelspacher, \& Gómez-López, 2013). Usually, a basic species list is what is first published for any region, so it always requires a critical evaluation. The reliability of the existing literature is an issue for any catalog and unreliable primary sources result in biases or difficulties in compiling lists; in addition, it is practically impossible to verify all species identifications, and the number of reviews or monographs consulted or available is relatively low. A further problem is the use of different taxonomic criteria; specialists do not always coincide in the circumscription of species, genera or even taxonomic categories of higher rank, and reconciling these different treatments is not trivial. Sometimes an inventory follows one of these criteria, while another may prefer an alternative approach. Expert opinions help clarify uncertainties, especially when the geographical distribution of species reveals errors of reference in a given regional inventory. Ultimately, it is up to catalog users to judge the reliability of names and additional information presented.

Many people are skeptical of the scientific value of catalogs, especially scholars of biodiversity that require information that catalogs do not provide directly. However, among the merits of catalogs is that they synthesize a wealth of information accumulated throughout the history of botany and exploration of any territory. Listing species names is key to accessing a world of additional information on species, including aspects of natural history and current and potential uses. For taxonomists, lists are certainly valuable in order to consider the number of species to study in a review or monograph, and facilitate the inclusion of many species that have been overlooked in previous treatments. For ecologists and phytogeographers, catalogs are the first step to forming an informed opinion on the relationships between certain floras, and attempting to explain the causes, origins and evolution of diversity.

The aim of this contribution is to provide an updated catalog of the native vascular plant species of Mexico. This catalog is expected to serve as a basis for a better understanding of the Mexican flora and to promote more comprehensive floristic and taxonomic studies of groups or regions that require more detailed inventory or systematic work. As Nimis (1996) points out, catalogs are catalysts for new research projects and questions, and their relevance is not limited to floristic or taxonomic studies. The intention of this list of species was 2-fold: first, to document the current state of floristic knowledge, and second, to provide a basic reference that specialists can use to compare their data and more efficiently perform future taxonomic reviews. By examining the information provided here, it will soon be possible to handle more precise information on the floristic richness of the country and progress toward the long-awaited goal of having a flora of Mexico.

\section{Materials and methods}

The catalog is the result of the review of over 2,500 references covering different aspects of the flora of Mexico. Among them are the numerous fascicles published by different regional taxonomic treatments for the country. They include, for example, Flora of El Bajío and adjacent regions ( $>190$ fascicles and $>30$ additional fascicles), Flora of Veracruz $(>150)$, Flora of the Tehuacán-Cuicatlán Valley (>130), Flora of Guerrero $(>60)$, Flora of Jalisco (>20), Flora Mesoamericana (5 volumes) and Flora Novo-Galiciana (17 volumes). The protologues of many species that had never been mentioned in previous inventories or floristic treatments were also consulted. For example, in the last decade (2006-2015) 924 brand new species occurring in Mexico have been described, and 656 species have undergone name changes due to the proposal of new taxonomic combinations. Very few of these 1,580 names had been mentioned in publications of inventories or vegetation studies, and their inclusion in the catalog derives directly from the publications where they were originally described.

The compilation of this catalog has also benefited from the large body of published state and regional inventories published. In his 2 early-century reviews, Villaseñor (2003, 2004) reported the major contributions of these publications to the flora of Mexico, and pointed out the 13 states lacking statewide inventories at the time. Interestingly, updated floristic lists have been published for 5 of them since (Ciudad de México - formerly known as Distrito Federal [Rivera-Hernández \& Flores-Hernández, 2013]; Jalisco [Ramírez-Delgadillo et al., 


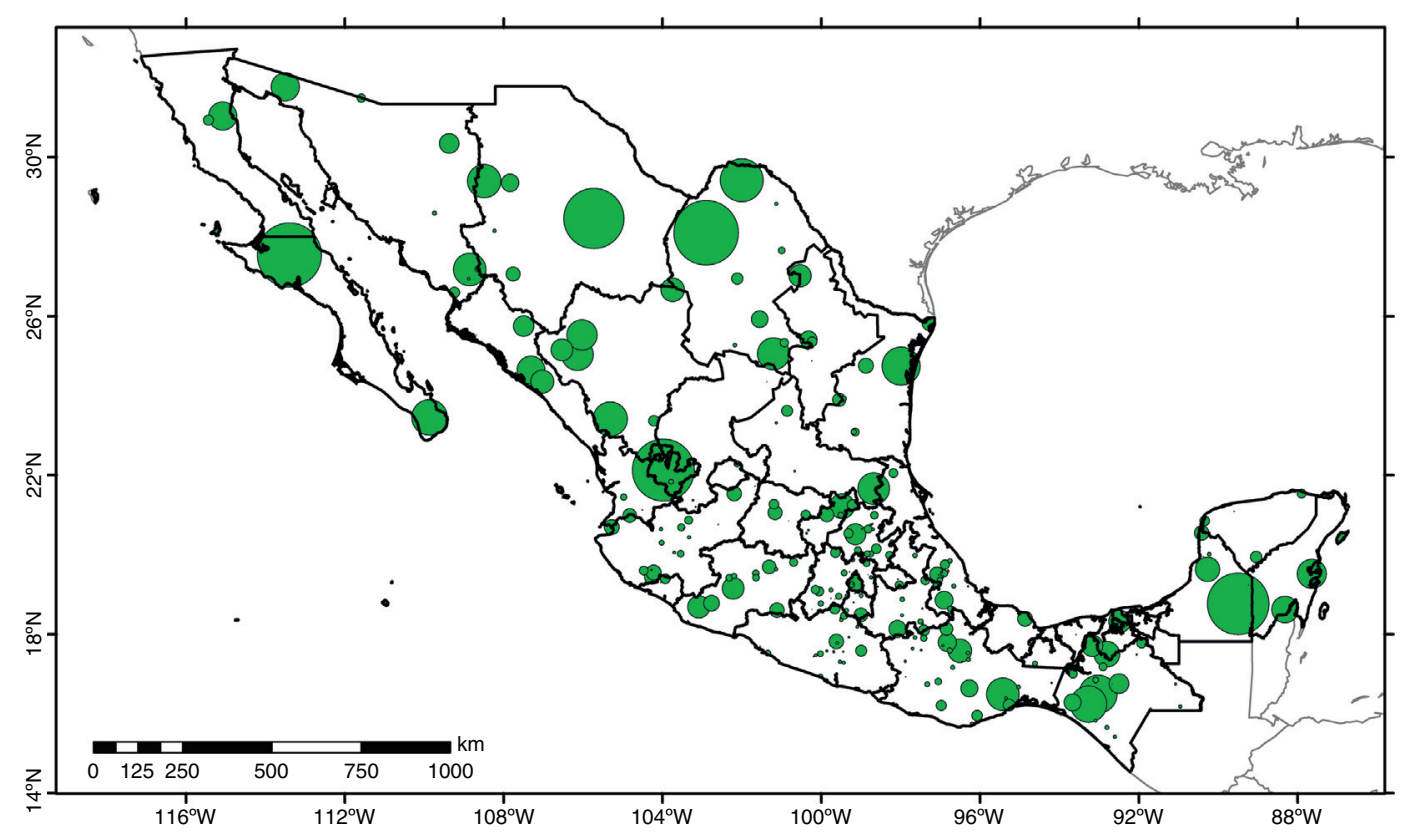

Figure 1. Mexican localities with floristic inventories $(N=284)$. The size of the circle is proportional to the studied surface in each inventory. Total surface covered by the studies is $378,887 \mathrm{~km}^{2}$ ( $19.2 \%$ of Mexico's territory).

2010]; Nuevo León [Villarreal \& Estrada, 2008]; Oaxaca [García-Mendoza \& Meave, 2011]; and Puebla [Rodríguez, Villaseñor, Coombes, \& Cerón, 2014]). Moreover, 284 local and regional inventories from all over the country have been reviewed (reference list available from the author upon request). It is unfortunate that many important works are available in libraries only as "gray literature" (e.g. technical reports, unpublished undergraduate and graduate theses; Corlett, 2011). Figure 1 illustrates the geographic distribution of these reviewed inventories; the area included in each inventory is represented by a circle centered at the intersection of the long and wide axes of the surveyed area. Thus, the size of the circle is proportional to the studied surface.

Undoubtedly, another invaluable source of data was the intense review of the library and specimens housed at the National Herbarium (MEXU) at the Instituto de Biología, UNAM and internet databases (IPNI, REMIB, The Plant List, Tropicos, Unibio, etc.). Finally, numerous experts also lent valuable support reviewing draft lists of their specialty and providing relevant information on species important literature that is difficult to access or not yet recorded in the consulted information sources. Many of them (Table 1) should be cited as responsible for these groups in the catalog, though any nomenclatural discrepancies and the accepted name included in the list are the sole responsibility of the author.

\section{Results}

\section{Floristic richness nationwide}

So far more than 54,000 names to compile the final list have been reviewed, which is included as Appendix 2. The number of accepted species is 23,314 , distributed in 73 orders, 297 families, and 2,854 genera (Table 2). In addition, there are 1,414 subspecific categories (subspecies, varieties or forms) that were not considered in the analysis; since there is uncertainty in assigning these taxa appropriately to the states, biomes or regions where they were reported, I preferred to document diversity to the species level. Considering these subspecific taxa would have increased floristic richness to about 24,728 native taxa. The higher taxonomic categories (families and orders) agree with the most recent classification proposals. For ferns, lycophytes, and gymnosperms, the arrangement follows the proposals of Christenhusz, Chun, and Scheider (2011) and Christenhusz, Reveal, et al. (2011), and angiosperms are arranged following the proposal of the APGIII (APG III, 2009; Chase \& Reveal, 2009; Haston, Richardson, Stevens, Chase, \& Harris, 2009; Wearn, Chase, Mabberley, \& Couch, 2013).

Appendix 1 contains the families of vascular plants in the accepted classification schemes. Families follow a numerical order, defining a linear sequence in accordance with recent classification proposals. For each family a summary of the number of genera and species accepted is presented. The genera and species are listed in Appendix 2, with species endemic to Mexico being denoted with an asterisk (*).

The list does not include naturalized exotic species, many of which escaped from cultivation. Villaseñor and EspinosaGarcía (2004) documented the presence in Mexico of 618 exotic species. Currently, this figure needs to be revised, because similar to the case of native species, in addition to recent taxonomic changes, some 300 additional species have been documented. Consequently, summing the richness of species, subspecific taxa, and naturalized exotics, the number 
Table 1

Specialists reviewers of preliminary lists of genera or families, or providers of relevant information crucial for elaborating the checklist.

\begin{tabular}{|c|c|}
\hline Specialist & Taxonomic group or region \\
\hline Leonardo Alvarado-Cárdenas & Apocynaceae \\
\hline William Anderson $^{\dagger}$ & Malpighiaceae \\
\hline Salvador Arias & Cactaceae \\
\hline Jesús Balleza-Cadengo & Flora of Zacatecas \\
\hline Theodore M. Barkley $^{\dagger}$ & Senecio, Asteraceae \\
\hline Attila Borhidi & Rubiaceae \\
\hline María Goreti Campos-Ríos & Boraginaceae \\
\hline Germán Carnevali & Flora of the Yucatán Peninsula \\
\hline Fernando Chiang-Cabrera & Lycium, nomenclatural aspects \\
\hline Thomas F. Daniel & Acanthaceae \\
\hline Arturo de Nova-Vázquez & Flora of San Luis Potosí \\
\hline Ricardo de Santiago & Melastomataceae \\
\hline María del Rosario García-Peña & Cunila, Lamiaceae \\
\hline José García-Pérez & Flora of San Luis Potosí \\
\hline Carlos Gómez-Hinostrosa & Cactaceae \\
\hline Socorro González-Elizondo & Flora of Durango \\
\hline Martha Gual-Díaz & Flora of the Humid Mountain Forest \\
\hline Ulises Guzmán & Cactaceae \\
\hline Héctor M. Hernández-Macías & Cactaceae \\
\hline Guillermo Ibarra-Manríquez & Ficus, Moraceae \\
\hline Jaime Jiménez-Ramírez & Flora of Guerrero \\
\hline Verónica Juárez-Jaimes & Asclepiadoideae \\
\hline Geoffrey A. Levin & $\begin{array}{l}\text { Acalypha, Drypetes, Euphorbiaceae, } \\
\text { Putranjivaceae }\end{array}$ \\
\hline Lucía G. Lohmann & Bignoniaceae \\
\hline José Antonio López-Sandoval & Flora of the state of Mexico \\
\hline Emily J. Lott & Flora of Jalisco, Flora of Oaxaca \\
\hline Martha Martínez-Gordillo & $\begin{array}{l}\text { Euphorbiaceae, Lamiaceae, } \\
\text { Peraceae, Phyllanthaceae }\end{array}$ \\
\hline Mahinda Martínez & Solanaceae \\
\hline Esteban Martínez-Salas & Arecaceae, Triuridaceae \\
\hline Angélica Ramírez-Roa & Gesneriaceae \\
\hline Jerónimo Reyes-Santiago & Crassulaceae, Flora of Oaxaca \\
\hline Lourdes Rico-Arce & Mimosoideae \\
\hline Gerardo Salazar-Chávez & Orchidaceae \\
\hline Mario Sousa-Sánchez & Fabaceae \\
\hline Rafael Torres-Colín & Caesalpinoideae \\
\hline Ivonne Sánchez del Pino & Amaranthaceae \\
\hline Victor W. Steinmann & Euphorbiaceae \\
\hline Jesús Valdés-Reyna & Poaceae \\
\hline Susana Valencia-Avalos & Quercus, Fagaceae \\
\hline Alejandra Vasco-Gutiérrez & Elaphoglossum, Dryopteridaceae \\
\hline Rito Vega-Aviña & Flora of Sinaloa \\
\hline Thomas L. Wendt & Polygalaceae \\
\hline Sergio Zamudio-Ruiz & Flora of the Bajío \\
\hline
\end{tabular}

$\dagger$ Deceased.

of vascular plants of the flora of Mexico totals more than 25,700 taxa of infrageneric rank. Finally, the list includes 48 species and 2 genera of hybrid origin (nothospecies and nothogenera).

Table 2

Taxonomic distribution of the native vascular flora of Mexico.

\begin{tabular}{lrrrr}
\hline & Orders & Families & Genera & Species \\
\hline Ferns and Monilophytes & 14 & 41 & 134 & 1,039 \\
Gymnosperms & 5 & 6 & 14 & 149 \\
Angiosperms & 54 & 250 & 2,706 & 22,126 \\
& 73 & 297 & 2,854 & 23,314 \\
\hline
\end{tabular}

Determining accepted names in the catalog required the revision of many additional names considered under synonymy. Considering that 31,263 synonyms were found, the average number of synonyms per accepted species is $1.3($ mode $=1)$; in other words, on average each species has been described more than once. It is important to emphasize that the synonymy search was not exhaustive and is almost certainly an underestimate. The ratio is based solely on the names retrieved from the floristic-taxonomic literature for the flora of Mexico, without considering relevant information for species which also occur outside the national territory. The numbers of species accepted and considered as synonyms in different taxonomic groups is variable; however, it should be noted that few orders comprise a lower number of synonyms than accepted species. Table 3 summarizes the number of accepted species and synonyms recorded in each order to date.

Table 4 shows the 25 families and genera with the most species. Comparing these lists with those provided by Villaseñor (2004), only Bignoniaceae, Cucurbitaceae and Scrophulariaceae are excluded, which were listed before among the families of angiosperms with the largest numbers of genera (Villaseñor, 2004). Notably, this list now also includes a family of ferns (Pteridaceae). Moreover, all genera with the largest numbers of species (Villaseñor, 2004) continue to be ranked highest; the only exception is the genus Opuntia, which was reduced from 90 to 72 species due to the segregation of the genus Cylindropuntia (18 species).

The 25 most diverse families include 15,306 species, representing $65.6 \%$ of Mexico's total floristic richness. The additional $34.4 \%$ is distributed unevenly among the remaining 272 families. It is worth noting that 44 families are represented in Mexico by a single species, 23 by 2 species, and 45 by 3 to 5 species; consequently, rare or poorly represented families in Mexico constitute an important fraction of the plant diversity of Mexico.

\section{Statewide floristic richness}

An important question is whether the floristic richness of Mexico is distributed evenly throughout the country or if there are areas hosting a disproportionately high richness, and if so, where are they located? Some studies have tried to answer this question, but without considering all of the flora of Mexico (for example, Luna-Vega, Espinosa, Rivas, \& Contreras-Medina, 2013; Rzedowski, 1991). The identification of extremely rich areas is the recurring theme of studies focused on conserving biodiversity. This paper provides a synthesis of floristic richness at the state level in order to identify the different levels of diversity by political entity (Table 5).

Table 5 presents an update of the vascular plant richness by state provided by Villaseñor and Ortiz (2014), supplemented with data for ferns and gymnosperms. Oaxaca, Chiapas, Veracruz, Jalisco and Guerrero respectively, continue to occupy the top 5 ranks of species richness. However, the accumulated information for other states, thanks to the collaboration with experts working with data on their floristic richness, has substantially changed compared to values recorded earlier. 
Table 3

Number of Mexican species accepted and number of synonyms recorded, by order of the 3 main groups of vascular plants.

\begin{tabular}{ll}
\hline Species accepted & Synonyms
\end{tabular}

Ferns and Lycophytes

Equisetale

Gleicheniales

Hymenophyllales

Isoetales

Lycopodiales

Marattiales

Ophioglossales

Osmundales

Polypodiales

Psilotales

Salviniales

Schizaeales

Selaginellales

Gymnosperms

Araucariales

Cupressales

Cycadales

Ephedrales

Pinales

Angiosperms

Alismatales

Apiales

Aquifoliales

Arecales

Asparagales

Asterales

Austrobaileyales

Brassicales

Buxales

Canellales

Caryophyllales

Celastrales

Ceratophyllales

Commelinales

Cornales

Crossosomatales

Cucurbitales

Chloranthales

Dilleniales

Dioscoreales

Dipsacales

Ericales

Fabales

Fagales

Garryales

Gentianales

Geraniales

Gunnerales

Huerteales

Icacinales

Lamiales

Laurales

Liliales

Magnoliales

Malpighiales

Malvales

Myrtales

Nymphaeales

Oxalidales

Pandanales

Picramniales
Table 3 (Continued)

\begin{tabular}{lcr}
\hline & Species accepted & Synonyms \\
\hline Piperales & 322 & 274 \\
Poales & 1,952 & 2,509 \\
Proteales & 8 & 20 \\
Ranunculales & 197 & 239 \\
Rosales & 476 & 636 \\
Sabiales & 12 & 5 \\
Santalales & 166 & 161 \\
Sapindales & 421 & 530 \\
Saxifragales & 427 & 233 \\
Solanales & 704 & 1,002 \\
Vitales & 38 & 50 \\
Zingiberales & 64 & 76 \\
Zygophyllales & 41 & 75 \\
\hline
\end{tabular}

Table 4

The 25 most diverse families and genera of Mexican vascular plants.

Family Species

\begin{tabular}{ll}
\hline Asteraceae & 3,057
\end{tabular}

Fabaceae

1,903

Orchidaceae

1,213

Poaceae

1,047

Euphorbiaceae 714

$\begin{array}{ll}\text { Rubiaceae } & 707\end{array}$

Cactaceae 677

Lamiaceae 601

Malvaceae $\quad 527$

Asparagaceae 445

Bromeliaceae $\quad 426$

Apocynaceae $\quad 418$

Cyperaceae 416

Solanaceae $\quad 407$

Acanthaceae 385

Crassulaceae 372

Convolvulaceae 295

Piperaceae 245

Pteridaceae 214

Amaranthaceae 211

Brassicaceae 210

Plantaginaceae 209

Apiaceae 208

Melastomataceae 204

Rosaceae 195

$\begin{array}{ll}\text { Species included } & 15,306\end{array}$

Genus Species

Salvia 328

Euphorbia 245

Tillandsia 237

Quercus 174

Mammillaria 169

Ageratina 165

Verbesina 165

Agave 160

Ipomoea 159

Dalea

Solanum 143

Piper 136

Sedum 133

Echeveria 132

Croton 127

Muhlenbergia $\quad 118$

Epidendrum 117 
Table 4 (Continued)

\begin{tabular}{lc}
\hline Genus & Species \\
\hline Stevia & 117 \\
Carex & 114 \\
Mimosa & 112 \\
Desmodium & 110 \\
Peperomia & 109 \\
Acalypha & 108 \\
Begonia & 106 \\
Bursera & 94 \\
Justicia & 94 \\
\hline
\end{tabular}

Figure 2 illustrates the distribution of species, genus and family richness for each state synthesized in Table 5. The values are divided by the logarithm $\left(\log _{10}\right)$ of the state's surface area, a procedure that essentially standardizes mean species richness per $10 \mathrm{~km}^{2}$. A diversity gradient from south to north is depicted, with some states of central Mexico breaking from this gradual shift of richness. Similarly, the 2 major peninsulas of the country (Baja California and Yucatán) had the lowest richness values for all 3 taxonomic levels evaluated.
Apparently, most states showed a roughly equal proportion of species per genus (species/genus ratio; mean $=3.5$, minimum $=2.3$, maximum =5.2), except Oaxaca, for which the largest ratio was recorded. The variation is more noticeable when comparing the proportion of species per family (minimum $=10.8$, maximum $=38.3$; average $=8.2$; Fig. 3 ).

\section{Endemism}

The total percentage of endemic species of Mexico among all vascular plants had not been previously reported. Most estimates are primarily for flowering plants. For this set, Rzedowski (1991) estimated their level of endemism at 52\%. Subsequently, Villaseñor and Ortiz (2014) estimated the value at 50.4\% The figures included in this paper suggested that the level of endemism for all vascular plants is $49.8 \%$ (Table 5). Given the consistency between Rzedowski's estimate and these updated figures, levels of endemism are likely around 50\%; in other words, about half of the flora of Mexico is endemic to its territory.

Another interesting finding from these analyses is that 3,167 species endemic to Mexico (13.6\% of the total floristic richness and $27.3 \%$ nationwide endemics) are found only in

Table 5

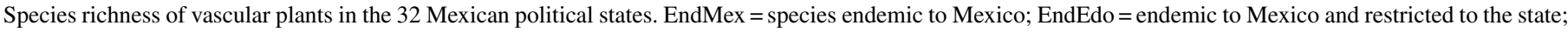
ExclEdo = non-endemic but restricted to the state.

\begin{tabular}{|c|c|c|c|c|c|c|}
\hline State & Families & Genera & Species & EndMex & EndEdo & ExclEdo \\
\hline Aguascalientes (AGS) & 147 & 690 & 1,871 & 658 & 2 & 1 \\
\hline Baja California (BCN) & 151 & 797 & 2,336 & 447 & 140 & 572 \\
\hline Baja California Sur (BCS) & 159 & 752 & 1,988 & 682 & 242 & 1 \\
\hline Campeche (CAM) & 172 & 917 & 2,369 & 177 & 4 & 2 \\
\hline Chiapas (CHIS) & 270 & 1,912 & 8,790 & 1,635 & 403 & 877 \\
\hline Chihuahua (CHIH) & 176 & 1,091 & 4,291 & 1,197 & 89 & 54 \\
\hline Coahuila (COAH) & 161 & 982 & 3,780 & 1,202 & 141 & 61 \\
\hline Colima (COL) & 219 & 1,267 & 4,333 & 1,734 & 32 & 0 \\
\hline Ciudad de México (CDMX) & 150 & 698 & 1,978 & 681 & 0 & 0 \\
\hline Durango (DGO) & 191 & 1,145 & 4,472 & 1,861 & 80 & 5 \\
\hline Guanajuato (GTO) & 176 & 965 & 3,206 & 1,350 & 7 & 0 \\
\hline Guerrero (GRO) & 238 & 1,507 & 6,551 & 2,715 & 237 & 13 \\
\hline Hidalgo (HGO) & 227 & 1,332 & 4,734 & 1,755 & 16 & 0 \\
\hline Jalisco (JAL) & 235 & 1,541 & 7,155 & 3,353 & 182 & 4 \\
\hline México (MEX) & 212 & 1,311 & 5,177 & 2,155 & 37 & 2 \\
\hline Michoacán (MICH) & 219 & 1,394 & 5,885 & 2,588 & 85 & 16 \\
\hline Morelos (MOR) & 197 & 1,063 & 3,491 & 1,242 & 23 & 1 \\
\hline Nayarit (NAY) & 209 & 1,160 & 3,964 & 1,571 & 27 & 2 \\
\hline Nuevo León (NLE) & 170 & 1,028 & 3,740 & 1,350 & 52 & 9 \\
\hline Oaxaca (OAX) & 266 & 1,946 & 10,229 & 4,071 & 760 & 90 \\
\hline Puebla (PUE) & 247 & 1,483 & 5,232 & 1,935 & 67 & 1 \\
\hline Querétaro (QRO) & 215 & 1,289 & 4,411 & 1,691 & 11 & 0 \\
\hline Quintana Roo (QROO) & 165 & 905 & 2,276 & 168 & 6 & 13 \\
\hline San Luis Potosí (SLP) & 226 & 1,441 & 5,413 & 1,994 & 42 & 2 \\
\hline Sinaloa (SIN) & 200 & 1,118 & 3,736 & 1,458 & 69 & 2 \\
\hline Sonora (SON) & 183 & 1,158 & 4,106 & 1,081 & 77 & 59 \\
\hline Tabasco (TAB) & 198 & 1,036 & 2,826 & 212 & 16 & 23 \\
\hline Tamaulipas (TAMS) & 218 & 1,309 & 4,278 & 1,267 & 65 & 43 \\
\hline Tlaxcala (TLAX) & 119 & 503 & 1,297 & 417 & 0 & 0 \\
\hline Veracruz (VER) & 271 & 1,956 & 8,497 & 2,498 & 238 & 59 \\
\hline Yucatán (YUC) & 150 & 803 & 1,900 & 157 & 6 & 7 \\
\hline Zacatecas (ZAC) & 179 & 1,045 & 3,705 & 1,599 & 12 & 0 \\
\hline MEXICO & 297 & 2,854 & 23,314 & 11,600 & 3,167 & 1,924 \\
\hline
\end{tabular}



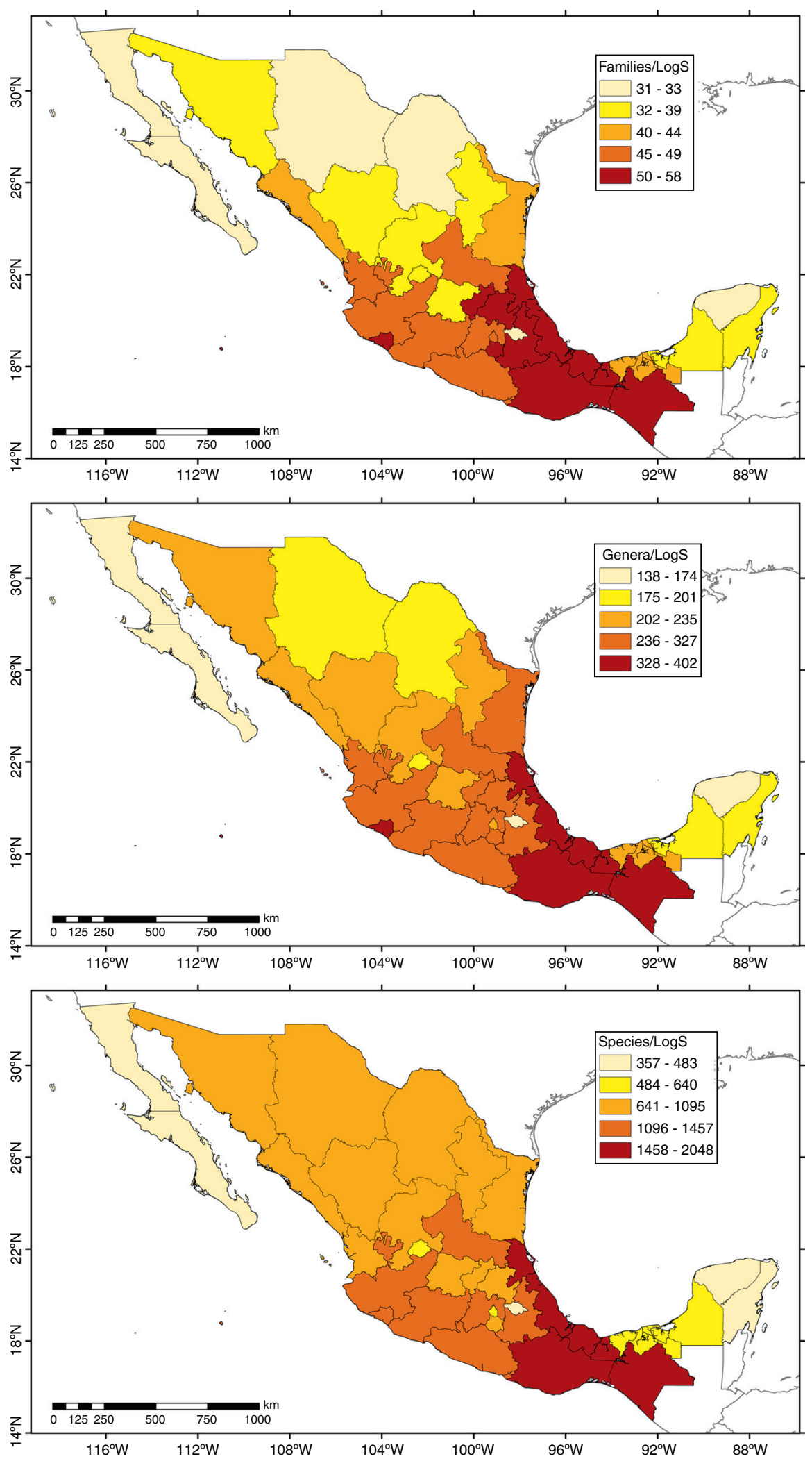

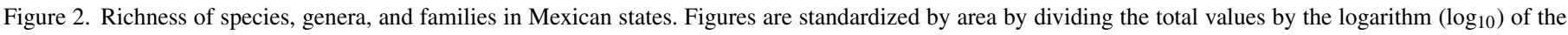
state's surface $(S)$. 


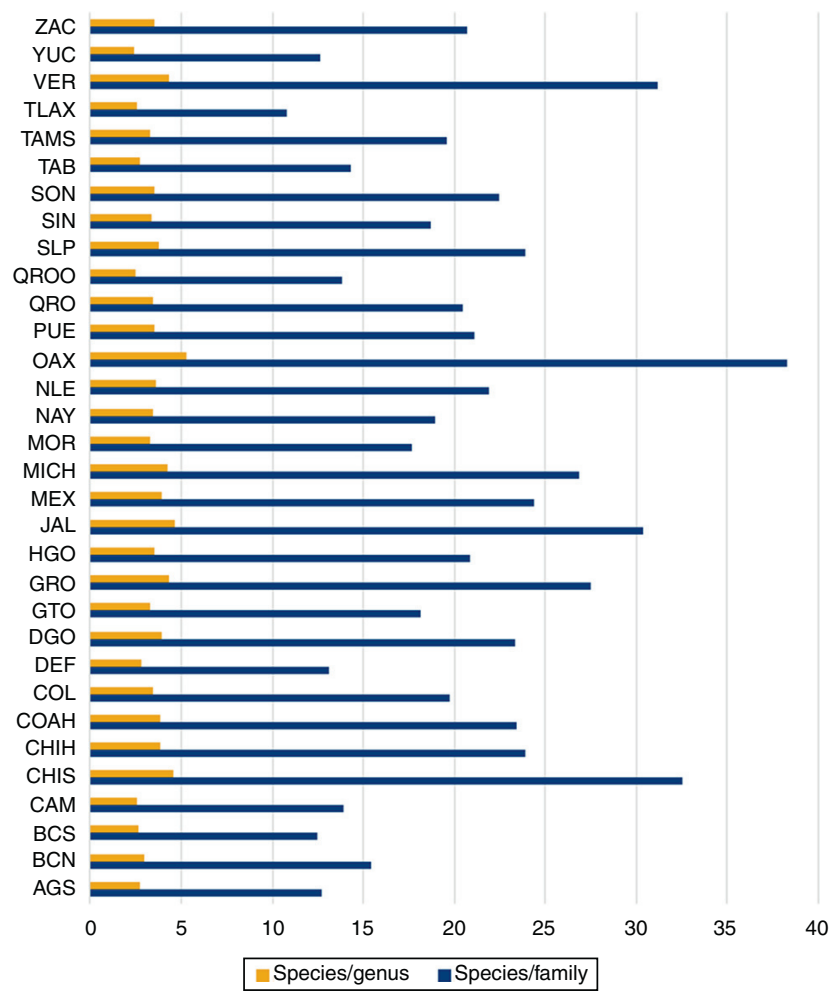

Figure 3. Proportion of species described by genera and families in the 32 political states of Mexico.

1 state. If we add to this figure those species that are not endemic to Mexico, but only found in a single Mexican state (classified here as restricted species), mostly at the northern or southern edge of the species' geographic range, the proportion of rare species (species found in a single state) reaches $21.8 \%$ of the national floristic richness. Table 5 indicates how many of these rare species are recorded in each one of the states of Mexico.

\section{Floristic richness in the major biomes of Mexico}

In agreement with the uneven distribution of floristic richness statewide, irregular distribution patterns are also evident at larger scales, although it is feasible to recognize areas with characteristic species assemblages. For example, Villaseñor and Ortiz (2014) described 5 major biomes into which the vegetation types in Mexico are grouped, ranging in value from 5,296 species in the tropical humid forest (THUF) to 8,824 species in the temperate forest (TEMF). Here, the species richness distribution is assessed by biome, with special consideration of the species characteristic to each one. Species are classified as being restricted to (or characteristic of) a biome when distributed in only 1 biome or in 2 contiguous biomes (e.g., humid mountain forest (HUMF) and TEMF, or HUMF and THUF).

Table 6 shows the numbers of characteristic species, recorded by biome in each Mexican state. Highlighted in bold typeface are the 5 states contributing with the largest richness values to each biome. There are states where 1 or more of the biomes are not represented; for example, in Aguascalientes there is neither humid mountain forest (HUMF) nor seasonally dry tropical forest (SDTF) and there in neither HUMF nor temperate forest (TEMF) in Quintana Roo. However, the richness of these biomes is recorded anyway in these states because many species are found in more than 1 biome and are therefore distributed even in states where their principle biome is lacking. For example, most species reported for the HUMF in Aguascalientes are shared between HUMF and TEMF, the latter of which occurs in the state. Other species, for example Acaciella angustissima (Mill.) Britton \& Rose (Fabaceae), Sambucus nigra L. (Adoxaceae), Tecoma stans (L.) Juss. ex Kunth (Bignoniaceae), or Zanthoxylumfagara (L.) Sarg. (Rutaceae), are widely distributed and have broad ecological tolerance, and are therefore found in almost every states and all 5 biomes in Mexico. On the other hand, of the 6,763 species reported by Villaseñor and Ortiz (2014) for the HUMF, 3,941 are characteristic of HUMF, found only in HUMF or in HUMF and only 1 more (adjacent) biome. Chiapas excells over all remaining states by having the largest number of HUMF characteristic species $(2,399)$, and it is followed by Oaxaca $(2,302)$ and Veracruz $(1,875)$. In contrast, of the 6,852 species reported for the xeric scrubland (XESC), 4,614 are characteristic of the biome, highlighting Coahuila with the largest number of XESC characteristic species $(1,764)$, followed by Chihuahua $(1,461)$ and Sonora $(1,411)$. Table 6 shows the values and percentages of characteristic species by biome in each of the states. In turn, Figure 4 shows the general distribution of characteristic species for each biome in Mexico as a whole. As in Figure 2, richness values are standardized per $10 \mathrm{~km}^{2}$ by dividing the total values by the logarithm $\left(\log _{10}\right)$ of the state's surface.

\section{Discussion}

Mexico's total floristic richness (23,314 species) places it fourth in the world, behind Brazil (32,000 species, BFG, 2015), China (29,000 species; Qian \& Ricklefs, 1999) and Colombia (24,000 species; Rangel, 2015). Villaseñor (2003) positioned Mexico in fifth place, below South Africa, although the most recent account for that country reported the existence of 20,000 native species (Germishuizen, Meyer, Steenkamp, \& Keith, 2006). Also noteworthy is the high level of endemism of the flora of Mexico; although recent values reduce the percentage of endemism to $49.8 \%$, it still occupies a privileged place among the world's countries, reporting a native flora that is very restricted to its political territories. Excluding archipelagos and other island territories that are characterized by large proportions of endemic species, Mexico is apparently surpassed only by South Africa among mainland countries in its proportion of endemic species $(57.1 \%)$.

Reviewing the literature, especially protologues, has been instrumental for the inclusion of many species that have been previously forgotten or overlooked in our floristic knowledge, and continuing searches among journal publications allows the inclusion of the many recently described species occurring in 
Table 6

Native vascular plant species characteristic of each of the 5 main biomes of Mexico (occurring in only 1 or 2 biomes). In bold the highest number of characteristic species. $\mathrm{HUMF}=$ humid mountain forest; TEMF = temperate forest, THUF = humid tropical forest; $\mathrm{SDTF}=$ seasonally dry tropical forest; XESC $=\mathrm{xeric}$ scrubland.

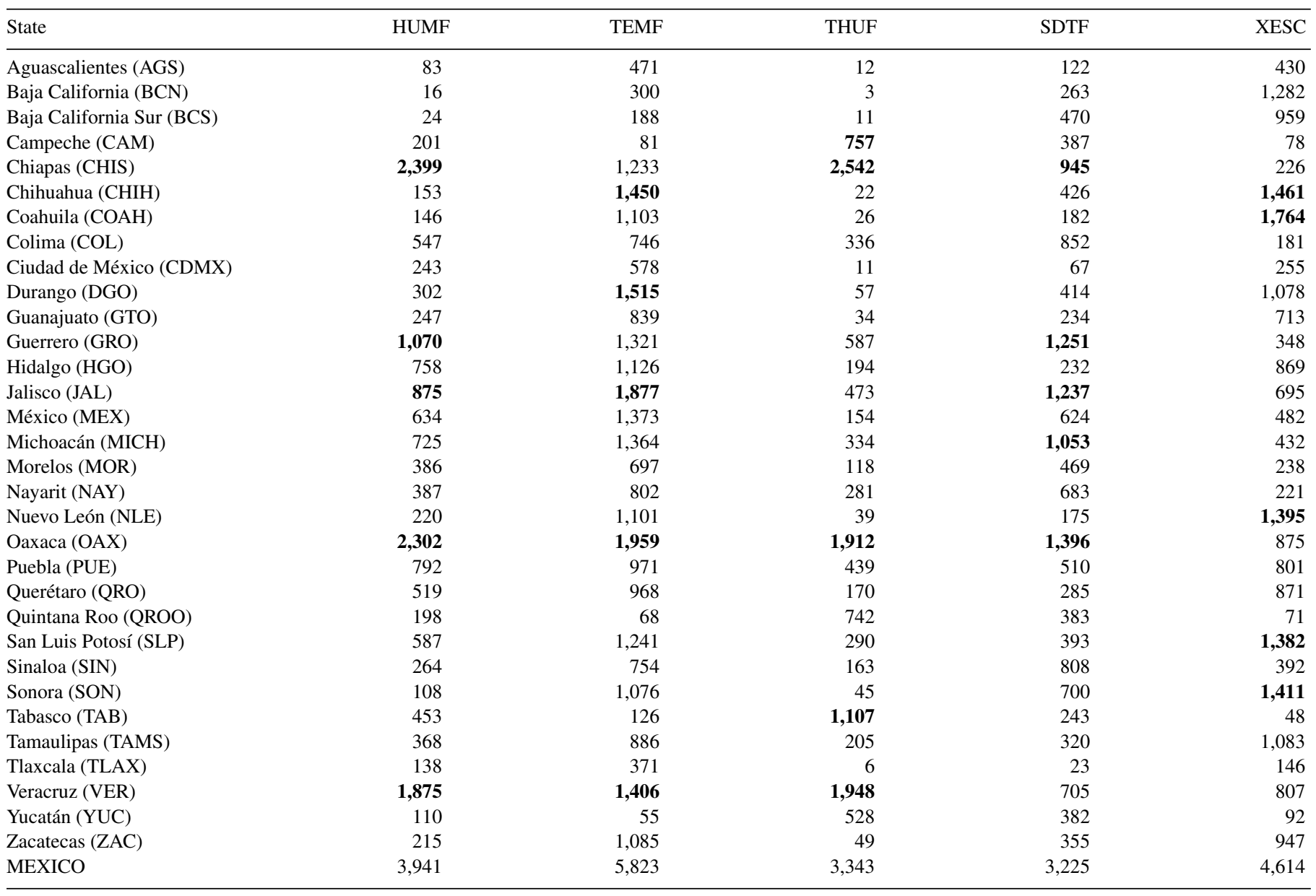

Mexico whose inclusion in international databases (IPNI, The Plant List, Tropicos, etc.) is not immediate, and in fact sometimes it never happens. Consequently, search protocols should be established to increase the efficacy in incorporating these taxa into national databases, as this would allow the continuous updating of catalogs like the one presented in Appendix 2. Moreover, dealing with the diverging species concepts used by different specialists has not been trivial; the decision to accept or reject a name often reflects experience in a particular group or personal preferences.

It is interesting to contrast the species richness between northern and southern halves of the country (Fig. 2); this contrast more or less identifies the latitude of contact between the Nearctic and Neotropical realms. In turn, the decrease in richness in extreme northwest and southeast of Mexico, on the Baja California and Yucatán peninsulas, respectively, is likely the result of the peninsular effect, which causes peninsular territories to be less diverse than the continental nearby mainland due to their geographic isolation and the lack of important biomes (Gaston \& Williams, 1996). Also interesting is the existence of some low-diversity states embedded within a matrix of high-diversity sites; this is the case of Tlaxcala, which had richness values well below its neighboring states at all 3 taxonomic levels. It would be revealing to further explore the cause of these low richness values; discerning whether this is due to lower collecting effort, land use change, or other factors would be informative and could modify the results reported here.

The data in Table 6 point out the large number of species that are found in only 1 or 2 biomes; thus, relatively few species actually show a wide ecological tolerance. Surely, this widely distributed component includes weedy species and those that tolerate disturbance, commonly occurring in successional plant communities known as secondary vegetation. For example, some 2,640 species are currently documented as having weedy and/or ruderal behavior (Villaseñor, unpublished data), of which $1,544(58.5 \%)$ are reported from more than 2 biomes. Similarly, of more than 1,000 species known to grow in secondary vegetation, over $700(70 \%)$ are also reported as occurring in 3 or more biomes.

Like other mega-diverse countries, the results reported here suggest that a large percentage of Mexico's flora tends to have high values of rarity, as indicated either by the breadth of its distribution (species recorded in few states; Table 5) or its habitat 

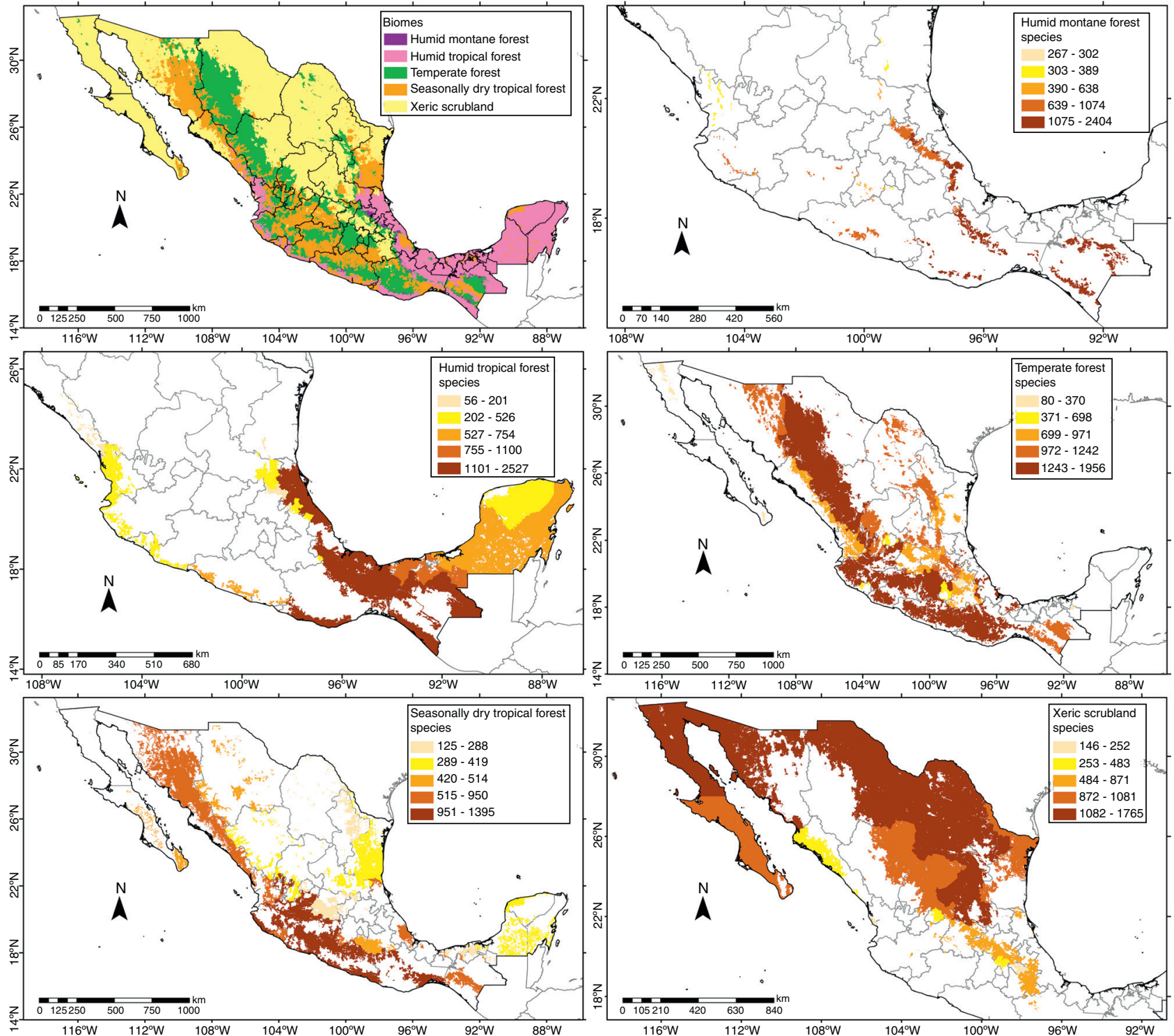

Figure 4. Map of the 5 main biomes of Mexico and richness values in each of them. Values were standardized by area by dividing the total values by the logarithm $\left(\log _{10}\right)$ of the state's surface. Biomes following the definition of Villaseñor and Ortiz (2014).

specificity (species recorded in few biomes; Table 6). Therefore, it should not come as a surprise to conclude that many species remain to be encountered before we can fully assess vascular plant diversity in Mexico (Villaseñor, 2015). With each new intensive exploration of a given territory (and sometimes by pure serendipity) anywhere in the country, new species are discovered and recorded, as a consequence of their narrow geographic and/or habitat distributions.

Concluding, any catalog, in the format of a flora, a taxonomic review, or a monograph, is constantly changing. The number of species reported here does not reflect the final number of different plants existing in Mexico. In fact, it may never be possible to explore any country in its entirety. This is particularly true for nations with orographies as complex as that of Mexico, so that each newly explored region will unavoidably reveal surprises. Even those regions that are better-researched are important sources of new floristic information (Ertter, 2000). Moreover, the dynamism of taxonomy and systematics, revived in recent decades by the implementation of new techniques and methods of analysis (cladistics, evolutionary biogeography, molecular biology, phylogeography, etc.) leads to constant changes in circumscriptions and taxonomic rearrangements, which requires periodic updates to the floristic information. The compilation of information in databases and the new tools provided by the informatics of biodiversity (Bisby, 2000; Graham, Ferrier, Huettman, Moritz, \& Peterson, 2004; Soberón \& Peterson, 2004), have made the curation and maintenance of taxonomic information more efficient, allowing for easier updates to catalogs like the one included here, and ultimately leading to the accumulation of more and better knowledge of our ever changing floristic richness. 
Importantly, the sources of information for scholars of biodiversity and its conservation comes from 4 main sources: (1) floras, reviews and monographs; (2) catalogs and inventories; (3) databases, and (4) unpublished experience of experts. These sources are not, however, independent of one another; floras, taxonomic revisions and monographs rely on catalogs, which rely on experts to carry them out, and databases rely on all 3 of these sources for their reliable development, curation, and management. For this reason, countries whose biodiversity is high but poorly known, such as Mexico, should continue to support inventory studies, so that these other sources, including personal intellectual enrichment of specialists, continue to improve and facilitate the exploration and proper evaluation of the country's natural capital. Scientific collections and databases with a reliable curatorial level are the basis for taxonomic and systematic work; they constitute what May (1990) calls the bricks and mortar for building the biodiversity knowledge on which ecologists, biogeographers and analysts of biodiversity and conservation depend.

Completing the flora of Mexico is a huge undertaking that requires the accumulation of a vast amount of information, from data derived from field explorations, inventories, preparation of regional floras and taxonomic reviews, to the creation of databases of well-curated data. Floristic checklists, as one of these components, report the numbers of species recorded in the area of study and reflect regional species richness on which many studies assessing patterns of diversity, endemism (biogeographic), conservation, and macroecological patterns, among others, depend. They all rely on the quality of data, which must be reported with the utmost seriousness and a deep understanding of the floristic and taxonomic aspects essential to the enterprise. The identification and correct application of the names of plants is central to many areas of research in natural sciences and since the coining of the concept of biodiversity, in government agencies as well. Producing lists of threatened species, such as the Mexican NOM-054 (Semarnat, 2010), the IUCN Red List of Threatened Species (Walter \& Gillett, 1998), and the international agreements regulating international trade of biological specimens (CITES), requires updated information on plants and their names.

The exercise carried out in this work, which aims to standardize the names used in the flora of Mexico, will hopefully serve as a useful framework for refining floristic information. Ultimately, this will provide benefits not only to the taxonomist community, but also to many other colleagues who use, require and routinely consult the kind of information presented here. Finally, paraphrasing the great botanist Arthur Cronquist, this catalog holds the work of our predecessors and will be the support of future floristic and taxonomic work.

\section{Acknowledgments}

Víctor Sánchez-Cordero, Director of the Institute of Biology, Universidad Nacional Autónoma de México (UNAM), and Gerardo Salazar, Head of its Department of Botany, supported this project with interest, energy and resources to bring it to its conclusion. The experienced and outstanding technical support from Enrique Ortiz, who prepared the maps and collaborated in the design, management and curation of the information in databases, facilitated the analysis and presentation of the data discussed in this work. Also, the technical assistance of Joselin Cadena, Guadalupe Segura, and Maribel Paniagua, allowed an efficient and accurate search of missing and necessary information to conduct better analysis and interpretation of results. Numerous colleagues, specialists of different plant groups (Table 1), revised preliminary or final versions of taxonomic groups included in this catalog; it was often difficult to reconcile their criteria with those used here to achieve homogeneity in integrating of the list. Thus, I assume full responsibility for what is quoted in the catalog, but thank them for their selfless support. Fernando Chiang critically reviewed the manuscript and the list of species, greatly improving the first and minimizing errors in the second. Emily J. Lott, Teresa Terrazas, Jorge Meave, and Claudio Delgadillo critically read the manuscript and their comments improved it considerably. Lynna M. Kiere reviewed and edited the English version of the paper. Some information was obtained through various projects that gave financial and human support; among the supporting institutions are Conabio (the Mexican Biodiversity Commission), Conacyt (the Mexican Council For Science And Technology), Senasica-Sagarpa (the National Service for Food Health and Quality of the Ministry for Agriculture, Animal Husbandry and Fisheries), Unibio (the Unit for Biodiversity Informatics), and the Institute of Biology, UNAM. Finally, thanks to all botanists (several thousand) not cited in the paper, collectors and taxonomists, ecologists and biogeographers, who with their contributions and publications, generated the combined wealth of information reflected in this study. 
Appendix 1. Systematic arrangement of the native vascular plants of Mexico. The number of the families corresponds to the linear arrangement proposed by APG III (2009), Chase and Reveal (2009), Christenhusz, Chun, et al. (2011), Christenhusz, Reveal, et al. (2011), Haston et al. (2009) and Wearn et al. (2013). In parentheses, the first number indicates the number of genera and the second the number of species recorded for the family in Mexico

Ferns and Lycophytes

Lycophytes

Subclass Lycopodiidae

Order Lycopodiales

1. Lycopodiaceae $(4 / 21)$

Order Isoetales

2. Isoetaceae (1/7)

Order Selaginellalles

3. Selaginellaceae (1/79)

\section{Pteridophytes}

\section{Subclass Equisetidae}

Order Equisetales

1. Equisetaceae (1/6)

Subclass Ophioglossidae

Order Ophioglossales

2. Ophioglossaceae (2/16)

Order Psilotales

3. Psilotaceae (1/2)

Subclass Marattiidae

Order Marattiales

4. Marattiaceae (2/6)

\section{Subclass Polypodiidae}

Order Osmundales

5. Osmundaceae $(1 / 2)$

Order Hymenophyllales

6. Hymenophyllaceae (5/51)

Order Gleicheniales

7. Gleicheniaceae (4/7)

Order Schizaeales

10. Lygodiaceae $(1 / 3)$

11. Schizaeaceae $(2 / 3)$

12. Anemiaceae $(1 / 21)$

Order Salviniales

13. Marsileaceae (2/8)

14. Salviniaceae (2/4)
Order Cyatheales

17. Culcitaceae (1/1)

18. Plagiogyriaceae (1/1)

19. Cibotiaceae (1/2)

20. Cyatheaceae (3/14)

21. Dicksoniaceae $(2 / 2)$

22. Metaxyaceae (1/1)

Order Polypodiales

23. Lonchitidaceae (1/1)

24. Saccolomataceae $(1 / 2)$

26. Lindsaeaceae (3/8)

27. Dennstaedtiaceae (4/23)

28. Pteridaceae (33/214)

29. Cystopteridaceae (1/4)

30. Aspleniaceae (4/89)

31. Diplaziopsidaceae (1/1)

32 . Thelypteridaceae $(1 / 70)$

33. Woodsiaceae $(1 / 8)$

35. Onocleaceae $(1 / 1)$

36. Blechnaceae $(2 / 20)$

37. Athyriaceae $(2 / 31)$

38. Hypodematiaceae (1/1)

39. Dryopteridaceae

(14/159)

40. Lomariopsidaceae $(2 / 3)$

41. Nephrolepidaceae (1/3)

42. Tectariaceae $(1 / 7)$

43. Oleandraceae (1/1)

45. Polypodiaceae

(20/136)

Gymnosperms

Subclass Cycadidae

Order Cycadales

2. Zamiaceae (3/50)

Subclass Gnetidae

Order Ephedrales

6. Ephedraceae (1/7)

\section{Subclass Pinidae}

Order Pinales

7. Pinaceae (4/59)

Order Araucaliales

9. Podocarpaceae (1/3)

Order Cupressales

11. Cupressaceae (4/29)
12. Taxaceae (1/1)

Angiosperms

Superorder Nymphaeanae

Orden Nymphaeales

3. Cabombaceae $(2 / 2)$

4. Nymphaeaceae $(2 / 12)$

Superorder Austrobaileyanae

Orden Austrobaileyales

7. Schisandraceae $(2 / 2)$

Superorder Chloranthanae

Orden Chloranthales

8. Chloranthaceae (1/1)

Superorder Magnolianae

Orden Canellales

9. Canellaceae (1/1)

10. Winteraceae $(1 / 1)$

Orden Piperales

11. Saururaceae $(2 / 2)$

12. Piperaceae $(2 / 245)$

15. Aristolochiaceae (1/75)

Orden Magnoliales

16. Myristicaceae (2/4)

17. Magnoliaceae $(1 / 22)$

21. Annonaceae (13/59)

Orden Laurales

23. Siparunaceae $(1 / 3)$

26. Hernandiaceae (3/8)

27. Monimiaceae (1/8)

28. Lauraceae (11/141)

Superorder Lilianae

Orden Alismatales

30. Araceae (16/140)

32. Alismataceae $(5 / 22)$

34 . Hydrocharitaceae $(5 / 8)$

37. Juncaginaceae $(1 / 3)$

38. Zosteraceae $(2 / 3)$

39. Potamogetonaceae (3/10)

41. Ruppiaceae (1/3)

42. Cymodoceaceae (2/2) 
Orden Dioscoreales 45. Burmanniaceae $(5 / 8)$

46. Dioscoreaceae $(1 / 75)$

Orden Pandanales

47. Triuridaceae $(2 / 2)$

50. Cyclanthaceae (4/7)

Orden Liliales

53. Melanthiaceae $(4 / 36)$

55. Alstroemeriaceae $(1 / 3)$

59. Smilacaceae $(1 / 20)$

61. Liliaceae (3/24)

Orden Asparagales

62. Orchidaceae $(162 / 1213)$

67 . Hypoxidaceae $(2 / 11)$

71. Iridaceae (16/111)

74. Amaryllidaceae (10/113)

75. Asparagaceae (28/445)

Orden Arecales

76. Arecaeae (21/105)

Orden Commelinales

78. Commelinaceae (11/126)

80. Pontederiaceae (3/13)

81. Haemodoraceae (1/1)

Orden Zingiberales

84. Heliconiaceae (1/19)

85. Musaceae (1/1)

86. Cannaceae $(1 / 3)$

87. Marantaceae $(6 / 26)$

88. Costaceae (1/10)

89. Zingiberaceae $(1 / 5)$

Orden Poales

91. Typhaceae (2/4)

92. Bromeliaceae (19/426)

94. Xyridaceae (1/3)

95. Eriocaulaceae (4/14)

96. Mayacaceae (1/1)

98. Juncaceae (2/41)

99. Cyperaceae (26/416)

106. Poaceae $(160 / 1,047)$

Superorder Ceratophyllanae

Orden Ceratophyllales

107. Ceratophyllaceae (1/2)

Superorder Ranunculanae

Orden Ranunculales

109. Papaveraceae $(10 / 45)$

112. Menispermaceae $(7 / 21)$

113. Berberidaceae $(1 / 34)$

114. Ranunculaceae (9/97)

Orden Sabiales

115. Sabiaceae (1/12)
Superorder Proteanae

162. Betulaceae (3/6)

Orden Proteales

116. Nelumbonaceae (1/1)

117. Platanaceae $(1 / 5)$

118. Proteaceae $(1 / 2)$

Superorder Buxanae

Orden Buxales

121. Buxaceae (2/7)

Superorder Myrothamnanae

Orden Gunnerales

123. Gunneraceae (1/3)

Superorder Dillenianae

Orden Dilleniales

124. Dilleniaceae (4/7)

Superorder Saxifraganae

Orden Saxifragales

127. Altingiaceae (1/1)

128. Hamamelidaceae (3/3)

131. Iteaceae $(1 / 3)$

132. Grossulariaceae $(1 / 23)$

133. Saxifragaceae $(5 / 18)$

135. Crassulaceae (13/372)

139. Haloragaceae (2/7)

Superorder Rosanae

Orden Vitales

140. Vitaceae $(5 / 38)$

Orden Zygophyllales

141. Krameriaceae (1/9)

142. Zygophyllaceae (8/32)

Orden Fabales

144. Fabaceae

(155/1,903)

145. Surianaceae $(2 / 5)$

146. Polygalaceae (6/104)

Orden Rosales

147. Rosaceae (30/195)

151. Rhamnaceae

(11/119)

152. Ulmaceae $(3 / 7)$

153. Cannabaceae (4/11)

154. Moraceae $(11 / 53)$

155. Urticaceae (17/91)

Orden Fagales

157 Fagaceae (2/175)

158. Myricaceae (1/3)

159. Juglandaceae $(4 / 15)$

161. Ticodendraceae (1/1)
Orden Cucurbitales

166. Coriariaceae (1/1)

167. Cucurbitaceae (33/151)

168. Apodanthaceae (2/4)

169. Datiscaceae (1/1)

170. Begoniaceae (1/106)

Orden Celastrales

172. Celastraceae (28/96)

Orden Oxalidales

174. Connaraceae (3/9)

175. Oxalidaceae $(2 / 37)$

176. Cunoniaceae (1/4)

177. Elaeocarpaceae $(1 / 10)$

179. Brunelliaceae (1/1)

Orden Malpighiales

181. Rhizophoracae (2/3)

182. Erythroxylaceae (1/10)

185. Euphorbiaceae (34/714)

188. Ochnaceae $(2 / 13)$

189. Picrodendraceae (2/4)

190. Phyllanthaceae (8/49)

191. Elatinaceae $(2 / 4)$

192. Malpighiaceae (24/169)

194. Trigoniaceae $(1 / 2)$

195. Dichapetalaceae (2/3)

197. Chrysobalanaceae (4/13)

199. Putranjivaceae $(1 / 3)$

200. Passifloraceae (4/84)

201. Lacistemataceae (1/1)

202. Salicaceae $(20 / 78)$

203. Violaceae $(6 / 56)$

205. Achariaceae (2/2)

209. Linaceae $(1 / 25)$

211. Calophyllaceae (3/3)

212. Clusiaceae $(4 / 18)$

214. Podostemaceae (4/9)

215 . Hypericaceae $(3 / 28)$

Orden Geraniales

216. Geraniaceae (4/45)

Orden Myrtales

219. Combretaceae (4/15)

220. Lythraceae $(9 / 111)$

221. Onagraceae (17/171)

222 . Vochysiaceae $(1 / 2)$

223. Myrtaceae (10/122)

224. Melastomataceae (27/204)

Orden Crossosomatales

231. Staphyleaceae (2/5)

232. Guamatelaceae (1/1)

234. Crossosomataceae (3/5) 
Orden Picramniales

235. Picramniaceae (2/12)

Orden Sapindales

237. Nitrariaceae $(1 / 1)$

239. Burseraceae (3/100)

240. Anacardiaceae (18/68)

241. Sapindaceae (22/124)

242. Rutaceae (19/93)

243. Simaroubaceae (4/9)

244. Meliaceae (4/26)

Orden Huerteales

245. Petenaeaceae $(1 / 1)$

246. Tapisciaceae (1/1)

247. Dipentodontaceae (1/2)

Orden Malvales

248. Cytinaceae (1/3)

249. Muntingiaceae $(2 / 2)$

251. Malvaceae $(83 / 527)$

253 . Thymelaeaceae $(2 / 16)$

254. Bixaceae (3/6)

256. Cistaceae (2/14)

Orden Brassicales

259. Tropaeolaceae (1/1)

261. Caricaceae (5/9)

263. Setchellanthaceae (1/1)

264. Koeberliniaceae (1/1)

265. Bataceae (1/1)

268. Tovariaceae $(1 / 1)$

271. Resedaceae (1/1)

272. Capparaceae (7/34)

273. Cleomaceae $(16 / 36)$

274. Brassicaceae (50/210)

Superorder Santalanae

Orden Santalales

277. Balanophoraceae $(2 / 2)$

278. Olacaceae $(1 / 3)$

278.1. Ximeniaceae (1/3)

279. Opiliaceae (1/3)

280. Santalaceae $(5 / 96)$

281. Loranthaceae $(5 / 55)$

283. Schoepfiaceae (1/4)

Superorder Caryophyllanae

Orden Caryophyllales 284. Frankeniaceae (1/6) 286. Plumbaginaceae (2/3) 287. Polygonaceae (20/157) 288. Droseraceae (1/2)
294. Simmondsiaceae (1/1)

297. Caryophyllaceae (20/117)

298. Achatocarpaceae (2/4)

299. Amaranthaceae (33/211)

300. Stegnospermataceae (1/3)

305. Aizoaceae (2/4)

306. Phytolaccaceae (7/12)

308. Nyctaginaceae $(18 / 109)$

309. Molluginaceae $(2 / 2)$

310. Montiaceae $(5 / 19)$

312. Basellaceae $(1 / 3)$

314. Talinaceae $(2 / 8)$

315. Portulacaceae $(2 / 20)$

315.1 Anacampserotaceae (2/2)

316. Cactaceae (62/677)

\section{Superorder Asteranae}

Orden Cornales

320. Cornaceae $(2 / 5)$

321 . Hydrangeaceae $(6 / 35)$

322. Loasaceae $(8 / 53)$

Orden Ericales

323. Balsaminaceae (1/1)

324. Marcgraviaceae (3/8)

326. Fouquieriaceae (1/11)

327. Polemoniaceae (17/105)

328. Lecythidaceae $(1 / 1)$

330. Pentaphylacaceae (4/18)

331. Sapotaceae $(5 / 45)$

332. Ebenaceae (1/30)

333. Primulaceae $(16 / 115)$

334. Theaceae $(1 / 1)$

335. Symplocaceae (1/19)

337. Styracaceae (1/14)

340. Actinidiaceae (1/21)

341. Clethraceae $(1 / 25)$

342. Cyrillaceae (1/1)

343. Mitrastemonaceae (1/1)

344. Ericaceae (25/96)

Orden Icacinales

347. Icacinaceae (4/10)

Orden Garryales

349. Garryaceae (1/9)

Orden Gentianales

350. Rubiaceae (107/707)

351. Gentianaceae $(17 / 88)$

352. Loganiaceae (4/24)

353. Gelsemiaceae (1/1)
354. Apocynaceae (58/418)

Orden Boraginales

356 . Boraginaceae (21/176)

356.1 Cordiaceae (3/49)

356.2 Ehretiaceae $(6 / 40)$

356.3 Heliotropiaceae (2/49)

356.4 Hydrophyllaceae

$(3 / 62)$

Orden Solanales

357. Convolvulaceae (18/295)

358. Solanaceae (34/407)

361. Hydroleaceae (1/2)

Orden Lamiales

362. Plocospermataceae (1/1)

364. Oleaceae $(6 / 48)$

365 . Tetrachondraceae $(1 / 1)$

366. Calceolariaceae (1/6)

367. Gesneriaceae $(29 / 115)$

368. Plantaginaceae (29/209)

369. Scrophulariaceae (11/45)

371. Linderniaceae $(2 / 8)$

373. Lamiaceae $(35 / 601)$

374. Phrymaceae $(6 / 47)$

376. Orobanchaceae $(16 / 171)$

377. Lentibulariaceae $(3 / 62)$

378. Acanthaceae (42/385)

379. Bignoniaceae (26/68)

381. Schlegeliaceae (2/3)

382. Verbenaceae $(16 / 169)$

384. Martyniaceae (2/7)

Orden Aquifoliales

387. Phyllonomaceae (1/1)

389. Aquifoliaceae (1/20)

Orden Asterales

391. Campanulaceae (15/89)

397. Menyanthaceae (1/3)

398. Goodeniaceae (1/1)

400. Asteraceae $(385 / 3,057)$

Orden Dipsacales

405. Adoxaceae (2/18)

406. Caprifoliaceae $(5 / 51)$

Orden Apiales

411. Araliaceae (6/35)

413. Apiaceae $(37 / 208)$ 
Appendix 2. Native species of vascular plants of Mexico. An asterisk (*) indicates those species endemic to the country. State acronyms as in Tables 5 and 6. ND= data not available.

\section{Ferns and Lycophytes}

\section{Family Anemiaceae}

Anemia adiantifolia (L.) Sw. CAM, CHIS, CHIH, COAH, COL, GRO, HGO, JAL, MEX, MICH, MOR, NAY, NLE, OAX, PUE, QRO, QROO, SLP, TAB, TAMS, VER, YUC

*Anemia affinis Hook. \& Baker COL, DGO, JAL, NAY, SIN, SON, ZAC

*Anemia brandegeea Davenp. SIN

Anemia cicutaria Poepp. ex Spreng. CHIS, QROO, YUC

*Anemia colimensis Mickel COL, GRO, OAX

*Anemia familiaris Mickel CHIS, OAX

Anemia hirsuta (L.) Sw. BCS, CHIS, COL, DGO, GRO, JAL, MEX, MICH, MOR, NAY, OAX, PUE, SIN, TAMS, VER, ZAC *Anemia intermedia Copel. DGO, NAY

*Anemia jaliscana Maxon BCN, BCS, COL, DGO, GRO, JAL, MEX, MICH, NAY, SIN, SON

*Anemia karwinskyana (C. Presl) Prantl COL, GRO, HGO, JAL, MEX, MICH, MOR, NAY, OAX, VER

Anemia mexicana Klotzsch COAH, COL, GTO, GRO, HGO, JAL, MEX, MOR, NLE, OAX, QRO, SLP, TAMS, VER

*Anemia multiplex Mickel COL, JAL, NAY, ZAC

*Anemia munchii Christ CHIS, OAX, VER

Anemia oblongifolia (Cav.) Sw. CHIS, COL, GRO, JAL, MEX, MICH, NAY, OAX, SIN, VER

Anemia pastinacaria Moritz ex Prantl CHIS, COL, GRO, JAL, MEX, MICH, NAY, OAX, SIN, VER

Anemia phyllitidis (L.) Sw. CHIS, COL, GRO, HGO, JAL, MEX, MICH, NAY, OAX, QRO, SLP, TAMS, VER

*Anemia recondita Mickel COL, GRO, JAL, MEX, MICH, MOR, NAY

*Anemia semihirsuta Mickel CHIS, GRO, OAX, VER

Anemia speciosa C. Presl CHIS, GRO, OAX

Anemia tomentosa (Savigny) Sw. BCN, CHIS, CHIH, DGO, GTO, GRO, HGO, JAL, MEX, NAY, OAX, PUE, SLP, SIN, SON, TAMS, VER, ZAC

Anemia $\times$ paraphyllitidis Mickel CHIS, OAX

\section{Family Aspleniaceae}

Asplenium abscissum Willd. CHIS, COL, HGO, JAL, MOR, NAY, OAX, PUE, SLP, TAB, VER

Asplenium achilleifolium (M. Martens \& Galeotti) Liebm. CHIS, COL, GRO, JAL, MICH, NAY, OAX, PUE, SIN, VER

Asplenium alatum Humb. \& Bonpl. ex Willd. CHIS, HGO, OAX, PUE, VER

*Asplenium arcanum A.R. Sm. DGO, NAY, SIN

Asplenium auriculatum Sw. CAM, CHIS, COL, GRO, HGO, JAL, MEX, MICH, OAX, PUE, QRO, SLP, TAMS, VER

Asplenium auritum Sw. CHIS, HGO, OAX, PUE, QRO, SLP, TAB, VER

Asplenium barbaense Hieron. CHIS, HGO, OAX, VER

*Asplenium blepharodes D.C. Eaton BCS

Asplenium blepharophorum Bertol. CHIS, COL, CDMX, GRO, HGO, JAL, MEX, MICH, MOR, OAX, PUE, QRO, SLP, VER
Asplenium breedlovei A.R. Sm. CHIS

Asplenium castaneum Schltdl. \& Cham. CHIS, CDMX, DGO, GTO, GRO, JAL, MEX, MICH, MOR, NLE, OAX, PUE, QRO, TLAX, VER

Asplenium cirrhatum Rich. ex Willd. CHIS, OAX

Asplenium cristatum Lam. CHIS, GRO, JAL, MEX, MICH, MOR, OAX, PUE, QRO, SLP, TAB, TAMS, VER

Asplenium cruegeri Hieron. CHIS

Asplenium cuspidatum Lam. CAM, CHIS, COL, GRO, HGO, JAL, MEX, MICH, MOR, NAY, OAX, PUE, QRO, SLP, SIN, TAMS, VER

Asplenium chihuahuense Baker CHIH, DGO, SON

Asplenium dalhousiae Hook. CHIH, SIN, SON

Asplenium delicatulum C. Presl CHIS, OAX

Asplenium delitescens (Maxon) L.D. Gómez CHIS, OAX, TAB, VER

Asplenium dentatum L. CAM, CHIS, VER, YUC

*Asplenium dianae A.R. Sm. TAMS

Asplenium dissectum $\mathrm{Sw}$. OAX

Asplenium divaricatum Kunze CHIS, OAX, VER

*Asplenium eatonii Davenp. COL, JAL, MICH, NAY, SIN

Asplenium exiguum Bedd. AGS, CHIS, CHIH, COAH, COL, CDMX, DGO, GTO, GRO, HGO, JAL, MEX, NLE, OAX, QRO, SLP, SON, TAMS

Asplenium feei Kunze ex Fée CHIS, OAX, VER

*Asplenium fibrillosum Pringle \& Davenp. ex Davenp. AGS, CDMX, DGO, GTO, GRO, JAL, MEX, MICH, MOR, QRO,

SLP

Asplenium flabellulatum Kunze CHIS, OAX, PUE, VER

Asplenium formosum Willd. CHIS, COL, CDMX, DGO, GRO, JAL, MEX, MICH, MOR, NAY, OAX, PUE, TAB, VER

Asplenium fragrans Sw. CHIS, COL, DGO, GRO, JAL, MEX, MICH, MOR, NAY, OAX, PUE, QRO, SLP, SIN, TAMS, VER *Asplenium gentryi A.R. Sm. AGS, BCS, CHIH, DGO, SIN, SON

Asplenium hallbergii Mickel \& Beitel AGS, CHIH, COL, CDMX, DGO, GTO, GRO, HGO, JAL, MEX, MICH, MOR, OAX, PUE, QRO, SLP, TLAX, VER, ZAC

Asplenium harpeodes Kunze CHIS, GRO, OAX, PUE, VER

Asplenium harrisii Jenman CHIS, HGO

Asplenium heterochroum Kunze CHIS, VER

Asplenium hoffmannii Hieron. CHIS, COL, GRO, JAL, NAY, OAX, QROO, VER

*Asplenium insolitum A.R. Sm. CHIS, GRO, OAX

Asplenium juglandifolium Lam. CHIS, OAX

Asplenium laetum Sw. CHIS, OAX, SLP, TAB, VER

Asplenium lamprocaulon Fée CHIS, OAX

Asplenium minimum M. Martens \& Galeotti CHIS, HGO, MEX, MOR, OAX, QRO, SLP, TAB, TAMS, VER

Asplenium miradorense Liebm. CHIS, GRO, HGO, OAX, PUE, SLP, VER

*Asplenium modestum Maxon CHIH

Asplenium monanthes L. AGS, BCN, BCS, CHIS, CHIH, 
COAH, COL, CDMX, DGO, GTO, GRO, HGO, JAL, MEX, MICH, MOR, NAY, NLE, OAX, PUE, QRO, SLP, SIN, SON, TAMS, TLAX, VER, ZAC

Asplenium monodon Liebm. CHIS, OAX, PUE, VER

*Asplenium munchii A.R. Sm. CHIS, COL, GRO, JAL, MEX, MICH, MOR, OAX

Asplenium myriophyllum (Sw.) C. Presl CHIS, GRO, OAX, VER

Asplenium nesioticum Maxon CDMX, GTO, MEX, NLE, SLP Asplenium obesum Baker GRO

*Asplenium oligosorum Mickel \& Beitel CHIS, OAX

Asplenium olivaceum A.R. Sm. CHIS, OAX

Asplenium palmeri Maxon AGS, CHIH, COAH, CDMX, DGO, GTO, HGO, JAL, MEX, MICH, NAY, NLE, OAX, PUE, QRO, SLP, SIN, SON, TAMS, VER, ZAC

Asplenium polyphyllum Bertol. CHIS, CDMX, GRO, HGO, MEX, MICH, MOR, OAX

Asplenium potosinum Hieron. CHIS, COL, GRO, JAL, MEX, NAY, OAX, SLP, SIN, VER

Asplenium praemorsum Sw. AGS, CHIS, COL, CDMX, DGO, GTO, GRO, HGO, JAL, MEX, MICH, MOR, NAY, OAX, PUE, QRO, QROO, SLP, SIN, TAMS, VER

*Asplenium pringlei Davenp. CHIH, COL, JAL, NAY

Asplenium pteropus Kaulf. CHIS, OAX, PUE, VER

Asplenium pulchellum Raddi CHIS, OAX, TAB, VER

Asplenium pumilum $\mathrm{Sw}$. BCN, BCS, CAM, CHIS, COL, GRO, HGO, JAL, MEX, MICH, MOR, NAY, NLE, OAX, QRO, QROO, SLP, SIN, SON, TAB, TAMS, VER, YUC, ZAC

Asplenium radicans $\mathrm{L}$. CHIS, GRO, OAX, PUE, VER

Asplenium resiliens Kunze CHIS, CHIH, COAH, GRO, HGO, JAL, MEX, MOR, NLE, OAX, PUE, QRO, SLP, SON, TAMS, TLAX, VER, ZAC

Asplenium riparium Liebm. CHIS, OAX, PUE, VER Asplenium rutaceum (Willd.) Mett. CHIS, OAX, VER

Asplenium salicifolium L. CHIS, OAX, VER

*Asplenium sanchezii A.R. Sm. CHIH, DGO, OAX, SIN

Asplenium scolopendrium L. CHIS, COAH, NLE, OAX

Asplenium seileri C.D. Adams CHIS

*Asplenium semipinnatum (Hieron.) A.R. Sm. SLP

Asplenium septentrionale (L.) Hoffm. BCN

Asplenium serra Langsd. \& Fisch. CHIS, GRO, HGO, OAX, PUE, VER

Asplenium serratum L. CHIS, OAX, TAB, VER

Asplenium sessilifolium Desv. CHIS, CHIH, COL, DGO, GRO, HGO, JAL, MEX, MICH, MOR, OAX, PUE, QRO, SLP, SIN, SON, VER

*Asplenium soleirolioides A.R. Sm. CHIS, COL, GRO, HGO, JAL, MICH, OAX, QRO

Asplenium solmsii Baker ex Hemsl. CHIS

Asplenium sphaerosporum A.R. Sm. CHIS, COL, GRO, HGO,

JAL, MEX, MICH, MOR, OAX, PUE, QRO, SLP, VER

*Asplenium stolonipes Mickel \& Beitel OAX

Asplenium tenerrimum Mett. ex Kuhn CHIS, COL, GRO, JAL,

MEX, MICH, NAY, OAX, SIN

Asplenium trichomanes L. CHIH

*Asplenium tryonii Correll CHIH

Asplenium tuerckheimii Maxon CHIS, HGO, OAX, VER
*Asplenium ultimum A.R. Sm. NLE

Asplenium uniseriale Raddi CHIS, OAX, PUE, VER

*Asplenium venturae A.R. Sm. VER

Asplenium vespertinum Maxon BCN

Asplenium virillae $\mathrm{H}$. Christ CHIS

*Asplenium yelagagense Mickel \& Beitel OAX

*Holodictyum ghiesbreghtii (E. Fourn.) Maxon HGO, OAX, PUE, QRO, SLP, TAMS, VER

Loxoscaphe thecifera (Kunth) T. Moore CHIS, OAX, VER

Schaffneria nigripes Fée CHIS, HGO, OAX, QRO, SLP, TAMS, VER

\section{Family Athyriaceae}

Athyrium arcuatum Liebm. CHIS, COL, CDMX, GTO, GRO, HGO, JAL, MEX, MICH, MOR, NAY, OAX, PUE, QRO, VER Athyrium filix-femina (L.) Roth BCN, BCS, CHIS, CHIH, COL, CDMX, DGO, GRO, HGO, JAL, MEX, MICH, MOR, NAY, NLE, OAX, PUE, QRO, SON, TAMS, VER, ZAC

Athyrium palmense (H. Christ) Lellinger CHIS, COL, JAL, MEX, MICH, NAY, OAX, SIN

Athyrium skinneri (Baker) Diels CHIS, COL, DGO, GRO, HGO, JAL, MEX, MICH, MOR, NAY, OAX, PUE, QRO, SLP, SIN

*Athyrium tejeroi Mickel \& Tejero GRO, MEX

Diplazium altissimum (Jenman) C. Chr. CHIS, OAX

Diplazium cristatum (Desr.) Alston CHIS, GRO, MOR, NAY, OAX, VER

*Diplazium diplazioides (Klotzsch \& H. Karts ex Klotzsch) Alston OAX, PUE

Diplazium donnell-smithii H. Christ CHIS, OAX, VER

Diplazium drepanolobium A.R. Sm. CHIS, GRO, MOR, OAX, TAB, VER

Diplazium expansum Willd. GRO, OAX, PUE, VER

Diplazium franconis Liebm. CHIS, CDMX, GRO, HGO, MEX, MICH, OAX, PUE, QRO, SLP, TAMS, VER

Diplazium gomezianum C.D. Adams CHIS

Diplazium grandifolium (Sw.) Sw. CHIS

*Diplazium hellwigii Mickel \& Beitel OAX

Diplazium herbaceum Fée CHIS

Diplazium hians Kunze ex Klotzsch CHIS, GRO, OAX, TAB, VER

Diplazium lindbergii (Mett.) H. Christ CHIS, GRO, OAX, PUE, VER

Diplazium lonchophyllum Kunze CHIS, COL, GRO, HGO, JAL, MEX, MICH, MOR, NAY, NLE, OAX, QRO, SLP, TAB, VER

Diplazium neglectum (H. Karst.) C. Chr. CHIS

Diplazium obscurum $\mathrm{H}$. Christ CHIS, GRO, OAX, PUE

Diplazium plantaginifolium (L.) Urb. CHIS, OAX, VER

Diplazium prominulum Maxon CHIS, TAMS

*Diplazium puberulentum Mickel \& Beitel OAX, VER

Diplazium striatastrum Lellinger CHIS, HGO, OAX, PUE, VER

Diplazium striatum (L.) C. Presl CHIS, GRO, HGO, OAX, PUE, TAB, VER

Diplazium ternatum Liebm. CHIS, HGO, OAX, PUE, VER

Diplazium urticifolium $\mathrm{H}$. Christ CHIS, GRO, OAX, PUE, VER Diplazium vera-pax (Donn. Sm.) Hieron. CHIS, OAX, VER 
*Diplazium wendtii Mickel \& A.R. Sm. OAX

Diplazium werckleanum $\mathrm{H}$. Christ CHIS, OAX, VER

\section{Family Blechnaceae}

Blechnum appendiculatum Willd. CHIS, COL, CDMX, GRO, HGO, JAL, MEX, MICH, MOR, NAY, OAX, PUE, QRO, SLP, SON, TAMS, TLAX, VER

Blechnum divergens (Kunze) Mett. CHIS, GRO, OAX

Blechnum ensiforme (Liebm.) C. Chr. CHIS, GRO, OAX, VER

Blechnum falciforme (Liebm.) C. Chr. CHIS, GRO, HGO, MEX, OAX, PUE, TAMS, VER

Blechnum fragile (Liebm.) C.V. Morton \& Lellinger CHIS, OAX, PUE, VER

Blechnum gracile Kaulf. CHIS, COL, GRO, JAL, NAY, OAX, PUE, QRO, TAB, VER

Blechnum lehmannii Hieron. CHIS, GRO, OAX

Blechnum lherminieri (Bory) C. Chr. CHIS, OAX

Blechnum meridense Klotzsch CHIS, VER

Blechnum occidentale L. CHIS, COL, GRO, HGO, JAL, MEX, MICH, MOR, NAY, OAX, PUE, QRO, SLP, TAB, TAMS, VER Blechnum polypodioides Raddi CHIS, DGO, GRO, JAL, MEX, MICH, NAY, OAX, PUE, SIN, VER

Blechnum schiedeanum (Schltdl. ex C. Presl) Hieron. CHIS, GRO, HGO, MEX, OAX, PUE, VER

Blechnum serrulatum Rich. CHIS, QROO, TAB, VER, YUC

Blechnum stoloniferum (Mett. ex E. Fourn.) C. Chr. CHIS, CHIH, DGO, GTO, GRO, HGO, MEX, MICH, MOR, OAX, PUE, QRO, VER

Blechnum wardiae Mickel \& Beitel CHIS, OAX, VER

Woodwardia fimbriata $\mathrm{Sm}$. BCN, SON

*Woodwardia martinezii Maxon ex Weath. HGO, PUE, VER

Woodwardia radicans (L.) Sm. DGO, HGO, JAL, MEX, MICH, VER

*Woodwardia semicordata Mickel \& Beitel HGO, OAX, PUE, VER

Woodwardia spinulosa M. Martens \& Galeotti CHIS, COL, CDMX, DGO, GTO, GRO, HGO, JAL, MEX, MICH, MOR, NAY, NLE, OAX, PUE, QRO, SLP, SIN, SON, TAMS, VER, ZAC

\section{Family Cibotiaceae}

Cibotium regale Verschaff. \& Lem. CHIS, PUE, VER

*Cibotium schiedei Schltdl. \& Cham. VER

\section{Family Culcitaceae}

Culcita coniifolia (Hook.) Maxon CHIS, GRO, OAX

\section{Family Cyatheaceae}

Alsophila firma (Baker) D.S. Conant CHIS, COL, GRO, HGO, JAL, MEX, OAX, PUE, QRO, SLP, TAMS, VER

Alsophila salvinii Hook. CHIS, OAX, VER

Alsophila tryoniana (Gastony) D.S. Conant VER

*Cyathea aristata Domin OAX, VER

Cyathea bicrenata Liebm. CHIS, GRO, OAX, PUE, VER

Cyathea costaricensis (Mett. ex Kuhn) Domin CHIS, COL, GRO, JAL, MICH, NAY, OAX, PUE, TAB, VER

Cyathea decurrentiloba Domin CHIS, OAX, VER

Cyathea divergens Kunze CHIS, GRO, HGO, OAX, PUE, VER
Cyathea fulva (M. Martens \& Galeotti) Fée CHIS, GRO, HGO, OAX, PUE, VER

Cyathea godmanii (Hook.) Domin CHIS, GRO, HGO, OAX, TAB, VER

Cyathea microdonta (Desv.) Domin CHIS, TAB, VER

Cyathea myosuroides (Liebm.) Domin CHIS, OAX, PUE, TAB, VER

Cyathea schiedeana (C. Presl) Domin CHIS, GRO, HGO, OAX, PUE, TAB, VER

Sphaeropteris horrida (Liebm.) R.M. Tryon CHIS, GRO, OAX, PUE, VER

\section{Family Cystopteridaceae}

Cystopteris fragilis (L.) Bernh. AGS, BCN, BCS, CHIS, CHIH, COAH, COL, CDMX, DGO, GTO, GRO, HGO, JAL, MEX, MICH, MOR, NAY, NLE, OAX, PUE, QRO, SLP, SIN, SON, TAMS, TLAX, VER, ZAC

Cystopteris membranifolia Mickel OAX, PUE, QRO, SLP, VER *Cystopteris millefolia Mickel \& Tejero MEX, MICH, MOR

Cystopteris reevesiana Lellinger $\mathrm{CHIH}, \mathrm{SON}$

\section{Family Dennstaedtiaceae}

Dennstaedtia auriculata H. Navarrete \& B. Ollg. CHIS

Dennstaedtia bipinnata (Cav.) Maxon CHIS, DGO, HGO, JAL, OAX, QRO, SLP, VER, YUC

Dennstaedtia cicutaria (Sw.) T. Moore CHIS, HGO, MICH, OAX, PUE, QRO, SLP, SON, TAMS, VER

Dennstaedtia cornuta (Kaulf.) Mett. CHIS, GRO, HGO, OAX, PUE, VER

Dennstaedtia coronata (Sodiro) C. Chr. CHIS

Dennstaedtia distenta (Kunze) T. Moore CHIS, CHIH, COL, DGO, GTO, GRO, HGO, JAL, MEX, MICH, MOR, OAX, PUE, QRO, SLP, SIN, SON, TAMS, VER

Dennstaedtia globulifera (Poir.) Hieron. CHIS, GRO, HGO, JAL, MICH, MOR, NLE, OAX, QRO, SLP, TAMS, VER

Dennstaedtia spinosa Mickel CHIS

Histiopteris incisa (Thunb.) J. Sm. CHIS, MICH, OAX, VER

Hypolepis blepharochlaena Mickel \& Beitel CHIS, HGO, OAX, PUE, VER

Hypolepis bogotensis H. Karst. CHIS, OAX

*Hypolepis melanochlaena A.R. Sm. CHIS

*Hypolepis microchlaena Mickel \& Beitel OAX

*Hypolepis munchii (Christ) Mickel CHIS, GRO, OAX

Hypolepis nigrescens Hook. CHIS, OAX, VER

Hypolepis pulcherrima Underw. \& Maxon CHIS

Hypolepis repens (L.) C. Presl CHIS, GRO, HGO, OAX, PUE, VER

Hypolepis stuebelii Hieron. VER

*Hypolepis thysanochlaena Mickel \& Beitel CHIS, MOR, OAX

*Hypolepis trichochlaena Mickel \& Beitel OAX

Pteridium aquilinum (L.) Kuhn AGS, CHIS, CHIH, COAH, COL, DGO, GTO, GRO, HGO, JAL, MEX, MICH, MOR, NAY, NLE, OAX, PUE, QRO, SLP, SIN, SON, TAMS, VER, ZAC

Pteridium arachnoideum (Kaulf.) Maxon CHIS, COL, CDMX, GRO, HGO, JAL, MEX, MICH, MOR, OAX, PUE, VER

Pteridium caudatum (L.) Maxon BCN, BCS, CAM, CHIS, CHIH, COAH, COL, DGO, GTO, GRO, HGO, JAL, MEX, 
MICH, NAY, NLE, OAX, PUE, QRO, QROO, SLP, SIN, SON, TAB, TAMS, VER, YUC

\section{Family Dicksoniaceae}

Dicksonia sellowiana Hook. CHIS, GRO, HGO, OAX, PUE, VER

Lophosoria quadripinnata (J.F. Gmel.) C. Chr. CHIS, GRO, HGO, MEX, MOR, OAX, PUE, QRO, SLP, TAMS, VER

\section{Family Diplaziopsidaceae}

Hemidictyum marginatum (L.) C. Presl CHIS, OAX, VER

\section{Family Dryopteridaceae}

Adenoderris sororia Maxon CHIS

Arachniodes denticulata (Sw.) Ching CHIS, GRO, HGO, MEX, MOR, OAX, PUE, VER

Bolbitis aliena (Sw.) Alston CHIS, VER

Bolbitis hastata (E. Fourn.) Hennipman CHIS, OAX, VER

Bolbitis portoricensis (Spreng.) Hennipman CHIS, COL, GRO,

JAL, MICH, NAY, OAX, PUE, TAB, VER

Bolbitis serratifolia (Mert. ex Kaulf.) Schott CHIS

Bolbitis umbrosa (Liebm.) Ching CHIS, HGO, VER

*Ctenitis baulensis A.R. Sm. CHIS

*Ctenitis bullata A.R. Sm. CHIS

*Ctenitis chiapasensis (H. Christ) A.R. Sm. CHIS

Ctenitis equestris (Kunze) Ching CHIS, COL, GRO, HGO, JAL,

MEX, MICH, MOR, NAY, OAX, SIN, TAMS, VER

Ctenitis erinacea A.R. Sm. CHIS, HGO, OAX, PUE, VER

Ctenitis excelsa (Desv.) Proctor CHIS, GRO, OAX, VER

Ctenitis grisebachii (Baker) Ching CHIS, GRO, NAY, VER

Ctenitis hemsleyana (Baker ex Hemsl.) Copel. CHIS, HGO,

OAX, PUE, VER

Ctenitis interjecta (C. Chr.) Ching CHIS, OAX, VER

Ctenitis lanceolata (Baker) A.R. Sm. CHIS

Ctenitis melanosticta (Kunze) Copel. CHIS, HGO, MOR, OAX,

PUE, QRO, SLP, TAB, TAMS, VER

*Ctenitis mexicana A.R. Sm. HGO, PUE, QRO, VER

Ctenitis microchlaena (Fée) Stolze CHIS, VER

Ctenitis nigrovenia (H. Christ) Copel. CHIS, HGO, OAX, PUE, QRO, SLP, VER

Ctenitis refulgens (Klotzsch ex Mett.) C. Chr. ex Vareschi CHIS

Ctenitis salvinii (Baker) Stolze CHIS, TAB, VER

Ctenitis sloanei (Poepp. ex Spreng.) C.V. Morton CHIS

Ctenitis strigilosa (Davenp.) Copel. CHIS, OAX, VER

Ctenitis submarginalis (Langsd. \& Fisch.) Ching CHIS, HGO,

OAX, PUE, QRO, SLP, VER

Ctenitis thelypteroides A.R. Sm. CHIS

*Ctenitis ursina A.R. Sm. CHIS

Dryopteris arguta (Kaulf.) Maxon BCN

Dryopteris cinnamomea (Cav.) C. Chr. AGS, CHIS, CHIH, COAH, COL, CDMX, DGO, GTO, GRO, HGO, JAL, MEX, MICH, MOR, NAY, OAX, PUE, QRO, SLP, SIN, SON, TAMS, TLAX, VER, ZAC

*Dryopteris feei C. Chr. CHIH, MEX, QRO, SLP, SON, VER Dryopteris filix-mas (L.) Schott COAH

Dryopteris futura A.R. Sm. CHIS, GRO, HGO, MEX, MICH, MOR, OAX
Dryopteris karwinskyana (Mett.) Kuntze CHIS, CDMX, DGO, GRO, JAL, MEX, MICH, MOR, NAY, OAX, SIN

Dryopteris knoblochii A.R. Sm. CHIH, DGO, SIN, SON

Dryopteris maxonii Underw. \& C. Chr. COL, GTO, GRO, JAL, MEX, MICH, MOR, NAY, SIN

*Dryopteris muenchii A.R. Sm. CHIS, HGO

Dryopteris nubigena Maxon \& C.V. Morton CHIS, GRO, MEX, OAX

*Dryopteris patens (Sw.) Kuntze JAL, MEX, MICH, NAY

Dryopteris patula (Sw.) Underw. CHIS, CHIH, COL, CDMX, DGO, GTO, HGO, JAL, MEX, MICH, MOR, NAY, NLE, OAX, PUE, QRO, SIN, VER

Dryopteris pseudofilix-mas (Fée) Rothm. CHIS, CDMX, HGO, MEX, MICH, MOR, NLE, OAX, PUE, QRO, SLP, SON, TAMS, VER

*Dryopteris rossii C. Chr. AGS, BCN, BCS, CHIH, COL, CDMX, DGO, GTO, GRO, HGO, JAL, MEX, MICH, MOR, NAY, OAX, QRO, SLP, SIN, ZAC

Dryopteris sugescens (Link) C. Chr. COAH

Dryopteris wallichiana (Spreng.) Hyl. CHIS, CHIH, COL, CDMX, GTO, GRO, HGO, JAL, MEX, MICH, MOR, OAX, PUE, QRO, SLP, TAMS, TLAX, VER

Elaphoglossum affine (M. Martens \& Galeotti) T. Moore CHIS, CDMX, GRO, MEX, MICH, MOR, OAX, VER

*Elaphoglossum alansmithii Mickel CHIS, OAX

Elaphoglossum albomarginatum A.R. Sm. CHIS

*Elaphoglossum angustifrons A. Rojas CHIS, OAX

Elaphoglossum auricomum (Kunze) T. Moore CHIS, OAX, VER

Elaphoglossum baquianorum A. Rojas CHIS, JAL, MEX, MICH, OAX

Elaphoglossum barbatum (H. Karst.) Hieron. CHIS, OAX

*Elaphoglossum decursivum Mickel OAX

*Elaphoglossum dissitifrons Mickel OAX

Elaphoglossum ellipticifolium A. Rojas CHIS, HGO, MEX, MICH, OAX, VER

Elaphoglossum engelii (H. Karst) H. Christ CHIS, GRO, MEX, MOR, OAX

Elaphoglossum erinaceum (Fée) T. Moore COL, GRO, HGO, JAL, MEX, MICH, MOR, OAX, PUE, QRO, SLP, SIN, VER

*Elaphoglossum eximium (Mett.) H. Christ CHIS, OAX, VER

Elaphoglossum glabellum J. Sm. OAX

Elaphoglossum glaucum T. Moore CHIS, COL, GRO, HGO, JAL, MEX, MICH, MOR, NAY, OAX, PUE, QRO, SLP, VER Elaphoglossum gratum (Fée) T. Moore AGS, CHIS, COL, CDMX, DGO, GTO, GRO, JAL, MEX, MICH, OAX, QRO, VER

Elaphoglossum guatemalense (Klotzsch) T. Moore CHIS, HGO, OAX, VER

Elaphoglossum hartwegii (Fée) T. Moore CHIS, CDMX, DGO, GRO, JAL, MEX, MICH, OAX, PUE, VER, ZAC

Elaphoglossum herrerae A. Rojas CHIS, GRO, OAX

Elaphoglossum huacsaro (Ruiz) Christ CHIS

*Elaphoglossum ipshookense Mickel OAX

*Elaphoglossum jaliscanum Mickel JAL

*Elaphoglossum laminarioides (Bory) T. Moore VER

Elaphoglossum lanceum Mickel CHIS, OAX 
Elaphoglossum latum (Mickel) L. Atehortúa ex Mickel CHIS

*Elaphoglossum laxipes Mickel CDMX, JAL, MEX

*Elaphoglossum leebrowniae Mickel CHIS

*Elaphoglossum leonardii Mickel OAX

*Elaphoglossum lepidopodum Mickel GRO, OAX

Elaphoglossum lindenii (Bory ex Fée) T. Moore CHIS, CDMX, GRO, HGO, JAL, MEX, MICH, MOR, OAX, VER

Elaphoglossum lonchophyllum (Fée) T. Moore CHIS, OAX, VER

Elaphoglossum longistipitatum A. Rojas CHIS, HGO, MEX, OAX, VER

*Elaphoglossum manantlanense Mickel COL, JAL, OAX

Elaphoglossum mcvaughii Mickel CHIS, MEX, MICH, OAX

Elaphoglossum mesoamericanum A. Rojas CHIS, HGO, OAX,

VER

Elaphoglossum mexicanum A. Rojas CHIS, OAX

*Elaphoglossum monicae Mickel COL, CDMX, DGO, GRO, JAL, MEX, MOR, SIN, VER

Elaphoglossum muelleri (E. Fourn.) C. Chr. CHIS, CHIH, COAH, COL, CDMX, DGO, GTO, GRO, HGO, JAL, MEX, MICH, NAY, NLE, OAX, PUE, QRO, SLP, SIN, SON, TAMS, VER, ZAC

Elaphoglossum muscosum (Sw.) T. Moore CHIS, OAX, PUE, VER

*Elaphoglossum obscurum (E. Fourn.) C. Chr. HGO, VER

Elaphoglossum paleaceum (Hook. \& Grev.) Sledge CHIS, COL, CDMX, GRO, HGO, JAL, MEX, MICH, OAX, QRO, SLP, VER

Elaphoglossum pallidum (Baker ex Jenman) C. Chr. CHIS, JAL, MICH, OAX

*Elaphoglossum parduei Mickel OAX

Elaphoglossum peltatum (Sw.) Urb. CHIS, COL, GRO, HGO, JAL, OAX, PUE, VER

Elaphoglossum petiolatum (Sw.) Urb. CHIS, COL, CDMX, DGO, GTO, GRO, HGO, JAL, MEX, MICH, MOR, NAY, NLE, OAX, PUE, QRO, SLP, SIN, VER, ZAC

Elaphoglossum piloselloides (C. Presl) T. Moore CHIS, COL, DGO, GRO, HGO, JAL, MEX, MICH, NAY, OAX, SIN, VER, ZAC

Elaphoglossum polypodium A. Rojas OAX

*Elaphoglossum potosianum C. Chr. CDMX, GTO, HGO, MEX, NLE, SLP, TAMS, VER

*Elaphoglossum pringlei (Davenp.) C. Chr. GRO, MEX, MICH, NAY, OAX, PUE, VER

Elaphoglossum pusillum (Mett. ex Kuhn) C. Chr. CHIS, OAX Elaphoglossum revolutum (Liebm.) T. Moore CHIS, OAX Elaphoglossum rigidum (Aubl.) Urb. CHIS, MEX, OAX Elaphoglossum rubescens H. Christ CHIS, VER

*Elaphoglossum rufescens (Liebm.) T. Moore JAL, MEX

*Elaphoglossum rzedowskii Mickel AGS, CHIH, DGO, GTO, JAL, MICH, NAY, SIN, SON, ZAC

Elaphoglossum sartorii (Liebm.) Mickel CHIS, COL, DGO, GRO, HGO, JAL, MEX, MOR, NAY, OAX, PUE, SIN, VER

*Elaphoglossum seminudum Mickel HGO, OAX, VER

Elaphoglossum setigerum (Sodiro) Diels CHIS, GRO, MOR, OAX

Elaphoglossum setosum (Liebm.) T. Moore CHIS, OAX, VER
Elaphoglossum squamipes (Hook.) T. Moore CHIS, GRO, OAX, VER

Elaphoglossum squarrosum (Klotzsch) T. Moore CHIS, GRO, MEX, OAX

Elaphoglossum tectum (Humb. \& Bonpl. ex Willd.) T. Moore CHIS, JAL, MICH, OAX, VER

Elaphoglossum tejeroanum A. Rojas CHIS, JAL, MICH, OAX, VER

Elaphoglossum tenuiculum (Fée) T. Moore ex C. Chr. OAX

Elaphoglossum tenuifolium (Liebm.) T. Moore CHIS, COL, CDMX, DGO, GRO, HGO, JAL, MEX, MICH, MOR, OAX, VER

*Elaphoglossum trichomallum (Rovirosa) C. Chr. CHIS

Elaphoglossum variable A. Rojas CHIS, OAX, VER

*Elaphoglossum vestitum (Schltdl. \& Cham.) Schott ex T.

Moore CHIS, HGO, OAX, PUE, VER

Elaphoglossum viride (E. Fourn.) C. Chr. OAX, VER

*Elaphoglossum xanthopodum Mickel CHIS, OAX, PUE, VER Lastreopsis effusa (Sw.) Tindale CHIS, GRO, MICH, OAX, PUE, SLP, VER

Lastreopsis exculta (Mett.) Tindale CHIS, OAX

Megalastrum atrogriseum (C. Chr.) A.R. Sm. \& R.C. Moran HGO, OAX, VER

Megalastrum galeottii (M. Martens) R.C. Moran \& J. Prado CHIS, GRO, HGO, MICH, OAX, VER

Megalastrum gompholepis R.C. Moran \& J. Prado CHIS

Megalastrum lunense (H. Christ) A.R. Sm. \& R.C. Moran CHIS

* Megalastrum mexicanum R.C. Moran \& J. Prado CHIS, HGO, OAX, SLP, VER

Megalastrum pulverulentum (Poir.) A.R. Sm. \& R.C. Moran CHIS, OAX, PUE, QRO, SLP, VER

Megalastrum sparsipilosum R.C. Moran \& J. Prado CHIS, HGO, PUE, SLP, VER

Megalastrum subincisum (Willd.) A.R. Sm. \& R.C. Moran CHIS, GRO, OAX, VER

Mickelia bernoullii (Kuhn ex H. Christ) R.C. Moran, Labiak \& Sundue CHIS, OAX, VER

Mickelia hemiotis (Maxon) R.C. Moran, Labiak \& Sundue OAX, VER

Mickelia pergamentacea (Maxon) R.C. Moran, Labiak \& Sundue CHIS, OAX, VER

Olfersia cervina (L.) Kunze CHIS, OAX, VER

Phanerophlebia auriculata Underw. CHIH, COAH, NLE, SON, TAMS

*Phanerophlebia gastonyi Yatsk. CHIS, GRO, HGO, OAX, VER

Phanerophlebia juglandifolia (Humb. \& Bonpl. ex Willd.) J. Sm. CHIS, HGO, OAX, VER

Phanerophlebia macrosora (Baker) Underw. CHIS, COL, CDMX, GRO, HGO, JAL, MICH, MEX, OAX, QRO, SLP, VER

Phanerophlebia nobilis (Schltdl. \& Cham.) C. Presl CHIS, CHIH, COL, CDMX, GTO, GRO, HGO, JAL, MEX, MICH, MOR, OAX, PUE, QRO, SLP, SIN, SON, TAMS, TLAX, VER Phanerophlebia pumila (M. Martens \& Galeotti) Fée CHIS, GRO, HGO, MICH, OAX, QRO, SLP, VER 
Phanerophlebia remotispora E. Fourn. CHIS, HGO, MEX, MICH, OAX, PUE, QRO, SLP, VER

Phanerophlebia umbonata Underw. CHIH, COAH, NLE, SLP, SON, TAMS

*Polybotrya caudata Kunze CHIS, OAX

Polybotrya polybotryoides (Baker) H. Christ CHIS, OAX, VER

Polystichum acrostichoides (Michx.) Schott NLE, TAMS

*Polystichum aculeatum (L.) Roth ex Mert. MEX, MICH, MOR, VER

Polystichum alfaroi (Christ) Barrington CHIS

Polystichum distans E. Fourn. CHIS, COL, GRO, HGO, JAL, MEX, MICH, MOR, OAX, PUE, QRO, SLP, VER

Polystichum erythrosorum A.R. Sm. CHIS, OAX

Polystichum fournieri A.R. Sm. CHIS, GRO, HGO, MEX, MICH, MOR, OAX, SLP, VER

Polystichum furfuraceum A.R. Sm. CHIS

Polystichum hartwegii (Klotzsch) Hieron. CHIS, GRO, HGO,

JAL, MEX, MOR, NLE, OAX, PUE, VER

Polystichum mickelii A.R. Sm. CHIS, OAX, PUE, VER

Polystichum muenchii (Christ) C. Chr. CHIS

Polystichum munitum (Kaulf.) C. Presl BCN

Polystichum muricatum (L.) Fée CHIS, COL, GRO, OAX, VER

Polystichum orbiculatum (Desv.) J. Rémy \& Fée CHIS

Polystichum ordinatum (Kunze) Liebm. CHIS, HGO, OAX, PUE, VER

Polystichum platyphyllum (Willd.) C. Presl CHIS, OAX, VER

Polystichum rachichlaena Fée CHIS, CDMX, GTO, GRO, JAL,

MEX, MICH, MOR, NAY, OAX, SIN

*Polystichum schizophyllum Lorea-Hern. \& A.R. Sm. GRO

Polystichum speciosissimum (A. Braun ex Kunze) Copel. CHIS, COL, CDMX, GTO, GRO, HGO, JAL, MEX, MICH, MOR, OAX, PUE, QRO, SLP, TLAX, VER

Polystichum turrialbae H. Chr. CHIS, CDMX, HGO, MEX, MICH, OAX, VER

Stigmatopteris longicaudata (Liebm.) C. Chr. CHIS, OAX, PUE, VER

Stigmatopteris sordida (Maxon) C. Chr. CHIS, OAX, VER

\section{Family Equisetaceae}

Equisetum giganteum L. CHIS, JAL, NAY, VER

Equisetum hyemale L. AGS, BCN, BCS, CHIS, CHIH, COAH, COL, CDMX, DGO, GTO, GRO, HGO, JAL, MEX, MICH, MOR, NAY, NLE, OAX, PUE, QRO, SLP, SIN, SON, TAMS, VER, ZAC

Equisetum laevigatum A. Braun BCN, CHIS, CHIH, COAH, COL, DGO, MEX, MOR, NLE, OAX, SON, TAMS

Equisetum myriochaetum Schltdl. \& Cham. CHIS, COL, GTO, GRO, HGO, JAL, MEX, MICH, NAY, NLE, OAX, PUE, QRO, SLP, SIN, TAMS, VER, ZAC

Equisetum $\times$ ferrisii Clute BCN, CHIH, COAH, DGO, NLE, SIN, SON

Equisetum $\times$ haukeanum Mickel \& A.R. Sm. CHIS, CDMX, GRO, HGO, MEX, MICH, MOR, NLE, OAX, PUE, QRO, SLP, SIN, VER

\section{Family Gleicheniaceae}

Dicranopteris flexuosa (Schrad.) Underw. CHIS, OAX, TAB, VER
Diplopterygium bancroftii (Hook.) A.R. Sm. CHIS, GRO, HGO, OAX, PUE, VER

Gleichenella pectinata (Willd.) Ching CHIS, GRO, HGO, JAL, MEX, OAX, PUE, VER

Sticherus bifidus (Willd.) Ching CHIS, COL, GRO, JAL, OAX, PUE, TAB, VER

Sticherus brevipubis (H. Christ) A.R. Sm. CHIS, HGO, OAX, VER

Sticherus palmatus (W. Schaffn. ex E. Fourn.) Copel. CHIS, CDMX, GRO, HGO, MEX, MOR, OAX, PUE, VER

Sticherus underwoodianus (Maxon) Nakai CHIS, GRO, HGO, OAX, PUE, VER

\section{Family Hymenophyllaceae}

Abrodictyum rigidum (Sw.) Ebihara \& Dubuisson CHIS, OAX, VER

Didymoglossum reptans (Sw.) C. Presl CHIS, COL, GRO, HGO, JAL, OAX, PUE, SLP, TAMS, VER

Hymenophyllum abruptum Hook. CHIS, OAX, VER

Hymenophyllum asplenioides (Sw.) Sw. CHIS, HGO, OAX, PUE, VER

Hymenophyllum brevistipes Liebm. CHIS, OAX, VER

Hymenophyllum crassipetiolatum Stolze CHIS

Hymenophyllum crispum Kunth CHIS, GRO, HGO, OAX, PUE, VER

Hymenophyllum ectocarpon Fée CHIS, HGO, OAX, VER Hymenophyllum fendlerianum J.W. Sturm CHIS, OAX, VER Hymenophyllum fragile (Hedw.) C.V. Morton CHIS, HGO, OAX, VER

Hymenophyllum fucoides (Sw.) Sw. CHIS, GRO, HGO, OAX, PUE, VER

Hymenophyllum hirsutum (L.) Sw. CHIS, GRO, OAX, PUE, VER

Hymenophyllum lanatum Fée CHIS, OAX

Hymenophyllum maxonii H. Christ ex C.V. Morton OAX, VER Hymenophyllum microcarpum Desv. CHIS, OAX

Hymenophyllum myriocarpum Hook. CHIS, COL, GRO, HGO, JAL, OAX, PUE, VER

Hymenophyllum paucicarpum Jenman CHIS, OAX

Hymenophyllum polyanthos (Sw.) Sw. CHIS, GRO, HGO, JAL, OAX, PUE, VER

Hymenophyllum pulchellum Schltdl. \& Cham. CHIS, OAX, VER

Hymenophyllum sieberi (C. Presl) Bosch CHIS

Hymenophyllum tegularis (Desv.) Proctor \& Lourteig CHIS, GRO, HGO, OAX, PUE, VER

Hymenophyllum trapezoidale Liebm. CHIS, GRO, MEX, MOR, OAX, PUE, VER

Hymenophyllum tunbrigense (L.) Sm. CHIS, CHIH, DGO, HGO, OAX, PUE, QRO, SLP, VER

Hymenophyllum undulatum (Sw.) Sw. CHIS, OAX, VER

Polyphlebium capillaceum (L.) Ebihara \& Dubuisson CHIS, GRO, HGO, MOR, OAX, PUE, VER

Polyphlebium hymenophylloides (Bosch) Ebihara \& Dubuisson CHIS, HGO, MOR, OAX, VER

Polyphlebium pyxidiferum (L.) Ebihara \& Dubuisson CHIS, OAX, SLP, VER 
*Trichomanes angustifrons (Fée) Wess. Boer CHIS

*Trichomanes bucinatum Mickel \& Beitel CHIS, HGO, OAX

Trichomanes capillaceum L. CHIS, GRO, MEX, MOR, OAX, PUE, TAB

Trichomanes collariatum Bosch CHIS, HGO, OAX, TAB, VER

Trichomanes crispum L. CHIS, OAX, VER

Trichomanes curtii Rosenst. CAM, CHIS, QROO

Trichomanes diversifrons (Bory) Mett. ex Sadeb. CHIS, OAX, TAB, VER

Trichomanes ekmanii Wess. Boer CHIS, OAX

Trichomanes galeottii E. Fourn. CHIS, OAX, VER

Trichomanes godmanii Hook. CHIS, OAX, TAB, VER

Trichomanes holopterum Kunze CHIS

Trichomanes hymenoides Hedw. CHIS, GRO, OAX, VER

Trichomanes hymenophylloides Bosch CHIS, HGO, MOR, OAX, VER

Trichomanes krausii Hook. \& Grev. CHIS, HGO, OAX, PUE, SLP, TAMS, VER

Trichomanes lucens Sw. OAX

Trichomanes membranaceum L. CHIS, OAX, TAB, VER

Trichomanes ovale (E. Fourn.) Wess. Boer CHIS, OAX, TAB

Trichomanes petersii A. Gray CHIS, OAX, PUE, VER

Trichomanes pinnatum Hedw. CHIS, GRO, MICH, OAX, TAB, VER

Trichomanes polypodioides L. CHIS, GRO, OAX, VER

Trichomanes pyxidiferum L. CHIS, OAX, PUE, QRO, SLP, VER

Trichomanes radicans Sw. CHIS, CHIH, COL, DGO, GRO, HGO, JAL, MEX, MICH, MOR, OAX, PUE, QRO, SLP, TAB, VER

Trichomanes reptans Sw. CHIS, GRO, HGO, JAL, OAX, PUE, VER

Trichomanes tuerckheimii H. Christ CHIS, OAX, VER

Family Hypodematiaceae

Didymochlaena truncatula (Sw.) J. Sm. CHIS, OAX, PUE, VER

Family Isoetaceae

Isoetes howellii Engelm. BCN, BCS

*Isoetes mexicana Underw. CHIH, CDMX, DGO, GTO, HGO, JAL, MEX, MICH, MOR, NAY, QRO, ZAC

Isoetes montezumae A.A. Eaton AGS, CHIS, GTO, JAL, MEX, MICH, MOR, NAY, ZAC

Isoetes orcuttii A.A. Eaton BCN

Isoetes pallida Hickey CAM, GRO, OAX

*Isoetes pringlei Underw. CHIS, JAL, MEX

Isoetes triangula $\mathrm{U}$. Weber OAX

Family Lindsaeaceae

Lindsaea arcuata Kunze CHIS, OAX, VER

Lindsaea klotzschiana Moritz VER

Lindsaea lancea (L.) Bedd. CHIS, GRO, OAX, VER

Lindsaea quadrangularis Raddi CAM, CHIS, JAL, OAX, QROO, VER

Lindsaea stricta (Sw.) Dryand. CAM, CHIS, GRO, JAL, NAY, OAX, VER

Odontosoria guatemalensis H. Christ CHIS, OAX
Odontosoria schlechtendalii (C. Presl) C. Chr. CHIS, GRO, MICH, OAX, PUE, VER

*Sphenomeris clavata (L.) Maxon CHIS

Family Lomariopsidaceae

Cyclopeltis semicordata (Sw.) J. Sm. CHIS, TAB, VER

Lomariopsis recurvata Fée CHIS, HGO, OAX, PUE, TAB, VER

Lomariopsis vestita E. Fourn. CHIS, OAX

Family Lonchitidaceae

Lonchitis hirsuta L. CHIS, OAX, PUE, VER

Family Lycopodiaceae

*Huperzia beiteliana Mickel OAX, SLP

Huperzia serrata (Thunb.) Trevis. HGO, OAX

Lycopodiella cernua (L.) Pic. Serm. CHIS, COL, GRO, HGO,

JAL, MEX, MICH, NAY, OAX, PUE, TAB, VER

Lycopodium clavatum L. CHIS, COL, GRO, HGO, JAL, MEX, MICH, OAX, PUE, QRO, SLP, VER

Lycopodium thyoides Humb. \& Bonpl. ex Willd. CHIS, HGO, OAX, PUE, VER

Phlegmariurus bradeorum (Christ) B. Øllg. CHIS

Phlegmariurus capillaris (Sodiro) B. Øllg. CHIS

Phlegmariurus crassus (Humb. \& Bonpl. ex Willd.) B. Øllg. OAX

Phlegmariurus cuernavacensis (Underw. \& F.E. Lloyd) B. Øllg. CHIS, COL, DGO, GRO, JAL, MEX, MICH, MOR, OAX, PUE, SIN, VER

Phlegmariurus dichotomus (Jacq.) W.H. Wagner CHIS, OAX, PUE, SLP, TAMS, VER

Phlegmariurus hartwegianus (Spring) B. Øllg. CHIS

Phlegmariurus hippurideus (Christ) B. Øllg. CHIS, OAX

Phlegmariurus linifolius (L.) B. Øllg. CHIS, GRO, OAX, PUE, VER

Phlegmariurus mexicanus (Herter) B. Øllg. CHIS, OAX

Phlegmariurus myrsinites (Lam.) B. Øllg. CHIS, GRO, OAX, VER

Phlegmariurus orizabae (Underw. \& F.E. Lloyd) B. Øllg. CHIS, OAX, VER

Phlegmariurus pithyoides (Schltdl. \& Cham.) B. Øllg. CHIS, COL, GRO, JAL, OAX, SLP, TAMS, VER

Phlegmariurus pringlei (Underw. \& F.E. Lloyd) B. Øllg. CHIS, COL, GRO, HGO, JAL, MEX, MICH, MOR, OAX, SIN, VER Phlegmariurus reflexus (Lam.) B. Øllg. CHIS, GRO, HGO, JAL, MEX, OAX, PUE, VER

Phlegmariurus taxifolius (Sw.) Á. Löve \& D. Löve CHIS, CDMX, GRO, HGO, JAL, MEX, MICH, OAX, PUE, SLP, SIN, TAMS, VER

Phlegmariurus wilsonii (Underw. \& F.E. Lloyd) B. Øllg. OAX

Family Lygodiaceae

Lygodium heterodoxum Kunze CHIS, OAX, PUE, TAB, VER

Lygodium venustum Sw. CAM, CHIS, COL, GTO, GRO, HGO,

JAL, MEX, MICH, NAY, OAX, PUE, QRO, QROO, SLP, SIN,

TAB, TAMS, VER

*Lygodium volubile Sw. CHIS, VER 


\section{Family Marattiaceae}

Danaea cuspidata Liebm. CHIS, OAX, PUE, VER

Danaea elliptica Sm. CHIS, OAX, VER

Danaea nodosa (L.) Sm. CHIS, OAX, VER

Marattia excavata Underw. CHIS

* Marattia laxa Kunze OAX, PUE, VER

Marattia weinmanniifolia Liebm. CHIS, COL, GRO, HGO, JAL, OAX, PUE, QRO, SLP, TAMS, VER

Family Marsileaceae

Marsilea ancylopoda A. Braun AGS, CHIH, COL, DGO, GRO, HGO, JAL, MEX, MICH, NAY, OAX, QRO, TAMS

Marsilea crotophora D.M. Johnson CAM, GRO, TAB, VER

Marsilea deflexa A. Braun JAL, NAY, OAX, PUE, VER

Marsilea macropoda Engelm. ex A. Braun COAH, HGO, NLE, TAMS

Marsilea mollis B.L. Rob. \& Fernald AGS, CHIS, CHIH, CDMX, DGO, GTO, GRO, HGO, JAL, MEX, MICH, MOR, NAY, OAX, PUE, QRO, SLP, SON, TAMS, VER, ZAC

Marsilea polycarpa Hook. \& Grev. GRO, JAL, NAY, TAB, VER Marsilea vestita Hook. \& Grev. AGS, BCN, BCS, CHIH, COAH, CDMX, DGO, GTO, JAL, NLE, PUE, QRO, SLP, SIN, SON, TAMS, ZAC

Pilularia americana A. Braun BCN

\section{Family Metaxyaceae}

Metaxya rostrata (Kunth) C. Presl CHIS

\section{Family Nephrolepidaceae}

Nephrolepis biserrata (Sw.) Schott CAM, CHIS, COL, JAL, NAY, OAX, PUE, QROO, TAB, TAMS, VER, YUC

Nephrolepis rivularis (Vahl) Mett. ex Krug CAM, CHIS, OAX, QROO, VER, YUC

Nephrolepis undulata (Afzel. ex Sw.) J. Sm. CHIS, COL, GRO, JAL, MEX, MICH, MOR, NAY, OAX, SIN, TAMS, VER

\section{Family Oleandraceae}

Oleandra articulata (Sw.) C. Presl CHIS, OAX, VER

\section{Family Onocleaceae}

Onocleopsis hintonii F. Ballard CHIS, COL, GRO, HGO, JAL, MEX, MICH, MOR, OAX, PUE, VER

\section{Family Ophioglossaceae}

Botrychium decompositum M. Martens \& Galeotti CHIS, GTO, HGO, MEX, MICH, MOR, NLE, OAX, PUE, QRO, SLP, TAMS, VER

Botrychium minganense Vict. NLE

*Botrychium pseudo-schaffneri W.H. Wagner COL, JAL

Botrychium schaffneri Underw. AGS, CHIS, CHIH, COL, CDMX, DGO, GRO, HGO, JAL, MEX, MICH, MOR, NAY, NLE, OAX, PUE, QRO, SLP, SIN, SON, TAMS, VER, ZAC

*Botrychium socorrense W.H. Wagner COL

*Botrychium tolucaense W.H. Wagner \& Mickel DGO, MEX Botrychium virginianum (L.) Sw. CHIS, CHIH, COL, CDMX, DGO, GTO, GRO, HGO, JAL, MEX, MICH, MOR, NAY, NLE, OAX, PUE, QRO, SLP, SIN, SON, TAMS, TLAX, VER

Ophioglossum californicum Prantl BCN, BCS, DGO
Ophioglossum crotalophoroides Walter CAM, CHIS, DGO, GRO, JAL, MEX, MICH, NAY, OAX, QRO, QROO, SLP, SIN, ZAC

Ophioglossum engelmannii Prantl BCN, BCS, CHIS, CHIH, COAH, COL, CDMX, HGO, JAL, MEX, MICH, NAY, NLE, OAX, SLP, SIN, SON

Ophioglossum nudicaule L. f. AGS, CHIS, CDMX, DGO, JAL, MEX, NAY, OAX, PUE, SLP, SIN, SON, TLAX, VER, ZAC Ophioglossum palmatum L. CHIS, OAX

Ophioglossum petiolatum Hook. CHIS, MEX, OAX

Ophioglossum polyphyllum A. Braun ex Schub. BCS, CHIH, CDMX, HGO, OAX, PUE, SLP, ZAC

Ophioglossum reticulatum L. CHIS, COL, DGO, JAL, MEX, NAY, OAX, QRO, SLP, SIN, TAMS, VER

Ophioglossum vulgatum L. CHIS, CHIH, CDMX, HGO, JAL, MICH, OAX, PUE, SON, VER

\section{Family Osmundaceae}

Osmunda cinnamomea L. CHIS, OAX, TAB, VER

Osmunda regalis L. AGS, CHIS, GTO, GRO, HGO, JAL, MEX, MICH, MOR, NAY, OAX, PUE, QRO, TAB, VER, ZAC

\section{Family Plagiogyriaceae}

Plagiogyria pectinata (Liebm.) Lellinger CHIS, CHIH, COL, DGO, GRO, HGO, JAL, MEX, MICH, MOR, OAX, PUE, SIN, VER

\section{Family Polypodiaceae}

Alansmia cultrata (Bory ex Willd.) Moguel \& M. Kessler CHIS, GRO, OAX, VER

Alansmia elastica (Bory ex Willd.) Moguel \& M. Kessler CHIS, OAX, VER

Alansmia senilis (Fée) Moguel \& M. Kessler CHIS, JAL, MICH, OAX

Alansmia spathulata (A.R. Sm.) Moguel \& M. Kessler CHIS, CDMX, JAL, MEX, MICH, OAX, PUE, VER

Ascogrammitis anfractuosa (Kunze ex Klotzsch) Sundue CHIS, HGO, OAX, VER

Campyloneurum amphostenon (Kunze ex Klotzsch) Fée CHIS, COL, GRO, HGO, JAL, MEX, MOR, OAX, PUE, SLP, VER

Campyloneurum angustifolium (Sw.) Fée CHIS, CHIH, COL, CDMX, DGO, GRO, HGO, JAL, MEX, MICH, MOR, NAY, NLE, OAX, PUE, QRO, SLP, SIN, SON, TAB, TAMS, VER

Campyloneurum costatum (Kunze) C. Presl CHIS, OAX, VER Campyloneurum ensifolium (Willd.) J. Sm. CHIS, CHIH, COL, DGO, JAL, MICH, NAY, OAX, SIN, SON, VER

Campyloneurum phyllitidis (L.) C. Presl CAM, CHIS, COL, GRO, HGO, JAL, MICH, NAY, OAX, PUE, QRO, QROO, SLP, TAB, VER, YUC

Campyloneurum repens (Aubl.) C. Presl CHIS, OAX, VER

Campyloneurum serpentinum (H. Christ) Ching CHIS, GRO, OAX, TAB, VER

Campyloneurum tenuipes Maxon CHIS, GRO, HGO, OAX, PUE, QRO, SLP, VER

Campyloneurum xalapense Fée CHIS, COL, GRO, HGO, JAL, NAY, OAX, PUE, QRO, SLP, TAB, VER

Ceradenia jungermannioides (Klotzsch) L.E. Bishop CHIS, OAX 
Ceradenia margaritata (A.R. Sm.) L.E. Bishop CHIS

*Ceradenia oidiophora (Mickel \& Beitel) A.R. Sm. GRO, OAX

Ceradenia pruinosa (Maxon) L.E. Bishop OAX

Cochlidium linearifolium (Desv.) Maxon ex C. Chr. CHIS, GRO, OAX, VER

Cochlidium rostratum (Hook.) Maxon ex C. Chr. CHIS, OAX, VER

Cochlidium serrulatum (Sw.) L.E. Bishop CHIS, OAX, PUE, VER

Enterosora ecostata (Sodiro) L.E. Bishop CHIS

Enterosora parietina (Klotzsch) L.E. Bishop OAX

Enterosora trichosora (Hook.) L.E. Bishop CHIS

Lellingeria apiculata (Kunze ex Klotzsch) A.R. Sm. \& R.C. Moran CHIS, OAX

Leucotrichum mitchellae (Baker) Labiak CHIS

Loxogramme mexicana (Fée) C. Chr. CHIS, COL, JAL, OAX, VER

Melpomene deltata (Mickel \& Beitel) A.R. Sm. \& R.C. Moran CHIS, OAX

Melpomene firma (J. Sm.) A.R. Sm. \& R.C. Moran CHIS, OAX Melpomene flabelliformis (Poir.) A.R. Sm. \& R.C. Moran CHIS, OAX

Melpomene leptostoma (Fée) A.R. Sm. \& R.C. Moran CHIS, GRO, HGO, MEX, OAX, PUE, VER

Melpomene melanosticta (Kunze) A.R. Sm. \& R.C. Moran CHIS

Melpomene moniliformis (Lag. ex Sw.) A.R. Sm. \& R.C. Moran CHIS, GRO, JAL, MEX, MICH, MOR, OAX, PUE, VER

Melpomene pilosissima (M. Martens \& Galeotti) A.R. Sm. \& R.C. Moran CHIS, COL, GRO, HGO, JAL, MEX, MICH, OAX, VER

Melpomene xiphopteroides (Liebm.) A.R. Sm. \& R.C. Moran CHIS, GRO, OAX, VER

*Melpomene zempoaltepetlensis (Mickel \& Beitel) A.R. Sm. CHIS, OAX

Microgramma lycopodioides (L.) Copel. CAM, CHIS, OAX, QROO, TAB, VER, YUC

Microgramma nitida (J. Sm.) A.R. Sm. CAM, CHIS, HGO, OAX, PUE, QRO, QROO, SLP, TAB, TAMS, VER, YUC

Microgramma percussa (Cav.) de la Sota CHIS, OAX, TAB, VER

*Microgramma piloselloides (L.) Copel. CHIS, TAB

* Microgramma reptans (Cav.) A.R. Sm. CHIS, TAB

Microgramma tecta (Kaulf.) Alston OAX

Moranopteris basiattenuata (Jenman) R.Y. Hirai \& J. Orado CHIS, OAX, VER

Moranopteris taenifolia (Jenman) R.Y. Hirai \& J. Prado CHIS, OAX, VER

Moranopteris trichomanoides (Sw.) R.Y. Hirai \& J. Prado CHIS, OAX, VER

Niphidium crassifolium (L.) Lellinger CAM, CHIS, GRO, HGO, OAX, PUE, QRO, QROO, SLP, VER

Pecluma alfredii (Rosenst.) M.G. Price CAM, CHIS, COL, CDMX, DGO, GTO, GRO, HGO, JAL, MEX, MICH, MOR, NAY, OAX, PUE, QRO, QROO, SLP, SIN, TAMS, VER

Pecluma atra (A.M. Evans) M.G. Price CHIS, OAX, PUE, QROO, TAB, VER
Pecluma consimilis (Mett.) M.G. Price CHIS, NAY, OAX, SIN, VER

Pecluma dispersa (A.M. Evans) M.G. Price CHIS, HGO, OAX, QROO, SLP, TAMS, VER

Pecluma divaricata (E. Fourn.) Mickel \& Beitel CHIS, VER

*Pecluma ferruginea (M. Martens \& Galeotti) M.G. Price CAM, CHIS, COL, GRO, HGO, JAL, MEX, MICH, MOR, NAY, OAX, QRO, SLP, SIN, YUC, ZAC

Pecluma hygrometrica (Splitg.) M.G. Price CHIS

Pecluma plumula (Humb. \& Bonpl. ex Willd.) M.G. Price CAM, CHIS, HGO, JAL, MICH, NAY, OAX, PUE, QRO, QROO, SLP, SIN, TAMS, VER

Pecluma ptilodon (Kunze) M.G. Price CHIS, MOR, OAX, QRO, SLP, TAMS, VER

*Pecluma sursumcurrens (Copel.) M.G. Price HGO, OAX, PUE, QRO, SLP, VER

Phlebodium areolatum (Humb. \& Bonpl. ex Willd.) J. Sm. AGS, CHIS, CHIH, COAH, COL, CDMX, DGO, GTO, GRO, HGO, JAL, MEX, MICH, MOR, NAY, NLE, OAX, PUE, QRO, SLP, SIN, SON, TAB, TAMS, VER, ZAC

Phlebodium decumanum (Willd.) J. Sm. CAM, CHIS, OAX, QRO, QROO, SLP, TAB, VER, YUC

Pleopeltis alan-smithii (R.C. Moran) A.R. Sm. \& Tejero CHIS, GRO, HGO, OAX, SLP, TAMS, VER

Pleopeltis angusta Humb. \& Bonpl. ex Willd. CHIS, COL, DGO, GTO, GRO, JAL, MICH, NAY, OAX, PUE, VER

Pleopeltis astrolepis (Liebm.) E. Fourn. CAM, CHIS, COL, GRO, HGO, JAL, MICH, NAY, OAX, QRO, QROO, SLP, TAB, TLAX, VER, YUC

Pleopeltis bradeorum (Rosenst.) A.R. Sm. \& Tejero CHIS, GRO, OAX, VER

*Pleopeltis collinsii (Maxon) A.R. Sm. \& Tejero CHIS, OAX, VER

* Pleopeltis conzattii (Weath.) R.M. Tryon \& A.F. Tryon GRO, OAX

Pleopeltis crassinervata (Fée) T. Moore CHIS, CHIH, DGO, GRO, HGO, MEX, MOR, OAX, PUE, QRO, SLP, SIN, TAMS, VER

Pleopeltis cryptocarpa (Fée) A.R. Sm. \& Tejero CHIS, DGO, GRO, HGO, JAL, MOR, NAY, OAX, PUE, QRO, SLP, VER

*Pleopeltis fallacissima (Maxon) A.R. Sm. \& Tejero COAH, NLE, TAMS

Pleopeltis fallax (Schltdl. \& Cham.) Mickel \& Beitel CHIS, HGO, OAX, PUE, QRO, SLP, VER

Pleopeltis furfuracea (Schltdl. \& Cham.) A.R. Sm. \& Tejero CHIS, COL, DGO, GRO, HGO, JAL, MEX, MICH, MOR, NAY, OAX, PUE, QRO, SLP, SIN, TAMS, VER, ZAC

*Pleopeltis lepidotricha (Fée) A.R. Sm. \& Tejero CHIS, HGO, OAX, PUE, VER

*Pleopeltis lindeniana (Kunze) A.R. Sm. \& Tejero CHIS

Pleopeltis madrensis (J. Sm.) A.R. Sm. \& Tejero AGS, COL, CDMX, DGO, GTO, GRO, HGO, JAL, MEX, MICH, MOR, NAY, OAX, PUE, QRO, SLP, SIN, VER, ZAC

Pleopeltis marginata A.R. Sm. \& Tejero CHIS, TAB

Pleopeltis mexicana (Fée) Mickel \& Beitel CHIS, COAH, COL, CDMX, GTO, GRO, HGO, JAL, MEX, MICH, MOR, NAY, NLE, OAX, PUE, QRO, SLP, SIN, TAMS, TLAX, VER, ZAC 
*Pleopeltis microgrammoides (Mickel \& A.R. Sm.) A.R. Sm. \& Tejero GTO, JAL

Pleopeltis percussa (Cav.) Hook. \& Grev. CHIS

Pleopeltis platylepis (Mett. ex Kuhn) A.R. Sm. \& Tejero CHIS, COL, GRO, HGO, JAL, MEX, MICH, OAX, QRO, SLP

Pleopeltis plebeia (Schltdl. \& Cham.) A.R. Sm. \& Tejero CHIS, CDMX, DGO, GTO, GRO, HGO, JAL, MEX, MICH, MOR, NLE, OAX, PUE, QRO, SLP, TAB, TAMS, TLAX, VER

Pleopeltis polylepis (Roem. ex Kuntze) T. Moore AGS, BCS, CHIS, CHIH, COAH, COL, CDMX, DGO, GTO, GRO, HGO, JAL, MEX, MICH, MOR, NLE, OAX, PUE, QRO, SLP, SIN, SON, TAMS, TLAX, VER, ZAC

Pleopeltis polypodioides (L.) E.G. Andrews \& Windham CHIH, HGO, SIN, SON

*Pleopeltis pyrrholepis (Fée) A.R. Sm. \& Tejero CHIS, OAX, VER

* Pleopeltis riograndense (T. Wendt) E.G. Andr. \& Windham $\mathrm{CHIH}, \mathrm{COAH}, \mathrm{SON}$

* Pleopeltis rosei (Maxon) A.R. Sm. \& Tejero CDMX, GRO, HGO, JAL, MEX, MICH, MOR, NAY, OAX, QRO, SLP, SIN, VER, ZAC

*Pleopeltis rzedowskiana (Mickel) A.R. Sm. \& Tejero GRO, JAL, OAX, SIN

Pleopeltis sanctae-rosae (Maxon) A.R. Sm. \& Tejero CHIS, COL, GRO, HGO, JAL, MEX, NAY, OAX, VER, ZAC

Pleopeltis thyssanolepis (A. Braun ex Klotzsch) E.G. Andrews \& Windham AGS, CHIS, CHIH, COAH, COL, CDMX, DGO, GTO, GRO, HGO, JAL, MEX, MICH, MOR, NAY, NLE, OAX, PUE, QRO, SLP, SIN, SON, VER, ZAC

*Pleopeltis villagranii (Copel.) A.R. Sm. \& Tejero GRO, HGO, OAX, PUE, QRO, SLP, VER

$*$ Pleopeltis $\times$ tricholepis (Mickel \& Beitel) A.R. Sm. \& Tejero OAX

Polypodium aequale Maxon CHIS, GRO

Polypodium alavae A.R. Sm. CHIS, HGO, OAX, VER

*Polypodium arcanum Maxon COAH, COL, GTO, GRO, HGO, JAL, MEX, MICH, NLE, OAX, PUE, QRO, SLP, TAMS, VER Polypodium californicum Kaulf. BCN, OAX

Polypodium calirhiza S.A. Whitmore \& A.R. Sm. COL, JAL, MEX, OAX, VER

Polypodium colpodes Kunze CHIS, COL, GRO, HGO, JAL, MEX, MICH, NAY, OAX, QRO, SLP, SIN, TAMS

*Polypodium colysoides Maxon \& Copel. ex Copel. CHIS, OAX, VER

*Polypodium conterminans Liebm. CHIS, HGO, OAX, PUE, QRO, SLP, VER

*Polypodium chiapense A.M. Evans \& A.R. Sm. CHIS, OAX Polypodium christensenii Maxon CHIS

*Polypodium diplotrichum Mickel \& Beitel CHIS, OAX

*Polypodium eatonii Baker CHIS, OAX, PUE, VER

Polypodium echinolepis Fée CHIS, HGO, JAL, MEX, MICH, OAX, PUE, VER

*Polypodium eperopeutes Mickel \& Beitel CHIS, OAX, VER Polypodium fissidens Maxon CHIS, OAX

Polypodium fraternum Schltdl. \& Cham. CHIS, GRO, HGO, JAL, MEX, MICH, NAY, OAX, PUE, QRO, SLP, SIN, VER, ZAC
*Polypodium fraxinifolium Jacq. CHIS

*Polypodium guttatum Maxon AGS, BCN, BCS, CHIH, COAH, DGO, GTO, HGO, JAL, MEX, MOR, NLE, PUE, QRO, SLP, SIN, SON, TAMS, ZAC

Polypodium hartwegianum Hook. CHIS, CHIH, COAH, COL, CDMX, DGO, GTO, GRO, HGO, JAL, MEX, MICH, MOR, NAY, NLE, OAX, PUE, SLP, TAMS, VER, ZAC

Polypodium hispidulum Bartlett CHIS, OAX, VER

Polypodium lanceolatum L. BCS, CDMX, JAL, MEX, MICH, TAMS, VER

*Polypodium liebmannii C. Chr. VER

Polypodium longepinnulatum E. Fourn. CHIS, COL, GRO, HGO, JAL, MICH, OAX, PUE, QRO, SLP, VER

*Polypodium martensii Mett. AGS, CHIH, COL, CDMX, GTO, GRO, HGO, JAL, MEX, MICH, MOR, NLE, OAX, PUE, QRO, SLP, TAMS, VER, ZAC

Polypodium muenchii $\mathrm{H}$. Christ CHIS, OAX

Polypodium plesiosorum Kunze CHIS, COAH, COL, GTO, GRO, HGO, JAL, MEX, MICH, MOR, NAY, NLE, OAX, PUE, QRO, SLP, SIN, TAMS, VER, ZAC

Polypodium pleurosorum Kunze ex Mett. CHIS, GRO, MEX, MICH, OAX, TAB

Polypodium polypodioides (L.) Watt AGS, CAM, CHIS, CHIH, COAH, COL, CDMX, DGO, GTO, GRO, HGO, JAL, MEX, MICH, MOR, NAY, NLE, OAX, PUE, QRO, QROO, SLP, SIN, SON, TAB, TAMS, VER, YUC, ZAC

*Polypodium praetermissum Mickel \& Tejero DGO, JAL, NAY, SIN, SON

Polypodium puberulum Schltdl. \& Cham. CHIS, GRO, MEX, OAX, PUE, QRO, SLP, VER

*Polypodium remotum Desv. CHIS

Polypodium rhachipterygium Liebm. CHIS, OAX, VER

Polypodium rhodopleuron Kunze CHIS, GRO, HGO, MEX, NLE, OAX, PUE, QRO, SLP, VER

Polypodium riograndense (T. Wendt) Mickel $\mathrm{CHIH}, \mathrm{COAH}$, SON

Polypodium scouleri Hook. \& Grev. BCN

Polypodium subpetiolatum Hook. AGS, CHIS, CHIH, COAH, COL, CDMX, DGO, GTO, GRO, HGO, JAL, MEX, MICH, MOR, NAY, NLE, OAX, PUE, QRO, SLP, SIN, SON, TAMS, VER, ZAC

Polypodium vulgare L. BCN, NLE, TAMS

Serpocaulon dissimile (L.) A.R. Sm. CHIS, GRO, OAX, VER Serpocaulon falcaria (Kunze) A.R. Sm. CHIS, GRO, OAX, PUE, TAMS, VER

Serpocaulon triseriale (Sw.) A.R. Sm. CAM, CHIS, MICH, OAX, PUE, QROO, TAB, VER

Terpsichore asplenifolia (L.) A.R. Sm. CHIS, GRO, MICH, OAX, VER

Stenogrammitis delitescens (Maxon) Labiak CHIS, OAX, VER

* Stenogrammitis hellwigii (Mickel \& Beitel) Labiak OAX Stenogrammitis limula (Christ) Labiak CHIS

Stenogrammitis prionodes (Mickel \& Beitel) Labiak CHIS, HGO, OAX, VER

Terpsichore delicaluta (M. Martens \& Galeotti) A.R. Sm. CHIS, HGO, OAX, VER

Terpsichore semihirsuta (Klotzsch) A.R. Sm. CHIS, GRO, OAX 
Terpsichore subtilis (Kunze ex Klotzsch) A.R. Sm. CHIS

\section{Family Psilotaceae}

Psilotum complanatum Sw. CHIS, CHIH, COL, GRO, HGO, JAL, MEX, MOR, NAY, OAX, PUE, SIN, SON, TAMS, VER Psilotum nudum (L.) P. Beauv. CAM, CHIS, COL, CDMX, GRO, JAL, MEX, MOR, OAX, SON, TAB, TAMS, VER, YUC

\section{Family Pteridaceae}

Acrostichum aureum L. CAM, CHIS, GRO, NAY, OAX, QROO, TAB, TAMS, VER, YUC

Acrostichum danaeifolium Langsd. \& Fisch. CAM, CHIS, COL, GRO, JAL, MICH, NAY, OAX, QRO, QROO, SLP, TAB, TAMS, VER, YUC

Adiantopsis radiata (L.) Fée CHIS, COL, GRO, HGO, OAX, PUE, QRO, SLP, TAB, TAMS, VER

Adiantopsis seemannii (Hook.) Maxon BCS, COL, GRO, JAL, NAY, OAX, SIN

Adiantum aleuticum (Rupr.) C.A. Paris CHIH, SIN, SON

*Adiantum amblyopteridium Mickel \& Beitel OAX, QROO

Adiantum amplum C. Presl CAM, CHIS, COL, DGO, GRO, JAL, MEX, MICH, NAY, OAX, SIN, TAB, VER, ZAC

Adiantum andicola Liebm. AGS, CHIS, CHIH, COL, CDMX, DGO, GTO, GRO, HGO, JAL, MEX, MICH, MOR, NAY, NLE, OAX, PUE, QRO, SLP, SIN, SON, TAMS, TLAX, VER

Adiantum braunii Mett. ex Kuhn AGS, CHIS, CHIH, COL, DGO, GRO, HGO, JAL, MEX, MICH, MOR, NAY, OAX, QRO, SLP, SIN, SON, VER, ZAC

Adiantum capillus-veneris $\mathrm{L}$. AGS, BCN, BCS, CHIS, CHIH, COAH, CDMX, DGO, GTO, GRO, HGO, JAL, MEX, MICH, MOR, NAY, NLE, OAX, PUE, QRO, SLP, SIN, SON, TAMS, TLAX, VER, ZAC

Adiantum caryotideum $\mathrm{H}$. Christ CHIS

Adiantum concinnum Humb. \& Bonpl. ex Willd. AGS, BCS, CHIS, CHIH, COL, CDMX, DGO, GRO, HGO, JAL, MEX, MICH, MOR, NAY, OAX, PUE, QRO, SLP, SIN, SON, TAMS, VER, ZAC

Adiantum decoratum Maxon \& Weath. CHIS, QROO, TAB, VER

Adiantum deflectens Mart. CHIS, OAX

Adiantumfeei $\mathrm{T}$. Moore ex Fée CHIS, GRO, OAX, QRO, TAMS, VER

*Adiantum galeottianum Hook. GRO, MICH, OAX

Adiantum jordanii Müll. Berol. BCN, BCS

Adiantum latifolium Lam. CAM, CHIS, NAY, OAX, QROO, TAB, VER

Adiantum lunulatum Burm. f. CHIS, COL, GRO, JAL, MEX, MICH, NAY, OAX, SIN, ZAC

Adiantum macrophyllum Sw. CHIS, GRO, OAX, PUE, VER

*Adiantum mcvaughii Mickel \& Beitel CHIS, COL, JAL, $\mathrm{MICH}, \mathrm{NAY}$

*Adiantum oaxacanum Mickel \& Beitel OAX, VER

Adiantum obliquum Willd. CHIS, OAX, TAB, VER

Adiantum patens Willd. CHIS, COL, DGO, GRO, JAL, MEX, MICH, MOR, NAY, OAX, SIN, SON, ZAC

Adiantum petiolatum Desv. CHIS, OAX, TAB, VER

Adiantum poiretii Wikstr. AGS, BCS, CHIS, CHIH, COAH,

COL, CDMX, DGO, GTO, GRO, HGO, JAL, MEX, MICH,
MOR, NAY, NLE, OAX, PUE, QRO, SLP, SIN, SON, TAMS, TLAX, VER, ZAC

Adiantum pulverulentum L. CHIS, GRO, OAX, QROO, SLP, TAB, VER

Adiantum seemannii Hook. CHIS

*Adiantum shepherdii Hook. GRO, JAL, MEX, MICH

Adiantum tenerum Sw. CAM, CHIS, HGO, MEX, MICH, NAY, NLE, OAX, PUE, QRO, QROO, SLP, TAB, TAMS, VER, YUC Adiantum terminatum Kunze ex Miq. CHIS

Adiantum tetraphyllum Humb. \& Bonpl. ex Willd. CHIS, GRO, NAY, OAX, PUE, QRO, SLP, TAB, VER

Adiantum trapeziforme L. CHIS, COL, GRO, HGO, JAL, MEX, MICH, NAY, NLE, OAX, PUE, QRO, SLP, TAB, TAMS, VER

Adiantum trichochlaenum Mickel \& Beitel CHIS, GRO, OAX Adiantum tricholepis Fée CAM, CHIS, CHIH, COAH, COL, DGO, GRO, HGO, JAL, MEX, MICH, MOR, NAY, NLE, OAX, PUE, QRO, QROO, SLP, SIN, SON, TAB, TAMS, VER, YUC, ZAC

Adiantum villosum L. CAM, CHIS, GRO, OAX, QROO, TAB, VER

Adiantum wilesianum Hook. CHIS, HGO, OAX, SLP, TAB, VER

Adiantum wilsonii Hook. CHIS, OAX, VER

Ananthacorus angustifolius (Sw.) Underw. \& Maxon CHIS, COL, GRO, JAL, NAY, OAX, VER

Anetium citrifolium (L.) Splitg. CHIS, VER

Anogramma chaerophylla (Desv.) Link CHIS, JAL, MEX, MICH, MOR, OAX, VER

Anogramma leptophylla (L.) Link AGS, CHIS, CHIH, COL, DGO, GTO, GRO, JAL, MEX, MICH, MOR, NAY, OAX, SIN, SON, TAMS, VER

*Anogramma novogaliciana Mickel JAL, MICH

Argyrochosma delicatula (Maxon \& Weath.) Windham CHIH, COAH, NLE

Argyrochosma fendleri (Kunze) Windham CHIH

Argyrochosma formosa (Liebm.) Windham AGS, CHIS, CHIH, COAH, GTO, GRO, HGO, MEX, NLE, OAX, PUE, QRO, SLP, TAMS, VER, ZAC

Argyrochosma incana (C. Presl) Windham AGS, BCN, BCS, CHIS, CHIH, COAH, COL, CDMX, DGO, GTO, GRO, HGO, JAL, MEX, MICH, MOR, NAY, NLE, OAX, PUE, QRO, SLP, SIN, SON, TAMS, TLAX, ZAC

Argyrochosma jonesii (Maxon) Windham BCN, SON

Argyrochosma limitanea (Maxon) Windham $\mathrm{CHIH}$, COAH, DGO, HGO, JAL, MICH, NLE, QRO, SLP, SIN, SON, TAMS, ZAC

*Argyrochosma lumholtzii (Maxon \& Weath.) Windham SON Argyrochosma microphylla (Mett. ex Kuhn) Windham CHIH, COAH, NLE, SON, TAMS, ZAC

*Argyrochosma palmeri (Baker) Windham DGO, GTO, SLP

*Argyrochosma pallens (Weath. ex R.M. Tryon) Windham AGS, CHIH, DGO, GTO, HGO, JAL, MEX, OAX, PUE, QRO

*Argyrochosma peninsularis (Maxon \& Weath.) Windham BCS *Argyrochosma pilifera (R.M. Tryon) Windham CHIH, DGO, MOR, OAX, PUE

Aspidotis californica (Hook.) Nutt. ex Copel. BCN 
*Aspidotis meifolia (D.C. Eaton) Pic. Serm. CHIH, COAH, DGO, HGO, NLE, QRO, SLP, TAMS, VER

Astrolepis cochisensis (Goodd.) D.M. Benham \& Windham AGS, BCN, CHIH, COAH, DGO, GTO, NLE, SON, TAMS

Astrolepis crassifolia (Houlston \& T. Moore) D.M. Benham \& Windham CHIS, CHIH, COAH, CDMX, DGO, GTO, GRO, HGO, MEX, MOR, NLE, OAX, PUE, QRO, SLP, TAMS, TLAX, VER

Astrolepis integerrima (Hook.) D.M. Benham \& Windham AGS, CHIS, CHIH, COAH, CDMX, DGO, GTO, GRO, HGO, JAL, MEX, NAY, NLE, OAX, PUE, QRO, SLP, SON, TAMS, TLAX, VER, ZAC

Astrolepis laevis (M. Martens \& Galeotti) Mickel AGS, CHIS, CHIH, COAH, COL, CDMX, DGO, GTO, GRO, HGO, JAL, MEX, MICH, NLE, OAX, PUE, QRO, SLP, SON, TAMS, TLAX, VER, ZAC

Astrolepis sinuata (Lag. ex Sw.) D.M. Benham \& Windham AGS, BCN, BCS, CHIS, CHIH, COAH, COL, CDMX, DGO, GTO, GRO, HGO, JAL, MEX, MICH, MOR, NAY, NLE, OAX, PUE, QRO, SLP, SIN, SON, TAMS, TLAX, VER, ZAC

*Bommeria ehrenbergiana (Klotzsch) Underw. DGO, GRO, HGO, JAL, MEX, NLE, OAX, PUE, QRO, SLP, TAMS, VER

*Bommeria elegans (Davenp.) Ranker \& Haufler COL, JAL, MEX, MICH, MOR, NAY, OAX, ZAC

Bommeria hispida (Mett. ex Kuhn) Underw. AGS, BCS, CHIH, COAH, CDMX, DGO, GTO, GRO, HGO, JAL, MEX, MICH, SLP, SON, ZAC

Bommeria pedata (Sw.) E. Fourn. AGS, BCS, CHIS, CHIH, COL, CDMX, DGO, GTO, GRO, HGO, JAL, MEX, MICH, MOR, NAY, OAX, PUE, QRO, SLP, SIN, SON, VER, ZAC

*Bommeria subpaleacea Maxon CHIH, CDMX, GTO, HGO, MEX, MOR, NLE, OAX, PUE, QRO, SLP, SIN, TAMS

Ceratopteris pteridoides (Hook.) Hieron. CAM, CHIS, GRO, NAY, QROO, SIN, TAB, VER, YUC

Cheilanthes bolborrhiza Mickel \& Beitel JAL, MEX, MOR, OAX

Cheilanthes brachypus (Kunze) Kunze AGS, CHIS, COL, GTO, GRO, JAL, MEX, MICH, MOR, NAY, OAX, PUE, SLP, SIN, SON, VER

*Cheilanthes brandegeei D.C. Eaton BCN, BCS

Cheilanthes clevelandii D.C. Eaton BCN

Cheilanthes covillei Maxon BCN, BCS, CHIH, COAH

Cheilanthes farinosa (Forssk.) Kaulf. AGS, CHIS, CHIH, COL, CDMX, DGO, GRO, HGO, JAL, MEX, MICH, MOR, NAY, OAX, PUE, QRO, SLP, SIN, TAMS, VER, ZAC

Cheilanthes feei $\mathrm{T}$. Moore CHIH, COAH, SON

Cheilanthes horridula Maxon CHIH, COAH, DGO, GTO, NLE, SLP, TAMS, ZAC

Cheilanthes leucopoda Link CHIH, COAH, DGO, GTO, HGO, JAL, NLE, QRO, SLP, SON, TAMS

*Cheilanthes lozanoi (Maxon) R.M. Tryon \& A.F. Tryon AGS, BCN, BCS, CHIS, CHIH, COL, CDMX, DGO, GTO, GRO, HGO, JAL, MEX, MICH, MOR, NAY, OAX, PUE, QRO, SLP, SIN, SON, TAMS, ZAC

Cheilanthes pyramidalis Fée AGS, BCS, CHIS, CHIH, COAH, COL, CDMX, DGO, GTO, GRO, HGO, JAL, MEX, MICH,
MOR, NAY, NLE, OAX, PUE, QRO, SLP, SIN, SON, TLAX, VER, ZAC

Cheilanthes skinneri (Hook.) R.M. Tryon \& A.F. Tryon BCN, BCS, CHIS, CHIH, COL, CDMX, DGO, GRO, JAL, MEX, MICH, MOR, NAY, OAX, SIN, SON, VER

*Cheilanthes spiculata Mickel MEX, MICH

Cheiloplecton rigidum (Sw.) Fée AGS, CHIS, CHIH, COL, GTO, GRO, HGO, JAL, MEX, MICH, MOR, NAY, NLE, OAX, PUE, QRO, SLP, SIN, SON, TAMS, VER, ZAC

Doryopteris concolor (Langsd. \& Fisch.) Kuhn CHIS, OAX, VER

Doryopteris pedata (L.) Fée CHIS, GRO, OAX, VER

Gaga angustifolia (Kunth) Fay W. Li \& Windham AGS, CHIS, CHIH, COL, CDMX, DGO, GTO, GRO, HGO, JAL, MEX, MICH, MOR, NAY, OAX, PUE, QRO, SLP, SIN, SON, TAMS, VER, ZAC

*Gaga apiacea (Mickel) Fay W. Li \& Windham NLE, TAMS Gaga arizonica (Maxon) Fay W. Li \& Windham CHIS, CHIH, COAH, COL, DGO, GRO, HGO, JAL, MEX, MICH, NAY, NLE, OAX, QRO, SIN, SON, VER, ZAC

Gaga complanata (A.R. Sm.) Fay W. Li \& Windham CHIS, GRO, OAX, VER

*Gaga cuneata (Kaulf. ex Link) F.W. Li \& Windham CHIS, CHIH, GRO, HGO, JAL, MEX, MICH, MOR, NAY, OAX, PUE, SIN, VER

Gaga chaerophylla (M. Martens \& Galeotti) F.W. Li \& Windham CHIS, COL, CDMX, DGO, GTO, GRO, JAL, MEX, MICH, MOR, NAY, OAX, PUE, SIN, SON

*Gaga decomposita (M. Martens \& Galeotti) F.W. Li \& Windham CDMX, DGO, GRO, JAL, MEX, MICH, NAY, SIN, OAX, VER, ZAC

Gaga decurrens (Mickel) F.W. Li \& Windham CHIS, OAX

*Gaga hintoniorum (M.G. Mendenh. \& G.L. Nesom) F.W. Li \& Windham NLE, TAMS

Gaga kaulfussii (Kunze) F.W. Li \& Windham AGS, CHIS, CHIH, COAH, COL, CDMX, DGO, GTO, GRO, HGO, JAL, MEX, MICH, MOR, NAY, NLE, OAX, PUE, QRO, SLP, SIN, SON, TAMS, TLAX, VER, ZAC

Gaga marginata (Kunth) F.W. Li \& Windham AGS, BCS, CHIS, CHIH, COAH, COL, CDMX, GTO, GRO, HGO, JAL, MEX, MICH, MOR, NAY, NLE, OAX, PUE, QRO, SLP, SON, TAMS, VER, ZAC

Gaga membranacea (Davenp.) F.W. Li \& Windham CHIS, COL, GTO, JAL, MEX, OAX, VER

*Gaga pellaeopsis (Mickel) F.W. Li \& Windham COL, GRO, JAL, OAX, TAMS

*Gaga purpusii (T. Reeves) F.W. Li \& Windham GTO, HGO, NLE, QRO, SLP, TAMS, VER

* Gymnopteris rufa (L.) Bernh. CHIS, TAB

Hecistopteris pumila (Spreng.) J. Sm. OAX

Hemionitis levyi E. Fourn. OAX

Hemionitis palmata L. CHIS, COL, DGO, GRO, JAL, NAY, OAX, PUE, QRO, SLP, SIN, TAB, TAMS, VER, YUC, ZAC

Hemionitis pinnatifida Baker CHIS, GRO, JAL, OAX

Hemionitis subcordata (D.C. Eaton ex Davenp.) Mickel CHIS, COL, DGO, JAL, MEX, MICH, NAY, OAX, SIN, ZAC

Jamesonia alstonii A.F. Tryon CHIS, OAX 
Jamesonia flexuosa (Kunth) Christenh.. CHIS, GRO, OAX Jamesonia hirta (Kunth) Christenh. CHIS, GRO, OAX Llavea cordifolia Lag. BCN, BCS, CHIS, COAH, DGO, GTO, GRO, HGO, MEX, NLE, OAX, PUE, QRO, SLP, TAMS, VER Mildella fallax (M. Martens \& Galeotti) G.L. Nesom AGS, CHIS, CHIH, COAH, COL, CDMX, GRO, HGO, JAL, MEX, MICH, MOR, NLE, OAX, PUE, QRO, SLP, SIN, SON, TAMS, TLAX, VER, ZAC

Mildella intramarginalis (Kaulf. ex Link) Trevis. CHIS, COAH, COL, GTO, GRO, HGO, JAL, MEX, MICH, MOR, NLE, OAX, PUE, QRO, SLP, SIN, TAMS, TLAX, VER

Myriopteris aemula (Maxon) Grusz \& Windham CHIS, CHIH, COAH, HGO, MICH, NLE, OAX, QRO, SLP, TAMS

Myriopteris alabamensis (Buckley) Grusz \& Windham CHIH, COAH, GTO, HGO, NLE, OAX, PUE, QRO, SLP, SON, TAMS, VER

*Myriopteris allosuroides (Mett.) Grusz \& Windham AGS, CHIH, CDMX, DGO, GTO, GRO, HGO, JAL, MEX, MICH, MOR, NAY, OAX, PUE, QRO, SLP, SIN, SON, VER, ZAC Myriopteris aurea (Poir.) Grusz \& Windham AGS, BCN, BCS, CHIS, CHIH, COAH, COL, CDMX, DGO, GTO, GRO, HGO, JAL, MEX, MICH, MOR, NAY, NLE, OAX, PUE, QRO, SLP, SIN, SON, TAMS, TLAX, VER, ZAC

Myriopteris cucullans (Fée) Grusz \& Windham AGS, CHIS, CHIH, COAH, CDMX, GTO, GRO, HGO, JAL, MEX, MICH, MOR, NLE, OAX, PUE, QRO, SLP, VER, ZAC

*Myriopteris chipinquensis (Knobloch \& Lellinger) Grusz \& Windham COAH, NLE

Myriopteris fendleri (Hook.) E. Fourn. BCN, CHIH, SON

Myriopteris fimbriata (A.R. Sm.) Grusz \& Windham CAM, CHIS, OAX, QROO, TAMS, VER, YUC

Myriopteris jamaicensis (Maxon) Grusz \& Windham CHIS, COAH, HGO, NLE, SLP, VER

Myriopteris lendigera (Cav.) J. Sm. AGS, CHIS, CHIH, COAH, COL, CDMX, DGO, GTO, GRO, HGO, JAL, MEX, MICH, MOR, NAY, OAX, PUE, QRO, SLP, SIN, SON, TLAX, VER, ZAC

Myriopteris lindheimeri (Hook.) J. Sm. AGS, BCN, BCS, CHIH, COAH, DGO, GTO, HGO, JAL, MICH, QRO, SLP, SON, ZAC *Myriopteris longipila (Baker) Grusz \& Windham GTO, GRO, JAL, MEX, NLE, OAX, SLP

Myriopteris marsupianthes Fée MEX, HGO, PUE, TLAX, VER *Myriopteris maxoniana (Mickel) Grusz \& Windham TAMS

Myriopteris mexicana (Davenp.) Grusz \& Windham AGS, CHIH, COAH, CDMX, DGO, GTO, HGO, JAL, MEX, QRO, SLP, ZAC

*Myriopteris mickelii (T. Reeves) Grusz \& Windham CHIS, OAX, VER

Myriopteris microphylla (Sw.) Grusz \& Windham BCS, CHIS, CHIH, COAH, CDMX, DGO, GTO, GRO, HGO, JAL, MEX, MICH, MOR, NLE, OAX, PUE, QRO, QROO, SLP, SON, TAMS, TLAX, VER, YUC, ZAC

Myriopteris myriophylla (Desv.) Sm. AGS, BCN, BCS, CHIH, CDMX, DGO, GTO, GRO, HGO, JAL, MEX, MICH, MOR, NAY, NLE, OAX, PUE, QRO, SLP, SIN, SON, TAMS, TLAX, VER, ZAC

Myriopteris newberryi (D.C. Eaton) Grusz \& Windham BCN
Myriopteris notholaenoides (Desv.) Grusz \& Windham AGS, CHIS, CHIH, COAH, COL, CDMX, GTO, GRO, HGO, JAL, MEX, MICH, MOR, NLE, OAX, PUE, QRO, QROO, SLP, TAMS, VER, ZAC

Myriopteris parryi (D.C. Eaton) Grusz \& Windham BCN, SON *Myriopteris peninsularis (Maxon) Grusz \& Windham BCS, COL

Myriopteris pringlei (Maxon) Grusz \& Windham BCN, BCS, CHIH, COAH, GTO, HGO, OAX, QRO, SLP, SON, ZAC

Myriopteris rufa Fée AGS, BCS, CHIH, COAH, CDMX, DGO, HGO, NLE, PUE, QRO, SLP, SON, TAMS, VER, ZAC

Myriopteris tomentosa (Link) Fée CHIH, COAH, HGO, NLE, PUE, QRO, SLP, SON, TAMS, VER

Myriopteris viscida (Davenp.) Grusz \& Windham BCN, BCS Myriopteris windhamii Grusz AGS, CHIH, COAH, DGO, GTO, HGO, NLE, SON, ZAC

Myriopteris wootonii (Maxon) Grusz \& Windham BCN, CHIH, SON

Myriopteris wrightii (Hook.) Grusz \& Windham BCN, BCS, $\mathrm{CHIH}, \mathrm{COAH}, \mathrm{DGO}, \mathrm{SON}$

*Myriopteris yatskievychiana (Mickel) Grusz \& Windham SON Myriopteris yavapensis (Mickel) Grusz \& Windham BCN, BCS

*Notholaena affinis (Mett.) Hook. ex T. Moore GTO, GRO, HGO, MICH, QRO, TAMS

Notholaena aliena Maxon CHIH, COAH, DGO

Notholaena aschenborniana Klotzsch AGS, CHIH, COAH, GRO, HGO, JAL, MEX, MOR, NLE, OAX, PUE, QRO, SON, TAMS, ZAC

*Notholaena aurantiaca D.C. Eaton AGS, JAL, NAY

Notholaena aureolina Yatsk. \& Arbeláez GRO, HGO, JAL, MEX, MOR, OAX, ZAC

*Notholaena brachycaulis Mickel NLE

*Notholaena brachypus (Kunze) J. Sm. CHIS, DGO, HGO, JAL, QRO, SLP

*Notholaena brevistipes Mickel TAMS

*Notholaena bryopoda Maxon CHIH, COAH, NLE, TAMS

Notholaena californica D.C. Eaton BCN, BCS, SON

Notholaena candida (M. Martens \& Galeotti) Hook. AGS, BCS, CHIS, CHIH, COAH, COL, DGO, GTO, GRO, HGO, JAL, MEX, MICH, MOR, NAY, NLE, OAX, PUE, QRO, SLP, SIN, SON, TAMS, VER, ZAC

Notholaena copelandii C.C. Hall COAH, HGO, MOR, NLE, QRO, SLP, TAMS, VER

*Notholaena ferruginea Hook. CHIH, SON

Notholaena galeottii Fée BCN, BCS, CHIS, CHIH, COL, CDMX, DGO, GTO, GRO, HGO, JAL, MEX, MICH, MOR, NAY, NLE, OAX, PUE, QRO, SLP, SIN, TLAX, VER

Notholaena grayi Davenp. CHIH, COAH, JAL, NLE, SIN, SON

Notholaena greggii (Mett. ex Kuhn) Maxon CHIH, COAH, DGO, NLE

*Notholaena jaliscana Yatsk. \& Arbeláez JAL, NAY

Notholaena lemmonii D.C. Eaton BCN, BCS, CHIH, GRO, JAL, MEX, MICH, OAX, PUE, SIN, SON

*Notholaena leonina Maxon COAH, NLE

Notholaena limitanea Maxon AGS, CHIH, COAH, DGO, NLE, SON, TAMS, ZAC 
Notholaena meridionalis Mickel OAX

Notholaena neglecta Maxon CHIH, COAH, NLE

*Notholaena ochracea (Hook.) Yatsk. \& Arbeláez DGO, GRO, HGO, JAL, MEX, MICH, MOR, NAY, OAX, SIN, ZAC

*Notholaena rigida Davenp. CHIS, COAH, NLE, TAMS

*Notholaena rosei Maxon JAL, MEX, OAX

Notholaena schaffneri (E. Fourn.) Underw. ex Davenp. AGS, BCS, CHIH, COAH, CDMX, GTO, GRO, JAL, MEX, MICH, MOR, NLE, OAX, PUE, SLP, TAMS, VER, ZAC

Notholaena standleyi Maxon BCN, BCS, CHIH, COAH, DGO, GTO, HGO, NLE, OAX, PUE, QRO, SLP, SIN, SON, TAMS

Notholaena sulphurea (Cav.) J. Sm. COAH, GTO, HGO, MICH, NLE, OAX, PUE, QRO, SLP, TAMS

Pellaea andromedifolia (Kaulf.) Fée BCN, BCS

* Pellaea arsenei $\mathrm{H}$. Christ CHIH, MEX

Pellaea atropurpurea (L.) Link CHIS, CHIH, COAH, COL, HGO, JAL, NLE, OAX, PUE, SON, TAMS, VER

Pellaea cordifolia (Sessé \& Moc.) A.R. Sm. AGS, CHIS, CHIH, COAH, CDMX, DGO, GTO, GRO, HGO, JAL, MEX, MICH, MOR, NAY, NLE, OAX, PUE, QRO, SLP, SON, TLAX, ZAC Pellaea intermedia Mett. ex Kuhn CHIH, COAH, NLE, SON, TAMS, ZAC

*Pellaea notabilis Maxon NLE, TAMS

Pellaea ovata (Desv.) Weath. AGS, BCN, BCS, CHIS, CHIH, COAH, COL, CDMX, DGO, GTO, GRO, HGO, JAL, MEX, MICH, MOR, NAY, NLE, OAX, PUE, QRO, SLP, SIN, SON, TAMS, VER, ZAC

*Pellaea pringlei Davenp. COL, GRO, JAL, MEX, MICH, MOR, NAY, SIN

* Pellaea ribae A. Mendoza \& Windham SLP

Pellaea sagittata (Cav.) Link AGS, CHIS, CHIH, COAH, COL, CDMX, DGO, GTO, GRO, HGO, JAL, MEX, MICH, MOR, NLE, OAX, PUE, QRO, SLP, SON, TLAX, VER, ZAC

*Pellaea seemanii Hook. GRO, NAY

Pellaea ternifolia (Cav.) Link. AGS, BCN, BCS, CHIS, CHIH, COAH, COL, CDMX, DGO, GTO, GRO, HGO, JAL, MEX, MICH, MOR, NAY, NLE, OAX, PUE, QRO, SLP, SIN, SON, TAMS, TLAX, VER, ZAC

Pellaea truncata Goodd. BCN, BCS, SON

*Pellaea villosa (Windham) Windham \& Mickel CHIH, COAH, GTO, NLE

*Pellaea $\times$ oaxacana Mickel \& Beitel COL, JAL, NAY, OAX

Pentagramma triangularis (Kaulf.) Yatsk., Windham \& E. Wollenw. BCN, BCS, CHIH, SON

Pityrogramma calomelanos (L.) Link CHIS, COL, GRO, HGO, JAL, MEX, MICH, NAY, OAX, PUE, QRO, SLP, SIN, SON, TAB, VER, ZAC

Pityrogramma dealbata (C. Presl) Domin CHIS, COL, HGO, JAL, MICH, NAY, OAX, QRO, SLP, VER

Pityrogramma ebenea (L.) Proctor CHIS, COL, GTO, GRO, HGO, JAL, MEX, MICH, MOR, NAY, OAX, PUE, QRO, SLP, SIN, VER

Pityrogramma trifoliata (L.) R.M. Tryon BCS, CHIS, COL, HGO, MEX, MICH, OAX, PUE, VER

Polytaenium cajenense (Desv.) Benedict CHIS, OAX, VER

Polytaenium chlorosporum (Mickel \& Beitel) E.H. Crane CHIS, OAX
Polytaenium feei (W. Schaffn. ex Fée) Maxon CHIS, OAX, TAMS, VER

Polytaenium lineatum (Sw.) J. Sm. CHIS, OAX, VER

Pteris altissima Poir. CHIS, HGO, OAX, PUE, QRO, SLP, TAB, VER

Pteris biaurita L. CHIS, JAL, MEX, NAY, OAX

*Pteris chiapensis A.R. Sm. CHIS, GRO, OAX

Pteris erosa Mickel \& Beitel COL, GRO, JAL, MEX, NAY, OAX, VER

Pteris grandifolia L. CAM, CHIS, HGO, MICH, OAX, PUE, QRO, QROO, SLP, TAB, VER

Pteris longifolia L. CAM, CHIS, COAH, COL, GRO, HGO, JAL, MEX, MICH, MOR, NAY, NLE, OAX, PUE, QRO, QROO, SLP, SIN, TAB, VER

Pteris muricata Hook. CHIS, OAX, VER

Pteris muricatopedata Arbeláez CHIS, OAX

Pteris muricella Fée CHIS, GRO, HGO, OAX, PUE, VER

Pteris orizabae M. Martens \& Galeotti CHIS, COL, GRO, HGO, JAL, MEX, MICH, MOR, OAX, PUE, QRO, SLP, TAMS, VER Pteris paucinervata Fée CHIS, GRO, OAX, VER

Pteris podophylla Sw. CHIS, GRO, JAL, OAX, PUE, VER

*Pteris pulchra Schltdl. \& Cham. CHIS, GRO, HGO, JAL, OAX, QRO, SLP, VER

Pteris pungens Willd. CHIS, OAX, VER

*Radiovittaria stipitata (Kunze) E.H. Crane CHIS

Scoliosorus ensiformis (Hook.) T. Moore CHIS, GRO, MEX, OAX, PUE, SLP, VER

Vittaria bradeorum Rosenst. CHIS, COL, GRO, OAX, VER Vittaria flavicosta Mickel \& Beitel CHIS, JAL, MEX, MICH, OAX

Vittaria graminifolia Kaulf. CHIS, COL, GRO, HGO, JAL, MEX, MICH, MOR, NAY, OAX, PUE, QRO, SLP, SIN, TAB, TAMS, VER

Vittaria lineata (L.) Sm. CAM, CHIS, HGO, OAX, QROO, TAB, VER

*x Hemionanthes gryphus (Mickel) Mickel COL, JAL, MEX, NAY

\section{Family Saccolomataceae}

Saccoloma elegans Kaulf. OAX, VER

Saccoloma inaequale (Kunze) Mett. CHIS, HGO, OAX, PUE, VER

\section{Family Salviniaceae}

Azolla filiculoides Lam. BCN, CHIH, COL, CDMX, GTO, GRO, HGO, JAL, MEX, MICH, MOR, OAX, PUE, QRO, SIN, TAB, TLAX, VER

Azolla microphylla Kaulf. AGS, BCN, BCS, CHIS, CHIH, COL, CDMX, GTO, GRO, HGO, JAL, MEX, MICH, MOR, NAY, OAX, PUE, QRO, SLP, SIN, SON, TAB, TAMS, TLAX, VER, ZAC

Salvinia auriculata Aubl. CAM, CHIS, GRO, MICH, OAX, QROO, TAB, TAMS, VER, YUC

Salvinia minima Baker CAM, CHIS, HGO, OAX, QRO, QROO, SLP, TAB, TAMS, VER, YUC

\section{Family Schizaeaceae}

Actinostachys pennula (Sw.) Hook. CHIS, QROO 
Schizaea elegans (Vahl) Sw. CHIS, OAX, VER

Schizaea poeppigiana J.W. Sturm CHIS

\section{Family Selaginellaceae}

Selaginella acutifolia (Stolze) Valdespino GTO, OAX, QRO

Selaginella apoda (L.) Spring CHIS, HGO, JAL, MEX, OAX, PUE, TAMS, VER

Selaginella arizonica Maxon BCN, BCS, CHIH, NLE, SON

* Selaginella arsenei Weath. CHIH, GTO, GRO, HGO, QRO, SLP, SON, VER

*Selaginella arsiclada Valdespino QRO, VER

Selaginella asprella Maxon BCN

* Selaginella barnebyana Valdespino CHIS

Selaginella basipilosa Valdespino OAX

Selaginella bernoullii Hieron. CHIS, OAX

Selaginella bigelovii Underw. BCN, BCS

*Selaginella breedlovei Valdespino CHIS

* Selaginella carnerosana Reeves COAH, NLE

Selaginella cinerascens Maxon BCN

Selaginella convoluta (Arn.) Spring CAM, YUC

*Selaginella corrugis Mickel \& Beitel OAX

* Selaginella cuneata Mickel \& Beitel OAX

*Selaginella chiapensis A.R. Sm. CHIS, OAX

Selaginella delicatissima Linden ex A. Braun AGS, CHIS, CHIH, COAH, COL, GTO, GRO, HGO, JAL, MEX, MICH, MOR, NAY, NLE, OAX, PUE, QRO, SLP, SIN, SON, TAMS, VER, ZAC

Selaginella eremophila Maxon BCN, COAH, NLE, SON, ZAC Selaginella eurynota A. Braun CHIS, COL, GRO, JAL, NAY, OAX

* Selaginella extensa Underw. HGO, JAL, MOR, OAX, PUE, QRO, SLP, TAMS, VER, ZAC

*Selaginella finitima Mickel \& Beitel CHIS, OAX, TAB, VER Selaginella flagellata Spring CHIS, COL, GRO, OAX, VER Selaginella flexuosa Spring CHIS, GRO, HGO, MICH, OAX, PUE, VER

Selaginella guatemalensis Baker CHIS, OAX

* Selaginella gypsophila A.R. Sm. \& Reeves NLE, TAMS

Selaginella harrisii Underw. \& Hieron. CAM, CHIS, GRO, HGO, MEX, MICH, MOR, OAX, QRO, SLP, TAMS, VER, YUC

*Selaginella hirtifolia Valdespino CHIS, OAX, TAB

Selaginella hoffmannii Hieron. CHIS, COL, GRO, HGO, JAL, MEX, MICH, MOR, NAY, OAX, PUE, QRO, SIN, VER

Selaginella huehuetenangensis Hieron. CHIS

Selaginella illecebrosa Alston CHIS, GRO, MOR, OAX, TAB, VER

*Selaginella landii Greenm. \& N. Pfeiff. JAL, MEX, MOR, NAY, OAX, PUE, ZAC

Selaginella lepidophylla (Hook. \& Grev.) Spring AGS, BCN, BCS, CHIS, CHIH, COAH, COL, CDMX, DGO, GTO, GRO, HGO, JAL, MEX, MICH, MOR, NAY, NLE, OAX, PUE, QRO, SLP, SIN, SON, TAMS, TLAX, VER, ZAC

*Selaginella lindenii Spring CHIS, OAX, TAB

* Selaginella lineolata Mickel \& Beitel COL, GRO, HGO, JAL, MEX, NAY, OAX, QRO, SLP, SIN, VER

* Selaginella macranthera Underw. CHIH, SON
Selaginella marginata (Willd.) Spring DGO, GRO, JAL, NAY, SIN, VER

Selaginella martensii Spring CHIS, HGO, MEX, OAX, PUE, QRO, SLP, VER

Selaginella mickelii Valdespino CHIS, OAX, TAB, VER

Selaginella minima Spring NAY, OAX

* Selaginella mixteca Mickel \& Beitel OAX, PUE

Selaginella moritziana Spring ex Klotzsch CHIS, OAX

*Selaginella mosorongensis Hieron. OAX, VER

Selaginella mutica D.C. Eaton ex Underw. CHIH

* Selaginella notohybrida Valdespino GRO, HGO, OAX

*Selaginella novoleonensis Hieron. CHIH, COAH, NLE, OAX, SON

Selaginella oaxacana Spring CHIS, OAX, VER

Selaginella pallescens (C. Presl) Spring AGS, BCN, BCS, CHIS, CHIH, COAH, COL, CDMX, DGO, GTO, GRO, HGO, JAL, MEX, MICH, MOR, NAY, NLE, OAX, PUE, QRO, SLP, SIN, SON, TAMS, TLAX, VER, YUC, ZAC

*Selaginella parishii Underw. COAH, NLE, PUE, ZAC

Selaginella peruviana (Milde) Hieron. CHIH, COAH, CDMX, DGO, GTO, HGO, JAL, MEX, MICH, OAX, NLE, SON, VER

Selaginella pilifera A. Braun CHIH, COAH, NLE, QRO, SLP, SON, TAMS

Selaginella polyptera Valdespino CHIS, HGO, OAX, SLP, VER

*Selaginella popayanensis Hieron. CHIS, GRO, OAX

Selaginella porphyrospora A. Braun CHIS, COL, GTO, GRO, HGO, JAL, MEX, MICH, MOR, NAY, OAX, PUE, SIN, SON, VER

Selaginella prolifera Valdespino CHIS, GRO, OAX

Selaginella pulcherrima Liebm. ex E. Fourn. CHIS, GRO, HGO, OAX, VER

Selaginella reflexa Underw. CHIS, GRO, HGO, JAL, NLE, QRO, SLP, TAMS, VER

*Selaginella ribae Valdespino DGO, GRO, NLE, TAMS

Selaginella rupincola Underw. AGS, CHIH, COAH, CDMX, DGO, GTO, GRO, HGO, JAL, MEX, MOR, OAX, PUE, QRO, SLP, SIN, SON, TAMS, ZAC

*Selaginella rzedowskii Lorea-Hern. GRO, MOR

Selaginella sartorii Hieron. CHIS, CHIH, DGO, GTO, GRO, JAL, MEX, MOR, NAY, NLE, OAX, PUE, SLP, SON, TAMS, VER, ZAC

* Selaginella schaffneri Hieron. GTO, JAL, MEX, MICH, MOR, NAY, SLP, ZAC

*Selaginella schiedeana A. Braun CHIS, GRO, HGO, MEX, MICH, OAX, PUE, QRO, SLP, TAMS, VER

Selaginella schizobasis Baker CHIS, OAX, TAB, VER

Selaginella sellowii Hieron. CDMX, DGO, GTO, HGO, JAL, MEX, MOR, OAX, PUE, QRO, SLP, SIN, SON, TAMS, VER, ZAC

Selaginella sertata Spring CAM, CHIS, COL, GRO, HGO, JAL, MEX, MICH, NAY, OAX, SIN, VER

Selaginella silvestris Aspl. CHIS, COL, HGO, JAL, MEX, OAX, PUE, VER

Selaginella simplex Baker GRO, NAY, OAX

Selaginella stellata Spring CHIS, COL, GRO, HGO, JAL, OAX, PUE, TAB, VER 
Selaginella stenophylla A. Braun HGO, NLE, PUE, QRO, SLP, TAMS, VER

Selaginella steyermarkii Alston CHIS

*Selaginella subrugosa Mickel \& Beitel OAX

* Selaginella tarda Mickel \& Beitel COL, DGO, GRO, JAL, MEX, MICH, NAY, OAX, SIN

Selaginella tenella (P. Beauv.) Spring CHIS, COL, DGO, GRO, HGO, JAL, MEX, NAY, OAX, SIN, VER

Selaginella tuberosa McAlpin \& Lellinger OAX

Selaginella umbrosa Lem. ex Hieron. CHIS, OAX, YUC

Selaginella underwoodii Hieron. CHIH, SON

Selaginella viridissima Weath. $\mathrm{CHIH}, \mathrm{COAH}$

Selaginella wrightii Hieron. CHIH, COAH, GTO, GRO, HGO,

JAL, MEX, NLE, OAX, PUE, QRO, SLP, SON, TAMS, TLAX,

VER, ZAC

\section{Family Tectariaceae}

Tectaria fimbriata (Willd.) Proctor \& Lourteig YUC

Tectaria heracleifolia (Willd.) Underw. CAM, CHIS, COL, GTO, GRO, HGO, JAL, MEX, MICH, MOR, NAY, NLE, OAX, PUE, QRO, QROO, SLP, TAB, TAMS, VER, YUC

Tectaria incisa Cav. CHIS, GRO, OAX, TAB, VER

Tectaria mexicana (Fée) C.V. Morton CHIS, COL, GRO, JAL,

MICH, NAY, OAX, PUE, SIN, VER

Tectaria panamensis (Hook.) R.M. Tryon \& A. F. Tryon CHIS, OAX, VER

Tectaria transiens (C.V. Morton) A.R. Sm. CHIS, HGO, OAX, QRO, SLP, VER

Tectaria trichodes (C.V. Morton) A.R. Sm. TAB

\section{Family Thelypteridaceae}

*Thelypteris albicaulis (Fée) A.R. Sm. CHIS, COL, GRO, JAL, MEX, MICH, MOR, OAX, PUE, VER

Thelypteris angustifolia (Willd.) Proctor CHIS, GRO, TAB, VER

Thelypteris atrovirens (C. Chr.) C.F. Reed CHIS, HGO, OAX, VER

Thelypteris augescens (Link) Munz \& I.M. Johnst. QROO

Thelypteris balbisii (Spreng.) Ching CHIS, OAX, PUE, TAB, VER

Thelypteris biolleyi (H. Christ) Proctor CHIS, OAX

Thelypteris blanda (Fée) C.F. Reed CHIS, HGO, OAX, QRO,

SLP, VER

Thelypteris blepharis A.R. Sm. CHIS

Thelypteris caucaensis (Hieron.) Alston CHIS

Thelypteris cinerea (Sodiro) A.R. Sm. CHIS, OAX

Thelypteris concinna (Willd.) Ching CHIS, COAH, HGO, JAL, MOR, NAY, NLE, OAX, PUE, QRO, SLP, VER

Thelypteris cretacea A.R. Sm. CHIS, OAX, VER

Thelypteris cheilanthoides (Kunze) Proctor CHIS, COL, GTO, GRO, JAL, MEX, MICH, OAX, PUE, VER

Thelypteris deflexa (C. Presl) R.M. Tryon CHIS, GRO, OAX

Thelypteris falcata (Liebm.) R.M. Tryon CHIS, OAX, PUE,

TAB, VER

Thelypteris ghiesbreghtii (Hook.) C.V. Morton CHIS, OAX, TAB

Thelypteris glandulosa (Desv.) Proctor CHIS

Thelypteris grandis A.R. Sm. GRO, JAL, OAX
Thelypteris guadalupensis (Wikstr.) Proctor QROO

Thelypteris hatchii A.R. Sm. CHIS, OAX, VER

Thelypteris hispidula (Decne.) C.F. Reed CHIS, COL, GRO, HGO, JAL, MEX, MICH, NAY, OAX, SLP, SIN, SON, TAB, TAMS, VER, ZAC

Thelypteris hondurensis L.D. Gómez TAB, VER

Thelypteris imbricata (Liebm.) C.F. Reed CHIS, COL, GRO, JAL, OAX, TAB, VER

Thelypteris kunthii (Desv.) C.V. Morton CAM, CHIS, GRO, HGO, MOR, OAX, PUE, QROO, SLP, TAMS, VER, YUC

*Thelypteris lanosa (C. Chr.) A.R. Sm. HGO, VER

Thelypteris leptocladia (Fée) Proctor QROO

Thelypteris linkiana (C. Presl) R.M. Tryon CHIS, MOR, OAX, PUE, QRO, SLP, VER

*Thelypteris loreae A.R. Sm. GRO, OAX

Thelypteris martinezii A.R. Sm. CAM, CHIS, QROO

Thelypteris melanochlaena (C. Chr.) C.F. Reed CHIS, GRO, OAX, PUE, VER

Thelypteris meniscioides (Liebm.) C.F. Reed CHIS, OAX, TAB, VER

*Thelypteris minor (C. Chr.) A.R. Sm. CHIS, GRO

*Thelypteris mortonii A.R. Sm. MEX, MICH, MOR

*Thelypteris muenchii A.R. Sm. CHIS

*Thelypteris nicaraguensis (E. Fourn.) C.V. Morton CHIS, QROO

Thelypteris nubigena A.R. Sm. CHIS, OAX

Thelypteris oaxacana A.R. Sm. CHIS, OAX

Thelypteris obliterata (Sw.) Proctor CHIS, OAX, PUE, VER

Thelypteris oligocarpa (Humb. \& Bonp. ex Willd.) Ching CHIS, CHIH, COL, GRO, HGO, JAL, MEX, MICH, NAY, OAX, PUE, QRO, SLP, TAMS, VER

Thelypteris ovata R.P. St. John CAM, CHIS, CHIH, COAH, GRO, HGO, MEX, MOR, NAY, NLE, OAX, PUE, QRO, QROO, SLP, TAMS, VER

Thelypteris palustris Schott CDMX, MICH

Thelypteris patens ( $\mathrm{Sw}$.) Small CAM, CHIS, GRO, HGO, MEX, MICH, OAX, PUE, QRO, SLP, TAB, VER

Thelypteris paucipinnata (Donn. Sm.) C.F. Reed CHIS, OAX, TAB, VER

Thelypteris pilosa (M. Martens \& Galeotti) Crawford CHIS, CHIH, COL, CDMX, DGO, GTO, GRO, HGO, JAL, MEX, MICH, MOR, NAY, OAX, PUE, QRO, SLP, SON, TAMS, TLAX, VER, ZAC

Thelypteris pilosohispida (Hook.) Alston CHIS, GRO, OAX, PUE, VER

Thelypteris pilosula (Klotzsch \& H. Karst. ex Mett.) R.M. Tryon CHIS, COL, GTO, GRO, HGO, JAL, MEX, MICH, MOR, OAX, PUE, TLAX, VER

Thelypteris poiteana (Bory) Proctor CAM, CHIS, OAX

Thelypteris praetermissa (Maxon) A.R. Sm. CHIS

Thelypteris puberula (Baker) C.V. Morton AGS, BCN, BCS, CHIS, CHIH, COAH, COL, DGO, GTO, GRO, HGO, JAL, MEX, MICH, MOR, NAY, NLE, OAX, PUE, QRO, QROO, SLP, SIN, SON, TAMS, VER, ZAC

*Thelypteris quadrangularis (Fée) Schelpe NAY, TAMS

Thelypteris reptans (J.F. Gmel.) C.V. Morton CAM, CHIS, OAX, VER, YUC 
Thelypteris resiliens (Maxon) A.R. Sm. CHIS, OAX, TAB, VER Thelypteris resinifera (Desv.) Proctor AGS, CAM, CHIS, CHIH, COL, GRO, JAL, MEX, MICH, MOR, NAY, OAX, PUE, QROO, SIN, TAB, TAMS, VER

Thelypteris reticulata (L.) Proctor CAM, CHIS, OAX, QROO

*Thelypteris rhachiflexuosa Riba CHIS, VER

Thelypteris rudis (Kunze) Proctor BCN, BCS, CHIS, CHIH, COL, DGO, GRO, HGO, JAL, MEX, MICH, MOR, NAY, OAX, PUE, SIN, SON, VER, ZAC

Thelypteris sancta (L.) Ching CHIS

Thelypteris scalaris (H. Christ) Alston CHIS, OAX, PUE, VER

*Thelypteris schaffneri (Fée) C.F. Reed QRO, SLP, TAMS, VER

Thelypteris serrata (Cav.) Alston CHIS, NAY, OAX, PUE, TAB, VER

Thelypteris standleyi (Maxon \& C.V. Morton) R.M. Tryon CHIS, JAL

*Thelypteris stolzeana A.R. Sm. CHIS

Thelypteris struthiopteroides (C. Chr.) C.F. Reed CHIS, COL, OAX

*Thelypteris tablana (H. Christ) A.R. Sm. CHIS

Thelypteris tetragona (Sw.) Small CAM, CHIS, COL, GRO, HGO, NAY, OAX, PUE, QRO, QROO, SLP, SIN, TAB, TAMS, VER, YUC

Thelypteris thompsonii (Jenman) Proctor CHIS, OAX

Thelypteris toganetra A.R. Sm. CHIS, OAX, SLP, VER

Thelypteris tuerckheimii (Donn. Sm.) C.F. Reed CHIS, OAX

*Thelypteris tuxtlensis T. Kromer, Acebey \& A.R. Sm. VER

*Thelypteris venturae A.R. Sm. VER

\section{Family Woodsiaceae}

*Woodsia canescens (Kunze) Mett. CDMX, MEX, OAX, PUE, SLP

*Woodsia cochisensis Windham CHIH, COAH, SIN, SON

*Woodsia cystopteroides Windham \& Mickel CHIH, DGO, SIN, SON

Woodsia mexicana Fée AGS, BCN, BCS, CHIH, COAH, CDMX, DGO, GTO, HGO, JAL, MEX, NLE, QRO, SLP, SIN, SON, VER, ZAC

Woodsia mollis (Kaulf.) J. Sm. AGS, BCN, BCS, CHIS, CHIH, COL, CDMX, DGO, GTO, GRO, HGO, JAL, MEX, MICH, MOR, NAY, NLE, OAX, PUE, QRO, SLP, SIN, SON, TAMS, TLAX, VER, ZAC

Woodsia neomexicana Windham COAH, NLE, TAMS, ZAC *Woodsia phillipsii Windham CHIH, COAH, DGO, JAL, SON Woodsia plummerae Lemmon BCN, BCS, CHIH, JAL, SON

\section{Gymnosperms}

\section{Family Cupressaceae}

Calocedrus decurrens (Torr.) Florin BCN, MEX

Cupressus arizonica Greene AGS, BCN, CHIH, COAH, COL, DGO, HGO, JAL, MEX, MICH, NAY, NLE, SLP, SIN, SON, TAMS, VER, ZAC

Cupressus guadalupensis $\mathrm{S}$. Watson BCN

Cupressus lusitanica Mill. AGS, CAM, CHIS, CHIH, COAH, COL, CDMX, DGO, GTO, GRO, HGO, JAL, MEX, MICH, MOR, NAY, NLE, OAX, PUE, QRO, QROO, SLP, SIN, SON, TAMS, TLAX, VER, YUC, ZAC
Cupressus montana Wiggins BCN

Cupressus stephensonii C.B. Wolf BCN

*Juniperus angosturana R.P. Adams COAH, GTO, HGO, JAL, NLE, QRO, SLP, TAMS, VER, ZAC

Juniperus ashei J. Buchholz COAH, NLE

*Juniperus blancoi Martínez DGO, JAL, MEX, MICH, SIN, SON, TLAX, ZAC

Juniperus californica Carrière BCN

Juniperus coahuilensis (Martínez) Gaussen ex R.P. Adams CHIH, COAH, DGO, JAL, NLE, SON, ZAC

Juniperus comitana Martínez CHIS

Juniperus compacta (Mart.) R.P. Adams CHIS, CHIH, COAH, COL, CDMX, JAL, MEX, NLE, TLAX, VER

Juniperus deppeana Steud. AGS, CHIS, CHIH, COAH, CDMX, DGO, GRO, HGO, JAL, MEX, MICH, MOR, NLE, OAX, PUE, QRO, SLP, SIN, SON, TAMS, TLAX, VER, ZAC

*Juniperus durangensis Martínez AGS, CHIH, DGO, JAL, SIN, SON, ZAC

Juniperus erythrocarpa Cory CHIH, COAH, DGO, GTO, JAL, NLE, SLP, SIN, SON, TAMS, ZAC

Juniperus flaccida Schltdl. AGS, CHIH, COAH, COL, CDMX, DGO, GTO, GRO, HGO, JAL, MEX, MICH, MOR, NAY, NLE, OAX, PUE, QRO, SLP, SIN, SON, TAMS, TLAX, VER, ZAC *Juniperus gamboana Martínez CHIS

*Juniperus jaliscana Martínez DGO, JAL, NAY

*Juniperus martinezii Pérez de la Rosa AGS, GTO, JAL, PUE, SLP, VER, ZAC

Juniperus monosperma (Engelm.) Sarg. CHIH, COAH, DGO, GTO, HGO, NLE, QRO, SLP, SON, TAMS, ZAC

*Juniperus monticola Martínez AGS, CHIH, COAH, COL, CDMX, DGO, GRO, HGO, JAL, MEX, MICH, MOR, NLE, PUE, QRO, SLP, TAMS, TLAX, VER, ZAC

Juniperus pinchotii Sudw. CHIH, COAH, DGO, NLE

*Juniperus poblana (Martínez) R.P. Adams DGO, JAL, OAX, PUE

Juniperus saltillensis M.T. Hall. CHIH, COAH, DGO, NLE, SLP, ZAC

Juniperus scopulorum Sarg. CHIH, COAH, SON

Juniperus standleyi Steyerm. CHIS

*Juniperus zanonii R.P. Adams NLE

Taxodium mucronatum Ten. AGS, BCS, CHIS, CHIH, COAH, COL, CDMX, DGO, GTO, GRO, HGO, JAL, MEX, MICH, MOR, NAY, NLE, OAX, PUE, QRO, SLP, SIN, SON, TAB, TAMS, TLAX, VER, ZAC

\section{Family Ephedraceae}

Ephedra antisyphilitica C.A. Mey. AGS, BCN, CHIH, COAH, DGO, NLE, SLP, TAMS, ZAC

Ephedra aspera Engelm. ex S. Watson BCN, BCS, CHIH, COAH, DGO, HGO, NLE, QRO, SLP, SON, TAMS, VER, ZAC

Ephedra californica $\mathrm{S}$. Watson BCN, SON

* Ephedra compacta Rose AGS, COAH, DGO, GTO, HGO, NLE, OAX, PUE, QRO, SLP, TAMS, VER, ZAC

Ephedra nevadensis S. Watson BCN, DGO, SON

Ephedra pedunculata Engelm. AGS, CHIH, COAH, DGO, HGO, NLE, QRO, SLP, TAMS, VER, ZAC 
Ephedra trifurca Torr. BCN, CHIH, COAH, DGO, SON

\section{Family Pinaceae}

*Abies coahuilensis I.M. Johnst. COAH

Abies concolor (Gordon \& Glend.) Lindl. ex Hildebr. BCN, $\mathrm{CHIH}, \mathrm{SON}$

*Abies durangensis Martínez CHIH, COAH, DGO, JAL, NLE, SIN, SON, TAMS, ZAC

Abies guatemalensis Rehder CAM, CHIS, COL, GTO, GRO, HGO, JAL, MEX, MICH, MOR, NLE, OAX, PUE, QRO, SLP, TAMS, VER, YUC

Abies hickelii Flous \& Gaussen CHIS, GRO, OAX, VER

*Abies hidalgensis Debreczy, I. Rácz \& Guízar HGO, VER

Abies religiosa (Kunth) Schltdl. \& Cham. AGS, CHIS, COL, CDMX, DGO, GTO, GRO, HGO, JAL, MEX, MICH, MOR, NLE, OAX, PUE, QRO, SLP, SIN, TAMS, TLAX, VER, ZAC *Abies vejarii Martínez COAH, HGO, NLE, TAMS, VER

* Picea chihuahuana Martínez CHIH, COAH, DGO, JAL, NLE, SIN, SON, TAMS, ZAC

Picea engelmannii J.P. Perry CHIH, COAH, NLE, SON, TAMS Pinus arizonica Engelm. CHIH, COAH, DGO, NAY, NLE, SLP, SIN, SON, TAMS, ZAC

Pinus attenuata Lemmon BCN

Pinus ayacahuite C. Ehrenb. ex Schltdl. AGS, CHIS, CHIH, COAH, CDMX, DGO, GTO, GRO, HGO, JAL, MEX, MICH, MOR, NLE, OAX, PUE, QRO, SLP, SIN, SON, TAMS, TLAX, VER, ZAC

Pinus caribaea Morelet CAM, QROO

Pinus cembroides Zucc. AGS, BCN, BCS, CHIH, COAH, CDMX, DGO, GTO, HGO, JAL, MEX, MICH, NAY, NLE, PUE, QRO, SLP, SIN, SON, TAMS, TLAX, VER, ZAC

Pinus contorta Douglas ex Loudon BCN, CAM, YUC

Pinus coulteri Lamb. ex D. Don BCN

* Pinus culminicola Andresen \& Beaman COAH, NLE, SLP

Pinus chiapensis (Martínez) Andresen CHIS, GRO, OAX, PUE, VER

Pinus devoniana Lindl. AGS, CHIS, COL, CDMX, DGO, GTO, GRO, HGO, JAL, MEX, MICH, MOR, NAY, NLE, OAX, PUE, QRO, SLP, SIN, TLAX, VER, ZAC

* Pinus douglasiana Martínez CHIS, CHIH, COL, DGO, GTO, GRO, JAL, MEX, MICH, MOR, NAY, OAX, QRO, SLP, SIN, SON, TLAX, ZAC

*Pinus durangensis Martínez AGS, CHIH, COL, DGO, GTO, JAL, MEX, MICH, NAY, QRO, SLP, SIN, SON, ZAC

Pinus edulis Engelm. CHIH

Pinus engelmannii Carrière AGS, CHIH, COAH, DGO, JAL, NLE, SIN, SON, TAMS, ZAC

Pinus flexilis E. James CHIH, COAH, DGO, NLE, SLP, TAMS

* Pinus georginae Pérez de la Rosa JAL

*Pinus greggii Engelm. ex Parl. COAH, HGO, NLE, PUE, QRO, SLP, TAMS, VER

Pinus hartwegii Lindl. AGS, CHIS, CHIH, COAH, COL, CDMX, DGO, GTO, GRO, HGO, JAL, MEX, MICH, MOR, NAY, NLE, OAX, PUE, QRO, SLP, TAMS, TLAX, VER, ZAC * Pinus herrerae Martínez CHIH, COL, DGO, GRO, JAL, MEX, MICH, OAX, SIN, SON

*Pinus jaliscana Pérez de la Rosa COL, JAL, NAY

\section{Pinus jeffreyi Balf. BCN}

Pinus lambertiana Douglas BCN

*Pinus lawsonii Roezl ex Gordon CDMX, GTO, GRO, JAL, MEX, MICH, MOR, NAY, OAX, PUE, QRO, VER

Pinus leiophylla Schiede ex Schltdl. \& Cham. AGS, CHIS, CHIH, COL, CDMX, DGO, GTO, GRO, HGO, JAL, MEX, MICH, MOR, NAY, OAX, PUE, QRO, SLP, SIN, SON, TLAX, VER, ZAC

*Pinus lumholtzii B.L. Rob. \& Fernald AGS, CHIH, COL, DGO, GTO, JAL, MICH, NAY, QRO, SIN, SON, ZAC

*Pinus luzmariae Pérez de la Rosa DGO, JAL, NAY, SIN, ZAC

*Pinus maximartinezii Rzed. DGO, JAL, ZAC

Pinus maximinoi H.E. Moore CHIS, CHIH, COL, DGO, GRO, HGO, JAL, MEX, MICH, MOR, NAY, OAX, PUE, SIN, SON, TLAX, VER

Pinus monophylla Torr. \& Frém. BCN

Pinus montezumae Lamb. CHIS, CHIH, COAH, COL, CDMX, DGO, GTO, GRO, HGO, JAL, MEX, MICH, MOR, NAY, NLE, OAX, PUE, QRO, SLP, SIN, SON, TAB, TAMS, TLAX, VER, ZAC

Pinus muricata D. Don BCN

*Pinus nelsonii Shaw COAH, NLE, QRO, SLP, TAMS, ZAC

Pinus oocarpa Schiede ex Schltdl. AGS, CHIS, CHIH, COL, CDMX, DGO, GTO, GRO, HGO, JAL, MEX, MICH, MOR, NAY, NLE, OAX, PUE, QRO, SLP, SIN, SON, TAMS, TLAX, VER, ZAC

Pinus patula Schiede ex Schltdl. \& Cham. CHIS, COAH, COL, CDMX, GRO, HGO, JAL, MEX, MICH, MOR, NAY, NLE, OAX, PUE, QRO, SLP, SIN, TAMS, TLAX, VER

*Pinus pinceana Gordon \& Glend. COAH, DGO, HGO, NLE, QRO, SLP, TAMS, VER, ZAC

Pinus ponderosa $\mathrm{P}$. Lawson \& C. Lawson CHIH, COAH, DGO, SON, ZAC

*Pinus praetermissa Styles \& McVaugh COL, DGO, JAL, MICH, NAY, SIN, ZAC

*Pinus pringlei Shaw DGO, GRO, HGO, JAL, MEX, MICH, MOR, NAY, OAX, PUE, QRO, TLAX

Pinus pseudostrobus Lindl. CHIS, CHIH, COAH, COL, CDMX, DGO, GTO, GRO, HGO, JAL, MEX, MICH, MOR, NAY, NLE, OAX, PUE, QRO, SLP, SIN, SON, TAMS, TLAX, VER

Pinus quadrifolia Parl. ex Sudw. BCN

Pinus radiata D. Don BCN, BCS

Pinus remota (Little) D.K. Bailey \& Hawksw. CHIH, COAH, DGO, NLE, SON

*Pinus rzedowskii Madrigal \& M. Caball. MICH

Pinus strobiformis Engelm. CHIH, COAH, DGO, GTO, JAL, NLE, SLP, SIN, SON, TAMS, ZAC

Pinus tecunumanii F. Schwerdtf. ex Eguiluz \& J.P.Perry CHIS, OAX

Pinus teocote Schiede ex Schltdl. \& Cham. AGS, CHIS, CHIH, COAH, CDMX, DGO, GTO, GRO, HGO, JAL, MEX, MICH, MOR, NAY, NLE, OAX, PUE, QRO, SLP, SIN, SON, TAMS, TLAX, VER, ZAC

* Pinus yecorensis Debreczy \& I. Rácz. CHIH, SON

Pseudotsuga macrocarpa (Vasey) Mayr BCN 
Pseudotsuga menziesii (Mirb.) Franco CHIH, COAH, DGO, GRO, HGO, MEX, NLE, OAX, PUE, QRO, SLP, SIN, SON, TAMS, TLAX, VER, ZAC

\section{Family Podocarpaceae}

Podocarpus guatemalensis Standl. CHIS, OAX, TAB, VER Podocarpus matudae Lundell AGS, CHIS, COL, GRO, HGO, JAL, MICH, NAY, OAX, PUE, QRO, SLP, TAMS, VER Podocarpus oleifolius D. Don ex Lamb. CHIS, OAX, VER

\section{Family Taxaceae}

Taxus globosa Schltdl. CHIS, COAH, HGO, NLE, OAX, PUE, QRO, SLP, TAMS, VER

\section{Family Zamiaceae}

*Ceratozamia alvarezii Pérez-Farrera, Vovides \& Iglesias CHIS

*Ceratozamia becerrae Pérez-Farrera, Vovides \& Schutzman CHIS, TAB

*Ceratozamia chimalapensis Pérez-Farrera \& Vovides OAX

*Ceratozamia decumbens Vovides, S. Avendaño, Pérez-Farrera \& González-Astorga VER

*Ceratozamia euryphyllidia Vázq. Torres, Sabato \& D.W. Stev. OAX, VER

*Ceratozamia fuscoviridis S. Moore HGO, VER

*Ceratozamia hildae G.P. Landry \& M.C. Wilson QRO, SLP, VER

*Ceratozamia kuesteriana Regel SLP, TAMS, VER

*Ceratozamia latifolia Miq. HGO, QRO, SLP, TAMS, VER

Ceratozamia matudae Lundell CHIS, OAX

Ceratozamia mexicana Brongn. CHIS, HGO, OAX, PUE, QRO, SLP, TAB, VER

*Ceratozamia microstrobila Vovides \& J.D. Rees QRO, SLP, TAMS, VER

*Ceratozamia miqueliana $\mathrm{H}$. Wendl. VER

*Ceratozamia mirandae Pérez-Farrera, Vovides \& Iglesias CHIS

*Ceratozamia mixeorum Chemnick, T.J. Greg. \& Salas-Mor. OAX

*Ceratozamia morettii Vázq. Torres \& Vovides VER

*Ceratozamia norstogii D.W. Stev. CHIS, OAX

*Ceratozamia sabatoi Vovides, Vázq. Torres, Schutzman \& Iglesias HGO, QRO, SLP

*Ceratozamia vovidesii Pérez-Farrera \& Iglesias CHIS

*Ceratozamia whitelockiana Chemnick \& T.J. Greg. OAX

*Ceratozamia zaragozae Medellín-Leal QRO, SLP, TAMS

*Ceratozamia zoquorum Pérez-Farrera, Vovides \& Iglesias CHIS

*Dioon angustifolium Miq. NLE, TAMS

*Dioon argenteum T.J. Greg., Chemnick, Salas-Mor. \& Vovides OAX

*Dioon califanoi De Luca \& Sabato OAX, PUE

*Dioon caputoi De Luca, Sabato \& Vázq. Torres OAX, PUE

*Dioon edule Lindl. CHIS, DGO, GTO, GRO, HGO, JAL, MEX, NAY, NLE, OAX, QRO, SLP, SIN, TAMS, VER

*Dioon holmgrenii De Luca, Sabato \& Vázq. Torres OAX

*Dioon merolae De Luca, Sabato \& Vázq. Torres CHIS, OAX

*Dioon planifolium Salas-Mor., Chemnick \& T.J.Greg. OAX

*Dioon purpusii Rose OAX, PUE
*Dioon rzedowskii De Luca, A. Moretti, Sabato \& Vázq. Torres OAX

*Dioon sonorense (De Luca, Sabato \& Vázq. Torres) Chemnick, T.J. Greg. \& Salas Mor. CHIH, JAL, NAY, SIN, SON

*Dioon spinulosum Dyer CAM, CHIS, OAX, QROO, TAB, VER, YUC

*Dioon tomasellii De Luca, Sabato \& Vázq. Torres DGO, GRO, JAL, MICH, NAY, SIN

*Zamia cremnophila Vovides, Schutzman \& Dehgan CHIS, TAB

*Zamia fischeri Miq. HGO, NLE, QRO, SLP, TAMS, VER

*Zamia furfuracea Aiton CAM, CHIS, QROO, TAB, VER, YUC

Zamia herrerae Calderón \& Standl. CHIS

*Zamia inermis Vovides, J.D. Rees \& Vázq. Torres VER

*Zamia katzeriana (Regel) E. Rettig CHIS, TAB, VER

*Zamia lacandona Schutzman \& Vovides CHIS

Zamia loddigesii Miq. CAM, CHIS, COL, GRO, HGO, JAL, MICH, NAY, OAX, PUE, QRO, QROO, SLP, TAB, TAMS, VER, YUC

Zamia monticola Chamb. CHIS, QROO, VER

Zamia muricata Willd. CHIS, OAX

Zamia prasina W. Bull. CAM, QROO, YUC

*Zamia purpurea Vovides, J.D. Rees \& Vázq. Torres OAX, VER

*Zamia soconuscensis Schutzman, Vovides \& Dehgan CHIS

Zamia spartea A. DC. OAX, TAB, VER

Zamia verschaffeltii Miq. ND

\section{Angiospermas}

\section{Family Acanthaceae}

*Anisacanthus andersonii T.F. Daniel CHIH, SON

Anisacanthus linearis (S.H. Hagen) Henr. \& E.J. Lott CHIH, COAH, NLE, SLP

Anisacanthus puberulus (Torr.) Henr. \& E.J. Lott AGS, CHIH, COAH, DGO, HGO, JAL, MEX, NLE, QRO, SON, VER, ZAC *Anisacanthus pumilus (F. Dietr.) Nees CHIH, GTO, JAL, MEX, MICH, QRO, SLP

Anisacanthus quadrifidus (Vahl) Nees CAM, COAH, CDMX, DGO, GTO, GRO, HGO, JAL, MEX, MICH, NLE, OAX, PUE, QRO, SLP, TAMS, VER, ZAC

Anisacanthus thurberi (Torr.) A. Gray CHIH, SIN, SON

*Anisacanthus tulensis Greenm. HGO, MOR, OAX, QRO

*Aphanosperma sinaloensis (Leonard \& Gentry) T.F. Daniel BCS, CHIH, SIN, SON

Aphelandra aurantiaca (Scheidw.) Lindl. CHIS, GRO, JAL, MEX, MICH, NAY, OAX, QROO, TAB, VER

Aphelandra gigantiflora Lindau CHIS, OAX, TAB

*Aphelandra guerrerensis Wassh. GRO, OAX

Aphelandra heydeana Donn. Sm. CHIS

*Aphelandra hintonii Wassh. MICH

Aphelandra leonardii McDade GRO

*Aphelandra lineariloba Leonard COL, GRO, JAL, MEX, $\mathrm{MICH}$

*Aphelandra madrensis Lindau COL, DGO, GRO, JAL, MICH, NAY, SIN

Aphelandra scabra (Vahl) Sm. CAM, CHIS, GRO, HGO, 
MICH, OAX, QRO, QROO, SLP, TAB, TAMS, VER, YUC

Aphelandra schiedeana Schltdl. \& Cham. CHIS, GRO, JAL, MEX, MICH, OAX, SIN, VER

Aphelandra speciosa Brandegee CHIS

*Aphelandra verticillata Nees ex Hemsl. GRO, MEX, MICH, MOR

*Aphelandra wendtii T.F. Daniel CHIS, TAB, VER

Avicennia bicolor Standl. CHIS

Avicennia germinans (L.) L. BCN, BCS, CAM, CHIS, COL, GRO, JAL, MICH, NAY, OAX, QROO, SLP, SIN, SON, TAB, TAMS, VER, YUC

Barleria oenotheroides Dum. Cours. CAM, CHIS, COL, CDMX, DGO, GRO, JAL, MEX, MICH, MOR, NAY, OAX, QROO, SLP, SIN, TAB, TAMS, VER, YUC, ZAC

*Beloperone fragilis B.L. Rob. GTO, HGO, QRO, SLP

Bravaisia berlandieriana (Nees) T.F. Daniel CAM, QROO, SLP, TAB, TAMS, VER, YUC

Bravaisia grandiflora Donn. Sm. CHIS

Bravaisia integerrima (Spreng.) Standl. CAM, CHIS, COL, GRO, JAL, MEX, MICH, OAX, QROO, TAB, TAMS, VER, YUC

*Buceragenia minutiflora Greenm. MOR

Carlowrightia arizonica A. Gray AGS, BCN, BCS, CHIS, CHIH, COAH, COL, DGO, GRO, JAL, MEX, MICH, MOR, NAY, OAX, PUE, QRO, SLP, SIN, SON, ZAC

*Carlowrightia fuertensis T.F. Daniel SIN

*Carlowrightia haplocarpa B.L. Rob. \& Greenm. SLP, TAMS, VER

Carlowrightia hintonii T.F. Daniel GRO, YUC

*Carlowrightia huicholiana T.F. Daniel JAL, NAY, ZAC

* Carlowrightia lesueurii Henr. \& T.F. Daniel CHIH

*Carlowrightia lindauiana Standl. HGO, QRO, SLP, SON, VER

Carlowrightia linearifolia (Torr.) A. Gray CHIH, COAH, DGO, QRO, SON, ZAC

*Carlowrightia mcvaughii T.F. Daniel COL, GRO, JAL, MICH, NAY

Carlowrightia mexicana Henr. \& T.F. Daniel CHIH, COAH, SIN

Carlowrightia myriantha (Standl.) Standl. CAM, QROO, YUC *Carlowrightia neesiana (Schauer ex Nees) T.F. Daniel AGS, DGO, GTO, GRO, HGO, JAL, MEX, MICH, MOR, NLE, OAX, PUE, QRO, SLP, TAMS, VER, ZAC

*Carlowrightia ovata A. Gray CHIH

Carlowrightia parviflora (Buckley) Wassh. COAH, GTO, HGO,

JAL, NLE, QRO, SLP, TAMS, ZAC

Carlowrightia parvifolia Brandegee CHIH, COAH, GTO, NLE, QRO

*Carlowrightia pectinata Brandegee BCS, CHIH, COL, DGO, GRO, MEX, MOR, OAX, SIN, SON

*Carlowrightia pringlei B.L. Rob. \& Greenm. MOR, OAX, PUE

*Carlowrightia purpurea T.F. Daniel QRO, SLP, TAMS

Carlowrightia serpyllifolia (Torr.) A. Gray CHIH, COAH, DGO, GTO, HGO, NLE, QRO, SLP, VER, ZAC

Carlowrightia texana Henr. \& T.F. Daniel CHIH, COAH, NLE, SLP, SON, TAMS
Carlowrightia torreyana Wassh. CHIH, COAH, NLE, SON

*Carlowrightia trichocarpa T.F. Daniel TAMS

*Carlowrightia venturae T.F. Daniel GTO, QRO, SLP

*Chalarothyrsus amplexicaulis Lindau COL, GRO, JAL, MEX, OAX

*Chileranthemum lottiae T.F. Daniel GRO, JAL

Chileranthemum pyramidatum (Lindau) T.F. Daniel CHIS,

OAX, VER

*Chileranthemum trifidum Oerst. HGO, OAX, PUE, VER

Dicliptera acuminata (Ruiz \& Pav.) Juss. SLP, SIN, VER

*Dicliptera anomala Leonard CHIS, OAX, VER

Dicliptera brachiata Spreng. NLE, QRO, SLP, VER

*Dicliptera haenkeana Nees COL, GRO, JAL, MEX, MICH, MOR, PUE, ZAC

*Dicliptera inaequalis Greenm. COL, GTO, GRO, JAL, MEX, MICH, MOR, NAY, QRO, ZAC

Dicliptera inutilis Leonard CHIS, GRO, JAL, OAX

Dicliptera membranacea Leonard CHIS, COL, GRO, JAL, OAX

*Dicliptera nervata Greenm. COL, DGO, JAL, MOR, NAY, SIN

*Dicliptera novogaliciana T.F. Daniel COL, JAL, MICH

*Dicliptera peduncularis Nees AGS, CHIS, COL, CDMX, GTO, GRO, HGO, JAL, MEX, MICH, MOR, OAX, PUE, QRO, SLP, VER, ZAC

Dicliptera resupinata (Vahl) A. Juss. BCS, CHIH, COL, DGO, GTO, GRO, JAL, MEX, MICH, NAY, NLE, SIN, SON, TAMS, ZAC

Dicliptera sciadephora Donn. Sm. CHIS, GRO, OAX

Dicliptera sexangularis (L.) Juss. CAM, CHIS, HGO, MOR, OAX, PUE, QRO, QROO, SLP, TAMS, VER, YUC

Dicliptera sumichrasti Lindau CHIS, OAX, VER

*Dicliptera thlaspioides Nees COL, GRO, JAL, MEX, MICH, MOR, NAY, OAX, PUE, SLP, ZAC

Dicliptera unguiculata Nees CHIS, GRO, OAX, VER

*Dyschoriste angustifolia (Hemsl.) Kuntze COL, JAL, MICH

Dyschoriste capitata (Oerst.) Kuntze CAM, CHIS, GRO, MOR, OAX, QROO

Dyschoriste crenulata Kobuski COAH, TAMS

* Dyschoriste greenmanii Kobuski COAH, NLE, TAMS

*Dyschoriste hirsutissima (Nees) Kuntze CHIS, CHIH, COL, DGO, GTO, GRO, HGO, JAL, MEX, MICH, MOR, NAY, OAX, PUE, SIN, SON, VER, ZAC

* Dyschoriste jaliscensis Kobuski JAL

Dyschoriste linearis (Torr. \& A. Gray) Kuntze CHIH, COAH, DGO, HGO, NAY, NLE, QRO, SLP, TAMS, ZAC

* Dyschoriste mcvaughii T.F. Daniel JAL

* Dyschoriste microphylla (Cav.) Kuntze AGS, GTO, HGO, JAL, MEX, MICH, OAX, PUE, QRO, SLP, TLAX, VER, ZAC *Dyschoriste novogaliciana T.F. Daniel DGO, JAL, NAY, SIN Dyschoriste ovata (Cav.) Kuntze CHIS, GRO, JAL, MEX, MICH, MOR, NAY, OAX, VER, ZAC

*Dyschoriste pinetorum Kobuski MICH, OAX

*Dyschoriste poliodes Leonard \& Gentry COAH, NLE, SLP, TAMS

*Dyschoriste pringlei Greenm. CHIH, JAL, NAY

*Dyschoriste purpusii Kobuski OAX, PUE 
Dyschoriste quadrangularis (Oerst.) Kuntze CAM, CHIS, DGO, GTO, GRO, HGO, MEX, MICH, MOR, NAY, NLE, OAX, QRO, QROO, SLP, SIN, TAMS, VER

*Dyschoriste rosei Kobuski DGO, NAY

*Dyschoriste saltuensis Fernald COL, GRO, JAL, MICH, OAX Dyschoriste schiedeana (Nees) Kuntze AGS, BCS, CHIH, COAH, DGO, GTO, GRO, HGO, JAL, MEX, MICH, NAY, NLE, OAX, PUE, QRO, SLP, SIN, SON, TAMS, TLAX, VER, ZAC

*Dyschoriste xylopoda Kobuski AGS, CHIH, DGO, JAL, NAY, SLP, SON

Elytraria bromoides Oerst. CAM, CHIS, CHIH, COAH, GRO, HGO, JAL, MEX, MICH, MOR, NAY, NLE, OAX, QRO, QROO, SLP, TAMS, VER, YUC

Elytraria imbricata (Vahl) Pers. AGS, BCN, BCS, CAM, CHIS, CHIH, COAH, COL, DGO, GTO, GRO, HGO, JAL, MEX, MICH, MOR, NAY, NLE, OAX, PUE, QRO, QROO, SLP, SIN, SON, TAB, VER, YUC, ZAC

* Elytraria macrophylla Leonard HGO, QRO, SLP, TAMS

*Elytraria mexicana Fryxell \& S.D. Koch CHIS, COL, GRO, JAL, MEX, MICH, SLP, SIN

*Glockeria glandulosa Oerst. OAX

*Gypsacanthus nelsonii E.J. Lott, V. Jaram. \& Rzed. GRO, MEX, MOR, OAX, PUE

*Habracanthus harleyi Wassh. GRO

Habracanthus ruberrimus D. Gibson CHIS

Henrya insularis Nees AGS, BCS, CHIS, CHIH, COL, DGO, GTO, GRO, HGO, JAL, MEX, MICH, MOR, NAY, OAX, PUE, QRO, QROO, SLP, SIN, SON, TAMS, VER, YUC, ZAC

*Henrya scorpioides Nees CHIS, JAL, MICH, QROO, YUC

*Henrya tuberculosperma T.F. Daniel COL, GRO, JAL, MEX, MOR, NAY, SIN

*Holographis anisophylla T.F. Daniel COL, JAL, MICH

*Holographis argyrea (Leonard) T.F. Daniel GRO, OAX

*Holographis caput-medusae T.F. Daniel GRO

*Holographis ehrenbergiana Nees GTO, HGO, JAL, NLE, OAX, PUE, QRO, SLP, TAMS, VER

*Holographis hintonii (Leonard) T.F. Daniel GRO, JAL, MICH

*Holographis ilicifolia Brandegee COAH, DGO

*Holographis leticiana T.F. Daniel OAX

*Holographis lizethae Cruz-Durán \& J. Jiménez Ram. GRO

*Holographis pallida Leonard \& H. Gentry SON

*Holographis parayana Miranda CHIS, OAX

*Holographis peloria (Leonard) T.F. Daniel DGO, JAL, NAY, ZAC

*Holographis pueblensis T.F. Daniel OAX, PUE

*Holographis tolantongensis T.F. Daniel HGO, VER

*Holographis velutifolia (House) T.F. Daniel OAX, PUE

*Holographis virgata (Harv. ex Benth. \& Hook.) T.F. Daniel BCN, BCS, SON

*Holographis websteri T.F. Daniel CAM, QROO, YUC

*Hoverdenia speciosa Nees HGO, QRO, SLP, TAMS, VER

Hygrophila costata Nees CHIS, OAX, GRO, VER

*Jacobinia aschenborniana (Nees) Hemsl. ND

*Jacobinia heterophylla (Cham. \& Schltdl.) Hemsl. VER

*Jacobinia incana (Nees) Hemsl. COAH, HGO, NLE, QRO, SLP, TAMS, VER
*Jacobinia leucothamna Standl. CAM, QROO, YUC

* Jacobinia roseana Leonard COL, JAL, MEX, MICH, MOR

* Justicia adenothyrsa (Lindau) T.F. Daniel GRO, OAX

*Justicia alopecuroidea T.F. Daniel GRO, OAX

Justicia americana (L.) Vahl CHIH, COAH

* Justicia anagallis Hemsl. CAM, TAB, VER

* Justicia angustiflora D.N. Gibson CHIS, OAX, PUE

*Justicia ardens T.F. Daniel OAX, VER

Justicia aurea Schltdl. CHIS, COL, JAL, MEX, MICH, NAY, OAX, PUE, QRO, SLP, TAB, VER

*Justicia austrocapensis T.F. Daniel BCS

Justicia bartlettii (Leonard) D.N. Gibson CHIS, GRO, OAX, VER

Justicia borrerae (Hemsl.) T.F. Daniel CHIS, OAX, PUE, VER * Justicia breedlovei T.F. Daniel CHIS

Justicia breviflora (Nees) Rusby CAM, CHIS, COL, GRO, HGO, JAL, OAX, PUE, QROO, SLP, TAB, TAMS, VER

Justicia californica (Benth.) D.N. Gibson BCN, BCS, SIN, SON

Justicia campechiana Standl. ex Lundell CAM, CHIS, MICH, OAX, QROO, YUC

Justicia candelariae (Oerst.) Leonard CHIS, GRO, OAX, PUE, TAB, VER, YUC

Justicia candicans (Nees) L.D. Benson AGS, BCS, CHIH, COL, DGO, GTO, GRO, JAL, MEX, MICH, MOR, NAY, OAX, PUE, QRO, SLP, SIN, SON, ZAC

Justicia carthaginensis Jacq. CAM, CHIS, GRO, HGO, JAL, MEX, MOR, OAX, QRO, QROO, SLP, SIN, TAB, VER, YUC Justicia caudata A. Gray CHIS, CHIH, COL, CDMX, DGO, GTO, GRO, HGO, JAL, MEX, MICH, MOR, NAY, OAX, PUE, QRO, SIN, SON, TAB, VER, ZAC

Justicia clinopodia A. Gray ex Greenm. CHIS, GRO, MEX, MICH, OAX, VER

* Justicia coahuilana T.F. Daniel COAH

Justicia colorifera V.A.W. Graham CHIS

Justicia comata (L.) Lam. CHIS, GRO, OAX, PUE, QRO, QROO, SLP, TAB, VER, YUC

* Justicia cuicatlana T.F. Daniel OAX

* Justicia chlorostachya Leonard CHIS, TAB

* Justicia chol T.F. Daniel CHIS, TAB

* Justicia decurvata Hilsenb. COAH, DGO

Justicia eburnea D.N. Gibson CHIS

Justicia ensiflora (Standl.) D. Gibson CHIS, VER

Justicia fimbriata (Nees) V.A.W. Graham CHIS, GRO, OAX, TAB, VER

Justicia fulvicoma Schltdl. \& Cham. CAM, CHIS, COAH, GTO, GRO, HGO, JAL, MEX, MOR, NLE, OAX, QRO, SLP, TAMS, VER, YUC

*Justicia furcata Jacq. CHIH, CDMX, DGO, GTO, GRO, HGO, JAL, MEX, MICH, MOR, NAY, NLE, OAX, PUE, QRO, QROO, SLP, SIN, VER, ZAC

*Justicia gonzalezii (Greenm.) Henr. \& Hiriart OAX, PUE

* Justicia henricksonii T.F. Daniel COAH, SLP, ZAC

* Justicia herpetacanthoides Leonard CHIS, YUC

* Justicia hians (Brandegee) Brandegee BCS

* Justicia hilsenbeckii T.F. Daniel COL, DGO, GRO, JAL, MEX, MICH, MOR, OAX, PUE, SLP, SIN, SON, VER 
*Justicia hintoniorum G.L. Nesom NLE, TAMS

* Justicia huacanensis T.F. Daniel \& V.W. Steinm. GRO, MICH

* Justicia hyssopus Lindau GTO, HGO, QRO

Justicia inaequalis Benth. CHIS, GRO, OAX, TAB

* Justicia insolita Brandegee BCS

*Justicia ixtlania T.F. Daniel COL, JAL, MICH, NAY, SIN

* Justicia jitotolana T.F. Daniel CHIS

Justicia kanal T.F. Daniel CHIS, VER

*Justicia laevilinguis (Nees) Lindau CAM, CHIS, TAB, VER

*Justicia leonardii Wassh. COAH, GTO, HGO, NLE, QRO, SLP, TAMS, VER

Justicia lindeniana (Nees.) J. Macbr. CHIS, TAB, VER

* Justicia linearis B.L. Rob. \& Greenm. DGO, SLP, TAMS

Justicia longii Hilsenb. CHIH, BCS, SON

*Justicia lundellii Leonard CAM, CHIS, QROO, TAB, YUC

Justicia macrantha Benth. CHIS, HGO, VER

* Justicia madrensis T.F. Daniel CHIS

* Justicia masiaca T.F. Daniel SIN, SON

* Justicia medranii Henr. \& Hiriart HGO, VER

Justicia metallica Lindau CHIS

* Justicia mirandae T.F. Daniel CHIS

Justicia multicaulis Donn. Sm. CHIS, GRO

*Justicia nelsonii Greenm. NAY

*Justicia nevlingii Wassh. \& T.F. Daniel CHIS, VER

*Justicia oaxacana (Greenm.) T.F. Daniel OAX, PUE

Justicia ovata (Walt.) Lindau TAMS

*Justicia palmeri Rose BCS

*Justicia paucifolia T.F. Daniel OAX, PUE

Justicia pectoralis Jacq. CHIS, OAX, PUE, QRO, SLP, TAB, VER, YUC

Justicia pedicellata D. Gibson CHIS

Justicia phlebodes Leonard \& Gentry CHIH, DGO, SIN, SON Justicia pilosella (Nees) Hilsenb. CHIH, COAH, DGO, GTO, HGO, NLE, OAX, PUE, SLP, TAMS, ZAC

Justicia pringlei B.L. Rob. CHIS, GTO, GRO, JAL, MEX, MICH, MOR, OAX

*Justicia purpusii (Brandegee) D.N. Gibson BCS

Justicia ramosa (Oerst.) V.A.W. Graham CAM, CHIS, GRO, OAX, PUE, QROO, YUC

*Justicia reflexiflora Rich. COL, GRO, JAL

Justicia reptans $\mathrm{Sw}$. CHIS

Justicia runyonii Small AGS, COAH, JAL, NLE, QRO, SLP, TAMS, VER, ZAC

*Justicia rzedowskii (Acosta) T.F. Daniel CHIS

* Justicia salasiae T.F. Daniel \& E.J. Lott OAX

* Justicia salviiflora Kunth CHIS, CHIH, COL, DGO, GRO,

JAL, MEX, MICH, MOR, NAY, OAX, SIN, SON, ZAC

* Justicia santelisiana Acosta \& T.F. Daniel OAX

Justicia soliana Standl. CHIS, SON

Justicia sonorae Wassh. SIN, SON

Justicia spicigera Schltdl. BCS, CAM, CHIS, COL, DGO, GTO, GRO, HGO, JAL, MEX, MICH, MOR, NAY, OAX, PUE, QRO, QROO, SLP, SIN, TAB, TAMS, VER, YUC

*Justicia stellata (B.L. Rob. \& Greenm.) T.F. Daniel JAL

*Justicia tabascina T.F. Daniel CHIS, TAB

*Justicia teletheca T.F. Daniel CHIS, OAX

Justicia tianguensis T.F. Daniel CHIS, OAX
* Justicia torresii T.F. Daniel OAX

* Justicia turipachensis T.F. Daniel CHIS, OAX

Justicia turneri Hilsenb. COAH, NLE, SLP, TAMS

*Justicia tuxtlensis T.F. Daniel VER

*Justicia uxpanapensis T.F. Daniel VER

Justicia valvata T.F. Daniel OAX, TAB, VER

* Justicia veracruzana T.F. Daniel PUE, VER

Justicia warnockii B.L. Turner CHIH, COAH

*Justicia zamudioi T.F. Daniel QRO, SLP

* Justicia zopilotensis Henr. \& Hiriart GRO, OAX, PUE

Lepidagathis alopecuroidea (Vahl) R. Br. ex Griseb. CHIS, OAX, PUE, TAB, VER

*Lepidagathis danielii Cruz-Durán \& J. Jiménez Ram. GRO

*Lophostachys chiapensis Acosta CHIS

Lophostachys guatemalensis Donn. Sm. CHIS, OAX

*Lophostachys soconuscana T.F. Daniel CHIS

*Lophostachys uxpanapensis Acosta OAX, VER

*Louteridium brevicalyx A.T. Richardson GRO, MICH

Louteridium donnell-smithii S. Watson CHIS, OAX, VER

*Louteridium koelzii Miranda \& McVaugh JAL

Louteridium mexicanum (Baill.) Standl. CHIS, JAL, NAY, OAX, TAB, VER

*Louteridium parayi Miranda CHIS, VER

Louteridium purpusii Brandegee CHIS

*Louteridium rzedowskii T.F. Daniel GRO

*Louteridium tamaulipense A.T. Richardson TAMS

Mendoncia guatemalensis Standl. \& Steyerm. CHIS, OAX, VER

Mendoncia lindavii Rusby CHIS

Mendoncia retusa Turrill CHIS, VER

* Mexacanthus mcvaughii T.F. Daniel COL, JAL, MICH, NAY

*Mirandea andradenia T.F. Daniel TAMS

* Mirandea grisea Rzed. COAH, GTO, JAL, SLP, TAMS, ZAC

*Mirandea huastecensis T.F. Daniel COAH, NLE, SLP

* Mirandea hyssopus (Nees) T.F. Daniel HGO, QRO, SLP

* Mirandea nutans (Nees) T.F. Daniel HGO, QRO

*Mirandea sylvatica Acosta CHIS, OAX, TAB, VER

Odontonema albiflorum Leonard CHIS

* Odontonema auriculatum (Rose) T.F. Daniel COL, GRO, JAL, $\mathrm{MICH}, \mathrm{NAY}, \mathrm{OAX}$

Odontonema callistachyum (Schltdl. \& Cham.) Kuntze CAM, CHIS, GTO, GRO, HGO, JAL, MEX, MICH, MOR, NLE, OAX, PUE, QRO, QROO, SLP, SIN, SON, TAB, TAMS, VER Odontonema glaberrimum (M.E. Jones) V.M. Baum CHIS, DGO, JAL, MICH, NAY, OAX, SIN

Odontonema glabrum Brandegee CHIS

Odontonema hondurense (Lindau) D. Gibson CHIS

Odontonema tubiforme (Bertol.) Kuntze CAM, CHIS, QROO, TAB, VER, YUC

*Poikilacanthus capitatus (Leonard) Ramamoorthy GRO, MEX Poikilacanthus macranthus Lindau CHIS, OAX

*Poikilacanthus novogalicianus T.F. Daniel COL, MICH, OAX Pseuderanthemum alatum (Nees) Radlk. CAM, CHIS, COL, CDMX, GRO, HGO, JAL, MEX, MICH, OAX, QRO, QROO, SLP, TAMS, VER, YUC

Pseuderanthemum cuspidatum (Nees) Radlk. CHIS, COL, GRO, JAL, MEX, OAX, PUE, QRO, SLP, VER 
Pseuderanthemum fasciculatum (Oerst.) Leonard CHIS, GRO, HGO, OAX, PUE, VER

*Pseuderanthemum floribundum T.F. Daniel OAX

* Pseuderanthemum guerrerense Cruz-Durán \& S. Valencia GRO

* Pseuderanthemum hispidulum (Nees) Radlk. CHIS, GRO, MEX, MOR, QRO, VER

*Pseuderanthemum pihuamoense T.F. Daniel COL, JAL

Pseuderanthemum praecox (Benth.) Leonard CHIS, CHIH, COL, CDMX, DGO, GTO, GRO, HGO, JAL, MEX, MICH, MOR, NAY, NLE, OAX, PUE, QRO, SLP, SIN, SON, TAB, TAMS, TLAX, VER, ZAC

Pseuderanthemum standleyi Leonard COL, GRO, JAL, MEX, OAX, QRO, SLP

Pseuderanthemum verapazense Donn. Sm. CHIS, TAB, VER

Razisea spicata Oerst. CHIS, OAX

*Ruellia alboviolacea Lindau GRO, JAL, MICH

* Ruellia amoena Sessé \& Moc. COL, GRO, JAL, MICH

Ruellia blechum L. BCS, CAM, CHIS, COL, GTO, GRO, HGO, JAL, MEX, MICH, MOR, NAY, NLE, OAX, PUE, QRO, QROO, SLP, SIN, SON, TAB, TAMS, VER, YUC

*Ruellia bourgaei Hemsl. COL, CDMX, GTO, GRO, HGO, JAL, MEX, NAY, OAX, PUE, VER, ZAC

*Ruellia breedlovei T.F. Daniel CHIS

* Ruellia californica (Rose) I.M. Johnst. BCN, BCS, SON

* Ruellia cedilloi Ramamoorthy OAX

*Ruellia comonduensis T.F. Daniel BCS

* Ruellia conzattii Standl. OAX

Ruellia corzoi Tharp \& F.A. Barkley COAH, NLE, TAMS

Ruellia davisiorum Tharp \& F.A. Barkley NLE, SLP, TAMS

*Ruellia discolor Nees GRO, MEX

Ruellia donnell-smithii Leonard CHIS, GRO, JAL, MICH, OAX, QRO, SLP

Ruellia erythropus (Nees) Lindau OAX, QRO, VER, YUC

* Ruellia eumorphantha Lindau GRO, OAX

Ruellia foetida Willd. CHIS, COL, GRO, JAL, MEX, MICH, MOR, NAY, OAX, SLP, TAMS, VER

*Ruellia foliosepala T.F. Daniel GRO

* Ruellia fruticosa Sessé \& Moc. GRO, MEX, MOR, OAX, PUE

Ruellia geminiflora Kunth CHIS, COL, GRO, HGO, JAL, NAY,

OAX, PUE, TAB, VER

*Ruellia guerrerensis T.F. Daniel GRO, MICH

Ruellia harveyana Stapf. CHIS, OAX, VER

Ruellia hookeriana (Nees) Hemsl. AGS, CHIS, COL, GTO, GRO, JAL, MEX, MICH, MOR, NAY, OAX, QRO, SLP, SIN, VER, ZAC

Ruellia humifusa (Oerst.) Hemsl. VER

*Ruellia intermedia Leonard BCS, CAM, CHIS, CHIH, COL, DGO, GRO, JAL, MEX, MICH, MOR, NAY, OAX, PUE, SIN, SON, TAMS, ZAC

Ruellia inundata Kunth BCS, CAM, CHIS, COL, DGO, GRO, JAL, MEX, MICH, MOR, NAY, OAX, PUE, QROO, SLP, SIN, SON, TAB, TAMS, VER, YUC, ZAC

*Ruellia jaliscana Standl. COL, GRO, JAL

*Ruellia jimulcensis Villarreal COAH, DGO

Ruellia jussieuoides Schltdl. \& Cham. CAM, CHIS, GRO, OAX, PUE, QROO, TAB, VER
Ruellia lactea Cav. AGS, CHIS, CHIH, COL, CDMX, DGO, GTO, GRO, HGO, JAL, MEX, MICH, MOR, OAX, PUE, QRO, SLP, SIN, SON, TAMS, TLAX, VER, ZAC

*Ruellia laslobasensis $\mathrm{E}$. Tripp OAX

Ruellia latibracteata D.N. Gibson CHIS, OAX

*Ruellia leucantha Brandegee BCS, SIN

*Ruellia longepetiolata (Oerst.) Hemsl. PUE, QRO

Ruellia macrophylla Vahl QROO, YUC

Ruellia malacosperma Greenm. AGS, COAH, GRO, MEX,

MICH, MOR, NLE, SLP, SON, TAMS, VER

Ruellia matagalpae Lindau CAM, CHIS, GRO, OAX

* Ruellia matudae Leonard CHIS

Ruellia maya T.F. Daniel CHIS, OAX, VER

* Ruellia mcvaughii T.F. Daniel COL, JAL, NAY

Ruellia megasphaera Lindau CHIS, GRO, MEX, MICH, MOR, OAX, VER

Ruellia metziae Tharp COAH, SLP

*Ruellia novogaliciana T.F. Daniel COL, JAL, MICH

Ruellia nudiflora (Engelm. \& A. Gray) Urb. AGS, BCS, CAM, CHIS, CHIH, COAH, COL, CDMX, DGO, GTO, GRO, HGO, JAL, MEX, MICH, MOR, NAY, NLE, OAX, PUE, QRO, QROO, SLP, SIN, SON, TAB, TAMS, TLAX, VER, YUC, ZAC

*Ruellia oaxacana Leonard GRO, JAL, NAY, OAX

* Ruellia palmeri Tharp \& F.A. Barkley COAH

Ruellia paniculata L. CAM, CHIS, GRO, JAL, NAY, OAX, QRO, QROO, SLP, SIN, TAMS, VER, YUC

Ruellia parryi A. Gray CHIH, COAH, DGO, NLE, SON, TAMS, ZAC

Ruellia parva (Nees) Hemsl. CHIS, GRO, MEX, MICH, MOR, OAX, VER

Ruellia pedunculata Torr. ex A. Gray TAMS, VER

Ruellia pereducta Standl. ex Lundell CAM, CHIS, JAL, OAX, QROO, TAB, VER, YUC

*Ruellia petiolaris (Nees) T.F. Daniel CHIS, GRO, MICH, OAX

*Ruellia pringlei Fernald GRO, MICH, OAX

Ruellia puberula (Leonard) Tharp \& F.A. Barkley CHIS, OAX, VER

*Ruellia pulcherrima T. Anderson ex Hemsl. CDMX, GRO, HGO, MEX, MOR, OAX, PUE

*Ruellia rosea (Nees) Hemsl. GTO, HGO, OAX, PUE, QRO, SLP, VER

Ruellia runyonii Tharp \& F.A. Barkley COAH, DGO, JAL, NAY, NLE, SLP, TAMS, VER, ZAC

*Ruellia sarukhaniana Ramamoorthy $\mathrm{MICH}$

Ruellia simplex C. Wright CAM, CHIS, HGO, MOR, OAX, PUE, QRO, QROO, SLP, TAB, TAMS, VER

$*$ Ruellia sororia Standl. GRO, OAX

*Ruellia speciosa (Nees) Lindau AGS, CAM, CDMX, HGO, JAL, MEX, MICH, OAX, PUE, QRO, SLP, VER

Ruellia spissa Leonard CHIS, COL, DGO, GTO, GRO, JAL, MEX, MICH, NAY

Ruellia stemonacanthoides (Oerst.) Hemsl. CAM, CHIS, COL, GRO, JAL, MICH, NAY, OAX, QROO, TAB, VER

Ruellia tuberosa L. CAM, CHIH, QROO, SON, SLP, VER, YUC

Ruellia tubiflora Kunth CHIS, YUC 
* Ruellia tuxtlensis Ramamoorthy \& Hornelas OAX, VER Schaueria parviflora (Leonard) T.F. Daniel OAX, VER

* Siphonoglossa bartletti Standl. GRO, VER

*Siphonoglossa canbyi (Greenm.) Hilsenb. COAH, GTO, HGO, NLE, QRO, SLP, TAMS, VER

*Siphonoglossa durangensis Henr. \& Hilsenb. COAH, DGO Siphonoglossa greggii Greenm. \& C.H. Thomps. COAH, NLE, TAMS, VER

* Siphonoglossa linearifolia Henr. \& Hilsenb. DGO

Siphonoglossa pilosella (Nees) Torr. CHIH, COAH, DGO, NLE, SLP, TAMS

Siphonoglossa sessilis (Jacq.) D. Gibson CHIS, GRO, MEX, MOR, OAX, PUE, QROO, SIN, VER, YUC

Spathacanthus hahnianus Baill. CHIS, OAX, PUE, VER

Spathacanthus parviflorus Leonard CHIS, OAX, TAB, VER

Staurogyne miqueliana Kuntze COL, JAL

Stenandrium barbatum Torr. \& A. Gray CHIH, COAH

*Stenandrium chameranthemoideum Oerst. CAM, CHIS, OAX, QROO, VER

Stenandrium dulce (Cav.) Nees AGS, CHIS, CHIH, COAH, CDMX, DGO, GTO, GRO, HGO, JAL, MEX, MICH, NAY, NLE, OAX, PUE, QRO, SLP, SIN, TAMS, VER, ZAC

*Stenandrium nanum (Standl.) T.F. Daniel QROO, YUC

Stenandrium pedunculatum (Donn. Sm.) Leonard CAM, CHIS, COL, GRO, JAL

*Stenandrium pilosulum (S.F. Blake) T.F. Daniel CHIH, SON

Stenandrium subcordatum Standl. CAM, CHIS, MOR, QROO, VER, YUC

*Stenandrium verticillatum Brandegee GTO, HGO, MEX, OAX, PUE, QRO

*Stenostephanus breedlovei T.F. Daniel CHIS

* Stenostephanus chiapensis T.F. Daniel CHIS

Stenostephanus glaber (Leonard) T.F. Daniel CHIS, HGO, OAX, QRO, SLP, VER

Stenostephanus gracilis (Oerst.) T.F. Daniel CHIS, OAX, VER

* Stenostephanus guerrerensis T.F. Daniel GRO

*Stenostephanus haematodes (Schltdl.) T.F. Daniel HGO, OAX, PUE, VER

Stenostephanus latilabris (D.N. Gibson) T.F. Daniel CHIS

Stenostephanus monolophus (Donn. Sm.) T.F. Daniel CHIS, GRO, OAX

* Stenostephanus purpusii (Brandegee) T.F. Daniel CHIS

Stenostephanus silvaticus (Nees) T.F. Daniel CHIS, OAX, TAB, VER

*Stenostephanus tacanensis (Acosta \& R. Fernández) T.F. Daniel CHIS

*Tetramerium abditum (Brandegee) T.F. Daniel CHIH, DGO, GRO, MICH, NAY, SIN, SON

*Tetramerium aureum Rose HGO, MOR, QRO, VER

*Tetramerium butterwickianum T.F. Daniel JAL, MICH

*Tetramerium carranzae T.F. Daniel GTO, HGO, QRO, SLP, VER

*Tetramerium crenatum T. Daniel OAX

*Tetramerium diffusum Rose COL, JAL, MICH

*Tetramerium emilyanum T.F. Daniel MICH

*Tetramerium fruticosum Brandegee BCN, BCS, SON
*Tetramerium glandulosum Oerst. COL, DGO, GRO, JAL, MEX, MICH, MOR, OAX, PUE, SIN, SON, ZAC

*Tetramerium glutinosum Lindau GRO, MEX, MICH, NAY, MOR, OAX, PUE

*Tetramerium guerrerense T.F. Daniel GRO

*Tetramerium langlassei Happ GRO, JAL, MEX, MICH, VER

*Tetramerium mcvaughii T.F. Daniel COL, JAL

Tetramerium nemorum Brandegee CHIS, VER, YUC

Tetramerium nervosum Nees AGS, BCS, CAM, CHIS, CHIH, COAH, COL, DGO, GTO, GRO, HGO, JAL, MEX, MICH, MOR, NAY, NLE, OAX, PUE, QRO, QROO, SLP, SIN, SON, TAMS, VER, YUC, ZAC

*Tetramerium oaxacanum T.F. Daniel CHIS, OAX

*Tetramerium obovatum T.F. Daniel OAX

*Tetramerium ochoterenae (Miranda) T.F. Daniel GRO, MEX, MOR, OAX, PUE

Tetramerium platystegium Torr. TAMS

*Tetramerium rubrum G. Happ GRO, MEX, MICH, NAY, OAX

*Tetramerium rzedowskii T.F. Daniel MICH

Tetramerium tenuissimum Rose CAM, CHIS, CHIH, COL, DGO, GTO, GRO, JAL, MEX, MICH, MOR, NAY, OAX, SIN, SON, VER, YUC

*Tetramerium yaquianum T.F. Daniel SON

*Yeatesia mabryi Hilsenb. NLE, TAMS

*Yeatesia platystegia (Torr.) Hilsenb. COAH, NLE, SLP

\section{Family Actinidiaceae}

Saurauia angustifolia Turcz. CHIS, OAX

Saurauia aspera Turcz. CHIS, HGO, OAX, QRO, SLP, TAB, TAMS, VER

* Saurauia comitis-rosea R.E. Schultes CHIS, OAX

Saurauia conzattii Buscal. OAX

Saurauia cuchumatanensis Standl. \& Steyerm. CHIS

Saurauia kegeliana Schltdl. CHIS

Saurauia leucocarpa Schltdl. CHIS, GRO, HGO, MICH, OAX, PUE, SLP, TAB, VER

* Saurauia madrensis B.T. Keller \& Breedlove CHIS

* Saurauia matudae Lundell CHIS, OAX

Saurauia oreophila Hemsl. CHIS, OAX, VER

Saurauia pringlei Rose CHIS, GRO, MICH, OAX, SLP, VER

Saurauia pseudoscabrida Buscal. CHIS

*Saurauia pustulata G.E. Hunter CHIS, TAB

* Saurauia radlkoferi Buscal. PUE, VER

Saurauia rubiformis Vatke CHIS, TAB

Saurauia scabrida Hemsl. CHIS, GRO, HGO, MEX, OAX, PUE, QRO, SLP, TAB, VER

Saurauia selerorum Buscal. CHIS

*Saurauia serrata DC. AGS, CHIS, COL, DGO, GRO, JAL, MEX, MICH, MOR, NAY, OAX, PUE, SLP, SIN, TAB, VER Saurauia villosa DC. CHIS, OAX, PUE, TAB, VER

Saurauia yasicae Loes. CHIS, HGO, MEX, OAX, PUE, QRO, SLP, TAB, VER

Saurauia zahlbruckneri Buscal. CHIS

Family Achariaceae

*Chiangiodendron mexicanum $\mathrm{T}$. Wendt CHIS, TAB, VER Mayna odorata Aubl. VER 


\section{Family Achatocarpaceae}

Achatocarpus gracilis $\mathrm{H}$. Walter COL, GRO, JAL, MICH, NAY, OAX, SIN, SON

Achatocarpus nigricans Triana CAM, CHIS, GRO, JAL, MICH, OAX, QRO, QROO, SLP, TAB, TAMS, VER, YUC

*Achatocarpus oaxacanus Standl. CHIS, GRO, MICH, OAX

Phaulothamnus spinescens A. Gray BCN, BCS, CHIH, COAH, NAY, NLE, PUE, SIN, SON, TAMS

\section{Family Adoxaceae}

Sambucus nigra L. AGS, BCN, CAM, CHIS, CHIH, COAH, COL, CDMX, DGO, GTO, GRO, HGO, JAL, MEX, MICH, MOR, NAY, NLE, OAX, PUE, QRO, QROO, SLP, SIN, SON, TAB, TAMS, TLAX, VER, YUC, ZAC

Viburnum acutifolium Benth. CHIS, COL, GRO, JAL, MEX, MICH, MOR.OAX

*Viburnum caudatum Greenm. GRO, HGO, PUE, SLP, VER

*Viburnum ciliatum Greenm. GRO, HGO, MEX, PUE, SLP, VER

Viburnum discolor Benth. CHIS, OAX

Viburnum disjunctum C.V. Morton CHIS, OAX

*Viburnum elatum Benth. CHIS, COAH, CDMX, GTO, GRO, HGO, JAL, MEX, MICH, NAY, NLE, OAX, QRO, SLP, SIN, TAMS, VER, ZAC

Viburnum hartwegii Benth. CHIS, COL, HGO, JAL, MICH, OAX, VER

Viburnum jucundum C.V. Morton CHIS, OAX

*Viburnum loeseneri Graebn. CHIS, GTO, GRO, JAL, MEX, MICH, OAX, SLP, VER

*Viburnum membranaceum (Oerst.) Hemsl. COAH, GRO, HGO, MICH, NLE, OAX, PUE, SLP, VER

*Viburnum microcarpum Schltdl. \& Cham. HGO, MEX, MICH, OAX, PUE, SLP, VER

*Viburnum obtusatum D.N. Gibson CHIS

Viburnum rafinesquianum Schult. COAH, NLE

Viburnum rufidulum Raf., COAH

*Viburnum stenocalyx (Oerst.) Hemsl. CDMX, GRO, HGO, MEX, MICH, MOR, OAX, PUE, QRO, VER

*Viburnum sulcatum (Oerst.) Hemsl. OAX

*Viburnum tiliifolium (Oerst.) Hemsl. GRO, HGO, MEX, OAX, PUE, SLP, VER

\section{Family Aizoaceae}

Sesuvium maritimum (Walt.) Britton, Stern. \& Pogg. TAMS, VER

Sesuvium portulacastrum L. CAM, CHIS, CHIH, COAH, COL, CDMX, DGO, GRO, HGO, JAL, MEX, MICH, NAY, OAX, QRO, QROO, SLP, SIN, SON, TAB, TAMS, VER, YUC Sesuvium verrucosum Raf. BCN, BCS, CHIH, COAH, COL, DGO, GTO, JAL, MICH, NLE, SLP, SIN, SON, TAMS

Trianthema portulacastrum L. AGS, BCN, BCS, CAM, CHIS, CHIH, COAH, COL, CDMX, DGO, GTO, GRO, HGO, JAL, MEX, MICH, NAY, NLE, OAX, PUE, QRO, QROO, SLP, SIN, SON, TAB, TAMS, VER, YUC, ZAC

\section{Family Alismataceae}

Echinodorus andrieuxii (Hook. \& Arn.) Small CAM, CHIS, COL, GRO, JAL, MEX, MICH, MOR, NAY, OAX, QROO,
SIN, TAB, VER, YUC

Echinodorus berteroi (Spreng.) Fassett BCN, BCS, CAM, CHIH, COAH, COL, DGO, GRO, JAL, MICH, NAY, NLE, OAX, QRO, QROO, SLP, SIN, SON, TAMS, VER, YUC Echinodorus cordifolius (L.) Griseb. CAM, SLP, TAMS, VER, YUC

Echinodorus grandiflorus (Cham. \& Schltdl.) Micheli CHIS, CDMX, GRO, MEX, TAB, TAMS, VER

Echinodorus nymphaeifolius (Griseb.) Buchenau CAM, QROO, YUC

Echinodorus paniculatus Micheli CAM, CHIS, SLP, TAB, TAMS, VER

Echinodorus tenellus (Mart. ex Schult. \& Schult. f.) Buchenau CHIS, GRO, VER

*Echinodorus virgatus (Hook. \& Arn.) Micheli NAY, VER

Helanthium bolivianum (Rusby) Lehtonen \& Myllys CHIS Hydrocleys nymphoides (Willd.) Buchenau VER

Hydrocleys parviflora Seub. CHIS, VER

Limnocharis flava (L.) Buchenau CAM, CHIS, GRO, NAY, OAX, SIN, SON, TAB, VER

Limnocharis laforestii Duchass. ex Griseb. CHIS, GRO, NAY, OAX, VER

Sagittaria demersa J.G. Sm. AGS, CHIH, DGO, GTO, HGO, JAL, QRO

Sagittaria guayanensis Kunth CAM, CHIS, GRO, JAL, MEX, MICH, NAY, OAX, PUE, SIN, TAB, VER

Sagittaria intermedia Micheli CAM, YUC

Sagittaria lancifolia L. AGS, CAM, CHIS, COL, GRO, JAL, MICH, NAY, OAX, PUE, QRO, QROO, TAB, TAMS, VER, YUC

Sagittaria latifolia Willd. CAM, CHIS, CDMX, DGO, GTO, HGO, JAL, MEX, MICH, MOR, NAY, OAX, PUE, QRO, SIN, TAB, TAMS, TLAX, VER

Sagittaria longiloba Engelm. ex J.G. Sm. AGS, BCN, BCS, DGO, GTO, GRO, HGO, JAL, MEX, MICH, MOR, NAY, OAX, PUE, QRO, SIN, SON, TAMS, VER

*Sagittaria macrophylla Zucc. COL, CDMX, HGO, JAL, MEX, MICH, SON, TLAX

Sagittaria montevidensis Cham. \& Schltdl. CHIS, CHIH, COAH, COL, JAL, MICH, NAY, SIN, SON, TAB

Sagittaria platyphylla (Engelm.) J.G. Smith MICH, NLE, TAMS

\section{Family Alstroemeriaceae}

Bomarea acutifolia (Link \& Otto) Herb. CHIS, CDMX, GRO, HGO, JAL, MEX, MICH, MOR, OAX, PUE, TAB, TAMS, VER, ZAC

Bomarea edulis (Tussac) Herb. CAM, CHIS, COL, CDMX, DGO, GTO, GRO, HGO, JAL, MEX, MICH, MOR, NAY, OAX, PUE, QRO, QROO, SLP, SIN, TAMS, TLAX, VER, $\mathrm{ZAC}$

*Bomarea gloriosa (Schltdl. \& Cham.) M. Roemer CHIS, VER

\section{Family Altingiaceae}

Liquidambar styraciflua L. CHIS, CDMX, GRO, HGO, MEX, MICH, MOR, NLE, OAX, PUE, QRO, SLP, TAB, TAMS, VER 


\section{Family Amaranthaceae}

Alternanthera bettzickiana (Regel) G. Nicholson MICH, NAY, TAB, VER

Alternanthera brasiliana (L.) Kuntze CAM, CHIS, PUE, QROO, VER, YUC

Alternanthera caracasana Kunth BCN, CHIH, COAH, COL, CDMX, DGO, GTO, GRO, HGO, JAL, MEX, MICH, NLE, OAX, PUE, QRO, SLP, SON, TAB, TAMS, TLAX, VER, ZAC

*Alternanthera cordobensis (Standl.) Standl. VER

*Alternanthera flava (L.) Mears CHIS, GTO, OAX, PUE, VER

Alternanthera flavescens Kunth CAM, QROO, YUC

Alternanthera flavogrisea (Urb.) Urb. QROO, VER, YUC

Alternanthera jacquini (Schrader) Alain CHIS

Alternanthera laguroides (Standl.) Standl. CHIS, SLP

Alternanthera lanceolata (Benth.) Schinz COL, JAL, MICH, OAX, SIN, VER

Alternanthera microcephala (Moq.) Schinz CAM, CHIS, HGO, OAX, QROO, VER, YUC

Alternanthera obovata (M. Martens \& Galeotti) Millsp. CHIS, MOR, OAX, SLP, TAB, TAMS, VER, YUC

Alternanthera philoxeroides (Mart.) Griseb. SLP, TAMS

Alternanthera polygonioides (L.) R. Br. CHIS, CHIH, COAH, GTO, QRO, SLP, TAB, TAMS, VER

Alternanthera pubiflora (Benth.) Kuntze VER

Alternanthera pungens Kunth AGS, BCN, BCS, CHIS, CHIH, COAH, COL, CDMX, DGO, GTO, GRO, HGO, JAL, MEX, MICH, MOR, NAY, NLE, OAX, PUE, QRO, SLP, SIN, SON, TAB, TAMS, TLAX, VER, ZAC

*Alternanthera pycnantha (Benth.) Standl. CHIS, COL, JAL, NAY, OAX, ZAC

Alternanthera sessilis (L.) R. Br. CHIH, COAH, NAY, OAX, TAB, VER

*Alternanthera stellata (S. Watson) Uline \& Bray CHIH, SIN, SON

Allenrolfea occidentalis (S. Watson) Kuntze BCN, BCS, CHIH, COAH, DGO, NLE, SLP, SIN, SON, TAMS

*Amaranthus acanthobracteatus $\mathrm{Henr}$. CHIH, COAH

Amaranthus acanthochiton J.D. Sauer CHIH, COAH

Amaranthus acutilobus Uline \& W.L. Bray HGO, MEX, PUE, TLAX

*Amaranthus annectens S.F. Blake CAM, VER, YUC

Amaranthus australis (A. Gray) Sauer CAM, QROO, TAB, TAMS, VER

Amaranthus berlandieri (Moq.) Uline \& Bray COAH, NLE, TAMS

*Amaranthus brandegei Standl. NAY, SON

Amaranthus californicus (Moq.) S. Watson BCN

Amaranthus crassipes Schltdl. COAH, DGO, NLE, QRO, SLP, SON, VER

Amaranthus cruentus L. BCS, CHIS, JAL, MICH, OAX, PUE, VER

Amaranthus chihuahuensis S. Watson CHIH, DGO, HGO, PUE, QRO, SLP

Amaranthus dubius C. Mart. ex Thell. CAM, CHIS, COL, GRO, JAL, MOR, NLE, QROO, SON, TAB, VER, YUC
Amaranthus fimbriatus (Torr.) Benth. ex S. Watson BCN, BCS, CHIH, COL, HGO, JAL, MICH, SIN, SON

Amaranthus graecizans L. BCN, BCS, CHIH, COAH, DGO, JAL, NLE, SLP, SON, TAMS, ZAC

Amaranthus greggii S. Watson CAM, QROO, TAB, TAMS, VER, YUC

Amaranthus hybridus L. AGS, BCN, BCS, CAM, CHIS, CHIH, COAH, COL, CDMX, DGO, GTO, GRO, HGO, JAL, MEX, MICH, MOR, NAY, NLE, OAX, PUE, QRO, QROO, SLP, SIN, SON, TAB, TAMS, TLAX, VER, YUC, ZAC

Amaranthus hypochondriacus L. CHIH, COL, CDMX, GRO, JAL, MEX, MICH, OAX, PUE, SLP, SON, TLAX, VER

*Amaranthus lepturus S.F. Blake BCN, BCS, GRO, SIN

*Amaranthus leucocarpus S. Watson CHIH, JAL, QRO, SLP, SON, VER

Amaranthus obcordatus (A. Gray) Standl. BCN, BCS, SIN, SON

Amaranthus palmeri S. Watson AGS, BCN, BCS, CHIH, COAH, COL, DGO, GRO, HGO, JAL, MEX, MICH, MOR, NLE, OAX, PUE, QRO, SLP, SIN, SON, TAMS, TLAX, VER, ZAC

Amaranthus polygonoides $\mathrm{L}$. AGS, CAM, CHIS, COAH, COL, GTO, GRO, JAL, MICH, NAY, NLE, SLP, SIN, TAMS, VER, YUC, ZAC

Amaranthus powellii S. Watson BCN, CHIH, COAH, DGO, GRO, HGO, JAL, MEX, MICH, MOR, NLE, OAX, PUE, SON, ZAC

Amaranthus retroflexus L. BCN, BCS, CHIS, CHIH, COAH, COL, DGO, JAL, MEX, MOR, NAY, NLE, OAX, SLP, SIN, SON, TAMS, VER

Amaranthus scariosus Benth. CAM, CHIS, GRO, OAX, SLP, TAB, VER

Amaranthus scleropoides Uline \& Bray CHIH, COAH, TAMS Amaranthus spinosus L. BCN, BCS, CAM, CHIS, CHIH, COAH, COL, DGO, GTO, GRO, HGO, JAL, MEX, MICH, MOR, NAY, NLE, OAX, PUE, QRO, QROO, SLP, SIN, SON, TAB, TAMS, VER, YUC, ZAC

Amaranthus torreyi (A. Gray) Benth. ex S. Watson BCN, BCS, CHIH, COAH, DGO, GTO, JAL, MICH, SLP, SON

Amaranthus watsonii Standl. BCN, BCS, SIN, SON

Aphanisma blitoides Nutt. ex Moq. BCN, BCS

*Atriplex abata I.M. Johnst. COAH, NLE, SLP

Atriplex acanthocarpa (Torr.) S. Watson CHIH, COAH, DGO, NLE, SLP, SON, TAMS, VER, ZAC

Atriplex argentea Nutt. TAMS

*Atriplex barclayana (Benth.) D. Dietr. BCN, BCS, SIN, SON Atriplex canescens (Pursh) Nutt. AGS, BCN, BCS, CAM, CHIH, COAH, DGO, NLE, HGO, PUE, QROO, SLP, SIN, SON, TAMS, VER, YUC, ZAC

Atriplex confertifolia (Torr. \& Frém.) S. Watson CHIH

Atriplex coulteri (Moq.) D. Dietr. BCN

Atriplex elegans (Moq.) D. Dietr. BCN, CHIH, DGO, SON

*Atriplex frankenioides Moran BCS

Atriplex hymenelytra (Torr.) S. Watson BCN, BCS, SON

*Atriplex julacea $\mathrm{S}$. Watson BCN, BCS

Atriplex lentiformis (Torr.) S. Watson BCN, BCS, SON

Atriplex leucophylla (Moq.) D. Dietr. BCN, BCS 
Atriplex lindleyi Moq. BCN

*Atriplex linifolia Humb. \& Bonpl. ex Willd. COL, CDMX, DGO, GTO, JAL, MEX, MICH, TLAX, VER

*Atriplex magdalenae Brandegee BCN, BCS

Atriplex matamorensis A. Nelson TAMS

*Atriplex monilifera S. Watson COAH, DGO

*Atriplex muricata Humb. \& Bonpl. ex Willd. AGS, COAH, CDMX, DGO, GTO, HGO, JAL, MEX, MICH, NLE, PUE, QRO, SLP, SON, VER, ZAC

Atriplex obovata Moq. CHIH, COAH, DGO, NLE, SLP, ZAC

Atriplex pacifica A. Nelson BCN, BCS, SON

Atriplex parishii $\mathrm{S}$. Watson $\mathrm{BCN}$

Atriplex pentandra (Jacq.) Standl. CAM, QROO, TAMS, VER, YUC

Atriplex polycarpa (Torr.) S. Watson BCN, BCS, SON

*Atriplex pringlei Standl. GTO, NLE, SLP

*Atriplex prosopidum I.M. Johnst. COAH

*Atriplex pueblensis Standl. PUE, VER

*Atriplex reptans I.M. Johnst. COAH, NLE, SLP

*Atriplex stewartii I.M. Johnst. COAH, DGO

Atriplex tampicensis Standl. CAM, QROO, TAMS, VER, YUC

Atriplex texana $\mathrm{S}$. Watson $\mathrm{CHIH}, \mathrm{COAH}, \mathrm{NLE}, \mathrm{SLP}, \mathrm{SON}$, TAMS

*Atriplex valdesii Flores-Olvera SLP, ZAC

Atriplex watsonii A. Nelson BCN, BCS

Atriplex wrightii S. Watson $\mathrm{CHIH}, \mathrm{SON}$

Blitum spathulatum (A. Gray) S. Fuentes, Uotila \& Borsch BCN

Blutaparon vermiculare (L.) Mears CAM, CHIS, GRO, QROO,

SLP, SIN, TAMS, VER, YUC

Celosia argentea L. CHIS, COL, HGO, JAL, MEX, MOR, NAY, OAX, SLP, SON, TAB, TAMS, VER, YUC

*Celosia chiapensis Brandegee CHIS

* Celosia floribunda A. Gray BCN, BCS

*Celosia moquini Guill. COL, MICH

Celosia nitida Vahl CAM, CHIS, COAH, GRO, MICH, NAY, NLE, OAX, QRO, QROO, SLP, SON, TAMS, VER, YUC

*Celosia orcuttii Greenm. COL, JAL

Celosia palmeri S. Watson CHIH, COAH, NLE, SLP, ZAC

Celosia virgata Jacq. CAM, OAX, QRO, SLP, TAMS, VER, YUC

Corispermum americanum (Nutt.) Nutt. CHIH

Cyathula achyranthoides (Kunth) Moq. CHIS, OAX, TAB, VER

Cycloloma atriplicifolium (Spreng.) J.M. Coult. CHIH

Chamissoa acuminata Mart. CHIS, OAX, PUE

Chamissoa altissima (Jacq.) Kunth CAM, CHIS, COL, GRO,

HGO, JAL, MEX, MICH, NAY, OAX, PUE, QRO, QROO, SLP,

SIN, TAB, TAMS, VER, YUC

Chamissoa maximiliani Moq. CHIS, OAX

Chenopodium berlandieri Moq. BCN, CAM, CHIH, COAH, CDMX, DGO, GTO, HGO, MEX, MICH, NLE, OAX, PUE, QRO, QROO, SON, TAMS, TLAX, VER, YUC

*Chenopodium bipinnatifidum Moric. ex Moq. MICH

Chenopodium californicum (S. Watson) S. Watson BCN

Chenopodium fremontii S. Watson AGS, BCN, BCS, CHIH, COAH, CDMX, GTO, GRO, HGO, JAL, MEX, MICH, NLE, QRO, SLP, SON, TLAX, VER, ZAC
Chenopodium hians Standl. BCN

Chenopodium inamoenum Standl. BCN, CHIH

Chenopodium incanum (S. Watson) Heller BCN, CHIH, SON

Chenopodium leptophyllum Nutt. ex S. Watson BCN, CHIH, NLE, SON

*Chenopodium mexicanum Moq. COL, CDMX, HGO, JAL, MEX

Chenopodium neomexicanum Standl. CHIH, COAH, SON

*Chenopodium nuttalliae Saff. AGS, CHIS, CDMX, HGO, MEX, MICH, MOR, PUE, VER

Chenopodium opulifolium Schrad. ex W.D.J. Koch \& Ziz BCN Chenopodium pratericola Rydb. MEX, NLE, SON

Dysphania ambrosioides (L.) Mosyakin \& Clemants AGS, $\mathrm{BCN}, \mathrm{BCS}, \mathrm{CAM}, \mathrm{CHIS}, \mathrm{CHIH}, \mathrm{COAH}, \mathrm{COL}, \mathrm{CDMX}$, DGO, GTO, GRO, HGO, JAL, MEX, MICH, MOR, NAY, NLE, OAX, PUE, QRO, QROO, SLP, SIN, SON, TAB, TAMS, TLAX, VER, YUC, ZAC

Dysphania graveolens (Willd.) Mosyakin \& Clemants AGS, BCN, BCS, CHIS, CHIH, COAH, COL, CDMX, DGO, GTO, GRO, HGO, JAL, MEX, MICH, MOR, NLE, OAX, PUE, QRO, SLP, SIN, SON, TAMS, TLAX, VER, ZAC

Eurotia lanata (Pursh) Moq. BCN, BCS, CHIH, COAH, NLE, SLP, SON

Extriplex californica (Moq.) E.H. Zacharias BCN, BCS

Froelichia arizonica Thornber ex Standl. CHIH, COAH, DGO, NLE, SON

Froelichia floridana (Nutt.) Moq. CHIH, COAH, VER

Froelichia gracilis (Hook.) Moq. CHIH, COAH, NLE, SON, TAMS

Froelichia interrupta (L.) Moq. AGS, BCN, BCS, CHIH, COAH, COL, CDMX, DGO, GTO, GRO, HGO, JAL, MEX, MICH, NAY, NLE, OAX, PUE, QRO, SLP, SIN, SON, TAMS, VER, ZAC

*Froelichia xantusii R.A. McCauley BCS

Gomphrena caespitosa Torr. CHIH, SIN, SON, SON

* Gomphrena crassicaulis Brandegee VER

Gomphrena dispersa Standl. CAM, CHIS, GRO, HGO, JAL, MEX, MICH, MOR, OAX, QROO, TAB, VER, YUC

Gomphrena elegans Mart. CHIS

Gomphrena filaginoides M. Martens \& Galeotti CAM, CHIS, GRO, HGO, OAX, QRO, SLP, SIN, TAMS, VER, YUC

Gomphrena haageana Klotzsch CHIH, COAH, NLE, OAX

Gomphrena nitida Rothr. AGS, CAM, CHIS, CHIH, COAH, COL, CDMX, DGO, GTO, GRO, JAL, MEX, MICH, MOR, NAY, NLE, OAX, QRO, QROO, SLP, SON, VER, ZAC

*Gomphrena parviceps Standl. CDMX, GTO, GRO, HGO, JAL, MEX, MICH, MOR, QRO

* Gomphrena pilosa (M. Martens \& Galeotti) Moq. CHIS, GTO, JAL, MICH, NAY, OAX, QRO, VER, YUC

*Gomphrena pringlei Coult. \& Fisher CDMX, GRO, HGO, JAL, MEX, MICH, MOR, OAX, PUE, TLAX, VER

*Gomphrena procumbens Jacq. GRO, JAL, MICH, VER

Gomphrena pulchella Mart. MICH, MOR

Gomphrena serrata L. AGS, CAM, CHIS, CHIH, COAH, COL, CDMX, DGO, GTO, GRO, HGO, JAL, MEX, MICH, MOR, NAY, NLE, OAX, PUE, QRO, QROO, SLP, SIN, SON, TAB, TAMS, TLAX, VER, YUC, ZAC 
Gomphrena sonorae Torr. BCN, BCS, CHIH, NAY, SIN, SON Gomphrena viridis Wooton \& Standl. CHIH, SON

Gossypianthus lanuginosus (Poir.) Moq. CHIH, COAH, DGO, NLE, SON, TAMS

Guilleminea densa (Humb. \& Bonpl. ex Schult.) Moq. AGS, CHIH, COAH, CDMX, DGO, GTO, HGO, JAL, MEX, MICH, NLE, OAX, PUE, QRO, SLP, SON, TAMS, TLAX, VER, ZAC Hebanthe grandiflora (Hook.) Borsch \& Pedersen CHIS, GTO, GRO, JAL, MICH, OAX, SLP, TAMS, VER

*Iresine ajuscana Suessenguth \& Beyerle CDMX, GRO, MEX, $\mathrm{MICH}, \mathrm{OAX}$

*Iresine alternifolia $\mathrm{S}$. Watson BCN, BCS, SON

Iresine angustifolia Euphrasén BCN, BCS, CHIS, COL, GRO, JAL, MEX, MICH, MOR, OAX, VER

Iresine arbuscula Uline \& W.L. Bray CHIS, MEX, OAX, PUE, QRO, SLP, TAB, TAMS, VER

*Iresine arenaria Standl. SIN

Iresine calea (Ibañez) Standl. AGS, BCS, CAM, CHIS, CHIH, COAH, COL, CDMX, DGO, GTO, GRO, HGO, JAL, MEX, MICH, MOR, NAY, NLE, OAX, PUE, QRO, SLP, SIN, SON, TAMS, VER, ZAC

*Iresine cassiniiformis Schauer COL, CDMX, GTO, HGO, JAL, MEX, MICH, NLE, PUE, QRO, SLP, TAMS, VER, ZAC Iresine diffusa Humb. \& Bonpl. ex Willd. AGS, CAM, CHIS, CHIH, COL, CDMX, DGO, GTO, GRO, HGO, JAL, MEX, MICH, MOR, NAY, NLE, OAX, PUE, QRO, QROO, SLP, SIN, SON, TAB, TAMS, TLAX, VER, YUC, ZAC

*Iresine discolor Greenm. OAX, PUE

*Iresine frutescens Moq. CHIS, VER

Iresine grandis Standl. AGS, CDMX, GTO, GRO, HGO, JAL, MEX, MICH, OAX, QRO, SLP, TLAX, VER, ZAC

*Iresine hartmanii Uline CHIH, COL, DGO, GTO, JAL, MICH, SIN, SON

*Iresine hebanthoides Suess. OAX, VER

Iresine herbstii Lindl. MEX, MICH, MOR, NAY, OAX, VER

*Iresine herrerae S.F. Blake CHIS, OAX, VER

Iresine heterophylla Standl. CAM, CHIS, CHIH, COAH, CDMX, DGO, GTO, GRO, HGO, JAL, MEX, MICH, MOR, NLE, QRO, QROO, SLP, SON, TAB, VER, YUC, ZAC

Iresine interrupta Benth. CHIS, COL, CDMX, DGO, GTO, GRO, HGO, JAL, MEX, MICH, MOR, NAY, OAX, PUE, QRO, SLP, SIN, SON, TAMS, VER, ZAC

Iresine leptoclada (Hook. f.) Henr. CHIH, COAH, DGO, NLE, SLP, ZAC

Iresine nigra Uline \& W.L. Bray CAM, CHIS, COL, DGO, GRO, HGO, JAL, MICH, NAY, OAX, PUE, QRO, QROO, SLP, SIN, TAB, VER

*Iresine nitens Standl. OAX, PUE

*Iresine orientalis G.L. Nesom COAH, GTO, HGO, NLE, QRO, SLP, TAMS, VER

*Iresine pacifica Standl. COL, GRO, JAL, SIN

Iresine palmeri (S. Watson) Standl. HGO, NLE, SLP, TAMS, VER

*Iresine paniculata L. CAM, CHIH, MOR, OAX, SON, VER

* Iresine papantlana Loes. PUE, VER

*Iresine pringlei S. Watson JAL, OAX, PUE

*Iresine rotundifolia Standl. OAX, PUE, QRO, VER
*Iresine rzedowskii Zumaya, Flores-Olvera \& Borsch CHIH, DGO, GTO, GRO, JAL, MICH, NAY, OAX, PUE, QRO, SIN

*Iresine schaffneri $\mathrm{S}$. Watson AGS, CHIH, COAH, DGO, GTO, GRO, HGO, JAL, MEX, OAX, PUE, QRO, SLP, SON, VER, ZAC

*Iresine stricta Standl. OAX, PUE

*Iresine tomentella Standl. GTO, MICH, NLE, TAMS, VER

*Iresine valdesii Zumaya, Flores-Olvera \& Borsch PUE

*Lagrezia monosperma (Rose) Standl. CHIS, COL, GRO, JAL, MICH, OAX

*Meiomeria stellata (S. Watson) Standl. COAH, NLE

Monolepis nuttalliana (Schult.) Greene BCN, CHIH, COAH, NLE, SON

Nitrophila occidentalis (Moq.) S. Watson BCN, SON

Pfaffia iresinoides (Kunth) Spreng. CHIS, OAX

Pleuropetalum sprucei (Hook. f.) Standl. CHIS, COL, HGO, JAL, QRO, SLP, TAB, VER

*Salicornia ambigua Michx. SLP, TAMS, VER

Salicornia bigelovii Torr. BCN, BCS, CAM, GRO, QROO, SLP, SIN, SON, TAMS, VER, YUC

Salicornia europea L. BCN, BCS, SIN, SON

Salicornia subterminalis Parish BCN, BCS, SIN, SON

Salicornia virginica $\mathrm{L}$. BCN, BCS, COAH, SON, TAMS

Sarcobatus vermiculatus (Hook.) Torrey SON

Sarcocornia pacifica (Standl.) A.J. Scott BCN, BCS, SIN, SON, VER

Sarcocornia perennis (Mill.) A.J. Scott QROO, VER, YUC

Suaeda californica $\mathrm{S}$. Watson BCN, BCS, SON

Suaeda conferta (Small) I.M. Johnst. SLP, TAMS

Suaeda esteroa Ferren \& S.A. Whitmore BCN, BCS, SON

Suaeda fruticosa (L.) Hook. \& Arn. BCN, BCS, CHIH, COAH, SLP, SIN, SON

*Suaeda jacoensis I.M. Johnst. COAH, NLE, SLP

Suaeda linearis (Elliott) Moq. CAM, QROO, SLP, TAMS, VER, YUC

Suaeda mexicana (Standl.) Standl. CAM, COAH, DGO, GTO, HGO, MEX, MICH, NLE, QRO, QROO, SLP, VER, YUC

Suaeda nigra (Raf.) J.F. Macbr. BCN, BCS, CHIH, COL, CDMX, HGO, JAL, MEX, PUE, SLP, SIN, SON, TAMS, VER, ZAC

Suaeda nigrescens I.M. Johnst. CHIH, COAH, DGO, NLE, TAMS

*Suaeda palmeri (Standl.) Standl. COAH, DGO, NLE, ZAC

* Suaeda puertopenascoa M.C. Watson \& Ferren SON

* Suaeda pulvinata Alvarado Reyes \& Flores-Olvera CDMX, MEX, PUE, TLAX

Suaeda ramosissima (Standl.) I.M. Johnst. BCN, BCS, SIN, SON

Suaeda suffrutescens $\mathrm{S}$. Watson CHIH, COAH, DGO, NLE

Suaeda tampicensis (Standl.) Standl. TAMS

Suaeda taxifolia (Standl.) Standl. BCN, BCS, DGO

*Tidestromia carnosa (Steyerm.) I.M. Johnst. CHIH, COAH

Tidestromia eliassoniana Sánchez-del Pino BCS, CHIH, SIN, SON

*Tidestromia gemmata I.M. Johnst. CHIH, COAH, DGO

Tidestromia lanuginosa (Nutt.) Standl. BCN, CHIH, COAH, DGO, NLE, SLP, SIN, SON, TAMS, ZAC 
Tidestromia oblongifolia (S. Watson) Standl. BCN, SON

*Tidestromia rhizomatosa I.M. Johnst. COAH, TAMS

Tidestromia suffruticosa (Torr.) Standl. CHIH, COAH, DGO, NLE

*Tidestromia tenella I.M. Johnst. COAH, DGO

*Tidestromia valdesiana Sánchez-del Pino \& Flores-Olvera $\mathrm{COAH}$

\section{Family Amaryllidaceae}

Allium cernuum Roth CHIH, COAH, NLE, SON

Allium drummondii Regel CHIH, COAH, DGO, NLE, TAMS

*Allium eurotophilum Wiggins BCN

*Allium fantasmosense Traub SLP

Allium fimbriatum $\mathrm{S}$. Watson BCN, GTO, GRO, HGO

Allium glandulosum Link \& Otto AGS, BCS, CHIS, CHIH, COAH, COL, CDMX, DGO, GTO, GRO, HGO, JAL, MEX, MICH, MOR, NAY, NLE, OAX, PUE, QRO, SLP, SIN, SON, TAMS, TLAX, VER, ZAC

Allium haematochiton S. Watson BCN, SON

*Allium hintoniorum B.L. Turner COAH, NLE, TAMS

*Allium huntiae Traub DGO, SIN

Allium kunthii G. Don AGS, CHIS, CHIH, COAH, CDMX, DGO, GTO, GRO, HGO, JAL, MEX, MOR, NAY, NLE, OAX, PUE, QRO, SLP, SON, TAB, TAMS, VER, ZAC

Allium macropetalum Rydb. SON

*Allium mannii Traub \& T. Howard DGO

*Allium melliferum Traub JAL

*Allium mexicanum Traub TAMS

*Allium michoacanum Traub MICH

*Allium ownbeyi Traub COAH, NLE

Allium palmeri $\mathrm{S}$. Watson SON, TAMS

Allium peninsulare Lemmon ex Greene BCN, BCS

Allium plummerae $\mathrm{S}$. Watson SON

*Allium potosiense Traub SLP

Allium praecox Brandegee $\mathrm{BCN}$

*Allium pueblanum Traub PUE, VER

Allium runyonii Ownbey NLE, TAMS

*Allium stoloniferum T.D. Jacobsen HGO, MEX, QRO, TLAX, VER

*Allium telaponense Traub MEX

*Allium traubii T.M. Howard COAH, NLE

Allium unifolium Kellog BCN

Crinum americanum L. CAM, MOR, NAY, QROO, SIN, TAB, VER

Crinum erubescens Sol. BCS, CAM, CHIS, COL, CDMX, GRO, JAL, MEX, MICH, MOR, NAY, OAX, PUE, QRO, QROO, SLP, TAB, TAMS, VER, YUC

*Chlidanthus ehrenbergii (Klotzsch) Kunth OAX

*Diphalangium graminifolium Schauer VER

*Habranthus arenicola (Brandegee) Flagg, G. Lom. Sm. \& Meerow BCS

*Habranthus concolor Lindl. AGS, CDMX, GTO, HGO, JAL, MEX, MOR, QRO, SLP, TAMS, ZAC

*Habranthus conzattii (Greenm.) Flagg, G. Lom. Sm. \& Meerow OAX

*Habranthus chichimeca (T.M. Howard \& S. Ogden) Flagg, G. Lom. Smith \& Meerow NLE, SLP, TAMS, ZAC
*Habranthus howardii (Traub) T.M. Howard COAH, NLE, SLP *Habranthus immaculatus Traub \& Clint GTO, OAX, QRO

Habranthus longifolius (Hemsl.) Flagg, G. Lom. Sm. \& Meerow BCN, BCS, CHIH, COAH, CDMX, DGO, GTO, HGO, JAL, MEX, MOR, NLE, QRO, SLP, SON, TAMS, ZAC

*Habranthus medinae L.O. Alvarado \& García-Mend. PUE

*Habranthus mexicanus T.M. Howard HGO, QRO, SLP

*Habranthus tepicensis Greenm. ex Flagg. \& G. Lom. Sm. JAL, NAY

*Habranthus vittatus T.M. Howard OAX

*Hymenocallis acutifolia (Herb.) Sweet CAM, CHIS, COL, CDMX, GRO, HGO, JAL, MEX, MICH, MOR, NAY, OAX, PUE, SIN, TAMS, TLAX, YUC

*Hymenocallis araniflora T.M. Howard NAY, SIN

*Hymenocallis astrostephana T.M. Howard GRO

*Hymenocallis azteciana Traub COL, JAL, NAY

*Hymenocallis baumlii Ravenna CHIS

Hymenocallis caribaea (L.) Herb. CAM, YUC

*Hymenocallis cleo Ravenna CHIS

*Hymenocallis clivorum Laferr. SON

*Hymenocallis concinna Baker COL, GTO, HGO, JAL, MICH, MOR, NAY, ZAC

*Hymenocallis cordifolia Micheli GRO

*Hymenocallis durangensis T.M. Howard DGO

*Hymenocallis eucharidifolia Baker GRO

*Hymenocallis glauca (Zucc.) M. Roem. GRO, MEX, MICH, MOR, OAX, PUE

*Hymenocallis graminifolia Greenm. MEX, MOR

*Hymenocallis guerreroensis T.M. Howard GRO

*Hymenocallis harrisiana Herb. CDMX, GRO, HGO, MEX, MICH, MOR, OAX, QRO

*Hymenocallis howardii Bauml GTO, JAL, NAY

*Hymenocallis imperialis T.M. Howard HGO, QRO, SLP, VER

*Hymenocallis jaliscensis M.E. Jones JAL, NAY

*Hymenocallis leavenworthii (Standl. \& Steyerm.) Bauml COL, JAL, MICH

*Hymenocallis lehmilleri T.M. Howard GRO

Hymenocallis littoralis (Jacq.) Salisb. CAM, CHIS, CDMX, GRO, JAL, MEX, MICH, MOR, OAX, QROO, SLP, SIN, TAB, TAMS, VER, YUC

*Hymenocallis longibracteata Hochr. VER

*Hymenocallis maximilianii T.M. Howard GRO

*Hymenocallis partita Ravenna CHIS

*Hymenocallis phalangidis Bauml NAY

*Hymenocallis pimana Laferr. CHIH, SON

*Hymenocallis portamonetensis Ravenna CHIS

*Hymenocallis proterantha Bauml COL, JAL, MICH, NAY

*Hymenocallis pumila Bauml COL, JAL

*Hymenocallis sonorensis Standl. NAY, SIN, SON

*Hymenocallis vasconcelosii García-Mend. OAX, PUE

*Hymenocallis woelfleana T.M. Howard DGO, NAY, SIN

*Jaimehintonia gypsophila B.L. Turner NLE, TAMS

Nothoscordum bivalve (L.) Britton AGS, BCN, BCS, CHIS, CHIH, COAH, COL, CDMX, DGO, GTO, GRO, HGO, JAL, MEX, MICH, MOR, NLE, OAX, PUE, QRO, SLP, SON, TAMS, TLAX, VER, ZAC

Nothoscordum gramineum (Sims) P. Beauv. CHIS 
* Sprekelia clintiae Traub COL, JAL, MICH

*Sprekelia formosissima (L.) Herb. AGS, CHIS, CHIH, COL, CDMX, DGO, GTO, GRO, HGO, JAL, MEX, MICH, MOR, NAY, OAX, PUE, QRO, SLP, SIN, TLAX, VER, ZAC

*Sprekelia howardii Lehmiller GRO, PUE

*Zephyranthes bella T.M. Howard \& Ogden SLP, ZAC

Zephyranthes brevipes (Baker ex Donn. Sm.) Standl. CHIS, COAH, CDMX, GTO, HGO, JAL, MEX, MICH, MOR, QRO, SLP, TAMS, TLAX, VER

Zephyranthes carinata Herb. CAM, CHIS, CDMX, DGO, GTO, HGO, MEX, MICH, MOR, OAX, PUE, QRO, SLP, TAMS, VER Zephyranthes citrina Baker CAM, CHIS, QROO, TAB, YUC *Zephyranthes clintiae Traub GTO, QRO, SLP, TAMS, VER

*Zephyranthes crociflora T.M. Howard \& Ogden COAH

Zephyranthes chlorosolen (Herb.) D. Dietr. CHIH, COAH, NLE, OAX, SLP, TAMS, VER, YUC, ZAC

Zephyranthes chrysantha Greenm. \& C.H. Thomps. NLE, TAMS

*Zephyranthes dichromantha T.M. Howard SLP, TAMS Zephyranthes drummondii D. Don COAH, NLE, SLP Zephyranthes erubescens S. Watson NLE, SLP, TAMS *Zephyranthes fosteri Traub AGS, COL, CDMX, GTO, GRO, HGO, JAL, MEX, MICH, MOR, NAY, OAX, PUE, QRO, TLAX, VER, ZAC

*Zephyranthes katheriniae L.B. Spencer HGO, QRO, SLP

*Zephyranthes latissimifolia L.B. Spencer GTO, JAL, MICH

*Zephyranthes leucantha T.M. Howard HGO, QRO

*Zephyranthes lilacina Liebm. ND

Zephyranthes lindleyana Herb. CHIS, GRO, HGO, MEX, MOR, NAY, NLE, OAX, PUE, QRO, SLP, TAMS, VER, YUC, ZAC

*Zephyranthes macrosiphon Baker HGO, OAX, SLP, TAMS

*Zephyranthes minuta (Kunth) D. Dietr. HGO, VER

*Zephyranthes miradorensis (Kraenzl.) Espejo \& López-Ferrari VER

*Zephyranthes moctezumae T.M. Howard SLP, VER

*Zephyranthes morrisclintii Traub \& T.M. Howard COAH, NLE, TAMS

*Zephyranthes nelsonii Greenm. CHIS, OAX, VER, YUC

*Zephyranthes nymphaea T.M. Howard \& Ogden SLP, TAMS

*Zephyranthes orellanae Carnevali, Duno \& Tapia-Muñoz YUC

*Zephyranthes primulina T.M. Howard \& S. Ogden SLP, TAMS, VER

Zephyranthes pulchella J.G. Sm. NLE, TAMS

*Zephyranthes reginae T.M. Howard \& S. Ogden SLP, VER

*Zephyranthes sessilis Herb. CDMX, GTO, GRO, HGO, JAL,

MEX, MICH, MOR, OAX, PUE, QRO, TLAX, VER

*Zephyranthes stellatorosea G.L. Sm., Spurrier, Flagg \& Espejo HGO, PUE

*Zephyranthes subflava L.B. Spencer SLP, TAMS, ZAC

Zephyranthes verecunda Herb. CHIS, CDMX, HGO, JAL, MEX, MICH, MOR, OAX, PUE, SLP

\section{Family Anacampserotaceae}

*Talinaria coahuilensis (S. Watson) P. Wilson COAH, NLE, SLP

Talinopsis frutescens A. Gray AGS, CHIH, COAH, DGO, GTO, HGO, JAL, MEX, MICH, NLE, PUE, QRO, SLP, TAMS, ZAC

\section{Family Anacardiaceae}

*Actinocheita filicina (DC.) F.A. Barkley GRO, MEX, MOR, OAX, PUE

*Amphipterygium adstringens (Schltdl.) Standl. AGS, COL, DGO, GRO, JAL, MEX, MICH, MOR, NAY, OAX, PUE, SLP, VER, ZAC

*Amphipterygium glaucum (Hemsl. \& Rose) Standl. COL, GRO, JAL, MICH, NAY

*Amphipterygium molle (Hemsl.) Hemsl. \& Rose AGS, DGO, JAL, NAY, ZAC

Amphipterygium simplicifolium (Standl.) Cuevas-Figueroa OAX

Astronium graveolens Jacq. CAM, CHIS, COL, GRO, HGO, JAL, MICH, NAY, OAX, QRO, QROO, TAB, VER, YUC

Attilaea abalak E. Martínez \& Ramos CAM, QROO, YUC

*Bonetiella anomala (I.M. Johnst.) Rzed. COAH, DGO, HGO, NLE, QRO, SLP, TAMS, ZAC

Comocladia guatemalensis Donn. Sm. CHIS

*Comocladia macrophylla (Hook. \& Arn.) Riley GRO

*Comocladia mollissima Kunth CHIS, COL, GRO, JAL, MEX, MICH, MOR, NAY, OAX, PUE, SLP, SIN, TAB, TAMS, VER

*Comocladia palmeri Rose COL, GRO, JAL, OAX

*Comocladia repanda S.F. Blake GRO, OAX

*Cotinus carranzae Rzed. \& Calderón QRO, SLP

*Cotinus chiangii (Young) Rzed. \& Calderón COAH, DGO

* Cyrtocarpa edulis (Bandegee) Standl. BCS, SON

* Cyrtocarpa kruseana Fonseca CHIS, COL, GRO, JAL, NAY

*Cyrtocarpa procera Kunth BCS, CHIS, COL, DGO, GRO, JAL, MEX, MICH, MOR, NAY, OAX, PUE, TAMS, ZAC

Malosma laurina (Nutt.) Nutt. ex Abrams BCN, BCS, CHIH, SON

Metopium brownei (Jacq.) Urb. CAM, CHIS, QROO, TAB, VER, YUC

Mosquitoxylum jamaicense Krug \& Urb. CAM, CHIS, OAX, QROO, TAB, VER

*Pachycormus discolor (Benth.) Coville BCN, BCS

Pistacia mexicana Kunth AGS, CHIS, CHIH, COAH, COL, GTO, GRO, HGO, JAL, MEX, MICH, NLE, OAX, PUE, QRO, SLP, TAMS, VER, ZAC

*Pseudosmodingium andrieuxii (Baill.) Engl. GRO, HGO, MEX, MOR, OAX, PUE, QRO, SLP, VER

*Pseudosmodingium barkleyi Miranda GRO

*Pseudosmodingium perniciosum (Kunth) Engl. COL, CDMX, GRO, JAL, MEX, MICH, MOR, NAY, OAX, PUE, QRO, SIN, TAMS

*Pseudosmodingium rhoifolium (DC.) F.A. Barkley ND

*Pseudosmodingium virletii (Baill.) Engl. GTO, HGO, MEX, QRO, SLP

*Rhus andrieuxii Engelm. COAH, HGO, NLE, OAX, PUE, QRO, SLP, VER, ZAC

Rhus aromatica Aiton CHIH, COAH, DGO, GTO, HGO, JAL, MEX, NLE, OAX, QRO, SLP, SIN, SON, TAMS

$*$ Rhus arsenei F.A. Barkley OAX, PUE

* Rhus barclayi (Hemsl.) Standl. COL, GRO, JAL, NAY

* Rhus ciliolata Turcz. OAX, PUE

* Rhus chiangii D.A. Young COAH, DGO 
* Rhus chondroloma Standl. GRO, OAX, PUE

Rhus diversiloba Torr. \& A. Gray BCN, CHIH, MICH, SON

* Rhus duckeri F.A. Barkley OAX

*Rhus eximia (Greene) Standl. DGO, TAMS

* Rhus galeottii Standl. GRO, OAX

Rhus glabra L. CHIH, SON

*Rhus hartmanii F.A. Barkley CHIH, SON

Rhus integrifolia (Nutt.) Benth. \& Hook. f. ex Rothr. BCN, BCS, SON

* Rhus jaliscana Standl. JAL, ZAC

Rhus kearneyi F.A. Barkley BCN, BCS, SON

Rhus lanceolata (A. Gray) Britton CHIH, COAH, NLE, SLP, TAMS

*Rhus lentii Kellogg BCN, BCS

Rhus microphylla Engelm. AGS, BCN, BCS, CHIH, COAH, DGO, GTO, HGO, JAL, NLE, QRO, SLP, SON, TAMS, VER, ZAC

*Rhus mollis Jacq. HGO, OAX, QRO, VER

*Rhus muelleri Standl. \& F.A. Barkley COAH, NLE

*Rhus nelsonii F.A. Barkley GRO

*Rhus oaxacana Loes. GRO, OAX

Rhus ovata $\mathrm{S}$. Watson BCN, BCS, CHIH, SON

* Rhus pachyrrhachis Hemsl. CHIS, COAH, COL, DGO, GTO, HGO, JAL, MICH, NLE, OAX, QRO, PUE, SLP, SIN, TAMS, VER, ZAC

Rhus schiedeana Schltdl. BCN, BCS, CHIS, COAH, DGO, GTO, GRO, HGO, JAL, MEX, NAY, OAX, PUE, QRO, SLP, VER, ZAC

*Rhus standleyi F.A. Barkley CHIS, CDMX, GRO, HGO, MEX, OAX, PUE, QRO, TLAX, VER

*Rhus tepetate Standl. \& F.A. Barkley BCS, CHIH, SON

Rhus terebinthifolia Schltdl. \& Cham. CHIS, CHIH, COL, DGO, GRO, HGO, JAL, MEX, MICH, MOR, NAY, OAX, PUE, QRO, SLP, SIN, SON, TAMS, VER, ZAC

Rhus trilobata Nutt. AGS, BCN, BCS, CHIH, COAH, COL, DGO, GTO, HGO, JAL, MICH, NAY, NLE, OAX, QRO, SLP, SIN, SON, TAMS, VER, ZAC

Rhus vestita Loes. CHIS

Rhus virens Lindh. ex A. Gray BCN, BCS, CHIH, COAH, DGO, HGO, JAL, MEX, NLE, OAX, PUE, QRO, SLP, SIN, SON, TAMS, VER, ZAC

Spondias mombin L. BCS, CAM, CHIS, COL, GTO, GRO, HGO, JAL, MEX, MICH, MOR, NAY, OAX, PUE, QRO, QROO, SLP, SIN, TAB, TAMS, VER, YUC

Spondias purpurea L. CAM, CHIS, CHIH, COL, DGO, GRO, HGO, JAL, MEX, MICH, MOR, NAY, OAX, PUE, QRO, QROO, SLP, SIN, SON, TAB, TAMS, VER, YUC, $\mathrm{ZAC}$

Spondias radlkoferi Donn. Sm. CAM, CHIS, GRO, NAY, OAX, QROO, TAB, VER, YUC

*Tapirira chimalapana T. Wendt \& J.D. Mitch. OAX, VER

Tapirira mexicana Marchand CHIS, OAX, PUE, VER

Toxicodendron radicans (L.) Kuntze AGS, BCN, BCS, CHIS, CHIH, COAH, COL, CDMX, DGO, GTO, GRO, HGO, JAL, MEX, MICH, MOR, NAY, NLE, OAX, PUE, QRO, SLP, SIN, SON, TAMS, VER, YUC, ZAC

Toxicodendron rydbergii (Rydb.) Greene CHIH, SON
Toxicodendron striatum (Ruiz \& Pav.) Kuntze CAM, CHIS, HGO, OAX, QROO, VER

\section{Family Annonaceae}

Anaxagorea guatemalensis Standl. OAX, VER

*Annona contrerasii J. Jiménez Ram. \& J.C. Soto GRO, MICH Annona diversifolia Saff. CHIS, COL, GRO, JAL, MEX, MICH, MOR, OAX, QROO, VER, YUC

Annona glabra L. CAM, CHIS, COL, GRO, JAL, NAY, OAX, QROO, TAB, TAMS, VER, YUC

*Annona globiflora Schltdl. CHIS, GTO, HGO, MEX, QRO, QROO, SLP, TAMS, VER

Annona liebmanniana Baill. CHIS, OAX, VER

*Annona longiflora S. Watson COL, GRO, JAL, MEX, MICH, MOR, NAY, OAX, PUE, VER, ZAC

*Annona longipes Saff. VER

Annona lutescens Saff. CHIS, JAL, VER

Annona macroprophyllata Donn. Sm. CHIS, VER

Annona mucosa Jacq. CHIS, OAX, PUE, TAB, VER

Annona palmeri Saff. COL, GRO, JAL, MICH, OAX, VER

Annona primigenia Standl. \& Steyerm. CAM, CHIS, QROO, VER, YUC

Annona purpurea Moc. \& Sessé ex Dunal CHIS, COL, JAL, MICH, OAX, PUE, QROO, TAB, VER, YUC

Annona rensoniana (Standl.) H. Rainer CHIS, OAX, PUE, TAB, VER

Annona reticulata L. CAM, CHIS, COL, DGO, GRO, HGO, JAL, MEX, MICH, MOR, NAY, OAX, PUE, QRO, QROO, SLP, SIN, TAB, TAMS, VER, YUC

Annona scleroderma Saff. CHIS

Annona spraguei Saff. CHIS

Annona squamosa L. CAM, CHIS, COL, GRO, JAL, MEX, MICH, MOR, OAX, PUE, QRO, QROO, SLP, SIN, SON, TAB, VER, YUC, ZAC

*Cymbopetalum baillonii R.E. Fr. CHIS, MEX, OAX, PUE, TAB, VER

*Cymbopetalum gracile R.E. Fr. GRO, OAX, VER

* Cymbopetalum hintonii Lundell JAL, MICH, NAY

* Cymbopetalum mayanum Lundell CAM, CHIS, QROO

Cymbopetalum mirabile R.E. Fr. CHIS, VER

* Cymbopetalum parviflorum N.A. Murray OAX

Cymbopetalum penduliflorum (Dunal) Baill. CAM, CHIS, GRO,

JAL, MICH, NAY, OAX, PUE, TAB, VER

Cymbopetalum stenophyllum Donn. Sm. CHIS, GRO, OAX

*Desmopsis erythrocarpa Lundell CHIS

*Desmopsis guerrerensis González-Martínez \& J. Jiménez Ram. GRO

*Desmopsis lanceolata Lundell CHIS, OAX

*Desmopsis mexicana R.E. Fr. MICH

Desmopsis schippii Standl. CHIS

Desmopsis stenopetala (Donn. Sm.) R.E. Fr. CHIS

*Desmopsis trunciflora (Schltdl. \& Cham.) G.E. Schatz CHIS, COL, JAL, NAY, OAX, TAB, VER

*Desmopsis uxpanapensis G.E. Schatz OAX, VER

Guatteria amplifolia Triana \& Planch. CHIS, GRO, OAX, PUE, TAB, VER

Guatteria anomala R.E. Fr. CHIS, GRO, OAX, TAB, VER 
Guatteria dolichopoda Donn. Sm. CHIS

* Guatteria galeottiana Baill. CAM, OAX, VER

*Guatteria jurgensenii Hemsl. OAX, TAB, VER

* Guatteria macrantha C. Presl ND

*Malmea gaumeri (Greenm.) Lundell CHIS, VER, YUC

Mosannona depressa (Baill.) Chatrou CAM, CHIS, GRO, JAL,

MICH, OAX, PUE, QROO, TAB, VER, YUC

Oxandra guatemalensis Lundell CHIS

Oxandra lanceolata (Sw.) Baill. CHIS, COL, GRO, JAL, MICH, QROO

Oxandra maya Miranda CHIS, TAB

Sapranthus campechianus (Kunth) Standl. CAM, CHIS, GRO, QROO, TAB, VER, YUC

*Sapranthus chiapensis G.E. Schatz CHIS, OAX

Sapranthus microcarpus (Donn. Sm.) R.E. Fr. CHIS, COL, GRO, JAL, MOR, OAX, SIN, VER

Sapranthus violaceus (Dunal) Saff. COL, GRO, JAL, NAY, OAX, SIN, VER

*Stenanona flagelliflora T. Wendt \& G.E. Schatz OAX, VER

*Stenanona humilis (Miranda) G.E. Schatz CHIS, OAX, VER

* Stenanona migueliana Ortiz-Rodr. \& G.E. Schatz CHIS

Stenanona stenopetala (Donn. Sm.) G.E. Schatz CHIS, TAB

*Stenanona wendtii G.E. Schatz \& Maas OAX, VER

*Tridimeris hahniana Baill. OAX, QRO, SLP, VER

*Unonopsis mexicana Maas \& Westra OAX

Unonopsis pittieri Saff. CHIS

Xylopia frutescens Aubl. CAM, CHIS, COL, OAX, QROO, TAB, VER

\section{Family Apiaceae}

Aletes acaulis (Torr.) J.M. Coult. \& Rose CHIH, COAH Ammoselinum giganteum J.M. Coult. \& Rose COAH, SON Ammoselinum popei Torr. \& A. Gray COAH, NLE, TAMS *Angelica nelsonii J.M. Coult. \& Rose AGS, CHIS, CDMX, GRO, HGO, JAL, MEX, MICH, OAX, PUE, VER, ZAC Apiastrum angustifolium Nutt. ex Torr. \& Gray BCN, BCS Arracacia aegopodioides (Kunth) J.M. Coult. \& Rose CHIS, CDMX, GTO, GRO, HGO, JAL, MEX, MICH, MOR, NLE, OAX, PUE, QRO, SLP, TAMS, VER

Arracacia atropurpurea (Lehm.) Benth. \& Hook. f. ex Hemsl. AGS, CHIS, COAH, CDMX, DGO, GTO, GRO, HGO, JAL, MEX, MICH, MOR, NLE, OAX, QRO, SLP, SIN, TAMS, TLAX, VER, ZAC

Arracacia bracteata J.M. Coult. \& Rose BCS, CHIS, OAX, TAB, VER

*Arracacia brandegeei J.M. Coult. \& Rose BCS

*Arracacia compacta Rose OAX, PUE

Arracacia donnell-smithii J.M. Coult. \& Rose CHIS, OAX

*Arracacia ebracteata (Rose) Mathias \& Constance CHIS, GRO

*Arracacia filipes Mathias \& Constance DGO, SIN

*Arracacia fruticosa Rose OAX, PUE

*Arracacia hemsleyana J.M. Coult. \& Rose GRO, OAX, PUE, TAMS, VER

*Arracacia hintonii Constance \& Affolter NLE, TAMS

*Arracacia longipedunculata J.M. Coult. \& Rose CHIS, MEX, $\mathrm{MICH}, \mathrm{MOR}$
*Arracacia macvaughii Mathias \& Constance GTO, JAL, MICH, QRO

*Arracacia molseedii Mathias \& Constance OAX

Arracacia nelsonii J.M. Coult. \& Rose CHIS, OAX, PUE, SLP, VER, ZAC

*Arracacia papillosa Mathias \& Constance JAL

*Arracacia pringlei J.M. Coult. \& Rose CHIS, OAX

*Arracacia purpusii Rose PUE

*Arracacia quadrifida Constance \& Affolter JAL, MEX, MOR, QRO, SLP, ZAC

*Arracacia ravenii Constance \& Affolter CHIS, OAX

*Arracacia rigida J.M. Coult. \& Rose CHIS, CDMX, GTO, HGO, JAL, MEX, MICH, MOR, QRO, VER

*Arracacia schiedei (H. Wolff) Mathias \& Constance VER

*Arracacia schneideri Mathias \& Constance COAH, NLE

*Arracacia ternata Mathias \& Constance COAH, NLE, TAMS Arracacia tolucensis (Kunth) Hemsl. COL, COAH, CDMX, DGO, GTO, GRO, HGO, JAL, MEX, MICH, MOR, NAY, NLE, OAX, QRO, SLP, SIN, TAMS, TLAX, VER, ZAC

Bowlesia flabilis J.F. Macbr. CDMX, GTO, GRO, HGO, MEX, $\mathrm{MICH}, \mathrm{QRO}$

Bowlesia incana Ruiz \& Pav. BCN, BCS, SON, TAMS

Cicuta douglasii (DC.) J.M. Coult. \& Rose CHIH

* Coaxana bambusioides Mathias \& Constance GRO, OAX

Coaxana purpurea J.M. Coult. \& Rose CHIS, GRO, MICH,

OAX

Conioselinum mexicanum J.M. Coult. \& Rose CHIH, JAL, SIN, SON

*Coulterophytum holwayi Rose JAL, MICH

*Coulterophytum laxum B.L. Rob. JAL

*Coulterophytum macrophyllum J.M. Coult. \& Rose DGO, NAY, SIN

Cyclospermum leptophyllum (Pers.) Sprague ex Britton \& P. Wilson AGS, BCN, BCS, CHIS, CHIH, COAH, COL, CDMX, GTO, GRO, HGO, JAL, MEX, MICH, MOR, NLE, OAX, PUE, QRO, SLP, SON, TAMS, TLAX, VER, ZAC

Cymopterus fendleri A. Gray TAMS

Cymopterus multinervatus (J.M. Coult. \& Rose) Tidestrom BCN, SON

*Chaerophyllum orizabae (I.M. Johnst.) K.F. Chung CDMX, HGO, MEX, PUE, VER

*Chaerophyllum tolucanum (I.M. Johnst.) K.F. Chung JAL, MEX, MICH, TLAX

*Dahliaphyllum almedae Constance \& Breedlove GRO

Daucus montanus Humb. \& Bonpl. ex Spreng. CHIS, CHIH, COAH, COL, CDMX, DGO, GTO, GRO, HGO, JAL, MEX, MICH, MOR, NLE, OAX, PUE, QRO, SLP, SIN, SON, TAMS, TLAX, VER, ZAC

Daucus pusillus Michx. BCN, BCS, CHIH, COAH, DGO, NLE, SON, TAMS

*Donnellsmithia ampulliformis Mathias \& Constance GRO, $\mathrm{MICH}$

*Donnellsmithia biennis (J.M. Coult. \& Rose) Mathias \& Constance GRO, MEX, MICH, MOR

*Donnellsmithia breedlovei Mathias \& Constance CHIS

*Donnellsmithia coahuilensis Mathias \& Constance COAH 
*Donnellsmithia cordata (J.M. Coult. \& Rose) Mathias \& Constance GRO, OAX

*Donnellsmithia dissecta (J.M. Coult. \& Rose) Mathias \& Constance CHIS, OAX

*Donnellsmithia glauca J.M. Coult. \& Rose GRO, OAX

Donnellsmithia guatemalensis J.M. Coult. \& Rose CHIS, OAX, VER

*Donnellsmithia hintonii Mathias \& Constance MEX, MICH

Donnellsmithia juncea (Spreng.) Mathias \& Constance CHIS, CHIH, COL, CDMX, DGO, GTO, GRO, HGO, JAL, MEX, MICH, MOR, NAY, NLE, OAX, PUE, QRO, SIN, SON, VER

*Donnellsmithia madrensis (J.M. Coult. \& Rose) Mathias \& Constance JAL, ZAC

*Donnellsmithia mexicana (B.L. Rob.) Mathias \& Constance COL, GTO, GRO, JAL, MEX, MICH, MOR, NAY, OAX, QRO

*Donnellsmithia ovata (J.M. Coult. \& Rose) Mathias \& Constance DGO, JAL, NAY, ZAC

*Donnellsmithia pinnatisecta (L. Riley) Mathias \& Constance JAL, MEX, MICH, NAY

*Donnellsmithia reticulata (J.M. Coult. \& Rose) Mathias \& Constance DGO, JAL, ZAC

*Donnellsmithia serrata (J.M. Coult. \& Rose) Mathias \& Constance CHIS, GRO, MEX, MICH, MOR, NAY, OAX, PUE

*Donnellsmithia silvicola Constance \& Bye CHIH

*Donnellsmithia submontana (J.M. Coult. \& Rose) Mathias \& Constance DGO, NAY

*Donnellsmithia ternata (S. Watson) Mathias \& Constance CHIH, COAH, DGO, JAL, NLE, OAX, SON, ZAC

*Donnellsmithia tuberosa (J.M. Coult. \& Rose) Mathias \& Constance GTO, GRO, HGO, JAL, MEX, MICH, OAX, QRO

Enantiophylla heydeana J.M. Coult. \& Rose COL, JAL, MEX, MICH, NAY, QRO, SLP, SIN

*Eryngium alternatum J.M. Coult. \& Rose CDMX, GRO, JAL, MEX, MICH, MOR, OAX, PUE

*Eryngium beecheyanum Hook. \& Arn. CHIH, COL, DGO, GTO, GRO, HGO, JAL, MEX, MICH, MOR, NAY, OAX, QRO, SIN, SON, TLAX, VER, ZAC

*Eryngium bonplandii F. Delaroche CDMX, GTO, HGO, MEX, MICH, MOR, OAX, QRO, SLP, VER

Eryngium carlinae F. Delaroche AGS, CHIS, CHIH, CDMX, DGO, GTO, GRO, HGO, JAL, MEX, MICH, MOR, OAX, PUE, QRO, SLP, TAB, TLAX, VER, ZAC

*Eryngium cervantesii F. Delaroche GTO, HGO, MEX, MICH, MOR, QRO

*Eryngium columnare Hemsl. CDMX, GRO, HGO, JAL, MEX, MICH, MOR, QRO, SLP, TAMS, TLAX, VER

*Eryngium comosum F. Delaroche CDMX, GTO, GRO, HGO, JAL, MEX, MICH, PUE, QRO, TLAX, VER, ZAC

*Eryngium compactum Small TAMS

*Eryngium crassisquamosum Hemsl. DGO, MEX, MICH, NAY, TAMS

Eryngium cymosum F. Delaroche AGS, CHIS, DGO, GTO, GRO, HGO, JAL, MEX, MICH, OAX, ZAC

*Eryngium deppeanum Schltdl. \& Cham. CDMX, HGO, MEX, OAX, PUE, VER

*Eryngium ferrisiae Constance JAL, NAY
Eryngium foetidum L. CHIS, OAX, PUE, QRO, SLP, TAB, VER

*Eryngium galeottii Hemsl. MEX, MICH, MOR, OAX

*Eryngium gentryi Constance \& Bye CHIH, SON

Eryngium ghiesbreghtii Decne. CHIS, COL, GRO, JAL, MEX, $\mathrm{MICH}, \mathrm{MOR}, \mathrm{OAX}$

*Eryngium globosum Hemsl. DGO, GRO, JAL, MEX, MICH, MOR, NAY, OAX

Eryngium gracile F. Delaroche CHIS, DGO, GTO, GRO, JAL, MEX, MICH, OAX, QRO, SLP

*Eryngium gramineum F. Delaroche COAH, GTO, MICH, NLE, OAX, SLP, SON, TAMS

Eryngium guatemalense Hemsl. CHIS, OAX

*Eryngium hemsleyanum $\mathrm{H}$. Wolff. COAH, NLE, OAX, SLP, TAMS

Eryngium heterophyllum Engelm. AGS, CHIH, COAH, DGO, GTO, GRO, JAL, MEX, MICH, MOR, NAY, NLE, OAX, QRO, SLP, SIN, SON, ZAC

Eryngium humile Cav. CHIH, CDMX, GTO, HGO, MEX, MICH, MOR, OAX, TLAX, VER

*Eryngium involucratum J.M. Coult. \& Rose OAX

*Eryngium jaliscense Mathias \& Constance COL, JAL

Eryngium lemmonii J.M. Coult. \& Rose CHIH, DGO, SIN, SON

*Eryngium longifolium Cav. CHIH, COAH, COL, GTO, GRO, HGO, JAL, MEX, MICH, NAY, NLE, OAX, SIN, SON, VER

Eryngium longirameum Turcz. CHIS, OAX

*Eryngium mexiae Constance COL, GTO, GRO, JAL, MICH, NAY, SIN, ZAC

*Eryngium mexicanum S. Watson CHIH, DGO, MEX, MICH, MOR

*Eryngium monocephalum Cav. COL, CDMX, HGO, JAL, MEX, MICH, MOR, OAX, PUE, TLAX, VER

Eryngium nasturtiifolium Juss. ex F. Delaroche BCN, BCS, CHIS, COAH, COL, JAL, NLE, OAX, QRO, SLP, SIN, SON, TAB, TAMS, VER, ZAC

*Eryngium palmeri Hemsl. COL, DGO, GRO, JAL, MEX, $\mathrm{MICH}, \mathrm{ZAC}$

Eryngium parishii J.M. Coult. \& Rose BCN

*Eryngium pectinatum C. Presl ex DC. AGS, GTO, GRO, HGO, JAL, MEX, MICH, MOR, OAX, PUE, QRO, SLP, TAMS, TLAX, ZAC

Eryngium phyteumae F. Delaroche CHIH, MEX, MICH, SON *Eryngium pilularioides Hemsl. \& Rose HGO, VER

*Eryngium pringlei Hemsl. \& Rose COAH, GTO, NLE, SLP

*Eryngium proteiflorum F. Delaroche CDMX, JAL, MEX, MICH, PUE, TLAX, VER

*Eryngium purpusii Hemsl. \& Rose OAX, PUE

Eryngium reptans Hemsl. CHIS

*Eryngium rosei Hemsl. DGO, JAL, NAY, ZAC

Eryngium scaposum Turcz. CHIS, GRO, OAX, PUE, VER

*Eryngium serratum Cav. COAH, CDMX, GTO, HGO, JAL, MEX, MICH, NLE, OAX, QRO, SLP, TAMS, TLAX, VER, ZAC

Eryngium sparganophyllum Hemsl. CHIH, DGO, JAL, MICH, NAY, SON, ZAC

*Eryngium spiculosum Hemsl. GRO, MEX, MICH, MOR

*Eryngium strotheri Constance \& Affolter CHIS 
*Eryngium subacaule Cav. MEX, MICH, MOR

*Eryngium tzeltal Constance CHIS, OAX

*Eryngium venustum Bartlett \& Constance NLE, TAMS, VER

*Ligusticum madrense J.M. Coult. \& Rose CHIH, SON

Ligusticum porteri J.M. Coult. \& Rose CHIH, DGO, SON

Lilaeopsis schaffneriana (Schltdl.) J.M. Coult. \& Rose AGS, CHIH, COAH, CDMX, DGO, HGO, JAL, MEX, MICH, MOR, NLE, PUE, QRO, SLP, SON, VER, ZAC

Lomatium insulare (Eastw.) Munz BCN, BCS

Lomatium mohavensis J.M. Coult. \& Rose BCN

Lomatium nevadense (S. Watson) J.M. Coult. \& Rose SON

* Mathiasella bupleuroides Constance \& Hitchc. NLE

Micropleura renifolia Lag. CHIS, COL, DGO, GRO, HGO,

JAL, MEX, MICH, MOR, NAY, OAX, PUE, QRO, SLP, SIN,

VER

Myrrhidendron donnellsmithii J.M. Coult. \& Rose CHIS

*Neogoezia breedlovei Constance GRO, JAL

*Neogoezia gracilipes (Hemsl.) Hemsl. GRO, MEX, MICH, OAX

*Neogoezia minor Hemsl. OAX

*Neogoezia planipetala (Hemsl.) Hemsl. DGO, JAL, MEX, MICH, NAY, VER, ZAC

Neonelsonia acuminata (Benth.) J.M. Coult. \& Rose ex Drude CHIS

Osmorhiza bipatriata Constance \& Shan COAH, NLE

*Osmorhiza geohintonii B.L. Turner NLE

Osmorhiza mexicana Griseb. CHIH, COAH, CDMX, HGO,

MEX, MICH, MOR, NLE, OAX, PUE, QRO, SLP, SON,

TAMS, TLAX, VER

Ottoa oenanthoides Kunth CHIS, OAX, VER

* Prionosciadium acuminatum B.L. Rob. GTO, GRO, JAL,

MEX, MICH, MOR, NAY, OAX, ZAC

*Prionosciadium bellii Mathias \& Constance MEX, MICH

* Prionosciadium cuneatum J.M. Coult. \& Rose JAL, MEX, MICH, MOR, QRO

*Prionosciadium dissectum J.M. Coult. \& Rose COL, DGO, JAL

*Prionosciadium diversifolium Rose GRO, HGO, JAL, MEX, MICH, MOR, NAY, OAX, PUE, QRO, VER, ZAC

*Prionosciadium filifolium J.M. Coult. \& Rose DGO, JAL, ZAC

*Prionosciadium gomez-pompai Lincoln \& Affolter VER

* Prionosciadium humile Rose COAH, NLE

* Prionosciadium lilacinum Mathias \& Constance JAL, NAY

*Prionosciadium linearifolium (S. Watson) J.M. Coult. \& Rose JAL, ZAC

*Prionosciadium macrophyllum J.M. Coult. \& Rose CHIH, DGO, JAL, SIN, ZAC

*Prionosciadium madrense S. Watson CHIH, DGO, SON

Prionosciadium megacarpum J.M. Coult. \& Rose MEX, MICH, OAX

*Prionosciadium nelsonii J.M. Coult. \& Rose CHIS, GRO, MEX, MICH, MOR, OAX, ZAC

*Prionosciadium palmeri Rose SLP

*Prionosciadium saraviki Laferr. CHIH

* Prionosciadium serratum J.M. Coult. \& Rose DGO, JAL, NAY

Prionosciadium thapsoides (DC.) Mathias AGS, CHIH, CDMX,
DGO, GTO, GRO, HGO, JAL, MEX, MICH, NAY, OAX, PUE, QRO, SLP, TAMS, TLAX, VER, ZAC

*Prionosciadium townsendii Rose CHIH, NAY, SON

*Prionosciadium watsoni J.M. Coult. \& Rose AGS, CHIH, DGO, GTO, HGO, MEX, QRO, SLP, SON, ZAC

Pseudocymopterus montanus (A. Gray) J.M. Coult. \& Rose CHIH, DGO, JAL, SON, ZAC

* Rhodosciadium argutum (Rose) Mathias \& Constance SLP Rhodosciadium diffusum (J.M. Coult. \& Rose) Mathias \& Constance CHIS, COL, GRO, MEX, MICH, MOR, OAX

*Rhodosciadium dissectum J.M. Coult. \& Rose OAX

Rhodosciadium glaucum J.M. Coult. \& Rose GRO, MEX, OAX, PUE

*Rhodosciadium longipes (Rose) Mathias \& Constance HGO, PUE, VER

*Rhodosciadium macrophyllum Mathias \& Constance DGO, JAL, NAY, SIN

*Rhodosciadium macvaughiae Mathias \& Constance JAL

*Rhodosciadium montanum (J.M. Coult. \& Rose) Mathias \& Constance DGO, GTO, HGO, JAL, MEX, MICH, QRO, SLP

*Rhodosciadium nelsonii (J.M. Coult. \& Rose) Mathias \& Constance CHIS

*Rhodosciadium nudicaule Mathias \& Constance OAX, QRO, VER

*Rhodosciadium pringlei $\mathrm{S}$. Watson CHIH, COL, DGO, GTO, GRO, JAL, MICH, OAX, SIN

*Rhodosciadium purpureum (Rose) Mathias \& Constance CDMX, DGO, JAL, HGO, MEX, MICH, TLAX

*Rhodosciadium rzedowskii Mathias \& Constance GTO, QRO, SLP, VER

*Rhodosciadium tolucense (Kunth) Mathias CHIS, CDMX, DGO, GTO, HGO, JAL, MEX, MICH, MOR, OAX, QRO, SLP, TLAX, VER

*Rhodosciadium tuberosum J.M. Coult. \& Rose CDMX, GTO, HGO, MEX, MICH, MOR, OAX, QRO

Sanicula arguta Greene ex J.M. Coult. \& Rose BCN

Sanicula bipinnatifida Dougl. ex Hook. BCN

Sanicula crassicaulis Poepp. ex DC. BCN

* Sanicula deserticola C.R. Bell BCN, BCS

Sanicula liberta Cham. \& Schltdl. CHIS, CHIH, COAH, COL, GTO, GRO, HGO, JAL, MEX, MICH, NLE, OAX, PUE, QRO, SLP, SON, TAMS, VER

* Sanicula moranii P. Vargas, Constance \& B.G. Baldwin BCN Spananthe paniculata Jacq. CHIS, COL, DGO, GRO, HGO, JAL, MEX, MICH, MOR, NAY, OAX, PUE, QRO, SLP, SIN, VER

Spermolepis divaricata (Walt.) Britton $\mathrm{COAH}$

Spermolepis echinata (Nutt. ex DC.) Heller BCN, COAH, SON, TAMS

Spermolepis inermis (Nutt. ex DC.) Mathias \& Constance COAH, QRO, SLP, VER

*Tauschia alpina (J.M. Coult. \& Rose) Mathias CDMX, GTO, MEX, MICH, PUE, QRO, TLAX, VER

*Tauschia allioides Bye \& Constance $\mathrm{CHIH}$

Tauschia arguta (Torr. \& Gray) J.F. Macbr. BCN

*Tauschia beruloides Constance \& Affolter MICH

*Tauschia bicolor Constance \& Bye CHIH, COAH, NLE 
*Tauschia decumbens (Benth.) J.M. Coult. \& Rose CDMX, GTO, HGO, JAL, MEX, MICH, QRO

*Tauschia edulis (S. Watson) J.M. Coult. \& Rose CHIH, SON *Tauschia ehrenbergii (H. Wolff) Mathias OAX, VER

Tauschia filiformis (J.M. Coult. \& Rose) J.M. Coult. \& Rose CHIS, OAX

*Tauschia hintoniorum Constance \& Affolter COAH, NLE

*Tauschia humilis J.M. Coult. \& Rose GTO, HGO, MEX, MICH, QRO, VER

*Tauschia infernicola Constance \& Affolter COAH, NLE, TAMS

*Tauschia johnstoniana Mathias \& Constance COAH, NLE

*Tauschia linearifolia J.M. Coult. \& Rose DGO, NAY

*Tauschia madrensis Rose CHIH, COAH, DGO, NAY, NLE, QRO, SIN, SON, TAMS

Tauschia mariana (S. Watson) J.M. Coult. \& Rose MICH

*Tauschia moorei Constance \& Affolter HGO, MEX, MICH, PUE, TLAX, VER

*Tauschia neglecta Calderón \& Constance MEX

Tauschia nudicaulis Schltdl. CDMX, DGO, GTO, HGO, JAL, MEX, MICH, MOR, NAY, OAX, PUE, QRO, SLP, SIN, VER

*Tauschia seatonii J.M. Coult. \& Rose GTO, MICH, PUE, QRO, VER

*Tauschia spellenbergii Constance \& Affolter CHIH

*Tauschia tarahumara Constance \& Bye CHIH

*Tauschia tenuifolia (S. Watson) Mathias \& Constance CHIH, COAH, SON

Tauschia vaginata (J.M. Coult. \& Rose) J.M. Coult. \& Rose CHIS, JAL, MICH, MOR, OAX

*Villarrealia calcicola (Mathias \& Constance) G.L. Nesom COAH, NLE

Yabea microcarpa (Hook. \& Arn.) Koso-Pol. BCN, SON

\section{Family Apocynaceae}

Alstonia longifolia (A. DC.) Pichon

CHIS, COL, GRO, HGO, JAL, MEX, MICH, MOR, NAY, OAX, PUE, VER

Allamanda cathartica L. CAM, CHIS, GRO, JAL, MEX, MOR, NAY, OAX, PUE, QROO, SIN, TAB, VER, YUC

Allotoonia parviflora (Sessé \& Moc.) J.F. Morales \& J.K. Williams GRO, JAL, MICH, QROO

Allotoonia turbinata (Woodson) J.F. Morales \& J.K. Williams CHIS

Allotoonia tuxtlensis (Standl.) J.F. Morales \& J.K. Williams CAM, CHIS, OAX, QRO, QROO, SLP, VER, YUC

Allotoonia woodsoniana (Monach.) J.F. Morales \& J.K. Williams GRO, JAL, MICH, OAX

Amsonia arenaria Standl. CHIH, COAH, SON

Amsonia grandiflora Alexander COAH, DGO, NLE, SON

Amsonia longiflora Torr. COAH, DGO, SON

Amsonia palmeri A. Gray CHIH, SON

Apocynum androsaemifolium L. CHIH, COAH, SON

Apocynum cannabinum L. BCN, CHIH, COAH, DGO, NAY,

NLE, QRO, SON, TAMS

Apocynum sibericum Jacq. BCN, COAH

Apocynum $\times$ floribundum Greene CHIH, NLE

Asclepias albicans S. Watson BCN, BCS, SIN, SON
Asclepias angustifolia Schweigg. AGS, CHIS, CHIH, COAH, COL, CDMX, DGO, GTO, GRO, HGO, JAL, MEX, MICH, NAY, NLE, OAX, PUE, QRO, SLP, SON, TAMS, VER, ZAC Asclepias arenaria Torr. CHIH, DGO

Asclepias asperula (Decne.) Woodson CHIH, COAH, DGO, NLE, QRO, SLP, SON, TAMS

*Asclepias atroviolacea Woodson CHIH, DGO, QRO, SLP, SON

Asclepias auriculata Kunth CHIS, COL, DGO, GTO, GRO, HGO, JAL, MEX, MICH, MOR, NAY, OAX, PUE, QRO, SLP, VER, ZAC

Asclepias brachystephana Engelm. ex Torr. AGS, CHIH, COAH, DGO, GTO, JAL, NLE, SLP, SON, ZAC

*Asclepias circinalis (Decne.) Woodson GRO, MEX, MICH, OAX, PUE, VER

*Asclepias conzattii Woodson OAX

*Asclepias coulteri A. Gray GTO, HGO, QRO, SLP, TAMS, VER

*Asclepias crocea Woodson COL, JAL

Asclepias curassavica L. AGS, BCN, BCS, CAM, CHIS, CHIH, COAH, COL, DGO, GTO, GRO, HGO, JAL, MEX, MICH, MOR, NAY, NLE, OAX, PUE, QRO, QROO, SLP, SIN, SON, TAB, TAMS, VER, YUC, ZAC

Asclepias elata Benth. CHIS, CHIH, COAH, DGO, GTO, JAL, MICH, NLE, OAX, SON, TAMS, VER, ZAC

*Asclepias elegantula Fishbein DGO, QRO, SLP, SIN

Asclepias emoryi (Greene) Vail ex Small DGO, NLE, SLP, TAMS

Asclepias engelmanniana Woodson COAH, DGO, SON

Asclepias eriocarpa Benth. BCN

Asclepias erosa Torr. BCN, SON

*Asclepias euphorbiifolia Engelm. ex A. Gray DGO, SLP

Asclepias fascicularis Decne. BCN

*Asclepias fournieri Woodson CHIH, GTO, HGO, JAL, MEX, MICH, NAY, QRO, SON, VER

*Asclepias gentryi Standl. CHIH, SON

Asclepias glaucescens Kunth AGS, CHIS, CHIH, COAH, COL, DGO, GRO, JAL, MEX, MICH, MOR, NAY, NLE, OAX, PUE, QRO, SLP, SIN, SON, TAMS, VER

Asclepias hypoleuca (A. Gray) Woodson CHIH, JAL, SON, ZAC

Asclepias involucrata Engelm. ex Torr. CHIH, COAH, DGO, NLE, SON

Asclepias jaliscana B.L. Rob. AGS, CHIS, CHIH, COL, DGO, GTO, GRO, HGO, JAL, MEX, MICH, MOR, NAY, NLE, OAX, PUE, QRO, SLP, SIN, SON, TAB, TAMS, VER, ZAC

*Asclepias jorgeana Fishbein \& S.P. Lynch CHIH, DGO, GTO, HGO, QRO, SLP, SIN, SON

*Asclepias laxiflora Colla OAX

Asclepias lemmonii A. Gray CHIH, DGO, JAL, NAY, SON

*Asclepias leptopus I.M. Johnst. CHIH, SIN, SON

Asclepias linaria Cav. AGS, CHIS, CHIH, COAH, COL, CDMX, DGO, GTO, GRO, HGO, JAL, MEX, MICH, MOR, NLE, OAX, PUE, QRO, SLP, SIN, SON, TAMS, TLAX, VER, ZAC

*Asclepias lynchiana Fishbein GRO, JAL, MEX, MICH, MOR, OAX, PUE 
Asclepias macrotis Torr. CHIH, COAH, SON

*Asclepias masonii Woodson BCS

*Asclepias mcvaughii Woodson AGS, COL, GRO, JAL, ZAC

*Asclepias mexicana Cav. CHIH, COAH, CDMX, GTO, GRO, HGO, JAL, MEX, MICH, NLE, OAX, PUE, QRO, SLP, SON, TAMS, VER

*Asclepias mirifica Woodson CHIH, SON

*Asclepias notha W.D. Stevens AGS, COAH, COL, CDMX, DGO, GRO, HGO, JAL, MEX, MICH, MOR, NAY, OAX, PUE, QRO, SLP, TLAX, VER, ZAC

Asclepias nummularia Torr. CHIH, COAH, DGO, GTO, MICH, SLP, SON

*Asclepias nummularioides W.D. Stevens GTO, JAL, MEX, SLP

Asclepias oenotheroides Schltdl. \& Cham. AGS, CAM, CHIS, CHIH, COAH, COL, DGO, GTO, GRO, HGO, JAL, MEX, MICH, MOR, NLE, OAX, PUE, QRO, QROO, SLP, SIN, SON, TAB, TAMS, TLAX, VER, YUC, ZAC

*Asclepias otarioides E. Fourn. AGS, COAH, CDMX, DGO, GTO, HGO, JAL, MEX, MICH, NAY, NLE, OAX, PUE, QRO, SLP, SIN, TLAX, VER, ZAC

*Asclepias ovata M. Martens \& Galeotti CHIH, COL, CDMX, DGO, GTO, GRO, HGO, JAL, MEX, MICH, MOR, NAY, OAX, QRO, SLP, SIN, SON, TAMS, VER

Asclepias pellucida E. Fourn. CHIS, COL, GTO, GRO, HGO, JAL, MEX, MICH, OAX, QRO, SLP, TAMS, VER

*Asclepias pratensis Benth. COL, GTO, JAL, MICH, NAY, QRO

*Asclepias pringlei (Greenm.) Woodson COL, CDMX, DGO, GTO, GRO, HGO, JAL, MEX, MICH, MOR, NAY, OAX, PUE, SLP, VER, ZAC

*Asclepias prostrata W.H. Blackw. TAMS

*Asclepias pseudorubricaulis Woodson NLE, SLP, TAMS, VER, ZAC

*Asclepias puberula A. Gray AGS, DGO, GTO, HGO, JAL, MEX, NAY, QRO, SLP, VER, ZAC

Asclepias quinquedentata A. Gray AGS, CHIH, CDMX, DGO, GTO, HGO, MEX, MICH, SLP, SIN, SON, ZAC

Asclepias rosea Kunth CHIS, COL, DGO, GTO, GRO, JAL, MEX, MICH, MOR, OAX, PUE, SLP, VER, ZAC

Asclepias scaposa Vail AGS, COAH, NLE, SLP, TAMS, ZAC

*Asclepias schaffneri A. Gray AGS, DGO, GTO, JAL, MICH, SLP, ZAC

*Asclepias scheryi Woodson DGO, GRO, JAL, MICH, NAY, OAX

Asclepias similis Hemsl. CHIS, COAH, GTO, GRO, HGO, JAL, MEX, MICH, NLE, OAX, PUE, QRO, SLP, TAMS, VER

Asclepias sperryi Woodson COAH

*Asclepias standleyi Woodson SON

*Asclepias subaphylla Woodson SIN, SON

Asclepias subulata Decne. BCN, BCS, SIN, SON

Asclepias subverticillata (A. Gray) Vail AGS, CHIS, CHIH, COAH, DGO, GTO, HGO, MICH, NLE, OAX, QRO, SLP, SON, ZAC

Asclepias texana A. Heller CHIH, COAH, NLE, SLP Asclepias tuberosa L. CHIH, COAH, NLE, SON, TAMS

*Asclepias vinosa (E. Fourn.) Woodson CDMX, MEX, OAX
Asclepias viridiflora Raf. CHIH, COAH, DGO, NLE

*Asclepias virletii E. Fourn. COAH, NLE, QRO, SLP, TAMS

Asclepias woodsoniana Standl. \& Steyerm. CHIS, OAX, PUE, VER

*Asclepias zanthodacryon (L.B. Sm.) Woodson COAH, NLE, TAMS

Aspidosperma desmanthum Benth. ex Müll. Arg. CAM, CHIS, QROO, TAB, VER

Aspidosperma megalocarpon Müll. Arg. CAM, CHIS, GRO, OAX, QROO, TAB, VER, YUC

Aspidosperma spruceanum Benth. ex Müll. Arg. CAM, CHIS, OAX, QROO, VER

Beaumontia grandiflora Wall. GRO

Blepharodon mucronatum (Schltdl.) Decne. CAM, CHIS, COL, DGO, GRO, HGO, JAL, MEX, MICH, MOR, NAY, OAX, PUE, QRO, QROO, SLP, SIN, TAB, VER, YUC

Bruceholstia magnifolia (Pittier) Morillo CHIS, JAL, OAX, PUE, TAB, VER, ZAC

Cameraria latifolia $\mathrm{L}$. CAM, QROO, TAB, VER, YUC

* Cascabela balsaensis L.O. Alvarado \& J.C. Soto GRO, MICH

Cascabela gaumeri (Hemsl.) Lippold CAM, GRO, QROO, VER, YUC

Cascabela ovata (Cav.) Lippold CAM, CHIS, COL, DGO, GRO, JAL, MEX, MICH, MOR, NAY, OAX, PUE, QROO, SIN, TAB, VER, ZAC

Cascabela pinifolia (Standl.\& Steyerm. ex Leavenw.) L.O. Alvarado \& Ochot.-Booth CAM, GRO, MEX, MICH, PUE, QROO, YUC

Cascabela thevetia (L.) Lippold BCS, CAM, CHIS, COL, GTO, GRO, HGO, JAL, MEX, MICH, MOR, NAY, NLE, OAX, PUE, QRO, QROO, SLP, SIN, SON, TAB, TAMS, VER, YUC

*Cascabela thevetioides (Kunth) Lippold AGS, CAM, CHIS, CHIH, CDMX, GTO, GRO, HGO, JAL, MEX, MICH, MOR, OAX, PUE, QRO, QROO, SLP, SIN, TAB, TAMS, VER, ZAC Cynanchum cyathiforme (Sundell) W.D. Stevens CHIS

*Cynanchum foetidum (Cav.) Kunth CAM, CHIS, COL, CDMX, GTO, GRO, JAL, MEX, MICH, MOR, OAX, PUE, QRO, QROO, SLP, TLAX, YUC

*Cynanchum jaliscanum (Vail.) Woodson COL, JAL, MICH, NAY, OAX, VER

Cynanchum ligulatum (Benth.) Woodson AGS, CHIH, COL, DGO, GTO, GRO, JAL, MEX, MICH, MOR, NAY, OAX, PUE, QRO, SIN, SON, ZAC

Cynanchum rensonii (Pittier) Woodson CAM, CHIS, OAX, QROO, YUC

*Cynanchum surrubiflorum W.D. Stevens TAB, VER

Cynanchum unifarium (Scheele) Woodson BCS, CAM, CHIH, COAH, COL, GTO, GRO, HGO, JAL, MICH, NLE, OAX, PUE, QRO, QROO, SLP, SIN, TAMS, VER, YUC

*Dictyanthus aeneus Woodson CAM, YUC

*Dictyanthus altatensis (Brandegee) W.D. Stevens JAL, SIN, SON

Dictyanthus asper (Mill.) W.D. Stevens CHIS, COL, GRO, JAL, MEX, MICH, OAX, PUE, VER

*Dictyanthus eximius (W.D. Stevens) W.D. Stevens CHIS

*Dictyanthus hamatus (W.D. Stevens) W.D. Stevens GRO, OAX

*Dictyanthus lautus (W.D. Stevens) W.D. Stevens COL, JAL 
*Dictyanthus macvaughianus (W.D. Stevens) W.D. Stevens AGS, GTO, JAL, MICH

Dictyanthus parviflorus Hemsl. AGS, CHIS, CDMX, DGO, GTO, GRO, JAL, MEX, MICH, MOR, OAX, PUE, VER, ZAC *Dictyanthus pavonii Decne. AGS, CHIS, COL, DGO, GTO, GRO, JAL, MEX, MICH, MOR, NAY, OAX, SIN, VER, ZAC *Dictyanthus reticulatus (Turcz.) Hemsl. COL, DGO, GRO, JAL, MEX, MICH, MOR, NAY, OAX, PUE, SIN, VER

*Dictyanthus sepicola (W.D. Stevens) W.D. Stevens CHIH, COL, JAL, NAY, SIN, SON

*Dictyanthus suffruticosus (W.D. Stevens) W.D. Stevens OAX

*Dictyanthus tigrinus Conz. \& Standl. CHIS, JAL, MEX, OAX, VER, ZAC

*Dictyanthus tuberosus B.L. Rob. COL, DGO, JAL, NAY, SIN, SON, ZAC

*Dictyanthus yucatanensis Standl. CAM, CHIS, QROO, VER, YUC

*Echites lanatus M. Martens \& Galeotti OAX

*Echites mexicana (Müll. Arg.) Miers TAMS, VER

Echites rosea A. DC. SLP, VER

Echites turrigera Woodson COL, GRO, JAL, MICH, OAX

Echites umbellatus Jacq. CAM, QROO, TAB, VER, YUC

Echites yucatanensis Millsp. CAM, COL, GRO, JAL, MICH, QROO, TAB, YUC

*Fernaldia asperoglottis Woodson GRO, MEX, MICH

Fernaldia pandurata (A. DC.) Woodson CAM, CHIS, COL, GRO, HGO, JAL, MEX, NAY, OAX, PUE, QRO, QROO, SLP, SIN, TAMS, VER, YUC

Fischeria scandens DC. CAM, CHIS, OAX, PUE, TAB, VER

Forsteronia acouci (Aubl.) A. DC CHIS, OAX, PUE, TAB, VER

Forsteronia myriantha Donn. Sm. CHIS, GRO, OAX, PUE, VER

Forsteronia spicata (Jacq.) G. Mey. CAM, CHIS, COL, DGO, GRO, JAL, MICH, NAY, OAX, VER

*Funastrum arenarium (Decne. ex Benth.) Liede BCN, BCS

Funastrum bilobum (Hook. \& Arn.) J.F. Macbr. CAM, CHIS, COL, GRO, JAL, MEX, MICH, MOR, OAX, PUE, QRO, QROO, SLP, TAMS, VER, YUC

Funastrum clausum (Jacq.) Schltr. BCN, BCS, CAM, CHIS, CHIH, COL, DGO, GRO, HGO, JAL, MEX, MICH, MOR, NAY, NLE, OAX, PUE, QRO, QROO, SLP, SIN, SON, TAB, TAMS, VER, YUC, ZAC

Funastrum crispum (Benth.) Schltr. AGS, BCN, CHIH, COAH, COL, DGO, JAL, NLE, QRO, SLP, SON, TAMS, ZAC

Funastrum cynanchoides (Decne.) Schltr. BCN, BCS, CHIS, CHIH, COAH, COL, DGO, GTO, HGO, JAL, MICH, NAY, NLE, QRO, SLP, SIN, SON, TAMS, ZAC

*Funastrum elegans (Decne.) Schltr. AGS, CAM, CHIS, CHIH, COAH, COL, CDMX, DGO, GTO, GRO, HGO, JAL, MEX, MICH, MOR, NLE, OAX, PUE, QRO, SLP, SIN, SON, TAMS, TLAX, VER, YUC, ZAC

Funastrum hirtellum (A. Gray) Schltr. BCN

Funastrum lindenianum (Decne.) Schltr. CAM, CHIS, SLP, VER, YUC

Funastrum odoratum (Hemsl.) Schltr. CAM, CHIS, NAY, OAX *Funastrum pannosum (Decne.) Schltr. AGS, BCN, BCS,
CHIS, CHIH, COL, DGO, GTO, GRO, JAL, MEX, MICH, MOR, NAY, NLE, OAX, PUE, QRO, SLP, SIN, SON, TAMS, VER, ZAC

Funastrum torreyi (A. Gray) Schltr. CHIH, COAH, DGO, GTO, NLE, SLP, TAMS, ZAC

*Gonolobus albiflorus W.D. Stevens COL, JAL

Gonolobus ancorifer W.D. Stevens CHIS, OAX, VER

Gonolobus aristolochiifolius (Brandegee) Woodson CHIS

Gonolobus arizonicus (A. Gray) Woodson SON

Gonolobus barbatus Kunth CAM, CHIS, DGO, GRO, JAL, MEX, MICH, MOR, NAY, OAX, PUE, QROO, SIN, TAB, VER, YUC

*Gonolobus bifidus Hemsl. SLP

* Gonolobus breedlovei L.O. Williams CHIS

*Gonolobus croceus W.D. Stevens NAY, SIN

Gonolobus cteniophorus (S.F. Blake) Woodson CAM, CHIS, OAX, QROO, TAB, VER, YUC

Gonolobus cuajayote W.D. Stevens CHIS

Gonolobus chiapensis (Brandegee) Woodson CHIS

*Gonolobus chloranthus Schltdl. CHIS, COL, GTO, GRO, HGO, JAL, MEX, MICH, OAX, PUE, QRO, SLP, TAB, VER

Gonolobus dasystephanus (S.F. Blake) Woodson CHIS

Gonolobus erianthus Decne. CHIS, COAH, COL, CDMX, GTO, GRO, HGO, JAL, MEX, MICH, MOR, NAY, NLE, OAX, PUE, QRO, SLP, SON, TAMS, VER

Gonolobus exannulatus W.D. Stevens CHIS

Gonolobus fraternus Schltdl. CAM, CHIS, GRO, HGO, MEX, MICH, OAX, PUE, QRO, QROO, SLP, TAB, TAMS, VER

*Gonolobus fuscus Decne. MEX, MICH

*Gonolobus gonoloboides (Greenm.) Woodson CHIH, DGO, JAL, NLE, SIN, SON

*Gonolobus grandiflorus (Cav.) R. Br. ex Roem. \& Schult. CHIS, CDMX, DGO, GTO, GRO, HGO, JAL, MEX, MICH, MOR, OAX, PUE, QRO, SLP, SIN, VER, ZAC

Gonolobus incerianus W.D. Stevens \& Montiel CHIS, GRO, OAX, VER

*Gonolobus jaliscensis B.L. Rob. \& Greenm. COL, DGO, GRO, JAL, MEX, MICH, MOR, NAY, OAX

Gonolobus leianthus Donn. Sm. CAM, CHIS, QROO, TAB, VER

*Gonolobus luridus Decne. HGO, VER

*Gonolobus megalocarpus Paul G. Wilson JAL, MEX, OAX

*Gonolobus nemorosus Decne. OAX, PUE, VER

Gonolobus niger (Cav.) R. Br. ex Schult. CHIS, GRO, HGO, OAX, PUE, QRO, SLP, TAB, TAMS, VER

*Gonolobus pallidus W.D. Stevens CHIS

*Gonolobus pectinatus Brandegee GRO, HGO, MEX, MICH, MOR, NAY, OAX, PUE, QRO, VER

Gonolobus sandersii W.D. Stevens COL, JAL

*Gonolobus sidaefolius M. Martens \& Galeotti HGO, VER

*Gonolobus sororius A. Gray DGO, GRO, JAL, MEX, MICH, OAX, SIN, VER, ZAC

*Gonolobus spiranthus Juárez-Jaimes, W.D. Stevens \& LozadaPérez COL, GRO, JAL, MEX, MICH, OAX

Gonolobus stenanthus (Standl.) Woodson CAM, QROO, TAB, YUC 
Gonolobus stenosepalus (Donn. Sm.) Woodson CAM, CHIS, QROO, VER, YUC

*Gonolobus striatus M. Martens \& Galeotti HGO, VER

*Gonolobus tingens Decne. HGO, QRO

*Gonolobus triflorus M. Martens \& Galeotti OAX

Gonolobus uniflorus Kunth AGS, CHIS, COL, CDMX, GTO, GRO, HGO, JAL, MEX, MICH, MOR, NLE, OAX, PUE, QRO, SLP, SIN, SON, TAMS, TLAX, VER, ZAC

Gonolobus versicolor Woodson CHIS, PUE

Gonolobus xanthotrichus Brandegee CHIS, OAX, PUE, VER

Haplophyton cimicidium A. DC. BCN, CHIS, CHIH, COL, DGO, GRO, JAL, MEX, MICH, MOR, OAX, PUE, SON, VER Haplophyton crooksii (L. Benson) L. Benson CHIH, COAH, SIN, SON

Jobinia eulaxiflora (Lundell) W.D. Stevens VER

* Laubertia contorta (M. Martens \& Galeotti) Woodson CHIS, COL, DGO, GRO, JAL, MEX, MICH, MOR, NAY, OAX, PUE, SLP, SIN

Macroscepis diademata (Ker Gawl.) W.D. Stevens CAM, CHIS, CHIH, COL, GRO, JAL, MEX, MICH, NAY, OAX, QRO, QROO, SLP, SIN, SON, TAMS, VER, YUC

Macroscepis pleistantha Donn. Sm. CHIS, QRO, SLP, TAMS, VER

*Macroscepis stevensii Morillo CHIS

*Macroscepis yucatanensis Morillo YUC

Mandevilla acutiloba (A. DC.) Woodson CHIS, GRO, OAX, QRO

*Mandevilla andrieuxii (Müll. Arg.) Hemsl. COL, GRO, JAL, MICH, OAX

* Mandevilla apocynifolia (A. Gray) Woodson JAL

* Mandevilla convolvulacea (A. DC.) Hemsl. OAX, PUE

*Mandevilla exilicaulis (Sessé \& Moc.) J.K. Williams COL, JAL, MICH

*Mandevilla foliosa (Müll. Arg.) Hemsl. CAM, CHIH, COAH, COL, CDMX, DGO, GTO, GRO, HGO, JAL, MEX, MICH, MOR, NAY, NLE, OAX, PUE, QRO, SLP, SIN, SON, VER, $\mathrm{ZAC}$

*Mandevilla guerrerensis Lozada-Pérez \& Diego GRO, OAX Mandevilla hirsuta (A. Rich.) K. Schum. CHIS, GRO, OAX, TAB, VER

*Mandevilla holosericea (Sessé \& Moc.) J.K. Williams AGS, CHIS, COL, DGO, GTO, GRO, HGO, JAL, MEX, MICH, MOR, NAY, OAX, PUE, QRO, SIN

Mandevilla karwinskii (Müll. Arg.) Hemsl. COAH, GTO, GRO, HGO, MEX, NLE, OAX, QRO, SLP, TAMS, VER, ZAC

*Mandevilla mexicana (Müll. Arg.) Woodson GRO, MEX, OAX

*Mandevilla oaxacana (A. DC.) Hemsl. GRO, OAX

Mandevilla platydactyla Woodson CHIS, OAX

* Mandevilla pringlei J.K. Williams JAL, NAY

*Mandevilla stans (A. Gray) J.K. Williams CHIH, DGO, GTO, MICH, QRO, SIN, SON

Mandevilla subsagittata (Ruiz \& Pav.) Woodson CAM, CHIS, COL, GRO, HGO, JAL, MEX, MICH, MOR, NAY, OAX, PUE, QRO, QROO, SLP, SIN, TAB, TAMS, VER, YUC

Mandevilla subsessilis (A. DC.) Woodson GRO, JAL, MICH, MOR, OAX, SLP, VER
Mandevilla torosa (Jacq.) Woodson CAM, CHIS, HGO, NLE, QRO, QROO, SLP, TAMS, VER, YUC

Mandevilla tubiflora (M. Martens \& Galeotti) Woodson CHIS, GRO, JAL, MEX, MICH, OAX, SIN, TAB, VER, YUC

Mandevilla villosa (Miers) Woodson CAM, CHIS, QROO, VER

*Marsdenia astephanoides (A. Gray) Woodson COL, GRO, JAL, MICH, NAY, OAX, SIN

Marsdenia bourgaeana (Baill.) W. Rothe CHIS, GRO, MEX, MOR, OAX, VER

*Marsdenia callosa Juárez-Jaimes \& W.D. Stevens GRO, JAL, MICH, OAX

* Marsdenia carterae W.D. Stevens \& Juárez-Jaimes BCS

* Marsdenia coulteri Hemsl. CAM, CHIS, COAH, GTO, GRO, HGO, JAL, MICH, NLE, OAX, PUE, QRO, QROO, SLP, SIN, SON, TAMS, VER, YUC, ZAC

*Marsdenia cuixmalensis Juárez-Jaimes \& L.O. Alvarado COL, JAL

*Marsdenia edulis S. Watson BCN, CHIS, CHIH, COL, DGO, GRO, HGO, JAL, MEX, NAY, OAX, QRO, SLP, SIN, SON

*Marsdenia gallardoae Lozada-Pérez CHIS, OAX

Marsdenia gualanensis Donn. Sm. CAM, CHIS, JAL, OAX, QROO, YUC

Marsdenia gymnemoides W. Rothe CHIS

Marsdenia hiriartiana Juárez-Jaimes \& W.D. Stevens CHIS, QROO

Marsdenia lanata (Paul G. Wilson) W.D. Stevens CHIS, COL, GRO, JAL, MEX, MICH, MOR, NAY, OAX, PUE, SIN, VER

Marsdenia laxiflora Donn. Sm. CHIS, OAX, TAB, VER

*Marsdenia ludani Juárez-Jaimes \& Saynes OAX

* Marsdenia magallanesiana Juárez-Jaimes JAL

Marsdenia mayana Lundell CHIS, GRO, JAL, NAY, QROO, YUC

Marsdenia mexicana Decne. CHIS, COL, GRO, JAL, MEX, MICH, MOR, OAX, PUE, VER, ZAC

*Marsdenia microcarpa Juárez-Jaimes \& Lozada-Pérez GRO, OAX

Marsdenia neriifolia (Decne.) Woodson CHIS, OAX, VER

* Marsdenia oaxacana Morillo OAX

* Marsdenia parvifolia Brandegee OAX, PUE

* Marsdenia popoluca Juárez-Jaimes \& A. Campos TAB, VER

* Marsdenia pringlei S. Watson CHIS, COAH, GRO, NLE, QRO, SLP, TAMS

Marsdenia propinqua Hemsl. CAM, CHIS, COL, GRO, HGO, JAL, MICH, NAY, NLE, OAX, PUE, QRO, QROO, SLP, TAB, TAMS, VER, YUC, ZAC

*Marsdenia purpusiana W.D. Stevens HGO, OAX, VER

*Marsdenia rzedowskiana Juárez-Jaimes \& W.D. Stevens OAX, PUE, VER

Marsdenia schlechteriana W. Rothe CHIS

Marsdenia stephanotidifolia Woodson CHIS

* Marsdenia stevensiana Juárez-Jaimes CHIS

Marsdenia steyermarkii Woodson CHIS

* Marsdenia tholiformis Juárez-Jaimes \& L.O. Alvarado NAY

Marsdenia trivirgulata Bartlett CAM, CHIS, COL, GRO, JAL, MEX, MICH, NAY, OAX, QROO, SIN, SON 
*Marsdenia tubularis L.O. Williams CHIS, GRO, MEX, MICH, OAX

Marsdenia veronicae W.D. Stevens CHIS, OAX

*Marsdenia zimapanica Hemsl. CHIS, GTO, GRO, HGO, JAL,

MEX, MICH, MOR, NAY, OAX, PUE, QRO, SLP, VER, ZAC

* Matelea adenocardia (Standl.) Woodson CHIH

*Matelea angustiloba (B.L. Rob. \& Greenm.) W.D. Stevens DGO, NAY

* Matelea atrocoronata (Brandegee) Woodson OAX, PUE

Matelea belizensis (Lundell \& Standl.) Woodson CAM, QROO, YUC

* Matelea calcarata Woodson COAH, NLE, SLP, TAMS

*Matelea calcicola (Greenm.) Woodson GRO, MEX, MOR,

$\mathrm{OAX}$

Matelea campechiana (Standl.) Woodson CAM, CHIS, QROO,

YUC

* Matelea camporum (Brandegee) Woodson COAH

* Matelea castanea (Brandegee) Woodson OAX, PUE

* Matelea caudata (A. Gray) Woodson BCS, CHIH, MOR, OAX, PUE, SON

*Matelea congesta (Decne.) Woodson GTO, JAL, MEX, MICH, OAX, QRO, ZAC

Matelea cordifolia (A. Gray) Woodson BCN, BCS, SON

* Matelea crassifolia (Standl.) Woodson CAM, CHIS, QROO, VER, YUC

*Matelea crenata (Vail) Woodson COL, CDMX, DGO, GTO, GRO, HGO, JAL, MEX, MICH, MOR, OAX, QRO, SLP, SIN, TLAX, ZAC

Matelea chihuahuensis (A. Gray) Woodson $\mathrm{CHIH}$, COAH, $\mathrm{HGO}, \mathrm{QRO}, \mathrm{SON}$

*Matelea chrysantha (Greenm.) Woodson COAH, COL, CDMX, DGO, GTO, GRO, HGO, JAL, MEX, MICH, MOR, OAX, PUE, QRO, SIN, SON, TLAX, VER, ZAC

* Matelea decumbens W.D. Stevens CDMX, DGO, HGO, MEX, QRO, TLAX

*Matelea emmartinezii W.D. Stevens CHIS

* Matelea fruticosa (Brandegee) Woodson BCS

Matelea gentlei (Lundell \& Standl.) Woodson CAM, CHIS, QROO, YUC

Matelea gonoloboides (B.L. Rob. \& Greenm.) Woodson CHIS, CHIH, GRO, JAL, MEX, MICH, OAX, SON

* Matelea gracilis (Decne.) W.D. Stevens OAX

* Matelea greggii (Vail) Woodson COAH, DGO

Matelea guatemalensis (Schum.) Woodson CHIS

* Matelea hastatula (A. Gray) Sundell BCN

* Matelea inconspicua (Brandegee) Woodson OAX, PUE, VER

* Matelea inops Woodson CHIS, OAX

* Matelea ionantha Woodson VER

* Matelea johnstonii Shinners CHIH, SON

Matelea lanceolata (Decne.) Woodson CHIS, OAX, PUE, VER

*Matelea lesueurii (Standl.) Woodson CHIH, SON

*Matelea magallanesii E.J. Lott COL, JAL

Matelea medusae Woodson CHIS

* Matelea meyeri Woodson TAMS

Matelea micrantha L.O. Williams CAM, CHIS, QROO

Matelea molinarum L.O. Williams CHIS

*Matelea nigrescens (Schltdl.) Woodson QRO, SLP, VER
*Matelea nummularia (Decne.) Woodson CHIH, DGO, HGO, JAL, MEX, MICH, MOR, PUE, VER

*Matelea oaxacana (Standl.) W.D. Stevens OAX

Matelea ocellata W.D. Stevens MICH, OAX

Matelea parviflora (Torr.) Woodson COAH

Matelea parvifolia (Torr.) Woodson BCN, BCS, CHIH, SON, TAMS

Matelea patalensis (Donn. Sm.) Woodson CHIS

*Matelea pedunculata (Decne.) Woodson CDMX, DGO, GTO, MEX, MICH, QRO, ZAC

*Matelea petiolaris (A. Gray) Woodson CHIH, SIN, SON Matelea picturata (Hemsl.) Woodson CHIS, QRO, SLP

*Matelea pilosa (Benth.) Woodson AGS, COAH, DGO, GTO, HGO, JAL, MEX, MICH, MOR, NAY, NLE, OAX, PUE, QRO, SLP, SIN, TAMS, VER, ZAC

Matelea pringlei (A. Gray) Woodson BCN, BCS, SIN, SON

Matelea producta (Torr.) Woodson CHIH, SON, TAMS, ZAC

*Matelea prostrata (Willd.) Woodson DGO, HGO, PUE, QRO

*Matelea pueblensis (Brandegee) Woodson OAX, PUE

*Matelea purpusii (Brandegee) Woodson GRO, OAX, PUE

Matelea pusilliflora L.O. Williams CAM, CHIS, QROO, YUC

*Matelea quercetorum (Standl.) W.D. Stevens CHIH, SON

Matelea reticulata (Engelm. ex A. Gray) Woodson CHIH, COAH, DGO, NLE, OAX, SLP, SIN, SON, TAMS

*Matelea rogersii Woodson NLE, TAMS

Matelea sagittifolia (A. Gray) Woodson ex Shinners NLE, TAMS

*Matelea schaffneri (Engelm. ex A. Gray) Woodson HGO, QRO, SLP, VER, ZAC

Matelea serpens Woodson COAH, NLE

* Matelea stenosepala Lundell CAM, QROO, YUC

* Matelea suberifera (B.L. Rob.) W.D. Stevens HGO, SLP, TAMS, VER

Matelea sugillata W.D. Stevens OAX, QRO, VER

Matelea trachyantha (Greenm.) W.D. Stevens CHIS, CHIH, COL, GRO, MEX, MICH, MOR, OAX, PUE, SON

*Matelea tristiflora (Standl.) Woodson CHIH, SON

Matelea tuerckheimii (Donn. Sm.) Woodson CHIS

* Matelea umbellata (Brandegee) Woodson BCS

* Matelea vailiana Woodson $\mathrm{CHIH}, \mathrm{SON}$

Matelea velutina (Schltdl.) Woodson CAM, CHIS, CHIH, GTO, HGO, MOR, OAX, PUE, QRO, QROO, SLP, SIN, SON, TAB, TAMS, VER, YUC, ZAC

Matelea velutinoides W.D. Stevens CHIS

*Matelea wootonii (Vail) Woodson CHIH, SON

Mesechites trifidus (Jacq.) Müll. Arg. CAM, CHIS, GRO, OAX,

TAB, TAMS, VER

Metalepis peraffinis (Woodson) Morillo CHIS, JAL, OAX, VER

Metastelma arizonicum A. Gray CHIH, SON

Metastelma barbigerum Scheele CAM, CHIS, CHIH, COAH, HGO, NLE, OAX, QRO, SLP, TAB, TAMS, VER, YUC, ZAC Metastelma brachymischum W.D. Stevens CHIS

*Metastelma californicum Benth. BCN, BCS, COL, NAY, SLP, SIN, SON, TAMS

* Metastelma cuneatum Brandegee SIN, SON

Metastelma chiapense A. Gray CHIS, OAX, PUE, VER

* Metastelma lanceolatum Schltr. CHIS, CDMX, GTO, GRO, 
HGO, JAL, MEX, MOR, OAX, PUE, QRO, SLP, YUC

*Metastelma latifolium Rose CHIH, COL, JAL, NLE, SIN, SON, TAMS, VER

*Metastelma longicoronatum (L.O. Williams) Liede CHIS

* Metastelma macropodum Greenm. OAX, PUE

Metastelma mexicanum (Brandegee) Fishbein \& R.A. Levin CHIH, COAH, DGO, HGO, MEX, NAY, NLE, OAX, QRO, SLP, SIN, SON, VER

* Metastelma minutiflorum Wiggins BCS, COL, SIN, SON

Metastelma palmeri S. Watson CHIS, COAH, GRO, HGO, NLE, OAX, PUE, QRO, SLP, TAMS, VER, YUC

Metastelma pringlei A. Gray BCN, BCS, CHIH, COAH, DGO, NLE, SLP, SIN, SON, TAMS, VER

* Metastelma schaffneri A. Gray BCS, CHIS, COL, DGO, GRO, JAL, MEX, MICH, MOR, NAY, OAX, PUE, SLP, SIN, SON, ZAC

Metastelma schlechtendalii Decne. CAM, CHIS, GRO, MEX, MOR, NAY, OAX, PUE, QRO, QROO, SLP, SIN, SON, TAB, VER, YUC

Metastelma sepium (Decne.) W.D. Stevens CHIS, HGO, MEX, MICH, OAX

Metastelma stenomeres (Standl. \& Steyerm.) W.D. Stevens OAX

Metastelma thalamosiphon W.D. Stevens CAM, CHIS, QROO, YUC

Metastelma trichophyllum (L.O. Williams) W.D. Stevens CAM, CHIS, OAX

*Metastelma turneri Liede \& Meve COAH, NLE, SLP, TAMS Metastelma yucatanense W.D. Stevens CAM, QROO, YUC

* Microdactylon cordatum Brandegee OAX, PUE

Odontadenia macrantha (Willd. ex Roem. \& Schult.) Markgr. CHIS, OAX, VER

* Orthosia angustifolia Turcz. CHIH, COAH, CDMX, GTO, HGO, JAL, MEX, MICH, NLE, OAX, PUE, QRO, SLP, TAMS, TLAX, VER

Orthosia cynanchoides W.D. Stevens CHIS

Orthosia glaberrima (Woodson) W.D. Stevens CHIS, VER

Orthosia misera (L.O. Williams) W.D. Stevens CHIS, VER

*Orthosia pubescens (Greenm.) Liede \& Meve CDMX, GRO,

MEX, MICH, MOR, OAX, PUE

*Orthosia stipitata W.D. Stevens CHIS

Oxypetalum cordifolium (Vent.) Schltr. CAM, CHIS, HGO, JAL, OAX, QRO, QROO, SLP, VER, YUC

Pentalinon andrieuxii (Müll. Arg.) B.F. Hansen \& Wunderlin CAM, CHIS, GRO, HGO, MEX, MOR, OAX, PUE, QRO, QROO, SLP, SIN, TAB, TAMS, VER, YUC

*Pherotrichis leptogenia B.L. Rob. JAL, MOR

*Pherotrichis mixtecana Brandegee GRO, MICH, OAX

Pherotrichis schaffneri A. Gray CHIH, DGO, GTO, SLP, SIN, SON

Pherotrichis villosa Meisn. CHIS, MEX, PUE

Pinochia peninsularis (Woodson) M.E. Endress \& B.F. Hansen CAM, TAB

Plumeria obtusa L. AGS, CAM, CHIS, CHIH, COL, HGO, JAL, MICH, NAY, QRO, QROO, SLP, SON, VER, YUC, ZAC Plumeria rubra L. AGS, BCN, BCS, CAM, CHIS, CHIH, COL, CDMX, DGO, GTO, GRO, HGO, JAL, MEX, MICH, MOR,
NAY, NLE, OAX, PUE, QRO, QROO, SLP, SIN, SON, TAB, TAMS, VER, YUC, ZAC

*Polystemma canisferum McDonnell \& Fishbein SON

Polystemma guatemalense (Schltr.) W.D. Stevens AGS, CHIS, COL, GRO, JAL, MEX, MICH, MOR, NAY, OAX, PUE, SIN

* Polystemma mirandae Lozada-Pérez GRO, OAX, PUE

Polystemma viridiflorum Decne. CHIS, GRO, MEX, MICH, MOR, OAX, VER

Prestonia amanuensis Woodson CAM, QROO, TAB, VER

*Prestonia clandestina J.F. Morales CHIS, OAX, VER

* Prestonia contorta (M. Martens \& Galeotti) Hemsl. CHIS, COL, DGO, GRO, MICH, JAL, NAY, OAX, ZAC

Prestonia guatemalensis Woodson CHIS, OAX, TAB, VER

Prestonia longifolia (Sessé \& Moc.) J.F. Morales CHIS, TAB, VER

Prestonia mexicana A. DC. CAM, CHIS, COL, GTO, GRO, HGO, JAL, MEX, MICH, MOR, NAY, OAX, QRO, QROO, SLP, SIN, TAB, TAMS, VER, YUC

Prestonia portobellensis (Beurl.) Woodson CHIS, HGO, OAX, QRO, QROO, SLP, TAB, VER, YUC

* Prestonia sericea M. Martens \& Galeotti OAX

Prestonia speciosa Donn. Sm. CHIS, OAX, VER

Prosthecidiscus guatemalensis Donn. Sm. CHIS, COL, GRO, JAL, MEX, MICH, NAY, OAX, VER

Rauvolfia ligustrina Willd. ex Roem. \& Schult. CHIS, COL, GRO, JAL, OAX, QROO, VER, YUC

Rauvolfia tetraphylla L. CAM, CHIS, COL, GTO, GRO, HGO, JAL, MEX, MICH, MOR, NAY, OAX, PUE, QRO, QROO, SLP, SIN, TAB, TAMS, VER, YUC

Rhabdadenia biflora (Jacq.) Müll. Arg. CAM, CHIS, QROO, TAB, TAMS, VER, YUC

* Rhabdadenia cordata (Mill.) Miers. QRO, TAB, TAMS, VER Rotundanthus fulvidus (F. Ballard) Morillo CHIS

Seutera angustifolia (Pers.) Fishbein \& W.D. Stevens AGS, CAM, CHIS, CHIH, COAH, COL, CDMX, DGO, GTO, HGO, JAL, MEX, MICH, NLE, OAX, PUE, QRO, QROO, SLP, SON, TAMS, TLAX, VER, YUC, ZAC

Seutera palmeri (S. Watson) Fishbein \& W.D. Stevens BCN, BCS

*Suberogerens cyclophylla (Standl.) Morillo CHIS, COL, GRO, JAL, MEX, MICH, OAX, PUE, SIN, VER

Tabernaemontana alba Mill. CAM, CHIS, COL, HGO, JAL, MEX, MICH, NAY, OAX, PUE, QRO, QROO, SLP, SIN, TAB, TAMS, VER, YUC

Tabernaemontana amygdalifolia Jacq. CAM, CHIS, COL, DGO, GRO, JAL, MICH, NAY, OAX, PUE, QRO, QROO, SLP, SIN, VER, YUC, ZAC

Tabernaemontana arborea Rose CHIS, OAX, TAB, VER

Tabernaemontana citrifolia L. CHIS, HGO, OAX, PUE, SLP, TAB, TAMS, VER

Tabernaemontana donnell-smithii Rose CAM, CHIS, COL, DGO, GRO, HGO, JAL, MEX, MICH, NAY, OAX, PUE, QRO, QROO, SLP, SIN, TAB, TAMS, VER, YUC

Tabernaemontana eubracteata (Woodson) A.O. Simões \& M.E. Endress CHIS, OAX, VER

Tabernaemontana grandiflora Jacq. CHIS, COL, GRO, JAL, MICH, NAY, ZAC 
Tabernaemontana hannae (M. Méndez \& J.F. Morales) A.O. Simões \& M.E. Endress CHIS, OAX

Tabernaemontana litoralis Kunth BCS, CAM, CHIS, GRO, HGO, JAL, MEX, MICH, MOR, NAY, OAX, PUE, QROO, TAB, VER, YUC

*Tabernaemontana mixtecana L.O. Alvarado \& Juárez-Jaimes OAX

*Tabernaemontana oaxacana (L.O. Alvarado) A.O. Simões \& M.E. Endress OAX

Tabernaemontana odontadeniiflora A.O. Simões \& M.E. Endress CHIS, CHIH, COL, DGO, GRO, JAL, MEX, MICH, MOR, NAY, OAX, PUE, SLP, VER

*Tabernaemontana stenoptera (Leeuwenb.) A.O. Simões \& M.E. Endress COL, JAL

Tabernaemontana tomentosa (Greenm.) A.O. Simões \& M.E. Endress BCS, CHIS, CHIH, COL, DGO, GRO, HGO, JAL, MEX, MICH, MOR, NAY, OAX, PUE, SIN, SON, VER, ZAC *Tabernaemontana venusta (J.F. Morales) A.O. Simões \& M.E. Endress OAX

Tassadia obovata Decne. CHIS, OAX, VER

Telosiphonia brachysiphon (Torr.) Henr. CHIH, DGO, SON

*Telosiphonia hesperia (I.M. Johnst.) Henr. BCS

Telosiphonia hypoleuca (Benth.) Henr. AGS, CHIH, COAH, DGO, GTO, GRO, HGO, JAL, MEX, MICH, MOR, NAY, NLE, OAX, PUE, QRO, SLP, SIN, SON, TAMS, ZAC

Telosiphonia lanuginosa (M. Martens \& Galeotti) Henr. COAH, GRO, HGO, NLE, OAX, PUE, QRO, SLP, TAMS, VER

Telosiphonia macrosiphon (Torr.) Henr. CHIH, COAH, DGO, NLE, SLP, TAMS, ZAC

*Telosiphonia nacapulensis Felger \& Henr. SON

*Thenardia chiapensis J.K. Williams CHIS, GRO, OAX

*Thenardia floribunda Kunth COL, GRO, JAL, MEX, MICH, MOR, NAY, OAX

*Thenardia galeottiana Baill. CHIS, GRO, JAL, MEX, OAX

Thevetia ahouai (L.) A. DC. BCS, CAM, CHIS, CDMX, GTO, GRO, MOR, NAY, OAX, PUE, QROO, SIN, SON, TAB, TAMS, VER, YUC

Thevetia gaumeri Hemsl. CAM, CHIS, GRO, MICH, MOR, QROO, SLP, VER, YUC

*Thevetia pinifolia (Standl. \& Steyerm.) J.K. Williams GRO, JAL, MICH

*Thoreauea aberrans J.F. Morales VER

*Thoreauea guerrerensis Diego \& Lozada-Pérez GRO

*Thoreauea paneroi J.K. Williams GRO, OAX

*Tintinnabularia gratissima J.F. Morales VER

Tintinnabularia mortonii Woodson CHIS, OAX

Trachelospermum difforme (Walt.) A. Gray AGS, JAL, TAMS, VER

Trichosacme lanata Zucc. NLE, SLP, TAMS

*Urechites microcalyx DC. CHIS, VER

Vallesia antillana Woodson QROO, TAB, VER, YUC

Vallesia aurantiaca (M. Martens \& Galeotti) J.F. Morales CHIS, CHIH, COL, GRO, JAL, OAX, SON, VER

*Vallesia conzattii Standl. OAX

Vallesia glabra (Cav.) Link BCN, BCS, CAM, CHIS, CHIH, CDMX, GTO, GRO, HGO, JAL, MICH, MOR, NAY, OAX, PUE, QRO, QROO, SLP, SIN, SON, TAB, TAMS, VER, YUC
*Vallesia laciniata Brandegee BCS, GRO, SON

*Vallesia spectabilis El. Mey. ex J.F. Morales COL, JAL

Vulcanoa steyermarkii (Woodson) Morillo CHIS

\section{Family Apodanthaceae}

*Apodanthes pringlei S. Watson ex B.L. Rob. COAH, HGO, NLE, PUE, QRO

Pilostyles blanchetii (Gardn.) R. Br. MICH

Pilostyles mexicana (Brandegee) Rose CAM, CHIS, MICH, QROO, VER

Pilostyles thurberi A. Gray AGS, BCN, BCS, CHIH, COAH, CDMX, DGO, GTO, HGO, JAL, MEX, MOR, NLE, PUE, QRO, SLP, SON, VER, ZAC

\section{Family Aquifoliaceae}

Ilex anodonta Standl. \& Steyerm. CHIS

Ilex belizensis Lundell CHIS, HGO, VER

Ilex brandegeana Loes. BCS, CHIS, COAH, COL, DGO, GTO, GRO, JAL, MEX, MICH, MOR, NAY, NLE, OAX, QRO, SLP, SIN, TAMS

*Ilex condensata Turcz. CHIS, OAX, PUE, QRO, SLP, TAB, VER

Ilex costaricensis Donn. Sm. CHIS, OAX, VER

Ilex decidua Walter COAH, NLE, QRO, SLP, TAMS

Ilex discolor Hemsl. BCS, CHIS, CHIH, COL, CDMX, DGO, GTO, GRO, HGO, JAL, MEX, MICH, MOR, NAY, NLE, OAX, PUE, QRO, SLP, SIN, SON, TAB, TAMS, VER

*Ilex dugesii Fernald GTO, DGO, JAL, MICH, NAY, SLP, SIN, ZAC

Ilex gracilipes I.M. Johnst. CHIS

Ilex guianensis (Aubl.) Kuntze CHIS, TAB, VER

Ilex johnstonii Merr. CHIS

*Ilex liebmannii Standl. CHIS, VER

*Ilex mexicana (Turcz.) Black ex Hemsl. HGO, JAL, MEX, MICH, MOR, NAY, NLE, SIN, VER

*Ilex pringlei Standl. CHIS, HGO, OAX, PUE, VER

Ilex quercetorum I.M. Johnst. CHIS, GRO, OAX, VER

*Ilex rubra S. Watson CHIH, COAH, DGO, GTO, JAL, NAY,

NLE, QRO, SLP, SIN, SON, TAMS, ZAC

*Ilex servinii E. Carranza QRO, SLP

*Ilex socorroensis Brandegee COL

*Ilex tonii Lundell CHIS

Ilex vomitoria Aiton CHIS

\section{Family Araceae}

*Anthurium andicola Liebm. CHIS, MEX, OAX, PUE, VER Anthurium armeniense Croat CHIS

Anthurium bakeri Hook. f. CHIS, TAB

*Anthurium berriozabalense Matuda CHIS, OAX

*Anthurium cerrobaulense Matuda CHIS, OAX

*Anthurium cerropelonense Matuda OAX

*Anthurium clarinervium Matuda CHIS, OAX

*Anthurium coicoyanense Croat \& de Avila OAX

*Anthurium cordatotriangulum Matuda CHIS, OAX

Anthurium cubense Engl. CAM, QROO, YUC

*Anthurium chamulense Matuda CHIS, OAX, VER

*Anthurium chiapasense Standl. CHIS, OAX

*Anthurium faustomirandae Pérez-Farrera \& Croat CHIS 
Anthurium flexile Schott CHIS, OAX, TAB, VER

*Anthurium hagsaterianum Haager GRO

*Anthurium halmoorei Croat COL, JAL, MICH, NAY, SIN, ZAC

*Anthurium huautlense Matuda OAX

*Anthurium huixtlense Matuda CHIS, OAX, VER

*Anthurium joseanum Engl. CHIS, TAB

*Anthurium leuconeurum Lem. CHIS

*Anthurium lezamae Matuda CHIS, OAX, TAB

*Anthurium longipeltatum Matuda OAX, VER

Anthurium lucens Standl. CHIS, OAX, VER

*Anthurium machetioides Matuda CHIS, OAX, VER

Anthurium microspadix Schott CHIS, OAX

Anthurium montanum Hemsl. CHIS, GRO, OAX, VER

Anthurium myosuroides (Kunth) Endl. CHIS, VER

*Anthurium nakamurae Matuda CHIS, OAX

*Anthurium nelsonii Croat OAX

*Anthurium nizandense Matuda GRO, OAX

Anthurium nymphaeifolium K. Koch \& Bouché CHIS

*Anthurium ocotepecense Matuda CHIS, OAX

*Anthurium parvifolium Hemsl. CHIS

Anthurium parvispathum Hemsl. CHIS

Anthurium pedatoradiatum Schott CHIS, OAX, TAB, VER

Anthurium pentaphyllum (Aubl.) G. Don CAM, CHIS, OAX,

PUE, QRO, QROO, SLP, TAB, VER

*Anthurium podophyllum (Cham. \& Schltdl.) Kunth OAX, TAB, VER

*Anthurium riograndicola Matuda OAX

*Anthurium rionegrense Matuda CHIS, OAX

*Anthurium rzedowskii Croat CHIS, GRO, OAX

*Anthurium sarukhanianum Croat \& Haager CHIS, GRO

Anthurium scandens (Aubl.) Engl. CHIS, COL, GRO, JAL, OAX, PUE, TAB, VER

Anthurium schlechtendalii Kunth CAM, CHIS, GRO, JAL, MEX, NAY, OAX, QROO, TAB, VER, YUC

Anthurium seleri Engl. CHIS, OAX

*Anthurium subovatum Matuda OAX

Anthurium titanium Standl. \& Steyerm. CHIS, VER

Anthurium trinerve Miq. CHIS

*Anthurium umbrosum Liebm. CHIS, OAX, VER

Anthurium verapazense Engl. CHIS, OAX, TAB, VER

*Anthurium yetlense Matuda OAX

Arisaema dracontium (L.) Schott COAH, GTO, GRO, HGO, MEX, MOR, NLE, PUE, QRO, SLP, TAMS, VER

*Arisaema macrospathum Benth. COL, GTO, GRO, HGO, JAL, MEX, MICH, MOR, NAY, NLE, OAX, PUE, QRO, SLP, SIN, TAMS, VER

Dieffenbachia oerstedii Schott CHIS, OAX, TAB, VER

Dieffenbachia seguine (Jacq.) Schott CAM, CHIS, CDMX, GRO, JAL, MEX, MICH, MOR, OAX, QROO, TAB, VER, YUC

Dieffenbachia wendlandii Schott CHIS, GTO, OAX, QRO, VER

Dracontium soconuscum Matuda CHIS

Lemna aequinoctialis Welw. AGS, BCN, BCS, CAM, CHIS, $\mathrm{CHIH}, \mathrm{COAH}, \mathrm{COL}, \mathrm{GTO}, \mathrm{GRO}, \mathrm{HGO}$, JAL, MEX, MICH,
MOR, NAY, OAX, QRO, QROO, SLP, SIN, SON, TAB, TAMS, VER, YUC, ZAC

Lemna gibba L. AGS, BCN, BCS, CHIH, COAH, COL, CDMX, DGO, GTO, HGO, JAL, MEX, MICH, MOR, OAX, PUE, QRO, SLP, SON, TAMS, TLAX, VER, ZAC

Lemna minuta Kunth AGS, BCN, BCS, CAM, CHIS, CHIH, COAH, CDMX, GTO, GRO, HGO, JAL, MEX, MICH, MOR, NLE, OAX, PUE, QRO, QROO, SLP, SON, ZAC

Lemna obscura (Austin) Daubs AGS, CHIS, CDMX, HGO, JAL, MEX, MICH, MOR, PUE, TAB, VER, ZAC

Lemna trisulca L. BCN, BCS, CDMX, HGO, MEX

Lemna turionifera Landolt $\mathrm{BCN}$

Lemna valdiviana Phil. BCN, BCS, CHIH, COAH, COL, CDMX, DGO, GRO, HGO, JAL, MEX, MICH, MOR, OAX, QROO, TAB

* Monstera acacoyaguensis Matuda CHIS

Monstera acuminata K. Koch CAM, CHIS, OAX, PUE, QRO, QROO, SLP, TAB, VER

Monstera adansonii Schott HGO, OAX

Monstera deliciosa Liebm. CAM, CHIS, COL, HGO, JAL, MEX, MOR, NAY, OAX, PUE, QRO, SLP, SIN, TAB, TAMS, VER, YUC

Monstera dubia (Kunth) Engl. \& K. Krause CHIS

*Monstera florescanoana Croat, T. Krömer \& Acebey VER

Monstera friedrichstahlii Schott CHIS, GRO, HGO, OAX, VER

Monstera lechleriana Schott OAX, PUE, VER

Monstera pertusa (L.) de Vriese CHIS, TAB, VER

Monstera punctulata (Schott) Schott ex Engl. CHIS, OAX, VER Monstera tuberculata Lundell CAM, CHIS, OAX, QROO, TAB, VER

*Philodendron advena Schott CHIS, OAX, VER

Philodendron aurantiifolium Schott CHIS

*Philodendron basii Matuda COL, GRO, JAL, MEX

*Philodendron breedlovei Croat CHIS

*Philodendron dressleri G.S. Bunting NAY, SIN

*Philodendron escuintlense Matuda CHIS, OAX, TAB, VER

* Philodendron glanduliferum Matuda CHIS, OAX

Philodendron guttiferum Kunth CHIS, TAB, VER

Philodendron hederaceum (Jacq.) Schott CAM, CHIS, COL, GRO, JAL, MEX, MICH, MOR, NAY, OAX, PUE, QRO, QROO, SLP, SIN, TAB, VER, YUC

Philodendron inaequilaterum Liebm. CHIS, OAX, PUE, SLP, TAB, TAMS, VER

*Philodendron jodavisianum G.S. Bunting CHIS, TAB

Philodendron mexicanum Engl. CHIS, GRO, MEX, MOR, TAB, VER

Philodendron purulhense Croat CHIS

Philodendron radiatum Schott CAM, CHIS, GRO, MOR, NAY, OAX, PUE, QRO, QROO, SLP, SIN, TAB, VER, YUC

Philodendron sagittifolium Liebm. CHIS, MEX, MOR, OAX, PUE, QRO, SLP, TAB, VER

Philodendron schottii $\mathrm{K}$. Koch CHIS, OAX, TAB, VER

Philodendron seguine Schott CHIS, OAX, PUE, VER

Philodendron smithii Engl. CHIS, OAX, QROO, TAB, VER

*Philodendron sousae Croat CHIS, OAX

Philodendron standleyi Grayum CHIS, OAX, VER

*Philodendron subincisum Schott PUE, VER 
Philodendron tripartitum (Jacq.) Schott CHIS, COL, GRO, JAL, MEX, MOR, NAY, OAX, PUE, TAB, VER

Philodendron tuerckheimii Grayum OAX, TAB, VER

Philodendron verapazense Croat CHIS

Philodendron warscewiczii K. Koch \& C.D. Bouché CHIS, COL, JAL, MICH, NAY, OAX, SIN

Pistia stratiotes L. BCN, CAM, CHIS, COL, CDMX, DGO, GTO, GRO, HGO, JAL, MEX, MICH, MOR, NAY, OAX, PUE, QROO, SLP, SIN, TAB, TAMS, VER, YUC

Rhodospatha wendlandii Schott CHIS, TAB, VER

Spathiphyllum blandum Schott CHIS, OAX, TAB

*Spathiphyllum brevirostre (Liebm.) Schott CHIS, OAX

*Spathiphyllum cochlearispathum (Liebm.) Engl. CHIS, CDMX, GTO, MEX, OAX, TAB, VER, YUC

Spathiphyllum friedrichsthalii Schott CHIS, OAX, VER

* Spathiphyllum matudae G.S. Bunting CHIS, OAX, VER

*Spathiphyllum ortgiesii Regel VER

Spathiphyllum phryniifolium Schott CHIS, OAX, TAB, VER

*Spathiphyllum uxpanapense Matuda VER

Spathiphyllum wallisii Regel OAX, VER

Spirodela intermedia Koch CHIS, TAB

Spirodela polyrrhiza (L.) Schleid. CHIS, CHIH, COL, CDMX, GRO, HGO, JAL, MEX, MICH, NAY, OAX, QRO, SIN, SON,

TAB, TAMS, VER, YUC

Stenospermation marantifolium Hemsl. CHIS

Stenospermation robustum Engl. CHIS

Syngonium angustatum Schott CAM, CHIS, GRO, OAX, PUE, QROO, SLP, SIN, TAB, TAMS, VER, YUC

*Syngonium chiapense Matuda CHIS, OAX, VER

Syngonium hoffmannii Schott JAL, NAY, SON, ZAC

Syngonium macrophyllum Engl. CHIS, OAX, TAB, VER

Syngonium neglectum Schott CHIS, COL, CDMX, GRO, HGO,

JAL, MEX, MICH, MOR, NAY, OAX, PUE, QRO, SLP, SIN, TAB, TAMS, VER

Syngonium podophyllum Schott CAM, CHIS, GRO, HGO, MOR, NAY, OAX, PUE, QRO, QROO, SLP, SIN, TAB, TAMS, VER, YUC

* Syngonium sagittatum G.S. Bunting OAX, VER

Syngonium salvadorense Schott CHIS, HGO, OAX, PUE, TAB, VER

Syngonium schottianum H. Wendl. ex Schott CHIS, VER

Syngonium steyermarkii Croat CHIS

Wolffia brasiliensis Wedd. AGS, CAM, CHIS, COL, CDMX, HGO, JAL, MEX, MICH, MOR, OAX, PUE, QROO, SLP, TAB, TAMS, YUC, ZAC

Wolffia columbiana $\mathrm{H}$. Karst. AGS, CDMX, GRO, HGO, JAL, MEX, MICH, MOR, OAX, PUE, QRO, SIN, TAB, TAMS, TLAX, YUC, ZAC

Wolffiella gladiata (Hegelm.) Hegelm. CDMX, HGO, MEX

Wolffiella lingulata (Hegelm.) Hegelm. CHIS, CDMX, GTO,

HGO, JAL, MEX, MICH, TAB, TAMS, VER

Wolffiella oblonga (Phil.) Hegelm. CDMX, HGO, MEX, TAB, VER

Wolffiella welwitschii (Hegelm.) Monod CHIS, MOR, TAB

Xanthosoma helleborifolium (Jacq.) Schott CHIS

*Xanthosoma kerberi Engl. VER

*Xanthosoma mendozae Matuda GRO, MEX, MICH
Xanthosoma mexicanum Liebm. CHIS, COL, GRO, JAL, MEX, MICH, NAY, OAX, SLP, SIN, VER

*Xanthosoma obtusilobum Engl. ND

Xanthosoma pedatum Hemsl. CHIS, COL

Xanthosoma robustum Schott AGS, CAM, CHIS, COL, CDMX, DGO, GRO, HGO, JAL, MEX, MICH, MOR, NAY, NLE, OAX, PUE, QRO, SLP, SIN, TAB, TAMS, VER, YUC, ZAC

Xanthosoma sagittifolium (L.) Schott CHIS, COAH, MICH, NAY, NLE, OAX, PUE, QRO, QROO, SLP, TAB, VER

Xanthosoma wendlandii (Schott) Schott CHIS, COL, GRO, JAL, MEX, MICH, MOR, NAY, OAX, SIN, SON, TAB

*Xanthosoma yucatanense Engl. CAM, QROO, YUC

\section{Family Araliaceae}

Aralia humilis Cav. AGS, CHIS, CHIH, COAH, COL, DGO, GTO, GRO, HGO, JAL, MEX, MICH, MOR, NAY, OAX, PUE, QRO, SIN, SON, VER

Aralia racemosa $\mathrm{L}$. CHIH, COAH, DGO, SON

*Aralia regeliana Marchal COAH, DGO, GTO, HGO, NLE, QRO, SLP, TAMS, VER, ZAC

*Aralia scopulorum Brandegee BCN, BCS

Dendropanax arboreus (L.) Decne. \& Planch. CAM, CHIS, CHIH, COAH, COL, DGO, GRO, HGO, JAL, MEX, MICH, NAY, OAX, PUE, QRO, QROO, SLP, SIN, TAB, TAMS, VER, YUC

Dendropanax gonatopodus (Donn. Sm.) A.C. Sm. CHIS

Dendropanax hondurensis M.J. Cannon \& Cannon CHIS

* Dendropanax insulare (Rose) R.C. Schneid. NAY

Dendropanax leptopodus (Donn. Sm.) A.C. Sm. CHIS, GRO, OAX, SLP, VER

Dendropanax oliganthus (A.C. Sm.) A.C. Sm. CHIS, OAX

Dendropanax pallidus M. Cannon \& Cannon CHIS

*Dendropanax populifolius (Marchal) A.C. Sm. CHIS, OAX

Dendropanax schippii (A.C. Sm.) A.C. Sm. CAM, CHIS, VER

Hydrocotyle bonariensis Lam. CAM, CHIS, HGO, MOR, OAX, PUE, QRO, QROO, TAB, TAMS, VER, YUC

Hydrocotyle leucocephala Cham. \& Schltdl. CHIS, OAX, VER Hydrocotyle mexicana Schltdl. \& Cham. CHIS, COL, GRO, HGO, JAL, MEX, NAY, OAX, PUE, QRO, SLP, TAMS, VER

Hydrocotyle pusilla A. Rich. CHIH, JAL, SON

Hydrocotyle ranunculoides L. f. AGS, BCN, BCS, CHIH, COAH, CDMX, DGO, GTO, GRO, HGO, JAL, MEX, MICH, MOR, NLE, OAX, PUE, QRO, SLP, SON, TLAX, VER, ZAC Hydrocotyle umbellata L. BCN, BCS, CAM, CHIS, CHIH, COAH, COL, CDMX, DGO, GTO, HGO, JAL, MEX, MICH, MOR, NAY, NLE, OAX, QRO, QROO, SLP, SIN, SON, TAB, TAMS, TLAX, VER

Hydrocotyle verticillata Thunb. AGS, BCN, BCS, CAM, CHIS, CHIH, COAH, COL, CDMX, DGO, GRO, HGO, JAL, MEX, MICH, MOR, NLE, OAX, PUE, QRO, SLP, SIN, SON, TAB, TAMS, TLAX, VER, ZAC

Oreopanax arcanus A.C. Sm. CHIS

Oreopanax capitatus (Jacq.) Decne. \& Planch. CHIS, HGO, OAX, PUE, TAB, VER

Oreopanax echinops (Cham. \& Schltdl.) Decne. \& Planch. CHIS, COL, DGO, GRO, HGO, JAL, MEX, MICH, OAX, PUE, SIN, VER 
* Oreopanax flaccidus Marchal HGO, OAX, PUE, VER

* Oreopanax geminatus Marchal CHIS

Oreopanax guatemalensis (Lem. ex Bosse) Decne. \& Planch.

CAM, CHIS, OAX, QROO, TAB, VER

Oreopanax lachnocephalus Standl. CHIS

Oreopanax langlassei Standl. CHIS, COL, GRO, JAL, MICH, OAX

Oreopanax liebmannii Marchal CHIS, OAX, PUE, TAB, VER Oreopanax peltatus Linden CHIS, CHIH, COL, DGO, GRO, JAL, MEX, MICH, MOR, NAY, OAX, PUE, SLP, SIN, SON, VER, ZAC

Oreopanax platyphyllus Marchal CHIS, OAX, VER

Oreopanax sanderianus Hemsl. CHIS, COL, GRO, JAL, OAX Oreopanax xalapensis (Kunth) Decne. \& Planch. CHIS, COL, DGO, GRO, HGO, JAL, MEX, MICH, MOR, NAY, OAX, PUE, QRO, SLP, SIN, TAB, VER

Sciadodendron excelsum Griseb. COL, GRO, JAL, NAY

Schefflera morototoni (Aubl.) Maguire, Steyerm. \& Frodin CHIS, OAX, TAB, VER

\section{Family Arecaceae}

Acoelorrhaphe wrightii (Griseb. \& H. Wendl.) H. Wendl. ex Becc. CAM, CHIS, QROO, TAB, TAMS, VER, YUC

Acrocomia aculeata (Jacq.) Lodd. ex Mart. CAM, CHIS, COL, GRO, HGO, JAL, MEX, MICH, NAY, OAX, PUE, QRO, QROO, SLP, SIN, TAB, TAMS, VER, YUC, ZAC

Astrocaryum mexicanum Liebm. ex Mart. CHIS, JAL, NAY, OAX, TAB, VER

Attalea butyracea (Mutis ex L. f.) Wess. Boer CAM, CHIS, HGO, OAX, PUE, QROO, TAB, TAMS, VER, YUC

Attalea cohune Mart. CAM, CHIS, COL, GRO, JAL, MICH, NAY, OAX, QROO, SIN, TAB, YUC

*Attalea guacuyule (Liebm. ex Mart.) Zona COL, GRO, JAL, $\mathrm{MICH}, \mathrm{NAY}, \mathrm{OAX}$

Attalea liebmannii (Becc.) Zona CAM, CHIS, OAX, TAB, TAMS, VER

Attalea lundellii (Bartlett) Zona CHIS

Bactris major Jacq. CAM, CHIS, GRO, OAX, QROO, TAB, VER

Bactris mexicana Liebm. ex Mart. CAM, CHIS, GRO, OAX, PUE, QROO, TAB, VER

*Brahea aculeata (Brandegee) H.E. Moore CHIH, DGO, NAY, SIN, SON

*Brahea armata $\mathrm{S}$. Watson BCN, BCS, SON

*Brahea berlandieri Bartlett COAH, DGO, GTO, HGO, NLE, QRO, SLP, TAMS, VER

*Brahea brandegeei (Purpus) H.E. Moore BCN, BCS, JAL, $\mathrm{MICH}$

* Brahea decumbens Rzed. NLE, QRO, SLP, TAMS

Brahea dulcis (Kunth) Mart. CHIS, CHIH, COAH, COL, DGO, GTO, GRO, HGO, JAL, MEX, MICH, MOR, NAY, NLE, OAX, PUE, QRO, SLP, SIN, SON, TAMS, VER

*Brahea edulis $\mathrm{H}$. Wendl. ex S. Watson BCN

*Brahea elegans (Franceschi ex Becc.) H.E. Moore CHIH, SON

*Brahea moorei L.H. Bailey HGO, NLE, QRO, SLP, TAMS

Brahea nitida André CHIS, CHIH, GRO, OAX, PUE, SIN, SON
*Brahea pimo Becc. COL, GRO, JAL, MEX, MICH, NAY, OAX, SIN, ZAC

*Brahea roezlii Linden SON

*Brahea sarukhanii H.J. Quero JAL, NAY

Calyptrogyne ghiesbreghtiana (Linden \& H. Wendl.) H. Wendl. CHIS, HGO, TAB, VER

*Coccothrinax readii H.J. Quero CAM, QROO, YUC

*Cryosophila nana (Kunth) Blume ex Salomon CHIS, COL, GRO, JAL, MICH, NAY, OAX, SIN

Cryosophila stauracantha (Heynh.) R. Evans CAM, CHIS, GRO, MICH, OAX, QROO, SLP, TAB, VER

Chamaedorea adscendens (Dammer) Burret CHIS

*Chamaedorea alternans H. Wendl. CHIS, VER

Chamaedorea arenbergiana $\mathrm{H}$. Wendl. CHIS, OAX, VER

*Chamaedorea atrovirens Mart. OAX

Chamaedorea carchensis Standl. \& Steyerm. CHIS

*Chamaedorea cataractarum Mart. CHIS, OAX, QRO, TAB

Chamaedorea costaricana Oerst. CHIS, OAX

Chamaedorea elatior Mart. CHIS, HGO, OAX, PUE, SLP, TAB, VER

Chamaedorea elegans Liebm. ex Mart. CAM, CHIS, HGO, MEX, MICH, OAX, PUE, QRO, QROO, SLP, TAB, TAMS, VER

Chamaedorea ernesti-augusti H. Wendl. CAM, CHIS, GRO, OAX, QRO, QROO, SLP, TAB, VER

Chamaedorea fractiflexa Hodel \& Cast. Mont CHIS

Chamaedorea geonomiformis $\mathrm{H}$. Wendl. CHIS, OAX, TAB

*Chamaedorea glaucifolia H. Wendl. CHIS, OAX

Chamaedorea graminifolia H. Wendl. CAM, CHIS, OAX, QROO, YUC

*Chamaedorea hooperiana Hodel VER

* Chamaedorea ibarrae Hodel CHIS

* Chamaedorea karwinskiana H. Wendl. OAX, VER

Chamaedorea keelerorum Hodel \& Cast. Mont CHIS

*Chamaedorea klotzschiana $\mathrm{H}$. Wendl. VER

Chamaedorea liebmannii Mart. CHIS, OAX, PUE, VER

*Chamaedorea metallica O.F. Cook ex H.E. Moore CHIS, OAX, VER

*Chamaedorea microspadix Burret HGO, QRO, SLP, VER

*Chamaedorea monostachys Burret OAX, VER

Chamaedorea neurochlamys Burret CAM, CHIS, OAX, QROO, $\mathrm{TAB}$

Chamaedorea nubium Standl. \& Steyerm. CHIS, GRO, OAX

Chamaedorea oblongata Mart. CAM, CHIS, OAX, PUE, QROO, TAB, VER

*Chamaedorea oreophila Mart. OAX, PUE, VER

Chamaedorea parvisecta Burret CHIS

Chamaedorea pinnatifrons (Jacq.) Oerst. CHIS, HGO, OAX, PUE, TAB, VER

*Chamaedorea plumosa Hodel CHIS

*Chamaedorea pochutlensis Liebm. CHIS, COL, DGO, GRO, JAL, MICH, NAY, OAX, SIN

*Chamaedorea pringlei $\mathrm{S}$. Watson HGO, QRO, SLP, TAMS

*Chamaedorea queroana Hodel OAX

Chamaedorea quezalteca Standl. \& Steyerm. CHIS

*Chamaedorea radicalis Mart. DGO, HGO, JAL, NAY, NLE, QRO, SLP, TAMS, VER 
* Chamaedorea rhizomatosa Hodel OAX

* Chamaedorea rigida $\mathrm{H}$. Wendl. ex Dammer CHIS, OAX

Chamaedorea rojasiana Standl. \& Steyerm. CHIS, OAX, VER

Chamaedorea sartorii Liebm. CAM, GRO, OAX, PUE, QRO, QROO, SLP, VER

Chamaedorea schiedeana Mart. CHIS, GRO, HGO, OAX, PUE, QRO, SLP, TAB, VER

Chamaedorea seifrizii Burret CAM, CHIS, QROO, TAB, VER, YUC

Chamaedorea simplex Burret CHIS, TAB

Chamaedorea stolonifera H. Wendl. ex Hook. f. CHIS, GRO

Chamaedorea stricta Standl. \& Steyerm. CHIS

Chamaedorea tenella $\mathrm{H}$. Wendl. CHIS, OAX, VER

Chamaedorea tenerrima Burret CHIS

Chamaedorea tepejilote Liebm. CHIS, GRO, HGO, OAX, PUE, TAB, VER

Chamaedorea tuerckheimii (Dammer) Burret CHIS, OAX, VER Chamaedorea vulgata Standl. \& Steyerm. CHIS, OAX

Chamaedorea whitelockiana Hodel \& N.W. Uhl CHIS, OAX

Chamaedorea woodsoniana L.H. Bailey CHIS, OAX, TAB, VER

Desmoncus ferox Bartlett CAM, CHIS, QROO, TAB, VER

Desmoncus leiorhachis Burret CHIS

Desmoncus orthacanthos Mart. CAM, CHIS, OAX, QROO, TAB, VER, YUC

Desmoncus quasillarius Bartlett CAM, CHIS, QROO, TAB, YUC

Desmoncus uaxactunensis Bartlett CHIS

*Gaussia gomez-pompae (H.J. Quero) H.J. Quero CHIS, OAX, TAB, VER

Gaussia maya (O.F. Cook) H.J. Quero \& Read CAM, CHIS, OAX, QROO, TAB, VER

Geonoma interrupta (Ruiz \& Pav.) Mart. CHIS, OAX, TAB, VER

Geonoma oxycarpa Mart. CHIS, OAX, TAB, VER

Pseudophoenix sargentii H. Wendl. ex Sarg. CAM, QROO, YUC

Reinhardtia elegans Liebm. CHIS, OAX, VER

Reinhardtia gracilis (H. Wendl.) Drude ex Dammer CHIS, OAX, TAB, VER

Reinhardtia simplex (H. Wendl.) Drude ex Dammer CHIS

Roystonea dunlapiana P.H. Allen CAM, CHIS, QROO, TAB, VER

Roystonea regia (Kunth) O.F. Cook CHIS, CAM, QROO, TAB, VER, YUC

*Sabal gretherae H.J. Quero CAM, QROO, YUC

Sabal mauritiiformis (H. Kars.) Griseb. \& H. Wendl. CAM, CHIS, OAX, QROO, TAB, VER, YUC

Sabal mexicana Mart. CAM, CHIS, COL, GRO, HGO, JAL, MEX, MICH, NAY, NLE, OAX, PUE, QRO, QROO, SLP, SIN, TAB, TAMS, VER, YUC, ZAC

*Sabal pumos (Kunth) Burret BCS, DGO, GTO, GRO, JAL, MEX, MICH, MOR, NAY, SIN, SON, ZAC

* Sabal rosei (O.F. Cook) Becc. COL, JAL, NAY, SIN

* Sabal uresana Trel. BCS, CHIH, SIN, SON

Sabal yapa C. Wright ex Becc. CAM, QROO, YUC

Schippia concolor Burret CHIS
Synechanthus fibrosus (H. Wendl.) H. Wendl. CHIS, OAX, TAB, VER

Thrinax radiata Lodd. ex Schult. \& Schult. f. CAM, QROO, YUC

Washingtonia filifera (Linden ex André) H. Wendl. ex de Bary BCN, BCS, CHIH, COAH, DGO, SIN, SON

Washingtonia robusta $\mathrm{H}$. Wendl. BCN, BCS, CHIH, SON

\section{Family Aristolochiaceae}

*Aristolochia acontophylla Pfeifer COL

Aristolochia anguicida Jacq. CHIS, MICH, OAX, QROO, YUC

Aristolochia arborea Linden CHIS, TAB, VER

*Aristolochia asclepiadifolia Brandegee VER

*Aristolochia bracteosa Duch. COL, JAL, NAY

*Aristolochia brevipes Benth. AGS, BCN, BCS, CHIH, COAH, CDMX, DGO, GTO, GRO, HGO, JAL, MEX, MICH, NAY, OAX, PUE, SIN, SON

Aristolochia bullata Pfeifer CHIS, GRO, VER

*Aristolochia buntingii Pfeifer COL, DGO, JAL, MICH, SIN

*Aristolochia cardiantha Pfeifer COL, GRO, MEX, MICH

*Aristolochia carterae Pfeifer JAL, NAY

*Aristolochia colimensis Santana Mich. COL, JAL

*Aristolochia conversiae Pfeifer COL, MEX, MICH, PUE

*Aristolochia cordata Eastw. DGO

Aristolochia coryi I.M. Johnst. CHIH, COAH, NLE

*Aristolochia chiapensis J.F. Ortega \& R.V. Ortega CHIS

*Aristolochia davilae Calzada, G. Flores \& O. Téllez NAY

*Aristolochia durangensis Pfeifer DGO, NAY

*Aristolochia emiliae Santana Mich. \& Solìs JAL

Aristolochia erecta L. COAH, VER

*Aristolochia flexuosa Duch. JAL, MICH

*Aristolochia foetida Kunth COL, DGO, GRO, JAL, MEX, MICH, MOR, OAX, ZAC

*Aristolochia glossa Pfeifer JAL, MICH, MOR

Aristolochia grandiflora Sw. CHIS, OAX, TAB, VER, YUC, ZAC

*Aristolochia impudica J.F. Ortega VER

Aristolochia inflata Kunth CHIS, GRO, MICH, VER

*Aristolochia islandica Pfeifer COL

*Aristolochia karwinskii Duch. VER

*Aristolochia lassa I.M. Johnst. COAH, NLE, SLP

Aristolochia leuconeura Linden VER

*Aristolochia luzmariana Santana Mich. COL, JAL

*Aristolochia manantlanensis Santana Mich. COL, JAL

Aristolochia maxima Jacq. CAM, CHIS, OAX, QROO, TAB, VER, YUC

*Aristolochia micrantha Duch. TAMS, VER

*Aristolochia monticola Brandegee BCS, COL, DGO, NAY

*Aristolochia mutabilis Pfeifer GRO, JAL, MICH

*Aristolochia mycteria Pfeifer GRO, MEX, MICH

*Aristolochia nahua Paizanni \& Santana Mich. JAL

*Aristolochia nana S. Watson HGO, SLP

*Aristolochia nelsonii Eastw. CHIS, OAX

*Aristolochia oaxacana Eastw. OAX

*Aristolochia occidentalis Santana Mich. \& S. Lemus COL, JAL 
Aristolochia odoratissima L. CHIS, COL, JAL, MICH, NAY, OAX, TAB, VER

*Aristolochia orbicularis Duch. GRO, JAL, MICH, NAY, OAX, QRO, SLP, VER

Aristolochia ovalifolia Duch. CHIS, OAX, TAB, VER, YUC

*Aristolochia pacifica Santana Mich. \& Paizanni COL, JAL

*Aristolochia palmeri S. Watson JAL

Aristolochia paracleta Pfeifer CHIS

Aristolochia pentandra Jacq. CAM, COAH, GRO, HGO, JAL, MICH, MOR, NAY, NLE, OAX, QROO, TAB, TAMS, VER, YUC

Aristolochia pilosa Kunth CHIS, GRO, JAL, MEX, MICH, MOR, OAX, TAB, VER

Aristolochia porphyrophylla Pfeifer BCN, BCS, NAY, SIN, SON

*Aristolochia pringlei Rose DGO, GRO, JAL, MEX, MICH, MOR, NAY, SIN, ZAC

*Aristolochia pueblana J.F. Ortega \& R.V. Ortega GRO, PUE

*Aristolochia rhizantha Lundell COL, JAL, SLP, VER

*Aristolochia rzedowskiana Santana Mich. \& GuzmánHernández JAL

*Aristolochia rostrata Pfeifer TAMS

*Aristolochia savannoidea Paizanni \& M. Ramírez NAY

*Aristolochia secunda Pfeifer COAH, NLE

Aristolochia sericea Benth. CHIS, COL, JAL, MICH

*Aristolochia sinaloae Brandegee CHIH, DGO, JAL, NAY,

SIN, SON

*Aristolochia socorroensis Pfeifer COL

*Aristolochia styloglossa Pfeifer COL, JAL, MEX, OAX, PUE, VER

*Aristolochia taliscana Hook. \& Arn. COL, GRO, JAL, MEX, MICH, MOR, NAY, SIN

*Aristolochia tentaculata O.C. Schmidt GRO, JAL, MEX, $\mathrm{MICH}$

*Aristolochia tequilana S. Watson COL, JAL, NAY

*Aristolochia teretiflora Pfeifer OAX

*Aristolochia tresmariae Ferris NAY

*Aristolochia tricaudata Lem. CHIS, OAX

Aristolochia trilobata L. QROO, TAB, YUC

*Aristolochia tuitensis Santana Mich. \& Paizanni JAL

*Aristolochia variifolia Duch. CHIS, GRO, JAL, MICH, OAX

*Aristolochia veracruzana J.F. Ortega VER

*Aristolochia versabilifolia Pfeifer AGS, CDMX, GTO, HGO,

MEX, MICH, PUE, QRO, SLP, VER, ZAC

Aristolochia watsonii Wooton \& Standl. BCN, BCS, CHIH,

COAH, COL, SIN, SON, ZAC

*Aristolochia whitei I.M. Johnst. CHIH

Aristolochia wrightii Seem. CHIH, COAH, DGO, GTO, NLE, SLP

\section{Family Asparagaceae}

*Agave abisaii A. Vázquez \& Nieves JAL

*Agave aktites Gentry SIN, SON

*Agave albicans Jacobi HGO, QRO, VER

*Agave albomarginata Gentry CHIH, COAH, HGO, NLE, QRO, SLP, TAMS
*Agave albopilosa I. Cabral, Villarreal \& E. Estrada COAH, NLE

Agave americana L. BCN, BCS, CHIS, CHIH, COAH, COL, DGO, GTO, HGO, JAL, MEX, MOR, NAY, NLE, OAX, PUE, QRO, SLP, SON, TAMS, TLAX, VER, ZAC

*Agave andreae Sahagún \& A. Vázquez MICH

*Agave angustiarum Trel. GRO, JAL, MEX, MICH, MOR, OAX, PUE, VER

Agave angustifolia Haw. AGS, CAM, CHIS, CHIH, COL, DGO, GRO, HGO, JAL, MEX, MICH, MOR, NAY, OAX, PUE, QRO, QROO, SLP, SIN, SON, TAMS, TLAX, VER, YUC, ZAC

*Agave applanata K. Koch ex Jacobi CHIH, DGO, GTO, HGO, JAL, MEX, OAX, PUE, QRO, TLAX, VER, ZAC

*Agave arcedianoensis Cházaro, $\mathrm{O}$. Valencia \& A. Vázquez JAL

*Agave asperrima Jacobi COAH, DGO, NLE, QRO, SLP, TAMS

*Agave atrovirens Karw. ex Salm-Dyck CDMX, HGO, MEX, MOR, NLE, OAX, PUE, QRO, SLP, TLAX, VER, ZAC

*Agave attenuata Salm-Dyck COL, GRO, HGO, JAL, MEX, MICH, MOR, QRO, VER

*Agave aurea Brandegee BCS

*Agave avellanidens Trel. BCN, BCS

*Agave bovicornuta Gentry CHIH, SIN, SON

*Agave bracteosa S. Watson ex Engelm. COAH, NLE

Agave brachystachys Cav. CHIS, GRO, JAL, MICH

*Agave breedlovei Gentry CHIS, OAX

*Agave calodonta A. Berger ND

Agave cantala Roxb. CHIS, JAL, ZAC

*Agave capensis Gentry BCS

*Agave celsii Hook. GTO, HGO, NLE, PUE, QRO, SLP, TAMS, VER

*Agave cerulata Trel. BCN, BCS

*Agave colimana Gentry COL, JAL, NAY

*Agave colorata Gentry SIN, SON

*Agave collina Greenm. GRO, MEX, MOR

*Agave congesta Gentry CHIS

Agave convallis Trel. OAX, PUE

*Agave cupreata Trel. \& A. Berger GRO, MICH, OAX

*Agave chazaroi A. Vázquez y O.M. Valencia JAL

*Agave chiapensis Jacobi CHIS, OAX

*Agave chrysoglossa I.M. Johnst. BCN, SON

Agave dasylirioides Jacobi \& C.D. Bouché MEX, MOR, OAX, SLP

*Agave datylio Simon ex Weber BCS

Agave deserti Engelm. BCN, SON

Agave desmettiana Jacobi QRO, SIN, VER

*Agave difformis Berger HGO, QRO, SLP, TAMS, VER

*Agave doctorensis L. Hern. \& Magallán QRO

*Agave durangensis Gentry DGO, JAL, ZAC

*Agave ellemeetiana Jacobi GRO, MEX, OAX, VER

*Agave ensifera Jacobi HGO, MEX, SLP

*Agave felgeri Gentry SON

*Agave filifera Salm-Dyck AGS, CDMX, DGO, GTO, HGO, JAL, MEX, MICH, QRO, SLP, SIN, VER, ZAC

* Agave flexispina Trel. CHIH, DGO, NAY, ZAC

*Agave fortiflora Gentry SON 
Agave fourcroydes Lem. BCN, BCS, CAM, SLP, SIN, SON, TAMS, VER, YUC

* Agave franzosinii P. Sewell OAX

*Agave funkiana K. Koch \& C.D. Bouché CHIS, HGO, NLE, QRO, SLP, TAMS

*Agave garciae-mendozae Galván \& L. Hern. HGO, QRO, SLP, VER

*Agave geminiflora (Tagl.) Ker Gawl. DGO, JAL, NAY, SIN, ZAC

*Agave gentryi B. Ullrich COAH, DGO, HGO, NLE, QRO, SLP, TAMS

Agave ghiesbreghtii Lem. ex Jacobi CHIS, GRO, MEX, OAX, PUE, VER

*Agave gigantensis Gentry BCS

*Agave gilbertii A. Berger MEX

Agave $\times$ glomeruliflora (Engelm.) A. Berger $\mathrm{CHIH}, \mathrm{COAH}$, QRO, SLP

*Agave gomezpompae Cházaro \& Jimeno-Sevilla VER

*Agave gracielae Galván \& Zamudio QRO, SLP

Agave gracilipes Trel. CHIH

*Agave grijalvensis $\mathrm{B}$. Ullrich CHIS

*Agave guadalajarana Trel. JAL, NAY, ZAC

*Agave guiengola Gentry OAX

*Agave gypsophila Gentry COL, GRO, JAL

Agave havardiana Trel. AGS, CHIH, COAH

Agave hiemiflora Gentry CHIS

*Agave hookeri Jacobi JAL, MICH, SIN, SON

*Agave horrida Lem. ex Jacobi CDMX, GRO, HGO, JAL, MEX, MICH, MOR, OAX, SLP, TLAX, VER

*Agave impressa Gentry NAY, SIN

*Agave inaequidens $\mathrm{K}$. Koch CDMX, DGO, HGO, JAL, MEX, MICH, MOR, SIN, TLAX

*Agave isthmensis García-Mend. \& F. Palma CHIS, OAX

*Agave jaiboli Gentry CHIH, SON

*Agave jimenoi Cházaro \& A. Vázquez VER

*Agave karwinskii Zucc. OAX, PUE, VER

*Agave kavandivi García-Mend. \& Chávez-Rendón OAX

*Agave kerchovei Lem. GRO, HGO, OAX, PUE, QRO

*Agave kewensis Jacobi CHIS

* Agave kirchneriana Berger GRO

*Agave kristenii A. Vázquez \& Cházaro MICH

Agave lechuguilla Torr. AGS, CHIH, COAH, CDMX, DGO, GTO, HGO, MEX, NLE, PUE, QRO, SLP, TAMS, VER, ZAC Agave lophantha Schiede COAH, HGO, NLE, QRO, SLP, TAMS, VER

*Agave lurida Aiton OAX, PUE, VER

*Agave macrantha Tod. CDMX, HGO, MEX, MOR, OAX, QRO

*Agave macroacantha Zucc. OAX, PUE

*Agave macroculmis Tod. COAH, CDMX, DGO, GTO, HGO, MEX, MICH, NLE, OAX, PUE, QRO, SLP, TAMS, VER

*Agave manantlanicola Cuevas \& Santana Mich. JAL

*Agave mapisaga Trel. CDMX, GTO, HGO, MEX, MOR, OAX, PUE, QRO, SLP, TAMS, TLAX, ZAC

*Agave margaritae Brandegee BCS

*Agave marmorata Roezl OAX, PUE

*Agave maximiliana Baker COL, DGO, JAL, NAY, SIN, ZAC
*Agave microceps (Kimnach) A. Vázquez \& Cházaro SIN

*Agave mitis Mart. HGO, SLP

*Agave montana Villarreal COAH, NLE

*Agave montium-sancticaroli García-Mend. NLE, TAMS

*Agave moranii Gentry BCN

*Agave multifilifera Gentry CHIH, DGO, SIN, SON

Agave murpheyi F. Gibson SON

*Agave nayaritensis Gentry JAL, NAY, ZAC

*Agave nickelsii Gosselin COAH, NLE

*Agave nizandensis Cutak OAX

*Agave nussaviorum García-Mend. GRO, OAX

*Agave obscura Schiede ex Schltdl. COAH, HGO, OAX, PUE, SLP, VER

*Agave ocahui Gentry SON

*Agave ornithobroma Gentry DGO, JAL, NAY, SIN, ZAC

*Agave oroensis Gentry COAH, ZAC

*Agave ovatifolia Starr \& Villarreal NLE

*Agave pablocarrilloi A. Vázquez, M. A. Muñiz \& Padilla-

Lepe COL

Agave pachycentra Trel. CHIS, OAX

Agave palmeri Engelm. CHIH, DGO, SON

*Agave parrasana A. Berger AGS, CHIH, COAH, DGO, NLE, SLP, ZAC

Agave parryi Engelm. AGS, CHIH, COAH, DGO, GTO, HGO, JAL, NLE, QRO, SLP, SON, TAMS, ZAC

Agave parviflora Torr. CHIH, SON

*Agave peacockii Croucher HGO, OAX, PUE

*Agave pedunculifera Trel. COL, GRO, JAL, MICH, NAY, SIN, ZAC

*Agave pelona Gentry BCN, SON

*Agave pendula Schnittsp. CHIS, OAX, VER

*Agave petrophila García-Mend. \& E. Martínez GRO, OAX, PUE

*Agave pintilla S. González, M. González \& L. Reséndiz DGO

*Agave polianthiflora Gentry CHIH, SON

*Agave polyacantha Haw. OAX, SLP, TAMS, VER

*Agave potatorum Zucc. CHIS, GRO, OAX, PUE, VER

*Agave potrerana Trel. CHIH, COAH, JAL, ZAC

*Agave promontorii Trel. BCS

Agave pumila De Smet ex Baker COAH

*Agave pygmaea Gentry CHIS, OAX

*Agave rhodacantha Trel. CHIH, COL, JAL, NAY, OAX, PUE, SIN, SON, ZAC

*Agave rzedowskiana P. Carrillo, R. Vega \& R. Ramírez-D. DGO, JAL, SIN, ZAC

*Agave salmiana Otto ex Salm-Dyck AGS, CHIS, COAH, COL, CDMX, DGO, GTO, HGO, JAL, MEX, MICH, MOR, NLE, OAX, PUE, QRO, SLP, TLAX, VER, ZAC

*Agave scaposa Gentry OAX, PUE

*Agave schidigera Lem. AGS, CHIH, DGO, GTO, GRO, JAL, MEX, MICH, NAY, QRO, SLP, SIN, SON, ZAC

*Agave schneideriana A. Berger PUE

Agave schottii Engelm. CHIH, SON

*Agave sebastiana Greene BCN, BCS

Agave seemanniana Jacobi CHIS, OAX

Agave shawii Engelm. BCN, BCS

*Agave shrevei Gentry CHIH, DGO, SIN, SON 
Agave sisalana Perrine BCS, CAM, CHIS, HGO, QROO, VER, YUC

*Agave sobria Brandegee BCS

*Agave striata Zucc. AGS, COAH, DGO, HGO, JAL, MEX, NLE, QRO, SLP, TAMS, VER, ZAC

*Agave stricta Salm-Dyck OAX, PUE

*Agave stringens Trel. JAL

*Agave subsimplex Trel. BCN, SON

*Agave temacapulinensis A. Vázquez \& Cházaro JAL

*Agave tenuifolia Zamudio \& E. Sánchez HGO, QRO, SLP, TAMS

*Agave tequilana F.A.C. Weber COL, GTO, JAL, MICH, NAY, OAX, PUE, SIN, SON, ZAC

*Agave titanota Gentry OAX, PUE

*Agave triangularis Jacobi OAX, PUE

* Agave turneri $\mathrm{R}$. Webb \& Salazar-Ceseña BCN

*Agave valenciana Cházaro \& A. Vázquez JAL

*Agave vazquezgarciae Cházaro \& J.A. Lomelí COL, JAL

*Agave victoriae-reginae T. Moore COAH, DGO, NLE

* Agave vilmoriniana A. Berger AGS, CHIH, COL, DGO, JAL, SIN, SON, ZAC

*Agave vizcainoensis Gentry BCS

*Agave warelliana Baker CHIS

Agave weberi Cels ex Poisson COAH, DGO, NLE, SLP, TAMS

*Agave wendtii Cházaro VER

*Agave wocomahi Gentry CHIH, DGO, JAL, SIN, SON, ZAC

*Agave xylonacantha Salm-Dyck HGO, QRO, SLP, TAMS, VER

*Agave yuccifolia DC. ex Redouté HGO

*Agave zebra Gentry SON

*Anthericum ciliatum Spreng. GRO, OAX

*Anthericum humboldtii Hemsl. MEX, MICH, MOR

* Beaucarnea compacta L. Hern. \& Zamudio GTO, QRO, SLP

*Beaucarnea glassiana (L. Hern. \& Zamudio) V. Rojas GTO, QRO, SLP

*Beaucarnea goldmanii Rose CHIS

* Beaucarnea gracilis Lem. OAX, PUE

* Beaucarnea hiriartiae L. Hern. GRO, OAX

*Beaucarnea hookeri (Lem.) Baker GTO, HGO, QRO, SLP, TAMS

*Beaucarnea inermis (S. Watson) Rose QRO, SLP, TAMS, VER

Beaucarnea pliabilis (Baker) Rose CAM, QROO, YUC

* Beaucarnea purpusii Rose PUE

*Beaucarnea recurvata Lem. OAX, PUE, TAMS, VER

* Beaucarnea sanctomariana L. Hern. OAX

*Beaucarnea stricta Lem. OAX, PUE

Beschorneria albiflora Matuda CHIS, OAX

*Beschorneria calcicola García-Mend. OAX, PUE, VER

*Beschorneria rigida Rose GTO, QRO, SLP, TAMS, VER

*Beschorneria septentrionalis García-Mend. NLE, TAMS

*Beschorneria tubiflora (Kunth \& C.D. Bouché) Kunth HGO, QRO, VER

*Beschorneria wrightii Hook. MEX, MICH

*Beschorneria yuccoides K. Koch HGO, PUE, SLP, VER

*Bessera elegans Schult. f. AGS, COL, DGO, GRO, JAL, MEX,

MICH, MOR, NAY, OAX, PUE, SIN, ZAC

*Bessera tenuiflora (Greene) J.F. Macbr. BCS
*Bessera tuitensis R. Ramírez-D. JAL, NAY

Bloomeria clevelandii $\mathrm{S}$. Watson $\mathrm{BCN}$

Bloomeria crocea (Torr.) Cov. BCN

Brodiaea jolonensis Eastw. BCN

Brodiaea orcuttii (Greene) Baker BCN

*Dandya balsensis López-Ferrari \& Espejo GRO, MEX, MICH, MOR

*Dandya hannibalii L.W. Lenz MICH

* Dandya purpusii (Brandegee) H.E. Moore COAH

*Dandya thadhowardii L.W. Lenz GRO, MICH

*Dasylirion acrotrichum (Schiede) Zucc. AGS, DGO, GTO, HGO, JAL, MEX, NAY, NLE, OAX, PUE, QRO, SLP, TAMS, VER, ZAC

*Dasylirion berlandieri S. Watson COAH, GTO, JAL, NLE, QRO, SLP, TAMS

*Dasylirion cedrosanum Trel. CHIH, COAH, DGO, JAL, NLE, QRO, SLP, ZAC

*Dasylirion gentryi Bogler CHIH, SIN, SON

*Dasylirion glaucophyllum Hook. HGO, QRO, VER

*Dasylirion graminifolium (Zucc.) Zucc. AGS, JAL, SLP, ZAC Dasylirion leiophyllum Engelm. ex Trel. CHIH, COAH, NLE

*Dasylirion longissimum Lem. HGO, NLE, QRO, SLP, TAMS, VER, ZAC

*Dasylirion longistylum J. Macbr. QRO, SLP

*Dasylirion lucidum Rose OAX, PUE, VER

*Dasylirion miquihuanense Bogler NLE, SLP, TAMS

*Dasylirion occidentalis Bogler ex Hochstätter AGS

*Dasylirion palaciosii Rzed. SLP

*Dasylirion palmeri Trel. COAH, NLE, TAMS, ZAC

*Dasylirion parryanum Trel. GTO, NLE, QRO, SLP

*Dasylirion quadrangulatum S. Watson NLE, SLP, TAMS

*Dasylirion sereke Bogler CHIH

*Dasylirion serratifolium (Karw. ex Schult. f.) Zucc. GRO, OAX, PUE, VER

*Dasylirion simplex Trel. DGO

*Dasylirion stewartii I.M. Johnst. COAH

Dasylirion texanum Scheele CHIH, COAH, DGO, NLE, SLP, TAMS

*Dasylirion treleasei (Bogler) Hochstätter SLP

Dasylirion wheeleri S. Watson ex Rothr. CHIH, COAH, DGO, SON, ZAC

Dichelostemma capitatum (Benth.) Alph. Wood BCN, BCS, COL, SON, COL

Dracaena americana Donn. Sm. CAM, CHIS, OAX, QROO, $\mathrm{TAB}, \mathrm{VER}, \mathrm{YUC}$

*Echeandia albiflora (Schltdl. \& Cham.) M. Martens \& Galeotti VER

*Echeandia atoyacana Cruden GRO, MEX

*Echeandia attenuata Cruden DGO, SIN

*Echeandia breedlovei Cruden CHIS, GRO, OAX

*Echeandia campechiana Cruden CAM, QROO, YUC

*Echeandia coalcomanensis Cruden MICH

*Echeandia confertiflora Cruden OAX

*Echeandia conzattii Cruden GRO, OAX

*Echeandia crudeniana Aarón Rodr. JAL, NAY

Echeandia chandleri (Greenm. \& C.H. Thomps.) Cruden CHIH, COAH, NLE, TAMS 
*Echeandia chiapensis Cruden CHIS, OAX

* Echeandia drepanoides (Greenm.) Cruden OAX

*Echeandia durangensis (Greenm.) Cruden AGS, CHIH, COAH, COL, CDMX, DGO, GTO, GRO, HGO, JAL, MEX, MICH, MOR, NLE, OAX, QRO, SLP, SIN, ZAC

Echeandia echeandioides (Schltdl.) Cruden CHIS, CDMX, GTO, GRO, MEX, MICH, MOR, OAX, PUE, SLP, VER

*Echeandia elegans Cruden GRO, MOR

* Echeandia falcata Cruden GTO, QRO

Echeandia flavescens (Schult. \& Schult. f.) Cruden AGS, CHIH, COAH, COL, CDMX, DGO, GTO, GRO, HGO, JAL, MEX, MICH, MOR, NAY, NLE, OAX, PUE, QRO, SLP, SIN, SON, TAMS, TLAX, VER, ZAC

*Echeandia flexuosa Greenm. AGS, GTO, GRO, HGO, JAL, MEX, MICH, MOR, NAY, OAX, QRO, SLP, SIN, ZAC

*Echeandia formosa (Weath.) Cruden CHIS, GRO, JAL, MEX, MICH, MOR, OAX, TAMS

*Echeandia gentryi Cruden DGO, JAL, NAY, SIN, ZAC

*Echeandia gracilis Cruden CDMX, GRO, HGO, MEX, MOR, OAX, PUE, QRO, VER

*Echeandia grandiflora Cruden OAX

*Echeandia hallbergii Cruden OAX

* Echeandia hintonii Cruden GRO

*Echeandia hirticaulis Cruden GRO, MEX, MICH, OAX

*Echeandia imbricata Cruden COL, GRO, JAL, MICH

*Echeandia longifolia (Weath.) Cruden CHIS, OAX, VER

Echeandia longipedicellata Cruden CHIS, COL, CDMX, DGO, GRO, HGO, JAL, MEX, MOR, OAX, SIN, VER

Echeandia luteola Cruden CAM, QROO, YUC

*Echeandia llanicola Cruden OAX

*Echeandia macrophylla Rose ex Weath. DGO, QRO, SLP

*Echeandia magnifica López-Ferrari, Espejo \& Ceja GRO

Echeandia matudae Cruden CHIS

*Echeandia mcvaughii Cruden COL, JAL, NAY, ZAC

*Echeandia mexiae Cruden GRO, MEX, MOR

*Echeandia mexicana Cruden AGS, CAM, CHIH, COL, CDMX, DGO, GTO, GRO, HGO, JAL, MEX, MICH, MOR, NAY, NLE, OAX, PUE, QRO, QROO, SLP, SIN, SON, TAMS, TLAX, VER, YUC, ZAC

*Echeandia michoacensis (Poelln.) Cruden COL, GTO, MICH, MOR

* Echeandia mirandae Cruden OAX, PUE

* Echeandia montealbanensis Cruden OAX

*Echeandia nana (Baker) Cruden CDMX, GTO, HGO, MEX, MICH, MOR, PUE, QRO, SLP, TLAX, VER

*Echeandia nayaritensis Cruden NAY, SIN

*Echeandia novogaliciana Aarón Rodr. \& Ortiz-Catedral JAL, NAY, ZAC

*Echeandia oaxacana Cruden OAX

*Echeandia occidentalis Cruden COL, GTO, GRO, JAL, MEX, MICH, NAY, OAX, SIN, SLP, ZAC

*Echeandia palmeri Cruden CHIH, DGO, SON

*Echeandia paniculata Rose CDMX, GTO, GRO, HGO, JAL, MEX, MICH, MOR, OAX, PUE, QRO

* Echeandia parva Cruden OAX, PUE

* Echeandia parvicapsulata Cruden COL, JAL, NAY

Echeandia parviflora Baker CHIS, COL, GRO, JAL, MEX,
MICH, MOR, OAX, PUE, SIN, VER

*Echeandia pihuamensis Cruden COL, JAL

*Echeandia platyphylla (Greenm.) Cruden PUE

*Echeandia pseudopetiolata Cruden GRO

*Echeandia pseudoreflexa Cruden CHIS

*Echeandia ramosissima (C. Presl) Cruden CHIH, COL, DGO, GRO, JAL, MICH, NAY, SIN, SON, ZAC

Echeandia reflexa (Cav.) Rose CAM, CHIS, CHIH, COL, CDMX, DGO, GTO, GRO, HGO, JAL, MEX, MICH, MOR, NAY, NLE, OAX, PUE, QRO, SLP, SIN, TAMS, TLAX, VER, ZAC

*Echeandia robusta Cruden COL, JAL, MICH

*Echeandia sanmiguelensis Cruden GTO, QRO

*Echeandia scabrella (Benth.) Cruden AGS, CHIH, COAH, DGO, GTO, JAL, MICH, NAY, QRO, ZAC

* Echeandia sinaloensis Cruden COL, JAL, NAY, SIN

Echeandia skinneri (Baker) Cruden CHIS, GRO, MICH, OAX

*Echeandia smithii Cruden OAX

* Echeandia tamaulipensis Cruden TAMS

*Echeandia taxacana Cruden GRO, MEX, MOR, OAX

*Echeandia tenuifolia Cruden OAX

*Echeandia tenuis (Weath.) Cruden GRO, JAL, MEX, MOR, OAX

*Echeandia udipratensis Cruden JAL, MICH

* Echeandia vaginata Cruden OAX

Echeandia vestita (Baker) Cruden CHIS, GTO, GRO, JAL, MICH, OAX, PUE, TLAX, VER

Furcraea cabuya Trel. CHIS, YUC

* Furcraea cahum Trel. CAM, QROO, YUC

* Furcraea elegans Tod. ND

* Furcraea flavo-viridis Hook. HGO

Furcraea guatemalensis Trel. CHIS, OAX

*Furcraea guerrerensis Matuda COL, GRO, JAL, MICH, NAY, OAX

Furcraea longaeva Karw. \& Zucc. OAX, PUE

*Furcraea macdougallii Matuda OAX, PUE

* Furcraea martinezii García-Mend. \& L. de la Rosa GRO

* Furcraea niquivilensis Matuda ex García-Mend. CHIS

*Furcraea parmentieri (Roezl ex Ortgies) García-Mend. COL, CDMX, GTO, GRO, HGO, JAL, MEX, MICH, MOR, OAX, QRO, VER

Furcraea pubescens Tod. COL, GRO, JAL, MEX, MICH, OAX, VER

Furcraea quicheensis Trel. CHIS

Furcraea samalana Trel. CHIS

*Furcraea undulata Jacobi CHIS, TAB

*Hemiphylacus alatostylus L. Hern. GTO, HGO, QRO, SLP

*Hemiphylacus hintoniorum L. Hern. NLE, TAMS

*Hemiphylacus latifolius S. Watson AGS, COAH, GTO, HGO, NLE, OAX, PUE, QRO, SLP, ZAC

*Hemiphylacus mahindae L. Hern., OAX, PUE

*Hemiphylacus novogalicianus L. Hern. AGS, JAL, ZAC

*Hesperaloe campanulata G.D. Starr NLE

*Hesperaloe chiangii (G.D. Starr) B.L. Turner SLP

Hesperaloe funifera (K. Koch) Trel. COAH, NLE, SLP, ZAC

*Hesperaloe malacophylla Hochstätter \& Mart.-Aval. TAMS

*Hesperaloe nocturna Gentry SON 
Hesperaloe parviflora (Torr.) J.M. Coult. COAH, SON, TAMS

*Hesperaloe tenuifolia G.D. Starr SON

Hesperocallis undulata A. Gray BCN, BCS, SON

*Hesperoyucca peninsularis (McKelvey) Clary BCN, BCS

Hesperoyucca whipplei (Torr.) Baker ex Trel. BCN, SON

Maianthemum amoenum (H.L. Wendl.) LaFrankie CHIS, OAX, VER

*Maianthemum comaltepecense Espejo, López-Ferrari \& Ceja OAX

Maianthemum flexuosum (Bertol.) La Frankie CHIS, GRO, HGO, MEX, MICH, OAX, VER

Maianthemum gigas (Woodson) La Frankie CHIS

*Maianthemum macrophyllum (M. Martens \& Galeotti)

LaFrankie OAX, VER

*Maianthemum mexicanum García-Arév. DGO, SIN

Maianthemum paniculatum (M. Martens \& Galeotti) LaFrankie CHIS, GRO, HGO, MEX, OAX, PUE, VER

Maianthemum scilloideum (M. Martens \& Galeotti) LaFrankie CHIS, COAH, GRO, MEX, MICH, NLE, OAX, PUE, VER

Maianthemum stellatum (L.) Link CHIH, COAH, NLE, SON

*Manfreda brunnea (S. Watson) Rose CHIH, COAH, DGO,

TAMS

*Manfreda bulbulifera Castillejos \& E. Solano GRO

*Manfreda chamelensis E.J. Lott \& Verh.-Will. COL, GRO, JAL, OAX

*Manfreda elongata Rose DGO, JAL, NAY, ZAC

* Manfreda galvaniae A. Castañeda, S. Franco \& García-Mend. MEX

* Manfreda guerrerensis Matuda GRO, MICH

* Manfreda guttata (Jacobi \& C.D. Bouché) Rose AGS, CHIH, DGO, GTO, HGO, JAL, MICH, NAY, QRO, SLP, TAMS, ZAC

*Manfreda hauniensis (J.B. Petersen) Verh.-Will. GRO, MEX, MICH, MOR, OAX

* Manfreda involuta McVaugh JAL, NAY, ZAC

*Manfreda jaliscana Rose CHIH, COL, DGO, GRO, JAL, MICH, NAY, SIN, SON, ZAC

*Manfreda littoralis García-Mend., A. Castañeda \& S. Franco GRO, OAX

*Manfreda longibracteata Verh.-Will. COL, JAL, MICH

Manfreda longiflora (Rose) Verh.-Will. NLE, TAMS

* Manfreda maculata (C. Mart.) Rose GRO, MEX, MICH, OAX

Manfreda maculosa (Hook.) Rose COAH, NLE, TAMS

* Manfreda malinaltenangensis Matuda MEX

* Manfreda nanchititlensis Matuda GRO, MEX

* Manfreda paniculata L. Hern., R.A. Orellana \& Carnevali CAM, QROO, YUC

* Manfreda parva Aarón Rodr. GRO

*Manfreda petskinil R.A. Orellana, L. Hern. \& Carnevali QROO, YUC

*Manfreda planifolia (S. Watson) Rose CHIH, NAY, SON

*Manfreda potosina (B.L. Rob. \& Greenm.) Rose COAH, DGO, SLP, ZAC

*Manfreda pringlei Rose CDMX, GRO, HGO, JAL, MEX, MICH, MOR, NAY, OAX, PUE, TLAX

*Manfreda pubescens (Regel \& Ortgies) Verh.-Will. ex Pina CHIS, GRO, MEX, MOR, OAX

*Manfreda revoluta (Klotzsch) Rose MEX
*Manfreda rubescens Rose DGO, JAL, NAY, VER, ZAC

Manfreda scabra (Ortega) McVaugh AGS, CHIS, COL, CDMX, DGO, GTO, GRO, HGO, JAL, MEX, MICH, MOR, NAY, OAX, PUE, QRO, SLP, SIN, TLAX, VER, ZAC

Manfreda sileri Verh.-Will. TAMS

*Manfreda singuliflora (S. Watson) Rose CHIH, DGO, JAL, SIN, SON, ZAC

Manfreda variegata (Jacobi) Rose CHIS, CHIH, COAH, DGO, GRO, HGO, MOR, NLE, PUE, QRO, SLP, TAMS, VER, YUC Manfreda virginica (L.) Salisb. ex Rose COAH, NLE, TAMS Milla biflora Cav. AGS, BCS, CHIS, CHIH, COAH, COL, CDMX, DGO, GTO, GRO, HGO, JAL, MEX, MICH, MOR, NAY, OAX, PUE, QRO, SLP, SIN, SON, TAMS, TLAX, VER, ZAC

*Milla bryantii I.M. Johnst. CHIH, COAH, DGO

* Milla delicata H.E. Moore GRO

* Milla filifolia T.M. Howard GRO, MEX, MOR

* Milla magnifica H.E. Moore GRO, MEX, MOR

* Milla mexicana T.M. Howard OAX, PUE

* Milla mortoniana H.E. Moore GRO

* Milla oaxacana Ravenna OAX

* Milla potosina T.M. Howard SLP

* Milla rosea H.E. Moore NLE

* Milla valliflora J.E. Gut. \& E. Solano OAX, PUE

Muilla maritima (Torr.) S. Watson BCN

Nolina affinis Trel. CHIH, SON

*Nolina azureogladiata D. Donati OAX

*Nolina beldingii Brandegee BCS

Nolina bigelovii (Torr.) S. Watson BCN, SON

* Nolina cespitifera Trel. COAH, NLE, SLP, ZAC

*Nolina durangensis Trel. CHIH, DGO

Nolina erumpens (Torr.) S. Watson CHIH, COAH, DGO

*Nolina excelsa García-Mend. \& E. Solano OAX

*Nolina hibernica Hochstätter ex D. Donati QRO, TAMS

*Nolina humilis S. Watson SLP

*Nolina juncea (Zucc.) J. Macbr. AGS, COAH, DGO, JAL, NAY, ZAC

*Nolina matapensis Wiggins CHIH, SON

Nolina micrantha I.M. Johnst. CHIH, COAH

Nolina microcarpa $\mathrm{S}$. Watson CHIH, COAH, DGO, NLE, SON

*Nolina nelsonii Rose HGO, NLE, QRO, TAMS, VER

*Nolina palmeri S. Watson BCN, BCS

Nolina parryi S. Watson BCN

*Nolina parviflora (Kunth) Hemsl. AGS, CHIH, COAH, COL, CDMX, DGO, GTO, HGO, JAL, MEX, MICH, MOR, NAY, NLE, OAX, PUE, QRO, SLP, SIN, TAMS, TLAX, VER, ZAC *Nolina pumila Rose DGO, NAY

*Nolina rigida (Brongn.) Trel. ND

Nolina texana S. Watson CHIH, COAH, DGO, NLE, SLP, SON, ZAC

*Nolina watsoni (Baker) Hemsl. GTO, SLP, TAMS

* Petronymphe decora H.E. Moore GRO

* Polianthes alboaustralis E. Solano \& Ríos-Gómez OAX

* Polianthes bicolor Solano \& García-Mend. OAX

*Polianthes cernua A. Castro, J. G. González \& Aarón Rodr. JAL

*Polianthes densiflora (B.L. Rob. \& Fernald) Shinners CHIH 
* Polianthes elongata Rose GRO

*Polianthes geminiflora (Lex.) Rose AGS, COL, CDMX, DGO, GTO, GRO, HGO, JAL, MEX, MICH, MOR, NAY, OAX, PUE, QRO, SLP, TLAX, VER, ZAC

*Polianthes howardii Verh. Will. COL, JAL

*Polianthes longiflora Rose DGO, JAL, MICH, ZAC

*Polianthes montana Rose GTO, JAL, NAY, SLP, ZAC

*Polianthes multicolor E. Solano \& Dávila GTO, JAL, QRO, SLP

*Polianthes nelsonii Rose DGO

*Polianthes oaxacana García-Mend. \& E. Solano OAX

*Polianthes palustris Rose DGO, JAL, NAY, SLP, ZAC

*Polianthes platyphylla Rose DGO, JAL, NAY, ZAC

*Polianthes pringlei Rose AGS, DGO, GTO, GRO, JAL, MEX, MICH, MOR, NAY, SLP, ZAC

*Polianthes sessiliflora (Hemsl.) Rose JAL, MICH, SLP, TAMS Polianthes tuberosa L. CAM, CHIS, GRO, MEX, MICH, MOR, OAX, QROO, VER, YUC

*Polianthes zapopanensis E. Solano \& Ríos-Gómez JAL

*Prochnyanthes mexicana (Zucc.) Rose AGS, COL, DGO, GTO, JAL, MEX, MICH, MOR, NAY, QRO, ZAC

*Triteleia guadalupensis L.W. Lenz BCN

Triteleiopsis palmeri (S. Watson) Hoover BCN, BCS, SON

Yucca aloifolia L. CHIS, GRO, MOR, OAX, PUE, VER, YUC Yucca arizonica McKelvey CHIH, SON

Yucca baccata Torr. CHIH, COAH, SON

Yucca brevifolia Engelm. BCN, SON

*Yucca capensis L.W. Lenz BCS

Yucca carnerosana (Trel.) McKelvey CHIH, COAH, DGO, GTO, NLE, SLP, SON, TAMS, ZAC

*Yucca coahuilensis Matuda \& Piña COAH

*Yucca decipiens Trel. AGS, DGO, GTO, JAL, NLE, SLP, ZAC

*Yucca declinata Laferr. SON

*Yucca desmetiana Baker CHIH

Yucca elata Engelm. CHIH, COAH, DGO, SON

*Yucca endlichiana Trel. COAH, NLE

Yucca faxoniana Sarg. CHIH, COAH

*Yucca filifera Chabaud AGS, CHIH, COAH, CDMX, DGO, GTO, HGO, JAL, MEX, MICH, NLE, OAX, PUE, QRO, SLP, TAMS, TLAX, VER, ZAC

*Yucca grandiflora Gentry CHIH, SON

Yucca guatemalensis Baker CAM, CHIS, CDMX, GRO, MEX, MOR, OAX, PUE, QRO, QROO, SLP, TLAX, VER, YUC

*Yucca jaliscensis Trel. COL, DGO, GTO, JAL, MEX, MICH, NAY, SIN, ZAC

*Yucca lacandonica Gómez-Pompa \& J. Valdés CAM, CHIS, OAX, TAB, VER

*Yucca linearifolia Clary COAH, NLE

*Yucca madrensis Gentry CHIH, SON

* Yucca mixtecana García-Mend. OAX, PUE

*Yucca periculosa Baker OAX, PUE, TLAX, VER

*Yucca potosina Rzed. SLP, TAMS

*Yucca queretaroensis Piña GTO, HGO, QRO, SLP

Yucca reverchonii Trel. COAH

* Yucca rigida (Engelm.) Trel. CHIH, COAH, DGO, NLE, SLP, SON, ZAC

Yucca rostrata Engelm. ex Trel. CHIH, COAH, DGO, NLE
Yucca rupicola Scheele CHIH, COAH, NLE, TAMS

Yucca schidigera Roezl ex Ortgies BCN, SON

Yucca schottii Engelm. CHIH, SON

Yucca torreyi Shafer CHIH, COAH, DGO, NLE, TAMS

Yucca treculeana Carrière AGS, COAH, DGO, NLE, QRO,

SLP, TAMS, VER

*Yucca valida Brandegee BCN, BCS

\section{Family Asteraceae}

Acmella filipes (Greenm.) R.K. Jansen CAM, QROO, YUC Acmella pilosa R.K. Jansen CAM, CHIS, QROO, YUC

Acmella radicans (Jacq.) R.K. Jansen CHIS, COL, DGO, GTO, GRO, JAL, MEX, MICH, MOR, NAY, OAX, PUE, QRO, QROO, SIN, SON, VER, YUC, ZAC

*Acmella ramosa (Hemsl.) R.K. Jansen PUE, VER

Acmella repens (Walter) Rich. AGS, CAM, CHIS, COAH, COL, CDMX, DGO, GTO, GRO, HGO, JAL, MEX, MICH, MOR, NAY, NLE, OAX, PUE, QRO, QROO, SLP, SIN, SON, TAB, TAMS, TLAX, VER, YUC, ZAC

*Acourtia bravohollisiana Rzed. GRO

*Acourtia butandae L. Cabrera CHIH, SON

*Acourtia caltepecana B.L. Turner PUE

*Acourtia carpholepis (Sch. Bip. ex A. Gray) Reveal \& R.M. King CHIS, HGO, OAX, PUE, QRO, SLP, VER

*Acourtia carranzae L. Cabrera QRO, SLP

*Acourtia ciprianoi Panero \& Villaseñor OAX

*Acourtia cordata (Cerv.) B.L. Turner CDMX, GRO, HGO, MEX, MOR, OAX, PUE, QRO, SLP, TLAX, VER

Acourtia coulteri (A. Gray) Reveal \& R.M. King CHIS, GTO, HGO, NLE, QRO, SLP, TAMS, VER, ZAC

*Acourtia cuernavacana (B.L. Rob. \& Greenm.) Reveal \& R.M. King GRO, MEX, MICH, MOR

*Acourtia dieringeri L. Cabrera CHIH, SON

*Acourtia discolor Rzed. GRO, OAX

*Acourtia dissiticeps (Bacig.) Reveal \& R.M. King AGS, SLP

*Acourtia dugesii (A. Gray) Reveal \& R.M. King AGS, COL, GTO, GRO, HGO, JAL, MEX, MICH, MOR, OAX, PUE, QRO, SLP, VER

*Acourtia durangensis B.L. Turner DGO

* Acourtia elizabethiae Rzed. \& Calderón HGO, QRO, SLP

*Acourtia erioloma (S.F. Blake) Reveal \& R.M. King OAX

*Acourtia fragrans Rzed. PUE

*Acourtia fruticosa (Lex.) B.L. Turner AGS, COL, CDMX, DGO, GTO, HGO, JAL, MEX, MICH, NAY, QRO, SLP, SIN, $\mathrm{ZAC}$

*Acourtia gentryi L. Cabrera SIN

Acourtia glandulifera (Nash) B.L. Turner CHIS

*Acourtia glomeriflora (A. Gray) Reveal \& R.M. King AGS, COL, GRO, JAL, MEX, MICH, MOR, OAX, ZAC

*Acourtia gracilis L. Cabrera PUE, VER

*Acourtia grandifolia (S. Watson) Reveal \& R.M. King JAL, MICH, NAY, ZAC

*Acourtia hidalgoana B.L. Turner GTO, HGO, NLE, QRO, SLP, TAMS, VER

*Acourtia hooveri (McVaugh) Reveal \& R.M. King COL, JAL, $\mathrm{MICH}$ 
*Acourtia huajuapana B.L. Turner GRO, MEX, MOR, OAX, PUE

*Acourtia humboldtii (Less.) B.L. Turner COL, CDMX, GTO, GRO, HGO, JAL, MEX, MICH, PUE, QRO, SLP, TLAX, VER

*Acourtia intermedia L. Cabrera DGO

*Acourtia joaquinensis L. Cabrera HGO, QRO

*Acourtia lepidopoda (B.L. Rob.) Reveal \& R.M. King JAL, MEX, MICH, MOR

*Acourtia lobulata (Bacig.) Reveal \& R.M. King OAX, PUE

*Acourtia longifolia (S.F. Blake) Reveal \& R.M. King DGO, JAL, NAY, ZAC

*Acourtia lozanoi (Greenm.) Reveal \& R.M. King CDMX, HGO, MEX, PUE, QRO, SLP, TLAX, VER

*Acourtia macrocephala Sch. Bip. DGO, NAY

*Acourtia macvaughii B.L. Turner GRO, MICH

*Acourtia mexiae L. Cabrera JAL

Acourtia mexicana (Lag. ex D. Don) H. Rob. AGS, CHIH, DGO, SIN, SON, ZAC

Acourtia microcephala DC. BCN

*Acourtia michoacana (B.L. Rob.) Reveal \& R.M. King COL, JAL, MICH

*Acourtia moctezumae Rzed. \& Calderón QRO, SLP

*Acourtia moschata (La Llave) DC. GTO, GRO, HGO, JAL, MEX, MICH, QRO, TLAX

Acourtia nana (A. Gray) Reveal \& R.M. King AGS, CHIH, COAH, DGO, GTO, JAL, NLE, QRO, SLP, SON, TAMS, ZAC

*Acourtia nelsonii (B.L. Rob.) Reveal \& R.M. King COL, JAL,

$\mathrm{MICH}, \mathrm{NAY}$

Acourtia nudicaulis (A. Gray) B.L. Turner CHIS

*Acourtia nudiuscula (B.L. Rob.) B.L. Turner JAL, NAY

*Acourtia oaxacana L. Cabrera OAX

*Acourtia ovatifolia L. Cabrera GRO, OAX, PUE

*Acourtia oxylepis (A. Gray) Reveal \& R.M. King GTO, HGO, QRO, SLP

*Acourtia palmeri (S. Watson) Reveal \& R.M. King BCN, BCS

*Acourtia parryi (A. Gray) Reveal \& R.M. King AGS, CHIH, COAH, DGO, GTO, HGO, JAL, NLE, QRO, SLP, TAMS, VER, ZAC

*Acourtia patens (A. Gray) Reveal \& R.M. King CHIH, COL, DGO, JAL, NAY, SIN, SON

*Acourtia pilulosa (Bacig.) B.L. Turner GRO, MOR, OAX, PUE

*Acourtia pinetorum (Brandegee) Reveal \& R.M. King BCS

*Acourtia platyphylla (A. Gray) Reveal \& R.M. King CHIH, DGO

*Acourtia platyptera (B.L. Rob.) Reveal \& R.M. King GRO, MEX, MICH

*Acourtia potosina L. Cabrera SLP

*Acourtia pringlei (B.L. Rob. \& Greenm.) Reveal \& R.M. King GRO, MEX, MICH

*Acourtia pulchella L. Cabrera AGS, ZAC

*Acourtia purpusii (Brandegee) Reveal \& R.M. King COAH, GTO, HGO, JAL, NLE, QRO, SLP, TAMS, VER, ZAC

*Acourtia queretarana B.L. Turner GTO, NLE, QRO, SLP

Acourtia reticulata (Lag. ex D. Don) Reveal \& R.M. King CHIS, DGO, GTO, GRO, HGO, JAL, MEX, MICH, MOR, OAX, PUE, QRO, SLP, VER
Acourtia runcinata (Lag. ex D. Don) B.L. Turner CHIH, COAH, HGO, NLE, QRO, SLP, TAMS, VER, ZAC

*Acourtia rzedowskii B.L. Turner PUE, VER

*Acourtia scapiformis (Bacig.) B.L. Turner GRO, OAX, PUE

*Acourtia scaposa (S.F. Blake) B.L. Turner MICH

*Acourtia simulata (S.F. Blake) Reveal \& R.M. King AGS, COL, GRO, JAL, MEX, MICH, MOR, ZAC

*Acourtia sinaloana B.L. Turner SIN

*Acourtia tenoriensis B.L. Turner OAX, PUE

*Acourtia tomentosa (Brandegee) Reveal \& R.M. King GTO, NLE, PUE, QRO, SLP, TAMS

*Acourtia turbinata (Lex.) DC. AGS, CAM, COL, CDMX, GTO, GRO, JAL, MEX, MICH, MOR, QRO, VER, ZAC

*Acourtia umbratilis (B.L. Rob. \& Greenm.) B.L. Turner OAX, PUE

*Acourtia venturae L. Cabrera GTO, SLP

*Acourtia veracruzana B.L. Turner CDMX, HGO, MEX, OAX, PUE, QRO, VER

*Acourtia wislizeni (A. Gray) Reveal \& R.M. King AGS, CHIH, COL, DGO, GTO, JAL, MEX, MICH, NAY, QRO, SLP, SIN, ZAC

Acourtia wrightii (A. Gray) Reveal \& R.M. King AGS, CHIH, COAH, DGO, GTO, JAL, NLE, SLP, SON, TAMS, ZAC

*Acourtia zacatecana B.L. Turner AGS, DGO, JAL, ZAC

Achillea millefolium L. AGS, BCN, CHIS, CHIH, COAH, CDMX, DGO, GTO, GRO, HGO, JAL, MEX, MICH, MOR, NAY, NLE, OAX, PUE, QRO, QROO, SLP, SIN, SON, TAMS, TLAX, VER, ZAC

Achyrachaena mollis Schauer BCN

Achyrocline deflexa B.L. Rob. \& Greenm. CHIS, OAX, VER

*Achyrocline guerreroana G.L. Nesom GRO

*Achyrocline oaxacana G.L. Nesom OAX

Achyrocline turneri G.L. Nesom CHIS

*Achyrocline ventosa Klatt HGO, VER

*Achyropappus anthemoides Kunth CDMX, GTO, HGO, MEX, QRO, SLP, TLAX, VER

*Achyropappus queretarensis B.L. Turner GTO, HGO, QRO Adenocaulon lyratum S.F. Blake CHIS

*Adenophyllum anomalum (Canby \& Rose) Strother CHIH, DGO, NAY, SIN, SON

*Adenophyllum aurantium (L.) Strother CHIS, COL, GRO, JAL, MEX, MICH, OAX, VER

*Adenophyllum cancellatum (Cass.) Villarreal AGS, CHIH, COL, DGO, GTO, GRO, HGO, JAL, MEX, MICH, MOR, NAY, NLE, OAX, QRO, SLP, SIN, SON, TAMS, VER, ZAC

*Adenophyllum glandulosum (Cav.) Strother GRO, MEX, MOR, OAX, PUE

Adenophyllum porophylloides (A. Gray) Strother BCN, BCS, SON

Adenophyllum porophyllum (Cav.) Hemsl. AGS, CAM, CHIS, COL, DGO, GTO, GRO, HGO, JAL, MEX, MICH, MOR, NAY, NLE, OAX, PUE, QRO, SLP, SIN, SON, TAB, TAMS, VER, YUC, ZAC

*Adenophyllum pulcherrimum (Strother) Villarreal AGS, COL, GTO, HGO, JAL, MEX, MICH, QRO, VER

*Adenophyllum speciosum (A. Gray) Strother BCS 
*Adenophyllum squamosum (A. Gray) Strother COL, JAL, NAY, SIN, ZAC

Adenophyllum wrightii A. Gray CHIH, COAH, NLE

*Adenophyllum yecoranum B.L. Turner CHIH, SON

Adenostemma hirtiflorum Benth. CHIS

*Adenothamnus validus (Brandegee) D.D. Keck BCN

*Ageratella microphylla (Sch. Bip.) A. Gray CHIH, DGO, JAL, NAY, SIN, SON, ZAC

Ageratina adenophora (Spreng.) R.M. King \& H. Rob. AGS, CHIS, COL, CDMX, DGO, GTO, GRO, HGO, JAL, MEX, MICH, MOR, NAY, OAX, PUE, QRO, SLP, SIN, VER, ZAC *Ageratina aegirophylla (B.L. Rob.) R.M. King \& H. Rob. OAX, VER

*Ageratina amblyolepis (B.L. Rob.) R.M. King \& H. Rob. GRO, MOR

Ageratina areolaris (DC.) Gage ex B.L. Turner AGS, CHIS, CHIH, COL, CDMX, DGO, GTO, GRO, HGO, JAL, MEX, MICH, MOR, NAY, NLE, OAX, PUE, QRO, SLP, SIN, SON, TAMS, TLAX, VER, ZAC

*Ageratina arsenei (B.L. Rob.) R.M. King \& H. Rob. COL, CDMX, GTO, JAL, MEX, MICH, NAY, OAX, QRO, SLP

*Ageratina astillera (B.L. Turner) B.L. Turner COAH, NLE, ZAC

Ageratina atrocordata (B.L. Rob.) R.M. King \& H. Rob. CHIS, COL, DGO, GTO, GRO, JAL, MEX, MICH, NLE, OAX, QRO, SIN, VER

*Ageratina ayerscottiana B.L. Turner CHIH

*Ageratina barriei B.L. Turner COL, JAL

*Ageratina beamanii B.L. Turner DGO, JAL, NAY

Ageratina bellidifolia (Benth.) R.M. King \& H. Rob. CHIS, COL, DGO, GRO, HGO, JAL, MEX, MICH, OAX, PUE, SIN, VER

*Ageratina blepharilepis (Sch. Bip.) R.M. King \& H. Rob. AGS, COL, DGO, JAL, MICH, NAY, QRO, SIN, ZAC

*Ageratina bobjansenii B.L. Turner DGO, SIN

*Ageratina brandegeana (B.L. Rob.) R.M. King \& H. Rob. GTO, HGO, QRO, SLP

*Ageratina brevipes (DC.) R.M. King \& H. Rob. AGS, CHIH, COL, CDMX, DGO, GTO, GRO, HGO, JAL, MEX, MICH, MOR, NLE, OAX, PUE, QRO, SLP, TAMS, VER, ZAC

*Ageratina calaminthifolia (Kunth) R.M. King \& H. Rob. AGS, CHIH, COAH, CDMX, DGO, GTO, GRO, HGO, JAL, MEX, MOR, NAY, NLE, OAX, PUE, QRO, SLP, SIN, SON, TAMS, TLAX, VER, ZAC

*Ageratina calophylla (Greene) R.M. King \& H. Rob. AGS, COAH, CDMX, GTO, HGO, MEX, NLE, OAX, PUE, QRO, SLP, TAMS, TLAX, VER, ZAC

*Ageratina campyloclada (B.L. Rob.) R.M. King \& H. Rob. AGS, COAH, NLE, TAMS, ZAC

Ageratina capillipes R.M. King \& H. Rob. CHIS

*Ageratina cardiophylla (B.L. Rob.) R.M. King \& H. Rob. CHIH, DGO, GTO, GRO, HGO, JAL, MEX, MICH, PUE, SIN, SON

*Ageratina cerifera (McVaugh) R.M. King \& H. Rob. COL, GRO, JAL, MEX, MICH

*Ageratina colimana B.L. Turner COL, JAL
*Ageratina collodes (B.L. Rob. \& Greenm.) R.M. King \& H. Rob. OAX

*Ageratina concordiana B.L. Turner DGO, SIN

Ageratina conspicua (Kunth \& C.D. Bouché) R.M. King \& H. Rob. BCS, CHIS, COL, CDMX, GTO, GRO, HGO, JAL, MEX, MICH, MOR, NAY, NLE, OAX, PUE, QRO, SLP, SIN, TAMS, VER

Ageratina crassiramea (B.L. Rob.) R.M. King \& H. Rob. CHIS, GRO, JAL, MEX, MICH, MOR, OAX, VER

*Ageratina cremasta (B.L. Rob.) R.M. King \& H. Rob. GRO, $\mathrm{MICH}, \mathrm{OAX}$

*Ageratina cronquistii R.M. King \& H. Rob. DGO, SIN

*Ageratina cruzii B.L. Turner OAX

*Ageratina cuicatlana B.L. Turner OAX

*Ageratina cylindrica (McVaugh) R.M. King \& H. Rob. COL, CDMX, GRO, JAL, MEX, MICH, MOR

*Ageratina chazaroana B.L. Turner CHIS, VER

Ageratina chiapensis (B.L. Rob.) R.M. King \& H. Rob. CHIS, COL, GRO, HGO, JAL, MEX, MICH, MOR, OAX, TLAX, VER

*Ageratina chimalapana B.L. Turner OAX

*Ageratina choricephala (B.L. Rob.) R.M. King \& H. Rob. CHIH, COL, CDMX, DGO, GTO, GRO, HGO, JAL, MEX, MICH, MOR, NAY, OAX, PUE, QRO, SLP, SIN, TLAX, VER, ZAC

*Ageratina deltoidea (Jacq.) R.M. King \& H. Rob. CDMX, GTO, HGO, JAL, MEX, MICH, MOR, OAX, PUE, QRO, SLP, TLAX, VER

*Ageratina desquamans (B.L. Rob.) R.M. King \& H. Rob. OAX *Ageratina dolichobasis (McVaugh) R.M. King \& H. Rob. COL, GRO, MEX, JAL, MICH, OAX

*Ageratina enixa (B.L. Rob.) R.M. King \& H. Rob. CDMX, HGO, MEX, PUE, TLAX, VER

*Ageratina espinosarum (A. Gray) R.M. King \& H. Rob. AGS, COAH, CDMX, DGO, GTO, HGO, JAL, MEX, NAY, NLE, OAX, PUE, QRO, SLP, TAB, TAMS, TLAX, VER, ZAC

*Ageratina etlana B.L. Turner OAX

*Ageratina etlensis (B.L. Rob.) R.M. King \& H. Rob. OAX

*Ageratina flourensifolia (B.L. Turner) R.M. King \& H. Rob. $\mathrm{COAH}$

*Ageratina geminata (McVaugh) R.M. King \& H. Rob. JAL, $\mathrm{MICH}$

*Ageratina gentryana B.L. Turner DGO, SIN

*Ageratina glabrata (Kunth) R.M. King \& H. Rob. CDMX, DGO, GTO, GRO, HGO, JAL, MEX, MICH, MOR, OAX, PUE, QRO, SLP, SIN, TAMS, TLAX, VER, ZAC

*Ageratina glauca (Sch. Bip. ex Klatt) R.M. King \& H. Rob. GRO, OAX, VER

*Ageratina glischra (B.L. Rob.) R.M. King \& H. Rob. COAH, DGO, SLP

*Ageratina gonzalezorum B.L. Turner DGO, NAY

*Ageratina grashoffii B.L. Turner DGO, NAY

*Ageratina gypsophila B.L. Turner COAH, NLE

*Ageratina halbertiana (McVaugh) R.M. King \& H. Rob. COL, JAL, MICH

*Ageratina hasegawana B.L. Turner OAX

Ageratina havanensis (Kunth) R.M. King \& H. Rob. CHIH, 
COAH, GTO, HGO, NLE, QRO, SLP, TAMS, VER

*Ageratina hederifolia (A. Gray) R.M. King \& H. Rob. CHIH, COAH, DGO, SIN

*Ageratina henzium B.L. Turner DGO, SIN

Ageratina herbacea (A. Gray) R.M. King \& H. Rob. BCN, CHIH, COAH, NLE, QRO, SON

*Ageratina hernandezii B.L. Turner COAH, NLE, TAMS

*Ageratina hidalgensis (B.L. Rob.) R.M. King \& H. Rob. GTO, HGO, NLE, QRO, SLP, TAMS, TLAX, VER

*Ageratina huahuapana B.L. Turner OAX

*Ageratina humochica B.L. Turner OAX

*Ageratina hyssopina (A. Gray) R.M. King \& H. Rob. CHIH, COAH, DGO, NLE, SLP, SIN, SON, ZAC

*Ageratina ilicifolia B.L. Turner NLE

*Ageratina iltisii B.L. Turner COL, JAL

*Ageratina irrasa (B.L. Rob.) R.M. King \& H. Rob. HGO, MEX, OAX, PUE, TLAX, VER

*Ageratina isolepis (B.L. Rob.) R.M. King \& H. Rob. COL, CDMX, GTO, GRO, JAL, MEX, MICH, OAX, QRO, SLP, TAMS, ZAC

*Ageratina jaliscensis (B.L. Rob.) Gage ex B.L. Turner COL, JAL, NAY

*Ageratina jalpana B.L. Turner JAL, ZAC

*Ageratina jalpanserra B.L. Turner QRO

*Ageratina jocotepecana B.L. Turner JAL

*Ageratina josepaneroi B.L. Turner GRO

*Ageratina juxtlahuacensis Panero \& Villaseñor GRO, OAX

* Ageratina kochiana B.L. Turner OAX

*Ageratina lapsensis B.L. Turner OAX

*Ageratina lasia (B.L. Rob.) R.M. King \& H. Rob. GRO, MICH Ageratina lasioneura (Hook. \& Arn.) R.M. King \& H. Rob. CHIH, COL, CDMX, DGO, JAL, MEX, MICH, MOR, NAY, SIN, ZAC

*Ageratina leiocarpa (B.L. Rob.) Gage ex B.L. Turner GRO, MEX, MOR

Ageratina lemmonii (B.L. Rob.) R.M. King \& H. Rob. CHIH, DGO, SIN, SON

*Ageratina leptodictyon (A. Gray) R.M. King \& H. Rob. COL, DGO, JAL, NAY, ZAC

*Ageratina liebmannii (Sch. Bip. ex Klatt) R.M. King \& H. Rob. GRO, MEX, MOR, OAX, PUE

Ageratina ligustrina (DC.) R.M. King \& H. Rob. CHIS, COAH, GTO, GRO, HGO, JAL, MEX, MICH, NAY, NLE, OAX, PUE, QRO, SLP, TAMS, VER, ZAC

*Ageratina lucida (Ortega) R.M. King \& H. Rob. CHIS, CDMX, GRO, HGO, MEX, MOR, OAX, PUE, TLAX, VER

*Ageratina macdonaldii B.L. Turner OAX

*Ageratina macvaughii R.M. King \& H. Rob. GRO, MEX, MICH, PUE

Ageratina mairetiana (DC.) R.M. King \& H. Rob. AGS, CHIS, COL, CDMX, DGO, GTO, GRO, HGO, JAL, MEX, MICH, MOR, NAY, OAX, PUE, QRO, SLP, SIN, TAMS, TLAX, VER, ZAC

*Ageratina malacolepis (B.L. Rob.) R.M. King \& H. Rob. AGS, CHIS, CHIH, COL, DGO, GRO, JAL, MEX, MICH, NAY, OAX, SIN, SON, ZAC

*Ageratina manantlana B.L. Turner COL, JAL
*Ageratina markporteri B.L. Turner OAX

*Ageratina mayajana B.L. Turner OAX

*Ageratina mazatecana B.L. Turner OAX

*Ageratina megacephala B.L. Turner OAX

*Ageratina megaphylla B.L. Turner OAX

*Ageratina miahuatlana B.L. Turner OAX

*Ageratina microcephala B.L. Turner GRO, OAX

*Ageratina miquihuana (B.L. Turner) R.M. King \& H. Rob. COAH, TAMS

*Ageratina moorei B.L. Turner MEX, MICH

Ageratina muelleri (Sch. Bip. ex Klatt) R.M. King \& H. Rob. CHIS, COL, GTO, GRO, HGO, JAL, MEX, MICH, MOR, NAY, OAX, QRO, SLP, SIN, VER, ZAC

*Ageratina neohintoniorum B.L. Turner MEX, MICH, OAX

*Ageratina nesomii B.L. Turner COAH, NLE, TAMS

*Ageratina oaxacana (Klatt) R.M. King \& H. Rob. CHIS, GRO, OAX

*Ageratina oligocephala (DC.) R.M. King \& H. Rob. AGS, CHIH, COAH, COL, CDMX, DGO, GTO, GRO, HGO, JAL, MEX, MICH, MOR, NAY, NLE, OAX, PUE, QRO, SLP, SON, TAMS, TLAX, VER, ZAC

*Ageratina oppositifolia (A. Gray) B.L. Turner SLP, ZAC

*Ageratina oreithales (Greenm.) B.L. Turner COAH, CDMX, GRO, HGO, MEX, MICH, MOR, NLE, PUE, QRO, SLP, TAMS, VER

Ageratina ovilla (Standl. \& Steyerm.) R.M. King \& H. Rob. CHIS, OAX

*Ageratina ozolotepecana B.L. Turner OAX

*Ageratina palmeri (A. Gray) Gage ex B.L. Turner AGS, CHIH, DGO, JAL, NAY, NLE, SIN, SON, ZAC

*Ageratina parayana (J. Espinosa) B.L. Turner CDMX, GRO, MEX, MICH, OAX

*Ageratina pauciflora B.L. Turner OAX

Ageratina paupercula (A. Gray) R.M. King \& H. Rob. CHIH, COAH, COL, DGO, JAL, NAY, SON

Ageratina pazcuarensis (Kunth) R.M. King \& H. Rob. BCS, CHIS, CHIH, COAH, COL, CDMX, DGO, GTO, GRO, HGO, JAL, MEX, MICH, MOR, NAY, NLE, OAX, PUE, QRO, SLP, SIN, SON, TAMS, TLAX, VER, ZAC

*Ageratina pelotropha (B.L. Rob.) R.M. King \& H. Rob. GRO, $\mathrm{MICH}$

*Ageratina pendula Panero \& Villaseñor OAX

*Ageratina peracuminata R.M. King \& H. Rob. OAX

*Ageratina perezii B.L. Turner MEX, MOR

*Ageratina petiolaris (Moc. \& Sessé ex DC.) R.M. King \& H. Rob. AGS, CHIH, CHIS, COAH, CDMX, DGO, GTO, GRO, HGO, JAL, MEX, MICH, MOR, NLE, OAX, PUE, QRO, SLP, TAMS, TLAX, VER, YUC, ZAC

*Ageratina photina (B.L. Rob.) R.M. King \& H. Rob. MOR Ageratina pichinchensis (Kunth) R.M. King \& H. Rob. AGS, CHIS, COAH, COL, CDMX, DGO, GTO, GRO, HGO, JAL, MEX, MICH, MOR, NAY, NLE, OAX, PUE, QRO, SLP, SIN, SON, TAB, TAMS, TLAX, VER, ZAC

*Ageratina pochutlana B.L. Turner OAX

*Ageratina potosina B.L. Turner COAH, NLE, SLP, TAMS Ageratina pringlei (B.L. Rob. \& Greenm.) R.M. King \& H. Rob. CHIS, OAX 
Ageratina prunellifolia (Kunth) R.M. King \& H. Rob. CHIS, COAH, COL, CDMX, GTO, HGO, JAL, MEX, MICH, MOR, NLE, OAX, PUE, QRO, SLP, TLAX, VER

*Ageratina purpusii (Brandegee) R.M. King \& H. Rob. BCS

*Ageratina queretaroana B.L. Turner HGO, QRO, SLP, YUC

*Ageratina ramireziorum (J. Espinosa) B.L. Turner CHIS, COL, CDMX, GRO, HGO, JAL, MEX, MICH, MOR, TLAX

*Ageratina ramonensis (B.L. Rob.) R.M. King \& H. Rob. DGO

*Ageratina reserva B.L. Turner CHIS

*Ageratina rhodopoda (B.L. Rob.) R.M. King \& H. Rob. SLP, ZAC

*Ageratina rhomboidea (Kunth) R.M. King \& H. Rob. COAH, COL, CDMX, GTO, HGO, JAL, MEX, MICH, MOR, NLE, OAX, PUE, QRO, SLP, TLAX, VER

Ageratina riparia (Regel) R.M. King \& H. Rob. MICH, MOR, OAX, PUE, VER

*Ageratina riskindii B.L. Turner COAH

*Ageratina robinsoniana (Greene) B.L. Turner GTO, JAL

Ageratina rothrockii (A. Gray) R.M. King \& H. Rob. CHIH, COAH, DGO, SON

*Ageratina rubricaulis (Kunth) R.M. King \& H. Rob. AGS, CHIH, COL, CDMX, DGO, GTO, GRO, HGO, JAL, MEX, MICH, MOR, NAY, OAX, PUE, QRO, SLP, VER, ZAC

*Ageratina rupicola (B.L. Rob. \& Greenm.) R.M. King \& H. Rob. OAX

*Ageratina salicifolia R.M. King \& H. Rob. DGO, SIN

*Ageratina saltillensis (B.L. Rob.) R.M. King \& H. Rob. COAH, HGO, NLE, QRO, SLP, TAMS, VER

*Ageratina sandersii B.L. Turner DGO, SIN, SON

Ageratina saxorum (Standl. \& Steyerm.) R.M. King \& H. Rob. CHIS

*Ageratina scorodonioides (A. Gray) R.M. King \& H. Rob. AGS, COAH, DGO, GTO, HGO, JAL, MEX, MICH, NLE, PUE, QRO, SLP, TAMS, TLAX, VER, ZAC

*Ageratina seleri B.L. Turner OAX

*Ageratina serboana B.L. Turner OAX

*Ageratina soejimana B.L. Turner OAX

*Ageratina solana B.L. Turner OAX

*Ageratina sousae B.L. Turner OAX

*Ageratina spooneri B.L. Turner VER

*Ageratina stricta (A. Gray) R.M. King \& H. Rob. CHIH, SIN, SON

*Ageratina sundbergii B.L. Turner DGO, SIN

*Ageratina tejalapana B.L. Turner OAX

*Ageratina textitlana B.L. Turner OAX

Ageratina thyrsiflora (Greene) R.M. King \& H. Rob. CHIH, DGO, JAL, NAY, SIN, SON, ZAC

Ageratina tomentella (Schrad.) R.M. King \& H. Rob. CHIS, GRO, HGO, MEX, OAX, PUE, QRO, TLAX, VER

*Ageratina triniona (McVaugh) R.M. King \& H. Rob. AGS, DGO, JAL, NAY, ZAC

*Ageratina venulosa (A. Gray) R.M. King \& H. Rob. AGS, CHIH, DGO, JAL, SON, ZAC

Ageratina vernalis (Vatke \& Kurtz) R.M. King \& H. Rob. CHIS, DGO, HGO, MEX, NLE, OAX, PUE, QRO, SLP, SIN, TAMS, VER

*Ageratina vernicosa (Sch. Bip. ex Greenm.) R.M. King \& H.
Rob. CDMX, HGO, MEX, MICH, MOR, VER

*Ageratina viburnoides (DC.) R.M. King \& H. Rob. COAH, NLE, QRO, SLP, TAMS

*Ageratina viejoana B.L. Turner NLE, TAMS

*Ageratina villarrealii B.L. Turner COAH, NLE

*Ageratina viscosissima (Rolfe) R.M. King \& H. Rob. BCS

Ageratina wrightii (A. Gray) R.M. King \& H. Rob. AGS, COAH,

DGO, GTO, HGO, JAL, MEX, NAY, NLE, OAX, PUE, QRO,

SLP, TAMS, TLAX, VER, ZAC

*Ageratina yaharana B.L. Turner GRO

*Ageratina yecorana B.L. Turner CHIH, SON

*Ageratina zapalinama B.L. Turner COAH, NLE

*Ageratina zaragozana B.L. Turner NLE

Ageratina zunilana (Standl. \& Steyerm.) R.M. King \& H. Rob. CHIS

*Ageratum albidum (DC.) Hemsl. OAX

Ageratum conyzoides $\mathrm{L}$. CAM, CHIS, CHIH, GRO, HGO, JAL, MEX, MICH, MOR, NAY, NLE, OAX, PUE, QROO, SIN, TAB, VER, YUC

Ageratum corymbosum Zuccagni AGS, BCN, CAM, CHIS, CHIH, COAH, COL, CDMX, DGO, GTO, GRO, HGO, JAL, MEX, MICH, MOR, NAY, NLE, OAX, PUE, QRO, QROO, SLP, SIN, SON, TAMS, TLAX, VER, YUC, ZAC

Ageratum echioides (Less.) Hemsl. CHIS, GRO, OAX, TAB, VER

Ageratum gaumeri B.L. Rob. CAM, QROO, YUC

Ageratum houstonianum Mill. CAM, CHIS, COL, GRO, HGO, JAL, MEX, MICH, MOR, NAY, NLE, OAX, PUE, QRO, QROO, SLP, TAB, VER, YUC, ZAC

Ageratum maritimum Kunth CAM, QROO, YUC

Ageratum microcephalum Hemsl. CHIS, GRO, OAX, VER

*Ageratum munaense R.M. King \& H. Rob. CAM, YUC

*Ageratum paleaceum (DC.) Hemsl. CHIS, GRO, MICH, OAX, PUE

*Ageratum platypodum B.L. Rob. COL, JAL

*Ageratum tehuacanum R.M. King \& H. Rob. OAX, PUE, VER Agnorhiza ovata (Torr. \& A. Gray) W.A. Weber BCN

Agoseris heterophylla (Nutt.) Greene BCN, SON

*Aldama angustifolia (Hook. \& Arn.) E.E. Schill. \& Panero DGO, JAL, NAY, SIN, ZAC

*Aldama apiculata (S.F. Blake) E.E. Schill. \& Panero GRO, $\mathrm{MICH}$

*Aldama brandegeei (A.M. Carter) E.E. Schill. \& Panero BCS *Aldama buddleiiformis (DC.) E.E. Schill. \& Panero COL, CDMX, DGO, GTO, GRO, HGO, JAL, MEX, MICH, NAY, OAX, PUE, QRO, VER, ZAC

*Aldama congesta (Rose ex Hoffm.) E.E. Schill. \& Panero CHIH, SIN, SON

Aldama cordifolia (A. Gray) E.E. Schill. \& Panero AGS, CHIH, COAH, DGO, GTO, JAL, NLE, SLP, SON, TAMS, ZAC

*Aldama cornifolia (Kunth) E.E. Schill. \& Panero GTO, MICH, QRO

Aldama dentata La Llave CAM, CHIS, COL, CDMX, DGO, GTO, GRO, HGO, JAL, MEX, MICH, MOR, NAY, OAX, PUE, QRO, QROO, SLP, SIN, SON, TAB, TAMS, VER, YUC, ZAC *Aldama ensifolia (Sch. Bip.) E.E. Schill. \& Panero COL, DGO, JAL, NAY, SIN, ZAC 
*Aldama excelsa (Willd.) E.E. Schill. \& Panero CDMX, GTO, GRO, HGO, JAL, MEX, MICH, MOR, OAX, PUE, QRO, SLP, TLAX, VER

*Aldama flava (Hemsl.) E.E. Schill. \& Panero COL, DGO, JAL, MEX, MICH, NAY, OAX, SIN, ZAC

*Aldama fruticosa (Brandegee) E.E. Schill. \& Panero BCS *Aldama gentryi (B.L. Turner) E.E. Schill. \& Panero SON

*Aldama ghiesbreghtii (Hemsl.) E.E. Schill. \& Panero GTO, GRO, HGO, JAL, MEX, MICH, MOR, NAY, OAX, QRO

*Aldama glomerata (Brandegee) E.E. Schill. \& Panero BCN, BCS

*Aldama goldmanii (Greenm.) E.E. Schill. \& Panero DGO

*Aldama grahamii (McVaugh) E.E. Schill. \& Panero COL, JAL, ZAC

*Aldama hispida (Kunth) E.E. Schill. \& Panero AGS, GTO, HGO, JAL, MEX, MICH, QRO, SLP, SIN, TAMS, ZAC

*Aldama hypochlora (S.F. Blake) E.E. Schill. \& Panero COL, GTO, JAL, MEX, MICH, NAY, ZAC

*Aldama kingii (H. Rob.) E.E. Schill. \& Panero MICH

*Aldama latibracteata (Hemsl.) E.E. Schill. \& Panero COL, DGO, JAL, NAY, SIN

*Aldama linearis (Cav.) E.E. Schill. \& Panero AGS, CHIH, COAH, CDMX, DGO, GTO, HGO, JAL, MEX, MICH, NAY, NLE, OAX, PUE, QRO, SLP, TAMS, TLAX, VER, ZAC

*Aldama michoacana (B.L. Turner \& F.G. Davies) E.E. Schill. \& Panero GRO, JAL, MEX, MICH

*Aldama montana (Rose) E.E. Schill. \& Panero CHIH, DGO, SIN, SON

*Aldama morelensis (Greenm.) E.E. Schill. \& Panero GRO, MEX, MICH, MOR, OAX, PUE

*Aldama nesomii (B.L. Turner) E.E. Schill. \& Panero NLE, TAMS

*Aldama pachycephala (DC.) E.E. Schill. \& Panero AGS, COL, DGO, GTO, JAL, MEX, MICH, MOR, NAY, SLP, ZAC

*Aldama palmeri (A. Gray) E.E. Schill. \& Panero AGS, GTO, GRO, JAL, MICH, OAX, ZAC

*Aldama parkinsonii (Hemsl.) E.E. Schill. \& Panero DGO, JAL, NAY, SIN, ZAC

*Aldama perennans (B.L. Turner \& F.G. Davies) E.E. Schill. \& Panero GRO, MICH

Aldama phenax (S.F. Blake) E.E. Schill. \& Panero CHIH, $\mathrm{COAH}, \mathrm{DGO}$

*Aldama pringlei (B.L. Rob. \& Greenm.) E.E. Schill. \& Panero COL, DGO, JAL, MICH, NAY, SIN

*Aldama purisimae (Brandegee) E.E. Schill. \& Panero BCN, BCS, SON

*Aldama seemannii (Sch. Bip.) E.E. Schill. \& Panero CHIH, DGO, JAL, SIN

*Aldama squarrosa (Sch. Bip.) E.E. Schill. \& Panero JAL, NAY, ZAC

*Aldama subcanescens (S.F. Blake) E.E. Schill. \& Panero MEX, $\mathrm{MICH}$

*Aldama torresii (B.L. Turner) E.E. Schill. \& Panero GRO, OAX

Alepidocline annua S.F. Blake CHIS

*Alepidocline breedlovei (B.L. Turner) B.L. Turner CHIS

*Alepidocline macdonaldana B.L. Turner OAX
*Alepidocline pochutlana B.L. Turner OAX

*Alepidocline trifida (J.J. Fay) B.L. Turner OAX

Almutaster pauciflorus (Nutt.) A. Löve \& D. Löve AGS, CHIH, CDMX, DGO, GTO, HGO, JAL, MEX, MICH, QRO, SIN, SON, ZAC

*Alomia ageratoides Kunth GRO, MEX, MICH, PUE, QRO, SLP

*Alomia alata Hemsl. GRO, MEX, MOR, OAX, PUE

*Alomia callosa (S. Watson) B.L. Rob. JAL

*Alomia stenolepis S.F. Blake SON

*Alloispermum colimense (McVaugh) H. Rob. COL, JAL, $\mathrm{MICH}$

*Alloispermum gonzalezae (B.L. Turner) B.L. Turner DGO

*Alloispermum guerreroanum B.L. Turner GRO, OAX

Alloispermum integrifolium (DC.) H. Rob. CHIS, COL, CDMX, DGO, GRO, HGO, JAL, MEX, MICH, MOR, OAX, PUE, QRO, SLP, SIN, TAMS, VER

*Alloispermum longiradiatum (Urbatsch \& B.L. Turner) B.L. Turner GRO, MICH

*Alloispermum michoacanum (B.L. Rob.) B.L. Turner DGO, GRO, JAL, MEX, MICH, NAY, OAX, PUE, VER

*Alloispermum palmeri (S. Watson ex A. Gray) C.F. Fernández \& Urbatsch CHIH, COL, DGO, GRO, JAL, MEX, MICH, NAY, SIN, SON, ZAC

*Alloispermum scabrifolium (Hook. \& Arn.) H. Rob. DGO, JAL, NAY, SIN, SON, ZAC

Alloispermum scabrum (Lag.) H. Rob. AGS, CHIS, COL, CDMX, DGO, GTO, GRO, HGO, JAL, MEX, MICH, MOR, NAY, NLE, OAX, PUE, QRO, SLP, SIN, TAMS, TLAX, VER, ZAC

*Alloispermum tridacoides (Urbatsch \& B.L. Turner) C.F. Fernández \& Urbatsch COL, DGO, JAL, NAY, SIN

*Amauria brandegeana (Rose) Rydb. BCN, BCS

*Amauria carterae A.M. Powell BCS

*Amauria rotundifolia Benth. BCN, BCS

*Amauriopsis autumnalis (W.L. Ellison) B.L. Turner COAH, NLE, TAMS

Amauriopsis dissecta (A. Gray) Rydb. BCN, CHIH, COAH, SON

*Amauriopsis glandulosa (Greenm.) B.L. Turner AGS, CHIH, DGO, GTO, JAL, NAY, SLP, ZAC

*Amauriopsis janakosana B.L. Turner CHIH

Amauriopsis pedata (A. Gray) B.L. Turner CHIH, COAH, DGO, NLE, SON, TAMS

Amblyolepis setigera DC. COAH, NLE, TAMS

Amblyopappus pusillus Hook. \& Arn. BCN, BCS

Ambrosia acanthicarpa Hook. BCN, BCS, CHIH, COAH, SON

*Ambrosia acuminata (Brandegee) W.W. Payne BCN, BCS, SON

Ambrosia ambrosioides (Cav.) W.W. Payne BCN, BCS, CHIH, COAH, DGO, SIN, SON

Ambrosia artemisiifolia L. AGS, CHIH, COAH, DGO, GTO, HGO, JAL, MEX, MICH, MOR, NAY, NLE, OAX, PUE, QRO, SLP, SIN, SON, TAMS, VER, ZAC

*Ambrosia bryantii (Curran) W.W. Payne BCN, BCS

*Ambrosia camphorata (Greene) W.W. Payne BCN, BCS, GTO, MICH, SLP, SON 
*Ambrosia canescens A. Gray AGS, BCS, CHIH, DGO, GTO, HGO, JAL, MEX, MICH, QRO, SLP, SON, ZAC

*Ambrosia carduacea (Greene) W.W. Payne BCN, BCS, SON Ambrosia confertiflora DC. AGS, BCN, BCS, CHIH, COAH, COL, CDMX, DGO, GTO, GRO, HGO, JAL, MEX, MICH, MOR, NLE, QRO, SLP, SIN, SON, TAMS, VER, ZAC Ambrosia cordifolia (A. Gray) W.W. Payne BCN, BCS, CHIH, GTO, HGO, QRO, SLP, SIN, SON

Ambrosia chamissonis (Less.) Greene BCN, BCS

Ambrosia cheiranthifolia A. Gray COAH, TAMS

Ambrosia chenopodiifolia (Benth.) W.W. Payne BCN, BCS, SON

Ambrosia deltoidea (Torr.) W.W. Payne BCN, BCS, SON

*Ambrosia divaricata (Brandegee) W.W. Payne BCN, BCS, SON

Ambrosia dumosa (A. Gray) W.W. Payne BCN, BCS, SON

*Ambrosia flexuosa (A. Gray) W.W. Payne BCN, SON

Ambrosia hispida Pursh CAM, QROO, VER, YUC

*Ambrosia humi León de la Luz \& Rebman BCS

Ambrosia ilicifolia (A. Gray) W.W. Payne BCN, SON

*Ambrosia johnstoniorum Henr. COAH

*Ambrosia magdalenae (Brandegee) W.W. Payne BCN, BCS, SON

Ambrosia monogyra (Torr. \& A. Gray) Strother \& B.G. Baldwin AGS, BCN, BCS, CHIH, COAH, DGO, JAL, SIN, SON, ZAC *Ambrosia nivea (B.L. Rob. \& Fernald) W.W. Payne CHIH Ambrosia peruviana Willd. AGS, BCN, BCS, CAM, CHIS, CHIH, COAH, CDMX, DGO, GTO, GRO, HGO, JAL, MEX, MICH, MOR, NAY, NLE, OAX, PUE, QRO, QROO, SLP, SIN, SON, TAB, TAMS, TLAX, VER, ZAC

Ambrosia pumila (Nutt.) A. Gray BCN

Ambrosia salsola (Torr. \& A. Gray) Strother \& B.G. Baldwin BCN, BCS, CHIH, SIN, SON

Ambrosia trifida L. CHIH, COAH, HGO, QRO, SON, TAMS, VER

*Ambrosia $\times$ platyspina (Seaman) Strother \& B.G. Baldwin $\mathrm{BCN}$

Amolinia heydeana (B.L. Rob.) R.M. King \& H. Rob. CHIS

Anaphalis margaritacea (L.) Benth. \& Hook. f. BCN, SON

Ancistrocarphus filagineus A. Gray BCN

Anisocoma acaulis Torr. \& A. Gray BCN

Antennaria marginata Greene CHIH, COAH, SON

Antennaria parvifolia Nutt. CHIH, NLE, SON

*Aphanactis macdonaldii B.L. Turner OAX

Aphanostephus ramosissimus DC. AGS, CHIH, COAH, CDMX, DGO, GTO, GRO, HGO, JAL, MEX, MICH, NLE, PUE, QRO, SLP, TAMS, TLAX, VER, ZAC

Aphanostephus riddellii Torr. \& A. Gray COAH, NLE, TAMS

Aphanostephus skirrhobasis (DC.) Trel. NLE, TAMS

*Archibaccharis albescens (J.D. Jacks.) G.L. Nesom OAX

*Archibaccharis almedana G.L. Nesom GRO

Archibaccharis androgyna (Brandegee) S.F. Blake CHIS

Archibaccharis asperifolia (Benth.) S.F. Blake CHIS, COL, CDMX, DGO, GRO, HGO, JAL, MEX, MICH, MOR, OAX, PUE, QRO, SLP, TLAX, VER

*Archibaccharis auriculata (Hemsl.) G.L. Nesom COL, CDMX, GTO, GRO, HGO, JAL, MEX, MICH, MOR, OAX,
PUE, QRO, SLP, TLAX, VER

Archibaccharis blakeana Standl. \& Steyerm. CHIS

*Archibaccharis breedlovei G.L. Nesom \& B.L. Turner OAX

*Archibaccharis campii S.F. Blake OAX

Archibaccharis corymbosa (Donn. Sm.) S.F. Blake CHIS

Archibaccharis flexilis (S.F. Blake) S.F. Blake CHIS

*Archibaccharis hieracioides (S.F. Blake) S.F. Blake COL, CDMX, GTO, GRO, HGO, JAL, MEX, MICH, MOR, OAX, PUE, SLP, TAMS, TLAX, VER

Archibaccharis hirtella (DC.) Heering CHIS, CDMX, DGO, GTO, GRO, HGO, JAL, MEX, MICH, MOR, NAY, OAX, PUE, QRO, VER

*Archibaccharis intermedia (S.F. Blake) B.L. Turner HGO, PUE, QRO, SLP, VER

*Archibaccharis macdonaldii G.L. Nesom OAX

*Archibaccharis nephocephala G.L. Nesom OAX

*Archibaccharis peninsularis S.F. Blake BCS

*Archibaccharis pringlei (Greenm.) S.F. Blake OAX

Archibaccharis salmeoides S.F. Blake CHIS, GRO, MICH, OAX, VER

Archibaccharis schiedeana (Benth.) J.D. Jacks. CHIS, COL, CDMX, DGO, GRO, HGO, JAL, MEX, MICH, MOR, NAY, OAX, PUE, QRO, SLP, SIN, VER

Archibaccharis serratifolia (Kunth) S.F. Blake AGS, CHIS, CHIH, COL, CDMX, DGO, GTO, GRO, HGO, JAL, MEX, MICH, MOR, NAY, NLE, OAX, PUE, QRO, SLP, SIN, SON, TAMS, TLAX, VER, ZAC

*Archibaccharis simplex S.F. Blake CAM, PUE, VER

Archibaccharis standleyi S.F. Blake CHIS

Archibaccharis subsessilis S.F. Blake CHIS, OAX

Archibaccharis taeniotricha (S.F. Blake) G.L. Nesom CHIS

*Archibaccharis trichotoma (Klatt) G.L. Nesom CHIS, OAX

*Archibaccharis tuxtlensis G.L. Nesom VER

*Archibaccharis venturana G.L. Nesom VER

*Archibaccharis veracruzana G.L. Nesom VER

*Archibaccharis vesticaulis G.L. Nesom CHIS

*Arnicastrum glandulosum Greenm. CHIH, DGO, SON

*Arnicastrum guerrerense Villaseñor GRO

Artemisia californica Less. BCN, BCS

Artemisia campestris L. CHIH

Artemisia carruthii Alph. Wood. ex Carruth BCN, CHIH, NLE, SON

Artemisia douglasiana Besser BCN

Artemisia filifolia Torr. CHIH, COAH, DGO, TAMS

Artemisia franserioides Greene $\mathrm{CHIH}$

*Artemisia klotzschiana Besser AGS, COAH, DGO, GTO, HGO, JAL, MEX, NLE, PUE, QRO, SLP, TAMS, TLAX, VER, ZAC

Artemisia ludoviciana Nutt. AGS, BCN, BCS, CAM, CHIS, CHIH, COAH, COL, CDMX, DGO, GTO, GRO, HGO, JAL, MEX, MICH, MOR, NAY, NLE, OAX, PUE, QRO, QROO, SLP, SIN, SON, TAB, TAMS, TLAX, VER, YUC, ZAC

Artemisia palmeri A. Gray BCN

*Artemisia pringlei Greenm. CHIH, COAH, DGO, NLE, SON Artemisia tridentata Nutt. BCN

*Asanthus solidaginifolius (A. Gray) R.M. King \& H. Rob. CHIH, COAH, DGO, NLE, SIN, ZAC 
Asanthus squamulosus (A. Gray) R.M. King \& H. Rob. CHIH, DGO, GTO, HGO, JAL, OAX, SLP, SON, ZAC

*Asanthus thyrsiflorus (A. Gray) R.M. King \& H. Rob. AGS, CHIH, COAH, DGO, GTO, HGO, JAL, MEX, MICH, NLE, QRO, SLP, SIN, TAMS, ZAC

*Astranthium beamanii De Jong COAH, NLE, SLP, TAMS Astranthium ciliatum (Raf.) G.L. Nesom COAH, NLE, TAMS

*Astranthium laetificum De Jong MEX, MICH

*Astranthium orthopodum (B.L. Rob. \& Fernald) Larsen AGS, CHIH, COL, CDMX, DGO, GTO, JAL, MEX, MICH, SIN, SON, TLAX, VER, ZAC

*Astranthium purpurascens (B.L. Rob.) Larsen CHIS, GTO, HGO, OAX, QRO, SLP, TAMS, VER

*Astranthium reichei Rzed. MEX

*Astranthium splendens De Jong COAH, NLE, TAMS

*Astranthium xanthocomoides (Less.) Larsen HGO, MICH, OAX, PUE, TLAX, VER

*Astranthium xylopodum Larsen COL, JAL, MICH, MOR, NAY Atrichoseris platyphylla (A. Gray) A. Gray BCN

*Axiniphyllum corymbosum Benth. GRO, OAX

*Axiniphyllum durangense B.L. Turner DGO, JAL, NAY, ZAC

*Axiniphyllum pinnatisectum (Paul G. Wilson) B.L. Turner GRO, MICH

*Axiniphyllum sagittalobum B.L. Turner GRO, OAX

*Axiniphyllum scabrum (Zucc.) S.F. Blake OAX

*Aztecaster matudae (Rzed.) G.L. Nesom COAH, HGO, NLE, QRO, SLP, TAMS, ZAC

*Aztecaster pyramidatus (B.L. Rob. \& Greenm.) G.L. Nesom GRO, OAX

Baccharis bigelovii A. Gray CHIH, COAH, DGO, NLE, SLP, SON, TAMS

Baccharis brachyphylla A. Gray BCN, BCS, SON

*Baccharis brevipappa (McVaugh) G.L. Nesom AGS, COL, DGO, GRO, JAL, MICH, ZAC

Baccharis conferta Kunth CHIS, CDMX, DGO, GTO, GRO, HGO, JAL, MEX, MICH, MOR, OAX, PUE, QRO, SLP, TLAX, VER

*Baccharis crassicuneata G.L. Nesom COAH, NLE, TAMS

Baccharis dioica Vahl CAM, QROO, YUC

*Baccharis erosoricola Rzed. CDMX, HGO, MEX, QRO

*Baccharis glandulifera G.L. Nesom CHIS, OAX

Baccharis heterophylla Kunth AGS, CHIS, CHIH, COL, CDMX, DGO, GTO, GRO, HGO, JAL, MEX, MICH, MOR, NAY, NLE, OAX, PUE, QRO, SLP, SIN, SON, TAMS, TLAX, VER, ZAC

Baccharis lancifolia Schltdl. CHIS, HGO, OAX, PUE, QRO, SLP, VER

*Baccharis macrocephala Sch. Bip. ex Greenm. CDMX, HGO, MEX, VER

*Baccharis mexicana Cuatrec. GRO, OAX, PUE, VER

Baccharis multiflora Kunth CHIS, CDMX, DGO, GTO, GRO, HGO, JAL, MEX, MICH, MOR, OAX, PUE, QRO, SLP, SIN, TLAX, VER, ZAC

Baccharis neglecta Britton CHIH, COAH, DGO, NLE, SLP, SON, TAMS, ZAC

*Baccharis occidentalis S.F. Blake COL, GTO, GRO, JAL, NAY, SIN, ZAC
*Baccharis palmeri Greenm. AGS, DGO, GTO, JAL, ZAC

Baccharis pedunculata (Mill.) Cabrera CHIS

Baccharis pteronioides DC. AGS, CHIH, COAH, COL, CDMX, DGO, GTO, GRO, HGO, JAL, MEX, MICH, MOR, NAY, NLE, OAX, PUE, QRO, SLP, SIN, SON, TAMS, TLAX, VER, ZAC

*Baccharis ramiflora A. Gray GTO, GRO, HGO, QRO, SLP, VER, YUC

Baccharis salicifolia (Ruiz \& Pav.) Pers. AGS, BCN, BCS, CHIS, CHIH, COAH, COL, CDMX, DGO, GTO, GRO, HGO, JAL, MEX, MICH, MOR, NAY, NLE, OAX, PUE, QRO, SLP, SIN, SON, TAMS, TLAX, VER, ZAC

Baccharis salicina Torr. \& A. Gray BCN, CHIH, COAH, DGO, NLE, SON, TAMS

Baccharis sarothroides A. Gray BCN, BCS, CHIH, SIN, SON

Baccharis sergiloides A. Gray BCN, BCS, SON

*Baccharis sordescens DC. CHIS, CDMX, GTO, GRO, HGO, MEX, MICH, MOR, OAX, PUE, QRO, SLP, TAMS, TLAX, VER

*Baccharis squarrosa Kunth AGS, DGO, GTO, JAL, NAY, SLP, ZAC

Baccharis sulcata DC. AGS, CHIH, COAH, DGO, GTO, HGO, JAL, NLE, QRO, SLP, TAMS, ZAC

Baccharis texana (Torr. \& A. Gray) A. Gray CHIH, COAH, DGO, NLE, TAMS

Baccharis thesioides Kunth AGS, CHIH, CDMX, DGO, GTO, HGO, JAL, MEX, MICH, MOR, NAY, NLE, QRO, SLP, SIN, SON, TAMS, TLAX, VER, ZAC

Baccharis trinervis (Lam.) Pers. CAM, CHIS, COL, GRO, HGO, JAL, MEX, MICH, MOR, NAY, OAX, PUE, QRO, QROO, SLP, SIN, TAB, TAMS, VER, YUC

Baccharis wrightii A. Gray CHIH, DGO, SON

*Baccharis zamoranensis Rzed. GTO, QRO

*Baeriopsis guadalupensis J.T. Howell BCN

Bahia absinthifolia Benth. AGS, BCS, CHIH, COAH, DGO, GTO, GRO, HGO, JAL, NLE, QRO, SLP, SON, TAMS, VER, ZAC

*Bahia pringlei Greenm. GTO, HGO, MEX, PUE, QRO, SLP, TLAX, VER

*Bahia schaffneri S. Watson AGS, COAH, GTO, HGO, JAL, NLE, QRO, SLP, ZAC

*Bahiopsis carterae (E.E. Schill.) E.E. Schill. \& Panero BCS

*Bahiopsis chenopodina (Greene) E.E. Schill. \& Panero BCN, BCS, COL, SON

*Bahiopsis deltoidea (A. Gray) E.E. Schill. \& Panero BCN, BCS

Bahiopsis laciniata (A. Gray) E.E. Schill. \& Panero BCN, BCS, SON

*Bahiopsis lanata Kellogg BCN, BCS

*Bahiopsis microphylla (Vasey \& Rose) E.E. Schill. \& Panero BCN, BCS

Bahiopsis parishii (Greene) E.E. Schill. \& Panero BCN, BCS, SON

*Bahiopsis similis (Brandegee) E.E. Schill. \& Panero BCS

*Bahiopsis subincisa (Benth.) E.E. Schill. \& Panero BCN, BCS, SON

*Bahiopsis tomentosa (A. Gray) E.E. Schill. \& Panero BCS 
*Bahiopsis triangularis (M.E. Jones) E.E. Schill. \& Panero BCN, BCS, SON

Baileya multiradiata Harv. \& A. Gray AGS, BCN, CHIH, COAH, DGO, JAL, SON, TAMS, ZAC

Baileya pauciradiata Harv. \& A. Gray BCN, SON

Baileya pleniradiata Harv. \& A. Gray BCN, SON

* Bajacalia crassifolia (S. Watson) Loockerman, B.L. Turner \&

R.K. Jansen BCN, BCS, SON

* Bajacalia moranii B.L. Turner BCS

*Bajacalia tridentata (Benth.) Loockerman, B.L. Turner \& R.K. Jansen BCS

Baltimora geminata (Brandegee) Stuessy COL, GRO, JAL, MEX, MICH, NLE, OAX, QRO, SLP, SIN, TAMS, VER

Baltimora recta L. CAM, CHIS, GRO, OAX, QROO, TAB, TAMS, VER, YUC

Barkleyanthus salicifolius (Kunth) H. Rob. \& Brettell AGS, CHIS, CHIH, COAH, COL, CDMX, DGO, GTO, GRO, HGO, JAL, MEX, MICH, MOR, NAY, NLE, OAX, PUE, QRO, SLP, SIN, SON, TAMS, TLAX, VER, ZAC

Bartlettia scaposa A. Gray CHIH, COAH, DGO, ZAC

Bartlettina breedlovei R.M. King \& H. Rob. CHIS, HGO, OAX, PUE, VER

*Bartlettina calderonii (B.L. Turner) B.L. Turner OAX

*Bartlettina constipatiflora (Klatt) R.M. King \& H. Rob. HGO, OAX, VER

*Bartlettina cronquistii R.M. King \& H. Rob. COL, GRO, JAL, MICH, OAX

*Bartlettina ehrenbergii (Hemsl.) R.M. King \& H. Rob. HGO, QRO, VER

* Bartlettina hintonii R.M. King \& H. Rob. GRO, OAX

*Bartlettina juxtlahuaca B.L. Turner OAX

*Bartlettina karvinskiana (DC.) R.M. King \& H. Rob. HGO, OAX, PUE, SLP, VER

Bartlettina lanicaulis (B.L. Rob.) B.L. Turner CHIS, TAB

*Bartlettina macdougallii R.M. King \& H. Rob. HGO, OAX, PUE, VER

Bartlettina oresbia (B.L. Rob.) R.M. King \& H. Rob. CHIS, COL, DGO, GRO, HGO, JAL, MEX, MICH, MOR, OAX, PUE, SIN, VER

Bartlettina ornata R.M. King \& H. Rob. CHIS

Bartlettina pansamalensis (B.L. Rob.) R.M. King \& H. Rob. CHIS

Bartlettina pinabetensis (B.L. Rob.) R.M. King \& H. Rob. CHIS

Bartlettina platyphylla (B.L. Rob.) R.M. King \& H. Rob. CHIS, HGO, OAX, PUE, VER

*Bartlettina serboana B.L. Turner OAX

*Bartlettina solavegana B.L. Turner OAX

Bartlettina sordida (Less.) R.M. King \& H. Rob. CHIS, GRO, HGO, MEX, MICH, MOR, OAX, PUE, SLP, TAMS, VER

*Bartlettina tamaulipana (B.L. Turner) R.M. King \& H. Rob. HGO, SLP, TAMS

*Bartlettina textitlana B.L. Turner OAX

Bartlettina tuerckheimii (Klatt) R.M. King \& H. Rob. CHIS, GRO, HGO, OAX, PUE, VER

*Bartlettina xalapana (B.L. Turner) B.L. Turner VER

* Bartlettina yaharana B.L. Turner OAX
*Batopilasia byei (S.D. Sundb. \& G.L. Nesom) G.L. Nesom \& Noyes CHIH

*Bebbia atriplicifolia (A. Gray) Greene BCS

Bebbia juncea (Benth.) Greene BCN, BCS, CHIH, SIN, SON

Berlandiera lyrata Benth. AGS, CHIH, COAH, DGO, JAL, NLE, SLP, SON, TAMS, ZAC

Berlandiera monocephala (B.L. Turner) Pinkava CHIH, SON

Berlandiera $\times$ macrophylla (A. Gray) M.E. Jones CHIH, NLE, SON, TAMS, ZAC

*Bidens acrifolia Sherff COL, DGO, GRO, JAL, MICH, NAY, OAX, SIN, ZAC

*Bidens aequisquama (Fernald) Sherff COL, GTO, GRO, HGO, JAL, MEX, MICH, QRO, SLP

Bidens alba (L.) DC. BCN, BCS, CAM, CHIS, COL, GRO, JAL, MEX, MICH, NAY, OAX, PUE, QRO, QROO, SLP, TAB,

TAMS, VER, YUC

*Bidens angustissima Kunth AGS, COAH, DGO, GTO, HGO, JAL, MEX, MICH, NAY, NLE, OAX, QRO, SLP, SIN, TAMS, VER, ZAC

*Bidens anthemoides (DC.) Sherff COL, CDMX, GRO, HGO, JAL, MEX, MICH, MOR, OAX, PUE, TLAX, VER

Bidens antiguensis J.M. Coult. CHIS

Bidens aurea (Aiton) Sherff AGS, BCN, BCS, CHIS, CHIH, COAH, COL, CDMX, DGO, GTO, GRO, HGO, JAL, MEX, MICH, MOR, NAY, NLE, OAX, PUE, QRO, SLP, SIN, SON, TAMS, TLAX, VER, ZAC

*Bidens balsana Melchert GRO

Bidens bicolor Greenm. CHIS, OAX

Bidens bigelovii A. Gray AGS, BCS, CHIS, CHIH, COAH, COL, CDMX, DGO, GTO, GRO, HGO, JAL, MEX, MICH, MOR, NLE, OAX, PUE, QRO, SLP, SIN, SON, TAMS, TLAX, VER, ZAC

*Bidens brandegeei Sherff PUE

*Bidens cabopulmensis León de la Luz \& B.L. Turner BCS

*Bidens carpodonta Sherff COAH, NLE, TAMS

*Bidens clavata R.E. Ballard QRO, SLP, TAMS

*Bidens colimana Melchert COL, JAL

*Bidens cordylocarpa (A. Gray) D.J. Crawford DGO, JAL, NAY

*Bidens cornuta Sherff CHIH, SON

*Bidens cronquistii (Sherff) Melchert GRO

Bidens cynapiifolia Kunth CAM, QROO, YUC

Bidens chiapensis Brandegee AGS, CHIS, COL, GTO, JAL, MICH, OAX, PUE, SIN

Bidens chrysanthemifolia (Kunth) Sherff CHIS

*Bidens esmartinezii Villaseñor GRO, MICH

Bidens ferulifolia (Jacq.) DC. AGS, CHIH, COAH, COL, DGO, GTO, HGO, JAL, MICH, NAY, NLE, QRO, SLP, SON, TAMS, ZAC

*Bidens gentryi Sherff CHIH, SON

*Bidens geraniifolia Brandegee CHIS

*Bidens gracillima Sherff GRO, MEX, MICH

*Bidens gypsophila Miranda PUE

Bidens heterosperma A. Gray BCN, BCS, CHIH, DGO, JAL, MICH, NAY, SIN, SON

*Bidens hintonii (Sherff) Melchert GRO, MICH

Bidens holwayi Sherff \& S.F. Blake CHIS 
*Bidens insolita Sherff CHIH, SON

Bidens izabalensis Roseman CHIS

Bidens laevis (L.) Britton, Sterns \& Pogg. BCN, BCS, CHIS, CHIH, COAH, CDMX, DGO, GTO, HGO, JAL, MEX, MICH, MOR, NAY, NLE, OAX, SLP, SON, TAB, TAMS, TLAX, VER Bidens lemmonii A. Gray AGS, BCN, BCS, CHIH, COL, CDMX, DGO, GTO, JAL, MEX, MICH, MOR, NAY, SLP, SIN, SON, VER, ZAC

Bidens leptocephala Sherff BCN, BCS, CHIH, COAH, HGO, JAL, QRO, SIN, SON, ZAC

*Bidens longistyla C.R. Hart COAH, NLE, TAMS

*Bidens melchertii B.L. Turner JAL

*Bidens mexicana Sherff CHIS, COL, DGO, GRO, JAL, MICH, NAY, OAX, SIN, VER

*Bidens minensis Sherff GRO

*Bidens mollifolia Sherff CHIH, COL, DGO, GRO, JAL, MEX, MICH, MOR, OAX, SIN, SON

Bidens nana M.O. Dillon CHIS

*Bidens nudata Brandegee BCS

*Bidens oaxacana Melchert OAX

*Bidens ocellatus (Greenm.) Melchert MEX, MICH, MOR

Bidens odorata Cav. AGS, BCS, CHIS, CHIH, COAH, COL, CDMX, DGO, GTO, GRO, HGO, JAL, MEX, MICH, MOR, NAY, NLE, OAX, PUE, QRO, SLP, SIN, SON, TAMS, TLAX, VER, YUC, ZAC

*Bidens oligantha Brandegee CHIH, DGO, JAL, SIN, SON

Bidens ostruthioides (DC.) Sch. Bip. CAM, CHIS, COL, CDMX, DGO, GTO, GRO, JAL, HGO, MEX, MICH, MOR, NLE, OAX, PUE, QRO, SLP, SIN, TLAX, VER, ZAC

Bidens pilosa L. AGS, BCN, BCS, CAM, CHIS, CHIH, COAH, COL, CDMX, DGO, GTO, GRO, HGO, JAL, MEX, MICH, MOR, NAY, NLE, OAX, PUE, QRO, QROO, SLP, SIN, SON, TAB, TAMS, TLAX, VER, YUC, ZAC

*Bidens pringlei Greenm. COL, JAL, MEX, MICH

*Bidens pseudalausensis Sherff MICH

*Bidens pueblensis (Sherff) Melchert CDMX, GRO, JAL, MEX, MICH, MOR, OAX, PUE

*Bidens purpusorum Bitter \& Petersen HGO, MEX, PUE, QRO, TLAX, VER

Bidens reptans (L.) G. Don CAM, QROO, YUC

Bidens riparia Kunth BCN, BCS, CAM, CHIS, COL, DGO, GRO, JAL, MEX, MICH, MOR, NAY, OAX, QROO, SIN, SON, TAMS, VER, YUC, ZAC

*Bidens rosemaniana B.L. Turner \& Melchert GRO

Bidens rostrata Melchert CHIS, CHIH, COL, DGO, GRO, JAL, MEX, MICH, NAY, NLE, OAX, SLP, SIN, SON, ZAC

*Bidens saltillensis Melchert COAH, NLE

*Bidens sambucifolia Cav. CHIH, SIN, SON

*Bidens schaffneri (A. Gray) Sherff AGS, BCS, CHIH, DGO, GTO, HGO, JAL, MEX, MICH, QRO, SLP, SON, VER, ZAC

*Bidens serboana B.L. Turner OAX

*Bidens serrulata (Poir.) Desf. CHIS, COAH, CDMX, DGO, GTO, GRO, HGO, JAL, MEX, MICH, MOR, NAY, NLE, OAX, PUE, QRO, TAMS, TLAX, VER, ZAC

*Bidens sharpii (Sherff) Melchert GRO, OAX, PUE

*Bidens socorrensis Moran \& G.A. Levin COL

Bidens squarrosa Kunth CAM, CHIS, COL, GRO, HGO, JAL,
MEX, MICH, MOR, NAY, NLE, OAX, PUE, QRO, QROO, SLP, SIN, SON, TAB, TAMS, VER, YUC, ZAC

*Bidens subspiralis McVaugh COL, JAL, MICH

Bidens tenuisecta A. Gray BCS, CHIH, COAH, DGO, NLE, SLP, SON

Bidens triplinervia Kunth CHIS, CHIH, COAH, COL, CDMX, DGO, GTO, GRO, HGO, JAL, MEX, MICH, MOR, NAY, NLE, OAX, PUE, QRO, SLP, SIN, TAMS, TLAX, VER, ZAC

*Bidens xanti (A. Gray) B.L. Turner BCS

Blumea viscosa (Mill.) V.M. Badillo BCS, CAM, CHIS, COL, DGO, GRO, JAL, MEX, MICH, MOR, NAY, OAX, QRO, QROO, SLP, SIN, SON, VER, YUC

*Boeberastrum anthemidifolium (Benth.) Rydb. BCN, BCS, SON

*Boeberastrum littoralis (Brandegee) Rydb. BCS

*Boeberoides grandiflora (DC.) Strother GRO, MEX, MICH, MOR

*Bolanosa coulteri A. Gray COL, DGO, GTO, JAL, MICH, NAY, ZAC

Borrichia arborescens (L.) DC. CAM, QROO, SLP, TAB, TAMS, VER, YUC

Borrichia frutescens (L.) DC. CAM, COAH, GTO, NLE, PUE, QROO, SLP, TAB, TAMS, VER, YUC

*Brickellia adenolepis (B.L. Rob.) Shinners AGS, COL, JAL, ZAC

Brickellia amplexicaulis B.L. Rob. CHIH, DGO, SIN, SON

*Brickellia aramberrana B.L. Turner NLE, TAMS

Brickellia argyrolepis B.L. Rob. CHIS, OAX

* Brickellia atarjea B.L. Turner GTO

Brickellia atractyloides A. Gray BCN, SON

Brickellia baccharidea A. Gray SON

Brickellia betonicifolia A. Gray BCN, CHIH, COAH, DGO, SON

*Brickellia brandegei B.L. Rob. BCS, SIN, SON

Brickellia californica (Torr. \& A. Gray) A. Gray BCN, BCS, CHIH, COAH, SON

*Brickellia cardiophylla B.L. Rob. AGS, COL, JAL, NAY, ZAC

*Brickellia cavanillesii (Cass.) A. Gray CHIS, DGO, GTO, GRO, HGO, JAL, MEX, MICH, MOR, OAX, PUE, SLP, VER, ZAC

*Brickellia coahuilensis (A. Gray) Harc. \& Beaman CHIH, COAH, NLE, SLP, TAMS, VER, ZAC

*Brickellia coixtlahuaca B.L. Turner OAX

Brickellia coulteri A. Gray AGS, BCN, BCS, CHIH, COAH, COL, DGO, GRO, JAL, MICH, NAY, OAX, PUE, SLP, SIN, SON, VER, ZAC

*Brickellia cuspidata A. Gray DGO, JAL, NAY

Brickellia cylindracea A. Gray \& Engelm. CHIH, COAH, NLE Brickellia diffusa (Vahl) A. Gray BCS, CAM, CHIS, CHIH, COL, DGO, GTO, GRO, HGO, JAL, MEX, MICH, MOR, NAY, OAX, PUE, QRO, QROO, SLP, SIN, SON, TAB, TAMS, VER, YUC, ZAC

*Brickellia enigmatica B.L. Turner CHIH, SON

Brickellia eupatorioides (L.) Shinners AGS, CHIH, COAH, CDMX, DGO, GTO, GRO, HGO, JAL, MEX, MICH, NAY, NLE, OAX, PUE, QRO, SLP, SON, TAMS, TLAX, VER, ZAC 
*Brickellia filipes B.L. Rob. COL, GRO, JAL, MICH, NAY, OAX

Brickellia floribunda A. Gray CHIH, SON

Brickellia frutescens A. Gray BCN, BCS

*Brickellia gentryi B.L. Turner DGO

*Brickellia glabrata (Rose) B.L. Rob. BCN, BCS

Brickellia glandulosa (La Llave) McVaugh CHIS, COL, DGO, GTO, GRO, HGO, JAL, MEX, MICH, MOR, OAX, PUE, QRO, SLP, VER

* Brickellia glomerata Fernald GRO, MEX, MICH, MOR, OAX

* Brickellia glutinosa A. Gray CHIH, COAH, DGO

Brickellia grandiflora (Hook.) Nutt. BCN, CHIH, COAH, JAL, NLE, SON, TAMS

*Brickellia hastata Benth. BCN, BCS

Brickellia hinckleyi Standl. CHIH, COAH, DGO

*Brickellia hintoniorum B.L. Turner COAH, JAL, NLE, SLP, TAMS, ZAC

*Brickellia huahuapana B.L. Turner OAX

*Brickellia hymenochlaena A. Gray GRO, HGO, NLE, OAX, PUE, SLP, TAMS, VER, ZAC

*Brickellia jaliscensis McVaugh AGS, COL, JAL, MICH, NAY, ZAC

*Brickellia jimenezii Hinojosa \& Cruz-Durán GRO

*Brickellia laccata Flyr COAH

Brickellia laciniata A. Gray CHIH, COAH, DGO, NLE, SLP, TAMS, ZAC

*Brickellia lanata (DC.) A. Gray AGS, COL, DGO, GTO, JAL, MICH, NAY, SIN, ZAC

*Brickellia laxiflora (Brandegee) B.L. Turner GRO, MEX, MICH, MOR, OAX, PUE, VER

Brickellia lemmonii A. Gray AGS, CHIH, COAH, JAL, NLE, SLP, SON, TAMS, ZAC

*Brickellia leonis Rzed. \& Calderón MICH

*Brickellia lewisii B.L. Turner CHIH, SON

*Brickellia magnifica McVaugh CHIH, COL, JAL, NAY

*Brickellia megaphylla M.E. Jones ex B.L. Rob. BCS

Brickellia microphylla (Nutt.) A. Gray BCN

*Brickellia monocephala B.L. Rob. AGS, DGO, GTO, HGO, JAL, MEX, MICH, OAX, QRO, SIN, TLAX, ZAC

*Brickellia nesomii B.L. Turner COAH, NLE, SLP, TAMS

*Brickellia nutanticeps S.F. Blake CDMX, GTO, GRO, HGO, JAL, MEX, MICH, MOR, OAX, PUE, QRO, SLP, TAMS, TLAX, VER

*Brickellia odontophylla A. Gray CHIH, COAH, DGO, GTO, NLE, SLP, ZAC

*Brickellia oligadena (B.L. Rob.) B.L. Turner COL, DGO, GRO, JAL, MEX, MICH

Brickellia oliganthes (Less.) A. Gray AGS, CHIS, CHIH, DGO, GTO, GRO, JAL, MEX, MICH, MOR, NAY, NLE, OAX, QRO, SLP, SIN, SON, TAMS, TLAX, VER, ZAC

* Brickellia oreithales (B.L. Rob.) Shinners AGS, CHIH, COAH, DGO, JAL, NAY, NLE, SIN, SON, TAMS, ZAC

Brickellia orizabaensis Klatt CHIS, GRO, OAX, VER

*Brickellia palmeri A. Gray AGS, COAH, DGO, JAL, NLE, SLP, TAMS, ZAC

Brickellia paniculata (Mill.) B.L. Rob. AGS, CHIS, COL, CDMX, GTO, GRO, HGO, JAL, MEX, MICH, MOR, NAY,
NLE, OAX, PUE, QRO, SLP, SIN, TAMS, VER

Brickellia parvula A. Gray CHIH

*Brickellia pavonii (A. Gray) B.L. Turner CDMX, GTO, GRO, HGO, JAL, MEX, MICH, MOR, OAX, PUE, QRO, SLP, TLAX *Brickellia pedunculosa (DC.) Harc. \& Beaman AGS, COAH, DGO, GTO, HGO, JAL, MEX, MICH, NLE, QRO, SLP, TAMS, VER, ZAC

*Brickellia pendula (Schrad.) A. Gray CDMX, DGO, GTO, GRO, HGO, JAL, MEX, MICH, MOR, OAX, PUE, QRO, TLAX, VER

*Brickellia peninsularis Brandegee BCN, BCS, COL

Brickellia pringlei A. Gray CHIH, DGO, NAY, SIN, SON

* Brickellia problematica B.L. Turner OAX, PUE

* Brickellia rhomboidea Greene SON

*Brickellia robinsoniana S.F. Blake DGO, ZAC

Brickellia rusbyi A. Gray CHIH, SON

Brickellia scoparia (DC.) A. Gray AGS, CHIS, CHIH, COL, CDMX, DGO, GTO, GRO, HGO, JAL, MEX, MICH, MOR, NAY, OAX, PUE, QRO, SLP, SIN, TAMS, VER, ZAC

*Brickellia secundiflora (Lag.) A. Gray AGS, COAH, COL, CDMX, DGO, GTO, GRO, HGO, JAL, MEX, MICH, NAY, NLE, OAX, PUE, QRO, SLP, SIN, TAMS, TLAX, VER, ZAC

*Brickellia seemannii A. Gray DGO, NAY, SIN

*Brickellia serboana B.L. Turner OAX

Brickellia simplex A. Gray CHIH, COAH, DGO, SON

* Brickellia sonorana B.L. Turner CHIH, SIN, SON

*Brickellia spinulosa (A. Gray) A. Gray AGS, CHIH, DGO, GTO, JAL, NLE, SLP, ZAC

*Brickellia squarrosa B.L. Rob. \& Seaton COL, GRO, JAL, MEX, MICH, MOR

*Brickellia stolonifera B.L. Turner COAH

*Brickellia subuligera (S. Schauer) B.L. Turner AGS, CHIH, COAH, COL, DGO, GTO, GRO, HGO, JAL, MEX, MICH, MOR, NAY, NLE, OAX, PUE, QRO, SLP, SIN, SON, TAMS, VER, ZAC

*Brickellia tomentella A. Gray CHIH, GTO, MEX, MICH, MOR, OAX, PUE, QRO, SLP, TLAX, ZAC

*Brickellia urolepis S.F. Blake CHIH, COAH

Brickellia venosa (Wooton \& Standl.) B.L. Rob. CHIH, SON

*Brickellia vernicosa B.L. Rob. AGS, CHIH, DGO, SON, ZAC

Brickellia veronicifolia (Kunth) A. Gray AGS, CHIH, COAH, CDMX, DGO, GTO, GRO, HGO, JAL, MEX, MICH, MOR, NAY, NLE, OAX, PUE, QRO, SLP, SON, TAMS, TLAX, VER, ZAC

*Brickellia vollmeri Wiggins BCN

* Brickellia wendtii B.L. Turner COAH

*Brickellia wislizeni A. Gray CHIH, DGO, ZAC

*Brickellia worthingtonii B.L. Turner DGO, JAL, NAY, SIN

*Calanticaria bicolor (S.F. Blake) E.E. Schill. \& Panero GTO, HGO, QRO, SLP, VER

*Calanticaria brevifolia (Greenm.) E.E. Schill. \& Panero CHIH, COAH, DGO, NLE, ZAC

*Calanticaria greggii (A. Gray) E.E. Schill. \& Panero COAH, DGO, HGO, NLE, SLP, TAMS, ZAC

*Calanticaria inegii (S. González, M. González \& Rzed.) E.E. Schill. \& Panero DGO 
*Calanticaria oligantha (S. González, M. González \& Rzed.) E.E. Schill. \& Panero DGO

*Calea crocinervosa Wussow, Urbatsch \& Sullivan CHIS, OAX Calea jamaicensis (L.) L. CAM, CHIS, GRO, JAL, NAY, OAX, QROO, TAB, VER, YUC

Calea longipedicellata B.L. Rob. \& Greenm. CHIS, OAX, VER

*Calea megacephala B.L. Rob. \& Greenm. CHIS, OAX

*Calea oaxacana (B.L. Turner) B.L. Turner OAX

Calea ternifolia Kunth AGS, CAM, CHIS, COAH, COL, CDMX, GTO, GRO, HGO, JAL, MEX, MICH, MOR, NAY, NLE, OAX, PUE, QRO, QROO, SLP, TAB, TAMS, VER, YUC, $\mathrm{ZAC}$

Calea urticifolia (Mill.) DC. AGS, CAM, CHIS, COL, DGO, GTO, GRO, HGO, JAL, MEX, MICH, MOR, NAY, OAX, PUE, QRO, QROO, SLP, SIN, SON, TAB, TAMS, VER, YUC, ZAC Calycadenia tenella (Nutt.) Torr. \& A. Gray BCN

Calycoseris wrightii A. Gray BCN, SON

Calyptocarpus vialis Less. AGS, BCS, CAM, COAH, COL, CDMX, DGO, GTO, GRO, HGO, JAL, MEX, MICH, MOR, NLE, OAX, PUE, QRO, QROO, SLP, SIN, SON, TAB, TAMS, VER, YUC, ZAC

Calyptocarpus wendlandii Sch. Bip. CHIS, GRO, MEX, MICH, MOR, VER

Campuloclinium macrocephalum (Less.) DC. CHIS, QRO, SLP, TAB, VER

*Carminatia alvarezii Rzed. \& Calderón GRO, HGO, MEX, MICH, OAX, PUE, QRO, SLP

*Carminatia papagayana B.L. Turner GRO

Carminatia recondita McVaugh CHIS, COL, GTO, GRO, HGO, JAL, MEX, MICH, MOR, NAY, OAX, QRO, SLP, SIN, VER, ZAC

Carminatia tenuiflora DC. AGS, BCN, BCS, CHIS, CHIH, COAH, COL, CDMX, DGO, GTO, GRO, HGO, JAL, MEX, MICH, MOR, NAY, NLE, OAX, PUE, QRO, SLP, SIN, SON, VER, ZAC

Carphochaete bigelovii A. Gray CHIH, COAH, SON

*Carphochaete durangensis Grashoff ex B.L. Turner DGO, SIN

*Carphochaete grahamii A. Gray AGS, DGO, GTO, HGO, JAL, MEX, MICH, NAY, QRO, SLP, ZAC

*Carphochaete macrocephala (Paray) Grashoff ex B.L. Turner \& Kerr GRO

*Carphochaete pringlei (S. Watson) Grashoff ex B.L. Turner $\mathrm{CHIH}, \mathrm{DGO}, \mathrm{SON}$

*Carphochaete schaffneri Greenm. SLP, ZAC

*Carphochaete wislizeni A. Gray CHIH, DGO, SIN, SON

Centaurea americana Nutt. CHIH, COAH, DGO, HGO, NLE, OAX, QRO, SLP, SON, TAMS

Centaurea rothrockii Greenm. CHIH, CDMX, DGO, GTO, GRO, HGO, JAL, MEX, MICH, MOR, NAY, OAX, QRO, SIN, SON, ZAC

Centromadia parryi (Greene) Greene BCN

*Centromadia perennis Greene BCN

Centromadia pungens (Hook. \& Arn.) Greene BCN

*Cirsium acantholepis (Hemsl.) Petr. CDMX, GTO, HGO, JAL, MEX, MICH, MOR, QRO, TLAX

*Cirsium acrolepis (Petr.) G.B. Ownbey COAH, GTO, HGO,
JAL, NLE, QRO, SLP, TAMS, ZAC

*Cirsium anartiolepis Petr. AGS, COL, DGO, GTO, GRO, HGO, JAL, MEX, MICH, NAY, NLE, OAX, PUE, TAMS, VER, ZAC

Cirsium arizonicum (A. Gray) Petr. CHIH, SON

*Cirsium bicentenariale Rzed. HGO, QRO, SLP

Cirsium californicum A. Gray BCN

*Cirsium cernuum Lag. MEX, VER

*Cirsium coahuilense G.B. Ownbey \& Pinkava COAH

*Cirsium conspicuum (G. Don) Sch. Bip. GRO, MEX, JAL, OAX, PUE, VER

*Cirsium durangense (Greenm.) G.B. Ownbey DGO, ZAC

*Cirsium ehrenbergii Sch. Bip. COAH, COL, CDMX, DGO, GTO, GRO, HGO, JAL, MEX, MICH, MOR, NAY, NLE, OAX, PUE, QRO, SLP, SIN, TAMS, TLAX, VER, ZAC

*Cirsium excelsior (B.L. Rob.) Petr. SLP

*Cirsium faucium Petr. MEX, OAX, PUE

Cirsium grahamii A. Gray CHIH, DGO, JAL, NAY, SON, ZAC

Cirsium horridulum Michx. CAM, CHIS, COL, DGO, GTO,

GRO, JAL, NLE, QRO, QROO

*Cirsium imbricatum (B.L. Rob. \& Greenm.) Petr. OAX

*Cirsium jorullense (Kunth) Spreng. CDMX, GTO, HGO, JAL, MEX, MICH, MOR, PUE, QRO, SLP, TAMS, TLAX, VER

*Cirsium lappoides (Less.) Sch. Bip. COAH, GTO, HGO, NLE, OAX, PUE, QRO, SLP, TAMS, VER

*Cirsium liebmannii Sch. Bip. ex Klatt VER

*Cirsium lomatolepis (Hemsl.) Petr. CDMX, MEX, MOR, PUE Cirsium mexicanum DC. BCN, BCS, CAM, CHIS, COAH, COL, DGO, GTO, GRO, HGO, JAL, MEX, MOR, NLE, OAX, PUE, QRO, QROO, SLP, TAB, TAMS, VER, YUC, ZAC

Cirsium mohavense (Greene) Petr. BCN

Cirsium neomexicanum A. Gray CHIH, SON

Cirsium nigriceps Standl. \& Steyerm. CHIS

*Cirsium nivale (Kunth) Sch. Bip. CDMX, JAL, MEX, MICH, MOR, PUE, TLAX, VER

Cirsium ochrocentrum A. Gray BCN, CHIH, COAH, DGO, SON, ZAC

*Cirsium orizabaensis Sch. Bip. ex Klatt VER

*Cirsium pascuarense (Kunth) Spreng. CDMX, JAL, MEX, $\mathrm{MICH}$

*Cirsium pinetorum Greenm. CDMX, GTO, GRO, HGO, JAL, MEX, MICH, MOR, OAX, PUE, QRO, SLP, VER, ZAC

*Cirsium pringlei (S. Watson) Petr. COAH, NLE, TAMS, ZAC Cirsium radians Benth. CHIS, VER

*Cirsium reglense (Klatt) Petr. CHIH, JAL, PUE

*Cirsium rhaphilepis (Hemsl.) Petr. COAH, CDMX, DGO, GTO, GRO, HGO, JAL, MEX, MICH, NAY, NLE, OAX, PUE, QRO, SLP, SIN, SON, TLAX, VER, ZAC

Cirsium scariosum Nutt. BCN

Cirsium subcoriaceum (Less.) Sch. Bip. CHIS, CDMX, GTO, GRO, HGO, JAL, MEX, MICH, MOR, OAX, PUE, QRO, QROO, SLP, TLAX, VER

*Cirsium subuliforme G.W. Ownbey CDMX, HGO, MEX, MOR, PUE, QRO, TLAX, VER

Cirsium texanum Buckley CHIH, COAH, DGO, NLE, SLP, TAMS

*Cirsium tolucanum (B.L. Rob. \& Seaton) Petr. COL, CDMX, 
JAL, MEX, MICH, MOR

*Cirsium trachylomum S.F. Blake BCN, BCS

Cirsium turneri M.J. Warnock CHIH, COAH

Cirsium undulatum (Nutt.) Spreng. BCN, CHIH, COAH, DGO, NLE, SON

*Cirsium velatum (S. Watson) Petr. COL, GTO, GRO, HGO, JAL, MEX, MICH, NAY, OAX

Cirsium wrightii A. Gray CHIH, SON

* Cirsium zamoranense Rzed. GTO, QRO

Clappia suaedifolia A. Gray NLE, TAMS

Clibadium arboreum Donn. Sm. CHIS, COL, GRO, HGO, JAL, MEX, NAY, OAX, PUE, QRO, SLP, TAB, VER

Comaclinium montanum (Benth.) Strother CHIS

Conoclinium betonicifolium (Mill.) R.M. King \& H. Rob. CAM, CHIS, CHIH, COAH, COL, DGO, GTO, JAL, MEX, MOR, NLE, OAX, PUE, QRO, QROO, SLP, SON, TAB, TAMS, VER, YUC, ZAC

Conoclinium dissectum A. Gray CHIH, COAH, DGO, NLE, SLP, SON, TAMS, ZAC

*Conoclinium mayfieldii T.F. Patterson CHIH, DGO, NAY, NLE, SON, TAMS

Conyza bonariensis (L.) Cronquist AGS, BCN, BCS, CAM, CHIS, COAH, COL, CDMX, DGO, GTO, GRO, HGO, JAL, MEX, MICH, MOR, NAY, NLE, OAX, PUE, QRO, QROO, SLP, SIN, SON, TAB, TAMS, TLAX, VER, YUC, ZAC

Conyza canadensis (L.) Cronquist AGS, BCN, BCS, CAM, CHIS, CHIH, COAH, COL, CDMX, DGO, GTO, GRO, HGO, JAL, MEX, MICH, MOR, NAY, NLE, OAX, PUE, QRO, QROO, SLP, SIN, SON, TAB, TAMS, TLAX, VER, YUC, ZAC

Conyza coronopifolia Kunth AGS, CHIS, CHIH, COL, CDMX, DGO, GTO, GRO, HGO, JAL, MEX, MICH, MOR, NAY, NLE, OAX, PUE, QRO, SLP, SIN, TAMS, TLAX, VER, ZAC Conyza laevigata (Rich.) Pruski CAM, CHIS, CHIH, COL, GRO, JAL, MEX, MICH, OAX, QRO, SLP, SIN, SON, TAB, VER, YUC

*Conyza microcephala Hemsl. AGS, CHIS, CHIH, COL, CDMX, DGO, GTO, GRO, HGO, JAL, MEX, MICH, NAY, OAX, QRO, SLP, SIN, TLAX, VER, ZAC

Conyza primulifolia (Lam.) Cuatrec. \& Lourteig CHIS, VER

Conyza sumatrensis (Retz.) E. Walker CAM, CHIS, COL, GRO, HGO, JAL, MEX, MICH, NAY, OAX, PUE, QROO, TAB, VER Coreocarpus arizonicus (A. Gray) S.F. Blake CHIH, SIN, SON * Coreocarpus congregatus (S.F. Blake) E.B. Sm. DGO, SIN

* Coreocarpus dissectus (Benth.) S.F. Blake BCS

* Coreocarpus ixtapanus B.L. Turner MEX

* Coreocarpus parthenioides Benth. BCN, BCS, SON

* Coreocarpus sanpedroensis E.B. Sm. SON

* Coreocarpus sonoranus Sherff BCN, SON

* Coreopsis bolanosana Panero \& Villaseñor JAL, ZAC

Coreopsis californica (Nutt.) H. Sharsm. BCN

* Coreopsis crawfordii Mesfin GTO, QRO

*Coreopsis cyclocarpa S.F. Blake AGS, COAH, DGO, GTO, JAL, NAY, NLE, QRO, SLP, ZAC

*Coreopsis davilae Panero \& Villaseñor PUE

Coreopsis gigantea (Kellogg) H.M. Hall BCN

*Coreopsis guanajuatensis B.L. Turner GTO, QRO, SLP
*Coreopsis insularis (Brandegee) S.F. Blake COL

Coreopsis maritima (Nutt.) Hook. f. BCN

*Coreopsis mcvaughii D.J. Crawford AGS, DGO, GTO, JAL, ZAC

Coreopsis nuecensis A. Heller TAMS

*Coreopsis oaxacensis B.L. Turner OAX, PUE

*Coreopsis paludosa M.E. Jones CHIH, DGO, GTO, SLP, SIN, $\mathrm{SON}$

*Coreopsis petrophila A. Gray CDMX, DGO, GRO, HGO, JAL, MEX, MICH, NAY, OAX, SIN, ZAC

*Coreopsis petrophiloides B.L. Rob. \& Greenm. COL, CDMX, DGO, GTO, GRO, HGO, JAL, MEX, MICH, VER

*Coreopsis pinnatisecta S. F. Blake GRO, OAX, PUE

*Coreopsis pringlei B.L. Rob. GTO, HGO, QRO

* Coreopsis queretarensis B.L. Turner QRO, SLP

*Coreopsis rhyacophila Greenm. GTO, HGO, MEX, MICH, MOR, QRO, SLP, TAMS, VER

*Coreopsis rudis (Benth.) Hemsl. DGO, GTO, JAL, NAY, ZAC Coreopsis tinctoria Nutt. CHIH, COAH, NLE, TAMS, VER

Corethrogyne filaginifolia (Hook. \& Arn.) Nutt. BCN

*Cosmos atrosanguineus (Hook. f.) Voss GTO, HGO, QRO, SLP

Cosmos bipinnatus Cav. AGS, CHIS, CHIH, COAH, COL, CDMX, DGO, GTO, GRO, HGO, JAL, MEX, MICH, MOR, NAY, NLE, OAX, PUE, QRO, SLP, SIN, SON, TAMS, TLAX, VER, ZAC

*Cosmos carvifolius Benth. COL, DGO, JAL, MICH, NAY, SIN, SON, ZAC

Cosmos caudatus Kunth CAM, CHIS, COL, GRO, JAL, MEX, MICH, OAX, QRO, QROO, SLP, SIN, TAB, TAMS, VER, YUC, ZAC

*Cosmos concolor Sherff DGO, JAL, NAY, SIN, ZAC

Cosmos crithmifolius Kunth AGS, CHIS, CHIH, COAH, COL, CDMX, DGO, GTO, GRO, HGO, JAL, MEX, MICH, MOR, NAY, NLE, OAX, PUE, QRO, SLP, SIN, SON, TAMS, VER, ZAC

*Cosmos deficiens (Sherff) Melchert JAL

Cosmos diversifolius Otto CHIS, CDMX, DGO, GTO, GRO, HGO, JAL, MEX, MICH, MOR, NAY, NLE, OAX, PUE, QRO, SLP, TAMS, TLAX, VER, ZAC

*Cosmos intercedens Sherff COL, JAL, NAY

* Cosmos jaliscensis Sherff JAL

* Cosmos juxtlahuacensis Panero \& Villaseñor GRO, OAX

* Cosmos landii Sherff JAL, ZAC

*Cosmos linearifolius (Sch. Bip.) Hemsl. CHIH, DGO, JAL, MICH, NAY, SIN, SON, ZAC

*Cosmos longipetiolatus Melchert JAL

*Cosmos mattfeldii Sherff GRO, MICH

*Cosmos mcvaughii Sherff JAL

*Cosmos modestus Sherff GRO, MEX, OAX

*Cosmos montanus Sherff DGO, JAL, MICH, NAY, ZAC

*Cosmos nelsonii B.L. Rob. \& Fernald OAX

*Cosmos nitidus Paray MEX

*Cosmos ochroleucoflorus T. Melchert CHIH, DGO, JAL, NAY, SIN, ZAC

*Cosmos pacificus Melchert CHIS, COL, GRO, JAL, MEX, MICH, SIN 
*Cosmos palmeri B.L. Rob. AGS, CHIH, DGO, GTO, JAL, NAY, SLP, SIN, SON, ZAC

Cosmos parviflorus (Jacq.) Pers. AGS, BCN, BCS, CHIH, COAH, CDMX, DGO, GTO, GRO, HGO, JAL, MEX, MICH, NAY, OAX, PUE, QRO, SLP, SIN, SON, TAMS, TLAX, VER, ZAC

*Cosmos pringlei B.L. Rob. \& Fernald CHIH, DGO, NAY, SIN, SON

*Cosmos pseudoperfoliatus A. Castro, M. Harker \& Aarón Rodr. JAL

*Cosmos purpureus (DC.) Hemsl. GRO, MEX, MICH, MOR, OAX, VER

*Cosmos ramirezianus A. Castro, M. Harker \& Aarón Rodr. JAL

*Cosmos scabiosoides Kunth AGS, CHIS, COL, CDMX, DGO, GTO, GRO, HGO, JAL, MEX, MICH, MOR, NAY, OAX, PUE, QRO, SLP, SIN, TLAX, VER, ZAC

*Cosmos schaffneri Sherff GRO, HGO, MEX, QRO

*Cosmos sessilis Sherff DGO, GRO, JAL, MICH, NAY, ZAC

*Cosmos sherffii Melchert OAX

Cosmos sulphureus Cav. AGS, BCS, CAM, CHIS, CHIH, COAH, COL, DGO, GTO, GRO, JAL, MEX, MICH, MOR, NAY, NLE, OAX, PUE, QRO, QROO, SLP, SIN, SON, TAMS, VER, YUC, ZAC

Cotula mexicana (DC.) Cabrera CDMX, HGO, MEX, MICH, MOR, PUE, QRO, SLP, VER

*Coulterella capitata Vasey \& Rose BCS

Crepis runcinata Torr. \& A. Gray CHIH, SON

Critonia aromatisans (DC.) R.M. King \& H. Rob. CAM, YUC Critonia billbergiana (Beurl.) R.M. King \& H. Rob. CHIS, OAX, VER

Critonia campechensis (B.L. Rob.) R.M. King \& H. Rob. CAM, QROO, YUC

*Critonia conzattii (Greenm.) R.M. King \& H. Rob. CHIS, PUE, VER

Critonia daleoides DC. CAM, CHIS, HGO, NLE, OAX, PUE, QRO, QROO, SLP, TAB, TAMS, VER, YUC

Critonia hebebotrya DC. CHIS, CHIH, COL, DGO, GRO, JAL, MEX, MICH, MOR, NAY, OAX, PUE, QRO, SLP, SIN, SON, ZAC

Critonia hospitalis (B.L. Rob.) R.M. King \& H. Rob. CHIS, GRO, OAX, PUE, TAMS, VER

*Critonia lozanoana (B.L. Rob.) R.M. King \& H. Rob. HGO, NLE, QRO, SLP, TAMS, VER

Critonia microdon (B.L. Rob.) B.L. Turner CHIS, OAX

Critonia morifolia (Mill.) R.M. King \& H. Rob. CAM, CHIS, COL, GRO, HGO, JAL, NLE, OAX, PUE, QRO, QROO, SLP, SIN, TAB, TAMS, VER

*Critonia paneroi B.L. Turner GRO

Critonia quadrangularis (DC.) R.M. King \& H. Rob. AGS, CHIS, CHIH, COAH, COL, DGO, GRO, JAL, MEX, MICH, MOR, NAY, NLE, OAX, PUE, QRO, SLP, SIN, SON, TAMS, VER, YUC

Critonia sexangularis (Klatt) R.M. King \& H. Rob. CHIS, OAX, TAB, VER

*Critonia siltepecana (B.L. Turner) R.M. King \& H. Rob. CHIS

*Critonia yashanalensis (A.T. Whittemore) R.M. King \& H.
Rob. CHIS

Critoniadelphus nubigenus (Benth.) R.M. King \& H. Rob. CHIS, GRO, OAX

*Critoniopsis autumnalis (McVaugh) H. Rob. COL, JAL

*Critoniopsis baadii (McVaugh) H. Rob. COL, JAL, MICH

*Critoniopsis barbinervis (Sch. Bip.) H. Rob. AGS, DGO, JAL, NAY, SIN, ZAC

*Critoniopsis feddemae (McVaugh) Villaseñor \& RedondaMartínez JAL, NAY

*Critoniopsis foliosa (Benth.) H. Rob. AGS, CHIH, COL, DGO, GTO, GRO, JAL, MICH, NAY, QRO, SLP, SIN, ZAC

Critoniopsis heydeana (J.M. Coult.) H. Rob. CHIS, GRO, HGO, JAL, OAX, PUE, QRO, SLP, VER

Critoniopsis leiocarpa (DC.) H. Rob. CHIS, GRO, HGO, MEX, MICH, OAX, VER

*Critoniopsis littoralis (Brandegee) H. Rob. COL

*Critoniopsis macphersonii (S.B. Jones \& Stutts) H. Rob. JAL

*Critoniopsis macvaughii (S.B. Jones) H. Rob. GRO, OAX, PUE

*Critoniopsis obtusa (Gleason) H. Rob. GTO, HGO, NLE, QRO, SLP, TAMS, VER, ZAC

*Critoniopsis oolepis (S.F. Blake) H. Rob. CAM, QROO, YUC

*Critoniopsis ovata (Gleason) H. Rob. CHIH, DGO, SIN

*Critoniopsis pugana (S.B. Jones \& Stutts) H. Rob. COL, JAL

*Critoniopsis salicifolia (DC.) H. Rob. AGS, CHIS, COL, CDMX, DGO, GRO, HGO, JAL, MEX, MICH, MOR, NAY, OAX, PUE, QRO, SIN, TAMS, VER, ZAC

Critoniopsis shannonii (J.M. Coult.) H. Rob. CHIS

*Critoniopsis solorzanoana (Rzed. \& Calderón) H. Rob. MEX, $\mathrm{MICH}$

*Critoniopsis tarchonanthifolia (DC.) H. Rob. GRO, OAX

*Critoniopsis tomentosa (Lex.) H. Rob. AGS, COL, DGO, GTO, GRO, HGO, JAL, MEX, MICH, MOR, OAX, QRO, SLP, SIN, ZAC

Critoniopsis triflosculosa (Kunth) H. Rob. AGS, CHIS, CHIH, COL, DGO, GRO, JAL, MEX, MICH, MOR, NAY, NLE, OAX, PUE, SIN, SON, TAMS, VER, ZAC

*Critoniopsis uniflora (Sch. Bip.) H. Rob. AGS, COL, CDMX, DGO, GRO, JAL, MEX, MICH, MOR, NAY, OAX, PUE, TAMS, TLAX, VER, ZAC

*Critoniopsis villaregalis (Carvajal) H. Rob. COL, JAL, MICH, ZAC

*Cymophora accedens (S.F. Blake) B.L. Turner \& A.M. Powell COL, GRO, JAL, MICH

*Cymophora hintonii B.L. Turner \& A.M. Powell COL, JAL, $\mathrm{MICH}$

*Cymophora luckowana B.L. Turner GRO

*Cymophora pringlei B.L. Rob. \& Greenm. COL, GRO, JAL

Cyrtocymura scorpioides (Lam.) H. Rob. CAM, CHIS, QROO, YUC

Chaenactis artemisiifolia (Harv. \& A. Gray) A. Gray BCN

Chaenactis carphoclinia A. Gray BCN, SON

Chaenactis fremontii A. Gray BCN, SON

* Chaenactis furcata Stockw. BCN, BCS

Chaenactis glabriuscula DC. BCN

Chaenactis lacera Greene BCN, BCS

Chaenactis parishii A. Gray BCN 
Chaenactis stevioides Hook. \& Arn. BCN, BCS, SON

Chaetopappa asteroides (Nutt.) DC. HGO, TAMS

Chaetopappa bellioides (A. Gray) Shinners AGS, CHIH, COAH, DGO, JAL, NLE, SLP, TAMS, ZAC

Chaetopappa ericoides (Torr.) G.L. Nesom AGS, CHIH, COAH, DGO, GTO, HGO, JAL, MEX, NLE, QRO, SLP, SON, TAMS, VER, ZAC

Chaetopappa parryi A. Gray COAH, NLE, SLP, TAMS

*Chaetopappa plomoensis B.L. Turner COAH

*Chaetopappa pulchella Shinners CHIH, COAH

*Chaetymenia peduncularis Hook. \& Arn. CHIH, COL, DGO, JAL, MICH, NAY, SIN, SON, ZAC

Chaptalia albicans (Sw.) Vent. ex Steud. CAM, CHIS, QROO, SLP, VER, YUC

*Chaptalia estribensis G.L. Nesom HGO, OAX, PUE, SLP, VER

*Chaptalia hidalgoensis L. Cabrera \& G.L. Nesom HGO, QRO

*Chaptalia hintonii Bullock GRO, MEX, MICH

*Chaptalia hololeuca Greene AGS, COAH, GTO, HGO, NLE, QRO, SLP, TAMS, VER, ZAC

*Chaptalia lyratifolia Burkart COAH, GTO, HGO, NLE, PUE, QRO, SLP, TAMS, VER

Chaptalia nutans (L.) Pol. AGS, BCS, CHIS, CHIH, COAH, CDMX, GTO, GRO, HGO, JAL, MEX, MICH, MOR, NAY, NLE, OAX, PUE, QRO, SLP, SON, TAB, TAMS, VER, YUC, $\mathrm{ZAC}$

*Chaptalia pringlei Greene GRO, OAX, PUE

Chaptalia runcinata Kunth CDMX, DGO, HGO, JAL, MEX, MICH, NAY, QRO, SIN, SON, ZAC

Chaptalia transiliens G.L. Nesom CHIS, COAH, CDMX, GTO, HGO, MEX, NLE, OAX, PUE, QRO, SLP, TAMS, TLAX, VER *Chihuahuana purpusii (Brandegee) Urbatsch \& R.P. Roberts $\mathrm{CHIH}, \mathrm{COAH}, \mathrm{DGO}$

*Chionolaena aecidiocephala (Grierson) Anderb. \& S.E. Freire CHIS, OAX

*Chionolaena concinna (A. Gray) Anderb. \& S.E. Freire DGO, HGO, MEX, MICH, SLP, TAMS, VER

*Chionolaena cryptocephala (G.L. Nesom) G.L. Nesom CHIS

*Chionolaena durangensis (G.L. Nesom) G.L. Nesom DGO

*Chionolaena eleagnoides Klatt OAX

*Chionolaena lavandulifolia (Kunth) Benth. \& Hook. f. ex B.D.

Jacks. MEX, MOR, PUE, TLAX, VER

*Chionolaena macdonaldii (G.L. Nesom) G.L. Nesom OAX

Chionolaena salicifolia (Bertol.) G.L. Nesom CHIS, CDMX, DGO, GTO, GRO, HGO, JAL, MEX, MICH, MOR, OAX, PUE, QRO, SLP, SIN, TLAX, VER, ZAC

*Chionolaena sartorii Klatt CDMX, OAX, TLAX

Chloracantha spinosa (Benth.) G.L. Nesom AGS, BCN, BCS, CHIS, CHIH, COAH, COL, DGO, GTO, GRO, HGO, JAL, MEX, MICH, NAY, NLE, OAX, QRO, SLP, SIN, SON, TAMS, VER, ZAC

Chromolaena bigelovii (A. Gray) R.M. King \& H. Rob. COAH, HGO, NLE, QRO, SLP, TAMS

*Chromolaena breedlovei R.M. King \& H. Rob. CHIS, OAX

Chromolaena collina (DC.) R.M. King \& H. Rob. AGS, CHIS, CHIH, COAH, COL, CDMX, DGO, GTO, GRO, HGO, JAL, MEX, MICH, MOR, NAY, NLE, OAX, PUE, QRO, SLP, SIN,
SON, TAMS, TLAX, VER, ZAC

Chromolaena glaberrima (DC.) R.M. King \& H. Rob. CHIS, COL, DGO, GRO, JAL, MICH, NAY, OAX, SIN, SON, TAB, VER

*Chromolaena guiengolense (L. Torres \& Villaseñor) B.L. Turner GRO, OAX

*Chromolaena haenkeana (DC.) R.M. King \& H. Rob. COL, DGO, GRO, JAL, MEX, MICH, NAY, OAX, SIN

Chromolaena ivifolia (L.) R.M. King \& H. Rob. CHIS, OAX, QRO, SLP, VER

Chromolaena laevigata (Lam.) R.M. King \& H. Rob. CAM, CHIS, QROO

Chromolaena lundellii R.M. King \& H. Rob. CAM, CHIS, QROO, TAB, YUC

*Chromolaena misella (McVaugh) R.M. King \& H. Rob. GRO, JAL

Chromolaena odorata (L.) R.M. King \& H. Rob. CAM, CHIS, CHIH, COAH, COL, DGO, GTO, GRO, HGO, JAL, MEX, MICH, MOR, NAY, NLE, OAX, PUE, QRO, QROO, SLP, SIN, SON, TAB, TAMS, VER, YUC, ZAC

*Chromolaena opadoclinia (S.F. Blake) R.M. King \& H. Rob. CHIS

*Chromolaena ovaliflora (Hook. \& Arn.) R.M. King \& H. Rob. CHIH, COL, DGO, GRO, JAL, MICH, NAY, OAX, PUE, SIN, SON, VER, ZAC

*Chromolaena pulchella (Kunth) R.M. King \& H. Rob. AGS, CHIH, CDMX, DGO, GTO, GRO, HGO, JAL, MEX, MICH, MOR, NAY, OAX, PUE, QRO, SLP, SIN, TLAX, VER, ZAC Chromolaena quercetorum (L.O. Williams) R.M. King \& H. Rob. CHIS, OAX, VER

*Chromolaena sagittata (A. Gray) R.M. King \& H. Rob. BCN, BCS, NAY, SIN, SON

*Chromolaena tamaulipasensis (R.M. King \& H. Rob.) B.L. Turner NLE, TAMS

*Chromolepis heterophylla Benth. DGO, GTO, MEX, MICH, QRO, SIN

*Chrysactinia acerosa S.F. Blake COAH, NLE, SLP, ZAC

*Chrysactinia lethoae D.J. Keil SIN

*Chrysactinia luzmariae Rzed. \& Calderón GTO, QRO, SLP

Chrysactinia mexicana A. Gray AGS, CHIH, COAH, CDMX, DGO, GTO, HGO, MEX, MICH, MOR, NLE, OAX, PUE, QRO, SLP, TAMS, TLAX, VER, ZAC

*Chrysactinia pinnata S. Watson COAH, NLE, QRO, SLP, TAMS

*Chrysactinia truncata S. Watson COAH, NLE, SLP, TAMS, ZAC

Chrysanthellum americanum (L.) Vatke CHIS, MICH

*Chrysanthellum filiforme McVaugh JAL, MICH

Chrysanthellum indicum DC. AGS, CHIS, COL, DGO, GTO, GRO, JAL, MEX, MICH, NAY, OAX, PUE, QRO, SLP, SON, TAMS, VER, ZAC

Chrysanthellum integrifolium Steetz CHIS, OAX, VER

*Chrysanthellum involutum Paul G. Wilson GRO, MEX, MICH

*Chrysanthellum keilii B.L. Turner JAL, MICH

*Chrysanthellum michoacanum B.L. Turner JAL, MICH

*Chrysanthellum perennans B.L. Turner CHIS, OAX

*Chrysanthellum pilzii Strother OAX 
* Chrysanthellum tamaulipense B.L. Turner TAMS

*Dahlia apiculata (Sherff) P.D. Sorensen GRO, OAX, PUE

* Dahlia atropurpurea P.D. Sorensen GRO, MEX

Dahlia australis (Sherff) P.D. Sorensen CHIS, GRO, OAX, PUE

*Dahlia barkeriae Knowles \& Westc. MICH

* Dahlia brevis P.D. Sorensen GTO, MEX, MICH, QRO

* Dahlia campanulata Saar, P.D. Sorensen \& Hjert. GRO, OAX

Dahlia coccinea Cav. AGS, CHIS, CHIH, COAH, COL, CDMX, DGO, GTO, GRO, HGO, JAL, MEX, MICH, MOR, NAY, NLE, OAX, PUE, QRO, SLP, SIN, SON, TAMS, TLAX, VER, ZAC

* Dahlia congestifolia P.D. Sorensen HGO

* Dahlia cordifolia (Sessé \& Moc.) McVaugh GRO

*Dahlia cuspidata Saar, P.D. Sorensen \& Hjert. GTO, HGO, QRO, SLP

*Dahlia dissecta S. Watson HGO, QRO, SLP, TAMS, ZAC

Dahlia excelsa Benth. CHIS, CDMX, HGO, MEX, MICH, QRO

*Dahlia foeniculifolia Sherff NLE, SLP, TAMS

*Dahlia hintonii Sherff GRO, OAX

*Dahlia hjertingii H.V. Hansen \& P.D. Sorensen HGO

Dahlia imperialis Roezl ex Ortgies CHIS, CDMX, GRO, HGO, MEX, MOR, OAX, QRO, VER

*Dahlia linearis Sherff GTO, QRO, SLP

*Dahlia macdougallii Sherff OAX

* Dahlia merckii Lehm. CDMX, GTO, GRO, HGO, JAL, MEX, MICH, MOR, NLE, OAX, PUE, QRO, SLP, TAMS, TLAX, VER, ZAC

*Dahlia mollis P.D. Sorensen GTO, HGO, QRO, SLP

* Dahlia moorei Sherff GTO, HGO, QRO, SLP, VER

*Dahlia neglecta Saar CDMX, GTO, HGO, MEX, MICH, QRO, VER

*Dahlia parvibracteata Saar \& P.D. Sorensen GRO

Dahlia pinnata Cav. CHIS, CDMX, HGO, MEX, MICH, OAX, QRO, SLP, VER

*Dahlia pteropoda Sherff OAX, PUE

* Dahlia pugana Aarón Rodr. \& A. Castro DGO, JAL, NAY

* Dahlia purpusii Brandegee CHIS

*Dahlia rudis P.D. Sorensen GRO, HGO, JAL, MEX, MICH, MOR, QRO

* Dahlia rupicola P.D. Sorensen DGO, SIN

*Dahlia scapigera (A. Dietr.) Knowles \& Westc. CDMX, GTO, GRO, HGO, JAL, MEX, MICH, MOR, PUE, QRO, TLAX, VER

*Dahlia scapigeroides Sherff GTO, HGO, QRO, SLP, VER

* Dahlia sherffii P.D. Sorensen CHIH, DGO, JAL, NAY, SIN, SON, ZAC

Dahlia sorensenii H.V. Hansen \& Hjert. CHIS, CHIH, CDMX, GTO, GRO, HGO, JAL, MEX, MICH, MOR, NLE, OAX, PUE, QRO, SLP, SON, TAMS, TLAX, VER, ZAC

*Dahlia spectabilis Saar \& P.D. Sorensen SLP

*Dahlia sublignosa (P.D. Sorensen) Saar \& P.D. Sorensen SLP, TAMS

*Dahlia tenuicaulis P.D. Sorensen COL, GRO, JAL, MICH, OAX, PUE, VER

*Dahlia tenuis B.L. Rob. \& Greenm. OAX, QRO

* Dahlia tubulata P.D. Sorensen COAH, NLE, SLP, TAMS,
ZAC

*Dahlia wixarika A. Castro, M. Carrasco-Ortiz \& Aarón Rodr. DGO, JAL

*Damnxanthodium calvum (Greenm.) Strother DGO, SIN

*Davilanthus davilae (Panero \& Villaseñor) E.E. Schill. \& Panero PUE

*Davilanthus hidalgoanus (E.E. Schill. \& Panero) E.E. Schill. \& Panero HGO, QRO, VER

*Davilanthus huajuapanus (Panero \& Villaseñor) E.E. Schill. \& Panero OAX

*Davilanthus hypargyreus (B.L. Rob. \& Greenm.) E.E. Schill. \& Panero OAX, PUE

*Davilanthus purpusii (Brandegee) E.E. Schill. \& Panero PUE

*Davilanthus sericeus (Klatt) E.E. Schill. \& Panero OAX, PUE, VER

* Davilanthus veracruzanus B.L. Turner VER

*Decachaeta haenkeana DC. CHIH, COL, DGO, GRO, JAL, MEX, MICH, MOR, NAY, OAX, SIN, SON, VER

Decachaeta incompta (DC.) R.M. King \& H. Rob. AGS, CHIS, COL, GTO, GRO, JAL, MEX, MICH, OAX, PUE, VER, ZAC *Decachaeta ovandensis (Grashoff \& Beaman) R.M. King \& H. Rob. CHIS

*Decachaeta ovatifolia (DC.) R.M. King \& H. Rob. COL, DGO, GRO, JAL, MEX, MICH, MOR, NAY, OAX, SIN

*Decachaeta perornata (Klatt) R.M. King \& H. Rob. CHIS, HGO, MEX, OAX, PUE, VER

*Decachaeta pyramidalis (B.L. Rob.) S.D. Sundb., C.P. Cowan \& B.L. Turner COL, GRO, JAL, MEX, MICH, MOR, OAX, PUE

*Decachaeta scabrella (B.L. Rob.) R.M. King \& H. Rob. CHIH, COL, DGO, GRO, JAL, MEX, MICH, NAY, SIN, SON, ZAC

*Decachaeta serboana B.L. Turner OAX

Deinandra conjugens (D.D. Keck) B.G. Baldwin BCN

Deinandra fasciculata (DC.) Greene BCN, BCS

Deinandra floribunda (A. Gray) Davidson \& Moxley BCN

Deinandra frutescens (A. Gray) B.G. Baldwin BCN

* Deinandra greeneana (Rose) B.G. Baldwin BCN

Deinandra kelloggii (Greene) Greene BCN

*Deinandra martirensis (D.D. Keck) B.G. Baldwin BCN

*Deinandra palmeri (Rose) B.G. Baldwin BCN

Deinandra paniculata (A. Gray) Davidson \& Moxley BCN

* Deinandra streetsii (A. Gray) B.G. Baldwin BCN

Delilia biflora (L.) Kuntze AGS, CAM, CHIS, CHIH, COL, DGO, GTO, GRO, JAL, MEX, MICH, MOR, NAY, OAX, PUE, QRO, QROO, SLP, SIN, SON, TAB, TAMS, VER, YUC, ZAC

*Dendroviguiera adenophylla (S.F. Blake) E.E. Schill. \& Panero COAH, HGO, NLE, QRO, SLP, TAMS, VER

*Dendroviguiera eriophora (Greenm.) E.E. Schill. \& Panero MOR, OAX, PUE

*Dendroviguiera guerrerana (Panero \& E.E. Schill.) E.E. Schill. \& Panero GRO, PUE

*Dendroviguiera insignis (Miranda) E.E. Schill. \& Panero GRO, OAX, PUE

*Dendroviguiera mirandae (Panero \& E.E. Schill.) E.E. Schill. \& Panero MOR

*Dendroviguiera neocronquistii (B.L. Turner) E.E. Schill. \& 
Panero OAX

*Dendroviguiera oaxacana (Greenm.) E.E. Schill. \& Panero GRO, OAX, PUE

*Dendroviguiera pringlei (Fernald) E.E. Schill. \& Panero GTO, HGO, MEX, MICH, QRO, SLP, VER

*Dendroviguiera puruana (Paray) E.E. Schill. \& Panero CHIS, GRO, MEX, MICH

*Dendroviguiera quinqueradiata (Cav.) E.E. Schill. \& Panero AGS, COL, DGO, GTO, GRO, JAL, MICH, NAY, QRO, ZAC

*Dendroviguiera sharpii (Panero \& E.E. Schill.) E.E. Schill. \& Panero GRO

*Dendroviguiera sphaerocephala (DC.) E.E. Schill. \& Panero GTO, GRO, HGO, JAL, MEX, MICH, MOR, OAX, PUE

*Dendroviguiera splendens (Panero \& E.E. Schill.) E.E. Schill. \& Panero GTO, GRO, JAL, MICH, QRO

*Desmanthodium fruticosum Greenm. COL, DGO, GRO, JAL, MEX, MICH, NAY, OAX

*Desmanthodium hintoniorum B.L. Turner OAX

*Desmanthodium ovatum Benth. GRO, MEX, MOR, OAX, PUE

*Desmanthodium perfoliatum Benth. CHIS, GRO, OAX

Desmanthodium tomentosum Brandegee CHIS, OAX

Diaperia verna (Raf.) Morefield CHIH, COAH, NLE, SON, TAMS

*Dicoria argentea Strother SON

Dicoria canescens A. Gray BCN, BCS, SON

Dicranocarpus parviflorus A. Gray CHIH, COAH, DGO, HGO,

NLE, QRO, SLP, TAMS, ZAC

Dichaetophora campestris A. Gray CHIH, COAH, NLE

Dieteria asteroides Torr. BCN, CHIH, DGO, SON

Dieteria canescens (Pursh) Nutt. BCN, CHIH, COAH, DGO, SON

*Digitacalia crypta B.L. Turner GRO, MEX, MOR, OAX

*Digitacalia chiapensis (Hemsl.) Pippen CHIS

*Digitacalia hintoniorum B.L. Turner MICH

*Digitacalia jatrophoides (Kunth) Pippen AGS, COL, GTO, GRO, HGO, JAL, MEX, MICH, OAX, PUE, QRO, SLP, SIN, ZAC

*Digitacalia napeifolia (DC.) Pippen GRO, OAX

*Dugesia mexicana (A. Gray) A. Gray CDMX, HGO, MEX, MICH, PUE, QRO, SLP, TLAX, VER

*Dyscritothamnus filifolius B.L. Rob. GTO, HGO, QRO, SLP, VER

* Dyscritothamnus mirandae Paray GTO, HGO, QRO, SLP, VER

Dyssodia decipiens (Bartl.) M.C. Johnst. CHIS, OAX

Dyssodia papposa (Vent.) Hitchc. AGS, CHIS, CHIH, COAH, CDMX, DGO, GTO, GRO, HGO, JAL, MEX, MICH, MOR, NAY, NLE, OAX, PUE, QRO, SLP, SON, TAMS, TLAX, VER, ZAC

*Dyssodia pinnata (Cav.) B.L. Rob. AGS, COAH, CDMX, DGO, GTO, GRO, HGO, JAL, MEX, MICH, MOR, NAY, NLE, OAX, PUE, QRO, SLP, TAMS, TLAX, VER, ZAC

*Dyssodia tagetiflora Lag. AGS, CHIS, COL, DGO, GTO, GRO, HGO, JAL, MEX, MICH, MOR, NAY, OAX, PUE, QRO, SLP, SIN, TAMS, TLAX, VER, ZAC

Eclipta prostrata (L.) L. AGS, BCN, BCS, CAM, CHIS, CHIH,
COAH, COL, DGO, GTO, GRO, HGO, JAL, MEX, MICH, MOR, NAY, NLE, OAX, PUE, QRO, QROO, SLP, SIN, SON, TAB, TAMS, VER, YUC, ZAC

Egletes liebmannii Sch. Bip. ex Klatt CAM, CHIS, COL, HGO, JAL, OAX, PUE, QROO, SLP, TAB, TAMS, VER, YUC, ZAC Egletes viscosa (L.) Less. CAM, CHIS, COL, GRO, JAL, NAY, OAX, QROO, SLP, SIN, SON, TAB, TAMS, VER, ZAC

*Electranthera cuneifolia (Greenm.) Mesfin, D.J. Crawford \& Pruski DGO, JAL, NAY

Electranthera mutica (DC.) Mesfin, D.J. Crawford \& Pruski AGS, CHIS, GTO, GRO, HGO, JAL, MEX, MICH, OAX, PUE, QRO, SLP, TAMS, VER

*Electranthera parvifolia S.F. Blake PUE, VER

Elephantopus mollis Kunth CHIS, COL, DGO, GRO, HGO, JAL, MEX, MICH, NAY, NLE, OAX, PUE, QRO, SLP, SIN,

TAB, TAMS, VER, ZAC

Encelia actoni Elmer BCN

*Encelia asperifolia (S.F. Blake) C. Clark \& Kyhos BCN, BCS

Encelia californica Nutt. BCN

*Encelia densifolia Gentry ex C. Clark \& Kyhos BCS

Encelia farinosa A. Gray ex Torr. BCN, BCS, CHIH, DGO, SIN, SON

Encelia frutescens (A. Gray) A. Gray BCN, BCS, CHIH, SON

*Encelia halimifolia Cav. BCN, BCS, SON

*Encelia laciniata Vasey \& Rose BCN, BCS

*Encelia palmeri Vasey \& Rose BCN, BCS

*Encelia ravenii Wiggins BCN, BCS, SON

Encelia scaposa (A. Gray) A. Gray CHIH

*Encelia stenophylla Greene BCN, BCS

*Encelia ventorum Brandegee $\mathrm{BCN}, \mathrm{BCS}$

Engelmannia peristenia (Raf.) Goodman \& C.A. Lawson CHIH, COAH, NLE, TAMS

Enydra sessilifolia (Ruiz \& Pav.) Cabrera TAB

Epaltes mexicana Less. CAM, CHIS, OAX, PUE, TAB, VER

Erechtites hieracifolius (L.) Raf. ex DC. CAM, CHIS, COL, GRO, HGO, JAL, MEX, MICH, NAY, OAX, PUE, QRO, QROO, SLP, TAB, TAMS, VER, YUC

Erechtites valerianifolius (Link ex Spreng.) DC. CHIS, COL, GRO, HGO, JAL, OAX, PUE, QRO, SLP, TAB, VER

Ericameria brachylepis (A. Gray) H.M. Hall BCN, BCS, SON

Ericameria cooperi (A. Gray) H.M. Hall BCN

Ericameria cuneata (A. Gray) McClatchie BCN, BCS

* Ericameria juarezensis (Moran) Urbatsch BCN

Ericameria laricifolia (A. Gray) Shinners CHIH, SON

Ericameria linearifolia (DC.) Urbatsch \& Wussow BCN

*Ericameria martirensis Wiggins BCN

Ericameria nauseosa (Pall. ex Pursh) G.L. Nesom \& G.I. Baird BCN, SON

Ericameria palmeri (A. Gray) H.M. Hall BCN

Ericameria paniculata (A. Gray) Rydb. SON

Ericameria parishii (Greene) H.M. Hall BCN

Ericameria pinifolia (A. Gray) H.M. Hall BCN

*Erigeron annuactis G.L. Nesom JAL, MICH

Erigeron arisolius G.L. Nesom SON

Erigeron arizonicus A. Gray SON

*Erigeron astranthioides De Jong \& G.L. Nesom DGO, SIN

*Erigeron barbarensis G.L. Nesom \& Van Devender CHIH, 
SON

*Erigeron basaseachensis G.L. Nesom CHIH, SON

*Erigeron basilobatus S.F. Blake COAH, NLE, HGO, TAMS, TLAX

Erigeron bigelovii A. Gray CHIH, COAH, GTO, JAL, NLE, SLP, SON, TAMS, ZAC

Erigeron breweri A. Gray BCN

*Erigeron caulinifolius G.L. Nesom CHIH

*Erigeron cieloensis G.L. Nesom TAMS

*Erigeron circulis G.L. Nesom CHIH

Erigeron colomexicanus A. Nelson CHIH, COAH, SON

*Erigeron coronarius Greene CHIH, COAH, DGO, JAL, NAY, QRO, SIN, SON, ZAC

*Erigeron coroniglandifer G.L. Nesom CHIH, DGO

*Erigeron crenatus Eastw. ex I.M. Johnst. COL

*Erigeron cuatrocienegensis G.L. Nesom COAH

*Erigeron chiangii G.L. Nesom COAH, NLE

*Erigeron dactyloides (Greenm.) G.L. Nesom CHIH, SON

Erigeron delphinifolius Willd. AGS, CHIH, COAH, CDMX, DGO, GTO, HGO, JAL, MEX, MICH, MOR, NAY, PUE, QRO, SLP, SIN, SON, TLAX, VER, ZAC

Erigeron divergens Torr. \& A. Gray BCN, BCS, CHIH, COAH, DGO, NLE, SON

*Erigeron dryophyllus A. Gray COAH, NLE, TAMS

*Erigeron eruptens G.L. Nesom CHIH, DGO, SON

*Erigeron exilis A. Gray JAL, NAY, ZAC

Erigeron flagellaris A. Gray CHIH, COAH, DGO, NLE, SLP, SON, TAMS, ZAC

Erigeron foliosus Nutt. BCN

*Erigeron forreri (Greene) Greene DGO

*Erigeron fraternus Greene CHIH, DGO, SON

*Erigeron fundus G.L. Nesom CHIH, SON

*Erigeron galeottii (A. Gray ex Hemsl.) Greene CHIH, CDMX, DGO, GTO, HGO, JAL, MEX, MICH, MOR, OAX, PUE, QRO, SLP, SIN, SON, TLAX, VER

*Erigeron griseus (Greenm.) G.L. Nesom AGS, DGO, NAY, SON

*Erigeron gypsoverus G.L. Nesom NLE, TAMS

*Erigeron heleniae G.L. Nesom NLE

*Erigeron heteromorphus B.L. Rob. SLP

*Erigeron hintoniorum G.L. Nesom COAH, NLE

*Erigeron inoptatus A. Gray CHIH

*Erigeron janivultus G.L. Nesom AGS, CHIH, COAH, DGO, GTO, HGO, JAL, MEX, MICH, QRO, SLP, TLAX, VER, ZAC

*Erigeron jenkinsii G.L. Nesom CHIH, SON

Erigeron karvinskianus DC. CHIS, CHIH, CDMX, DGO, GTO, GRO, HGO, JAL, MEX, MICH, MOR, NAY, NLE, OAX, PUE, QRO, SLP, TAMS, TLAX, VER, ZAC

*Erigeron lepidopodus (B.L. Rob. \& Fernald) G.L. Nesom CHIH, DGO, SON

Erigeron lobatus A. Nelson SON

Erigeron longipes DC. AGS, CHIS, COAH, COL, CDMX, DGO, GTO, GRO, HGO, JAL, MEX, MICH, MOR, NAY, NLE, OAX, PUE, QRO, SLP, TAMS, TLAX, VER, ZAC

*Erigeron macdonaldii G.L. Nesom CHIH

*Erigeron mayoensis G.L. Nesom CHIH, SON

*Erigeron metrius S.F. Blake CHIH, COAH, NLE, TAMS
*Erigeron mimus (S.F. Blake) G.L. Nesom DGO

Erigeron modestus A. Gray CHIH, COAH, NLE, SLP, SON, TAMS, ZAC

*Erigeron mohinorensis G.L. Nesom CHIH

*Erigeron morelensis Greenm. MOR

*Erigeron nacoriensis G.L. Nesom SON

*Erigeron narcissus G.L. Nesom TAMS

Erigeron neomexicanus A. Gray CHIH, DGO, SON

*Erigeron nitens G.L. Nesom CHIH, SON

*Erigeron oaxacanus Greenm. OAX

*Erigeron onofrensis G.L. Nesom NLE, TAMS

Erigeron oreophilus Greenm. CHIH, DGO, SIN, SON

Erigeron oxyphyllus Greene SON

Erigeron pacayensis Greenm. CHIS

*Erigeron palmeri A. Gray HGO, NLE, PUE, SLP, TAMS, TLAX, VER

*Erigeron pattersonii G.L. Nesom COAH, NLE

*Erigeron pinkavii B.L. Turner CHIH, COAH, NLE

*Erigeron podophyllus G.L. Nesom CHIH, DGO, SON

*Erigeron polycephalus (Larsen) G.L. Nesom COL, DGO, GRO, HGO, JAL, MEX, MICH, NAY, QRO, SIN, ZAC

*Erigeron potosinus Standl. COAH, NLE, SLP, TAMS

Erigeron procumbens (Mill.) G.L. Nesom SLP, TAMS, VER

Erigeron pubescens Kunth AGS, CHIH, COAH, CDMX, DGO, GTO, GRO, HGO, JAL, MEX, MICH, NLE, OAX, PUE, QRO, SLP, TAMS, TLAX, VER, ZAC

*Erigeron quiexobrensis G.L. Nesom OAX

*Erigeron rhizomactis G.L. Nesom CHIH, SIN

Erigeron sceptrifer G.L. Nesom CHIH, SON

*Erigeron scoparioides G.L. Nesom TAMS

*Erigeron seemannii (Sch. Bip.) Greene CHIH, DGO, SIN

*Erigeron socorrensis Brandegee COL

Erigeron speciosus (Lindl.) DC. BCN, SON

*Erigeron stanfordii G.L. Nesom TAMS

*Erigeron strigulosus Greene CHIH, SON

*Erigeron subacaulis (McVaugh) G.L. Nesom AGS, DGO, JAL, SLP, ZAC

Erigeron tenellus DC. COAH, MICH, NLE, TAMS

*Erigeron tephropodus G.L. Nesom GRO, MEX, MOR, OAX

*Erigeron turnerorum G.L. Nesom NLE, TAMS

*Erigeron unguiphyllus G.L. Nesom QRO, SLP, ZAC

*Erigeron velutipes Hook. \& Arn. AGS, CHIH, COL, DGO, GTO, GRO, JAL, MEX, MICH, NAY, OAX, QRO, SLP, SIN, SON, VER, ZAC

*Erigeron veracrucensis G.L. Nesom COAH, NLE, OAX, PUE, TAMS, VER

Erigeron versicolor (Greenm.) G.L. Nesom CHIH, COAH, HGO, MEX, MICH, NLE, QRO, SON, TAMS, VER, ZAC

*Erigeron vicinus G.L. Nesom COAH

*Erigeron wellsii G.L. Nesom NLE, TAMS

*Erigeron wislizenii (A. Gray) Greene CHIH, DGO, GTO, JAL, SLP, SIN, SON, ZAC

*Erigeron zacatensis G.L. Nesom JAL, ZAC

Eriophyllum confertiflorum (DC.) A. Gray BCN

Eriophyllum lanatum (Pursh) Forbes BCN

Eriophyllum lanosum (A. Gray) A. Gray BCN, BCS, SON

Eriophyllum wallacei (A. Gray) A. Gray BCN, SON 
*Eryngiophyllum pinnatisectum P.G. Wilson GRO, NAY

*Eryngiophyllum rosei Greenm. DGO, SIN

Espejoa mexicana DC. CHIS, GRO, OAX, VER

Eupatoriastrum angulifolium (B.L. Rob.) R.M. King \& H. Rob. CHIS

*Eupatoriastrum corvi (McVaugh) B.L. Turner CHIS

*Eupatoriastrum chlorostylum B.L. Turner OAX

*Eupatoriastrum johnbeamanii B.L. Turner VER

Eupatoriastrum nelsonii Greenm. CHIS, OAX, VER

* Eupatoriastrum pochutlanum B.L. Turner OAX

*Eupatoriastrum triangulare (DC.) B.L. Rob. COL, GRO, JAL, MEX, MICH, NAY, OAX, ZAC

Eupatorium serotinum Michx. COAH

*Euphrosyne partheniifolia DC. AGS, COAH, COL, CDMX, DGO, GTO, HGO, JAL, MEX, MICH, MOR, NAY, PUE, QRO, SLP, ZAC

*Eutetras palmeri A. Gray AGS, GTO, JAL, SLP, ZAC

*Eutetras pringlei Greenm. GTO, HGO, QRO, SLP, VER

Euthamia occidentalis Nutt. BCN

* Faxonia pusilla Brandegee BCS

* Flaveria angustifolia (Cav.) Pers. CDMX, GRO, MEX, MICH, MOR, OAX, PUE

*Flaveria anomala B.L. Rob. AGS, COAH, HGO, NLE, QRO, SLP, TAMS, ZAC

*Flaveria cronquistii A.M. Powell OAX, PUE

Flaveria chlorifolia A. Gray CHIH, COAH, DGO, NLE, TAMS

* Flaveria intermedia J.R. Johnst. COAH, DGO

* Flaveria kochiana B.L. Turner GRO, OAX

Flaveria linearis Lag. CAM, PUE, QROO, VER, YUC

* Flaveria oppositifolia (DC.) Rydb. AGS, COAH, DGO, GTO, HGO, NLE, QRO, SLP, TAMS, VER, ZAC

*Flaveria palmeri J.R. Johnst. CHIH, COAH, DGO, NLE, SLP

* Flaveria pringlei Gand. GRO, MOR, OAX, PUE, SLP

*Flaveria pubescens Rydb. GTO, HGO, QRO, SLP, TAMS

* Flaveria ramosissima Klatt OAX, PUE

* Flaveria robusta Rose COL, JAL, MICH

* Flaveria sonorensis A.M. Powell CHIH, SON

Flaveria trinervia (Spreng.) C. Mohr AGS, BCS, CAM, CHIS, CHIH, COAH, COL, CDMX, DGO, GTO, GRO, HGO, JAL, MEX, MICH, MOR, NAY, NLE, OAX, PUE, QRO, QROO, SLP, SON, TAMS, VER, YUC, ZAC

* Flaveria vaginata B.L. Rob. \& Greenm. GRO, OAX, PUE

Fleischmannia arguta (Kunth) B.L. Rob. COL, CDMX, GRO, JAL, MEX, MICH, MOR, OAX, TAMS

Fleischmannia blakei (B.L. Rob.) R.M. King \& H. Rob. CHIS Fleischmannia bohlmanniana R.M. King \& H. Rob. CHIS, OAX

Fleischmannia capillipes (Benth. ex Oerst.) R.M. King \& H. Rob. CHIS, GRO, OAX, VER

Fleischmannia crocodilia (Standl. \& Steyerm.) R.M. King \& H. Rob. CHIS

*Fleischmannia gonzalezii (B.L. Rob.) R.M. King \& H. Rob. CHIS, OAX

Fleischmannia guatemalensis R.M. King \& H. Rob. CHIS, OAX

*Fleischmannia holwayana (B.L. Rob.) R.M. King \& H. Rob. GRO, MEX, OAX
Fleischmannia imitans (B.L. Rob.) R.M. King \& H. Rob. CAM, CHIS, OAX, YUC

Fleischmannia incarnata (Walter) R.M. King \& H. Rob. NLE, TAMS, VER

Fleischmannia matudae R.M. King \& H. Rob. CHIS, OAX, VER

Fleischmannia microstemon (Cass.) R.M. King \& H. Rob. CHIS, QROO, YUC

Fleischmannia multinervis (Benth.) R.M. King \& H. Rob. CHIS *Fleischmannia porphyranthema (A. Gray) R.M. King \& H. Rob. COAH, GTO, HGO, NLE, OAX, PUE, QRO, SLP, TAMS *Fleischmannia purpusii R.M. King \& H. Rob. HGO, VER Fleischmannia pycnocephala (Less.) R.M. King \& H. Rob. AGS, CAM, CHIS, CHIH, COAH, COL, CDMX, DGO, GTO, GRO, HGO, JAL, MEX, MICH, MOR, NAY, NLE, OAX, PUE, QRO, QROO, SLP, SON, TAB, TAMS, VER, YUC, ZAC

Fleischmannia pycnocephaloides (B.L. Rob.) R.M. King \& H. Rob. CHIS

Fleischmannia seleriana (B.L. Rob.) R.M. King \& H. Rob. CHIS, QROO, YUC

*Fleischmannia sideritidis (Benth.) R.M. King \& H. Rob. CHIS, TAB

Fleischmannia sinclairii (Benth. ex Oerst.) R.M. King \& H. Rob. CHIS, GRO, OAX

Fleischmannia sonorae (A. Gray) R.M. King \& H. Rob. AGS, BCS, CHIH, COL, DGO, GTO, GRO, JAL, MEX, MICH, MOR, NAY, OAX, QRO, SIN, SON

*Fleischmannia trinervia (Sch. Bip.) R.M. King \& H. Rob. DGO, JAL, NAY, SIN

Fleischmanniopsis leucocephala (Benth.) R.M. King \& H. Rob. CHIS, GRO, HGO, MICH, OAX, PUE, VER

Fleischmanniopsis nubigenoides (B.L. Rob.) R.M. King \& H. Rob. CHIS

Florestina latifolia (DC.) Rydb. CHIS, OAX

*Florestina liebmannii Sch. Bip. ex Greenm. HGO, QRO, SLP, VER

*Florestina lobata B.L. Turner GRO, MEX, MICH, OAX Florestina pedata (Cav.) Cass. AGS, CHIS, CHIH, COAH, COL, CDMX, DGO, GTO, GRO, HGO, JAL, MEX, MICH, MOR, NLE, OAX, PUE, QRO, SLP, TAMS, TLAX, VER, ZAC

*Florestina platyphylla (B.L. Rob. \& Greenm.) B.L. Rob. \& Greenm. CHIS, OAX

* Florestina purpurea (Brandegee) Rydb. OAX, PUE

*Florestina simplicifolia B.L. Turner GRO, OAX, PUE

Florestina tripteris DC. CHIH, COAH, DGO, HGO, NLE, QRO, SLP, TAMS, VER, ZAC

Flourensia cernua DC. AGS, CHIH, COAH, DGO, GTO, HGO, JAL, NLE, QRO, SLP, SON, TAMS, VER, ZAC

* Flourensia collodes (Greenm.) S.F. Blake CHIS, OAX

* Flourensia dentata S.F. Blake COAH, DGO, SLP, ZAC

*Flourensia glutinosa (B.L. Rob. \& Greenm.) S.F. Blake GRO, OAX, PUE

*Flourensia ilicifolia Brandegee COAH, DGO

*Flourensia laurifolia DC. GTO, HGO, NLE, QRO, SLP, TAMS, VER, ZAC

*Flourensia microphylla (A. Gray) S.F. Blake COAH, NLE 
*Flourensia monticola M.O. Dillon COAH, NLE

Flourensia pringlei (A. Gray) S.F. Blake CHIH, DGO

*Flourensia pulcherrima M.O. Dillon CHIH, COAH, DGO

*Flourensia resinosa (Brandegee) S.F. Blake HGO, QRO, SLP, TAMS, VER

*Flourensia retinophylla S.F. Blake COAH

*Flourensia solitaria S.F. Blake COAH

*Flyriella harrimanii R.M. King \& H. Rob. TAMS

*Flyriella leonensis (B.L. Rob.) R.M. King \& H. Rob. COAH, NLE

Flyriella parryi (A. Gray) R.M. King \& H. Rob. CHIH, COAH, NLE

* Flyriella stanfordii R.M. King \& H. Rob. NLE, TAMS

Gaillardia arizonica A. Gray SON

* Gaillardia candelaria B.L. Turner COAH

Gaillardia coahuilensis B.L. Turner COAH, NLE

* Gaillardia comosa A. Gray AGS, CHIH, COAH, DGO, NLE,

SLP, TAMS, ZAC

* Gaillardia gypsophila B.L. Turner COAH

* Gaillardia henricksonii B.L. Turner COAH

* Gaillardia mexicana A. Gray CHIH, COAH, CDMX, DGO, MEX, MOR, NLE, SLP, SIN, TAMS, VER, ZAC

Gaillardia pinnatifida Torr. CHIH, COAH, DGO, NLE, SLP, SON, TAMS, ZAC

* Gaillardia powellii B.L. Turner COAH, NLE, SLP, TAMS

Gaillardia pulchella Foug. CHIS, CHIH, COAH, DGO, NLE, SON, TAMS

Gaillardia suavis (A. Gray \& Engelm.) Britton \& Rusby COAH, NLE, TAMS

* Gaillardia turneri Averett \& A.M. Powell CHIH

Galeana pratensis (Kunth) Rydb. AGS, CHIS, COL, DGO, GTO, GRO, JAL, MEX, MICH, MOR, NAY, OAX, PUE, QRO, VER, ZAC

* Galinsoga crozierae Panero DGO, SIN

* Galinsoga durangensis (Longpre) Canne DGO, SIN

*Galinsoga longipes Canne GTO, GRO, JAL, MEX, MICH, MOR, OAX, SLP

* Galinsoga mollis McVaugh JAL, ZAC

Galinsoga parviflora Cav. AGS, BCS, CHIS, CHIH, COAH, COL, CDMX, DGO, GTO, GRO, HGO, JAL, MEX, MICH, MOR, NAY, NLE, OAX, PUE, QRO, QROO, SLP, SIN, SON, TAMS, TLAX, VER, YUC, ZAC

* Galinsoga spellenbergii B.L. Turner DGO, SIN

* Galinsoga subdiscoidea Cronquist DGO, SIN

* Galinsoga triradiata Canne GRO, JAL, MICH, OAX

Gamochaeta americana (Mill.) Wedd. AGS, BCS, CHIS, CHIH, COAH, COL, CDMX, DGO, GTO, GRO, HGO, JAL, MEX, MICH, MOR, NAY, NLE, OAX, PUE, QRO, SLP, SIN, TAB, TAMS, TLAX, VER, ZAC

Gamochaeta coarctata (Willd.) Kreguélen GRO, OAX

Gamochaeta falcata (Lam.) Cabrera CHIH, COAH, CDMX, DGO, GTO, HGO, JAL, MEX, MICH, NLE, OAX, QRO, SLP, SIN, SON, TAMS, TLAX, VER, ZAC

Gamochaeta pensylvanica (Willd.) Cabrera BCS, CHIS, COL, DGO, JAL, MICH, NLE, PUE, SIN, SON, TAMS, VER, YUC Gamochaeta purpurea (L.) Cabrera BCS, CHIS, CHIH, DGO, GTO, GRO, HGO, JAL, MEX, MICH, MOR, NLE, OAX, PUE,
QRO, SLP, SIN, SON, TAMS, TLAX, VER

Gamochaeta sphacelata (Kunth) Cabrera AGS, BCS, CHIH, COL, CDMX, DGO, GTO, GRO, HGO, JAL, MEX, NAY, QRO, SLP, SIN, SON, TLAX, VER, ZAC

Gamochaeta standleyi (Steyerm.) G.L. Nesom CHIS

*Geissolepis suaedifolia B.L. Rob. GTO, QRO, SLP, TAMS

Geraea canescens Torr. \& A. Gray BCN, SON

Geraea viscida (A. Gray) S.F. Blake BCN

Gnaphalium palustre Nutt. BCN, BCS, HGO, SON

* Gochnatia arborescens Brandegee BCS

* Gochnatia hiriartiana Medrano, Villaseñor \& Medina HGO

Gochnatia hypoleuca (DC.) A. Gray COAH, DGO, GTO, HGO, MEX, NLE, OAX, PUE, QRO, SLP, SON, TAMS, VER, YUC, ZAC

*Gochnatia magna M.C. Johnst. GTO, HGO, QRO, SLP, TAMS, VER

* Gochnatia purpusii Brandegee PUE

* Gochnatia smithii B.L. Rob. \& Greenm. OAX, PUE

Goldmanella sarmentosa Greenm. CAM, CHIS, QROO, TAB, YUC

*Gonzalezia decurrens (A. Gray) E.E. Schill. \& Panero CHIH, DGO, SON

*Gonzalezia hypargyrea (Greenm.) E.E. Schill. \& Panero DGO, SIN, ZAC

*Gonzalezia rosei (Greenm.) E.E. Schill. \& Panero AGS, GTO, JAL, ZAC

*Greenmaniella resinosa (S. Watson) W.M. Sharp COAH, NLE, TAMS

Grindelia arizonica A. Gray CHIH, COAH, DGO

* Grindelia confusa Steyerm. CHIH, DGO, SON, TAMS

Grindelia grandiflora Hook. COAH, NLE

*Grindelia greenmanii Steyerm. COAH, NLE, SLP, TAMS

Grindelia havardii Steyerm. COAH, SLP

* Grindelia hintoniorum G.L. Nesom NLE, TAMS

* Grindelia hirtella (B.L. Rob. \& Greenm.) A. Bartoli \& Tortosa OAX, PUE

*Grindelia inuloides Willd. COAH, CDMX, GTO, HGO, JAL, MEX, MICH, NLE, OAX, PUE, QRO, SLP, VER, YUC

Grindelia lanceolata Nutt. COAH, NLE

* Grindelia leptocarpa (De Jong \& Beaman) A. Bartoli \& Tortosa DGO

* Grindelia macvaughii G.L. Nesom AGS, JAL, ZAC

* Grindelia nelsonii Steyerm. JAL, MICH

* Grindelia oaxacana G.L. Nesom OAX, PUE

* Grindelia obovatifolia S.F. Blake COAH, NLE, TAMS

* Grindelia oxylepis Greene CHIS, COAH, DGO, SLP

* Grindelia palmeri Steyerm. COL, MICH, SLP

Grindelia pusilla (Steyerm.) G.L. Nesom COAH

* Grindelia robinsonii Steyerm. HGO, SLP

Grindelia squarrosa (Pursh) Dunal CHIH, COAH, NLE

*Grindelia subdecurrens DC. AGS, COL, GTO, JAL, OAX, QRO, SLP, ZAC

* Grindelia sublanuginosa Steyerm. JAL

Grindelia tenella Steyerm. COAH, NLE, SLP, TAMS

*Grindelia tricuspis (Sch. Bip.) A. Bartoli \& Tortosa AGS, COL, GTO, JAL, MEX, MICH

* Grindelia turneri G.L. Nesom COAH, NLE 
* Grindelia villarrealii G.L. Nesom NLE, TAMS

* Guardiola arguta (A. Gray) B.L. Rob. CHIH, DGO, SIN, SON

* Guardiola carinata B.L. Rob. DGO, JAL, NAY, SIN, ZAC

* Guardiola mexicana Bonpl. AGS, CHIH, COL, DGO, GTO, GRO, HGO, JAL, MEX, MICH, MOR, NAY, OAX, PUE, QRO, SIN, SON, ZAC

* Guardiola odontophylla B.L. Rob. DGO

* Guardiola pappifera P.G. Wilson GRO, JAL, MICH

Guardiola platyphylla A. Gray CHIH, DGO, SIN, SON

* Guardiola rosei B.L. Rob. CHIH, COAH, DGO, NAY, SIN

* Guardiola rotundifolia B.L. Rob. JAL, SIN

* Guardiola stenodonta S.F. Blake SIN

* Guardiola thompsonii Van Faasen JAL, MICH

*Guardiola tulocarpus A. Gray CHIH, COL, GRO, JAL, MICH, NAY, OAX, SIN, TAMS, ZAC

*Gundlachia diffusa (Benth.) Urbatsch \& R.P. Roberts BCN, BCS, SIN, SON

*Gundlachia riskindii (B.L. Turner \& G. Langford) Urbatsch \& R.P. Roberts COAH, NLE, TAMS

Gundlachia triantha (S.F. Blake) Urbatsch \& R.P. Roberts CHIH, COAH, DGO, NLE, SLP

*Gundlachia truncata (G.L. Nesom) Urbatsch \& R.P. Roberts $\mathrm{COAH}$

*Gutierrezia alamanii A. Gray CHIH, CDMX, DGO, MEX, MOR, SIN, SON

* Gutierrezia argyrocarpa Greenm. HGO, QRO, VER

Gutierrezia arizonica (A. Gray) M.A. Lane SON

Gutierrezia californica (DC.) Torr. \& A. Gray BCN

*Gutierrezia conoidea (Hemsl.) M.A. Lane AGS, CHIH, COAH, DGO, JAL, NAY, SLP, SIN, SON, ZAC

*Gutierrezia grandis S.F. Blake COAH, NLE, QRO, SLP, ZAC Gutierrezia microcephala (DC.) A. Gray BCN, CHIH, COAH, DGO, NLE, SLP, SON, TAMS, VER, ZAC

* Gutierrezia ramulosa (Greene) M.A. Lane BCN, BCS

Gutierrezia sarothrae (Pursh) Britton \& Rusby BCN, BCS, CHIH, COAH, DGO, HGO, NLE, SLP, SON, ZAC

*Gutierrezia sericocarpa (A. Gray) M.A. Lane AGS, CHIH, COAH, DGO, GTO, HGO, JAL, NAY, QRO, SLP, SON, TAMS, ZAC

Gutierrezia sphaerocephala A. Gray CHIH, COAH, DGO, HGO, NLE, QRO, SLP, TAMS, ZAC

Gutierrezia texana (DC.) Torr. \& A. Gray COAH, DGO, GTO, HGO, JAL, NLE, QRO, SLP, TAMS

Gutierrezia wrightii A. Gray CHIH, DGO, SIN, SON

Gymnocoronis latifolia Hook. \& Arn. CAM, CHIS, NAY, TAB, TAMS, VER

* Gymnolaena chiapasana Strother CHIS, OAX

* Gymnolaena oaxacana (Greenm.) Rydb. OAX, PUE

* Gymnolaena serratifolia (DC.) Rydb. OAX

Gymnosperma glutinosum (Spreng.) Less. AGS, CHIS, CHIH, COAH, CDMX, DGO, GTO, GRO, HGO, JAL, MEX, MOR, NLE, OAX, PUE, QRO, SLP, SON, TAMS, TLAX, VER, ZAC *Haploesthes fruticosa B.L. Turner COAH, NLE

Haploesthes greggii A. Gray CHIH, COAH, NLE, SLP, TAMS

*Haploesthes hintoniana B.L. Turner COAH

*Haploesthes robusta I.M. Johnst. COAH

Harleya oxylepis (Benth.) S.F. Blake CAM, CHIS, QROO, TAB,
YUC

*Hazardia berberidis (A. Gray) Greene BCN

Hazardia cana (A. Gray) Greene BCN

*Hazardia enormidens (Moran) W.D. Clark BCN

*Hazardia ferrisiae (S.F. Blake) W.D. Clark BCN

* Hazardia odontolepis (Moran) W.D. Clark BCN

Hazardia orcuttii (A. Gray) Greene BCN, BCS

*Hazardia rosarica (Moran) W.D. Clark BCN

Hazardia squarrosa (Hook. \& Arn.) Greene BCN

*Hazardia vernicosa (Brandegee) W.D. Clark BCN

Hebeclinium macrophyllum (L.) DC. CAM, CHIS, GRO,

MICH, MOR, OAX, PUE, QROO, SLP, TAB, VER

Hedosyne ambrosiifolia (A. Gray) Strother $\mathrm{CHIH}, \mathrm{COAH}$, DGO, GTO, HGO, JAL, NAY, NLE, QRO, SLP, SON, ZAC

Helenium amarum (Raf.) H. Rock COAH, NLE, TAMS

*Helenium apterum (S.F. Blake) Bierner DGO, NAY

*Helenium chihuahuensis Bierner CHIH, SON

Helenium elegans DC. COAH, GTO, NLE, QRO, SLP, TAMS, ZAC

Helenium laciniatum A. Gray SIN, SON

Helenium mexicanum Kunth AGS, CAM, CHIS, CHIH, COAH, CDMX, DGO, GTO, GRO, HGO, JAL, MEX, MICH, NLE, OAX, PUE, QRO, SLP, SON, TAMS, TLAX, VER, YUC, ZAC Helenium microcephalum DC. CHIH, COAH, DGO, GTO, NLE, QRO, SLP, TAMS, ZAC

Helenium puberulum DC. BCN

Helenium quadridentatum Labill. CHIH, COAH, DGO, GTO, GRO, HGO, JAL, MEX, MICH, NLE, PUE, QRO, SLP, TAB, TAMS, VER, ZAC

Helenium scorzonerifolium (DC.) A. Gray CHIS, COL, CDMX, JAL, MEX, MICH, MOR

Helenium thurberi A. Gray BCN, BCS, CHIH, NAY, SIN, SON

*Helianthella ciliata S.F. Blake CHIH, ZAC

*Helianthella durangensis B.L. Turner DGO, SIN

*Helianthella gypsophila B.L. Turner COAH, NLE, TAMS

*Helianthella mexicana A. Gray COAH, GTO, JAL, NLE, OAX, SLP, TAMS, ZAC

Helianthella quinquenervis (Hook.) A. Gray $\mathrm{CHIH}, \mathrm{COAH}$, NLE, SON

Helianthus annuus L. AGS, BCN, BCS, CAM, CHIS, CHIH, COAH, COL, CDMX, DGO, GTO, GRO, HGO, JAL, MEX, MICH, MOR, NAY, NLE, OAX, PUE, QRO, SLP, SIN, SON, TAB, TAMS, VER, YUC, ZAC

Helianthus californicus DC. BCN

Helianthus ciliaris DC. CHIH, COAH, DGO, NLE, SLP, SON, TAMS, ZAC

Helianthus gracilentus A. Gray BCN

Helianthus hirsutus Raf. COAH, NLE

Helianthus laciniatus A. Gray AGS, CHIH, COAH, CDMX, DGO, GTO, HGO, JAL, MEX, MICH, NLE, QRO, SLP, SON, TAMS, ZAC

Helianthus maximilianii Schrad. CHIH, COAH, NLE

Helianthus niveus (Benth.) Brandegee BCN, BCS, CHIH, SON Helianthus petiolaris Nutt. CHIH, COAH, DGO, SON, TAMS Heliomeris annua (M.E. Jones) Cockerell CHIH, COAH, DGO, SLP, SON

Heliomeris hispida (A. Gray) Cockerell CHIH, DGO, SIN, SON 
Heliomeris multiflora Nutt. CHIS, CHIH, COAH, DGO, GTO, HGO, JAL, MICH, MOR, NAY, NLE, QRO, SLP, SIN, SON, TAMS, ZAC

*Heliomeris obscura (S.F. Blake) Cockerell OAX, PUE, VER

*Heliomeris serboana B.L. Turner OAX

*Heliopsis anпиа Hemsl. AGS, CHIH, COAH, DGO, GTO, GRO, HGO, JAL, MEX, MICH, MOR, NLE, OAX, PUE, QRO, SLP, SON, ZAC

*Heliopsis anomala (M.E. Jones) B.L. Turner BCN, BCS, SON Heliopsis buphthalmoides (Jacq.) Dunal CHIS, COL, GTO, GRO, HGO, JAL, MEX, MICH, OAX, PUE, QRO, SLP, SIN, TAMS, VER, ZAC

*Heliopsis filifolia $\mathrm{S}$. Watson COAH

*Heliopsis longipes (A. Gray) S.F. Blake DGO, GTO, HGO, QRO, SLP, TAMS, ZAC

*Heliopsis novogaliciana B.L. Turner DGO, JAL, NAY, SIN, ZAC

*Heliopsis parviceps S.F. Blake GRO, JAL, MEX, MICH, NAY, SIN

Heliopsis parvifolia A. Gray CHIH, COAH, DGO, GTO, NLE, QRO, SLP, SON, TAMS

*Heliopsis procumbens Hemsl. COL, CDMX, DGO, GRO, HGO, JAL, MEX, MICH, MOR, NAY, QRO, SIN, ZAC

*Heliopsis sinaloensis B.L. Turner SIN

*Heliopsis suffruticosa Ramírez-Noya \& S. González ZAC

*Henricksonia mexicana B.L. Turner COAH, DGO

Heterosperma pinnatum Cav. AGS, BCS, CHIS, CHIH, COAH, COL, CDMX, DGO, GTO, GRO, HGO, JAL, MEX, MICH, MOR, NAY, NLE, OAX, PUE, QRO, SLP, SIN, SON, TAMS, VER, ZAC

*Heterotheca chihuahuana (B.L. Turner \& Sundberg) B.L. Turner CHIH, DGO, NAY, SIN

Heterotheca fulcrata (Greene) Shinners CHIH, COAH, NLE, SON, TAMS, ZAC

*Heterotheca gypsophila B.L. Turner NLE, TAMS

*Heterotheca inuloides Cass. AGS, BCN, CHIH, COAH, COL, CDMX, DGO, GTO, GRO, HGO, JAL, MEX, MICH, MOR, NAY, NLE, OAX, PUE, QRO, SLP, SON, TAMS, TLAX, VER, ZAC

*Heterotheca leptoglossa DC. AGS, GTO, JAL, MEX, MICH, QRO, SLP, ZAC

*Heterotheca martirensis Moran BCN

*Heterotheca mexicana V.L. Harms DGO, SIN

*Heterotheca mucronata V.L. Harms COAH, NLE, SLP, TAMS, ZAC

Heterotheca sessiliflora (Nutt.) Shinners BCN

Heterotheca subaxillaris (Lam.) Britton \& Rusby AGS, CHIH, COAH, DGO, JAL, MICH, NLE, SLP, SIN, SON, TAMS, VER, ZAC

*Heterotheca thiniicola (Rzed. \& E. Ezcurra) B.L. Turner SON

*Hidalgoa pentamera Sherff PUE, VER

Hidalgoa ternata La Llave CHIS, OAX, TAB, VER

*Hidalgoa uspanapa B.L. Turner CHIS, OAX, VER

Hieracium abscissum Less. AGS, CHIS, CHIH, COAH, COL, CDMX, DGO, GTO, GRO, HGO, JAL, MEX, MICH, MOR, NAY, NLE, OAX, PUE, QRO, SLP, SIN, SON, TAMS, TLAX, VER, ZAC
Hieracium albiflorum Hook. BCN, CHIH, SON

*Hieracium beamanii B.L. Turner HGO, PUE, VER

Hieracium bolanderi A. Gray BCN

Hieracium carneum Greene CHIH, COAH, SLP, SON

Hieracium crepidispermum Fr. AGS, CHIH, COAH, CDMX, DGO, GTO, HGO, JAL, MEX, MICH, MOR, NAY, NLE, OAX, PUE, QRO, SLP, SIN, SON, TAMS, TLAX, VER, ZAC

*Hieracium dysonymum S.F. Blake COAH, CDMX, GTO, GRO, HGO, MEX, MICH, NLE, OAX, PUE, QRO, SLP, TAMS, TLAX, VER, ZAC

Hieracium fendleri Sch. Bip. BCN, BCS, CHIH, COL, DGO, GTO, JAL, MEX, MICH, NAY, NLE, QRO, SIN, SON, VER, ZAC

Hieracium gronovii L. CHIS, COAH, NLE, OAX

*Hieracium gypsophilum B.L. Turner COAH, NLE, TAMS

*Hieracium herrerae S.F. Blake DGO, SIN

*Hieracium hintonii Beaman GRO, MEX, MICH

Hieracium irasuense Benth. CHIS

*Hieracium macdonaldii Beaman \& B.L. Turner OAX

Hieracium mexicanum Less. CHIS, COAH, CDMX, DGO, GRO, HGO, MEX, MICH, MOR, NLE, OAX, PUE, QRO, TLAX, VER

Hieracium pringlei A. Gray AGS, CHIS, CHIH, COAH, COL, CDMX, DGO, GTO, HGO, JAL, MEX, MICH, NAY, NLE, OAX, PUE, QRO, SLP, SIN, SON, TAMS, VER, ZAC

Hieracium schultzii Fr. BCS, CHIH, CHIS, COAH, CDMX, DGO, GTO, HGO, JAL, MEX, MICH, NAY, NLE, OAX, PUE, QRO, SLP, SIN, TAMS, TLAX, VER, ZAC

*Hofmeisteria anomalochaeta (R.M. King) B.L. Turner BCS

*Hofmeisteria crassifolia S. Watson BCS, SON

*Hofmeisteria dissecta (Hook. \& Arn.) R.M. King \& H. Rob. COL, GRO, JAL, MEX, MICH, NAY, SIN

*Hofmeisteria fasciculata (Benth.) Walp. BCN, BCS, SON

*Hofmeisteria filifolia I.M. Johnst. BCN

*Hofmeisteria gayleana B.L. Turner DGO, SIN

*Hofmeisteria malvifolia (B.L. Rob. \& Greenm.) B.L. Turner OAX, PUE

*Hofmeisteria mexiae (B.L. Rob.) B.L. Turner JAL, NAY

*Hofmeisteria schaffneri (A. Gray) R.M. King \& H. Rob. COL, DGO, GTO, HGO, JAL, MEX, MICH, QRO, SLP, ZAC

*Hofmeisteria sinaloensis Gentry SIN

*Hofmeisteria standleyi (S.F. Blake) R.M. King \& H. Rob. DGO, NAY, SIN, SON

*Hofmeisteria urenifolia (Hook. \& Arn.) Walp. CHIS, COL, DGO, GRO, JAL, MICH, NAY, OAX, SIN, SON, ZAC

Holocarpha virgata (A. Gray) D.D. Keck BCN

Hulsea californica Torr. \& A. Gray BCN

Hulsea mexicana Rydb. BCN

Hulsea vestita A. Gray BCN

*Hybridella anthemidifolia (B.L. Rob. \& Greenm.) Olsen JAL

*Hybridella globosa (Ortega) Cass. AGS, CDMX, DGO, GTO, HGO, JAL, MEX, PUE, QRO, SLP, TLAX, VER, ZAC

*Hydropectis aquatica (S. Watson) Rydb. CHIH, DGO, SIN

*Hydropectis estradii B.L. Turner CHIH, SON

*Hydropectis stevensii McVaugh AGS, GTO, JAL

Hymenopappus artemisiifolius DC. TAMS

Hymenopappus filifolius Hook. BCN 
Hymenopappus flavescens A. Gray CHIH

*Hymenopappus flavomarginatus I.M. Johnst. COAH, NLE, SLP, TAMS, ZAC

*Hymenopappus hintoniorum B.L. Turner COAH, NLE

Hymenopappus mexicanus A. Gray CHIH, SLP, SON, TAMS, $\mathrm{ZAC}$

Hymenopappus scabiosaeus L'Hér. COAH

*Hymenostephium brandegei (S.F. Blake) E.E. Schill. \& Panero PUE

Hymenostephium cordatum (Hook. \& Arn.) S.F. Blake CHIS, COL, CDMX, DGO, GRO, HGO, JAL, MEX, MICH, MOR, NAY, NLE, OAX, PUE, QRO, SLP, SIN, TAMS, VER, ZAC Hymenostephium gracillimum (Brandegee) E.E. Schill. \& Panero GRO, OAX

Hymenostephium guatemalense (B.L. Rob. \& Greenm.) S.F. Blake CHIS, GRO

*Hymenostephium hintonii (H. Rob.) E.E. Schill. \& Panero GRO, MICH

*Hymenostephium kingii (McVaugh) E.E. Schill. \& Panero JAL, NAY

*Hymenostephium superaxillare S.F. Blake CHIH, DGO

Hymenostephium tenuis (A. Gray) E.E. Schill. \& Panero CHIS, COL, DGO, GRO, JAL, MEX, MICH, MOR, NAY, OAX, SLP, SIN, ZAC

*Hymenostephium uniseriatum E.E. Schill. \& Panero GRO, MEX, MICH, MOR, NAY, OAX, PUE, VER

*Hymenostephium websteri (B.L. Turner) E.E. Schill. \& Panero COL, GRO, JAL, MICH, NAY

*Hymenostephium woronowii (S.F. Blake) E.E. Schill. \& Panero $\mathrm{MICH}$

*Hymenothrix greenmanii (Heiser) B.L. Turner GRO, MEX

*Hymenothrix palmeri A. Gray CHIH, DGO, SON

Hymenothrix wislizenii A. Gray CHIH, DGO, SON

Hymenothrix wrightii A. Gray BCN, CHIH, SON

*Hymenoxys chrysanthemoides (Kunth) DC. COAH, HGO, MEX, NLE, OAX, PUE, SLP, TLAX, VER, ZAC

*Hymenoxys insignis (A. Gray ex S. Watson) Cockerell CHIH, COAH, NLE, SLP

Hymenoxys integrifolia (Kunth) Bierner CHIS, CDMX, DGO, GRO, HGO, MEX, MOR, PUE, TLAX, VER

Hymenoxys odorata DC. BCN, BCS, CHIH, COAH, NLE, SLP, SON, TAMS, ZAC

*Hymenoxys pinetorum (Standl.) Bierner COAH, NLE

*Iostephane heterophylla (Cav.) Hemsl. AGS, CHIH, COL, CDMX, DGO, GTO, GRO, HGO, JAL, MEX, MICH, MOR, NAY, NLE, OAX, PUE, QRO, SLP, SIN, SON, TAMS, TLAX, VER, ZAC

*Iostephane madrensis (S. Watson) Strother AGS, CHIH, DGO, GTO, JAL, NAY, SLP, SIN, SON, ZAC

*Iostephane trilobata Hemsl. CHIS, GRO, MEX, MICH, OAX, PUE

Isocarpha atriplicifolia (L.) R. Br. ex DC. CHIS, GRO

Isocarpha oppositifolia (L.) Cass. CAM, CHIS, COL, GRO, JAL, MICH, NLE, OAX, QRO, QROO, SLP, TAMS, VER, YUC

Isocoma acradenia (Greene) Greene BCN, BCS, SON

Isocoma coronopifolia (A. Gray) Greene CHIH, COAH, NLE,
SON, TAMS

Isocoma drummondii (Torr. \& A. Gray) Greene COAH, NLE, SLP, TAMS

*Isocoma felgeri G.L. Nesom SON

*Isocoma gypsophila B.L. Turner AGS, COAH, NLE, ZAC

*Isocoma hartwegii (A. Gray) Greene AGS, GTO, HGO, JAL, SLP, TLAX, VER, ZAC

Isocoma menziesii (Hook. \& Arn.) G.L. Nesom BCN, BCS

Isocoma pluriflora (Torr. \& A. Gray) Greene BCN, CHIH, COAH, DGO, SON

*Isocoma tehuacana G.L. Nesom PUE Isocoma tenuisecta Greene $\mathrm{CHIH}, \mathrm{SON}$

*Isocoma tomentosa G.L. Nesom CHIH

* Isocoma veneta (Kunth) Greene AGS, BCN, COAH, CDMX, GTO, GRO, HGO, JAL, MEX, MOR, NLE, OAX, PUE, QRO, SLP, TAMS, TLAX, VER, ZAC

Iva annua L. SLP, TAMS, VER

Iva asperifolia Less. PUE, TAMS, VER

Iva hayesiana A. Gray BCN, BCS

*Jaegeria bellidiflora (Sessé \& Moc. ex DC.) A.M. Torres \& Beaman CDMX, HGO, JAL, MEX, MICH, MOR, PUE, QRO, SLP, TLAX, VER, ZAC

*Jaegeria glabra (S. Watson) B.L. Rob. AGS, CHIH, CDMX, DGO, GTO, HGO, JAL, MEX, MICH, QRO, SLP, TLAX, VER, ZAC

Jaegeria hirta (Lag.) Less. AGS, CHIS, CHIH, COL, CDMX, DGO, GTO, GRO, HGO, JAL, MEX, MICH, MOR, NAY, OAX, PUE, QRO, SLP, SIN, SON, TAMS, TLAX, VER, ZAC

*Jaegeria macrocephala Less. COL, DGO, GRO, JAL, MEX, MICH, NAY, OAX, PUE, VER

*Jaegeria pedunculata Hook. \& Arn. GTO, GRO, HGO, JAL, MEX, MICH, NAY

*Jaegeria purpurascens B.L. Rob. DGO, GTO, JAL, MICH, QRO, SIN

Jaegeria standleyi (Steyerm.) B.L. Turner CHIS

*Jaegeria sterilis McVaugh COL, JAL

*Jaliscoa goldmanii (B.L. Rob.) R.M. King \& H. Rob. CHIH, DGO, JAL

*Jaliscoa paleacea (Cronquist) R.M. King \& H. Rob. COL, JAL

*Jaliscoa pringlei $\mathrm{S}$. Watson COL, GRO, JAL, MEX, MICH, MOR

Jaumea carnosa (Less.) A. Gray BCN, BCS

Jefea brevifolia (A. Gray) Strother AGS, CHIH, COAH, DGO, NLE, SLP, TAMS, ZAC

*Jefea gnaphalioides (A. Gray) Strother COAH, HGO, NLE, SLP, TAMS, VER

* Jefea lantanifolia (S. Schauer) Strother GTO, HGO, NLE, QRO, SLP, TAMS, VER, ZAC

*Jefea pringlei (Greenm.) Strother OAX, PUE

Jungia ferruginea L. f. CHIS

*Jungia pringlei Greenm. GRO, JAL, MICH

Koanophyllon albicaule (Sch. Bip. ex Klatt) R.M. King \& H. Rob. CAM, CHIS, COL, GRO, HGO, JAL, MEX, MICH, MOR, NAY, OAX, PUE, QRO, QROO, SLP, SIN, TAB, TAMS, VER, YUC, ZAC

*Koanophyllon coixtlahuacum B.L. Turner OAX 
*Koanophyllon concordianum B.L. Turner DGO, SIN Koanophyllon coulteri (B.L. Rob.) R.M. King \& H. Rob. CHIS *Koanophyllon galeana (B.L. Turner) B.L. Turner NLE, TAMS *Koanophyllon gracilicaule (Sch. Bip. ex B.L. Rob.) R.M. King \& H. Rob. GRO, MEX, MICH, OAX, PUE, SLP, VER

*Koanophyllon hintoniorum (B.L. Turner) Velazco NLE, TAMS *Koanophyllon longifolium (B.L. Rob.) R.M. King \& H. Rob. COAH, GTO, HGO, NLE, QRO, SLP, TAMS, VER

* Koanophyllon monanthum (Sch. Bip.) T.J. Ayers \& B.L. Turner CHIH, COL, DGO, GRO, JAL, MEX, MICH, NAY, OAX, SIN, SON

*Koanophyllon palmeri (A. Gray) R.M. King \& H. Rob. AGS, CHIH, COL, DGO, GRO, JAL, MEX, MICH, NAY, SIN, SON, ZAC

*Koanophyllon peninsulare (Brandegee) B.L. Turner BCS

Koanophyllon pittieri (Klatt) R.M. King \& H. Rob. CHIS, OAX, QROO, TAB, VER

*Koanophyllon pochutlana B.L. Turner OAX

*Koanophyllon pseudoperfoliatum (Sch. Bip. ex Klatt) R.M. King \& H. Rob. VER

*Koanophyllon ravenii R.M. King \& H. Rob. CHIS

*Koanophyllon revealii B.L. Turner GRO, OAX

*Koanophyllon reyrobinsonii B.L. Turner COAH, NLE, TAMS

*Koanophyllon richardsonii B.L. Turner HGO, NLE, QRO, SLP, TAMS, ZAC

*Koanophyllon rzedowskii B.L. Turner GTO, SLP

*Koanophyllon sinaloense B.L. Turner CHIH, COL, JAL, SIN, SON

Koanophyllon solidaginifolium (A. Gray) R.M. King \& H. Rob. AGS, CHIH, COAH, COL, DGO, GRO, HGO, JAL, MICH, NAY, NLE, SIN, SON, VER, ZAC

Koanophyllon solidaginoides (Kunth) R.M. King \& H. Rob. CHIS, GRO, HGO, OAX, SLP, TAB, VER

* Koanophyllon tripartitum B.L. Turner CHIS

Krigia cespitosa (Raf.) K.L. Chambers TAMS

*Kyrsteniopsis cymulifera (B.L. Rob.) R.M. King \& H. Rob. GTO, SLP

* Kyrsteniopsis chiapasana B.L. Turner CHIS

*Kyrsteniopsis dibollii R.M. King \& H. Rob. GRO, OAX, PUE, VER

*Kyrsteniopsis eriocarpa (B.L. Rob. \& Greenm.) B.L. Turner OAX, PUE

*Kyrsteniopsis heathiae (B.L. Turner) B.L. Turner CHIS

Kyrsteniopsis iltisii (R.M. King \& H. Rob.) B.L. Turner CHIS

*Kyrsteniopsis nelsonii (B.L. Rob.) R.M. King \& H. Rob. CHIS, COL, DGO, GTO, GRO, JAL, MEX, MICH, NAY, OAX, QRO, SLP, ZAC

Kyrsteniopsis perpetiolata (R.M. King \& H. Rob.) B.L. Turner CHIS

*Kyrsteniopsis spinaciifolia (DC.) B.L. Turner GTO, HGO, QRO, SLP, TAMS, VER, ZAC

*Lactuca brachyrrhyncha Greenm. MEX, MICH

Lactuca graminifolia Michx. CHIS, CHIH, COAH, GTO, HGO, MICH, NLE, OAX, PUE, QRO, SLP, SON, TAMS, VER

Lactuca intybacea Jacq. CAM, CHIS, COL, GRO, JAL, MICH, MOR, NAY, OAX, PUE, QROO, SLP, SIN, TAMS, VER, YUC Lactuca ludoviciana (Nutt.) DC. COAH, NLE, SLP, TAMS
Lactuca pulchella (Pursh) DC. CHIH, COAH, SON

Laennecia confusa (Cronquist) G.L. Nesom AGS, CHIS, CHIH, COL, DGO, GTO, GRO, HGO, JAL, MEX, MICH, NAY, OAX, QRO, SLP, SIN, SON, TLAX, ZAC

Laennecia coulteri (A. Gray) G.L. Nesom BCN, BCS, CHIH, COAH, COL, DGO, GTO, HGO, JAL, NLE, SLP, SON, TAMS, VER, ZAC

*Laennecia chihuahuana G.L. Nesom CHIH, SON

Laennecia eriophylla (A. Gray) G.L. Nesom CHIH, SON

Laennecia filaginoides DC. AGS, BCS, CHIS, CHIH, CDMX, DGO, GTO, GRO, HGO, JAL, MEX, MICH, MOR, NLE, OAX, PUE, QRO, SLP, SIN, SON, TLAX, VER, ZAC

Laennecia gnaphalioides (Kunth) Cass. AGS, CHIH, COAH, CDMX, DGO, GTO, HGO, JAL, MEX, MICH, NAY, NLE, OAX, QRO, SLP, TLAX, VER, ZAC

* Laennecia mapimiana G.L. Nesom CHIH, DGO

*Laennecia microglossa (S.F. Blake) G.L. Nesom AGS, BCS, CHIH, COAH, DGO, GTO, JAL, NLE, QRO, SLP, ZAC

*Laennecia pimana G.L. Nesom \& Laferr. CHIH, SON

Laennecia schiedeana (Less.) G.L. Nesom BCN, BCS, CHIS, CHIH, COAH, CDMX, DGO, GTO, GRO, HGO, JAL, MEX, MICH, MOR, NAY, NLE, OAX, PUE, QRO, SLP, SON, TAMS, TLAX, VER, ZAC

Laennecia sophiifolia (Kunth) G.L. Nesom AGS, BCS, CHIS, CHIH, COAH, COL, CDMX, DGO, GTO, GRO, HGO, JAL, MEX, MICH, NAY, NLE, OAX, PUE, QRO, SLP, SIN, SON, TAMS, TLAX, VER, ZAC

*Laennecia spellenbergii G.L. Nesom CHIH, DGO, SON

*Lagascea angustifolia DC. DGO, GTO, JAL, NAY, SLP, SIN, ZAC

*Lagascea aurea Stuessy COL, GTO, GRO, JAL, MICH, QRO Lagascea decipiens Hemsl. CHIH, COL, DGO, GRO, JAL, MEX, MICH, NAY, OAX, SIN, SON, ZAC

Lagascea helianthifolia Kunth AGS, CAM, CHIS, CHIH, COL, DGO, GRO, HGO, JAL, MEX, MICH, MOR, NAY, OAX, PUE, QRO, QROO, SLP, SIN, SON, TAMS, VER, ZAC

*Lagascea heteropappus Hemsl. GRO, JAL, MEX, MICH

Lagascea mollis Cav. CAM, CHIS, COL, GRO, JAL, MEX, MICH, MOR, OAX, PUE, QROO, TAB, TAMS, VER, YUC

*Lagascea palmeri (B.L. Rob.) B.L. Rob. COL, GRO, JAL, MICH, NAY

*Lagascea rigida (Cav.) Stuessy CDMX, GRO, HGO, MEX, MICH, MOR, OAX, PUE, QRO, TLAX, VER

*Lasianthaea aurea (D. Don) K.M. Becker AGS, CHIH, COL, CDMX, DGO, GTO, GRO, HGO, JAL, MEX, MICH, OAX, QRO, SLP, TLAX, VER, ZAC

*Lasianthaea beckeri B.L. Turner COL, JAL

*Lasianthaea ceanothifolia (Willd.) K.M. Becker AGS, CHIS, CHIH, COL, DGO, GTO, GRO, HGO, JAL, MEX, MICH, MOR, NAY, OAX, PUE, QRO, SLP, SIN, SON, VER, ZAC

*Lasianthaea crocea (A. Gray) K.M. Becker AGS, GTO, GRO, JAL, MEX, MICH, MOR, OAX, PUE

Lasianthaea fruticosa (L.) K.M. Becker CAM, CHIS, CHIH, COL, DGO, GTO, GRO, HGO, JAL, MEX, MICH, MOR, NAY, OAX, PUE, QRO, QROO, SLP, SIN, SON, TAB, TAMS, VER, YUC, ZAC

*Lasianthaea gentryi B.L. Turner SIN 
*Lasianthaea helianthoides DC. COL, DGO, GRO, JAL, MEX, MICH, MOR, NAY, OAX, PUE, SIN

*Lasianthaea macrocephala (Hook. \& Arn.) K.M. Becker COL, DGO, GRO, JAL, MEX, MICH, MOR, NAY, OAX, SIN

*Lasianthaea machucana B.L. Turner JAL

*Lasianthaea palmeri (Greenm.) K.M. Becker AGS, COL, DGO, GTO, JAL, MICH, NAY, QRO, SIN, ZAC

Lasianthaea podocephala (A. Gray) K.M. Becker CHIH, DGO, SON

*Lasianthaea ritovegana B.L. Turner SIN

*Lasianthaea rosei (Greenm.) McVaugh DGO, JAL, NAY, SIN, ZAC

*Lasianthaea seemannii (A. Gray) K.M. Becker CHIH, DGO, NAY, SIN, SON

*Lasianthaea squarrosa (Greenm.) K.M. Becker GRO, MEX, MOR, PUE

*Lasianthaea zinnioides (Hemsl.) K.M. Becker DGO, JAL, NAY, SIN, ZAC

Lasthenia coronaria (Nutt.) Ornduff BCN

Lasthenia glabrata Lindl. BCN

Lasthenia gracilis (DC.) Greene BCN, SON

Layia glandulosa (Hook.) Hook. \& Arn. BCN

Layia platyglossa (Fisch. \& C.A. Mey.) A. Gray BCN

Leibnitzia lyrata (D. Don) G.L. Nesom AGS, CHIH, COAH, COL, CDMX, DGO, GTO, GRO, HGO, JAL, MEX, MICH, MOR, NAY, NLE, OAX, PUE, QRO, SLP, SIN, SON, TAMS, TLAX, VER, ZAC

*Leibnitzia occimadrensis G.L. Nesom CHIH, DGO, SIN, SON

*Leiboldia guerreroana (S.B. Jones) H. Rob. GRO, OAX

*Leiboldia serrata (D. Don) Gleason HGO, OAX, PUE, QRO, SLP, TAMS, VER

*Lepidaploa boquerona (B.L. Turner) H. Rob. CHIS

Lepidaploa canescens (Kunth) H. Rob. CAM, CHIS, COL, GRO, HGO, JAL, MICH, NAY, OAX, PUE, QRO, QROO, SLP, TAB, VER, YUC, ZAC

*Lepidaploa diazlunana (B.L. Turner) H. Rob. COL, GRO, JAL

*Lepidaploa koelzii (McVaugh) H. Rob. COL, JAL

Lepidaploa polypleura (S.F. Blake) H. Rob. CHIS, OAX

Lepidaploa remotiflora (Rich.) H. Rob. CHIS, TAB

Lepidaploa salzmannii (DC.) H. Rob. CAM, CHIS, GRO, HGO,

OAX, PUE, QRO, QROO, SLP, TAB, VER

Lepidaploa tortuosa (L.) H. Rob. CAM, CHIS, HGO, OAX, PUE, QRO, SLP, TAB, TAMS, VER

Lepidaploa uniflora (Mill.) H. Rob. CAM, CHIS, QROO, TAB, YUC

* Lepidonia alba Redonda-Martínez \& E. Martínez CHIS

*Lepidonia callilepis (Gleason) H. Rob. \& V.A. Funk GRO, $\mathrm{MICH}, \mathrm{OAX}$

*Lepidonia jonesii (B.L. Turner) H. Rob. \& V.A. Funk OAX

*Lepidonia mexicana (Less.) H. Rob. \& V.A. Funk PUE, VER

Lepidonia salvinae (Hemsl.) H. Rob. \& V.A. Funk CHIS

*Lepidonia wendtiana (B.L. Turner) Redonda-Martínez \& Villaseñor OAX, VER

Lepidospartum squamatum (A. Gray) A. Gray BCN, BCS

Lessingia glandulifera A. Gray BCN

*Leucactinia bracteata (S. Watson) Rydb. AGS, COAH, NLE,

SLP, TAMS, ZAC
Leuciva dealbata (A. Gray) Rydb. CHIH, COAH, DGO, GTO, JAL, NLE, SLP, SON, TAMS, ZAC

Leucosyris arida (B.L. Turner \& D.B. Horne) Pruski \& R.L. Hartm. SON

Leucosyris blapharophylla (A. Gray) Pruski \& R.L. Hartm. $\mathrm{CHIH}, \mathrm{COAH}$

Leucosyris carnosa (A. Gray) Greene SON

Leucosyris coulteri (A. Gray) Pruski \& R.L. Hartm. CHIH, SON

*Leucosyris crispa (Brandegee) Pruski \& R.L. Hartm. BCN, BCS, SON

Leucosyris parviflora (A. Gray) Pruski \& R.L. Hartm. CHIH, $\mathrm{COAH}, \mathrm{DGO}, \mathrm{SON}$

Leucosyris riparia (Kunth) Pruski \& R.L. Hartm. CHIH, MEX, COAH, GTO, MICH, SON, ZAC

*Leucosyris turneri (M.L. Arnold \& R.C. Jacks.) Pruski \& R.L. Hartm. CHIH, COAH

*Liabellum angustissimum (A. Gray) Rydb. DGO, GTO, JAL, MEX, MICH, NAY, ZAC

*Liabellum cervinum (B.L. Rob.) Rydb. COL, JAL, NAY, SIN

*Liabellum gentryi $\mathrm{H}$. Rob. JAL, NAY

*Liabellum hintoniorum (B.L. Turner) H. Rob. MEX

*Liabellum palmeri A. Gray AGS, CHIH, DGO, JAL, MEX, NAY, SIN, ZAC

Liabum bourgeaui Hieron. CHIS, OAX, VER

Liatris punctata Hook. CHIH, COAH, NLE, SLP, TAMS

Lindheimera texana A. Gray \& Engelm. $\mathrm{COAH}$

Logfia arizonica (A. Gray) Holub BCN, BCS, SON

Logfia depressa (A. Gray) Holub BCN, BCS, SON

Logfia filaginoides (Hook. \& Arn.) Morefield BCN, BCS, SON Lorandersonia baileyi (Wooton \& Standl.) Urbatsch, R.P. Roberts \& Neubig COAH, NLE, SLP, TAMS

Lorandersonia pulchella (A. Gray) Urbatsch, R.P. Roberts \& Neubig CHIH, COAH

*Loxothysanus pedunculatus Rydb. HGO, NLE, QRO, SLP, TAMS, VER

*Loxothysanus sinuatus (Less.) B.L. Rob. CHIS, HGO, OAX, PUE, QRO, SLP, TAMS, VER

*Lundellianthus breedlovei (B.L. Turner) Strother CHIS

Lundellianthus guatemalensis (Donn. Sm.) Strother CAM, CHIS, QROO

*Lundellianthus jaliscensis (McVaugh) Strother COL, JAL

Lundellianthus salvinii (Hemsl.) Strother CHIS

Lygodesmia ramosissima Greenm. CHIH, COAH, DGO, NLE, SLP, SON

Lygodesmia texana (Torr. \& A. Gray) Greene ex Small CHIH, $\mathrm{COAH}, \mathrm{DGO}$

Macvaughiella chiapensis R.M. King \& H. Rob. CHIS, OAX

*Macvaughiella mexicana (Sch. Bip.) R.M. King \& H. Rob. CHIS, OAX, VER

Machaeranthera tagetina Greene CHIH, SON

Machaeranthera tanacetifolia (Kunth) Nees AGS, CHIH, COAH, DGO, GTO, JAL, NLE, QRO, SLP, SIN, SON, TAMS, ZAC

Madia elegans D. Don ex Lindl. BCN

Madia exigua (Sm.) A. Gray BCN

Madia gracilis (Sm.) D.D. Keck BCN 
Malacothrix californica DC. BCN, BCS, SON

Malacothrix clevelandii A. Gray BCN, SON

Malacothrix coulteri Harv. \& A. Gray BCN

Malacothrix fendleri A. Gray CHIH, SON

Malacothrix glabrata (A. Gray ex D.C. Eaton) A. Gray BCN,

BCS, SON

* Malacothrix insularis Greene BCN

Malacothrix similis W.S. Davis \& P.H. Raven BCN

Malacothrix sonorae W.S. Davis \& P.H. Raven SON

Malacothrix stebbinsii W.S. Davis \& P.H. Raven SON

* Malacothrix xanti A. Gray BCS

Malperia tenuis $\mathrm{S}$. Watson BCN, BCS, SON

* Marshalljohnstonia gypsophila Henr. COAH

* Medranoa parrasana (S.F. Blake) Urbatsch \& R.P. Roberts COAH, DGO, ZAC

Megaliabum andrieuxii (DC.) Rydb. CHIS, MOR, OAX, PUE, VER

*Megaliabum pringlei (B.L. Rob. \& Greenm.) Rydb. DGO, JAL, NAY, MICH, SIN, ZAC

Melampodium americanum L. CAM, CHIS, COL, DGO, GRO, JAL, MEX, MICH, NAY, OAX, QRO, SLP, SIN, TAMS, VER, YUC, ZAC

Melampodium appendiculatum B.L. Rob. CHIH, DGO, NAY, SIN, SON

*Melampodium argophyllum (A. Gray ex B.L. Rob.) S.F. Blake COAH, DGO, NLE

*Melampodium aureum Brandegee MEX, MICH, NLE, OAX, PUE, TAMS

Melampodium bibracteatum S. Watson CHIH, DGO, GTO, HGO, MEX, MICH, PUE, QRO, SIN, TLAX, VER

Melampodium cinereum DC. CHIH, COAH, GTO, NLE, SLP, SON, TAMS

*Melampodium cupulatum A. Gray BCS, CHIH, DGO, JAL, MICH, NAY, SIN, SON, ZAC

*Melampodium dicoelocarpum B.L. Rob. COL, GRO, JAL, MEX, MICH

Melampodium diffusum Cass. CHIS, COL, GRO, MEX, MICH, OAX, VER

Melampodium divaricatum (Rich.) DC. BCS, CAM, CHIS, COAH, COL, CDMX, DGO, GTO, GRO, HGO, JAL, MEX, MICH, MOR, NAY, NLE, OAX, PUE, QRO, QROO, SLP, SIN, SON, TAB, TAMS, TLAX, VER, YUC, ZAC

*Melampodium ellottianum B.L. Turner JAL

* Melampodium glabribracteatum Stuessy OAX

*Melampodium glabrum S. Watson GTO, GRO, HGO, JAL, MEX, MICH, QRO

Melampodium gracile Less. CAM, CHIS, COL, GRO, HGO, JAL, MEX, MICH, MOR, NAY, OAX, PUE, QRO, QROO, SLP, SIN, TAMS, VER, YUC

Melampodium leucanthum Torr. \& A. Gray CHIH, COAH, SON

Melampodium linearilobum DC. CHIS, COL, DGO, GTO, GRO, JAL, MEX, MICH, MOR, OAX, PUE, SIN, VER

Melampodium longicorne A. Gray CHIH, SIN, SON

* Melampodium longifolium Cerv. ex Cav. CHIH, CDMX, GTO, HGO, JAL, MEX, MICH, NAY, NLE, OAX, PUE, QRO, SLP, TAMS, VER
*Melampodium longipes (A. Gray) B.L. Rob. DGO, JAL, MICH, NAY, SIN

Melampodium longipilum B.L. Rob. CHIS, GTO, GRO, HGO, MICH, OAX, PUE, QRO, SLP, TAMS, VER

*Melampodium mayfieldii B.L. Turner JAL

Melampodium microcephalum Less. AGS, CHIS, COL, DGO, GTO, GRO, HGO, JAL, MEX, MICH, MOR, NAY, OAX, PUE, QRO, SLP, SIN, TAMS, VER

*Melampodium mimulifolium B.L. Rob. OAX

Melampodium moctezumum B.L. Turner SON

Melampodium montanum Benth. CHIS, COL, GTO, GRO, HGO, JAL, MEX, MICH, NLE, OAX, PUE, QRO, SLP, TAMS * Melampodium nayaritense Stuessy DGO, JAL, NAY, SIN

*Melampodium northingtonii B.L. Turner CHIS, OAX

* Melampodium nutans Stuessy COL, GRO, JAL, MICH, OAX Melampodium paniculatum Gardner CHIS, GRO, MEX, MICH, MOR, OAX

Melampodium perfoliatum (Cav.) Kunth AGS, CHIS, CHIH, COL, CDMX, DGO, GTO, GRO, HGO, JAL, MEX, MICH, MOR, NAY, NLE, OAX, PUE, QRO, SLP, SIN, SON, TAMS, TLAX, VER, ZAC

*Melampodium pilosum Stuessy GRO, JAL, MEX, MICH, OAX

*Melampodium pringlei B.L. Rob. OAX, PUE

*Melampodium repens Sessé \& Moc. CDMX, DGO, GTO, GRO, HGO, JAL, MEX, MICH, MOR, OAX, PUE, QRO, SIN, TLAX, VER

*Melampodium rosei B.L. Rob. DGO, JAL, NAY, SIN

Melampodium sericeum Lag. AGS, CHIS, CHIH, COL, CDMX, DGO, GTO, GRO, HGO, JAL, MEX, MICH, MOR, NAY, OAX, PUE, QRO, SLP, SIN, SON, TAMS, VER, ZAC

* Melampodium sinaloense Stuessy DGO, JAL, SIN

* Melampodium sinuatum Brandegee BCS

Melampodium strigosum Stuessy AGS, CHIH, COAH, CDMX, DGO, GTO, HGO, JAL, MEX, MICH, MOR, NAY, OAX, PUE, QRO, SLP, SON, TAMS, TLAX, VER, ZAC

*Melampodium tenellum Hook. \& Arn. COL, DGO, GRO, JAL, MEX, MICH, MOR, NAY, OAX, SIN

*Melampodium tepicense B.L. Rob. COL, JAL, MICH, NAY, SIN

Melanthera angustifolia A. Rich. CAM, CHIS, QROO, TAB, VER, YUC

Melanthera nivea (L.) Small CAM, CHIS, COL, GRO, JAL, MEX, MICH, NAY, OAX, PUE, QRO, QROO, SLP, TAB, TAMS, VER, YUC, ZAC

*Mexerion mexicanum G.L. Nesom CHIH, DGO, SIN

*Mexerion sarmentosum (Klatt) G.L. Nesom CDMX, MEX, MICH, MOR, PUE, TLAX, VER

*Mexianthus mexicanus B.L. Rob. COL, JAL, NAY

Micropus californicus Fisch. \& C.A. Mey. BCN

Microseris douglasii (DC.) Sch. Bip. BCN

Microseris elegans Greene ex A. Gray BCN

*Microspermum debile Benth. CHIS, COL, GRO, JAL, MEX, MICH, OAX

*Microspermum flaccidum Paul G. Wilson GRO, MEX

*Microspermum gonzalezii Rzed. JAL

* Microspermum gracillimum Rzed. JAL 
* Microspermum hintonii Rzed. GRO

*Microspermum michoacanum (R.M. King) B.L. Turner COL, JAL, MICH, NAY

*Microspermum nummulariifolium Lag. GRO, JAL, MEX, $\mathrm{MICH}, \mathrm{OAX}$

*Microspermum tenue Paul G. Wilson GRO, MICH

Mikania amblyolepis B.L. Rob. CHIS

Mikania cordifolia (L.f.) Willd. CAM, CHIS, COL, GRO, HGO, JAL, MEX, MICH, MOR, NAY, OAX, PUE, QRO, QROO, SLP, SIN, TAB, TAMS, VER, YUC, ZAC

Mikania globosa Coult. CHIS

Mikania gonzalezii B.L. Rob. \& Greenm. CHIS, OAX, TAB, VER

Mikania guaco Bonpl. CHIS, OAX, TAMS, VER

Mikania hookeriana DC. CHIS, OAX, VER

Mikania houstoniana (L.) B.L. Rob. CAM, CHIS, OAX, PUE, QROO, TAB, VER

Mikania leiostachya Benth. CHIS, OAX, VER

Mikania micrantha Kunth CAM, CHIS, COL, GRO, HGO, JAL, MICH, MOR, NAY, NLE, OAX, PUE, QRO, QROO, SLP, SIN, TAB, TAMS, VER, YUC

*Mikania neei W.C. Holmes VER

* Mikania pascualii B.L. Turner OAX

* Mikania pooleana W.C. Holmes \& Vodopich OAX

Mikania pterocaula Sch. Bip. ex Klatt CHIS, OAX, VER

Mikania pyramidata Donn. Sm. CHIS, GRO, HGO, OAX, PUE, QRO, SLP, VER

Mikania scandens (L.) Willd. COAH, NLE, PUE, TAMS, VER

*Mikania tehuacanensis W.C. Holmes OAX, PUE

Mikania tonduzii B.L. Rob. CHIS, GRO, OAX, PUE, VER

Mikania vitifolia DC. CAM, CHIS, OAX, VER, YUC

Milleria perfoliata B.L. Turner JAL

Milleria quinqueflora L. AGS, BCS, CAM, CHIS, CHIH, COL, DGO, GTO, GRO, JAL, MEX, MICH, MOR, NAY, OAX, PUE, QRO, QROO, SLP, SIN, SON, VER, YUC, ZAC

Monoptilon bellioides (A. Gray) H.M. Hall BCN, SON

*Montanoa andersonii McVaugh COL, GRO, JAL, MICH, NAY Montanoa atriplicifolia (Pers.) Sch. Bip. CAM, CHIS, QROO, YUC

*Montanoa bipinnatifida (Kunth) K. Koch COL, DGO, GTO, GRO, JAL, MEX, MICH, MOR, NAY, OAX, PUE, SIN

Montanoa echinacea S.F. Blake CHIS, OAX

*Montanoa frutescens (Mairet ex DC.) Hemsl. CHIS, CDMX, DGO, GTO, GRO, HGO, JAL, MEX, MICH, MOR, OAX, PUE, QRO, TLAX

*Montanoa grandiflora Alamán ex DC. CAM, CHIS, COL, CDMX, GTO, GRO, HGO, JAL, MEX, MICH, MOR, OAX, PUE, QRO, QROO, SLP, SIN, TAB, TAMS, VER, YUC, ZAC Montanoa hexagona B.L. Rob. \& Greenm. CHIS

Montanoa hibiscifolia Benth. CHIS, MICH, OAX, VER

*Montanoa imbricata V.A. Funk GRO, MICH

*Montanoa karwinskii DC. CHIS, COL, DGO, GRO, JAL, MEX, MICH, MOR, NAY, OAX, ZAC

*Montanoa laskowskii McVaugh COL, JAL

Montanoa leucantha (Lag.) S.F. Blake AGS, CHIS, CHIH, COL, CDMX, DGO, GTO, GRO, HGO, JAL, MEX, MICH, MOR, NAY, NLE, OAX, PUE, QRO, SLP, SIN, SON, TAMS,
TLAX, ZAC

*Montanoa liebmannii (Sch. Bip.) S.F. Blake OAX

*Montanoa mollissima Brongn. ex Groenl. GTO, HGO, OAX, PUE, QRO, SLP, VER

Montanoa pteropoda S.F. Blake CHIS

*Montanoa revealii $\mathrm{H}$. Rob. GRO, OAX

* Montanoa serboana B.L. Turner OAX

*Montanoa standleyi V.A. Funk CHIS, GRO, OAX

Montanoa tomentosa Cerv. CHIS, CHIH, COL, CDMX, DGO, GTO, GRO, HGO, JAL, MEX, MICH, MOR, NAY, NLE, OAX, PUE, QRO, SLP, SIN, SON, TAMS, TLAX, VER, ZAC

Nelsonianthus epiphyticus H. Rob. \& Brettell CHIS

*Nelsonianthus tapianus (B.L. Turner) C. Jeffrey VER

Neomirandea araliifolia (Less.) R.M. King \& H. Rob. CHIS, COL, GRO, JAL, OAX, PUE, TAB, VER

Neomirandea ovandensis R.M. King \& H. Rob. CHIS

*Neonesomia johnstonii (G.L. Nesom) Urbatsch \& R.P. Roberts GTO, HGO, SLP, VER

Neonesomia palmeri (A. Gray) Urbatsch \& R.P. Roberts COAH, GTO, HGO, NLE, QRO, SLP, TAMS

*Nesomia chiapensis B.L. Turner CHIS

*Neurolaena balsana B.L. Turner GRO

Neurolaena cobanensis Greenm. ex Donn. Sm. CHIS

*Neurolaena fulva B.L. Turner CHIS, OAX

* Neurolaena jannaweissana B.L. Turner OAX

* Neurolaena lamina B.L. Turner OAX, VER

Neurolaena lobata (L.) Cass. CAM, CHIS, GTO, GRO, HGO, NLE, OAX, PUE, QRO, QROO, SLP, TAB, TAMS, VER, YUC *Neurolaena macrocephala Sch. Bip. ex Hemsl. CHIS, OAX, VER

Neurolaena macrophylla Greenm. CHIS, VER

*Neurolaena oaxacana B.L. Turner OAX

* Neurolaena venturana B.L. Turner VER

*Neurolaena wendtii B.L. Turner VER

Nicolletia edwardsii A. Gray CHIH, COAH, DGO, GTO, JAL, NLE, SLP, SON, ZAC

Nicolletia occidentalis A. Gray BCN

* Nicolletia trifida Rydb. BCN, BCS

Onoseris onoseroides (Kunth) B.L. Rob. CHIS, COL, GRO, JAL, MICH, NAY, OAX, TAB

Oreochrysum parryi (A. Gray) Rydb. CHIH, DGO, SON

*Oritrophium durangense G.L. Nesom DGO, SIN

* Oritrophium orizabense G.L. Nesom VER

Orthopappus angustifolius (Sw.) Gleason CHIS, GRO, OAX, QRO, SLP, TAB, VER

*Osbertia bartlettii (S.F. Blake) G.L. Nesom COAH, NLE, SON, TAMS

Osbertia stolonifera (DC.) Greene CHIS, COL, CDMX, GRO, HGO, JAL, MEX, MICH, OAX, TAMS, TLAX, VER

* Oteiza acuminata La Llave CDMX, GTO, HGO, JAL, MEX, MICH, MOR, OAX, PUE, QRO, SLP, ZAC

* Oteiza mixtecana Villaseñor \& Panero OAX

* Oteiza scandens Panero \& Villaseñor OAX

*Otopappus acuminatus S. Watson COL, JAL, MEX, ZAC

Otopappus brevipes B.L. Rob. CHIS

Otopappus curviflorus (R. Br.) Hemsl. CAM, CHIS, OAX, QROO, TAB, VER, YUC 
*Otopappus epaleaceus Hemsl. COL, GRO, JAL, MEX, MICH, MOR, OAX, PUE

Otopappus guatemalensis (Urb.) R.L. Hartm. \& Stuessy CAM, CHIS, QROO, YUC

*Otopappus imbricatus (Sch. Bip.) S.F. Blake GRO, MEX, MOR, OAX, PUE

*Otopappus koelzii McVaugh COL, JAL, MICH

*Otopappus mexicanus (Rzed.) H. Rob. GRO, MICH, OAX

*Otopappus microcephalus S.F. Blake COL, GRO, JAL, MICH, NAY, OAX

*Otopappus robustus Hemsl. VER

Otopappus scaber S.F. Blake CAM, CHIS, QROO, YUC

* Otopappus serboana B.L. Turner OAX

*Otopappus tequilanus (A. Gray) B.L. Rob. COL, DGO, GRO, JAL, MEX, MICH, NAY, SIN, ZAC

Otopappus verbesinoides Benth. CHIS, GRO, OAX, VER

Oxylobus adscendens (Sch. Bip. ex Hemsl.) B.L. Rob. \& Greenm. CHIS, CDMX, GRO, HGO, MEX, MICH, MOR, OAX, PUE, SLP, TAMS, TLAX, VER

Oxylobus arbutifolius (Kunth) A. Gray CDMX, MEX, OAX, PUE, TLAX, VER

Oxylobus glanduliferus (Sch. Bip. ex Hemsl.) A. Gray CHIS, OAX

* Oxylobus juarezensis Rzed. \& Calderón OAX

*Oxylobus oaxacanus S.F. Blake CHIS, OAX

* Oxylobus preecei B.L. Turner OAX, PUE

* Oxylobus subglabrus R.M. King \& H. Rob. OAX

* Oxypappus scaber Benth. COL, DGO, GRO, JAL, MICH, NAY, OAX, SIN, ZAC

Packera actinella (Greene) W.A. Weber \& A. Löve CHIH, DGO, SIN, SON

* Packera bellidifolia (Kunth) W.A. Weber \& A. Löve AGS, CHIH, COAH, COL, CDMX, DGO, GRO, HGO, JAL, MEX, MICH, MOR, NAY, NLE, OAX, PUE, SIN, TAMS, TLAX, VER, ZAC

* Packera candidissima (Greene) W.A. Weber \& A. Löve CHIH, COAH, DGO, NLE, SON

*Packera coahuilensis (Greenm.) C. Jeffrey COAH, DGO, GTO, HGO, NLE, PUE, QRO, SLP, TAMS, VER, ZAC

*Packera hintoniorum (B.L. Turner) C. Jeffrey COAH, NLE, SLP, TAMS

* Packera loratifolia (Greenm.) W.A. Weber \& A. Löve COAH, NLE, SLP, TAMS, ZAC

Packera millelobata (Rydb.) W.A. Weber \& A. Löve CHIH, $\mathrm{COAH}$

*Packera montereyana (S. Watson) C. Jeffrey COAH, NLE

* Packera moranii (T.M. Barkley) C. Jeffrey BCN, BCS

Packera neomexicana (A. Gray) W.A. Weber \& A. Löve CHIH, $\mathrm{COAH}, \mathrm{SON}$

Packera obovata (Muhl. ex Willd.) W.A. Weber \& A. Löve $\mathrm{COAH}$

*Packera quebradensis (Greenm.) W.A. Weber \& A. Löve CHIH, DGO, JAL, SIN

* Packera rosei (Greenm.) W.A. Weber \& A. Löve DGO, JAL, NAY, SIN, ZAC

*Packera sanguisorbae (DC.) C. Jeffrey COL, CDMX, GTO, GRO, HGO, JAL, MEX, MICH, MOR, OAX, PUE, QRO, SLP,
TAMS, TLAX, VER, ZAC

*Packera scalaris (Greene) C. Jeffrey CHIH, COAH, DGO, SIN, SON

Packera tampicana (DC.) C. Jeffrey CHIH, COAH, HGO, NLE, PUE, QRO, SLP, TAMS, VER

* Packera toluccana (DC.) W.A. Weber \& A. Löve AGS, CHIH, COL, CDMX, DGO, GTO, GRO, HGO, JAL, MEX, MICH, MOR, NAY, NLE, OAX, PUE, QRO, SLP, SIN, SON, TAMS, TLAX, VER, ZAC

* Packera umbraculifera (S. Watson) W.A. Weber \& A. Löve CHIH, DGO

*Packera zimapanica (Hemsl.) C.C. Freeman \& T.M. Barkley COAH, HGO, NLE, QRO, SLP, TAMS

Palafoxia arida B.L. Turner \& M.I. Morris BCN, BCS, CHIH, SON

Palafoxia callosa (Nutt.) Torr. \& A. Gray COAH

*Palafoxia lindenii A. Gray VER

Palafoxia linearis (Cav.) Lag. BCN, BCS, SIN, SON

Palafoxia riograndensis Cory CHIH, COAH, DGO

Palafoxia sphacelata (Nutt. ex Torr.) Cory CHIH, COAH, DGO, SON

Palafoxia texana DC. CHIH, COAH, NLE, PUE, SLP, TAMS, VER

*Paneroa stachyofolia (B.L. Rob.) E.E. Schill. OAX

Parthenice mollis A. Gray BCS, CHIH, SIN, SON

Parthenium argentatum A. Gray AGS, CHIH, COAH, DGO, GTO, HGO, JAL, NLE, QRO, SLP, TAMS, ZAC

Parthenium bipinnatifidum (Ortega) Rollins AGS, CHIS, CHIH, COAH, CDMX, DGO, GTO, GRO, HGO, JAL, MEX, MICH, MOR, NAY, NLE, OAX, PUE, QRO, SLP, SIN, TAMS, TLAX, VER, ZAC

Parthenium confertum A. Gray BCS, CHIH, COAH, DGO, HGO, NLE, QRO, SLP, TAMS, VER, ZAC

*Parthenium densipilum S.F. Blake TAMS

* Parthenium fruticosum Less. CHIS, COAH, HGO, NLE, QRO, SLP, TAMS, VER

Parthenium hysterophorus L. AGS, CAM, CHIS, CHIH, COAH, COL, CDMX, DGO, GTO, GRO, HGO, JAL, MEX, MICH, MOR, NAY, NLE, OAX, PUE, QRO, QROO, SLP, SIN, SON, TAB, TAMS, TLAX, VER, YUC, ZAC

Parthenium incanum Kunth AGS, CHIH, COAH, DGO, GTO, HGO, JAL, MEX, MICH, NLE, PUE, QRO, SLP, SON, TAMS, VER, ZAC

* Parthenium lozanoanum Bartlett COAH, NLE, TAMS

*Parthenium rollinsianum Rzed. GTO, JAL, SLP, ZAC

*Parthenium schottii Greenm. YUC

*Parthenium tomentosum DC. CHIH, DGO, JAL, NAY, OAX, PUE, SON, ZAC

*Pectis amplifolia D.J. Keil OAX

Pectis angustifolia Torr. AGS, CHIH, COAH, DGO, NLE, SLP, SON, TAMS, VER, ZAC

*Pectis barberi Greenm. CHIH, SON

*Pectis berlandieri DC. SLP, TAMS, VER

Pectis bonplandiana Kunth CHIS, GRO, HGO, OAX, QRO, SLP, TAB, TAMS, VER

Pectis brachycephala Urb. CHIS, OAX

*Pectis canescens Kunth GRO, OAX 
Pectis coulteri Harv. \& A. Gray BCS, SIN, SON

Pectis cylindrica (Fernald) Rydb. BCN, BCS, CHIH, COAH, DGO, NLE, SIN, SON, TAMS

*Pectis decemcarinata McVaugh GRO, JAL, MICH

*Pectis depressa Fernald GRO

*Pectis diffusa Hook. \& Arn. COL, GRO, JAL, MEX, MICH, NAY, SIN

Pectis elongata Kunth CHIS, GRO, OAX, QROO, SLP, TAMS, VER, YUC

*Pectis exilis D.J. Keil GRO, JAL, MEX, MICH, MOR, OAX, PUE

* Pectis exserta McVaugh COL, JAL

Pectis filipes Harv. \& A. Gray CHIH, COAH, DGO, SON

*Pectis haenkeana (DC.) Sch. Bip. GRO, MEX, MICH, MOR, OAX, PUE

*Pectis holochaeta (S.F. Blake) D.J. Keil GRO, MEX, MICH

Pectis imberbis A. Gray CHIH, SON

*Pectis incisifolia I.M. Johnst. CHIH, COAH, DGO

*Pectis latisquama Sch. Bip. ex Greenm. CDMX, MEX, MICH, PUE, QRO, VER

*Pectis leavenworthii Standl. JAL, MEX, MICH, SIN

*Pectis liebmannii Sch. Bip. ex Hemsl. GRO, JAL, OAX

Pectis linearis La Llave CAM, CHIS, OAX, VER, YUC

Pectis linifolia L. BCN, BCS, CAM, CHIH, COL, DGO, GRO, JAL, MEX, MICH, MOR, NAY, OAX, QROO, SON, YUC

Pectis longipes A. Gray CHIH, DGO, SON

*Pectis luckoviae D.J. Keil GRO, MEX, MICH

Pectis multiflosculosa (DC.) Sch. Bip. CHIS, COL, DGO, GRO, JAL, MICH, NAY, OAX, SIN

*Pectis multiseta Benth. BCS

Pectis papposa Harv. \& A. Gray BCN, BCS, CHIH, COAH, DGO, SIN, SON

* Pectis pimana Laferr. \& Keil CHIH, SON

*Pectis pringlei Fernald CHIH, COAH, DGO

* Pectis propetes Greenm. DGO, JAL, NAY, ZAC

Pectis prostrata Cav. AGS, BCS, CAM, CHIS, CHIH, COAH, COL, CDMX, DGO, GTO, GRO, HGO, JAL, MEX, MICH, MOR, NAY, NLE, OAX, PUE, QRO, SLP, SIN, SON, TAB, TAMS, VER, YUC, ZAC

*Pectis purpurea Brandegee NAY, SIN, SON

* Pectis repens Brandegee COAH, COL, GTO, GRO, HGO, JAL, MEX, MICH, MOR, NAY, NLE, OAX, QRO, SLP, SIN, VER, ZAC

Pectis rusbyi Greene ex A. Gray BCN, BCS, SIN, SON

Pectis saturejoides (Mill.) Sch. Bip. CHIS, GRO, MEX, MOR, OAX, SLP, SIN, TAB, VER

*Pectis schaffneri Sch. Bip. ex Fernald CDMX, DGO, HGO, MEX

*Pectis sinaloensis Fernald JAL, SIN

*Pectis stenophylla A. Gray CHIH, DGO, SIN, SON

Pectis uniaristata DC. AGS, BCS, CHIS, CHIH, COL, DGO, GRO, JAL, MEX, MICH, MOR, NAY, OAX, SIN, SON, VER, ZAC

*Pectis vandevenderi B.L. Turner CHIH, SON

* Pectis vollmeri Wiggins BCS

* Pelucha trifida $\mathrm{S}$. Watson BCN, BCS, SON

Pentacalia epidendra (L.O Williams) H. Rob. \& Cuatrec. CHIS
* Pentacalia guerrerensis (T.M. Barkley) C. Jeffrey GRO

Pentacalia parasitica (Hemsl.) H. Rob. \& Cuatrec. CHIS, OAX, PUE, VER

*Pentacalia venturae (T.M. Barkley) C. Jeffrey CHIS, OAX, VER

Pentachaeta aurea Nutt. BCN

Pericome caudata A. Gray CHIH, COAH, SON

* Pericome macrocephala B.L. Rob. DGO, JAL

Perityle aglossa A. Gray CHIH, COAH

*Perityle aurea Rose BCN, BCS, SON

*Perityle batopilensis A.M. Powell CHIH

Perityle bisetosa (Torr. ex A. Gray) Shinners CHIH, COAH

* Perityle californica Benth. BCN, BCS, SIN, SON

* Perityle canescens Everly SIN

* Perityle carmenensis A.M. Powell COAH

* Perityle castillonii I.M. Johnst. CHIH, COAH, JAL, ZAC

*Perityle coahuilensis A.M. Powell COAH

*Perityle cordifolia (Rydb.) S.F. Blake SIN, SON

Perityle coronopifolia A. Gray CHIH, SON

*Perityle crassifolia Brandegee $\mathrm{BCN}, \mathrm{BCS}$

*Perityle cuneata Brandegee BCS

Perityle dissecta (Torr.) A. Gray CHIH, COAH, SON

Perityle emoryi Torr. BCN, BCS, CHIH, COL, SIN, SON

* Perityle feddemae McVaugh JAL, ZAC

* Perityle gentryi A.M. Powell CHIH, SON

*Perityle glaucescens B.L. Turner JAL, ZAC

* Perityle grandifolia Brandegee SIN

* Perityle harkerae P. Carrillo JAL, ZAC

* Perityle hofmeisteria Rydb. DGO

Perityle huecoensis A.M. Powell CHIH

*Perityle incana A. Gray BCN

*Perityle jaliscana A. Gray JAL, ZAC

* Perityle leptoglossa Harv. \& A. Gray SIN, SON

* Perityle lineariloba Rydb. DGO

*Perityle lobata (Rydb.) I.M. Johnst. BCS

* Perityle microcephala A. Gray CHIH, DGO, SON

Perityle microglossa Benth. AGS, BCN, BCS, CHIS, CHIH, COAH, COL, DGO, GTO, GRO, HGO, JAL, MICH, NAY, QRO, SLP, SIN, SON, TAMS, ZAC

*Perityle montana (A.M. Powell) B.G. Baldwin CHIH

*Perityle palmeri $\mathrm{S}$. Watson SIN, SON

Perityle parryi A. Gray $\mathrm{CHIH}, \mathrm{COAH}$

* Perityle pennellii B.L. Turner DGO

* Perityle reinana $\mathrm{B} . \mathrm{L}$. Turner SON

*Perityle rosei Greenm. AGS, DGO, GTO, JAL, NAY, SIN, ZAC

*Perityle scopulorum (M.E. Jones) A.M. Powell \& B.L. Turner $\mathrm{CHIH}, \mathrm{SON}$

*Perityle socorrosensis Rose COL

*Perityle stevensii B.L. Turner SIN

*Perityle trichodonta S.F. Blake DGO, JAL, MICH, NAY, ZAC

*Perityle turneri A.M. Powell CHIH, DGO, SIN

*Perityle vandevenderi B.L. Turner SON

Perityle vaseyi J.M. Coult. CHIH, COAH

*Perityle vigilans Spellenb. \& A.M. Powell CHIH

* Perymenium alticola McVaugh COL, JAL, MICH

*Perymenium arriagae Rzed. \& Calderón HGO, QRO, SLP 
*Perymenium asperifolium Sch. Bip. ex Klatt OAX, PUE

*Perymenium basaseachicanum B.L. Turner CHIH

*Perymenium beckeri J.J. Fay DGO, SIN

*Perymenium berlandieri DC. CDMX, GTO, GRO, HGO, JAL, MEX, MICH, MOR, OAX, PUE, QRO, VER

*Perymenium buphthalmoides DC. AGS, CHIH, COL, CDMX, DGO, GTO, GRO, HGO, JAL, MEX, MICH, MOR, NAY, OAX, PUE, QRO, SLP, SIN, SON, TLAX, VER, ZAC

*Perymenium cornutum Brandegee HGO, SLP, TAMS, VER

*Perymenium cualense B.L. Turner JAL

Perymenium chloroleucum S.F. Blake CHIS

*Perymenium discolor Schrad. GRO, OAX, PUE, VER

*Perymenium episcopale J.J. Fay GRO, MICH, OAX

*Perymenium fayi B.L. Turner DGO, SIN

Perymenium ghiesbreghtii B.L. Rob. \& Greenm. CHIS, QROO

* Perymenium glandulosum Brandegee PUE

*Perymenium globosum B.L. Rob. GRO, JAL, MEX, MICH, MOR, OAX

Perymenium gracile Hemsl. CHIS, GRO, OAX, PUE, VER

Perymenium grande Hemsl. CHIS, OAX, TAB

Perymenium gymnolomoides (Less.) DC. CAM, CHIS, PUE, QROO, SLP, TAMS, VER, YUC

*Perymenium gypsophilum (B.L. Turner) B.L. Turner NLE

*Perymenium hintonii McVaugh GRO, JAL, MEX, MICH

*Perymenium hintoniorum B.L. Turner NLE, TAMS

*Perymenium huentitanum B.L. Turner JAL

*Perymenium ibarrarum Rzed. \& Calderón MEX, MICH

*Perymenium jaliscense B.L. Rob. \& Greenm. COL, JAL, ZAC

*Perymenium klattianum J.J. Fay CHIS, OAX, VER

*Perymenium macrocephalum Greenm. GRO, MEX, MICH, MOR

*Perymenium mendezii DC. AGS, CHIH, COAH, COL, CDMX, DGO, GTO, GRO, HGO, JAL, MEX, MICH, MOR, OAX, PUE, QRO, SLP, SON, TAMS, VER, ZAC

*Perymenium moctezumae Rzed. \& Calderón HGO, QRO

*Perymenium oaxacanum B.L. Turner OAX

*Perymenium ovalifolium (A. Gray) B.L. Turner HGO, MOR, OAX, PUE, QRO, SLP, TAMS, VER

*Perymenium ovatum Brandegee OAX, PUE

*Perymenium oxycarphum S.F. Blake CHIH, DGO, GRO, JAL,

MICH, NAY, OAX, SIN, ZAC

*Perymenium paneroi B.L. Turner MEX, MICH

* Perymenium pinetorum Brandegee CHIS, OAX

*Perymenium pringlei B.L. Rob. \& Greenm. CHIH, DGO, JAL,

NAY, SIN, SON, ZAC

*Perymenium reticulatum J.J. Fay GTO, HGO, MEX, MICH, QRO

*Perymenium rogmacvaughii Rzed. \& Calderón MEX, MICH

*Perymenium sedasanum J.J. Fay OAX

*Perymenium sonoranum (B.L. Turner) B.L. Turner CHIH, SON

*Perymenium sotoarenasii Rzed. \& Calderón QRO, SLP

*Perymenium stenophyllum S.F. Blake CHIH, DGO, NAY, SIN, SON

*Perymenium subsquarrosum B.L. Rob. \& Greenm. AGS, JAL, $\mathrm{MICH}, \mathrm{ZAC}$

*Perymenium tamaulipense B.L. Turner NLE, TAMS
* Perymenium tehuacanum Villaseñor \& Panero PUE

*Perymenium uxoris McVaugh COL, DGO, JAL, NAY

*Perymenium wilburorum McVaugh COL, JAL

*Perymenium yanezii B.L. Turner TAMS

*Peteravenia malvifolia (DC.) R.M. King \& H. Rob. COAH, GTO, HGO, NLE, QRO, SLP, TAMS, VER

Peteravenia phoenicolepis (B.L. Rob.) R.M. King \& H. Rob. CHIS

Peteravenia schultzii (Schnittsp.) R.M. King \& H. Rob. CHIS, GTO, GRO, HGO, MICH, NLE, OAX, QRO, SLP, TAMS, VER Peucephyllum schottii A. Gray BCN, BCS, SON

*Philactis fayi A.M. Torres JAL, MICH

Philactis zinnioides Schrad. CHIS, OAX, PUE

*Pinaropappus diguetii McVaugh COL, DGO, JAL, MICH, NAY, ZAC

*Pinaropappus junceus A. Gray CHIH, SIN, SON

*Pinaropappus mojadanus B.L. Turner COAH

*Pinaropappus multicaulis Brandegee GTO, NLE, SLP, TAMS, ZAC

Pinaropappus parvus S.F. Blake COAH

*Pinaropappus pattersonii B.L. Turner COAH, NLE

*Pinaropappus pooleanus B.L. Turner CHIH, SON

*Pinaropappus powellii B.L. Turner $\mathrm{COAH}$

Pinaropappus roseus (Less.) Less. AGS, CHIH, COAH, COL, CDMX, DGO, GTO, GRO, HGO, JAL, MEX, MICH, MOR, NAY, NLE, OAX, PUE, QRO, SLP, SON, TAMS, TLAX, VER, ZAC

Pinaropappus spathulatus Brandegee CHIS, OAX, PUE, VER * Pippenalia delphinifolia (Rydb.) McVaugh AGS, CHIH, DGO, JAL, NAY, SIN, ZAC

Piptocarpha poeppigiana (DC.) Baker CHIS, OAX, VER

*Piqueria glandulosa B.L. Turner JAL, MICH

*Piqueria laxiflora B.L. Rob. \& Seaton AGS, COL, DGO, JAL, MEX, MICH, SLP, ZAC

*Piqueria pilosa Kunth CHIS, CDMX, GTO, GRO, HGO, JAL, MEX, MICH, MOR, OAX, PUE, QRO, SLP, TAMS, TLAX, VER

* Piqueria serrata A. Gray GTO, NLE, SLP, TAMS

*Piqueria triflora Hemsl. AGS, COL, DGO, GTO, GRO, JAL, MEX, MICH, NAY, OAX, SLP, SIN, ZAC

Piqueria trinervia Cav. AGS, BCN, CHIS, CHIH, COAH, COL, CDMX, DGO, GTO, GRO, HGO, JAL, MEX, MICH, MOR, NAY, NLE, OAX, PUE, QRO, SLP, TAMS, TLAX, VER, ZAC *Pittocaulon bombycophole (Bullock) H. Rob. \& Brettell GRO, JAL, MEX, MICH, MOR, PUE

*Pittocaulon filare (McVaugh) H. Rob. \& Brettell AGS, COL, JAL, MICH, NAY, ZAC

*Pittocaulon hintonii $\mathrm{H}$. Rob. \& Brettell MICH

*Pittocaulon praecox (Cav.) H. Rob. \& Brettell AGS, CDMX, GTO, HGO, JAL, MEX, MICH, NLE, OAX, PUE, QRO, SLP, TAMS, VER, ZAC

Pittocaulon velatum (Greenm.) H. Rob. \& Brettell CHIS, COL, GRO, JAL, MEX, MICH, MOR, NAY, OAX, PUE, VER, ZAC Pityopsis graminifolia (Michx.) Nutt. CHIS, OAX, PUE, TAMS, VER

*Plagiolophus millspaughii Greenm. CAM, QROO, YUC

Plateilema palmeri (A. Gray) Cockerell COAH, NLE 
*Pleurocoronis gentryi (Wiggins) R.M. King \& H. Rob. BCS *Pleurocoronis laphamioides (Rose) R.M. King \& H. Rob. BCN, BCS, SON

Pleurocoronis pluriseta (A. Gray) R.M. King \& H. Rob. BCN, BCS, SON

Pluchea baccharis (Mill.) Pruski CAM, OAX, QROO, VER, YUC

Pluchea carolinensis (Jacq.) G. Don BCN, BCS, CAM, CHIS, CHIH, COAH, COL, CDMX, GTO, GRO, HGO, JAL, MEX, MICH, MOR, NAY, NLE, OAX, PUE, QRO, QROO, SLP, SIN, SON, TAB, TAMS, VER, YUC, ZAC

Pluchea foetida (L.) DC. OAX, VER

*Pluchea mexicana (Godfrey) G.L. Nesom GTO, SLP, ZAC

Pluchea odorata (L.) Cass. BCN, BCS, CAM, CHIS, CHIH, COAH, COL, GRO, JAL, MICH, MOR, NAY, NLE, OAX, QROO, SLP, SIN, SON, TAB, TAMS, VER, YUC

*Pluchea parvifolia (A. Gray) Godfrey BCS

Pluchea salicifolia (Mill.) S.F. Blake AGS, CAM, CHIS, CHIH, COAH, COL, DGO, GTO, GRO, HGO, JAL, MEX, MICH, MOR, NAY, NLE, OAX, PUE, QRO, SLP, SIN, SON, TAMS, VER, YUC, ZAC

Pluchea sericea (Nutt.) Coville BCN, CHIH, COAH, SON

Pluchea yucatanensis G.L. Nesom CAM, QROO

*Podachaenium chiapanum B.L. Turner \& Panero CHIS

* Podachaenium chimalapanum B.L. Turner OAX, VER

Podachaenium eminens (Lag.) Sch. Bip. CHIS, COL, DGO, GRO, HGO, JAL, MICH, NAY, OAX, PUE, QRO, SLP, SIN, TAB, VER

Podachaenium pachyphyllum (Sch. Bip. ex Klatt) R.K. Jansen, N.A. Harriman \& Urbatsch CHIS, OAX

*Porophyllum amplexicaule Engelm. ex A. Gray COAH, NLE, SLP

*Porophyllum calcicola B.L. Rob. \& Greenm. GRO, MEX, MOR, OAX, PUE

*Porophyllum coloratum (Kunth) DC. AGS, CHIH, COAH, COL, DGO, GTO, GRO, HGO, JAL, MEX, MICH, NAY, NLE, OAX, QRO, SLP, SIN, SON, TAMS, VER, ZAC

*Porophyllum filiforme Rydb. COAH, NLE, SLP, TAMS, ZAC

Porophyllum gracile Benth. BCN, BCS, CHIH, SIN, SON

Porophyllum greggii A. Gray CHIH, COAH

*Porophyllum linaria (Cav.) DC. AGS, CHIS, CHIH, COAH, CDMX, DGO, GTO, GRO, HGO, JAL, MEX, MICH, MOR, NAY, OAX, PUE, QRO, SLP, SIN, TAMS, TLAX, VER, ZAC Porophyllum macrocephalum DC. AGS, BCS, CAM, CHIS, CHIH, COAH, COL, DGO, GTO, GRO, HGO, JAL, MEX, MICH, MOR, NAY, NLE, OAX, PUE, QRO, QROO, SLP, SIN, SON, TAB, TAMS, VER, YUC, ZAC

*Porophyllum maritimum Brandegee BCN, BCS

*Porophyllum pausodynum B.L. Rob. \& Greenm. SON

*Porophyllum pringlei B.L. Rob. CHIS, COL, GRO, JAL, MEX, MICH, MOR, NAY, OAX, SIN, ZAC

Porophyllum punctatum (Mill.) S.F. Blake CAM, CHIS, COL, DGO, GRO, HGO, JAL, MEX, MICH, MOR, NAY, OAX, PUE, QRO, QROO, SLP, SIN, TAB, VER, YUC, ZAC

Porophyllum scoparium A. Gray BCN, BCS, CHIH, COAH, DGO, NLE, TAMS, ZAC

*Porophyllum viridiflorum (Kunth) DC. AGS, COL, DGO,
GTO, GRO, HGO, JAL, MEX, MICH, MOR, NAY, OAX, PUE, QRO, SLP, TAMS, VER, ZAC

*Porophyllum warnockii R.R. Johnson GRO, MEX, MICH

*Porophyllum zimapanum B.L. Turner HGO, QRO

Prenanthella exigua (A. Gray) Tomb BCN, SON

* Psacaliopsis macdonaldii (B.L. Turner) C. Jeffrey OAX

* Psacaliopsis paneroi (B.L. Turner) C. Jeffrey GRO, OAX

*Psacaliopsis pinetorum (Hemsl.) Funston \& Villaseñor GRO, OAX

*Psacaliopsis purpusii (Greenm.) H. Rob. \& Brettell OAX, PUE

*Psacalium amplifolium (DC.) H. Rob. \& Brettell GRO, MEX, MICH, MOR, OAX, PUE

*Psacalium amplum (Rydb.) H. Rob. \& Brettell AGS, DGO, JAL, NAY, ZAC

* Psacalium beamanii H. Rob. OAX

*Psacalium brachycomum (S.F. Blake) H. Rob. \& Brettell JAL, MEX, MICH

* Psacalium calvum (Brandegee) Pippen OAX, PUE

* Psacalium cervinum (Rydb.) H. Rob. \& Brettell NAY, SIN

*Psacalium cirsiifolium (Zucc.) H. Rob. \& Brettell CHIS, GRO,

JAL, MEX, MICH, MOR, OAX, VER

* Psacalium cronquistiorum B.L. Turner DGO, SIN, ZAC

Psacalium decompositum (A. Gray) H. Rob. \& Brettell CHIH, DGO, SON

*Psacalium eriocarpum (S.F. Blake) S.F. Blake COL, DGO, JAL, NAY

*Psacalium filicifolium (Rydb.) H. Rob. \& Brettell DGO, JAL, NAY, ZAC

*Psacalium globosum (B.L. Rob. \& Fernald) H. Rob. \& Brettell CHIH, DGO, NAY, SIN

*Psacalium goldsmithii (B.L. Rob.) H. Rob. \& Brettell COL, GRO, JAL, MEX, MICH, NAY, OAX, SIN

*Psacalium guerreroanum B.L. Turner GRO, OAX

* Psacalium hintonii (Pippen) H. Rob. \& Brettell MEX

* Psacalium hintoniorum B.L. Turner OAX

*Psacalium holwayanum (B.L. Rob.) Rydb. GRO, JAL, MEX, MICH

*Psacalium latilobum (Pippen) B.L. Turner GRO, OAX

*Psacalium laxiflorum Benth. DGO, JAL, MEX, MICH, NAY, ZAC

*Psacalium matudae H. Rob. \& Brettell GRO, MEX, MICH

* Psacalium megaphyllum (B.L. Rob. \& Greenm.) Rydb. AGS, COL, DGO, GTO, GRO, JAL, MEX, MICH, MOR, NAY, OAX, PUE, QRO, SIN, ZAC

*Psacalium mollifolium S.F. Blake GRO

* Psacalium multilobum (Pippen) H. Rob. \& Brettell COL, JAL

* Psacalium nanum Pippen GRO

* Psacalium napellifolium (Schauer) H. Rob. \& Brettell GTO, GRO, HGO, QRO, VER

* Psacalium nelsonii Rydb. OAX

*Psacalium nephrophyllum (Rydb.) H. Rob. \& Brettell DGO, NAY

*Psacalium pachyphyllum (Sch. Bip.) H. Rob. \& Brettell DGO, JAL, NAY, SIN, ZAC

*Psacalium palmeri (Greene) H. Rob. \& Brettell AGS, COL, DGO, JAL, MEX, MICH, NAY, ZAC 
*Psacalium paucicapitatum (B.L. Rob. \& Greenm.) H. Rob. \& Brettell GRO, OAX

*Psacalium peltatum (Kunth) Cass. AGS, CHIH, COAH, COL, CDMX, DGO, GTO, GRO, HGO, JAL, MEX, MICH, MOR, NLE, OAX, PUE, QRO, SLP, SIN, TAMS, TLAX, VER, ZAC * Psacalium peltigerum (B.L. Rob. \& Seaton) Rydb. COL, DGO, JAL, NAY, SIN, ZAC

*Psacalium pentaflorum B.L. Turner COL, JAL

* Psacalium perezii B.L. Turner COL, JAL

* Psacalium platylepis (B.L. Rob. \& Seaton) H. Rob. \& Brettell AGS, DGO, GTO, HGO, JAL, MEX, MICH, NAY, QRO, ZAC *Psacalium poculiferum (S. Watson) Rydb. AGS, COL, GTO, JAL, NAY, OAX, QRO, SIN, ZAC

* Psacalium pringlei (S. Watson) H. Rob. \& Brettell AGS, COL, DGO, JAL, MEX, MICH, NAY, SIN, ZAC

*Psacalium purpusii (Greenm.) H. Rob. \& Brettell GRO, OAX, PUE

*Psacalium putlanum B.L. Turner OAX

*Psacalium quercifolium $\mathrm{H}$. Rob. \& Brettell DGO, SIN

* Psacalium radulifolium (Kunth) H. Rob. \& Brettell AGS, COL, DGO, GTO, HGO, JAL, MICH, NLE, QRO, SLP, SIN, TAMS, ZAC

*Psacalium schillingii Panero \& Villaseñor GRO, OAX

*Psacalium sharpii B.L. Turner GRO

*Psacalium silphiifolium (B.L. Rob. \& Greenm.) H. Rob. \& Brettell CDMX, GRO, HGO, MEX, MICH, MOR, OAX, PUE, QRO, TLAX, VER

*Psacalium sinuatum (Cerv.) H. Rob. \& Brettell AGS, CHIH, COAH, CDMX, DGO, GTO, GRO, HGO, JAL, MEX, MICH, NAY, OAX, PUE, QRO, SLP, SIN, TAMS, ZAC

* Psacalium tabulare (Hemsl.) Rydb. CHIS, GRO, MEX, MICH, OAX, PUE, VER

*Psacalium tussilaginoides (Kunth) H. Rob. \& Brettell AGS, GTO, HGO, MEX, MICH, SLP, VER

Psathyrotes ramosissima (Torr.) A. Gray BCN, SON

* Psathyrotopsis hintoniorum B.L. Turner COAH

*Psathyrotopsis purpusii (Brandegee) Rydb. COAH

Psathyrotopsis scaposa (A. Gray) H. Rob. CHIH, COAH, DGO Pseudelephantopus spicatus (Juss. ex Aubl.) C.F. Baker CAM, CHIS, COL, DGO, GTO, GRO, HGO, JAL, MEX, MICH, MOR, NAY, OAX, PUE, QRO, QROO, SLP, SIN, SON, TAB, TAMS, VER, YUC, ZAC

Pseudoclappia arenaria Rydb. CHIH, COAH

*Pseudognaphalium altamiranum (Greenm.) Anderb. MEX, MOR

Pseudognaphalium attenuatum (DC.) Anderb. AGS, CHIS, CHIH, COL, CDMX, DGO, GTO, GRO, HGO, JAL, MEX, MICH, MOR, NAY, OAX, PUE, QRO, QROO, SLP, SIN, SON, TAB, TAMS, TLAX, VER, ZAC

Pseudognaphalium austrotexanum G.L. Nesom COAH, NLE

Pseudognaphalium beneolens (Davidson) Anderb. BCN

Pseudognaphalium biolettii Anderb. BCN, BCS, CHIH, SIN, SON

*Pseudognaphalium bourgovii (A. Gray) Anderb. CHIS, CHIH, COL, CDMX, DGO, GRO, JAL, MEX, MICH, MOR, NAY, SIN, SON, TLAX, VER, ZAC

Pseudognaphalium brachyphyllum (Greenm.) Anderb. CHIS
Pseudognaphalium brachypterum (DC.) Anderb. AGS, CHIS, COAH, CDMX, GTO, HGO, JAL, MEX, NLE, PUE, OAX, QRO, SLP, TAMS, VER, ZAC

Pseudognaphalium californicum (DC.) Anderb. BCN

Pseudognaphalium canescens (DC.) Anderb. AGS, BCN, CHIH, COAH, COL, CDMX, DGO, GTO, GRO, HGO, JAL, MEX, MICH, MOR, NAY, NLE, OAX, PUE, QRO, SLP, SIN, SON, TAMS, TLAX, VER, ZAC

*Pseudognaphalium conoideum (Kunth) Anderb. AGS, COAH, CDMX, DGO, GTO, HGO, JAL, MEX, MICH, MOR, NLE, OAX, PUE, QRO, SIN, TLAX, VER, ZAC

*Pseudognaphalium crenatum (Greenm.) Anderb. JAL

*Pseudognaphalium chartaceum (Greenm.) Anderb. AGS, CHIS, COL, CDMX, GTO, GRO, HGO, JAL, MEX, MICH, MOR, NAY, NLE, OAX, PUE, QRO, SLP, TLAX, VER, ZAC *Pseudognaphalium ehrenbergianum (Sch. Bip. ex Klatt) Hinojosa \& Villaseñor HGO, NLE, PUE, TAMS, VER

Pseudognaphalium elegans (Kunth) Kartesz CHIS, HGO, NLE, OAX, PUE, SLP, TAMS, VER

Pseudognaphalium greenmanii (S.F. Blake) Anderb. CHIS, CHIH, COL, DGO, GRO, JAL, MEX, NAY, OAX, SON, ZAC *Pseudognaphalium hintoniorum (G.L. Nesom) Hinojosa \& Villaseñor COAH, DGO, NLE, TAMS

*Pseudognaphalium inornatum (DC.) Anderb. AGS, BCN, CHIS, CHIH, COAH, CDMX, DGO, GTO, GRO, HGO, JAL, MEX, MICH, MOR, NAY, NLE, OAX, PUE, QRO, SLP, SIN, SON, TLAX, VER, ZAC

Pseudognaphalium jaliscense (Greenm.) Anderb. AGS, COL, JAL, NAY, ZAC

Pseudognaphalium leucocephalum (A. Gray) Anderb. BCN, BCS, CHIH, COAH, DGO, NAY, SLP, SIN, SON

Pseudognaphalium liebmannii (Sch. Bip. ex Klatt) Anderb. CHIS, CHIH, COL, CDMX, GTO, GRO, HGO, JAL, MEX, MICH, MOR, NLE, OAX, PUE, QRO, SLP, TAMS, TLAX, VER

Pseudognaphalium microcephalum (Nutt.) Anderb. BCN, $\mathrm{CHIH}$

*Pseudognaphalium nubicola (I.M. Johnst.) Anderb. CDMX, JAL, MEX, MICH, QRO, SLP, TLAX

*Pseudognaphalium oaxacanum (Greenm.) G.L. Nesom OAX Pseudognaphalium oxyphyllum (DC.) Kirp. AGS, BCS, CHIS, CHIH, COAH, COL, CDMX, DGO, GTO, GRO, HGO, JAL, MEX, MICH, MOR, NLE, OAX, PUE, QRO, SLP, SON, TLAX, VER, ZAC

Pseudognaphalium pringlei (A. Gray) Anderb. CHIH, COAH, DGO, GTO, NLE, SLP, SON, TAMS

*Pseudognaphalium purpurascens (DC.) Anderb. AGS, CDMX, GTO, HGO, JAL, MEX, MICH, MOR, NLE, OAX, PUE, QRO, SLP, TAMS, TLAX, VER, ZAC

Pseudognaphalium roseum (Kunth) Anderb. AGS, CHIS, COAH, COL, CDMX, DGO, GTO, GRO, HGO, JAL, MEX, MICH, MOR, NAY, NLE, OAX, QRO, SLP, TAMS, TLAX, VER, ZAC

Pseudognaphalium semiamplexicaule (DC.) Anderb. AGS, BCS, CHIS, COAH, COL, CDMX, DGO, GTO, GRO, HGO, JAL, MEX, MICH, MOR, NLE, OAX, PUE, QRO, SLP, SIN, TAMS, TLAX, VER, ZAC 
*Pseudognaphalium semilanatum (DC.) Anderb. AGS, BCN, CHIS, CHIH, COAH, COL, CDMX, GTO, HGO, JAL, MEX, MICH, NAY, NLE, OAX, PUE, QRO, SLP, SON, TAMS, TLAX, VER, ZAC

Pseudognaphalium stramineum (Kunth) Anderb. AGS, BCN, BCS, CHIS, CHIH, COAH, CDMX, DGO, GTO, GRO, HGO, JAL, MEX, MICH, MOR, NAY, NLE, OAX, PUE, QRO, SLP, SIN, SON, TLAX, VER, ZAC

Pseudognaphalium viscosum (Kunth) Anderb. AGS, BCS, CHIS, CHIH, COAH, COL, CDMX, DGO, GTO, GRO, HGO, JAL, MEX, MICH, MOR, NAY, NLE, OAX, PUE, QRO, SLP, SIN, SON, TAMS, TLAX, VER, ZAC

Pseudogynoxys chenopodioides (Kunth) Cabrera BCS, CAM, CHIS, COL, GRO, HGO, JAL, MOR, NAY, OAX, PUE, QRO, QROO, SLP, TAB, TAMS, VER, YUC, ZAC

Pseudogynoxys haenkei (DC.) Cabrera CHIS, OAX, VER

Psilactis asteroides A. Gray AGS, CHIH, COAH, CDMX, DGO, GTO, HGO, JAL, MEX, MICH, NLE, PUE, QRO, SLP, SIN, SON, TAMS, ZAC

Psilactis brevilingulata Sch. Bip. ex Hemsl. AGS, CHIS, CHIH, COAH, CDMX, DGO, GTO, GRO, HGO, JAL, MEX, MICH, MOR, NAY, NLE, OAX, PUE, QRO, SLP, TAMS, TLAX, VER, ZAC

Psilactis gentryi (Standl.) D.R. Morgan CHIH, DGO, GTO, HGO, MEX, QRO, SIN, SON

*Psilactis odysseus (G.L. Nesom) D.R. Morgan NLE, TAMS

Psilactis tenuis S. Watson CHIH, COAH, NLE, SLP, SON, TAMS, ZAC

Psilocarphus brevissimus Nutt. BCN

Psilocarphus tenellus Nutt. BCN, MEX

Psilostrophe cooperi (A. Gray) Greene BCN, BCS, SON

Psilostrophe gnaphalodes DC. CHIH, COAH, DGO, NLE, SLP, SON, TAMS, ZAC

Psilostrophe tagetina (Nutt.) Greene CHIH, COAH, SON

Psilostrophe villosa Rydb. COAH

Pterocaulon virgatum (L.) DC. PUE, TAMS, VER

Pyrrhopappus pauciflorus (D. Don) DC. AGS, CHIH, COAH, DGO, GTO, JAL, MEX, NLE, PUE, QRO, SLP, SIN, SON, TAMS, ZAC

Rafinesquia californica Nutt. BCN, SON

Rafinesquia neomexicana A. Gray BCN, BCS, CHIH, SON

* Ratibida coahuilensis B.L. Turner COAH, NLE

Ratibida columnifera (Nutt.) Wooton \& Standl. CHIH, COAH, HGO, NLE, PUE, SLP, SON, TAMS, VER, ZAC

* Ratibida latipalearis E.L. Richards CHIH, SON

* Ratibida mexicana (S. Watson) W.M. Sharp CHIH, COAH, DGO, NLE, SON

Ratibida peduncularis (Torr. \& A. Gray) Barnhart COAH, TAMS

Ratibida tagetes (E. James) Barnhart CHIH, SON

Rayjacksonia phyllocephala (DC.) R.L. Hartman \& M.A. Lane TAMS

Rensonia salvadorica S.F. Blake CHIS, OAX, VER

Robinsonecio gerberifolius (Sch. Bip.) T.M. Barkley \& J.P. Janovec CHIS, MEX, PUE, VER

*Robinsonecio porphyresthes (T.M. Barkley) T.M. Barkley \& J.P. Janovec TAMS
Rojasianthe superba Standl. \& Steyerm. CHIS

Roldana acutangula (Bertol.) Funston CHIS

*Roldana albonervia (Greenm.) H. Rob. \& Brettell AGS, COL, CDMX, GTO, GRO, HGO, JAL, MEX, MICH, MOR, PUE, QRO, SLP, TAMS, TLAX, VER, ZAC

Roldana angulifolia (DC.) H. Rob. \& Brettell COL, CDMX, DGO, GTO, GRO, HGO, JAL, MEX, MICH, MOR, NAY, NLE, OAX, PUE, QRO, SLP, SIN, TAMS, TLAX, VER, ZAC

*Roldana anisophylla (Klatt) Funston OAX

Roldana aschenborniana (S. Schauer) H. Rob. \& Brettell GTO, HGO, MEX, MICH, MOR, NLE, OAX, PUE, QRO, SLP, TAMS, VER, YUC

Roldana barba-johannis (DC.) H. Rob. \& Brettell CHIS, COL, CDMX, DGO, GTO, GRO, HGO, JAL, MEX, MICH, MOR, OAX, PUE, QRO, SLP, SIN, TLAX, VER

* Roldana calzadana B.L. Turner GRO, OAX

*Roldana cordovensis (Hemsl.) H. Rob. \& Brettell CHIS, HGO, OAX, PUE, SLP, VER

Roldana cristobalensis (Greenm.) H. Rob. \& Brettell CHIS, OAX

*Roldana chapalensis (S. Watson) H. Rob. \& Brettell AGS, COL, DGO, GTO, GRO, JAL, MEX, MICH, MOR, NAY, OAX, PUE, QRO, SLP, SIN, ZAC

* Roldana chiapensis H. Rob. \& Brettell CHIS

*Roldana ehrenbergiana (Klatt) H. Rob. \& Brettell GRO, HGO, MEX, MOR, OAX, PUE, QRO, SLP, VER

*Roldana eriophylla (Greenm.) H. Rob. \& Brettell CHIS, GRO, OAX, PUE

*Roldana gentryi H. Rob. \& Brettell CHIH, DGO, JAL, NAY, SIN, SON, ZAC

*Roldana gesneriifola (B.L. Turner) C. Jeffrey DGO, JAL, ZAC Roldana gilgii (Greenm.) H. Rob. \& Brettell CHIS, MICH

*Roldana glinophylla H. Rob. \& Brettell JAL, MICH

*Roldana gonzalezae (B.L. Turner) B.L. Turner DGO, JAL, NAY, ZAC

Roldana greenmanii H. Rob. \& Brettell CHIS

*Roldana grimesii (B.L. Turner) C. Jeffrey HGO, PUE, QRO, VER

*Roldana guadalajarensis (B.L. Rob.) H. Rob. \& Brettell COL, DGO, GTO, JAL, MICH, NAY

Roldana hartwegii (Benth.) H. Rob. \& Brettell AGS, CHIH, COAH, COL, DGO, GTO, HGO, JAL, NAY, QRO, SLP, SIN, SON, ZAC

*Roldana hederifolia (Hemsl.) H. Rob. \& Brettell GRO, MEX, MICH, MOR, OAX

*Roldana heracleifolia (Hemsl.) H. Rob. \& Brettell AGS, COL, GTO, HGO, JAL, MEX, MICH, OAX, QRO, SLP, ZAC

Roldana heterogama (Benth.) H. Rob. \& Brettell CHIS

*Roldana heteroidea (Klatt) H. Rob. \& Brettell OAX

*Roldana hintonii H. Rob. \& Brettell MEX, MICH

*Roldana hirsuticaulis (Greenm.) Funston SLP, TAMS

Roldana jurgensenii (Hemsl.) H. Rob. \& Brettell CHIS, OAX

*Roldana kerberi (Greenm.) H. Rob. \& Brettell COL, JAL, OAX

*Roldana langlassei (Greenm.) H. Rob. \& Brettell GRO, MEX, $\mathrm{MICH}$

Roldana lanicaulis (Greenm.) H. Rob. \& Brettell CHIS, HGO, 
OAX, PUE, QRO, SLP, TAMS, VER

*Roldana lineolata (DC.) H. Rob. \& Brettell AGS, COL, CDMX, GTO, GRO, HGO, JAL, MEX, MICH, MOR, OAX, PUE, QRO, SLP, TAMS, TLAX, VER, ZAC

*Roldana lobata La Llave COL, CDMX, DGO, GTO, GRO, HGO, JAL, MEX, MICH, MOR, NAY, NLE, OAX, QRO, SLP, TLAX, VER, ZAC

*Roldana marquesii (B.L. Turner) C. Jeffrey HGO, PUE, SLP, VER

*Roldana metepecus (B.L. Turner) C. Jeffrey HGO, PUE, VER *Roldana mexicana (McVaugh) H. Rob. \& Brettell GRO, JAL, MEX, MICH, OAX

*Roldana michoacana (B.L. Rob.) H. Rob. \& Brettell COL, DGO, GTO, GRO, JAL, MEX, MICH, NAY, QRO, ZAC

*Roldana mixtecana Panero \& Villaseñor GRO, OAX

*Roldana neogibsonii (B.L. Turner) B.L. Turner HGO, PUE, VER

*Roldana nesomiorum (B.L. Turner) C. Jeffrey NLE, TAMS

*Roldana oaxacana (Hemsl.) H. Rob. \& Brettell GRO, OAX, PUE, VER

*Roldana pennellii $\mathrm{H}$. Rob. \& Brettell AGS, CHIH, COAH, COL, DGO, JAL, NAY, NLE, SIN, SON, TAMS, ZAC

*Roldana platanifolia (Benth.) H. Rob. \& Brettell CDMX, GTO, HGO, JAL, MEX, MICH, MOR, PUE, SLP, TAMS, TLAX, VER

*Roldana reticulata (DC.) H. Rob. \& Brettell COL, CDMX, GTO, GRO, HGO, JAL, MEX, MICH, MOR, PUE, QRO, SLP, TLAX, VER

*Roldana robinsoniana (Greenm.) H. Rob. \& Brettell COL, GRO, JAL, MOR, OAX

*Roldana sartorii (Sch. Bip. ex Hemsl.) H. Rob. \& Brettell GRO, HGO, OAX, PUE, VER

Roldana schaffneri (Sch. Bip. ex Klatt) H. Rob. \& Brettell CHIS, GRO, OAX, VER

*Roldana sessilifolia (Hook. \& Arn.) H. Rob. \& Brettell AGS, CHIH, COL, CDMX, DGO, GTO, GRO, HGO, JAL, MEX, MICH, MOR, NAY, NLE, QRO, SLP, SIN, TAMS, VER, ZAC *Roldana subcymosa $\mathrm{H}$. Rob. CHIS, OAX

*Roldana subpeltata (Sch. Bip.) H. Rob. \& Brettell JAL, MICH, DGO, SIN

*Roldana suffulta (Greenm.) H. Rob. \& Brettell COL, DGO, GTO, HGO, JAL, MEX, MICH, MOR, NAY, QRO, SIN, VER *Roldana sundbergii (B.L. Turner) B.L. Turner COAH, NLE, TAMS

*Roldana tlacotepecana Funston GRO

*Roldana uxordecora Quedensley \& Villaseñor OAX

*Rumfordia alcortae Rzed. NLE, QRO, SLP, TAMS

*Rumfordia connata Brandegee BCS

*Rumfordia exauriculata B.L. Turner COAH, NLE, TAMS

*Rumfordia floribunda DC. COL, CDMX, DGO, GRO, JAL, MEX, MICH, MOR, NAY, OAX, SIN, VER, ZAC

Rumfordia guatemalensis (J.M. Coult.) S.F. Blake CHIS, GRO, HGO, OAX, VER

Rumfordia penninervis S.F. Blake CHIS, OAX

* Rumfordia revealii $\mathrm{H}$. Rob. GRO

* Sabazia elata (Canne) B.L. Turner HGO, QRO, SLP

*Sabazia glandulosa (Canne) B.L. Turner HGO, QRO, SLP
*Sabazia humilis (Kunth) Cass. CDMX, HGO, JAL, MEX, MICH, MOR, OAX, PUE, QRO, TLAX, VER

* Sabazia lapsensis B.L. Turner GRO

*Sabazia leavenworthii Longpre GRO, JAL, MICH

*Sabazia microspermoides Longpre GRO

*Sabazia multiradiata (Seaton) Longpre CDMX, DGO, GTO, GRO, HGO, MEX, MOR, OAX, PUE, QRO, TLAX, VER

*Sabazia mullerae S.F. Blake COAH, NLE, TAMS

* Sabazia purpusii Brandegee BCS

Sabazia sarmentosa Less. CHIS, GRO, HGO, JAL, MEX, MICH, OAX, PUE, VER

*Salmea oligocephala Hemsl. GRO, HGO, MEX, MICH, MOR, OAX, QRO, SLP

Salmea orthocephala Standl. \& Steyerm. CHIS

*Salmea palmeri S. Watson COL, DGO, GRO, JAL, MEX, MICH, NAY, QRO

Salmea scandens (L.) DC. CHIS, GRO, HGO, JAL, MEX, MICH, OAX, PUE, QRO, QROO, SLP, TAB, TAMS, VER, YUC

Sanvitalia abertii A. Gray BCN, CHIH, COAH, DGO, SON, ZAC

*Sanvitalia angustifolia Engelm. ex A. Gray AGS, CHIH, COAH, DGO, GTO, GRO, HGO, JAL, MEX, MICH, MOR, NLE, OAX, QRO, SLP, TAMS, ZAC

* Sanvitalia fruticosa Hemsl. OAX, PUE

Sanvitalia ocymoides DC. AGS, CHIH, COAH, COL, DGO, GTO, HGO, JAL, MEX, MICH, NLE, OAX, QRO, SLP, TAMS, ZAC

Sanvitalia procumbens Lam. AGS, CAM, CHIS, CHIH, COAH, CDMX, DGO, GTO, GRO, HGO, JAL, MEX, MICH, MOR, NAY, NLE, OAX, PUE, QRO, QROO, SLP, TAMS, TLAX, VER, YUC, ZAC

*Sartwellia gypsophila A.M. Powell \& B.L. Turner CHIH, $\mathrm{COAH}$

* Sartwellia mexicana A. Gray COAH, NLE, SLP, TAMS, ZAC *Sartwellia puberula Rydb. CHIH, COAH, DGO, NLE, SLP, ZAC

Sclerocarpus divaricatus (Benth.) Hemsl. BCS, CAM, CHIS, CHIH, COL, DGO, GRO, HGO, JAL, MEX, MICH, MOR, NAY, OAX, QROO, SIN, TAB, VER, YUC, ZAC

*Sclerocarpus multifidus Greenm. GRO, MEX, MICH, MOR, OAX

*Sclerocarpus papposus (Greenm.) Feddema GRO, MEX, MICH, MOR, OAX, PUE

*Sclerocarpus sessilifolius Greenm. COL, DGO, JAL, NAY, SIN, ZAC

*Sclerocarpus spatulatus Rose CHIH, SIN, SON

Sclerocarpus uniserialis (Hook.) Hemsl. CAM, CHIS, COAH, COL, GTO, GRO, HGO, JAL, MEX, MICH, MOR, NAY, NLE, OAX, PUE, QRO, QROO, SLP, SIN, TAMS, VER, YUC, ZAC *Schistocarpha bicolor Less. CHIS, HGO, MEX, OAX, PUE, QRO, SLP, TAMS, VER

*Schistocarpha calzadana B.L. Turner GRO, OAX

Schistocarpha eupatorioides (Fenzl) Kuntze CAM, CHIS, COAH, GRO, HGO, NLE, OAX, PUE, QRO, QROO, SLP, TAB, VER

*Schistocarpha liebmannii Klatt CHIS, OAX, VER 
Schistocarpha longiligula Rydb. CHIS, TAB

* Schistocarpha matudae H. Rob. CHIS

* Schistocarpha pedicellata Klatt CHIS, OAX, VER

Schistocarpha platyphylla Greenm. CHIS, OAX

Schkuhria multiflora Hook. \& Arn. CHIH, COAH, SON

Schkuhria pinnata (Lam.) Kuntze ex Thell. AGS, CHIS, CHIH, COAH, COL, CDMX, DGO, GTO, GRO, HGO, JAL, MEX, MICH, MOR, NAY, NLE, OAX, PUE, QRO, SLP, SIN, SON, TAMS, TLAX, VER, ZAC

*Schkuhria schkuhrioides (Link. \& Otto) Thell. AGS, CDMX, DGO, GTO, GRO, HGO, JAL, MEX, MICH, NAY, OAX, QRO, SLP, ZAC

*Selloa plantaginea Kunth CDMX, MEX, MICH, MOR, PUE, VER

*Senecio alvarezensis Greenm. GTO, SLP

Senecio aphanactis Greene BCN, BCS

*Senecio argutus Kunth COL, CDMX, HGO, JAL, MEX, MICH, MOR, PUE, QRO, SLP, TLAX, VER

*Senecio barkleyi B.L. Turner NLE, TAMS

*Senecio bartlettii Greenm. SLP, TAMS

*Senecio billieturneri T.M. Barkley DGO, SIN

*Senecio bracteatus Klatt GRO, OAX

Senecio californicus DC. BCN, BCS

Senecio callosus Sch. Bip. CHIS, COL, CDMX, GTO, GRO, HGO, JAL, MEX, MICH, MOR, OAX, PUE, QRO, TLAX, VER

*Senecio carnerensis Greenm. COAH, NLE, TAMS

* Senecio cedrosensis Greene BCN

* Senecio cinerarioides Kunth COL, CDMX, GTO, GRO, HGO, JAL, MEX, MICH, MOR, PUE, QRO, SLP, TLAX, VER

* Senecio claryae B.L. Turner COAH

*Senecio conzattii Greenm. GRO, OAX, PUE

* Senecio chihuahuensis S. Watson CHIH, DGO, SON

*Senecio deformis Klatt AGS, COL, GTO, HGO, JAL, MEX, MICH, MOR, PUE, QRO, VER, ZAC

Senecio deppeanus Hemsl. CHIS, GRO, OAX, VER

Senecio doratophyllus Benth. CHIS

* Senecio durangensis Greenm. CHIH, DGO, SIN

Senecio flaccidus Less. AGS, BCN, CHIH, COAH, DGO, GTO, HGO, JAL, MEX, MICH, NLE, PUE, QRO, SLP, SON, TAMS, TLAX, VER, ZAC

Senecio godmanii Hemsl. CHIS

* Senecio helodes Benth. HGO, JAL, MEX, MICH, VER

*Senecio heterodontus Greenm. SLP, ZAC

*Senecio iodanthus Greenm. COL, CDMX, GTO, JAL, MEX, MICH, MOR, QRO

* Senecio jacalensis Greenm. HGO, MEX, VER

*Senecio lasiocaulon T.M. Barkley COAH, DGO, SIN

Senecio lemmonii A. Gray BCN, BCS, CHIH, SON

* Senecio lithophilus Greenm. NLE, TAMS

Senecio lyonii A. Gray BCN

*Senecio madrensis A. Gray COAH, NLE, SLP, TAMS

Senecio mairetianus DC. CDMX, MEX, JAL, MICH, PUE, TLAX, VER

*Senecio martirensis T.M. Barkley BCN

Senecio mohavensis A. Gray BCN, BCS, SON

* Senecio mohinorensis Greenm. CHIH
*Senecio mulgediifolius S. Schauer CDMX, MEX, MOR, PUE Senecio multidentatus Sch. Bip. ex Hemsl. CHIH, COAH, COL, CDMX, GTO, HGO, JAL, MEX, MICH, NLE, PUE, QRO, SLP, SON, TAMS, TLAX, VER, ZAC

Senecio oerstedianus Benth. ex Oerst. CHIS

* Senecio orizabensis Sch. Bip. ex Hemsl. MEX, PUE, VER

* Senecio palmeri A. Gray BCN

Senecio parryi A. Gray CHIH, COAH, SIN, SON

* Senecio pattersonii B.L. Turner COAH, NLE

Senecio picridis S. Schauer COAH, DGO, GTO, GRO, HGO,

MICH, NLE, OAX, QRO, SLP, TAMS, VER, ZAC

*Senecio pinacatensis Felger SON

* Senecio platypus Greenm. COAH, NLE

* Senecio polypodioides (Greene) Greene GRO, OAX

* Senecio powellii B.L. Turner COAH, NLE

*Senecio prionopterus B.L. Rob. \& Greenm. GRO, MEX, MICH, MOR, OAX, PUE

*Senecio procumbens Kunth MEX, PUE, TLAX, VER

*Senecio pseudopicridis T.M. Barkley GTO, NLE, QRO, SLP, TAMS, ZAC

*Senecio richardsonii B.L. Turner COAH, NLE, QRO, SLP, TAMS

Senecio riddellii Torr. \& A. Gray COAH, NLE, TAMS

*Senecio riomayensis B.L. Turner CHIH, SON

* Senecio riskindii B.L. Turner \& T.M. Barkley COAH

*Senecio roseus Sch. Bip. MEX, MOR, PUE, TLAX, VER

* Senecio runcinatus Less. HGO, PUE, VER

* Senecio sandersiana B.L. Turner DGO, SIN

* Senecio sandersii B.L. Turner CHIH, SON

Senecio spartioides Torr. \& A. Gray BCN

*Senecio stoechadiformis DC. AGS, COL, CDMX, DGO, GTO, GRO, HGO, JAL, MEX, MICH, MOR, NAY, NLE, OAX, PUE, QRO, SLP, SIN, VER, ZAC

*Senecio subauriculatus Greenm. OAX

*Senecio viejoanus B.L. Turner NLE, TAMS

Senecio warszewiczii A. Braun \& C.D. Bouché CHIS

Senecio wootonii Greene CHIH, SON

Shinnersia rivularis (A. Gray) R.M. King \& H. Rob. COAH, NLE

*Sidneya pinnatilobata (Sch. Bip.) E.E. Schill. \& Panero OAX, PUE

Sidneya tenuifolia (A. Gray) E.E. Schill. \& Panero CHIH, COAH, DGO, NAY, NLE, SLP, SON, TAMS, ZAC

Sigesbeckia agrestis Poepp. CHIS, COL, CDMX, DGO, GTO, GRO, HGO, JAL, MEX, MICH, MOR, OAX, PUE, QRO, SLP, SIN, TAMS, VER

Sigesbeckia jorullensis Kunth AGS, CHIS, CHIH, CDMX, DGO, GTO, GRO, HGO, JAL, MEX, MICH, MOR, NAY, OAX, PUE, QRO, TLAX, VER, ZAC

*Sigesbeckia repens B.L. Rob. \& Greenm. OAX

Simsia amplexicaulis (Cav.) Pers. AGS, CHIS, CHIH, COAH, COL, CDMX, DGO, GTO, GRO, HGO, JAL, MEX, MICH, MOR, NAY, NLE, OAX, PUE, QRO, SLP, SIN, SON, TAMS, TLAX, VER, ZAC

*Simsia annectens S.F. Blake CHIS, COL, GRO, JAL, MEX, MICH, NAY, OAX, ZAC

*Simsia benziorum (B.L. Turner) E.E. Schill. \& Panero OAX 
*Simsia bicentenarialis Rzed. \& Calderón GTO, HGO, QRO, SLP

Simsia calva (A. Gray \& Engelm.) A. Gray CHIH, COAH, NLE, TAMS, VER

* Simsia chaseae (Millsp.) S.F. Blake CAM, QROO, VER, YUC * Simsia eurylepis S.F. Blake CAM, COAH, NLE, QRO, QROO, SLP, TAMS, VER, YUC

Simsia foetida (Cav.) S.F. Blake AGS, CHIS, CHIH, COAH, COL, CDMX, DGO, GTO, GRO, HGO, JAL, MEX, MICH, MOR, NAY, OAX, PUE, QRO, SLP, TLAX, VER, ZAC

Simsia ghiesbreghtii (A. Gray) S.F. Blake CHIS

Simsia lagasciformis DC. AGS, CHIH, COAH, COL, DGO, GTO, GRO, HGO, JAL, MEX, MICH, MOR, OAX, PUE, QRO, SLP, SIN, SON, TLAX, VER, ZAC

*Simsia ovata (A. Gray) E.E. Schill. \& Panero CHIS, GRO, OAX

*Simsia rhombifolia (B.L. Rob. \& Greenm.) E.E. Schill. \& Panero OAX, PUE

Simsia sanguinea A. Gray CHIS, DGO, GRO, JAL, MEX, MICH, MOR, NAY, OAX, PUE, SLP, VER

*Simsia setosa S.F. Blake CHIH, DGO, NAY, SON

* Simsia spooneri Panero \& E.E. Schill. GRO

*Simsia sylvicola Panero \& E.E. Schill. OAX

*Simsia tenuis (Fernald) S.F. Blake GRO, MICH

*Simsia villasenorii D.M. Spooner CHIS, OAX

Sinclairia andromachioides (Less.) Sch. Bip. ex Rydb. OAX, VER

* Sinclairia broomeae H. Rob. COL, GRO, JAL

Sinclairia deamii (B.L. Rob. \& Bartlett) Rydb. CAM, CHIS, QROO, TAB

*Sinclairia deppeana (Less.) Rydb. HGO, OAX, PUE, TAMS, VER

Sinclairia discolor Hook. \& Arn. CAM, CHIS, GRO, JAL, MICH, OAX, PUE, QROO, VER

Sinclairia glabra (Hemsl.) Rydb. CHIS, COL, DGO, GRO, JAL, MEX, MICH, MOR, NAY, OAX, SIN, ZAC

Sinclairia hypochlora (S.F. Blake) Rydb. CHIS

*Sinclairia liebmannii (Klatt) Sch. Bip. ex Rydb. CHIS, COL, GRO, JAL, MEX, MICH, MOR, OAX, PUE

* Sinclairia manriquei Panero \& Villaseñor GRO, OAX

*Sinclairia moorei (H. Rob. \& Brettell) H. Rob. \& Brettell GRO, MOR, OAX, PUE

Sinclairia polyantha (Klatt) Rydb. CHIS, OAX, VER

*Sinclairia sericolepis (Hemsl.) Rydb. CHIS, OAX, TAB, VER

* Sinclairia similis (McVaugh) H. Rob. \& Brettell JAL, NAY

Sinclairia sublobata (B.L. Rob.) Rydb. CHIS, OAX, VER

Sinclairia vagans (S.F. Blake) H. Rob. \& Brettell CHIS

* Sinclairiopsis ismaelis (Panero \& Villaseñor) V.A. Funk OAX

*Sinclairiopsis klattii (B.L. Rob. \& Greenm.) Rydb. GRO, MICH, OAX, PUE, VER

*Smallanthus apus (S.F. Blake) H. Rob. DGO, JAL, NAY, ZAC Smallanthus maculatus (Cav.) H. Rob. CHIS, CHIH, COAH, COL, CDMX, GTO, GRO, HGO, JAL, MEX, MICH, MOR, NAY, NLE, OAX, PUE, QRO, SLP, SIN, SON, TAB, TAMS, VER, ZAC

*Smallanthus mcvaughii (J.R. Wells) H. Rob. COL, JAL, NAY Smallanthus oaxacanus (Sch. Bip. ex Klatt) H. Rob. CHIS,
GRO, MICH, OAX, VER

*Smallanthus putlanus B.L. Turner OAX

Smallanthus riparius (Kunth) H. Rob. CHIS

Solidago altissima L. COAH, NLE, OAX, SLP, TAMS, VER

Solidago confinis A. Gray BCN

*Solidago durangensis G.L. Nesom DGO

*Solidago ericamerioides G.L. Nesom NLE

Solidago gigantea Aiton NLE, TAMS

*Solidago gypsophila G.L. Nesom COAH

*Solidago hintoniorum G.L. Nesom COAH, DGO, NLE, TAMS

Solidago juliae G.L. Nesom CHIH, COAH, NLE

*Solidago macvaughii G.L. Nesom AGS, ZAC

Solidago missouriensis Nutt. BCN, COAH, NLE, TAMS

*Solidago orientalis (G.L. Nesom) G.L. Nesom COAH, NLE, TAMS

*Solidago paniculata DC. CHIH, CDMX, MEX, MICH, MOR, TLAX

Solidago petiolaris Aiton AGS, COAH, NLE, TAMS

Solidago sempervirens L. TAB, TAMS, VER

Solidago simplex Kunth GTO, HGO, MEX, NLE, QRO, SLP, TAMS, VER, ZAC

Solidago stricta Aiton CHIS, VER

Solidago velutina DC. AGS, BCN, CHIH, COAH, CDMX, DGO, HGO, JAL, MEX, MOR, NLE, OAX, SLP, SON, TAMS, VER, ZAC

Solidago wrightii A. Gray CHIH, COAH, DGO, NLE, SON

* Sphaeromeria martirensis (Wiggins) A.H. Holmgren, L.M. Schultz \& Lowrey BCN

Sphagneticola trilobata (L.) Pruski CAM, CHIS, GRO, OAX, QROO, SIN, TAB, VER, YUC

Spilanthes urens Jacq. JAL, NAY, SIN

Spiracantha cornifolia Kunth CAM, MEX, MICH, QROO, TAB, YUC

Squamopappus skutchii (S.F. Blake) R.K. Jansen, N.A. Harriman \& Urbatsch CHIS

*Stachycephalum mexicanum Sch. Bip. ex Benth. OAX

Stebbinsoseris heterocarpa (Nutt.) K.L. Chambers BCN

*Stenocarpha filiformis (Hemsl.) S.F. Blake DGO, SIN

* Stenocarpha ritovegana B.L. Turner SIN

Stenocephalum jucundum (Gleason) H. Rob. CHIS, OAX

*Stenotus pulvinatus (Moran) G.L. Nesom BCN

*Stephanodoria tomentella (B.L. Rob.) Greene GTO, SLP, TAMS

Stephanomeria diegensis Gottlieb BCN

Stephanomeria exigua Nutt. BCN, CHIH, COAH, SON

*Stephanomeria guadalupensis Brandegee BCN

*Stephanomeria monocephala Moran BCN

Stephanomeria pauciflora (Torr.) A. Nelson BCN, BCS, CHIH, $\mathrm{COAH}, \mathrm{SON}$

Stephanomeria schottii (A Gray) A. Gray SON

Stephanomeria tenuifolia (Torr.) H.M. Hall CHIH, COAH, NLE, SON

Stephanomeria thurberi A. Gray CHIH, SON

Stephanomeria virgata Benth. BCN

*Stevia amblyolepis (B.L. Rob.) B.L. Rob. CHIH, DGO

* Stevia anadenotricha (B.L. Rob.) Grashoff CHIH, DGO, NAY,

SIN, SON 
* Stevia aschenborniana Sch. Bip. CHIH, COAH, COL, CDMX, GRO, HGO, JAL, MEX, MICH, MOR, NAY, NLE, OAX, PUE, QRO, SIN, SON

*Stevia baccharifolia B.L. Turner JAL, ZAC

* Stevia berlandieri A. Gray COAH, DGO, GTO, HGO, NLE, OAX, QRO, SLP, SIN, TAMS, VER, ZAC

*Stevia burrana B.L. Turner COAH

*Stevia caltepecana B.L. Turner GRO, OAX, PUE

* Stevia calzadana B.L. Turner GRO, OAX

Stevia caracasana DC. AGS, CHIS, CHIH, COAH, COL, DGO, GTO, GRO, HGO, JAL, MEX, MICH, MOR, NAY, OAX, PUE, QRO, SLP, SIN, SON, TAMS, TLAX, VER, ZAC

*Stevia clinopodioides Greenm. CDMX, MEX, MICH, MOR, OAX, PUE, TLAX, VER

*Stevia coahuilensis Soejima \& Yahara COAH, NLE

* Stevia concordiana B.L. Turner SIN

Stevia connata Lag. CHIS, CDMX, GRO, HGO, MEX, MICH, MOR, OAX, PUE, QRO, VER

* Stevia constricta (Grashoff) Soejima, Yahara \& K. Watanabe SIN

*Stevia cordifolia Benth. HGO, VER

* Stevia crassifolia Soejima \& Yahara OAX

*Stevia cruzii Grashoff OAX, PUE

* Stevia chiapensis Grashoff CHIS, OAX

* Stevia chilapensis Soejima \& Yahara GRO

* Stevia decumbens (B.L. Rob. \& Greenm.) Greene OAX, PUE

Stevia deltoidea Greene AGS, CDMX, GTO, GRO, HGO, JAL,

MEX, MICH, MOR, OAX, PUE, QRO, SLP, TLAX, VER

* Stevia dictyophylla B.L. Rob. AGS, GTO, JAL, MEX, MICH,

NAY, SLP, ZAC

* Stevia ecatepecana Soejima, Yahara \& K. Watanabe OAX

Stevia elatior Kunth CHIS, CDMX, GTO, GRO, HGO, JAL, MEX, MICH, MOR, NAY, NLE, OAX, PUE, QRO, SLP, SIN, TAMS, TLAX, VER, ZAC

* Stevia enigmatica B.L. Turner GRO, OAX

* Stevia ephemera Grashoff GRO, OAX, PUE

*Stevia eupatoria (Spreng.) Willd. COAH, CDMX, GTO, GRO, HGO, JAL, MEX, MICH, MOR, NAY, NLE, PUE, QRO, SLP, TAMS, TLAX, VER, ZAC

* Stevia filodecaballoana Soejima, Yahara \& K. Watanabe GRO

* Stevia glandulosa Hook. \& Arn. CHIH, DGO, JAL, NAY, SIN, SON

*Stevia hintonii (Grashoff) B.L. Turner GRO, MEX

*Stevia hintoniorum B.L. Turner COAH, NLE, TAMS

* Stevia hypomalaca B.L. Rob. GRO, MEX, MICH, MOR

* Stevia iltisiana Grashoff CDMX, GRO, HGO, MEX, MOR, NLE, PUE, QRO, SLP, TLAX, VER

Stevia incognita Grashoff CHIS, COAH, CDMX, GTO, GRO, HGO, MEX, MICH, MOR, NLE, OAX, PUE, QRO, TAMS

*Stevia isomeca Grashoff HGO, MEX, PUE, QRO, VER

*Stevia jaliscensis B.L. Rob. DGO, JAL, MICH, NAY, SIN, ZAC

Stevia jorullensis Kunth AGS, CHIS, COAH, CDMX, DGO, GTO, GRO, HGO, JAL, MEX, MICH, MOR, NLE, OAX, PUE, QRO, SLP, SIN, TAMS, TLAX, VER, ZAC

*Stevia karwinskyana Steud. VER

*Stevia lasioclada Grashoff COL, JAL, NAY, SIN
*Stevia latifolia Benth. COL, GTO, GRO, HGO, JAL, MEX, MICH, MOR, OAX, PUE, QRO, SLP, VER

Stevia lehmannii Hieron. GRO, OAX, PUE

Stevia lemmonii A. Gray CHIH, DGO, SIN, SON

*Stevia liebmannii Sch. Bip. ex Klatt HGO, OAX, PUE, VER

*Stevia lita Grashoff GRO, OAX

Stevia lucida Lag. AGS, CAM, CHIS, COL, DGO, GTO, GRO, HGO, JAL, MEX, MICH, MOR, NAY, NLE, OAX, PUE, QRO,

SLP, SIN, VER, ZAC

*Stevia macvaughii Grashoff COL, JAL

*Stevia martinii B.L. Turner CHIH, SON

*Stevia mascotensis Soejima \& Yahara JAL

* Stevia miahuatlana B.L. Turner OAX

* Stevia micradenia B.L. Rob. COL, DGO, GRO, JAL, MEX, MICH, MOR, NAY, SIN, ZAC

Stevia micrantha Lag. AGS, BCN, BCS, CHIH, COAH, COL, CDMX, DGO, GTO, GRO, HGO, JAL, MEX, MICH, MOR, NAY, NLE, PUE, QRO, SLP, SIN, SON, TLAX, VER, ZAC

Stevia microchaeta Sch. Bip. CHIS, GRO, OAX, PUE, VER

*Stevia mitopoda B.L. Rob. GRO, OAX, PUE

*Stevia monardifolia Kunth CHIS, COL, CDMX, DGO, GTO, GRO, HGO, JAL, MEX, MICH, MOR, NAY, OAX, PUE, QRO, SLP, SIN, TAMS, TLAX, VER

*Stevia myricoides McVaugh COL, DGO, JAL, MICH, NAY, SIN, ZAC

* Stevia nelsonii B.L. Rob. COL, GTO, JAL, MEX, MICH, QRO

*Stevia neurophylla B.L. Rob. \& Greenm. GRO, OAX

* Stevia oaxacana Soejima \& Yahara OAX

* Stevia occidentalis (Grashoff) Soejima, Yahara \& K. Watanabe COL, DGO, GRO, JAL, MICH, SIN

*Stevia oligophylla Soejima \& Yahara DGO, GRO, MEX, OAX, SIN

*Stevia origanoides Kunth AGS, BCN, BCS, CHIS, CHIH, COL, CDMX, DGO, GTO, GRO, HGO, JAL, MEX, MICH, MOR, NAY, NLE, OAX, PUE, QRO, SLP, SIN, SON, TAMS, TLAX, VER, YUC, ZAC

*Stevia orizabensis B.L. Rob. MEX, TAMS, VER

*Stevia ovalis (B.L. Rob.) B.L. Rob. JAL, NAY

Stevia ovata Willd. AGS, BCS, CHIS, CHIH, COAH, COL, CDMX, DGO, GTO, GRO, HGO, JAL, MEX, MICH, MOR, NAY, NLE, OAX, PUE, QRO, SLP, SIN, SON, TAMS, TLAX, VER, ZAC

*Stevia palmeri A. Gray CHIH, DGO, JAL, SIN, SON, ZAC

*Stevia pelophila S.F. Blake DGO, SIN

* Stevia perfoliata Cronquist GRO, MICH, OAX

*Stevia phlebophylla A. Gray JAL, ZAC

* Stevia pilosa Lag. AGS, CHIH, COAH, CDMX, DGO, GTO, HGO, JAL, MEX, MICH, MOR, NLE, PUE, QRO, SLP, TAMS, TLAX, VER, ZAC

Stevia plummerae A. Gray CHIH, DGO, SON

Stevia polycephala Bertol. CHIS, GRO, OAX, VER

* Stevia porphyrea McVaugh AGS, CHIH, COAH, CDMX, DGO, GTO, GRO, HGO, JAL, MEX, MICH, MOR, NLE, OAX, PUE, QRO, SLP, TAMS, TLAX, VER, ZAC

* Stevia porphyreoides Yahara \& Soejima CHIH, DGO, JAL

* Stevia potosina Soejima, Yahara \& K. Watanabe COAH, NLE

* Stevia pratheri B.L. Turner CHIS 
* Stevia puricana B.L. Turner SON

* Stevia purpusii B.L. Rob. AGS, COAH, CDMX, GTO, GRO, HGO, JAL, MEX, MICH, MOR, NLE, OAX, PUE, QRO, SLP, TLAX, VER, ZAC

*Stevia pyrolifolia Schltdl. HGO, NLE, OAX, PUE, QRO, SLP, VER

* Stevia quiexobra B.L. Turner OAX

* Stevia reinana B.L. Turner SON

* Stevia reticulata Grashoff DGO, JAL, NAY, ZAC

* Stevia revoluta B.L. Rob. OAX, PUE

* Stevia rosei B.L. Rob. AGS, DGO, JAL, NAY, SIN, ZAC

* Stevia rotundifolia Soejima, Yahara \& K. Watanabe JAL, ZAC

* Stevia rzedowskii McVaugh JAL, ZAC

* Stevia sahuaribana B.L. Turner SON

Stevia salicifolia Cav. AGS, CHIH, COAH, COL, CDMX, DGO, GTO, HGO, JAL, MEX, MICH, MOR, NLE, OAX, PUE, QRO, SLP, SIN, SON, TAMS, TLAX, VER, ZAC

*Stevia scabrella Benth. CHIH, DGO, JAL, MEX, MOR, NAY, SIN, SON, ZAC

* Stevia scabrelloides Soejima \& Yahara DGO, SIN

*Stevia schiblii B.L. Turner OAX

Stevia seemannii Sch. Bip. CHIS, COL, DGO, GRO, JAL, MEX,

MICH, NAY, OAX, SIN, VER, ZAC

*Stevia seemannioides Grashoff GRO, OAX

* Stevia seleriana B.L. Rob. NAY, OAX

* Stevia serboana B.L. Turner OAX

Stevia serrata Cav. AGS, CHIS, CHIH, COAH, COL, CDMX, DGO, GTO, GRO, HGO, JAL, MEX, MICH, MOR, NAY, NLE, OAX, PUE, QRO, SLP, SIN, SON, TAMS, TLAX, VER, ZAC *Stevia stolonifera Yahara \& Soejima AGS, COL, JAL, MICH, ZAC

* Stevia strotheriana B.L. Turner CHIH, DGO, SIN, SON

Stevia suaveolens Lag. AGS, BCS, CHIS, CDMX, DGO, GTO, GRO, HGO, JAL, MEX, MICH, MOR, OAX, PUE, QRO, SLP, SIN, TAMS, TLAX, VER, ZAC

*Stevia subpubescens Lag. AGS, CHIS, COL, CDMX, DGO, GTO, GRO, HGO, JAL, MEX, MICH, MOR, NAY, OAX, PUE, SLP, SIN, SON, TAMS, TLAX, VER, ZAC

*Stevia talpensis Grashoff JAL

* Stevia tephra B.L. Rob. AGS, CHIH, COAH, CDMX, DGO, GTO, GRO, HGO, JAL, MEX, MOR, NLE, PUE, QRO, SLP, TAMS, TLAX, VER, ZAC

Stevia tephrophylla S.F. Blake CHIS, OAX

* Stevia tomentosa Kunth AGS, CHIS, COAH, CDMX, DGO, GTO, HGO, JAL, MEX, MICH, MOR, NLE, OAX, PUE, QRO,

SLP, TAMS, TLAX, VER, ZAC

* Stevia totalcoana B.L. Turner VER

* Stevia triangularis Grashoff GRO

* Stevia trifida Lag. AGS, COL, DGO, GTO, GRO, JAL, MEX, MICH, MOR, NAY, OAX, PUE, SIN, SON, ZAC

Stevia triflora DC. CHIS, DGO, GRO, HGO, JAL, MEX, MICH, MOR, OAX, PUE, SIN

* Stevia urceolata Grashoff JAL

* Stevia vacana B.L. Turner COAH

* Stevia velutinella Grashoff GRO

* Stevia vernicosa Greenm. COL, JAL, MEX, MOR

* Stevia viejoana Soejima, Yahara \& K. Watanabe NLE, TAMS
* Stevia villaregalis McVaugh DGO, JAL, NAY, ZAC

Stevia viscida Kunth AGS, CHIH, COAH, COL, CDMX, DGO, GTO, GRO, HGO, JAL, MEX, MICH, MOR, NAY, NLE, OAX, PUE, QRO, SLP, SIN, SON, TAMS, TLAX, VER, ZAC

* Stevia zacatecana McVaugh JAL, ZAC

* Stevia zaragozana B.L. Turner NLE

* Stevia zephyrantha Grashoff GRO

*Steviopsis adenosperma (Sch. Bip.) B.L. Turner DGO, GTO, GRO, JAL, MEX, MICH, MOR, NAY, OAX, QRO, SIN, ZAC

*Steviopsis amblyolepis (B.L. Rob.) R.M. King \& H. Rob. GRO, MOR, PUE

*Steviopsis dryophila (B.L. Rob.) B.L. Turner DGO, GTO, JAL, NAY, NLE, SLP, SIN, ZAC

Steviopsis fendleri (A. Gray) B.L. Turner CHIH, COAH, DGO, SON

*Steviopsis nesomii B.L. Turner NLE, TAMS

*Steviopsis rapunculoides (DC.) R.M. King \& H. Rob. DGO, GTO, JAL, MICH, NAY, QRO, ZAC

*Steviopsis vigintiseta (DC.) R.M. King \& H. Rob. GRO, MEX, MOR, OAX, PUE

*Stramentopappus congestiflorus Redonda-Martínez \& Villaseñor OAX

*Stramentopappus pooleae (B.L. Turner) H. Rob. \& V.A. Funk OAX

*Strotheria gypsophila B.L. Turner COAH, NLE, SLP

Struchium sparganophorum (L.) Kuntze CHIS, TAB

Stylocline gnaphaloides Nutt. BCN, BCS, SON

Stylocline micropoides A. Gray BCN, CHIH, SON

Stylocline sonorensis Wiggins SON

Symphyotrichum bullatum (Sch. Bip. ex Klatt) G.L. Nesom CAM, CHIS, CHIH, COAH, OAX, QROO, VER, YUC

*Symphyotrichum carnerosanum (S. Watson) G.L. Nesom COAH, NLE, TAMS

Symphyotrichum drummondii (Lindl.) G.L. Nesom COAH

Symphyotrichum ericoides (L.) G.L. Nesom CHIH, COAH, HGO, NLE, VER

Symphyotrichum expansum (Poepp. ex Spreng.) G.L. Nesom AGS, BCN, BCS, CAM, CHIS, CHIH, COAH, COL, CDMX, DGO, GTO, GRO, HGO, JAL, MEX, MICH, MOR, NAY, NLE, OAX, PUE, QRO, QROO, SLP, SIN, SON, TAB, TAMS, TLAX, VER, YUC, ZAC

Symphyotrichum falcatum (Lindl.) G.L. Nesom CHIH, COAH, DGO, NLE, SLP, SON

Symphyotrichum frondosum (Nutt.) G.L. Nesom BCN

*Symphyotrichum gypsophilum (B.L. Turner) G.L. Nesom COAH, NLE

*Symphyotrichum hintonii (G.L. Nesom) G.L. Nesom GRO, $\mathrm{MICH}$

Symphyotrichum laeve (L.) A. Löve \& D. Löve COAH, OAX, QRO, VER, YUC

Symphyotrichum moranense (Kunth) G.L. Nesom AGS, CHIS, CHIH, COL, CDMX, DGO, GTO, GRO, HGO, JAL, MEX, MICH, MOR, NAY, OAX, PUE, QRO, SLP, SIN, TLAX, VER, ZAC

Symphyotrichum oblongifolium (Nutt.) G.L. Nesom COAH, TAMS

Symphyotrichum oolentangiense (Riddell) G.L. Nesom COAH, 


\section{NLE, TAMS}

*Symphyotrichum potosinum (A. Gray) G.L. Nesom AGS, CHIH, DGO, GTO, GRO, HGO, JAL, MEX, MICH, OAX, PUE, SLP, SON, VER, ZAC

Symphyotrichum praealtum (Poir.) G.L. Nesom CHIH, COAH, NLE, TAMS

*Symphyotrichum schaffneri (Sch. Bip. ex S.D. Sundb. \& A.G. Jones) G.L. Nesom HGO, PUE, VER

Symphyotrichum spathulatum (Lindl.) G.L. Nesom BCN

Symphyotrichum trilineatum (Sch. Bip. ex Klatt) G.L. Nesom CHIS, GTO, GRO, HGO, MEX, NLE, OAX, PUE, QRO, SLP, TAMS

Synedrella nodiflora (L.) Gaertn. CAM, CHIS, COL, DGO, GRO, JAL, MEX, MICH, MOR, NAY, OAX, QROO, TAB, VER, YUC

*Tagetes arenicola Panero \& Villaseñor GRO, OAX

*Tagetes coronopifolia Willd. CHIS, COAH, CDMX, DGO, HGO, MEX, MOR, PUE, QRO, TLAX, VER

*Tagetes epapposa B.L. Turner DGO, SIN

Tagetes erecta L. AGS, BCS, CAM, CHIS, COAH, COL, CDMX, DGO, GTO, GRO, HGO, JAL, MEX, MICH, MOR, NAY, OAX, PUE, QRO, QROO, SLP, SIN, SON, TAB, TAMS, TLAX, VER, YUC, ZAC

Tagetes filifolia Lag. BCS, CHIS, CHIH, COL, CDMX, DGO, GTO, GRO, HGO, JAL, MEX, MICH, MOR, NAY, OAX, PUE, QRO, SLP, SIN, SON, TAB, TAMS, TLAX, VER, ZAC

Tagetes foetidissima DC. CHIS, CHIH, COL, CDMX, DGO, GTO, GRO, HGO, JAL, MEX, MICH, MOR, NAY, OAX, PUE, QRO, SIN, TAMS, TLAX, VER, ZAC

*Tagetes hartwegii Greenm. JAL, NAY, SLP, ZAC

Tagetes jaliscensis Greenm. AGS, CHIS, CHIH, CDMX, DGO, GRO, JAL, MEX, MICH, MOR, NAY, OAX, PUE, SIN, SON, VER, ZAC

*Tagetes lacera Brandegee BCS

Tagetes lemmonii A. Gray CHIH, SON

*Tagetes linifolia Seaton OAX, PUE, VER

Tagetes lucida Cav. AGS, CAM, CHIS, CHIH, COAH, COL, CDMX, DGO, GTO, GRO, HGO, JAL, MEX, MICH, MOR, NAY, NLE, OAX, PUE, QRO, QROO, SLP, SIN, SON, TAB, TAMS, TLAX, VER, ZAC

*Tagetes lunulata Ortega AGS, COL, CDMX, DGO, GTO, GRO, HGO, JAL, MEX, MICH, MOR, NAY, OAX, PUE, QRO, SLP, SIN, TLAX, VER, ZAC

Tagetes micrantha Cav. AGS, BCS, CHIS, CHIH, COAH, COL, CDMX, DGO, GTO, GRO, HGO, JAL, MEX, MICH, MOR, NAY, OAX, PUE, QRO, SLP, SIN, SON, TAMS, TLAX, VER, ZAC

Tagetes minuta L. SON

*Tagetes moorei $\mathrm{H}$. Rob. GTO, HGO, QRO, SLP

*Tagetes mulleri S.F. Blake COAH, NLE

Tagetes nelsonii Greenm. CHIS

*Tagetes oaxacana B.L. Turner OAX

*Tagetes palmeri A. Gray CHIH, DGO, SLP, SIN, SON

*Tagetes parryi A. Gray HGO, QRO, SLP

*Tagetes persicifolius (Benth.) B.L. Turner COL, DGO, GTO, HGO, JAL, MEX, MICH, NAY, OAX, QRO

*Tagetes pringlei $\mathrm{S}$. Watson AGS, CHIH, COL, DGO, GTO,
JAL, MEX, MICH, QRO, SON, ZAC

*Tagetes stenophylla B.L. Rob. COL, GRO, JAL, MEX, MICH, MOR, OAX, SIN

Tagetes subulata Cerv. AGS, BCS, CHIS, CHIH, COL, CDMX, DGO, GTO, GRO, JAL, MEX, MICH, MOR, NAY, OAX, QRO, SIN, SON, ZAC

Tagetes tenuifolia Cav. AGS, CAM, CHIS, CHIH, COL, CDMX, DGO, GTO, GRO, HGO, JAL, MEX, MICH, MOR, NAY, OAX, PUE, QRO, QROO, SLP, SIN, TLAX, VER, YUC, ZAC

Tamaulipa azurea (DC.) R.M. King \& H. Rob. COAH, HGO, NLE, QRO, SLP, TAMS, VER

*Tehuana calzadae Panero \& Villaseñor OAX

*Telanthophora andrieuxii (DC.) H. Rob. \& Brettell CDMX, GRO, HGO, MEX, MICH, OAX, QRO, SLP, TAMS, VER

Telanthophora cobanensis (J.M. Coult.) H. Rob. \& Brettell CHIS, HGO, OAX, VER

Telanthophora grandifolia (Less.) H. Rob. \& Brettell CHIS, COL, GRO, HGO, JAL, OAX, PUE, QRO, SLP, VER

*Telanthophora liebmannii (Buchinger ex Klatt) H. Rob. \& Brettell OAX

*Telanthophora standleyi (Greenm.) H. Rob. \& Brettell COL, GRO, JAL, MICH

Telanthophora uspantanensis (J.M. Coult.) H. Rob. \& Brettell CHIS, OAX, PUE, VER

*Tetrachyron brandegeei (Greenm.) Wussow \& Urbatsch OAX, PUE

*Tetrachyron chimalapanum B.L. Turner OAX, VER

*Tetrachyron discolor (A. Gray) Wussow \& Urbatsch HGO, QRO, SLP, VER

*Tetrachyron grayi (Klatt) Wussow \& Urbatsch NLE, SLP, TAMS

*Tetrachyron manicatum Schltdl. OAX, VER

*Tetrachyron omissum Rzed. \& Calderón QRO, SLP

Tetrachyron orizabaensis Sch. Bip. ex Klatt CAM, CHIS, HGO,

OAX, PUE, QRO, SLP, VER

*Tetrachyron torresii B.L. Turner OAX

*Tetrachyron websteri (Wussow \& Urbatsch) B.L. Turner HGO, QRO, SLP, TAMS, VER

Tetradymia comosa A. Gray BCN

Tetragonotheca texana A. Gray \& Engelm. CHIH, COAH, NLE, TAMS

Tetraneuris linearifolia (Hook.) Greene COAH, NLE, SLP, TAMS

Tetraneuris scaposa (DC.) Greene CHIH, COAH, DGO, NLE, SLP, TAMS, ZAC

Tetraneuris turneri (K.F. Parker) K.F. Parker COAH

Thelesperma filifolium (Hook.) A. Gray COAH, NLE, QRO, SLP

*Thelesperma graminiformis (Sherff) Melchert NLE, TAMS

Thelesperma longipes A. Gray AGS, CHIH, COAH, DGO, NLE, SLP, SON, TAMS, ZAC

Thelesperma megapotamicum (Spreng.) Kuntze AGS, CHIH, COAH, DGO, NLE, SLP, SON, TAMS, ZAC

*Thelesperma muelleri (Sherff) Melchert NLE, TAMS

*Thelesperma scabridulum S.F. Blake COAH, NLE, TAMS

*Thelesperma shinnersii Flyr COAH

Thelesperma simplicifolium A. Gray CHIH, COAH, DGO, JAL, 
NLE, SLP, TAMS, ZAC

*Thelesperma subaequale S.F. Blake COAH, NLE

Thymophylla acerosa (DC.) Strother AGS, CHIH, COAH, DGO, GTO, HGO, JAL, MICH, NLE, QRO, SLP, SIN, SON, TAMS, VER, ZAC

*Thymophylla aurantiaca (Brandegee) Rydb. OAX, PUE Thymophylla aurea (A. Gray) Greene AGS, CHIH, COAH, DGO, NLE, SLP, ZAC

Thymophylla concinna (A. Gray) Strother BCN, SON

*Thymophylla gentryi (M.C. Johnst.) Strother COAH, DGO, SIN

Thymophylla greggii A. Gray CHIH, COAH, NLE, SLP

*Thymophylla gypsophila (B.L. Turner) Strother COAH

Thymophylla micropoides (DC.) Strother CHIH, COAH, NLE, SLP, TAMS

*Thymophylla mutica (M.C. Johnst.) Strother SLP, TAMS, VER

Thymophylla pentachaeta (DC.) Small AGS, BCN, CHIH, COAH, DGO, GTO, HGO, JAL, MEX, MICH, NAY, NLE, QRO, SLP, SON, TAMS, VER, ZAC

Thymophylla setifolia Lag. AGS, CHIH, COAH, DGO, GTO, HGO, JAL, MICH, NLE, PUE, QRO, SLP, SON, TAMS, VER, ZAC

*Thymophylla tenuifolia (Cass.) Rydb. AGS, CHIH, COAH, CDMX, DGO, GTO, HGO, JAL, MEX, MICH, NLE, PUE, QRO, SLP, TAMS, TLAX, VER, ZAC

Thymophylla tenuiloba (DC.) Small COAH, NLE, TAMS

Thymophylla tephroleuca (S.F. Blake) Strother TAMS

*Tithonia auriculata (Brandegee) S.F. Blake CHIH, COL, JAL, NAY, SIN, SON

*Tithonia brachypappa B.L. Rob. SLP, TAMS, VER

*Tithonia calva Sch. Bip. CHIH, DGO, JAL, NAY, SIN, SON, ZAC

Tithonia diversifolia (Hemsl.) A. Gray CAM, CHIS, COL, GRO, JAL, MEX, MICH, NAY, OAX, PUE, QRO, QROO, SLP, TAB, VER, YUC

*Tithonia fruticosa Canby \& Rose CHIH, DGO, SIN, SON

*Tithonia koelzii McVaugh COL, JAL

Tithonia longiradiata (Bertol.) S.F. Blake CHIS, HGO, OAX, PUE, QRO, SLP, VER

*Tithonia paneroi (B.L. Turner) E.E. Schill. \& Panero OAX

*Tithonia pedunculata Cronquist OAX

Tithonia rotundifolia (Mill.) S.F. Blake CAM, CHIS, COAH, COL, DGO, GTO, GRO, JAL, MEX, MICH, MOR, NAY, OAX, PUE, QROO, VER, YUC, ZAC

Tithonia thurberi A. Gray CHIH, SON

Tithonia tubiformis (Jacq.) Cass. AGS, CAM, CHIS, CHIH, COAH, COL, CDMX, DGO, GTO, GRO, HGO, JAL, MEX, MICH, MOR, NAY, NLE, OAX, PUE, QRO, SLP, SIN, SON, TAB, TAMS, TLAX, VER, ZAC

*Tomentaurum niveum (S. Watson) G.L. Nesom CHIH, SON Townsendia exscapa (Richardson) Porter CHIH, COAH, DGO, SON

*Townsendia mexicana A. Gray AGS, CHIH, COAH, CDMX, HGO, MEX, MICH, MOR, NLE, QRO, SLP, TAMS, TLAX, VER, ZAC

*Trichocoronis sessilifolia (Schauer) B.L. Rob. GTO, JAL,
MEX, MICH, OAX, QRO

Trichocoronis wrightii (A. Gray) A. Gray BCS, SLP, TAMS

*Trichocoryne connata S.F. Blake DGO, SIN

Trichoptilium incisum (A. Gray) A. Gray BCN, BCS, SON

Trichospira verticillata (L.) S.F. Blake CHIS, OAX, TAB, VER

*Tridax balbisioides (Kunth) A. Gray AGS, CHIH, DGO, GTO, GRO, HGO, JAL, MEX, MICH, MOR, OAX, QRO, SLP, TAMS, ZAC

*Tridax bicolor $\mathrm{A}$. Gray $\mathrm{CHIH}, \mathrm{COAH}$

*Tridax bilabiata A.M. Powell GRO

*Tridax brachylepis Hemsl. GRO, MEX, MICH, MOR, OAX

*Tridax candidissima A. Gray SLP

*Tridax coronopifolia (Kunth) Hemsl. AGS, CHIS, COAH, COL, CDMX, DGO, GTO, GRO, HGO, JAL, MEX, MICH, MOR, NAY, NLE, OAX, PUE, QRO, SLP, TAMS, TLAX, VER, ZAC

*Tridax dubia Rose COL, JAL, MICH, NAY

*Tridax durangensis García-Arév. DGO

*Tridax erecta A. Gray CHIH, SON

*Tridax hintoniorum B.L. Turner NLE, TAMS

*Tridax luisana Brandegee OAX, PUE

*Tridax mexicana A.M. Powell AGS, COL, DGO, GTO, GRO, JAL, MEX, MICH, MOR, NAY, OAX, PUE

*Tridax moore $i \mathrm{H}$. Rob. HGO

*Tridax oaxacana B.L. Turner OAX

*Tridax obovata Turcz. GRO, OAX

*Tridax palmeri A. Gray AGS, GTO, HGO, JAL, MEX, MICH, OAX, PUE, QRO, SLP, VER, ZAC

*Tridax paneroi B.L. Turner OAX

*Tridax petrophila B.L. Rob. \& Greenm. AGS, GTO, JAL, ZAC Tridax platyphylla B.L. Rob. CHIS, GRO, JAL, MEX, MICH, NAY, OAX, SIN

Tridax procumbens L. CAM, CHIS, COAH, COL, CDMX, DGO, GTO, GRO, HGO, JAL, MEX, MICH, MOR, NAY, NLE, OAX, PUE, QRO, QROO, SLP, SIN, SON, TAB, TAMS, TLAX, VER, YUC, ZAC

*Tridax purpusii Brandegee GRO, VER

*Tridax rosea Sch. Bip. ex B.L. Rob. \& Greenm. CDMX, GTO, HGO, MEX, QRO, SLP, TAMS, VER

*Tridax serboana B.L. Turner OAX

*Tridax tenuifolia Rose CHIH, SIN, SON

*Tridax trilobata (Cav.) Hemsl. CDMX, GTO, GRO, HGO, MEX, MICH, QRO, TLAX

*Tridax yecorana B.L. Turner CHIH, SON

*Trigonospermum adenostemmoides Less. CHIS, VER

*Trigonospermum alexandri Rzed., Calderón \& Pérez-Calix $\mathrm{MICH}$

Trigonospermum annuum McVaugh \& Lask. AGS, CHIH, CDMX, DGO, GTO, GRO, HGO, JAL, MEX, MICH, NLE, QRO, SLP, SON, ZAC

*Trigonospermum auriculatum B.L. Turner GRO

*Trigonospermum hintoniorum B.L. Turner NLE, TAMS

Trigonospermum melampodioides DC. CHIS, CHIH, COL, DGO, GTO, GRO, HGO, JAL, MEX, MICH, MOR, NAY, OAX, SLP, SIN, SON, VER

*Trixis alata D. Don GRO, MEX, MICH, MOR, OAX

*Trixis angustifolia DC. AGS, CHIH, COAH, DGO, GTO, 
HGO, JAL, MEX, MICH, NAY, NLE, QRO, SLP, SON, TAMS, ZAC

*Trixis anomala B.L. Turner CHIS

*Trixis calcicola B.L. Rob. GRO, MICH, PUE

Trixis californica Kellogg AGS, BCN, BCS, CHIH, COAH, DGO, NLE, SLP, SIN, SON, TAMS, ZAC

Trixis chiapensis C.E. Anderson CHIS

*Trixis grandibracteata C.E. Anderson GRO, OAX

*Trixis haenkei Sch. Bip. AGS, DGO, JAL, MICH, NAY, SIN, ZAC

*Trixis hintoniorum B.L. Turner OAX

*Trixis hyposericea $\mathrm{S}$. Watson AGS, COL, DGO, JAL, MICH, NAY, SIN, ZAC

Trixis inula Crantz CAM, CHIS, COAH, COL, GTO, GRO, HGO, JAL, MEX, MICH, NAY, NLE, OAX, PUE, QRO, QROO, SLP, TAMS, VER, YUC, ZAC

*Trixis jaliscana B.L. Turner JAL

*Trixis megalophylla Greenm. GRO, MEX, MICH, MOR, OAX, PUE

*Trixis mexicana Lex. COL, GTO, GRO, HGO, JAL, MEX, MICH, NAY, OAX, QRO, SLP, ZAC

*Trixis michuacana Lex. AGS, COL, CDMX, DGO, GTO, GRO, HGO, JAL, MEX, MICH, MOR, NAY, OAX, QRO, SLP, SIN, SON, TAMS, ZAC

Trixis nelsonii Greenm. CHIS, OAX

*Trixis parviflora C.E. Anderson GRO, OAX

*Trixis pringlei B.L. Rob. \& Greenm. GRO, MEX, MOR, OAX, PUE, VER

*Trixis pterocaulis B.L. Rob. \& Greenm. BCS, COL, GRO, JAL, MICH, NAY, SIN, SON

*Trixis silvatica B.L. Rob. \& Greenm. OAX

Tuxtla pittieri (Greenm.) Villaseñor \& Strother VER

*Urbinella palmeri Greenm. DGO, SIN

Uropappus lindleyi (DC.) Nutt. BCN, BCS, SON

*Varilla mexicana A. Gray CHIH, COAH, DGO, ZAC

Varilla texana A. Gray COAH, NLE, TAMS

Venegasia carpesioides DC. BCN

*Verbesina abietifolia Rzed. \& Calderón QRO, SLP

*Verbesina abscondita Klatt GRO, MEX, MICH, OAX, PUE

*Verbesina acapulcensis B.L. Rob. \& Greenm. GRO

Verbesina agricolarum Standl. \& Steyerm. CHIS

*Verbesina alcabrerae Rzed. GRO

*Verbesina angustifolia (Benth.) S.F. Blake AGS, CHIH, COL, DGO, GTO, GRO, JAL, MEX, MICH, NAY, OAX, QRO, SIN, $\mathrm{ZAC}$

*Verbesina angustissima S.F. Blake DGO, JAL, NAY

*Verbesina apiculata J.R. Coleman SIN

Verbesina apleura S.F. Blake CHIS

*Verbesina aramberrana B.L. Turner NLE, TAMS

*Verbesina auriculata DC. GRO, OAX, PUE

*Verbesina barrancae M. Harker \& N. Jiménez-R. JAL

*Verbesina bolanosana B.L. Turner JAL, ZAC

*Verbesina breedlovei B.L. Turner CHIS, COL, GRO, JAL, MEX, MICH, OAX

*Verbesina calzadae Panero \& Villaseñor GRO, OAX

*Verbesina callilepis S.F. Blake CHIH, SON

*Verbesina carranzae P. Carrrillo QRO, SLP
*Verbesina coahuilensis A. Gray COAH, NLE, TAMS

*Verbesina corral-diazii B.L. Turner DGO

*Verbesina coulteri A. Gray GTO, HGO, QRO, SLP, TAMS

*Verbesina crassipes B.L. Rob. \& Greenm. OAX

Verbesina crocata (Cav.) Less. CHIS, COAH, COL, DGO, GRO, HGO, JAL, MEX, MICH, MOR, NAY, NLE, OAX, PUE, TAMS, VER, ZAC

*Verbesina cronquistii B.L. Turner CHIS

*Verbesina cuautlensis McVaugh JAL

*Verbesina culminicola McVaugh COL, JAL

*Verbesina curatella McVaugh JAL

*Verbesina chiapensis B.L. Rob. \& Greenm. CHIS, OAX

*Verbesina chihuahuensis A. Gray CHIH, COAH, DGO, GTO,

HGO, NLE, QRO, SLP, SON, VER, ZAC

*Verbesina chilapana B.L. Turner GRO

*Verbesina daviesiae B.L. Turner COAH, NLE, TAMS

Verbesina dissita A. Gray BCN

*Verbesina durangensis B.L. Turner DGO, NAY

*Verbesina elgalloana B.L. Turner GRO

Verbesina encelioides (Cav.) A. Gray AGS, BCN, BCS, CHIH, COAH, DGO, GTO, GRO, HGO, JAL, MEX, MICH, MOR, NLE, OAX, PUE, QRO, SLP, SIN, SON, TAMS, VER, ZAC

*Verbesina erosa Brandegee BCS

*Verbesina etlana B.L. Turner OAX

*Verbesina fastigiata B.L. Rob. \& Greenm. AGS, CHIS, COL, DGO, GRO, HGO, JAL, MEX, MICH, MOR, NAY, OAX, PUE, SIN, TAB, VER, ZAC

*Verbesina fayii B.L. Turner GRO, OAX

*Verbesina felgeri B.L. Turner SON

Verbesina fraseri Hemsl. VER

*Verbesina furfuracea McVaugh COL, JAL, MICH

*Verbesina fusiformis McVaugh JAL, NAY

*Verbesina gentryi Standl. CHIH

Verbesina gigantea Jacq. CAM, CHIS, GRO, MEX, MICH, OAX, QROO, VER, YUC

*Verbesina glaucophylla S.F. Blake JAL

*Verbesina gracilipes B.L. Rob. OAX, PUE

*Verbesina grayii (Sch. Bip.) Benth. ex Hemsl. COL, CDMX, DGO, GRO, JAL, MEX, MICH, MOR, SLP, SIN, TAMS

Verbesina guatemalensis B.L. Rob. \& Greenm. CHIS

*Verbesina guerreroana B.L. Turner GRO

*Verbesina hidalgoana B.L. Turner GTO, HGO, PUE, QRO, VER

*Verbesina hintoniorum B.L. Turner NLE, TAMS

*Verbesina hispida McVaugh JAL, NAY, ZAC

*Verbesina hygrophila Panero \& Villaseñor DGO

Verbesina hypargyrea B.L. Rob. \& Greenm. CHIS, OAX

Verbesina hypoglauca Sch. Bip. ex Klatt CHIS, GRO, HGO,

MEX, MICH, NLE, OAX, PUE, TAMS, VER

*Verbesina hypoleuca A. Gray SLP, ZAC

* Verbesina hypomalaca B.L. Rob. \& Greenm. AGS, COAH, GTO, GRO, HGO, JAL, MEX, MICH, MOR, NLE, OAX, PUE,

QRO, SLP, TAMS, TLAX, VER, ZAC

Verbesina hypsela B.L. Rob. CHIS

*Verbesina jacksonii B.L. Turner DGO, SIN

*Verbesina jimrobbinsi B.L. Turner COL, JAL, MICH

*Verbesina juxtlahuacensis Panero \& Villaseñor GRO, OAX 
*Verbesina kimii B.L. Turner PUE, VER

*Verbesina klattii B.L. Rob. \& Greenm. COL, GRO, JAL, MEX, MICH, MOR

*Verbesina langfordae B.L. Turner NLE

*Verbesina langlassei B.L. Rob. COL, GRO, JAL, MEX, MICH

*Verbesina lanulosa Villarreal \& A.E. Estrada NLE

*Verbesina leptochaeta A. Gray CHIH, SIN

*Verbesina liebmannii Sch. Bip. ex Klatt CDMX, GRO, MEX, OAX

Verbesina lindheimeri B.L. Rob. \& Greenm. COAH

*Verbesina linearis (McVaugh) B.L. Turner JAL

Verbesina longifolia (A. Gray) A. Gray CHIH, COAH, DGO, SON

Verbesina longipes Hemsl. AGS, CHIH, COAH, DGO, HGO, MEX, MICH, NLE, QRO, SLP, SON, TAMS, VER, ZAC

*Verbesina lottiana B.L. Turner \& Olsen COL, DGO, JAL, NAY

*Verbesina lundellii S.F. Blake CHIH, SLP

*Verbesina macdonaldii B.L. Turner OAX

Verbesina macvaughii B.L. Turner OAX

*Verbesina machucana B.L. Turner JAL

*Verbesina madrensis Greenm. DGO, JAL, NAY, ZAC

*Verbesina mexiae B.L. Turner GRO, MEX, MICH, OAX

*Verbesina miahuatlana B.L. Turner OAX

*Verbesina mickelii McVaugh COL, JAL

*Verbesina microcarpa S.F. Blake DGO, SIN

Verbesina microptera DC. COAH, HGO, NLE, QRO, SLP,

TAMS, VER

Verbesina minarum Standl. \& Steyerm. CHIS

*Verbesina mixtecana Brandegee OAX

*Verbesina mollis Kunth AGS, COAH, GTO, HGO, JAL, MICH, NAY, NLE, QRO, SLP, TAMS, ZAC

*Verbesina montanoifolia B.L. Rob. \& Greenm. DGO, GTO, JAL, MEX, MICH, QRO, SLP, SIN

Verbesina myriocephala Sch. Bip. ex Klatt CAM, CHIS, GRO, MEX, MICH, OAX, QROO, TAB, VER

Verbesina nana (A. Gray) B.L. Rob. \& Greenm. COAH, NLE

*Verbesina nayaritensis B.L. Turner NAY, SIN

*Verbesina nelsonii B.L. Rob. \& Greenm. GRO, OAX

*Verbesina neotenoriensis B.L. Turner OAX, PUE

*Verbesina neriifolia Hemsl. CHIS, OAX

*Verbesina oaxacana DC. GRO, MOR, OAX, PUE, VER

Verbesina oerstediana Benth. CHIS, OAX

*Verbesina oligantha B.L. Rob. BCS, CHIS, COL, GRO, JAL, MICH, OAX, PUE, SON

*Verbesina oligocephala I.M. Johnst. BCN, BCS, SON

*Verbesina olsenii B.L. Turner COAH, NLE, TAMS

*Verbesina oncophora B.L. Rob. \& Seaton COL, CDMX, DGO, GTO, GRO, HGO, JAL, MEX, MICH, MOR, NAY, OAX, PUE, QRO, SIN, TLAX, VER

Verbesina oreophila Wooton \& Standl. CHIH, COAH

*Verbesina ortegae S.F. Blake SIN

*Verbesina otophylla S.F. Blake TAMS

*Verbesina ovata (Cav.) A. Gray CDMX, HGO, MEX, MOR, PUE, SLP, TLAX, VER

*Verbesina oxylepis S.F. Blake COL, JAL, MICH, NAY, ZAC

*Verbesina palmeri $\mathrm{S}$. Watson $\mathrm{BCN}$
*Verbesina paneroi B.L. Turner COL, JAL

*Verbesina pantoptera S.F. Blake AGS, COL, DGO, JAL, MEX, MICH, NAY, SIN, ZAC

*Verbesina papasquiara Panero \& Villaseñor DGO

*Verbesina parviflora (Kunth) S.F. Blake AGS, CHIH, COAH, COL, DGO, GTO, HGO, JAL, MEX, MICH, MOR, NAY, PUE, QRO, SLP, SIN, SON, VER, ZAC

*Verbesina pauciflora Hemsl. CHIH, DGO, SIN, SON

*Verbesina pedunculosa (DC.) B.L. Rob. AGS, CDMX, DGO, GTO, HGO, JAL, MEX, MICH, MOR, NAY, QRO, SLP, ZAC

*Verbesina pellucida Villaseñor \& Panero OAX

*Verbesina peninsularis S.F. Blake BCN, BCS

Verbesina persicifolia DC. CHIS, COAH, DGO, GTO, HGO, NLE, OAX, PUE, QRO, SLP, TAB, TAMS, VER

Verbesina perymenioides Sch. Bip. ex Klatt CHIS, GRO, MICH, OAX, PUE, TLAX

*Verbesina petrophila Brandegee OAX, PUE

Verbesina petzalensis Standl. \& Steyerm. CHIS

*Verbesina phyllolepis S.F. Blake CHIS

*Verbesina pietatis McVaugh JAL, MICH, QRO

*Verbesina platanara B.L. Turner JAL, ZAC

*Verbesina platyptera Sch. Bip. ex Klatt COL, GRO, JAL, $\mathrm{MICH}, \mathrm{OAX}$

Verbesina pleistocephala (Donn. Sm.) B.L. Rob. CHIS

*Verbesina potosina B.L. Rob. QRO, SLP, VER

*Verbesina pterocarpha S.F. Blake JAL, MEX, MICH

*Verbesina pterocaula DC. HGO, MEX, MOR, VER

Verbesina punctata B.L. Rob. \& Greenm. CHIS, OAX, VER

*Verbesina purpusii Brandegee PUE, VER

*Verbesina pustulata M.E. Jones BCS

*Verbesina resinosa Klatt OAX

*Verbesina reyesii Panero \& Villaseñor OAX

*Verbesina richardsonii B.L. Turner TAMS

*Verbesina robinsonii (Klatt) Fernald ex B.L. Rob. \& Greenm. GTO, HGO, QRO, SLP, TAMS, VER

*Verbesina rosei B.L. Rob. \& Greenm. DGO, NAY, SIN

*Verbesina rumicifolia B.L. Rob. \& Greenm. COAH

*Verbesina scabrida Rzed. GRO, OAX

*Verbesina scotiodonta S.F. Blake CHIH

*Verbesina seatonii S.F. Blake GTO, MEX, MICH, MOR, PUE, QRO

*Verbesina sericea Kunth \& C.D. Bouché OAX

*Verbesina serrata Cav. AGS, CAM, CHIS, COAH, CDMX, DGO, GTO, GRO, HGO, JAL, MEX, MICH, NAY, OAX, PUE, QRO, SLP, TAB, VER, YUC, ZAC

*Verbesina sinaloensis B.L. Turner SIN

*Verbesina sororia A. Gray SLP

*Verbesina sousae J.J. Fay CHIS, OAX

*Verbesina sphaerocephala A. Gray COL, DGO, GTO, GRO, JAL, MEX, MICH, MOR, NAY, QRO, ZAC

*Verbesina steinmannii P. Carrrillo GTO, QRO, SLP

*Verbesina stenophylla Greenm. GRO, MEX, MOR

*Verbesina strotheri Panero \& Villaseñor CHIS

*Verbesina suberosa P. Carrrillo GTO, JAL

*Verbesina synotis S.F. Blake CHIH, DGO, SON

*Verbesina tamaunuevana B.L. Turner NLE, TAMS

*Verbesina tecolotlana B.L. Turner COL, JAL 
*Verbesina tequilana J.R. Coleman JAL, NAY, ZAC

*Verbesina tetraptera (Ortega) A. Gray COL, CDMX, DGO, GTO, GRO, HGO, JAL, MEX, MICH, MOR, NAY, OAX, PUE, QRO, SLP, TLAX, VER

*Verbesina textitlana B.L. Turner OAX

*Verbesina tiburonensis B.L. Turner SON

*Verbesina torresii B.L. Turner DGO

*Verbesina trilobata B.L. Rob. \& Greenm. CHIS, MICH, OAX, PUE

Verbesina turbacensis Kunth CHIS, COL, DGO, GRO, HGO, JAL, MICH, MOR, NLE, OAX, PUE, QRO, QROO, SLP, SIN, TAB, TAMS, VER

*Verbesina vallartana B.L. Turner \& Olsen COL, JAL, NAY

*Verbesina venosa Greene BCN, BCS

*Verbesina villaregalis McVaugh JAL

*Verbesina villasenorii B.L. Turner OAX

*Verbesina virgata Cav. AGS, CDMX, GTO, GRO, HGO, JAL, MEX, MICH, MOR, OAX, PUE, QRO, SLP, TLAX, VER, ZAC

*Verbesina xanthochlora B.L. Rob. \& Greenm. PUE

*Verbesina xicoana B.L. Turner VER

*Verbesina zaragozana B.L. Turner COAH, NLE

*Vernonanthura cordata (Kunth) H. Rob. COL, GRO, JAL, MEX, MICH, MOR, NAY, PUE

*Vernonanthura cronquistii (S.B. Jones) H. Rob. GRO, OAX

*Vernonanthura hintoniorum (B.L. Turner) H. Rob. NLE, TAMS

*Vernonanthura liatroides (DC.) H. Rob. CHIH, COL, DGO, GTO, GRO, HGO, JAL, MEX, MICH, MOR, NAY, OAX, PUE, QRO, SLP, SIN, SON, TAMS, VER, ZAC

Vernonanthura oaxacana (Sch. Bip. ex Klatt) H. Rob. CHIS, OAX

Vernonanthura patens (Kunth) H. Rob. CAM, CHIS, COL, GRO, HGO, JAL, MEX, MICH, MOR, NAY, OAX, PUE, QRO, QROO, SLP, SIN, TAB, TAMS, VER, YUC, ZAC

*Vernonanthura serratuloides (Kunth) H. Rob. AGS, CHIH, COL, DGO, GTO, JAL, MEX, MICH, MOR, NAY, QRO, SLP, SIN, SON, ZAC

*Vernonanthura sinclairii (Benth.) H. Rob. CDMX, GRO, JAL, MEX, MICH, MOR, NAY, OAX, PUE, SLP, SIN, VER, ZAC

*Vernonia alamanii DC. CAM, COL, CDMX, GTO, GRO,

HGO, JAL, MEX, MICH, MOR, OAX, PUE, QRO, SLP, TAMS,

VER

*Vernonia barclayi H. Rob. \& C.F. Reed SON

*Vernonia bealliae McVaugh AGS, COL, JAL, MICH, ZAC

*Vernonia bolleana Sch. Bip. DGO, NAY, SIN

*Vernonia faustiana G.C. Chapman \& S.B. Jones COAH, SLP

*Vernonia greggii A. Gray COAH, DGO, GTO, HGO, MICH, NLE, QRO, SLP, TAMS, VER, ZAC

*Vernonia joyaliae B.L. Turner SON

*Vernonia karvinskiana DC. GRO, OAX, PUE

Vernonia larseniae B.L. King \& S.B. Jones COAH

Vernonia lindheimeri A. Gray \& Engelm. COAH

Vernonia marginata (Torr.) Raf. CHIH, COAH

*Vigethia mexicana (S. Watson) W.A. Weber COAH, NLE, TAMS

*Viguiera ayutlana B.L. Turner JAL
Viguiera dentata (Cav.) Spreng. AGS, BCN, CAM, CHIS, CHIH, COAH, COL, CDMX, DGO, GTO, GRO, HGO, JAL, MEX, MICH, MOR, NAY, NLE, OAX, PUE, QRO, QROO, SLP, SIN, SON, TAMS, TLAX, VER, YUC, ZAC

*Viguiera huajicoria B.L. Turner NAY, SIN

*Viguiera iltisii B.L. Turner COL, JAL

*Viguiera moreliana B.L. Turner MICH

*Viguiera paneroana Rzed. \& Calderón QRO, SLP

*Viguiera potosina S.F. Blake GTO, NLE, SLP, TAMS, VER

*Viguiera sultepecana Paray MEX

*Viguiera tepoxtlensis Paray MOR

*Villanova achillaeoides (Less.) Less. CDMX, MEX, PUE, TLAX, VER

*Villasenoria orcuttii (Greenm.) B.L. Clark CHIS, OAX, TAB, VER

*Wamalchitamia appressipila (S.F. Blake) Strother CHIS

Wamalchitamia aurantiaca (Klatt) Strother CHIS, OAX

*Wamalchitamia dionysi Strother CHIS

*Wamalchitamia strigosa (DC.) Strother COL, GRO, JAL, MEX, MICH, NAY, OAX, SIN

Wedelia acapulcensis Kunth CAM, CHIS, CHIH, COAH, COL, CDMX, DGO, GTO, GRO, HGO, JAL, MEX, MICH, NAY, NLE, OAX, PUE, QRO, QROO, SLP, SIN, TAB, TAMS, TLAX, VER, YUC

*Wedelia aggregata (Greenm.) B.L. Turner JAL, NAY, SIN, ZAC

*Wedelia cordiformis McVaugh JAL, MICH

*Wedelia chihuahuana B.L. Turner CHIH, SON

*Wedelia gonzaleziarum B.L. Turner DGO, NAY

*Wedelia grayi McVaugh DGO, JAL, NAY, ZAC

*Wedelia greenmanii B.L. Turner CHIH, JAL, SIN, SON

*Wedelia hintoniorum B.L. Turner GRO, MEX, OAX

Wedelia iners (S.F. Blake) Strother CHIS

*Wedelia keilii B.L. Turner JAL, MICH

*Wedelia mexicana (Sch. Bip.) McVaugh DGO, GTO, JAL, NAY, QRO, SIN, ZAC

*Wedelia pimana B.L. Turner CHIH, SON

*Wedelia purpurea (Greenm.) B.L. Turner CHIS, GRO, OAX, PUE

*Wedelia rosei (Greenm.) McVaugh AGS, DGO, JAL, NAY, SIN, ZAC

*Wedelia simsioides McVaugh JAL, NAY

*Wedelia strigosa Hook. \& Arn. COL, GRO, JAL, MICH

*Wedelia talpana B.L. Turner JAL

*Wedelia tegetis Strother DGO, JAL, NAY, SIN

*Wedelia vexata Strother COL, JAL, NAY, SIN

Werneria nubigena Kunth CHIS

*Xanthisma arenarium (Benth.) D.R. Morgan \& R.L. Hartm. BCS

*Xanthisma crutchfieldii (B.L. Turner) D.R. Morgan \& R.L. Hartm. COAH, NLE, SLP, ZAC

Xanthisma gracile (Nutt.) D.R. Morgan \& R.L. Hartm. BCN, CHIH, COAH, DGO, SON

Xanthisma gymnocephalum (DC.) D.R. Morgan \& R.L. Hartm. AGS, CHIH, COAH, CDMX, DGO, GTO, HGO, JAL, MEX, MICH, NAY, NLE, PUE, QRO, SLP, TAMS, TLAX, VER, ZAC 
Xanthisma gypsophilum (B.L. Turner) D.R. Morgan \& R.L. Hartm. CHIH, COAH, DGO, SLP

*Xanthisma johnstonii (S.F. Blake) D.R. Morgan \& R.L. Hartm. COAH, NLE

Xanthisma junceum (Greene) D.R. Morgan \& R.L. Hartm. BCN, SON

*Xanthisma pseudorestiforme B.L. Turner NLE

*Xanthisma restiforme (B.L. Turner) D.R. Morgan \& R.L. Hartm. COAH

*Xanthisma rhizomatum (M.C. Johnst.) D.R. Morgan \& R.L. Hartm. NLE

Xanthisma scabrellum (Greene) G.L. Nesom \& B.L. Turner $\mathrm{BCN}, \mathrm{BCS}, \mathrm{SON}$

Xanthisma spinulosum (Pursh) D.R. Morgan \& R.L. Hartm. AGS, BCN, BCS, CHIH, COAH, DGO, GTO, GRO, HGO, JAL, MICH, NLE, QRO, SLP, SON, TAMS, ZAC

*Xanthisma stenolobum (Greene) D.R. Morgan \& R.L. Hartm. CHIH, DGO

*Xanthisma wigginsii (S.F. Blake) D.R. Morgan \& R.L. Hartm. $\mathrm{BCN}$

Xanthium australe Millsp. \& Sherff TAMS

Xanthium spinosum L. BCN, JAL, SLP, SIN, SON

Xanthium strumarium L. AGS, BCN, BCS, CHIS, CHIH, COAH, COL, CDMX, DGO, GTO, GRO, HGO, JAL, MEX, MICH, MOR, NAY, NLE, OAX, PUE, QRO, SLP, SIN, SON, TAMS, VER, YUC, ZAC

*Xanthocephalum benthamianum Hemsl. AGS, CHIH, DGO, GTO, JAL, MEX, MICH, NLE, SLP, SIN, ZAC

*Xanthocephalum centauroides Willd. CDMX, DGO, GTO, HGO, MEX, MICH, QRO, SLP, VER

*Xanthocephalum durangense M.A. Lane DGO

*Xanthocephalum eradiatum (M.A. Lane) G.L. Nesom CHIH, SON

Xanthocephalum gymnospermoides (A. Gray) Benth. AGS, CHIH, DGO, GTO, JAL, NLE, SLP, SIN, SON, ZAC

*Xanthocephalum humile (Kunth) Benth. \& Hook. f. CDMX, DGO, HGO, MEX, PUE, SLP, TLAX, VER

Xylorhiza frutescens (S. Watson) Greene BCN, BCS

Xylorhiza orcuttii (Vasey \& Rose) Greene BCN

Xylorhiza wrightii (A. Gray) Greene $\mathrm{CHIH}, \mathrm{COAH}$

*Xylovirgata pseudobaccharis (S.F. Blake) Urbatsch \& R.P. Roberts COAH, DGO

*Zaluzania augusta (Lag.) Sch. Bip. AGS, CDMX, DGO, GTO, HGO, JAL, MEX, MICH, MOR, NAY, QRO, SLP, TLAX, VER, ZAC

*Zaluzania delgadoana B.L. Turner DGO, JAL, ZAC

*Zaluzania discoidea A. Gray CHIH, DGO

*Zaluzania durangensis B.L. Turner CHIH, DGO

Zaluzania grayana B.L. Rob. \& Greenm. CHIH, SON

*Zaluzania megacephala Sch. Bip. COAH, CDMX, DGO, GTO, HGO, MEX, MOR, NAY, NLE, QRO, SLP, TAMS, VER *Zaluzania mollissima A. Gray AGS, COAH, DGO, NLE, SLP, ZAC

*Zaluzania montagnifolia (Sch. Bip.) Sch. Bip. GRO, MEX, MICH, MOR, OAX, PUE, VER

*Zaluzania pringlei Greenm. GRO, MEX, MOR, OAX, PUE

*Zaluzania subcordata W.M. Sharp OAX, PUE, VER
*Zaluzania triloba (Ortega) Pers. AGS, COAH, CDMX, GTO, HGO, JAL, MEX, NLE, OAX, PUE, QRO, SLP, SON, TAMS, TLAX, VER, ZAC

*Zandera andersoniae (B.L. Turner) D.L. Schulz MEX, MICH, MOR, OAX

*Zandera blakei (McVaugh \& Lask.) D.L. Schulz GRO, MOR, OAX

*Zandera hartmanii (B.L. Turner) D.L. Schulz DGO, SIN Zexmenia serrata La Llave CAM, CHIS, OAX, TAB, VER Zexmenia virgulta Klatt CHIS, TAB

Zinnia acerosa (DC.) A. Gray AGS, CHIH, COAH, DGO, HGO, JAL, NLE, QRO, SLP, SON, TAMS, ZAC

Zinnia americana (Mill.) Olorode \& A.M. Torres CHIS, COL, DGO, GTO, GRO, JAL, MEX, MICH, MOR, NAY, OAX, QRO, SLP, VER, ZAC

*Zinnia angustifolia Kunth AGS, CHIH, COAH, COL, DGO, GTO, GRO, JAL, MEX, MICH, NAY, QRO, SLP, SIN, SON, ZAC

Zinnia anomala A. Gray COAH, NLE, SLP, ZAC

*Zinnia bicolor (DC.) Hemsl. AGS, CHIH, COL, DGO, GTO, JAL, MICH, NAY, QRO, SLP, ZAC

*Zinnia citrea A.M. Torres COAH, SLP, TAMS

*Zinnia coahuilana B.L. Turner COAH

Zinnia elegans Jacq. CAM, CHIS, CHIH, COAH, COL, DGO, GRO, HGO, JAL, MEX, MICH, MOR, NAY, NLE, OAX, PUE, QRO, QROO, SLP, SIN, SON, TAB, TAMS, VER, YUC, ZAC *Zinnia flavicoma (DC.) Olorode \& A.M. Torres CHIS, COL, GRO, JAL, MEX, MICH, MOR, OAX

Zinnia grandiflora Nutt. CHIH, COAH, DGO, NAY, NLE, SON, TAMS, ZAC

*Zinnia guanajuatensis (Calderón \& Rzed.) B.L. Turner GTO, QRO, SLP

*Zinnia haageana Regel AGS, DGO, GTO, GRO, JAL, MEX, MICH, NAY, OAX, QRO, SLP, ZAC

*Zinnia juniperifolia (DC.) A. Gray COAH, DGO, NLE, SLP, SON, TAMS, ZAC

*Zinnia leucoglossa S.F. Blake COL, DGO, JAL, NAY, SIN, ZAC

*Zinnia maritima Kunth COL, GRO, JAL, MICH, NAY, SIN, ZAC

*Zinnia microglossa (DC.) McVaugh GTO, JAL, MICH, ZAC

*Zinnia oligantha I.M. Johnst. CHIH, COAH

Zinnia peruviana (L.) L. AGS, CHIS, CHIH, COAH, COL, CDMX, DGO, GTO, GRO, HGO, JAL, MEX, MICH, MOR, NAY, NLE, OAX, PUE, QRO, SLP, SIN, SON, TAMS, TLAX, VER, ZAC

*Zinnia purpusii Brandegee CHIS, COL, GRO, JAL, MEX, $\mathrm{MICH}, \mathrm{OAX}$

*Zinnia tenuis (S. Watson) Strother CHIH, DGO, SON

*Zinnia venusta (A.M. Torres) Olorode \& A.M. Torres GRO, $\mathrm{MICH}$

*Zinnia zamudiana Calderón \& Rzed. HGO, QRO

*Zinnia zinnioides (Kunth) Olorode \& A.M. Torres CHIS, CHIH, COL, DGO, GRO, JAL, MEX, MICH, MOR, NAY, OAX, SIN, SON, ZAC

Zyzyxia lundellii (H. Rob.) Strother CAM, CHIS 


\section{Family Balanophoraceae}

Helosis cayennensis (Sw.) Spreng. CAM, CHIS, HGO, MOR, OAX, QRO, SLP, TAB, TAMS, VER

Langsdorffia hypogaea Mart. OAX

Family Balsaminaceae

*Impatiens mexicana Rydb. OAX, VER

\section{Family Basellaceae}

Anredera baselloides (Kunth) Baill. MICH, MOR, QRO

Anredera ramosa (Moq.) Eliasson CHIS, GTO, GRO, HGO, MEX, MICH, MOR, OAX, PUE, QRO, QROO, SLP, TAMS, VER

Anredera vesicaria (Lam.) C.F. Gaertn. BCS, CAM, CHIS, COAH, COL, DGO, GTO, GRO, HGO, JAL, MEX, MICH, MOR, NAY, NLE, OAX, PUE, QRO, QROO, SLP, SIN, TAMS, VER, YUC

\section{Family Bataceae}

Batis maritima L. BCN, BCS, CAM, CHIS, COL, GRO, JAL, NAY, OAX, QROO, SLP, SIN, SON, TAB, TAMS, VER, YUC

\section{Family Begoniaceae}

Begonia acutiloba Liebm. CHIS, OAX

*Begonia alice-clarkae Ziesenh. CHIS

* Begonia almedana Burt-Utley \& Utley CHIS

*Begonia angustiloba DC. CHIH, DGO, GRO, JAL, NAY, OAX, SIN, SON, ZAC

*Begonia asteroides L.B. Smith \& B.G. Schub. JAL, MEX, $\mathrm{MICH}, \mathrm{MOR}, \mathrm{NAY}$

*Begonia balmisiana Ruiz ex Klotzsch AGS, COL, DGO, GRO, JAL, MEX, MICH, MOR, NAY, OAX, SIN, ZAC

*Begonia barkeri Knowles \& Westc. HGO, PUE, QRO, SLP, TAMS, VER

*Begonia bettinae Ziesenh. CHIS

Begonia biserrata Lindl. CHIS, COL, DGO, GRO, JAL, MEX, MICH, MOR, NAY, OAX, PUE, SIN, ZAC

*Begonia boissieri DC. GRO, MOR, OAX

Begonia boqueronensis Burt-Utley \& Utley CHIS

*Begonia bowerae Ziesenh. CHIS, OAX

*Begonia breedlovei Burt-Utley CHIS, OAX

Begonia calderonii Standl. CHIS, COL, GRO, JAL, NAY, OAX

*Begonia calzadae Burt-Utley \& Utley CHIS

Begonia caroliniifolia Regel CHIS, OAX, PUE, VER

* Begonia carrieae Ziesenh. CHIS

Begonia cebadillensis Houghton ex L.B. Sm. \& B.G. Schub. CHIS

Begonia convallariodora C. DC. CHIS

*Begonia corzoensis Ziesenh. CHIS

Begonia crassicaulis Lindl. CHIS, GRO, OAX

*Begonia cristobalensis Ziesenh. CHIS, GRO, MEX, MICH

*Begonia cucullata Willd. CAM, CHIS, OAX, SLP, VER, YUC

*Begonia cylindrata L.B. Sm. \& B.G. Schub. GRO, MEX

*Begonia chiapensis Burt-Utley CHIS

*Begonia chivatoa Ziesenh. GRO, OAX

*Begonia extranea L.B. Sm. \& B.G. Schub. GRO, JAL, MEX, $\mathrm{MICH}$

*Begonia falciloba Liebm. CHIS, GRO, JAL, MEX, MICH, OAX, VER
*Begonia faustinoi Burt-Utley \& Utley CHIS

* Begonia fimbriata Liebm. OAX

Begonia fischeri Schrank CHIS, HGO, OAX, PUE, SLP, QROO,

TAB, VER

Begonia franconis Liebm. CHIS, GTO, HGO, OAX, PUE, QRO, SLP, TAB, TAMS, VER

Begonia fusca Liebm. CHIS, MEX, MOR, OAX, PUE, VER

*Begonia fusibulba C. DC. COL, DGO, GRO, JAL, MICH, NAY, SIN

Begonia glabra Aubl. CHIS, HGO, NAY, OAX, PUE, QRO, SLP, TAB, VER

*Begonia glandulosa Hook. HGO, PUE, QRO, SLP, VER

*Begonia gracilior Burt-Utley \& McVaugh COL, JAL

Begonia gracilis Kunth AGS, CHIS, CHIH, COL, CDMX, DGO, GTO, GRO, HGO, JAL, MEX, MICH, MOR, NAY, NLE, OAX, PUE, QRO, SLP, SIN, SON, TAMS, TLAX, VER, ZAC

Begonia heracleifolia Schltdl. \& Cham. CHIS, COL, DGO, GRO, HGO, JAL, MEX, MOR, NAY, OAX, PUE, QRO, SLP, SIN, TAB, TAMS, VER, YUC

Begonia heydei C. DC. CHIS

*Begonia hintoniana L.B. Sm. \& B.G. Schub. GRO, MEX, $\mathrm{MICH}$

*Begonia hispidavillosa Ziesenh. CHIS, OAX

*Begonia hubertii Ziesenh. CHIS

*Begonia humilis Aiton DGO, SIN

*Begonia hydrocotylifolia Otto ex Hook. OAX, VER

*Begonia imperialis Lem. CHIS, OAX, VER

* Begonia incarnata Link \& Otto CHIS, GRO, HGO, MEX, MOR, OAX, PUE, QRO, SLP, TAMS, VER

*Begonia jaliscana Burt-Utley JAL

*Begonia karwinskyana A. DC. HGO, QRO, VER

*Begonia kenworthyae Ziesehn. CHIS

*Begonia knoopii Ziesenh. CHIS

*Begonia lachaoensis Ziesenh. COL, JAL, OAX

Begonia lindleyana Walp. CHIS, VER

Begonia ludicra A. DC. CHIS, OAX, PUE, TAB, VER

*Begonia lyman-smithii Burt-Utley \& Utley OAX

*Begonia lyniceorum Burt-Utley CHIS, OAX, VER

*Begonia maculata Raddi CHIS, HGO, SLP, TAMS, VER

*Begonia macvaughii Burt-Utley \& Utley GRO, OAX

*Begonia makrinii Morton ex Burt-Utley \& Utley OAX

Begonia manicata Brongn. ex F. Cels. CHIS, HGO, MEX, MICH, OAX, PUE, VER

*Begonia mariti Burt-Utley OAX

*Begonia martinezii Burt-Utley \& Utley GRO

*Begonia matudae Burt-Utley \& Utley CHIS

*Begonia mazae Ziesenh. CHIS

*Begonia mexicana G. Karst. ex Fotsch CHIS

Begonia militaris L.B. Sm. \& B.G. Schub. CHIS

*Begonia monophylla A. DC. COL, GRO, JAL, MEX, MICH, MOR, OAX, PUE

Begonia motozintlensis Burt-Utley \& Utley CHIS

*Begonia multistaminea Burt-Utley HGO, PUE, VER

Begonia nelumbonifolia Schltdl. \& Cham. CHIS, CDMX, HGO, MEX, MOR, OAX, PUE, SLP, TAB, VER

*Begonia nemoralis L.B. Sm. \& B.G. Schub. COL, JAL, OAX 
Begonia oaxacana A. DC. CHIS, COL, GRO, HGO, JAL, MEX, OAX, PUE, VER

*Begonia ornithocarpa Standl. COL, DGO, GRO, JAL, MEX, NAY, SIN, ZAC

*Begonia palmeri S. Watson BCS, CHIS, CHIH, COL, DGO, GRO, HGO, JAL, MICH, NAY, OAX, SIN, SON, VER, ZAC

*Begonia pedata Liebm. GRO, OAX

Begonia peltata Otto \& D. Dietr. CHIS, OAX, VER

*Begonia philodendroides Ziesenh. CHIS, OAX

Begonia pinetorum A. DC. CHIS, HGO, OAX, QRO, SLP, VER

*Begonia plantaginea L.B. Sm. \& B.G. Schub. CHIS

Begonia plebeja Liebm. CHIS, COL, GRO, HGO, JAL, MEX,

MICH, NAY, OAX, VER, ZAC

Begonia polygonata Liebm. VER

*Begonia portillana S. Watson CHIH, DGO, GRO, JAL, NAY,

OAX, SIN, SON, ZAC

*Begonia pudica L.B. Sm. \& B.G. Schub. HGO, VER

Begonia purpusii Houghton ex Ziesenh. CHIS

Begonia pustulata Liebm. CHIS, OAX, TAB, VER

*Begonia rafael-torresii Burt-Utley OAX

*Begonia rhodochlamys L.B. Sm. \& B.G. Schub. COL, GRO, JAL, MICH, OAX

*Begonia sandtii Houghton ex Ziesenh. CHIS, CHIH, NAY, OAX

Begonia sartorii Liebm. CHIS, OAX, VER

Begonia sciadiophora L.B. Smith \& B.G. Schub. CHIS

Begonia sericoneura Liebm. CAM, CHIS, OAX, QROO, VER

*Begonia sousae Burt-Utley OAX, VER

*Begonia squarrosa Liebm. MEX, OAX

Begonia stigmosa Lindl. COL, GRO, JAL, MEX, MICH, NAY, OAX, VER

Begonia strigillosa A. Dietr. CHIS

*Begonia tacana Ziesenh. CHIS

*Begonia tapatia Burt-Utley \& McVaugh CHIH, DGO, GTO, GRO, JAL, NAY, SIN, ZAC

*Begonia tenuis Burt-Utley \& Utley CHIS

Begonia thiemei C. DC. CHIS, TAB, VER

*Begonia tlapensis Burt-Utley \& Utley GRO, OAX

Begonia trichosepala C. DC. CHIS

*Begonia uniflora S. Watson COAH, NLE, TAMS

*Begonia uruapensis Sessé \& Moc. COL, GRO, JAL, MICH, NAY

*Begonia violifolia A. DC. CHIS, TAB

*Begonia xilitlensis Burt-Utley QRO, SLP, VER

Begonia yunckeri Standl. CHIS

\section{Family Berberidaceae}

* Berberis albicans Zamudio \& Marroq. HGO, QRO

*Berberis alpina Zamudio CDMX, GTO, HGO, MEX, MICH, PUE, QRO, SLP, VER

*Berberis andrieuxii Hook. \& Arn. OAX

* Berberis angustifolia Hartw. HGO, QRO, VER

* Berberis berriozabalensis (Miranda) Marroq. CHIS

*Berberis chiapensis (Lundell) Lundell CHIS

*Berberis chochoco Schltdl. HGO, NLE, QRO, SLP, TAMS, VER

Berberis dictyota Jeps. BCN
*Berberis ehrenbergii Kunze CHIS, TAMS, VER

*Berberis eutriphylla (Fedde) C.H. Müll. COAH, DGO, MEX, NLE, QRO, SLP

Berberis fremontii Torr. BCN, BCS, SON

*Berberis gracilis Benth. CHIH, COAH, GTO, HGO, NLE, OAX, PUE, QRO, SLP, TAMS, VER

Berberis haematocarpa Wooton CHIH, COAH, SON

*Berberis hartwegii Benth. HGO, QRO, SLP, TAMS, VER

Berberis hemsleyi Donn. Sm. CHIS, COL, GRO, HGO, JAL, MICH, OAX, QRO, SLP

Berberis higginsiae Munz BCN

* Berberis ilicina (Schltdl.) Hemsl. HGO, QRO, TAMS

*Berberis incerta (Fedde) Marroq. COL, HGO, JAL, VER

Berberis johnstonii Standl. \& Steyerm. CHIS

*Berberis lanceolata Benth. GRO, HGO, MEX, OAX, PUE, QRO, SLP, VER

*Berberis longipes (Standl.) Marroq. CHIH, DGO, SON

Berberis moranensis Schult. \& Schult. f. CHIH, COL, CDMX, DGO, GTO, HGO, JAL, MEX, MICH, MOR, NAY, OAX, PUE, QRO, SLP, SIN, SON, TAMS, TLAX, VER

*Berberis muelleri (I.M. Johnst.) Marroq. ex Laferrière \& Marroq. COAH, GTO, NLE, SLP, TAMS

*Berberis pallida Hartw. ex Benth. GTO, HGO, OAX, PUE, QRO, TAMS, VER

*Berberis pimana Laferr. \& Marroq. CHIH, SON

*Berberis pinifolia (Lundell) C.H. Müll. COAH, DGO, NLE, SLP, ZAC

Berberis pinnata Lag. BCN

*Berberis quinquefolia (Standl.) Marroq. \& Laferrière CAM, GRO, HGO, OAX, PUE, QRO, VER

*Berberis russellii (N.P. Taylor) Marroq. \& Laferr. VER

Berberis tenuifolia Lindl. CHIS, HGO, OAX, VER

*Berberis trifolia (Schltdl. \& Cham.) Schult. \& Schult. f. PUE, VER

Berberis trifoliolata Moric. CHIH, COAH, DGO, HGO, JAL, NLE, SLP, SON, TAMS, ZAC

Berberis volcania (Standl. \& Steyerm.) Marroq. \& Laferr. CHIS Berberis wilcoxii Kearney CHIH, SON

Family Betulaceae

Alnus acuminata Kunth AGS, CHIS, CHIH, COL, CDMX, DGO, GTO, GRO, HGO, JAL, MEX, MICH, MOR, NAY, OAX, PUE, QRO, SLP, SIN, SON, TAMS, TLAX, VER, ZAC Alnus jorullensis Kunth AGS, CHIS, CHIH, COL, CDMX, DGO, GTO, GRO, HGO, JAL, MEX, MICH, MOR, NAY, OAX, PUE, QRO, SLP, SIN, SON, TLAX, VER, ZAC Alnus oblongifolia Torr. CHIH, DGO, NAY, SIN, SON Alnus rhombifolia Nutt. BCN

Carpinus tropicalis (Donn. Sm.) Lundell AGS, CHIS, CHIH, COAH, COL, DGO, GTO, GRO, HGO, JAL, MEX, MICH, MOR, NAY, NLE, OAX, PUE, QRO, SLP, SIN, TAMS, TLAX, VER

Ostrya virginiana (Mill.) K. Koch CHIS, CHIH, COAH, COL, DGO, GTO, GRO, HGO, JAL, MEX, MICH, NAY, NLE, OAX, PUE, QRO, SLP, SIN, SON, TAB, TAMS, TLAX, VER

\section{Family Bignoniaceae}

Adenocalymma inundatum Mart. ex DC. CAM, CHIS, COL, 
GRO, JAL, MEX, MICH, MOR, NAY, OAX, PUE, QROO, SIN, TAB, VER

*Adenocalymma sousae A.H. Gentry TAB, VER

Amphilophium buccinatorium (DC.) L.G. Lohmann CHIS, COL, GTO, JAL, MEX, MICH, MOR, OAX, PUE, QRO, SLP Amphilophium crucigerum (L.) L.G. Lohmann CAM, CHIS, COL, GTO, GRO, HGO, JAL, MEX, MICH, MOR, NAY, OAX, PUE, QRO, QROO, SLP, SIN, TAB, TAMS, VER, YUC Amphilophium laxiflorum (DC.) L.G. Lohmann CHIS, GTO, GRO, JAL, MEX, MOR, OAX, PUE, TAB, VER

Amphilophium paniculatum (L.) Kunth CAM, CHIS, COL, DGO, GRO, HGO, JAL, MEX, MICH, MOR, NAY, OAX, PUE, QRO, QROO, SLP, SIN, TAB, TAMS, VER, YUC

Amphitecna apiculata A.H. Gentry CAM, CHIS, GRO, OAX, PUE, QROO, TAB, VER, YUC

Amphitecna breedlovei A.H. Gentry CHIS

Amphitecna donnell-smithii (Sprague) L.O. Williams CHIS, TAB

Amphitecna latifolia (Mill.) A.H. Gentry CAM, CHIS, OAX, TAB, VER, YUC

Amphitecna macrophylla (Seem.) Miers ex Baill. CHIS, OAX, TAB, VER

Amphitecna montana L.O. Williams CHIS

*Amphitecna regalis (Linden) A.H. Gentry CHIS, OAX, TAB, VER

Amphitecna silvicola L.O. Williams CHIS

Amphitecna steyermarkii (A.H. Gentry) A.H. Gentry CHIS, OAX, VER

*Amphitecna tuxtlensis A.H. Gentry OAX, VER

Anemopaegma chrysanthum Dugand CHIS, OAX, TAB, VER

Anemopaegma chrysoleucum (Kunth) Sandwith CAM, CHIS, GRO, VER

Anemopaegma puberulum (Seibert) Miranda CHIS, HGO, OAX, VER

Astianthus viminalis (Kunth) Baill. CHIS, COL, GRO, HGO, JAL, MEX, MICH, MOR, NAY, OAX, PUE, QRO, SLP, TAMS, VER

Bignonia aequinoctialis L. CHIS, COL, GRO, JAL, MICH, MOR, NAY, OAX, QROO, SLP, SIN, TAB, TAMS, VER, YUC Bignonia binata Thunb. CAM, CHIS, COL, GRO, JAL, MICH, OAX, QRO, QROO, SLP, TAB, VER

Bignonia diversifolia Kunth CAM, CHIS, COL, GRO, JAL, MICH, OAX, QROO, TAB, YUC

Bignonia hyacinthina (Standl.) L.G. Lohmann CAM, CHIS, GRO, OAX, PUE, VER, YUC

Bignonia neoheterophylla L.G. Lohmann CAM, CHIS, GRO, JAL, MICH, NAY, OAX, QROO, TAB, VER, YUC

Bignonia potosina (K. Schum. \& Loes.) L.G. Lohmann CAM, CHIS, GRO, HGO, MICH, NAY, OAX, PUE, QRO, QROO, SLP, SIN, TAB, TAMS, VER, YUC

Callichlamys latifolia (Rich.) K. Schum. CHIS, OAX, VER Crescentia alata Kunth BCN, BCS, CHIS, CHIH, COL, DGO, GRO, HGO, JAL, MEX, MICH, MOR, NAY, OAX, PUE, QRO, SLP, SIN, SON, TAB, TAMS, VER, ZAC

Crescentia cujete L. CAM, CHIS, GRO, HGO, MOR, NAY, OAX, PUE, QROO, SIN, TAB, TAMS, VER, YUC

Cuspidaria inaequalis (DC. ex Splitg.) L.G. Lohmann CAM,
CHIS, TAB, VER

Chilopsis linearis (Cav.) Sweet BCN, CHIH, COAH, DGO, NLE, SLP, SON, TAMS, ZAC

Dolichandra quadrivalvis (Jacq.) L.G. Lohmann CAM, CHIS, COL, GRO, HGO, JAL, MEX, MICH, OAX, PUE, QRO, QROO, SLP, TAMS, VER, YUC

Dolichandra uncata (Andrews) L.G. Lohmann CHIS, MOR, QROO, TAB, VER

Dolichandra unguis-cati (L.) L.G. Lohmann BCS, CAM, CHIS, CHIH, COL, GTO, GRO, HGO, JAL, MEX, MICH, MOR, NAY, OAX, PUE, QRO, QROO, SLP, SIN, SON, TAB, TAMS, VER, YUC

Fridericia candicans (Rich.) L.G. Lohmann CHIS, OAX, VER Fridericia costaricensis (Kraenzl.) L.G. Lohmann CAM, CHIS, GRO, MICH, MOR, OAX, QROO, SON, VER

Fridericia chica (Bonpl.) L.G. Lohmann CAM, CHIS, OAX, TAB, VER

Fridericia dichotoma (Jacq.) L.G. Lohmann CHIS, COL, GRO, JAL, MEX, OAX, VER

Fridericia floribunda (Kunth) L.G. Lohmann CAM, CHIS, GRO, OAX, QROO, YUC

Fridericia florida (DC.) L.G. Lohmann CHIS, OAX, VER

Fridericia mollissima (Kunth) L.G. Lohmann CHIS, GRO, MICH, OAX

Fridericia patellifera (Schltdl.) L.G. Lohmann CAM, CHIS, COL, GRO, JAL, MEX, MICH, MOR, NAY, OAX, QROO, SLP, TAB, TAMS, VER, YUC

Fridericia podopogon (DC.) L.G. Lohmann CAM, CHIS, OAX, QROO, VER, YUC

Fridericia pubescens (L.) L.G. Lohmann CAM, CHIS, GRO, HGO, OAX, PUE, QRO, QROO, SLP, TAB, TAMS, VER, YUC Fridericia schumanniana (Loes.) L.G. Lohmann CAM, CHIS, OAX, QROO, TAB, VER, YUC

Fridericia viscida (Donn. Sm.) L.G. Lohmann CHIS, COL, GRO, JAL, MEX, MOR, OAX, VER

Godmania aesculifolia (Kunth) Standl. CAM, CHIS, GRO, JAL, MEX, MICH, NAY, OAX, QROO, TAB, VER, YUC

Handroanthus chrysanthus (Jacq.) S.O. Grose CAM, CHIS, CHIH, COL, DGO, GRO, JAL, MEX, MICH, NAY, OAX, PUE, QROO, SIN, SON, TAB, TAMS, VER, YUC, ZAC

Handroanthus guayacan (Seem.) S.O. Grose CAM, CHIS, OAX, QROO, TAB, VER

Handroanthus impetiginosus (Mart. ex DC.) Mattos AGS, CAM, CHIS, CHIH, COAH, COL, DGO, GRO, JAL, MEX, MICH, MOR, NAY, OAX, PUE, SIN, SON, YUC

Lundia puberula Pittier CAM, CHIS, OAX, QROO, VER

Mansoa hymenaea (DC.) A.H. Gentry CAM, CHIS, DGO, GRO, JAL, MEX, MOR, NAY, OAX, PUE, QRO, QROO, SLP, SIN, TAB, TAMS, VER, YUC

Mansoa verrucifera (Schltdl.) A.H. Gentry CAM, CHIS, GRO, OAX, QROO, TAB, VER, YUC

Martinella obovata (Kunth) Bureau \& K. Schum. CHIS, OAX, TAB, VER

Parmentiera aculeata (Kunth) Seem. BCS, CAM, CHIS, COL, GRO, HGO, JAL, MEX, MICH, MOR, NAY, OAX, PUE, QRO, QROO, SLP, SIN, TAB, TAMS, VER, YUC

Parmentiera millspaughiana L.O. Williams CAM, COL, 


\section{QROO, YUC}

Parmentiera parviflora Lundell CHIS, TAB

Roseodendron donnell-smithii (Rose) Miranda CAM, CHIS, COL, GRO, JAL, MEX, MICH, MOR, NAY, OAX, SIN, TAB, VER

Stizophyllum perforatum (Cham.) Miers CAM, OAX, YUC

Stizophyllum riparium (Kunth) Sandwith CAM, CHIS, OAX, QROO, TAB, VER, YUC

Tabebuia rosea (Bertol.) A. DC. CAM, CHIS, COL, DGO, GRO, HGO, JAL, MEX, MICH, MOR, NAY, OAX, PUE, QRO, QROO, SLP, SIN, SON, TAB, TAMS, VER, YUC, ZAC Tanaecium caudiculatum (Standl.) L.G. Lohmann CHIS, OAX, VER

Tanaecium pyramidatum (Rich.) L.G. Lohmann CAM, CHIS, COL, GRO, JAL, OAX, QROO, TAB, VER, YUC

Tanaecium tetragonolobum (Jacq.) L.G. Lohmann CAM, CHIS, OAX, QROO, TAB, VER, YUC

Tecoma stans (L.) Juss ex Kunth AGS, BCS, CAM, CHIS, CHIH, COAH, COL, CDMX, DGO, GTO, GRO, HGO, JAL, MEX, MICH, MOR, NAY, NLE, OAX, PUE, QRO, QROO, SLP, SIN, SON, TAB, TAMS, TLAX, VER, YUC, ZAC

Tourrettia lappacea (L'Hér.) Willd. CHIS, JAL, OAX

Tynanthus guatemalensis Donn. Sm. CAM, CHIS, OAX, QROO, TAB, VER, YUC

Xylophragma seemannianum (Kuntze) Sandwith CAM, CHIS, COL, GRO, JAL, MICH, OAX, QROO, TAB, TAMS, VER, YUC

\section{Family Bixaceae}

Amoreuxia gonzalezii Sprague \& L. Riley JAL, MICH, SIN, SON

*Amoreuxia malvifolia A. Gray CHIH, DGO

Amoreuxia palmatifida Moc. \& Sessé ex DC. BCN, BCS, CAM, CHIS, CHIH, COAH, COL, DGO, GRO, JAL, MEX, MICH, MOR, NAY, NLE, OAX, QRO, SIN, SON, VER, YUC, ZAC

Amoreuxia wrightii A. Gray CAM, CHIH, COAH, DGO, GRO, HGO, JAL, NLE, QRO, SLP, TAMS, VER, YUC

Bixa orellana L. CAM, CHIS, COL, GRO, JAL, MEX, MICH, MOR, NAY, OAX, PUE, QROO, SIN, TAB, VER, YUC

Cochlospermum vitifolium (Willd.) Spreng. BCS, CAM, CHIS, CHIH, COL, DGO, GRO, HGO, JAL, MEX, MICH, MOR, NAY, OAX, PUE, QROO, SLP, SIN, SON, TAB, TAMS, VER, YUC, ZAC

\section{Family Boraginaceae}

Amsinckia intermedia Fisch. \& C.A. Mey. BCN, BCS, SON Amsinckia menziesii (Lehm.) A. Nelson \& J.F. Macbr. BCN, BCS, CHIH, SON

Amsinckia spectabilis Fisch. \& C.A. Mey. BCN

Amsinckia tessellata A. Gray BCN, SON

*Antiphytum caespitosum I.M. Johnst. GRO, OAX, PUE

Antiphytum floribundum (Torr.) A. Gray CHIH, COAH, DGO, JAL, MICH, ZAC

Antiphytum heliotropioides A. DC. CHIH, COAH, DGO, GTO, GRO, HGO, NLE, OAX, PUE, QRO, SLP, TAMS, TLAX, VER, ZAC

*Antiphytum hintoniorum Higgins \& B.L. Turner COAH, NLE
*Antiphytum nudicalces I.M. Johnst. OAX

*Antiphytum paniculatum I.M. Johnst. OAX, PUE

*Antiphytum parryi S. Watson GTO, HGO, MEX, MICH, NLE, OAX, PUE, QRO, SLP, TAMS

*Antiphytum peninsulare (Rose) I.M. Johnst. BCN, BCS

Cryptantha albida (Kunth) I.M. Johnst. AGS, BCN, CHIH, COAH, CDMX, DGO, GTO, GRO, HGO, JAL, MEX, MICH, NLE, PUE, QRO, SLP, SON, VER, ZAC

*Cryptantha angelica I.M. Johnst. BCN, BCS, SON

Cryptantha angustifolia (Torr.) Greene BCN, BCS, CHIH, COAH, SON

Cryptantha barbigera (A. Gray) Greene BCN, BCS, SON

Cryptantha cinerea (Greene) Cronquist CHIH, SON

Cryptantha circumscissa (Hook. \& Arn.) I.M. Johnst. BCN

Cryptantha clevelandii Greene BCN

Cryptantha costata Brandegee BCN, SON

Cryptantha crassisepala (Torr. \& A. Gray) Greene CHIH, COAH, SON

Cryptantha decipiens (M.E. Jones) Heller BCN, BCS

*Cryptantha echinosepala J.F. Macbr. BCS, DGO

*Cryptantha fastigiata I.M. Johnst. BCN, BCS, SON

*Cryptantha foliosa (Greene) Greene BCN, COL

Cryptantha ganderi I.M. Johnst. BCN, SON

*Cryptantha geohintonii B.L. Turner NLE

*Cryptantha grayi (Vasey \& Rose) J.F. Macbr. BCN, BCS, SON

*Cryptantha gypsites I.M. Johnst. NLE

Cryptantha holoptera (A. Gray) J.F. Macbr. BCN, SON

Cryptantha intermedia (A. Gray) Greene BCN, SON

Cryptantha jamesii (Torr.) Payson $\mathrm{CHIH}$

Cryptantha maritima (Greene) Greene BCN, BCS, SON

Cryptantha mexicana (Brandegee) I.M. Johnst. AGS, BCN, CHIH, COAH, JAL, NLE, SLP, SON, ZAC

Cryptantha micrantha (Torr.) I.M. Johnst. BCN, BCS, CHIH, SON

Cryptantha micromeres (A. Gray) Greene BCN

Cryptantha muricata (Hook. \& Arn.) A. Nelson \& J.F. Macbr. $\mathrm{BCN}$

Cryptantha nevadensis A. Nelson \& P.B. Kenn. BCN, SON

Cryptantha oblata (M.E. Jones) Payson CHIH

Cryptantha palmeri (A. Gray) Payson CHIH, COAH, NLE

* Cryptantha patula Greene BCS

*Cryptantha pondii Greene BCN, BCS

Cryptantha pterocarya (Torr.) Greene BCN, BCS, SON

Cryptantha pusilla (Torr. \& A. Gray) Greene CHIH, COAH, DGO, SON

Cryptantha racemosa (S. Watson ex A. Gray) Greene BCN, BCS, SON

*Cryptantha wigginsii I.M. Johnst. BCN

*Cynoglossum henricksonii L.C. Higgins COAH, DGO, NLE, SLP, ZAC

Emmenanthe penduliflora Benth. BCN

Eriodictyon angustifolium Nutt. BCN

Eriodictyon lanatum (Brand) Abrams BCN

*Eriodictyon sessilifolium Greene BCN

Eriodictyon trichocalyx Heller BCN

Eucrypta chrysanthemifolia (Benth.) Greene BCN, BCS, SON

Eucrypta micrantha (Torr.) A. Heller BCN, BCS, SON 
*Hackelia hintoniorum (B.L. Turner) Sutorý OAX

*Hackelia leonotis I.M. Johnst. COAH, NLE

Hackelia mexicana (Schltdl. \& Cham.) I.M. Johnst. CHIS, CDMX, GRO, HGO, JAL, MEX, MICH, MOR, NLE, OAX, PUE, TLAX, VER

Hackelia pinetorum (Greene) I.M. Johnst. CHIH, SON

*Hackelia stricta I.M. Johnst. GRO, JAL, MICH, MOR, OAX, PUE, VER

Hackelia ursina (Greene) I.M. Johnst. SON

Harpagonella palmeri A. Gray BCN, SON

Lappula redowskii (Hornem.) Greene $\mathrm{CHIH}, \mathrm{COAH}, \mathrm{SON}$

Lappula texana (Scheele) Britton COAH, SON

*Lasiarrhenum confundum B.L. Turner DGO, SIN

*Lithospermum album (G.L. Nesom) J. Cohen TAMS

*Lithospermum barbigerum (I.M. Johnst.) J. Cohen COAH, NLE

*Lithospermum berlandieri I.M. Johnst. COAH, NLE, TAMS

*Lithospermum calcicola B.L. Rob. COAH, GTO, GRO, HGO, MEX, MICH, NLE, OAX, PUE, SLP, TAB, TAMS, TLAX, VER

Lithospermum calycosum (J.F. Macbr.) I.M. Johnst. CHIS, COAH, GTO, GRO, HGO, MEX, MICH, NLE, OAX, PUE, QRO, SLP, TAMS, VER, ZAC

Lithospermum cobrense Greene AGS, CHIH, COAH, DGO, SIN, SON, ZAC

Lithospermum confine I.M. Johnst. COAH, NLE

Lithospermum chiapense J. Cohen CHIS

*Lithospermum discolor M. Martens \& Galeotti CHIH, COAH, COL, GRO, JAL, MEX, MICH, NAY, NLE, OAX, PUE, SON, ZAC

Lithospermum distichum Ortega CHIS, COAH, CDMX, DGO, GTO, GRO, HGO, JAL, MEX, MICH, MOR, NAY, NLE, OAX, PUE, QRO, SLP, SIN, TAMS, TLAX, VER, ZAC

*Lithospermum dodrantale (I.M. Johnst.) J. Cohen COAH, NLE, OAX

*Lithospermum exsertum (D. Don) J. Cohen CHIH, COL, CDMX, DGO, GTO, GRO, HGO, JAL, MEX, MICH, MOR, NAY, NLE, OAX, PUE, SIN, TAMS, VER, ZAC

Lithospermum guatemalense Donn. Sm. CHIS

*Lithospermum hintoniorum B.L. Turner NLE, TAMS

Lithospermum incisum Lehm. CHIH, COAH, MICH, NLE, OAX, SON, TAMS

*Lithospermum indecorum I.M. Johnst. COAH, NLE, SLP, TAMS

*Lithospermum jimulcense I.M. Johnst. COAH, DGO

*Lithospermum johnstonii J. Cohen MICH

*Lithospermum leonotis (I.M. Johnst.) J. Cohen COAH, NLE, SLP, TAMS

*Lithospermum linifolium M. Martens \& Galeotti OAX, PUE

Lithospermum macromeria J. Cohen CHIH, DGO, SON

Lithospermum matamorense DC. COAH, NLE, SLP, TAMS

*Lithospermum mirabile Small COAH, NLE

*Lithospermum muelleri I.M. Johnst. OAX, NLE, TAMS

Lithospermum multiflorum Torr. ex A. Gray CHIH, COAH, SON

*Lithospermum nelsonii Greenm. COAH, NLE
*Lithospermum notatum (I.M. Johnst.) J. Cohen COAH, NLE, SLP

*Lithospermum oaxacanum (B.L. Turner) J. Cohen OAX

*Lithospermum oblongifolium Greenm. CDMX, GRO, HGO, MEX, MICH, MOR, NLE, OAX, PUE, TLAX

*Lithospermum obovatum J.F. Macbr. CHIH, DGO

Lithospermum onosmodium J. Cohen CHIH, TAMS

Lithospermum parksii I.M. Johnst. CHIH, COAH, GTO, NLE, QRO

* Lithospermum pinetorum (I.M. Johnst.) J. Cohen OAX

Lithospermum pringlei I.M. Johnst. CHIH, COAH, CDMX, GRO, HGO, MEX, MICH, NLE, OAX, PUE, TLAX, VER

*Lithospermum rosei (I.M. Johnst.) J. Cohen DGO, NAY

*Lithospermum rzedowskii J. Cohen GTO, GRO, HGO, JAL, MEX, MICH, OAX, PUE, QRO, SLP, TAMS, VER, ZAC

*Lithospermum strictum Lehm. AGS, CDMX, GTO, GRO, HGO, JAL, MEX, MICH, MOR, OAX, PUE, SLP, TLAX, VER, ZAC

*Lithospermum trinervium (Lehm.) J. Cohen COL, CDMX, GRO, HGO, JAL, MEX, MICH, MOR, NAY, OAX, PUE, TLAX, VER, ZAC

*Lithospermum tubiliflorum Greene CHIH, COAH, DGO, SON

* Lithospermum tubuliflorum Greene CHIH, DGO, SON

* Lithospermum turneri J. Cohen GRO, OAX

*Lithospermum unicum (J.F. Macbr.) J. Cohen HGO, NLE, OAX, QRO, SLP

Lithospermum viride Greene CHIH, COAH, NLE, SLP, ZAC

*Lithospermum worthingtonii B.L. Turner DGO, SIN

*Mimophytum benitomartinezii Pérez-Calix \& Patiño-Siciliano QRO, SLP, VER

*Mimophytum omphalodoides Greenm. HGO, PUE, QRO, SLP, VER

* Nama bartlettii Standl. NLE, SLP, TAMS

*Nama biflora Choisy COAH, GTO, HGO, MOR, NLE, QRO, SLP, TAMS, VER, ZAC

Nama californica (A. Gray) J. Bacon BCN

*Nama canescens C.L. Hitchc. COAH, NLE, SLP, TAMS, ZAC Nama carnosa (Wooton) C.L. Hitchc. AGS, CHIH, COAH, DGO, TAMS

* Nama coulteri A. Gray AGS, BCS, PUE, SIN, SON, ZAC

*Nama cuatrocienegense G.L. Nesom COAH

Nama demissa A. Gray BCN, BCS, CHIH, SON

Nama dichotoma (Ruiz \& Pav.) Choisy BCN, BCS, CHIS, CHIH, COAH, COL, CDMX, DGO, GTO, GRO, HGO, JAL, MEX, MICH, NLE, OAX, PUE, QRO, SLP, SON, TAMS, TLAX, VER, ZAC

* Nama flavescens Brandegee COAH, NLE, ZAC

Nama havardii A. Gray CHIH, COAH, NLE

* Nama hintoniorum G.L. Nesom COAH, NLE, TAMS

Nama hirsuta M. Martens \& Galeotti CHIS, OAX, SLP

Nama hispida A. Gray BCN, BCS, CHIH, COAH, DGO, HGO, NLE, PUE, QRO, SLP, SIN, SON, TAMS, VER, ZAC

*Nama hitchcockii J.D. Bacon COAH, NLE, SLP, TAMS

Nama jamaicensis L. BCS, CAM, CHIS, CHIH, COAH, COL, GTO, HGO, JAL, MICH, NLE, OAX, PUE, QRO, QROO, SLP, SIN, SON, TAMS, VER, YUC, ZAC

*Nama johnstonii C.L. Hitchc. COAH, DGO, NLE, SLP 
* Nama linearis D.L. Nash PUE, VER

*Nama marshii (Standl.) I.M. Johnst. COAH, SLP

* Nama origanifolia Kunth AGS, COAH, CDMX, GTO, HGO, JAL, MEX, MICH, MOR, NLE, PUE, QRO, SLP, TAMS, VER, ZAC

*Nama orizabensis D.L. Nash VER

*Nama palmeri A. Gray ex Hemsl. AGS, COAH, DGO, GRO, HGO, NLE, QRO, SLP, TAMS, VER, ZAC

Nama parvifolia (Torr.) Greenm. CHIH, COAH, DGO, NLE, TAMS

* Nama propinqua Morton \& C.L. Hitchc. COAH

* Nama prostrata Brand JAL, MEX, MICH, VER

*Nama rotundifolia (A. Gray) J.F Macbr. COAH, DGO, NLE

* Nama rzedowskii J.D. Bacon QRO, SLP

* Nama schaffneri A. Gray ex Hemsl. COAH, NLE

*Nama sericea Willd. ex Roem. \& Schult. GTO, HGO, NLE, OAX, QRO, SLP

Nama serpylloides A. Gray ex Hemsl. COAH, NLE

*Nama spathulata Brandegee GTO, HGO, NLE, PUE, QRO, TAMS

Nama stenocarpa A. Gray BCN, BCS, CHIH, COAH, DGO, JAL, SON

*Nama stenophylla A. Gray ex Hemsl. CHIH, COAH, DGO, GTO, JAL, NLE, SLP, ZAC

Nama stevensii C.L. Hitchc. COAH, NLE, SLP, ZAC

*Nama stewartii I.M. Johnst. COAH, NLE

Nama torynophylla Greenm. CHIH, COAH, DGO

* Nama turneri J.D. Bacon COAH, NLE, SLP

Nama undulata Kunth AGS, CHIH, COAH, COL, CDMX, DGO, GTO, HGO, JAL, MEX, MOR, NLE, OAX, PUE, QRO, SLP, SIN, SON, TAMS, VER, ZAC

Nama xylopoda (Wooton \& Standl.) C.L. Hitchc. CHIH, COAH, DGO

Omphalodes aliena A. Gray COAH, NLE, TAMS

* Omphalodes cardiophylla A. Gray ex Hemsl. COAH, NLE, PUE, TAMS

* Omphalodes chiangii Higgins $\mathrm{COAH}$

*Omphalodes erecta I.M. Johnst. NLE, TAMS

*Omphalodes mexicana S. Watson COAH, NLE, TAMS

*Omphalodes richardsonii G.L. Nesom TAMS

*Oncaglossum pringlei (Greenm.) Sutorý COL, GTO, JAL, MEX, MICH

Pectocarya heterocarpa (I.M. Johnst.) I.M. Johnst. BCN, SON Pectocarya linearis DC. BCN, BCS

Pectocarya penicillata (Hook. \& Arn.) DC. BCN

Pectocarya peninsularis I.M. Johnst. BCN, BCS

Pectocarya platycarpa (Munz \& I.M. Johnst.) Munz \& I.M. Johnst. BCN, SON

Pectocarya recurvata I.M. Johnst. BCN, SON

Pectocarya setosa A. Gray BCN

Plagiobothrys acanthocarpus (Piper) I.M. Johnst. BCN

Plagiobothrys arizonicus (A. Gray) Greene ex A. Gray BCN, SON

Plagiobothrys bracteatus (Howell) I.M. Johnst. BCN

Plagiobothrys collinus (Phil.) I.M. Johnst. BCN, BCS

Plagiobothrys jonesii A. Gray BCN

Plagiobothrys leptocladus (Greene) I.M. Johnst. BCN
Plagiobothrys nothofulvus (A. Gray) A. Gray BCN

Plagiobothrys pringlei Greene SON

Plagiobothrys tenellus (Nutt.) A. Gray BCN

Plagiobothrys undulatus (Piper) I.M. Johnst. BCN

*Psilolaemus revolutus (B.L. Rob.) I.M. Johnst. GTO, SLP, TAMS

Turricula parryi (A. Gray) J.F. Macbr. BCN

Wigandia urens (Ruiz \& Pav.) Kunth AGS, CHIS, COL, CDMX, DGO, GTO, GRO, HGO, JAL, MEX, MICH, MOR, NAY, OAX, PUE, QRO, SLP, SIN, SON, TLAX, VER, YUC, ZAC

\section{Family Brassicaceae}

*Asta schaffneri (S. Watson) O.E. Schulz AGS, COAH, NLE, SLP, TAMS, ZAC

*Asta stricta Rollins HGO, QRO

Athysanus pusillus (Hook.) Greene BCN, BCS

Athysanus unilateralis (M.E. Jones) Jeps. BCN

Boechera californica (Rollins) Windham \& Al-Shehbaz BCN

Boechera fendleri (S. Watson) W.A. Weber CHIH, COAH, SLP, $\mathrm{ZAC}$

Boechera perennans (S. Watson) W.A. Weber BCN, SON

Boechera pulchra (M.E. Jones ex S. Watson) W.A. Weber BCN Cakile edentula (Bigelow) Hook. BCN

Cakile geniculata (B.L. Rob.) Millsp. TAMS, VER

Cakile lanceolata (Willd.) O.E. Schulz CAM, QROO, TAMS, VER, YUC

*Cardamine auriculata S. Watson COAH, HGO, NLE, SLP, TAMS

Cardamine bonariensis Pers. CHIS, COAH, CDMX, GRO, HGO, JAL, MEX, MICH, MOR, OAX, PUE, QRO, SLP, VER Cardamine breweri $\mathrm{S}$. Watson $\mathrm{CHIH}, \mathrm{SON}$

Cardamine californica (Nutt.) Greene BCN

* Cardamine cebollana B.L. Turner NLE

Cardamine fulcrata Greene CHIS, COL, JAL, MEX, MICH, OAX, PUE, VER

Cardamine longipedicellata Rollins CHIS, CDMX, DGO, HGO, NLE, PUE, QRO, SLP, VER

Cardamine macrocarpa Brandegee CHIH, COAH, NLE, SLP

* Cardamine mexicana O.E. Schulz COAH, NLE, SLP

Cardamine obliqua Hochst. CDMX, GRO, HGO, MEX, SON, VER

Caulanthus cooperi (S. Watson) Payson BCN

Caulanthus hallii Payson BCN

Caulanthus heterophyllus (Nutt.) Payson BCN

Caulanthus simulans Payson BCN

Caulanthus stenocarpus Payson BCN

*Cibotarium macrum (Stand1.) Rollins COAH, NLE

*Cibotarium stellatum (S. Watson) O.E. Schulz AGS, COAH, NLE, ZAC

Coelophragmus auriculatus (A. Gray) O.E. Schulz CHIH, COAH, NLE, SIN, SON, ZAC

*Chaunanthus acuminatus (Rollins) R.A. Price \& Al-Shehbaz COL, JAL

* Chaunanthus gracielae M. Martìnez \& L. Hern. GTO, QRO

* Chaunanthus mexicanus (Rollins) R.A. Price \& Al-Shehbaz PUE

*Chaunanthus petiolatus (Hemsl.) O.E. Schulz CDMX, GTO, 
HGO, MEX, PUE, QRO

*Dentaria californica Nutt. BCN

* Descurainia hartwegiana Britton OAX

Descurainia impatiens (Cham. \& Schltdl.) O.E. Schulz AGS, BCS, CHIH, CDMX, HGO, JAL, MEX, MICH, OAX, PUE, SLP, SON, TLAX, VER, ZAC

Descurainia incisa (A. Gray) Britton BCN, BCS, NLE

Descurainia obtusa (Greene) O.E. Schulz BCN, BCS, CHIH, COAH, SON

Descurainia pinnata (Walter) Britton AGS, BCN, BCS, CHIH, COAH, DGO, JAL, NLE, QRO, SLP, SIN, SON, TAMS, ZAC Descurainia richardsonii (Sweet) O.E. Schulz BCN, BCS, $\mathrm{CHIH}, \mathrm{COAH}$

Descurainia streptocarpa (Fourn.) O.E. Schulz CHIS, JAL, VER

*Descurainia virletii (Fourn.) O.E. Schulz AGS, CHIS, CHIH, COAH, CDMX, GTO, HGO, JAL, MEX, NLE, OAX, PUE, QRO, SLP, TLAX, VER, ZAC

Dithyrea californica Harv. BCN, BCS, SON

Dithyrea maritima (Davidson ex B.L. Rob.) Davidson BCN

Dithyrea wislizenii Engelm. BCN, CHIH, COAH, DGO, SON

Draba corrugata $\mathrm{S}$. Watson $\mathrm{BCN}$

Draba helleriana Greene CHIH, COAH, NLE, SON

* Draba hidalgensis Calderón HGO, QRO, VER

*Draba implexa Rollins DGO, SIN

Draba jorullensis Kunth CDMX, JAL, MEX, MICH, MOR, PUE, TLAX, VER

* Draba myosotidioides Hemsl. VER

Draba nivicola Rose CDMX, MEX, PUE, TLAX, VER

Draba petrophila Greene CHIH, SON

* Draba rubricaulis Heller CHIH

Draba standleyi J. Macbr. \& Payson COAH, DGO, SON

Draba volcanica Benth. CHIS

*Dryopetalon breedlovei (Rollins) Al-Shehbaz SIN

*Dryopetalon byei (Rollins) Al-Shehbaz CHIH

*Dryopetalon crenatum (Brandegee) Rollins BCS

*Dryopetalon membranifolium Rollins DGO, SIN

*Dryopetalon palmeri (S. Watson) O.E. Schulz BCN, BCS, $\mathrm{SON}$

*Dryopetalon paysonii (Rollins) Al-Shehbaz COAH, DGO

* Dryopetalon purpureum Rollins BCS

Dryopetalon runcinatum A. Gray CHIH, SIN, SON

Erysimum asperum (Nutt.) DC. AGS, BCN, BCS, CHIH, COAH, CDMX, DGO, GTO, HGO, JAL, MEX, NLE, PUE, QRO, SLP, SON, VER, ZAC

*Erysimum canescens Roth OAX, PUE, VER

Erysimum ghiesbreghtii Donn. Sm. CHIS, OAX, VER

Erysimum insulare Greene BCN

*Erysimum macradenium A. Gray MEX, VER

*Erysimum moranii Rollins BCN

*Exhalimolobos berlandieri (E. Fourn.) Al-Shehbaz \& C.D. Bailey AGS, CDMX, GTO, HGO, JAL, MEX, MICH, PUE, QRO, TLAX, VER, ZAC

Exhalimolobos hispidulus (DC.) Al-Shehbaz \& C.D. Bailey CDMX, GTO, HGO, MEX, PUE, QRO, SLP, VER, ZAC

*Exhalimolobos palmeri (Hemsl.) Al-Shehbaz \& C.D. Bailey AGS, GTO, HGO, JAL, NLE, QRO, SLP, ZAC
*Exhalimolobos parryi (Hemsl.) Al-Shehbaz \& C.D. Bailey SLP, ZAC

*Exhalimolobos polyspermus (E. Fourn.) Al-Shehbaz \& C.D. Bailey CDMX, GTO, GRO, HGO, MEX, MICH, NLE, PUE, QRO, SLP, VER, ZAC

Guillenia lasiophylla (Hook. \& Arn.) Greene BCN, SON

*Halimolobos elatus (Rollins) Al-Shehbaz \& C.D. Bailey DGO

*Halimolobos henricksonii (Rollins) Al-Shehbaz \& C.D. Bailey ZAC

*Halimolobos multiracemosus (S. Watson) Rollins QRO, SLP

*Halimolobos pedicellata (Rollins) Rollins COAH, DGO

*Halimolobos pubens (A. Gray) Al-Shehbaz \& C.D. Bailey $\mathrm{CHIH}, \mathrm{COAH}$

*Halimolobos rigidus Rollins COAH, NLE, SLP

*Halimolobos stylosus (Rollins) Al-Shehbaz \& C.D. Bailey COAH, DGO

Hesperidanthus linearifolius (A. Gray) Rydb. AGS, CHIH, COAH, DGO, MEX, MICH, MOR, NLE, SON

Lepidium alyssoides A. Gray CHIH, COAH, NLE, SLP, SON, TAMS, ZAC

Lepidium austrinum Small CHIH, COAH, NLE, TAMS

Lepidium bipinnatifidum Desv. JAL, MEX, MOR, SIN

Lepidium costaricense Thell. CAM, CHIS, HGO, PUE, QROO,

VER, YUC

Lepidium densiflorum Schrad. CHIH, DGO, SON, TAMS

Lepidium flavum Torr. BCN, BCS

Lepidium fremontii $\mathrm{S}$. Watson CHIH, COAH, DGO

Lepidium lasiocarpum Nutt. BCN, BCS, CHIH, COAH, COL, CDMX, DGO, GTO, JAL, MEX, MICH, MOR, NLE, OAX, QRO, SLP, SIN, SON, TAMS

Lepidium montanum Nutt. BCN, CHIH, COAH, NLE, SLP

Lepidium nitidum Nutt. ex Torr. \& A. Gray BCN, BCS

Lepidium oblongum Small BCN, BCS, CHIS, COAH, COL, CDMX, HGO, JAL, MEX, MICH, MOR, NLE, OAX, QRO, SLP, SON, TAMS, TLAX, VER

*Lepidium schaffneri Thell. CDMX, GTO, HGO, MEX, MICH, MOR, PUE, QRO, SLP, TLAX, VER

Lepidium sordidum A. Gray CHIH, COAH, CDMX, DGO, GTO, HGO, MEX, MICH, QRO, SON, TLAX, VER

Lepidium thurberi Wooton BCS, COAH, SON

Lepidium virginicum $\mathrm{L}$. AGS, BCN, BCS, CAM, CHIS, CHIH, COAH, COL, CDMX, DGO, GTO, GRO, HGO, JAL, MEX, MICH, MOR, NAY, NLE, OAX, PUE, QRO, QROO, SLP, SIN, SON, TAMS, TLAX, VER, YUC, ZAC

*Lexarzanthe mexicana (Al-Shehbaz \& Iltis) N. Diego \& Calderón GRO

Lyrocarpa coulteri Hook. \& Harv. ex Harv. BCN, BCS, SON

* Lyrocarpa xanti Brandegee BCS

* Mancoa bracteata (S. Watson) Rollins AGS, DGO, JAL, ZAC

* Mancoa laxa Rollins CHIH

*Mancoa mexicana Gilg \& Muschl. COAH, GTO, JAL, SLP

*Mancoa perennis L. Hern. \& M. Martínez MEX, MICH

* Mancoa rollinsiana Calderón HGO, VER

*Mostacillastrum arcuatum (Rollins) Al-Shehbaz COAH, NLE, TAMS

Mostacillastrum purpusii (Brandegee) Al-Shehbaz COAH, NLE 
*Mostacillastrum versicolor (Brandegee) Al-Shehbaz COAH, NLE, PUE, ZAC

Nasturtium officinale R. Br. AGS, BCN, BCS, CHIS, CHIH, COAH, COL, CDMX, DGO, GTO, GRO, HGO, JAL, MEX, MICH, MOR, NLE, OAX, PUE, QRO, SLP, SON, TAMS, TLAX, VER, ZAC

*Nerisyrenia baconiana B.L. Turner COAH

Nerisyrenia camporum (A. Gray) Greene CHIH, COAH, DGO,

NLE, SLP, TAMS, ZAC

*Nerisyrenia castillonii Rollins COAH

*Nerisyrenia gracilis I.M. Johnst. COAH, NLE, SLP, ZAC

*Nerisyrenia gypsophila J.D. Bacon CHIH, TAMS

*Nerisyrenia incana Rollins COAH

*Nerisyrenia johnstonii J.D. Bacon COAH

Nerisyrenia linearifolia (S. Watson) Greene CHIH, COAH, DGO, NLE, TAMS

*Nerisyrenia mexicana (J.D. Bacon) B.L. Turner COAH, DGO, NLE, SLP, ZAC

*Nerisyrenia powellii J.D. Bacon COAH

Noccaea fendleri (A. Gray) Holub CHIH, COAH, SON

Noccaea mexicana (Standl.) Holub COAH, MEX, NLE, PUE, SON

* Ornithocarpa fimbriata Rose JAL, MICH

* Ornithocarpa torulosa Rollins CHIH, DGO, SIN

Paysonia lasiocarpa (Hook. ex A. Gray) O'Kane \& Al-Shehbaz COAH, NLE, SLP, TAMS, VER

*Pennellia lasiocalycina (O.E. Schulz) Rollins COAH, HGO, NLE

Pennellia longifolia (Benth.) Rollins CHIS, CHIH, COAH, CDMX, DGO, GTO, HGO, JAL, MEX, MICH, MOR, NLE, OAX, PUE, QRO, SLP, SIN, SON, TLAX, VER, ZAC

Pennellia micrantha (A. Gray) Nieuwl. AGS, CHIH, COAH, CDMX, GTO, HGO, JAL, MEX, MICH, NLE, QRO, SON, ZAC

*Pennellia microsperma (Rollins) R.A. Price, C.D. Bailey \& Al-Shehbaz CHIH, SON

*Pennellia patens (O.E. Schulz) Rollins CHIS, CDMX, DGO, GTO, HGO, MEX, MICH, OAX, PUE, VER

Phravenia viereckii (O.E. Schulz) Al-Shehbaz \& S.I. Warwick COAH, HGO, NLE, PUE, SLP, ZAC

* Physaria argentea (S. Schauer) O'Kane \& Al-Shehbaz CHIH, CDMX, GTO, HGO, MEX, PUE, QRO, VER, ZAC

Physaria argyraea (A. Gray) O'Kane \& Al-Shehbaz AGS, CHIH, COAH, GTO, JAL, NLE, QRO, SLP, TAMS, ZAC

*Physaria berlandieri (S. Watson) O'Kane \& Al-Shehbaz COAH, NLE, SLP, TAMS

Physaria densiflora (A. Gray) O'Kane \& Al-Shehbaz TAMS

Physariafendleri (A. Gray) O'Kane \& Al-Shehbaz AGS, CHIH, COAH, DGO, HGO, NAY, NLE, QRO, SLP, SON, ZAC

Physaria gordonii (A. Gray) O'Kane \& Al-Shehbaz AGS, CHIH, SON

* Physaria inflata (Rollins \& E.A. Shaw) O'Kane \& Al-Shehbaz COAH, NLE

* Physaria johnstonii (Rollins) O' Kane \& Al-Shehbaz COAH Physaria kingii (S. Watson) O'Kane \& Al-Shehbaz BCN

Physaria mcvaughiana (Rollins) O'Kane \& Al-Shehbaz CHIH, $\mathrm{COAH}$
*Physaria mexicana (Rollins) O'Kane \& Al-Shehbaz COAH

* Physaria mirandiana (Rollins) O'Kane \& Al-Shehbaz COAH, NLE, SLP, TAMS, ZAC

Physaria palmeri (S. Watson) O'Kane \& Al-Shehbaz BCN, SON

*Physaria peninsularis (Wiggins) O'Kane \& Al-Shehbaz BCN *Physaria pueblensis (Payson) O'Kane \& Al-Shehbaz OAX, PUE

Physaria purpurea (A. Gray) O'Kane \& Al-Shehbaz CHIH, COAH, NLE, SLP, SON, VER, ZAC

*Physaria rosei (Rollins) O'Kane \& Al-Shehbaz CDMX, HGO, MEX, PUE, VER

*Physaria schaffneri (S. Watson) O'Kane \& Al-Shehbaz GTO, HGO, QRO, SLP, TAMS

* Physaria sinuosa (Rollins) O'Kane \& Al-Shehbaz PUE

* Physaria vigana B.L. Turner COAH, NLE

*Physaria wyndii (Rollins \& E.A. Shaw) O'Kane \& Al-Shehbaz $\mathrm{COAH}$

*Planodes mexicanum (S. Watson) Al-Shehbaz GTO, JAL

Planodes virginicum (L.) Greene BCN

* Rhaphanorhyncha crassa Rollins CHIH

Romanschulzia arabiformis (DC. ex Kunth) Rollins AGS, CDMX, GTO, HGO, JAL, MEX, MICH, MOR, PUE, VER, ZAC

*Romanschulzia correllii Rollins CHIH

* Romanschulzia guerrerensis Bustamante \& Fonseca GRO

* Romanschulzia meyeri Rollins NLE, TAMS

*Romanschulzia orizabae (Schltdl. \& Cham.) O.E. Schulz JAL, MEX, MOR, VER

*Romanschulzia rzedowskii Rollins JAL

*Romanschulzia subclavata Rollins COL, JAL, MICH

*Romanschulzia turritoides (Loes.) O.E. Schulz OAX

Rorippa curvisiliqua (Hook.) Bessey BCN

Rorippa intermedia (Kuntze) Stuckey DGO

Rorippa islandica (Oeder) Borbás BCN, BCS

Rorippa mexicana (Moc. \& Sessé ex DC.) Standl. \& Steyerm. AGS, CHIS, CHIH, COAH, CDMX, DGO, GTO, HGO, JAL, MEX, MICH, MOR, NLE, OAX, PUE, QRO, SLP, SIN, TLAX, VER, ZAC

*Rorippa microtites (B.L. Rob.) Rollins CHIH, SON

Rorippa pinnata (Moc. \& Sessé) Rollins AGS, COL, CDMX, GTO, GRO, HGO, JAL, MEX, MICH, TLAX, ZAC

Rorippa ramosa Rollins COAH, DGO

Rorippa sinuata (Nutt.) Hitchc. COAH

Rorippa teres (Michx.) Stuckey CHIH, COAH, COL, GTO, JAL, NAY, NLE, SLP, SON, TAMS, VER

*Scoliaxon mexicanus (S. Watson) Payson CHIH, COAH, NLE Selenia dissecta Torr. \& A. Gray CHIH, COAH, NLE

* Sibara angelorum (S. Watson) Greene BCN, BCS

* Sibara brandegeana (Rose) Greene BCN, BCS

* Sibara davidsonii Al-Shehbaz BCS

* Sibara laxa (S. Watson) Greene BCN, BCS

* Sibara pectinata (Greene) Greene BCN, BCS

Sibara runcinata (S. Watson) Rollins COAH, NLE, TAMS

Sisymbrium diffusum A. Gray CHIH, COAH

* Sphaerocardamum compressum (Rollins) Rollins COAH

*Sphaerocardamum divaricatum (Rollins) Rollins COAH, 
NLE, SLP

*Sphaerocardamum macropetalum (Rollins) Rollins COAH, NLE, ZAC

*Sphaerocardamum stellatum (S. Watson) Rollins COAH, NLE, SLP

Stanleya pinnata (Pursh) Britton BCN, CHIH, COAH, DGO

Streptanthella longirostris (S. Watson) Rydb. BCN, SON

Streptanthus arizonicus S. Watson BCN, BCS, CHIH, COAH, SON

Streptanthus bernardinus (Greene) Parish BCN

Streptanthus campestris S. Watson BCN

Streptanthus coulteri (S. Watson) Greene CHIH, COAH

Streptanthus hyacinthoides Hook. SLP, ZAC

Streptanthus platycarpus A. Gray CHIH, COAH

* Synthlipsis densiflora Rollins COAH

Synthlipsis greggii A. Gray CHIH, COAH, DGO, NLE, SLP,

TAMS, ZAC

*Thelypodiopsis incisa Rollins COAH

*Thelypodiopsis retrofracta (Rollins) Rollins COAH, NLE, ZAC

Thelypodiopsis shinnersii (M.C. Johnst.) Rollins TAMS

*Thelypodiopsis wootonii (B.L. Rob.) Rollins CHIH

*Thelypodium lobatum Brandegee COAH

*Thelypodium longipes (Rollins) Rollins COAH, NLE

Thelypodium wrightii (A. Gray) Rydb. BCN, BCS, CHIH, COAH, CDMX, GTO, HGO, MEX, NLE, SLP, SON, ZAC

Thysanocarpus curvipes Hook. BCN, SON

*Thysanocarpus erectus $\mathrm{S}$. Watson $\mathrm{BCN}$

Thysanocarpus laciniatus Nutt. ex Torr. \& A. Gray BCN

Thysanocarpus rigidus (Munz) P.J. Alexander \& Windham $\mathrm{BCN}$

Tomostima cuneifolia (Nutt. ex Torr. \& A. Gray) Al-Shehbaz, M. Koch \& Jordon-Thaden BCN, BCS, CHIH, COAH, NLE, SON

Tomostima platycarpa (Torr. \& A. Gray) Al-Shehbaz, M. Koch \& Jordon-Thaden $\mathrm{COAH}$

*Tomostima sonorae (Greene) Al-Shehbaz, M. Koch \& JordonThaden SON

Tropidocarpum gracile Hook. BCN

\section{Family Bromeliaceae}

*Aechmea aenigmatica López-Ferrari, Espejo, Ceja \& A. Mendoza OAX

Aechmea bracteata (Sw.) Griseb. CAM, CHIS, COL, DGO, GRO, HGO, JAL, MICH, NAY, OAX, PUE, QRO, QROO, SLP, SIN, TAB, TAMS, VER, YUC

Aechmea bromeliifolia (Rudge) Baker CAM, QROO

Aechmea cylindrica Mez VER

Aechmea lueddemanniana (K. Koch) Brongn. ex Mez CHIS, OAX, PUE, VER

Aechmea magdalenae (André) André ex Baker CHIS, OAX, QROO, VER

Aechmea matudae L.B. Sm. CHIS

Aechmea mexicana Baker CHIS, COL, GRO, HGO, JAL, OAX, PUE, SLP, TAB, VER

Aechmea nudicaulis (L.) Griseb. CHIS, HGO, OAX, PUE, SLP, TAB, VER
Aechmea tillandsioides (Mart. ex Schult. \& Schult. f.) Baker CAM, CHIS, OAX, QROO, TAB, VER

Androlepis skinneri (K. Koch) Brongn. ex Houllet CHIS, TAB, VER

Billbergia pallidiflora Liebm. CHIS, COL, GRO, JAL, MICH, NAY, OAX, SIN, VER

Billbergia viridiflora $\mathrm{H}$. Wendl. CHIS, TAB

Bromelia alsodes H. St. John CHIS, NAY, OAX, QRO, QROO, SLP, SIN, SON, TAB, TAMS, VER, YUC

Bromelia hemisphaerica Lam. CHIS, GTO, GRO, MEX, MICH, MOR, OAX, YUC

Bromelia karatas L. CAM, CHIS, COL, GRO, JAL, MICH, MOR, NAY, OAX, QRO, QROO, SLP, SIN, TAB, VER, YUC *Bromelia palmeri Mez COL, GRO, JAL, MICH, NAY, OAX Bromelia pinguin L. CAM, CHIS, COL, DGO, GRO, JAL, MEX, MICH, MOR, NAY, OAX, PUE, QRO, QROO, SLP, SIN, SON, TAB, TAMS, VER, YUC

Catopsis berteroniana (Schult. \& Schult. f.) Mez CAM, CHIS, GRO, OAX, QROO, TAB, VER, YUC

*Catopsis compacta Mez CHIS, COL, GRO, JAL, OAX, PUE, VER

Catopsis delicatula L.B. Sm. CHIS, OAX

Catopsis floribunda L.B. Sm. OAX, TAB, VER, YUC

Catopsis juncifolia Mez \& Wercklé ex Mez CHIS, VER

*Catopsis minimiflora Matuda CHIS, OAX

Catopsis montana L.B. Sm. CHIS, OAX

Catopsis morreniana Mez CHIS, HGO, OAX, TAB, VER

Catopsis nitida (Hook.) Griseb. CHIS, OAX, VER

Catopsis nutans (Sw.) Griseb. CAM, CHIS, COL, GRO, JAL, MEX, NAY, OAX, PUE, QROO, TAB, TAMS, VER, YUC

*Catopsis occulta Mart.-Correa, Espejo \& López-Ferrari CHIS, OAX, VER

Catopsis paniculata E. Morren CHIS, COL, GRO, JAL, MEX, MICH, NAY, OAX, PUE, VER, ZAC

Catopsis sessiliflora (Ruiz \& Pav.) Mez CAM, CHIS, GRO, HGO, JAL, MEX, MICH, OAX, PUE, TAB, VER

Catopsis subulata L.B. Sm. CHIS, OAX, VER

Catopsis wangerinii Mez \& Wercklé ex Mez CHIS, VER

Catopsis wawranea Mez CHIS, OAX, VER

Fosterella micrantha (Lindl.) L.B. Sm. CHIS, GRO, JAL, MEX, NAY, NLE, OAX, VER

* Greigia juareziana L.B. Sm. CHIS, OAX, VER

* Greigia van-hyningii L.B. Sm. CHIS, OAX, VER

Guzmania lingulata (L.) Mez CHIS

Guzmania nicaraguensis Mez \& C.F. Baker CHIS, OAX, VER

*Hechtia aquamarina I. Ramírez \& C.F. Jiménez PUE

*Hechtia argentea Baker HGO, QRO, SLP, VER

*Hechtia caerulea (Matuda) L.B. Sm. GRO, MEX, MICH

*Hechtia capituligera Mez SLP

*Hechtia carlsoniae Burt-Utley \& Utley GRO

*Hechtia caudata L.B. Sm. OAX

*Hechtia caulescens López-Ferrari, Espejo \& Martinez-Correa OAX, PUE, QRO

*Hechtia colossa Martínez-Correa, Espejo \& López-Ferrari GRO, OAX, PUE

*Hechtia complanata Burt-Utley OAX

*Hechtia confusa L.B. Sm. OAX, PUE 
*Hechtia conzattiana L.B. Sm. OAX, PUE

*Hechtia chichinautzensis Martínez-Correa, Espejo \& LópezFerrari MEX, MOR

*Hechtia deceptrix I. Ramírez \& Hornung HGO

*Hechtia edulis I. Ramírez, Espejo \& López-Ferrari CHIH

*Hechtia elliptica L.B. Sm. COAH, NLE, ZAC

*Hechtia epigyna Harms TAMS

*Hechtia flexilifolia I. Ramírez \& Carnevali OAX

*Hechtia fosteriana L.B. Sm. OAX

*Hechtia fragilis Burt-Utley \& Utley OAX, PUE

*Hechtia galeottii Mez OAX

*Hechtia gayorum L.W. Lenz BCS

*Hechtia glabra Brandegee VER

*Hechtia glauca Burt-Utley \& Utley GRO, MICH

Hechtia glomerata Zucc. AGS, CHIS, CHIH, COAH, DGO, GTO, GRO, HGO, JAL, MEX, NLE, OAX, QRO, SLP, TAMS, ZAC

*Hechtia hernandez-sandovalii I. Ramírez, C.F. Jiménez \& Treviño TAMS

*Hechtia hintoniana Burt-Utley, Utley \& García Mend. GRO, MEX, MICH

*Hechtia huamelulaensis I. Ramírez \& Carnevali OAX

*Hechtia iltisii Burt-Utley \& Utley JAL

*Hechtia isthmusiana Burt-Utley OAX

*Hechtia ixtlanensis Burt-Utley OAX

*Hechtia jaliscana L.B. Sm. COL, JAL

*Hechtia laevis L.B. Sm. COL, JAL

*Hechtia lanata L.B. Sm. OAX

*Hechtia laxissima L.B. Sm. JAL, MICH

*Hechtia lepidophylla I. Ramírez HGO, QRO, VER

*Hechtia liebmannii Mez PUE

*Hechtia lundelliorum L.B. Sm. HGO, QRO, SLP, VER

*Hechtia lyman-smithii Burt-Utley \& Utley OAX

*Hechtia macrophylla Greenm. VER

*Hechtia mapimiana López-Ferrari \& Espejo COAH, DGO

*Hechtia marnier-lapostollei L.B. Sm. MEX, OAX

*Hechtia matudae L.B. Sm. MEX, MOR

*Hechtia melanocarpa L.B. Sm. GRO, OAX

*Hechtia mexicana L.B. Sm. SLP, TAMS, VER

*Hechtia michoacana Burt-Utley, Utley \& García Mend. MICH

*Hechtia montana Brandegee BCS, CHIH, SIN, SON

*Hechtia montis-frigidi Gonz.-Rocha, Espejo, López-Ferrari \& Cerros MOR

*Hechtia mooreana L.B. Sm. GRO

*Hechtia myriantha Mez VER

*Hechtia nivea I. Ramírez \& C.F. Jiménez OAX

*Hechtia nuusaviorum Espejo \& López-Ferrari OAX

*Hechtia oaxacana Burt-Utley, Utley \& García Mend. OAX

*Hechtia pedicellata S. Watson JAL

*Hechtia perotensis I. Ramírez \& Martínez-Correa PUE, VER

*Hechtia podantha Mez AGS, COAH, DGO, GTO, GRO, HGO, JAL, MEX, MICH, MOR, NLE, OAX, PUE, QRO, SLP, TLAX, VER, ZAC

*Hechtia pretiosa Espejo \& López-Ferrari GTO, QRO, SLP

*Hechtia pringlei B.L. Rob. \& Greenm. GRO, HGO, MEX, MICH, MOR, OAX, PUE, VER

*Hechtia pueblensis Burt-Utley, Utley \& García-Mend. PUE
*Hechtia pumila Burt-Utley \& Utley GRO

*Hechtia purhepecha I. García, Espejo \& López-Ferrari JAL, $\mathrm{MICH}$

*Hechtia purpusii Brandegee VER

*Hechtia reflexa L.B. Sm. GRO, MICH

*Hechtia reticulata L.B. Sm. COL, JAL, MICH

*Hechtia rosea E. Morren ex Baker CHIS, GRO, OAX, VER

*Hechtia roseana L.B. Sm. OAX, PUE, VER

*Hechtia rubicunda López-Ferrari \& Espejo OAX

*Hechtia schottii Baker CAM, CHIS, PUE, QROO, SLP, VER, YUC

*Hechtia sphaeroblasta B.L. Rob. GRO, OAX, PUE

*Hechtia stenopetala Klotzsch VER

*Hechtia suaveolens E. Morren ex Mez ND

*Hechtia subalata L.B. Sm. CHIH, DGO, JAL, NAY, SIN, ZAC

Hechtia texensis S. Watson $\mathrm{CHIH}, \mathrm{COAH}, \mathrm{DGO}, \mathrm{HGO}, \mathrm{JAL}$, NLE, QRO, SLP, TAMS, VER, ZAC

*Hechtia tillandsioides (André) L.B. Sm. HGO, MEX, QRO, SLP, VER

*Hechtia zamudioi Espejo, López-Ferrari \& I. Ramírez QRO, SLP

*Hohenbergia mesoamericana I. Ramírez, Carnevali \& CetzalIx QROO

Hohenbergiopsis guatemalensis (L.B. Sm.) L.B. Sm. \& Read CHIS, OAX

*Pepinia amblyosperma (L.B. Sm.) G.S. Varad. \& Gilmartin HGO, PUE, QRO, SLP, VER

Pepinia punicea (Scheidw.) Brongn. ex André CHIS, TAB, VER

*Pitcairnia abundans L.B. Sm. JAL, NAY, OAX

Pitcairnia atrorubens (Beer) Baker CHIS, NAY

*Pitcairnia breedlovei L.B. Sm. CHIS, OAX

Pitcairnia calderonii Standl. \& L.B. Sm. CHIS

Pitcairnia carioana Wittm. CHIS

* Pitcairnia colimensis L.B. Sm. COL, JAL, MICH

*Pitcairnia compostelae McVaugh DGO, JAL, NAY

*Pitcairnia cylindrostachya L.B. Sm. GRO, JAL, MEX, MICH, NAY, ZAC

*Pitcairnia chiapensis Miranda CHIS, VER

* Pitcairnia densiflora Brongn. ex Lem. CHIS, GRO, PUE, VER

* Pitcairnia flavescentia Matuda GRO

*Pitcairnia flexuosa L.B. Sm. GRO, MEX, MICH

* Pitcairnia foliacea L.B. Sm. MICH

Pitcairnia heterophylla (Lindl.) Beer CHIS, COL, GRO, HGO, JAL, MEX, MOR, NAY, OAX, PUE, SIN, VER

*Pitcairnia hintoniana L.B. Sm. GRO, MEX

Pitcairnia imbricata (Brong.) Regel CHIS, COL, GRO, JAL, NAY, OAX, PUE, VER

* Pitcairnia jaliscana $\mathrm{S}$. Watson COL, JAL, ZAC

*Pitcairnia karwinskyana Schult. \& Schult. f. AGS, CHIS, COL, GRO, HGO, JAL, MEX, MICH, MOR, NAY, OAX, PUE, SLP, SIN, TAMS, VER, ZAC

*Pitcairnia lanosisepala Matuda GRO

* Pitcairnia leprosa L.B. Sm. GRO, MICH

*Pitcairnia loki-schmidtii Rauh \& Barthlott JAL

* Pitcairnia matudae L.B. Sm. CHIS

* Pitcairnia micropoda L.B. Sm. GRO, MEX

*Pitcairnia micheliana André COL, JAL, MICH, ZAC 
* Pitcairnia mirandae Utley \& Burt-Utley CHIS

* Pitcairnia modesta L.B. Sm. GRO

* Pitcairnia monticola Brandegee DGO, SIN

*Pitcairnia mooreana L.B. Sm. GRO

*Pitcairnia oaxacana L.B. Sm. COL, GRO, JAL, MICH, NAY, OAX, SIN

*Pitcairnia ocotensis Beutelsp. \& G. Lopez V. CHIS

*Pitcairnia palmeri S. Watson AGS, CHIH, COL, DGO, GRO, JAL, MEX, MICH, MOR, NAY, SIN, SON

*Pitcairnia pteropoda L.B. Sm. GRO, HGO, JAL, MEX, MICH, MOR, VER

Pitcairnia puberula Mez \& Donn. Sm. CHIS, GRO, OAX

* Pitcairnia queroana Espejo \& López-Ferrari CHIS

Pitcairnia recurvata (Scheidw.) K. Koch CHIS, OAX, TAB, VER

*Pitcairnia ringens Klotzsch $\mathrm{HGO}$, MICH, MOR, OAX, PUE, QRO, SLP, TAMS, VER

*Pitcairnia roseana L.B. Sm. DGO, GRO, JAL, MEX, MICH, MOR, NAY, PUE, ZAC

Pitcairnia saxicola L.B. Sm. CHIS

*Pitcairnia schiedeana Baker JAL, MICH, OAX, VER

*Pitcairnia secundiflora L.B. Sm. CHIS, OAX

*Pitcairnia sordida L.B. Sm. GRO

*Pitcairnia tabuliformis Linden CHIS, OAX, TAB, VER

*Pitcairnia tillandsioides L.B. Sm. GRO

Pitcairnia tuerckheimii Donn. Sm. CHIS, OAX

* Pitcairnia undulata Scheidw. CHIS, OAX, TAB, VER

* Pitcairnia vallisoletana Lex. JAL, MEX, MICH

* Pitcairnia virginalis Utley \& Burt-Utley OAX

* Pitcairnia xanthocalyx Mart. QRO, SLP, VER

*Pitcairnia yocupitziae Espejo \& López-Ferrari GRO

Racinaea adscendens (L.B. Sm.) M.A. Spencer \& L.B. Sm. CHIS, MOR, OAX, VER

Racinaea ghiesbreghtii (Baker) M.A. Spencer \& L.B. Sm. CHIS, GRO, OAX, VER

Racinaea rothschuhiana (Mez) M.A. Spencer \& L.B. Sm. CHIS *Tillandsia achyrostachys E. Morren ex Baker CHIS, CHIH, COL, DGO, GTO, GRO, HGO, JAL, MEX, MICH, MOR, NAY, OAX, PUE, QRO, SLP, SIN, SON, VER, ZAC

*Tillandsia aesii I. Ramírez \& Carnevali GRO, JAL, OAX

*Tillandsia aguascalientensis C.S. Gardner AGS, JAL, SLP, TAMS

*Tillandsia albida Mez \& Purpus GTO, HGO, QRO, SLP, VER

*Tillandsia alfredo-lauii Rauh \& J. Lehm. HGO

*Tillandsia alvareziae Rauh VER

*Tillandsia andrieuxii (Mez) L.B. Sm. AGS, CHIS, CDMX, GRO, HGO, MEX, MICH, MOR, OAX, PUE, QRO, VER

Tillandsia argentea Griseb. CHIS, GRO, OAX, SIN

*Tillandsia arroyoensis (W. Weber \& Ehlers) Espejo \& López-

Ferrari CHIH, COAH, NLE, SLP, TAMS

*Tillandsia atenangoensis Ehlers \& Wülfinghoff GRO, OAX

*Tillandsia atroviolacea Ehlers \& Koide OAX

Tillandsia baileyi Rose ex Small CHIS, JAL, OAX, QROO, TAMS

Tillandsia balbisiana Schult. \& Schult. f. CAM, CHIS, COL, GRO, JAL, MEX, MICH, NAY, OAX, QROO, SLP, SIN, TAB, TAMS, VER, YUC
Tillandsia bartramii Elliott CHIS, COAH, COL, GTO, GRO, HGO, JAL, MICH, NAY, NLE, OAX, QRO, SLP, TAMS, VER, ZAC

Tillandsia belloensis W. Weber CHIS, OAX, VER

Tillandsia beutelspacheri Matuda CHIS, OAX

*Tillandsia bochilensis Ehlers CHIS

*Tillandsia boqueronensis Ehlers OAX

*Tillandsia borealis López-Ferrari \& Espejo-Serna DGO, SIN

*Tillandsia botterii E. Morren ex Baker VER

*Tillandsia bourgaei Baker CHIS, COL, CDMX, DGO, GTO, GRO, HGO, JAL, MEX, MICH, MOR, NAY, OAX, PUE, SIN, VER

Tillandsia brachycaulos Schltdl. CAM, CHIS, MEX, MICH, MOR, OAX, PUE, QRO, QROO, SLP, TAB, VER, YUC

Tillandsia bulbosa Hook. CAM, CHIS, MOR, QROO, TAB, VER, YUC

Tillandsia butzii Mez CHIS, CDMX, GRO, HGO, MEX, OAX, PUE, VER

*Tillandsia caballosensis Ehlers GRO

*Tillandsia califani Rauh OAX, PUE

*Tillandsia calothyrsus Mez COL, GRO, JAL, NAY, OAX

*Tillandsia callichroma L. Hrom. OAX

*Tillandsia capistranoensis Ehlers \& W. Weber JAL, ZAC

Tillandsia capitata Griseb. CHIS, COL, GRO, JAL, MEX, MICH, NAY, OAX, PUE, SIN, SON, VER, ZAC

Tillandsia caput-medusae E. Morren CAM, CHIS, CHIH, COL, CDMX, DGO, GRO, JAL, MEX, MICH, MOR, NAY, OAX, PUE, QROO, SIN, SON, TAB, VER, ZAC

*Tillandsia carlos-hankii Matuda GRO, OAX

*Tillandsia carlsoniae L.B. Sm. CHIS

*Tillandsia celata Ehlers \& Lautner OAX

*Tillandsia circinnatoides Matuda GRO, MEX, MOR, OAX, PUE, VER

*Tillandsia coalcomanensis Ehlers MICH

*Tillandsia comitanensis Ehlers CHIS

Tillandsia compressa Bertero ex Schult. \& Schult. f. CHIS, COL, GTO, JAL, MEX, MICH, NAY, OAX, PUE, SLP, VER

*Tillandsia concolor L.B. Sm. CHIS, GRO, OAX, VER

*Tillandsia copalaensis Ehlers GRO, OAX

*Tillandsia cossoni Baker COL, CDMX, GTO, GRO, JAL, MEX, MICH, MOR, NAY, OAX, SIN, ZAC

*Tillandsia cretacea L.B. Sm. CHIH, SON

*Tillandsia crista-galli Ehlers CHIS

*Tillandsia chaetophylla Mez GRO, JAL, MEX, MICH, MOR, NAY, OAX, VER

*Tillandsia chalcatzingensis Gonz.-Rocha, Cerros, LópezFerrari \& Espejo MOR

*Tillandsia chapalillaensis Ehlers \& Lautner JAL, NAY

*Tillandsia chiapensis C.S. Gardner CHIS

Tillandsia chlorophylla L.B. Sm. CHIS, OAX, VER

Tillandsia dasyliriifolia Baker CAM, CHIS, COL, GRO, HGO, JAL, MEX, MICH, MOR, NAY, OAX, PUE, QROO, SLP, TAB,

TAMS, VER, YUC

Tillandsia deflexa L.B. Sm. CHIS

*Tillandsia delicata Ehlers OAX

Tillandsia deppeana Steud. GTO, HGO, OAX, PUE, QRO, SLP, TAMS, VER 
*Tillandsia diguettii Mez \& Rol.-Goss. COL, JAL

*Tillandsia dugesii Baker AGS, CHIS, GTO, GRO, JAL, MEX, MICH, OAX, PUE, QRO

*Tillandsia durangensis Rauh \& Ehlers DGO, SIN

*Tillandsia ehlersiana Rauh CHIS

*Tillandsia eistetteri Ehlers CHIS, COL, DGO, GRO, JAL, MICH, NAY, OAX, SIN

*Tillandsia eizii L.B. Sm. CHIS

Tillandsia elizabethae Rauh CHIH, SON

Tillandsia elongata Kunth CAM, QROO, TAB, YUC

*Tillandsia elusiva Pinzón, I. Ramírez \& Carnevali CHIS

*Tillandsia erubescens Schltdl. AGS, CHIH, COAH, CDMX, DGO, GTO, GRO, HGO, JAL, MEX, MICH, NLE, OAX, PUE, QRO, SLP, SIN, SON, TAMS, TLAX, VER, ZAC

*Tillandsia escahuascensis Espejo, López-Ferrari, Ceja \& A.

Mend. PUE

Tillandsia excelsa Griseb. CHIS, SON

*Tillandsia exserta Fernald SIN, SON

Tillandsia fasciculata Sw. CAM, CHIS, COL, DGO, GTO, GRO, HGO, JAL, MEX, MICH, MOR, NAY, OAX, PUE, QRO, QROO, SLP, SIN, TAB, TAMS, TLAX, VER, YUC, ZAC

*Tillandsia fascifolia Flores Cruz \& Diego-Esc. GRO

*Tillandsia ferrisiana L.B. Sm. BCS, DGO, NAY, SIN

Tillandsia festucoides Brongn. ex Mez CAM, CHIS, COL, JAL, OAX, QROO, TAB, TAMS, VER

Tillandsia filifolia Schltdl. \& Cham. CHIS, HGO, OAX, PUE, TAB, VER

Tillandsia flabellata Baker CHIS, OAX, VER

*Tillandsia flavobracteata Matuda OAX, VER

Tillandsia flexuosa Sw. CAM, QROO, YUC

*Tillandsia floresensis Ehlers CHIS

*Tillandsia foliosa M. Martens \& Galeotti CHIS, OAX, TAB, VER

*Tillandsia fresnilloensis W. Weber \& Ehlers DGO, ZAC

Tillandsia fuchsii W. Till CAM, CHIS, COL, GRO, JAL, MICH,

NAY, OAX, SIN, TAB, VER, YUC

*Tillandsia glabrior (L.B. Sm.) López-Ferrari, Espejo \& I. Ramírez OAX

*Tillandsia gracillima L.B. Sm. PUE

*Tillandsia graebeneri Mez VER

Tillandsia grandis Schltdl. CHIS, GTO, GRO, HGO, MEX,

MOR, OAX, PUE, QRO, SLP, VER

*Tillandsia grandispica Ehlers GRO

*Tillandsia grossispicata Espejo, López-Ferrari \& W. Till COL, GTO, GRO, JAL, MICH

Tillandsia guatemalensis L.B. Sm. CHIS, GRO, HGO, OAX, PUE, VER

*Tillandsia guenther-nolleri Ehlers COL, JAL, MICH

*Tillandsia guerreroensis Rauh GRO

*Tillandsia gymnobotrya Baker CHIS, GRO, HGO, OAX, PUE, VER

*Tillandsia hammeri Rauh \& Ehlers OAX, PUE

*Tillandsia heterophylla E. Morren CHIS, HGO, OAX, PUE, VER

*Tillandsia hintoniana L.B. Sm. GRO, MEX, MICH, MOR

*Tillandsia huajuapanensis Ehlers \& Lautner OAX

*Tillandsia huamelulaensis Ehlers OAX
*Tillandsia hubertiana Matuda GRO, MEX, MOR

*Tillandsia ilseana W. Till, Halbritter \& Zecher GRO

Tillandsia imperialis E. Morren ex Mez CHIS, HGO, JAL, NLE, OAX, PUE, QRO, SLP, VER

*Tillandsia inopinata Espejo, López-Ferrari \& W. Till GTO, HGO, QRO, SLP, TAMS, VER

*Tillandsia intermedia Mez COL, GRO, JAL, MICH, NAY, SIN

*Tillandsia intumescens L.B. Sm. GRO, JAL, MEX, MICH

Tillandsia ionantha Planch. CHIS, COL, GTO, GRO, HGO, JAL, MEX, MICH, MOR, NAY, OAX, QRO, SLP, SIN, TAMS, VER

*Tillandsia jaliscomonticola Matuda COL, JAL, MICH

*Tillandsia jaliscopinicola L. Hrom \& P. Schneid. JAL

*Tillandsia juerg-rutschmannii Rauh CHIS

Tillandsia juncea (Ruiz \& Pav.) Poir. CAM, CHIS, COL, CDMX, GTO, GRO, HGO, JAL, MEX, MICH, NAY, OAX, PUE, QRO, QROO, SLP, SIN, TAB, TLAX, VER, ZAC

*Tillandsia kalmbacheri Matuda GRO, OAX

*Tillandsia karwinskyana Schult. \& Schult. f. AGS, GTO, GRO, HGO, MEX, NLE, PUE, QRO, SLP, TAMS

*Tillandsia kirchhoffiana Wittm. MEX, OAX, PUE, VER

*Tillandsia klausii Ehlers CHIS

Tillandsia kolbii W. Till \& Schatzl CHIS, OAX

*Tillandsia lagunaensis Ehlers OAX

Tillandsia lampropoda L.B. Sm. CHIS, OAX, TAB, VER

*Tillandsia langlasseana Mez COL, GTO, GRO, JAL, MEX, $\mathrm{MICH}$

*Tillandsia laui Matuda GRO, OAX

Tillandsia lautneri Ehlers CHIS, OAX

Tillandsia leiboldiana Schltdl. CHIS, OAX, PUE, TAMS, VER

*Tillandsia leucolepis L.B. Sm. OAX

*Tillandsia limbata Schltdl. CAM, CHIS, CHIH, GRO, HGO, JAL, MEX, MICH, OAX, PUE, QRO, QROO, SLP, TAB, VER

*Tillandsia loma-blancae Ehlers \& Lautner JAL

*Tillandsia loxichaensis Ehlers OAX

Tillandsia lucida E. Morren ex Baker CHIS, OAX, PUE, VER

*Tillandsia lydiae Ehlers GRO

*Tillandsia macdougallii L.B. Sm. COL, CDMX, DGO, GTO, GRO, HGO, JAL, MEX, MICH, NAY, OAX, PUE, QRO, SIN, TLAX, VER, ZAC

*Tillandsia macrochlamys Baker DGO, NAY, PUE, SIN, VER, ZAC

*Tillandsia macropetala Wawra CHIS, OAX, PUE, VER

*Tillandsia macvaughii Espejo \& López-Ferrari COL, JAL, $\mathrm{MICH}$

*Tillandsia magnispica Espejo \& López-Ferrari CHIS, OAX Tillandsia magnusiana Wittm. CHIS, COL, GRO, JAL, MOR, OAX, PUE

Tillandsia makoyana Baker CAM, CHIS, COL, DGO, GTO, GRO, JAL, MEX, MICH, MOR, NAY, OAX, PUE, QROO, SIN

*Tillandsia makrinii L. Hrom. OAX

*Tillandsia marabascoensis Ehlers \& Lautner COL, GRO, JAL

Tillandsia mateoensis Ehlers CHIS

Tillandsia matudae L.B. Sm. CHIS, OAX

*Tillandsia maya I. Ramírez \& Carnevali YUC

*Tillandsia may-patii I. Ramírez \& Carnevali QROO

*Tillandsia mazatlanensis Rauh SIN 
*Tillandsia mirabilis L. Hrom. GRO, OAX

*Tillandsia mitlaensis W. Weber \& Ehlers OAX

*Tillandsia mixtecorum Ehlers \& Koide OAX

*Tillandsia mooreana L.B. Sm. CHIH, DGO, GRO, JAL, NAY, OAX, SIN, SON

*Tillandsia moronensis Ehlers JAL, ZAC

Tillandsia multicaulis Steud. CHIS, OAX, PUE, VER

*Tillandsia nicolasensis Ehlers COL, JAL, OAX

*Tillandsia nolleriana Ehlers OAX, VER

*Tillandsia novakii $\mathrm{H}$. Luther VER

*Tillandsia nuyooensis Ehlers OAX

*Tillandsia oaxacana L.B. Sm. COL, GRO, JAL, NAY, OAX

*Tillandsia occulta $\mathrm{H}$. Luther DGO, SIN

Tillandsia orogenes Standl. \& L.O. Williams CHIS

*Tillandsia ortgiesiana E. Morren ex Mez COL, GRO, JAL, OAX

*Tillandsia pacifica Ehlers JAL

*Tillandsia palmasolana Matuda VER

*Tillandsia pamelae Rauh COL, JAL

*Tillandsia paraisoensis Ehlers GRO

*Tillandsia parryi Baker COAH, GTO, HGO, MEX, NLE, PUE, QRO, SLP, TAMS, TLAX, VER

Tillandsia paucifolia Baker CAM, CHIS, GRO, JAL, OAX, QROO, VER, YUC

*Tillandsia penascoensis Ehlers \& Lautner OAX

*Tillandsia pentasticha Rauh \& Wülfinghoff GRO, OAX

*Tillandsia pinicola I. Ramírez \& Carnevali OAX

Tillandsia polita L.B. Sm. CHIS, OAX, PUE

Tillandsia polystachia (L.) L. AGS, CAM, CHIS, COL, GRO, HGO, JAL, MEX, MICH, NAY, OAX, PUE, QRO, QROO, SLP, SIN, TAB, TAMS, VER, ZAC

Tillandsia ponderosa L.B. Sm. CHIS, OAX

*Tillandsia pringlei $\mathrm{S}$. Watson GTO, HGO, MEX, NLE, QRO, SLP, TAMS, VER

*Tillandsia prodigiosa (Lem.) Baker CHIS, COL, CDMX, DGO, GRO, HGO, JAL, MEX, MICH, MOR, NAY, OAX, PUE, SIN, TLAX, VER

Tillandsia pruinosa Sw. CHIS, OAX, TAB, VER

Tillandsia pseudobaileyi C.S. Gardner CHIS, COL, GRO, JAL, MICH, NAY, OAX, QROO, VER, YUC

*Tillandsia pseudooaxacana Ehlers OAX

*Tillandsia pseudosetacea Ehlers \& Rauh COL, DGO, GRO, JAL, NAY, OAX, SIN

*Tillandsia pueblensis L.B. Sm. MICH, MOR, OAX, PUE

Tillandsia punctulata Schltdl. \& Cham. CHIS, HGO, OAX, PUE, VER

*Tillandsia quaquaflorifera Matuda COL, GRO, JAL, OAX

*Tillandsia rayonensis Ehlers COAH, NLE

Tillandsia recurvata (L.) L. AGS, BCN, BCS, CAM, CHIS, CHIH, COAH, COL, CDMX, DGO, GTO, GRO, HGO, JAL, MEX, MICH, MOR, NAY, NLE, OAX, PUE, QRO, QROO, SLP, SIN, SON, TAMS, TLAX, VER, YUC, ZAC

*Tillandsia religiosa Hern.-Cárdenas, Gonz.-Rocha, Espejo, López-Ferrari, Cerros \& Ehlers MOR

Tillandsia remota Wittm. OAX, SIN, VER

* Tillandsia rhodocephala Ehlers \& Koide OAX

Tillandsia rhomboidea André CHIS
Tillandsia rodrigueziana Mez CHIS, OAX, VER

*Tillandsia roland-gosselinii Mez COL, GRO, JAL, MICH, OAX

*Tillandsia roseoscapa Matuda PUE

*Tillandsia roseospicata Matuda GRO, MEX, TLAX

*Tillandsia rothii Rauh COL, JAL

Tillandsia rotundata (L.B. Sm.) C.S. Gardner CHIS

*Tillandsia rubrispica Ehlers \& Koide OAX

*Tillandsia salmonea Ehlers CHIS

*Tillandsia santosiae Ehlers OAX

*Tillandsia schatzlii Rauh OAX, PUE

Tillandsia schiedeana Steud. AGS, BCN, BCS, CAM, CHIS, COL, DGO, GTO, GRO, HGO, JAL, MEX, MICH, MOR, NAY, OAX, PUE, QRO, QROO, SLP, SIN, TAB, TAMS, VER, YUC, ZAC

*Tillandsia schusteri Rauh OAX

Tillandsia seleriana Mez CHIS, COL, JAL, MICH, NAY, OAX, VER

*Tillandsia sessemocinoi López-Ferrari, Espejo \& P. Blanco JAL, MEX, MICH, MOR

*Tillandsia setiformis Ehlers OAX

*Tillandsia sierrahalensis Espejo \& López-Ferrari COL, JAL

*Tillandsia socialis L.B. Sm. CHIS, OAX

Tillandsia standleyi L.B. Sm. CHIS

Tillandsia streptophylla Scheidw. ex E. Morren CAM, CHIS, COL, GRO, HGO, JAL, MEX, MICH, MOR, NAY, OAX, PUE, QROO, SIN, TAB, VER, YUC

*Tillandsia subinflata L.B. Sm. ZAC

*Tillandsia sueae Ehlers HGO, MEX, NLE, PUE, QRO, SLP, TAMS, TLAX, VER

*Tillandsia suesilliae Espejo, López-Ferrari \& W. Till HGO, SLP

*Tillandsia superinsignis Matuda GRO, MEX

*Tillandsia supermexicana Matuda GRO, MEX, MOR, OAX

*Tillandsia takizawae Ehlers \& H. Luther PUE

*Tillandsia taxcoensis Ehlers GRO

*Tillandsia tehuacana I. Ramírez \& Carnevali PUE

*Tillandsia teloloapanensis Ehlers \& Lautner GRO

*Tillandsia thyrsigera E. Morren ex Baker GRO, MEX, MICH

*Tillandsia tillii Ehlers JAL

*Tillandsia tonalaensis Ehlers GRO, OAX

*Tillandsia trauneri L. Hrom. GRO

Tillandsia tricolor Schltdl. \& Cham. CHIS, GRO, JAL, OAX, PUE, VER

*Tillandsia tuxtlensis Matuda SLP, TAMS, VER

*Tillandsia ulrici Ehlers OAX

Tillandsia usneoides (L.) L. AGS, BCN, BCS, CAM, CHIS, COAH, COL, CDMX, DGO, GTO, GRO, HGO, JAL, MEX, MICH, MOR, NAY, NLE, OAX, PUE, QRO, QROO, SLP, SIN, TAB, TAMS, TLAX, VER, YUC, ZAC

Tillandsia utriculata L. CAM, CHIS, OAX, QROO, SLP, TAB, TAMS, VER, YUC

Tillandsia variabilis Schltdl. CAM, CHIS, COL, JAL, MICH, OAX, PUE, QRO, QROO, SLP, TAB, VER, ZAC

*Tillandsia velutina Ehlers CHIS

*Tillandsia ventanaensis Ehlers \& Koide DGO, SIN

Tillandsia verapazana Ehlers CHIS 
*Tillandsia vernardoi Rauh OAX

Tillandsia vicentina Standl. CHIS, OAX, PUE

*Tillandsia violacea Baker CHIS, CDMX, GRO, HGO, JAL, MEX, MICH, MOR, OAX, PUE, QRO, VER

Tillandsia viridiflora (Beer) Baker CHIS, GRO, HGO, OAX, PUE, QRO, SLP, TAMS, VER

*Tillandsia vriesioides Matuda CHIS

*Tillandsia weberi L. Hrom. \& P. Schneid. COL, JAL, MICH

*Tillandsia wuelfinghoffii Ehlers OAX

*Tillandsia $\times$ alfredo-lauii Rauh \& J. Lehm. HGO, VER

$*$ Tillandsia $\times$ cuchnichim R. Guess \& V. Guess CHIS

*Tillandsia $\times$ jaguactalensis I. Ramírez, Carnevali \& Chi CAM, QROO

*Tillandsia $\times$ jaliscopinicola L. Hrom. \& P. Schneid. COL, JAL

$*$ Tillandsia $\times$ marceloi Takizawa \& P. Koide OAX

*Tillandsia $\times$ nidus Rauh \& J. Lehm. ND

Tillandsia $\times$ rectifolia C.A. Wiley ND

Tillandsia xerographica Rohweder CHIS, GRO, OAX

*Tillandsia yerba-santae Ehlers OAX

Tillandsia yunckeri L.B. Sm. OAX

*Tillandsia yutaninoensis Ehlers \& Lautner OAX

*Tillandsia zacualpanensis Ehlers \& R. Wülfinghoff MEX

*Tillandsia zaragozaensis Ehlers NLE, TAMS

*Tillandsia zoquensis Ehlers CHIS, OAX

*Ursulaea macvaughii (L.B. Sm.) Read \& H.U. Baensch COL, JAL, MICH

*Ursulaea tuitensis (Magaña \& E.J. Lott) Read \& H.U. Baensch DGO, GRO, JAL, NAY, SIN

*Viridantha atroviridipetala (Matuda) Espejo COL, GTO, GRO, HGO, JAL, MEX, MICH, MOR, OAX, PUE, VER, ZAC

*Viridantha curvifolia (Ehlers \& Rauh) López-Ferrari \& Espejo GTO, JAL, QRO, SLP, ZAC

*Viridantha ignesiae (Mez) Espejo COL, GTO, GRO, JAL, MEX, MICH, MOR, QRO

*Viridantha lepidosepala (L.B. Sm.) Espejo GTO, GRO, HGO, JAL, MEX, MICH, PUE, QRO, SLP, VER

*Viridantha mauryana (L.B. Sm.) Espejo AGS, GRO, HGO, JAL, OAX, QRO, VER, ZAC

*Viridantha penascoensis (Ehlers \& Lautner) Espejo \& LópezFerrari OAX

Viridantha plumosa (Baker) Espejo CHIS, COL, DGO, GTO, GRO, HGO, JAL, MEX, MICH, MOR, NAY, OAX, PUE, TAB, VER, ZAC

*Viridantha tortilis (Klotzsch ex Baker) Espejo AGS, CDMX, DGO, GTO, HGO, JAL, MEX, MICH, QRO, SLP, TAMS, VER, ZAC

Vriesea heliconioides (Kunth) Hook. ex Walp. CAM, CHIS, OAX, QROO, TAB, VER

*Vriesea malzinei E. Morren CHIS, OAX, TAB, VER

Werauhia gladioliflora (H. Wendl.) J.R. Grant CHIS, OAX, TAB, VER

Werauhia noctiflorens Krömer, Espejo, López-Ferrari \& Acebey CHIS, VER

*Werauhia nocturna (Matuda) J.R. Grant OAX, VER

Werauhia pectinata (L.B. Sm.) J.R. Grant CHIS, VER

Werauhia pycnantha (L.B. Sm.) J.R. Grant CHIS, OAX

*Werauhia vanhyningii (L.B. Sm.) J.R. Grant CHIS, OAX,
PUE, VER

Werauhia viridiflora (Regel) J.R. Grant CHIS, OAX

Werauhia werckleana (Mez) J.R. Grant CHIS, OAX, VER

\section{Family Brunelliaceae}

Brunellia mexicana Standl. CHIS, GRO, HGO, MEX, OAX, PUE, VER

\section{Family Burmanniaceae}

Apteria aphylla (Nutt.) Barnhart ex Small CHIS, OAX, PUE, QROO, VER

Burmannia capitata (Walter ex J.F. Gmel.) Mart. CAM, CHIS, QROO, TAB, VER

Burmannia flava Mart. CHIS, QROO

Dictyostega orobanchoides (Hook.) Miers CHIS, HGO, OAX, QROO, VER

Gymnosiphon divaricatus (Benth.) Benth. \& Hook. f. CAM, CHIS, OAX, QROO, YUC

Gymnosiphon panamensis Jonker CAM, CHIS, OAX, QROO, VER

Gymnosiphon suaveolens (H. Karst.) Urb. CAM, CHIS, HGO, OAX, QRO, QROO, SLP, VER

Thismia panamensis (Standl.) Jonker CHIS

\section{Family Burseraceae}

* Beiselia mexicana Forman MICH

*Bursera altijuga Rzed., Calderón \& Medina OAX, PUE

*Bursera aptera Ramírez COL, GRO, HGO, JAL, MEX, MICH, MOR, OAX, PUE, VER

*Bursera arborea (Rose) L. Riley CHIS, CHIH, COL, DGO, GRO, JAL, MEX, MICH, MOR, NAY, OAX, SIN, SON

*Bursera arida (Rose) Standl. OAX, PUE

*Bursera ariensis (Kunth) McVaugh \& Rzed. CHIS, COL, GRO, JAL, MEX, MICH, MOR, NAY, OAX, PUE

*Bursera aspleniifolia Brandegee COL, JAL, MEX, OAX, PUE, VER

*Bursera attenuata (Rose) L. Riley DGO, GRO, JAL, NAY, SIN, SON

*Bursera bicolor (Willd. ex Schltdl.) Engl. GRO, JAL, MEX, MICH, MOR, OAX, PUE

* Bursera biflora (Rose) Standl. OAX, PUE

Bursera bipinnata (DC.) Engl. AGS, CHIS, CHIH, COL, DGO, GTO, GRO, HGO, JAL, MEX, MICH, MOR, NAY, OAX, PUE, SIN, SON, VER, ZAC

* Bursera bolivarii Rzed. GRO, OAX, PUE

* Bursera bonetii Rzed. GRO, OAX

* Bursera cerasifolia Brandegee BCN, BCS

*Bursera cinerea Engl. OAX, PUE, VER

*Bursera citronella McVaugh \& Rzed. COL, GRO, JAL, MICH, OAX

* Bursera collina Brandegee DGO, SIN

*Bursera confusa (Rose) Engl. CHIH, COL, GRO, JAL, MEX, MICH, NAY, SON

*Bursera copallifera (Sessé \& Moc. ex DC.) Bullock COL, DGO, GTO, GRO, HGO, JAL, MEX, MICH, MOR, NAY, OAX, PUE, VER, ZAC

*Bursera coyucensis Bullock GRO, JAL, MEX, MICH

*Bursera crenata Paul G. Wilson COL, GRO, JAL, MEX, MICH 
*Bursera cuneata (Schltdl.) Engl. CAM, CDMX, GTO, GRO, HGO, JAL, MEX, MICH, MOR, OAX, PUE, QRO, SLP, ZAC *Bursera chemapodicta Rzed. \& E. Ortiz GRO

*Bursera denticulata McVaugh \& Rzed. COL, GRO, JAL, $\mathrm{MICH}, \mathrm{NAY}$

*Bursera discolor Rzed. CHIH, COL, GRO, JAL, MEX, MICH, MOR, NAY, OAX, PUE, SON

Bursera diversifolia Rose CHIS, COL, GTO, GRO, JAL, MEX, $\mathrm{MICH}, \mathrm{MOR}, \mathrm{OAX}$, PUE

*Bursera epinnata (Rose) Engl. BCN, BCS, COL, JAL

* Bursera esparzae Rzed., Calderón \& Medina OAX

Bursera excelsa (Kunth) Engl. AGS, CHIS, COL, DGO, GTO, GRO, JAL, MEX, MICH, MOR, NAY, OAX, QRO, SIN, VER, ZAC

Bursera fagaroides (Kunth) Engl. AGS, BCN, BCS, CHIS, CHIH, COAH, COL, CDMX, DGO, GTO, GRO, HGO, JAL, MEX, MICH, MOR, NAY, NLE, OAX, PUE, QRO, SLP, SIN, SON, TAMS, VER, ZAC

*Bursera filicifolia Brandegee BCS, SON

* Bursera fragilis S. Watson CHIH, DGO, SIN, SON

*Bursera fragrantissima Bullock COL, GRO, MICH

*Bursera galeottiana Engl. GTO, GRO, OAX, PUE, QRO, SLP

* Bursera glabrifolia (Kunth) Engl. CHIS, COL, GRO, JAL, MEX, MICH, MOR, NAY, OAX, PUE, SON, VER

*Bursera grandifolia (Schltdl.) Engl. BCS, CHIS, CHIH, COL, DGO, GTO, GRO, JAL, MEX, MICH, MOR, NAY, OAX, PUE, SIN, SON, VER, ZAC

Bursera graveolens (Kunth) Triana \& Planch. CAM, CHIS, HGO, MEX, OAX, PUE, QRO, QROO, SLP, TAB, VER, YUC * Bursera heliae Rzed. \& Calderón OAX

Bursera heteresthes Bullock CAM, CHIS, COL, GRO, JAL, MEX, MICH, OAX, QROO

*Bursera hindsiana (Benth.) Engl. BCN, BCS, COL, JAL, SON, ZAC

* Bursera hintonii Bullock GRO, MEX, MICH, OAX

*Bursera infernidialis Guevara \& Rzed. GRO, JAL, MEX, MICH, OAX

* Bursera instabilis McVaugh \& Rzed. COL, GRO, JAL, MICH, NAY, OAX, PUE, ZAC

*Bursera isthmica Rzed. \& Calderón OAX

*Bursera jerzyi Medina OAX

*Bursera kerberi Engl. BCS, CHIS, COL, GRO, JAL, MEX, $\mathrm{MICH}, \mathrm{NAY}, \mathrm{OAX}, \mathrm{ZAC}$

*Bursera krusei Rzed. COL, GRO, JAL, MEX, MICH, MOR, NAY, OAX, ZAC

*Bursera lancifolia (Schltdl.) Engl. BCS, CHIH, GRO, HGO, JAL, MEX, MICH, MOR, NAY, OAX, PUE, QRO, SLP, SIN, SON, VER

* Bursera laurihuertae Rzed. \& Calderón OAX

*Bursera laxiflora S. Watson BCN, BCS, CHIH, GRO, SIN, SON

*Bursera linanoe (La Llave) Rzed., Calderón \& Medina GRO, HGO, HGO, MEX, MICH, MOR, OAX, PUE, VER

*Bursera littoralis León de la Luz \& Pérez Navarro BCS

*Bursera longipes (Rose) Standl. CHIS, GRO, MEX, MICH, MOR, OAX, PUE

* Bursera macvaughiana Cuevas \& Rzed. COL, JAL
* Bursera madrigalii Rzed. \& Calderón MEX, MICH

* Bursera martae J. Jiménez Ram. \& Cruz-Durán GRO

* Bursera medranoana Rzed. \& E. Ortiz HGO, QRO, VER

Bursera microphylla A. Gray BCN, BCS, CHIH, SON, ZAC

*Bursera mirandae C.A. Toledo CHIS, GRO, MEX, OAX,

PUE

Bursera morelensis Ramírez DGO, GTO, GRO, HGO, JAL, MEX, MICH, MOR, OAX, PUE, QRO, SLP, VER, ZAC

* Bursera multifolia (Rose) Engl. JAL, ZAC

*Bursera multijuga Engl. AGS, COL, DGO, GRO, JAL, MICH, NAY, SIN, SON, ZAC

*Bursera occulta McVaugh \& Rzed. COL, MICH

Bursera odorata Brandegee BCN, BCS, CHIH, OAX, SIN, SON, ZAC

Bursera ovalifolia (Schltdl.) Engl. CHIS, COL, GRO, JAL, MEX, MICH, OAX

*Bursera palaciosii Rzed. \& Calderón JAL

*Bursera palmeri S. Watson AGS, COL, DGO, GTO, GRO, JAL, MEX, MICH, NAY, OAX, QRO, SLP, SIN, ZAC

*Bursera paradoxa Guevara \& Rzed. GRO, MICH

*Bursera penicillata (Sessé \& Moc. ex DC.) Engl. AGS, CHIH, COL, DGO, GTO, GRO, HGO, JAL, MEX, MICH, MOR, NAY, OAX, QROO, SLP, SIN, SON, VER, YUC, ZAC

*Bursera pontiveteris Rzed., Calderón \& Medina OAX, PUE

* Bursera ribana Rzed. \& Calderón COL, JAL, MICH

* Bursera roseana Rzed., Calderón \& Medina AGS, COL, DGO, GRO, JAL, MEX, MICH, NAY, OAX, ZAC

* Bursera rupicola León de la Luz BCS

* Bursera rzedowskii C.A. Toledo GRO

*Bursera sarcopoda Paul G. Wilson COL, GRO, JAL, MICH, OAX

*Bursera sarukhanii Guevara \& Rzed. GRO, JAL, MICH

Bursera schlechtendalii Engl. CAM, CHIS, COAH, COL, DGO, GTO, GRO, HGO, JAL, MEX, MICH, MOR, NAY, NLE, OAX, PUE, QRO, QROO, SLP, TAMS, VER, YUC, ZAC

*Bursera silviae Rzed. \& Calderón OAX

Bursera simaruba (L.) Sarg. AGS, CAM, CHIS, CHIH, COL, DGO, GTO, GRO, HGO, JAL, MEX, MICH, MOR, NAY, OAX, PUE, QRO, QROO, SLP, SIN, SON, TAB, TAMS, VER, YUC, ZAC

*Bursera simplex Rzed. \& Calderón OAX

Bursera staphyleoides McVaugh \& Rzed. GRO, MICH

*Bursera stenophylla Sprague \& L. Riley CHIH, DGO, SIN, SON

*Bursera submoniliformis Engl. GRO, HGO, MEX, MICH, MOR, OAX, PUE, VER

*Bursera subtrifoliata (Rose) Standl. JAL, MICH, NAY, ZAC

* Bursera suntui C.A. Toledo GRO, OAX, PUE

*Bursera tecomaca (DC.) Standl. COL, CDMX, GRO, JAL, MEX, MICH

* Bursera toledoana Rzed. \& Calderón GRO, MICH

Bursera tomentosa (Jacq.) Triana \& Planch. CAM, CHIS, GRO, OAX, QROO, YUC

*Bursera trifoliolata Bullock DGO, GRO, JAL, MEX, MICH, NAY

*Bursera trimera Bullock COL, GRO, JAL, MEX, MICH

*Bursera vazquezyanesii Rzed. \& Calderón JAL 
*Bursera vejar-vazquezii Miranda GRO, MEX, MICH, MOR, OAX, PUE

*Bursera velutina Bullock GRO, MEX, MICH, OAX, PUE

*Bursera xochipalensis Rzed. GRO, HGO, MICH, OAX, PUE, VER

*Bursera xolocotzii Guevara GRO, MICH

Protium confusum (Rose) Pittier CHIS, QROO, YUC

Protium copal (Schltdl. \& Cham.) Engl. CAM, CHIS, GRO, HGO, OAX, PUE, QRO, QROO, SLP, TAB, TAMS, VER, YUC

Protium glabrum (Rose) Engl. CHIS, OAX, VER

Protium multiramiflorum Lundell CAM, CHIS, OAX, QROO, VER

Protium pittieri (Rose) Engl. OAX

\section{Family Buxaceae}

Buxus bartlettii Standl. HGO, OAX, QROO, VER

*Buxus lancifolia Brandegee QRO, SLP

*Buxus mexicana Brandegee OAX, PUE

*Buxus microphylla Siebold \& Zucc. VER

*Buxus moctezumae Eg. Köhler, R. Fernández \& Zamudio QRO, SLP

*Buxus pubescens Greenm. NAY

Sarcococca conzattii (Standl.) I.M. Johnst. COL, GRO, JAL, OAX

\section{Family Cabombaceae}

Brasenia schreberi J.F. Gmel. CHIS, JAL, MICH, SLP, TAB, VER

Cabomba palaeformis Fassett CAM, CHIS, MEX, MOR, OAX, QRO, QROO, SLP, TAB, TAMS, VER

\section{Family Cactaceae}

Acanthocereus horridus Britton \& Rose CHIS, OAX

*Acanthocereus occidentalis Britton \& Rose COL, DGO, GRO, JAL, MICH, NAY, OAX, SIN, SON

*Acanthocereus subinermis Britton \& Rose GRO, MOR, OAX, PUE, VER

Acanthocereus tetragonus (L.) Hummelinck CAM, CHIS, GRO, HGO, JAL, MICH, NLE, OAX, QRO, QROO, SLP, TAB, TAMS, VER, YUC

*Acharagma aguirreanum (Glass \& R.A. Foster) Glass COAH

*Acharagma roseanum (Boed.) E.F. Anderson COAH, NLE

*Ariocarpus agavoides (Castañeda) E.F. Anderson COAH, NLE, SLP, TAMS

Ariocarpus fissuratus (Engelm.) K. Schum. CHIH, COAH, DGO, GTO, JAL, NLE, SLP, TAMS, ZAC

*Ariocarpus kotschoubeyanus (Lem.) K. Schum. COAH, DGO, HGO, NLE, QRO, SLP, TAMS, ZAC

*Ariocarpus retusus Scheidw. COAH, DGO, NLE, SLP, TAMS, ZAC

*Ariocarpus scaphirostris Boed. NLE

Astrophytum asterias (Zucc.) Lem. NLE, TAMS

*Astrophytum capricorne (A. Dietr.) Britton \& Rose COAH, DGO, NLE, SLP

*Astrophytum caput-medusae (Velazco \& Nevárez) D.R. Hunt NLE, TAMS

*Astrophytum myriostigma Lem. COAH, DGO, NLE, SLP,
TAMS, ZAC

*Astrophytum ornatum (DC.) F.A.C. Weber ex Britton \& Rose COAH, GTO, HGO, QRO, SLP, TAMS, VER

*Aztekium hintonii Glass \& Fitz Maurice NLE, TAMS

*Aztekium ritteri (Boed.) Boed. ex A. Berger COAH, NLE, SLP

*Backebergia militaris (Audot) Bravo ex Sánchez-Mej. GRO, JAL, MICH

Bergerocactus emoryi (Engelm.) Britton \& Rose BCN, BCS

Carnegiea gigantea (Engelm.) Britton \& Rose BCN, CHIH, SON

*Cephalocereus apicicephalium E.Y. Dawson CHIS, OAX

*Cephalocereus columna-trajani (Karw. ex Pfeiff.) K. Schum.

OAX, PUE

*Cephalocereus nizandensis (Bravo \& T. MacDoug.) Buxb. CHIS, OAX

*Cephalocereus senilis (Haw.) Pfeiff. GTO, HGO, QRO, SLP, TAMS, VER

*Cephalocereus totolapensis (Bravo \& T. MacDoug.) Buxb. CHIS, OAX

*Cochemiea halei (Brandegee) Walton BCN, BCS

*Cochemiea pondii (Greene) Walton BCN, BCS

*Cochemiea poselgeri (Hildm.) Britton \& Rose BCS

*Corynopuntia bulbispina (Engelm.) F.M. Knuth COAH, DGO, NLE, ZAC

Corynopuntia emoryi M.P. Griff. BCN, CHIH, COAH, SON

Corynopuntia grahamii (Engelm.) F.M. Knuth AGS, CHIH, COAH, DGO, JAL, NLE, SLP, ZAC

*Corynopuntia invicta (Brandegee) F.M. Knuth BCN, BCS

*Corynopuntia moelleri (A. Berger) F.M. Knuth COAH, DGO, SLP

*Corynopuntia reflexispina (Wiggins \& Rollins) Backeb. SON Corynopuntia schottii (Engelm.) F.M. Knuth CHIH, COAH, DGO, NLE, SLP, TAMS

*Corynopuntia vilis (Rose) F.M. Knuth AGS, COAH, DGO, NLE, SLP, ZAC

Coryphantha clavata (Scheidw.) Backeb. AGS, GTO, HGO, JAL, MICH, QRO, SLP, VER, ZAC

*Coryphantha compacta (Engelm.) Britton \& Rose CHIH, COAH, DGO, NLE, SLP, SON, TAMS, ZAC

*Coryphantha delaetiana (Quehl) A. Berger CHIH, COAH, DGO, HGO, NLE, QRO, SLP, TAMS

*Coryphantha delicata L. Bremer COAH, DGO, NLE, QRO, SLP, TAMS, ZAC

*Coryphantha difficilis (Quehl) A. Berger COAH, NLE, SLP

*Coryphantha durangensis (Runge ex K. Schum.) Britton \& Rose COAH, DGO

*Coryphantha echinoidea (Quehl) Britton \& Rose DGO, GTO, NLE, SLP, ZAC

Coryphantha echinus (Engelm.) Britton \& Rose CHIH, COAH, GTO, HGO, NLE, QRO, SLP

*Coryphantha elephantidens (Lem.) Lem. AGS, GTO, GRO, HGO, JAL, MEX, MICH, MOR, OAX, PUE, QRO, SLP, VER, ZAC

*Coryphantha erecta (Lem.) Lem. GTO, HGO, QRO, SLP, VER

*Coryphantha georgii Boed. NLE, SLP, TAMS, ZAC

*Coryphantha glanduligera (Otto \& A. Dietr.) Lem. COAH, 
DGO, GTO, JAL, NLE, SLP, TAMS, ZAC

*Coryphantha glassii Dicht. \& A. Lüthy GTO, SLP

* Coryphantha gracilis L. Bremer \& A.B. Lau CHIH

* Coryphantha guerkeana (Boed.) Britton \& Rose AGS, COAH, DGO, JAL, NLE, SLP, TAMS, ZAC

*Coryphantha hintoniorum Dicht \& A. Lüthy COAH, NLE, SLP

*Coryphantha jalpanensis Buchenau GTO, HGO, QRO, SLP

* Coryphantha kracikii Halda, Chalupa \& Kupcak DGO

* Coryphantha longicornis Boed. CHIH, COAH, DGO

Coryphantha macromeris (Engelm.) Lem. CHIH, COAH, DGO,

NLE, SLP, TAMS, ZAC

*Coryphantha maiz-tablasensis Backeb. GTO, JAL, SLP, TAMS, ZAC

* Coryphantha neglecta L. Bremer COAH, NLE

Coryphantha nickelsiae (K. Brandegee) Britton \& Rose COAH, NLE, TAMS

*Coryphantha octacantha (DC.) Britton \& Rose AGS, GTO, HGO, JAL, NLE, QRO, SLP, TAMS, VER, ZAC

*Coryphantha ottonis (Pfeiff.) Lem. AGS, DGO, GTO, HGO, JAL, MEX, MICH, PUE, QRO, SLP, VER, ZAC

* Coryphantha pallida Britton \& Rose OAX, PUE, VER

* Coryphantha poselgeriana (A. Dietr.) Britton \& Rose CHIH, COAH, DGO, NLE, SLP, ZAC

*Coryphantha potosiana (Jacobi) Glass \& R.A. Foster NLE, SLP, ZAC

*Coryphantha pseudoechinus Boed. COAH, NLE, SLP, ZAC

* Coryphantha pseudonickelsiae Backeb. CHIH, COAH, DGO

* Coryphantha pulleineana (Backeb.) Glass SLP, TAMS

* Coryphantha pycnacantha (Mart.) Lem. AGS, CDMX, HGO,

JAL, MEX, OAX, PUE, QRO, VER

Coryphantha radians (DC.) Britton \& Rose AGS, CHIH, COAH, DGO, GTO, HGO, JAL, MEX, NLE, OAX, PUE, QRO, SLP, TAMS, VER, ZAC

Coryphantha ramillosa Cutak CHIH, COAH, NLE

Coryphantha recurvata (Engelm.) Britton \& Rose DGO, SON

* Coryphantha reduncispina Boed. OAX, PUE

* Coryphantha retusa (Pfeiff.) Britton \& Rose OAX, PUE

Coryphantha robustispina (Ant. Schott ex Engelm.) Britton \&

Rose CHIH, COAH, DGO, NLE, SON, ZAC

* Coryphantha salinensis (Poselg.) A.D. Zimmerman ex Dicht. \& A. Lüthy CHIH, COAH, NLE, TAMS

Coryphantha sulcata (Engelm.) Britton \& Rose COAH, NLE, TAMS

* Coryphantha tripugionacantha A.B. Lau JAL, ZAC

* Coryphantha unicornis Boed. COAH, DGO, GTO, QRO

*Coryphantha vaupeliana Boed. DGO, TAMS, ZAC

*Coryphantha voghtherriana Werderm. \& Boed. GTO, JAL, SLP, ZAC

* Coryphantha werdermannii Boed. CHIH, COAH, DGO

* Coryphantha wohlschlageri Holzeis SLP, TAMS

*Cumarinia odorata (Boed.) Buxb. COAH, GTO, JAL, NLE, SLP, TAMS, ZAC

Cylindropuntia acanthocarpa (Engelm. \& Bigelow) F.M. Knuth BCN, SON

*Cylindropuntia alcahes (F.A.C. Weber) F.M. Knuth BCN, BCS, SIN
* Cylindropuntia anteojoensis (Pinkava) E.F. Anderson COAH Cylindropuntia arbuscula (Engelm.) F.M. Knuth CHIH, SIN, SON

Cylindropuntia bigelovii (Engelm.) F.M. Knuth BCN, BCS, SON

Cylindropuntia californica (Torr. \& A. Gray) F.M. Knuth BCN, SON

*Cylindropuntia calmalliana (J.M. Coult.) F.M. Knuth BCN, BCS

* Cylindropuntia cedrosensis Rebman BCN

*Cylindropuntia cholla (F.A.C. Weber) F.M. Knuth BCN, BCS Cylindropuntia echinocarpa (Engelm. \& Bigelow) F.M. Knuth BCN, BCS, SON

Cylindropuntia fulgida (Engelm.) F.M. Knuth BCN, BCS, CHIH, SIN, SON

Cylindropuntia ganderi (C.B. Wolf) Rebman BCN

Cylindropuntia imbricata (Haw.) F.M. Knuth AGS, BCS, CHIH, COAH, CDMX, DGO, GTO, HGO, JAL, MEX, MICH, NLE, PUE, QRO, SLP, SON, TAMS, VER, ZAC

Cylindropuntia kleiniae (DC.) F.M. Knuth CHIH, COAH, DGO, GTO, HGO, NLE, PUE, QRO, SLP, SIN, SON, TAMS, VER, ZAC

Cylindropuntia leptocaulis (DC.) F.M. Knuth BCN, BCS, CHIH, COAH, DGO, GTO, HGO, JAL, NLE, PUE, QRO, SLP, SIN, SON, TAMS, VER, ZAC

*Cylindropuntia libertadensis Rebman BCN

* Cylindropuntia lindsayi (Rebman) Rebman BCN, BCS

*Cylindropuntia molesta (K. Brandegee) F.M. Knuth BCN, BCS, SON

Cylindropuntia munzii (C.B. Wolf) Backeb. BCN, SON

Cylindropuntia prolifera (Engelm.) F.M. Knuth BCN, BCS, SON

Cylindropuntia ramosissima (Engelm.) F.M. Knuth BCN, BCS, SON

Cylindropuntia rosea (DC.) Backeb. CDMX, GTO, HGO, MEX, PUE, QRO, SLP, TLAX, VER

*Cylindropuntia sanfelipensis (Rebman) Rebman BCN

* Cylindropuntia santamaria (E.M. Baxter) Rebman BCS

Cylindropuntia spinosior (Engelm.) F.M. Knuth CHIH, DGO, SON

*Cylindropuntia tesajo (Engelm. ex J.M. Coult.) F.M. Knuth BCN, BCS, SON

Cylindropuntia thurberi (Engelm.) F.M. Knuth CHIH, SIN, SON

Cylindropuntia tunicata (Lehm.) F.M. Knuth AGS, COAH, CDMX, DGO, GTO, HGO, JAL, MEX, NLE, PUE, QRO, SLP, TAMS, TLAX, VER, ZAC

Cylindropuntia versicolor (Engelm. ex J.M. Coult.) F.M. Knuth BCN, CHIH, SON

Cylindropuntia wolfii (L.D. Benson) Rebman BCN

*Cylindropuntia waltoniorum Rebman BCN

*Disocactus ackermannii (Haw.) Ralf Bauer CHIS, MEX, MICH, OAX, VER

Disocactus cinnabarinus (Eichlam ex Weing.) Barthlott CHIS, GRO, OAX

*Disocactus flagelliformis (L.) Barthlott GTO, HGO, MEX, OAX, PUE, QRO, SLP, VER 
*Disocactus macdougallii (Alexander) Barthlot CHIS

*Disocactus macranthus (Alexander) Kimnach \& Hutchison CHIS, OAX, VER

*Disocactus martianus (Zucc.) Barthlott OAX

Disocactus nelsonii (Britton \& Rose) Linding. CHIS, GRO, OAX

*Disocactus phyllanthoides (DC.) Barthlot HGO, OAX, PUE, VER

Disocactus speciosus (Cav.) Barthlott CHIS, COL, CDMX, DGO, GRO, HGO, JAL, MEX, MICH, MOR, OAX, SIN, ZAC *Echinocactus grusonii Hildm. GTO, HGO, QRO, SLP, ZAC Echinocactus horizonthalonius Lem. AGS, CHIH, COAH, CDMX, DGO, GTO, HGO, MEX, NLE, QRO, SLP, SON, TAMS, ZAC

*Echinocactus parryi Engelm. CHIH, COAH, SON

* Echinocactus platyacanthus Link \& Otto COAH, DGO, GTO, HGO, JAL, NLE, OAX, PUE, QRO, SLP, TAMS, VER, ZAC Echinocactus polycephalus Engelm. \& Bigelow BCS, SON

Echinocactus texensis Hopffer CHIH, COAH, DGO, NLE, TAMS, ZAC

*Echinocereus adustus Engelm. CHIH, DGO

*Echinocereus barthelowanus Britton \& Rose BCS

Echinocereus berlandieri (Engelm.) Haage COAH, HGO, NLE, QRO, SLP, TAMS

*Echinocereus brandegeei (J.M. Coult.) K. Schum. BCN, BCS Echinocereus bristolii W.T. Marshall SON

*Echinocereus carmenensis W. Blum, Mich. Lange \& E. Scherer $\mathrm{COAH}$

Echinocereus cinerascens (DC.) Lem. COAH, CDMX, GTO, HGO, JAL, MEX, NLE, QRO, SLP, TAMS, VER, ZAC

Echinocereus coccineus Engelm. CHIH, COAH, SON

Echinocereus chisoensis W.T. Marshall COAH, DGO

Echinocereus dasyacanthus Engelm. CHIH, COAH, SON

Echinocereus engelmannii (Parry ex Engelm.) Lem. BCN, BCS, SON

Echinocereus enneacanthus Engelm. CHIH, COAH, DGO, NLE, QRO, SLP, TAMS, ZAC

Echinocereus fasciculatus (Engelm. ex S. Watson) L.D. Benson SIN, SON

Echinocereus fendleri (Engelm.) Rümpler CHIH, COAH, SON

*Echinocereus ferreirianus H.E. Gates BCN

*Echinocereus grandis Britton \& Rose BCN, BCS, SON

* Echinocereus klapperi W. Blum SON

* Echinocereus knippelianus Liebner COAH, NLE, SLP, TAMS

*Echinocereus laui G. Frank CHIH, SON

*Echinocereus leucanthus N.P. Taylor SIN, SON

*Echinocereus lindsayi J. Meyrán BCN

*Echinocereus longisetus (Engelm.) Lem. CHIH, COAH, NLE

*Echinocereus mapimiensis E.F. Anderson, W. Hodgs. \& P. Quirk COAH, DGO

*Echinocereus maritimus (M.E. Jones) K. Schum. BCN, BCS

Echinocereus nicholii (L.D. Benson) B.D. Parfitt SON

*Echinocereus nivosus Glass \& R.A. Foster COAH, NLE

*Echinocereus ortegae Rose ex J.G. Ortega DGO, SIN, SON

* Echinocereus palmeri Britton \& Rose CHIH, DGO, ZAC

* Echinocereus pamanesiorum A.B. Lau DGO, JAL, NAY, ZAC

Echinocereus papillosus Linke ex Rümpler NLE, SLP, TAMS
*Echinocereus parkeri N.P. Taylor COAH, NLE, SLP, TAMS, ZAC

Echinocereus pectinatus (Scheidw.) Engelm. AGS, CHIH, COAH, DGO, GTO, NLE, SLP, SON, TAMS, ZAC

*Echinocereus pensilis (K. Brandegee) Purpus BCS

Echinocereus pentalophus (DC.) Lem. COAH, GTO, HGO, JAL, NLE, QRO, SLP, TAMS, VER, ZAC

Echinocereus polyacanthus Engelm. BCN, CHIH, DGO, GTO, JAL, SLP, ZAC

Echinocereus poselgeri Lem. COAH, DGO, NLE, TAMS

*Echinocereus primolanatus A.F. Schwarz ex N.P. Taylor COAH, GTO, JAL, SLP, ZAC

Echinocereus pseudopectinatus (N.P. Taylor) N.P. Taylor SON

*Echinocereus pulchellus (Mart.) C.F. Först. ex F. Seitz AGS, COAH, GTO, HGO, JAL, NLE, OAX, PUE, QRO, SLP, TAMS, VER, ZAC

*Echinocereus rayonesensis N.P. Taylor COAH, NLE, TAMS

Echinocereus reichenbachii (Terscheck ex Walp.) Haage CHIH, COAH, NLE, TAMS

Echinocereus rigidissimus (Engelm.) F. Haage AGS, CHIH, COAH, DGO, SON

Echinocereus russanthus Weniger CHIH, COAH

Echinocereus sciurus (K. Brandegee) Dams BCN, BCS, SIN, SON

*Echinocereus scopulorum Britton \& Rose BCN, NAY, SIN, SON

*Echinocereus scheeri (Salm-Dyck) Scheer CHIH, DGO, JAL, NAY, SON, ZAC

*Echinocereus schereri G. Frank DGO

*Echinocereus schmollii (Weing.) N.P. Taylor HGO, QRO, SLP

*Echinocereus spinigemmatus A.B. Lau JAL, ZAC

*Echinocereus stoloniferus W.T. Marshall CHIH, SIN, SON

Echinocereus stramineus (Engelm.) Engelm. ex F. Seitz CHIH, COAH, DGO, GTO, HGO, JAL, NLE, QRO, SLP, TAMS, ZAC *Echinocereus subinermis Salm-Dyck ex Scheer CHIH, DGO, SIN, SON

*Echinocereus topiensis Rischer \& Trocha DGO

Echinocereus triglochidiatus Engelm. AGS, BCN, CHIH, COAH, DGO, GTO, JAL, NAY, NLE, SLP, SIN, SON, TAMS, ZAC

Echinocereus viereckii Werderm. COAH, NLE, TAMS

Echinocereus viridiflorus Engelm. CHIH, COAH

* Echinocereus websterianus G.E. Linds. SON

Echinocereus $\times$ roetteri (Engelm.) Rümpler $\mathrm{CHIH}$

*Echinomastus erectocentrus (J.M. Coult.) Britton \& Rose SON *Echinomastus gautii (L.D. Benson) Mosco \& Zanovello COAH, NLE, SLP, TAMS

Echinomastus intertextus (Engelm.) Britton \& Rose BCN, CHIH, COAH, SON

Echinomastus mariposensis Hester COAH, NLE

Echinomastus unguispinus Engelm. CHIH, COAH, DGO, SLP, ZAC

Echinomastus warnockii (L. Benson) Glass \& Foster CHIH, $\mathrm{COAH}$

*Epiphyllum anguliger (Lem.) G. Don COL, GRO, HGO, JAL, MEX, MICH, NAY, OAX, PUE

*Epiphyllum caudatum Britton \& Rose CAM, CHIS, OAX, 
QROO, TAB, VER

Epiphyllum crenatum (Lindl.) G. Don CHIS, HGO, OAX, PUE, TAB, VER

Epiphyllum hookeri Haw. CAM, CHIS, OAX, GRO, JAL, MOR, OAX, QRO, QROO, SLP, TAB, VER, YUC

*Epiphyllum laui Kimnach CHIS

Epiphyllum oxypetalum (DC.) Haw. CHIS, OAX, TAB, VER

Epiphyllum phyllanthus (L.) Haw. CAM, CHIS, MEX, MICH, OAX, QROO, TAB, VER, YUC

Epiphyllum thomasianum (K. Schum.) Britton \& Rose CHIS, VER

Epithelantha micromeris (Engelm.) F.A.C. Weber CHIH, COAH, DGO, NLE, SLP, TAMS, ZAC

*Escobaria chihuahuensis Britton \& Rose CHIH, DGO, NLE

Escobaria dasyacantha (Engelm.) Britton \& Rose CHIH, COAH, DGO, GTO, JAL, NLE, SLP, ZAC

Escobaria emskoetteriana (Quehl) Borg COAH, NLE, TAMS

*Escobaria henricksonii Glass \& R. Foster CHIH

* Escobaria laredoi (Glass \& Foster) N.P. Taylor COAH, ZAC

*Escobaria lloydii Britton \& Rose COAH, NLE, ZAC

Escobaria missouriensis (Sweet) D.R. Hunt COAH, NLE, SLP

Escobaria sneedii Britton \& Rose CHIH, COAH, NLE

Escobaria tuberculosa (Engelm.) Britton \& Rose CHIH, COAH,

DGO, HGO, NLE, QRO, SLP, SON, ZAC

Escobaria vivipara (Nutt.) Buxb. CHIH, COAH, NLE, SON, ZAC

*Escobaria zilziana (Boed.) Backeb. COAH, DGO, NLE

*Escontria chiotilla (F.A.C. Weber) Rose GRO, MICH, OAX, PUE

*Ferocactus alamosanus Britton \& Rose CHIH, DGO, SON

Ferocactus cylindraceus (Engelm.) Orcutt BCN, SON

* Ferocactus chrysacanthus (Orcutt) Britton \& Rose BCN, BCS

*Ferocactus diguetii (F.A.C. Weber) Britton \& Rose BCN, BCS

Ferocactus echidne (DC.) Britton \& Rose GTO, HGO, NLE, QRO, SLP, TAMS, VER

Ferocactus emoryi (Engelm.) Orcutt SON

*Ferocactus flavovirens (Scheidw.) Britton \& Rose OAX, PUE

Ferocactus fordii (Orcutt) Britton \& Rose BCN, BCS

*Ferocactus glaucescens (DC.) Britton \& Rose GTO, HGO,

NLE, QRO, SLP, TAMS, VER

*Ferocactus gracilis Gates BCN, BCS

Ferocactus hamatacanthus (Muehlenpf.) Britton \& Rose CHIH, COAH, DGO, GTO, NLE, PUE, QRO, SLP, TAMS, VER, ZAC *Ferocactus histrix (DC.) Lindsay AGS, DGO, GTO, HGO, JAL, MICH, NLE, PUE, QRO, SLP, TAMS, VER, ZAC

*Ferocactus johnstonianus Britton \& Rose BCN

*Ferocactus latispinus (Haw.) Britton \& Rose AGS, COAH, CDMX, DGO, GTO, GRO, HGO, JAL, MEX, MOR, NLE, OAX, PUE, QRO, SLP, VER, ZAC

*Ferocactus lindsayi Bravo GRO, MICH

*Ferocactus macrodiscus (Mart.) Britton \& Rose GTO, OAX, QRO, SLP, TAMS

*Ferocactus peninsulae (Engelm. ex F.A.C. Weber) Britton \& Rose BCN, BCS

*Ferocactus pilosus (Galeotti ex Salm Dyck) Werderm. COAH, DGO, GTO, JAL, NLE, SLP, TAMS, ZAC

*Ferocactus pottsii (Salm-Dyck) Backeb. CHIH, SIN, SON
Ferocactus rectispinus (Engelm.) Britton \& Rose BCN, BCS, $\mathrm{CHIH}, \mathrm{SON}$

*Ferocactus recurvus (Mill.) Y. Ito ex G.E. Linds. OAX, PUE

*Ferocactus reppenhagenii $\mathrm{G}$. Unger COL, JAL, MICH, OAX

* Ferocactus robustus (Link \& Otto) Britton \& Rose OAX, PUE

*Ferocactus schwarzii Lindsay SIN

*Ferocactus townsendianus Britton \& Rose BCS

Ferocactus viridescens (Torr. \& A. Gray) Britton \& Rose BCN

Ferocactus wislizenii (Engelm.) Britton \& Rose BCN, BCS, CHIH, COAH, DGO, SIN, SON

*Geohintonia mexicana Glass \& Fitz Maurice NLE, TAMS

*Glandulicactus crassihamathus (F.A.C. Weber) Backeb. GTO, QRO, SLP

Glandulicactus uncinatus (Pfeiff \& Otto) Backeb. CHIH, COAH, DGO, GTO, JAL, NLE, QRO, SLP, SON, TAMS, VER, ZAC

*Grusonia bradtiana (J.M. Coult.) Britton \& Rose CHIH, COAH, DGO, NLE

Grusonia kunzei (Rose) Pinkava BCN, SON

*Grusonia marenae (W.E. Parsons) E.F. Anderson SON

*Hamatocactus crassihamatus (F.A.C. Weber) Buxb. GTO, QRO, SLP

Hylocereus ocamponis (Salm-Dyck) Britton \& Rose COL, GRO, JAL, MEX, MICH, MOR, NAY, OAX, QRO, SIN

*Hylocereus purpusii (Weing.) Britton \& Rose COL, DGO, GRO, JAL, MEX, MICH, NAY, OAX, QRO

Hylocereus undatus (Haw.) Britton \& Rose AGS, CAM, CHIS, COL, DGO, GTO, GRO, HGO, JAL, MEX, MICH, MOR, NAY, OAX, PUE, QRO, QROO, SLP, TAB, TAMS, VER, YUC

*Lemaireocereus hollianus (F.A.C. Weber) Britton \& Rose OAX, PUE

*Leuchtenbergia principis Hook. COAH, DGO, GTO, HGO, JAL, NLE, SLP, TAMS, ZAC

*Lophocereus gatesii M.E. Jones BCS

*Lophocereus marginatus (DC.) S. Arias \& Terrazas AGS, COL, CDMX, DGO, GTO, GRO, HGO, JAL, MEX, MICH, MOR, NLE, OAX, PUE, QRO, SLP, TAMS, TLAX, VER, ZAC

Lophocereus schottii (Engelm.) Britton \& Rose BCN, BCS, SON

*Lophophora diffusa (Croizat) Bravo HGO, QRO, SLP

Lophophora williamsii (Lem.ex Salm-Dyck) J.M. Coult. CHIH, COAH, DGO, JAL, NLE, SLP, TAMS, ZAC

* Mammillaria albicans (Britton \& Rose) A. Berger BCN, BCS

* Mammillaria albicoma Boed. NLE, SLP, TAMS

*Mammillaria albiflora (Werderm.) Backeb. GTO, QRO, SLP Mammillaria albilanata Backeb. CHIS, COL, GRO, JAL, OAX *Mammillaria amajacensis C. Brachet \& M. Lacoste HGO, VER

*Mammillaria anniana Glass \& R. Foster SLP, TAMS, VER

* Mammillaria armillata $\mathrm{K}$. Brandegee BCS

*Mammillaria aureilanata Backeb. COAH, GTO, JAL, NLE, SLP, TAMS, ZAC

*Mammillaria backebergiana Buchenau GRO, MEX

Mammillaria barbata Engelm. CHIH, DGO

* Mammillaria baumii Boed. BCS, TAMS

*Mammillaria beneckei C. Ehrenb. COL, GRO, JAL, MEX, MICH, MOR, NAY, OAX, ZAC 
*Mammillaria berkiana A.B. Lau DGO, JAL, NAY, ZAC

* Mammillaria blossfeldiana Boed. BCN, BCS

* Mammillaria bocasana Poselg. GTO, JAL, QRO, SLP, ZAC

* Mammillaria bocensis Craig SIN, SON

* Mammillaria boelderliana Wohlschl. ZAC

* Mammillaria bombycina Quehl AGS, COAH, GTO, JAL, SLP

* Mammillaria boolii Lindsay SON

Mammillaria brandegeei (J.M. Coult.) K. Brandegee BCN, BCS

*Mammillaria capensis (Gates) Craig BCS, SON

* Mammillaria carmenae Castañeda TAMS

*Mammillaria carnea Zucc. ex Pfeiff. OAX, PUE, VER

* Mammillaria carretii Rebut ex K. Schum. COAH, NLE

* Mammillaria cerralboa (Britton \& Rose) Orcutt BCS

*Mammillaria cielensis Martínez-Avalos, Golubov, S. Arias \& Villarreal TAMS

* Mammillaria coahuilensis (Boed.) Moran COAH, DGO, NLE, ZAC

Mammillaria columbiana Salm-Dyck CHIS, YUC

Mammillaria compressa DC. GTO, HGO, JAL, MEX, NLE, QRO, SLP, TAMS, VER, ZAC

*Mammillaria crinita DC. AGS, GTO, HGO, JAL, MEX, MICH, NLE, QRO, SLP, VER, ZAC

*Mammillaria crucigera Mart. OAX, PUE

* Mammillaria chionocephala Purpus COAH, DGO, NLE, SLP, ZAC

*Mammillaria decipiens Scheidw. DGO, GTO, HGO, JAL, QRO, SLP, VER, ZAC

* Mammillaria deherdtiana Farwig OAX

*Mammillaria densispina (J.M. Coult.) Vaupel AGS, DGO, GTO, JAL, MICH, QRO, SLP, TAMS, ZAC

Mammillaria dioica $\mathrm{K}$. Brandegee BCN, BCS, SIN

*Mammillaria discolor Haw. CDMX, HGO, MEX, MOR, OAX, PUE, TLAX, VER

* Mammillaria dixanthocentron Backeb. ex Mottram OAX, PUE

*Mammillaria duoformis R.T. Craig \& E.Y. Dawson GRO, OAX, PUE

*Mammillaria duwei Rogoz. \& P.J. Braun GTO, SLP

* Mammillaria elongata DC. GTO, HGO, QRO, SLP, VER

* Mammillaria eriacantha Link \& Otto ex Pfeiff. VER

*Mammillaria erythrosperma Boed. GTO, JAL, QRO, SLP, TAMS, ZAC

*Mammillaria evermanniana (Britton \& Rose) Orcutt BCS, SON

* Mammillaria fittkaui Glass \& R.A. Foster GTO, JAL, MICH

*Mammillaria formosa Galeotti ex Scheidw. COAH, GTO, HGO, MICH, NLE, SLP, TAMS, ZAC

*Mammillaria gaumeri Orcutt QROO, VER, YUC

Mammillaria geminispina Haw. GTO, HGO, QRO, SLP, TAMS, VER

*Mammillaria gigantea Hildm. ex K. Schum. GTO, JAL, QRO, SLP

*Mammillaria glassii R.A. Foster COAH, NLE, SLP, TAMS

*Mammillaria goodridgii Scheer ex Salm-Dyck BCN, BCS

*Mammillaria gracilis Pfeiff. HGO, QRO, VER

Mammillaria grahamii Engelm. BCN, CHIH, SON

* Mammillaria grusonii Runge CHIH, COAH, DGO

*Mammillaria guelzowiana Werderm. COAH, DGO, NLE
*Mammillaria guerreronis (Bravo) Boed. ex Backeb. \& F.M. Knuth GRO

* Mammillaria guillauminiana Backeb. DGO, SIN

*Mammillaria haageana Pfeiff. CDMX, GTO, GRO, HGO, JAL, MEX, MOR, OAX, PUE, QRO, SLP, TLAX, VER

*Mammillaria hahniana Werderm. GTO, HGO, JAL, QRO, SLP

*Mammillaria heidiae Krainz PUE

* Mammillaria hernandezii Glass \& R.A. Foster OAX

* Mammillaria herrerae Werderm. GTO, HGO, QRO, SLP

*Mammillaria hertrichiana R.T. Craig CHIH, SON

Mammillaria heyderi Muehlenpf. CHIH, COAH, DGO, NLE, SLP, SON, TAMS, VER, YUC, ZAC

*Mammillaria huitzilopochtli D.R. Hunt OAX

*Mammillaria humboldtii Ehrenb. HGO, QRO

* Mammillaria hutchisoniana (Gates) Boed. BCN, BCS

* Mammillaria insularis Gates BCN, BCS

*Mammillaria jaliscana (Britton \& Rose) Boed. DGO, GTO, JAL, MICH, QRO, SLP, ZAC

* Mammillaria johnstonii (Britton \& Rose) Orcutt SON

*Mammillaria karwinskiana Mart. COL, GRO, JAL, MEX, MICH, MOR, NAY, OAX, PUE

*Mammillaria klissingiana Boed. GTO, NLE, SLP, TAMS

*Mammillaria knippeliana Quehl MOR

* Mammillaria kraehenbuehlii (Krainz) Krainz OAX, PUE

* Mammillaria lanata (Britton \& Rose) Orcutt OAX, PUE

Mammillaria lasiacantha Engelm. CHIH, COAH, DGO, NLE, SLP, SON, ZAC

*Mammillaria laui D.R. Hunt TAMS

* Mammillaria lenta K. Brandegee COAH

* Mammillaria lindsayi R.T. Craig CHIH, DGO

*Mammillaria longiflora (Britton \& Rose) Berger CHIH, DGO, SIN, ZAC

Mammillaria longimamma DC. GTO, HGO, QRO, SLP, TAMS, VER

*Mammillaria luethyi G.S. Hinton COAH

*Mammillaria lloydii (Britton \& Rose) Orcutt GTO, SLP, ZAC

*Mammillaria magallanii Schmoll ex R.T. Craig COAH, ZAC

*Mammillaria magnifica Buchenau MOR, PUE

* Mammillaria magnimamma Haw. CDMX, GTO, HGO, JAL, MEX, MICH, NLE, PUE, QRO, SLP, TAMS, TLAX, VER, ZAC

Mammillaria mainiae K. Brandegee SIN, SON

* Mammillaria marksiana Krainz DGO, SIN, SON, ZAC

* Mammillaria mathildae Kraehenb. \& Krainz GTO, QRO

* Mammillaria matudae Bravo GRO, MEX, MICH

*Mammillaria mazatlanensis K. Schum. COL, JAL, MICH, NAY, SIN, SON

*Mammillaria melaleuca Karw. ex Salm-Dyck TAMS

Mammillaria melanocentra Posel. COAH, NLE, TAMS

*Mammillaria mercadensis Patoni AGS, COAH, DGO, JAL, MICH, NAY, SLP, SIN, ZAC

* Mammillaria meyranii Bravo CDMX, MEX, MICH

* Mammillaria microhelia Werdermann GTO, HGO, QRO, SLP

* Mammillaria miegiana W.H. Earle SON

* Mammillaria mieheana Tiegel QRO

*Mammillaria moelleriana Boed. AGS, DGO, ZAC 
*Mammillaria muehlenpfordtii C.F. Först. GTO, HGO, JAL, QRO, SLP

*Mammillaria multidigitata W.T. Marshall ex G.E. Linds. SON

*Mammillaria multihamata Boed. GTO, QRO, SLP

*Mammillaria mystax Mart. OAX, PUE

*Mammillaria nana Backeb. GTO, QRO, SLP

* Mammillaria napina J.A. Purpus OAX, PUE

Mammillaria neopalmeri Craig BCN, BCS

*Mammillaria nunezii (Britton \& Rose) Orcutt COL, GRO, JAL, MEX, MICH, MOR

*Mammillaria orcuttii Boed. GTO, QRO, SLP, TAMS

* Mammillaria oteroi Glass \& R.A. Foster OAX

Mammillaria pachycylindrica Backeb. COAH, DGO, ZAC

*Mammillaria parkinsonii Ehrenb. GTO, HGO, QRO, SLP, VER

* Mammillaria pectinifera (Stein) F.A.C. Weber OAX, PUE

*Mammillaria peninsularis (Britton \& Rose) Orcutt BCS

* Mammillaria pennispinosa Krainz COAH, DGO, SLP

* Mammillaria perbella Hildm. GTO, HGO, OAX, QRO, SLP

* Mammillaria perezdelarosae Bravo \& Scheinvar AGS, GTO, JAL, SLP, ZAC

*Mammillaria petrophila K. Brandegee BCS

* Mammillaria petterssonii Hildm. AGS, DGO, GTO, JAL, QRO, SLP, ZAC

*Mammillaria picta Meinsh. COAH, NLE, SLP, TAMS, ZAC

*Mammillaria pilispina J.A. Purpus COAH, NLE, SLP, TAMS

* Mammillaria plumosa F.A.C. Weber COAH, NLE, TAMS

* Mammillaria polyedra Mart. GRO, OAX, PUE

*Mammillaria polythele Mart. GTO, HGO, MICH, QRO, SLP, VER

Mammillaria pottsii Scheer ex Salm-Dyck CHIH, COAH, DGO, NLE, SLP, ZAC

Mammillaria prolifera (Mill.) Haw. COAH, GTO, HGO, NLE, OAX, QRO, SLP, TAMS

*Mammillaria pseudocrucigera R.T. Craig GTO, HGO, QRO

* Mammillaria rekoi (Britton \& Rose) Vaupel MOR, OAX, PUE

* Mammillaria rettigiana Boed. AGS, GTO, HGO, JAL, MEX, MICH, QRO, SLP, ZAC

*Mammillaria rhodantha Link \& Otto AGS, CDMX, GTO, HGO, JAL, MEX, MICH, MOR, PUE, QRO, SLP, TLAX, VER, ZAC

* Mammillaria roseoalba Boed. NLE, TAMS

* Mammillaria saboae Glass CHIH, SON

* Mammillaria sanchez-mejoradae R. González G. NLE

*Mammillaria sartorii J.A. Purpus QRO, SLP, VER

* Mammillaria scrippsiana (Britton \& Rose) Orcutt AGS, COL,

DGO, JAL, NAY, SIN, ZAC

*Mammillaria schiedeana C. Ehrenb. GTO, HGO, QRO, SLP, TAMS, VER

* Mammillaria schumannii Hildm. BCS

* Mammillaria schwarzii Shurly GTO, JAL, MICH, SLP

*Mammillaria sempervivi DC. GTO, HGO, NLE, QRO, SLP, VER, ZAC

*Mammillaria senilis Lodd. CHIH, DGO, JAL, NAY, SIN, ZAC

*Mammillaria sheldonii (Britton \& Rose) Boed. CHIH, SIN, SON

*Mammillaria sinistrohamata Boed. COAH, DGO, GTO, QRO,
SLP, ZAC

*Mammillaria solisioides Backeb. OAX, PUE

* Mammillaria sphacelata Mart. OAX, PUE

Mammillaria sphaerica A. Dietr. NLE, SLP, TAMS

*Mammillaria spinosissima Lem. DGO, GRO, MEX, MOR

*Mammillaria standleyi (Britton \& Rose) Gentry AGS, CHIH, DGO, JAL, SIN, SON

*Mammillaria stella-de-tacubaya Heese COAH, CDMX, DGO, MEX, ZAC

* Mammillaria supertexta Mart. ex Pfeiff. OAX, PUE

* Mammillaria surculosa Boed. SLP, TAMS

* Mammillaria tayloriorum Glass \& R. Foster SON

* Mammillaria tepexicensis J. Meyrán OAX

Mammillaria tetrancistra Engelm. BCN, SON

* Mammillaria theresae Cutak DGO, ZAC

Mammillaria thornberi Orcutt SON

*Mammillaria tonalensis D.R. Hunt OAX

*Mammillaria uncinata Zucc. ex Pfeiff. AGS, CHIH, COAH, CDMX, DGO, GTO, HGO, JAL, MEX, MICH, NLE, OAX, PUE, QRO, SLP, TLAX, VER, ZAC

*Mammillaria varieaculeata Buchenau PUE

* Mammillaria verhaertiana Boed. BCN, BCS

* Mammillaria vetula Mart. GTO, HGO, QRO, SLP

Mammillaria voburnensis Scheer CHIS, COL, DGO, HGO, JAL, OAX, QRO, SIN

*Mammillaria wagneriana Boed. AGS, DGO, JAL, ZAC

*Mammillaria weingartiana Boed. COAH, NLE, SLP, TAMS

Mammillaria wiesingeri Boed. CDMX, HGO, MEX, QRO, VER

Mammillaria winterae Boed. COAH, NLE, SLP, TAMS

Mammillaria wrightii Engelm. CHIH, SON

*Mammillaria xaltianguensis Sánchez-Mej. COL, GRO, JAL, $\mathrm{MICH}$

* Mammillaria zeilmanniana Boed. GTO, QRO

*Mammillaria zephyranthoides Scheidw. GTO, HGO, MEX, OAX, PUE, QRO, SLP

*Mammillaria zublerae Repp. TAMS

*Mammilloydia candida (Scheidw.) Buxb. CHIH, COAH, DGO, GTO, NLE, QRO, SLP, TAMS, ZAC

Melocactus curvispinus Pfeiff. CHIS, COL, JAL, MICH, OAX, VER

* Myrtillocactus cochal (Orcutt) Britton \& Rose BCN, BCS

* Myrtillocactus geometrizans (Mart. ex Pfeiff.) Console AGS, CDMX, DGO, GTO, GRO, HGO, JAL, MEX, MICH, MOR, NAY, NLE, OAX, PUE, QRO, SLP, SIN, TAMS, TLAX, VER, ZAC

*Myrtillocactus schenckii (J.A. Purpus) Britton \& Rose OAX, PUE

*Neobuxbaumia euphorbioides (Haw.) Buxb. TAMS, VER

*Neobuxbaumia laui (P.V. Heath) D.R. Hunt OAX

*Neobuxbaumia macrocephala (F.A.C. Weber) Dawson OAX, PUE

*Neobuxbaumia mezcalaensis (Bravo) Backeb. COL, GRO, JAL, MEX, MICH, MOR, OAX, PUE

*Neobuxbaumia multiareolata Bravo GRO

*Neobuxbaumia polylopha (DC.) Backeb. GTO, HGO, QRO, SLP, VER 
*Neobuxbaumia scoparia (Poselg.) Backeb. OAX, VER

*Neobuxbaumia squamulosa Scheinvar \& Sánchez-Mej. COL, GRO, JAL, MICH, OAX

*Neobuxbaumia tetetzo (F.A.C. Weber) Backeb. OAX, PUE Neolloydia conoidea (DC.) Britton \& Rose AGS, CHIH, COAH, DGO, GTO, HGO, NLE, QRO, SLP, TAMS, VER, ZAC

*Neolloydia matehualensis Backeb. COAH, GTO, JAL, NLE, SLP, ZAC

* Obregonia denegrii Fric NLE, TAMS

* Opuntia amarilla Griffiths GTO, HGO, QRO, SLP

Opuntia arenaria Engelm. CHIH

Opuntia atrispina Griffiths $\mathrm{CHIH}, \mathrm{COAH}$

*Opuntia atropes Rose COL, GTO, GRO, HGO, JAL, MEX, MICH, MOR, OAX, QRO, SLP, VER

*Opuntia auberi Pfeiff. CAM, CHIS, COL, GRO, HGO, JAL, MEX, MOR, OAX, PUE, QRO, SLP, VER

* Opuntia azurea Rose CHIH, COAH, DGO, GTO, HGO, JAL, QRO, SLP, VER, ZAC

Opuntia basilaris Engelm. \& J.M. Bigelow BCN, SON

*Opuntia bensonii Sánchez-Mej. GRO, JAL, MICH

* Opuntia bravoana E.M. Baxter BCS

*Opuntia cantabrigiensis Lynch AGS, COAH, DGO, GTO, HGO, JAL, MICH, NLE, QRO, SLP, TAMS, VER, ZAC

*Opuntia clarkiorum Rebman $\mathrm{BCN}$

Opuntia cochenillifera (L.) Mill. CAM, CHIS, COL, GTO, GRO, HGO, JAL, MICH, NAY, OAX, PUE, QRO, QROO, SLP, TAMS, VER, YUC

*Opuntia cochinera Griffiths GTO, HGO, JAL, MEX, MICH, QRO, SLP, VER, ZAC

* Opuntia crassa Haworth CDMX, GTO, MEX

* Opuntia crystalenia Griffiths TAB

* Opuntia chaffeyi Britton \& Rose COAH, DGO, NLE, SLP, ZAC

* Opuntia chiangiana Scheinvar \& Manzanero OAX

Opuntia chlorotica Engelm. \& J.M. Bigelow BCN, BCS, CHIH, COAH, SON

Opuntia deamii Rose CHIS

Opuntia decumbens Salm-Dyck CHIS, COL, DGO, GRO, HGO, JAL, MEX, MICH, MOR, NAY, OAX, PUE, QRO, SLP, SIN, SON, TAMS, VER

Opuntia dejecta Salm-Dyck CHIS, COL, GTO, GRO, JAL, MICH, OAX, QRO, SLP, TAMS, VER

*Opuntia delafuentiana Martínez-Gonz., Luna-Vega, Gallegos \& García-Sand.

* Opuntia depressa Rose GRO, MOR, OAX, PUE, TLAX, VER Opuntia dillenii (Ker-Gaw.) Haw. CAM, GRO, MICH, OAX, QRO, QROO, SLP, TAB, TAMS, VER, YUC

*Opuntia durangensis Britton \& Rose AGS, CHIH, COAH, DGO, GTO, GRO, HGO, JAL, QRO, SLP, SON, ZAC

Opuntia eichlamii Rose CHIS

*Opuntia elizondoana E. Sánchez \& Villaseñor GTO, QRO, SLP

Opuntia engelmannii Salm-Dyck AGS, BCN, CHIH, COAH, DGO, GTO, HGO, JAL, MEX, NLE, QRO, SLP, SIN, SON, TAMS, TLAX, VER, ZAC

Opuntia erinacea Engelm. \& J.M. Bigelow CHIH, SON

*Opuntia excelsa Sánchez-Mej. COL, GRO, JAL, MICH, NAY,
SIN

* Opuntia feroacantha Britton \& Rose JAL, MICH, NAY, SIN

* Opuntia fuliginosa Griffiths AGS, COL, DGO, GTO, GRO, HGO, JAL, MEX, MICH, MOR, NAY, OAX, QRO, SLP, ZAC * Opuntia gallegiana Scheinvar \& Olalde ZAC

Opuntia guatemalensis Britton \& Rose OAX, VER

Opuntia guilanchi Griffiths AGS, GTO, JAL, MEX, MICH, NLE, QRO, SLP, ZAC

*Opuntia hitchcockii J.G. Ortega NAY, SIN

* Opuntia howeyi J.A. Purpus ZAC

* Opuntia huajuapensis Bravo OAX, PUE, VER

Opuntia humifusa (Raf.) Raf. SLP, TAMS

* Opuntia hyptiacantha F.A.C. Weber AGS, CDMX, GTO, HGO, JAL, MEX, NLE, OAX, PUE, QRO, SLP, TLAX, VER, ZAC

* Opuntia inaperta (Schott ex Griffiths) D.R. Hunt CAM, CHIS, QROO, TAB, VER, YUC

*Opuntia jaliscana Bravo AGS, COL, DGO, GTO, JAL, MEX, $\mathrm{MICH}, \mathrm{QRO}, \mathrm{ZAC}$

*Opuntia joconostle F.A.C. Weber AGS, COL, GTO, HGO, JAL, MEX, MICH, QRO, SLP, TLAX, VER, ZAC

*Opuntia karwinskiana Salm-Dyck CHIS, COL, DGO, GRO, HGO, JAL, MEX, MICH, MOR, NAY, OAX, QRO, SLP, SIN, SON, TAMS, VER

*Opuntia lagunae $\mathrm{K}$. Brandegee BCS

* Opuntia lasiacantha Pfeiff. AGS, CDMX, GTO, GRO, HGO, JAL, MEX, MICH, NLE, OAX, PUE, QRO, SLP, TAMS, TLAX, VER, ZAC

* Opuntia leiascheinvariana Martínez-González \& GallegosVázquez HGO

*Opuntia leucotricha DC. AGS, COAH, DGO, GTO, HGO, JAL, MICH, NLE, QRO, SLP, TAMS, ZAC

Opuntia littoralis (Engelm.) Cockerell BCN

Opuntia lutea (Rose) D.R. Hunt CHIS, OAX

Opuntia macrocentra Engelm. BCN, CHIH, COAH, DGO, NLE, OAX, SON, ZAC

Opuntia macrorhiza Engelm. CHIH, COAH, NLE, SLP, SON, TAMS

Opuntia megacantha Salm-Dyck AGS, BCN, COAH, CDMX, DGO, GTO, HGO, JAL, MEX, MICH, MOR, NLE, QRO, SLP, SON, TAMS, TLAX, VER, ZAC

Opuntia megarhiza Rose SLP, TAMS

Opuntia microdasys (Lehm.) Pfeiff. CHIH, COAH, DGO, GTO, HGO, JAL, NAY, NLE, QRO, SLP, SON, TAMS, VER, ZAC

*Opuntia nejapensis Bravo OAX

* Opuntia neochrysacantha Bravo AGS, COAH, SLP, ZAC

*Opuntia olmeca Pérez-Crisanto, J. Reyes \& Brachet OAX

* Opuntia orbiculata Salm-Dyck ex Pfeiff. QRO, SLP

Opuntia oricola Philbrick BCN

*Opuntia pachyrrhiza H.M. Hern., Gómez-Hin. \& R.T. Bárcenas NLE, QRO, SLP

* Opuntia pailana Weingart COAH

* Opuntia parviclada S. Arias \& S. Gama OAX, PUE

* Opuntia perotensis Scheinvar, Olalde \& Gallegos VER

Opuntia phaeacantha Engelm. AGS, BCN, CHIH, COAH, DGO, GTO, NLE, SLP, SIN, SON, TAMS, ZAC

*Opuntia pilifera F.A.C. Weber OAX, PUE, TLAX 
Opuntia polyacantha Haw. CHIH, COAH, DGO

Opuntia pubescens J.C. Wendl. ex Pfeiff. AGS, BCS, CHIS, CHIH, COL, DGO, GTO, GRO, HGO, JAL, MEX, MICH, MOR, NLE, OAX, PUE, QRO, SLP, SIN, SON, TAMS, VER, YUC, ZAC

*Opuntia pycnacantha Engelm. BCS

* Opuntia pyriformis Rose DGO, OAX, ZAC

* Opuntia rastrera F.A.C. Weber AGS, CHIH, COAH, DGO, GTO, HGO, JAL, NLE, QRO, SLP, TAMS, VER, ZAC

*Opuntia rileyi J.G. Ortega SIN

* Opuntia ritteri A. Berger ZAC

* Opuntia robinsonii J.G. Ortega CHIH, JAL, MICH, NAY, SIN, SON, ZAC

Opuntia robusta $\mathrm{H}$. Wendl. AGS, CHIH, COAH, CDMX, DGO, GTO, HGO, JAL, MEX, MICH, MOR, NLE, PUE, QRO, SLP, SON, TAMS, TLAX, VER, ZAC

*Opuntia scheeri F.A.C. Weber AGS, DGO, GTO, HGO, JAL, QRO

*Opuntia spinulifera Salm-Dyck AGS, CDMX, GTO, HGO, MEX, QRO, SLP, TLAX, VER, ZAC

*Opuntia spraguei J.G. Ortega SIN

*Opuntia stenopetala Engelm. AGS, COAH, DGO, GTO, HGO, NLE, QRO, SLP, TAMS, VER, ZAC

* Opuntia streptacantha Lem. AGS, CHIH, COAH, CDMX, DGO, GTO, GRO, HGO, JAL, MEX, MICH, MOR, NLE, OAX, PUE, QRO, SLP, TAMS, TLAX, VER, ZAC

Opuntia stricta Haw. CAM, NLE, QRO, SLP, YUC

Opuntia strigil Engelm. CHIH, COAH, NLE, TAMS

* Opuntia tapona Engelm. BCS

* Opuntia tehuacana S. Arias \& U. Guzmán OAX, PUE

* Opuntia tehuantepecana (Bravo) Bravo CHIS, OAX

* Opuntia tezontepecana Gallegos-Vázquez \& Scheinvar HGO

* Opuntia tomentosa Salm-Dyck AGS, COL, CDMX, GTO, GRO, HGO, JAL, MEX, MICH, MOR, NLE, OAX, PUE, QRO, SLP, SIN, SON, TAMS, TLAX, VER, ZAC

*Opuntia undulata Griffiths COL, CDMX, GTO, JAL, MEX, MICH, MOR, NAY, SLP

*Opuntia velutina F.A.C. Weber GTO, GRO, HGO, JAL, MEX, MICH, MOR, OAX, PUE, QRO, SLP, TLAX, VER, ZAC

* Opuntia wilcoxii Britton \& Rose BCS, CHIH, DGO, GRO, JAL, MEX, MICH, NAY, OAX, SIN, SON, ZAC

*Opuntia $\times$ andersonii H.M. Hern., Gómez-Hin. \& R.T. Bárcenas SLP, TAMS

* Opuntia zamudioi Scheinvar QRO, SLP

* Ortegocactus macdougallii Alexander OAX

*Pachycereus grandis Rose MEX, MICH, MOR, OAX, PUE

* Pachycereus pecten-aboriginum (Engelm. ex S. Watson) Britton \& Rose BCS, CHIH, COL, DGO, GRO, JAL, MEX, MICH, NAY, OAX, PUE, SIN, SON, ZAC

*Pachycereus pringlei (S. Watson) Britton \& Rose BCN, BCS, DGO, SON

*Pachycereus tepamo S. Gama \& S. Arias GRO, MICH

*Pachycereus weberi (J.M. Coult.) Backeb. GRO, HGO, MEX, MICH, MOR, OAX, PUE, QRO, VER

*Pelecyphora aselliformis Ehrenb. GTO, JAL, NLE, SLP, TAMS, ZAC

*Pelecyphora strobiliformis (Werderm.) Fric \& Schelle COAH,
NLE, SLP, TAMS

*Peniocereus castellae Sánchez-Mej. COL, GRO, JAL, MICH

*Peniocereus cuixmalensis Sánchez-Mej. COL, JAL, MICH

Peniocereus chiapensis (Bravo) Gómez-Hin. \& H.M. Hern. CHIS

*Peniocereus fosterianus Cutak CHIS, GRO, OAX

Peniocereus greggii (Engelm.) Britton \& Rose CHIH, COAH, DGO, NLE, SON, TAMS, ZAC

*Peniocereus haackianus Backeb. ND

*Peniocereus johnstonii Britton \& Rose BCS

*Peniocereus lazaro-cardenasii (J.L. Contr., J. Jiménez Ram., Sánchez-Mej. \& C.A. Toledo) D.R. Hunt GRO, MICH

*Peniocereus macdougallii Cutak CHIS, OAX

*Peniocereus maculatus (Weing.) Cutak GRO, MEX, MICH, OAX, PUE

*Peniocereus marianus (Gentry) Sánchez-Mej. SIN, SON

*Peniocereus oaxacensis (Britton \& Rose) D.R. Hunt OAX

*Peniocereus occidentalis Bravo OAX

* Peniocereus papillosus (Britton \& Rose) U. Guzmán SIN

*Peniocereus rosei J.G. Ortega COL, JAL, MICH, NAY, SIN

*Peniocereus serpentinus (Lag. \& Rodr.) N.P. Taylor AGS, CHIH, COAH, COL, CDMX, DGO, GTO, GRO, HGO, JAL, MEX, MICH, MOR, NLE, OAX, PUE, QRO, SLP, SIN, SON, VER, ZAC

Peniocereus striatus Brandegee BCN, BCS, CHIH, SIN, SON

*Peniocereus tepalcatepecanus Sánchez-Mej. GRO, JAL, $\mathrm{MICH}$

*Peniocereus viperinus (F.A.C. Weber) Buxb. MOR, OAX, PUE *Peniocereus zopilotensis (J. Meyrán) Sánchez-Mej. GRO, JAL, MICH, OAX

Pereskia lychnidiflora DC. GRO, OAX

*Pereskiopsis aquosa (F.A.C. Weber) Britton \& Rose COL, DGO, GTO, JAL, MICH, NAY, QRO, SLP, SIN, TAMS, VER, ZAC

*Pereskiopsis blakeana J.G. Ortega DGO, MICH, SIN

*Pereskiopsis diguetii (F.A.C. Weber) Britton \& Rose COL, GTO, GRO, HGO, JAL, MEX, MICH, MOR, NAY, OAX, QRO, ZAC

*Pereskiopsis kellermanii Rose CAM, OAX, VER, YUC

*Pereskiopsis porteri (Brandegee) Britton \& Rose BCS, DGO, NAY, SIN, SON

*Pereskiopsis rotundifolia (DC.) Britton \& Rose GRO, MEX, MICH, MOR, OAX, PUE, QRO

*Pilosocereus alensis (F.A.C. Weber) Byles \& G.D. Rowley CHIH, COL, GRO, JAL, MEX, MICH, NAY, SIN, SON, ZAC *Pilosocereus collinsii (Britton \& Rose) Byles \& G.D. Rowley OAX

*Pilosocereus cometes (Scheidw.) Byles \& G.D. Rowley DGO, HGO, QRO, SLP

*Pilosocereus chrysacanthus (F.A.C. Weber ex Schum.) Byles $\&$ G.D. Rowley GRO, MEX, MICH, MOR, OAX, PUE, VER

*Pilosocereus gaumeri (Britton \& Rose) Backeb. CAM, QROO, YUC

Pilosocereus leucocephalus (Poselg.) Byles \& G.D. Rowley CHIS, CHIH, DGO, GRO, HGO, MICH, QRO, SLP, SON, TAMS, VER

*Pilosocereus purpusii (Britton \& Rose) Byles \& G.D. Rowley 
COL, GRO, JAL, MICH, NAY, OAX, SIN, ZAC

*Pilosocereus quadricentralis (E.Y. Dawson) Byles \& G.D. Rowley CHIS, OAX

*Polaskia chende (Gosselin) A.C. Gibson \& K.E. Horak OAX, PUE

*Polaskia chichipe (Gosselin) Backeb. MICH, OAX, PUE

*Pseudomitrocereus fulviceps (F.A.C. Weber ex K. Schum.)

Bravo \& Buxb. OAX, PUE

Pseudorhipsalis ramulosa (Salm-Dyck) Barthlott CHIS, JAL, OAX, PUE, TAB, VER

*Pterocereus gaumeri (Britton \& Rose) T. MacDoug. \& Miranda CHIS, QROO, VER, YUC

Rhipsalis baccifera (J.S. Muell.) Stearn CAM, CHIS, COL, HGO, JAL, OAX, PUE, QRO, QROO, SLP, SIN, TAB, TAMS, VER

Sclerocactus scheeri (Salm-Dyck) N.P. Taylor CHIH, COAH, NLE, TAMS

*Selenicereus anthonyanus (Alexander) D.R. Hunt CHIS, OAX, TAB, VER

*Selenicereus atropilosus Kimnach COL, JAL

Selenicereus boeckmannii (Otto) Britton \& Rose CHIS, TAB, TAMS

Selenicereus coniflorus (Weing.) Britton \& Rose CHIS, OAX, TAB, TAMS, VER

*Selenicereus chontalensis (Alexander) Kimnach OAX

*Selenicereus chrysocardium (Alexander) Kimnach CHIS, TAB Selenicereus donkelaarii (Salm-Dyck) Britton \& Rose CAM, QROO, TAB, YUC

Selenicereus grandiflorus (L.) Britton \& Rose CAM, CHIS, OAX, QROO, TAB, TAMS, VER, YUC

*Selenicereus hamatus (Scheidw.) Britton \& Rose OAX, PUE, VER

Selenicereus inermis (Pfeiff.) Britton \& Rose CHIS, OAX

*Selenicereus nelsonii (Weingart) Britton \& Rose HGO, VER

Selenicereus pteranthus Britton \& Rose CAM, CHIS, QROO,

TAB, VER

Selenicereus spinulosus (DC.) Britton \& Rose CHIS, COAH, GTO, HGO, NLE, OAX, PUE, QRO, SLP, TAMS, VER

Selenicereus testudo (Karw. ex Zucc.) Buxb. CAM, CHIS, OAX, QROO, TAB, VER, YUC

*Selenicereus vagans (Brandegee) Britton \& Rose CHIS, COL, JAL, MICH, NAY, OAX, PUE, SIN, SON, VER

*Stenocactus anfractuosus (C. Mart. ex Pfeiff.) Berger ex Backeb. \& Knuth HGO, MEX, OAX, QRO, VER

*Stenocactus arrigens (Link ex A. Dietr.) Berger ex Backeb. \& Knuth HGO, SLP

*Stenocactus boedekerianus Berger ex Backeb. \& Knuth COAH, NLE, ZAC

*Stenocactus coptonogonus (Lem.) Berger ex Backeb. \& Knuth GTO, HGO, JAL, MEX, SLP, VER, ZAC

*Stenocactus crispatus (DC.) A. Berger ex A.W. Hill GTO, HGO, MEX, NLE, OAX, PUE, QRO, SLP, TAMS, TLAX, VER

*Stenocactus dichroacanthus (C. Mart. ex Pfeiff.) Berger ex Backeb. \& Knuth AGS, DGO, GTO, HGO, JAL, MEX, NLE, QRO, SLP, TAMS, VER, ZAC

*Stenocactus heteracanthus (Muehlenpf.) Berger ex Backeb. \&
Knuth AGS, DGO, GTO, HGO, MEX, OAX, QRO, VER, ZAC *Stenocactus lamellosus (A. Dietr.) Berger ex Backeb. \& Knuth GTO, HGO, QRO, SLP, VER

*Stenocactus lancifer (A. Dietr.) A. Berger ex Backeb. \& F.M. Knuth HGO

*Stenocactus multicostatus (Hildm. ex Schum.) Berger ex Backeb. \& Knuth AGS, CHIH, COAH, DGO, GTO, JAL, MICH, NLE, QRO, SLP, TAMS, ZAC

*Stenocactus obvallatus (DC.) Berger ex Backeb. \& Knuth AGS, CDMX, DGO, GTO, HGO, JAL, MEX, OAX, PUE, QRO, SLP, TAMS, VER

*Stenocactus ochoterenianus Tiegel AGS, GTO, HGO, JAL, QRO, SLP, ZAC

*Stenocactus pentacanthus (Lem.) Berger ex Backeb. \& Knuth GTO, HGO, JAL, QRO, SLP

*Stenocactus phyllacanthus (C. Mart. ex A. Dietr. \& Otto) Berger ex Backeb. \& Knuth AGS, GTO, HGO, JAL, MEX, NLE, QRO, SLP, VER, ZAC

*Stenocactus sulphureus (A. Dietr.) Bravo HGO, QRO

*Stenocactus tetraxiphus (Otto ex K. Schum.) A. Berger ex A.W. Hill HGO, ZAC

*Stenocactus vaupelianus (Werderm.) F.M. Knuth DGO, HGO, NLE, QRO

*Stenocactus wippermannii (Muehlenpf.) Berger ex Backeb. \& Knuth GTO, HGO, JAL, SLP, ZAC

*Stenocereus alamosensis (J.M. Coult.) A.C. Gibson \& K.E. Horak COL, JAL, MICH, NAY, SIN, SON

*Stenocereus beneckei (Ehrenb.) F. Buxb. GRO, MEX, MOR, OAX, PUE

*Stenocereus chacalapensis (Bravo \& T. Macdoug.) Buxb. OAX *Stenocereus chrysocarpus Sánchez-Mej. GRO, JAL, MICH

*Stenocereus dumortieri (Scheidw.) Buxb. AGS, COL, CDMX, GTO, GRO, HGO, JAL, MEX, MICH, MOR, NAY, OAX, PUE, QRO, SLP, VER, ZAC

Stenocereus eichlamii (Britton \& Rose) Buxb. CHIS, YUC

*Stenocereus eruca (Brandegee) Gibson \& Horak BCS

*Stenocereus fricii Sánchez-Mej. COL, GRO, JAL, MICH Stenocereus griseus (Haw.) Buxb. CHIS, GTO, GRO, HGO, JAL, MICH, MOR, NLE, OAX, PUE, QRO, SLP, TAMS, VER, YUC, ZAC

*Stenocereus gummosus (Engelm.) Gibson \& Horak BCN, BCS, SON

*Stenocereus kerberi (K. Schum.) A.C. Gibson \& K.E. Horak COL, DGO, JAL, NAY, SIN

*Stenocereus martinezii (J.G. Ortega) Bravo SIN

*Stenocereus montanus (Britton \& Rose) Buxb. CHIH, COL, DGO, JAL, NAY, SIN, SON, ZAC

*Stenocereus pruinosus (Otto ex Pfeiff.) Buxb. CAM, CHIS, GTO, GRO, HGO, JAL, MEX, MICH, OAX, PUE, QRO, QROO, SLP, TAMS, VER, YUC

*Stenocereus queretaroensis (F.A.C. Weber) Buxb. AGS, COL, GTO, HGO, JAL, MEX, MICH, NAY, PUE, QRO, SLP, ZAC

*Stenocereus quevedonis (J.G. Ortega) Buxb. COL, DGO, GRO, JAL, MICH, NAY, SIN

*Stenocereus standleyi (J.G. Ortega) Buxb. COL, JAL, MICH, NAY, SIN

*Stenocereus stellatus (Pfeiff.) Riccob. GRO, MOR, OAX, PUE 
Stenocereus thurberi (Engelm.) Buxb. BCN, BCS, CHIH, DGO, SIN, SON

*Stenocereus treleasei (Vaupel) Backeb. OAX, PUE

*Strombocactus corregidorae S. Arias \& E. Sánchez HGO, QRO

*Strombocactus disciformis (DC.) Britton \& Rose GTO, HGO, QRO, SLP

Thelocactus bicolor (Galeotti) Britton \& Rose CHIH, COAH, DGO, NLE, SLP, TAMS, ZAC

*Thelocactus conothelos (Regel \& Klein) Knuth COAH, DGO, NLE, SLP, TAMS, ZAC

*Thelocactus hastifer (Wenderm. \& Boed.) F.M. Knuth HGO, QRO

*Thelocactus heterocromus (F.A.C. Weber) Van Oost. CHIH, COAH, DGO, ZAC

Thelocactus hexaedrophorus (Lem.) Britton \& Rose COAH, DGO, GTO, JAL, NLE, QRO, SLP, TAMS, ZAC

*Thelocactus lausseri Ríha \& Busek COAH

Thelocactus leucacanthus (Zucc.) Britton \& Rose GTO, HGO, QRO, SLP

*Thelocactus macdowellii (Rebut ex Quehl) W. Marshall COAH, NLE

Thelocactus rinconensis (Poselg.) Britton \& Rose $\mathrm{CHIH}$, COAH, DGO, NLE, SLP

Thelocactus setispinus (Engelm.) E.F. Anderson CHIH, COAH, NLE, SLP, TAMS

*Thelocactus tulensis (Poselg.) Britton \& Rose COAH, GTO, JAL, NLE, SLP, TAMS, ZAC

*Turbinicarpus alonsoi Glass \& S. Arias GTO, SLP

*Turbinicarpus beguinii (N.P. Taylor) Mosco \& Zanovello CHIH, COAH, NLE, SLP, TAMS, ZAC

*Turbinicarpus booleanus G.S. Hinton NLE

*Turbinicarpus gielsdorfianus (Werderm.) Vác. John \& Riha GTO, JAL, NLE, SLP, TAMS, ZAC

*Turbinicarpus heliae García-Mor., Díaz-Salim \& Gonz.-Bot. HGO

*Turbinicarpus hoferi Lüthy \& A.B. Lau NLE, TAMS

*Turbinicarpus horripilus (Lem.) Vác. John \& Riha HGO, QRO, VER

*Turbinicarpus jauernigii G. Frank SLP, TAMS

*Turbinicarpus laui Glass \& R.C. Foster GTO, JAL, NLE, SLP, ZAC

*Turbinicarpus lophophoroides (Werderm.) F. Buxb. \& Backeb. GTO, JAL, NLE, SLP, ZAC

*Turbinicarpus mandragora (Fric ex A. Berger) A.D. Zimmerman COAH, DGO, NLE

Turbinicarpus pseudomacrochele (Backeb.) Buxb. \& Backeberg HGO, QRO, NLE, SLP

*Turbinicarpus pseudopectinatus (Backeb.) Glass \& R. Foster NLE, SLP, TAMS

*Turbinicarpus roseiflorus Backeb. ND

*Turbinicarpus saueri (Boed.) Vác. John \& Ríha SLP, TAMS

*Turbinicarpus schmiedickeanus (Boed.) Buxb. \& Backeb. COAH, GTO, JAL, NLE, SLP, TAMS, ZAC

*Turbinicarpus subterraneus (Backeb.) A.D. Zimmerman NLE, SLP, TAMS

*Turbinicarpus swobodae L. Diers COAH, NLE
*Turbinicarpus valdezianus (Moell.) Glass \& R. Foster COAH, NLE, SLP, ZAC

*Turbinicarpus viereckii (Werderm.) Vác. John \& Riha COAH, NLE, SLP, TAMS

*Turbinicarpus zaragozae (Glass \& R.A. Foster) Glass \& A. Hofer NLE, TAMS

*Weberocereus alliodorus Gómez-Hin. \& H.M. Hern. OAX

Weberocereus glaber (Eichlam) G.D. Rowley CHIS

*X Pachebergia apicicostata S. Arias \& Terrazas GRO

*X Pachgerocereus orcuttii (K. Brandegee) Moran BCN

\section{Family Calceolariaceae}

Calceolaria chelidonioides Kunth CHIS, OAX, SLP, VER

Calceolaria dichotoma Lam. COL, JAL

Calceolaria mexicana Benth. CHIS, COL, DGO, GRO, HGO, JAL, MEX, MICH, MOR, NLE, OAX, PUE, QRO, SLP, SIN, TAMS, TLAX, VER

Calceolaria trilobata Hemsl. CHIS, OAX

Calceolaria tripartita Ruiz \& Pav. CHIS, GTO, GRO, HGO,

JAL, MEX, MICH, OAX, PUE, SLP, VER

Calceolaria virgata Ruiz \& Pav. OAX

\section{Family Calophyllaceae}

Calophyllum brasiliense Cambess. CAM, CHIS, COL, GRO, JAL, MICH, NAY, OAX, PUE, QROO, TAB, VER, YUC

Mammea americana L. CAM, CHIS, HGO, MOR, QROO, TAB, VER, YUC

Marila laxiflora Rusby VER

\section{Family Campanulaceae}

Burmeistera virescens (Benth.) Benth. \& Hook. f. ex Hemsl. CHIS

* Calcaratolobelia aurita (Brandegee) Wilbur BCS

Calcaratolobelia cordifolia (Hook. \& Arn.) Wilbur CHIS, CHIH, COL, GRO, JAL, MEX, MICH, NAY, OAX, SIN, SON, VER

*Calcaratolobelia flexuosa (C. Presl) Wilbur DGO, GRO, JAL, NAY, OAX, ZAC

* Calcaratolobelia gibbosa (S. Watson) Wilbur CHIH, NAY

*Calcaratolobelia goldmannii (Fernald) Wilbur CHIH, DGO, SIN, SON

*Calcaratolobelia knoblochii (T.J. Ayers) Wilbur CHIH

*Calcaratolobelia macrocentron (Benth.) Wilbur DGO, JAL, NAY

* Calcaratolobelia margarita (E. Wimm.) Wilbur NLE, TAMS

* Calcaratolobelia mcvaughii (T.J. Ayers) Wilbur DGO, SIN

*Calcaratolobelia pringlei (B.L. Rob.) Wilbur NLE, SLP, TAMS

*Calcaratolobelia tenella (Turcz.) Wilbur GRO, HGO, JAL, MEX, MICH, MOR, VER

*Calcaratolobelia villaregalis (T.J. Ayers) Wilbur JAL

Campanula rotundifolia L. CHIH, COAH, NLE

Centropogon cordifolius Benth. CHIS, OAX

Centropogon grandidentatus (Schltdl.) Zahlbr. CHIS, GRO, HGO, OAX, PUE, VER

*Centropogon wilburii Lammers OAX

*Diastatea expansa McVaugh GRO, JAL, MEX, MICH, NAY, OAX 
Diastatea micrantha (Kunth) McVaugh AGS, CAM, CHIS, COAH, COL, CDMX, DGO, GTO, GRO, HGO, JAL, MEX, MICH, MOR, NAY, NLE, OAX, PUE, QRO, QROO, SLP, SIN, TAB, TLAX, VER

Diastatea tenera (A. Gray) McVaugh AGS, CHIS, CHIH, COL, CDMX, DGO, GTO, GRO, HGO, JAL, MEX, MICH, MOR, NAY, OAX, PUE, QRO, SON, VER, ZAC

*Diastatea virgata Scheidw. GRO, JAL, MEX, MICH, MOR, OAX, PUE, ZAC

Downingia cuspidata (Greene) Greene BCN

Githopsis diffusa A. Gray BCN

Heterotoma lobelioides Zucc. CHIS, COL, GRO, JAL, MEX, MICH, NAY, OAX, SLP, ZAC

Hippobroma longiflora (L.) G. Don CAM, CHIS, COL, GRO, JAL, MICH, OAX, PUE, QRO, QROO, SLP, SON, TAB, VER, YUC

*Laurentia insignis Brandegee VER

*Laurentia ramosissima (Mart. \& Galeotti) Benth. \& Hook. f. ex Hemsl. GRO, OAX

Lobelia aguana E. Wimm. CHIS, GRO, MEX, OAX

Lobelia anatina Wimmer CHIH, COAH, DGO, QRO, SON, VER

Lobelia berlandieri A. DC. AGS, CHIS, CHIH, COAH, CDMX, DGO, GTO, GRO, HGO, JAL, MEX, MICH, NLE, OAX, PUE, QRO, QROO, SLP, SIN, TAB, TAMS, TLAX, VER, ZAC

Lobelia bryophila E. Wimm. CHIS, GRO

*Lobelia caeciliae E. Wimm. CHIS, HGO

Lobelia cardinalis L. BCN, CHIS, CHIH, COAH, CDMX, DGO, GTO, GRO, HGO, JAL, MEX, MICH, MOR, NAY, NLE, OAX, PUE, QRO, QROO, SLP, SIN, SON, TAB, TAMS, VER, ZAC

Lobelia cliffortiana L. GRO, HGO, MEX, MOR, VER

*Lobelia diastateoides McVaugh CHIS, OAX

*Lobelia diazlunae Rzed. \& Calderón DGO, NAY

*Lobelia divaricata Hook. \& Arn. MICH, NLE, QRO, SLP

Lobelia dunnii Greene BCN

Lobelia ehrenbergii Vatke CHIH, COAH, DGO, HGO, NLE, OAX, QRO, SLP, TAMS

Lobelia fenestralis Cav. AGS, CHIH, COAH, COL, CDMX, DGO, GTO, GRO, HGO, JAL, MEX, MICH, MOR, NAY, NLE, OAX, PUE, QRO, SLP, SON, TLAX, VER, ZAC

*Lobelia flexicaulis Rzed. \& Calderón GRO, MEX

*Lobelia ghiesbreghtii Decne. OAX

*Lobelia gruina Cav. AGS, CHIH, COAH, CDMX, DGO, GTO, GRO, HGO, JAL, MEX, MICH, MOR, NLE, OAX, PUE, QRO, SLP, SIN, TAMS, TLAX, VER, ZAC

*Lobelia guerrerensis Eakes \& Lammers GRO

*Lobelia hartwegii A. DC. DGO, JAL, OAX, VER, ZAC

*Lobelia henricksonii M.C. Johnst. COAH

*Lobelia hintoniorum B.L. Turner OAX

*Lobelia hypnodes E. Wimm. ex McVaugh VER

*Lobelia illota McVaugh CHIS, VER

Lobelia irasuensis Planch. \& Oerst. AGS, CDMX, DGO, GTO, JAL, MEX, MICH, TLAX, ZAC

*Lobelia jaliscensis McVaugh COL, JAL, MICH, NAY, ZAC Lobelia laxiflora Kunth AGS, BCN, BCS, CHIS, CHIH, COL, CDMX, DGO, GTO, GRO, HGO, JAL, MEX, MICH, MOR,
NAY, OAX, PUE, QRO, SLP, SIN, SON, TLAX, VER, ZAC

*Lobelia lithophila Senterre \& Cast.-Campos VER

Lobelia longicaulis Brandegee CHIS, COL, GRO, JAL, MEX, MICH, MOR, OAX, VER

*Lobelia macdonaldii B.L. Turner OAX

Lobelia mexicana E. Wimm. CHIS, GRO, JAL, VER, ZAC

Lobelia nana Kunth CHIS, CHIH, CDMX, DGO, GTO, GRO, HGO, JAL, MEX, MICH, MOR, OAX, PUE, QRO, TLAX, VER

*Lobelia occidentalis McVaugh \& Huft COL, GRO, JAL

*Lobelia orientalis Rzed. \& Calderón QRO, SLP

*Lobelia picta B.L. Rob. \& Seaton AGS, JAL, VER

*Lobelia poetica E. Wimm. DGO

*Lobelia porphyrea Rzed. \& Calderón HGO, QRO

*Lobelia pringlei S. Watson COAH, NLE

*Lobelia pulchella Vatke GRO, OAX

*Lobelia purpusii Brandegee PUE, QRO, SLP, TAMS, VER

Lobelia sartorii Vatke CHIS, GTO, GRO, HGO, OAX, PUE, QRO, SLP, TAMS, VER

*Lobelia setulosa E. Wimm. GRO, OAX

*Lobelia sinaloae Sprague DGO, NAY, SIN

*Lobelia stenodonta (Fernald) McVaugh CHIS, HGO, VER

Lobelia stolonifera Donn. Sm. CHIS

*Lobelia sublibera $\mathrm{S}$. Watson COAH, NLE, TAMS

*Lobelia subnuda Benth. AGS, HGO, JAL, VER, ZAC

*Lobelia tarsophora Seaton ex Greenm. HGO, OAX, PUE, QRO, SLP, VER

Lobelia tatea (E. Wimm.) E. Wimm. OAX

*Lobelia trivialis E. Wimm. NLE, TAMS

Lobelia umbellifera McVaugh CHIS

Lobelia xalapensis Kunth CHIS, COL, GRO, HGO, JAL, MOR, NAY, OAX, PUE, QRO, SLP, TAMS, VER

Lobelia yucatana E. Wimm. CHIS, YUC

Nemacladus glanduliferus Jepson BCN, BCS, SON

Nemacladus longiflorus A. Gray BCN

Nemacladus pinnatifidus Greene BCN

Nemacladus ramosissimus Nutt. BCN

Nemacladus rubescens Greene BCN

Nemacladus sigmoideus G.T. Robbins BCN

Pratia calochlamys (Donn. Sm.) Wimmer CHIS

*Pseudonemacladus oppositifolius (B.L. Rob.) McVaugh HGO, QRO, SLP, VER

Triodanis biflora (Ruiz \& Pav.) Greene AGS, BCN, BCS, CHIH, GTO, JAL, MICH, NLE, OAX, SLP, SON, TAMS

Triodanis coloradoensis (Buckley) McVaugh COAH, NLE

Triodanis perfoliata (L.) Nieuwl. CHIH, COAH, COL, CDMX, HGO, MEX, OAX, PUE, QRO, SLP, SON, TAMS, VER

Family Canellaceae

Canella winterana (L.) Gaertn. CAM, QROO, YUC

Family Cannabaceae

Aphananthe monoica (Hemsl.) J.F. Leroy AGS, CHIS, CHIH, COL, DGO, GTO, GRO, HGO, JAL, MEX, MICH, MOR, NAY, NLE, OAX, PUE, QRO, SLP, SIN, SON, TAB, TAMS, VER, ZAC

Celtis caudata Planch. CHIS, COL, DGO, GTO, GRO, HGO, JAL, MEX, MICH, MOR, NLE, OAX, PUE, QRO, SLP, SIN, 
TAMS, VER, ZAC

Celtis ehrenbergiana (Klotzsch) Liebm. AGS, BCN, BCS, CHIH, COAH, COL, DGO, GTO, GRO, HGO, JAL, MICH, NLE, OAX, PUE, QRO, SLP, SIN, SON, TAMS, VER, ZAC Celtis iguanaea (Jacq.) Sarg. AGS, BCN, BCS, CAM, CHIS, CHIH, COL, DGO, GTO, GRO, HGO, JAL, MEX, MICH, MOR, NAY, NLE, OAX, PUE, QRO, QROO, SLP, SIN, SON, TAB, TAMS, VER, YUC

Celtis laevigata Willd. BCS, CHIH, COAH, DGO, GTO, MICH, NLE, QRO, SLP, SON, TAMS, VER

*Celtis lindheimeri Engelm. COAH, NLE

Celtis reticulata Torr. AGS, BCN, BCS, CHIH, COAH, COL, DGO, GTO, HGO, JAL, MEX, MICH, MOR, NLE, OAX, QRO, SLP, SIN, SON, TAMS, ZAC

Celtis trinervia Lam. CAM, QROO, YUC

Lozanella enantiophylla (Donn. Sm.) Killip \& C.V. Morton CHIS, GRO, HGO, OAX, PUE, VER

*Trema laxiflora Lundell CHIS

Trema micrantha (L.) Blume CAM, CHIS, COL, DGO, GTO, GRO, HGO, JAL, MEX, MICH, MOR, NAY, OAX, PUE, QRO, QROO, SLP, SIN, SON, TAB, TAMS, TLAX, VER, YUC, ZAC

\section{Family Cannaceae}

Canna discolor Lindl. CHIS

Canna glauca L. CAM, CHIS, COL, GRO, JAL, MOR, NAY, PUE, QROO, SLP, TAB, TAMS, VER, YUC

Canna tuerckheimii Kraenzl. CHIS, HGO, OAX, PUE, TAB, TAMS, VER

\section{Family Capparaceae}

Caphexandra heydeana (Donn. Sm.) H.H. Iltis \& X. Cornejo CHIS, OAX, TAB

Capparidastrum baducca (L.) Hutch. CAM, CHIS, COL, GRO, JAL, OAX, QROO, SLP, TAB, TAMS, VER

Capparidastrum discolor (Donn. Sm.) X. Cornejo \& H.H. Iltis CHIS, GRO, TAB, VER

Capparidastrum frondosum (Jacq.) X. Cornejo \& H.H. Iltis CHIS, GRO, OAX, QRO, SLP, TAB, TAMS, VER, YUC

Capparidastrum mollicellum (Standl.) X. Cornejo \& H.H. Iltis CHIS, COL, JAL, NAY, OAX, PUE, TAB, VER

Capparidastrum pachaca (Kunth) Hutch. CAM, GRO, QROO, YUC

Capparidastrum quiriguense (Standl.) X. Cornejo \& H.H. Iltis CHIS, COL, JAL, TAB, VER

*Capparidastrum tuxtlense X. Cornejo \& H.H. Iltis VER

Crateva palmeri Rose CHIS, COL, DGO, GRO, JAL, MEX, MICH, MOR, NAY, SIN, SON, ZAC

Crateva tapia L. BCS, CAM, CHIS, COL, DGO, GRO, HGO, JAL, MEX, MICH, MOR, NAY, OAX, PUE, QRO, QROO, SLP, SIN, TAB, TAMS, VER, YUC

Cynophalla flexuosa (L.) J. Presl CAM, CHIS, COL, GRO, JAL, MICH, NAY, OAX, QROO, SLP, SIN, SON, TAMS, VER, YUC Cynophalla verrucosa (Jacq.) J. Presl CAM, CHIS, COL, GRO, JAL, MICH, NAY, OAX, QROO, SIN, VER

*Forchhammeria hintonii Paul G. Wilson GRO, OAX

*Forchhammeria longifolia Standl. SLP, TAMS, VER

* Forchhammeria macrocarpa Standl. GRO, MOR, OAX, PUE

Forchhammeria matudae Lundell CHIS, SLP, TAMS, VER
*Forchhammeria pallida Liebm. COL, GRO, JAL, MEX, $\mathrm{MICH}, \mathrm{OAX}$

*Forchhammeria sessilifolia Standl. COL, JAL, MICH, NAY

*Forchhammeria tamaulipana B.F. Hansen, Iltis \& Carlquist TAMS

Forchhammeria trifoliata Radlk. CAM, CHIS, OAX, QROO, SLP, TAB, TAMS, VER, YUC

*Forchhammeria watsonii Rose BCN, BCS, SIN, SON

*Quadrella angustifolia (Kunth) H.H. Iltis \& X. Cornejo COL, GRO, JAL, MICH, OAX

*Quadrella asperifolia (C. Presl) H.H. Iltis \& X. Cornejo COL, GRO, JAL, MICH, NAY

Quadrella calciphila (Standl. \& Steyerm.) H.H. Iltis \& X. Cornejo CHIS

Quadrella incana (Kunth) H.H. Iltis \& X. Cornejo CAM, CHIS, COL, GTO, GRO, HGO, JAL, MEX, MICH, NLE, OAX, PUE, QRO, QROO, SLP, TAMS, VER, YUC

Quadrella indica (L.) H.H. Iltis \& X. Cornejo CAM, CHIS, COL, GTO, GRO, JAL, MICH, NAY, OAX, QRO, QROO, SLP, SIN, VER, YUC, ZAC

Quadrella isthmensis (Eichler) Hutch. CAM, QROO, YUC

Quadrella lindeniana Cornejo \& H.H. Iltis CAM, QROO, YUC Quadrella lundellii (Standl.) H.H. Iltis \& X. Cornejo CAM, CHIS, OAX, QROO

Quadrella morenoi X. Cornejo \& H.H. Iltis MICH, OAX

Quadrella odoratissima (Jacq.) Hutch. CHIS, OAX

Quadrella pringlei (Briq.) H.H. Iltis \& X. Cornejo CHIS, OAX, PUE, VER

*Quadrella quintanarooensis H.H. Iltis \& X. Cornejo QROO

Steriphoma clara Standl. CHIS

\section{Family Caprifoliaceae}

*Abelia coriacea Hemsl. CHIH, COAH, DGO, NLE, SLP, VER

*Abelia floribunda (M. Martens \& Galeotti) Decne. AGS, CHIS, DGO, OAX, PUE, QRO, VER

*Abelia grandifolia Villarreal QRO, SLP

*Abelia mexicana Villarreal GRO, OAX

*Abelia occidentalis Villarreal DGO, JAL

Lonicera albiflora Torr. \& A. Gray CHIH, COAH, NLE, SLP, SON, TAMS

Lonicera arizonica Rehder CHIH, SON

*Lonicera cerviculata S.S. White CHIH, SON

Lonicera involucrata (Richardson) Banks ex Spreng. CHIH, SON

*Lonicera mexicana (Kunth) Rehder AGS, CHIS, CDMX, HGO, MEX, OAX, PUE, QRO, SLP, TLAX, VER

*Lonicera pilosa (Kunth) Willd. ex Kunth AGS, CHIS, CHIH, COAH, CDMX, DGO, GTO, HGO, JAL, MEX, MICH, MOR, NAY, NLE, OAX, PUE, QRO, SLP, SIN, SON, TAMS, VER, ZAC

Lonicera subspicata Hook. \& Arn. BCN, BCS

Plectritis ciliosa (Greene) Jepson BCN

*Symphoricarpos longiflorus A. Gray BCN, CHIH

Symphoricarpos microphyllus Kunth CHIS, CHIH, COAH, COL, CDMX, DGO, GTO, GRO, HGO, JAL, MEX, MICH, MOR, NLE, OAX, PUE, QRO, SLP, TAMS, TLAX, VER, ZAC Symphoricarpos mollis Nutt. BCN 
Symphoricarpos orbiculatus Moench COAH, NLE

Symphoricarpos oreophilus A. Gray BCN, SON

Symphoricarpos palmeri G.N. Jones CHIH, COAH, DGO, SON

Symphoricarpos parishii Rydb. BCN

Symphoricarpos rotundifolius A. Gray BCN, CHIH, COAH, SON

*Valeriana albonervata B.L. Rob. NLE, QRO, SLP

*Valeriana apiifolia A. Gray CHIS, CHIH, DGO, JAL, MOR, NAY, SIN, SON

*Valeriana ceratophylla Kunth CDMX, GTO, GRO, HGO, MEX, MOR, OAX, PUE, QRO, SLP, VER

Valeriana clematitis Kunth CHIS, COAH, CDMX, GRO, HGO, JAL, MEX, MICH, MOR, NLE, OAX, PUE, QRO, SLP, TAMS,

TLAX, VER, ZAC

Valeriana cucurbitifolia Standl. CHIS, OAX

*Valeriana chiapensis Barrie CHIS

Valeriana deltoidea F.G. Mey. CHIS, GRO, JAL, OAX

*Valeriana densiflora Benth. CHIS, COL, CDMX, DGO, GTO, GRO, HGO, JAL, MEX, MICH, MOR, NLE, OAX, PUE, QRO, SLP, SIN, TLAX, VER

Valeriana edulis Nutt. AGS, CHIH, COAH, COL, CDMX, DGO, HGO, JAL, MEX, MICH, MOR, NAY, OAX, PUE, SON, TLAX, VER

*Valeriana emmanuelii Rzed. \& Calderón MICH

*Valeriana laciniosa M. Martens \& Galeotti DGO, GTO, HGO, MEX, MICH, OAX, PUE, QRO, SLP

*Valeriana moorei Barrie HGO, QRO

*Valeriana naidae Barrie CDMX, GRO, JAL, MEX, MICH, MOR, OAX, PUE, TLAX, VER

Valeriana oaxacana Barrie CHIS, OAX, VER

*Valeriana otomiana Barrie GTO, HGO, QRO, SLP

*Valeriana palmatiloba F.G. Mey. GRO

*Valeriana palmeri A. Gray CHIS, COL, DGO, GTO, GRO, JAL, MEX, MICH, MOR, NAY, OAX, PUE, QRO, VER, ZAC

*Valeriana pratensis (Benth.) Steud. DGO, GTO, JAL, MEX, MICH, NAY, VER

Valeriana prionophylla Standl. CHIS

Valeriana pulchella M. Martens \& Galeotti CHIS, GRO, OAX Valeriana robertianifolia Briq. CHIS, COL, CDMX, DGO, GTO, GRO, JAL, MEX, MICH, MOR, OAX, QRO, TLAX, VER

* Valeriana rzedowskiorum Barrie CDMX, MEX, PUE

Valeriana scandens L. CHIS, COAH, COL, GTO, GRO, HGO, JAL, MEX, MICH, NAY, NLE, OAX, PUE, QRO, SLP, TAMS, VER

*Valeriana selerorum Graebn. \& Loesen. COL, DGO, JAL, MEX, MICH, OAX, ZAC

Valeriana sorbifolia Kunth AGS, BCS, CHIS, CHIH, COAH, COL, CDMX, DGO, GTO, GRO, HGO, JAL, MEX, MICH, MOR, NAY, NLE, OAX, PUE, QRO, SLP, SIN, SON, TAMS, TLAX, VER, ZAC

*Valeriana totzilana Barrie CHIS

Valeriana urticifolia Kunth CHIS, CHIH, COL, CDMX, DGO, GTO, GRO, HGO, JAL, MEX, MICH, MOR, NAY, OAX, PUE, SLP, SIN, TAMS, TLAX, VER, ZAC

*Valeriana vaginata Kunth COL, CDMX, GTO, HGO, JAL, MEX, MICH, MOR, VER
* Valeriana zamoranensis Rzed. \& Calderón GTO, QRO

*Valeriana zapotecana Barrie OAX

\section{Family Caricaceae}

Carica papaya L. AGS, BCS, CAM, CHIS, CHIH, COL, GRO, HGO, JAL, MEX, MICH, MOR, NAY, OAX, PUE, QRO, QROO, SLP, SIN, SON, TAB, TAMS, VER, YUC, ZAC

*Horovitzia cnidoscoloides (Lorence \& R. Torres) V.M. Badillo OAX

Jacaratia dolichaula (Donn. Sm.) Woodson CHIS, GRO, OAX, TAB, VER, YUC

Jacaratia mexicana A. DC. CAM, CHIS, COL, GRO, JAL, MEX, MICH, MOR, NAY, OAX, PUE, QROO, SIN, TAB, VER, YUC, ZAC

* Jarilla caudata (Brandegee) Standl. BCS, GTO, HGO, JAL, MICH, QRO, SLP, SIN

Jarilla chocola Standl. CHIS, CHIH, COL, JAL, MICH, NAY, OAX, SIN, SON

*Jarilla heterophylla (Cerv. ex Llave) Rusby COL, CDMX, GTO, GRO, HGO, JAL, MEX, MICH, OAX, PUE, QRO, SLP, ZAC

*Jarilla nana (Benth.) McVaugh CDMX, GTO, GRO, HGO, JAL, MEX, MICH, NAY, QRO, ZAC

Vasconcellea cauliflora (Jacq.) A. DC. CHIS, HGO, OAX, PUE, SIN, VER

\section{Family Caryophyllaceae}

Achyronychia cooperi Torr. \& A. Gray BCN, BCS, SLP, SON, VER

Arenaria altorum Standl. \& Steyerm. CHIS

Arenaria benthamii Fenzl CHIH, COAH, NLE

Arenaria bourgaei Hemsl. AGS, CHIS, CHIH, CDMX, DGO, GTO, GRO, HGO, JAL, MEX, MICH, MOR, NLE, OAX, QRO, SLP, SON, VER, ZAC

Arenaria bryoides Willd. ex Schltdl. CHIS, COL, GRO, JAL, MEX, PUE, TLAX, VER

Arenaria douglasii Fenzl ex Torr. \& A. Gray BCN, BCS

Arenaria eastwoodiae Rydb. SON

Arenaria guatemalensis Standl. \& Steyerm. CHIS, GRO, OAX, VER

*Arenaria gypsostrata B.L. Turner NLE

*Arenaria hintoniorum B.L. Turner NLE, TAMS

Arenaria lanuginosa (Michx.) Rohrb. AGS, BCN, BCS, CHIS, CHIH, COAH, COL, CDMX, DGO, GTO, GRO, HGO, JAL, MEX, MICH, MOR, NAY, NLE, OAX, PUE, QRO, SLP, SIN, SON, TAMS, TLAX, VER, ZAC

Arenaria ludens Shinners CHIH, COAH

Arenaria lycopodioides Willd. ex Schltdl. AGS, CHIS, COAH, CDMX, DGO, GTO, GRO, HGO, JAL, MEX, MICH, MOR, NLE, OAX, QRO, PUE, SLP, TAMS, TLAX, VER, ZAC

Arenaria macrotheca (Hornem.) Heynh. BCN

Arenaria megalantha (Rohrb.) F.N. Williams COL, DGO, JAL

*Arenaria mexicana Bartl. HGO, QRO

*Arenaria oresbia Greenm. COAH, COL, CDMX, HGO, JAL, MEX, MICH, NLE, PUE, VER

Arenaria paludicola B.L. Rob. CHIS, CHIH, CDMX, GTO, HGO, JAL, MEX, MICH, MOR, SON, VER

Arenaria parvifolia Benth. VER 
*Arenaria patula Michx. MICH

Arenaria reptans Hemsl. CHIS, CDMX, GTO, GRO, HGO, JAL, MEX, MICH, MOR, OAX, PUE, QRO, TLAX, VER

Arenaria serpens Kunth CHIH, JAL, VER

Cardionema ramosissimum (Weinm.) A. Nelson \& J.F. Macbr. AGS, BCN, BCS, CDMX, GTO, HGO, JAL, MEX, OAX, PUE, SLP, TLAX, VER

Cerastium arvense L. OAX

Cerastium axillare Correll $\mathrm{CHIH}, \mathrm{COAH}$

Cerastium brachypodum (Engelm. ex A. Gray) B.L. Rob. ex Britton CHIS, CHIH, COAH, DGO, GRO, HGO, JAL, MEX, MICH, MOR, NAY, NLE, QRO, SLP, SON, TLAX, VER Cerastium guatemalense Standl. CHIS, GRO, MOR

* Cerastium hintoniorum B.L. Turner OAX

* Cerastium madrense S. Watson CHIH, DGO

Cerastium nutans Raf. AGS, CHIS, CHIH, COL, CDMX, DGO, GTO, GRO, HGO, JAL, MEX, MICH, MOR, OAX, PUE, QRO, SLP, SIN, SON, TLAX, VER, ZAC

*Cerastium orithales Schltdl. CDMX, MEX, PUE, TLAX, VER

*Cerastium purpusii Greenm. CDMX, HGO, JAL, MEX, MICH, MOR, PUE, QRO, VER

*Cerastium ramigerum Bartl. MEX, TLAX, VER

*Cerastium sinaloense D.A. Good COL, JAL, SIN

Cerastium sordidum B.L. Rob. CHIH, SON

Cerastium texanum Britton BCS, CHIH, SIN, SON

*Cerastium tolucense D.A. Good CDMX, JAL, MEX, MICH, PUE

Cerastium vulcanicum Schltdl. CDMX, DGO, GRO, MEX, MICH, MOR, PUE, TLAX, VER

*Cerdia congestiflora Hemsl. AGS, CDMX, DGO, GTO, HGO,

JAL, MEX, MICH, QRO, SLP, VER, ZAC

* Cerdia glauca Hemsl. DGO, SLP, ZAC

Colobanthus quitensis (Kunth) Bartl. MEX, PUE, VER

Corrigiola andina Triana \& Planch. CHIH, CDMX, DGO, GTO, HGO, JAL, MEX, MICH, PUE, SLP, SON, ZAC

*Drymaria anomala S. Watson AGS, COAH, NLE, SLP, ZAC

Drymaria arenarioides Willd. AGS, BCS, CHIH, CDMX, DGO, GTO, HGO, JAL, MEX, NLE, QRO, SLP, SON, VER, ZAC

*Drymaria axillaris Brandegee CHIH, COAH, DGO

* Drymaria barkleyi J.A. Duke \& Steyerm. COAH, NLE

* Drymaria coahuilana (I.M. Johnst.) B.L. Turner CHIH, COAH, NLE, SLP, ZAC

*Drymaria confusa Rose DGO

* Drymaria conzattii J.A. Duke OAX

Drymaria cordata (L.) Willd. ex Roem. \& Schult. CAM, CHIS, CHIH, COL, CDMX, GRO, JAL, MEX, MICH, MOR, NAY, OAX, PUE, SIN, TAB, VER, YUC

* Drymaria crassifolia Benth. BCN, BCS

*Drymaria debilis Brandegee BCS

Drymaria effusa A. Gray CHIH, CDMX, DGO, GTO, HGO, JAL, MEX, MICH, QRO, SON, VER

* Drymaria elata I.M. Johnst. COAH

* Drymaria excisa Standl COL, JAL, MEX, MICH, MOR

Drymaria fendleri S. Watson BCS, HGO, QRO, SLP, SON, VER

Drymaria glandulosa Bartl. AGS, BCS, CHIS, CHIH, COAH, COL, CDMX, GTO, GRO, HGO, JAL, MEX, MICH, MOR,
NAY, NLE, OAX, PUE, QRO, SLP, SIN, SON, TLAX, VER, YUC, ZAC

Drymaria gracilis Schltdl. \& Cham. AGS, BCS, CHIS, CHIH, COAH, COL, DGO, GTO, GRO, HGO, JAL, MEX, MICH, MOR, NLE, OAX, QRO, SLP, SON, TAB, TLAX, VER, ZAC *Drymaria holosteoides Benth. BCN, BCS, SON

Drymaria hypericifolia Briq. CHIS

Drymaria ladewii Rusby CHIS, OAX

Drymaria laxiflora Benth. AGS, CHIH, COAH, CDMX, GTO, GRO, HGO, JAL, MEX, MICH, MOR, NLE, OAX, PUE, QRO, SLP, SON, TAMS, TLAX, VER, ZAC

Drymaria leptophylla (Cham. \& Schltdl.) Fenzl ex Rohrb. AGS, BCS, CHIH, COL, CDMX, DGO, GTO, HGO, JAL, MEX, MICH, PUE, SLP, SON, TLAX, VER, ZAC

*Drymaria longepedunculata $\mathrm{S}$. Watson COL, GRO, JAL, MEX

*Drymaria lyropetala I.M. Johnst. CHIH, COAH, NLE, SLP, ZAC

*Drymaria malachioides Briq. CDMX, GTO, HGO, JAL, MEX, MICH, OAX, PUE, TLAX, VER

Drymaria minuscula Standl. \& Steyerm. CHIS, COL, JAL, MEX

Drymaria molluginea (Lag.) Didr. BCS, CHIH, COAH, CDMX, DGO, HGO, JAL, MEX, PUE, QRO, SLP, SON, TLAX

Drymaria multiflora Brandegee CHIH, COL, CDMX, GTO, HGO, JAL, MEX, MICH, OAX, QRO

*Drymaria nodosa Engelm. CHIH, SON

Drymaria pachyphylla Wooton \& Standl. CHIH, COAH, DGO, NLE

* Drymaria pattersonii B.L. Turner COAH

* Drymaria polycarpoides A. Gray COAH

Drymaria polystachya Brandegee BCS

*Drymaria stipitata Fosberg COAH, NLE

* Drymaria subumbellata I.M. Johnst. COAH, NLE

* Drymaria suffruticosa A. Gray COAH

*Drymaria tenuis S. Watson AGS, CDMX, GTO, GRO, HGO, JAL, MEX, MICH, NLE, QRO, TLAX, VER, ZAC

*Drymaria townsendii B.L. Rob. CHIH, SON

Drymaria villosa Schltdl. \& Cham. CHIS, CHIH, COAH, COL, CDMX, DGO, GTO, GRO, HGO, JAL, MEX, MICH, MOR, NLE, OAX, PUE, SLP, SIN, SON, TLAX, VER

Drymaria viscosa S. Watson BCN, BCS, SON

*Drymaria xerophylla A. Gray AGS, GTO, HGO, JAL, MEX, MICH, OAX, QRO, SLP, VER, ZAC

Krascheninnikovia lanata (Pursh) A. Meeuse \& A. Smit BCN, COAH, NLE

Loeflingia squarrosa Nutt. BCN, BCS, SON

*Lychnis mexicana Rose CDMX, MEX, MOR, NLE

* Minuartia moehringioides (Moc. \& Sessé ex Ser.) Mattf. AGS, COL, CDMX, DGO, GTO, GRO, HGO, JAL, MEX, MICH, MOR, NAY, OAX, PUE, TLAX, ZAC

*Paronychia albomarginata Core COAH, NLE

* Paronychia congesta Correll COAH

*Paronychia hintoniorum B.L. Turner NLE, VER

Paronychia jamesii Torr. \& A. Gray CHIH, COAH, NLE, SON *Paronychia mexicana Hemsl. AGS, BCS, CDMX, DGO, GTO, HGO, JAL, MEX, MICH, NLE, OAX, QRO, SLP, TAMS, TLAX, VER, ZAC 
Paronychia monticola Cory COAH

Paronychia virginica Spreng. COAH

Paronychia wilkinsoni S. Watson CHIH, COAH

*Pentacaena polycnemoides Bartl. VER

Polycarpon tetraphyllum (L.) L. BCN, BCS

Sagina decumbens (Elliott) Torr. \& A. Gray BCN

Sagina procumbens L. CHIS, CDMX, HGO, MEX, MICH, PUE, VER

Sagina saginoides (L.) Karst. AGS, BCN, BCS, COAH, GRO, HGO, JAL, MEX, MICH, NAY, NLE, OAX, QRO, TLAX, VER, ZAC

*Scopulophila parryi (Hemsl.) I.M. Johnst. COAH, GTO, HGO, MEX, NLE, PUE, SLP, VER

Silene antirrhina $\mathrm{L}$. BCN, CHIH, COAH, SON, TAMS

* Silene chihuahuensis Standl. CHIH, SON

Silene laciniata Cav. AGS, BCN, BCS, CHIH, COAH, CDMX, DGO, GTO, HGO, JAL, MEX, MICH, NLE, OAX, PUE, QRO, SLP, SON, TAMS, TLAX, VER, ZAC

Silene multinervia $\mathrm{S}$. Watson $\mathrm{BCN}$

Silene scouleri Hook. CHIH, DGO, SON

Silene thurberi $\mathrm{S}$. Watson $\mathrm{CHIH}, \mathrm{SON}$

Silene verecunda $\mathrm{S}$. Watson $\mathrm{BCN}$

Spergularia macrotheca (Hornem. ex Cham. \& Schltdl.) Heynh. $\mathrm{BCN}$

Spergularia marina (L.) Griseb. BCN, SON

*Spergularia mexicana Hemsl. AGS, GTO, HGO, JAL, MEX,

MICH, QRO, SLP, TLAX, VER, ZAC

Stellaria cuspidata Willd. ex Schltdl. BCS, CHIS, CHIH, COAH, COL, CDMX, GTO, GRO, HGO, JAL, MEX, MICH, MOR, NAY, NLE, OAX, PUE, QRO, SLP, TAMS, TLAX, VER *Stellaria hintoniorum B.L. Turner COAH, NLE, TAMS

Stellaria irazuensis Donn. Sm. CHIS, GRO

*Stellaria miahuatlana B.L. Turner OAX

Stellaria nitens Nutt. BCN, BCS

Stellaria ovata Willd. ex Schltdl. CHIS, DGO, GRO, HGO, JAL, MICH, NAY, OAX, PUE, QRO, SLP, SIN, TAMS, VER, YUC

Stellaria umbellata Turcz. AGS, CHIH, GTO, JAL, MEX, PUE, QRO, ZAC

\section{Family Celastraceae}

*Acanthothamnus aphyllus (Schltdl.) Standl. HGO, NLE, OAX, PUE, QRO, SLP, TAMS

Canotia holacantha Torr. BCN, SON

*Canotia wendtii M.C. Johnst. CHIH

*Celastrus lenticellatus Lundell CHIS

*Celastrus liebmannii Standl. OAX, VER

*Celastrus pringlei Rose CHIS, COL, DGO, GRO, HGO, JAL, MEX, MICH, MOR, OAX, PUE, SLP, VER

*Celastrus tetramerus Standl. GRO

Celastrus vulcanicola Donn. Sm. CHIS, GRO, MOR, OAX, PUE, VER

*Crossopetalum densiflorum Lundell OAX, VER

Crossopetalum filipes (Sprague) Lundell CAM, CHIS, GRO, QROO, TAB, VER, YUC

Crossopetalum gaumeri (Loes.) Lundell CAM, QROO, VER, YUC
*Crossopetalum glabrum Lundell VER

*Crossopetalum managuatillo (Loes.) Lundell COL, GRO, JAL, $\mathrm{MICH}$

*Crossopetalum minimiflorum Lundell OAX, VER

*Crossopetalum oxyphyllum (S.F. Blake) Lundell OAX, SLP, TAB, TAMS, VER

Crossopetalum parviflorum (Hemsl.) Lundell CAM, CHIS, COL, GRO, JAL, NAY, OAX, QROO, SLP, SIN, TAB, TAMS, VER, YUC

Crossopetalum rhacoma Crantz CAM, QROO, VER, YUC

Crossopetalum uragoga (Jacq.) Kuntze CAM, CHIS, COL, GRO, HGO, JAL, NAY, OAX, QRO, SLP, TAMS, VER

Cuervea kappleriana (Miq.) A.C. Sm. CHIS, OAX, TAB, VER

Cheiloclinium belizense (Standl.) A.C. Sm. CHIS, VER

Elachyptera floribunda (Benth.) A.C. Sm. CAM, CHIS, QROO,

TAB, VER

Elaeodendron laneanum A.H. Moore VER

Elaeodendron xylocarpum (Vent.) DC. CAM, CHIS, COL, GRO, HGO, JAL, MICH, NAY, OAX, QRO, QROO, SLP, TAB, VER, YUC

Euonymus benthamii Lundell CHIS, OAX, QRO, SLP, VER

*Euonymus calzadae Lundell VER

*Euonymus corymbosus Sprague \& Bullock GRO, MEX, OAX

*Euonymus chiapensis Lundell CHIS, VER

*Euonymus mexicanus Benth. GRO, HGO, OAX, VER

*Euonymus platyphyllus Lundell VER

Gyminda latifolia (Sw.) Urb. QROO, TAMS, VER, YUC

Gyminda tonduzii Loes. CHIS, OAX, QROO

Gymnosporia haberiana Hammel VER

Hippocratea volubilis L. CAM, CHIS, COL, DGO, GRO, JAL, MEX, MICH, MOR, OAX, QROO, SLP, SIN, TAB, TAMS, VER, YUC, ZAC

Maytenus belizensis Standl. CAM, QROO, VER, YUC

*Maytenus chiapensis Lundell CHIS

Maytenus guatemalensis Lundell CAM, CHIS, QROO

Maytenus matudae Lundell CHIS, GRO

Maytenus phyllanthoides Benth. BCN, BCS, CAM, COAH, HGO, JAL, NLE, PUE, QRO, QROO, SLP, SIN, SON, TAMS, VER, YUC, ZAC

*Maytenus purpusii Lundell CHIS, TAB, VER

*Maytenus repanda Turcz. CHIS, OAX, VER

Maytenus schippii Lundell CAM, CHIS, COL, GRO, OAX, QROO, TAB, VER

*Maytenus stipitata Lundell CHIS

Maytenus texana Lundell COAH, NLE, TAMS

*Maytenus wendtii Lundell OAX, VER

* Mortonia diffusa Rose \& Standl. OAX, PUE

Mortonia greggii A. Gray CHIH, COAH, DGO, HGO, NLE, QRO, SLP, SON, TAMS, VER

*Mortonia latisepala I.M. Johnst. COAH, HGO, NLE, QRO, SLP, TAMS, VER, ZAC

*Mortonia palmeri Hemsl. AGS, CHIH, COAH, DGO, NLE, SLP, SON, ZAC

Mortonia scabrella A. Gray CHIH, COAH, SLP, SON

Mortonia sempervirens A. Gray CHIH, COAH, SON

*Myginda crossopetalum Schult. VER

*Orthosphenia mexicana Standl. NLE, SLP, TAMS, ZAC 
Paxistima myrsinites (Pursh) Raf. COAH, DGO, NLE, SON, TAMS

Prionostemma asperum (Lam.) Miers NAY

Pristimera celastroides (Kunth) A.C. Sm CAM, CHIS, COL, DGO, GTO, GRO, HGO, JAL, MEX, MICH, MOR, NAY, OAX, PUE, QRO, QROO, SLP, SIN, TAB, TAMS, VER, YUC, ZAC Quetzalia contracta (Lundell) Lundell CHIS, GRO, OAX, VER Quetzalia guatemalensis (Sprague) Lundell CHIS, OAX

Quetzalia occidentalis (Loes. ex Donn. Sm.) Lundell HGO, OAX, VER

*Quetzalia pauciflora Lundell OAX, VER

*Quetzalia schiedeana (Loes.) Lundell GRO, HGO, OAX, PUE, SLP, VER

*Quetzalia stipitata (Lundell) Lundell HGO, PUE, VER

* Rhacoma macrocarpa (Brandegee) Standl. CHIS

Rhacoma standleyi (Lundell) Standl. \& Steyerm. CHIS

*Rzedowskia tolantongensis Medrano GTO, HGO, QRO, SLP, TAMS, VER

Salacia cordata (Miers) Mennega CHIS, OAX, TAB, VER

Salacia impressifolia (Miers) A.C. Sm. CHIS, OAX, PUE, TAB, VER

Schaefferia cuneifolia A. Gray BCN, BCS, CHIH, COAH, NLE, SLP, SON, TAMS

Schaefferia frutescens Jacq. CAM, CHIS, COL, GRO, JAL, MICH, NLE, OAX, QROO, SLP, TAMS, VER

*Schaefferia lottiae Lundell COL, JAL, MICH

* Schaefferia oaxacana Standl. OAX, PUE

* Schaefferia ovatifolia Lundell VER

* Schaefferia pilosa Standl. BCS, COL, GRO, HGO, JAL, OAX,

PUE, QRO, SLP

*Schaefferia shrevei Lundell BCS, SON

* Schaefferia stenophylla Standl. GRO, OAX, PUE

Semialarium mexicanum (Miers) Mennega CAM, CHIS, COL, DGO, GRO, JAL, MEX, MICH, MOR, NAY, OAX, PUE, QROO, SIN, TAB, VER, YUC

Tontelea hondurensis A.C. Sm. CHIS

*Wimmeria acuminata L.O. Williams CHIS

Wimmeria bartlettii Lundell CHIS, HGO, MICH, OAX, PUE, QRO, SLP, TAB, VER

Wimmeria concolor Schltdl. \& Cham. CHIS, HGO, OAX, PUE, QRO, SLP, TAMS, VER

*Wimmeria chiapensis Lundell CHIS

*Wimmeria lanceolata Rose COL, GRO, JAL, MEX, MICH, MOR, OAX, TLAX

*Wimmeria lundelliana Carnevali, R. Duno, Tapia-Muñoz \& I. Ramírez CAM, QROO

*Wimmeria mexicana (DC.) Lundell AGS, CHIH, DGO, GRO, JAL, NAY, OAX, SIN, SON, ZAC

*Wimmeria microphylla Radlk. AGS, DGO, GRO, JAL, NAY, OAX, PUE, VER, ZAC

*Wimmeria montana Lundell CHIS

*Wimmeria obtusifolia Standl. CAM, CHIS, QROO, VER

*Wimmeria persicifolia Radlk. CHIS, COL, CDMX, GRO, HGO, JAL, MEX, MICH, MOR, OAX, PUE, VER, ZAC

Wimmeria pubescens Radlk. CHIS, GRO, NAY, OAX, PUE, VER

*Wimmeria serrulata (DC.) Radlk. CHIS, GRO, MICH, MOR,
OAX

Wimmeria sternii Lundell CHIS, OAX

*Zinowiewia concinna Lundell COL, GRO, HGO, JAL, MEX, MICH, MOR, OAX, VER

Zinowiewia costaricensis Lundell HGO, VER

Zinowiewia integerrima (Turcz.) Turcz. CHIS, GRO, MEX,

MICH, MOR, OAX, PUE, SLP, VER

*Zinowiewia matudae Lundell CHIS

*Zinowiewia pauciflora Lundell VER

Zinowiewia rubra Lundell CHIS

Zinowiewia tacanensis Lundell CHIS

\section{Family Ceratophyllaceae}

Ceratophyllum demersum $\mathrm{L}$. BCN, BCS, CAM, CHIS, COAH, COL, CDMX, DGO, GRO, HGO, JAL, MEX, MICH, MOR, OAX, PUE, QRO, SLP, SIN, SON, TAB, TAMS, TLAX, VER, ZAC

Ceratophyllum muricatum Cham. CHIS, COL, CDMX, GRO, JAL, MEX, OAX, TAB, VER

\section{Family Cistaceae}

*Helianthemum argenteum Hemsl. DGO, GTO, HGO, JAL, QRO, SLP, VER, ZAC

*Helianthemum concolor (L. Riley) J.G. Ortega COL, GRO, JAL, MEX, MICH, MOR, NAY, OAX, SIN

Helianthemum coulteri S. Watson COL, CDMX, DGO, GTO, GRO, HGO, JAL, MEX, MICH, NLE, PUE, QRO, SLP, TAMS, VER, ZAC

Helianthemum chihuahuense S. Watson AGS, CHIH, DGO, GTO, HGO, JAL, MICH, NAY, SIN, SON, TAMS, ZAC

Helianthemum glomeratum (Lag.) Lag. ex DC. AGS, BCS, CHIS, CHIH, COAH, COL, CDMX, DGO, GTO, GRO, HGO, JAL, MEX, MICH, MOR, NAY, NLE, OAX, PUE, QRO, SLP, SIN, SON, TAMS, TLAX, VER, ZAC

*Helianthemum majus (L.) Britton, Stearn \& Pogg. CHIH, SON

*Helianthemum nutans Brandegee BCN, BCS

*Helianthemum patens Hemsl. AGS, GTO, HGO, JAL, MEX, PUE, QRO, SLP, VER, ZAC

Helianthemum pringlei S. Watson AGS, CHIH, DGO, GTO, HGO, JAL, SLP, SON, VER, ZAC

*Helianthemum pugae Calderón AGS, GTO, JAL, QRO, SLP

Helianthemum scoparium Nutt. BCN

Lechea mensalis Hodgdon COAH

Lechea mucronata Raf. COAH, QRO, SLP, TAMS, VER

Lechea tripetala (Moc. \& Sessé ex Dunal) Britton BCN, BCS, CHIS, CHIH, DGO, GTO, HGO, JAL, MEX, MICH, OAX, PUE, QRO, SLP, SIN, SON, VER

\section{Family Cleomaceae}

*Andinocleome magnifica (Briq.) Iltis \& Cochrane CHIS, GRO, MICH, OAX, PUE, QRO, SLP, VER

Andinocleome pilosa (Benth.) Iltis \& Cochrane CHIS, COL, GRO, JAL, MICH, OAX, PUE, TAB, VER

Atamisquea emarginata Miers BCN, BCS, SIN, SON

*Cleome chapalensis H.H. Iltis JAL, MICH

* Cleome fosteriana $\mathrm{H}$.H. Iltis GRO, MEX

Cleome parvisepala Heilborn CHIS, GRO, OAX, QROO, TAB, VER 
* Cleome potosina B.L. Rob. QRO, SLP

* Cleome rutidosperma DC. SIN

*Cleome tenuis S. Watson BCN, BCS, GRO, MICH, NAY, OAX, SIN, SON

*Cleomella jaliscensis Villegas \& R. Ramírez-D. COL, JAL

Cleomella longipes Torr. CHIH

*Cleomella mexicana Moc. \& Sessé CDMX, MEX, PUE, SLP, VER, ZAC

*Cleomella perennis H.H. Iltis DGO, GTO, SLP, ZAC

Cleoserrata serrata (Jacq.) H.H. Iltis CAM, CHIS, COL, GRO,

JAL, MICH, OAX, QROO, SLP, TAB, VER, YUC

Cleoserrata speciosa (Raf.) H.H. Iltis CHIS, COL, DGO, GTO, GRO, HGO, JAL, MEX, MICH, MOR, NAY, NLE, OAX, PUE, QRO, SIN, TAB, TAMS, VER, YUC

Corynandra viscosa (L.) Cochrane \& Iltis BCS, CAM, CHIS, COL, GRO, JAL, MICH, NAY, OAX, QROO, SIN, SON, TAB, VER

Gynandropsis gynandra (L.) Briq. CAM, CHIS, COAH, GRO, MICH, MOR, OAX, QROO, SLP, SIN, SON, TAB, TAMS, VER, YUC

Hemiscola aculeata (L.) Raf. BCS, CAM, CHIS, COL, HGO, JAL, NAY, OAX, QRO, SLP, SIN, SON, TAB, TAMS, VER, YUC

Isomeris arborea Nutt. BCN, BCS, SON

Melidiscus giganteus (L.) Raf. CHIS, SLP

Melidiscus viridiflorus (Schreb.) Raf. OAX, QRO, SLP, VER

Morisonia americana L. CHIS, COL, GRO, JAL, MICH, NAY, OAX, QRO, SLP, SIN, TAMS, VER

Peritoma jonesii (J.F. Macbr.) H.H. Iltis BCN, BCS

Peritoma multicaulis (DC.) H.H. Iltis CHIH, CDMX, DGO, MEX, MICH, SON

Physostemon guianense (Aubl.) Malme BCS, CHIS, GRO, NAY, OAX, SON, VER

*Physostemon hemsleyanum (Bullock) R.C. Foster CHIS, COL, GRO, JAL, MEX, MICH, OAX, VER

Physostemon humilis (Rose) H.H. Iltis COL, JAL, ZAC

Physostemon lanceolatum Mart. \& Zucc. CHIS, OAX

*Physostemon melanospermum (S. Watson) Pax \& K. Hoffm. CHIH, NAY, SIN, SON

Physostemon stenophyllum (Klotzsch ex Urb.) Iltis CHIS, QROO, VER

Polanisia dodecandra (L.) DC. CHIH, COAH, DGO, HGO, NLE, OAX, QRO, SLP, SIN, SON, TAMS

Polanisia trachysperma Torr. \& A. Gray CHIH, SIN, SON

Polanisia uniglandulosa (Cav.) DC. CHIH, COAH, CDMX, DGO, GTO, GRO, HGO, JAL, MEX, MICH, NLE, OAX, PUE, QRO, SLP, SIN, SON, VER

Tarenaya parviflora (Kunth) H.H. Iltis CHIS, OAX, TAB, VER Tarenaya spinosa (Jacq.) Raf. CAM, CHIS, COL, GRO, JAL, NAY, OAX, TAB, VER, YUC

Wislizenia refracta Engelm. BCN, BCS, CHIH, SON

\section{Family Clethraceae}

* Clethra alcoceri Greenm. CHIS, COL, GRO, HGO, JAL, MICH, OAX, PUE, TAB, VER

* Clethra breedlovei C.W. Ham. GRO

* Clethra conzattiana L.M. González OAX
* Clethra chiapensis L.M. González CHIS

* Clethra fragrans L.M. González \& R. Ramírez-D. COL, JAL

* Clethra hartwegii Britton AGS, CHIS, CHIH, COL, DGO, GTO, GRO, JAL, MEX, MICH, MOR, NAY, OAX, QRO, SIN, SON, ZAC

*Clethra hirsutovillosa S. Valencia \& Cruz-Durán GRO

Clethra hondurensis Britton CAM, CHIS, TAB

* Clethra integerrima (Turcz.) L.M. González OAX, VER

* Clethra kenoyeri Lundell HGO, OAX, PUE, QRO, SLP, TAMS, VER

Clethra lanata M. Martens \& Galeotti CHIS, CHIH, COL, DGO, GRO, HGO, JAL, MEX, MICH, NAY, OAX, PUE, SLP, SIN, SON, VER

* Clethra luzmariae L.M. González OAX

Clethra macrophylla M. Martens \& Galeotti CHIS, COL, GRO, HGO, JAL, MICH, MOR, OAX, PUE, QRO, SLP, TAMS, VER Clethra mexicana DC. AGS, CHIS, CHIH, COL, CDMX, DGO, GTO, GRO, HGO, JAL, MEX, MICH, MOR, NAY, OAX, PUE, QRO, SLP, SIN, SON, TAB, TAMS, VER

Clethra nicaraguensis C.W. Ham. CHIS

Clethra occidentalis (L.) Kuntze CHIS, GRO, JAL, MEX, MICH, OAX, SLP, VER

Clethra oleoides L.O. Williams CHIS

Clethra pachecoana Standl. \& Steyerm. CHIS, OAX

* Clethra parvifolia Lundell CHIS

*Clethra pringlei S. Watson CHIS, GRO, HGO, JAL, MEX, MICH, NLE, OAX, PUE, QRO, SLP, TAMS, VER

* Clethra purpusii L.M. González CHIS, OAX

* Clethra rosei Britton CHIH, COL, DGO, GRO, JAL, MEX, MICH, NAY, OAX, SIN, SON, ZAC

Clethra suaveolens Turcz. CHIS, OAX, QRO, SLP, VER

* Clethra tuxtlensis L.M. González VER

Clethra vicentina Standl. CHIS, COL, JAL, OAX, TAB

\section{Family Clusiaceae}

Clusia belizensis Standl. CHIS

Clusia flava Jacq. CAM, CHIS, OAX, QROO, TAB, TAMS, VER, YUC

Clusia gentlei Lundell CHIS

Clusia guatemalensis Hemsl. CHIS, OAX, PUE, TAB, VER

Clusia lundellii Standl. CAM, CHIS, OAX, TAB, VER

Clusia lusoria Standl. \& Steyerm. CHIS, GRO, OAX, VER

Clusia massoniana Lundell CHIS, MEX, MICH, OAX

Clusia minor L. CHIS, OAX, PUE, TAB, VER

Clusia ovigera Triana \& Planch. VER

*Clusia pringlei Lundell COL, GRO, MICH

Clusia quadrangula Bartlett CHIS, OAX, VER

Clusia rosea Jacq. CAM, CHIS, GRO, MICH, QROO, VER

Clusia salvinii Donn. Sm. CAM, CHIS, COL, DGO, GRO, JAL, MEX, MICH, MOR, NAY, OAX, QROO, SIN, TAB, TAMS, VER, YUC, ZAC

Clusia suborbicularis Lundell CHIS

Chrysochlamys nicaraguensis (Oerst., Planch. \& Triana) Hemsl. CHIS, OAX, TAB, VER

Garcinia intermedia (Pittier) Hammel CHIS, COL, GRO, JAL, MICH, NAY, OAX, PUE, QROO, TAB, VER

Garcinia macrophylla Mart. CHIS, JAL, OAX, TAB, VER 
Symphonia globulifera L. f. OAX, TAB, VER

\section{Family Combretaceae}

Combretum argenteum Bertol. CAM, CHIS, GRO, MEX, OAX, VER

Combretum decandrum Jacq. CHIS, GRO, JAL, MICH, NAY, OAX, TAB, VER

Combretum formosum G. Don. CAM, CHIS, OAX, QROO

Combretum fruticosum (Loefl.) Stuntz CAM, CHIS, CHIH, COL, DGO, GRO, HGO, JAL, MEX, MICH, MOR, NAY, OAX, PUE, QROO, SLP, SIN, TAB, TAMS, VER, YUC

*Combretum igneiflorum Rendón \& R. Delgad. COL, JAL, NAY, OAX

Combretum laxum Jacq. CAM, CHIS, COL, GRO, JAL, MEX, MICH, MOR, NAY, OAX, QROO, TAB, VER

*Combretum rovirosae Exell CAM, CHIS, OAX, TAB, VER

Conocarpus erectus L. BCN, BCS, CAM, CHIS, COL, GRO, JAL, MICH, NAY, OAX, PUE, QROO, SIN, SON, TAB, TAMS, VER, YUC

Laguncularia racemosa (L.) C.F. Gaertn. BCN, BCS, CAM, CHIS, COL, GRO, JAL, MICH, NAY, OAX, PUE, QROO, SLP, SIN, SON, TAB, TAMS, VER, YUC

Terminalia amazonia (J.G. Gmel.) Exell CAM, CHIS, OAX, QROO, TAB, VER

Terminalia buceras (L.) C. Wright CAM, CHIS, GRO, MICH, OAX, PUE, QROO, SIN, TAB, VER, YUC

*Terminalia lucida Hoffmanns. ex Mart. CHIS

Terminalia macrostachya (Standl.) Stace CHIS, OAX, VER

Terminalia oblonga (Ruiz \& Pav.) Steud. CHIS

Terminalia spinosa Northr. CAM, QROO, VER

\section{Family Commelinaceae}

Callisia cordifolia (Sw.) E.S. Anderson \& Woodson CAM, QROO, TAMS

Callisia filiformis (M. Martens \& Galeotti) D.R. Hunt CHIS, COL, GRO, JAL, MEX, MICH, MOR, NAY, OAX, SIN, SON, VER

Callisia fragrans (Lindl.) Woodson GRO, HGO, MOR, OAX, QRO, SLP, TAMS, VER, YUC

Callisia gentlei Matuda CHIS, OAX

*Callisia hintoniorum B.L. Turner NLE, TAMS

*Callisia insignis C.B. Clarke CDMX, GTO, GRO, HGO, JAL, MEX, OAX, PUE, QRO, SLP, VER

*Callisia laui (D.R. Hunt) D.R. Hunt GRO, MOR, OAX

Callisia micrantha (Torr.) D.R. Hunt TAMS

Callisia monandra (Sw.) Schult. \& Schult. f. BCS, CAM, CHIS, COL, GRO, HGO, JAL, MICH, OAX, PUE, SLP, SIN, SON, VER

Callisia multiflora (M. Martens \& Galeotti) Standl. CHIS, GRO, HGO, JAL, OAX, PUE, SLP, TAB, VER

*Callisia navicularis (Ortgies) D.R. Hunt COAH, NLE, OAX, PUE, SLP, VER

Callisia repens L. CAM, CHIS, COL, GTO, GRO, HGO, JAL, MEX, MICH, MOR, NLE, OAX, PUE, QRO, QROO, SLP, SIN, SON, TAB, TAMS, VER, YUC, ZAC

*Callisia soconuscensis Matuda CHIS, GRO, OAX

* Callisia tehuantepecana Matuda CHIS, OAX

Callisia warszewicziana (Kunth \& C.D. Bouché) D.R. Hunth
CHIS, VER

*Commelina bambusifolia Matuda MEX

* Commelina bravoa Matuda PUE

Commelina coelestis Willd. AGS, BCS, CHIS, CHIH, COAH, COL, CDMX, DGO, GTO, GRO, HGO, JAL, MEX, MICH, MOR, NAY, NLE, OAX, PUE, QRO, SLP, SIN, SON, TAMS, TLAX, VER, ZAC

Commelina communis L. MOR, QRO, TAMS

*Commelina congestispatha López-Ferrari, Espejo \& Ceja GTO, MEX, MICH, QRO, SLP

Commelina dianthifolia Delile AGS, BCS, CAM, CHIS, CHIH, COAH, COL, CDMX, DGO, GTO, GRO, HGO, JAL, MEX, MICH, MOR, NAY, NLE, OAX, PUE, QRO, QROO, SLP, SIN, SON, TAMS, VER, YUC, ZAC

Commelina diffusa Burm. f. AGS, BCS, CAM, CHIS, COAH, COL, CDMX, DGO, GTO, GRO, HGO, JAL, MEX, MICH, MOR, NAY, NLE, OAX, PUE, QRO, QROO, SLP, SIN, SON, TAB, TAMS, TLAX, VER, YUC, ZAC

Commelina elliptica Kunth CHIS, CDMX, HGO, MEX, MICH, MOR, NLE, OAX, TAMS, VER

Commelina erecta L. AGS, BCS, CAM, CHIS, CHIH, COAH, COL, CDMX, DGO, GTO, GRO, HGO, JAL, MEX, MICH, MOR, NAY, NLE, OAX, PUE, QRO, QROO, SLP, SIN, SON, TAB, TAMS, VER, YUC, ZAC

*Commelina jaliscana Matuda COL, DGO, JAL, MICH, NAY, $\mathrm{ZAC}$

Commelina leiocarpa Benth. AGS, CHIS, CHIH, COL, DGO, GRO, HGO, JAL, MEX, MICH, MOR, NAY, OAX, QRO, SLP, SIN, SON, TAB, VER

*Commelina nivea López-Ferrari, Espejo \& Ceja DGO Commelina obliqua Vahl CHIS, COL, GRO, JAL, OAX

Commelina orchioides Booth ex Lindl. AGS, CDMX, DGO, GTO, GRO, HGO, MEX, MICH, MOR, OAX, QRO, VER, $\mathrm{ZAC}$

*Commelina queretarensis López-Ferrari, Espejo \& Ceja HGO, QRO, SLP

*Commelina ramosissima López-Ferrari, Espejo \& Ceja GTO, HGO, JAL, MICH, QRO, VER

Commelina rufipes Seub. CAM, CHIS, COL, GRO, JAL, MEX, NAY, OAX, PUE, QROO, TAB, VER

*Commelina rzedowskii López-Ferrari, Espejo \& Ceja GRO, MEX

*Commelina scabra Benth. AGS, CHIH, DGO, GTO, HGO, JAL, MEX, MICH, MOR, NAY, SLP, TAMS, VER, ZAC

*Commelina socorrogonzaleziae Espejo \& López-Ferrari CHIH, DGO, GTO, MEX, MICH, QRO, SIN

Commelina standleyi Steyerm. CHIS, COL, DGO, GRO, HGO, JAL, MEX, MICH, MOR, NAY, OAX, QRO, SIN, TLAX, VER Commelina texcocana Matuda CDMX, HGO, JAL, MEX, MICH, MOR, PUE, VER

Commelina tuberosa L. BCS, CHIS, CHIH, COL, CDMX, DGO, GTO, GRO, HGO, JAL, MEX, MICH, MOR, NAY, NLE, OAX, PUE, QRO, SLP, SIN, TAMS, TLAX, VER, ZAC Commelina virginica L. CHIS, MEX

Dichorisandra amabilis J.R. Grant CHIS, OAX, VER

Dichorisandra hexandra (Aubl.) Standl. CAM, CHIS, GRO, OAX, TAB, VER 
*Gibasis consobrina D.R. Hunt GRO, MEX, MOR, OAX, PUE, VER

*Gibasis chihuahuensis (Standl.) Rohweder CHIH, COAH, DGO, JAL, MICH, NAY, SIN, SON, ZAC

Gibasis geniculata (Jacq.) Rohweder AGS, CAM, CHIS, COAH, GRO, HGO, MEX, MICH, MOR, OAX, QRO, QROO, SLP, TAB, TAMS, VER, ZAC

*Gibasis gypsophila B.L. Turner NLE, TAMS

* Gibasis hintoniorum B.L. Turner COAH, NLE, TAMS

*Gibasis karwinskyana (Schult. f.) Rohweder COAH, DGO, GTO, HGO, JAL, MEX, MOR, NLE, QRO, SLP, TAMS, VER, ZAC

*Gibasis linearis (Benth.) Rohweder AGS, BCS, CHIH, COAH, CDMX, DGO, GTO, GRO, HGO, JAL, MEX, MICH, MOR, NAY, NLE, OAX, PUE, QRO, SLP, SIN, SON, VER, ZAC

*Gibasis matudae D.R. Hunt OAX, VER

* Gibasis oaxacana D.R. Hunt OAX, VER

Gibasis pellucida (M. Martens \& Galeotti) D.R. Hunt CHIS, COAH, COL, GRO, HGO, JAL, MEX, MICH, MOR, NAY, NLE, OAX, PUE, QRO, SLP, SIN, TAMS, VER

Gibasis pulchella (Kunth) Raf. CHIH, COL, CDMX, GTO, GRO, HGO, JAL, MEX, MICH, MOR, OAX, PUE, QRO, SLP, SON, TLAX, VER

Gibasis triflora (M. Martens \& Galeotti) D.R. Hunt COL, GRO, JAL, MICH, NAY, OAX

*Gibasis venustula (Kunth) D.R. Hunt BCS, CHIH, COAH, DGO, GTO, HGO, JAL, MEX, MICH, NAY, NLE, PUE, SLP, SON, ZAC

*Gibasoides laxiflora (C.B. Clarke) D.R. Hunt MEX, MOR, OAX, PUE

*Matudanthus nanus (M. Martens \& Galeotti) D.R. Hunt OAX *Thyrsanthemum floribundum (M. Martens \& Galeotti) Pichon COL, GTO, GRO, HGO, JAL, MEX, MICH, MOR, NAY, OAX, PUE, QRO, SLP

*Thyrsanthemum goldianum D.R. Hunt DGO, GRO, JAL, MEX, MICH, NAY, OAX, VER, ZAC

*Thyrsanthemum macrophyllum (Greenm.) Rohweder CHIS, GTO, GRO, MEX, MOR, OAX, QRO

Tinantia erecta (Jacq.) Schltdl. BCS, CHIS, CHIH, COL, CDMX, DGO, GTO, GRO, HGO, JAL, MEX, MICH, MOR, NAY, OAX, PUE, QRO, SLP, SIN, SON, TAB, TAMS, TLAX, VER, ZAC

Tinantia glabra (Standl. \& Steyerm.) Rohweder BCS, COL, CDMX, MEX, JAL, SIN, VER

Tinantia leiocalyx C.B. Clarke ex Donn. Sm. CHIS, COL, DGO, GRO, JAL, MEX, MICH, MOR, NAY, OAX, SIN, SON, TAB, VER

Tinantia longipedunculata Standl. \& Steyerm. BCS, CHIS, COL, DGO, JAL, MEX, MICH, MOR, NAY, OAX, SIN, SON, TAB, VER, ZAC

*Tinantia macrophylla S. Watson BCS, CHIH, COL, DGO, JAL, NAY, SIN, SON

Tinantia parviflora Rohweder COL, JAL, MEX, MICH, NAY, OAX

*Tinantia pringlei (S. Watson) Rohweder COAH, JAL, NLE, SLP, TAMS, VER

Tinantia standleyi Steyerm. CHIS, COL, GRO, JAL, MEX,
MICH, OAX, SIN, VER

Tinantia violacea Rohweder CHIS, COL, GRO, JAL, MICH, NAY, OAX, VER

*Tradescantia andrieuxii C.B. Clarke AGS, CHIS, CHIH, COL, GRO, JAL, MICH, MOR, NAY, OAX, PUE, SIN, SON, ZAC

*Tradescantia brachyphylla Greenm. CHIH, COAH, DGO, GTO, HGO, NLE, OAX, QRO, SLP, TAMS, VER

Tradescantia brevifolia (Torr.) Rose CHIH, COAH, NLE

Tradescantia buckleyi (I.M. Johnst.) D.R. Hunt TAMS

*Tradescantia burchii D.R. Hunt COL, JAL

*Tradescantia cirrifera Mart. DGO, GTO, HGO, QRO, SLP, SIN

Tradescantia commelinoides Schult. \& Schult. f. CHIS, COL, CDMX, DGO, GRO, HGO, JAL, MEX, MICH, MOR, OAX, PUE, SIN, TLAX, VER, ZAC

Tradescantia crassifolia Cav. AGS, CHIS, CHIH, COAH, COL, CDMX, DGO, GTO, GRO, HGO, JAL, MEX, MICH, MOR, NAY, NLE, OAX, PUE, QRO, SLP, SIN, SON, TAMS, TLAX, VER, ZAC

*Tradescantia deficiens Brandegee CHIS

*Tradescantia exaltata D.R. Hunt OAX

*Tradescantia gentryi D.R. Hunt SIN, SON

Tradescantia gracillima Standl. CHIS, GRO, MOR, OAX

Tradescantia guatemalensis C.B. Clarke CHIS, MOR, OAX

*Tradescantia guiengolensis Matuda OAX

*Tradescantia gypsophila B.L. Turner CHIH, COAH

*Tradescantia hirta D.R. Hunt COAH, NLE, TAMS

*Tradescantia huehueteca (Standl. \& Steyerm.) D.R. Hunt CHIS

*Tradescantia llamasii Matuda GRO, MEX, MOR, OAX

*Tradescantia masonii Matuda SIN

*Tradescantia mcvaughii D.R. Hunt COL, JAL, NAY, ZAC

*Tradescantia mirandae Matuda GRO

*Tradescantia monosperma Brandegee OAX, PUE

*Tradescantia murilloae Zamudio, Espejo, López-Ferrari \& Ceja HGO, QRO, SLP

*Tradescantia nuevoleonensis Matuda COAH, GTO, NLE, QRO, SLP

Tradescantia occidentalis Britton SON

*Tradescantia orchidophylla Rose \& Hemsl. COL, GRO, JAL, $\mathrm{MICH}$

Tradescantia pallida (Rose) D.R. Hunt CAM, CHIS, COAH, GRO, JAL, MOR, NAY, NLE, OAX, PUE, QROO, SLP, TAMS, VER, YUC

*Tradescantia peninsularis Brandegee BCS

Tradescantia pinetorum Greene BCS, CHIH, COAH, DGO, SON

*Tradescantia plusiantha Standl. CHIS, OAX

Tradescantia poelliae D.R. Hunt CHIS, JAL, OAX, VER

*Tradescantia pygmaea (Greene) D.R. Hunt DGO, SIN

*Tradescantia rozynskii Matuda SLP, TAMS

*Tradescantia schippii D.R. Hunt GRO, OAX

*Tradescantia sillamontana Matuda NLE, TAMS

*Tradescantia soconuscana Matuda CHIS, GRO, OAX, VER

Tradescantia spathacea Sw. CAM, CHIS, QROO, TAB, VER, YUC

Tradescantia standleyi Steyerm. CHIS, GRO, MEX, OAX, TAB 
*Tradescantia subtilis Matuda DGO

*Tradescantia tepoxtlana Matuda MEX, MOR, OAX, PUE

*Tradescantia velutina Kunth CHIS, COAH, HGO, QRO

Tradescantia wrightii Rose \& Bush COAH

Tradescantia zanonia (L.) Sw. CHIS, COL, GRO, HGO, JAL, MOR, OAX, PUE, QRO, SLP, TAB, TAMS, VER

Tradescantia zebrina Heynh. AGS, BCS, CAM, CHIS, CHIH, COAH, COL, CDMX, DGO, GTO, GRO, HGO, JAL, MEX, MICH, MOR, NAY, NLE, OAX, PUE, QRO, QROO, SLP, SIN, SON, TAB, TAMS, TLAX, VER, YUC, ZAC

*Tripogandra amplexans Handlos CHIS, COL, GRO, JAL, MEX, MICH, MOR, OAX, PUE

Tripogandra amplexicaulis (Klotzsch ex C.B. Clarke) Woodson CHIS, CHIH, COL, DGO, GTO, GRO, HGO, JAL, MEX, MICH, MOR, NAY, OAX, PUE, QRO, SIN, SON, TLAX, VER, ZAC

Tripogandra angustifolia (B.L. Rob.) Woodson CHIS, COAH, COL, GRO, HGO, MEX, MICH, MOR, NLE, OAX, PUE, QRO, SLP, TAMS, VER

Tripogandra disgrega (Kunth) Woodson CHIS, COL, CDMX, DGO, GTO, GRO, HGO, JAL, MEX, MICH, MOR, OAX, QRO, SIN, SON, TLAX, VER

Tripogandra grandiflora (Donn. Sm.) Woodson CAM, CHIS, GRO, OAX, QROO, TAB, VER

*Tripogandra guerrerensis Matuda COL, GRO, JAL

*Tripogandra kruseana Matuda GRO

Tripogandra montana Handlos CHIS, OAX

*Tripogandra palmeri (Rose) Woodson COL, DGO, GRO, JAL, MEX, MICH, NAY, PUE, SIN, SON, ZAC

Tripogandra purpurascens (S. Schauer) Handlos AGS, CHIS, CHIH, COL, CDMX, DGO, GTO, GRO, HGO, JAL, MEX, MICH, MOR, NAY, OAX, PUE, QRO, SLP, SIN, SON, TAMS, TLAX, VER, ZAC

*Tripogandra saxicola (Greenm.) Woodson GRO, MEX, MOR, PUE

Tripogandra serrulata (Vahl) Handlos CAM, CHIS, COL, CDMX, GRO, HGO, JAL, MEX, MICH, MOR, NAY, OAX, PUE, QRO, QROO, SLP, TAB, VER

*Tripogandra silvatica Handlos VER

Weldenia candida Schult. f. CHIS, COL, CDMX, DGO, GRO, HGO, JAL, MEX, MICH, MOR, OAX, VER, ZAC

\section{Family Connaraceae}

Cnestidium rufescens Planch. CAM, CHIS, OAX, PUE, QROO, TAB, VER

Connarus lambertii (DC.) Sagot CHIS

Connarus lentiginosus Brandegee CHIS, GRO, OAX

* Connarus schultesii Standl. CHIS, OAX, VER

*Connarus stenophyllus Standl. \& L.O. Williams ex A. Molina

R. CHIS, GRO, OAX

Rourea glabra Kunth CAM, CHIS, COL, GRO, HGO, JAL, MICH, NAY, OAX, PUE, QRO, QROO, SLP, SIN, TAB, TAMS, VER, YUC

Rourea schippii Standl. CHIS, PUE, TAB, VER

Rourea suerrensis Donn. Sm. TAB

Rourea vulcanicola Forero VER

\section{Family Convolvulaceae}

Aniseia cernua Moric. CAM, OAX, QROO, TAB, VER, YUC Aniseia martinicensis (Jacq.) Choisy CAM, CHIS, GRO, OAX, QROO, TAB, VER, YUC

*Bonamia elliptica (L.B. Sm. \& B.G. Schub.) Myint \& D.B. Ward MEX, MICH

*Bonamia mexicana J.A. McDonald COL, JAL

*Bonamia multicaulis (Brandegee) House CHIH, COAH, DGO Bonamia ovalifolia (Torr.) Hallier f. COAH

Bonamia repens (I.M. Johnst.) D.F. Austin \& Staples CHIH, COAH, DGO, GTO, HGO, JAL, NLE, SLP, TAMS, ZAC

Bonamia sulphurea (Brandegee) Myint \& Ward CAM, CHIS, GRO, JAL, MEX, OAX, QROO, SIN, VER, ZAC

*Calycobolus nutans (Choisy) D.F. Austin COL, GRO, MEX, MICH, MOR, OAX, PUE

*Calycobolus velutinus (M. Martens \& Galeotti) House GRO, MEX, MICH, MOR, OAX, PUE

Calystegia macrostegia (Greene) Brummitt BCN, BCS

Calystegia sepium (L.) R. Br. COAH, SLP

Calystegia soldanella (L.) R. Br. BCN

Convolvulus equitans Benth. AGS, CHIH, COAH, CDMX, GTO, HGO, JAL, MEX, MICH, NLE, OAX, PUE, QRO, SLP, SON, TAMS, ZAC

Convolvulus nodiflorus Desr. CAM, CHIS, COL, GRO, JAL, MEX, MICH, OAX, PUE, QROO, SLP, SIN, TAMS, VER, YUC

Convolvulus simulans L.M. Perry BCN

Cressa truxillensis Kunth BCN, BCS, CHIS, CHIH, COAH, COL, DGO, GTO, JAL, NAY, NLE, OAX, SLP, SIN, SON, TAMS

Cuscuta americana L. CAM, CHIH, DGO, GTO, GRO, HGO, MEX, MOR, NAY, OAX, PUE, QRO, QROO, SIN, SON, TAB, VER, YUC

Cuscuta applanata Engelm. CHIH, COAH, DGO, JAL, MEX, MICH, NLE, OAX, PUE, SLP, SON, TAMS, ZAC

Cuscuta boldinghii Urb. GRO, HGO, OAX, QRO, SLP, SIN, SON, TAB, VER, YUC

Cuscuta californica Hook. \& Arn. BCN, BCS, SON

Cuscuta ceanothii Behr BCN

Cuscuta cockerellii Yunck. CDMX, GTO, HGO, JAL, MEX, MICH, OAX, QRO, SLP, SON, VER, ZAC

Cuscuta corymbosa Ruiz \& Pav. BCN, BCS, COL, CDMX, GTO, GRO, HGO, JAL, MEX, MICH, MOR, OAX, PUE, QRO, SLP, SIN, SON, TAMS, TLAX, VER, YUC

Cuscuta costaricensis Yunck. DGO, GTO, GRO, JAL, MICH, SLP

* Cuscuta cotijana Costea \& I. García COL, JAL, MICH

Cuscuta cozumeliensis Yunck. QROO

* Cuscuta chapalana Yunck. JAL

* Cuscuta choisiana Yunck. MICH, NAY, NLE, SLP

Cuscuta decipiens Yunck. COAH, DGO, ZAC

* Cuscuta deltoidea Yunck. COL

Cuscuta denticulata Engelm. BCN, HGO, QRO, VER

* Cuscuta desmouliniana Yunck. BCS, SON

*Cuscuta durangana Yunck. DGO, HGO, QRO, SLP

Cuscuta erosa Yunck. SON, VER

Cuscuta globulosa Benth. GRO, YUC

*Cuscuta gracillima Engelm. BCS, COL, GRO, JAL, MEX, 
MICH, MOR, OAX, SIN, SON

*Cuscuta iguanella Costea \& I. García JAL, NAY

Cuscuta indecora Choisy CHIH, COAH, HGO, NLE, SON, TAMS

*Cuscuta insolita Costea \& I. García NAY

Cuscuta jalapensis Schltdl. CHIS, CHIH, GTO, GRO, HGO,

MICH, NLE, OAX, QRO, SLP, VER

*Cuscuta lacerata Yunck. OAX

Cuscuta legitima Costea \& Stefanov. SON

Cuscuta leptantha Engelm. BCN, BCS, SIN, SON

*Cuscuta lindsayi Wiggins DGO, JAL, SIN

*Cuscuta macrocephala W. Schaffner ex Yunck. BCS, NAY, SIN, TAMS

*Cuscuta mexicana Yunck. DGO, JAL, NAY

Cuscuta mitriformis Engelm. ex Hemsl. CHIH, COAH, COL, GTO, GRO, HGO, JAL, MEX, MICH, NLE, OAX, PUE, QRO,

SLP, SON, TAMS, VER, ZAC

*Cuscuta modesta Costea \& Stefanov. COAH

* Cuscuta montana Costea \& Stefanov. DGO, SIN

Cuscuta obtusiflora Kunth CDMX, DGO, GTO, GRO, HGO,

JAL, MEX, MICH, NAY, QRO, VER

Cuscuta occidentalis Millsp. BCN, BCS

Cuscuta odontolepis Engelm. CHIH, SON

*Cuscuta ortegana Yunck. DGO, JAL, MICH, SIN

Cuscuta pentagona Engelm. BCN, BCS, CHIH, COAH, CDMX, GTO, GRO, HGO, JAL, MEX, MICH, NLE, SLP, SIN, SON, TAMS, TLAX, ZAC

*Cuscuta polyanthemos Schaffner ex Yunck. BCS, SIN, SON

*Cuscuta potosina W. Schaffn. ex Engelm. CDMX, MEX, MOR, SLP

*Cuscuta purpusii Yunck. NLE, SLP

Cuscuta rugosiceps Yunck. COAH, CDMX, DGO, GTO, GRO, HGO, JAL, MEX, MICH, NAY, NLE, OAX, QRO, SLP, SIN, VER

Cuscuta saccharata (Engelm.) Yunck. GRO, NAY, OAX

Cuscuta salina Engelm. BCN, BCS, SON, TAMS, VER

*Cuscuta serruloba Yunck. COL, OAX

Cuscuta sidarum Liebm. NAY, OAX

Cuscuta squamata Engelm. CHIH, COAH, DGO

*Cuscuta strobilacea Liebm. JAL, OAX, VER

*Cuscuta timida Costea \& Stefanov. HGO, QRO, SLP, VER

Cuscuta tinctoria Mart. ex Engelm. AGS, BCS, CHIS, CHIH, COAH, COL, CDMX, GTO, GRO, HGO, JAL, MEX, MICH, MOR, NAY, NLE, OAX, PUE, QRO, SLP, SIN, SON, TAMS, VER

Cuscuta tolteca Costea \& Stefanov. GTO, HGO, QRO

Cuscuta tuberculata Brandegee BCN, BCS, SON

Cuscuta umbellata Kunth BCS, CHIH, COAH, COL, CDMX, DGO, GTO, GRO, HGO, JAL, MEX, MICH, MOR, NAY, NLE, OAX, PUE, QRO, SLP, SIN, SON, TAMS, VER

*Cuscuta veatchii Brandegee BCN, BCS

*Cuscuta volcanica Costea \& I. García GRO, JAL, MICH

Cuscuta woodsonii Yunck. CHIS

Cuscuta yucatana Yunck. GRO, OAX, PUE, YUC

Dichondra argentea Humb. \& Bonpl. ex Willd. AGS, CHIS, CHIH, COAH, CDMX, DGO, GTO, GRO, HGO, JAL, MEX, MICH, NLE, OAX, PUE, QRO, SLP, TAMS, TLAX, VER,
ZAC

Dichondra brachypoda Wooton \& Standl. AGS, CHIH, COAH, GTO, MEX, MICH, NLE, OAX, SLP, SON, TAMS, ZAC

Dichondra micrantha Urb. COAH, GTO, NLE, OAX, SLP, TAMS

*Dichondra nivea (Brandegee) Tharp \& M.C. Johnst. OAX, PUE, VER

Dichondra occidentalis House BCN

Dichondra sericea Sw. BCS, CHIS, COL, CHIH, COAH, CDMX, DGO, GTO, GRO, HGO, JAL, MEX, MICH, NLE, OAX, PUE, QRO, SLP, SON, TAMS, VER

Evolvulus alsinoides (L.) L. AGS, BCN, BCS, CAM, CHIS, CHIH, COAH, COL, CDMX, DGO, GTO, GRO, HGO, JAL, MEX, MICH, MOR, NAY, NLE, OAX, PUE, QRO, QROO, SLP, SIN, SON, TAB, TAMS, TLAX, VER, YUC, ZAC

Evolvulus arizonicus A. Gray BCS, CHIH, SON

Evolvulus cardiophyllus Schltdl. CHIS, COL, GRO, JAL, OAX, SIN, VER, YUC

Evolvulus convolvuloides (Willd.) Stearn CAM, OAX, QROO, YUC

*Evolvulus choapanus J.A. McDonald VER

Evolvulus filipes Mart. CHIS, YUC

*Evolvulus hallierii Ooststr. TAMS

Evolvulus nummularius (L.) L. CAM, CHIS, COL, GRO, HGO, JAL, NAY, OAX, QRO, QROO, SLP, SIN, TAB, VER, YUC

Evolvulus nuttallianus Roem. \& Schult. CHIH, COAH, DGO, HGO, QRO, SLP, SON

Evolvulus ovatus Fernald CHIS, DGO, GRO, OAX

*Evolvulus palmeri House DGO

Evolvulus pohlii Meisn. CHIS, VER

*Evolvulus prostratus B.L. Rob. AGS, CHIH, CDMX, DGO, GTO, GRO, HGO, JAL, MEX, MICH, MOR, NLE, OAX, PUE, QRO, SIN, SON, TLAX, VER, ZAC

*Evolvulus purpusii Meisn. CHIH, COAH, SLP

*Evolvulus rotundifolius (S. Watson) Hall. f. CHIH, DGO, GTO, JAL, NLE, SLP, SIN, SON

Evolvulus sericeus $\mathrm{Sw}$. AGS, BCN, BCS, CAM, CHIS, CHIH, COAH, DGO, GTO, GRO, HGO, JAL, MEX, MICH, MOR, NAY, NLE, OAX, PUE, QRO, QROO, SLP, SON, TAB, TAMS, VER, YUC, ZAC

Evolvulus tenuis Mart. ex Choisy CHIS, COL, JAL, MEX, YUC Ipomoea alba L. BCS, CAM, CHIS, COL, GTO, GRO, HGO, JAL, MEX, MICH, MOR, NAY, OAX, PUE, QRO, QROO, SLP, SIN, SON, TAB, TAMS, VER, YUC

*Ipomoea ampullacea Fernald CHIH, COL, GRO, JAL, MEX, MICH, OAX, SIN, SON

*Ipomoea angustata Brandegee SIN

Ipomoea anisomeres B.L. Rob. \& Bartlett CAM, CHIS, OAX, PUE, QROO, SLP, SIN, TAB, TAMS, VER, YUC

Ipomoea aquatica Forssk. QROO

Ipomoea arborescens (Humb. \& Bonpl. ex Willd.) G. Don AGS, CHIS, CHIH, COL, DGO, GTO, GRO, HGO, JAL, MEX, MICH, MOR, NAY, OAX, PUE, QRO, SLP, SIN, SON, TAB, VER

Ipomoea argentifolia A. Rich. MOR, OAX, PUE

Ipomoea aristolochiifolia (Kunth) G. Don AGS, BCS, CHIH, COAH, COL, DGO, GTO, GRO, JAL, MEX, MICH, MOR, 
NLE, OAX, QRO, SLP, SIN, SON, VER

Ipomoea asarifolia (Desr.) Roem. \& Schult. CAM, CHIS, QROO, TAB, VER

Ipomoea aurantiaca L.O. Williams CHIS

Ipomoea barbatisepala A. Gray BCS, CHIH, COAH, GRO, MICH, OAX, SLP, SIN, SON, TAMS, VER

Ipomoea batatas (L.) Lam. AGS, CAM, CHIS, CHIH, COL, CDMX, GRO, HGO, JAL, MEX, MICH, MOR, NAY, OAX, PUE, QRO, QROO, SLP, SIN, TAB, TAMS, VER, YUC, ZAC Ipomoea batatoides Choisy CHIS, COL, JAL, MICH, NAY, OAX, PUE, QRO, VER

Ipomoea bernoulliana Peter CHIS

*Ipomoea bombycina (Choisy) Hemsl. COL, GRO, JAL

*Ipomoea bracteata Cav. BCS, CHIH, COL, DGO, GTO, GRO, JAL, MEX, MICH, MOR, NAY, OAX, PUE, SLP, SIN, SON, VER, ZAC

Ipomoea capillacea (Kunth) G. Don AGS, BCS, CHIS, CHIH, COAH, COL, CDMX, DGO, GTO, GRO, HGO, JAL, MEX, MICH, MOR, NAY, NLE, OAX, PUE, QRO, QROO, SLP, SIN, SON, TLAX, VER, ZAC

Ipomoea carnea Jacq. CAM, CHIS, CHIH, COAH, COL, GTO, GRO, HGO, JAL, MEX, MOR, NLE, OAX, PUE, QRO, QROO, SLP, SIN, SON, TAB, TAMS, VER, YUC

*Ipomoea ciervensis Painter GTO, HGO, QRO, SLP

Ipomoea clavata (G. Don) J.F. Macbr. CAM, CHIS, CHIH, COL, GRO, JAL, MICH, NAY, OAX, QROO, SON, VER, YUC

*Ipomoea concolora (Matuda) D.F. Austin GRO, OAX

Ipomoea contrerasii L.O. Williams CHIS

*Ipomoea conzattii Greenm. GRO, MEX, MOR, OAX, PUE, VER

Ipomoea cordatotriloba Dennst. MOR, NLE, SLP, TAMS

Ipomoea costellata Torr. AGS, BCN, BCS, CHIS, CHIH, COAH, CDMX, DGO, GTO, GRO, HGO, JAL, MEX, MICH, MOR, NAY, NLE, OAX, PUE, QRO, SLP, SIN, SON, TAMS, VER, ZAC

Ipomoea crinicalyx S. Moore CAM, CHIS, COL, JAL, MICH, OAX, QROO, YUC

Ipomoea cristulata Hallier f. BCS, CHIH, COAH, CDMX, DGO, GTO, HGO, JAL, MEX, MICH, NLE, QRO, SLP, SIN, SON, TAMS

*Ipomoea cuernavacensis House MOR

*Ipomoea cuprinacoma E. Carranza \& J.A. McDonald GRO, JAL, MICH

*Ipomoea chamelana J.A. McDonald COL, GRO, JAL

Ipomoea chenopodifolia (M. Martens \& Galeotti) Hemsl. CHIS, GRO, JAL, OAX

*Ipomoea chilopsidis Standl. CHIH, DGO, SON

Ipomoea cholulensis Kunth AGS, BCS, CHIS, COL, CDMX, GTO, GRO, HGO, JAL, MEX, MICH, MOR, NAY, OAX, PUE, QRO, QROO, SLP, SIN, SON, TAMS, VER, YUC

*Ipomoea decemcornuta O'Donell COL, JAL, OAX

*Ipomoea diegoae M.C. Lara GRO

*Ipomoea dimorphophylla Greenm. CHIS, COL, DGO, GRO, HGO, JAL, MEX, MICH, MOR, NAY, OAX, QRO, SIN, ZAC *Ipomoea discoidea Gozález-Martínez \& J. Jiménez Ram. GRO, JAL
Ipomoea dumetorum Willd. ex Roem. \& Schult. CAM, CHIS, CDMX, GTO, GRO, HGO, MEX, MICH, OAX, PUE, QRO, SLP, SON, VER

Ipomoea dumosa (Benth.) L.O. Williams CHIS, COL, DGO, GRO, HGO, JAL, MEX, MICH, MOR, OAX, PUE, QRO, SLP, SIN, VER

*Ipomoea durangensis House DGO, ZAC

*Ipomoea electrina D.F. Austin \& J.A. McDonald CHIS, OAX Ipomoea elongata Choisy GRO, HGO, JAL, MEX, MICH, MOR, OAX, PUE, QRO, ZAC

*Ipomoea emetica Choisy AGS, CHIS, COAH, CDMX, DGO, GTO, GRO, HGO, JAL, MEX, MICH, MOR, OAX, QRO, SIN, VER, ZAC

*Ipomoea eximia House CHIH, JAL, VER

*Ipomoea expansa J.A. McDonald GRO, OAX

*Ipomoea fissifolia (McPherson) Eckenw. MICH

*Ipomoea funis Schltdl. \& Cham. BCS, COL, GTO, GRO, HGO, JAL, MEX, MICH, OAX, PUE, SIN, VER

*Ipomoea gesnerioides J.A. McDonald OAX

*Ipomoea gloverae J.A. McDonald MICH

*Ipomoea hartwegii Benth. AGS, CHIH, COL, GTO, JAL, MEX, MICH, OAX, QRO, SLP, ZAC

*Ipomoea hastigera Kunth CHIS, GRO, JAL, MICH, NAY, OAX, PUE, VER

Ipomoea hederacea (L.) Jacq. BCS, CHIS, CHIH, DGO, GTO, HGO, JAL, MEX, MICH, NAY, OAX, QRO, SLP, SIN, SON, TAMS, VER

Ipomoea hederifolia L. AGS, BCS, CAM, CHIS, CHIH, COAH, COL, CDMX, DGO, GTO, GRO, HGO, JAL, MEX, MICH, MOR, NAY, NLE, OAX, PUE, QRO, QROO, SLP, SIN, SON, TAB, TAMS, VER, YUC, ZAC

Ipomoea heterodoxa Standl. \& Steyerm. CAM, CHIS, OAX, QROO, TAB, YUC

*Ipomoea hirta M. Martens \& Galeotti MOR, SIN, TAMS

*Ipomoea ignava House GRO, OAX, VER

*Ipomoea igualensis Weath. COL, GRO, JAL, MEX, MICH, NAY, VER

Ipomoea imperati (Vahl) Griseb. BCS, CAM, COL, GRO, JAL, OAX, QROO, SLP, SIN, SON, TAB, TAMS, VER, YUC

Ipomoea indica (Burm.) Merr. BCN, CAM, CHIS, COAH, COL, GTO, GRO, HGO, JAL, MEX, MICH, OAX, PUE, QRO, QROO, SLP, TAB, TAMS, VER, YUC, ZAC

*Ipomoea intrapilosa Rose AGS, CHIH, COL, DGO, GTO, JAL, MEX, MICH, MOR, NAY, OAX, PUE, SIN, SON, ZAC

*Ipomoea invicta House COL, JAL

*Ipomoea jacalana Matuda AGS, HGO, QRO, SLP

*Ipomoea jalapa (L.) Pursh CAM, GTO, GRO, HGO, JAL, MEX, NLE, OAX, QRO, QROO, SLP, SON, TAMS, VER, YUC

*Ipomoea jicama Brandegee BCS

*Ipomoea kruseana Matuda GRO

*Ipomoea laeta A. Gray CHIS, CHIH, COL, GRO, HGO, JAL, NAY, QRO, SLP, SIN, SON, VER, ZAC

*Ipomoea lambii Fernald DGO, NAY

*Ipomoea lenis House AGS, DGO, GTO, JAL, ZAC

*Ipomoea leonensis B.L. Rob. NLE

Ipomoea lindenii M. Martens \& Galeotti CAM, CHIS, COL, 
GRO, JAL, MEX, MICH, OAX, PUE, QRO, SIN, VER

Ipomoea lindheimeri A. Gray CHIH, COAH, NLE, SLP, SON, TAMS

Ipomoea lobata (Cerv.) Thell. GRO, JAL, MEX, MICH, OAX, SLP, VER

Ipomoea longifolia Benth. AGS, CHIH, DGO, GTO, JAL, QRO, SLP, SON, ZAC

*Ipomoea lottiae J.A. McDonald COL, GRO, JAL, MICH

*Ipomoea lozanii Painter GTO, HGO, QRO, SLP

Ipomoea lutea Hemsl. CHIS, OAX

*Ipomoea macdonaldii E. Carranza OAX

*Ipomoea madrensis S. Watson AGS, CHIH, DGO, GTO, GRO, HGO, JAL, MEX, MICH, QRO, SON, ZAC

Ipomoea mairetii Choisy CHIS, COL, CDMX, DGO, GRO, JAL, MEX, MICH, NAY, OAX, SIN, TAB, VER

*Ipomoea mcphersonii D.F. Austin OAX

*Ipomoea mcvaughii McPherson OAX

Ipomoea meyeri (Spreng.) G. Don BCS, CHIS, COL, GRO, HGO, JAL, MEX, MICH, NAY, OAX, QRO, SLP, SIN, SON, TAB, VER

Ipomoea microsepala Benth. CHIS, COL, DGO, GRO, JAL, MEX, MICH, NAY, OAX, SLP, SIN, VER

Ipomoea minutiflora (M. Martens \& Galeotti) House BCS, CHIS, CHIH, COL, DGO, GRO, JAL, MICH, NAY, OAX, PUE, SLP, SIN, SON, TAMS, VER

*Ipomoea miquihuanensis J.A. McDonald NLE, TAMS

Ipomoea muricata (L.) Jacq. BCS, CHIH, COAH, COL, CDMX, GTO, JAL, MEX, SLP, SIN, SON, YUC

Ipomoea murucoides Roem. \& Schult. AGS, CHIS, COL, CDMX, DGO, GTO, GRO, HGO, JAL, MEX, MICH, MOR, NAY, OAX, PUE, QRO, SLP, SIN, ZAC

Ipomoea neei (Spreng.) O'Donell CHIS, CHIH, COL, GRO, JAL, MEX, MICH, MOR, NAY, OAX, SIN, TAB, VER, ZAC

Ipomoea nil (L.) Roth BCS, CAM, CHIS, CHIH, COAH, COL, CDMX, DGO, GTO, GRO, JAL, MEX, MICH, MOR, NAY, NLE, OAX, PUE, QRO, QROO, SLP, SIN, SON, TAMS, VER, YUC, ZAC

*Ipomoea noctulifolia McPherson COL, JAL, MICH

Ipomoea oocarpa Benth. BCS, GRO, JAL, MEX, MICH, MOR, OAX, SIN, SON, TAB, VER, ZAC

Ipomoea orizabensis (Pelletan) Ledeb. ex Steud. AGS, CHIS, CHIH, COAH, COL, CDMX, DGO, GTO, GRO, HGO, JAL, MEX, MICH, MOR, NAY, NLE, OAX, PUE, QRO, SLP, SIN, SON, TAB, TAMS, TLAX, VER, ZAC

Ipomoea parasitica (Kunth) G. Don BCS, CHIS, CHIH, COL, GTO, GRO, JAL, MICH, MOR, OAX, SIN, SON, VER

Ipomoea pauciflora $\mathrm{M}$. Martens \& Galeotti CHIS, COL, CDMX, GRO, HGO, JAL, MEX, MICH, MOR, OAX, PUE, QRO, ZAC Ipomoea pedicellaris Benth. AGS, CHIS, CHIH, COL, DGO, GRO, HGO, JAL, MEX, MICH, MOR, NAY, OAX, PUE, QRO, SLP, SIN, SON, TAB, TAMS, VER

*Ipomoea perpartita McPherson JAL

Ipomoea pes-caprae (L.) R. Br. BCN, BCS, CAM, CHIS, COL, GRO, JAL, MICH, NAY, OAX, QROO, SLP, SIN, SON, TAB, TAMS, VER, YUC

*Ipomoea petrophila House CHIH

Ipomoea philomega (Vell.) House CHIS, HGO, OAX, TAB,
VER

Ipomoea plummerae A. Gray CHIH, COAH, DGO, HGO, JAL, MICH, NAY, QRO, SON, VER, ZAC

Ipomoea populina House CHIS, CHIH, COL, GRO, HGO, JAL, MEX, MICH, OAX, PUE, QRO, SLP, SON

Ipomoea praecaena House CHIS, COL, GRO, JAL, MEX, MICH, MOR, OAX, PUE, QROO

*Ipomoea proxima M. Martens \& Galeotti OAX, PUE

*Ipomoea pruinosa McPherson GRO, MICH

*Ipomoea pseudoracemosa McPherson COL, JAL, MICH, NAY, ZAC

Ipomoea pubescens Lam. AGS, CHIH, COAH, CDMX, DGO, GTO, GRO, HGO, JAL, MEX, MICH, NLE, OAX, PUE, QRO, SLP, SON, TAMS, TLAX, VER, ZAC

*Ipomoea punticulata Benth. COL, GRO, JAL, MICH, NAY, OAX

Ipomoea purga (Wender.) Hayne CHIS, HGO, JAL, MEX, MICH, MOR, OAX, PUE, VER

Ipomoea purpurea (L.) Roth AGS, BCS, CAM, CHIS, CHIH, COAH, COL, CDMX, DGO, GTO, GRO, HGO, JAL, MEX, MICH, MOR, NAY, NLE, OAX, PUE, QRO, QROO, SLP, SIN, SON, TAB, TAMS, TLAX, VER, YUC, ZAC

Ipomoea quamoclit L. BCS, CAM, CHIS, CHIH, COL, DGO, GRO, JAL, MEX, MICH, NAY, OAX, QROO, SLP, SIN, SON, TAB, TAMS, VER, YUC

Ipomoea ramosissima (Poir.) Choisy CHIS, GRO

Ipomoea reticulata O'Donell CHIS, QRO, TAMS, VER

*Ipomoea rhomboidea House SIN

*Ipomoea robinsonii House GRO, JAL, MEX, MICH, MOR, OAX, PUE

*Ipomoea rubens Choisy JAL, TAB, TAMS

Ipomoea rupicola House COAH, NLE, SLP, TAMS

*Ipomoea rzedowskii E. Carranza, Zamudio \& G. Murguía GTO, HGO, QRO

Ipomoea sagittata Poir. CAM, CHIS, COAH, GRO, NLE, OAX, QROO, SLP, TAB, TAMS, VER, YUC

Ipomoea santillanii O'Donell CHIS, COL, GRO, JAL, MEX, MICH, OAX, VER

*Ipomoea scopulorum Brandegee BCS, SIN, SON

*Ipomoea schaffneri S. Watson SLP, ZAC

Ipomoea seducta House CHIS, COL, GRO, JAL, MICH, NAY, OAX, PUE, SIN, TAB, VER

Ipomoea sepacuitensis Donn. Sm. CHIS, QROO

*Ipomoea sescossiana Baill. AGS, CHIH, COAH, DGO, GRO,

NLE, OAX, SLP, SIN, SON, ZAC

Ipomoea setifera Poir. GRO, TAB

Ipomoea setosa Ker Gawl. AGS, CHIS, GRO, JAL, MICH, OAX, SLP, TAMS, VER

Ipomoea silvicola House CHIS, GRO, OAX, TAB, VER

*Ipomoea simulans T. Hanb. COAH, GTO, GRO, JAL, MEX, MICH, MOR, NAY, NLE, OAX, SLP

*Ipomoea sororia D.F. Austin \& Tapia-Muñoz CAM, YUC

*Ipomoea spectata J.A. McDonald COL, JAL, MICH Ipomoea splendor-sylvae House CAM, CHIS, OAX, QROO Ipomoea squamosa Choisy CAM, CHIS, GRO, HGO, MOR, OAX, PUE, QRO, QROO, SLP, TAB, TAMS, VER, YUC

*Ipomoea stans Cav. AGS, COAH, CDMX, DGO, GTO, GRO, 
HGO, JAL, MEX, MICH, MOR, OAX, PUE, QRO, SLP, TLAX, VER, ZAC

Ipomoea steerei (Standl.) L.O. Williams CAM, CHIS, QROO, YUC

*Ipomoea suaveolens (M. Martens \& Galeotti) Hemsl. CHIS, GTO, GRO, HGO, MEX, MICH, MOR, NAY, OAX, PUE, QRO, SIN, VER

Ipomoea suffulta (Kunth) G. Don CHIS, COL, GRO, JAL, MEX, MICH, NAY, OAX

*Ipomoea tabascana J.A. McDonald \& D.F. Austin TAB

*Ipomoea tacambarensis E. Carranza MICH, OAX

*Ipomoea tastensis Brandegee BCS

*Ipomoea tehuantepecensis L. Torres, R. Torres, M.P. Ramírez \& J.A. McDonald OAX

*Ipomoea temascaltepecensis P. Wilkin MEX, MICH

*Ipomoea tentaculifera Greenm. GRO, MICH, OAX

Ipomoea tenuiloba Torr. CHIH, DGO, JAL, SIN, SON, ZAC

*Ipomoea teotitlanica McPherson OAX

Ipomoea ternifolia Cav. BCS, CHIS, CHIH, COL, CDMX, DGO, GTO, GRO, HGO, JAL, MEX, MICH, MOR, NAY, NLE, OAX, PUE, QRO, SIN, SON, TLAX

Ipomoea thurberi $\mathrm{A}$. Gray $\mathrm{CHIH}, \mathrm{DGO}, \mathrm{SON}$

Ipomoea tiliacea (Willd.) Choisy CAM, CHIS, GRO, JAL, MICH, NAY, OAX, QRO, QROO, SLP, TAB, TAMS, VER, YUC

Ipomoea tricolor Cav. AGS, CAM, CHIS, CDMX, GTO, GRO, HGO, JAL, MEX, MICH, MOR, OAX, PUE, QRO, QROO, SLP, SIN, TAB, TAMS, VER, YUC, ZAC

Ipomoea trifida (Kunth) G. Don BCN, CAM, CHIS, COL, CDMX, GTO, GRO, HGO, JAL, MEX, MICH, MOR, NAY, NLE, OAX, PUE, QRO, QROO, SLP, SIN, TAB, TAMS, VER Ipomoea triloba $\mathrm{L}$. BCS, CAM, CHIS, CHIH, COAH, COL, GRO, HGO, JAL, MEX, MICH, MOR, NAY, NLE, OAX, QRO, QROO, SLP, SIN, SON, TAB, TAMS, VER, YUC, ZAC

Ipomoea tuxtlensis House CAM, CHIS, OAX, QROO, TAB, TAMS, VER, YUC

*Ipomoea urbinei House CHIS, COL, GRO, JAL, MICH, PUE, SLP

Ipomoea variabilis (Burm.) Merr. CAM, CHIS, GRO, JAL, OAX, PUE, QROO, SLP, TAB, VER

*Ipomoea venusta (M. Martens \& Galeotti) Hemsl. CHIS, TAB Ipomoea villifera House CHIS

Ipomoea violacea $\mathrm{L}$. CAM, DGO, JAL, MOR, QROO, YUC, VER, ZAC

Ipomoea wolcottiana Rose CHIS, COL, GRO, HGO, JAL, MEX, MICH, MOR, OAX, PUE, QRO, VER

Ipomoea $\times$ leucantha Jacq. SON

*Ipomoea zimmermanii J.A. McDonald COAH

Iseia luxurians (Moric.) O’Donell CAM

Itzaea sericea (Standl.) Standl. \& Steyerm. CAM, CHIS, OAX, PUE, QROO, TAB, VER, YUC

*Jacquemontia abutiloides Benth. BCN, BCS, DGO, NAY, SLP, SIN, SON

Jacquemontia agrestis (Mart. ex Choisy) Meissn. BCS, CAM, CHIH, COL, DGO, GRO, JAL, MEX, MICH, MOR, NAY, OAX, SIN, SON, VER, YUC

*Jacquemontia albida Wiggins \& Rollins CHIH, SON
Jacquemontia confusa Meisn. CAM, CHIS, OAX, QROO, YUC Jacquemontia havanensis (Jacq.) Urb. QROO, YUC

Jacquemontia mexicana (Loes.) Standl. \& Steyerm. CHIS, GRO, OAX

*Jacquemontia nelsonii House COL, JAL, OAX

*Jacquemontia oaxacana (Meisn.) Hallier f. BCS, CAM, CHIH, GRO, HGO, MICH, OAX, QRO, QROO, SLP, SIN, SON, TAMS, VER, YUC

Jacquemontia ovalifolia (Vahl ex West) Hallier f. HGO, SLP, TAMS, VER, YUC

Jacquemontia pentanthos (Jacq.) G. Don BCS, CAM, CHIS, CHIH, COL, DGO, GTO, GRO, HGO, JAL, MEX, MICH, NAY, NLE, OAX, PUE, QRO, QROO, SLP, SIN, SON, TAB, TAMS, VER, YUC

Jacquemontia polyantha (Schltdl. \& Cham.) Hallier f. CAM, CHIS, CHIH, GRO, MICH, OAX, QROO, SIN, SON, TAMS, VER, YUC

Jacquemontia pringlei A. Gray BCS, CHIH, GRO, MICH, OAX, SIN, SON

*Jacquemontia pycnocephala Benth. GRO, OAX

*Jacquemontia smithii B.L. Rob. \& Greenm. OAX, PUE

Jacquemontia sphaerostigma (Cav.) Rusby CAM, CHIS, COL, GRO, JAL, MEX, MICH, MOR, NAY, OAX, QROO, VER

Jacquemontia tamnifolia (L.) Griseb. CAM, CHIS, COL, GRO, JAL, MEX, MICH, MOR, OAX, QROO, SLP, SIN, TAB, TAMS, VER, YUC

Jacquemontia verticillata (L.) Urb. CAM, QROO, SLP, TAMS, VER, YUC

Maripa nicaraguensis Hemsl. CHIS

Merremia aegyptia (L.) Urb. CAM, CHIS, COL, GRO, JAL, MEX, MICH, NAY, OAX, QROO, SIN, TAB, VER, YUC

Merremia aturensis (Kunth) Hallier f. CHIS

*Merremia aurea (Kellogg) O'Donell BCS

*Merremia austinii J.A. McDonald TAMS

*Merremia cieloensis J.A. McDonald TAMS

Merremia cissoides (Lam.) Hallier f. CAM, CHIS, DGO, GRO, MEX, MICH, NAY, OAX, QROO, SLP, SIN, TAB, TAMS, VER, YUC

Merremia discoidesperma (Donn. Sm.) O’Donell CHIS, COAH, GTO, VER

Merremia dissecta (Jacq.) Hallier f. BCN, BCS, CAM, CHIS, COAH, COL, DGO, GTO, GRO, HGO, JAL, MEX, MICH, MOR, NAY, NLE, OAX, PUE, QRO, QROO, SLP, SIN, SON, TAB, TAMS, VER, YUC

*Merremia macdonaldii Valencia-Avalos \& Mart. Gord. GRO, OAX

*Merremia palmeri (S. Watson) Hallier f. CHIH, GRO, SIN, SON

*Merremia platyphylla (Fernald) O'Donell COL, GRO, JAL, $\mathrm{MICH}, \mathrm{MOR}, \mathrm{OAX}$, PUE, ZAC

Merremia quinquefolia (L.) Hallier f. BCS, CAM, CHIS, CHIH, COL, GRO, JAL, MEX, MICH, MOR, NAY, OAX, PUE, QRO, SLP, SIN, SON, TAB, TAMS, VER

Merremia tuberosa (L.) Rendle CAM, CHIS, COL, GRO, HGO, OAX, PUE, QRO, QROO, SLP, TAB, VER, YUC

Merremia umbellata (L.) Hallier f. CAM, CHIS, COL, DGO, GTO, GRO, HGO, JAL, MEX, MICH, MOR, NAY, OAX, PUE, 
QRO, QROO, SLP, SIN, TAB, TAMS, VER, YUC

Odonellia hirtiflora (M. Martens \& Galeotti) K.R. Robertson CHIS, GRO, OAX, TAB, VER

Operculina pinnatifida (Kunth) O'Donell CAM, CHIS, COAH, COL, GRO, HGO, JAL, MEX, MICH, MOR, NAY, NLE, OAX, PUE, QRO, QROO, SLP, SIN, SON, TAB, TAMS, VER, YUC * Operculina platyphylla (Fernald) House GRO, MOR

Operculina pteripes (G. Don) O'Donell CAM, CHIS, CHIH, COL, CDMX, GRO, JAL, MEX, MICH, MOR, NAY, OAX, PUE, SIN, SON, ZAC

Turbina corymbosa (L.) Raf. CAM, CHIS, COL, DGO, GTO, GRO, HGO, JAL, MEX, MICH, MOR, NAY, OAX, PUE, QRO, QROO, SLP, SIN, TAB, TAMS, VER, YUC

\section{Family Cordiaceae}

Cordia alliodora (Ruiz \& Pav.) Oken CAM, CHIS, COAH, COL, DGO, GTO, GRO, HGO, JAL, MEX, MICH, MOR, NAY, OAX, PUE, QRO, QROO, SLP, SIN, SON, TAB, TAMS, VER, YUC

Cordia bicolor A. DC. CHIS, OAX, VER

Cordia boissieri A. DC. AGS, COAH, GTO, GRO, HGO, MOR, NLE, QRO, SLP, SIN, TAMS, VER

*Cordia colimensis I.M. Johnst. COL, JAL

Cordia collococca L. GRO, JAL, OAX, TAB, VER, YUC

Cordia cordiformis I.M. Johnst. CHIS, COL, JAL

Cordia coyucana I.M. Johnst. GRO, NAY, OAX

Cordia dentata Poir. CAM, CHIS, COL, GRO, JAL, MEX, MICH, MOR, NAY, OAX, PUE, QRO, QROO, SLP, SIN, TAB, TAMS, VER, YUC

Cordia diversifolia Pav. ex DC. CAM, CHIS, COL, GRO, JAL, NAY, OAX, QROO, SIN, TAB, VER, YUC

Cordia dodecandra DC. CAM, CHIS, COL, GRO, MICH, MOR, OAX, QROO, TAB, VER, YUC

*Cordia elaeagnoides DC. CHIS, COL, GRO, JAL, MEX, MICH, MOR, OAX, QROO, SIN

Cordia eriostigma Pittier CHIS, COL, NAY, OAX, TAB, VER Cordia gerascanthus L. CAM, CHIS, COL, GRO, JAL, MICH, NAY, OAX, PUE, QROO, SLP, TAB, TAMS, VER, YUC

*Cordia globulifera I.M. Johnst. GRO, JAL, MICH, SIN

*Cordia gracilipes I.M. Johnst. GRO, OAX

*Cordia guerckeana Loes. CAM, OAX, QROO

*Cordia igualensis Bartlett GRO, MOR

*Cordia macvaughii J.S. Miller GRO, JAL, MICH

Cordia megalantha S.F. Blake CHIS, OAX, TAB, VER

*Cordia morelosana Standl. COL, GRO, JAL, MEX, MICH, MOR, OAX, PUE

Cordia obliqua Willd. CAM, TAB

Cordia panamensis L. Riley CHIS

Cordia parvifolia A. DC. BCN, BCS, CHIH, COAH, DGO, SIN, SON, ZAC

Cordia prunifolia I.M. Johnst. CHIS, COL, GRO, JAL, NAY, OAX, VER

Cordia salvadorensis Standl. CAM, CHIS, COL, GRO, JAL, MEX, MICH, NAY, OAX, SIN, VER

Cordia sebestena L. CAM, CHIS, GRO, QROO, SIN, YUC *Cordia seleriana Fernald CHIS, COL, GRO, JAL, MICH, OAX
*Cordia sonorae Rose CHIH, DGO, GRO, JAL, MICH, NAY, OAX, PUE, SIN, SON

Cordia stellifera I.M. Johnst. CAM, CHIS, OAX, QROO, SLP, TAB, VER, YUC

Cordia stenoclada I.M. Johnst. CAM, CHIS, OAX, TAB, VER *Cordia tinifolia Willd. ex Roem. \& Schult. GRO, MEX, NAY, OAX

Cordia truncatifolia Bartlett CHIS, GRO, JAL, MEX, OAX

Lepidocordia williamsii (I.M. Johnst.) J.S. Mill. CHIS, MICH, QROO

*Varronia ambigua (Schltdl. \& Cham.) Bohr. CAM, CHIS, OAX, VER, YUC

Varronia bullata L. CAM, CHIS, JAL, MICH, MOR, QROO, YUC

Varronia curassavica Jacq. BCN, BCS, CAM, CHIS, COL, DGO, GRO, HGO, JAL, MEX, MICH, MOR, NAY, OAX, PUE, QRO, QROO, SLP, SIN, SON, TAB, VER, YUC

Varronia foliosa (M. Martens \& Galeotti) Bohr. CHIS, GRO, HGO, MOR, NAY, OAX, VER

Varronia globosa Jacq. AGS, CAM, CHIS, COL, DGO, GTO, GRO, HGO, JAL, MEX, MICH, MOR, NAY, OAX, PUE, QRO, QROO, SLP, SIN, SON, VER, YUC, ZAC

Varronia guanacastensis (Standl.) J.S. Mill. CHIS, DGO, GRO, NAY, OAX

Varronia inermis (Mill.) Bohr. CAM, CHIS, COL, GRO, HGO, JAL, MEX, MICH, NAY, OAX, QROO, SIN, TAB, VER, YUC *Varronia lauta (I.M. Johnst.) J.S. Mill. JAL, NAY

*Varronia limicola (Brandegee) Friesen HGO, VER

Varronia linnaei (Stearn) J.S. Mill.

HGO, NAY, OAX, PUE, SIN, VER

Varronia macrocephala Desv. CHIS, COAH, COL, DGO, GTO, GRO, MICH, NAY, OAX, QRO, SLP, SIN, TAMS, VER

Varronia oaxacana (DC.) Friesen AGS, CAM, CHIS, COL, DGO, GTO, GRO, JAL, MICH, NAY, OAX, PUE, QRO, QROO, SLP, SIN, TAMS, VER, YUC, ZAC

Varronia podocephala (Torr.) Bohr. COAH, GRO, HGO, JAL, NAY, NLE, QRO, SLP, TAMS, VER

Varronia spinescens $(\mathrm{L}$.$) Bohr. BCN, BCS, CAM, CHIS, COL,$ DGO, GRO, HGO, JAL, MICH, NAY, OAX, PUE, QRO, QROO, SLP, SIN, TAB, VER

*Varronia stellata (Greenm.) Bohr. OAX, PUE, SIN, VER

*Varronia urticacea Standl. GRO, MICH

\section{Family Coriariaceae}

Coriaria ruscifolia L. CHIS, COL, DGO, GRO, JAL, MEX, MICH, MOR, NAY, OAX, PUE, SLP, SIN

\section{Family Cornaceae}

Cornus disciflora Moc. \& Sessé ex DC. CHIS, CHIH, COL, CDMX, DGO, GTO, GRO, HGO, JAL, MEX, MICH, MOR, NAY, NLE, OAX, PUE, QRO, SLP, SIN, SON, TAMS, VER, ZAC

Cornus excelsa Kunth CHIS, CHIH, COL, CDMX, DGO, GTO, GRO, HGO, JAL, MEX, MICH, MOR, NAY, NLE, OAX, PUE, QRO, SLP, SIN, TAMS, TLAX, VER

Cornus florida L. COAH, NLE, SLP, TAMS, VER

Cornus stolonifera Michx. CHIH, COAH, DGO, NLE, OAX, SON, TAMS 
Nyssa sylvatica Marshall CHIS, HGO, NLE, PUE, TAMS, VER

\section{Family Costaceae}

Costus comosus (Jacq.) Roscoe CHIS, OAX, VER

Costus congestus Rowlee GRO, OAX

* Costus dirzoi García-Mend. \& Ibarra-Manríquez OAX, VER

Costus guanaiensis Rusby CHIS, OAX

Costus laevis Ruiz \& Pav. CHIS, OAX

Costus pictus D. Don CHIS, COL, GRO, HGO, JAL, MICH,

NAY, OAX, PUE, SLP, SIN, TAB, TAMS, VER

Costus pulverulentus C. Presl CHIS, HGO, OAX, PUE, QRO,

QROO, SLP, TAB, TAMS, VER, YUC

Costus scaber Ruiz \& Pav. CHIS, HGO, NAY, OAX, PUE, QRO, TAMS, VER

Costus spicatus (Jacq.) Sw. CHIS, SLP, SIN, TAB, VER

Costus villosissimus Jacq. CHIS, SLP, TAB, VER

\section{Family Crassulaceae}

* Byrnesia bernalensis (Kimnach \& Moran) P.V. Heath TAMS

Crassula aquatica (L.) Schönland AGS, BCN, BCS, CDMX, GTO, GRO, HGO, JAL, MEX, MICH, MOR, OAX, QRO, SLP, VER, ZAC

Crassula longipes (Rose) M. Bywater \& Wickens GTO, HGO, $\mathrm{MICH}$

Crassula viridis (S. Watson) Fedde CHIH, GTO, HGO, MEX, QRO, SLP, VER

*Cremnophila linguifolia (Lem.) Moran MEX

* Cremnophila nutans (Rose) Rose

Dudleya abramsii Rose BCN

*Dudleya acuminata Rose BCN, BCS

* Dudleya albiflora Rose BCS

*Dudleya anomala (Davidson) Moran BCN

* Dudleya anthonyi Rose BCN

Dudleya attenuata (S. Watson) Moran BCN

Dudleya blochmanae (Eastw.) Moran BCN

*Dudleya brittonii D.A. Johansen BCN

*Dudleya campanulata Moran BCN

Dudleya candida Britton BCN

Dudleya edulis (Nutt.) Moran BCN

Dudleya formosa Moran BCN

* Dudleya gatesii Johansen BCN, BCS

*Dudleya guadalupensis Moran BCN

*Dudleya ingens Rose BCN

Dudleya lanceolata (Nutt.) Britton \& Rose BCN

Dudleya linearis (Greene) Britton \& Rose BCN

*Dudleya nubigena (Brandegee) Britton \& Rose BCS

*Dudleya pachyphytum Moran \& M. Benedict BCN

*Dudleya pauciflora Rose BCN, BCS

Dudleya pulverulenta (Nutt.) Britton \& Rose BCN, BCS, SON

* Dudleya rigidiflora Rose BCS

* Dudleya rubens (Brandegee) Britton \& Rose BCS

Dudleya saxosa Britton \& Rose BCN

Dudleya variegata (S. Watson) Moran BCN

Dudleya virens (Rose) Moran $\mathrm{BCN}$

Dudleya $\times$ semiteres (Rose) Moran BCN

*Echeveria acutifolia Lindl. CHIS, MICH, OAX

*Echeveria affinis $\mathrm{E}$. Walther CHIH, DGO, SIN

*Echeveria agavoides Lem. AGS, COAH, GTO, HGO, JAL,
MICH, SLP, ZAC

* Echeveria alata Alexander OAX

*Echeveria amoena L. de Smet. PUE, VER

*Echeveria amphoralis $\mathrm{E}$. Walther OAX

* Echeveria angustifolia E. Walther SLP

* Echeveria bella Alexander CHIS

*Echeveria bifida (Hemsl.) Schltdl. COAH, GTO, HGO, NLE, QRO, SLP, TAMS, VER

*Echeveria brachetii J. Reyes \& González-Zorzano OAX

*Echeveria calderoniae Pérez-Calix GTO, JAL

* Echeveria calycosa Moran GRO, MICH

*Echeveria canaliculata Hook. f. CHIS, HGO, OAX

*Echeveria cante Glass \& Mend.-Garc. ZAC

* Echeveria carminea Alexander OAX

* Echeveria carnicolor (Baker) E. Morren PUE, VER

*Echeveria cerrograndensis A. Vázquez \& G. Nieves COL, JAL

*Echeveria coccinea (Cav.) DC. CHIS, CDMX, GTO, GRO, HGO, JAL, MEX, NLE, OAX, PUE, QRO, SLP, TAMS, TLAX, VER, ZAC

*Echeveria colorata $\mathrm{E}$. Walther JAL

*Echeveria craigiana E. Walther CHIH, SIN

* Echeveria crenulata Rose MOR

*Echeveria cuicatecana J. Reyes, Pérez-Crisanto \& Brachet $\mathrm{OAX}$

* Echeveria cuspidata Rose COAH

* Echeveria chazaroi Kimnach OAX

Echeveria chiapensis Rose ex Poelln. CHIS, OAX, PUE, VER

* Echeveria chihuahuansis Poelln. CHIH

* Echeveria dactylifera E. Walther DGO, JAL, SLP, ZAC

* Echeveria derenbergii J.A. Purpus OAX

*Echeveria diffractens Kimnach \& A.B. Lau VER

*Echeveria elegans Rose CHIH, COAH, HGO, JAL, NLE, QRO, VER, ZAC

* Echeveria fimbriata C.H. Thomps. MOR

* Echeveria fulgens Lem. DGO, GRO, JAL, MEX, MICH, OAX Echeveria gibbiflora DC. CDMX, GRO, HGO, MEX, MICH, MOR, OAX, PUE, QRO, TLAX

*Echeveria gigantea Rose \& Purpus OAX, PUE

* Echeveria globuliflora E. Walther OAX

* Echeveria globulosa Moran OAX

* Echeveria goldmani Rose CHIS, GRO, MEX, MICH, OAX

* Echeveria gracilis Rose ex E. Walther OAX, PUE

* Echeveria grandiflora Haw. CDMX, MEX, MICH, MOR

*Echeveria grisea E. Walther GRO, MEX, MICH, MOR

* Echeveria halbingeri E. Walther HGO, QRO

*Echeveria harmsii J.F. Macbr. OAX

* Echeveria helmutiana Kimnach OAX

*Echeveria heterosepala Rose OAX, PUE, VER

*Echeveria humilis Rose HGO, SLP

*Echeveria hyalina E. Walther GTO, JAL, QRO, SLP, VER

* Echeveria juarezensis E. Walther OAX

*Echeveria kimnachii J. Meyrán \& R. Vega SIN

*Echeveria laui Moran \& J. Meyrán OAX

*Echeveria leucotricha J.A. Purpus OAX, PUE

*Echeveria lilacina Kimnach \& Moran NLE

* Echeveria longiflora E. Walther GRO, OAX 
*Echeveria longissima E. Walther OAX, PUE

* Echeveria lozanii Rose JAL

*Echeveria lurida Haw. VER

Echeveria lutea Rose HGO, QRO, SLP

* Echeveria macdougallii $\mathrm{E}$. Walther OAX

*Echeveria marianae I. García \& Costea JAL

*Echeveria maxoni Rose HGO, OAX, VER

*Echeveria megacalyx $\mathrm{E}$. Walther OAX, PUE

*Echeveria meyraniana E. Walther PUE

*Echeveria microcalyx Britton \& Rose PUE, VER

*Echeveria minima J. Meyrán HGO

*Echeveria mondragoniana J. Reyes \& Brachet OAX

*Echeveria moranii E. Walther OAX

*Echeveria mucronata Schltdl. AGS, CHIS, CHIH, COAH, CDMX, DGO, GTO, GRO, HGO, JAL, MEX, MICH, NLE, OAX, PUE, QRO, SLP, TAMS, TLAX, VER, ZAC

*Echeveria multicaulis Rose GRO

*Echeveria munizii Padilla Lepe \& A. Vázquez COL

*Echeveria nayaritensis Kimnach JAL, NAY

* Echeveria nebularum Moran \& Kimnach OAX

*Echeveria nodulosa (Baker) Otto OAX, PUE

*Echeveria novogaliciana J. Reyes, Brachet \& GonzálezZorzano AGS, DGO, JAL

Echeveria nuda Lindl. CHIS, OAX, PUE, QRO, VER

*Echeveria nuyooensis J. Reyes \& Islas OAX

*Echeveria obtusifolia Rose MEX, MOR

* Echeveria olivacea Moran OAX

*Echeveria palmeri Rose HGO, SLP

* Echeveria paniculata A. Gray CHIH, COAH, DGO, SLP, ZAC

*Echeveria parrasana $\mathrm{E}$. Walther $\mathrm{COAH}$

*Echeveria patriotica I. García \& Pérez-Calix JAL, MICH

*Echeveria peacockii Croucher COAH, PUE

*Echeveria penduliflora $\mathrm{E}$. Walther OAX

* Echeveria perezcalixii Jimeno-Sevilla \& P. Carrillo JAL, NAY, ZAC

*Echeveria pilosa J.A. Purpus OAX, PUE

*Echeveria potosina $\mathrm{E}$. Walther SLP

*Echeveria pringlei (S. Watson) Rose DGO, JAL, ZAC

*Echeveria procera Moran OAX

* Echeveria prunina Kimnach \& Moran CHIS

*Echeveria pulidonis $\mathrm{E}$. Walther HGO, PUE, VER

* Echeveria pulvinata Rose OAX, PUE

*Echeveria purpusorum Berger OAX, PUE

*Echeveria racemosa Schltdl. OAX, VER

*Echeveria reglensis E. Walther HGO, VER

*Echeveria rodolfoi Martínez-Avalos \& Mora-Olivo NLE, TAMS

*Echeveria rosea Lindl. CHIS, GRO, HGO, OAX, PUE, QRO, SLP, TAMS, TLAX, VER

*Echeveria roseiflora J. Reyes \& González-Zorzano JAL

*Echeveria rubromarginata Rose PUE, VER

*Echeveria rulfiana Jimeno-Sevilla, Santana Mich. \& P. Carrillo

JAL

*Echeveria sanchez-mejoradae E. Walther HGO, QRO, VER

*Echeveria schaffneri (S. Watson) Rose HGO, NLE, QRO, SLP

*Echeveria scheerii Lindl. OAX

* Echeveria secunda Booth ex Lindl. CDMX, GTO, GRO, HGO,
JAL, MEX, MICH, MOR, NAY, NLE, PUE, QRO, SLP, TLAX, VER, ZAC

*Echeveria semivestita Moran HGO, NLE, QRO, SLP

*Echeveria sessiliflora Rose CHIS, OAX

* Echeveria setosa Rose \& J.A. Purpus OAX, PUE

* Echeveria shaviana $\mathrm{E}$. Walther COAH, NLE, TAMS

* Echeveria skinneri $\mathrm{E}$. Walter OAX

*Echeveria spectabilis Alexander OAX, PUE

Echeveria steyermarkii Standl. CHIS

Echeveria strictiflora A. Gray CHIH, COAH, NLE

*Echeveria subalpina Rose \& Purpus PUE, VER

* Echeveria subcorymbosa Kimnach \& Moran OAX

* Echeveria subrigida (B.L. Rob. \& Seaton) Rose GTO, HGO,

MEX, MICH, PUE, QRO, SLP, SIN, TLAX, VER

*Echeveria subsessilis Rose OAX, PUE

* Echeveria tamaulipana Martínez-Avalos, Mora-Olivo \& M.

Terry SLP, TAMS

*Echeveria tencho Moran \& C.H. Uhl OAX, VER

* Echeveria tenuifolia $\mathrm{E}$. Walther HGO, QRO

*Echeveria tenuis Rose ZAC

*Echeveria tobarensis Berger DGO

* Echeveria tolimanensis Matuda HGO, QRO

*Echeveria trianthina Rose HGO, QRO

* Echeveria triquiana J. Reyes \& Brachet OAX

*Echeveria turgida Rose COAH, DGO, NLE

* Echeveria uhlii J. Meyrán OAX

* Echeveria valvata Moran MEX

* Echeveria violescens Walt. COAH

* Echeveria viridissima $\mathrm{E}$. Walther OAX

*Echeveria walpoleana Rose COAH, NLE, QRO, SLP

* Echeveria waltheri Moran \& Meyrán GTO, JAL, MEX, MICH, MOR

*Echeveria xichuensis López-Chávez \& J. Reyes GTO, SLP

*Echeveria yalmanantlanensis A. Vázquez \& Cházaro COL, JAL

*Echeveria zorzaniana J. Reyes \& Brachet OAX

* Graptopetalum amethystinum (Rose) E. Walther DGO, JAL, SIN, ZAC

*Graptopetalum bellum (Moran \& J. Meyrán) D.R. Hunt CHIH, SON

* Graptopetalum craigii (R.T. Clausen) R.T. Clausen CHIH

* Graptopetalum filiferum (S. Watson) J. Whitehead CHIH, SIN, SON

* Graptopetalum fruticosum R. Moran COL, JAL

* Graptopetalum grande Alexander CHIS, OAX

*Graptopetalum macdougallii Alexander OAX

*Graptopetalum mendozae Glass \& Cházaro GTO

* Graptopetalum mexicanum Matuda MEX, OAX, PUE, QRO

*Graptopetalum occidentale Rose CHIH, SIN, SON

*Graptopetalum pachyphyllum Rose GTO, HGO, JAL, QRO, SLP, ZAC

Graptopetalum pentandrum Moran JAL, MICH

*Graptopetalum pusillum Rose CHIH, DGO, SON

Graptopetalum rusbyi (Greene) Rose CHIH, SIN, SON

*Graptopetalum saxifragoides Kimnach DGO

* Lenophyllum acutifolium Rose COAH, NLE

* Lenophyllum guttatum (Rose) Rose COAH, NLE 
*Lenophyllum latum Moran NLE, TAMS

*Lenophyllum obtusum Moran COAH, NLE

*Lenophyllum reflexum S.S. White TAMS

Lenophyllum texanum (J.G. Sm.) Rose NLE, TAMS

*Pachyphytum bracteosum Link, Klotzsch \& Otto HGO, QRO, VER

*Pachyphytum brachetii J. Reyes, González-Zorzano \& A. Gutiérrez HGO, VER

*Pachyphytum brevifolium Rose GTO, JAL

* Pachyphytum caesium Kimnach \& Moran AGS

*Pachyphytum compactum Rose GTO, HGO, QRO

*Pachyphytum contrerasii Pérez-Calix, I. García \& Cházaro JAL

*Pachyphytum fittkaui Moran AGS, GTO, JAL, SLP

*Pachyphytum garciae Pérez-Calix \& Glass QRO

*Pachyphytum glutinicaule Moran HGO, QRO

*Pachyphytum hookeri (Salm-Dyck) Berger AGS, GTO, HGO, QRO, SLP, ZAC

*Pachyphytum kimnachii Moran GTO, SLP

*Pachyphytum longifolium Rose HGO, QRO, VER

*Pachyphytum machucae I. García, Glass \& Cházaro MICH

*Pachyphytum oviferum Purpus SLP

*Pachyphytum rzedowskii I. García, Pérez-Calix \& J.Meyrán $\mathrm{MICH}$

Pachyphytum saltensis Brachet, J. Reyes \& Mondragón JAL, ZAC

*Pachyphytum viride E. Walther GTO, HGO, QRO

* Pachyphytum werdermannii Poelln. TAMS

* Sedum alamosanum S. Watson BCS, CHIH, SIN, SON

* Sedum alexanderi Eggli OAX

*Sedum allantoides Rose OAX, PUE

Sedum australe Rose CHIS

* Sedum batallae Barocio CDMX, HGO, MEX

Sedum batesii Hemsl. CHIS, MEX, OAX

Sedum bellum Rose ND

Sedum booleanum B.L. Turner COAH, NLE

* Sedum botterii Hemsl. CHIS, QRO, SLP, VER

*Sedum bourgaei Hemsl. AGS, CDMX, DGO, GRO, HGO, JAL, MEX, MICH, MOR, NAY, OAX, QRO, SIN, TLAX, ZAC *Sedum burrito Moran VER

*Sedum caducum R.T. Clausen TAMS

*Sedum calcaratum Rose AGS, CDMX, HGO, JAL, MEX, QRO, ZAC

*Sedum calcicola B.L. Rob. \& Greenm. COAH, GTO, HGO, NLE, QRO, SLP, TAMS

*Sedum carinatifolium (R.T. Clausen) E. Pérez-Calix HGO, QRO

*Sedum catorce G.L. Nesom NLE, SLP

*Sedum clausenii Pérez-Calix GTO, QRO, SLP, TAMS

*Sedum clavatum R.T. Clausen MEX

*Sedum clavifolium Rose MEX, PUE

Sedum cockerellii Britton CHIH

*Sedum commixtum Moran \& Hutchison OAX

*Sedum compactum Rose OAX, PUE, VER

*Sedum confusum Hemsl. HGO, PUE, VER

* Sedum conzattii Rose MOR, OAX

* Sedum copalense Kimnach SIN
*Sedum cormiferum R.T. Clausen MEX

*Sedum corynephyllum (Rose) Frod. GTO, HGO, QRO, SLP, VER

*Sedum cremnophyla R.T. Clausen MOR

*Sedum cupressoides Hemsl. OAX, PUE

*Sedum cuspidatum E.J. Alexander CHIS, OAX

*Sedum chihuahuense S. Watson CHIH

*Sedum chloropetalum R.T. Clausen OAX

*Sedum chrysicaulum J.A. McDonald COAH, NLE

*Sedum dendroideum Moc. \& Sessé ex DC. CHIS, CDMX, GTO, HGO, MEX, MOR, OAX, PUE, QRO, TAMS, VER

*Sedum diffusum $\mathrm{S}$. Watson COAH, NLE, SLP

*Sedum diminutum (R.T. Clausen) G.L. Nesom COAH

*Sedum dispermum Fröd. JAL

*Sedum dulcinomen G.L. Nesom NLE, TAMS

Sedum ebracteatum Moc. \& Sessé ex DC. AGS, COAH, CDMX, DGO, GTO, GRO, HGO, JAL, MEX, MICH, MOR, OAX, PUE, QRO, SLP, TAMS, TLAX, VER, ZAC

*Sedum farinosum Rose DGO

*Sedum flaccidum Rose DGO

*Sedum forreri Greene DGO

*Sedum frutescens Rose MEX, MICH, MOR, PUE

* Sedum furfuraceum Moran SLP

*Sedum fuscum Hemsl. AGS, DGO, GTO, JAL, SLP

*Sedum glabrum (Rose) Praeger COAH, NLE, SLP, ZAC

*Sedum glassii E. Pérez-Calix GTO, SLP

*Sedum globuliflorum R.T. Clausen HGO

Sedum goldmanii (Rose) Moran CHIS, COAH, CDMX, JAL, MEX, MICH, MOR, OAX, PUE, SIN, TLAX

* Sedum grandipetalum Fröd. COL, JAL

*Sedum grandisepalum R.T. Clausen OAX

*Sedum greggii Hemsl. CHIH, COAH, COL, CDMX, GTO, HGO, JAL, MEX, MICH, NLE, PUE, QRO, SLP, TLAX, VER, ZAC

*Sedum griseum Praeger GTO, JAL, MICH, QRO

*Sedum guadalajaranum S. Watson JAL, MOR, NAY, ZAC

Sedum guatemalense Hemsl. CHIS, OAX

*Sedum gypsophilum B.L. Turner COAH, NLE, TAMS

Sedum havardii Rose CHIH, COAH

*Sedum hemsleyanum Rose CHIS, GRO, HGO, JAL, MEX, MICH, MOR, OAX, PUE, QRO, SLP, TLAX, VER

*Sedum hernandezii J. Meyrán PUE

*Sedum hintonii R.T. Clausen COL, DGO, JAL, MICH, ZAC

*Sedum hintoniorum B.L. Turner NLE, TAMS

*Sedum hultenii Frod. HGO, PUE, QRO, SLP, VER

*Sedum humifusum Rose GTO, HGO, QRO

*Sedum hypogaeum J. Reyes, Brachet \& González-Zorzano OAX

*Sedum jaliscanum S. Watson COL, CDMX, DGO, GTO, GRO, JAL, MEX, MICH, MOR, NAY, OAX, SIN, ZAC

*Sedum jerzedowskii E. Pérez-Calix QRO, SLP

*Sedum jurgensenii (Hemsl.) Moran CDMX, GTO, HGO, MEX, PUE, QRO, SLP, VER

*Sedum latifilamentum R.T. Clausen HGO, QRO

*Sedum liebmannianum Hemsl. HGO, OAX, PUE, QRO, VER

*Sedum longipes Rose CDMX, GTO, HGO, MEX, MICH, MOR 
*Sedum lucidum R.T. Clausen VER

*Sedum lumholtzii B.L. Rob. \& Fernald SON

*Sedum macdonaldii G.L. Nesom COAH, NLE

*Sedum macdougallii Moran OAX

*Sedum madrense S. Watson CHIH

* Sedum mellitulum Rose CHIH

Sedum mexicanum Britton CHIS, PUE

*Sedum meyranianum J. Metzg. \& Acevedo JAL

*Sedum minimum Rose AGS, CDMX, DGO, GTO, GRO, HGO,

MEX, MICH, NAY, PUE, QRO, VER, ZAC

*Sedum mocinianum Pérez-Calix GTO, QRO

*Sedum moniliforme I. García \& Costea MICH

Sedum moranense Kunth CHIH, COAH, CDMX, GTO, HGO,

JAL, MEX, MICH, MOR, OAX, QRO, SLP, TAMS, TLAX,

VER, ZAC

*Sedum morganianum E. Walther CHIS, PUE, VER

*Sedum multiflorum R.T. Clausen COL, JAL

*Sedum muscoideum Rose OAX, PUE

Sedum nanifolium Frod. CHIH, COAH, NLE

*Sedum napiferum Peyr. AGS, CDMX, MEX

*Sedum neovolcanicum Pérez-Calix \& I. García COL, JAL, $\mathrm{MICH}$

*Sedum niveum Davidson BCN

*Sedum nussbaumerianum Bitter VER

* Sedum oaxacanum Rose OAX, PUE

*Sedum obcordatum R.T. Clausen VER

*Sedum ocuilense J. Meyrán MEX

*Sedum orbatum Moran \& J. Meyrán PUE

* Sedum oteroi Moran OAX, PUE

*Sedum oxycoccoides Rose DGO, JAL, NAY, ZAC

*Sedum oxypetalum Kunth CDMX, GTO, GRO, HGO, MEX,

MICH, MOR, PUE, QRO, TLAX

*Sedum pachucense (C.H. Thomps.) Praeger HGO, QRO

* Sedum pachyphyllum Rose OAX, PUE

*Sedum palmeri S. Watson COAH, GTO, HGO, NLE, SLP,

TAMS, VER

*Sedum papillicaulum G.L. Nesom COAH, NLE, TAMS

Sedum parvum Hemsl. COAH, GTO, JAL, NLE, SLP, ZAC

*Sedum pentastamineum R.T. Clausen MEX

*Sedum perezdelarosae Jimeno-Sevilla PUE

*Sedum piaxtlaense Reyes, Etter \& Kristen DGO, SIN

*Sedum porphyranthes J. Reyes, Brachet \& O. González OAX

* Sedum potosinum Rose SLP, ZAC

Sedum praealtum A. DC. AGS, CHIS, CDMX, GRO, HGO,

JAL, MEX, MICH, OAX, PUE, QRO, SLP, TLAX, VER

*Sedum pringlei $\mathrm{S}$. Watson $\mathrm{CHIH}$

* Sedum pulvinatum R.T. Clausen OAX

*Sedum quadripetalum R.T. Clausen DGO

*Sedum quevae Raym.-Hamet AGS, CDMX, GRO, HGO, JAL, MEX, MICH, MOR, OAX, PUE, QRO, SLP, TLAX

*Sedum raramuri J. Metzg. CHIH

*Sedum reptans R.T. Clausen NLE, QRO, SLP

*Sedum retusum Hemsl. HGO, QRO, SLP, TAMS

*Sedum rhodocarpum Rose COAH, NLE, TAMS

*Sedum scopulinum (Rose) Moran PUE

* Sedum semiteres Rose DGO

*Sedum spathulisepalum R.T. Clausen DGO
*Sedum stahlii Solms OAX, PUE, VER

Sedum stelliforme S. Watson $\mathrm{CHIH}, \mathrm{SON}$

*Sedum suaveolens Kimnach DGO

*Sedum submontanum Rose JAL, ZAC

*Sedum tamaulipensis G.L. Nesom SLP, TAMS

* Sedum tehuaztlense Moran \& J. Meyrán MEX

*Sedum tortuosum Hemsl. COL, GRO, JAL, MEX, MICH, NAY, OAX, ZAC

*Sedum torulosum R.T. Clausen OAX, PUE

*Sedum treleasei Rose OAX, PUE

*Sedum trichromum R.T. Clausen DGO

*Sedum tuberculatum Rose OAX

*Sedum versadense C.H. Thomps. MEX, MOR, OAX

*Sedum vinicolor $\mathrm{S}$. Watson CHIH, COAH, SON

Sedum wrightii A. Gray CHIH, COAH, NLE

*Tacitus bellus Moran \& J. Meyrán CHIH, SON

*Thompsonella colliculosa Moran MICH

*Thompsonella garcia-mendozae P. Carrillo \& Pérez-Calix $\mathrm{MICH}$

*Thompsonella minutiflora (Rose) Britton \& Rose GRO, MEX, MOR, OAX, PUE

*Thompsonella mixtecana J. Reyes \& López-Chávez OAX

*Thompsonella nellydiegoae P. Carrillo \& Pérez-Calix GRO, OAX, PUE

*Thompsonella platyphylla Rose GRO

*Thompsonella spathulata Kimnach OAX

*Thompsonella xochipalensis Gual, Peralta \& Pérez-Calix GRO

Tillaea erecta Hook. \& Arn. BCN, BCS, SON

*Villadia acuta Moran \& Uhl QRO, SLP

* Villadia albiflora (Hemsl.) Rose CHIS, OAX, PUE

*Villadia aperta Moran \& C.H. Uhl DGO

*Villadia aristata Moran COAH, NLE, SLP, TAMS

*Villadia batesii (Hemsl.) Baehni \& J.F Macbr. CDMX, GTO, GRO, HGO, JAL, MEX, MICH, MOR, OAX, QRO, SLP, TLAX, VER

*Villadia cucullata Rose COAH, NLE, SLP, VER, ZAC

* Villadia diffusa Rose CHIS

*Villadia grandisepala (R.T. Clausen) R.T. Clausen OAX

Villadia guatemalensis Rose OAX

*Villadia imbricata Rose OAX

*Villadia laxa Moran \& C.H. Uhl CHIH, SON

*Villadia levis Rose OAX

*Villadia mexicana (Schltdl.) Jacobs CDMX, GTO, GRO, HGO, JAL, MEX, MICH, OAX, QRO, TLAX

*Villadia minutiflora Rose OAX

*Villadia misera (Lindl.) R.T. Clausen AGS, CHIH, COAH, CDMX, GTO, GRO, HGO, JAL, MEX, NLE, OAX, QRO, SLP, TAMS, TLAX, VER, ZAC

*Villadia nelsonii Rose CHIS, OAX

*Villadia painteri Rose JAL, ZAC

*Villadia patula Moran \& C.H. Uhl GTO, QRO, SLP

*Villadia platystyla (Fröd) R.T. Clausen JAL

*Villadia pringlei Rose CHIH

*Villadia ramirezii $\mathrm{P}$. Carrillo JAL

*Villadia ramosissima Rose MEX, OAX, PUE, TLAX, VER

*Villadia recurva Moran, Kimnach \& C.H. Uhl GRO, OAX

Villadia squamulosa (S. Watson) Rose CHIH, COAH, NLE, 
SON

*Villadia stricta Rose COAH, ZAC

\section{Family Crossosomataceae}

Crossosoma bigelovii S. Watson BCN, CHIH, SON

Crossosoma californicum Nutt. BCN

Crossosoma parviflora B.L. Rob. \& Fernald SON

Forsellesia spinescens (A. Gray) Greene AGS, CHIH, COAH,

NLE, SON, ZAC

*Velascoa recondita Calderón \& Rzed. QRO

\section{Family Cucurbitaceae}

*Apatzingania arachoidea Dieterle JAL, MICH

*Apodanthera aspera Cogn. DGO, OAX, PUE

*Apodanthera crispa Cogn. COAH, DGO, SLP, ZAC

*Apodanthera cucurbitoides Lundell SLP, ZAC

*Apodanthera palmeri A. Gray BCS, SON

*Apodanthera roseana A. Gray SIN

Apodanthera undulata A. Gray AGS, CHIH, COAH, COL, DGO, GTO, HGO, JAL, MEX, MICH, NAY, NLE, OAX, QRO, SLP, SIN, SON, ZAC

Brandegea bigelovii (S. Watson) Cogn. BCN, BCS, JAL, SON, ZAC

Cayaponia attenuata (Hook. \& Arn.) Cogn. CHIS, COL, DGO, GRO, JAL, MEX, MICH, NAY, OAX, SIN, TAB, VER

Cayaponia leucosticta Standl. CHIS

Cayaponia longiloba A.K. Monro CHIS, OAX, TAB, VER

Cayaponia racemosa (Mill.) Cogn. CAM, CHIS, COL, GRO, HGO, JAL, NAY, OAX, QRO, QROO, SLP, TAMS, VER, YUC Cionosicyos excisus (Griseb.) C. Jeffrey CAM, CHIS, QROO, YUC

Cionosicyos macranthus (Pittier) C. Jeffrey CAM, CHIS, GRO, HGO, MEX, MOR, OAX, PUE, SLP, TAB, TAMS, VER

Cucurbita argyrosperma K. Koch CAM, CHIS, COL, GRO, JAL, MEX, MICH, MOR, NAY, OAX, PUE, SLP, SIN, SON, TAMS, VER, YUC, ZAC

* Cucurbita cordata S. Watson BCN, BCS

* Cucurbita cylindrata L.H. Bailey BCS

Cucurbita digitata A. Gray BCN, CHIH, SON

Cucurbita ficifolia Bouché CHIS, COL, CDMX, GRO, HGO, JAL, MEX, MICH, MOR, OAX, PUE, QRO, SLP, VER

Cucurbita foetidissima Kunth AGS, BCN, CHIH, COAH, COL, DGO, GTO, HGO, JAL, MEX, MICH, NLE, QRO, SLP, SIN, SON, TAMS, VER, ZAC

*Cucurbita fraterna L.H. Bailey NLE, TAMS

*Cucurbita galeotii Cogn. CHIS

Cucurbita lundelliana L.H. Bailey CAM, CHIS, QROO, TAB, YUC

Cucurbita mixta Pangalo CAM, CHIS, JAL, QRO, QROO, SLP, VER, YUC

Cucurbita moschata Duchesne CAM, CHIS, GRO, HGO, JAL, NAY, OAX, PUE, QROO, SLP, TAB, TAMS, VER, YUC, ZAC Cucurbita okeechobeensis (Small) L.H. Bailey CHIS, HGO, OAX, PUE, QRO, SLP, TAMS, VER, ZAC

Cucurbita palmata S. Watson BCN, SON

*Cucurbita pedatifolia L.H. Bailey GTO, HGO, MEX, MICH, OAX, PUE, QRO, SLP, VER, ZAC

Cucurbita pepo L. AGS, CAM, CHIS, MICH, COAH, COL,
GRO, HGO, JAL, MEX, MICH, MOR, NAY, NLE, OAX, PUE, QROO, SLP, SIN, TAB, TAMS, TLAX, VER, YUC, ZAC

*Cucurbita radicans Naudin AGS, COL, CDMX, GTO, GRO, HGO, JAL, MEX, MICH, MOR, NAY, QRO, SLP, ZAC

* Cucurbita $\times$ scabridifolia L.H. Bailey COAH, GTO, HGO, NLE, QRO, SLP, TAMS, VER

* Cyclanthera dieterleana C.E. Jones \& Kearns CHIH, DGO, $\mathrm{MICH}, \mathrm{SIN}$

Cyclanthera dioscoreoides C.E. Jones \& Kearns MEX, MICH

* Cyclanthera entata C.E. Jones \& Kearns CHIS

Cyclanthera explodens Naudin CHIS, VER

Cyclanthera gracillima Cogn. AGS, CHIS, CHIH, COAH, COL, CDMX, DGO, GTO, GRO, HGO, JAL, MEX, MICH,

MOR, NAY, NLE, OAX, PUE, QRO, SLP, SIN, SON, TAMS, VER, ZAC

* Cyclanthera heiseri C.E. Jones \& Kearns DGO, JAL, SIN

Cyclanthera integrifolia Cogn. CHIS, GTO, GRO, HGO, JAL,

MEX, MICH, MOR, NLE, OAX, QRO, SLP, TAMS, VER, ZAC

* Cyclanthera jeffreyi Lira \& Rodr.-Arévalo HGO, QRO, SLP

* Cyclanthera jonesii McVaugh COL, JAL, MICH, NAY

Cyclanthera langaei Cogn. CHIS, COL, GRO, HGO, JAL, MEX, MICH, NLE, OAX, PUE, QRO, SLP, TAMS, VER

* Cyclanthera micrantha Cogn. SON

* Cyclanthera minima (S. Watson) C.E. Jones \& Kearns CHIH, DGO, JAL, NAY, SIN, SON

*Cyclanthera monticola Gentry SIN

Cyclanthera multifoliola Cogn. CHIS, COL, DGO, GRO, HGO, JAL, MEX, MICH, NAY, NLE, OAX, QRO, SLP, SIN, TAMS, VER, ZAC

Cyclanthera pedata (L.) Schrad. CHIS, GRO, JAL, MICH

Cyclanthera ribiflora (Schltdl.) Cogn. AGS, CHIS, CHIH, CDMX, DGO, GTO, GRO, HGO, JAL, MEX, MICH, MOR, OAX, PUE, QRO, SLP, SIN, VER

* Cyclanthera rostrata (P.G. Wilson) Kearns \& C.E. Jones COL, GRO, JAL, MEX, MICH

Cyclanthera steyermarkii Standl. CHIS, COL, GRO, JAL

* Cyclanthera tamnoides (Willd.) Cogn. AGS, BCN, BCS, CHIS, COL, CDMX, DGO, GRO, HGO, JAL, MEX, MICH, MOR, NAY, OAX, PUE, SIN, VER

Cyclanthera tenuisepala Cogn. JAL

* Cyclanthera testudinea Brandegee BCS

* Chalema synanthera Dieterle COL, GRO, JAL, MICH, OAX, SIN

*Dieterlea fusiformis E.J. Lott COL, GRO, JAL, MICH, OAX, SIN, SON

*Dieterlea maxima (Lira \& Kearns) McVaugh COL, DGO, GTO, GRO, JAL, MICH, NAY, SIN

Doyerea emetocathartica Grosourdy CAM, CHIS, COL, GRO, JAL, MICH, NAY, OAX, PUE, QRO, QROO, SLP, SIN, SON, TAMS, VER, YUC

*Echinopepon cirrhopedunculatus Rose BCS, CHIH, COL, GRO, JAL, MEX, MICH, MOR, NAY, SIN, SON

Echinopepon coulteri (A. Gray) Rose CHIS, CHIH, COAH, CDMX, DGO, GTO, GRO, HGO, JAL, MEX, MICH, MOR, OAX, PUE, QRO, SLP, TLAX, VER, ZAC

Echinopepon jaliscanus Rose AGS, COL, GTO, GRO, JAL, 
MEX, MICH, NAY, SIN, ZAC

*Echinopepon milleflorus Naudin CHIS, COAH, COL, CDMX, DGO, GTO, GRO, HGO, JAL, MEX, MICH, MOR, NAY, OAX, PUE, QRO, SLP, TLAX, VER

*Echinopepon minimus (Kellogg) S. Watson BCN, BCS, SIN Echinopepon paniculatus (Cogn.) Dieterle CAM, CHIS, COL, GRO, JAL, MEX, OAX, QROO

*Echinopepon peninsularis Gentry BCS

Echinopepon pubescens (Benth.) Cogn. AGS, DGO, GTO, GRO, JAL, MEX, MICH, MOR, OAX, PUE, VER, ZAC

Echinopepon racemosus (Steud.) C. Jeffrey CHIS, CHIH, COL, DGO, GTO, GRO, HGO, JAL, MEX, MICH, MOR, NAY, OAX, PUE, QRO, SIN, SON, VER, ZAC

Echinopepon torquatus (Ser.) Rose BCS, CHIS, CDMX, HGO, JAL, MEX, MICH, NAY, OAX, SLP, VER

*Echinopepon tultitlanapaensis A.K. Monro \& P.J. Stafford PUE

Echinopepon wrightii (A. Gray) S. Watson BCS, CAM, CHIS, CHIH, COL, GRO, JAL, MICH, MOR, OAX, SON

Gurania makoyana (Lem.) Cogn. CHIS, TAB

*Hanburia mexicana Seem. HGO, OAX, PUE, VER

Hanburia parviflora Donn. Sm. CHIS

Ibervillea guatemalensis (Standl. \& Steyerm.) Kearns CHIS

*Ibervillea hypoleuca (Standl.) C. Jeffrey COL, GRO, JAL, $\mathrm{MICH}, \mathrm{OAX}$

Ibervillea lindheimeri (A. Gray) Greene CHIH, GTO, HGO, NLE, OAX, QRO, SLP, TAMS, VER

Ibervillea millspaughii (Cogn.) C. Jeffrey CAM, OAX, QROO, TAMS, VER, YUC

*Ibervillea sonorae (S. Watson) Greene BCN, BCS, SIN, SON Ibervillea tenuisecta (A. Gray) Small CHIH, COAH, DGO, NLE, SLP, SON, ZAC

Ibervillea tripartita (Cogn.) Greene CAM, OAX, QROO, YUC Luffa operculata (L.) Cogn. CHIS, COL, GRO, JAL, MICH, NAY, OAX, SIN, SON, TAB, VER

*Luffa quinquefida (Hook. \& Arn.) Seemann COL, JAL

Marah gilensis (S. Watson) Greene SON

Marah guadalupensis (S. Watson) Greene BCN

Marah macrocarpus (Greene) Greene BCN, BCS, SON

Melothria pendula L. BCS, CAM, CHIS, COAH, COL, GTO, GRO, HGO, JAL, MEX, MICH, MOR, NAY, NLE, OAX, PUE, QRO, QROO, SLP, SIN, SON, TAB, TAMS, VER, YUC, ZAC * Melothria pringlei (S. Watson) Mart. Crov. CHIH, COL, GRO, JAL, MEX, MICH, MOR, PUE, SON

Melothria trilobata Cogn. CHIS, GRO, JAL, MICH, OAX, VER

*Microsechium gonzalo-palomae Lira OAX

Microsechium palmatum (Ser.) Cogn. CHIS, CHIH, COAH, COL, CDMX, DGO, GTO, GRO, HGO, JAL, MEX, MICH, MOR, OAX, PUE, QRO, SLP, SIN, SON, TAMS, TLAX, VER, ZAC

*Parasicyos dieterleae Lira \& R. Torres CHIS, OAX

Parasicyos maculatus Dieterle QRO, SLP, TAB

*Peponopsis adhaerens Naudin HGO, OAX, PUE, QRO, SLP, TAMS, VER, ZAC

Polyclathra albiflora (Cogn.) C. Jeffrey BCS, COL, DGO, GTO, GRO, JAL, MEX, MICH, MOR, NAY, OAX, SIN, VER.ZAC
Polyclathra cucumerina Bertol. CAM, CHIS, COL, JAL, MICH, NAY, OAX, QROO, VER, YUC

Posadaea sphaerocarpa Cogn. CHIS

* Psiguria tabascensis Donn. Sm. CHIS, TAB, VER

Psiguria triphylla (Miq.) C. Jeffrey CAM, CHIS, DGO, HGO, OAX, PUE, QROO, TAB, VER

Psiguria warscewiczii (Hook. f.) Wunderlin CHIS, OAX, TAB, VER

Rytidostylis gracilis Hook. \& Arn. CAM, CHIS, COL, DGO, GRO, JAL, MEX, MICH, MOR, NAY, OAX, QROO, SIN, VER *Rytidostylis longisepala (Cogn.) C. Jeffrey COL, DGO, GRO, JAL, MEX, MICH, MOR, NAY, SIN, ZAC

Rytidostylis macrophylla (Standl. \& Steyerm.) Dieterle CHIS

*Schizocarpum attenuatum Cogn. \& Rose ex Rose CHIS, GRO, MICH, NAY

*Schizocarpum dieterleae Kearns COL, JAL, MICH

Schizocarpum filiforme Schrad. CHIS, GTO, GRO, JAL, MEX, MICH, OAX, PUE

*Schizocarpum liebmannii Cogn. OAX

*Schizocarpum longisepalum C. Jeffrey COL, GRO, JAL, $\mathrm{MICH}$

*Schizocarpum palmeri Cogn. \& Rose CHIS, COL, DGO, GTO, GRO, JAL, MEX, MICH, NAY, OAX, PUE, SIN, SON, VER, ZAC

*Schizocarpum parviflorum B.L. Rob. \& Greenm. AGS, CHIS, GTO, GRO, JAL, MEX, MICH, MOR, NAY, QRO, ZAC

*Schizocarpum reflexum Rose COL, GRO, JAL, MEX, MICH, MOR, NAY, OAX, PUE, QRO, SIN

* Schizocarpum tripodum Kearns COL, JAL

* Sechiopsis diptera Kearns CHIS

* Sechiopsis distincta Kearns CHIS, OAX

Sechiopsis laciniata (Brandegee) Kearns CHIS

*Sechiopsis tetraptera Dieterle COL, GRO, JAL, MICH

* Sechiopsis triquetra (Moc. \& Sessé ex Ser.) Naudin AGS, COL, DGO, GTO, GRO, JAL, MEX, MICH, MOR, NAY, OAX, PUE, QRO, SLP, SIN, SON, ZAC

Sechium compositum (Donn. Sm.) C. Jeffrey CHIS, COL, GRO, JAL, MEX, QRO

* Sechium chinantlense Lira \& F. Chiang OAX

Sechium edule (Jacq.) Sw. AGS, CAM, CHIS, COAH, COL, GRO, HGO, JAL, MEX, MICH, MOR, NAY, NLE, OAX, PUE, QRO, QROO, SLP, SIN, SON, TAB, VER, YUC

*Sechium hintonii (P.G. Wilson) C. Jeffrey COL, GRO, JAL, MEX, MICH

*Sechium mexicanum Lira \& M. Nee HGO, PUE, QRO, SLP, VER

* Sicydium daviliae Lira CHIS

Sicydium glabrum Standl. \& Steyerm. CHIS, OAX, VER Sicydium schiedeanum Schltdl. CHIS, OAX, PUE, SLP, VER Sicydium tamnifolium (Kunth) Cogn. CAM, CHIS, HGO, OAX, PUE, QRO, QROO, SLP, TAB, TAMS, VER, YUC, ZAC

Sicydium tuerckheimii Donn. Sm. CHIS, OAX, PUE, QRO, SLP, TAB, VER

* Sicyos acerifolius Brandegee SIN

Sicyos ampelophyllus Wooton \& Standl. CHIH, SON

Sicyos barbatus (Gentry) C. Jeffrey CHIS, COL, JAL, MICH, NAY, SIN 
* Sicyos bulbosus Rodr.-Arévalo, Lira \& Dávila OAX

* Sicyos collinus B.L. Rob. \& Fernald CHIH

* Sicyos cordifolius Rodr.-Arévalo, Lira \& Dávila GRO

Sicyos chiriquensis Hammel \& D’Arcy CHIS

Sicyos davilae Rodr.-Arévalo \& Lira CHIS, OAX

*Sicyos dieterleae Rodr.-Arévalo \& Lira GTO, JAL, MICH

Sicyos galeottii Cogn. CHIS, OAX

Sicyos guatemalensis Standl. \& Steyerm. CHIS, GTO, GRO,

HGO, OAX, PUE, QRO, SLP

Sicyos kunthii Cogn. CHIS, OAX

Sicyos laciniatus L. AGS, CHIS, CHIH, COAH, COL, CDMX, DGO, GTO, HGO, JAL, MEX, MOR, NLE, OAX, PUE, QRO, SLP, SIN, SON, TAMS, TLAX, VER, ZAC

Sicyos lirae Rodrìguez-Arévalo CHIS

Sicyos longisepalus Cogn. CAM, CHIS, COL, DGO, GRO, HGO, JAL, MEX, MICH, NAY, OAX, QRO, QROO, SLP, SIN, TAMS, TLAX, VER, YUC

*Sicyos mcvaughii Rodr.-Arévalo, Lira \& Calzada OAX

*Sicyos microphyllus Kunth AGS, CHIS, COAH, COL, CDMX, DGO, GTO, GRO, HGO, JAL, MEX, MICH, MOR, NAY, NLE, OAX, PUE, QRO, SLP, SIN, TAMS, TLAX, VER, ZAC

Sicyos parviflorus Willd. AGS, BCS, CHIS, CHIH, COAH, CDMX, GTO, GRO, HGO, JAL, MEX, MICH, NLE, OAX, PUE, QRO, SLP, SON, TAMS, TLAX, VER, ZAC

*Sicyos peninsularis Brandegee BCN, BCS, CHIH, COL, JAL, NAY, SIN, SON

Sicyos sertulifer Cogn. COL, GRO, JAL, MICH

* Sicyos sinaloae Brandegee CHIH, SIN, SON

Sicyosperma gracile A. Gray BCS, CHIH, SON

Tecunumania quetzalteca Standl. \& Steyerm. CHIS, OAX

Tumamoca macdougallii Rose BCN, SON

*Tumamoca mucronata Kearns ZAC

*Vaseyanthus brandegeei (Cogn.) Rose BCN, BCS, SON

*Vaseyanthus insularis (S. Watson) Rose BCN, BCS, SON

\section{Family Cunoniaceae}

Weinmannia balbisiana Kunth CHIS, OAX

Weinmannia glabra L. f. CHIS, HGO, OAX, VER

Weinmannia intermedia Schltdl. \& Cham. HGO, MEX, OAX, PUE, VER

Weinmannia pinnata L. CHIS, GRO, HGO, OAX, TAMS, VER

\section{Family Cyclanthaceae}

*Asplundia labela (R.E. Schult.) Harling CHIS, OAX, PUE, TAB, VER

Asplundia liebmannii Harling CHIS, HGO, OAX, VER

Asplundia utilis (Oerst.) Harling OAX, VER

Carludovica drudei Mast. CHIS, OAX, TAB, VER

Carludovica palmata Ruiz \& Pav. CAM, CHIS, TAB, VER

Cyclanthus bipartitus Poit. ex A. Rich. CHIS

Dicranopygium gracile (Liebm. ex Matuda) Harling OAX, VER

Family Cymodoceaceae

Halodule wrightii Asch. BCS, CAM, QROO, SIN, SON, TAB, TAMS, VER, YUC

Syringodium filiforme Kuntze CAM, QROO, TAMS, VER, YUC

Family Cyperaceae
*Abildgaardia mexicana (Palla) Kral AGS, CHIS, CDMX, DGO, GTO, GRO, HGO, JAL, MEX, MICH, NLE, OAX, PUE, QRO, SLP, ZAC

Abildgaardia ovata (Burm. f.) Kral CAM, CHIS, OAX, QROO, TAB, TAMS, VER, YUC

Amphiscirpus nevadensis (S. Watson) Oteng-Yeb. COL, JAL, MEX

Bolboschoenus maritimus (L.) Palla BCN, BCS, CHIH, COAH, COL, CDMX, DGO, HGO, MEX, MICH, QRO, QROO, SIN, SON, TAB, TAMS

Bolboschoenus robustus (Pursh) Soják BCN, SIN, SON, TAB, TAMS, VER

*Bulbostylis arcuata Kral AGS, BCS, DGO, JAL, SIN, ZAC

Bulbostylis capillaris (L.) C.B. Clarke AGS, CHIS, CHIH, COAH, CDMX, GTO, GRO, HGO, JAL, MEX, MICH, MOR, OAX, PUE, QRO, QROO, SLP, SON, TAMS, VER, ZAC

Bulbostylis curassavica (Britton) Kük. ex Ekman COL, JAL

Bulbostylis funckii (Steud.) C.B. Clarke AGS, CHIH, COL, CDMX, DGO, GRO, HGO, JAL, MEX, MICH, NAY, OAX, SLP, SIN, SON, VER, ZAC

Bulbostylis hirta (Thunb.) Svenson CHIS, CHIH, COL, JAL, NAY, OAX, VER

Bulbostylis junciformis (Kunth) C.B. Clarke CAM, CHIS, JAL, MEX, MICH, OAX, QROO, TAB, VER

Bulbostylis juncoides (Vahl) Kük. ex Osten AGS, BCN, CAM, CHIS, CHIH, COAH, COL, CDMX, DGO, GTO, GRO, HGO, JAL, MEX, MICH, MOR, NAY, NLE, OAX, PUE, QRO, QROO, SLP, SIN, SON, TAB, TAMS, TLAX, VER, ZAC

*Bulbostylis nesiotica (I.M. Johnst.) Fernald COL

Bulbostylis paradoxa (Spreng.) Lindm. CHIS, OAX, VER

Bulbostylis pubescens (J. Presl \& C. Presl) Svenson CHIS, GRO, JAL, MEX, MICH, MOR, NAY, OAX, SIN, VER

Bulbostylis schaffneri (Boeck.) C.B. Clarke CHIH, DGO, SLP Bulbostylis tenuifolia (Rudge) J.F. Macbr. CHIS, COL, GRO, HGO, JAL, MEX, MOR, NAY, OAX, PUE, SIN, SON, TAB, VER

Bulbostylis trilobata Kral CHIS, GRO, OAX, VER

Bulbostylis vestita (Kunth) C.B. Clarke CAM, CHIS, COL, GRO, JAL, MICH, NAY, OAX, QROO, SIN, VER

Calyptrocarya glomerulata (Brongn.) Urb. CHIS

Carex albicans Willd. ex Spreng. HGO, PUE, VER

* Carex albolutescens Schwein. MICH

Carex alma L.H. Bailey BCN, BCS, CHIH, DGO, GRO, SON

* Carex angustispica Reznicek \& S. González OAX

Carex anisostachys Liebm. CHIS, CDMX, DGO, GRO, HGO,

MEX, MICH, OAX, QRO, VER

* Carex arsenei Kük. JAL, MEX, MICH

* Carex asynchrona Naczi QRO, SLP, TAMS

Carex athrostachya Olney BCN, COAH, CDMX, DGO, HGO, MEX, VER

* Carex atractodes F.J. Herm. CHIS

* Carex austromexicana Reznicek OAX

* Carex aztecica Mack. CHIS, GRO, MEX, MOR, OAX

* Carex ballsii Nelmes VER

Carex bella L.H. Bailey NLE

Carex boliviensis van Heurck \& Müll. Arg. CHIH, COL, CDMX, DGO, GRO, HGO, JAL, MEX, MICH, OAX, PUE, 
VER

Carex bonplandii Kunth VER

Carex brevior (Dewey) Mackenzie ex Lundell MICH, TAMS

Carex brunnipes Reznicek CHIS, CDMX, HGO, MEX, MICH, MOR

* Carex caeligena Reznicek TAMS

Carex caxinensis F.J. Herm. CHIS

* Carex ciliaris Fernald AGS, DGO, HGO, MEX, OAX, ZAC

* Carex cochranei Reznicek JAL, MEX, MICH

Carex comosa W. Boott MICH

Carex complanata Torr. \& Hook. CHIS, OAX

* Carex congestiflora Reznicek \& S. González CHIS

* Carex conspecta Mack. GRO, MEX, MICH, PUE, VER

Carex cortesii Liebm. CHIS, COL, GRO, HGO, JAL, MEX, MOR, OAX, PUE, TLAX, VER

* Carex coulteri W. Boott CDMX, GTO, HGO, MEX, SLP, TAMS, VER

* Carex curviculmis Reznicek CHIS, GTO, MEX, PUE

* Carex chiapensis F.J. Herm. CHIS, OAX

Carex chihuahuensis Mack. BCS, CHIH, SON

Carex chordalis Liebm. CHIS, COL, CDMX, GTO, GRO, HGO,

JAL, MEX, MICH, OAX, VER

* Carex distentiformis F.J. Herm. CHIS, HGO, VER

Carex donnell-smithii L.H. Bailey CHIS, HGO, OAX, VER

Carex douglasii W. Boott BCN

* Carex durangensis Reznicek \& S. González DGO, SIN

Carex echinata Murray CHIH, CDMX, GRO, HGO, MEX,

MICH, MOR, OAX, SON, VER

Carex emoryi Dewey COAH, NLE

Carex endlichii Kük. CHIH, DGO, SON

* Carex evadens S. González \& Reznicek QRO, SLP

* Carex festivelloides Reznicek CHIH

Carex fissa Mack. TAMS

* Carex flexirostris Reznicek OAX

Carex frankii Kunth COAH

* Carex fructus Reznicek TAMS

Carex geophila Mackenzie HGO, MEX, PUE, SON, VER

Carex hassei L.H. Bailey BCN

Carex hermannii Cochrane MEX, MOR, PUE

Carex huehueteca Standl. \& Steyerm. CHIS

* Carex hultenii Aspl. CHIS, HGO, PUE, QRO

Carex humboldtiana Steud. CDMX, GRO, HGO, MEX, OAX, PUE

Carex hystericina Muhlenb. ex Willd. COAH, VER

* Carex interjecta Reznicek MEX, MOR

* Carex involucratella Mackenzie CDMX, MEX, QRO, SLP

* Carex ixtapalucensis Reznicek MEX

Carex jamesonii Boott CHIS, HGO, OAX, VER

*Carex lagunensis M.E. Jones BCS, CHIH, CDMX, DGO, MEX, MOR

Carex laxiflora Lam. CHIS

Carex leptopoda Mackenzie CHIH, DGO

Carex leucodonta T. Holm CHIH, COAH, DGO, GTO, HGO,

JAL, NLE, SLP, SON, TAMS

*Carex longicaulis Boeck. CHIH, CDMX, DGO, GTO, HGO,

JAL, MEX, MOR, OAX, PUE, QRO, TLAX, VER

Carex longii Mack. CHIS, CDMX, HGO, JAL, MEX, MICH,
OAX, PUE, SLP, VER

* Carex longiligula Reznicek \& S. González CHIS

* Carex longissima M.E. Jones BCS

Carex lurida Wahlenb. COAH, MEX, MOR, VER

* Carex mackenziana Weath. NLE, TAMS

*Carex madrensis L.H. Bailey COL, DGO, GRO, JAL, MEX, $\mathrm{MICH}, \mathrm{MOR}, \mathrm{ZAC}$

*Carex marianensis Stacey AGS, CHIS, CHIH, COL, CDMX, DGO, GRO, HGO, JAL, MEX, MICH, MOR, OAX, PUE, TLAX, VER, ZAC

*Carex mcvaughii Reznicek COL, JAL

Carex meadii Dewey CHIH

Carex melanosperma Liebm. CHIS, MEX, MOR, OAX, PUE, TAB, VER

Carex microptera Mack. COAH, DGO, JAL, MEX, MICH, SON

* Carex michoacana Reznicek, Hipp \& S. González MICH

Carex nebraskensis Dewey SON

* Carex novogaliciana Reznicek JAL

Carex oreocharis T. Holm COAH, DGO

Carex orizabae Liebm. CDMX, MEX, MICH, MOR, NLE, OAX, PUE, VER

* Carex percostata F.J. Herm. CHIH, DGO, SON

* Carex perlonga Fernald CHIS, HGO, OAX, VER

Carex pertenuis L.H. Bailey VER

Carex peucophila T. Holm BCS, CHIS, CHIH, CDMX, DGO, GTO, HGO, JAL, MEX, MICH, MOR, NLE, OAX, PUE, QRO, SLP, VER

*Carex pinophila Reznicek \& S. González QRO, SLP

Carex planostachys Kunze CHIS, CHIH, COAH, DGO, GTO, MEX, NLE, OAX, PUE, SLP, TAMS, TLAX, ZAC

Carex polystachya Sw. ex Wahlenb. CAM, CHIS, COL, DGO, GTO, GRO, HGO, JAL, MEX, MICH, MOR, NAY, OAX, QRO, QROO, SLP, SIN, TAMS, VER, YUC, ZAC

Carex polysticha Boeck. VER

* Carex potosina Hemsl. COAH, NLE, SLP, TAMS, ZAC

Carex praegracilis W. Boott AGS, BCN, CHIH, COAH, CDMX, DGO, HGO, JAL, MEX, MICH, SON, VER

* Carex pringlei L.H. Bailey COAH, SLP

Carex psilocarpa Steud. CHIS, CDMX, HGO, JAL, MEX, MICH, MOR, OAX, TLAX, VER

* Carex pubigluma Reznicek CHIS

* Carex queretarensis Reznicek \& S. González QRO, SLP

Carex quichensis F.J. Herm. OAX

* Carex rhynchoperigynium S.D. Jones \& Reznicek HGO, NLE

* Carex rzedowskii Reznicek \& S. González MICH

Carex scabrella Vahl CHIS, OAX

* Carex schaffneri Boeck CDMX, MEX

Carex schiedeana Kuntze BCS, CHIH, COAH, DGO, GTO, GRO, HGO, JAL, MEX, MICH, NLE, OAX, PUE, QRO, SLP, TAMS, ZAC

Carex schottii Dewey BCN

Carex senta W. Boott BCN, CHIH, SON

Carex spissa L.H. Bailey BCN, BCS, CHIH, COAH, HGO, JAL, NLE, OAX, SON

Carex standleyana Steyerm. CAM, CHIS, GRO, OAX, QROO

Carex steyermarkii Standl. CHIS 
Carex subfusca W. Boott BCN

* Carex tenejapensis Reznicek \& S. González CHIS

Carex tetrastachya Scheele COAH, TAMS

Carex thurberi Dewey CHIS, DGO, SIN, VER

* Carex tolucensis (F.J. Herm.) Reznicek MEX

Carex triquetra W. Boott BCN

*Carex tuberculata Liebm. CDMX, HGO, JAL, MEX, MICH, OAX, PUE, VER

Carex tunimanensis Standl. \& Steyerm. CHIS, OAX

*Carex turbinata Liebm. AGS, CHIH, COL, CDMX, DGO, GTO, HGO, JAL, MEX, MICH, MOR, NAY, OAX, PUE, SLP, SIN, SON, VER, ZAC

Carex vallicola Dewey HGO, MEX, QRO, SON

Carex vulpinoidea Michx. MICH, OAX

Carex wootonii Mackenzie CHIH, COAH, CDMX, DGO, MEX, NLE, SIN, SON

Carex xalapensis Kunth CHIS, CHIH, DGO, GTO, HGO, JAL, MEX, MICH, OAX, QRO, SLP, TAMS, VER

Cladium jamaicense Crantz CAM, CHIS, CHIH, COAH, COL, HGO, NLE, OAX, QRO, QROO, SLP, TAB, TAMS, VER, YUC

Cyperus acuminatus Torr. \& Hook. ex Hook. COAH, NLE, TAMS

Cyperus aggregatus (Willd.) Endl. CAM, CHIS, CHIH, COAH, COL, CDMX, DGO, GTO, GRO, HGO, JAL, MEX, MICH, MOR, NAY, NLE, OAX, PUE, QRO, QROO, SLP, SIN, SON, TAB, TAMS, TLAX, VER, YUC, ZAC

Cyperus amabilis Vahl BCS, CHIS, CHIH, COL, DGO, GRO, JAL, MEX, MICH, NAY, OAX, SLP, SIN, SON, TAB, VER, ZAC

Cyperus arsenei O’Neill \& Ben. Ayers BCS, CDMX, GRO, JAL, MEX, MICH, MOR, NAY, SIN

Cyperus articulatus L. BCS, CAM, CHIS, COL, CDMX, GTO, GRO, HGO, JAL, MEX, MICH, MOR, NAY, NLE, OAX, PUE, QROO, SLP, SIN, SON, TAB, TAMS, VER, YUC, ZAC

*Cyperus aschenbornianus Boeck. COL, CDMX, DGO, JAL, MEX, MICH, MOR, NAY, OAX, SIN, SON, VER, ZAC

Cyperus bipartitus Torr. CHIS, CHIH, DGO, GRO, MEX, OAX, SON

* Cyperus breedlovei G.C. Tucker CHIS

*Cyperus calderoniae S. González CDMX, DGO, GTO, HGO, JAL, MEX, QRO, SLP, VER

Cyperus camphoratus Liebm. CHIS, OAX, TAB, VER

Cyperus canus J. Presl \& C. Presl BCN, CAM, CHIS, COAH, COL, DGO, GRO, HGO, JAL, MEX, MICH, MOR, NAY, NLE, OAX, QRO, QROO, SLP, SIN, SON, TAB, TAMS, VER, YUC * Cyperus ciliatus Jungh. CHIS, GRO, OAX, VER

Cyperus compressus L. CAM, CHIS, COL, DGO, GRO, JAL, MEX, MICH, MOR, NAY, OAX, QROO, SIN, SON, TAB, TAMS, VER, YUC

Cyperus costaricensis Gómez Laur. CAM, QROO

Cyperus cuspidatus Kunth BCN, BCS, CHIS, CHIH, COL, GRO, JAL, MEX, MICH, MOR, NAY, OAX, SIN, SON, VER Cyperus chorisanthos C.B. Clarke CAM, CHIS

Cyperus dentoniae G.C. Tucker CHIS, CHIH, COL, DGO, GRO, JAL, MICH, NAY, OAX, SIN, SON, YUC, VER, ZAC Cyperus digitatus Roxb. CAM, CHIS, CHIH, COL, GRO, HGO,
JAL, MEX, MICH, MOR, OAX, PUE, QROO, SLP, SIN, TAB, TAMS, VER, YUC

*Cyperus dioicus I.M. Johnst. BCS

Cyperus dipsaceus Liebm. BCS, CHIH, JAL, MICH, NAY, SIN, SON, VER, ZAC

* Cyperus duripes I.M. Johnst. COL, GRO

Cyperus elegans L. BCN, BCS, CAM, CHIS, CHIH, COAH, COL, GRO, HGO, JAL, MICH, MOR, NAY, NLE, OAX, PUE, QRO, QROO, SLP, SIN, SON, TAB, TAMS, VER, YUC

Cyperus entrerianus Boeck. BCS, CHIS, COL, GTO, JAL, MICH, NAY, OAX, SLP, SIN, SON, TAMS, VER, ZAC

Cyperus eragrostis Lam. MICH

Cyperus erythrorhizos Muhl. BCN, TAB, TAMS

Cyperus esculentus L. AGS, BCN, BCS, CAM, CHIS, CHIH, COAH, COL, CDMX, DGO, GTO, GRO, HGO, JAL, MEX, MICH, MOR, NAY, OAX, PUE, QRO, QROO, SLP, SIN, SON, TAB, TAMS, TLAX, VER, YUC, ZAC

Cyperus fendlerianus Boeck. BCN, BCS, CHIH, COAH, CDMX, DGO, HGO, MEX, MICH, NLE, OAX, QRO, SLP, SON, TLAX, VER

Cyperus flavescens L. AGS, CHIS, CHIH, COL, CDMX, DGO, GTO, GRO, HGO, JAL, MEX, MICH, MOR, NAY, NLE, OAX, PUE, QRO, QROO, SLP, SIN, SON, TLAX, VER, ZAC

Cyperus flavicomus Michx. BCN, CHIS, CHIH, COAH, GTO, GRO, JAL, MEX, MICH, MOR, NAY, OAX, QROO, SIN, SON, TAB, TAMS, VER, ZAC

Cyperus floribundus (Kük) R.N. Carter \& S.D. Jones TAMS

Cyperus fugax Liebm. CHIS, CHIH, COL, DGO, GRO, JAL, NAY, OAX, SIN, VER

Cyperus gardneri Nees CAM, QROO, TAB

Cyperus giganteus Vahl CAM, CHIS, COL, JAL, MICH, OAX, PUE, QROO, SLP, TAB, TAMS, VER

Cyperus globulosus Aubl. CAM, CHIS, QROO, SIN, TAB, TAMS, VER, YUC

Cyperus haspan L. BCS, CAM, CHIS, GRO, JAL, MICH, NAY, OAX, PUE, QROO, TAB, TAMS, VER, YUC

Cyperus hermaphroditus (Jacq.) Standl. AGS, BCN, BCS, CAM, CHIS, CHIH, COAH, COL, CDMX, DGO, GTO, GRO, HGO, JAL, MEX, MICH, MOR, NAY, NLE, OAX, PUE, QRO, QROO, SLP, SIN, SON, TAB, TAMS, TLAX, VER, YUC, ZAC Cyperus humilis Kunth CAM, CHIS, GRO, MEX, MICH, MOR, OAX, QRO, QROO, SLP, TAB, TAMS, VER, YUC

Cyperus hypopitys G.C. Tucker CHIH, DGO, SIN, SON

Cyperus imbricatus Retz. CAM, CHIS, COL, GTO, GRO, JAL, MEX, MICH, MOR, NAY, OAX, QRO, QROO, SLP, SIN, TAB, VER, YUC

Cyperus ischnos Schltdl. CHIS, CHIH, COL, DGO, GRO, JAL, MEX, MICH, NAY, OAX, SIN, SON, VER, ZAC

Cyperus laevigatus L. AGS, BCN, BCS, CHIS, CHIH, COAH, CDMX, DGO, GTO, GRO, HGO, JAL, MEX, MICH, OAX, PUE, QRO, SLP, SIN, SON, TAMS, TLAX, VER, ZAC

Cyperus lanceolatus Poir. BCN, BCS, CHIS, CHIH, COL, DGO, GRO, HGO, JAL, MEX, MOR, NAY, NLE, OAX, PUE, SLP, SIN, SON, TAB, TAMS, TLAX, VER, ZAC

Cyperus laxus Lam. CAM, CHIS, COL, GRO, JAL, MEX, MICH, MOR, NAY, OAX, QROO, SIN, TAB, TAMS, VER, YUC 
Cyperus lentiginosus Millsp. \& Chase CAM, CHIS, COAH, GRO, JAL, MICH, OAX, QROO, SLP, SIN, TAB, TAMS, VER, YUC

Cyperus ligularis L. BCN, BCS, CAM, CHIS, COL, DGO, GRO, JAL, MICH, NAY, OAX, QROO, SIN, SON, TAB, TAMS, VER, YUC

Cyperus lundellii O'Neill CAM, CHIS, QROO, TAMS

Cyperus luzulae (L.) Rottb. ex Retz. CAM, CHIS, COAH, DGO, GTO, GRO, HGO, JAL, MICH, NAY, OAX, QRO, QROO, SLP, TAB, TAMS, VER, YUC

Cyperus macrostachyos Lam. BCN, CHIH, GTO, MICH, NAY, SLP, SON

Cyperus manimae Kunth AGS, BCS, CHIS, CHIH, COAH, COL, CDMX, DGO, GTO, GRO, HGO, JAL, MEX, MICH, MOR, NAY, NLE, OAX, PUE, QRO, SLP, SIN, SON, TAMS, TLAX, VER, ZAC

*Cyperus matudae G.C. Tucker CHIS

Cyperus megalanthus (Kük.) G.C. Tucker QRO, QROO, SLP, TAMS, VER

Cyperus microbrunneus G.C. Tucker OAX

*Cyperus michoacanensis Britton ex C.B. Clarke COL, JAL, $\mathrm{MICH}, \mathrm{NAY}$

Cyperus mutisii (Kunth) Andersson AGS, BCS, CHIS, CHIH, COL, CDMX, DGO, GRO, HGO, JAL, MEX, MICH, MOR, NAY, NLE, OAX, PUE, QRO, SIN, SON, TAB, TAMS, TLAX, VER, YUC, ZAC

Cyperus nanus Willd. VER

*Cyperus nayaritensis Tucker COL, JAL, MICH, NAY

Cyperus niger Ruiz \& Pav. AGS, BCN, BCS, CHIS, CHIH, COAH, COL, CDMX, DGO, GTO, GRO, HGO, JAL, MEX, MICH, MOR, NAY, NLE, OAX, PUE, QRO, SLP, QROO, SIN, SON, TAMS, TLAX, VER, ZAC

Cyperus ochraceus Vahl AGS, BCS, CAM, CHIS, COAH, COL, DGO, GTO, GRO, HGO, JAL, MEX, MICH, MOR, NAY, NLE, OAX, PUE, QRO, QROO, SLP, SIN, SON, TAB, TAMS, VER, YUC, ZAC

Cyperus odoratus L. AGS, BCN, BCS, CAM, CHIS, CHIH, COAH, COL, CDMX, DGO, GTO, GRO, HGO, JAL, MEX, MICH, MOR, NAY, NLE, OAX, PUE, QRO, QROO, SLP, SIN, SON, TAB, TAMS, VER, YUC, ZAC

Cyperus pallidicolor (Kük.) G.C. Tucker AGS, BCS, CHIS, CHIH, COAH, CDMX, DGO, GTO, HGO, JAL, MEX, MICH, MOR, NAY, NLE, OAX, PUE, SON, TLAX, VER, ZAC

Cyperus panamensis (C.B. Clarke) Britton ex Standl. CHIS, GRO, OAX, SIN

*Cyperus penicillatus Conz. OAX

*Cyperus pennellii O'Neill \& Ben. Ayers CDMX, DGO, MEX, $\mathrm{MICH}$

*Cyperus perennis (M.E. Jones) O'Neill BCN, BCS, CHIH, QROO, SON, YUC

Cyperus planifolius A. Rich. CAM, NAY, OAX, QROO, YUC Cyperus polystachyos Rottb. CAM, CHIS, GRO, NAY, OAX, QROO, SIN, SON, TAB, TAMS, VER, YUC

Cyperus prolixus Kunth CHIS, CDMX, DGO, HGO, JAL, MEX, MICH, NAY, OAX, SON, TAMS, VER, ZAC

Cyperus pseudovegetus Steud. CHIS, JAL, NLE, OAX, TAB, TAMS, TLAX, YUC
Cyperus pychnostachyus (Kunth) Kunth COL, CDMX, HGO, JAL, MEX, MICH, OAX, PUE, SON, TLAX

Cyperus reflexus Vahl CHIS, CDMX, GTO, GRO, HGO, JAL, MEX, MICH, NLE, OAX, QRO, SLP, TAMS, VER

Cyperus regiomontanus Britton CHIS, COL, GRO, JAL, MICH, NAY, OAX, SIN, SON

Cyperus retroflexus Buckley CHIS, CHIH, COAH, GRO, NLE, QRO, SLP, SON, TAB, TAMS, VER

Cyperus sanguineo-ater Boeck. CHIH, COL, CDMX, DGO, GRO, HGO, JAL, MEX, MICH, MOR, NAY, OAX, PUE, SIN, SON, ZAC

*Cyperus schaffneri Boeck CDMX, MEX

Cyperus schweinitzii Torr. CHIH, COAH, DGO, JAL, ZAC

Cyperus semiochraceus Boeck. BCS, CAM, CHIH, COL, CDMX, GTO, GRO, HGO, JAL, MEX, MICH, MOR, NAY, OAX, PUE, SON, TAB, TAMS, VER

Cyperus seslerioides Kunth AGS, CHIS, CHIH, COAH, COL, CDMX, DGO, GTO, GRO, HGO, JAL, MEX, MICH, MOR, NAY, OAX, PUE, QRO, QROO, SLP, SIN, SON, TAB, TAMS, TLAX, VER, YUC, ZAC

Cyperus simplex Kunth CHIS, OAX

*Cyperus sordidus J. Presl \& C. Presl COL, GRO, JAL, NAY, SIN

Cyperus spectabilis Link AGS, CHIH, COAH, COL, CDMX, DGO, GTO, GRO, HGO, JAL, MEX, MICH, MOR, NAY, NLE, OAX, PUE, QRO, SLP, SIN, SON, TAMS, TLAX, VER, ZAC Cyperus sphaerolepis Boeck. CHIH, COAH, CDMX, DGO, GTO, HGO, MEX, NLE, PUE, SLP, SON, ZAC

Cyperus squarrosus L. AGS, BCN, BCS, CAM, CHIS, CHIH, COAH, CDMX, DGO, GTO, GRO, HGO, JAL, MEX, MICH, MOR, NLE, OAX, PUE, QRO, QROO, SLP, SIN, SON, TAMS, TLAX, VER, YUC, ZAC

Cyperus surinamensis Rottb. AGS, BCS, CAM, CHIS, COL, DGO, GTO, GRO, JAL, MEX, MICH, MOR, NAY, OAX, PUE, QRO, QROO, SLP, SIN, SON, TAB, TAMS, VER, YUC, ZAC Cyperus svensonii G.C. Tucker CHIS

*Cyperus tempeae G.C. Tucker SLP, TAMS, VER

Cyperus tenerrimus J. Presl \& C. Presl CHIS, CHIH, COL, DGO, GRO, JAL, MEX, MICH, MOR, NAY, OAX, SIN, SON, VER, ZAC

Cyperus tenuis Sw. BCS, CAM, CHIS, COAH, COL, DGO, GTO, GRO, HGO, JAL, MEX, MICH, NAY, NLE, OAX, PUE, QRO, SLP, SON, TAB, TAMS, VER, YUC, ZAC

Cyperus tetragonus Elliott CHIS

Cyperus thyrsiflorus Jungh. CAM, CHIS, COAH, GRO, HGO, MICH, MOR, NAY, NLE, OAX, PUE, SLP, SIN, TAB, TAMS, VER, YUC

Cyperus uncinulatus Schrad. ex Nees CHIS, OAX

Cyperus unioloides R. Br. CHIS, MEX, MICH, OAX, TAB, VER

Cyperus virens Michx. AGS, CHIS, COAH, COL, CDMX, DGO, GTO, GRO, HGO, JAL, MEX, MICH, MOR, NAY, OAX, PUE, QRO, SLP, SON, TAB, TAMS, VER, ZAC

*Cyperus wilburii G.C. Tucker OAX

*Cypringlea analecta (Beetle) M.T. Strong HGO, NLE, OAX, QRO, SLP, TAMS, VER

*Cypringlea coahuilensis (Svenson) M.T. Strong COAH, NLE 
*Cypringlea evadens (C.D. Adams) Reznicek \& S. González CHIS, GRO, OAX, PUE

Eleocharis acicularis (L.) Roem. \& Schult. AGS, BCN, CAM, CHIS, CHIH, CDMX, DGO, GTO, GRO, HGO, JAL, MEX, MICH, MOR, OAX, PUE, QRO, QROO, SLP, SIN, SON, TAMS, TLAX, VER, ZAC

Eleocharis acutangula (Roxb.) Schult. CAM, CHIS, DGO, GRO, JAL, NAY, OAX, QROO, TAB, TAMS, VER, YUC

Eleocharis albibracteata Kunth GRO

Eleocharis albida Torr. NLE, TAMS

*Eleocharis arsenifera S. González, Tena \& T.Alarcón CHIH

Eleocharis atropurpurea (Retz.) J. Presl \& C. Presl CAM, CHIS, GRO, JAL, OAX, SON, TAMS, YUC

Eleocharis bella (Piper) Svenson BCN, BCS, SON

Eleocharis bonariensis Nees CDMX, DGO, GTO, JAL, MEX, MICH, MOR, OAX, QRO

Eleocharis brachycarpa Svenson TAMS

Eleocharis cancellata S. Watson GTO, JAL, MEX, SLP, ZAC

Eleocharis cellulosa Torr. CAM, CHIS, COAH, COL, GRO, JAL, MEX, NLE, QROO, TAB, TAMS, VER, YUC

Eleocharis coloradoensis (Britton) Gilly TAMS

*Eleocharis cryptica Saarela, P.M. Peterson, González, S. \& D.J. Rosen DGO

*Eleocharis densa Benth. AGS, CDMX, DGO, GTO, HGO, JAL, MEX, MICH, MOR, OAX, PUE, QRO, SLP, SIN, VER, ZAC

Eleocharis dombeyana Kunth CHIS, COAH, CDMX, DGO, HGO, JAL, MEX, MICH, MOR, OAX, PUE, QRO, SLP, TAB, TLAX, VER, ZAC

Eleocharis elegans (Kunth) Roem. \& Schult. CAM, CHIS, COL, DGO, GTO, GRO, HGO, JAL, MEX, MICH, MOR, NAY, OAX, PUE, QRO, QROO, SLP, SIN, SON, TAB, TAMS, VER, YUC, ZAC

Eleocharis filiculmis Kunth CAM, CHIS, COAH, GTO, GRO, JAL, MEX, MOR, NAY, OAX, PUE, QRO, QROO, TAB, VER, YUC

Eleocharis flavescens (Poir.) Urb. BCS, CHIS, COAH, GRO, JAL, NAY, OAX, QROO, SLP, TAMS

Eleocharis geniculata (L.) Roem. \& Schult. BCN, BCS, CAM, CHIS, CHIH, COAH, COL, DGO, GTO, GRO, HGO, JAL, MEX, MICH, MOR, NAY, NLE, OAX, PUE, QRO, QROO, SLP, SIN, SON, TAB, TAMS, VER, YUC, ZAC

*Eleocharis gonzaleziae D.J. Rosen DGO

*Eleocharis ignota S. González \& Reznicek AGS, GTO, JAL Eleocharis interstincta (Vahl) Roem. \& Schult. CAM, CHIS, CHIH, COAH, COL, HGO, MOR, OAX, QRO, QROO, SLP, TAB, TAMS, VER, YUC

Eleocharis macrostachya Britton AGS, BCN, CHIS, CHIH, COAH, COL, CDMX, DGO, GTO, GRO, HGO, JAL, MEX, MICH, MOR, NLE, OAX, PUE, QRO, SLP, SIN, SON, TAMS, TLAX, TAMS, VER, ZAC

Eleocharis maculosa (Vahl) Roem. \& Schult. MICH

Eleocharis minima Kunth AGS, CAM, CHIS, DGO, GRO, JAL, MEX, MICH, NAY, OAX, QROO, SIN, TAB, TAMS, VER, ZAC

Eleocharis minutissima Britton CHIS, OAX

Eleocharis mitrata (Griseb.) C.B. Clarke CAM, CHIS, OAX
Eleocharis montana (Kunth) Roem. \& Schult. AGS, CAM, CHIS, CHIH, COAH, CDMX, DGO, GTO, GRO, HGO, JAL, MEX, MICH, MOR, NAY, OAX, PUE, QRO, QROO, SLP, SIN, SON, VER, YUC, ZAC

Eleocharis montevidensis Kunth AGS, BCN, BCS, CHIS, CHIH, COAH, CDMX, DGO, GTO, GRO, HGO, JAL, MEX, MICH, MOR, NLE, OAX, PUE, QRO, SLP, SIN, SON, TAMS, TLAX, VER, ZAC

*Eleocharis moorei M. Strong \& S. González HGO, VER Eleocharis mutata (L.) Roem. \& Schult. CAM, CHIS, COL, DGO, GRO, JAL, MEX, NAY, OAX, QROO, TAB, TAMS, VER, YUC

Eleocharis nigrescens (Nees) Kunth AGS, CAM, CHIS, GRO, JAL, OAX, QROO, TAB, VER

Eleocharis obtusa (Willd.) Schult. MOR, TAMS

Eleocharis obtusetrigona (Lindl. \& Nees) Steud. TAMS, VER Eleocharis pachystyla (C. Wright) C.B. Clarke TAB

Eleocharis palustris (L.) Roem. \& Schult. BCN, CHIH, COAH, DGO, SON, TAMS

Eleocharis parishii Britton BCN, BCS, CHIH, COAH, DGO, NLE, SON

Eleocharis parvula (Roem. \& Schult.) Link ex Bluff, Nees \& Schauer BCN, CAM, CHIH, COAH, DGO, OAX, PUE, QROO, SLP, SIN, TAB, TAMS, VER

Eleocharis pauciflora (Lightf.) Link BCN

Eleocharis plicarhachis (Griseb.) Svenson CAM, CHIS, QROO, TAB, VER, YUC

Eleocharis quadrangulata (Michx.) Roem. \& Schult. COL, DGO, JAL, MICH, NAY, TAMS

Eleocharis radicans (Poir.) Kunth BCN, CHIH, CDMX, DGO, GRO, HGO, JAL, MEX, MICH, MOR, OAX, QRO, SLP, SON, TAMS, SON, ZAC

Eleocharis retroflexa (Poir.) Urb. CAM, CHIS, OAX, TAB, TLAX, VER

*Eleocharis reznicekii S. González, D.J. Rosen, R. Carter \& P.M. Peterson DGO

Eleocharis rostellata (Torr.) Torr. AGS, BCN, CHIS, CHIH, COAH, DGO, MEX, MICH, NLE, QRO, SLP, SON, TAMS, ZAC

*Eleocharis rzedowskii S. González COAH, NLE, SLP

Eleocharis schaffneri Boeck. AGS, CHIS, CHIH, DGO, GTO, JAL, MICH, OAX, SLP, ZAC

*Eleocharis subcancellata C.B. Clarke DGO, JAL

*Eleocharis svensoniana S. González DGO, JAL, ZAC

*Eleocharis tenarum S. González \& M. González Elizond. DGO, ZAC

Eleocharis tenuis (Willd.) Schult. CHIH, SON

Eleocharis urceolata (Liebm.) Svenson CAM, CHIS, QROO, VER

Eleocharis yecorensis Roalson CHIH, DGO, JAL, SON

*Fimbristylis argillicola Kral AGS, CDMX, DGO, GTO, GRO, HGO, JAL, MEX, MICH, MOR, PUE, ZAC

Fimbristylis autumnalis (L.) Roem. \& Schult. CAM, CHIS, CHIH, COAH, GRO, NLE, OAX, QROO, SLP, TAB, VER, YUC, ZAC

Fimbristylis caroliniana (Lam.) Fernald OAX, SLP, TAMS, VER 
Fimbristylis castanea (Michx.) Vahl QROO, TAMS, VER, YUC Fimbristylis complanata (Retz.) Link BCS, CAM, CHIS, CHIH, COL, DGO, GRO, HGO, JAL, MEX, MICH, NAY, NLE, OAX, PUE, QROO, SLP, TAB, TAMS, TLAX, VER, ZAC

Fimbristylis cymosa R. Br. CAM, CHIS, COL, GRO, JAL, MICH, NAY, OAX, QROO, SIN, SON, TAB, TAMS, VER, YUC

Fimbristylis dichotoma (L.) Vahl AGS, BCN, BCS, CAM, CHIS, CHIH, COAH, COL, DGO, GTO, GRO, HGO, JAL, MEX, MICH, MOR, NAY, OAX, PUE, QRO, QROO, SLP, SIN, SON, TAB, TAMS, VER, YUC, ZAC

Fimbristylis ferruginea (L.) Vahl CAM, OAX, QROO, YUC

Fimbristylis miliacea (L.) Vahl CAM, CHIS, COL, GRO, JAL, MEX, MICH, MOR, NAY, NLE, OAX, SIN, TAB, VER

*Fimbristylis pentastachya Boeck. CHIH, COL, GRO, JAL, MICH, NAY, OAX, SIN, SON, VER

Fimbristylis puberula (Michx.) Vahl CAM, CHIH, COAH, MICH, SON, TAMS, VER

Fimbristylis spadicea (L.) Vahl CAM, CHIS, COL, GRO, JAL, NAY, OAX, QROO, SLP, SIN, SON, TAB, TAMS, VER, YUC Fimbristylis thermalis S. Watson BCN, CHIH, COAH, SLP Fimbristylis vahlii (Lam.) Link COAH, TAB, TAMS, VER Fuirena camptotricha C. Wright CAM, CHIS, GRO, OAX, QROO, TAB, TAMS, VER, YUC

Fuirena incompleta Nees CHIS, DGO, GTO, JAL, MEX, MICH, NAY, PUE, SON, VER, ZAC

*Fuirena repens Boeck. DGO, QRO, SLP

Fuirena robusta Kunth CHIS, TAB

Fuirena simplex Vahl BCN, BCS, CAM, CHIS, CHIH, COAH, DGO, GRO, HGO, JAL, MEX, MICH, MOR, NLE, OAX, PUE, QRO, QROO, SLP, SIN, SON, TAB, TAMS, VER, YUC

Fuirena stephani Ramos \& N. Diego CAM, QROO

Fuirena umbellata Rottb. CAM, CHIS, COL, GRO, JAL, NAY, OAX, PUE, QROO, TAB, VER, YUC

Hypolytrum longifolium (Rich.) Nees CHIS, TAB, VER

Isolepis carinata Hook. \& Arn. ex Torr. BCN

Isolepis cernua (Vahl) Roem. \& Schult. BCN, BCS, SIN

* Karinia mexicana (C.B. Clarke ex Britton) Reznicek \& McVaugh AGS, CAM, DGO, GTO, JAL, MEX, MICH, MOR, PUE, QRO, SLP, SLP, TLAX, VER, ZAC

Kyllinga brevifolia Rottb. CHIS, COL, GTO, GRO, JAL, MEX, MICH, MOR, NAY, OAX, PUE, TAB, TAMS, VER

Kyllinga odorata Vahl AGS, CHIS, CHIH, COL, CDMX, DGO, GTO, GRO, HGO, JAL, MEX, MICH, MOR, NAY, OAX, PUE, QRO, SLP, SIN, SON, TAMS, TLAX, VER, ZAC

Kyllinga pumila Michx. CAM, CHIS, COL, CDMX, DGO, GRO, HGO, JAL, MEX, MICH, MOR, NAY, OAX, PUE, QRO, QROO, SLP, SIN, TAB, TAMS, TLAX, VER, ZAC

Lipocarpha humboldtiana Nees CHIS, GRO

Lipocarpha maculata (Michx.) Torr. CHIS, GRO, JAL, NAY, VER

*Lipocarpha mexicana Liebm. CHIS, DGO, GRO, OAX, VER Lipocarpha micrantha (Vahl) G.C. Tucker AGS, BCN, BCS, CHIS, CHIH, COL, GRO, JAL, MEX, MICH, MOR, NAY, OAX, PUE, SLP, SIN, SON, TAMS, VER, ZAC

Oxycaryum cubense (Poepp. \& Kunth) Palla CAM, CHIS, GTO, GRO, JAL, MICH, NAY, QROO, SLP, TAB, TAMS, VER, YUC
Remirea maritima Aubl. QROO, YUC

*Rhynchospora angosturensis W.W. Thomas GTO, SLP

Rhynchospora aristata Boeck. CHIS, COL, CDMX, DGO, GRO, HGO, JAL, MEX, MICH, NAY, OAX, SIN, TAB, TAMS, VER, ZAC

Rhynchospora barbata (Vahl) Kunth CAM, CHIS, OAX, QROO, TAB, VER

Rhynchospora berteroi (Spreng.) C.B. Clarke CAM, QROO, YUC

Rhynchospora boliviensis C.B. Clarke OAX

Rhynchospora brevirostris Griseb. JAL, MICH, MOR, NAY, OAX, TAB

Rhynchospora brittonii Gale TAB

Rhynchospora brownii Roem. \& Schult. CHIS

Rhynchospora cephalotes (L.) Vahl CAM, CHIS, GRO, JAL, OAX, QROO, TAB, VER

Rhynchospora colorata (L.) H. Pfeiff. CAM, CHIS, COAH, COL, DGO, GRO, JAL, MEX, MOR, NAY, NLE, OAX, QRO, QROO, SLP, TAB, TAMS, VER, YUC

Rhynchospora consanguinea (Kunth) Boeck. CHIS, OAX, TAB Rhynchospora contracta (Nees) J. Raynal AGS, CAM, CHIS, COL, GRO, JAL, MEX, MOR, NAY, OAX, QROO, SIN, SON, TAB, TAMS, VER, YUC

Rhynchospora corymbosa (L.) Britton CAM, CHIS, COL, GRO, JAL, MICH, NAY, OAX, QROO, SLP, TAB, VER, YUC Rhynchospora divergens Chapm. ex M.A. Curtis CHIS Rhynchospora dives Standl. CHIS, GRO, OAX, VER

*Rhynchospora durangensis Kral \& W.W. Thomas DGO, SIN Rhynchospora eximia (Nees) Boeck. CAM, CHIS, COL, GRO, JAL, NAY, OAX, SIN, TAB, VER

Rhynchospora fascicularis (Michx.) Vahl TAB, VER

Rhynchospora filifolia A. Gray CAM, QROO, TAB

Rhynchospora filiformis Vahl CAM, CHIS, QROO, TAB

Rhynchospora floridensis (Britton ex Small) H. Pfeiff. CAM, CHIS, OAX, QROO, TAB, VER, YUC

Rhynchospora gigantea Link CAM, QROO, TAB, VER, YUC Rhynchospora globosa (Kunth) Roem. \& Schult. CHIS, OAX, TAB, VER

Rhynchospora globularis (Chapm.) Small CHIS, OAX, QROO, TAB, VER

Rhynchospora gracilenta A. Gray CHIS, QROO, TAB Rhynchospora hirsuta (Vahl) Vahl CAM, CHIS, OAX, QROO, $\mathrm{TAB}$

Rhynchospora holoschoenoides (Rich.) Herter CAM, CHIS, JAL, MEX, NAY, OAX, PUE, QROO, SIN, TAB, VER, YUC Rhynchospora inundata (Oakes) Fernald TAB

*Rhynchospora jaliscensis McVaugh COL, JAL, ZAC

Rhynchospora jubata Liebm. CHIS, DGO, VER

Rhynchospora kunthii Nees ex Kunth CHIS, CHIH, CDMX, DGO, HGO, JAL, MEX, MICH, NAY, OAX, PUE, SLP, SON, VER, ZAC

Rhynchospora lindeniana Griseb. CAM, QROO, YUC Rhynchospora marisculus Lindl. ex Nees CHIS, OAX, VER Rhynchospora mexicana (Liebm.) Steud. CHIS, GRO, JAL, OAX

Rhynchospora nervosa (Vahl) Boeck. CAM, CHIS, CHIH, COL, DGO, GRO, JAL, MEX, MICH, NAY, OAX, QROO, SLP, SIN, 
SON, TAB, TAMS, VER, YUC, ZAC

Rhynchospora nivea Boeck. COAH

* Rhynchospora oaxacana Kral \& W.W. Thomas CHIS, OAX

Rhynchospora plumosa Elliott QROO, TAB

Rhynchospora polyphylla (Vahl) Vahl CHIS, GRO, OAX, PUE, VER

Rhynchospora pusilla Chapm. ex M.A. Curtis CAM, CHIS, QROO, TAB

Rhynchospora radicans (Schltdl. \& Cham.) H. Pfeiff. CAM, CHIS, GRO, HGO, OAX, PUE, QRO, QROO, SLP, TAB, TAMS, VER

Rhynchospora robusta (Kunth) Boeck. CHIS, VER

* Rhynchospora rosae W.W. Thomas CHIS

Rhynchospora rugosa (Vahl) Gale CHIS, JAL, OAX, PUE, QROO, TAB, VER

Rhynchospora ruizana Boeck. CHIS, HGO, MEX, OAX, VER Rhynchospora scutellata Griseb. CAM, CHIS, OAX, PUE, QROO, TAB, VER, YUC

Rhynchospora schiedeana Kunth CHIS, GRO, JAL, MEX, MICH, MOR, NAY, OAX, PUE, TAB, TAMS, VER

Rhynchospora tenerrima Nees \& Spreng. CAM, CHIS, GRO, OAX, QROO, TAB

Rhynchospora tenuis Willd. ex Link CHIS, DGO, JAL, NAY, OAX, SIN, TAB, VER, ZAC

Rhynchospora tracyi Britton QROO

Rhynchospora triflora Vahl CAM, CHIS, TAB, VER

Rhynchospora trispicata (Nees) Schrad. ex Steud. CAM, CHIS, OAX, QROO, TAB, VER

*Rhynchospora velutina (Kunth) Boeck. CAM, QROO, VER

Rhynchospora vulcanii Boeck. CHIS

Rhynchospora watsonii (Britton) Davidse CAM, VER

*Rhynchospora zacualtipanensis M. Strong HGO, VER

Scirpus cyperinus (L.) Kunth HGO, VER

Scirpus microcarpus J. Presl \& C. Presl BCN, DGO, OAX, SIN

Scirpus pendulus Muhl. COAH

Scleria anceps Liebm. CHIS, VER

*Scleria bourgeaui Boeck. CHIS, CHIH, DGO, JAL, NAY, VER, ZAC

Scleria bracteata Cav. CAM, CHIS, GRO, OAX, PUE, QROO, TAB, VER, YUC

Scleria ciliata Michx. CHIS, DGO, GRO, JAL, MEX, NAY, OAX, TAB, VER, ZAC

Scleria distans Poir. CHIS, NAY, OAX, TAB, VER

Scleria eggersiana Boeck. CAM, CHIS, NAY, OAX, QROO,

TAB, VER, YUC

Scleria flagellum-nigrorum Bergius TAB, VER

* Scleria hirta Boeck. VER

Scleria interrupta L. CAM, CHIS, QROO

Scleria latifolia Sw. CAM, CHIS, GRO, OAX

Scleria lithosperma (L.) Sw. CAM, CHIS, COL, GRO, MICH, OAX, QRO, QROO, SLP, TAB, TAMS, VER, YUC

Scleria macrophylla J. Presl \& C. Presl BCN, CAM, GRO, OAX, QROO, TAB, VER, YUC

Scleria melaleuca Rchb. ex Schltdl. \& Cham. CAM, CHIS, GRO, OAX, PUE, QRO, QROO, SLP, TAB, TAMS, VER, YUC

*Scleria mexicana (Liebm.) Boeck. CHIS, GRO, OAX, VER
Scleria microcarpa Nees ex Kunth CAM, CHIS, GRO, JAL, OAX, QROO, TAB, VER

Scleria oligantha Michx. CHIS, COAH, DGO, HGO, NLE, QRO, SLP, TAMS, VER

Scleria phylloptera C. Wright ex Griseb. CAM, CHIS, TAB, VER

Scleria reticularis Michx. CAM, CHIS, COL, DGO, GTO, GRO, HGO, JAL, MEX, MICH, NAY, NLE, OAX, QRO, SIN, SON, TAB, TAMS, VER, YUC, ZAC

*Scleria scabriuscula Schltdl. CHIS, COL, JAL, NAY, VER Scleria scindens Nees TAB

Scleria schiedeana Schltdl. COL, GRO, JAL, NAY, OAX, VER Scleria secans (L.) Urb. CHIS, OAX, PUE, QROO, TAB, VER Scleria setuloso-ciliata Boeck. CAM, CHIS, GRO, MOR, OAX, PUE, QROO, SLP, TAB, TAMS, VER, YUC

Scleria tenella Kunth CHIS, COL, GRO, JAL, NAY, OAX, VER Scleria triglomerata Michx. NLE

Scleria vaginata Steud. CAM, CHIS, OAX, QROO, TAB

Scleria verticillata Muhl. ex Willd. CHIS, GRO, JAL, NLE

Schoenoplectiella erecta (Poir.) Lye CHIS, CHIH, COAH, NLE, OAX, QROO, TAMS, YUC

Schoenoplectiella saximontana (Fernald) Lye BCN, BCS, CHIH, COAH, NLE, QROO, SLP, TAMS

Schoenoplectus acutus (Muhl. ex Bigelow) A. Löve \& D. Löve AGS, BCN, CHIH, COAH, MEX, MICH, MOR, NLE, OAX, SON, ZAC

Schoenoplectus americanus (Pers.) Volkart ex Schinz \& R. Keller BCN, BCS, CHIS, CHIH, COAH, COL, CDMX, DGO, GTO, HGO, JAL, MEX, MICH, MOR, NLE, OAX, PUE, SIN, SON, TAMS, TLAX, VER, ZAC

Schoenoplectus californicus (C.A. Mey.) Soják AGS, BCN, BCS, CHIS, CHIH, COAH, COL, CDMX, DGO, GTO, HGO, JAL, MEX, MICH, MOR, NAY, NLE, OAX, PUE, QRO, SLP, SON, TAMS, TLAX, VER, YUC

Schoenoplectus pungens (Vahl) Palla BCN, BCS, COAH, COL, GTO, JAL, MICH, SON, TAMS, VER

Schoenoplectus tabernaemontani (C.C. Gmel.) Palla AGS, CAM, CHIS, CHIH, COAH, COL, CDMX, DGO, GTO, GRO, HGO, JAL, MEX, MICH, MOR, NAY, NLE, OAX, PUE, QRO, QROO, SLP, SIN, SON, TAB, TAMS, TLAX, VER, YUC

Schoenus nigricans L. CHIS, COAH, NLE, OAX, QROO, SLP, TAB, TAMS

Uncinia hamata (Sw.) Urb. CHIS, CDMX, GRO, HGO, MEX, OAX, VER

Uncinia koyamae Gómez-Laur. CHIS, OAX, VER

Uncinia phleoides (Cav.) Pers. CDMX, MEX

*Uncinia tenuis Poepp. ex Kunth OAX

Family Cyrillaceae

Cyrilla racemiflora $\mathrm{L}$. OAX

Family Cytinaceae

Bdallophytum americanum (R. Br.) Eichler ex Solms AGS, BCN, CHIS, COL, DGO, GRO, JAL, MEX, MICH, MOR, OAX, PUE, QRO, SLP, SIN, TAMS, VER, ZAC

*Bdallophytum andrieuxii Eichler GRO, JAL, MICH, MOR, OAX, PUE

*Bdallophytum oxylepis (B.L. Rob.) Harms CHIS, GRO, JAL, 


\section{MICH, OAX}

\section{Family Chloranthaceae}

Hedyosmum mexicanum Cordem. CHIS, COL, DGO, GRO, JAL, MEX, MICH, MOR, NAY, OAX, PUE, SIN, VER

\section{Family Chrysobalanaceae}

Couepia polyandra (Kunth) Rose CAM, CHIS, COL, GTO, GRO, JAL, MICH, NAY, OAX, PUE, QRO, QROO, SIN, TAB, VER, YUC

Chrysobalanus icaco L. CAM, CHIS, GRO, MICH, OAX, QROO, TAB, TAMS, VER, YUC

Hirtella americana L. CAM, CHIS, GRO, OAX, QROO, SLP, TAB, VER

Hirtella paniculata $\mathrm{Sw}$. CHIS

Hirtella racemosa Lam. CHIS, GRO, JAL, MICH, NAY, OAX, TAB, VER

Hirtella triandra Sw. CHIS, OAX, PUE, TAB, VER

Licania arborea Seem. CHIS, GRO, JAL, MEX, MICH, MOR, OAX, PUE

*Licania gonzalezii Miranda COL, GRO, JAL, NAY

Licania hypoleuca Benth. CHIS, GRO, NAY, OAX, TAB, VER

*Licania mexicana Lundell SIN

Licania platypus (Hemsl.) Fritsch CHIS, GTO, GRO, HGO, JAL, OAX, PUE, TAB, VER

*Licania retifolia S.F. Blake COL, GRO, JAL, MICH, OAX

Licania sparsipilis S.F. Blake CHIS, GRO, OAX, VER

\section{Family Datiscaceae}

Datisca glomerata (Presl) Baill. BCN

\section{Family Dichapetalaceae}

Dichapetalum donnell-smithii Engl. CHIS, JAL, OAX, QRO, QROO, TAB, VER

* Dichapetalum mexicanum Prance VER

*Tapura mexicana Prance COL, GRO, JAL, OAX

\section{Family Dilleniaceae}

Curatella americana L. CAM, CHIS, COL, GRO, HGO, JAL, MEX, MICH, NAY, OAX, PUE, QROO, SIN, TAB, VER, YUC Davilla kunthii A. St.-Hil. CAM, CHIS, COL, JAL, OAX, PUE, QROO, TAB, VER

Davilla nitida (Vahl) Kubitzki CHIS, VER

Doliocarpus dentatus (Aubl.) Standl. CAM, CHIS, OAX, PUE, QROO, TAB, VER

Tetracera portobellensis Beurl. CHIS, COL, GRO, JAL, MICH, NAY, OAX

Tetracera sessiliflora Triana \& Planch. CHIS, GRO, JAL, MICH, NAY, OAX

Tetracera volubilis L. CAM, CHIS, GRO, NAY, OAX, PUE, QROO, SLP, TAB, VER, YUC

\section{Family Dioscoreaceae}

Dioscorea bartlettii C.V. Morton CAM, CHIS, OAX, QROO, TAB, VER, YUC

*Dioscorea berenicea McVaugh JAL

Dioscorea carpomaculata O. Téllez \& B.G. Schub. CAM, CHIS, CHIH, COL, DGO, GRO, JAL, MEX, MICH, MOR, NAY, OAX, SIN

Dioscorea composita Hemsl. CAM, CHIS, COL, GRO, JAL,
MEX, MICH, OAX, PUE, TAB, VER

Dioscorea convolvulacea Schltdl. \& Cham. CAM, CHIS, COL, DGO, GTO, GRO, HGO, JAL, MEX, MICH, MOR, NAY, NLE, OAX, PUE, QRO, QROO, SLP, SIN, SON, TAB, TAMS, VER, YUC, ZAC

*Dioscorea conzattii R. Knuth MEX, MICH, OAX

*Dioscorea cruzensis R. Knuth VER

Dioscorea cyanisticta Donn. Sm. CAM, CHIS, QROO, VER, YUC

*Dioscorea cyphocarpa B.L. Rob. ex R. Knuth GRO, OAX Dioscorea chiapasensis Matuda CHIS

Dioscorea densiflora Hemsl. CAM, CHIS, GRO, OAX, PUE, QROO, TAB, VER, YUC

*Dioscorea dugesii B.L. Rob. CHIS, COL, GTO, GRO, JAL, MEX, MICH, MOR, OAX, QRO, SLP

*Dioscorea fasciculocongesta (Sosa \& B.G. Schub.) O. Téllez PUE, VER

Dioscorea floribunda M. Martens \& Galeotti CAM, CHIS, GRO, OAX, PUE, QROO, TAB, VER, YUC

*Dioscorea galeottiana Kunth COL, CDMX, GTO, GRO, HGO, JAL, MEX, MICH, MOR, OAX, PUE, QRO, TLAX, VER

*Dioscorea gallegosii Matuda CHIS, GRO, JAL, MEX, MICH, MOR, OAX

Dioscorea gaumeri R. Knuth CAM, QROO, YUC

Dioscorea gomez-pompae O. Téllez CHIS, OAX, VER

*Dioscorea guerrerensis Matuda GRO, JAL, MEX, MICH

*Dioscorea hintonii R. Knuth GRO, JAL, MEX, MICH, MOR

Dioscorea hondurensis R. Knuth CHIS, OAX, TAB

Dioscorea howardiana O. Téllez, B.G. Schub. \& R. Geeta CHIS

* Dioscorea igualamontana Matuda GRO

* Dioscorea insignis C.V. Morton \& Schub. GRO, OAX

*Dioscorea jaliscana $\mathrm{S}$. Watson CHIH, COL, DGO, GRO, JAL, MEX, MICH, NAY, OAX, QRO, SLP, SIN, SON, TAMS, VER, ZAC

*Dioscorea juxtlahuacensis (O. Téllez \& Dávila) Caddick \& Wilkin GRO, OAX

Dioscorea lepida C.V. Morton CHIS

*Dioscorea liebmannii Uline AGS, CHIS, COL, GRO, JAL, MEX, MICH, MOR, NAY, OAX, SIN, VER, ZAC

*Dioscorea longirhiza Caddick \& Wilkin GRO, OAX

*Dioscorea longituba Uline GRO, MEX, MICH, NAY

Dioscorea matagalpensis Uline CAM, CHIS, QROO, TAB, YUC

*Dioscorea matudae O. Téllez \& B.G. Schub. HGO, QRO, SLP

*Dioscorea mcvaughii B.G. Schub. JAL, NAY, ZAC

*Dioscorea megaphylla Ramírez-Amezcua, O. Téllez \& V.W. Steinm. MICH

*Dioscorea mesoamericana O. Téllez \& Mart.-Rodr. CHIS, OAX

Dioscorea mexicana Scheidw. CAM, CHIS, COL, GRO, HGO, JAL, MEX, MICH, NLE, OAX, PUE, QRO, SLP, TAB, VER, YUC, ZAC

*Dioscorea militaris B.L. Rob. AGS, COL, DGO, GTO, HGO, JAL, MEX, MICH, MOR, NAY, OAX, PUE, QRO, SLP, TAMS, VER, ZAC

*Dioscorea minima B.L. Rob. \& Seaton COL, DGO, GRO, JAL, MICH, NAY, OAX, QRO, SIN, ZAC 
*Dioscorea mitis C.V. Morton COL, JAL, MICH

*Dioscorea morelosana (Uline) Matuda COL, GRO, JAL, MEX, MICH, MOR

Dioscorea nelsonii Uline ex R. Knuth CHIS, DGO, GRO, HGO, JAL, MEX, MICH, MOR, NAY, OAX, SIN, VER, ZAC

*Dioscorea nematodes Uline ex R. Knuth COL, GTO, JAL

*Dioscorea oaxacensis Uline OAX

*Dioscorea omiltemensis O. Téllez GRO

*Dioscorea oreodoxa B.G. Schub. COL, JAL

*Dioscorea orizabensis Uline COL, JAL, PUE, VER

*Dioscorea palmeri R. Knuth COL, JAL, MICH, NAY

*Dioscorea pallens Schltdl. PUE, SLP, TAMS, VER

*Dioscorea pantojensis R. Knuth GRO, MEX

Dioscorea pilosiuscula Bertero ex Spreng. CAM, CHIS, MICH, OAX, QROO, VER, YUC

*Dioscorea platycolpota Uline GRO, MEX, MICH, MOR, OAX

*Dioscorea plumifera B.L. Rob. COL, DGO, GRO, JAL, MEX, MICH, NAY, OAX, SIN

Dioscorea polygonioides Humb. \& Bonpl. ex Willd. CAM, CHIS, GRO, JAL, MEX, MICH, OAX, QROO, VER, YUC

*Dioscorea preslii Steud. GRO, JAL, MICH, OAX

*Dioscorea pringlei B.L. Rob. COL, JAL, MEX, MICH

* Dioscorea pumicicola Uline MEX, MOR

Dioscorea racemosa (Klotzsch) Uline CHIS, OAX

*Dioscorea remotiflora Kunth CHIS, CHIH, COL, DGO, GTO, GRO, HGO, JAL, MEX, MICH, MOR, NAY, OAX, PUE, SLP, SIN, SON, TAB, TAMS, ZAC

* Dioscorea reversiflora Uline DGO, NAY, SIN

*Dioscorea sanchez-colinii Matuda MEX, OAX

*Dioscorea sessiliflora McVaugh JAL, NAY

*Dioscorea sparsiflora Uline CHIS, COL, GRO, JAL, MEX, MICH, MOR, NAY, OAX, SIN, ZAC

Dioscorea spiculiflora Hemsl. CAM, CHIS, DGO, MICH, NAY, OAX, PUE, QROO, SLP, TAB, TAMS, VER, YUC

*Dioscorea spiculoides Matuda OAX

Dioscorea subtomentosa Miranda CHIS, COL, GRO, JAL, MEX, MICH, MOR, NAY, OAX, SIN

Dioscorea sumiderensis B.G. Schub. \& O. Téllez CHIS

*Dioscorea tacanensis Lundell CHIS

*Dioscorea tancitarensis Matuda MICH

*Dioscorea temascaltepecensis R. Knuth GRO, MEX, MICH

*Dioscorea tolucana (Matuda) Caddick \& Wilkin CHIH, COL, DGO, GTO, JAL, MEX, MICH, NAY, OAX, SIN, ZAC

*Dioscorea tubiperianthia Matuda GRO, MEX, MICH

*Dioscorea ulinei Greene COL, GTO, GRO, HGO, JAL, MEX, MICH, MOR, NAY, OAX, QRO, SIN, TAMS, VER

*Dioscorea urceolata Uline GRO, MEX, MICH, MOR, OAX, PUE

Dioscorea urophylla Hemsl. OAX, VER

*Dioscorea uruapanensis Matuda MEX, MICH, SIN

\section{Family Dipentodontaceae}

Perrottetia longistylis Rose CHIS, COL, DGO, GRO, HGO, JAL, MEX, MICH, MOR, NAY, OAX, PUE, SIN, VER

Perrottetia ovata Hemsl. CHIS, COL, DGO, GRO, HGO, JAL, OAX, PUE, QRO, SLP, SIN, VER

\section{Family Droseraceae}

Drosera brevifolia Pursh CHIS, OAX

Drosera capillaris Poir. CHIS, OAX, QROO, TAB, VER

\section{Family Ebenaceae}

*Diospyros acapulcensis Kunth CAM, GRO, QROO, VER

*Diospyros aequoris Standl. COL, DGO, JAL, MICH, NAY, OAX, SIN

*Diospyros alisu B. Walln. OAX

Diospyros anisandra S.F. Blake CAM, CHIS, QROO, TAB, YUC

*Diospyros blepharophylla Standl. ND

Diospyros bumelioides Standl. CAM, CHIS, QROO, TAB, YUC

Diospyros californica (Brandegee) I.M. Johnst. BCS, DGO, SIN, SON

Diospyros campechiana Lundell CAM, CHIS, COL, JAL, OAX, QROO, TAB, VER, YUC

*Diospyros camposii Provance \& A.C. Sanders OAX

Diospyros conzattii Standl. CHIS, HGO, OAX, PUE, QRO,

SLP, TAMS, VER

Diospyros cuneata Standl. CAM, CHIS, QROO, YUC

Diospyros ebenaster Retz. CHIS, GRO, HGO, JAL, MEX,

MICH, MOR, OAX, QRO, TAB, VER, YUC

*Diospyros intricata (A. Gray) Standl. BCS

* Diospyros johnstoniana Standl. \& Steyerm. GRO

*Diospyros latifolia Gürke COL, DGO, JAL, MICH, OAX, SIN

Diospyros morenoi A. Pool OAX

*Diospyros oaxacana Standl. CAM, COL, GRO, JAL, MEX, MICH, OAX, QROO, VER, YUC

*Diospyros palmeri Eastw. GTO, HGO, NLE, QRO, SLP, TAMS, VER

*Diospyros reinae B. Walln. SON

* Diospyros rekoi Standl. GRO, MEXOAX

*Diospyros riojae Gómez-Pompa GRO, HGO, MICH, PUE, QRO, SLP, TAMS, VER

Diospyros salicifolia Humb. \& Bonpl. ex Willd. CAM, CHIS, COL, GRO, JAL, MEX, MICH, MOR, NAY, OAX, QROO, TAB, VER, YUC

*Diospyros sonorae Standl. CHIH, SIN, SON

*Diospyros sphaerantha Standl. COL, GRO, JAL, MICH, NAY, SIN

Diospyros tetrasperma Sw. CAM, CHIS, QROO, YUC

Diospyros texana Scheele CHIH, COAH, DGO, NLE, SLP, TAMS

*Diospyros torresii Provance \& A.C. Sanders OAX

*Diospyros tuxtlensis Provance \& A.C. Sanders VER

*Diospyros xolocotzii Madrigal \& Rzed. GTO, MEX, MICH, QRO, SLP

Diospyros yatesiana Standl. CAM, CHIS, MICH, QROO, TAB, YUC

\section{Family Ehretiaceae}

*Bourreria andrieuxii (DC.) Hemsl. CHIS, GRO, MEX, MICH, MOR, OAX, PUE

Bourreria formosa (DC.) Hemsl. OAX

*Bourreria hintonii I.M. Johnst. GRO, JAL, MICH, OAX

Bourreria huanita (Lex.) Hemsl. CAM, CHIS, COL, GRO, JAL, MEX, MICH, OAX, QROO, TAB 
*Bourreria longiflora I.M. Johnst. MICH

Bourreria motaguensis Véliz, Campos-Ríos \& J.S. Mill. CHIS, OAX

*Bourreria obovata Eastw. GRO, OAX, PUE

Bourreria ovata Miers OAX, QROO, YUC

Bourreria oxyphylla Standl. CAM, CHIS, QROO, TAB, YUC

Bourreria pulchra (Millsp.) Greenm. CAM, CHIS, QROO, YUC

Bourreria purpusii Brandegee CHIS, COL, GRO, JAL, MICH, OAX

*Bourreria rekoi Standl. OAX

* Bourreria rowellii I.M. Johnst. GRO

* Bourreria rubra E.J. Lott \& J.S. Miller COL, JAL

* Bourreria sonorae S. Watson BCS, GRO, SON

* Bourreria spathulata (Miers) Hemsl. GRO, JAL, MEX, MICH,

NAY, OAX, PUE, ZAC

Bourreria succulenta Jacq. QROO

Bourreria superba I.M. Johnst. COL, GRO, JAL, MICH, NAY, SIN

Bourreria tuxtlae Campos-Ríos \& F. Chiang CHIS

*Bourreria veracruzana Campos-Ríos \& F. Chiang VER

Ehretia anacua (Terán \& Berland.) I.M. Johnst. COAH, GTO, GRO, HGO, JAL, MICH, NLE, QRO, SLP, TAMS, VER

Ehretia latifolia DC. CAM, CHIS, COL, GTO, GRO, HGO, JAL, MEX, MICH, MOR, OAX, PUE, QRO, QROO, TLAX, VER

Ehretia tinifolia L. CAM, CHIS, CHIH, GRO, HGO, JAL, MEX, MICH, MOR, NAY, OAX, PUE, QRO, QROO, SLP, SIN, SON, TAB, TAMS, VER, YUC

Lennoa madreporoides Lex. AGS, CHIS, CHIH, COL, CDMX, GTO, GRO, HGO, JAL, MEX, MICH, MOR, NAY, OAX, PUE, QRO, SLP, SIN, SON, TLAX, VER, YUC, ZAC

Pholisma arenarium Nutt. ex Hook. BCN

*Pholisma culiacanum (Dressler \& Kuijt) Yatskievych CHIH, SIN, SON

Pholisma sonorae (Torr. ex A. Gray) Yatskievych BCN, SIN, SON

Rochefortia lundellii Camp CAM, CHIS, HGO, QROO, VER

Rochefortia spinosa (Jacq.) Urb. CAM, CHIS, NAY, QROO, TAMS, VER

Tiquilia canescens (DC.) A.T. Richardson BCN, BCS, CHIH, COAH, DGO, GTO, HGO, NLE, OAX, PUE, QRO, SLP, SON, TAMS, VER, ZAC

*Tiquilia cuspidata (I.M. Johnst.) A.T. Richardson BCN, BCS, SON

Tiquilia gossypina (Wooton \& Standl.) A.T. Richardson CHIH, COAH, DGO, NLE

Tiquilia greggii (Torr. \& A. Gray) A.T. Richardson $\mathrm{CHIH}$, COAH, DGO, GTO, JAL, NLE, SLP, SON, ZAC

Tiquilia hispidissima (Torr. \& A. Gray) A.T. Richardson CHIH, COAH, DGO, SLP, TAMS

Tiquilia mexicana (S. Watson) A.T. Richardson CHIH, COAH, GTO, HGO, JAL, NLE, QRO, SLP, SON, ZAC

Tiquilia palmeri (A. Gray) A.T. Richardson BCN, SON

Tiquilia plicata (Torr.) A.T. Richardson BCN, SON

*Tiquilia purpusii (Brandegee) A.T. Richardson COAH, GTO, HGO, NLE, QRO, SLP, TAMS, ZAC
*Tiquilia tuberculata A.T. Richardson COAH, NLE

*Tiquilia turneri A.T. Richardson COAH, NLE

\section{Family Elaeocarpaceae}

*Sloanea ahuatoso Lozada-Pérez CHIS, GRO

Sloanea ampla I.M. Johnst. CHIS

Sloanea cruenta Lundell CHIS, OAX

Sloanea medusula K. Schum. \& Pittier CHIS, OAX, VER

Sloanea meianthera Donn. Sm. CHIS, OAX, VER

* Sloanea mexicana Standl. COL, GRO, JAL, MICH, TAB

Sloanea petenensis Standl. \& Steyerm. CHIS, OAX, TAB, VER

Sloanea schippii Standl. CHIS

Sloanea terniflora (Sessé \& Moc. ex DC.) Standl. CHIS, COL, DGO, GRO, JAL, NAY, OAX, TAB, VER

Sloanea tuerckheimii Donn. Sm. CHIS, OAX, TAB, VER

\section{Family Elatinaceae}

Bergia capensis L. OAX

Bergia texana (Hook.) Seub. BCN, BCS, NLE, SIN, SON, TAMS

Elatine brachysperma A. Gray AGS, BCN, CHIS, GTO, HGO, JAL, MEX, MICH, OAX, QRO, SLP, ZAC Elatine californica A. Gray BCN

\section{Family Ericaceae}

Agarista mexicana (Hemsl.) Judd AGS, CHIS, COAH, GRO, HGO, JAL, MEX, MICH, NAY, NLE, OAX, PUE, QRO, SLP, TAMS, VER, ZAC

*Agarista sleumeri Judd HGO, PUE, VER

*Agarista villarrealana L.M. González JAL

*Andromeda squamulosa D. Don CHIS, VER

Arbutus arizonica (A. Gray) Sarg. AGS, CHIH, COAH, COL, DGO, GTO, JAL, MEX, MICH, NAY, NLE, QRO, SLP, SIN, SON, TAMS, ZAC

*Arbutus bicolor S. González, M. González \& P.D. Sorensen CHIH, CDMX, DGO, GTO, HGO, MEX, MICH, PUE, QRO, SLP, SIN

*Arbutus madrensis S. González DGO, JAL, NAY, SLP, SIN, ZAC

*Arbutus occidentalis McVaugh \& Rosatti AGS, CHIH, COL, DGO, GTO, JAL, MICH, NAY, SIN, ZAC

*Arbutus tessellata P.D. Sorensen CHIH, COL, CDMX, DGO, GTO, HGO, JAL, MEX, MICH, NAY, QRO, SLP, SIN, TAMS, TLAX, VER, ZAC

Arbutus xalapensis Kunth AGS, BCS, CHIS, CHIH, COAH, COL, CDMX, DGO, GTO, GRO, HGO, JAL, MEX, MICH, MOR, NAY, NLE, OAX, PUE, QRO, SLP, SIN, SON, TAMS, TLAX, VER, ZAC

*Arctostaphylos angustifolia (Klotzsch) Hemsl. JAL, MICH

*Arctostaphylos australis Eastw. BCN

*Arctostaphylos bolensis P.V. Wells BCN

Arctostaphylos drupacea (Parry) Standl. BCN, SON

Arctostaphylos glandulosa Eastw. BCN, MOR, OAX

Arctostaphylos glauca Lindl. BCN

Arctostaphylos incognita J. E. Keeley, Massihi, J. Delgad. \& Hirales BCN

*Arctostaphylos lanata (Small) Standl. DGO, QRO, TAMS

*Arctostaphylos lucida (Small) Standl. OAX, VER 
*Arctostaphylos moranii P.V. Wells BCN

Arctostaphylos otayensis Wiesl. \& Schreiber BCN

Arctostaphylos patula Greene BCN

Arctostaphylos peninsularis Wells BCN

Arctostaphylos pungens Kunth AGS, BCN, CHIS, CHIH, COAH, COL, CDMX, DGO, GTO, HGO, JAL, MEX, MICH, MOR, NAY, NLE, OAX, PUE, QRO, SLP, SIN, SON, TAMS, TLAX, VER, ZAC

Arctostaphylos pyrifolia (Donn. Sm.) Standl. \& Steyerm. CHIS, GRO, OAX

Bejaria aestuans Mutis ex L. CHIS, COL, DGO, GRO, HGO, JAL, MICH, NAY, OAX, PUE, QRO, SIN, VER, ZAC

Bejaria guatemalensis Camp CHIS, OAX

Bejaria mexicana Benth. CHIS, DGO, GRO, JAL, MICH, NAY, OAX, SIN, VER, ZAC

Cavendishia bracteata (Ruiz \& Pav. ex J. St.-Hil.) Hoerold CHIS, OAX, VER

Cavendishia callista Donn. Sm. CHIS

Cavendishia crassifolia (Benth.) Hemsl. CHIS, OAX, VER

Cavendishia guatemalensis Loes. CHIS

* Cavendishia latifolia Hemsl. CHIS, TAB

Cavendishia laurifolia (Klotzsch) Benth. \& Hook. CHIS

Comarostaphylis arbutoides Lindl. CHIS, GRO, JAL, OAX, QRO, TAMS

Comarostaphylis discolor (Hook.) Diggs CHIS, COL, CDMX, DGO, GRO, HGO, JAL, MEX, MICH, MOR, OAX, PUE, QRO, SLP, TLAX, VER, ZAC

Comarostaphylis diversifolia (Parry) Greene BCN

*Comarostaphylis glaucescens (Kunth) Zucc. ex Klotzsch AGS, COL, DGO, GTO, GRO, HGO, JAL, MEX, MICH, MOR, NAY, OAX, QRO, SLP, SIN, ZAC

*Comarostaphylis longifolia (Benth.) Klotzsch CHIS, COL, GRO, HGO, JAL, MEX, MICH, OAX, QRO

*Comarostaphylis macvaughii (Diggs) L.M. González COL, JAL

*Comarostaphylis mucronata Klotzsch GTO, HGO, QRO, SLP

*Comarostaphylis polifolia (Kunth) Zucc. ex Klotzsch AGS, CHIH, COAH, DGO, GTO, GRO, HGO, JAL, MEX, MICH, MOR, NAY, NLE, OAX, PUE, QRO, SLP, SIN, SON, TAMS, TLAX, VER, ZAC

* Comarostaphylis sharpii Dorr \& Diggs TAMS

* Comarostaphylis spinulosa (M. Martens \& Galeotti) Diggs GRO, OAX, PUE, QRO, SLP

Chimaphila maculata (L.) Pursh CHIS, CHIH, COAH, COL, DGO, GTO, GRO, HGO, JAL, MEX, MOR, NLE, OAX, PUE, QRO, SLP, SIN, SON, TAMS, VER, ZAC

Chimaphila mensiezii (R. Br.) Spreng. BCN

Chimaphila umbellata (L.) W.P.C. Barton CHIS, CHIH, COAH, CDMX, DGO, GTO, GRO, HGO, JAL, MEX, MICH, NLE, OAX, PUE, QRO, SLP, SIN, SON, TAMS, TLAX, VER

Disterigma humboldtii (Klotzsch) Nied. CHIS, OAX

Gaultheria acuminata Schltdl. \& Cham. CHIS, GRO, HGO, MOR, OAX, PUE, QRO, VER

* Gaultheria angustifolia Brandegee JAL, MEX, MICH

Gaultheria erecta Vent. AGS, CHIS, CHIH, COL, CDMX, DGO, GTO, GRO, HGO, JAL, MEX, MICH, MOR, OAX, PUE, QRO, SLP, SIN, SON, TLAX, VER
*Gaultheria glaucifolia Hemsl. CHIS, DGO, JAL, NAY, SON, ZAC

Gaultheria hartwegiana Klotzsch ex Loes. CHIS

* Gaultheria ovata DC. HGO, VER

* Gaultheria parvifolia Small OAX, PUE

* Gaultheria pringlei Camp VER

* Gaultheria rigida Kunth GRO

* Gaultheria schultesii Camp OAX

* Leucothoe acuminata (Aiton) D. Don HGO, VER

Leucothoe pinetorum Standl. \& L.O. Williams CHIS, GRO, JAL

Lyonia ferruginea (Walt.) Nutt. CHIS, HGO, OAX, PUE, VER Lyonia squamulosa M. Martens \& Galeotti CHIS, COAH, GRO, HGO, NLE, OAX, PUE, QRO, SLP, TAMS, VER

Macleania insignis M. Martens \& Galeotti CHIS, OAX, VER Monotropa hypopitys L. AGS, CHIS, CHIH, COAH, COL, CDMX, DGO, GTO, GRO, HGO, JAL, MEX, MICH, NLE, OAX, PUE, QRO, SLP, SIN, SON, TAMS, TLAX, VER, ZAC Monotropa uniflora L. CHIS, CHIH, CDMX, DGO, HGO, JAL, MEX, MICH, MOR, OAX, PUE, SLP, SIN, TLAX, VER

Ornithostaphylos oppositifolia (Parry) Small BCN

Orthaea brachysiphon (Sleumer) Luteyn OAX

* Orthaea stipitata (Luteyn) Luteyn OAX

Orthilia secunda (L.) House CHIS, CHIH, COAH, CDMX, DGO, GTO, HGO, JAL, MEX, MICH, MOR, NLE, SON, TLAX, VER

Pernettya ciliata (Schltdl. \& Cham.) Small CHIS, CDMX, DGO, GTO, GRO, HGO, JAL, MEX, MICH, MOR, NAY, OAX, QRO, SLP, SIN, TAMS, TLAX, VER, ZAC

Pernettya prostrata (Cav.) DC. CHIS, COL, CDMX, DGO, GTO, GRO, HGO, JAL, MEX, MICH, MOR, OAX, PUE, QRO, SLP, SIN, TLAX, VER, ZAC

Pernettya saxicola Standl. \& Steyerm. CHIS

Pterospora andromedea Nutt. BCN, CHIH, COAH, CDMX, DGO, HGO, MEX, MOR, NLE, OAX, PUE, SON, TLAX, VER

Pyrola angustifolia (Alef.) Hemsl. CHIS, CDMX, JAL, MEX, OAX, PUE, TLAX, VER, ZAC

Pyrola picta $\mathrm{Sm}$. BCN

Sarcodes sanguinea Torr. BCN

Satyria elongata A.C. Sm. CHIS, OAX

Satyria meiantha Donn. Sm. CHIS, VER

Satyria panurensis (Benth. ex Meisn.) Hook. f. ex Nied. CHIS, OAX, VER

Satyria warszewiczii Klotzsch CHIS, OAX, VER Sphyrospermum cordifolium Benth. CHIS, OAX

Vaccinium breedlovei L.O. Williams CHIS

Vaccinium caespitosum Michx. CHIH, GTO, QRO

Vaccinium confertum Kunth CHIS, CHIH, COL, DGO, GTO, GRO, HGO, JAL, MEX, MICH, OAX, PUE, QRO, SLP, SIN, SON, TLAX, VER

Vaccinium consanguineum Klotzsch CHIS, GRO, OAX

Vaccinium cordifolium (M. Martens \& Galeotti) Hemsl. CHIS, OAX, VER

Vaccinium geminiflorum Kunth CHIS, CHIH, CDMX, DGO, GTO, HGO, JAL, MEX, MICH, MOR, NLE, OAX, PUE, TAMS, VER, ZAC 
*Vaccinium kunthianum Klotzsch COAH, GTO, HGO, NLE, PUE, SLP, TAMS, VER

Vaccinium leucanthum Schltdl. CHIS, GTO, GRO, HGO, JAL, MEX, MICH, OAX, PUE, QRO, SLP, VER, ZAC

Vaccinium lundellianum L.O. Williams CHIS

Vaccinium minarum Standl. \& Steyerm. CHIS

Vaccinium selerianum (Loes.) Sleumer CHIS, OAX

Vaccinium stamineum L. GTO, QRO, SLP

Vaccinium stenophyllum Steud. CHIS, COL, DGO, GRO, HGO,

JAL, MICH, NAY, OAX, SIN, VER, ZAC

*Vaccinium wilburii Almeda \& Breedlove GRO, OAX

Xylococcus bicolor Nutt. BCN, BCS

\section{Family Eriocaulaceae}

Eriocaulon benthamii Kunth CHIS, CHIH, CDMX, DGO, GTO, GRO, HGO, JAL, MEX, MICH, MOR, NAY, OAX, PUE, TAB, TLAX, VER, ZAC

Eriocaulon bilobatum Morong CAM, CHIS, CHIH, DGO, GTO, JAL, QRO, QROO

*Eriocaulon capitulatum Moldenke HGO, VER

Eriocaulon decangulare L. CAM, MICH, TAB, VER

*Eriocaulon jaliscanum S. Watson AGS, DGO, JAL, MEX, $\mathrm{MICH}, \mathrm{NAY}, \mathrm{QRO}, \mathrm{ZAC}$

Eriocaulon microcephalum Kunth BCN, BCS, CDMX, DGO, HGO, MEX, MICH, MOR, PUE, TAMS, VER

*Eriocaulon pringlei $\mathrm{S}$. Watson $\mathrm{CHIH}$

*Eriocaulon schiedeanum Körn. AGS, CHIS, DGO, JAL, MEX, MICH, NAY, QRO, VER, ZAC

Eriocaulon schippii Standl. ex Moldenke CAM, QROO, TAB

Eriocaulon seemannii Moldenke CAM, TAB, VER

*Paepalanthus chiapensis Moldenke CHIS

*Paepalanthus mellii Moldenke VER

*Syngonanthus davidsei Huft CHIS

Tonina fluviatilis Aubl. TAB, VER

\section{Family Erythroxylaceae}

Erythroxylum areolatum L. CAM, CHIS, HGO, QRO, QROO, SLP, TAB, VER, YUC

Erythroxylum becquaertii Standl. CAM, CHIS, GRO, OAX, QROO, YUC

Erythroxylum confusum Britton CAM, CHIS, QROO, YUC

Erythroxylum guatemalense Lundell CAM, CHIS, QROO, TAB, YUC

Erythroxylum havanense Jacq. CHIS, COL, GRO, HGO, JAL, MEX, MICH, MOR, NAY, OAX, PUE, SLP, SIN, SON, VER, YUC

Erythroxylum macrophyllum Cav. CHIS, OAX, TAB, VER

*Erythroxylum mexicanum Kunth CHIS, CHIH, COL, DGO, GRO, JAL, MEX, MICH, NAY, OAX, PUE, SLP, SIN, SON, TAMS, VER

Erythroxylum panamense Turcz. VER

*Erythroxylum pringlei Rose COL, GRO, JAL, MEX, MICH, MOR, OAX

Erythroxylum rotundifolium Lunan CAM, CHIS, CHIH, COL, DGO, GRO, JAL, MEX, MICH, MOR, NAY, OAX, PUE, QROO, SLP, VER, YUC, ZAC

\section{Family Euphorbiaceae}

Acalypha adenostachya Müll. Arg. AGS, BCS, CAM, CHIS, CHIH, COAH, COL, CDMX, DGO, GTO, GRO, HGO, JAL, MEX, MICH, MOR, NAY, NLE, OAX, PUE, QRO, SLP, SIN, SON, TAMS, TLAX, VER, YUC, ZAC

Acalypha aliena Brandegee BCS, CHIH, GTO, OAX, QROO, SIN, SON, YUC

Acalypha alopecuroidea Jacq. CAM, CHIS, CHIH, COL, GRO, JAL, MEX, MICH, MOR, NAY, OAX, QROO, SLP, SIN, SON, TAB, VER, YUC, ZAC

*Acalypha anadenia Standl. GRO

Acalypha arvensis Poepp. \& Endl. CAM, CHIS, COL, GRO, JAL, MEX, MOR, OAX, PUE, QRO, QROO, SLP, TAB, TAMS, VER, YUC

*Acalypha botteriana Müll. Arg. CAM, CHIS, HGO, MEX, MICH, MOR, OAX, PUE, QRO, VER

*Acalypha brevicaulis Müll. Arg. AGS, HGO, JAL, MICH, QRO, SLP, VER, ZAC

*Acalypha burquezii V.W. Steinm. \& Felger CHIH, SIN, SON

Acalypha californica Benth. BCN, BCS, SIN, SON

*Acalypha capitellata Brandegee CHIS

*Acalypha cincta Müll. Arg. CHIH, COL, GRO, JAL, MEX, MICH, NAY, SIN, SON, ZAC

*Acalypha cinerea Pax \& K. Hoffm. OAX

*Acalypha coleispica Pax \& K. Hoffm. ND

*Acalypha comonduana Millsp. BCN, BCS, JAL, MICH, SON

*Acalypha confertiflora Pax \& K. Hoffm. OAX

Acalypha conspicua Müll. Arg. VER

Acalypha costaricensis (Kuntze) Knobl. ex Pax \& K. Hoffm. CAM, CHIS, OAX

Acalypha cuspidata Jacq. GRO, MICH, OAX

*Acalypha chiapensis Brandegee CHIS

*Acalypha delgadoana McVaugh JAL

*Acalypha depauperata Müll. Arg. MEX, OAX

Acalypha depressa Sessé \& Moc. ND

*Acalypha dioica $\mathrm{S}$. Watson COAH, NLE, SLP, TAMS, ZAC

Acalypha diversifolia Jacq. CAM, CHIS, HGO, OAX, QRO, QROO, TAB, TAMS, VER, YUC

Acalypha euphrasiostachys Bartlett GRO, MEX

Acalypha ferdinandii K. Hoffm. CHIS, GRO, TAB

*Acalypha filipes (S. Watson) McVaugh BCS, COL, GRO, JAL, MICH, PUE, SIN, SON

Acalypha firmula Müll. Arg. CHIS, OAX

*Acalypha fournieri Müll. Arg. CHIS, COL, JAL, OAX, SLP, TAMS, VER

*Acalypha frederici Müll. Arg. CHIS, OAX, VER

*Acalypha gaumeri Pax \& K. Hoffm. CAM, QROO, YUC

*Acalypha gigantesca McVaugh JAL

*Acalypha glandulosa Cav. CHIS

*Acalypha grisea Pax \& K. Hoffm. COL, JAL, MICH, NAY

Acalypha gummifera Lundell CHIS

*Acalypha haploclada Pax \& K. Hoffm. OAX, VER

Acalypha havanensis Müll. Arg. CHIS, COL, GRO, JAL, MICH,

OAX, PUE, QRO, QROO, SLP, SIN, SON, VER, YUC

*Acalypha hypogaea S. Watson COL, GTO, JAL, MEX, MICH, PUE

*Acalypha karwinskii Müll. Arg. DGO, OAX

*Acalypha katharinae Pax OAX 
*Acalypha lagascana Müll. Arg. OAX

*Acalypha lagopus McVaugh MICH

Acalypha lancetillae Standl. QROO, VER

Acalypha langiana Müll. Arg. CHIS, COL, DGO, GRO, HGO, JAL, MEX, MICH, MOR, NAY, OAX, PUE, QRO, SLP, SIN, SON, VER

Acalypha laxiflora Müll. Arg. CHIS, OAX, PUE, SLP, TAMS, VER

Acalypha leptopoda Müll. Arg. CAM, CHIS, MICH, OAX, QROO, VER, YUC

*Acalypha liebmanniana Müll. Arg. OAX, VER

*Acalypha lignosa Brandegee GRO, OAX

*Acalypha lindeniana Müll. Arg. CHIS, PUE, VER

*Acalypha longestipularis Müll. Arg. OAX, VER

*Acalypha longispicata Müll. Arg. SLP, TAMS

Acalypha macrostachya Jacq. CAM, CHIS, CHIH, GRO, HGO, JAL, MEX, NLE, OAX, QRO, QROO, SLP, TAB, TAMS, VER, YUC

*Acalypha macrostachyoides Müll. Arg. CHIS, QRO, SLP, TAB, VER

*Acalypha melochiifolia Müll. Arg. PUE, VER

Acalypha mexicana Müll. Arg. CHIS, CHIH, CDMX, GTO, GRO, HGO, JAL, MEX, MICH, OAX, PUE, QRO, SON, TAB, TLAX, VER

*Acalypha microcephala Müll. Arg. HGO, OAX, VER

Acalypha microphylla Klotzsch CHIS, COL, JAL, MICH, NAY, OAX, SIN

*Acalypha microstachya Benth. OAX, VER

*Acalypha michoacanensis Sessé \& Moc. MICH

Acalypha mollis Kunth CHIS, COL, CDMX, GTO, GRO, HGO, JAL, MEX, MICH, MOR, NAY, OAX, QRO, SLP, TAMS, TLAX, VER, ZAC

Acalypha monostachya Cav. AGS, CHIS, CHIH, COAH, CDMX, DGO, GTO, GRO, HGO, JAL, MEX, MICH, NAY, NLE, OAX, PUE, QRO, SLP, TAMS, TLAX, VER, ZAC

Acalypha mortoniana Lundell CHIS, VER

*Acalypha multiflora (Standl.) Radcl. Sm. BCS, COL, GRO, JAL, MOR, NAY, OAX, PUE, QRO, SIN, SON, VER, ZAC

*Acalypha multispicata S. Watson JAL, MICH

Acalypha neomexicana Müll. Arg. AGS, CHIH, COAH, DGO, GTO, HGO, JAL, MEX, MICH, MOR, NLE, OAX, QRO, SLP, SIN, SON, VER, ZAC

*Acalypha nubicola McVaugh JAL, MICH, NAY

*Acalypha ocymoides Kunth COL, DGO, JAL, MICH, NAY, ZAC

*Acalypha oligantha Müll. Arg. VER

*Acalypha oligodonta Müll. Arg. OAX, VER

*Acalypha oreopola Greenm. GRO, VER

*Acalypha papillosa Rose CHIH, SIN, SON

Acalypha persimilis Müll. Arg. AGS, BCS, CHIH, COAH, COL, DGO, GTO, GRO, HGO, JAL, MEX, MICH, MOR, NAY, NLE, OAX, PUE, QRO, SLP, SIN, SON, TAB, TAMS, VER, ZAC Acalypha phleoides Cav. AGS, CHIS, CHIH, COAH, COL, CDMX, DGO, GTO, GRO, HGO, JAL, MEX, MICH, MOR, NAY, NLE, OAX, PUE, QRO, SLP, SIN, SON, TAMS, TLAX, VER, ZAC

*Acalypha pippenii McVaugh GRO, JAL, MICH
Acalypha poiretii Spreng. CAM, CHIS, COL, GRO, MEX, MICH, MOR, NLE, OAX, PUE, SLP, TAMS, VER, YUC Acalypha polystachya Jacq. CAM, CHIS, CHIH, GRO, JAL, NAY, OAX, QROO, SIN, SON, TAB, VER

*Acalypha pseudovagans Pax \& K. Hoffm. ND

*Acalypha purpurascens Kunth GTO, GRO, NLE, OAX, PUE, TAMS

*Acalypha purpusii Brandegee OAX

Acalypha radians Torr. SLP, TAMS, VER

*Acalypha rafaelensis Standl. SLP, TAMS

Acalypha rhombifolia Schltdl. MICH, VER

*Acalypha sabulicola Brandegee OAX

Acalypha salvadorensis Standl. COL, JAL, MICH, NAY

*Acalypha saxicola Wiggins BCS

Acalypha schiedeana Schltdl. CHIS, COL, GRO, JAL, MEX, MICH, NAY, OAX, QRO, SLP, SIN, TAMS, VER

Acalypha schlechtendaliana Müll. Arg. CAM, CHIS, HGO, OAX, PUE, QRO, QROO, SLP, TAB, TAMS, VER, YUC

*Acalypha schlumbergeri Müll. Arg. CHIS, VER

*Acalypha seleriana Greenm. CAM, OAX, QROO, TAB, YUC

*Acalypha sessilifolia $\mathrm{S}$. Watson JAL

Acalypha setosa A. Rich. CAM, CHIS, COL, GRO, JAL, MEX, MICH, MOR, NAY, OAX, QROO, SLP, TAB, TAMS, VER, YUC, ZAC

Acalypha skutchii I.M. Johnst. CHIS, OAX, SLP, TAB, VER

*Acalypha subterranea Paul G. Wilson GRO, MEX

*Acalypha subtomentosa Lag. MEX

*Acalypha synoica Pax \& K. Hoffm. HGO, OAX, PUE, QRO, VER

*Acalypha tacanensis Lundell CHIS

*Acalypha tamaulipasensis Lundell TAMS

Acalypha tenuicauda Pax \& Hoffm. CHIS, TAB

Acalypha trachyloba Müll. Arg. CHIS, GRO, OAX

Acalypha tricholoba Müll. Arg. CHIS, GRO, JAL, OAX

*Acalypha trilaciniata Paul G. Wilson JAL, MICH

Acalypha triloba Müll. Arg. COL, GRO, JAL, MEX, MOR, OAX

*Acalypha umbrosa Brandegee BCS, COL, JAL, NAY

*Acalypha vagans Cav. CHIS, CHIH, COL, GRO, JAL, MEX, MICH, NAY, OAX, QRO, SLP, TAMS, VER, ZAC

*Acalypha vallartae McVaugh JAL, NAY

*Acalypha verbenacea Standl. NAY

*Acalypha veronicoides Pax \& K. Hoffm. GRO, OAX

Acalypha villosa Jacq. CAM, CHIS, JAL, OAX, QROO, TAB,

TAMS, VER, YUC

*Acidocroton madrigalensis Hanan-Alipi \& V.W. Steinm. TAB Acidocroton spinosus (Standl.) G.L. Webster CHIS, COL, JAL, OAX, TAB, VER

Acidocroton steyermarkii (Standl.) G.L. Webster CHIS

Adelia barbinervis Schltdl. \& Cham. CAM, CHIS, COL, GTO, GRO, HGO, JAL, OAX, PUE, QRO, QROO, SLP, SIN, TAB, TAMS, VER, YUC

*Adelia brandegeei V.W. Steinm. BCS, SIN, SON

*Adelia cinerea (Wiggins \& Rollins) A. Cerv., V.W. Steinm. \& Flores-Olvera SON

*Adelia oaxacana (Müll. Arg.) Hemsl. CAM, CHIS, COL, GRO, HGO, JAL, MICH, OAX, PUE, QRO, QROO, SLP, SIN, 
TAMS, VER, YUC

*Adelia obovata Wiggins \& Rollins SON

Adelia triloba Hemsl. VER

Adelia vaseyi (J.M. Coult.) Pax \& Hoffm. NLE, SIN, SLP, TAMS, VER

*Alchornea chiapasana Miranda CHIS, VER

Alchornea latifolia $\mathrm{Sw}$. CHIS, COL, GTO, GRO, HGO, JAL,

NAY, OAX, PUE, QRO, QROO, SLP, TAB, TAMS, VER

*Argythamnia argentea Millsp. SLP

*Argythamnia coatepensis (Brandegee) Croizat OAX, PUE, VER

*Argythamnia lottiae J.W. Ingram. COL, JAL

*Argythamnia lundellii J.W. Ingram QROO, YUC

Argythamnia mercurialina (Nutt.) Müll. Arg. COAH

*Argythamnia moorei J.W. Ingram GRO

*Argythamnia sitiens (Brandegee) J.W. Ingram VER

Argythamnia tinctoria Millsp. CAM, QROO, YUC

*Argythamnia wheeleri J.W. Ingram QROO, YUC

Astraea lobata (L.) Klotzsch CAM, CHIS, COL, GRO, JAL, MICH, NAY, OAX, PUE, QRO, QROO, SLP, SIN, TAB, TAMS, VER, YUC

*Bernardia albida Lundell GTO, HGO, QRO, SLP, VER

*Bernardia chiangii A. Cerv. \& Flores-Olvera OAX, PUE

*Bernardia chiapensis Lundell CHIS

*Bernardia chinantlensis A. Cerv. \& Flores-Olvera OAX, VER Bernardia dodecandra (Sessé ex Cavagnaro) McVaugh CAM, CHIS, GRO, HGO, OAX, PUE, QRO, QROO, SLP, TAB, TAMS, VER

*Bernardia fonsecae A. Cerv. \& J. Jiménez Ram. GRO

* Bernardia gentryana Croizat COL, JAL, MICH, NAY, OAX, SIN, SON

*Bernardia heteropilosa McVaugh NAY

Bernardia incana C.V. Morton BCN

*Bernardia kochii McVaugh COL, JAL

*Bernardia lagunensis (M.E. Jones) L.C. Wheeler BCS

* Bernardia macrocarpa A. Cerv. \& Flores-Olvera PUE, VER

*Bernardia mcvaughii A. Cerv. \& Flores-Olvera JAL

Bernardia mexicana (Hook. \& Arn.) Müll. Arg. AGS, BCS, CAM, CHIS, COL, DGO, GTO, GRO, HGO, JAL, MEX, MICH, NAY, OAX, PUE, QRO, QROO, SLP, SIN, TAB, TAMS, VER, YUC

Bernardia mollis Lundell CHIS

Bernardia myricifolia (Scheele) S. Watson BCS, CHIH, COAH, DGO, JAL, NLE, OAX, PUE, SLP, SON, TAMS, VER, ZAC

Bernardia oblanceolata Lundell CHIS, OAX, QROO

Bernardia obovata I.M. Johnst. CHIH, COAH, DGO

*Bernardia ovalifolia Lundell DGO

* Bernardia rzedowskii A. Cerv. \& Flores-Olvera DGO

*Bernardia santanae McVaugh COL, JAL, MEX

Bernardia sidoides (Klotzsch) Müll. Arg. TAB, VER

*Bernardia spongiosa McVaugh COL, JAL, SIN

*Bernardia valdesii A. Cerv. \& Flores-Olvera COL, JAL

*Bernardia viridis Millsp. BCS, CHIH, COAH, SIN, SON

*Bernardia wilburii McVaugh COL, JAL

Bernardia yucatanensis Lundell CAM, CHIS, QROO, YUC Bia cordata (Baill.) G.L. Webster CHIS, OAX, TAB, VER

*Bia manuelii V.W. Steinm. \& Ramírez-Amezcua MICH
Caperonia castaneifolia (L.) A. St.-Hil. CAM, CHIS, JAL, MICH, OAX, PUE, QROO, TAB, TAMS, VER

*Caperonia chiltepecensis Croizat OAX

Caperonia palustris (L.) A. St.-Hil. CAM, CHIS, COL, GRO, JAL, MEX, MICH, MOR, NAY, OAX, PUE, QROO, SLP, SIN, TAB, TAMS, VER, YUC

Cleidion castaneifolium Müll. Arg. CHIS

Cnidoscolus aconitifolius (Mill.) I.M. Johnst. CAM, CHIS, GRO, HGO, JAL, MEX, MICH, MOR, OAX, PUE, QRO, QROO, SLP, TAB, TAMS, VER, YUC

*Cnidoscolus albidus Lundell HGO, QRO, SLP

Cnidoscolus angustidens Torr. BCN, BCS, CHIH, DGO, GRO, JAL, MEX, MICH, MOR, OAX, PUE, SLP, SIN, SON, TAMS, ZAC

*Cnidoscolus autlanensis Breckon COL, GRO, JAL

*Cnidoscolus egregius Breckon ex Fern. Casas OAX

*Cnidoscolus elasticus Lundell DGO, NAY, SIN

*Cnidoscolus liebmannii (Müll. Arg.) Lundell GRO, OAX, PUE, VER

*Cnidoscolus maculatus (Brandegee) Pax \& K. Hoffm. BCS

Cnidoscolus megacanthus Breckon OAX

*Cnidoscolus monicanus J.A. Lomelí, Sahagun \& V.W. Steinm. $\mathrm{MICH}$

Cnidoscolus multilobus (Pax) I.M. Johnst. CAM, CHIS, COAH, COL, DGO, GRO, HGO, JAL, MEX, MICH, MOR, NLE, OAX, PUE, QRO, QROO, SLP, SIN, TAB, TAMS, VER, YUC

*Cnidoscolus palmeri (S. Watson) Rose BCN, BCS, SON

*Cnidoscolus rostratus Lundell GRO, HGO, MEX, MICH, MOR, OAX, PUE, VER

*Cnidoscolus rotundifolius (Müll. Arg.) McVaugh COAH, NLE, SLP, TAMS, VER

*Cnidoscolus shrevei I.M. Johnst. COAH, DGO, ZAC

*Cnidoscolus sinaloensis Breckon ex Fern. Casas DGO, NAY, SIN

Cnidoscolus souzae McVaugh CAM, QROO, YUC

*Cnidoscolus spinosus Lundell COL, JAL, MICH, NAY, ZAC

*Cnidoscolus tehuacanensis Breckon OAX, PUE

*Cnidoscolus tepiquensis (Costantin \& Gallaud) McVaugh COL, JAL, NAY

Cnidoscolus texanus (Müll. Arg.) Small NLE, TAMS

Cnidoscolus tubulosus (Müll. Arg.) I.M. Johnst. CAM, CHIS, GRO, HGO, JAL, MICH, NAY, OAX, PUE, QRO, QROO, SLP, SIN, TAB, VER

Cnidoscolus urens (L.) Arthur CAM, CHIS, COL, GRO, JAL, MEX, MICH, MOR, OAX, PUE, QROO, SLP, TAB, TAMS, VER

*Croton acapulcensis Mart. Gord. \& J. Jiménez Ram. COL, GRO, JAL

Croton adspersus Benth. CHIS, COL, CDMX, GTO, GRO, HGO, JAL, MEX, MICH, MOR, NAY, OAX, QRO, SLP, VER *Croton alamosanus Rose CHIH, COL, DGO, GRO, JAL, MICH, NAY, OAX, SIN, SON, ZAC

*Croton ameliae Lundell CAM, QROO, YUC

*Croton amphileucus Briq. HGO, VER

Croton arboreus Millsp. CAM, CHIS, OAX, QROO, SLP, VER, YUC

Croton argenteus L. CAM, CHIS, GRO, HGO, MOR, NAY, 
OAX, PUE, QRO, QROO, SLP, SIN, TAB, TAMS, VER

Croton argyranthemus Michx. NLE, TAMS

*Croton atrostellatus V.W. Steinm. GTO, MICH

Croton axillaris Müll. Arg. CAM, CHIS, OAX, QRO, SLP, SIN, TAMS, VER, YUC

*Croton balsensis V.W. Steinm. \& Mart. Gord. GRO, MOR, OAX, PUE

Croton billbergianus Müll. Arg. CAM, CHIS, COL, JAL, NAY, OAX, TAB, VER

*Croton boregensis M.E. Jones BCS

*Croton breedlovei van Ee \& P.E. Berry CHIS

Croton californicus Müll. Arg. BCN, BCS, COAH, SIN, SON

Croton capitatus Michx. TAMS

*Croton carpostellatus B.L. León \& Mart. Gord. CHIS

Croton ciliatoglandulifer Ortega AGS, BCS, CAM, CHIS, CHIH, COAH, COL, DGO, GTO, GRO, HGO, JAL, MEX, MICH, MOR, NAY, NLE, OAX, PUE, QRO, QROO, SLP, SIN, SON, TAMS, VER, ZAC

Croton conspurcatus Schltdl. CAM, CHIS, COL, JAL, MEX, VER

Croton cortesianus Kunth CAM, CHIS, COAH, COL, GTO, GRO, HGO, MEX, NLE, OAX, PUE, QRO, QROO, SLP, SIN, TAMS, VER, YUC, ZAC

*Croton culiacanensis Croizat COL, JAL, SIN

*Croton cupuliferus McVaugh COL, JAL, NAY

*Croton chamelensis E.J. Lott COL, JAL, NAY

*Croton chiapensis Lundell CHIS

*Croton chichenensis Lundell CAM, QROO, YUC

Croton decalobus Müll. Arg. CHIS, OAX, VER

Croton dioicus Cav. AGS, CHIH, COAH, DGO, HGO, JAL, MEX, NAY, NLE, OAX, PUE, QRO, SLP, SIN, TAMS, TLAX, VER, ZAC

*Croton disjunctus V.W. Steinm. AGS, CHIH, ZAC

Croton draco Schltdl. \& Cham. CAM, CHIS, COL, DGO, GRO, HGO, JAL, MICH, MOR, NAY, OAX, PUE, QRO, QROO, SLP, SIN, TAB, TAMS, VER, YUC

*Croton ehrenbergii Schltdl. GTO, HGO, MEX, QRO, SLP, VER

Croton fantzianus F. Seym. CHIH, JAL, MICH, OAX, SON

Croton flavens L. CAM, CHIS, COAH, NLE, OAX, QROO, VER, YUC

*Croton flaviglandulosus Lundell TAB

Croton francoanus Müll. Arg. CHIS, GRO, OAX

Croton fruticulosus Torr. CHIH, COAH, DGO, HGO, NLE, QRO, SLP, TAMS

*Croton gaumeri Millsp. YUC

Croton glabellus L. CAM, CHIS, JAL, NAY, OAX, PUE, QROO, SLP, TAB, TAMS, VER, YUC

Croton glandulosepalus Millsp. CAM, CHIS, QROO, VER, YUC

Croton glandulosus L. CHIS, GRO, HGO, JAL, MEX, MICH, OAX, PUE, SIN, TAB, TAMS, VER

*Croton gomezii G.L. Webster OAX, PUE, QRO, SLP, VER

Croton gossypifolius Vahl CHIS, HGO, OAX, TAB, VER

*Croton grewiifolius Müll. Arg. CHIS, GRO, OAX

Croton guatemalensis Lotsy CAM, CHIS, COL, GRO, JAL, OAX, SLP, VER, YUC
*Croton guerreroanus Mart. Gord. \& Cruz-Durán GRO

*Croton gynopetalus Croizat OAX

*Croton heptalon (Kuntze) B.W. van Ee \& P.E. Berry TAMS

Croton hirtus L'Hér. CAM, CHIS, COL, GRO, JAL, MICH,

NAY, OAX, SIN, VER

Croton hoffmannii Müll. Arg. TAB

*Croton huajuapensis Mart. Gord. \& Cruz-Durán OAX

Croton humilis L. CAM, CHIS, HGO, NLE, QRO, QROO,

TAMS, VER, YUC

*Croton hypoleucus Schltdl. COAH, HGO, NLE, OAX, PUE, QRO, SLP, TAMS, VER

*Croton icche Lundell CAM, QROO, YUC

Croton incanus Kunth AGS, CHIH, COAH, DGO, GTO, HGO, JAL, MICH, NLE, OAX, PUE, QRO, SLP, TAMS, VER, ZAC

*Croton itzaeus Lundell CAM, QROO, YUC

*Croton jucundus Brandegee HGO, QRO, SLP, SIN, SON

Croton jutiapensis Croizat CAM, CHIS, QROO

Croton lasiopetaloides Croizat CHIS, OAX, PUE

Croton leucophyllus Müll. Arg. CHIH, COAH, DGO, NLE, TAMS

*Croton liebmannii Müll. Arg. OAX, PUE, VER

Croton lindheimerianus Scheele COAH, NLE, TAMS

*Croton lindquistii V.W. Steinm. JAL, MICH, OAX, SIN, SON

*Croton lucidus L. CAM, QROO, YUC

*Croton macrodontus Müll. Arg. OAX, PUE, SLP, VER

*Croton magdalenae Millsp. BCN, BCS, SON

Croton malvaviscifolius Millsp. CAM, CHIS, QROO, YUC

*Croton martinianus V.W. Steinm. JAL, SIN, SON, ZAC

*Croton masonii I.M. Johnst. COL

*Croton mayanus B.L. León \& Vester CAM, QROO

*Croton mazapensis Lundell CHIS, GTO, GRO, HGO, JAL, OAX, PUE, QRO, SLP, YUC, VER, ZAC

*Croton mcvaughii G.L. Webster JAL

*Croton meissneri Müll. Arg. VER

Croton mexicanus Müll. Arg. CHIS, OAX, TAB

*Croton michaelii V.W. Steinm. AGS, DGO, JAL, ZAC

*Croton millspaughii Standl. QROO, YUC

*Croton miradorensis Müll. Arg. CHIS, HGO, VER

Croton monanthogynus Michx. COAH, NLE, SLP, TAMS

Croton morifolius Willd. BCS, CAM, CHIS, CHIH, COL, DGO, GTO, GRO, HGO, JAL, MEX, MICH, MOR, NAY, NLE, OAX, PUE, QRO, QROO, SLP, SIN, SON, TAB, TAMS, VER, YUC, $\mathrm{ZAC}$

Croton neomexicanus Müll. Arg. CHIH, COAH, NLE

Croton nitens Sw. CAM, CHIS, QROO, TAB, VER, YUC

Croton niveus Jacq. CAM, CHIS, CHIH, COL, DGO, GTO,

GRO, HGO, JAL, MEX, MICH, MOR, NAY, NLE, OAX, PUE,

QRO, QROO, SLP, SIN, SON, TAMS, VER, YUC, ZAC

Croton oerstedianus Müll. Arg. CAM, CHIS, OAX, QROO,

YUC

Croton ortholobus Müll. Arg. CHIS, QROO

Croton ovalifolius Vahl OAX

*Croton palmeri $\mathrm{S}$. Watson COAH

*Croton pascualii E.J. Lott \& M. Mart.-Gord. OAX

Croton pedicellatus Kunth CAM, CHIS, CHIH, COL, DGO,

JAL, MEX, MICH, NAY, QRO, SLP, SIN, SON

*Croton pendens Lundell CHIS 
*Croton peraeruginosus Croizat CAM, QROO, YUC

Croton pottsii (Klotzsch) Müll. Arg. AGS, CHIH, COAH, DGO, GTO, HGO, JAL, NLE, QRO, SLP, SIN, SON, TAMS, VER, ZAC

*Croton pseudoglabellus Lundell QROO, YUC

Croton pseudoniveus Lundell CHIS, CHIH, COL, JAL, OAX, SIN, SON

*Croton pulcher Müll. Arg. OAX, PUE

Croton punctatus Jacq. CAM, QROO, SLP, SIN, TAB, TAMS, VER, YUC

Croton quercetorum Croizat CHIS

*Croton ramillatus Croizat CAM, GRO, MEX, MOR, OAX, VER

Croton reflexifolius Kunth CAM, CHIS, COL, CDMX, GTO, GRO, HGO, JAL, MEX, NAY, OAX, PUE, QRO, QROO, SLP, SIN, TAB, TAMS, VER, YUC

Croton repens Schltdl. CAM, CHIS, DGO, GRO, JAL, MEX, MICH, NAY, OAX, QROO, SIN, TAB, VER

*Croton rosarianus Mart.-Gord. \& Cruz-Durán PUE, VER

*Croton roxanae Croizat COL, GRO, JAL, MICH, NAY, SIN

*Croton sancti-lazari Croizat CHIH, COAH, DGO, SLP, ZAC

Croton schiedeanus Schltdl. CAM, CHIS, NAY, OAX, QROO,

SLP, TAB, VER, YUC

*Croton siltepecensis Lundell CHIS

Croton soliman Cham. \& Schltdl. HGO, OAX, PUE, QRO, SLP,

TAB, TAMS, VER

Croton sonorae Torr. BCN, BCS, DGO, GRO, NAY, OAX,

PUE, SIN, SON, VER

*Croton sousae Mart. Gord. \& Cruz-Durán VER

*Croton stenopetalus G.L. Webster COL, JAL

*Croton stipulaceus Kunth MEX, OAX, PUE, SIN, VER

*Croton stylosus Müll. Arg. ND

Croton suaveolens Torr. CHIH, COAH, DGO, HGO, NLE,

QRO, SLP, TAMS

*Croton suberosus Kunth COL, GRO, JAL, MICH, NAY, OAX,

PUE

Croton subfragilis Müll. Arg. CAM, CHIS, QROO

*Croton sutup Lundell CAM, QROO, YUC

Croton tabascensis Lundell CHIS, TAB

Croton texensis (Klotzsch) Müll. Arg. CHIH, SON

*Croton tremulifolius Croizat COL, GRO

Croton trinitatis Millsp. CHIS, OAX, SIN, TAB, VER

*Croton varelae V.W. Steinm. DGO, NAY, SIN

Croton verapazensis Donn. Sm. CHIS

*Croton virletianus Müll. Arg. NLE, SLP, TAMS

*Croton volubilis L. NAY

*Croton watsonii Standl. GRO, NAY, OAX, SLP, SIN, TAMS, VER

*Croton websterii Mart. Gord. \& J. Jiménez Ram. GRO

Croton wigginsii L.C. Wheeler BCN, SON

Croton xalapensis Kunth CHIS, JAL, MICH, OAX, PUE, SLP,

TAB, TAMS, VER

*Croton yecorensis V.W. Steinm. \& Felger CHIH, SON

*Croton ynesae Croizat COL, JAL, NAY

Croton yucatanensis Lundell CHIS, OAX, YUC

*Chiropetalum astroplethos (J.W. Ingram) Radcl. Sm. \& R.

Govaerts COAH, COL, HGO, JAL, NLE, QRO, SLP, SIN,
TAMS, VER

*Chiropetalum schiedeanum (Müll. Arg.) Pax COAH, DGO, GTO, HGO, PUE, QRO, SLP, TAMS

Dalechampia cissifolia Poepp. \& Endl. CAM, CHIS, OAX, PUE, QROO, VER

Dalechampia laevigata Standl. VER

*Dalechampia magnistipulata G.L. Webster \& Armbr. OAX, VER

Dalechampia scandens L. BCS, CAM, CHIS, CHIH, COL, GRO, JAL, MICH, MOR, NAY, OAX, PUE, QRO, QROO, SLP, SIN, SON, TAB, TAMS, VER, YUC, ZAC

Dalechampia schottii Greenm. CAM, QROO, YUC

Dalechampia spathulata (Scheidw.) Baill. CHIS, OAX, TAB, VER

Dalechampia tiliifolia Lam. CHIS, GRO, TAB, VER

*Dalembertia populifolia Baill. CHIS, COL, GTO, GRO, JAL, MEX, MICH, MOR, NAY, OAX, PUE, QRO, SLP, SIN, SON, ZAC

Dalembertia triangularis Müll. Arg. CHIS, OAX, PUE

Ditaxis adenophora (A. Gray) Pax \& K. Hoffm. BCN, SIN, SON

*Ditaxis arlynniana (J.W. Ingram) Radcl. Sm. \& Govaerts $\mathrm{COAH}$

Ditaxis brandegeei (Millsp.) Rose \& Standl. BCN, BCS, SON

*Ditaxis depressa (Greenm.) Pax \& K. Hoffm. BCS, OAX, PUE

Ditaxis guatemalensis (Müll. Arg.) Pax \& K. Hoffm. CAM, CHIS, COL, DGO, GTO, GRO, HGO, JAL, MEX, MICH, MOR, NAY, OAX, PUE, QRO, QROO, SLP, SIN, SON, TAMS, VER, ZAC

*Ditaxis heterantha Zucc. BCS, COL, GTO, GRO, HGO, JAL, MEX, MICH, MOR, QRO, SLP, SIN, TAMS

Ditaxis humilis (Engelm. \& A. Gray) Pax COAH, NLE, TAMS, ZAC

Ditaxis lanceolata (Benth.) Pax \& K. Hoffm. BCN, BCS, COAH, NAY, SIN, SON

*Ditaxis manzanilloana (Rose) Pax \& K. Hoffm. COL, GRO, JAL, NAY, OAX, SIN, SON

*Ditaxis pringlei (Greenm.) Pax \& K. Hoffm. CDMX, GRO, HGO, MEX, MICH, MOR, OAX, PUE, QRO

Ditaxis serrata (Torr.) A. Heller BCN, BCS, CHIS, CHIH, COAH, DGO, GTO, GRO, JAL, MEX, MICH, NLE, OAX, PUE, QRO, SLP, SIN, SON, TAMS, VER, ZAC

*Enriquebeltrania crenatifolia (Miranda) Rzed. CAM, QROO, YUC

*Enriquebeltrania disjuncta De-Nova \& Sosa COL, JAL, SIN

Euphorbia abramsiana L.C. Wheeler BCN, BCS, CHIH, SIN, SON

*Euphorbia adenoptera Bertol. CDMX, JAL, MEX, NAY, SON, PUE, VER, ZAC

*Euphorbia alatocaulis V.W. Steinm. \& Felger SON

Euphorbia albomarginata Torr. \& A. Gray BCN, BCS, CHIH, COAH, DGO, GTO, HGO, MICH, MOR, NLE, OAX, QRO, SLP, SIN, SON, TAMS

Euphorbia alta Norton CHIH, DGO, HGO, MEX, PUE, SON, TLAX, VER

Euphorbia angusta Engelm. COAH 
*Euphorbia anthonyi Brandegee BCN, BCS, COL, SIN Euphorbia antisiphylitica Zucc. AGS, CHIH, COAH, DGO, GTO, GRO, HGO, JAL, NLE, OAX, PUE, QRO, SLP, TAMS, VER, ZAC

Euphorbia anychioides Boiss. AGS, CAM, CHIS, CHIH, CDMX, DGO, GTO, GRO, HGO, JAL, MEX, MICH, NAY, OAX, PUE, QRO, QROO, SLP, SIN, SON, TAMS, TLAX, VER, YUC, ZAC

*Euphorbia apatzingania McVaugh COL, JAL, MICH

* Euphorbia apicata L.C. Wheeler BCS

Euphorbia ariensis Kunth CHIS, COL, DGO, GRO, JAL, MEX, MICH, MOR, OAX, PUE, SIN, VER

Euphorbia arizonica Engelm. BCN, BCS, CHIH, COAH, DGO, SON

Euphorbia armourii Millsp. CAM, QROO, VER, YUC

*Euphorbia arteagae W.R. Buck \& Huft JAL, MICH

Euphorbia astyla Engelm. ex Boiss. COAH, DGO, NLE

*Euphorbia barnesii (Millsp.) Oudejans GRO

*Euphorbia barrancana Mayfield \& V.M. Steinman CHIH, SON

*Euphorbia bartolomaei Greene BCN, BCS

*Euphorbia beamanii M.C. Johnst. COAH, NLE

Euphorbia berteroana Balb. ex Spreng. AGS, COL, DGO, GTO, GRO, JAL, MOR, NLE, OAX, PUE, QRO, SLP, VER

Euphorbia bifurcata Engelm. CHIH, COAH, NLE, TAMS

Euphorbia bilobata Engelm. CHIH, DGO, SON

Euphorbia blodgettii Engelm. Ex Hitchc. OAX, QROO, TAB

Euphorbia bombensis Jacq. CAM, QROO, TAB, TAMS, VER,

YUC

*Euphorbia bracteata Jacq. COL, GRO, HGO, JAL, MEX, MICH, NAY, OAX, PUE, QRO, SLP, SIN, SON, ZAC

Euphorbia brachycera Engelm. CHIH, COAH, NLE, SIN, SON *Euphorbia brandegeei Millsp. BCS

*Euphorbia calcarata (Schltdl.) V.W. Steinm. CHIS, COL, GRO, HGO, JAL, MEX, MICH, NAY, OAX, PUE, QRO, QROO, SIN, TAB, VER

* Euphorbia calcicola Fernald GRO

*Euphorbia calderoniae V.W. Steinm. MICH

*Euphorbia californica Benth. BCS, COL, JAL, SIN, SON, ZAC

*Euphorbia calyculata Kunth COL, CDMX, GTO, GRO, JAL, MEX, MICH, MOR, OAX, PUE, SLP

*Euphorbia caperata McVaugh JAL, MICH, OAX

Euphorbia capitellata Engelm. BCN, BCS, CHIH, COAH, DGO, GRO, HGO, MICH, NLE, QRO, SLP, SIN, SON, TAMS Euphorbia carunculata Waterf. CHIH

*Euphorbia ceroderma I.M. Johnst. BCS, SON

* Euphorbia cerralvensis Maya Lastra \& V.W. Steinm. BCS

Euphorbia cinerascens Engelm. CHIH, COAH, GTO, NLE, SLP, TAMS

*Euphorbia coalcomanensis (Croizat) V.W. Steinm. MICH, NAY

*Euphorbia colorata Engelm. CHIH, DGO, JAL, SIN, SON, ZAC

Euphorbia colletioides Benth. CHIS, CHIH, COL, DGO, GTO, GRO, HGO, JAL, MEX, MICH, NAY, NLE, OAX, PUE, QRO, SLP, SIN, SON, TAMS, VER, YUC, ZAC
* Euphorbia colligata V.W. Steinm. JAL

* Euphorbia conzattii V.W. Steinm. OAX

* Euphorbia cornastra (Dressler) Radcl. Sm. GRO

*Euphorbia correllii M.C. Johnst. COAH, NLE

Euphorbia cotinifolia L. CHIS, COL, GRO, JAL, MEX, MICH, OAX, PUE, SIN, VER

*Euphorbia creberrima McVaugh AGS, DGO, JAL, ZAC

Euphorbia crepitata L.C. Wheeler COAH

*Euphorbia crepuscula (L.C. Wheeler) V.W. Steinm. \& Felger SIN, SON

*Euphorbia cressoides M.C. Johnst. COAH, DGO

Euphorbia cuchumatanensis Standl. \& Steyerm. CHIS

Euphorbia cumbrae Boiss. COAH, GTO, GRO, HGO, NLE, OAX, PUE, QRO, SLP

Euphorbia cuphosperma (Engelm.) Boiss. AGS, CHIH, COAH, GTO, JAL, MEX, MOR, OAX, SON

Euphorbia cyathophora Murray BCN, BCS, CAM, CHIS, CHIH, COAH, COL, DGO, GTO, GRO, HGO, JAL, MEX, MICH, MOR, NAY, NLE, OAX, PUE, QRO, QROO, SLP, SIN, SON, TAB, TAMS, TLAX, VER, YUC, ZAC

*Euphorbia cymbifera (Schltdl.) V.W. Steinm. OAX, PUE, VER

* Euphorbia cymosa Poir. GRO, MICH, QROO, YUC

* Euphorbia cyri V.W. Steinm. OAX

Euphorbia chaetocalyx (Boiss.) Tidestr. COAH

Euphorbia chamaesula Boiss. CHIH, CDMX, COAH, DGO, HGO, JAL, MEX, MICH, SLP, SON, TAMS, VER

*Euphorbia chamberlinii I.M. Johnst. BCS

* Euphorbia chersonesa Huft BCS

* Euphorbia chiribensis V.W. Steinm. \& Felger SON

Euphorbia davidii Subils CHIH, COAH, SON

Euphorbia delicatula Boiss. CHIS, COL, GRO, JAL, MEX, MICH, MOR, NAY, OAX, PUE, QRO, SIN, ZAC

Euphorbia densiflora (Klotzsch \& Garcke) Klotzsch CHIS, CHIH, COL, GTO, GRO, HGO, JAL, MEX, MICH, MOR, NAY, OAX, QRO, SLP, SIN, SON, VER, ZAC

Euphorbia dentata Michx. AGS, BCS, CHIS, CHIH, COAH, COL, CDMX, DGO, GTO, GRO, HGO, JAL, MEX, MICH, MOR, NLE, OAX, PUE, QRO, QROO, SLP, SIN, SON, TAMS, TLAX, VER, YUC, ZAC

*Euphorbia dentosa I.M. Johnst. BCN, BCS, DGO, SON

*Euphorbia derickii V.W. Steinm. GTO, GRO, MEX, MICH, MOR

*Euphorbia diazlunana (J.A. Lomelí \& Sahagún) V.W. Steinm. COL, JAL

Euphorbia dioeca Kunth CAM, CHIS, COL, GRO, JAL, MICH, NAY, OAX, PUE, QRO, QROO, SIN, SON, TAB, VER, YUC *Euphorbia dioscoreoides Boiss. CHIH, COL, DGO, GTO, GRO, JAL, MEX, MICH, MOR, NAY, OAX, QRO, SLP, SIN, SON, VER, ZAC

* Euphorbia dressleri V.W. Steinm. GRO

*Euphorbia eglandulosa V.W. Steinm. CHIS, GRO, MEX, MOR

Euphorbia eriantha Benth. BCN, BCS, CHIH, COAH, DGO, HGO, JAL, NLE, QRO, SLP, SIN, SON, ZAC

*Euphorbia esuliformis S. Schauer ex Nees \& S. Schauer CHIS, COAH, COL, CDMX, GTO, HGO, JAL, MEX, MICH, NLE, OAX, SLP, SIN, TAMS, ZAC 
Euphorbia exstipulata Engelm. CHIH, COAH, GTO, SLP, SON, ZAC

*Euphorbia feddemae McVaugh AGS, GTO, JAL

Euphorbia fendleri Torr. \& A. Gray CHIH, COAH, NLE

*Euphorbia finkii (Boiss.) V.W. Steinm. GRO, OAX, PUE, VER

* Euphorbia floribunda Engelm. ex Boiss. JAL

Euphorbia florida Engelm. BCN, CHIH, JAL, SIN, SON

Euphorbia francoana Boiss. CAM, CHIS, COL, GRO, JAL, MEX, MICH, OAX, QROO, TAB, VER

Euphorbia fruticulosa Engelm. ex Boiss. COAH, DGO, NLE

* Euphorbia fulgens Karw. ex Klotzsch OAX

Euphorbia furcillata Kunth AGS, CHIS, CHIH, COAH, COL, CDMX, DGO, GTO, HGO, JAL, MEX, MICH, MOR, NAY, NLE, QRO, SLP, SIN, TAMS, TLAX, VER, ZAC

*Euphorbia gentryi V.W. Steinm. \& T.F. Daniel SIN, SON

Euphorbia georgei Oudejans COAH

Euphorbia geyeri Engelm. CHIH

Euphorbia glyptosperma Engelm. CHIH, COAH, NLE, TAMS, ZAC

Euphorbia golondrina L.C. Wheeler CHIH, COAH, NLE

Euphorbia gracillima S. Watson CHIH, JAL, SIN, SON, ZAC

*Euphorbia gradyi V.W. Steinm. \& Ramírez-Roa OAX, PUE

Euphorbia graminea Jacq. AGS, CAM, CHIS, CHIH, COAH, COL, CDMX, DGO, GTO, GRO, HGO, JAL, MEX, MICH, MOR, NAY, NLE, OAX, PUE, QRO, QROO, SLP, SIN, SON, TAMS, TLAX, VER, YUC, ZAC

*Euphorbia grammata (McVaugh) Oudejans MICH

* Euphorbia greggii Engelm. ex Boiss. COAH, HGO, NLE, SLP,

TAMS, VER

*Euphorbia guadalajarana S. Watson JAL, NAY, SLP, TAMS, ZAC

Euphorbia guatemalensis Standl. \& Steyerm. CHIS

* Euphorbia guiengola W.R. Buck \& Huft CHIS, OAX

Euphorbia helleri Millsp. COAH, NLE, TAMS

*Euphorbia henricksonii M.C. Johnst. CHIH

Euphorbia heterophylla L. BCN, BCS, CAM, CHIS, CHIH, COAH, COL, DGO, GTO, GRO, HGO, JAL, MEX, MICH, MOR, NAY, NLE, OAX, PUE, QRO, QROO, SLP, SIN, SON, TAB, TAMS, TLAX, VER, YUC, ZAC

*Euphorbia hexagonoides S. Watson CHIH, SIN, SON

* Euphorbia hindsiana Benth. BCS, SIN, SON

*Euphorbia hintonii L.C. Wheeler MEX, MICH

Euphorbia hirta L. CAM, CHIS, CHIH, COL, DGO, GTO, GRO, HGO, JAL, MEX, MICH, MOR, NAY, NLE, OAX, PUE, QRO, QROO, SLP, SIN, SON, TAB, TAMS, VER, YUC, ZAC *Euphorbia hormorrhiza (Dressler) Radcl. Sm. TAMS

* Euphorbia huajuapensis Mart. Gord. \& Cruz-Durán OAX

Euphorbia humayensis Brandegee BCS, COL, GRO, JAL, MEX, MICH, MOR, OAX, SIN, SON, YUC

Euphorbia hypericifolia L. CAM, CHIS, COL, DGO, GRO, HGO, JAL, MEX, MICH, MOR, NAY, OAX, PUE, QRO, QROO, SLP, SIN, SON, TAB, TAMS, VER, YUC

Euphorbia hyssopifolia L. BCS, CAM, CHIS, CHIH, COAH, COL, CDMX, DGO, GTO, GRO, HGO, JAL, MEX, MICH, MOR, NAY, NLE, OAX, PUE, QRO, QROO, SLP, SIN, SON, TAB, TAMS, TLAX, VER, YUC, ZAC

*Euphorbia incerta Brandegee BCS, COL, NAY, SIN, SON
Euphorbia indivisa (Engelm.) Tidestr. AGS, CHIH, COAH, COL, CDMX, DGO, GTO, GRO, HGO, JAL, MEX, MICH, MOR, OAX, PUE, QRO, SLP, SON, TAMS, TLAX, VER, ZAC *Euphorbia infernidialis V.W. Steinm. GRO, MICH

* Euphorbia ivanjohnstonii M.C. Johnst. COAH

*Euphorbia ixtlana Huft OAX, PUE, VER

*Euphorbia jaliscensis B.L. Rob. \& Greenm. CHIS, COL, DGO, JAL, OAX, PUE

Euphorbia jejuna Warnock \& M.C. Johnst. COAH

* Euphorbia johnstonii Mayfield NLE, TAMS

*Euphorbia lacera Boiss. CDMX, GTO, HGO, JAL, MEX, OAX, PUE, QRO, SLP, VER

*Euphorbia lagunensis Huft BCS

Euphorbia lancifolia Schltdl. CHIS, OAX, QRO, SLP, VER

Euphorbia laredana Millsp. NLE, TAMS

Euphorbia lasiocarpa Klotzsch CAM, CHIS, GRO, HGO, JAL, NLE, OAX, QRO, SLP, TAB, TAMS, VER

Euphorbia lata Engelm. CHIH, COAH

*Euphorbia leucantha (Klotzsch \& Garcke) Boiss. MOR, VER Euphorbia leucocephala Lotsy CHIS, OAX

*Euphorbia leucophylla Benth. BCN, BCS, SON

*Euphorbia lineata S. Watson COL, DGO, GRO, JAL, MOR, NAY

*Euphorbia linguiformis McVaugh COL, GRO, MICH

*Euphorbia lomelii V.W. Steinm. BCN, BCS, COL, JAL, NAY, SIN, SON

*Euphorbia longecornuta S. Watson COAH, NLE

*Euphorbia lottiae V.W. Steinm. COL, GRO, JAL, MICH

Euphorbia luciismithii B.L. Rob. \& Greenm. CHIS, GRO, MICH, OAX, PUE

*Euphorbia lundelliana Croizat CHIS, OAX

Euphorbia lurida Engelm. SON

Euphorbia macropodoides B.L. Rob. \& Greenm. CHIS, OAX

Euphorbia macropus (Klotzsch \& Garcke) Boiss. AGS, CAM, CHIS, CHIH, COAH, COL, CDMX, DGO, GTO, GRO, HGO, JAL, MEX, MICH, MOR, NAY, NLE, OAX, PUE, QRO, QROO, SLP, SIN, SON, TAMS, TLAX, VER, ZAC

Euphorbia maculata L. BCN, BCS, CHIH, COAH, GTO, GRO, HGO, JAL, MEX, MICH, MOR, NLE, QRO, SLP, SON, TAMS, VER

*Euphorbia macvaughii Carvajal \& J.A. Lomelí COL, GRO, JAL

* Euphorbia magdalenae Benth. BCN, BCS, SON

Euphorbia marginata Pursh CAM, COAH, OAX, SLP, SIN, TAB, TAMS, VER

*Euphorbia maysillesii McVaugh DGO, JAL, SLP, ZAC

*Euphorbia mcvaughiana M.C. Johnst. COAH, DGO, NLE, ZAC

Euphorbia melanadenia Torr. BCN, BCS, CHIH, COAH, SON Euphorbia mendezii Boiss. CAM, CHIS, COL, CDMX, DGO, GTO, JAL, MEX, MOR, NLE, OAX, PUE, QRO, QROO, SLP, TAMS, VER, YUC, ZAC

Euphorbia mesembryanthemifolia Jacq. CAM, QROO, VER, YUC

*Euphorbia mexiae Standl. COL, JAL, NAY

Euphorbia micromera Boiss. BCN, BCS, CHIH, COAH, DGO, MICH, NLE, SLP, SIN, SON, VER 
*Euphorbia misella S. Watson CHIS, CHIH, CDMX, GTO, GRO, HGO, JAL, MEX, OAX, PUE, QRO, VER

Euphorbia misera Benth. BCN, BCS, SLP, SON

*Euphorbia montereyana Millsp. COAH, NLE, TAMS

Euphorbia multiseta Benth. COL, CDMX, GRO, JAL, MEX, MICH, MOR, NAY, OAX, PUE, TLAX

*Euphorbia muscicola Fernald MOR

*Euphorbia nayarensis V.W. Steinm. DGO, NAY

*Euphorbia neilmulleri M.C. Johnst. NLE, TAMS

*Euphorbia nesomii Mayfield NLE

*Euphorbia nocens (L.C. Wheeler) V.W. Steinm. CHIH, DGO, GTO, HGO, JAL, MEX, MICH, NAY, PUE, QRO, SLP, SON

Euphorbia nutans Lag. AGS, CHIS, CHIH, COAH, COL, CDMX, DGO, GTO, GRO, HGO, JAL, MEX, MICH, MOR, NAY, NLE, OAX, PUE, QRO, SLP, SON, TAB, TAMS, TLAX, VER, ZAC

*Euphorbia oaxacana B.L. Rob. \& Greenm. CHIS, COL, GRO, JAL, MICH, OAX, PUE, VER

Euphorbia ocymoidea L. CAM, CHIS, COL, CDMX, GTO, GRO, HGO, JAL, MEX, MICH, MOR, NAY, OAX, PUE, QRO, QROO, SLP, SIN, SON, TAMS, VER, YUC, ZAC

Euphorbia ophthalmica Pers. CHIS, COL, CDMX, DGO, GTO, GRO, HGO, JAL, MEX, MICH, MOR, NAY, OAX, PUE, QRO, SIN, TLAX

*Euphorbia oppositifolia McVaugh JAL

Euphorbia orizabae Boiss. CHIS, HGO, OAX, PUE, VER

Euphorbia palmeri Engelm. ex S. Watson BCN

Euphorbia parryi Engelm. CHIH

Euphorbia pediculifera Engelm. BCN, BCS, SIN, SON

*Euphorbia peninsularis I.M. Johnst. BCS

Euphorbia peplidion Engelm. TAMS

Euphorbia perennans (Shinners) Warnock \& M.C. Johnst. $\mathrm{CHIH}, \mathrm{COAH}$

*Euphorbia peritropoides (Millsp.) V.W. Steinm. COL, GRO, HGO, JAL, MICH, NAY, OAX, VER

*Euphorbia perlignea McVaugh COL, GRO, JAL

*Euphorbia personata (Croizat) V.W. Steinm. QROO, YUC

*Euphorbia petrina S. Watson BCN, BCS, SLP, SIN, SON

*Euphorbia picachensis Brandegee OAX

*Euphorbia pinkavana M.C. Johnst. COAH

*Euphorbia pionosperma V.W. Steinm. \& Felger CHIH, SON

Euphorbia platysperma Engelm. ex S. Watson BCN, SON

Euphorbia polycarpa Benth. BCN, BCS, COAH, SON, ZAC

*Euphorbia pondii Millsp. BCN

*Euphorbia potosina Fernald AGS, CDMX, GTO, HGO, JAL, MEX, MICH, PUE, QRO, SLP, TAMS, TLAX, VER, ZAC

Euphorbia prostrata Aiton AGS, CAM, CHIS, CHIH, COAH, COL, CDMX, DGO, GTO, GRO, HGO, JAL, MEX, MICH, MOR, NAY, NLE, OAX, PUE, QRO, QROO, SLP, SIN, SON, TAB, TAMS, TLAX, VER, ZAC

*Euphorbia pseudofulva Miranda CHIS

Euphorbia pteroneura A. Berger CHIS, OAX, QROO, VER, YUC

Euphorbia pulcherrima Willd. ex Klotzsch CAM, CHIS, CHIH, COL, DGO, GTO, GRO, HGO, JAL, MEX, MICH, MOR, NAY, OAX, PUE, QRO, QROO, SLP, SIN, TAMS, VER, YUC

*Euphorbia pumicicola Huft BCS
Euphorbia radians Benth. AGS, CHIH, COAH, CDMX, DGO, GTO, HGO, JAL, MEX, MICH, MOR, OAX, PUE, QRO, SLP, SON, TLAX, VER, ZAC

*Euphorbia radioloides Boiss. GRO, JAL, NAY, OAX, SIN, SON

*Euphorbia rayturneri V.W. Steinm. \& Jercinovic CHIH, SON

*Euphorbia restiacea Benth. DGO, JAL, NAY

Euphorbia revoluta Engelm. BCN, CHIH, COAH, NLE, SON, ZAC

*Euphorbia rossiana Pax GRO, OAX, PUE, VER

Euphorbia rutilis (Millsp.) Standl. \& Steyerm. YUC

*Euphorbia rzedowskii McVaugh MICH

*Euphorbia scandens Kunth CHIS, GRO, OAX, VER

*Euphorbia scopulorum Brandegee COAH, ZAC

Euphorbia schiedeana (Klotzsch \& Garcke) Mayfield CHIS, COAH, COL, GTO, GRO, HGO, JAL, MEX, MICH, MOR, NLE, OAX, PUE, QRO, SIN, TAMS, VER

Euphorbia schlechtendalii Boiss. CAM, CHIS, COL, DGO, GRO, HGO, JAL, MEX, MICH, MOR, NAY, NLE, OAX, PUE, QRO, QROO, SLP, SIN, TAMS, VER, YUC, ZAC

Euphorbia segoviensis (Klotzsch \& Garcke) Boiss. CHIS, OAX, PUE

Euphorbia seleri Donn. Sm. MOR, OAX, PUE

Euphorbia serpens Kunth BCN, BCS, CAM, CHIS, CHIH, COAH, GTO, GRO, HGO, JAL, MEX, MICH, MOR, NLE, OAX, QRO, QROO, SLP, SIN, SON, TAB, TAMS, TLAX, VER

Euphorbia serpyllifolia Pers. AGS, BCN, CHIH, COAH, CDMX, DGO, GTO, HGO, JAL, MEX, MICH, NLE, OAX, PUE, QRO, SLP, SIN, SON, TAMS, VER, ZAC

Euphorbia serrula Engelm. CHIH, COAH, DGO, GTO, GRO, MICH, NLE, OAX, PUE, SLP, SON, TAMS, ZAC

Euphorbia setiloba Engelm. ex Torr. BCN, BCS, CHIH, COAH, SIN, SON

Euphorbia simulans (L.C. Wheeler) Warnock \& M.C. Johnst. $\mathrm{CHIH}, \mathrm{COAH}$

*Euphorbia sinaloensis Brandegee DGO, SIN, SON

Euphorbia sinclairiana Benth. CHIS, OAX, VER

Euphorbia sonorae Rose COL, GRO, HGO, JAL, MEX, MICH, MOR, NAY, OAX, PUE, QRO, SLP, SIN, SON

*Euphorbia soobyi McVaugh JAL

Euphorbia spathulata Lam. BCN, CHIH, COAH, DGO, NLE, SON, TAMS

*Euphorbia sphaerorhiza Benth. AGS, CHIH, COL, CDMX, DGO, GTO, GRO, JAL, MEX, MICH, MOR, NAY, OAX, PUE, SLP, SON, TLAX, ZAC

*Euphorbia standleyi (Millsp.) Oudejans NAY, SIN

Euphorbia stictospora Engelm. AGS, CHIS, CHIH, COAH, CDMX, DGO, GTO, GRO, HGO, JAL, MEX, MICH, NLE, OAX, PUE, QRO, SLP, SON, VER, ZAC

*Euphorbia strigosa Hook. \& Arn. CHIH, COL, JAL, NAY, SIN, SON, ZAC

*Euphorbia subpeltata S. Watson COAH, GTO, GRO, HGO, MOR, NLE, OAX, QRO, SLP, TAMS, VER

*Euphorbia subreniformis S. Watson CHIS, CHIH, COL, CDMX, GTO, GRO, JAL, MEX, MICH, NAY, OAX, SIN, SON, VER 
*Euphorbia succedanea L.C. Wheeler GTO, JAL, NAY, SIN, ZAC

*Euphorbia taluticola Wiggins BCS

*Euphorbia tanquahuete Sessé \& Moc. CHIS, COL, GTO, GRO, JAL, MEX, MICH, MOR, OAX, PUE, QRO, ZAC

*Euphorbia tehuacana (Brandegee) V.W. Steinm. OAX, PUE Euphorbia theriaca L.C. Wheeler CHIH, COAH, SON

Euphorbia thymifolia L. CAM, CHIS, COL, GRO, HGO, JAL, MEX, MICH, MOR, NAY, OAX, PUE, QRO, QROO, SLP, SIN, SON, TAB, VER, ZAC

Euphorbia tithymaloides L. CAM, CHIS, GRO, HGO, MEX, MICH, MOR, OAX, QRO, QROO, SLP, TAB, TAMS, VER, YUC

*Euphorbia tomentella Engelm. ex Boiss. SLP

*Euphorbia tomentulosa S. Watson BCN, BCS, SIN, SON

Euphorbia trachysperma Engelm. BCN, BCS, SIN, SON

*Euphorbia tresmariae (Millsp.) Standl. COL, JAL, NAY, OAX, SIN

*Euphorbia trialata (Huft)V.W. Steinm. COL, GRO, MICH

*Euphorbia tricolor Greenm. OAX, PUE

Euphorbia trichotoma Kunth COL, QROO

*Euphorbia umbellulata Engelm. ex Boiss. GTO, GRO, JAL, MICH, MOR, NAY

Euphorbia velleriflora (Klotzsch \& Garcke) Boiss. AGS, CHIS, COL, GTO, JAL, MICH, NLE, OAX, PUE, QRO, SLP, TAMS, ZAC

Euphorbia vermiculata Raf. DGO, GTO, JAL, MICH

*Euphorbia vestita Boiss. GRO, MOR

Euphorbia villifera Scheele AGS, CHIS, CHIH, COAH, CDMX, DGO, GTO, GRO, HGO, MEX, MOR, NLE, OAX, PUE, QRO, SLP, SON, TAMS, VER, YUC, ZAC

*Euphorbia violacea Greenm. PUE

*Euphorbia vizcainensis Maya Lastra \& V.W. Steinm. BCS

*Euphorbia whitei L.C. Wheeler GRO, MICH, OAX

Euphorbia wrightii Torr. \& A. Gray CHIH, COAH, DGO, ZAC

Euphorbia xalapensis Kunth CHIS, CHIH, COL, GTO, JAL, OAX, SON, VER

*Euphorbia xanti Engelm. ex Boiss. BCN, BCS, SIN, SON

Euphorbia xbacensis Millsp. CAM, QROO, YUC

*Euphorbia xylopoda Greenm. OAX

*Euphorbia yucatanensis (Millsp.) Standl. CAM, QROO, YUC

*Euphorbia zierioides Boiss. GRO, OAX, VER

Garcia nutans Vahl CAM, CHIS, COL, GTO, HGO, JAL, NAY, OAX, PUE, QRO, QROO, SLP, SIN, TAB, TAMS, VER, YUC *Garcia parviflora Lundell CHIS, PUE, SLP, TAB, VER, YUC Gymnanthes actinostemoides Müll. Arg. CHIS, COL, JAL, MEX, MICH, NAY, OAX, PUE, TAMS, VER

* Gymnanthes insolita R.S. Ferris NAY

*Gymnanthes longipes Müll. Arg. COAH, GTO, JAL, NLE, OAX, PUE, QRO, SLP, TAMS, VER

Gymnanthes lucida Sw. CAM, CHIS, GRO, HGO, QROO, TAB, VER, YUC

Gymnanthes riparia (Schltdl.) Klotzsch CHIS, HGO, MEX, OAX, PUE, QRO, SLP, TAMS, VER

Hippomane mancinella L. BCN, CAM, CHIS, COL, GRO, JAL, MEX, MICH, NAY, OAX, PUE, QROO, VER, YUC

Hura polyandra Baill. CAM, CHIS, COL, DGO, GRO, HGO,
JAL, MEX, MICH, MOR, NAY, OAX, PUE, QRO, QROO, SLP, SIN, SON, TAB, VER, YUC

*Jatropha alamanii Müll. Arg. CAM, CHIS, NAY, OAX, YUC

*Jatropha andrieuxii Müll. Arg. GRO, JAL, OAX, PUE

*Jatropha bartlettii Wilbur COL, JAL

*Jatropha bullockii E.J. Lott COL, JAL

Jatropha cardiophylla (Torr.) Müll. Arg. CHIH, SON

Jatropha cathartica Terán \& Berland. CHIH, COAH, NLE, SON, TAMS

*Jatropha ciliata Sessé ex Cerv. GRO, MEX, MOR, OAX, PUE, SIN, VER

Jatropha cinerea (Ortega) Müll. Arg. BCN, BCS, DGO, JAL, SIN, SON

* Jatropha contrerasii J. Jiménez Ram. \& Mart. Gord. GRO

* Jatropha conzattii J. Jiménez Ram. OAX

* Jatropha cordata (Ortega) Müll. Arg. AGS, BCN, BCS, CHIH, COL, DGO, JAL, MEX, MICH, NAY, PUE, SLP, SIN, SON, ZAC

Jatropha cuneata Wiggins \& Rollins BCN, BCS, SIN, SON

Jatropha curcas L. CAM, CHIS, COL, DGO, GRO, HGO, JAL, MEX, MICH, MOR, NAY, OAX, PUE, QRO, QROO, SLP, SIN, TAB, TAMS, VER, YUC

*Jatropha chamelensis Pérez-Jiménez COL, JAL, MICH, NAY

*Jatropha dehganii J. Jiménez Ram. JAL, MICH

Jatropha dioica Sessé ex Cerv. AGS, BCS, CHIH, COAH, CDMX, DGO, GTO, HGO, JAL, MEX, MICH, MOR, NLE, OAX, PUE, QRO, SLP, SIN, SON, TAMS, VER, ZAC

*Jatropha elbae J. Jiménez Ram. GRO, OAX, PUE

* Jatropha fremontioides Standl. OAX

* Jatropha galvanii J. Jiménez Ram. \& L.M. Contr. GRO, JAL, $\mathrm{MICH}$

Jatropha gaumeri Greenm. CAM, CHIS, OAX, QROO, TAB, YUC

*Jatropha giffordiana Dehgan \& G.L. Webster BCS

Jatropha gossypiifolia L. COL, GRO, JAL, MICH, OAX, TAMS, VER

Jatropha integerrima Jacq. CHIS

*Jatropha jaimejimenezii V.W. Steinm. GRO, MICH

* Jatropha krusei J. Jiménez Ram. \& Mart. Gord. GRO

Jatropha macrorhiza Benth. AGS, CHIH, SON, ZAC

*Jatropha malacophylla Standl. CHIS, CHIH, COL, JAL, NAY, OAX, SIN, SON

*Jatropha mcvaughii Dehgan \& G.L. Webster COL, DGO, JAL, NAY, SIN

*Jatropha mirandana J. Jiménez Ram. \& K. Vega GRO, OAX, PUE

*Jatropha moranii Dehgan \& G.L. Webster BCS

* Jatropha neopauciflora Pax OAX, PUE

*Jatropha oaxacana J. Jiménez Ram. \& R. Torres OAX, PUE

*Jatropha olivacea Müll. Arg. CDMX, MEX, MOR, OAX, PUE, SIN

*Jatropha ortegae Standl. COL, JAL, SIN

*Jatropha peltata Cerv. COL, DGO, JAL, MICH, NAY, SIN

*Jatropha pereziae J. Jiménez Ram. JAL, MICH

*Jatropha platyphylla Müll. Arg. COL, DGO, JAL, MICH, NAY, OAX, SIN, ZAC

Jatropha podagrica Hook. CHIS, GRO, OAX, PUE, TAB, VER 
*Jatropha pseudocurcas Müll. Arg. CHIS, COL, GRO, JAL, OAX, PUE, TAB, VER

* Jatropha purpurea Rose BCS, SIN, SON

* Jatropha riojae Miranda PUE

*Jatropha rufescens Brandegee OAX, PUE

* Jatropha rzedowskii J. Jiménez Ram. OAX, PUE

*Jatropha sotoi-nunyezii Fernández Casas \& E. Martínez TAMS, VER

*Jatropha stephanii J. Jiménez Ram. \& Mart. Gord. GRO, JAL, $\mathrm{MICH}$

*Jatropha sympetala S.F. Blake \& Standl. COL, GRO, JAL, NAY, OAX

*Jatropha tlalcozotitlanensis J. Jiménez Ram. GRO, OAX

* Jatropha vernicosa Brandegee BCS

*Jatropha websteri J. Jiménez Ram. GRO, OAX, PUE

Mabea excelsa Standl. \& Steyerm. CHIS

Mabea occidentalis Benth. CHIS, COL, GRO, JAL, NAY, OAX, PUE, TAB, VER

*Mabea tenorioi Mart. Gord., J. Jiménez Ram. \& Cruz-Durán OAX, VER

Manihot aesculifolia (Kunth) Pohl CAM, CHIS, CHIH, COL, GRO, JAL, MEX, MICH, MOR, NAY, OAX, QRO, QROO, SLP, SIN, SON, VER, YUC

Manihot angustiloba (Torr.) Müll. Arg. BCS, CHIH, COAH, COL, DGO, GRO, JAL, MEX, MICH, MOR, NAY, OAX, SIN, SON, ZAC

*Manihot auriculata McVaugh JAL, NAY

*Manihot caudata Greenm. AGS, CHIH, COAH, DGO, GTO, GRO, JAL, MICH, NAY, SIN, SON, ZAC

*Manihot crassisepala Pax \& K. Hoffm. COL, JAL, MEX, MICH, MOR

* Manihot chlorosticta Standl. \& Goldman BCN, BCS, CHIS, COL, GRO, JAL, MEX, MICH, NAY, OAX, SIN, SON, VER

Manihot davisiae Croizat CHIH, SIN, SON

Manihot esculenta Crantz CAM, CHIS, COL, GRO, JAL, MEX, MOR, OAX, PUE, QRO, QROO, SLP, TAB, TAMS, VER, YUC

*Manihot mcvaughii V.W. Steinm. GRO, JAL, MEX, MICH

*Manihot michaelis McVaugh COL, JAL, MICH, ZAC

* Manihot oaxacana D.J. Rogers \& Appan OAX

* Manihot obovata J. Jiménez Ram. GRO

* Manihot pauciflora Brandegee JAL, OAX, PUE

*Manihot pringlei $\mathrm{S}$. Watson GTO, HGO, QRO, SLP, TAMS

Manihot rhomboidea Müll. Arg. AGS, CHIS, COL, DGO, GTO, GRO, JAL, MEX, MICH, MOR, NAY, OAX, PUE, QRO, SIN, YUC, ZAC

*Manihot rubricaulis I.M. Johnst. CHIH, DGO, NAY, SIN, SON

*Manihot subspicata D.J. Rogers \& Appan COAH, NLE, TAMS

*Manihot tomatophylla Standl. GRO, JAL, MICH, MOR, OAX

*Manihot triloba (Sessé ex Cerv.) McVaugh ex Miranda CHIS, COL, GRO, JAL, MEX, MICH, MOR, OAX, PUE

Manihot walkerae Croizat SLP, TAMS, VER

*Manihot websteri D.J. Rogers \& Appan OAX, PUE

Microstachys corniculata (Vahl) Griseb. COL, JAL, NAY, SIN

Omphalea diandra L. CHIS, OAX, TAB, TAMS, VER

Pleradenophora bilocularis (S. Watson) Esser \& A. L. Melo $\mathrm{BCN}, \mathrm{BCS}, \mathrm{CHIH}, \mathrm{SON}$
Pleradenophora longicuspis (Standl.) Esser CAM, CHIS, QROO, TAB, VER

*Pleradenophora lottiae (McVaugh) A. L. Melo \& Esser COL, GRO, JAL, MICH, MOR, OAX

Pleradenophora tikalana (Lundell) A. L. Melo \& Esser CAM, CHIS, OAX, QROO, YUC

Pleradenophora tuerckheimiana (Pax \& K. Hoffm.) A. L. Melo \& Esser CHIS

* Plukenetia carabiasiae J. Jiménez Ram. OAX

Plukenetia penninervia Müll. Arg. CAM, CHIS, OAX, QROO,

TAB

Plukenetia stipellata L.J. Gillespie CHIS, OAX, VER

Plukenetia volubilis L. CHIS, TAB, VER

Sapium glandulosum (L.) Morong CAM, CHIS, OAX, QROO, TAB, VER, YUC

Sapium lateriflorum Hemsl. CAM, CHIS, DGO, GRO, JAL, MEX, NAY, OAX, PUE, QROO, SIN, TAB, VER

Sapium macrocarpum Müll. Arg. CAM, CHIS, COL, DGO, GTO, GRO, JAL, MEX, MICH, MOR, NAY, OAX, PUE, QRO, SLP, SIN, SON, TAMS, VER

Sebastiania adenophora Pax \& K. Hoffm. CAM, CHIS, PUE, QROO, VER, YUC

*Sebastiania appendiculata (Müll. Arg.) Jabl. CHIS, CHIH, DGO, GRO, MICH, NAY, OAX, PUE, SIN, SON

Sebastiania confusa Lundell CAM, CHIS, QROO, TAB, VER

*Sebastiania cornuta McVaugh CHIH, DGO, NAY, SON

Sebastiania cruenta (Standl. \& Steyerm.) Miranda CHIS

*Sebastiania hintonii Lundell COL, GRO, JAL, MICH, MOR, NAY, OAX

*Sebastiania jaliscensis McVaugh COL, GRO, JAL, NAY, OAX, SIN

Sebastiania pavoniana (Müll. Arg.) Müll. Arg. BCS, CHIS, CHIH, COL, DGO, GTO, GRO, HGO, JAL, MEX, MICH, MOR, NAY, OAX, PUE, QRO, SLP, SIN, SON, TAMS, VER, ZAC

Stillingia acutifolia (Benth.) Benth. ex Hemsl. CHIS, HGO, QRO, SLP, VER, YUC

*Stillingia bicarpellaris S. Watson COAH, GTO, HGO, NLE, SLP

Stillingia diphtherina D.J. Rogers CHIS

Stillingia linearifolia S. Watson BCN, BCS, SON

*Stillingia pietatis McVaugh JAL, MICH

* Stillingia querceticola McVaugh NAY, SIN

Stillingia sanguinolenta Müll. Arg. COAH, GTO, GRO, HGO, JAL, NLE, OAX, PUE, QRO, SLP, TAMS, VER, ZAC

Stillingia spinulosa Torr. BCN, NLE, SON

Stillingia sylvatica L. COAH, MICH, TAMS

Stillingia texana I.M. Johnst. COAH

Stillingia treculiana (Müll. Arg.) I.M. Johnst. CHIH, COAH, NLE, SLP, TAMS

Stillingia zelayensis (Kunth) Müll. Arg. CHIS, CDMX, GTO, GRO, HGO, JAL, MEX, MICH, MOR, NAY, OAX, PUE, QRO, SLP, TAMS, TLAX

Tetrorchidium brevifolium Standl. \& Steyerm. CHIS, OAX Tetrorchidium rotundatum Standl. CHIS, OAX, TAB, VER

*Tragia affinis B.L. Rob. \& Greenm. COL, GRO, JAL, MEX, MOR, OAX, VER 
Tragia amblyodonta (Müll. Arg.) Pax \& K. Hoffm. BCN, BCS, CHIH, COAH, NLE, SLP, SON, TAMS, ZAC

Tragia brevispica Engelm. \& A. Gray COAH, TAMS

*Tragia chiltepecana Urtecho ex Mart. Gord. \& R. Torres OAX Tragia glanduligera Pax \& K. Hoffm. CAM, DGO, GTO, HGO, NAY, OAX, QRO, QROO, SLP, TAMS, VER, YUC

*Tragia jonesii Radcl. Sm. \& Govaerts BCS, SON

Tragia laciniata (Torr.) Müll. Arg. CHIH, SON

Tragia mexicana Müll. Arg. CAM, CHIS, JAL, OAX, PUE, QRO, QROO, SLP, SIN, TAMS, VER

Tragia nepetifolia Cav. AGS, BCS, CAM, CHIS, CHIH, COAH, CDMX, DGO, GTO, GRO, HGO, JAL, MEX, MICH, NAY, NLE, OAX, PUE, QRO, QROO, SLP, SIN, SON, TAMS, VER, YUC, ZAC

*Tragia pacifica McVaugh COL, JAL, NAY, OAX, SIN

* Tragia potosina Lundell SLP, VER

Tragia ramosa Torr. BCN, CHIH, COAH, HGO, NLE, QRO, SLP, SON, TAMS, VER

Tragia volubilis L. CAM, CHIS, COL, GRO, JAL, MEX, OAX, QROO, SIN, VER, YUC

Tragia yucatanensis Millsp. CAM, CHIS, QROO, YUC

\section{Family Fabaceae}

Abarema idiopoda (S.F. Blake) Barneby \& J.W. Grimes CAM, CHIS, OAX, TAB, VER, YUC

Abarema zolleriana (Standl. \& Steyerm.) Barneby \& J.W. Grimes CHIS, OAX, VER

* Acacia rotundata Benth. ND

Acaciella angustissima (Mill.) Britton \& Rose AGS, BCS, CAM, CHIS, CHIH, COAH, COL, CDMX, DGO, GTO, GRO, HGO, JAL, MEX, MICH, MOR, NAY, NLE, OAX, PUE, QRO, QROO, SLP, SIN, SON, TAB, TAMS, TLAX, VER, YUC, ZAC *Acaciella barrancana (Gentry) L. Rico DGO, SIN

*Acaciella bicolor Britton \& Rose COL, GRO, JAL, MICH, OAX, PUE

*Acaciella chamelensis (L. Rico) L. Rico COL, JAL

*Acaciella goldmanii Britton \& Rose BCN, BCS, NAY

*Acaciella hartwegii (Benth.) Britton \& Rose AGS, DGO, GTO, GRO, JAL, MEX, MICH, TAMS, ZAC

*Acaciella igualensis Britton \& Rose COL, GRO, MEX, OAX, PUE

Acaciella lemmonii Britton \& Rose CHIH, SIN, SON

*Acaciella painteri Britton \& Rose CHIS, COL, DGO, GTO, GRO, JAL, MEX, MICH, MOR, NAY, OAX, PUE, SIN, SON, $\mathrm{ZAC}$

*Acaciella rosei (Standl.) Britton \& Rose COL, DGO, JAL, MICH, NAY, PUE, SIN, SON

*Acaciella sousae (L. Rico) L. Rico GRO, OAX, PUE

*Acaciella tequilana (S. Watson) Britton \& Rose CHIH, COL, DGO, GTO, GRO, HGO, JAL, MEX, MOR, NAY, OAX, PUE, QRO, SLP, SIN, SON, TAMS, ZAC

*Acaciella velutina Britton \& Rose NAY, SIN

Acaciella villosa (Sw.) Britton \& Rose CHIS, CHIH, COAH, DGO, GRO, JAL, MEX, MICH, NAY, NLE, OAX, PUE, SLP, SON, TAMS, VER

Acmispon brachycarpus (Benth.) D.D. Sokoloff BCN, SON

*Acmispon distichus (Greene) Brouillet BCN, BCS
Acmispon grandiflorus (Benth.) Brouillet BCN

Acmispon greenei (Wooton \& Standl.) Brouillet CHIH, DGO, SON

Acmispon haydonii (Orcutt) Brouillet BCN

Acmispon heermannii (Durand \& Hilg.) Brouillet BCN

Acmispon nevadensis (S. Watson) Brouillet BCN

*Acmispon nudatus (Greene) Brouillet BCN, BCS

Acmispon oroboides (Kunth) Ottley ex Kearney \& Peebles AGS, BCN, BCS, CHIH, COAH, COL, CDMX, DGO, GTO, HGO, JAL, MEX, NAY, OAX, PUE, QRO, SLP, SIN, SON, VER, ZAC

Acmispon rigidus (Benth.) Brouillet BCN, BCS, SON Acmispon strigosus (Nutt.) Brouillet BCN, BCS, CHIH, SON Acmispon wrightii (A. Gray) Greene CHIH, DGO, SON

*Adenopodia gymnantha Brenan COL, GRO, JAL, MICH, NAY

*Adenopodia oaxacana M. Sousa OAX

*Adenopodia patens (Hook. \& Arn.) J.R. Dixon ex Brenan CHIS, COL, GRO, JAL, MICH, NAY, OAX

Adenopodia polystachya (L.) J.R. Dixon ex Croat OAX

*Aeschynomene acapulcensis Rose GRO, OAX

Aeschynomene americana L. CAM, CHIS, CHIH, COL, DGO, GTO, GRO, JAL, MEX, MICH, MOR, NAY, OAX, PUE, QRO, QROO, SLP, SIN, SON, TAB, TAMS, VER, YUC, ZAC

*Aeschynomene amorphoides (S. Watson) Rose ex B.L. Rob. COL, DGO, GRO, JAL, MICH, NAY, SIN, ZAC

Aeschynomene brasiliana (Poir.) DC. CHIS, GRO, JAL, MICH, OAX, TAB, NAY

Aeschynomene ciliata Vogel CHIS, GRO, OAX, TAB

Aeschynomene compacta Rose CHIS, GRO, MICH, OAX, PUE, VER

Aeschynomene deamii B.L. Rob. TAB, VER

Aeschynomene elegans Schltdl. \& Cham. CHIS, JAL, NAY, MICH, OAX, VER

Aeschynomene fascicularis Schltdl. \& Cham. CAM, CHIS, CHIH, DGO, GRO, JAL, MEX, MICH, NAY, OAX, PUE, QROO, SLP, SIN, SON, TAB, TAMS, VER, YUC, ZAC

*Aeschynomene hintonii Sandwith GRO, MEX, MICH

Aeschynomene hystrix Poir. COL, JAL, MEX, SIN

Aeschynomene indica L. TAB, TAMS

*Aeschynomene langlassei Micheli ex Rudd COL, GRO, JAL

*Aeschynomene lyonnetii Rudd GRO

Aeschynomene nicaraguensis (Oerst.) Standl. OAX

*Aeschynomene nivea Brandegee BCS

*Aeschynomene palmeri Rose GRO, MICH, OAX

Aeschynomene paniculata Willd. ex Vogel CHIS, COL, GRO, MEX, NAY, OAX, TAB, VER

*Aeschynomene paucifoliolata Micheli COL, GRO, JAL, MEX, $\mathrm{MICH}$

*Aeschynomene petraea B.L. Rob. CHIH, COL, DGO, GRO, JAL, MEX, MICH, MOR, NAY, OAX, SIN, SON, ZAC

*Aeschynomene pinetorum Brandegee CHIS, GRO, NAY, OAX, PUE

*Aeschynomene purpusii Brandegee CHIS, GRO, OAX, PUE, SIN, VER

*Aeschynomene rosei C.V. Morton SIN

Aeschynomene rudis Benth. CAM, CHIS, COL, DGO, GRO, JAL, MEX, MICH, NAY, OAX, SON, SON, TAB, TAMS, VER, 
YUC, ZAC

Aeschynomene scabra G. Don CHIS, GRO, JAL, MEX, MICH, MOR, NAY, OAX, SIN, TAB, TAMS, VER

Aeschynomene sensitiva Sw. CAM, CHIS, GRO, MEX, MICH, OAX, TAB, VER

*Aeschynomene simulans Rose DGO, GRO, JAL, NAY, SIN, ZAC

*Aeschynomene sousae Rudd ex A. Delgado \& Sotuyo OAX

*Aeschynomene unijuga (M.E. Jones) Rudd GRO, JAL, NAY, OAX, SIN

*Aeschynomene vigil Brandegee BCS

Aeschynomene villosa Poir. AGS, CHIS, CHIH, COL, DGO, GTO, GRO, JAL, MEX, MICH, MOR, NAY, NLE, OAX, PUE, SIN, SON, TAMS, VER, ZAC

Aeschynomene virginica (L.) Britton, Stearn \& Pogg. CAM, CHIS, DGO, GRO, MEX, MOR, OAX, SIN, TAB, VER

Aeschynomene viscidula Michx. OAX, SIN, TAMS

Albizia adinocephala (Donn. Sm.) Britton \& Rose ex Record CHIS, GRO, OAX

Albizia guachapele (Kunth) Dugand CAM, CHIS, OAX, TAB, VER

Albizia leucocalyx (Britton \& Rose) L. Rico CHIS, TAB

Albizia niopoides (Spruce ex Benth.) Burkart CAM, CHIS, OAX, QROO, TAB, VER, YUC

*Albizia occidentalis Brandegee AGS, BCS, CHIS, COL, DGO, GTO, GRO, HGO, JAL, MEX, MICH, MOR, NAY, OAX, PUE, QRO, SLP, SIN, ZAC

*Albizia sinaloensis Britton \& Rose BCS, CHIH, DGO, NAY, SIN, SON

Albizia tomentosa (Micheli) Standl. CAM, CHIS, COL, GTO, GRO, HGO, JAL, MEX, MICH, NAY, OAX, PUE, QRO, QROO, SLP, SIN, TAB, VER, YUC

*Amicia zygomeris DC. CHIS, CHIH, COAH, DGO, GTO, GRO, HGO, JAL, MEX, MICH, NAY, NLE, OAX, PUE, QRO, SLP, SIN, SON, TAMS, VER, ZAC

*Amorpha apiculata Wiggins BCN

Amorpha californica Nutt. BCN

Amorpha fruticosa L. BCN, CHIH, SON

Amorpha roemeriana Scheele COAH

*Andira galeottiana Standl. CAM, CHIS, OAX, PUE, QROO,

TAB, VER, YUC

Andira inermis (W. Wright) Kunth ex DC. CAM, CHIS, COL, DGO, GRO, JAL, MEX, MICH, NAY, OAX, SIN, TAB, VER, YUC

*Andira jaliscensis R.T. Penn. COL, JAL, MICH

Apoplanesia paniculata C. Presl CAM, CHIS, COL, GRO, JAL, MICH, NAY, OAX, QROO, VER, YUC

Astragalus acutirostris $\mathrm{S}$. Watson $\mathrm{BCN}$

Astragalus allochrous A. Gray CHIH, SON

Astragalus anemophilus Greene BCN

Astragalus aridus A. Gray BCN, SON

Astragalus arizonicus A. Gray SON

Astragalus brazoensis Buckley COAH, DGO, SLP, TAMS, ZAC

*Astragalus carminis Barneby COAH

*Astragalus cenorrhynchus Barneby OAX

*Astragalus circumdatus Greene BCN

*Astragalus coahuilae M.E. Jones COAH, NLE, ZAC
Astragalus cobrensis A. Gray CHIH, SON

Astragalus coccineus (Parry) Brandegee BCN

*Astragalus coriaceus Hemsl. AGS, CHIH, COAH, DGO, NLE,

SLP, ZAC

Astragalus crassicarpus Nutt. COAH

Astragalus crotalariae (Benth.) A. Gray BCN

*Astragalus daleae Greene CHIH, DGO

Astragalus didymocarpus Hook. \& Arn. BCN, SON

*Astragalus diphacus $\mathrm{S}$. Watson AGS, DGO, JAL, MEX, MOR, NLE, SLP, ZAC

Astragalus douglasii (Torr. \& A. Gray) A. Gray BCN

Astragalus emoryanus (Rydb.) Cory $\mathrm{CHIH}$, COAH, NLE, TAMS

Astragalus ervoides Hook. \& Arn. COL, DGO, GTO, JAL, MICH, NAY, SIN

*Astragalus esperanzae M.E. Jones DGO, HGO, JAL, MEX, MICH, MOR, OAX, PUE, SLP, ZAC

*Astragalus fastidius (Kellogg) M.E. Jones BCN, BCS

Astragalus filipes Torr. ex A. Gray BCN

*Astragalus francisquitensis M.E. Jones BCS

Astragalus gambelianus Sheldon BCN

*Astragalus gentryi Standl. CHIH, SON

Astragalus giganteus $\mathrm{S}$. Watson $\mathrm{CHIH}, \mathrm{SON}$

*Astragalus goldmanii M.E. Jones AGS, CHIH, DGO, JAL, NAY

*Astragalus greggii S. Watson COAH, NLE, SLP

*Astragalus gruinus Barneby BCN, BCS

*Astragalus guanajuatensis Rzed. \& Calderón GTO

Astragalus guatemalensis Hemsl. AGS, CHIS, COL, CDMX, GTO, GRO, HGO, JAL, MEX, MICH, MOR, NAY, OAX, QRO, SLP, TLAX, VER, ZAC

*Astragalus harbisonii Barneby BCN

*Astragalus hartwegii Benth. AGS, CHIH, DGO, GTO, HGO, JAL, MICH, SLP, SON, VER, ZAC

*Astragalus helleri Fenzl PUE, TAMS, VER

*Astragalus hidalgensis Rydb. HGO, MEX, MOR, QRO, SLP

*Astragalus hintonii Barneby VER

Astragalus hornii A. Gray BCN, BCS

Astragalus humistratus A. Gray CHIH, SON

*Astragalus hypoleucus S. Schauer AGS, CHIH, COAH, DGO, GTO, GRO, HGO, JAL, MEX, MICH, NLE, OAX, PUE, QRO, SLP, TAMS, VER, ZAC

*Astragalus idrietorum Barneby BCN

Astragalus insularis Kellogg BCN, BCS, SON

*Astragalus jaliscensis (Rydb.) Barneby AGS, JAL, ZAC

*Astragalus legionensis Barneby COAH, NLE

Astragalus lentiginosus Douglas BCN, CHIH, SON

*Astragalus longissimus (Torr.) Barneby CHIH, SON

*Astragalus lyonnetii Barneby CDMX, HGO, JAL, MEX, MICH, MOR, VER

Astragalus magdalenae Greene BCN, BCS, SON

*Astragalus mario-sousae A.E. Estrada, Villarreal \& YenMendez COAH, NLE

*Astragalus martinii Spellenb., Van Devender \& P.D. Jenkins CHIH, SON

*Astragalus micranthus Desv. CDMX, GTO, HGO, MEX, MICH, MOR, OAX, PUE, QRO, TLAX, VER 
Astragalus mollissimus Torr. AGS, CHIH, COAH, CDMX, DGO, GTO, GRO, HGO, JAL, MEX, NLE, PUE, QRO, SLP, TLAX, VER, ZAC

*Astragalus moranii Barneby BCN

Astragalus nothoxys A. Gray CHIH, SON

Astragalus nuttallianus DC. BCN, BCS, CHIH, COAH, DGO, GTO, GRO, HGO, MEX, MICH, NLE, PUE, QRO, SLP, SON, TAMS, VER

*Astragalus orcuttianus $\mathrm{S}$. Watson BCN

*Astragalus oxyphysopsis Barneby BCN

Astragalus oxyphysus A. Gray BCN

*Astragalus oxyrhynchus Hemsl. CDMX, HGO, MEX, MICH, MOR, QRO, VER

Astragalus palmeri A. Gray BCN, SON

*Astragalus parvus Hemsl. JAL, NLE, SLP, ZAC

*Astragalus pennellianus Barneby DGO

*Astragalus piscinus (M.E. Jones) Barneby BCN

Astragalus pomonensis M.E. Jones BCN

*Astragalus pomphocalyx Villarreal \& M.A. Carranza COAH,

NLE

*Astragalus potosinus Barneby DGO, JAL, SLP, ZAC

*Astragalus pringlei $\mathrm{S}$. Watson CHIH, DGO

*Astragalus prorifer M.E. Jones BCN, BCS

*Astragalus pueblae M.E. Jones PUE

*Astragalus purpusii M.E. Jones COAH, NLE, ZAC

*Astragalus quinqueflorus S. Watson AGS, CHIH, COAH, CDMX, DGO, HGO, JAL, MEX, VER, ZAC

Astragalus racemosus Pursh NLE, SLP

*Astragalus radicans Hornem. CDMX, HGO, MEX, MICH

*Astragalus regiomontanus Barneby COAH, NLE

*Astragalus rupertii Villarreal \& M.A. Carranza COAH, NLE

Astragalus sabulonum A. Gray BCN, SON

*Astragalus sanctorum Barneby BCN

*Astragalus sanguineus Rydb. COAH, DGO, NLE, SLP, TAMS

*Astragalus scalaris $\mathrm{S}$. Watson $\mathrm{CHIH}, \mathrm{SON}$

*Astragalus scutaneus Barneby COL, JAL

*Astragalus sinaloae Barneby SIN, SON

*Astragalus strigulosus Kunth CDMX, DGO, GRO, HGO, JAL, MEX, MICH, MOR, OAX, PUE, QRO, TLAX, VER

Astragalus tephrodes A. Gray CHIH, SON

Astragalus thurberi A. Gray CHIH, SON

*Astragalus tioides (Rydb.) Barneby SLP

*Astragalus tolucanus B.L. Rob. \& Seaton CDMX, HGO, MEX, MOR, VER

Astragalus trichopodus (Nutt.) A. Gray BCN

Astragalus vaccarum A. Gray CHIH, COAH, DGO, SON

Astragalus waterfallii Barneby $\mathrm{COAH}$

Astragalus wootonii E. Sheld. AGS, CHIH, COAH, CDMX, DGO, GRO, HGO, JAL, MEX, MICH, NLE, PUE, QRO, SLP, SON, TAMS, VER, ZAC

*Astragalus zacatecanus (Rydb.) Barneby DGO, JAL, ZAC

*Ateleia albolutescens Mohlenbr. CHIS, OAX

*Ateleia arsenii Standl. MEX, MICH

Ateleia cubensis Griseb. CAM, QROO, YUC

*Ateleia chiangii J. Linares SLP, ZAC

*Ateleia chicoasensis J. Linares CHIS

*Ateleia glabrata J. Linares CHIS, OAX
Ateleia hexandra J. Linares CHIS

*Ateleia insularis Standl. NAY

*Ateleia mcvaughii Rudd OAX, PUE

Ateleia pterocarpa Moc. \& Sessé ex D. Dietr. CAM, CHIS, COL, JAL, MICH, NAY, OAX, TAB, VER

*Ateleia sousae J. Linares OAX

*Ateleia standleyana Mohlenbr. NAY, SIN

*Ateleia tenorioi J. Linares CHIS

Ateleia tomentosa Rudd CHIS

*Ateleia truncata Mohlenbr. GRO, MICH

Barbieria pinnata (Pers.) Baill. CHIS, NAY, OAX, VER

* Bauhinia amatlana Wunderlin OAX

*Bauhinia andrieuxii Hemsl. COL, GRO, JAL, MEX, MICH,

MOR, NAY, OAX, PUE, SIN, VER

*Bauhinia bartlettii B.L. Turner NLE, TAMS

Bauhinia cookii Rose CHIS, COL, GRO, JAL, MICH, NAY, OAX, SIN, VER

*Bauhinia coulteri J.F. Macbr. GTO, HGO, QRO, SLP, TAMS, VER

*Bauhinia chapulhuacania Wunderlin HGO, PUE, QRO, SLP, TAMS, VER

*Bauhinia deserti (Britton \& Rose) Lundell GRO, OAX, PUE

Bauhinia dipetala Hemsl. CHIS, HGO, MOR, OAX, PUE, QRO, SLP, VER

Bauhinia divaricata L. AGS, BCS, CAM, CHIS, COL, DGO, GRO, HGO, JAL, MEX, MICH, MOR, NAY, NLE, OAX, PUE, QRO, QROO, SLP, SIN, TAB, TAMS, VER, YUC

Bauhinia erythrocalyx Wunderlin CAM, QROO, YUC

*Bauhinia fryxellii Wunderlin SLP, TAMS

Bauhinia goldmanii Rose CHIS, OAX

*Bauhinia gypsicola McVaugh COL, JAL, MICH

Bauhinia jenningsii $\mathrm{P}$. Wilson CAM, CHIS, QROO, TAB, YUC *Bauhinia jucunda Brandegee VER

Bauhinia lunarioides A. Gray ex S. Watson COAH, NLE, SLP

*Bauhinia macranthera Benth. ex Hemsl. COAH, GTO, HGO, NLE, QRO, SLP, TAMS, VER

*Bauhinia melastomatoidea $\mathrm{R}$. Torres CHIS, OAX, TAB

*Bauhinia miriamae R. Torres OAX

Bauhinia pansamalana Donn. Sm. CHIS, TAB, VER

Bauhinia pauletia Pers. CHIS, COL, DGO, GRO, JAL, MEX, MICH, NAY, OAX, SIN, VER

*Bauhinia pes-caprae Cav. GRO, OAX

*Bauhinia pringlei $\mathrm{S}$. Watson CHIH, COL, GRO, JAL, MEX, MICH, NAY, SIN, SON, TAMS, ZAC

*Bauhinia ramirezii Reynoso GRO, JAL, NAY

*Bauhinia ramosissima Benth. ex Hemsl. CHIS, CHIH, COAH, DGO, HGO, MEX, NLE, QRO, SLP, TAMS, VER, ZAC

Bauhinia rubeleruziana Donn. Sm. CHIS, VER

Bauhinia seleriana Harms CHIS, MOR, OAX

*Bauhinia subrotundifolia Cav. COL, GRO, JAL, MEX, MICH, OAX

Bauhinia ungulata L. CAM, CHIS, CHIH, COL, DGO, GRO, HGO, JAL, MEX, MICH, NAY, OAX, QROO, SIN, TAB, VER, YUC

Bauhinia wunderlinii R. Torres CHIS, OAX, VER

* Brongniartia abbottiae I.M. Johnst. GRO

*Brongniartia alamosana Rydb. CHIH, JAL, MICH, OAX, 
SIN, SON

*Brongniartia argentea Rydb. GTO, HGO, JAL, QRO

*Brongniartia argyrophylla McVaugh DGO, JAL, NAY, ZAC

* Brongniartia capitata Hemsl. VER

* Brongniartia cordata McVaugh COL, JAL

*Brongniartia cuneata L.B. Sm. \& B.G. Schub. GRO, MEX

* Brongniartia diffusa Rose NAY, SIN

*Brongniartia discolor Brandegee HGO, MEX, MOR, NLE, OAX, PUE, QRO, SLP, TAMS, VER

*Brongniartia foliolosa Benth. ex Hemsl. HGO, MEX, MOR, NLE, OAX, PUE, QRO, SLP, TAMS, VER

*Brongniartia funiculata L.B. Sm. \& B.G. Schub. GRO, MEX, $\mathrm{MICH}$

*Brongniartia glabrata Hook. \& Arn. CHIS, COL, GRO, HGO, JAL, NAY, OAX, QRO, SLP, SIN

*Brongniartia goldmanii Rose JAL, NAY, SIN

*Brongniartia guerrerensis J. Jiménez Ram. \& J.L. Contr. GRO

*Brongniartia guiengolensis Dorado \& L. Torres OAX

*Brongniartia harrisii McVaugh DGO, JAL, ZAC

*Brongniartia herbacea R. Grether \& Rzed. MICH

*Brongniartia hirsuta Rydb. GTO, HGO, QRO, SLP

*Brongniartia imitator McVaugh JAL, NAY, ZAC

*Brongniartia inconstans S. Watson COL, GRO, JAL, MICH, NAY, ZAC

*Brongniartia intermedia Moric. CDMX, GTO, GRO, HGO, JAL, MEX, MICH, MOR, NLE, OAX, QRO, SLP, TAMS, TLAX, VER

*Brongniartia luisana Brandegee OAX, PUE

*Brongniartia lunata Rose DGO, JAL, NAY, SON, ZAC

*Brongniartia lupinoides (Kunth) Taub. AGS, CDMX, GTO, GRO, HGO, JAL, MEX, MICH, MOR, OAX, PUE, TLAX, $\mathrm{ZAC}$

*Brongniartia magnibracteata Schltdl. CHIS, CDMX, HGO, NLE, QRO, SLP, TAMS, VER

*Brongniartia minima McVaugh JAL

Brongniartia minutifolia S. Watson CHIH, COAH, SLP, SON

* Brongniartia mollicula Brandegee OAX, PUE

*Brongniartia mollis Kunth MEX, MOR, OAX, PUE

*Brongniartia montalvoana Dorado \& D.M. Arias GRO, MOR, PUE

*Brongniartia mortonii McVaugh COL, GRO, JAL, MICH

*Brongniartia norrisii McVaugh DGO, JAL, NAY, ZAC

*Brongniartia nudiflora S. Watson CHIH, COL, JAL, MEX, MICH, MOR, SON

*Brongniartia oligosperma Baill. MICH, OAX, PUE

* Brongniartia pacifica McVaugh COL, JAL, MICH

*Brongniartia papyracea Dorado \& D.M. Arias COL, JAL, $\mathrm{MICH}$

*Brongniartia parryi Hemsl. GTO, HGO, QRO, SLP

* Brongniartia parvifolia Rose OAX

*Brongniartia peninsularis Rose BCS

*Brongniartia podalyrioides Kunth GRO, HGO, JAL, MEX,

MICH, MOR, OAX, PUE, QRO, SON, VER, ZAC

*Brongniartia pringlei Rydb. HGO, MEX, MICH, QRO

*Brongniartia proteranthera L.B. Sm. \& B.G. Schub. GRO, $\mathrm{MICH}$

*Brongniartia riesebergii O. Dorado DGO
Brongniartia robinioides Kunth COAH, DGO, OAX, PUE

* Brongniartia shrevei Wiggins SON

* Brongniartia sousae Dorado OAX

*Brongniartia suberea Rose GTO, GRO, OAX, PUE

*Brongniartia tenuifolia Standl. JAL, SON

* Brongniartia trifoliata Brandegee BCS

*Brongniartia vazquezii O. Dorado GRO, MOR

*Brongniartia vicioides M. Martens \& Galeotti OAX, PUE

* Brongniartia yahualica Dorado \& D.M. Arias JAL

Caesalpinia cacalaco Bonpl. CAM, CHIS, CHIH, COL, CDMX, DGO, GRO, HGO, JAL, MEX, MICH, MOR, NAY, OAX, PUE, SIN, VER, YUC

* Caesalpinia coccinea G.P. Lewis \& J.L. Contr. OAX

*Caesalpinia colimensis F.J. Herm. COL, GRO, JAL, MICH, OAX, SIN

Caesalpinia drummondii (Torr. \& A. Gray) E.M. Fisher CHIH * Caesalpinia epifanioi J.L. Contr. GRO

Caesalpinia exostemma DC. SLP, TAMS, VER

* Caesalpinia hintonii Sandwith GRO, MICH, OAX, PUE

*Caesalpinia hughesii G.P. Lewis JAL, OAX

Caesalpinia jayabo M. Gómez VER

* Caesalpinia macvaughii J.L. Contr. \& G.P. Lewis GRO, MICH

* Caesalpinia major (Medik.) Dandy \& Exell CHIS, PUE

* Caesalpinia nelsonii (Britton \& Rose) J.L. Contr. Ex G.P. Lewis GRO, OAX

Caesalpinia oyamae Sotuyo \& G.P. Lewis GRO, OAX, PUE

* Caesalpinia pannosa Brandegee BCN, BCS

Caesalpinia phyllanthoides Standl. TAMS

* Caesalpinia placida Brandegee BCS

*Caesalpinia pringlei (Britton \& Rose) Standl. GTO, HGO, OAX, PUE, QRO, SLP, TAMS

* Caesalpinia pumila (Britton \& Rose) F.J. Herm. SON

*Caesalpinia sessilifolia S. Watson CHIH, COAH, DGO, NLE, SIN

Caesalpinia velutina (Britton \& Rose) Standl. CHIS, COL, GRO, JAL, MICH, NAY, OAX, PUE, VER, YUC

Caesalpinia vesicaria L. CAM, CHIS, QROO, TAB, VER, YUC

Calopogonium caeruleum (Benth.) C. Wight CHIS, COL, GRO, JAL, MEX, MICH, NAY, OAX, PUE, QRO, SLP, TAB, VER, YUC

Calopogonium galactioides (Kunth) Benth. ex Hemsl. CHIS, COL, JAL, MEX, MICH, NAY, OAX, PUE, SIN, TAB, VER

Calopogonium lanceolatum Brandegee CHIS

Calliandra arborea Standl. CHIS, GRO, OAX

Calliandra belizensis (Stand1.) Standl. CAM, QROO, YUC

Calliandra biflora Tharp COAH, DGO, HGO, QRO, SLP, TAMS

* Calliandra bifoliolata H.M. Hern. \& Ortiz-Rodr. CHIS

*Calliandra bijuga Rose CHIS, COL, GRO, JAL, MEX, MICH, NAY, OAX, VER

Calliandra caeciliae Harms CHIS, COL, GRO, JAL, MEX, MICH, MOR, NAY, OAX, PUE

* Calliandra californica Benth. BCN, BCS, SON

Calliandra calothyrsus Meisn. CAM, CHIS, COL, DGO, GRO,

JAL, MICH, NAY, OAX, SIN, VER

* Calliandra cualensis H.M. Hern. JAL 
* Calliandra dolichopoda H.M. Hern. GRO

Calliandra eriophylla Benth. AGS, BCN, BCS, CHIS, CHIH, COAH, COL, DGO, GTO, GRO, HGO, JAL, MEX, MOR, NAY, NLE, OAX, PUE, QRO, SLP, SIN, SON, TAMS, VER, ZAC

*Calliandra erythrocephala H.M. Hern. \& M. Sousa GRO, OAX

Calliandra grandiflora (L'Hér.) Benth. AGS, CHIS, CHIH, COL, CDMX, DGO, GTO, GRO, HGO, JAL, MEX, MICH, MOR, NAY, NLE, OAX, PUE, QRO, SLP, SIN, SON, TAMS, TLAX, VER, ZAC

*Calliandra hirsuta (G. Don) Benth. CHIS, COL, GRO, JAL, NAY, OAX, PUE, ZAC

Calliandra houstoniana (Mill.) Standl. CAM, CHIS, CHIH, COL, GRO, HGO, JAL, MEX, MICH, MOR, NAY, OAX, PUE, QRO, QROO, SLP, SIN, SON, TAB, TAMS, VER, YUC, ZAC Calliandra humilis (Schltdl.) Benth. AGS, CHIH, COAH, CDMX, DGO, GTO, GRO, HGO, JAL, MEX, NLE, OAX, PUE, QRO, SIN, SON, TAMS, VER, ZAC

* Calliandra juzepczukii Standl. CHIS, OAX, TAB, VER

* Calliandra laevis Rose COL, JAL, MICH, NAY, SIN, ZAC

Calliandra laxa (Willd.) Benth. CHIS, GRO, OAX, PUE, VER

* Calliandra longipedicellata (McVaugh) Macqueen \& H.M. Hern. COL, DGO, JAL

Calliandra magdalenae (Bertero ex DC.) Benth. CHIS, GRO, MICH, NAY, OAX, VER

*Calliandra palmeri S. Watson GRO, JAL, NAY, SIN, OAX, ZAC

* Calliandra peninsularis Rose BCS

* Calliandra physocalyx H.M. Hern. \& M. Sousa GRO, OAX

* Calliandra ricoana H. Hern. \& R. Duno CHIS

* Calliandra rubescens (M. Martens \& Galeotti) Standl. CHIS, MICH, OAX, TAB, VER

* Calliandra sesquipedalis McVaugh JAL

* Calliandra sinaloana (Britton \& Rose) Standl. SIN

* Calliandra strigillosa (Britton \& Rose) Standl. ex Leavenw. JAL, MICH, VER

Calliandra tergemina (L.) Benth. CAM, CHIS, CHIH, COL, GRO, JAL, MICH, NAY, OAX, PUE, QROO, SLP, SIN, SON, TAB, TAMS, VER, YUC

*Calliandropsis nervosus (Britton \& Rose) H.M. Hern. \& P. Guinet HGO, OAX, PUE

*Canavalia acuminata Rose COL, GRO, JAL, MICH, NAY, OAX, PUE, SIN, VER

Canavalia brasiliensis Mart. ex Benth. CAM, CHIS, COL, GRO, JAL, MEX, MICH, NAY, OAX, QROO, SIN, SON, TAMS, VER, YUC

* Canavalia dura J.D. Sauer CHIS, GRO

Canavalia ensiformis (L.) DC. CAM, CHIS, NLE, OAX, QROO, TAB, VER, YUC

Canavalia glabra (M. Martens \& Galeotti) J.D. Sauer CHIS, HGO, MOR, NAY, OAX, PUE, QRO, TAB, VER

Canavalia hirsutissima J.D. Sauer CHIS, COL, GRO, HGO, JAL, MEX, MICH, OAX, PUE, QRO, SIN, VER

Canavalia matudae J.D. Sauer CHIS

Canavalia oxyphylla Standl. \& L.O. Williams CHIS, OAX, PUE, TAB, VER
* Canavalia palmeri (Piper) Standl. CHIS, MICH, NAY, OAX Canavalia rosea (Sw.) DC. CAM, CHIS, COL, GRO, JAL, MICH, NAY, OAX, PUE, QROO, SIN, TAB, TAMS, VER, YUC

* Canavalia septentrionalis J.D. Sauer COAH, COL, DGO, HGO, JAL, NAY, NLE, PUE, QRO, SLP, TAMS, VER

Canavalia villosa Benth. AGS, CAM, CHIS, COAH, COL, CDMX, DGO, GTO, GRO, HGO, JAL, MEX, MICH, MOR, NAY, NLE, OAX, PUE, QRO, QROO, SLP, SIN, SON, TAB, TAMS, VER, YUC, ZAC

Cassia grandis L. f. CAM, CHIS, COL, GRO, MEX, MICH, OAX, QROO, TAB, VER, YUC

* Cassia hintonii Sandwith COL, GRO, JAL, MEX, MICH

Cassia moschata Kunth CHIS, OAX, TAB, VER

Centrosema angustifolium (Kunth) Benth. CAM, CHIS, OAX, SLP, TAB, TAMS, VER

*Centrosema galeottii Fantz CAM, CHIS, GRO, NAY, OAX, QROO

Centrosema macrocarpum Benth. CHIS, GRO, JAL, NAY, OAX, PUE, QROO, TAB, VER

Centrosema pascuorum Mart. ex Benth. CHIS, OAX, TAB

Centrosema plumieri (Turpin ex Pers.) Benth. CAM, CHIS, COL, GRO, JAL, MEX, MICH, MOR, NAY, OAX, PUE, QRO, QROO, SLP, TAB, TAMS, VER, YUC

Centrosema pubescens Benth. CAM, CHIS, COL, CDMX, GRO, HGO, JAL, MEX, MICH, MOR, NAY, OAX, PUE, QRO, QROO, SLP, TAB, VER, YUC

Centrosema sagittatum (Willd.) Brandegee ex L. Riley CAM, CHIS, COAH, COL, GRO, JAL, MICH, NAY, NLE, OAX, QRO, SLP, SIN, TAB, TAMS, VER, YUC

*Centrosema schottii (Millsp.) K. Schum CAM, CHIS, QROO, VER, YUC

*Centrosema unifoliatum (Rose) Lundell CHIS, QROO, TAB

Centrosema virginianum (L.) Benth. CAM, CHIS, COAH, COL, GTO, GRO, HGO, JAL, MEX, MICH, MOR, NAY, NLE, OAX, PUE, QRO, QROO, SLP, SIN, SON, TAB, TAMS, VER, YUC, ZAC

Cercidium $\times$ sonorae Rose \& I.M. Johnst. BCS, SIN, SON

Cercis canadensis L. CHIH, COAH, HGO, NLE, PUE, QRO, SLP, TAMS, VER

Clitoria falcata Lam. CHIS, NAY, OAX, SLP, TAB, VER, ZAC Clitoria glaberrima Pittier CHIS, TAB

Clitoria guianensis (Aubl.) Benth. CHIS, OAX, TAB, VER

* Clitoria humilis Rose DGO

Clitoria mariana L. CHIH, NLE, SON

Clitoria mexicana Link CAM, CHIS, COAH, COL, GRO, HGO, JAL, MEX, MICH, MOR, NLE, OAX, PUE, TAMS, VER, ZAC * Clitoria monticola Brandegee BCS

Clitoria polystachya Benth. CHIS, COL, GRO, JAL, MEX, MICH, MOR, NAY, OAX, PUE, SIN, SON, VER, ZAC

*Clitoria triflora S. Watson DGO, JAL, MICH, NAY, OAX, ZAC

Cojoba arborea (L.) Britton \& Rose CAM, CHIS, GRO, HGO, JAL, MICH, MOR, NLE, OAX, PUE, QRO, QROO, SLP, SIN, TAB, TAMS, VER

Cojoba graciliflora (S.F. Blake) Britton \& Rose CAM, CHIS, OAX, QROO, TAB, VER 
* Cojoba haematoloba L. Rico CHIS, TAB

* Cojoba mariaelenae L. Rico CHIS, OAX

Cojoba sophorocarpa (Benth.) Britton \& Rose CHIS, TAB, VER

Cologania angustifolia Kunth AGS, CHIS, CHIH, COAH, CDMX, DGO, GTO, GRO, HGO, JAL, MEX, MICH, MOR, NAY, NLE, OAX, PUE, QRO, SLP, SIN, SON, TAMS, TLAX, VER, ZAC

Cologania biloba (Lindl.) G. Nicholson CDMX, GRO, HGO, MEX, MICH, MOR, OAX, PUE, QRO, TLAX, VER

Cologania broussonetii (Balb.) DC. AGS, CHIS, CHIH, COAH, COL, CDMX, DGO, GTO, GRO, HGO, JAL, MEX, MICH, MOR, NAY, NLE, OAX, PUE, QRO, SLP, SIN, SON, TAMS, TLAX, VER, ZAC

* Cologania capitata Rose JAL, NAY, ZAC

* Cologania cordata Fearing ex McVaugh COL, DGO, JAL, NAY, SIN, SON, ZAC

Cologania glabrior Rose CHIS, HGO, MEX, MICH, TLAX, VER

*Cologania hintoniorum B.L. Turner GRO, MICH

*Cologania hirta (M. Martens \& Galeotti) Rose GRO, MICH, NAY, OAX

*Cologania intermedia Kunth CHIS, CHIH, DGO, MICH, QRO, SON, ZAC

*Cologania jaliscana S. Watson AGS, CHIH, JAL, NAY, ZAC Cologania obovata Schltdl. AGS, CHIH, CDMX, DGO, GTO, GRO, HGO, JAL, MEX, MICH, MOR, NAY, OAX, PUE, QRO, SLP, SIN, SON, TLAX, VER, ZAC

Cologania pallida Rose COAH, DGO, NLE, TAMS

Cologania procumbens Kunth CHIS, CHIH, COL, GTO, GRO, JAL, MEX, MICH, MOR, NAY, OAX, PUE, SIN, SON, VER, ZAC

*Cologania racemosa (B.L. Rob.) Rose AGS, COL, DGO, JAL, MICH, NAY, SIN, ZAC

Cologania rufescens Rose CHIH, CDMX, GTO, HGO, JAL, MEX, MICH, OAX, QRO, SON, VER

* Cologania tenuis Rose GRO, MOR, OAX

* Conzattia chiapensis Miranda CHIS

*Conzattia multiflora (B.L. Rob.) Standl. AGS, BCS, CHIH, COL, DGO, GTO, GRO, JAL, MEX, MICH, MOR, NAY, OAX, PUE, QRO, SLP, SIN, SON, TAB, TAMS, VER, ZAC

Coulteria mollis Kunth CAM, CHIS, QROO, YUC

* Coulteria platyloba (S. Watson) N. Zamora CAM, CHIH, COL, DGO, GRO, HGO, JAL, MEX, MICH, MOR, NAY, OAX, PUE, QRO, QROO, SLP, SIN, SON, TAMS, VER, YUC, ZAC

Coursetia axillaris J.M. Coult. \& Rose SLP, TAMS, VER

* Coursetia barrancana Lavin CHIH, SON

Coursetia caribaea (Jacq.) Lavin AGS, BCN, BCS, CAM, CHIS, CHIH, COAH, COL, DGO, GTO, GRO, HGO, JAL, MEX, MICH, MOR, NAY, NLE, OAX, PUE, QRO, QROO, SLP, SIN, SON, TAMS, VER, YUC, ZAC

* Coursetia chiapensis Lavin \& M. Sousa CHIS

Coursetia glabella (A. Gray) Lavin CHIH, COAH, DGO, NLE, SON

Coursetia glandulosa A. Gray AGS, BCN, BCS, CAM, CHIH, COL, DGO, GRO, HGO, JAL, MEX, MICH, MOR, NAY, OAX,
PUE, QRO, SLP, SIN, SON, ZAC

Coursetia greenmanii (Millsp.) R. Duno \& Carnevali CAM, QROO, VER, YUC

Coursetia guatemalensis Rudd CHIS

*Coursetia hidalgoana Lavin HGO, MEX, QRO, VER

* Coursetia hintonii Rudd GRO, MEX, NAY

* Coursetia insomniifolia Lavin COAH

*Coursetia madrensis Micheli GRO, MEX, MICH, MOR, OAX, PUE

*Coursetia mollis B.L. Rob. \& Greenm. COL, DGO, GRO, JAL, MEX, MICH, NAY, OAX, SIN, ZAC

*Coursetia oaxacensis M. Sousa \& Rudd GRO, OAX

* Coursetia paniculata M. Sousa \& Lavin OAX

* Coursetia planipetiolata Micheli GRO, OAX

Coursetia polyphylla Brandegee GRO, OAX, VER

Coursetia pumila (Rose) Lavin CHIH, COL, CDMX, DGO, GTO, GRO, JAL, MEX, MICH, NAY, NLE, OAX, PUE, QRO, SLP, SON, TAMS, VER, ZAC

*Coursetia robinioides M. Sousa \& Lavin OAX

Crotalaria angulata Mill. BCS, GRO, JAL, MEX, MICH, MOR, OAX, SON, VER

*Crotalaria brevipedunculata Windler CHIH, DGO, JAL, NAY, SIN

*Crotalaria bupleurifolia Cham. \& Schltdl. CHIS, COL, GRO, JAL, MEX, MICH, MOR, NAY, OAX, PUE, SLP, SIN, SON, TAMS, VER

Crotalaria cajanifolia Kunth BCN, BCS, CHIS, CHIH, COL, DGO, GRO, JAL, MEX, MICH, MOR, NAY, NLE, OAX, PUE, QROO, SIN, SON, TAB, TAMS, VER, YUC, ZAC

* Crotalaria chiapensis Brandegee CHIS

*Crotalaria filifolia Rose CHIS, CHIH, COL, DGO, GRO, JAL, MEX, MICH, MOR, NAY, NLE, OAX, PUE, SIN, SON

* Crotalaria gloriosa Rose GRO, OAX

Crotalaria incana L. BCS, CAM, CHIS, CHIH, COAH, COL, GTO, GRO, HGO, JAL, MEX, MICH, MOR, NAY, NLE, OAX, PUE, QRO, QROO, SLP, SIN, SON, TAB, TAMS, VER, YUC, ZAC

Crotalaria longirostrata Hook. \& Arn. CAM, CHIS, CHIH, COL, DGO, GRO, JAL, MEX, MICH, MOR, NAY, OAX, PUE, QROO, SIN, SON, TAB, VER, ZAC

Crotalaria maypurensis Kunth CHIS, CHIH, COL, GRO, HGO, JAL, MEX, MICH, MOR, NAY, OAX, PUE, SLP, SIN, SON, TAB, VER

* Crotalaria mexicana Windler GRO, JAL, OAX, SIN, ZAC Crotalaria micans Link CHIS, COL, DGO, GRO, JAL, MEX, MICH, MOR, NAY, OAX, PUE, QRO, SIN, VER, ZAC

Crotalaria mollicula Kunth AGS, CAM, CHIS, CHIH, COL, DGO, GTO, GRO, JAL, MEX, MICH, MOR, NAY, NLE, OAX, PUE, QRO, SLP, SIN, SON, TAMS, VER, YUC, ZAC

*Crotalaria monticola Brandegee CHIS

*Crotalaria nayaritensis Windler JAL, MICH, NAY, OAX, PUE, SIN, ZAC

Crotalaria nitens Kunth CHIS, COL, JAL, MICH, OAX, PUE, VER

Crotalaria pallida Aiton SLP, SIN, TAB, VER

*Crotalaria polyphylla L. Riley CHIS, CHIH, COAH, COL, DGO, GRO, JAL, MEX, MICH, MOR, NAY, NLE, OAX, PUE, 
SIN, SON, TAB, TAMS, VER, ZAC

Crotalaria pumila Ortega AGS, BCS, CAM, CHIS, CHIH, COAH, COL, CDMX, DGO, GTO, GRO, HGO, JAL, MEX, MICH, MOR, NAY, NLE, OAX, PUE, QRO, QROO, SLP, SIN, SON, TAMS, TLAX, VER, YUC, ZAC

Crotalaria purdiana Senn. CAM, CHIS, QROO, YUC

Crotalaria purshii DC. CHIH, JAL, SIN, SON, VER

Crotalaria rotundifolia (Walter) Walter ex J.F. Gmel. AGS, BCN, BCS, CAM, CHIS, CHIH, COAH, COL, CDMX, DGO, GTO, GRO, HGO, JAL, MEX, MICH, MOR, NAY, NLE, OAX, PUE, QRO, SLP, SIN, SON, VER, ZAC

*Crotalaria rzedowskii J. Espinosa CDMX, MEX, VER

Crotalaria sagittalis L. BCS, CAM, CHIS, CHIH, COAH, COL, DGO, GTO, GRO, HGO, JAL, MEX, MICH, MOR, NAY, NLE, OAX, PUE, QRO, SLP, SIN, SON, TAB, TAMS, VER, ZAC Crotalaria spectabilis Roth CHIS, OAX, VER

Crotalaria vitellina Ker Gawl. CHIS, CHIH, COL, GRO, JAL, MEX, MICH, MOR, NAY, OAX, QRO, SLP, SIN, SON, TAB, TAMS, VER, YUC

Cymbosema roseum Benth. CHIS

Cynometra hemitomophylla (Donn. Sm.) Rose CAM, VER

Cynometra oaxacana Brandegee CAM, CHIS, COL, GRO, JAL, MICH, OAX, TAB, VER

Cynometra retusa Britton \& Rose CAM, CHIS, OAX, TAB, VER

Chaetocalyx brasiliensis (Vogel) Benth. CHIS, JAL, NAY, OAX, $\mathrm{TAB}, \mathrm{VER}$

Chaetocalyx scandens (L.) Urb. CAM, CHIS, GRO, OAX, QROO, YUC

Chamaecrista absus (L.) H.S. Irwin \& Barneby BCS, CHIS, CHIH, COL, GRO, JAL, MEX, MICH, MOR, NAY, OAX, SIN, SON, VER

Chamaecrista calycioides (DC. ex Collad.) Greene OAX

Chamaecrista chamaecristoides (Collad.) Greene CAM, COL, GRO, HGO, JAL, MICH, NAY, OAX, PUE, SIN, TAB, TAMS, VER

Chamaecrista desvauxii (Collad.) Killip CHIS, GRO, JAL, NAY, OAX, SLP, TAB, TAMS, VER

Chamaecrista diphylla (L.) Greene CAM, CHIS, DGO, GRO, MEX, MOR, OAX, PUE, SIN, TAB, VER, YUC

Chamaecrista fagonioides (Vogel) H.S. Irwin \& Barneby CHIS, GRO, MICH, NAY, OAX, TAB, VER

Chamaecrista flexuosa (L.) Greene CAM, CHIS, COL, GRO, JAL, MEX, MICH, MOR, OAX, TAB, TAMS, VER, YUC

Chamaecrista glandulosa (L.) Greene AGS, CAM, CHIS, CHIH, COL, DGO, GTO, GRO, HGO, JAL, MICH, OAX, QRO, QROO, SLP, VER, YUC, ZAC

Chamaecrista greggii (A. Gray) Pollard ex A.H. Heller CHIH, COAH, GTO, HGO, NLE, OAX, PUE, QRO, SLP, TAMS

Chamaecrista hispidula (Vahl) H.S. Irwin \& Barneby CHIS, DGO, GRO, JAL, MICH, NAY, OAX, SIN, TAB, VER

Chamaecrista kunthiana (Schltdl. \& Cham.) H.S. Irwin \& Barneby CHIS, GRO, OAX, TAB, VER

Chamaecrista lineata (Sw.) Greene CHIS, OAX

Chamaecrista nictitans (L.) Moench AGS, BCN, BCS, CAM, CHIS, CHIH, COAH, COL, DGO, GTO, GRO, HGO, JAL, MEX, MICH, MOR, NAY, NLE, OAX, PUE, QRO, QROO,
SLP, SIN, SON, TAB, TAMS, VER, YUC, ZAC

Chamaecrista pilosa (L.) Greene BCS, CHIS, MOR, OAX, QRO, SLP, VER

*Chamaecrista punctulata (Hook. \& Arn.) H.S. Irwin \& Barneby COL, DGO, GRO, JAL, MEX, MICH, NAY, OAX, SIN, ZAC

Chamaecrista rotundifolia (Pers.) Greene COL, DGO, GRO, JAL, MEX, MICH, MOR, NAY, OAX, SIN, TAB, VER, ZAC

Chamaecrista rufa (M. Martens \& Galeotti) Britton \& Rose CHIS, GRO, HGO, MICH, OAX, PUE, QRO, SLP, TAB, TAMS, VER

Chamaecrista serpens (L.) Greene AGS, CHIS, CHIH, COL, DGO, GRO, JAL, MEX, MICH, NAY, OAX, PUE, SLP, SIN, SON, TAB, ZAC

Chamaecrista viscosa (Kunth) H.S. Irwin \& Barneby GRO, JAL, NAY, OAX, SIN

Chamaecrista zygophylloides (Taub.) H.S. Irwin \& Barneby CHIS, GRO, OAX, PUE

Chloroleucon mangense (Jacq.) Britton \& Rose BCS, CAM, CHIS, CHIH, COL, DGO, GRO, JAL, MICH, NAY, OAX, QROO, SIN, SON, VER, YUC

Dalbergia brownei (Jacq.) Schinz CAM, CHIS, OAX, QROO, SLP, TAB, TAMS, VER

Dalbergia calderonii Standl. CHIS, OAX

Dalbergia calycina Benth. CHIS, MEX, MICH, OAX

*Dalbergia congestiflora Pittier CHIS, COL, GRO, JAL, MEX, MICH, MOR, OAX, PUE, QRO, SLP

Dalbergia ecastaphyllum (L.) Taub. TAB, VER

Dalbergia glabra (Mill.) Standl. CAM, CHIS, GRO, HGO, MEX, MICH, MOR, OAX, QRO, QROO, SLP, TAB, VER, YUC

*Dalbergia glomerata Hemsl. CHIS, GRO, HGO, MEX, MICH, MOR, OAX, TAB, VER

*Dalbergia granadillo Pittier CHIS, COL, GRO, JAL, MEX, MICH, NAY, OAX, VER

Dalbergia longepedunculata J. Linares \& M. Sousa CHIS, OAX Dalbergia luteola J. Linares \& M. Sousa CHIS

Dalbergia melanocardium Pittier CHIS, VER

*Dalbergia modesta J. Linares \& M. Sousa CHIS, OAX

Dalbergia monetaria L. f. CHIS, TAB, VER

*Dalbergia palo-escrito Rzed. \& Guridi-Gómez HGO, QRO, SLP, VER

Dalbergia retusa Hemsl. CHIS

*Dalbergia rhachiflexa J. Linares \& M. Sousa GRO, MICH

Dalbergia ruddae J. Linares \& M. Sousa CHIS

Dalbergia stevensonii Standl. CHIS

*Dalea abietifolia (Rydb.) Bullock JAL, MICH, NAY, ZAC

* Dalea acracarpica Barneby CHIS

*Dalea aenigma Barneby CDMX, HGO, MEX, PUE, TLAX

*Dalea alamosana (Rose) Bullock SIN, SON

Dalea albiflora A. Gray CHIH, DGO, SIN, SON

Dalea alopecuroides Willd. MEX, MOR, SON, VER

*Dalea ananassa Barneby SIN, SON

Dalea aurea Nutt. ex Pursh CHIH, COAH, DGO, NLE, TAMS

*Dalea bacchantum Barneby MOR

Dalea bicolor Humb. \& Bonpl. ex Willd. AGS, BCN, BCS, CHIS, CHIH, COAH, CDMX, DGO, GTO, GRO, HGO, JAL, 
MEX, MICH, MOR, NAY, NLE, OAX, PUE, QRO, SLP, SIN, SON, TAMS, VER, ZAC

* Dalea boraginea Barneby COAH

*Dalea botterii (Rydb.) Barneby NLE, OAX, PUE, TAMS, VER

Dalea brachystachys A. Gray AGS, CHIH, COAH, DGO, HGO, JAL, MEX, MICH, NLE, OAX, PUE, QRO, SLP, SON, TAMS, VER, ZAC

*Dalea brandegeei (Rose) Bullock BCS

* Dalea caeciliae Harms OAX, PUE

Dalea candida Michx. ex Willd. CHIH, SON

*Dalea capitata S. Watson AGS, CHIH, COAH, DGO, NLE, SLP, TAMS, ZAC

Dalea carthagenensis (Jacq.) J.F. Macbr. CAM, CHIS, COL, HGO, JAL, MEX, MICH, NAY, OAX, PUE, QRO, QROO, SLP, TAMS, VER, YUC

Dalea caudata (Rydb.) Bullock HGO, QRO, SLP

*Dalea cinnamomea Barneby PUE

Dalea citriodora Willd. CDMX, GRO, MEX, OAX, VER

Dale a cliffortiana Willd. CHIS, CHIH, COL, DGO, GRO, HGO, JAL, MEX, MICH, MOR, NAY, OAX, QRO, SLP, SIN, SON, TAMS, VER, ZAC

*Dalea conetensis E. Estrada \& Villarreal DGO

*Dalea confusa (Rydb.) Barneby AGS, CHIH, JAL, MICH, ZAC

*Dalea cora Barneby DGO, JAL, NAY, ZAC

* Dalea crassifolia Hemsl. DGO, JAL, NAY, SIN

Dalea cuniculo-caudata Paul G. Wilson GRO, MEX, MICH

*Dalea cyanea Greene CHIH, DGO, JAL, MICH, NAY, SON, ZAC

* Dalea choanosema Barneby GRO

*Dalea daucosma Barneby JAL, NAY

* Dalea dipsacea Barneby JAL, MICH, NAY

*Dalea divaricata Benth. BCS

Dalea domingensis DC. CHIS, VER, YUC

*Dalea dorycnioides DC. CHIH, DGO, HGO, MEX, NLE, OAX, PUE, QRO, SLP, TAMS, VER, ZAC

*Dalea elata Hook. \& Arn. CHIS, COL, DGO, GRO, JAL, MEX, MICH, MOR, NAY, OAX, PUE, SIN, SON, VER

Dalea emarginata (Torr. \& A. Gray) Shinners TAMS, VER

* Dalea emmae Rzed. \& Calderón GTO

*Dalea eriophylla S. Watson COAH, GTO, JAL, NLE, SLP, TAMS, ZAC

*Dalea erythrorhiza Greenm. AGS, CHIH, DGO, GTO, JAL, SLP, ZAC

*Dalea escobilla Barneby JAL, NAY, ZAC

* Dalea estoraxana Rzed. \& Calderón QRO

Dalea exigua Barneby AGS, CHIH, JAL, SON, ZAC

Dalea exserta (Rydb.) Gentry CHIS, CHIH, COL, GRO, JAL, MEX, MICH, MOR, OAX, SIN, SON, VER

*Dalea filiciformis B.L. Rob. \& Greenm. AGS, CDMX, DGO, GTO, HGO, JAL, MEX, MICH, MOR, NLE, OAX, PUE, QRO, SLP, TAMS, VER, ZAC

Dalea filiformis A. Gray CHIH, DGO, SON

Dalea foliolosa (Aiton) Barneby AGS, CHIS, CHIH, COAH, COL, CDMX, DGO, GTO, GRO, HGO, JAL, MEX, MICH, MOR, NAY, OAX, PUE, QRO, SLP, TAMS, TLAX, VER, ZAC
Dalea formosa Torr. CHIH, COAH, SON

Dalea frutescens A. Gray CHIH, COAH, NLE, SLP, TAMS, ZAC

Dalea grayi (Vail) L.O. Williams CHIH, DGO, SON

Dalea greggii A. Gray CHIH, COAH, DGO, GTO, HGO, MOR,

NLE, OAX, PUE, QRO, SLP, SIN, SON, TAMS, VER, ZAC

*Dalea gymnocodon Barneby GRO

*Dalea gypsophila Barneby NLE

*Dalea hegewischiana Steud. CDMX, HGO, MEX, OAX, PUE, QRO, VER

*Dalea hemsleyana (Rose) Bullock AGS, DGO, GTO, JAL, SLP, ZAC

*Dalea hintonii Sandwith GRO, MEX

*Dalea holwayi Rose GRO, MOR

Dalea hospes (Rose) Bullock COAH, NLE, TAMS, ZAC

Dalea humilis G. Don AGS, CHIH, CDMX, DGO, GTO, GRO, HGO, JAL, MEX, MICH, MOR, NAY, OAX, PUE, QRO, SLP,

TAMS, TLAX, VER, YUC, ZAC

*Dalea illustris Barneby OAX

*Dalea insignis Hemsl. OAX, PUE, VER

Dalea jamesii (Torr.) Torr. \& A. Gray CHIH, DGO

*Dalea janosensis A.E. Estrada \& Villarreal CHIH

*Dalea juncea (Rydb.) Wiggins BCN

Dalea lachnostachys A. Gray CHIH, COAH, SON

*Dalea lagopina $\mathrm{Rydb}$. CHIH, SON

*Dalea lamprostachya Barneby DGO, NAY, SIN

Dalea lanata Spreng. CHIH, COAH, DGO, NLE, TAMS

Dalea laniceps Barneby COAH, DGO, NLE, SLP, ZAC

Dalea lasiathera A. Gray COAH, NLE, TAMS

Dalea leporina (Aiton) Bullock AGS, CHIS, CHIH, COAH, COL, CDMX, DGO, GTO, GRO, HGO, JAL, MEX, MICH, MOR, NAY, NLE, OAX, PUE, QRO, SLP, SIN, SON, TLAX, VER, YUC, ZAC

*Dalea leptostachya DC. GRO, MEX, MICH, MOR, OAX, PUE

Dalea leucosericea (Rydb.) Standl. \& Steyerm. GRO, HGO, MOR, OAX, PUE, QRO, VER

*Dalea leucostachya A. Gray CHIS, CHIH, COL, DGO, GTO, GRO, JAL, MEX, MICH, MOR, NAY, OAX, SIN, SON, ZAC

*Dalea luisana S. Watson AGS, COAH, DGO, NLE, PUE, QRO, SLP, TAMS, ZAC

Dalea lumholtzii B.L. Rob. \& Fernald CHIH, SON

Dalea lutea (Cav.) Willd. AGS, CHIS, CHIH, COAH, CDMX, DGO, GTO, GRO, HGO, JAL, MEX, MICH, NLE, OAX, PUE, QRO, SLP, TAMS, TLAX, VER, ZAC

*Dalea maritima Brandegee BCS

* Dalea mcvaughii Barneby COL, JAL

*Dalea megalostachys (Rose) Wiggins BCN, BCS

*Dalea melantha S. Schauer COAH, DGO, GTO, HGO, NLE, OAX, PUE, QRO, SLP, TAMS, VER, ZAC

*Dalea mexiae Barneby JAL, ZAC

*Dalea minutifolia (Rydb.) Harms CDMX, HGO, MEX, MICH, OAX, PUE, QRO, SLP, TLAX, VER, ZAC

*Dalea mixteca Barneby OAX

Dalea mollis Benth. BCN, BCS, CHIH, SON

*Dalea mucronata DC. AGS, CHIH, CDMX, GTO, GRO, HGO, JAL, MEX, MICH, NAY, OAX, SON, ZAC 
Dalea multiflora (Nutt.) Shinners COAH, NLE

Dalea nana Torr. ex A. Gray AGS, CHIH, COAH, DGO, NLE, SON, TAMS, ZAC

*Dalea nelsonii (Rydb.) Barneby CHIH, DGO

* Dalea nemaphyllidia Barneby JAL, NAY

Dalea neomexicana (A. Gray) Cory BCN, CHIH, COAH, DGO, GTO, JAL, NLE, SON, TAMS

*Dalea nobilis Barneby JAL, ZAC

Dalea nutans (Cav.) Willd. CHIS, MEX, MICH, MOR, SON, YUC

Dalea obovata (Torr. \& A. Gray) Shinners COAH, TAMS

Dalea obovatifolia Ortega AGS, CDMX, GTO, GRO, HGO, JAL, MEX, MICH, MOR, NLE, OAX, PUE, QRO, SLP, TLAX, VER, ZAC

*Dalea obreniformis (Rydb.) Barneby CHIH, COL, DGO, JAL, MEX, MICH, MOR, OAX, SIN, SON

Dalea ordiae A. Gray CHIH, SON

*Dalea pectinata Kunth AGS, DGO, GTO, JAL, MICH, NAY, SLP, ZAC

Dalea phleoides (Torr. \& A. Gray) Shinners MICH

* Dalea pinetorum Gentry CHIH, DGO, SON

*Dalea piptostegia Barneby OAX, PUE

* Dalea plantaginoides Barneby AGS, JAL, MICH

Dalea pogonathera A. Gray AGS, CHIH, COAH, DGO, JAL, NLE, SLP, SON, TAMS, ZAC

*Dalea polycephala (Rydb.) Bullock HGO, SLP, VER

Dalea polygonoides A. Gray AGS, CHIH, DGO, SON, ZAC

*Dalea polystachya (Sessé \& Moc.) Barneby AGS, COL, DGO, GRO, JAL, MEX, MICH, NAY, OAX, SIN, ZAC

Dalea pringlei A. Gray $\mathrm{CHIH}, \mathrm{SON}$

*Dalea prostrata Ortega AGS, CHIH, CDMX, DGO, GTO, GRO, HGO, JAL, MEX, MICH, NLE, OAX, QRO, SLP, TAMS, VER, ZAC

*Dalea pulchella G. Don COL, DGO, JAL, NAY, SLP, SIN

Dalea pulchra Gentry SON

*Dalea purpusii Brandegee BCN, BCS

Dalea quercetorum Standl. \& L.O. Williams CHIS, OAX

* Dalea radicans $\mathrm{S}$. Watson COAH, NLE, SLP

* Dalea reclinata (Cav.) Willd. AGS, CHIH, CDMX, DGO, GTO, HGO, JAL, MEX, MICH, MOR, OAX, PUE, QRO, SON, TLAX, VER, ZAC

* Dalea revoluta $\mathrm{S}$. Watson CHIH, DGO, JAL, SIN, SON, ZAC

* Dalea rosarum Rzed. \& Calderón QRO

*Dalea rubrolutea Barneby OAX

*Dalea rupertii E. Estrada, Villarreal \& M. González DGO, NAY

*Dalea rupertiana Rzed. \& Calderón GTO

*Dalea rzedowskii Barneby SLP, TAMS

*Dalea saffordii (Rose) Bullock COAH, DGO, NLE

Dalea scandens (Mill.) R.T. Clausen CAM, CHIS, COAH, HGO, JAL, NLE, OAX, QRO, QROO, SLP, SIN, SON, TAMS, VER, YUC

Dalea scoparia A. Gray CHIH, COAH, DGO

*Dalea schiblii Medina \& M. Sousa OAX

Dalea sericea Lag. CHIS, COL, CDMX, DGO, GTO, GRO, HGO, JAL, MEX, MICH, MOR, OAX, PUE, QRO, TLAX, VER, ZAC
* Dalea sericocalyx (Rydb.) L. Riley DGO, NAY, SIN

* Dalea similis Hemsl. DGO, SIN

*Dalea simulatrix Barneby CHIH, DGO

*Dalea sousae Barneby OAX

Dalea tentaculoides Gentry SON

*Dalea thouinii Schrank CDMX, DGO, GTO, HGO, JAL, MEX, MICH, MOR

*Dalea tinctoria Brandegee BCS

* Dalea tolteca Barneby OAX, PUE

Dalea tomentosa (Cav.) Willd. CHIS, CHIH, DGO, GTO, GRO, JAL, MEX, MICH, MOR, NAY, OAX, SLP, SIN, SON, TAMS, VER, ZAC

* Dalea transiens Barneby DGO, SIN

* Dalea trifoliata Zucc. GRO, OAX

* Dalea trochilina Brandegee BCN, BCS

*Dalea uniflora (Barneby) G.L. Nesom NLE, TAMS

Dalea urceolata Greene CHIH, DGO, JAL, MEX, MICH, MOR, SON

* Dalea verna Barneby CHIH, DGO, ZAC

Dalea versicolor Zucc. AGS, CHIS, CHIH, COL, CDMX, DGO, GTO, GRO, HGO, JAL, MEX, MICH, MOR, NAY, OAX, PUE, QRO, SLP, SIN, SON, TAMS, TLAX, VER, ZAC

*Dalea vetula Brandegee BCN, BCS, SON

* Dalea virgata Lag. OAX

*Dalea viridiflora $\mathrm{S}$. Watson CHIH, DGO, HGO

* Dalea wigginsii Barneby DGO, SIN

Dalea wrightii A. Gray CHIH, COAH, NLE, SLP, SON, ZAC

*Dalea zimapanica S. Schauer CDMX, GRO, HGO, MEX, MICH, MOR, NLE, OAX, PUE, QRO, TLAX, VER

Dermatophyllum gypsophilum (B.L. Turner \& A.M. Powell) Vincent CHIH

*Dermatophyllum juanhintonianum (B.L. Turner) B.L. Turner NLE, TAMS

*Dermatophyllum purpusii (Brandegee) Vincent COAH, ZAC Dermatophyllum secundiflorum (Ortega) Gandhi \& Reveal CHIH, COAH, DGO, GTO, HGO, NLE, OAX, PUE, QRO, SLP, TAMS, VER, ZAC

*Desmanthus balsensis J.L. Contr. GRO

Desmanthus bicornutus S. Watson BCS, CHIH, COL, GRO, JAL, MEX, MICH, NAY, SIN, SON, VER, ZAC

Desmanthus cooleyi (Eaton) Trel. CHIH, COAH, DGO, SON

Desmanthus covillei (Britton \& Rose) Wiggins ex B.L. Turner BCN, BCS, SIN, SON

*Desmanthus fruticosus Rose BCN, BCS, SON

Desmanthus glandulosus (B.L. Turner) Luckow COAH, NLE

*Desmanthus interior (Britton \& Rose) Bullock COL, DGO, GTO, GRO, JAL, MEX, MICH, MOR, ZAC

Desmanthus leptophyllus Kunth CAM, CHIS, GRO, MEX, MICH, MOR, OAX, PUE, QROO, TAB, VER, YUC

Desmanthus obtusus S. Watson COAH, NLE, TAMS

*Desmanthus oligospermus Brandegee BCS

*Desmanthus painteri (Britton \& Rose) Standl. CHIH, COAH, DGO, GTO, GRO, HGO, MEX, NLE, OAX, PUE, QRO, SLP, ZAC

Desmanthus paspalaceus (Lindm.) Burkart CAM, TAMS, VER *Desmanthus pringlei (Britton \& Rose) F.J. Herm. COAH, NLE Desmanthus pubescens B.L. Turner CAM, PUE, QROO, TAB, 
VER, YUC

*Desmanthus pumilus (Schltdl.) J.F. Macbr. AGS, CDMX, DGO, GTO, HGO, JAL, MEX, MICH, OAX, PUE, QRO, SLP, ZAC

Desmanthus velutinus Scheele CHIH, COAH, NLE

Desmanthus virgatus (L.) Willd. CAM, CHIS, CHIH, COAH, COL, DGO, GTO, GRO, HGO, JAL, MEX, MICH, MOR, NAY, NLE, OAX, PUE, QRO, QROO, SLP, SIN, SON, TAB, TAMS, VER, YUC, ZAC

Desmodium adscendens (Sw.) DC. CAM, CHIS, GRO, HGO, OAX, QRO, QROO, SLP, TAB, TAMS, VER

Desmodium affine Schltdl. CAM, CHIS, COL, GRO, JAL, MEX, MICH, MOR, NAY, OAX, QRO, SLP, TAB, TAMS, VER, ZAC *Desmodium alamanii DC. CDMX, GTO, HGO, JAL, MEX, MICH, MOR, TLAX

*Desmodium ambiguum Hemsl. COL, GRO, HGO, JAL, MICH, NAY, OAX, VER

Desmodium amplifolium Hemsl. CHIS, COL, DGO, GTO, GRO, JAL, MEX, MICH, OAX, TAB, ZAC

*Desmodium angustatum (Rose \& Standl.) Standl. JAL, NAY, ZAC

Desmodium angustifolium (Kunth) DC. AGS, CHIS, CHIH, COAH, COL, DGO, GRO, HGO, JAL, MEX, MICH, MOR, NAY, NLE, OAX, PUE, SLP, SIN, SON, TAMS, VER, ZAC Desmodium aparines (Link) DC. AGS, CHIS, CHIH, COL, CDMX, DGO, GTO, HGO, JAL, MEX, MICH, MOR, NLE, OAX, PUE, QRO, SON, VER, ZAC

Desmodium arizonicum $\mathrm{S}$. Watson $\mathrm{CHIH}, \mathrm{SON}$

*Desmodium asperum (Poir.) Desv. MOR, NAY, SIN, VER

Desmodium axillare (Sw.) DC. CHIS, OAX, PUE, QRO, SLP, TAB, VER

Desmodium barbatum (L.) Benth. CHIS, COL, GRO, JAL, MEX, MICH, NAY, OAX, SIN, TAB, VER

Desmodium batocaulon A. Gray BCS, CHIH, MOR, SON

*Desmodium bellum (S.F. Blake) B.G. Schub. COL, GRO, JAL, MEX, MICH, MOR, SIN, ZAC

*Desmodium bioculatum S. Watson $\mathrm{CHIH}$

Desmodium cajanifolium (Kunth) DC. CHIS, CHIH, OAX, $\mathrm{SON}$

*Desmodium callilepis Hemsl. CDMX, DGO, HGO, JAL, MEX, MICH, PUE, SLP, VER, ZAC

*Desmodium canaliculatum B.G. Schub. GRO, MEX, OAX

Desmodium canescens (L.) DC. CHIH, SON, TAMS, VER

Desmodium caripense (Kunth) G. Don CHIS, COAH, GRO, HGO, MICH, NLE, OAX, PUE, QRO, SLP, TAMS, VER

Desmodium cinerascens A. Gray CHIS, CHIH, OAX, SON

Desmodium cinereum (Kunth) DC. CHIS, COL, DGO, GRO, HGO, JAL, MEX, MICH, MOR, NAY, OAX, PUE, QRO, SLP, SIN, SON, TAMS, VER

*Desmodium conzattii Greenm. GRO, JAL, MICH, MOR, NAY, OAX, PUE, VER

*Desmodium cordistipulum Hemsl. CHIS, COL, GRO, JAL, MICH, NAY, OAX, SIN, ZAC

*Desmodium crassum M.E. Jones CHIH

* Desmodium chartaceum Brandegee SIN

*Desmodium decipiens Schindl. OAX, VER

*Desmodium densiflorum Hemsl. CDMX, DGO, GTO, GRO,
HGO, JAL, MEX, MICH, MOR, QRO, SIN, ZAC

Desmodium distortum (Aubl.) J.F. Macbr. CAM, CHIS, CHIH, COL, DGO, GRO, JAL, MEX, MICH, MOR, NAY, OAX, QROO, SIN, SON, TAB, VER, YUC, ZAC

*Desmodium foliosum Hemsl. OAX, VER

*Desmodium ghiesbreghtii Hemsl. COL, GRO, JAL, MEX, MICH, NAY, OAX, ZAC

Desmodium glabrum (Mill.) DC. BCS, CAM, CHIS, CHIH, COL, GRO, JAL, MICH, NAY, OAX, PUE, QROO, SIN, SON, TAMS, VER, YUC

Desmodium glutinosum (Muhl. ex Willd.) Wood COAH, NLE, PUE, QRO, SLP, TAMS, VER, ZAC

Desmodium grahamii A. Gray AGS, BCS, CHIH, COAH, CDMX, DGO, GTO, GRO, HGO, JAL, MEX, MICH, MOR, NLE, OAX, PUE, QRO, SLP, SIN, SON, TAMS, TLAX, VER, $\mathrm{ZAC}$

*Desmodium guadalajaranum S. Watson CHIH, COL, GRO, JAL, MEX, MICH, MOR, NAY, OAX, SON, ZAC

Desmodium hartwegianum Hemsl. AGS, CHIH, COL, DGO, GTO, GRO, HGO, JAL, MEX, MICH, MOR, NAY, NLE, OAX, PUE, QRO, SLP, SON, VER, ZAC

*Desmodium helleri Peyr. CAM, CHIS, OAX, TAB, TAMS, VER

*Desmodium hookerianum D. Dietr. CHIS, GRO, MICH, OAX Desmodium incanum DC. CAM, CHIS, CHIH, COL, GRO, HGO, JAL, NAY, NLE, OAX, QRO, QROO, SLP, SIN, TAB, TAMS, VER, YUC, ZAC

Desmodium infractum DC. CHIS, COL, GRO, JAL, MEX, MICH, NAY, OAX, PUE, SIN, TAB, VER

Desmodium intortum (Mill.) Urb. CHIS, COL, GRO, HGO, JAL, MEX, MICH, MOR, NAY, NLE, OAX, PUE, QRO, SON, VER

*Desmodium jaliscanum S. Watson COL, GTO, GRO, JAL, MEX, MICH, MOR, NAY, OAX, ZAC

Desmodium johnstonii Standl. ex B.G. Schub. MEX, OAX

Desmodium lavanduliflorum Standl. CHIH, MEX

Desmodium lempirae C. Nelson CHIS

*Desmodium leptoclados Hemsl. CAM, CHIH, COAH, DGO, GTO, HGO, MICH, NLE, OAX, PUE, SLP, SON, VER

*Desmodium leptomeres (S.F. Blake) B.G. Schub. \& McVaugh CHIH, DGO, JAL, NAY, ZAC

Desmodium lindheimeri Vail COAH, NLE, SLP, TAMS

Desmodium lineatum (Michx.) DC. NLE

*Desmodium lupulinum Schltdl. VER

Desmodium macrodesmum (S.F. Blake) Standl. \& Steyerm. CHIS, OAX, TAB, VER

*Desmodium macropodium Hemsl. CDMX, HGO, MEX, QRO *Desmodium macrostachyum Hemsl. CHIS, CHIH, COAH, COL, DGO, GTO, GRO, HGO, JAL, MEX, MICH, MOR, NAY, NLE, OAX, SLP, SIN, SON, ZAC

*Desmodium madrense Hemsl. CHIH, COL, DGO, JAL, NAY, OAX, SIN, SON

Desmodium maxonii (Standl.) Standl. CHIS

Desmodium metallicum (Rose \& Standl.) Standl. CHIS, TAB

*Desmodium mexiae B.G. Schub. GRO

*Desmodium michelianum (Schindl.) B.G. Schub. \& McVaugh GRO, MEX, OAX 
*Desmodium michoacanum B.G. Schub. \& McVaugh COL, GRO, JAL, MEX, MICH, NAY

Desmodium molliculum (Kunth) DC. BCS, CHIS, CHIH, COAH, COL, CDMX, DGO, GTO, GRO, HGO, JAL, MEX, MICH, MOR, NAY, NLE, OAX, PUE, QRO, SLP, SIN, SON, TAMS, TLAX, VER, ZAC

*Desmodium monticola Brandegee CHIS

Desmodium neomexicanum A. Gray AGS, BCS, CHIH, COAH, CDMX, DGO, GTO, GRO, HGO, JAL, MEX, MICH, MOR, NLE, OAX, PUE, QRO, SLP, SIN, SON, TAMS, VER, ZAC

Desmodium nicaraguense Oerst. CHIS, CHIH, COL, DGO, GRO, HGO, JAL, MEX, MICH, MOR, NAY, OAX, PUE, QRO, SLP, SON, VER

*Desmodium nitidum M. Martens \& Galeotti GRO, MEX, MOR, OAX, PUE, VER

*Desmodium novogalicianum B.G. Schub. \& McVaugh COL, JAL, MICH, OAX

*Desmodium occidentale (C.V. Morton) Standl. COL, JAL

Desmodium orbiculare Schltdl. CHIS, COL, CDMX, DGO, GTO, GRO, HGO, JAL, MEX, MICH, NAY, OAX, PUE, QRO, SLP, SIN, SON, TAMS, VER, ZAC

*Desmodium orizabanum Hemsl. OAX, VER

*Desmodium painteri Standl. GRO, MICH

Desmodium palmeri Hemsl. CHIS, SLP

*Desmodium pallidum Standl. OAX

*Desmodium plectocarpum Hemsl. OAX, SLP, VER

Desmodium plicatum Schltdl. \& Cham. BCS, CHIS, COL, CDMX, DGO, GRO, HGO, JAL, MEX, MICH, NAY, OAX, PUE, QRO, SIN, SON, TAMS, VER, ZAC

Desmodium polystachyum Schltdl. CHIS, GRO, JAL, MICH, NAY, OAX, SLP, TAMS, VER, ZAC

Desmodium prehensile Schltdl. AGS, BCS, CHIS, CHIH, COL, DGO, GRO, JAL, MEX, MICH, MOR, NAY, NLE, OAX, PUE, QRO, SLP, SIN, SON, TAMS, VER, ZAC

Desmodium pringlei $\mathrm{S}$. Watson AGS, BCS, CHIS, CHIH, GRO, HGO, JAL, MEX, MICH, NAY, OAX, PUE, SLP, VER, ZAC

Desmodium procumbens (Mill.) Hitchc. AGS, BCS, CAM, CHIS, CHIH, COAH, COL, DGO, GTO, GRO, HGO, JAL, MEX, MICH, MOR, NAY, NLE, OAX, PUE, QRO, QROO, SLP, SIN, SON, VER, YUC, ZAC

*Desmodium prodigum (Schindl.) Standl. CHIH

*Desmodium prostratum Brandegee BCS, CHIH, SON

*Desmodium pseudoamplifolium Micheli COL, GRO, JAL, $\mathrm{MICH}$

Desmodium psilocarpum A. Gray CHIH, SIN, SON

Desmodium psilophyllum Schltdl. CHIS, CHIH, COAH, DGO, NLE, PUE, QRO, SLP, SON, TAMS, VER

Desmodium purpusianum (Schindl.) B.G. Schub. PUE, VER

Desmodium purpusii Brandegee CHIS, OAX, TAB, VER

Desmodium raymundoramirezii L. Torres \& A. Delgado GRO, OAX

Desmodium retinens Schltdl. AGS, CHIH, COAH, GTO, HGO, JAL, MEX, MICH, NLE, OAX, PUE, QRO, SLP, SIN, SON, TAMS, ZAC

Desmodium rosei B.G. Schub. CHIH, SON, ZAC

*Desmodium saxatile (Morton) B.G. Schub. \& McVaugh JAL

*Desmodium scalare B.G. Schub. \& McVaugh JAL
Desmodium scopulorum S. Watson BCS, SON

Desmodium scorpiurus (Sw.) Desv. BCS, CAM, CHIS, COL, DGO, GRO, JAL, MEX, MICH, MOR, NAY, OAX, PUE, QRO, SLP, SIN, SON, TAB, TAMS, VER, YUC, ZAC

*Desmodium schindleri B.G. Schub. OAX

*Desmodium sericocarpum Hemsl. SLP, VER

Desmodium sericophyllum Schltdl. CHIS, COL, DGO, GRO, JAL, MEX, MICH, MOR, NAY, NLE, OAX, PUE, SIN, TAMS, TLAX, VER, ZAC

Desmodium sessilifolium (Torr.) Torr. \& A. Gray OAX, VER

Desmodium skinneri Benth. ex Hemsl. CHIS, COL, GRO, JAL, MEX, MICH, MOR, NAY, OAX, ZAC

Desmodium strobilaceum Schltdl. CHIS, COL, GRO, HGO, JAL, MEX, MICH, MOR, NAY, OAX, QRO, SLP, TAMS, VER *Desmodium subrosum G.L. Nesom COAH, NLE, SLP, ZAC

*Desmodium subsessile Schltdl. COAH, CDMX, GRO, HGO, JAL, MEX, MICH, MOR, NLE, OAX, PUE, SLP, TAMS

*Desmodium subspicatum S. Watson QRO, SLP

*Desmodium sumichrastii (Schindl.) Standl. CHIS, COL, GRO, JAL, MEX, MICH, MOR, NAY, OAX

*Desmodium sylvicola Brandegee VER

*Desmodium tastense Brandegee BCS

Desmodium tortuosum (Sw.) DC. AGS, BCS, CAM, CHIS, CHIH, COL, DGO, GTO, GRO, HGO, JAL, MEX, MICH, MOR, NAY, NLE, OAX, PUE, QRO, QROO, SLP, SIN, SON, TAB, TAMS, VER, YUC, ZAC

Desmodium triflorum (L.) DC. CHIS, COL, GRO, JAL, MICH, NAY, OAX, PUE, SLP, SIN, TAB, TAMS, VER, ZAC

Desmodium uncinatum (Jacq.) DC. CDMX, GTO, GRO, HGO, JAL, MEX, MICH, MOR, OAX, PUE, QRO, TAB, TLAX, VER, ZAC

*Desmodium urarioides (S.F. Blake) B.G. Schub. \& McVaugh COL, GRO, JAL, MEX, MICH, NAY, OAX, SON

Desmodium venustum Steud. CHIS, DGO, JAL, MEX, MICH, MOR

*Desmodium volubile (Schindl.) B.G. Schub. \& McVaugh AGS, COL, GRO, JAL, MICH, OAX

*Desmodium xylopodium Greenm. JAL, OAX

Dialium guianense (Aubl.) Sandwith CAM, CHIS, OAX, TAB, VER

Dioclea hexandra (Roxb.) Mabb. CHIS, QROO, TAB, YUC

Dioclea holtiana Pittier ex R.H. Maxwell CHIS, TAB

Dioclea violacea Mart. ex Benth. CAM, CHIS, OAX, QROO, TAB, VER

Dioclea virgata (Rich.) Amshoff CHIS, TAB

Diphysa americana (Mill.) M. Sousa CAM, CHIS, COL, GRO, HGO, JAL, MEX, MICH, MOR, NLE, OAX, PUE, QRO, QROO, SLP, TAB, TAMS, VER, YUC

Diphysa carthagenensis Jacq. CAM, CHIS, GRO, MEX, MICH, MOR, OAX, PUE, QROO, TAB, VER, YUC

Diphysa floribunda Peyr. CHIS, COL, GRO, JAL, MEX, MICH, MOR, OAX, PUE, VER

Diphysa humilis Oerst. ex Benth. \& Oerst. CHIS, MOR, OAX, QROO, VER

*Diphysa macrocarpa Standl. GRO, HGO, JAL, MEX, MICH, OAX, QRO, SLP, SIN, VER

*Diphysa microphylla Rydb. NLE, TAMS 
*Diphysa minutifolia Rose CHIS, GRO, MEX, MICH, MOR, NLE, OAX, PUE, SLP, TAMS, VER, YUC

*Diphysa occidentalis Rose BCS, CHIH, COL, DGO, GRO, JAL, MICH, NAY, NLE, SIN, SON

*Diphysa ormocarpoides (Rudd) M. Sousa \& R. Antonio CHIS, GRO, MICH, OAX, PUE

Diphysa paucifoliolata R. Antonio \& M. Sousa CAM, QROO

*Diphysa puberulenta Rydb. AGS, CHIS, CHIH, COL, DGO, GRO, JAL, MEX, MICH, MOR, NAY, OAX, SIN, ZAC

*Diphysa punctata Rydb. MOR, OAX, PUE

*Diphysa racemosa Rose CHIS, CHIH, DGO, GRO, MEX,

MICH, MOR, NAY, OAX, PUE, SIN, SON

Diphysa sennoides Benth. GRO, HGO, JAL, OAX, PUE, SLP, VER

Diphysa spinosa Rydb. CHIS, MOR, OAX, PUE

*Diphysa suberosa S. Watson AGS, CHIH, COL, DGO, GTO, GRO, HGO, JAL, MEX, MICH, MOR, NAY, OAX, PUE, QRO, SLP, SIN, SON, TAB, VER, ZAC

Diphysa thurberi (A. Gray) Rydb. ex Standl. CHIH, COL, GRO, JAL, NAY, OAX, PUE, SIN, SON

*Diphysa villosa Rydb. JAL, MEX, MICH, MOR, OAX, PUE

Diphysa yucatanensis Hanan-Alipi \& M. Sousa CAM, CHIS, QROO, TAB, YUC

Dussia cuscatlanica (Standl.) Standl. \& Steyerm. CHIS

Dussia mexicana (Standl.) Harms CHIS, OAX, PUE, VER

*Ebenopsis confinis (Standl.) Barneby \& J.W. Grimes BCN, BCS, SON

Ebenopsis ebano (Berland.) Barneby \& J.W. Grimes COAH, DGO, JAL, NLE, QRO, QROO, SLP, SIN, TAMS, VER, YUC, ZAC

Entada gigas (L.) Fawc. \& Rendle OAX, PUE, TAB, VER

Entada monostachya DC. CHIS, VER

Entadopsis polystachya (L.) Britton CHIS, COL, GRO, JAL, MEX, MICH, NAY, OAX, SIN, TAB, VER

Enterolobium cyclocarpum (Jacq.) Griseb. BCS, CAM, CHIS, COL, DGO, GRO, HGO, JAL, MEX, MICH, MOR, NAY, OAX, PUE, QRO, QROO, SLP, SIN, SON, TAB, TAMS, VER, YUC, ZAC

Enterolobium schomburgkii (Benth.) Benth. CHIS, OAX, TAB, VER

*Eriosema corymboides M. Sousa \& Fortunato OAX

Eriosema crinitum (Kunth) G. Don CHIS, NAY, OAX, TAB

Eriosema diffusum (Kunth) G. Don CHIS, CHIH, COL, GRO, JAL, MEX, MICH, MOR, NAY, OAX, PUE, SIN, SON, TAB, TAMS, VER, ZAC

Eriosema grandiflorum (Schltdl. \& Cham.) G. Don BCN, BCS, CHIS, CHIH, DGO, GRO, JAL, MEX, MICH, MOR, NAY, OAX, SIN, SON, TAMS, VER, ZAC

*Eriosema laetum I.M. Johnst. CHIH, SON

*Eriosema longicalyx Grear COL, GRO, JAL, MEX, MICH, MOR, NAY, SIN, ZAC

*Eriosema multiflorum B.L. Rob. COL, GRO, JAL, MEX, MICH, NAY, OAX, SIN

*Eriosema palmeri S. Watson CHIH, DGO, GRO, JAL, MEX, MICH, NAY, SLP, SIN, SON, ZAC

Eriosema pulchellum (Kunth) G. Don AGS, CHIS, CHIH, COL, DGO, GRO, JAL, MEX, MICH, MOR, NAY, OAX, SLP, SIN,
SON, TAB, TAMS, VER, ZAC

Eriosema rufum (Kunth) G. Don MICH

*Eriosema rugosum M. Sousa \& O. Téllez GRO, JAL, NAY, OAX

Eriosema simplicifolium (DC.) G. Don CAM, TAB, VER

Eriosema violaceum (Aubl.) G. Don CHIS, TAB, VER

*Errazurizia benthamii (Brandegee) I.M. Johnst. BCN, BCS

*Errazurizia megacarpa (S. Watson) I.M. Johnst. BCN, BCS, SON

*Erythrina americana Mill. CAM, CHIS, CHIH, COL, CDMX, GTO, GRO, HGO, JAL, MEX, MICH, MOR, NLE, OAX, PUE, QRO, QROO, SLP, SIN, TAB, TAMS, TLAX, VER, YUC, ZAC *Erythrina batolobium Barneby \& Krukoff GRO, MEX, OAX *Erythrina berenices Krukoff \& Barneby VER

Erythrina berteroana Urb. CAM, CHIS, OAX, TAB, VER

*Erythrina breviflora DC. COL, CDMX, GTO, GRO, HGO,

JAL, MEX, MICH, MOR, OAX, PUE

Erythrina caribaea Krukoff \& Barneby CAM, CHIS, OAX, PUE, TAB, VER

*Erythrina chiapasana Krukoff CHIS, OAX, VER

Erythrina flabelliformis Kearney AGS, BCS, CHIH, COL, DGO, HGO, JAL, MEX, MICH, MOR, NLE, PUE, QRO, SLP, SIN, SON, TAMS, VER, ZAC

*Erythrina florenciae Krukoff \& Barneby CHIS, OAX

Erythrina folkersii Krukoff \& Moldenke CHIS, GRO, OAX, TAB, VER

*Erythrina goldmanii Standl. CHIS, OAX

Erythrina herbacea L. CHIS, HGO, JAL, MICH, NLE, OAX, PUE, QRO, SLP, TAB, TAMS, VER

*Erythrina horrida DC. OAX

*Erythrina lanata Rose CHIS, COL, DGO, GTO, GRO, JAL, MEX, MICH, MOR, NAY, OAX, SIN, VER, ZAC

*Erythrina leptorhiza Moc. \& Sessé ex DC. CDMX, GTO, GRO, HGO, JAL, MEX, MICH, MOR, OAX, PUE, QRO, SLP, TLAX, VER, ZAC

Erythrina macrophylla DC. CHIS, TAB, VER

Erythrina mexicana Krukoff CHIS, GRO, MEX, OAX, PUE, SLP, TAB, VER

*Erythrina montana Rose \& Standl. AGS, DGO, HGO, JAL, NAY, QRO, SLP, SIN, ZAC

*Erythrina oaxacana (Krukoff) Barneby OAX

*Erythrina oliviae Krukoff CHIS, MICH, MOR, OAX, PUE

*Erythrina pudica Krukoff \& Barneby CHIS

Erythrina rubrinervia Kunth CHIS, OAX, TAB, VER

*Erythrina sousae Krukoff \& Barneby CHIS, OAX

Erythrina standleyana Krukoff CAM, CHIS, HGO, OAX, PUE, QRO, QROO, SLP, TAB, TAMS, VER, YUC

Erythrina tajumulcensis Krukoff \& Barneby CHIS

* Erythrina tuxtlana Krukoff \& Barneby CHIS, OAX, VER

Eysenhardtia adenostylis Baill. CHIS, OAX

*Eysenhardtia byei Cruz Durán \& M. Sousa CHIH, SON

*Eysenhardtia officinalis R. Cruz \& M. Sousa HGO, SLP, TAMS, VER

Eysenhardtia orthocarpa (A. Gray) S. Watson CHIH, DGO, JAL, SIN, SON

*Eysenhardtia parvifolia Brandegee CHIH, COAH, NLE, SLP, ZAC 
*Eysenhardtia peninsularis Brandegee BCN, BCS

* Eysenhardtia platycarpa Pennell \& Saff. CHIS, COL, DGO, GTO, GRO, JAL, MEX, MICH, NAY, OAX, PUE, QRO, SIN, TAMS

Eysenhardtia polystachya (Ortega) Sarg. AGS, CHIS, CHIH, COAH, COL, CDMX, DGO, GTO, GRO, HGO, JAL, MEX, MICH, MOR, NAY, NLE, OAX, PUE, QRO, SLP, SIN, SON, TAMS, TLAX, VER, ZAC

* Eysenhardtia punctata Pennell AGS, CHIH, COL, DGO, GTO, GRO, HGO, JAL, MEX, MICH, NAY, OAX, PUE, QRO, SON, ZAC

*Eysenhardtia schizocalyx Pennell CHIH, COAH, DGO, SLP Eysenhardtia spinosa Engelm. ex A. Gray CHIH, DGO, NLE * Eysenhardtia subcoriacea Pennell HGO, OAX, PUE, QRO, VER

Eysenhardtia texana Scheele CHIH, COAH, DGO, HGO, NLE, QRO, SLP, TAMS, VER

Galactia acapulcensis Rose BCS, CHIS, COL, GRO, JAL, MEX, MICH, MOR, NAY, OAX, SIN

Galactia argentea Brandegee CHIS, OAX, QRO, SLP

Galactia belizensis Standl. CHIS

*Galactia brachystachya Benth. AGS, COAH, DGO, GTO, GRO, HGO, JAL, NLE, OAX, PUE, QRO, SLP, SON, TAB,

TAMS, ZAC

Galactia canescens Benth. COAH, NLE, TAMS, VER

* Galactia densiflora Germán \& M. Sousa JAL

Galactia discolor Donn. Sm. CAM, CHIS, QROO, YUC

*Galactia dubia DC. GRO, MOR, TAB, VER

* Galactia incana (Rose) Standl. CHIS, COL, GRO, HGO, JAL, MEX, MICH, NAY, OAX, SIN, VER

Galactia joselyniae G.L. Nesom COAH

Galactia marginalis Benth. DGO, NLE, OAX

Galactia multiflora B.L. Rob. \& Greenm. CHIS, GRO, JAL,

MEX, MICH, MOR, NAY, NLE, OAX, PUE, ZAC

Galactia spiciformis Torr. \& A. Gray CAM, QROO, YUC

Galactia striata (Jacq.) Urb. CAM, CHIS, CHIH, COAH, COL, DGO, GRO, JAL, MEX, MICH, MOR, NAY, NLE, OAX, PUE, QROO, SLP, SIN, SON, TAB, TAMS, VER, YUC

Galactia texana (Scheele) A. Gray COAH, NLE, TAMS

*Galactia viridiflora (Rose) Standl. COL, GRO, MEX, MICH, MOR, OAX, PUE

Galactia wrightii A. Gray CHIH, COAH, COL, GTO, JAL, NAY, NLE, QRO, SLP, SIN, SON, VER, ZAC

Genistidium dumosum I.M. Johnst. CHIH, COAH, NLE

Gleditsia aquatica Marshall TAMS

Gleditsia triacanthos L. COAH, NLE, SON, TAMS

Gliricidia maculata (Kunth) Kunth ex Walp. CAM, CHIS, QROO, TAB, YUC

* Gliricidia robusta (M. Sousa \& Lavin) Lavin CHIS

Gliricidia sepium (Jacq.) Kunth ex Walp. CAM, CHIS, COL, DGO, GRO, HGO, JAL, MEX, MICH, MOR, NAY, OAX, PUE, QRO, QROO, SLP, SIN, SON, TAB, TAMS, VER, YUC, ZAC Glycyrrhiza lepidota Nutt. CHIH

*Guinetia tehuantepecensis L. Rico \& M. Sousa OAX

Haematoxylum brasiletto $\mathrm{H}$. Karst. BCS, CAM, CHIS, CHIH, COL, DGO, GRO, JAL, MEX, MICH, MOR, NAY, OAX, PUE, QROO, SIN, SON, TAB, TAMS, VER, YUC, ZAC
*Haematoxylum calakmulense Cruz-Durán \& M. Sousa CAM, QROO, YUC

Haematoxylum campechianum L. CAM, CHIS, QROO, TAB, VER, YUC

*Haematoxylum sousanum Cruz-Durán \& J. Jiménez Ram. GRO, OAX, PUE

*Harpalyce arborescens A. Gray GTO, GRO, HGO, OAX, QRO, QROO, SLP, TAB, TAMS, VER, YUC

Harpalyce formosa Moc. \& Sessé ex DC. CHIS, GRO, MEX, MOR, OAX, PUE

*Harpalyce macrobotrya Harms CHIS

*Harpalyce mexicana Rose JAL, NAY, ZAC

*Harpalyce pringlei Rose MICH, MOR, OAX, QRO

Harpalyce rupicola Donn. Sm. CAM, QROO, TAB, YUC

*Harpalyce sousae Arroyo GRO, MEX, OAX, PUE

Havardia albicans (Kunth) Britton \& Rose CAM, CHIS, GRO, QROO, TAB, VER, YUC

Havardia campylacantha (L. Rico \& M. Sousa) Barneby \& J.W. Grimes GRO, JAL, MICH, OAX

*Havardia mexicana (Rose) Britton \& Rose BCS, CHIH, SIN, SON

Havardia pallens (Benth.) Britton \& Rose BCS, CHIS, CHIH, COAH, DGO, GTO, HGO, MEX, MICH, MOR, NLE, OAX, PUE, QRO, QROO, SLP, SIN, SON, TAMS, VER, YUC

*Havardia sonorae (S. Watson) Britton \& Rose BCS, DGO, SIN, SON

Helicotropis linearis (Kunth) A. Delgado CHIS, COL, GRO, JAL, MEX, MOR, NAY, OAX, PUE, TAB, VER

Helicotropis spectabilis (Standl.) A. Delgado CHIS, OAX

*Hesperothamnus ehrenbergii (Harms) Rydb. HGO, QRO, VER

*Hesperothamnus littoralis (Brandegee) Brandegee BCS

*Hesperothamnus pentaphyllus (Harms) Rydb. OAX, PUE

*Hesperothamnus purpusii (Harms) Harms OAX, PUE

*Heteroflorum sclerocapum M. Sousa GRO, MICH, OAX

Hoffmannseggia drepanocarpa A. Gray CHIH, COAH, SON

Hoffmannseggia drumondii Torr. \& A. Gray TAMS

Hoffmannseggia glauca (Ortega) Eifert AGS, BCN, BCS, CHIH, COAH, DGO, GTO, HGO, JAL, MICH, NLE, PUE, QRO, SLP, SON, TAMS, VER, ZAC

*Hoffmannseggia humilis (M. Martens \& Galeotti) Hemsl. HGO, NLE, OAX, PUE, QRO, SLP, TAMS, VER

*Hoffmannseggia intricata Brandegee BCN, BCS, SON

Hoffmannseggia microphylla Torr. BCN, SON

*Hoffmannseggia montana (Britton) McVaugh AGS, JAL, ZAC Hoffmannseggia oxycarpa Benth. COAH, HGO, NLE, QRO, TAMS

*Hoffmannseggia peninsularis (Britton) Wiggins BCN

*Hoffmannseggia watsonii (Fisher) Rose COAH, GTO, HGO, NLE, QRO, SLP

Hoita macrostachya (DC.) Rydb. BCN

Hoita orbicularis (Lindl.) Rydb. BCN

Hybosema ehrenbergii (Schltdl.) Harms CHIS, GRO, HGO, OAX

*Hybosema robustum M. Sousa \& Lavin CHIS

Hymenaea courbaril L. CAM, CHIS, CHIH, COL, GRO, JAL, MEX, MICH, MOR, NAY, OAX, SIN, TAB, VER

*Indigofera acasonicae Brandegee VER 
*Indigofera constricta Rydb. COL, GRO, JAL, NAY, OAX, SIN, ZAC

*Indigofera conzattii Rose OAX, PUE

*Indigofera coronilloides M. Martens \& Galeotti OAX

* Indigofera cuernavacana Rose COL, GRO, JAL, MEX, MICH, MOR, NAY, OAX, PUE, QRO, SLP, SIN

*Indigofera densiflora M. Martens \& Galeotti CHIS, CHIH, COL, CDMX, DGO, GTO, GRO, HGO, JAL, MEX, MICH, MOR, OAX, PUE, SIN, SON, TLAX, VER

*Indigofera discolor Rydb. DGO, SIN, VER

* Indigofera fruticosa Rose BCS, NAY, VER

Indigofera guatemalensis Prain \& Baker CHIS, OAX

*Indigofera incompta McVaugh JAL

*Indigofera indica Mill. QROO

*Indigofera jaliscensis Rose CHIS, COL, GRO, JAL, MEX,

MICH, MOR, NAY, OAX, SIN

Indigofera jamaicensis Spreng. CAM, CHIS, CHIH, COL, DGO, GRO, JAL, MEX, MICH, MOR, NAY, OAX, PUE, QRO, QROO, SLP, SIN, SON, TAB, TAMS, VER, YUC, ZAC *Indigofera lancifolia Rydb. CHIS, OAX

Indigofera lespedezioides Kunth CAM, CHIS, GRO, MICH, OAX, PUE, SIN, TAB, VER

*Indigofera matudae Lundell CHIS

Indigofera microcarpa Desv. OAX, VER

Indigofera miniata Ortega AGS, CHIS, CHIH, COAH, COL, CDMX, DGO, GTO, GRO, HGO, JAL, MEX, MICH, MOR, NAY, NLE, OAX, PUE, QRO, SLP, SIN, TAB, TAMS, VER, ZAC

Indigofera montana Rose AGS, CHIS, CHIH, DGO, GTO, JAL, NAY, SON, ZAC

*Indigofera nana Rydb. MICH

*Indigofera nelsonii Rydb. BCS

Indigofera ornithopodioides Schltdl. \& Cham. CHIS, CHIH, COAH, GRO, NLE, OAX, SLP, TAMS, VER

*Indigofera palmeri S. Watson COL, DGO, GRO, JAL, MICH, NAY, SLP, SIN, TAMS, VER, ZAC

*Indigofera platycarpa Rose GRO, MEX, MICH, MOR, OAX

* Indigofera pueblensis Rydb. PUE

*Indigofera purpusii Brandegee OAX, VER

Indigofera sabulicola Benth. COL, GRO, JAL, OAX, TAB, VER

*Indigofera salmoniflora Rose JAL, NAY, OAX, SIN

Indigofera sphaerocarpa A. Gray CHIH, SON

*Indigofera sphinctosperma Standl. VER

Indigofera suffruticosa Mill. AGS, BCS, CAM, CHIS, CHIH, COAH, COL, CDMX, GTO, GRO, HGO, JAL, MEX, MICH, MOR, NAY, NLE, OAX, PUE, QRO, QROO, SLP, SIN, SON, TAB, TAMS, VER, YUC, ZAC

Indigofera thibaudiana DC. CAM, CHIS, COL, DGO, GTO, GRO, HGO, JAL, MEX, MICH, MOR, NAY, NLE, OAX, PUE, QRO, SLP, SIN, VER

*Indigofera tumidula Rose GRO

Inga acrocephala Steud. CHIS, OAX, VER

Inga affinis DC. CAM, CHIS, OAX, SLP, TAB, TAMS, VER

Inga alba (Sw.) Willd. CHIS, OAX, VER

*Inga andersonii McVaugh JAL, NAY

*Inga appendiculata $\mathrm{M}$. Sousa VER
Inga barbourii Standl. CHIS, OAX, VER

Inga belizensis Standl. CHIS, TAB

Inga cabrerae M. Sousa CHIS

*Inga calcicola $\mathrm{M}$. Sousa OAX

Inga calderonii Standl. CHIS

* Inga colimana E. Padilla, Cuevas \& A. Solís COL

*Inga chiapensis Miranda ex M. Sousa CHIS, VER

Inga dasycarpa $\mathrm{M}$. Sousa CHIS

Inga densiflora Benth. CHIS

*Inga eriocarpa Benth. CHIS, COL, DGO, GRO, HGO, JAL, MEX, MICH, MOR, NAY, OAX, PUE, SLP, SIN, TAB, VER, ZAC

*Inga huastecana M. Sousa HGO, PUE, QRO, SLP, VER

Inga inicuil Schltdl. \& Cham. ex G. Don CHIS, MEX, MICH, MOR, OAX, PUE, QROO, TAB, VER, YUC

*Inga ismaelis $\mathrm{M}$. Sousa OAX, VER

Inga lactifera M. Sousa CHIS, OAX, VER

*Inga lacustris $\mathrm{M}$. Sousa VER

*Inga latibracteata Harms CHIS, OAX, PUE, QRO, SLP, VER Inga laurina (Sw.) Willd. CHIS, COL, GRO, JAL, MICH, NAY, OAX, SIN, TAB, VER

Inga leiocalycina Benth. OAX, VER

Inga marginata Willd. CHIS, VER

Inga mexicana (T.D. Penn.) M. Sousa CHIS, OAX, PUE, TAB, VER

Inga multijuga Benth. CHIS, OAX

Inga nobilis Willd. CHIS, OAX, VER

Inga oerstediana Benth. ex Seem. CHIS, GRO, HGO, OAX, PUE, QRO, SLP, TAB, VER

Inga paterno Harms CHIS, GRO, OAX, PUE, QROO, TAB, VER

Inga pinetorum Pittier CHIS, OAX, TAB, VER

Inga punctata Willd. CAM, CHIS, GRO, HGO, OAX, PUE, TAB, VER

Inga sapindoides Willd. CHIS, GRO, NAY, OAX, PUE, TAB, VER

Inga schiedeana Steud. CHIS, COL, DGO, GRO, JAL, MEX, MICH, MOR, NAY, OAX, PUE, QRO, SLP, SIN, VER

*Inga sinacae M. Sousa \& Ibarra-Manríquez CHIS, OAX, TAB, VER

Inga spectabilis (Vahl) Willd. OAX, TAB

Inga thibaudiana DC. CHIS

Inga vera Willd. CAM, CHIS, COAH, COL, DGO, GRO, HGO, JAL, MEX, MICH, MOR, NAY, NLE, OAX, PUE, QRO, QROO, SLP, SIN, TAB, TAMS, VER

Lathyrus arizonicus Britton CHIH, SON

Lathyrus eucosmus Butters \& H. St. John COAH, DGO

Lathyrus graminifolius (S. Watson) T.G. White CHIH, SON

Lathyrus parvifolius S. Watson AGS, CHIH, COAH, CDMX, DGO, GTO, HGO, JAL, MEX, MICH, NAY, NLE, OAX, PUE, QRO, SLP, SIN, TAMS, VER, ZAC

Lathyrus polymorphus Nutt. DGO, NLE

*Lathyrus schaffneri Rydb. HGO, OAX, QRO, VER

Lathyrus splendens Kellogg BCN

Lathyrus vestitus Nutt. BCN

*Lathyrus whitei Kupicha BCN, BCS, CHIH, COAH, NLE, OAX 
Lecointea amazonica Ducke CHIS

Lennea melanocarpa (Schltdl.) Vatke ex Harms CAM, OAX, PUE, QROO, VER

Lennea modesta (Standl. \& Steyerm.) Standl. \& Steyerm. CHIS, OAX, TAB, VER

Lennea viridiflora Seem. CHIS, COL, JAL, MICH, OAX, VER Leptolobium panamense (Benth.) Sch. Rodr. \& A.M.G. Azevedo CAM, CHIS, GRO, HGO, MICH, OAX, QROO, TAB, VER, YUC

Leptospron adenanthum (G. Mey.) A. Delgado CAM, CHIS, COL, GTO, GRO, JAL, MEX, MICH, MOR, NAY, OAX, QRO, SLP, SIN, TAB, TAMS, VER

*Leptospron gentryi (Standl.) A. Delgado CHIH, NAY, SIN, SON

Lespedeza repens (L.) W.P.C. Barton NLE

Lespedeza texana Britton COAH, NLE, TAMS

Lespedeza virginica (L.) Britton COAH, NLE, TAMS

Leucaena collinsii Britton \& Rose CHIS, OAX

* Leucaena confertiflora Zárate OAX, PUE

*Leucaena cuspidata Standl. CHIS, HGO, OAX, PUE, QRO, SLP, VER

Leucaena diversifolia (Schltdl.) Benth. CHIS, GTO, GRO, JAL, MEX, MOR, OAX, PUE, SLP, SIN, TAB, VER

Leucaena esculenta (Moc. \& Sessé ex DC.) Benth. AGS, CAM, CHIS, CHIH, COL, GTO, GRO, HGO, JAL, MEX, MICH, MOR, OAX, PUE, QRO, SLP, VER, YUC, ZAC

Leucaena greggii $\mathrm{S}$. Watson COAH, NLE

* Leucaena involucrata Zárate SON

*Leucaena lanceolata S. Watson BCS, CAM, CHIS, CHIH, COL, DGO, GRO, JAL, MICH, MOR, NAY, OAX, PUE, SIN, SON, TAB, VER

Leucaena leucocephala (Lam.) de Wit AGS, BCN, BCS, CAM, CHIS, CHIH, COAH, COL, CDMX, DGO, GRO, HGO, JAL, MEX, MICH, MOR, NAY, NLE, OAX, PUE, QRO, QROO, SLP, SIN, SON, TAB, TAMS, TLAX, VER, YUC, ZAC

*Leucaena macrophylla Benth. CHIS, COL, DGO, GTO, GRO, JAL, MEX, MICH, MOR, NAY, OAX, PUE, SIN, VER, ZAC

* Leucaena matudae (Zárate) C.E. Hughes GRO

Leucaena pulverulenta (Schltdl.) Benth. COAH, GTO, HGO, NLE, OAX, PUE, QRO, SLP, TAMS, VER

Leucaena retusa Benth. CHIH, COAH

Leucaena shannoni Donn. Sm. CAM, CHIS

Leucaena $\times$ brachycarpa Urb. OAX, VER

$*$ Leucaena $\times$ mixtec C.E. Hughes \& S.A. Harris PUE

Libidibia coriaria (Jacq.) Schltdl. BCS, CHIS, COL, GRO, JAL, MEX, MICH, MOR, NAY, OAX, PUE, SIN, VER

*Libidibia sclerocarpa (Standl.) Britton \& Rose COL, DGO, GRO, JAL, MICH, NAY, OAX, QROO, SIN, SON

Lonchocarpus acuminatus (Schltdl.) M. Sousa CHIS, GRO, HGO, JAL, OAX, PUE, TAB, VER

*Lonchocarpus andrieuxii M. Sousa MEX, MOR, PUE

*Lonchocarpus angusticarpus M. Sousa CHIS, OAX

*Lonchocarpus argyrotrichus Harms GRO, MEX, MICH, MOR, OAX, PUE

Lonchocarpus atropurpureus Benth. CHIS, GRO, JAL, MICH, NAY, OAX, VER

*Lonchocarpus balsensis M. Sousa \& J.C. Soto GRO, MICH
*Lonchocarpus barbatus M. Sousa, E. Martínez \& Ramos VER Lonchocarpus berriozabalensis Miranda ex M. Sousa CHIS *Lonchocarpus brachyanthus M. Sousa MICH

Lonchocarpus brenesii M. Sousa CHIS

Lonchocarpus castilloi Standl. CAM, CHIS, QROO, TAB, YUC *Lonchocarpus caudatus Pittier CHIS, COL, GRO, HGO, JAL, MEX, MICH, MOR, OAX, PUE, QRO, SLP, VER

*Lonchocarpus cochleatus Pittier CHIS, COL, GRO, JAL, $\mathrm{MICH}, \mathrm{NAY}, \mathrm{OAX}, \mathrm{QROO}$

*Lonchocarpus comitensis Pittier CHIS

Lonchocarpus congestiflorus M. Sousa \& J. Linares CHIS

*Lonchocarpus constrictus Pittier CHIS, COL, GRO, JAL, MICH, OAX

*Lonchocarpus crassicalyx M. Sousa OAX

Lonchocarpus cruentus Lundell CHIS, OAX, TAB, VER

*Lonchocarpus dumetorum Brandegee VER

*Lonchocarpus emarginatus Pittier GRO, MOR, OAX

*Lonchocarpus epigaeus M. Sousa GRO, MEX, MICH

*Lonchocarpus eriocarinalis Micheli COL, GRO, JAL, MICH, OAX, PUE

*Lonchocarpus eriophyllus Benth. GRO, JAL, MEX, MICH, MOR, OAX, PUE

*Lonchocarpus foveolatus M. Sousa CHIS

* Lonchocarpus fuscopurpureus Brandegee VER

*Lonchocarpus galeottianus Harms OAX

*Lonchocarpus grandifoliolatus M. Sousa OAX

Lonchocarpus guatemalensis Benth. CAM, CHIS, COL, DGO, GRO, JAL, MICH, NAY, OAX, PUE, QROO, SLP, SIN, TAB, VER, YUC

*Lonchocarpus gyroides M. Sousa GRO

Lonchocarpus heptaphyllus (Poir.) Kunth ex DC. CHIS, TAB, VER

*Lonchocarpus hermannii M. Sousa CHIH, COL, GRO, JAL, MICH, MOR, NAY, OAX, PUE, SIN, SON

*Lonchocarpus hidalgensis Lundell HGO, NLE, PUE, QRO, SLP, TAMS, VER

*Lonchocarpus hintonii Sandwith COL, GRO, JAL, MEX, MICH, NAY, OAX, SIN, VER

Lonchocarpus hondurensis Benth. CAM, CHIS, OAX, QROO, TAB, VER, YUC

*Lonchocarpus huetamoensis M. Sousa \& J.C. Soto GRO, $\mathrm{MICH}$

Lonchocarpus hydrophilus M. Sousa \& J. Morales CHIS

*Lonchocarpus isthmensis M. Sousa CHIS, OAX

*Lonchocarpus jaliscensis Pittier JAL, NAY, ZAC

Lonchocarpus lanceolatus Benth. CHIS, COL, DGO, GRO, JAL, MICH, NAY, OAX, SIN, VER

Lonchocarpus lasiotropis F.J. Herm. CHIS, TAB, VER

*Lonchocarpus latimarginatus M. Sousa CHIS, OAX, TAB, VER

Lonchocarpus lineatus Pittier CAM, CHIS, OAX, PUE, TAB, VER

*Lonchocarpus longipedicellatus Pittier CHIS, COL, GRO, JAL, MICH, NAY, OAX

*Lonchocarpus longipedunculatus M. Sousa \& J.C. Soto GRO, JAL, MICH

Lonchocarpus luteomaculatus Pittier CAM, CHIS, OAX, 
QROO, TAB, VER, YUC

*Lonchocarpus magallanesii M. Sousa COL, JAL, MICH

*Lonchocarpus major M. Sousa COL, MICH

*Lonchocarpus martinezii M. Sousa CHIS

Lonchocarpus michelianus Pittier CHIS

*Lonchocarpus michoacanicus M. Sousa MICH

Lonchocarpus minimiflorus Donn. Sm. CHIS, OAX

*Lonchocarpus minor M. Sousa COL, JAL, NAY

*Lonchocarpus molinae Standl. \& L.O. Williams CHIS, OAX, VER

Lonchocarpus morenoi M. Sousa CHIS

*Lonchocarpus multifoliolatus M. Sousa CHIS, OAX, TAB, VER

*Lonchocarpus mutans M. Sousa COL, JAL, MICH, NAY, OAX, SIN

*Lonchocarpus oaxacensis Pittier OAX, PUE

*Lonchocarpus obovatus Benth. GRO, OAX, PUE

Lonchocarpus oliganthus F.J. Herm. CHIS, VER

*Lonchocarpus orizabensis Lundell OAX, VER

*Lonchocarpus parviflorus Benth. CAM, CHIS, JAL, MICH, OAX, QROO, SIN

*Lonchocarpus pedunculatus M. Sousa CHIS

Lonchocarpus pentaphyllus (Poir.) Kunth ex DC. CAM, CHIS, OAX, TAB, VER, YUC

Lonchocarpus phaseolifolius Benth. CHIS, OAX

*Lonchocarpus pittieri M. Sousa COL, JAL, MICH, OAX

*Lonchocarpus plicatus M. Sousa VER

Lonchocarpus punctatus Kunth CAM, CHIS, CHIH, COL, DGO, GRO, HGO, JAL, MICH, MOR, NAY, OAX, PUE, QROO, SIN, SON, TAB, TAMS, VER, YUC

Lonchocarpus purpureus Pittier CHIS, VER

Lonchocarpus robustus Pittier CHIS, OAX, TAB, VER

Lonchocarpus rugosus Benth. CAM, CHIS, COL, GRO, HGO,

JAL, MEX, MICH, MOR, OAX, PUE, QRO, QROO, SLP, TAB, TAMS, VER, YUC

Lonchocarpus salvadorensis Pittier CHIS, COL, GTO, GRO, JAL, MICH, NAY, OAX, TAB

Lonchocarpus sanctuarii Standl. \& L.O. Williams CHIS

Lonchocarpus santarosanus Donn. Sm. CHIS, OAX, QRO, SLP, TAB, VER

Lonchocarpus schiedeanus (Schltdl.) Harms CHIS, COL, JAL, NAY, OAX, SIN, TAB, VER

*Lonchocarpus schubertiae M. Sousa GRO, MEX, MICH

*Lonchocarpus septentrionalis M. Sousa OAX, VER

Lonchocarpus sericeus (Poir.) Kunth ex DC. CHIS, COL, GRO, JAL, MICH, NAY, OAX, SIN

*Lonchocarpus sinaloensis (Gentry) F.J. Herm. COL, DGO, JAL, SIN

*Lonchocarpus spectabilis F.J. Herm. GRO, MOR, OAX

*Lonchocarpus sumiderensis M. Sousa CHIS

*Lonchocarpus sylvicola M. Sousa CHIS

*Lonchocarpus tenorioi M. Sousa GRO, MICH

*Lonchocarpus tuxtepecensis M. Sousa OAX, VER

Lonchocarpus verrucosus M. Sousa CHIS, OAX, VER

Lonchocarpus vittatus M. Sousa CHIS, VER

Lonchocarpus wendtii M. Sousa OAX, TAB, VER

Lonchocarpus xuul Lundell CAM, CHIS, QROO, YUC
Lonchocarpus yucatanensis Pittier CAM, QROO, YUC

*Lotus alamosanus (Rose) Gentry CHIH, DGO, SIN, SON

Lotus argophyllus (A. Gray) Greene BCN

*Lotus argyraeus (Greene) Greene BCN

*Lotus bryantii (Brandegee) Ottley BCN, BCS

*Lotus cedrosensis Greene BCN, BCS

*Lotus chihuahuanus (S. Watson) Greene CHIH, DGO, SON

Lotus hamatus Greene BCN, BCS

*Lotus hintoniorum B.L. Turner NLE, TAMS

Lotus nuttalianus Greene BCN

Lotus oblongifolius (Benth.) Greene BCN

Lotus plebeius (Brandegee) Barneby BCN, BCS, CHIH, COAH, SON

Lotus repens (G. Don) Sessé \& Moc. ex Standl. \& Steyerm. AGS, CHIS, CHIH, COL, CDMX, DGO, GTO, GRO, HGO, JAL, MEX, MICH, NAY, OAX, PUE, QRO, SIN, SON, TAMS, VER, ZAC

Lotus salsuginosus Greene BCN, BCS, SON

Lotus scoparius (Nutt.) Ottley BCN, BCS

*Lotus watsonii (Vasey \& Rose) Greene BCN

Lupinus albifrons Benth. BCN

Lupinus andersonii S. Watson BCN

*Lupinus aralloius C.P. Sm. JAL, MICH

Lupinus aschenbornii Schauer CHIS, CDMX, GTO, JAL, MEX, MICH, MOR, PUE, TLAX, VER

*Lupinus barberi C.P. Sm. CHIH, SON

*Lupinus barkeri Lindl. CDMX, DGO, HGO, JAL, MEX, MICH, TLAX, VER

Lupinus bicolor Lindl. BCN, SON

*Lupinus caballoanus B.L. Turner COAH, NLE, TAMS

*Lupinus cacuminis Standl. COAH, NLE, TAMS

*Lupinus campestris Schltdl. \& Cham. AGS, CDMX, DGO, GTO, GRO, HGO, JAL, MEX, MICH, MOR, OAX, PUE, QRO, SIN, VER, ZAC

*Lupinus compactiflorus Rose MEX, MICH

Lupinus concinnus J. Agardh BCN, BCS, SON

Lupinus chiapensis Rose CHIS, OAX

* Lupinus chihuahuensis S. Watson CHIH, DGO

*Lupinus decaschistus C.P. Sm. GRO

*Lupinus delicatulus Sprague \& Riley DGO, SIN

Lupinus densiflorus Benth. BCN

*Lupinus diehlii M.E. Jones CHIH, MEX, MICH, NAY, OAX, SLP

*Lupinus dissimulans C.P. Sm. GRO

* Lupinus durangensis C.P. Sm. DGO

Lupinus elegans Kunth AGS, CHIS, CHIH, COL, CDMX, DGO, GRO, HGO, JAL, MEX, MICH, MOR, NAY, OAX, PUE, QRO, TLAX, VER, ZAC

*Lupinus ermineus S. Watson AGS, DGO, ZAC

*Lupinus exaltatus Zucc. COL, CDMX, GRO, HGO, JAL, MEX, MICH, MOR, OAX, PUE, TLAX, VER

Lupinus excubitus M.E. Jones BCN

* Lupinus filicaulis C.P. Sm. CHIS, HGO, SLP

Lupinus formosus Greene BCN

*Lupinus gentryanus C.P. Sm. SIN

*Lupinus geophilus Rose CDMX, JAL, MEX, MICH, MOR, PUE, TLAX 
*Lupinus glabratus J. Agardh CDMX, HGO, MEX, MICH, PUE

Lupinus grayi (S. Watson) S. Watson BCN

Lupinus guadalupensis Greene BCN

*Lupinus hintonii C.P. Sm. GRO, MEX, MICH, PUE

*Lupinus hintoniorum B.L. Turner NLE, TAMS

Lupinus hirsutissimus Benth. BCN

*Lupinus howardii M.E. Jones CHIH, DGO, JAL, NAY, ZAC

*Lupinus howard-scottii C.P. Sm. SIN

Lupinus huachucanus M.E. Jones CHIH, DGO

Lupinus idoneus C.P. Sm. MICH

*Lupinus jaimehintoniana B.L. Turner OAX

Lupinus latifolius Lindley ex J. Agardh BCN, CHIH, DGO

Lupinus leonensis S. Watson CHIH, COAH, NLE, TAMS

*Lupinus leptocarpus Benth. AGS, JAL, NAY, ZAC

*Lupinus leptophyllus Schltdl. \& Cham. CDMX, HGO, JAL,

MEX, OAX, PUE, QRO, TLAX, VER

*Lupinus lesueurii Standl. CHIH

Lupinus longifolius (S. Watson) Abrams BCN

*Lupinus macranthus Rose OAX

*Lupinus madrensis Seem. COL, DGO, GRO, JAL, MICH, NAY, SIN, ZAC

*Lupinus marschallianus Sweet HGO, JAL, MEX, MICH, SLP

*Lupinus mearnsii C.P. Sm. SON

Lupinus mexicanus Cerv. ex Lag. AGS, CHIH, CDMX, DGO, GTO, GRO, HGO, JAL, MEX, MICH, MOR, OAX, QRO, SLP, TLAX, VER, ZAC

Lupinus montanus Kunth CHIS, CHIH, CDMX, DGO, GTO, GRO, HGO, JAL, MEX, MICH, NAY, NLE, OAX, PUE, QRO,

SLP, SIN, TAMS, TLAX, VER, ZAC

*Lupinus muelleri Standl. COAH, NLE

*Lupinus niveus $\mathrm{S}$. Watson BCN

*Lupinus octablomus C.P. Sm. GRO

*Lupinus perbonus C.P. Sm. JAL, MICH

*Lupinus persistens Rose CDMX, HGO, JAL, MEX, MICH, MOR, OAX, QRO, TLAX

*Lupinus pilosissimus M. Martens \& Galeotti ND

*Lupinus platamodes C.P. Sm. NLE, TAMS

*Lupinus potosinus Rose COAH, NLE, SLP, TAMS

Lupinus quercuum C.P. Sm. MEX, MICH

*Lupinus reflexus Rose COL, CDMX, JAL, MEX, MICH, OAX, SLP

*Lupinus rhodanthus C.P. Sm. MEX, MICH

*Lupinus rotundiflorus M.E. Jones JAL, NAY

Lupinus ruber Heller BCN

*Lupinus semiaequus C.P. Sm. JAL, MICH

*Lupinus simulans Rose HGO, JAL, MEX, MICH, MOR, OAX, VER

*Lupinus sinaloensis C.P. Sm. SIN

Lupinus sitgreavesii $\mathrm{S}$. Watson $\mathrm{CHIH}$, SON

Lupinus sparsiflorus Benth. BCN, BCS, SON

*Lupinus splendens Rose CDMX, GRO, HGO, JAL, MEX, MICH, MOR, OAX, QRO, ZAC

*Lupinus stipulatus J. Agardh COL, CDMX, GRO, HGO, JAL, MEX, MICH, MOR, NLE, OAX, PUE, QRO, SLP, TLAX

Lupinus subcarnosus Hook. COAH, NLE

Lupinus subvexus C.P. Sm. BCN
Lupinus succulentus Dougl. ex K. Koch BCN, BCS

*Lupinus taurimortuus C.P. Sm. GRO

Lupinus texensis Hook. COAH, NLE, TAMS

Lupinus truncatus Nutt. ex Hook. \& Arn. BCN

*Lupinus uncinatus Schltdl. CDMX, HGO, MEX, MICH, OAX, VER

*Lupinus vernicius Rose CDMX, HGO, MEX

*Lupinus versicolor Sweet CDMX, HGO, MEX, PUE, TLAX, VER

Lysiloma acapulcense (Kunth) Benth. AGS, CAM, CHIS, CHIH, COL, DGO, GTO, GRO, HGO, JAL, MEX, MICH, MOR, NAY, OAX, PUE, QRO, QROO, SLP, SIN, SON, TAB, TAMS, VER, YUC, ZAC

Lysiloma auritum (Schltdl.) Benth. CHIS, GRO, OAX, TAB, VER

*Lysiloma candidum Brandegee BCN, BCS, SON

Lysiloma divaricatum (Jacq.) J.F. Macbr. AGS, BCN, BCS, CAM, CHIS, CHIH, COL, DGO, GTO, GRO, HGO, JAL, MEX, MICH, MOR, NAY, OAX, PUE, QRO, SLP, SIN, SON, TAB, TAMS, VER, ZAC

Lysiloma latisiliquum (L.) Benth. CAM, CHIS, QROO, TAB, VER, YUC

*Lysiloma tergeminum Benth. COL, GRO, JAL, MEX, MICH, MOR, NAY, OAX, PUE

Lysiloma watsonii Rose CHIH, SIN, SON

Macroptilium atropurpureum (Sessé \& Moc. ex DC.) Urb. AGS, BCN, BCS, CAM, CHIS, CHIH, COAH, COL, CDMX, DGO, GTO, GRO, HGO, JAL, MEX, MICH, MOR, NAY, NLE, OAX, PUE, QRO, QROO, SLP, SIN, SON, TAB, TAMS, VER, YUC, ZAC

Macroptilium gibbosifolium (Ortega) A. Delgado AGS, CHIS, CHIH, COAH, COL, CDMX, DGO, GTO, GRO, HGO, JAL, MEX, MICH, MOR, NAY, NLE, OAX, PUE, QRO, SLP, SIN, SON, TAMS, TLAX, VER, ZAC

Macroptilium gracile (Poepp. ex Benth.) Urb. CHIS, OAX, TAB Macroptilium lathyroides (L.) Urb. CAM, CHIS, HGO, JAL, MICH, OAX, QRO, QROO, SIN, TAB, TAMS, VER, YUC Macroptilium longepedunculatum (Mart. ex Benth.) Urb. BCS, CAM, CHIS, COL, GRO, JAL, MEX, MICH, MOR, NAY, NLE, OAX, QROO, SIN, VER, YUC

*Macroptilium pedatum (Rose) Maréchal \& Baudet GRO, MEX, MICH, MOR, NAY

*Macroptilium supinum (Wiggins \& Rollins) A. Delgado \& L. Torres NAY, SON

Machaerium arboreum (Jacq.) Vogel CHIS, COL, OAX, SLP, TAB, VER

Machaerium biovulatum Micheli CAM, CHIS, HGO, MEX, MICH, OAX, QRO, SLP, TAB, VER

Machaerium cirrhiferum Pittier CAM, CHIS, OAX, QROO, TAB, VER

Machaerium cobanense Donn. Sm. CHIS, OAX, VER

* Machaerium conzattii Rudd OAX

Machaerium chiapense Brandegee CHIS, OAX, TAB, VER

Machaerium falciforme Rudd CAM, CHIS, TAB, VER

Machaerium floribundum Benth. CHIS, OAX, TAB, VER

Machaerium inundatum (C. Mart. ex Benth.) Ducke CHIS, VER Machaerium isadelphum (E. Mey.) Amshoff CHIS, MEX, OAX, 
QROO, TAB, VER

Machaerium kegelii Meisn. CAM, CHIS, COL, GRO, JAL, NAY, OAX, TAB

Machaerium lunatum (L. f.) Ducke QRO, SLP, TAB, VER Machaerium pittieri J.F. Macbr. CHIS, GRO, HGO, OAX, VER * Machaerium ramosiae J. Linares CAM

Machaerium riparium Brandegee CAM, CHIS, OAX, QRO, QROO, SLP, TAB, VER

Machaerium salvadorense (Donn. Sm.) Rudd CHIS, COAH, COL, GRO, JAL, MICH, NAY, OAX, SLP, VER

Machaerium seemannii Benth. CAM, CHIS, QROO, TAB

Machaerium setulosum Pittier CAM, CHIS, TAB, VER

*Marina brevis León de la Luz BCS

Marina calycosa (A. Gray) Barneby CHIH, NLE, SON

* Marina capensis Barneby BCS

* Marina catalinae Barneby BCS

* Marina crenulata (Hook. \& Arn.) Barneby CHIH, COL, DGO, GRO, JAL, MICH, NAY, OAX, SIN, SON, ZAC

* Marina chrysorrhiza (A. Gray) Barneby BCS

Marina diffusa (Moric.) Barneby CHIS, CHIH, COL, DGO, GRO, JAL, MEX, MICH, MOR, NAY, OAX, PUE, SLP, SIN, SON, VER, ZAC

* Marina dispansa (Rydb.) Barneby JAL

* Marina divaricata (Benth.) Barneby BCS

* Marina evanescens (Brandegee) Barneby BCN, BCS

* Marina gemmea Barneby JAL, MICH

* Marina ghiesbreghtii Barneby CHIS, GRO, OAX

* Marina goldmanii (Rose) Barneby CHIH, SIN, SON

Marina gracilis Liebm. GRO, JAL, MEX, MICH, MOR, NAY, OAX

*Marina gracillima (S. Watson) Barneby GRO, JAL, MEX, $\mathrm{MICH}$

* Marina grammadenia Barneby COL, DGO, GRO, JAL, MICH, NAY, ZAC

* Marina greenmaniana (Rose) Barneby MEX, OAX, PUE

* Marina holwayi (Rose) Barneby GRO, MOR, OAX, PUE

* Marina interstes Barneby BCS

* Marina maritima (Brandegee) Barneby BCS

* Marina melilotina Barneby GRO, PUE

* Marina minor (Rose) Barneby DGO, SIN

* Marina minutiflora (Rose) Barneby GRO, MEX, MOR, OAX, PUE

*Marina neglecta (B.L. Rob.) Barneby AGS, BCS, COL, GTO, GRO, JAL, MICH, MOR, NAY, OAX, PUE, QRO, SLP, ZAC

*Marina nutans (Cav.) Barneby AGS, CHIS, CHIH, COL, CDMX, DGO, GTO, GRO, HGO, JAL, MEX, MICH, MOR, NAY, NLE, OAX, PUE, QRO, SLP, SIN, TAMS, VER, ZAC

* Marina oculata (Rydb.) Barneby BCS

Marina orcuttii (S. Watson) Barneby BCN, BCS

*Marina palmeri (Rose) Barneby CHIH, SIN, SON

Marina parryi (Torr. \& A. Gray) Barneby BCN, BCS, CHIH, SON

*Marina peninsularis (Rose) Barneby BCS, SIN, SON

*Marina procumbens (DC.) Barneby CHIS, GTO, GRO, JAL, MEX, MICH, MOR, NAY, OAX, QRO, ZAC

*Marina pueblensis (Brandegee) Barneby GRO, MOR, OAX, PUE
*Marina sarodes Barneby JAL, NAY

Marina scopa Barneby CHIS, COAH, COL, GTO, GRO, JAL, MEX, MICH, MOR, NAY, NLE, OAX, PUE, QRO, SLP, SIN, SON, TAMS, VER, YUC, ZAC

*Marina spiciformis (Rose) Barneby CHIS, GTO, GRO, JAL, MEX, MICH, MOR, OAX

*Marina stilligera Barneby COL, GRO, JAL, MEX, MICH, MOR, OAX, PUE

*Marina unifoliata (B.L. Rob. \& Greenm.) Barneby COL, GRO, JAL, MEX, MICH, MOR, OAX, PUE, ZAC

Marina vetula (Brandegee) Barneby BCN, BCS, COAH, SON

* Marina victoriae León de la Luz BCS

* Mariosousa acatlensis (Benth.) Seigler \& Ebinger AGS, CHIS, COL, DGO, GTO, GRO, JAL, MEX, MICH, MOR, NAY, OAX, PUE, SIN, VER, ZAC

Mariosousa centralis (Britton \& Rose) Seigler \& Ebinger CAM, CHIS, OAX, QROO, TAB, YUC

*Mariosousa compacta (Rose) Seigler \& Ebinger MEX, OAX, PUE, VER

Mariosousa coulteri (Benth.) Seigler \& Ebinger BCS, CHIS, CHIH, COAH, COL, DGO, GTO, GRO, HGO, JAL, MEX, MICH, MOR, NLE, OAX, PUE, QRO, SLP, SIN, SON, TAMS, VER, ZAC

Mariosousa dolichostachya (S.F. Blake) Seigler \& Ebinger CAM, QROO, YUC

*Mariosousa durangensis (Britton \& Rose) Seigler \& Ebinger DGO, SLP

*Mariosousa mammifera (Schltdl.) Seigler \& Ebinger COL, GTO, HGO, JAL, MICH, MOR, NLE, OAX, PUE, QRO, SLP, TAMS

Mariosousa millefolia (S. Watson) Seigler \& Ebinger CHIH, SIN, SON

*Mariosousa russelliana (Britton \& Rose) Seigler \& Ebinger NAY, SIN, SON

*Mariosousa salazarii (Britton \& Rose) Seigler \& Ebinger GRO, JAL, MEX, MICH, MOR, OAX, PUE

*Mariosousa sericea (M. Martens \& Galeotti) Seigler \& Ebinger OAX, PUE

Mariosousa usumacintensis (Lundell) Seigler \& Ebinger CAM, CHIS, OAX, QROO, TAB, VER, YUC

Mariosousa willardiana (Rose) Seigler \& Ebinger BCN, BCS, SIN, SON

*Microlobius foetidus (Jacq.) M. Sousa \& G. Andrade GRO, JAL, MEX, MICH, MOR, OAX, PUE, SIN

Mimosa acantholoba (Humb. \& Bonpl. ex Willd.) Poir. CHIS, COL, GRO, JAL, MICH, NAY, OAX, SIN

*Mimosa acapulcensis B.L. Rob. GRO, OAX

Mimosa aculeaticarpa Ortega AGS, BCN, BCS, CHIS, CHIH, COAH, COL, CDMX, DGO, GTO, GRO, HGO, JAL, MEX, MICH, MOR, NAY, NLE, OAX, PUE, QRO, SLP, SIN, SON, TAMS, TLAX, VER, ZAC

*Mimosa adenantheroides (M. Martens \& Galeotti) Benth. CHIS, COL, GRO, JAL, MEX, MICH, OAX, PUE

*Mimosa affinis B.L. Rob. COL, GRO, JAL, MEX, MICH, MOR, NAY, OAX, SIN, ZAC

Mimosa albida Humb. \& Bonpl. ex Willd. CAM, CHIS, COL, CDMX, DGO, GTO, GRO, HGO, JAL, MEX, MICH, MOR, 
NAY, OAX, PUE, QRO, QROO, SLP, SIN, TAB, TAMS, VER, YUC, ZAC

Mimosa antioquensis Killip ex Rudd CHIS, OAX, VER

Mimosa arenosa (Humb. \& Bonpl. ex Willd.) Poir. COL, GRO,

JAL, MICH, OAX

*Mimosa argillotropha B.L. Rob. GRO

*Mimosa aspera M.E. Jones JAL, NAY

Mimosa bahamensis Benth. CAM, CHIS, QROO, TAB, VER, YUC

* Mimosa barrancana Gentry DGO, SIN

*Mimosa benthamii J.F. Macbr. AGS, COL, DGO, GTO, GRO, JAL, MEX, MICH, MOR, NAY, OAX, PUE, SLP, SIN, ZAC

Mimosa borealis A. Gray CHIH, COAH, SLP

* Mimosa brandegei B.L. Rob. BCS, COL, JAL, MICH, SIN

* Mimosa brevispicata Britton OAX, PUE

* Mimosa caerulea Rose COL, GRO, JAL, MEX, MOR, OAX, VER

* Mimosa calcicola B.L. Rob. HGO, OAX, PUE, VER

Mimosa camporum Benth. CHIS, COL, GRO, JAL, NAY, OAX, SIN, TAB, VER

Mimosa candollei $\mathrm{R}$. Grether CHIS, OAX, TAB

*Mimosa coelocarpa B.L. Rob. SIN

Mimosa corynadenia Britton \& Rose CHIS

* Mimosa costenya McVaugh COL, JAL, NAY

* Mimosa chaetocarpa Brandegee MOR, VER

* Mimosa deamii B.L. Rob. OAX

* Mimosa depauperata Benth. HGO, MEX, OAX, QRO, SLP, VER

Mimosa diplotricha $\mathrm{C}$. Wright ex Sauvalle CHIS, CHIH, COL, DGO, GRO, JAL, MEX, MICH, MOR, NAY, OAX, PUE, SIN, SON, TAB, VER

Mimosa distachya Cav. BCN, BCS, CHIH, COL, DGO, GRO, JAL, MICH, NAY, OAX, PUE, QROO, SIN, SON, TAB, TAMS, VER, YUC, ZAC

Mimosa dormiens Humb. \& Bonpl. ex Willd. CHIS, GRO, OAX, TAB, VER

Mimosa dysocarpa Benth. AGS, CHIH, COAH, DGO, JAL, SIN, SON, ZAC

*Mimosa egregia Sandwith GRO, MEX, MICH

Mimosa emoryana Benth. CHIH, COAH, DGO, NLE, TAMS

*Mimosa epitropica Barneby \& León de la Luz BCS

Mimosa ervendbergii A. Gray CHIS, COL, GRO, JAL, OAX, PUE, TAB, VER

* Mimosa ferrisiae Britton \& Rose NAY, SIN

*Mimosa galeottii Benth. AGS, COL, DGO, GTO, GRO, JAL, MEX, MICH, MOR, NAY, OAX, PUE, SLP

* Mimosa gentryi Barneby CHIH, SIN, SON

*Mimosa goldmanii B.L. Rob. CHIS, GRO, MOR, OAX, PUE Mimosa grahamii A. Gray CHIH, DGO, SON

Mimosa guatemalensis (Hook. \& Arn.) Benth. COL, DGO, GRO, JAL, MEX, MICH, NAY, QROO, SIN, YUC, ZAC

Mimosa guirocobensis Gentry CHIH, SON

Mimosa hexandra Micheli OAX

Mimosa hondurana Britton CHIS

*Mimosa ionema B.L. Rob. GRO, MEX, MICH, MOR

*Mimosa lacerata Rose DGO, GTO, GRO, HGO, JAL, MEX,

MICH, MOR, OAX, PUE, QRO, SLP, TLAX, VER
Mimosa lactiflua Delile ex Benth. CHIS, GRO, MOR, OAX, PUE, VER

Mimosa latidens (Small) B.L. Turner COAH, NLE, TAMS

*Mimosa leptocarpa Rose COL, GRO, JAL, MICH

*Mimosa leucaenoides Benth. GTO, HGO, QRO, SLP, TAMS, VER

*Mimosa luisana Brandegee COAH, OAX, PUE

Mimosa malacophylla A. Gray CHIH, COAH, NLE, QRO, SLP,

TAMS, VER

* Mimosa margaritae Rose BCS

*Mimosa martindelcampoi Medrano SLP, TAMS

* Mimosa mellii Britton \& Rose CHIS, OAX

* Mimosa minutifolia B.L. Rob. \& Greenm. AGS, GTO, JAL, SLP, ZAC

* Mimosa mollis Benth. DGO, GRO, OAX, PUE

Mimosa monancistra Benth. AGS, CHIH, COAH, DGO, GTO, JAL, MICH, NLE, OAX, QRO, SLP, TAMS, ZAC

*Mimosa moniliformis (Britton \& Rose) R. Grether \& Barneby SON

*Mimosa nanchititlana R. Grether \& Barneby DGO, GRO, MEX, NAY

Mimosa occidentalis Britton \& Rose CHIS, JAL, NAY, OAX, TAB, VER

Mimosa orthocarpa Spruce ex Benth. CHIS, GRO, JAL, NAY, OAX, SIN, TAB, VER

*Mimosa palmeri Rose CHIH, GRO, JAL, MICH, NAY, SIN, SON

Mimosa paludosa Benth. GRO, MEX

* Mimosa pauli Barneby CHIH, SON

Mimosa pellita Humb. \& Bonpl. ex Willd. COL, JAL

Mimosa pigra L. CAM, CHIS, COL, GRO, JAL, MEX, MICH, MOR, NAY, OAX, QRO, QROO, SLP, SIN, SON, TAB, TAMS, VER, YUC

Mimosa platycarpa Benth. CHIS, OAX

* Mimosa polyancistra Benth. COL, JAL, OAX, PUE

Mimosa polyantha Benth. CHIS, DGO, GRO, JAL, MEX, MICH, MOR, NAY, OAX, PUE, SIN, SON, TAMS, VER, ZAC

*Mimosa polyanthoides B.L. Rob. GRO, MICH

Mimosa polydactyla Humb. \& Bonpl. ex Willd. CHIS, OAX

*Mimosa potosina (Britton \& Rose) B.L. Turner NLE, SLP, TAMS

Mimosa pringlei $\mathrm{S}$. Watson CHIH, COAH, DGO, HGO, QRO, SLP, TAMS

*Mimosa psilocarpa B.L. Rob. CHIS, OAX

* Mimosa puberula Benth. HGO, QRO, VER

Mimosa pudica L. CAM, CHIS, COL, GRO, HGO, JAL, MEX, MICH, MOR, NAY, OAX, PUE, QROO, SIN, TAB, VER, YUC *Mimosa purpusii Brandegee DGO, GTO, HGO, OAX, PUE Mimosa quadrivalvis L. CHIH, COAH, COL, DGO, JAL, MICH, NAY, NLE, SLP, SIN, VER, ZAC

Mimosa recordii Britton \& Rose CHIS, OAX

* Mimosa rhodocarpa (Britton \& Rose) R. Grether CHIS, CHIH, COAH, DGO, GTO, GRO, HGO, JAL, MEX, MICH, NLE, OAX, PUE, QRO, SLP, TAMS, TLAX, VER, ZAC

* Mimosa rhododactyla B.L. Rob. DGO, GRO, MICH, MOR, NAY, PUE, SIN 
*Mimosa robusta $\mathrm{R}$. Grether COL, GRO, JAL, MICH, NAY, OAX, SIN

*Mimosa rosei B.L. Rob. COL, GRO, JAL, MICH, NAY, ZAC Mimosa scalpens Standl. CHIS, VER

*Mimosa setuliseta Villarreal COAH, DGO, GTO, JAL, SLP, TAMS, ZAC

*Mimosa sicyocarpa B.L. Rob. CHIS, COL, GRO, JAL, MICH, NAY, OAX

*Mimosa similis Britton \& Rose GTO, HGO, QRO, SLP

* Mimosa sinaloensis Britton \& Rose COL, JAL, SIN

Mimosa skinneri Benth. CHIS, NAY, OAX, TAB

Mimosa somnians Humb. \& Bonpl. ex Willd. CAM, CHIS, GRO, JAL, NAY, OAX, PUE, TAB, VER, YUC

* Mimosa sotoi R. Grether \& V.W. Steinm. MICH

* Mimosa sousae R. Grether OAX

* Mimosa spirocarpa Rose COL, JAL, MICH, SIN

Mimosa strigillosa Torr. \& A. Gray CHIH, SLP, TAMS

*Mimosa subinermis (S. Watson) B.L. Turner COAH

Mimosa tarda Barneby OAX

*Mimosa tejupilcana R. Grether \& A. Martínez-Bernal GRO, MEX

Mimosa teledactyla Donn. Sm. CHIS

Mimosa tenuiflora (Willd.) Poir. CHIS, HGO, OAX

*Mimosa tequilana $\mathrm{S}$. Watson COL, JAL, NAY, ZAC

Mimosa texana (A. Gray) Small AGS, CHIH, COAH, DGO, GTO, HGO, MICH, NLE, OAX, PUE, QRO, SLP, SIN, SON, TAMS

*Mimosa torresiae R. Grether OAX

Mimosa tricephala Schltdl. \& Cham. BCS, CHIS, DGO, GRO, HGO, JAL, MICH, MOR, NAY, OAX, PUE, TAB, TAMS, VER, ZAC

Mimosa turneri Barneby CHIH, COAH, NLE

*Mimosa unipinnata Parfitt \& Pinkava COAH

Mimosa ursina Mart. CHIS, OAX, TAB

Mimosa velloziana Mart. CHIS, NAY, OAX, TAB, VER

Mimosa watsonii B.L. Rob. CHIS, GRO, OAX, TAB, VER

Mimosa wootonii Standl. TAMS

* Mimosa xochipalensis R. Grether GRO

*Mimosa zimapanensis Britton \& Rose HGO, QRO

Mimosa zygophylla Benth. AGS, CHIH, COAH, DGO, GTO,

HGO, JAL, NLE, QRO, SLP, TAMS, ZAC

*Minkelersia multiflora Rose CDMX, DGO, HGO, MEX, MOR, OAX

Mucuna argyrophylla Standl. CHIS, OAX, PUE, QRO, SLP, TAB, VER

*Mucuna aterrima Holland TAB

* Mucuna chiapaneca M. Sousa \& T.M. Moura CHIS

Mucuna holtonii (Kuntze) Moldenke CAM, CHIS, QROO, VER

Mucuna pruriens (L.) DC. CAM, CHIS, GRO, HGO, MOR, OAX, PUE, QRO, QROO, SLP, TAB, TAMS, VER, YUC

Mucuna sloanei Fawc. \& Rendle CHIS, COL, GRO, JAL, MICH, NAY, OAX, TAB, VER, YUC

Muellera frutescens (Aubl.) Standl. TAB, VER

*Muellera unifoliolata (Benth.) M. Sousa OAX, PUE, TAB, VER

Myrospermum frutescens Jacq. CAM, CHIS, COL, GRO, JAL,
MEX, MICH, MOR, OAX

Myrospermum sousanum A. Delgado \& M.C. Johnst. NLE

Myroxylon balsamum (L.) Harms CAM, CHIS, DGO, GRO, HGO, MEX, MICH, MOR, NAY, OAX, QROO, SIN, TAB, VER, YUC

Myroxylon peruiferum L. f. CHIS, COL, OAX

Neptunia olerecea Lour. CAM, CHIS, COL, GRO, JAL, MICH, MOR, NAY, OAX, SIN, TAB, TAMS, VER

Neptunia plena (L.) Benth. BCS, CAM, CHIS, COL, GRO, JAL, MICH, MOR, NAY, OAX, SIN, SON, TAB, TAMS, VER Neptunia pubescens Benth. CHIS, COAH, COL, GTO, HGO, JAL, MICH, MOR, NAY, NLE, OAX, QRO, SLP, SIN, TAB, TAMS, VER

*Nissolia chiapensis Rudd CHIS, OAX

Nissolia fruticosa Jacq. CAM, CHIS, COL, GTO, GRO, HGO, JAL, MEX, MICH, MOR, NAY, OAX, PUE, QRO, QROO, SLP, SIN, TAMS, VER, YUC, ZAC

*Nissolia gentryi Rudd SIN, SON

*Nissolia hintonii Sandwith GRO, MEX

*Nissolia laxior (B.L. Rob.) Rose COL, DGO, GTO, GRO, JAL, MEX, MICH, MOR, NAY, OAX, PUE, QRO

*Nissolia leiogyne Sandwith COL, GTO, GRO, JAL, MICH, NAY, OAX, QRO, SIN

*Nissolia microptera Poir. AGS, CAM, CHIS, CHIH, COL, DGO, GTO, GRO, JAL, MEX, MICH, MOR, NAY, OAX, PUE, QRO, SIN, SON, ZAC

Nissolia platycalyx $\mathrm{S}$. Watson CHIH, COAH, NLE, SLP, TAMS, VER

*Nissolia platycarpa Benth. COAH, GRO, HGO, NLE, OAX, PUE, QRO, SLP, SIN, SON, TAMS, VER

*Nissolia pringlei Rose CHIH, COAH, DGO, GTO, GRO, HGO, JAL, MEX, MICH, MOR, NLE, OAX, PUE, QRO, SLP, SIN, SON, ZAC

*Nissolia ruddiae R. Cruz \& M. Sousa GRO, MOR, OAX, PUE Nissolia schottii (Torr.) A. Gray BCN, BCS, CHIH, DGO, SIN, SON

*Nissolia setosa Brandegee BCS

Nissolia wislizenii (A. Gray) A. Gray AGS, CHIH, DGO, GTO, HGO, JAL, OAX, QRO, SLP, SON, ZAC

Olneya tesota A. Gray BCN, BCS, SIN, SON

*Orbexilum chiapasanum B.L. Turner CHIS

*Orbexilum melanocarpum (Benth.) Rydb. CHIS, COAH, HGO, NLE, QRO, TAMS, ZAC

Ormosia carinata N. Zamora GRO, OAX, VER

Ormosia isthmensis Standl. CHIS, OAX, PUE, TAB, VER

Ormosia macrocalyx Ducke CHIS, TAB, VER

*Ormosia oaxacana Rudd CHIS, GRO, OAX

Ormosia schippii Pierce ex Standl. \& Steyerm. CAM, CHIS, $\mathrm{TAB}$

*Orobus argenteus Mill. VER

*Oxyrhynchus populneus (Piper) Norvell ex A. Delgado \& E. Estrada COAH, NLE, TAMS

Oxyrhynchus trinervius (Donn. Sm.) Rudd CHIS, VER

Oxyrhynchus volubilis Brandegee CAM, COAH, NLE, PUE, QRO, QROO, SLP, TAMS, VER, YUC

Pachecoa prismatica (Sessé \& Moc.) Standl. \& B.G. Schub. COL, GRO, JAL, MEX, MICH, MOR, OAX, VER 
Pachyrhizus erosus (L.) Urb. AGS, CAM, CHIS, COL, GTO, GRO, HGO, JAL, MEX, MICH, MOR, NAY, OAX, PUE, QRO, QROO, SLP, SIN, TAB, TAMS, VER, YUC, ZAC

Pachyrhizus ferrugineus (Piper) M. Sørensen CAM, CHIS, GRO, OAX, QROO, VER

*Painteria elachistophylla (S. Watson) Britton \& Rose CHIH, COAH, DGO, GTO, JAL, HGO, NLE, OAX, PUE, QRO, SLP, TAMS, VER, ZAC

*Painteria leptophylla (DC.) Britton \& Rose AGS, CHIH, COL, CDMX, DGO, GTO, GRO, HGO, JAL, MEX, MICH, NLE, OAX, PUE, QRO, SLP, SON, TAMS, TLAX, ZAC

* Painteria revoluta (Rose) Britton \& Rose HGO, QRO, SLP, VER

Parkinsonia aculeata L. BCN, BCS, CAM, CHIS, CHIH, COAH, COL, DGO, GTO, GRO, HGO, JAL, MEX, MICH, MOR, NAY, NLE, OAX, PUE, QRO, QROO, SLP, SIN, SON, TAB, TAMS, VER, YUC, ZAC

Parkinsonia florida (Benth. ex A. Gray) S. Watson BCN, BCS, COAH, NAY, SIN, SON, TAMS

Parkinsonia microphylla Torr. BCN, BCS, SIN, SON

Parkinsonia praecox (Ruiz \& Pav. ex Hook.) J. Hawkins BCS, CHIS, CHIH, COL, DGO, GTO, GRO, JAL, MEX, MICH, MOR, NAY, OAX, PUE, SIN, SON, TAMS, VER, ZAC

Parkinsonia texana (A. Gray) S. Watson COAH, DGO, NLE, SLP, TAMS

Parkinsonia $\times$ carterae J. Hawkins BCS, GRO, MICH, OAX, PUE, SON

Pediomelum califonicum (S. Watson) Rydb. BCN

Pediomelum humile Rydb. COAH

*Pediomelum palmeri (Ockendon) J.W. Grimes AGS, CHIH, COAH, DGO, GTO, HGO, JAL, JAL, OAX, PUE, QRO, SLP, ZAC

Pediomelum pentaphyllum (L.) Grimes CHIH, COAH, GTO, JAL, MEX, SLP, SON

Pediomelum rhombifolium (Torr. \& A. Gray) Rydb. BCN, BCS, COAH, CDMX, GRO, HGO, JAL, MEX, MOR, NLE, OAX, PUE, QRO, SLP, SON, TAMS, TLAX, VER, ZAC

Peltogyne mexicana Martínez GRO

Petalostemon exile A. Gray CHIH, SON

*Peteria glandulosa (A. Gray) Rydb. CDMX, GTO, HGO, MEX, NLE, PUE, SLP, VER, ZAC

* Peteria pinetorum Ced. Porter CHIH

Peteria scoparia A. Gray CHIH, COAH

*Phaseolus acinaciformis Freytag \& Debouck OAX

Phaseolus acutifolius A. Gray BCN, BCS, CHIH, COAH, COL, DGO, GTO, GRO, HGO, JAL, MICH, NAY, OAX, QRO, SIN, SON, ZAC

*Phaseolus albescens McVaugh ex Ramírez-Delgadillo \& A. Delgado COL, JAL, MICH

*Phaseolus albiflorus Freytag \& Debouck COAH, NLE, TAMS

*Phaseolus albinervus Freytag \& Debouck CHIH

*Phaseolus albiviolaceus Freytag \& Debouck NLE, TAMS

*Phaseolus altimontanus Freytag \& Debouck COAH, NLE, TAMS

*Phaseolus amabilis Standl. CHIH, SON

*Phaseolus amblyosepalus (Piper) C.V. Morton DGO, MICH, SIN
Phaseolus angustissimus A. Gray CHIH, COAH, SON

*Phaseolus anisophyllus (Piper) Freytag \& Debouck CHIH, DGO

Phaseolus anisotrichos Schltdl. AGS, CHIS, CHIH, COAH, COL, CDMX, DGO, GTO, GRO, HGO, JAL, MEX, MICH, MOR, NAY, NLE, OAX, PUE, QRO, SLP, SIN, SON, TAMS, VER, ZAC

*Phaseolus campanulatus Freytag \& Debouck JAL, NAY, SLP

*Phaseolus carteri Freytag \& Debouck BCS

Phaseolus coccineus L. AGS, CHIS, CHIH, COAH, COL, CDMX, DGO, GTO, GRO, HGO, JAL, MEX, MICH, MOR, NAY, NLE, OAX, PUE, QRO, SLP, SIN, TAMS, TLAX, VER, ZAC

*Phaseolus chiapasanus Piper CHIS, OAX

*Phaseolus dasycarpus Freytag \& Debouck HGO, VER

Phaseolus dumosus Macfad. CHIS, HGO, OAX, VER

Phaseolus elegans Piper YUC

*Phaseolus esperanzae Seaton HGO, MEX, MICH, PUE, SLP

*Phaseolus esquincensis Freytag CHIS

Phaseolus filiformis Benth. BCN, BCS, CHIH, COAH, HGO, NLE, QRO, SLP, SON

*Phaseolus glabellus Piper COAH, NLE, OAX, PUE, SLP, TAMS, VER

*Phaseolus gladiolatus Freytag \& Debouck HGO, SLP

Phaseolus grayanus Wooton \& Standl. AGS, CHIH, COAH, DGO, JAL, NLE, SON, ZAC

*Phaseolus hintonii A. Delgado COL, DGO, GRO, HGO, JAL, MEX, MICH, NAY, OAX

*Phaseolus jaliscanus Piper COL, DGO, JAL, MICH, NAY, SIN

*Phaseolus juquilensis A. Delgado OAX

*Phaseolus longiplacentifer Freytag VER

Phaseolus lunatus L. BCS, CAM, CHIS, COL, CDMX, DGO, GRO, HGO, JAL, MEX, MICH, MOR, NAY, OAX, QRO, QROO, SLP, SIN, SON, TAB, TAMS, VER, YUC

*Phaseolus maculatifolius Freytag \& Debouck NLE, TAMS

Phaseolus maculatus Scheele AGS, CHIH, COAH, DGO, GTO, GRO, HGO, JAL, MEX, NAY, NLE, OAX, PUE, QRO, SLP, SIN, SON, TLAX, ZAC

*Phaseolus macvaughii A. Delgado COL, GRO, JAL, MICH, SIN

*Phaseolus magnilobatus Freytag \& Debouck COL, DGO, JAL

*Phaseolus marechalii A. Delgado MOR, PUE

*Phaseolus micranthus Hook. \& Arn. CHIS, COL, GRO, JAL, MICH, NAY, OAX, QRO, SLP, SIN, ZAC

*Phaseolus microcarpus Mart. CHIS, COL, DGO, GTO, GRO, JAL, MEX, MICH, MOR, NAY, OAX, PUE, ZAC

*Phaseolus neglectus F.J. Herm. COAH, NLE, TAMS

*Phaseolus nelsonii Maréchal, Mascherpa \& Stainier CHIS, GRO, JAL, MICH, OAX, PUE, ZAC

*Phaseolus nodosus Freytag \& Debouck COL, JAL

*Phaseolus novoleonensis Debouck NLE, TAMS

* Phaseolus oaxacanus Rose OAX

Phaseolus oligospermus Piper CHIS, NAY, OAX

*Phaseolus ovatifolius Piper NAY, SON

* Phaseolus pachycarpus Standl. CHIH, SON

*Phaseolus parvifolius Freytag BCN, CHIS, CHIH, COL, DGO, 
GRO, JAL, MICH, NAY, OAX, SIN, SON

Phaseolus parvulus Greene CHIH, DGO, NAY, SON

Phaseolus pauciflorus Sessé \& Moc. ex G. Don CHIS, CHIH, COL, CDMX, DGO, GRO, HGO, JAL, MEX, MICH, MOR, NAY, OAX, SIN, SON, TLAX, ZAC

Phaseolus pedicellatus Benth. BCS, CHIH, COAH, COL, CDMX, DGO, GTO, GRO, HGO, JAL, MEX, MICH, MOR, NLE, OAX, PUE, QRO, SLP, SIN, SON, TAB, TAMS, TLAX, VER

*Phaseolus perplexus A. Delgado COL, DGO, GRO, JAL, MEX, MICH, NAY, OAX, SIN

*Phaseolus plagiocalyx Harms COAH, NLE

*Phaseolus pluriflorus Maréchal, Mascherpa \& Stainier CDMX, DGO, GTO, HGO, JAL, MEX, MICH, MOR, NAY, OAX, QRO, SIN, ZAC

*Phaseolus polymorphus $\mathrm{S}$. Watson AGS, CHIH, COAH, DGO, GTO, GRO, HGO, JAL, MICH, NLE, QRO, SLP, SON, TAMS, ZAC

*Phaseolus pyramidalis Freytag $\mathrm{CHIH}$

* Phaseolus reticulatus Freytag \& Debouck DGO

*Phaseolus rotundatus Freytag \& Debouck JAL, MICH, OAX

*Phaseolus salicifolius Piper DGO, SIN

*Phaseolus scabrellus Benth. ex S. Watson COAH, DGO, HGO, NLE, QRO, SLP, SIN, SON

*Phaseolus scrobiculatifolius Freytag MICH

* Phaseolus sonorensis Standl. CHIH, SIN, SON

*Phaseolus tenellus Piper MEX, MICH, ZAC

*Phaseolus teulensis Freytag ZAC

* Phaseolus trifidus Freytag NLE, TAMS

Phaseolus tuerckheimii Donn. Sm. CHIS

Phaseolus vulgaris L. AGS, BCN, BCS, CAM, CHIS, CHIH, COAH, COL, DGO, GTO, GRO, HGO, JAL, MEX, MICH, MOR, NAY, NLE, OAX, PUE, QRO, QROO, SLP, SIN, SON, TAB, TAMS, VER, YUC, ZAC

Phaseolus xanthotrichus Piper CHIS, COAH, SLP

*Phaseolus xolocotzii A. Delgado GRO, JAL, MEX, MOR, OAX

*Phaseolus zimapanensis A. Delgado AGS, COAH, DGO, HGO, NLE, QRO, SLP, TAMS

Pickeringia montana Nutt. BCN

Piptadenia flava (Spreng. ex DC.) Benth. CAM, CHIS, COL, GRO, JAL, MEX, MICH, MOR, OAX, PUE, QROO, YUC

Piptadenia obliqua (Pers.) J.F. Macbr. CHIS, COL, GRO, JAL, MICH, NAY, OAX, SIN

Piptadenia viridiflora (Kunth) Benth. CAM, CHIS, GRO, OAX, PUE, QROO

Piscidia carthagenensis Jacq. CHIS, COL, GRO, JAL, MEX, MICH, OAX

Piscidia grandifolia (Donn. Sm.) I.M. Johnst. CHIS, COL, GRO, JAL, MEX, MICH, MOR, OAX, PUE, VER

* Piscidia mollis Rose CHIH, SIN, SON

Piscidia piscipula (L.) Sarg. CAM, CHIS, GRO, HGO, JAL, MEX, MICH, NAY, NLE, OAX, PUE, QRO, QROO, SLP, TAB, TAMS, VER, YUC

Pithecellobium dulce (Roxb.) Benth. AGS, BCN, BCS, CAM, CHIS, CHIH, COAH, COL, DGO, GTO, GRO, HGO, JAL, MEX, MICH, MOR, NAY, NLE, OAX, PUE, QRO, QROO,
SLP, SIN, SON, TAB, TAMS, VER, YUC, ZAC

Pithecellobium furcatum Benth. CHIS, OAX, TAB, VER

Pithecellobium hymenaeifolium (Humb. \& Bonpl. ex Willd.)

Benth. CHIS, OAX, TAB, VER

Pithecellobium insigne Micheli ex Donn. Sm. CAM, CHIS, GRO, HGO, OAX, PUE, QRO, QROO, SLP, TAB, TAMS, VER

Pithecellobium keyense Britton CAM, QROO, VER, YUC

Pithecellobium lanceolatum (Humb. \& Bonpl. ex Willd.) Benth. CAM, CHIS, COL, GRO, HGO, JAL, MICH, NAY, OAX, PUE, QRO, QROO, SLP, SIN, TAB, TAMS, VER, YUC

Pithecellobium macrandrium Donn. Sm. CHIS, TAB

Pithecellobium oblongum Benth. GRO, JAL, MICH, OAX

Pithecellobium unguis-cati (L.) Benth. CAM, COL, GRO, JAL, MICH, MOR, NAY, OAX, QRO, QROO, SLP, SIN, SON, TAB, TAMS, VER, YUC

*Pithecellobium velutinum Britton \& Rose JAL, MICH, SIN Pithecellobium winzerlingii Britton \& Rose CAM, QROO, TAB, VER

* Pityrocarpa viridiflora (Kunth) Brenan OAX

*Platymiscium calyptratum M. Sousa \& Klitg. HGO, VER

Platymiscium dimorphandrum Donn. Sm. CHIS, GRO, HGO, OAX, TAB, VER

Platymiscium jejunum Klitg. GRO, OAX

*Platymiscium lasiocarpum Sandwith COL, GRO, JAL, MEX, MICH, OAX

*Platymiscium trifoliolatum Benth. CHIH, JAL, NAY, SIN, SON, ZAC

Platymiscium yucatanum Standl. CAM, CHIS, OAX, QROO, TAB, VER, YUC

Poeppigia procera C. Presl CHIS, COL, GRO, JAL, MEX, MICH, OAX

*Poincianella acapulcensis (Standl.) Britton \& Rose GRO, JAL, $\mathrm{MICH}$

* Poincianella caladenia (Standl.) Britton \& Rose CHIH, COL, GRO, JAL, MICH, NAY, OAX, SIN, SON, TAMS, ZAC

Poincianella eriostachys (Benth.) Britton \& Rose CHIS, COL, DGO, GRO, JAL, MICH, NAY, OAX, SIN

Poincianella exostemma (DC.) Britton \& Rose CHIS, COL, GRO, JAL, MICH, MOR, OAX, TAB, VER

Poincianella gaumeri (Greenm.) Britton \& Rose CAM, OAX, QROO, TAB, VER, YUC

*Poincianella laxa (Benth.) Britton \& Rose GRO, MICH, NLE, OAX, PUE

* Poincianella melanadenia (Rose) Britton \& Rose OAX, PUE Poincianella mexicana (A. Gray) Britton \& Rose COAH, COL, DGO, GRO, HGO, JAL, MICH, NAY, NLE, OAX, QRO, SLP, SIN, TAMS, VER

*Poincianella palmeri (S. Watson) Britton \& Rose BCS, CHIH, SIN, SON

*Poincianella robinsoniana Britton \& Rose COL, JAL

*Poincianella standleyi Britton \& Rose COL, JAL, NAY, SIN, SON

Poincianella yucatanensis (Greenm.) Britton \& Rose CAM, CHIS, QROO, TAB, VER, YUC

Poiretia punctata (Willd.) Desv. CHIS, COL, JAL, MICH, NAY, OAX, VER 
Pomaria austrotexana B.B. Simpson TAMS

* Pomaria canescens (Fisher) B.B. Simpson CHIH, COAH, DGO, NLE, SLP, ZAC

*Pomaria fruticosa (S. Watson) B.B. Simpson COAH, DGO

* Pomaria glandulosa Cav. GTO, HGO, JAL, QRO, SLP

Pomaria jamesii (Torr. \& A. Gray) Walp. CHIH, COAH, DGO

Pomaria melanosticta S. Schauer CHIH, COAH, DGO, GTO, HGO, NLE, QRO, SLP, TAMS, ZAC

* Pomaria multijuga (S. Watson) B.B. Simpson AGS, CHIH, DGO, NAY, SIN, SON, ZAC

*Pomaria wootonii (Britton) Eifert. COAH, NLE, SLP, TAMS

* Prosopidastrum mexicanum (Dressler) Burkart BCN

Prosopis articulata $\mathrm{S}$. Watson BCN, BCS, SON

Prosopis glandulosa Torr. BCN, BCS, CHIH, COAH, DGO, NAY, NLE, QROO, SLP, SIN, SON, TAMS, VER, YUC, ZAC Prosopis juliflora (Sw.) DC. AGS, BCS, CHIS, CHIH, COAH, COL, GTO, GRO, HGO, JAL, MEX, MICH, MOR, NAY, NLE, OAX, PUE, QRO, QROO, SLP, SIN, SON, TAMS, VER, YUC, ZAC

Prosopis laevigata (Humb. \& Bonpl. ex Willd.) M.C. Johnst. AGS, CHIS, CHIH, COAH, COL, CDMX, DGO, GTO, GRO, HGO, JAL, MEX, MICH, MOR, NAY, NLE, OAX, PUE, QRO, SLP, TAMS, VER, ZAC

*Prosopis palmeri $\mathrm{S}$. Watson BCS

Prosopis pubescens Benth. BCN, CHIH, SON

Prosopis reptans Benth. COAH, NLE, SLP, TAMS, VER

* Prosopis tamaulipana Burkart NLE, SLP, TAMS, VER

Prosopis velutina Wooton $\mathrm{BCN}, \mathrm{BCS}, \mathrm{CHIH}, \mathrm{COAH}, \mathrm{MICH}$, SON

Psoralidium tenuiflorum (Pursh) Rydb. CHIH, NLE, SON

Psorothamnus arborescens (Torr. \& A. Gray) Barneby SON

*Psorothamnus dentatus Rydb. BCN, BCS

Psorothamnus emoryi (A. Gray) Rydb. BCN, BCS, SON

Psorothamnus scoparius (A. Gray) Rydb. CHIH, COAH, DGO,

VER

Psorothamnus schottii (Torr.) Barneby BCN, SON

Psorothamnus spinosus (A. Gray) Barneby BCN, BCS, SON

*Pterocarpus acapulcensis Rose CHIS, GRO, JAL, MICH, NAY, OAX, PUE, TAB, VER

Pterocarpus michelianus N. Zamora GRO, OAX

Pterocarpus officinalis Jacq. CHIS, OAX, VER, YUC

* Pterocarpus orbiculatus DC. COL, GRO, JAL, MEX, MICH, MOR, NAY, OAX, PUE, VER

Pterocarpus rohrii Vahl CHIS, MICH, OAX, QROO, TAB, VER

*Ramirezella calcoma Ochoterena-Booth \& A. Delgado GRO, MEX

* Ramirezella crassa (McVaugh) Ochoterena-Booth \& A. Delgado AGS, COL, JAL, MICH, NAY, SIN, ZAC

*Ramirezella lozanii (Rose) Piper COL, GRO, JAL, MICH, NAY

* Ramirezella micrantha A. Delgado \& Ochoterena-Booth COL, GRO, JAL, NAY, OAX

* Ramirezella nitida Piper SLP, TAMS, VER

Ramirezella occidentalis Rose GRO, JAL

Ramirezella penduliflora A. Delgado \& Ochoterena-Booth CHIS
Ramirezella strobilophora (B.L. Rob.) Rose AGS, CHIS, CHIH, COL, GTO, GRO, JAL, MEX, MICH, NAY, OAX, SIN, SON, VER, ZAC

*Rhynchosia amabilis Grear CHIS, COL, JAL, OAX

Rhynchosia americana (Mill.) Metz CAM, CHIS, COL, DGO, GRO, HGO, MICH, OAX, SLP, SIN, TAB, TAMS, VER

*Rhynchosia caribaea (Jacq.) DC. VER

*Rhynchosia delicatula O. Téllez \& M. Sousa COL, JAL

Rhynchosia difformis (Elliott) DC. COAH, NLE, TAMS

Rhynchosia discolor M. Martens \& Galeotti CHIS, CHIH, COL, DGO, GRO, JAL, MEX, MICH, MOR, NAY, OAX, PUE, SIN, SON, ZAC

Rhynchosia edulis Griseb. CAM, CHIS, CHIH, COL, DGO, GRO, HGO, JAL, MEX, MICH, NAY, OAX, QRO, QROO, SLP, SIN, SON, VER, YUC, ZAC

*Rhynchosia elisae O. Téllez COL, JAL, NAY

Rhynchosia erythrinoides Schltdl. \& Cham. CHIS, OAX, PUE, VER

Rhynchosia latifolia (Nutt.) Torr. \& A. Gray CHIS, SIN

Rhynchosia longeracemosa M. Martens \& Galeotti CAM, CHIS, COAH, GRO, HGO, JAL, MEX, MOR, NLE, OAX, PUE, QRO, QROO, SLP, TAB, TAMS, VER, YUC

*Rhynchosia macrocarpa Benth. AGS, CHIH, DGO, GTO, GRO, HGO, JAL, MICH, NAY, OAX, SLP, SIN, SON, ZAC

Rhynchosia minima (L.) DC. AGS, BCS, CAM, CHIS, CHIH, COAH, COL, DGO, GTO, GRO, HGO, JAL, MEX, MICH, MOR, NAY, NLE, OAX, PUE, QRO, QROO, SLP, SIN, SON, TAB, TAMS, VER, YUC, ZAC

Rhynchosia monticola Grear CHIS

* Rhynchosia nelsonii (Rose) Grear CHIS, GRO, OAX, VER

*Rhynchosia potosina Brandegee SLP, TAMS

Rhynchosia precatoria (Humb. \& Bonpl. ex Willd.) DC. BCN, BCS, CHIS, CHIH, COL, DGO, GRO, JAL, MEX, MICH, MOR, NAY, OAX, SLP, SIN, SON, TAB, VER, ZAC

*Rhynchosia pringlei Rose OAX, PUE

*Rhynchosia prostrata Brandegee CDMX, HGO, MEX, PUE, QRO, SLP

Rhynchosia pyramidalis (Lam.) Urb. BCS, CAM, CHIS, CHIH, COL, DGO, GRO, MEX, MICH, MOR, NAY, OAX, SLP, SIN, SON, TAB, TAMS, VER

Rhynchosia reticulata (Sw.) DC. CAM, CHIS, COL, GRO, HGO, JAL, MICH, NAY, OAX, QROO, SLP, TAB, VER, YUC Rhynchosia senna Gillies ex Hook. \& Arn. AGS, CHIH, COAH, DGO, GTO, GRO, HGO, JAL, MEX, NLE, OAX, PUE, QRO, SLP, SIN, SON, TAMS, ZAC

*Rhynchosia swartzii (Vail) Urb. CAM, QROO, YUC

*Rhynchosia tamaulipensis Grear NLE, TAMS

* Rhynchosia tarphantha Standl. COL, GRO, JAL, MICH, NAY, OAX

*Rhynchosia yucatanensis Grear CAM, QROO, YUC

Robinia neomexicana A. Gray CHIH, SON

Rupertia rigida (Parish) Grimes BCN

Schizolobium parahyba (Vell.) S.F. Blake CHIS, OAX, QROO, TAB, VER

Schnella glabra (Jacq.) Dugand CHIS, COL, OAX, TAB, VER Schnella guianensis (Aubl.) Wunderlin OAX, TAB, VER

Schnella herrerae Britton \& Rose CAM, CHIS, MEX, MOR, 
NAY, OAX, QROO, TAB, VER, YUC

Schnella microstachya Raddi CHIS, OAX, VER

*Schrankia heterocarpa Standl. CHIH

* Senegalia anisophylla (S. Watson) Britton \& Rose CHIS, COAH, DGO, QRO, SLP, TAMS, ZAC

Senegalia aristeguietana (L. Cárdenas) Seigler \& Ebinger CHIS Senegalia berlandieri (Benth.) Britton \& Rose AGS, CHIH, COAH, DGO, GTO, HGO, JAL, MEX, NLE, PUE, QRO, SLP, SIN, TAMS, VER, ZAC

* Senegalia crassifolia (A. Gray) Britton \& Rose COAH, DGO, HGO, QRO, SLP, TAMS, ZAC

Senegalia emoryana (Benth.) Britton \& Rose COAH

Senegalia gaumeri (S.F. Blake) Britton \& Rose CAM, QROO, YUC

Senegalia greggii (A. Gray) Britton \& Rose BCN, BCS, CHIH, COAH, DGO, HGO, NLE, SON, TAMS, ZAC

* Senegalia interior Britton \& Rose AGS, DGO, JAL, SLP, ZAC *Senegalia kelloggiana (A.M. Carter \& Rudd) C.E. Glass \& Seigler BCS

* Senegalia lozanoi Britton \& Rose COAH, NLE

* Senegalia macilenta (Rose) Britton \& Rose COAH, COL, DGO, GRO, JAL, MEX, MICH, MOR, NAY, NLE, OAX, PUE, SIN

* Senegalia micrantha Britton \& Rose GTO, HGO, NLE, QRO, SLP, TAMS, VER

* Senegalia mirandae (L. Rico) Seigler \& Ebinger CHIS, OAX

* Senegalia occidentalis (Rose) Britton \& Rose BCN, BCS, CHIH, HGO, QRO, SLP, SIN, SON, ZAC

* Senegalia painteri Britton \& Rose GTO, HGO, QRO

* Senegalia peninsularis Britton \& Rose BCS

Senegalia picachensis (Brandegee) Britton \& Rose CHIS, CHIH, COL, DGO, GRO, JAL, MEX, MICH, MOR, OAX, PUE

*Senegalia purpusii (Brandegee) Britton \& Rose OAX, PUE

* Senegalia reniformis (Benth.) Britton \& Rose HGO, QRO, SLP

Senegalia riparia (Kunth) Britton CAM, CHIS, COL, DGO, GRO, JAL, MEX, MICH, MOR, NAY, OAX, PUE, QROO, SLP, SIN, TAB, TAMS, VER, YUC, ZAC

Senegalia roemeriana (Scheele) Britton \& Rose BCN, BCS, CHIH, COAH, DGO, HGO, NLE, QRO, SLP, SON, TAMS, VER

* Senegalia sororia (Standl.) Britton \& Rose HGO, QRO, SLP * Senegalia subangulata (Rose) Britton \& Rose GRO, HGO, OAX, PUE, QRO, SLP

*Senegalia subsessilis Britton \& Rose HGO, QRO

Senegalia tenuifolia (L.) Britton \& Rose CHIS, COL, GRO, HGO, JAL, MEX, MICH, NAY, OAX, QRO, SLP, ZAC

Senegalia wrightii (Benth.) Britton \& Rose BCN, BCS, CHIH, COAH, NLE, SON, TAMS

* Senna andrieuxii (Benth.) H.S. Irwin \& Barneby GRO, OAX, PUE

*Senna apiculata (M. Martens \& Galeotti) H.S. Irwin \& Barneby HGO, OAX, PUE, SON, VER

* Senna argentea (Kunth) H.S. Irwin \& Barneby GRO, MEX, MICH, MOR, OAX, PUE

* Senna arida (Rose) H.S. Irwin \& Barneby DGO, GTO, HGO,
JAL, NAY, OAX, PUE, QRO, SLP, ZAC

Senna armata (S. Watson) H.S. Irwin \& Barneby BCN, SON Senna atomaria (L.) H.S. Irwin \& Barneby BCS, CAM, CHIS, CHIH, COL, DGO, GTO, GRO, HGO, JAL, MEX, MICH, MOR, NAY, OAX, PUE, QRO, QROO, SLP, SIN, SON, TAB, TAMS, VER, YUC

Senna bauhinoides (A. Gray) H.S. Irwin \& Barneby AGS, CHIH, COAH, DGO, HGO, JAL, NLE, SLP, SON, TAMS, ZAC

*Senna centranthera H.S. Irwin \& Barneby CHIS, COL, GRO, JAL, MICH, OAX

Senna cobanensis (Britton) H.S. Irwin \& Barneby CHIS, COL, GRO, JAL, MICH, NAY, OAX, PUE, QRO, QROO, SLP, TAB, VER

*Senna confinis (Greene) H.S. Irwin \& Barneby BCN, BCS, SON

Senna covesii (A. Gray) H.S. Irwin \& Barneby BCN, BCS, CHIH, COAH, DGO, NLE, SIN, SON

* Senna crotalarioides (Kunth) H.S. Irwin \& Barneby AGS, DGO, GTO, HGO, JAL, NLE, PUE, QRO, SLP, TAMS, VER, ZAC

* Senna demissa (Rose) H.S. Irwin \& Barneby COAH, HGO, NLE, QRO, TAMS, ZAC

Senna durangensis (Rose) H.S. Irwin \& Barneby CHIH, COAH, DGO, NLE, SLP, TAMS

* Senna foetidissima (G. Don) H.S. Irwin \& Barneby CHIS, COL, GRO, JAL, MEX, MICH, OAX, PUE, SIN, VER

Senna fruticosa (Mill.) H.S. Irwin \& Barneby CAM, CHIS, COL, DGO, GRO, JAL, MEX, MICH, NAY, OAX, PUE, QROO, SLP, SIN, TAB, TAMS, VER, YUC, ZAC

Senna galegifolia (L.) Barneby \& Lourteig CAM, CHIS, CHIH, COL, DGO, GRO, HGO, JAL, MEX, MICH, NAY, OAX, QROO, SIN, SON, VER, YUC

*Senna galeottiana (M. Martens) H.S. Irwin \& Barneby OAX, PUE

Senna guatemalensis (Donn. Sm.) H.S. Irwin \& Barneby CHIS, GTO, HGO, OAX, PUE, QRO, SLP, TAMS, VER

Senna hayesiana (Britton \& Rose) H.S. Irwin \& Barneby CAM, CHIS, OAX, QROO, TAB

Senna hirsuta (L.) H.S. Irwin \& Barneby AGS, CAM, CHIS, CHIH, COL, DGO, GTO, GRO, HGO, JAL, MEX, MICH, MOR, NAY, OAX, PUE, QRO, QROO, SLP, SIN, SON, TAMS, VER, YUC, ZAC

Senna holwayana (Rose) H.S. Irwin \& Barneby CHIS, COL, GRO, MEX, MICH, MOR, OAX, PUE, QROO

*Senna koelziana H.S. Irwin \& Barneby COL, JAL

Senna lindheimeriana (Scheele) H.S. Irwin \& Barneby CHIH, COAH, DGO, GTO, HGO, NLE, QRO, SLP, SON, TAMS, VER, ZAC

* Senna mensicola (H.S. Irwin \& Barneby) H.S. Irwin \& Barneby COAH, GTO, HGO, MEX, NLE, SLP, TAMS, ZAC

Senna mexicana (Jacq.) H.S. Irwin \& Barneby COL, JAL, VER Senna mollissima (Willd.) H.S. Irwin \& Barneby COL, GRO, JAL, MICH, NAY, OAX, SIN, TAB

* Senna monozyx (H.S. Irwin \& Barneby) H.S. Irwin \& Barneby COAH, NLE

*Senna multifoliolata (Paul G. Wilson) H.S. Irwin \& Barneby 
COL, GRO, JAL, MICH, OAX

Senna multiglandulosa (Jacq.) H.S. Irwin \& Barneby BCS, CHIS, CDMX, GTO, GRO, HGO, JAL, MEX, MICH, MOR, OAX, PUE, QRO, SLP, TLAX, VER, ZAC

*Senna multijuga (Rich.) H.S. Irwin \& Barneby CHIS, HGO, MEX, MICH, MOR, OAX, PUE, TAB, VER

Senna nicaraguensis (Benth.) H.S. Irwin \& Barneby CHIS, COL, GRO, HGO, JAL, MEX, MICH, MOR, OAX, SIN, VER Senna obtusifolia (L.) H.S. Irwin \& Barneby BCS, CAM, CHIS, CHIH, COL, GRO, HGO, JAL, MEX, MICH, MOR, NAY, NLE, OAX, QRO, QROO, SLP, SIN, SON, TAB, TAMS, VER, YUC, ZAC

Senna occidentalis (L.) Link AGS, BCS, CAM, CHIS, CHIH, COAH, COL, GRO, HGO, JAL, MEX, MICH, MOR, NAY, NLE, OAX, PUE, QRO, QROO, SLP, SIN, SON, TAB, TAMS, VER, YUC, ZAC

Senna orcuttii (Britton \& Rose) H.S. Irwin \& Barneby COAH Senna oxyphylla (Kunth) H.S. Irwin \& Barneby CAM, CHIS, QROO, SIN, VER

Senna pallida (Vahl) H.S. Irwin \& Barneby AGS, BCS, CAM, CHIS, CHIH, COL, DGO, GRO, HGO, JAL, MEX, MICH, MOR, NAY, OAX, PUE, QROO, SLP, SIN, SON, TAB, TAMS, VER, YUC, ZAC, ZAC

Senna papillosa (Britton \& Rose) H.S. Irwin \& Barneby CAM, CHIS, OAX, PUE, QROO, TAB, VER

Senna pendula (Humb. \& Bonpl. ex Willd.) H.S. Irwin \& Barneby CAM, CHIS, COL, CDMX, GRO, HGO, JAL, MEX, MICH, NAY, OAX, PUE, QRO, SLP, SIN, SON, TAB, TAMS, VER, YUC

Senna pentagonia (Mill.) H.S. Irwin \& Barneby CAM, CHIS, GRO, OAX, QROO

Senna peralteana (Kunth) H.S. Irwin \& Barneby CAM, CHIS, OAX, QROO, TAB, YUC

Senna pilifera (Vogel) H.S. Irwin \& Barneby CHIS, COL, DGO, GRO, JAL, MEX, MICH, NAY, OAX, SLP, SIN, VER, ZAC

Senna pilosior (J.F. Macbr.) H.S. Irwin \& Barneby CHIH, COAH, DGO

Senna polyphylla (Jacq.) H.S. Irwin \& Barneby CAM, GTO, $\mathrm{MICH}, \mathrm{YUC}$

Senna pumilio (A. Gray) H.S. Irwin \& Barneby COAH, DGO, NLE, SLP

*Senna purpusii (Brandegee) H.S. Irwin \& Barneby BCN, BCS Senna quinquangulata (Rich.) H.S. Irwin \& Barneby CHIS, COL, DGO, GRO, JAL, MICH, NAY, OAX, QROO, SIN, TAB, VER

Senna racemosa (Mill.) H.S. Irwin \& Barneby CAM, CHIS, COL, GRO, HGO, JAL, MEX, MICH, MOR, OAX, QRO, QROO, SLP, VER, YUC

Senna reticulata (Willd.) H.S. Irwin \& Barneby CHIS, GRO, JAL, MOR, OAX, QRO, SLP, TAB, VER, YUC

Senna ripleyana (H.S. Irwin \& Barneby) H.S. Irwin \& Barneby CHIH, COAH, NLE, ZAC

Senna roemeriana (Scheele) H.S. Irwin \& Barneby CHIH, COAH, HGO, NLE, QRO, SLP, SON

Senna septemtrionalis (Viv.) H.S. Irwin \& Barneby AGS, CHIS, COAH, COL, CDMX, DGO, GTO, GRO, HGO, JAL, MEX, MICH, NLE, OAX, PUE, QRO, SLP, SIN, TAMS, VER, ZAC
Senna skinneri (Benth.) H.S. Irwin \& Barneby CHIS, COL, GRO, JAL, MEX, MICH, MOR, OAX, PUE

*Senna sousana H.S. Irwin \& Barneby OAX

*Senna talpana H.S. Irwin \& Barneby COL, JAL

Senna tonduzii (Standl.) H.S. Irwin \& Barneby CHIS

Senna undulata (Benth.) H.S. Irwin \& Barneby CAM, CHIS, GRO, MICH, QROO, TAB, YUC

Senna uniflora (Mill.) H.S. Irwin \& Barneby AGS, CAM, CHIS, COAH, COL, GRO, HGO, JAL, MEX, MICH, MOR, NAY, NLE, OAX, PUE, QRO, QROO, SLP, SIN, SON, TAMS, VER, YUC, ZAC

* Senna unijuga (Rose) H.S. Irwin \& Barneby OAX, PUE, VER * Senna villosa (Mill.) H.S. Irwin \& Barneby BCS, CAM, CHIS, COL, GRO, JAL, NAY, OAX, QROO, VER, YUC

Senna wislizenii (A. Gray) H.S. Irwin \& Barneby CHIH, COAH, COL, DGO, GTO, GRO, HGO, JAL, MEX, MICH, MOR, NAY, NLE, OAX, PUE, QRO, SLP, SON, TAMS, VER, ZAC

*Senna $\times$ floribunda (Cav.) H.S. Irwin \& Barneby GTO, HGO, MICH, OAX, PUE, SLP, VER

Sesbania drummondii (Rydb.) Cory TAMS

Sesbania herbacea (Mill.) McVaugh BCN, BCS, CAM, CHIS, CHIH, COL, GTO, GRO, JAL, MEX, MICH, MOR, NAY, OAX, QROO, SLP, SIN, SON, TAB, TAMS, VER, YUC, ZAC Sesbania longifolia DC. GTO, JAL, MICH, QRO, SLP, TAMS Sesbania macrocarpa Muhl. BCN, BCS, CAM, COAH, GRO, MICH, MOR, NAY, QROO, SIN, SON, TAMS, VER, YUC Sesbania virgata (Cav.) Pers. VER

Sigmoidotropis speciosa (Kunth) A. Delgado BCS, CAM, CHIS, COL, GRO, JAL, MEX, MICH, NAY, NLE, OAX, PUE, QRO, SLP, SIN, SON, TAB, TAMS, VER, ZAC

Sophora nuttalliana B.L. Turner CHIH, COAH, NLE, SLP, SON

Sophora tomentosa L. BCS, CAM, COAH, COL, QROO, TAMS, VER, YUC

Sphinctospermum constrictum (S. Watson) Rose BCS, COL, GRO, JAL, MEX, MICH, NAY, OAX, SIN, SON, ZAC

*Sphinga acatlensis (Benth.) Barneby \& J.W. Grimes COL, DGO, GRO, JAL, MEX, MICH, MOR, NAY, OAX, PUE, SIN, ZAC

Sphinga platyloba (DC.) Barneby \& J.W. Grimes CAM, CHIS, COL, GRO, JAL, OAX, QROO, TAB, VER, YUC

Stenolobium brachycarpum Benth. CAM, CHIS, COL, GRO, JAL, MEX, MICH, NAY, OAX, PUE, QRO, SLP, SIN, TAB, VER, ZAC

Strophostyles leiosperma (Torr. \& A. Gray) Piper ND

Strophostyles umbellata (Willd.) Britton COAH

Stylosanthes guianensis (Aubl.) Sw. CHIS, COL, GRO, JAL, MICH, NAY, OAX, PUE, SON, TAB, VER, ZAC

Stylosanthes hamata (L.) Taub. CAM, CHIS, OAX, QROO, TAB, YUC

Stylosanthes humilis Kunth CAM, CHIS, COL, GTO, GRO, HGO, JAL, MEX, MICH, MOR, NAY, OAX, QRO, QROO, SLP, SIN, SON, TAB, TAMS, VER, YUC

* Stylosanthes macrocarpa S.F. Blake OAX, PUE

*Stylosanthes mexicana Taub. GTO, MICH, NLE, QRO, SLP, TAMS

*Stylosanthes quintanarooensis S. Gama \& Dávila QROO 
Stylosanthes subsericea S.F. Blake NAY, OAX

Stylosanthes viscosa (L.) Sw. BCS, CHIS, COL, DGO, GRO, JAL, NAY, NLE, OAX, PUE, SIN, SON, TAB, TAMS, VER, ZAC

*Styphnolobium burseroides M. Sousa, Rudd \& Medrano MOR, OAX, PUE

*Styphnolobium conzattii (Standl.) M. Sousa \& Rudd OAX

*Styphnolobium parviflorum M. Sousa \& Rudd VER

*Styphnolobium protantherum M. Sousa \& Rudd JAL Styphnolobium sporadicum M. Sousa \& Rudd CHIS, OAX Swartzia cubensis (Britton \& P. Wilson) Standl. CAM, CHIS, QROO, TAB, VER, YUC

Swartzia guatemalensis (Donn. Sm.) Pittier CHIS, OAX, TAB, VER

Swartzia mexicana M. Sousa \& R. Grether OAX

Swartzia myrtifolia Sm. CHIS, OAX, TAB, VER

Swartzia simplex (Sw.) Spreng. CHIS, COL, GRO, JAL, MICH, NAY, OAX, TAB

*Tephrosia abbottiae C.E. Wood GRO

Tephrosia adunca Benth. TAB

Tephrosia belizensis Lundell CHIS, OAX, PUE, TAB, VER

*Tephrosia cana Brandegee BCN, BCS

*Tephrosia carrollii O. Téllez GRO, NAY, OAX

Tephrosia cinerea (L.) Pers. CAM, CHIS, CHIH, GRO, JAL, MEX, MICH, MOR, OAX, QROO, TAB, TAMS, VER, YUC

*Tephrosia conzattii (Rydb.) Standl. COL, GRO, JAL, MEX, $\mathrm{MICH}, \mathrm{NAY}, \mathrm{OAX}$

*Tephrosia crassifolia Benth. CHIS, GRO, JAL, MICH, NAY, OAX, SIN

*Tephrosia cuernavacana (Rose) J.F. Macbr. GRO, JAL, MEX, MICH, MOR

*Tephrosia diversifolia (Rose) J.F. Macbr. JAL, MICH

*Tephrosia foliolosa (Rydb.) L. Riley AGS, CHIH, DGO, SIN, TAMS

*Tephrosia hypoleuca L. Riley DGO, NAY, SIN

Tephrosia lanata M. Martens \& Galeotti CHIS, GRO, NAY, OAX, VER

*Tephrosia langlassei Micheli CHIS, COL, GRO, JAL, MICH, NAY, OAX, VER

Tephrosia leiocarpa A. Gray CHIH, COL, JAL, NAY, OAX, SIN, SON, ZAC

*Tephrosia leucantha Kunth AGS, CHIH, DGO, GTO, HGO, JAL, MICH, NAY, QRO, SLP, SIN, SON, TAMS, ZAC

Tephrosia lindheimeri A. Gray TAMS

*Tephrosia macrantha B.L. Rob. \& Greenm. ex Pringle COL, GRO, JAL, MEX, MICH, MOR, NAY, OAX, TLAX, ZAC

*Tephrosia madrensis Seem. DGO, JAL, NAY, ZAC

*Tephrosia major Micheli COL, GRO, JAL, MICH

*Tephrosia mexicana C.E. Wood GRO, MEX, MICH

*Tephrosia microcarpa O. Téllez JAL, NAY

Tephrosia multifolia Rose CHIS, CHIH, COL, GRO, JAL, MICH, NAY, OAX, PUE, SIN, SON, VER

Tephrosia nicaraguensis Oerst. AGS, CHIS, CHIH, COL, DGO, GTO, GRO, JAL, MEX, MICH, MOR, NAY, OAX, PUE, QRO, SIN, SON, VER, ZAC

Tephrosia nitens Benth. ex Seem. CHIS, GRO, MICH, NAY, OAX, TAB, VER
*Tephrosia pachypoda L. Riley JAL, NAY, SIN

*Tephrosia palmeri S. Watson BCN, BCS, SIN, SON

*Tephrosia platyphylla (Rose) Standl. JAL

*Tephrosia pogonocalyx C.E. Wood GRO, MEX

Tephrosia potosina Brandegee COAH, NLE, SLP, TAMS

*Tephrosia pringlei (Rose) J.F. Macbr. GRO, OAX

Tephrosia purpurea (L.) Pers. BCS, CHIH, COAH, DGO, HGO,

JAL, NLE, OAX, SIN, SON, VER

*Tephrosia quercetorum C.E. Wood GRO, JAL, OAX

Tephrosia rhodantha Brandegee CHIS, COL, GRO, JAL, MEX, MICH, NAY, OAX, SIN, SON, TAB, VER

*Tephrosia saxicola C.E. Wood JAL, SIN, SLP, SON, ZAC

*Tephrosia seemannii (Britten \& Baker f.) K. Schum. DGO, NAY, SIN

Tephrosia senna Kunth YUC

*Tephrosia simulans C.E. Wood JAL, MEX, SIN

*Tephrosia sousae O. Téllez DGO, JAL, NAY

*Tephrosia submontana (Rose) L. Riley COL, JAL, NAY, SIN

*Tephrosia tepicana Standl. JAL, NAY, SIN, ZAC

Tephrosia thurberi (Rydb.) Standl. CHIH, SIN, SON

*Tephrosia tuitoensis O. Téllez JAL

*Tephrosia vernicosa C.E. Wood GRO, JAL, MICH

Tephrosia vicioides Schltdl. BCN, BCS, CHIS, CHIH, COAH, COL, DGO, GRO, HGO, JAL, MEX, MICH, MOR, NAY, OAX, PUE, QRO, SLP, SIN, SON, TAB, TAMS, VER

*Tephrosia viridiflora O. Téllez COL, JAL, NAY

*Tephrosia watsoniana (Standl.) J.F. Macbr. GRO, JAL, NAY, ZAC

Teramnus labialis (L. f.) Spreng. CHIS, MICH, MOR, NAY, OAX, TAB, VER

Teramnus uncinatus (L.) Sw. CHIS, COL, GRO, JAL, MEX, MICH, MOR, NAY, NLE, OAX, PUE, QRO, SLP, SIN, TAB, TAMS, VER, YUC

Trifolium albopurpureum Torr. \& A. Gray BCN

Trifolium amabile Kunth AGS, CHIS, CHIH, COAH, COL, CDMX, DGO, GTO, GRO, HGO, JAL, MEX, MICH, MOR, NAY, NLE, OAX, PUE, QRO, SLP, SIN, SON, TAMS, TLAX, VER, ZAC

Trifolium amplectens Torr. \& A. Gray BCN

Trifolium depauperatum Desv. BCN

Trifolium gracilentum Torr. \& A. Gray BCN

Trifolium microcephalum Pursh BCN, BCS

Trifolium palmeri $\mathrm{S}$. Watson $\mathrm{BCN}$

Trifolium pinetorum Greene CHIH, SON

Trifolium rusbyi Greenm. DGO

*Trifolium schneideri Standl. NLE

Trifolium tridentatum Lindl. BCN

Trifolium variegatum Nutt. ex Torr. \& A. Gray BCN, SON

*Trifolium wigginsii J.M. Gillet BCN

Trifolium wormskioldii Lehm. BCN, BCS, CHIH, COAH, CDMX, DGO, GTO, HGO, JAL, MEX, MICH, MOR, NLE, PUE, QRO, SLP, SON, TLAX, VER, ZAC

*Vachellia biaciculata (S. Watson) Seigler \& Ebinger CHIH

*Vachellia bilimekii (J.F. Macbr.) Seigler \& Ebinger GRO, MEX, MOR, OAX, PUE

*Vachellia brandegeana (I.M. Johnst.) Seigler \& Ebinger BCS Vachellia bravoensis (Isely) Seigler \& Ebinger AGS, CHIH, 
COAH, COL, DGO, HGO, JAL, NLE, SLP, TAMS, VER, ZAC *Vachellia californica (Brandegee) Seigler \& Ebinger BCS, CAM, CHIS, CHIH, QROO, SON, YUC

Vachellia campechiana (Mill.) Seigler \& Ebinger AGS, BCN, BCS, CAM, CHIS, CHIH, COAH, COL, DGO, GRO, JAL, MEX, MICH, MOR, NAY, OAX, PUE, QROO, SLP, SIN, SON, TAMS, VER, YUC, ZAC

Vachellia collinsii (Saff.) Seigler \& Ebinger CAM, CHIS, GRO, MICH, NAY, OAX, QROO, TAB, VER, YUC

Vachellia constricta (Benth.) Seigler \& Ebinger AGS, BCN, BCS, CHIH, COAH, DGO, GTO, HGO, JAL, NLE, OAX, PUE, QRO, SLP, SON, TAMS, ZAC

Vachellia cookii (Saff.) Seigler \& Ebinger CHIS, TAB

Vachellia cornigera (L.) Seigler \& Ebinger CAM, CHIS, COL, GRO, HGO, JAL, MOR, OAX, PUE, QRO, QROO, SLP, SIN, TAB, TAMS, VER, YUC, ZAC

Vachellia chiapensis (Saff.) Seigler \& Ebinger CAM, CHIS, OAX, QROO, TAB, VER

Vachellia farnesiana $(\mathrm{L}$.) Wight \& Arn. AGS, BCN, BCS, CAM, CHIS, CHIH, COAH, COL, DGO, GTO, GRO, HGO, JAL, MEX, MICH, MOR, NAY, NLE, OAX, PUE, QRO, QROO, SLP, SIN, SON, TAB, TAMS, VER, YUC, ZAC

Vachellia gentlei (Standl.) Seigler \& Ebinger CAM, CHIS, QROO, TAB, YUC

*Vachellia glandulifera (S. Watson) Seigler \& Ebinger CHIH, COAH, DGO, NLE, ZAC

Vachellia globulifera (Saff.) Seigler \& Ebinger CAM, CHIS, OAX, QROO, VER, YUC

Vachellia guanacastensis (H.D. Clarke, Seigler \& Ebinger) Seigler \& Ebinger OAX, VER

Vachellia hindsii (Benth.) Seigler \& Ebinger CHIS, COL, DGO, GRO, JAL, MEX, MICH, NAY, OAX, PUE, SIN, YUC

*Vachellia janzenii (Ebinger \& Seigler) Seigler \& Ebinger CHIS, TAB

Vachellia macracantha (Humb. \& Bonpl. ex Willd.) Seigler \& Ebinger CAM, CHIS, COL, GRO, JAL, MEX, MICH, NAY, OAX, PUE, QROO, SIN, VER, YUC

Vachellia mayana (Lundell) Seigler \& Ebinger CHIS, OAX, TAB, VER

*Vachellia pacensis (Rudd \& A.M. Carter) Seigler \& Ebinger BCS, SON

Vachellia pennatula (Schltdl. \& Cham.) Seigler \& Ebinger AGS, CAM, CHIS, CHIH, COL, DGO, GTO, GRO, HGO, JAL, MEX, MICH, MOR, NAY, OAX, PUE, QRO, QROO, SLP, SIN, SON, TAMS, VER, YUC, ZAC

*Vachellia pringlei (Rose) Seigler \& Ebinger BCS, CHIS, GRO, HGO, MICH, OAX, PUE, QRO, QROO, SLP, SON, TAMS, VER, YUC

Vachellia rigidula (Benth.) Seigler \& Ebinger $\mathrm{CHIH}$, COAH, GTO, GRO, HGO, JAL, MEX, MICH, MOR, NAY, NLE, QRO, SLP, SON, TAMS, VER, ZAC

Vachellia schaffneri (S. Watson) Seigler \& Ebinger AGS, BCN, BCS, CHIS, CHIH, COAH, COL, CDMX, DGO, GTO, HGO, JAL, MEX, MICH, NLE, OAX, PUE, QRO, SLP, SON, TAMS, TLAX, VER, ZAC

*Vachellia sphaerocephala (Cham. \& Schltdl.) Seigler \& Ebinger COL, GRO, MICH, OAX, SLP, TAB, TAMS, VER,
YUC

Vachellia vernicosa (Britton \& Rose) Seigler \& Ebinger CHIH, COAH, DGO, HGO, JAL, NAY, NLE, QRO, SLP, SON, TAMS, ZAC

*Vachellia villaregalis (McVaugh) Seigler \& Ebinger JAL, NAY

*Vachellia $\times$ cedilloi (L. Rico) Seigler \& Ebinger QROO, YUC Vachellia $\times$ standleyi (Saff.) Seigler \& Ebinger CHIS, COL, GRO, JAL, NAY, OAX

*Vachellia $\times$ ziggyi Seigler \& Ebinger OAX

Vatairea lundellii (Standl.) Killip ex Record CAM, CHIS, OAX, QROO, TAB, VER

Vicia americana Muhl. ex Willd. BCN, COAH, CDMX, HGO, MEX, MICH, NLE, OAX, QRO, SLP, SON, VER

Vicia exigua Nutt. ex Torr. \& A. Gray BCN, CHIH, COAH, SON

Vicia hassei S. Watson BCN

Vicia humilis Kunth CHIS, COAH, COL, CDMX, GTO, HGO, JAL, MEX, MICH, MOR, NLE, OAX, PUE, QRO, SLP, SIN, TAMS, TLAX, VER

Vicia leucophaea Greene CHIH, DGO, SON

Vicia ludoviciana Nutt. ex Torr. \& A. Gray BCN, BCS, CHIH, COAH, DGO, GTO, HGO, MEX, NLE, PUE, QRO, SON, TAMS, VER

Vicia mullerana B.L. Turner COAH, NLE

Vicia pulchella Kunth AGS, CHIS, CHIH, COAH, COL, CDMX, DGO, GTO, GRO, HGO, JAL, MEX, MICH, MOR, NLE, OAX, PUE, SLP, SIN, SON, TAMS, TLAX, VER, ZAC Vicia sessei G. Don COL, CDMX, HGO, JAL, MEX, MICH, MOR, OAX, PUE, TLAX

*Vigna angularis (Willd.) Ohwi \& Ohashi TAB

Vigna candida (Vell.) Maréchal, Mascherpa \& Stainier CAM, QROO, YUC

Vigna caracalla (L.) Verdc. CHIS, CHIH, DGO, OAX, SON, VER

*Vigna elegans (Piper) Maréchal, Mascherpa \& Stainier CAM, QROO

Vigna lasiocarpa (Mart. ex Benth.) Verdc. OAX, TAB

*Vigna lonchophylla Piper JAL, MICH

Vigna longifolia (Benth.) Verdc. CHIS, TAB

Vigna luteola (Jacq.) Benth. AGS, CAM, CHIS, COL, GRO, JAL, MEX, MOR, OAX, PUE, QROO, SLP, TAB, TAMS, VER, YUC

*Vigna mungo (L.) Hepper TAB

Vigna peduncularis (Kunth) Fawc. \& Rendle CHIS, GRO, MICH, NAY, OAX, SIN, TAB

Vigna trichocarpa (C. Wright ex Sauvalle) A. Delgado TAB

*Vigna umbellata (Thunb.) Ohwi \& Ohashi CAM, CHIS, OAX, TAB, VER

Vigna vexillata (L.) A. Rich. CAM, CHIS, GRO, HGO, JAL, MICH, MOR, NAY, OAX, QROO, TAB, TAMS, VER, YUC

*Zapoteca alinae H.M. Hern. GRO, OAX

*Zapoteca balsasensis H.M. Hern. GRO

*Zapoteca cruzii H.M. Hern. GRO

Zapoteca formosa (Kunth) H.M. Hern. BCN, BCS, CAM, CHIS, CHIH, COL, DGO, GTO, GRO, HGO, JAL, MEX, MICH, MOR, NAY, OAX, PUE, QRO, QROO, SLP, SIN, SON, TAB, TAMS, VER, YUC, ZAC 
*Zapoteca lambertiana (G. Don) H.M. Hern. CHIS, OAX, SLP, TAMS, VER

Zapoteca media (M. Martens \& Galeotti) H.M. Hern. AGS, CHIH, COAH, DGO, GTO, HGO, JAL, NLE, OAX, PUE, QRO, SLP, SIN, TAMS, VER, ZAC

Zapoteca portoricensis (Jacq.) H.M. Hern. CHIS, COL, GTO, GRO, HGO, JAL, MEX, OAX, PUE, QRO, SLP, SIN, TAB, TAMS, VER, YUC, ZAC

*Zapoteca quichoi H.M. Hern. \& Hanan-Alipi TAB

Zapoteca ravenii H.M. Hern. CHIS

*Zapoteca sousae H.M. Hern. \& A. Campos COL, OAX

*Zapoteca tehuana H.M. Hern. OAX

Zapoteca tetragona (Willd.) H.M. Hern. CHIS, COL, GRO, JAL, MEX, MICH, MOR, NAY, OAX, PUE, SIN, TAB, TAMS, VER

Zornia diphylla (L.) Pers. AGS, CHIS, CHIH, DGO, GRO, JAL, MEX, MICH, MOR, OAX, PUE, QRO, SLP, SIN, SON, TAB, TAMS, VER, ZAC

*Zornia gemella (Willd.) Vogel CHIH, TAMS

*Zornia laevis Schltdl. \& Cham. JAL, MOR, VER

Zornia lasiocarpa A. Molina CHIS, SIN, TAMS

*Zornia megistocarpa Mohlenbr. OAX

Zornia reticulata Sm. AGS, BCS, CHIS, CHIH, COAH, COL, GTO, GRO, JAL, MEX, MICH, MOR, NAY, NLE, OAX, PUE, QRO, SLP, SIN, SON, TAMS, VER, ZAC

*Zornia sinaloensis Mohlenbr. DGO, SIN

Zornia thymifolia Kunth AGS, CHIS, CHIH, COL, CDMX, DGO, GTO, GRO, HGO, JAL, MEX, MICH, MOR, NAY, OAX, PUE, QRO, SLP, TAMS, TLAX, VER, ZAC

*Zornia venosa Mohlenbr. CHIH, DGO, JAL, SON

Zygia cognata (Schltdl. \& Cham.) Britton \& Rose CAM, CHIS OAX, QROO, TAB, VER, YUC

Zygia conzattii (Standl.) Britton \& Rose CAM, CHIS, GRO, OAX, QROO, TAB, VER, YUC

Zygia inaequalis (Humb. \& Bonpl. ex Willd.) Pittier TAB

Zygia latifolia (L.) Fawc. \& Rendle TAB, VER

Zygia longifolia (Humb. \& Bonpl. ex Willd.) Britton \& Rose CHIS, OAX, TAB, VER

Zygia paucijugata (Lundell) L. Rico OAX, TAB, VER

Zygia peckii (B.L. Rob.) Britton \& Rose CHIS, TAB, VER

Zygia stevensonii (Standl.) Record CAM, OAX, QROO, TAB, YUC

*Zygia turneri (McVaugh) Barneby \& J.W. Grimes COL, JAL, $\mathrm{MICH}$

Zygia unifoliolata (Benth.) Pittier CHIS, OAX, TAB, VER

\section{Family Fagaceae}

Fagus grandifolia Ehrenb. HGO, PUE, SLP, TAMS, VER

Quercus acutifolia Née CHIS, COAH, COL, CDMX, DGO, GTO, GRO, HGO, JAL, MEX, MICH, MOR, NAY, NLE, OAX, PUE, QRO, SLP, SIN, TAMS, TLAX, VER, ZAC

*Quercus acherdophylla Trel. HGO, OAX, PUE, VER

*Quercus affinis Scheidw. CHIS, COAH, CDMX, GTO, GRO, HGO, JAL, MEX, MICH, MOR, NLE, OAX, PUE, QRO, SLP, TAMS, VER

Quercus agrifolia Née BCN

Quercus ajoensis C.H. Müll. BCN, SON
*Quercus albifolia C.H. Müll. CHIH, SON

*Quercus albocincta Trel. BCS, CHIH, DGO, SIN, SON

*Quercus alpescens Trel. HGO, NLE, QRO, VER

Quercus anglohondurensis C.H. Müll. CHIS

* Quercus aristata Hook. \& Arn. AGS, COL, DGO, GTO, GRO,

JAL, MEX, NAY, OAX, QRO, SIN, SON, VER, ZAC

Quercus arizonica Sarg. BCS, CHIH, COAH, DGO, NAY, NLE, SIN, SON, TAMS

*Quercus barrancana Spellenb. CHIH, SON

Quercus benthamii A. DC. CHIS, CHIH, COAH, GRO, OAX

*Quercus bonplandiana Sweet HGO, VER

*Quercus brandegeei Goldman BCS

*Quercus canbyi Trel. CHIH, COAH, NLE, SLP, TAMS

Quercus candicans Née CHIS, CHIH, COL, CDMX, DGO, GTO, GRO, HGO, JAL, MEX, MICH, MOR, NAY, OAX, PUE, QRO, SLP, SIN, SON, TAMS, TLAX, VER, ZAC

*Quercus carmenensis C.H. Müll. COAH

Quercus castanea Née AGS, CHIS, CHIH, COAH, COL, CDMX, DGO, GTO, GRO, HGO, JAL, MEX, MICH, MOR, NAY, NLE, OAX, PUE, QRO, SLP, SIN, SON, TAMS, TLAX, VER, ZAC

*Quercus cedrosensis C.H. Müll. BCN

*Quercus clivicola Trel. \& C.H. Müll. NLE, TAMS

*Quercus coahuilensis Nixon \& C.H. Müll. CHIH, COAH

*Quercus coccolobifolia Trel. AGS, CHIH, COAH, DGO, GTO, JAL, NAY, NLE, OAX, SLP, SIN, SON, ZAC

*Quercus convallata Trel. DGO, JAL, NAY, ZAC

*Quercus conzattii Trel. DGO, GRO, JAL, NAY, OAX, ZAC

*Quercus cordifolia Trel. COAH, DGO, GTO, NLE, SLP, ZAC Quercus cornelius-mulleri Nixon \& K.P. Steele BCN

Quercus corrugata Hook. CHIS, GRO, HGO, JAL, OAX, PUE, SLP, VER

Quercus cortesii Liebm. CHIS, COL, GRO, JAL, OAX, PUE, VER

Quercus crassifolia Bonpl. AGS, CHIS, CHIH, COAH, COL, CDMX, DGO, GTO, GRO, HGO, JAL, MEX, MICH, MOR, NAY, NLE, OAX, PUE, QRO, SLP, SIN, SON, TAMS, TLAX, VER, ZAC

*Quercus crassipes Bonpl. AGS, CHIS, COL, COAH, CDMX, DGO, GTO, GRO, HGO, JAL, MEX, MICH, MOR, NAY, NLE, OAX, PUE, QRO, SLP, TAMS, TLAX, VER, ZAC

Quercus crispifolia Trel. CHIS, OAX

Quercus crispipilis Trel. CHIS

*Quercus cualensis L.M. González JAL

*Quercus cupreata Trel. \& C.H. Müll. NLE, TAMS

*Quercus charcasana Trel. ex A. Camus SLP, ZAC

*Quercus chartacea Trel. CHIS, OAX

Quercus chesosensis (Sarg.) C.H. Müll. CHIH, COAH

*Quercus chihuahuensis Trel. AGS, CHIH, DGO, GTO, HGO,

JAL, NAY, NLE, QRO, SLP, SIN, SON, ZAC

Quercus chrysolepis Liebm. BCN, CHIH, SON

*Quercus chuhuichupensis C.H. Müll. CHIH, SON

*Quercus delgadoana Valencia-Avalos, Nixon \& L.M. Kelly CHIS, HGO, OAX, PUE, QRO, SLP, TAB, VER

*Quercus deliquescens C.H. Müll. CHIH

*Quercus depressa Bonpl. CDMX, HGO, MEX, NLE, OAX, PUE, TAMS, VER 
Quercus depressipes Trel. CHIH, DGO, GTO, HGO, JAL, NLE, QRO, SLP, SIN, SON, TAMS, ZAC

*Quercus deserticola Trel. AGS, CHIS, CHIH, COL, CDMX, DGO, GTO, GRO, HGO, JAL, MEX, MICH, MOR, OAX, PUE, QRO, SLP, SIN, SON, TAMS, TLAX, VER, ZAC

*Quercus devia Goldman BCS

*Quercus diversifolia Née COAH, HGO, MEX, MOR, NLE, OAX, SLP, TAMS, VER

Quercus dumosa Nutt. BCN, CHIH, JAL, MICH

*Quercus duratifolia C.H. Müll. CHIS

*Quercus durifolia Seemen CHIH, COAH, DGO, NLE, OAX, QRO, SLP, SIN, SON, TAMS, ZAC

*Quercus eduardi Trel. AGS, CHIH, COAH, DGO, GTO, HGO,

JAL, MEX, MICH, NAY, NLE, OAX, PUE, QRO, SLP, SIN,

TAMS, VER, ZAC

*Quercus edwardsiae C.H. Müll. NLE

Quercus elliptica Née CHIS, COL, DGO, GRO, HGO, JAL,

MEX, MICH, NAY, OAX, PUE, QRO, SLP, SIN, VER

Quercus emoryi Torr. BCN, CHIH, COAH, DGO, JAL, NLE,

SLP, SON, ZAC

Quercus engelmannii Greene BCN

*Quercus filiformis C.H. Müll. COAH

*Quercus frutex Trel. CHIH, CDMX, GTO, HGO, JAL, MEX, MICH, OAX, PUE, QRO, SLP, SON, TLAX, VER, ZAC

*Quercus fulva Liebm. AGS, CHIH, COAH, DGO, GTO, JAL, MICH, NAY, NLE, OAX, QRO, SLP, SIN, SON, ZAC

*Quercus furfuracea Liebm. MICH, OAX, PUE, QRO, SLP, TAMS

Quercus fusiformis Small COAH, NLE, TAMS, VER

*Quercus galeanensis C.H. Müll. COAH, NLE, SLP, TAMS

Quercus gambelii Nutt. CHIH, COAH, NLE, SON, VER

*Quercus gentryi C.H. Müll. AGS, CHIH, COL, DGO, GTO,

JAL, MICH, NAY, QRO, SIN, ZAC

*Quercus germana Schltdl. \& Cham. COAH, GTO, HGO, JAL, NLE, OAX, PUE, QRO, SLP, TAMS, VER

*Quercus ghiesbreghtii M. Martens \& Galeotti CHIS, VER

Quercus glabrescens Benth. CHIS, CDMX, GTO, GRO, HGO,

JAL, MEX, MICH, MOR, OAX, PUE, QRO, SLP, SIN, TLAX,

VER, ZAC

*Quercus glaucescens Bonpl. COL, GRO, JAL, MEX, MICH,

NAY, OAX, QRO, SLP, SIN, TAMS, VER

Quercus glaucoides M. Martens \& Galeotti CHIS, CHIH, COAH, COL, CDMX, DGO, GTO, GRO, HGO, JAL, MEX, MICH, MOR, NLE, OAX, PUE, QRO, SLP, SIN, SON, TAMS, TLAX, VER

*Quercus graciliramis C.H. Müll. COAH, NLE

*Quercus grahamii Benth. GRO, HGO, JAL, MEX, MICH, OAX, PUE

Quercus gravesii Sudw. CHIH, COAH, COL, JAL, NLE

*Quercus greggii (A. DC.) Trel. COAH, DGO, HGO, JAL, MEX, MICH, NAY, NLE, OAX, PUE, QRO, SLP, TAMS, TLAX, VER

Quercus grisea Liebm. AGS, CHIH, COAH, DGO, GTO, HGO,

JAL, NLE, OAX, QRO, SLP, SIN, SON, TAMS, VER, ZAC

Quercus hinckleyii C.H. Müll. CHIH

*Quercus hintonii E.F. Warb. GRO, MEX, MICH

*Quercus hintoniorum Nixon \& C.H. Müll. COAH, NLE, SLP,
TAMS

*Quercus hirtifolia M.L. Vázquez, Valencia-Avalos \& Nixon HGO, PUE, VER

Quercus hypoleucoides A. Camus CHIH, COAH, DGO, MEX, SIN, SON, ZAC

*Quercus hypoxantha Trel. CHIH, COAH, GTO, NLE, QRO, SLP

*Quercus ignaciensis C.H. Müll. SON

*Quercus iltisii L.M. González COL, JAL

Quercus insignis M. Martens \& Galeotti CHIS, COL, GTO,

GRO, HGO, JAL, MEX, MICH, NAY, OAX, PUE, VER

Quercus intricata Trel. CHIH, COAH, DGO, NLE, OAX, SLP,

TAMS, ZAC

*Quercus invaginata Trel. COAH, NLE, SLP

Quercus jaralensis Trel. CHIH, COAH, DGO, GTO, SLP

*Quercus juergensenii Liebm. OAX

Quercus kelloggii Newb. BCN

*Quercus laceyi Small COAH, NLE, SLP, TAMS

*Quercus laeta Liebm. AGS, CHIH, COAH, COL, CDMX, DGO, GTO, GRO, HGO, JAL, MEX, MICH, MOR, NAY, NLE, OAX, PUE, QRO, SLP, SIN, SON, TAMS, TLAX, VER, ZAC

Quercus lancifolia Schltdl. \& Cham. CHIS, COL, HGO, JAL, MEX, NAY, OAX, PUE, SLP, TAMS, VER

Quercus laurina Bonpl. AGS, CHIS, COAH, COL, CDMX, DGO, GTO, GRO, HGO, JAL, MEX, MICH, MOR, NAY, NLE, OAX, PUE, QRO, SLP, SIN, TAMS, TLAX, VER, ZAC *Quercus laxa Liebm. COL, CDMX, DGO, GTO, HGO, JAL, MEX, MICH, NAY, SLP, SIN

*Quercus liebmannii Oerst. ex Trel. GRO, MEX, MICH, OAX, PUE

*Quercus macdougallii Martínez OAX

*Quercus macvaughii Spellenb. CHIH, DGO, JAL, SLP, SIN, SON

Quercus magnoliifolia Née AGS, BCS, CHIS, COAH, COL, CDMX, DGO, GTO, GRO, HGO, JAL, MEX, MICH, MOR, NAY, OAX, PUE, QRO, SLP, SIN, TAMS, VER, ZAC

*Quercus martinezii C.H. Müll. AGS, CHIS, COL, GTO, GRO, JAL, MEX, MICH, NAY, OAX, QRO, SLP

*Quercus mexicana Bonpl. CHIS, COAH, CDMX, GTO, HGO, JAL, MEX, MICH, MOR, NAY, NLE, OAX, PUE, QRO, SLP, SIN, TAMS, TLAX, VER

*Quercus microlepis Trel. \& C.H. Müll. COAH, NLE, OAX, TAMS

*Quercus microphylla Née AGS, CHIH, COAH, CDMX, DGO, GTO, HGO, JAL, MEX, NAY, NLE, OAX, PUE, QRO, SLP, SIN, TAMS, TLAX, VER, ZAC

*Quercus miquihuanensis Nixon \& C.H. Müll. NLE, TAMS

Quercus mohriana Buckley ex Rydb. CHIH, COAH, NLE

${ }^{*}$ Quercus monserratensis C.H. Müll. CHIS

Quercus muehlenbergii Engelm. CHIH, COAH, NLE, SON, TAMS

*Quercus mulleri Martínez OAX

*Quercus nixoniana Valencia-Avalos \& Lozada-Pérez COL, GRO, JAL, OAX

Quercus oblongifolia Torr. BCN, BCS, CHIH, COAH, DGO, SIN, SON, ZAC 
*Quercus obtusata Bonpl. AGS, CHIS, COL, CDMX, DGO, GTO, GRO, HGO, JAL, MEX, MICH, MOR, NAY, NLE, OAX, PUE, QRO, SLP, SIN, TAMS, TLAX, VER, ZAC

Quercus oleoides Schltdl. \& Cham. CHIS, HGO, NLE, OAX, PUE, QRO, SLP, TAB, TAMS, VER, YUC

*Quercus opaca Trel. HGO, NLE, SLP, TAMS, VER

Quercus palmeri Engelm. BCN, BCS, CHIH, SON

*Quercus paxtalensis C.H. Müll. CHIS, GRO, HGO, OAX, PUE, TAMS, VER

Quercus peduncularis Née CHIS, COL, GTO, GRO, HGO, JAL, MEX, MICH, NAY, OAX, PUE, QRO, SLP, SIN, VER, ZAC

*Quercus peninsularis Trel. BCN

*Quercus perpallida Trel. CHIH, SON

Quercus pilarius Trel. CHIS, VER

*Quercus pinnativenulosa C.H. Müll. COAH, HGO, NLE, OAX, QRO, SLP, TAMS, VER

*Quercus planipocula Trel. COL, GRO, JAL, MEX, MICH, NAY, OAX, SLP, SIN, TAMS

*Quercus poculifer Trel. OAX

Quercus polymorpha Schltdl. \& Cham. CHIS, COAH, GTO, HGO, JAL, MEX, MICH, MOR, NLE, OAX, PUE, QRO, SLP, SIN, TAMS, VER

*Quercus potosina Trel. AGS, CHIH, COAH, COL, DGO, GTO, HGO, JAL, MEX, NLE, OAX, PUE, QRO, SLP, TAMS, VER, ZAC

*Quercus praeco Trel. COL, DGO, HGO, JAL, NAY, QRO, SLP, ZAC

*Quercus praineana Trel. COL, DGO, HGO, JAL, NAY, QRO, SLP, SIN, ZAC

*Quercus pringlei Seemen CHIH, COAH, DGO, HGO, NLE, QRO, SLP, TAMS, VER, ZAC

Quercus pungens Liebm. CHIH, COAH, DGO, GTO, NLE, QRO, SLP, SON, TAMS

Quercus purulhana Trel. CHIS

*Quercus radiata Trel. DGO, JAL, ZAC

*Quercus rekonis Trel. DGO, JAL, OAX, SIN

*Quercus repanda Bonpl. CDMX, GTO, HGO, JAL, MEX, MICH, NLE, OAX, PUE, QRO, SLP, TAMS, TLAX, VER, $\mathrm{ZAC}$

*Quercus resinosa Liebm. AGS, COL, DGO, GTO, GRO, HGO, JAL, MEX, MICH, NAY, OAX, PUE, QRO, SLP, SIN, SON, TAMS, ZAC

*Quercus rhysophylla Weath. COAH, HGO, NLE, OAX, QRO, SLP, TAMS, VER

*Quercus rubramenta Trel. CHIS, GRO, OAX

Quercus rugosa Née AGS, BCS, CHIS, CHIH, COAH, COL, CDMX, DGO, GTO, GRO, HGO, JAL, MEX, MICH, MOR, NAY, NLE, OAX, PUE, QRO, SLP, SIN, SON, TAMS, TLAX, VER, ZAC

*Quercus runcinatifolia Trel. \& C.H. Müll. NLE

Quercus salicifolia Née CHIS, COL, DGO, GRO, HGO, JAL,

MEX, MICH, MOR, NAY, OAX, SLP, SIN, VER, ZAC

*Quercus saltillensis Trel. COAH, NLE, SLP, TAMS, ZAC

*Quercus sanchez-colinii Martínez MEX

Quercus sapotifolia Liebm. CHIS, GRO, HGO, OAX, PUE, SLP, TAMS, VER
*Quercus sartorii Liebm. CHIS, COAH, GRO, HGO, MEX, NLE, OAX, PUE, QRO, SLP, TAMS, VER

*Quercus scytophylla Liebm. CHIS, CHIH, COL, DGO, GRO, HGO, JAL, MEX, MICH, NAY, OAX, PUE, SIN, SON, VER, ZAC

*Quercus sebifera Trel. CHIS, CHIH, DGO, HGO, NLE, OAX, PUE, QRO, SLP, SON, TAMS, VER

Quercus segoviensis Liebm. CHIS, MICH, OAX

*Quercus sideroxyla Bonpl. AGS, CHIH, COAH, DGO, GTO, HGO, JAL, MEX, MICH, MOR, NAY, NLE, OAX, PUE, QRO, SLP, SIN, SON, TAMS, VER, ZAC

Quercus sinuata Walter COAH, NLE, TAMS

*Quercus sipuraca Trel. CHIH, SON

Quercus skinneri Benth. CHIS, HGO, OAX, TAMS, VER

*Quercus splendens Née COL, DGO, GRO, JAL, MEX, MICH, MOR, NAY, OAX, SIN, TAMS, VER, ZAC

*Quercus subspathulata Trel. CHIH, COL, DGO, GTO, GRO, HGO, JAL, MEX, MICH, NAY, OAX, PUE, QRO, SIN, SON, ZAC

*Quercus supranitida C.H. Müll. NLE, TAMS

*Quercus tarahumara Spellenb., J.D. Bacon \& Breedlove CHIH, DGO, SIN, SON

*Quercus tenuiloba C.H. Müll. NLE, TAMS

*Quercus tinkhami C.H. Müll. COAH, DGO, GTO, HGO, JAL, NLE, QRO, SLP, TAMS, VER, ZAC

Quercus tomentella Engelm. BCN

Quercus toumeyi Sarg. CHIH, DGO, NLE, SIN, SON

*Quercus trinidadensis C.H. Müll. COAH, NLE, TAMS

Quercus tristis Liebm. CHIS

*Quercus tuberculata Liebm. BCS, CHIH, COAH, DGO, JAL, MEX, MICH, NAY, NLE, SIN, SON, ZAC

*Quercus tuitensis L.M. González JAL

Quercus turbinella Greene BCN, BCS, SON

*Quercus urbanii Trel. CHIH, DGO, GRO, JAL, MEX, MICH, NAY, OAX, QRO, SIN, SON, ZAC

*Quercus uxoris McVaugh AGS, COL, GRO, JAL, MICH, OAX

*Quercus vaseyana Buckley COAH, NLE, TAMS

*Quercus verde C.H. Müll. CHIH, COAH, NLE

*Quercus vicentensis Trel. CHIS, COL, GRO, JAL, MICH, OAX

*Quercus viminea Trel. AGS, CHIH, DGO, GTO, JAL, NAY, SLP, SIN, SON, ZAC

Quercus virginiana Mill. CHIH, COAH, NLE, SLP, TAMS, VER

Quercus wislizenii A. DC. BCN

*Quercus $\times$ basaseachicensis C.H. Müll. CHIH, SON

$*$ Quercus $\times$ dysophylla Benth. CHIS, CDMX, DGO, GTO, GRO, HGO, JAL, MEX, MICH, MOR, NLE, OAX, PUE, QRO, SLP, TAMS, TLAX, VER, ZAC

$*$ Quercus $\times$ knoblochii C.H. Müll. CHIH, SON

*Quercus $\times$ lesueurii C.H. Müll. CHIH

*Quercus $\times$ pastorensis C.H. Müll. NLE

*Quercus xalapensis Bonpl. CHIS, CHIH, COL, DGO, GRO, HGO, JAL, MEX, MICH, NAY, OAX, PUE, QRO, SLP, SIN, SON, TAMS, VER

\section{Family Fouquieriaceae}


*Fouquieria burragei Rose BCS

*Fouquieria columnaris (Kellogg) Kellogg ex Curran BCN, $\mathrm{BCS}, \mathrm{SON}$

*Fouquieria diguetii (Tiegh.) I.M. Johnst. BCN, BCS, SIN, SON

*Fouquieria fasciculata (Willd. ex Roem. \& Schult.) Nash HGO, QRO, SLP, VER

* Fouquieria formosa Kunth CHIS, COL, CDMX, GRO, HGO, JAL, MEX, MICH, MOR, OAX, PUE, QRO

* Fouquieria leonilae Miranda GRO, OAX

*Fouquieria macdougalii Nash CHIH, SIN, SON

*Fouquieria ochoterenae Miranda OAX, PUE

*Fouquieria purpusii Brandegee OAX, PUE

* Fouquieria shrevei I.M. Johnst. CHIH, COAH, DGO

Fouquieria splendens Engelm. BCN, BCS, CHIH, COAH, DGO, GTO, HGO, JAL, NLE, QRO, SLP, SON, TAMS, VER, ZAC

\section{Family Frankeniaceae}

Frankenia grandifolia Cham. \& Schltdl. BCN, BCS, SON

*Frankenia gypsophila I.M. Johnst. BCN, COAH, NLE, SLP

Frankenia johnstonii Correll COAH, NLE

*Frankenia margaritae Medrano COAH, NLE

Frankenia palmeri S. Watson BCN, BCS, SON

Frankenia salina (Molina) I.M. Johnst. BCN, BCS, COAH, SON

\section{Family Garryaceae}

Garrya flavescens S. Watson BCN, CHIH, SON

*Garrya glaberrima Wangerin COAH, GTO, JAL, NLE, QRO, SLP, TAMS

*Garrya grisea Wigg. BCN

Garrya laurifolia Hartw. ex Benth. AGS, CHIS, CHIH, COAH, COL, CDMX, DGO, GTO, GRO, HGO, JAL, MEX, MICH, MOR, NAY, NLE, OAX, PUE, QRO, SLP, SIN, TAMS, SON, TLAX, VER, ZAC

*Garrya longifolia Rose CHIS, COL, CDMX, DGO, GTO, GRO, JAL, MEX, MICH, MOR, NAY, OAX, PUE, SIN, ZAC Garrya ovata Benth. AGS, CHIH, COAH, DGO, GTO, HGO, JAL, NLE, OAX, PUE, QRO, SLP, SON, TAMS, VER, ZAC

*Garrya salicifolia Eastw. BCN, BCS

Garrya veatchii Kellogg BCN, BCS

Garrya wrightii Torr. CHIH, COAH, DGO, NLE, SON, TAMS, ZAC

\section{Family Gelsemiaceae}

Gelsemium sempervirens (L.) J. St.-Hil. CHIS, GRO, HGO, JAL, MICH, NAY, OAX, PUE, QRO, SLP, SIN, SON, TAB, TAMS, VER

\section{Family Gentianaceae}

*Centaurium capense C.R. Broome BCS, COL

*Centaurium macranthum (Hook. \& Arn.) B.L. Rob. NLE, TAMS, VER

*Centaurium petrocaule C.R. Broome GTO, QRO, SLP Centaurium pringleanum (Wittr.) B.L. Rob. JAL, QRO, SLP

Coutoubea spicata Aubl. CAM, CHIS, QROO, TAB, VER

Curtia tenella (Mart.) Cham. TAB

*Eustoma barkleyi Shinners COAH, NLE
Eustoma exaltatum (L.) Salisb. ex G. Don BCN, BCS, CAM, CHIS, CHIH, COAH, COL, GRO, HGO, JAL, MEX, MICH, MOR, NAY, NLE, OAX, PUE, QRO, QROO, SLP, SIN, SON, TAB, TAMS, VER, YUC

Eustoma russellianum (Hook.) G. Don CHIH, COAH, TAMS

*Geniostemon coulteri Engelm. \& A. Gray GTO, HGO, QRO, SLP

*Geniostemon gypsophilum B.L. Turner NLE

*Geniostemon rotundifolius Rzed. \& Calderón QRO, SLP

*Geniostemon schaffneri Engelm. \& A. Gray NLE, SLP, TAMS

*Gentiana bicuspidata (G. Don) Briq. CHIS, CHIH, CDMX, DGO, GRO, HGO, JAL, MEX, MICH, MOR, OAX, SLP, SIN, TAMS, TLAX, VER

*Gentiana calyculata Lex. COL, GRO, JAL, MEX, MICH, MOR

*Gentiana chazaroi H.H. Iltis DGO, SIN

* Gentiana durangensis Villarreal DGO

*Gentiana hintoniorum B.L. Turner NLE, TAMS

*Gentiana hooperi J.S. Pringle DGO, SIN

*Gentiana laevigata M. Martens \& Galeotti CHIS, OAX

*Gentiana longicollis G.L. Nesom DGO, JAL, NAY

*Gentiana mirandae Paray HGO, VER

Gentiana ovatiloba Kusn. CDMX, DGO, GTO, MEX, MICH, OAX, PUE, QRO, VER

*Gentiana perpusilla Brandegee MEX, VER

* Gentiana porphyrio J.F. Gmel. AGS, ZAC

Gentiana pumilio Standl. \& Steyerm. CHIS

Gentiana sedifolia Kunth CHIS

*Gentiana spathacea Kunth AGS, COL, CDMX, DGO, GTO, GRO, HGO, JAL, MEX, MICH, MOR, NAY, NLE, OAX, PUE, QRO, SLP, TAMS, TLAX, VER, ZAC

*Gentiana superba Greene AGS, CHIH, DGO, JAL, NAY, ZAC Gentianella amarella (L.) Börner AGS, BCN, CHIH, COAH, CDMX, DGO, GRO, HGO, JAL, MEX, MICH, NLE, OAX, PUE, SLP, SIN, SON, TLAX, VER, ZAC

*Gentianella canosoi G.L. Nesom \& B.L. Turner DGO, SIN

Gentianella heterospela (Engelm.) Holub DGO

Gentianella microcalyx (Lemmon) J.M. Gillett CHIH, COAH, DGO, SON

Gentianella wislizenii (Engelm.) J.M. Gillett CHIH, DGO, SON

*Gentianopsis lanceolata (Benth.) H.H. Iltis CHIH, DGO, GTO, JAL, MICH, NAY, NLE, QRO, SIN, VER, ZAC

Gyrandra brachycalyx (Standl. \& L.O. Williams) G. Mans. CHIS, CDMX, GTO, HGO, JAL, MEX, MICH, NAY, OAX, VER

*Gyrandra chironioides Griseb. CDMX, HGO, JAL, MEX, MICH, NLE, OAX, SLP, TAMS, TLAX, VER, ZAC

Gyrandra pauciflora (M. Martens \& Galeotti) G. Mans. GRO, HGO, MICH, OAX, PUE, VER

*Gyrandra pterocaulis (C.R. Broome) G. Mans. GTO, HGO, QRO, SLP

*Gyrandra tenuifolia (M. Martens \& Galeotti) G. Mans. COL, GRO, JAL, MICH, NAY, NLE, SLP

Halenia alata (M. Martens \& Galeotti) Hemsl. CHIS, MEX, MICH, VER

*Halenia alleniana Standl. ex Wilbur COAH, MICH, MOR, NLE, QRO, SLP, TAMS 
Halenia brevicornis (Kunth) G. Don AGS, CHIS, CHIH, COAH, COL, CDMX, DGO, GTO, GRO, HGO, JAL, MEX, MICH, MOR, NAY, NLE, OAX, PUE, QRO, SLP, SIN, SON, TLAX, VER, ZAC

*Halenia conzattii Greenm. GRO, OAX, PUE

Halenia crassiuscula B.L. Rob. \& Seaton CHIS, CDMX, HGO, JAL, MEX, MICH, TLAX, VER

*Halenia crumiana Wilbur COL, JAL

Halenia decumbens Benth. CHIS, OAX

*Halenia hintonii Bullock GRO, MEX, MICH

*Halenia palmeri A. Gray CHIH, DGO, SIN, SON

*Halenia plantaginea (Kunth) G. Don CDMX, GTO, HGO, JAL, MEX, MICH, MOR, NAY, NLE, OAX, PUE, QRO, SLP, TAMS, TLAX, VER

Halenia recurva (J.E. Sm.) C.K. Allen CHIH, COAH, DGO, JAL, NLE, SIN, SON

*Halenia schiedeana Griseb. DGO, OAX, SIN, VER

Irlbachia alata (Aubl.) Maas CHIS, GRO, MICH, OAX, TAB, VER

Lisianthius axillaris (Hemsl.) Kuntze CAM, CHIS, NLE, QROO, VER, YUC

Lisianthius brevidentatus (Hemsl.) Kuntze CHIS, VER

Lisianthius nigrescens Schltdl. \& Cham. CHIS, OAX, VER

*Lisianthius oreopolus B.L. Rob. CHIS

Lisianthius perkinsiae Struwe \& R.E. Weaver VER

*Lisianthius quichensis Donn. Sm. CHIS, OAX

Lisianthius saponarioides Schltdl. \& Cham. CHIS, VER

Lisianthius viscidiflorus B.L. Rob. CHIS, OAX, VER

Sabatia arenicola Greenm. TAMS

Sabatia stellaris Pursh COAH, DGO, GTO, JAL, MICH, NLE, QRO, SLP, TAMS

* Sabatia tuberculata J.E. Williams COAH

Schultesia brachyptera Cham. CHIS, TAB, VER

Schultesia guianensis (Aubl.) Malme CHIS, DGO, GRO, JAL,

OAX, SIN, TAB, VER, YUC

Schultesia heterophylla Miq. CHIS

Schultesia lisianthoides (Griseb.) Benth. \& Hook. ex Hemsl. CHIS, OAX, VER

Swertia parryi (Torr.) Kuntze BCN

Swertia radiata (Kellogg) Kuntze COAH, NLE, SON, TAMS

Voyria aphylla (Jacq.) Pers. CAM, CHIS, OAX, QROO, YUC

Voyria flavescens Griseb. CHIS, OAX

Voyria parasitica (Schltdl. \& Cham.) Ruyters \& P. Maas CAM,

CHIS, OAX, QROO, TAB, VER, YUC

Voyria tenella Hook. CAM, CHIS, OAX, QROO, VER

Voyria truncata (Standl.) Standl. \& Steyerm. CHIS

Zeltnera calycosa (Buckley) G. Mans. BCN, BCS, CHIH, COAH, COL, DGO, JAL, NLE, OAX, PUE, SLP, SIN, SON, TAMS

Zeltnera exaltata (Griseb.) G. Mans. BCN, BCS, SON

*Zeltnera gentryi (C.R. Broome) G. Mans. CHIH, SON

*Zeltnera madrensis (Hemsl.) G. Mans. DGO, JAL, NAY, SIN

Zeltnera martinii (C.R. Broome) G. Mans. COL, JAL

*Zeltnera nesomii B.L. Turner TAMS

Zeltnera nudicaule (Engelm.) G. Mans. AGS, BCS, CHIH, COL, DGO, JAL, NAY, PUE, SIN, SON

*Zeltnera pusilla (Eastw.) G. Mans. JAL, MICH, SLP
Zeltnera quitensis (Kunth) G. Mans. AGS, CHIS, COL, CDMX, DGO, GTO, GRO, HGO, JAL, MEX, MICH, MOR, NAY, OAX, PUE, QRO, QROO, SLP, SIN, TAB, TLAX, VER, YUC

Zeltnera setacea (Benth.) G. Mans. CHIS, CHIH, COL, DGO, JAL, MICH, NAY, SON

Zeltnera stricta (Schiede) Druce CHIS, COL, JAL, MICH, OAX, VER

Zeltnera venusta (A. Gray) G. Mans. BCN, CHIH, SON

*Zeltnera wigginsii (Broome) G. Mans. COL, DGO, NAY, SIN

\section{Family Geraniaceae}

California macrophylla (Hook. \& Arn.) Aldasoro, C. Navarro,

P. Vargas, L. Sáez \& Aedo BCN

*Erodium moranense Willd. ex Kunth VER

Erodium texanum A. Gray BCN, BCS, CHIH, COAH, NLE, SON, TAMS

*Geranium albidum Rydb. CHIH, DGO, SIN, SON

Geranium alpicola Loes. CHIS, OAX, VER

Geranium andicola Loes. CHIS, OAX

*Geranium bellum Rose CDMX, GTO, HGO, JAL, MEX, QRO,

VER

Geranium caespitosum James BCN, CHIH, COAH, DGO, NLE, SON

*Geranium campanulatum Paray DGO, HGO, JAL, QRO, SLP, ZAC

*Geranium carmineum $\mathrm{R}$. Knuth OAX

Geranium carolinianum L. BCN, CHIH, OAX, SON, TAMS

*Geranium clarum Small GRO, OAX

*Geranium crenatifolium H.E. Moore COAH, DGO, JAL, NAY, NLE, OAX, SIN

*Geranium cruceroense Knuth CDMX, GRO, HGO, JAL, MEX, MICH, PUE, TLAX, VER

*Geranium charucanum Standl. CHIH, SON

*Geranium deltoideum Rydb. AGS, CHIH, DGO, GTO, GRO, JAL, MEX, MICH, MOR, OAX, QRO, SIN, SON, VER, ZAC Geranium dissectum L. JAL, MICH, NLE

*Geranium flaccidum Small BCS

*Geranium geoides Small CHIH, SON

*Geranium goldmanii Rose ex Hanks \& Small CHIS

*Geranium gracile Engelm. BCN, CHIH

Geranium guatemalense R. Knuth CHIS

*Geranium hernandesii DC. COL, CDMX, DGO, GTO, HGO,

JAL, MEX, MICH, MOR, OAX, ZAC

*Geranium hintonii H.E. Moore GRO, OAX

*Geranium latilobum H.E. Moore JAL

*Geranium latum Small CDMX, DGO, GTO, HGO, JAL, MEX, MICH, MOR, OAX, PUE, QRO, SLP, SIN, TLAX, ZAC

Geranium lentum Wooton \& Standl. ND

*Geranium lilacinum R. Knuth COL, CDMX, GRO, HGO, JAL, MEX, MICH, MOR, OAX, QRO, SLP, TLAX

*Geranium lozanoi Rose CDMX, HGO, MEX, MICH, VER

* Geranium madrense Rose ex Hanks \& Small DGO, NAY

*Geranium mexicanum Kunth AGS, CHIS, COAH, COL, DGO, GRO, GTO, JAL, MEX, MICH, MOR, NAY, OAX, SIN, SON, TLAX, VER, ZAC

*Geranium miahuatlanum B.L. Turner OAX

*Geranium monanthum Small OAX 
* Geranium mooreanum Aedo COL, JAL

* Geranium niveum $\mathrm{S}$. Watson $\mathrm{CHIH}$, SON

*Geranium oaxacanum H.E. Moore OAX

*Geranium potentillifolium DC. CDMX, GTO, GRO, HGO, JAL, MEX, MICH, MOR, OAX, PUE, QRO, TLAX, VER

* Geranium potosinum H.E. Moore NLE

Geranium repens H.E. Moore CHIS, GRO, OAX

Geranium richardsonii Fisch. \& Traut. CHIH, SON

*Geranium schiedeanum Schltdl. CHIS, CDMX, HGO, JAL, MEX, MOR, OAX, PUE, QRO, SLP, TLAX, VER

Geranium seemannii Peyr. AGS, CHIS, CHIH, COAH, COL, CDMX, DGO, GTO, GRO, HGO, JAL, MEX, MICH, MOR, NLE, OAX, PUE, QRO, SLP, SIN, SON, TAMS, TLAX, VER, $\mathrm{ZAC}$

* Geranium tenue Hanks COAH, NLE, SLP, ZAC

*Geranium trollifolium Small CHIH, DGO, JAL, SLP

Geranium wislizenii S. Watson CHIH, DGO, SIN, SON

\section{Family Gesneriaceae}

Achimenes admirabilis Wiehler OAX, VER, TAB

Achimenes antirrhina (DC.) C.V. Morton CHIS, COL, DGO, GRO, HGO, JAL, MEX, MICH, MOR, NAY, NLE, OAX, QRO, SLP, SIN, TAMS, VER

*Achimenes bella C.V. Morton MICH

*Achimenes brevifolia C.V. Morton GRO

Achimenes candida Lindl. CHIS, OAX

*Achimenes cettoana H.E. Moore CHIS

*Achimenes dulcis C.V. Morton GRO, MEX, MICH

Achimenes erecta (Lam.) H.P. Fuchs CAM, CHIS, GTO, GRO, MEX, MICH, OAX, PUE, QRO, QROO, SLP, VER

*Achimenes fimbriata Rose ex C.V. Morton GRO, DGO, JAL, MEX, MICH, MOR, NAY, SIN

*Achimenes flava C.V. Morton CHIS, COL, GRO, JAL, MEX, $\mathrm{MICH}, \mathrm{NAY}$, OAX, SIN, ZAC

*Achimenes glabrata (Zucc.) Fritsch COL, GRO, DGO, JAL, MEX, MICH, MOR, NAY, OAX, SIN, ZAC

Achimenes grandiflora (Schiede) DC. CHIS, CHIH, COL, DGO, GRO, HGO, JAL, MEX, MICH, MOR, OAX, PUE, QRO, SLP, SIN, SON, TAB, TAMS, VER

Achimenes heterophylla (M. Martens) DC. CHIS, COL, DGO, GRO, HGO, JAL, MEX, MICH, MOR, NAY, SIN

*Achimenes hintoniana Ramírez-Roa \& L.E. Skog DGO, JAL, $\mathrm{MICH}, \mathrm{NAY}$

Achimenes longiflora DC. CHIS, COL, GRO, JAL, MICH, NAY, OAX

*Achimenes mexicana (Seem.) Benth. \& Hook. f. ex Fritsch CHIH, COL, DGO, JAL, SIN, SON, ZAC

Achimenes misera Lindl. CHIS, GRO, OAX

*Achimenes nayaritensis L.E. Skog NAY

*Achimenes obscura C.V. Morton CHIS, COL, GRO, JAL, MEX, MICH, OAX

*Achimenes occidentalis C.V. Morton JAL, ZAC

*Achimenes patens Benth. CHIS, GRO, JAL, MEX, MICH, MOR, NAY, OAX

Achimenes pedunculata Benth. CHIS, COL, JAL, MEX, OAX, TAB, VER

Achimenes skinneri Gordon \& Lindl. CHIS, OAX, QRO, TAMS
Achimenes warszewicziana (Regel) H.E. Moore CHIS, GRO, OAX

*Achimenes woodii C.V. Morton GRO, OAX

Alsobia dianthiflora (H.E. Moore \& Paul G. Wilson) Wiehler GRO

Alsobia punctata (Lindl.) Hanst. CHIS, COL, JAL, NAY

Alloplectus calochlamys Donn. Sm. CHIS

*Alloplectus strigosus (Oerst.) Hanst. CHIS, OAX, VER

Amalophyllon parviflorum (Brongn. \& C.D. Bouché) Boggan,

L.E. Skog \& E.H. Roalson CHIS

Amalophyllon rupestre Brandegee CHIS, OAX

Besleria conspecta C.V. Morton CHIS, OAX, PUE, VER

*Besleria cyrtanthemum Hanst. OAX, PUE, VER

*Besleria deflexa (Oerst.) Hanst. OAX, VER

Besleria flava C. V.Morton CHIS

* Besleria glabra (Oerst.) Hanst. CHIS, OAX, VER

Besleria hirsuta (Oerst.) Hanst. CHIS, OAX

Besleria laxiflora Benth. CHIS, OAX, PUE, TAB, VER

Codonanthe crassifolia (Focke) C.V. Morton CHIS, TAB, VER

Codonanthe macradenia Donn. Sm. CAM, CHIS, QROO, TAB, VER

Codonanthe uleana Fritsch CAM, CHIS, OAX, QROO, TAB, VER, YUC

Columnea crassifolia Brongn. ex Lem. CHIS, OAX

* Columnea erythrophaea Decne. ex Houllet CHIS, VER

Columnea guatemalensis Sprague CHIS, VER

Columnea hirta Klotzsch \& Hanst. CHIS, OAX

Columnea nervosa (Klotzsch ex Oerst.) Hanst. CHIS, GRO, OAX, VER

Columnea purpurata Hanst. CHIS

* Columnea purpusii Standl. CHIS, OAX, TAB, VER

*Columnea schiedeana Schltdl. CHIS, HGO, OAX, PUE, TAB, VER

*Columnea septentrionalis C.V. Morton COL, JAL, NAY

Columnea sulfurea Donn. Sm. CHIS, PUE, TAB, VER

Columnea tuerckheimii Sprague CHIS

*Corytoplectus oaxacensis Ramírez-Roa, C. Chávez \& Rodr.Flores OAX

Chrysothemis pulchella (Donn) Decne. CHIS

Diastema racemiferum Benth. CHIS, OAX, VER

Drymonia macrantha (Donn. Sm.) D.N. Gibson CHIS

Drymonia macrophylla (Oerst.) H.E. Moore CHIS

Drymonia oinochrophylla (Donn. Sm.) D.N. Gibson CHIS, OAX, TAB, VER

Drymonia serrulata (Jacq.) Mart. CHIS, COL, GRO, JAL, MICH, NAY, OAX, TAB, VER

*Drymonia strigosa (Oerst.) Wiehler CHIS, GRO, OAX, VER Episcia lilacina Hanst. CHIS

Episcia purpusii (Brandegee) Brandegee CHIS

*Eucodonia andrieuxii (DC.) Wiehler CHIS, GRO, JAL, MICH, OAX

*Eucodonia verticillata (M. Martens \& Galeotti) Wiehler GRO, HGO, MEX, MICH, OAX, VER

Gasteranthus pansamalanus (Donn. Sm.) Wiehler CHIS

Glossoloma cucullatum (C.V. Morton) J.L. Clark CHIS, OAX

Glossoloma tetragonum Hanst. CHIS, OAX, VER

*Kohleria longifolia (Lindl.) Hanst. CHIS 
*Kohleria rugata (Scheidw.) L.P. Kvist \& L.E. Skog CHIS, GRO, OAX

Kohleria spicata (Kunth) Oerst. CHIS, OAX, TAB, VER

Kohleria tigridia (Ohlend.) Roalson \& Boggan CHIS

Monopyle puberula C.V. Morton CHIS

Moussonia adpressipilosa D.L. Denham ex Ramírez-Roa CHIS

Moussonia deppeana (Schltdl. \& Cham.) Hanst. CHIS, GRO,

HGO, MOR, OAX, PUE, VER

Moussonia elegans Decne. CHIS, COL, DGO, HGO, JAL, MEX, MICH, MOR, NAY, OAX, PUE, VER, ZAC

Moussonia fruticosa (Brandegee) Wiehler CHIS, OAX, VER

*Moussonia hirsutissima (C.V. Morton) Wiehler OAX

*Moussonia larryskogii Ramìrez-Roa OAX, VER

*Moussonia papillosa Oerst. ex Hanst. JAL, MICH, NAY, OAX, PUE, VER

Moussonia rupicola (Standl. \& Steyerm.) Wiehler CHIS

*Moussonia septentrionalis (D.L. Denham) Wiehler DGO, SIN

Moussonia skutchii (C.V. Morton \& D.N. Gibson) Wiehler CHIS

*Moussonia triflora (M. Martens \& Galeotti) Hanst. OAX, VER

*Moussonia viminalis (Brandegee) Wiehler CHIS

Napeanthus bracteatus C.V. Morton CHIS, OAX, PUE, TAB,

VER

Nautilocalyx panamensis (Seem.) Seem. CHIS

Neomortonia nummularia (Hanst.) Wiehler CHIS

Niphaea mexicana C.V. Morton GRO, MEX, OAX

Niphaea oblonga Lindl. CHIS

*Niphaea pumila Boggan \& L.E. Skog DGO, SIN

* Oerstedina mexicana Wiehler GRO

* Paradrymonia congesta (Oerst.) Wiehler OAX

* Phinaea multiflora C.V. Morton COL, GRO, JAL, MEX, MICH, NAY, OAX, SIN

Phinaea repens (Donn. Sm.) Soler. CHIS

Rhynchoglossum azureum (Schltdl.) B.L. Burtt CHIS, HGO,

OAX, PUE, VER

Sinningia incarnata (Aubl.) D.L. Denham CHIS, GRO, OAX, VER

*Sinningia richii Clayberg VER

*Smithiantha aurantiaca Wiehler OAX, VER

* Smithiantha canarina Wiehler OAX

* Smithiantha cinnabarina (Linden) Kunze CHIS, OAX, TAB,

VER

*Smithiantha laui Wiehler OAX

* Smithiantha multiflora (M. Martens \& Galeotti) Fritsch OAX, PUE, VER

* Smithiantha zebrina (Regel) Kuntze CHIS, HGO, MOR, QRO, SLP, TAMS, VER

*Solenophora coccinea Benth. OAX

* Solenophora chiapasensis D.N. Gibson CHIS

* Solenophora glomerata Weigend \& Förther CHIS, OAX, VER

*Solenophora insignis (M. Martens \& Galeotti) Hanst. CHIS, GRO, OAX, VER

Solenophora maculata D.N. Gibson VER

* Solenophora modesta Weigend \& Forther CHIS

Solenophora obliqua D.L. Denham \& D.N. Gibson CHIS

Solenophora obscura Hanst. CHIS, OAX, VER

Solenophora pirana C.V. Morton CHIS

Solenophora toucana D.L. Denham \& D.N. Gibson CHIS, OAX
*Solenophora tuxtlensis Ramírez-Roa \& Ibarra-Manríquez OAX, VER

Solenophora wilsonii Standl. CHIS

\section{Family Goodeniaceae}

Scaevola plumieri (L.) Vahl BCS, CAM, COL, PUE, QROO, SIN, TAMS, VER, YUC

Family Grossulariaceae

*Ribes affine Kunth COAH, CDMX, DGO, GTO, HGO, JAL, MEX, MICH, MOR, NLE, OAX, PUE, QRO, SLP, TLAX, VER, ZAC

*Ribes brandegeei Eastw. BCS

Ribes canthariforme Wiggins BCN

* Ribes ceriferum Coville \& Rose CHIH, DGO, SON

Ribes ciliatum Humb. \& Bonpl. ex Roem. \& Schult. CHIS, COL, CDMX, DGO, HGO, JAL, MEX, MICH, MOR, NLE, OAX, PUE, SLP, SIN, TAMS, TLAX, VER

*Ribes chihuahuense Britton CHIH

* Ribes dugesii Greenm. GTO, JAL

*Ribes fontinale Britton ex Coville \& Britton CHIH

*Ribes grandisepalum Durán-Esp. \& S. Avendaño MICH

Ribes indecorum Eastw. BCN

Ribes leptanthum A. Gray COAH

* Ribes madrense Coville \& Rose DGO

Ribes malvaceum $\mathrm{Sm}$. $\mathrm{BCN}$

Ribes mescalerium Coville CHIH, SON

Ribes microphyllum Kunth CHIS, COAH, CDMX, MEX, MICH, MOR, NLE, PUE, VER

* Ribes neglectum Rose COAH, DGO, NLE, SLP, SIN, TAMS, ZAC

*Ribes nelsonii Coville \& Rose CHIH, SON

* Ribes orizabae Rose VER

Ribes quercetorum Greene BCN, BCS

Ribes sanguineum Pursh BCN

*Ribes speciosum Pursh $\mathrm{BCN}$

*Ribes tortuosum Benth. BCN, BCS

Ribes viburnifolium A. Gray BCN

Family Guamatelaceae

Guamatela tuerckheimii Donn. Sm. CHIS, OAX

Family Gunneraceae

Gunnera insignis (Oerst.) A. DC. CHIS

Gunnera killipiana Lundell CHIS, VER

Gunnera mexicana Brandegee CHIS, VER

\section{Family Haemodoraceae}

Xiphidium caeruleum Aubl. CHIS, GRO, OAX, PUE, TAB, VER

\section{Family Haloragaceae}

Myriophyllum farwellii Morong MICH

Myriophyllum heterophyllum Michx. CAM, CHIS, HGO, JAL, MEX, MICH, MOR, VER

Myriophyllum hippuroides Nutt. CHIH, MEX, MICH, MOR, QRO, TAMS

Myriophyllum pinnatum (Walter) Britton, Stearn \& Pogg. CHIH, $\mathrm{MICH}$

Myriophyllum quitense Kunth MEX 
Proserpinaca palustris L. CHIS, VER

Proserpinaca pectinata Lam. CHIS, TAB

\section{Family Hamamelidaceae}

*Hamamelis mexicana Standl. COAH, NLE

Matudaea trinervia Lundell CHIS, COL, JAL, MEX, OAX, PUE, VER

*Molinadendron sinaloense (Standl. \& Gentry) P.K. Endress SIN

\section{Family Heliconiaceae}

Heliconia adflexa (Griggs) Standl. CHIS, CDMX, MEX, OAX, VER

Heliconia aurantiaca Ghiesbr. ex Lem. CHIS, TAB

Heliconia bihai L. CHIS, GRO, OAX, PUE, TAB, VER

Heliconia bourgaeana Petersen CAM, CHIS, OAX, PUE, TAB, VER

Heliconia collinsiana Griggs CAM, CHIS, OAX, TAB, VER

Heliconia dielsiana Loes. CHIS

Heliconia latispatha Benth. CAM, CHIS, GRO, HGO, OAX, QRO, QROO, SLP, TAB, VER

Heliconia librata Griggs CHIS, OAX, TAB, VER

Heliconia lophocarpa G.S. Daniels \& F.G. Stiles OAX

*Heliconia mooreana R.R. Sm. COL, GRO, JAL, NAY

Heliconia psittacorum L. f. CHIS, OAX, TAB

Heliconia rostrata Ruiz \& Pav. CHIS, OAX

Heliconia schiedeana Klotzsch CHIS, OAX, PUE, QRO, SLP, TAB, TAMS, VER

Heliconia spissa Griggs CHIS, COL, JAL, OAX, TAB, VER

Heliconia subulata Ruiz \& Pav. CHIS, TAB, VER

Heliconia tortuosa Griggs OAX, VER

*Heliconia uxpanapensis Gutiérrez-Báez CAM, CHIS, OAX, VER

Heliconia vaginalis Benth. CHIS, OAX, TAB, VER

*Heliconia wagneriana Peterson CHIS

\section{Family Heliotropaceae}

Heliotropium angiospermum Murray AGS, BCN, BCS, CAM, CHIS, COAH, COL, DGO, GRO, HGO, JAL, MEX, MICH, MOR, NAY, NLE, OAX, PUE, QRO, QROO, SLP, SIN, SON, TAB, TAMS, VER, YUC, ZAC

Heliotropium angustifolium Torr. CHIH, COAH, HGO, NLE, QRO, SLP, TAMS, VER

*Heliotropium axillare Greenm. HGO, PUE, QRO, VER

*Heliotropium calcicola Fernald CHIS, COAH, GTO, GRO, HGO, NLE, OAX, PUE, QRO, SLP, TAMS, VER

Heliotropium confertifolium (Torr.) A. Gray $\mathrm{CHIH}, \mathrm{COAH}$, HGO, NLE, OAX, PUE, QRO, SLP, TAMS, ZAC

Heliotropium convolvulaceum (Nutt.) A. Gray BCN, CHIH, COAH, DGO, SLP, SON

Heliotropium coriaceum Lehm. CHIS, OAX

Heliotropium curassavicum L. AGS, BCN, BCS, CAM, CHIS, CHIH, COAH, COL, CDMX, DGO, GTO, GRO, HGO, JAL, MEX, MICH, MOR, NAY, NLE, OAX, PUE, QRO, QROO, SLP, SIN, SON, TAB, TAMS, TLAX, VER, YUC, ZAC Heliotropium fallax I.M. Johnst. BCS, CHIS, COL, DGO, GRO, HGO, JAL, MEX, MICH, NAY, OAX, QRO, SLP, TAMS Heliotropium filiforme Lehm. CAM, CHIS, OAX, QROO, TAB,
VER, YUC

Heliotropium foliosissimum J.F. Macbr. GTO, HGO, JAL, MEX, MICH, OAX, PUE, QRO, SLP

Heliotropium fruticosum L. AGS, BCS, CAM, CHIS, CHIH, COAH, COL, DGO, GRO, HGO, JAL, MEX, MICH, MOR, NAY, NLE, OAX, PUE, QRO, QROO, SLP, SIN, SON, TAB, VER, YUC, ZAC

Heliotropium glabriusculum (Torr.) A. Gray $\mathrm{CHIH}, \mathrm{COAH}$, DGO, NLE, SLP, ZAC

Heliotropium greggii Torr. AGS, CHIH, COAH, DGO, HGO, JAL, NLE, SON, ZAC

Heliotropium indicum L. CAM, CHIS, COL, GRO, JAL, MEX, MICH, MOR, NAY, OAX, PUE, QROO, SLP, SIN, TAB, TAMS, VER, YUC, ZAC

*Heliotropium karwinskyi I.M. Johnst. TAMS

Heliotropium lagoense (Warm.) Gürke CHIS, GRO, MEX, OAX, VER

*Heliotropium limbatum Benth. AGS, CHIH, DGO, GTO, GRO, HGO, JAL, MEX, MICH, MOR, NAY, OAX, PUE, QRO, SIN, SON, VER, ZAC

Heliotropium macrostachyum (DC.) Hemsl. BCS, CHIS, CHIH, JAL, OAX, PUE, SIN, SON, TAB, TAMS, VER

Heliotropium molle (Torr.) I.M. Johnst. CHIH, COAH, DGO, NAY, NLE

Heliotropium pringlei B.L. Rob. AGS, CAM, CHIS, CHIH, CDMX, GTO, GRO, HGO, JAL, MEX, MICH, MOR, NAY, OAX, PUE, QRO, QROO, SLP, VER, YUC, ZAC

Heliotropium procumbens Mill. AGS, BCN, BCS, CAM, CHIS, CHIH, COAH, COL, DGO, GTO, GRO, HGO, JAL, MEX, MICH, MOR, NAY, NLE, OAX, PUE, QRO, QROO, SLP, SIN, SON, TAB, TAMS, VER, YUC, ZAC

*Heliotropium queretaroanum I.M. Johnst. GTO, QRO, SLP Heliotropium racemosum (Rose \& Standl.) I.M. Johnst. TAMS Heliotropium rufipilum (Benth.) I.M. Johnst. CHIS, COL, GRO, JAL, MEX, MICH, NAY, OAX, VER

*Heliotropium sessei I.M. Johnst. BCS, COL, HGO, JAL, OAX, QRO, VER, ZAC

Heliotropium ternatum Vahl CAM, CHIS, CHIH, COL, DGO, GTO, GRO, HGO, JAL, MICH, NAY, OAX, PUE, QRO, QROO, SLP, SIN, SON, VER, YUC

Heliotropium torreyi I.M. Johnst. CHIH, COAH, DGO, HGO, NLE, QRO, SLP, TAMS, VER, ZAC

Heliotropium transalpinum Vell. SLP, VER

*Heliotropium wigginsii I.M. Johnst. BCN, BCS, SON

Tournefortia acutiflora M. Martens \& Galeotti CAM, CHIS, COL, GRO, HGO, JAL, MEX, MICH, MOR, NAY, OAX, PUE, SLP, QROO, VER, YUC

Tournefortia belizensis Lundell CAM, GRO, QROO, VER Tournefortia bicolor Sw. CHIS, GRO, MICH, OAX, PUE, TAB, VER

*Tournefortia caeciliana Loes. CHIS

*Tournefortia calycina Benth. CHIS, GRO, JAL, MEX, MICH, OAX, SLP

Tournefortia calycosa (Donn. Sm.) D. Nash CHIS, SLP, VER Tournefortia elongata D.N. Gibson CAM, CHIS, GRO, HGO, JAL, NAY, OAX, PUE, QROO, VER

Tournefortia glabra L. CAM, CHIS, COL, GRO, HGO, JAL, 
MEX, MICH, NAY, OAX, PUE, QRO, QROO, SLP, TAB, VER, YUC

Tournefortia gnaphalodes (L.) R. Br. ex Roem. \& Schult. CAM, QROO, VER, YUC

*Tournefortia hartwegiana Steud. BCN, BCS, CAM, CHIS, CHIH, COL, DGO, GTO, GRO, HGO, JAL, MEX, MICH, MOR, NAY, OAX, PUE, QRO, QROO, SLP, SIN, SON, TAMS, VER, ZAC

Tournefortia hirsutissima L. CAM, CHIS, COL, DGO, GTO, GRO, HGO, JAL, MEX, MICH, MOR, NAY, OAX, PUE, QRO, QROO, SLP, SIN, TAB, TAMS, VER

Tournefortia johnstonii Standl. GRO

Tournefortia longiloba D.N. Gibson CHIS

Tournefortia maculata Jacq. CAM, CHIS, GRO, HGO, JAL, MEX, MICH, MOR, NAY, OAX, PUE, QRO, QROO, SLP, SIN, VER, YUC

Tournefortia mutabilis Vent. BCS, CHIS, COL, DGO, GRO, HGO, JAL, MEX, MICH, MOR, NAY, OAX, PUE, QRO, QROO, SLP, SIN, TAMS, VER

*Tournefortia pedicellata D. Nash GRO, PUE, QRO, VER

Tournefortia petiolaris DC. CHIS, COL, GRO, JAL, MEX, MICH, MOR, NAY, OAX, PUE, TAB, VER

*Tournefortia umbellata Kunth CAM, CHIS, GRO, OAX, QROO, TAB, YUC

Tournefortia volubilis L. BCS, CAM, CHIS, CHIH, COL, DGO, GTO, GRO, HGO, JAL, MEX, MICH, MOR, NAY, NLE, OAX, PUE, QRO, QROO, SLP, SIN, SON, TAMS, VER, YUC

\section{Family Hernandiaceae}

Gyrocarpus americanus Jacq. CHIS, OAX, PUE

Gyrocarpus jatrophifolius Domin CAM, CHIS, COL, DGO, GRO, JAL, MEX, MICH, MOR, NAY, OAX, PUE, QROO, SIN, VER, YUC, ZAC

Gyrocarpus mocinnoi Espejo CHIS, OAX, PUE

Hernandia didymantha Donn. Sm. CHIS

Hernandia sonora L. NAY, PUE, SLP, VER

Hernandia stenura Standl. CHIS, VER

Hernandia wendtii Espejo CHIS, OAX, PUE, VER

Sparattanthelium amazonum Mart. CHIS, OAX, TAB, VER, YUC

\section{Family Hydrangeaceae}

*Deutzia mexicana Hemsl. OAX, PUE, VER

*Deutzia occidentalis Standl. OAX, PUE

*Deutzia pringlei C.K. Schneid. NLE, SLP, TAMS

Fendlera falcata Thornber $\mathrm{CHIH}$

Fendlera linearis Rehder CHIH, COAH, DGO, NLE, SLP, TAMS

*Fendlera tamaulipana B.L. Turner TAMS

Fendlera wrightii (Engelm. \& A. Gray) A. Heller CHIH, COAH, NLE, SON

*Fendlerella lasiopetala Standl. COAH, NLE, SLP

* Fendlerella mexicana Brandegee HGO, PUE, QRO, SLP

* Fendlerella queretana B.L. Turner QRO

Fendlerella utahensis (S. Watson) Heller CHIH, COAH, NLE, SLP, SON

Hydrangea albostellata Samain, Najarro \& E. Martínez CHIS

*Hydrangea nebulicola Nevlin \& Gómez-Pompa HGO, PUE,
QRO, SLP, VER

*Hydrangea seemannii L. Riley CHIH, DGO, JAL, NAY, SIN, SON, ZAC

Hydrangea steyermarkii Standl. CHIS

Jamesia americana Torr. \& A. Gray CHIH, NLE

*Philadelphus affinis Schltdl. HGO, OAX, QRO, SLP, VER

*Philadelphus asperifolius Körn. CHIH, SLP

*Philadelphus bifidus (C.L. Hitchc.) S.Y. Hu COAH, NLE, TAMS

*Philadelphus calcicolus S.Y. Hu COAH, NLE, TAMS

*Philadelphus coulteri S. Watson COAH, GTO, HGO, NLE, QRO, SLP, TAMS, VER

Philadelphus hitchcockianus S.Y. Hu CHIH, COAH, NLE

*Philadelphus karwinskyanus Koehne MEX, MICH, OAX, VER

Philadelphus maculatus (C.L. Hitchc.) S.Y. Hu COAH, NLE, TAMS

Philadelphus mearnsii W.H. Evans ex Rydb. CHIH

Philadelphus mexicanus Schltdl. CHIS, COL, CDMX, GTO, GRO, HGO, JAL, MEX, MICH, MOR, OAX, PUE, QRO, SLP, VER

Philadelphus microphyllus A. Gray BCN, CHIH, COAH, DGO, NLE, SLP, SON

Philadelphus myrtoides Bertol. CHIS

Philadelphus occidentalis A. Nelson CHIH, COAH

*Philadelphus pringlei $\mathrm{S}$.Y. Hu COAH, NLE

*Philadelphus pueblanus S.Y. Hu HGO, MEX, MICH, MOR, PUE, SLP, TLAX, VER

Philadelphus pumilus Rydb. BCN

*Philadelphus sargentianus S.Y. Hu COAH, NLE

Philadelphus serpyllifolius A. Gray CHIH, COAH, SON

Philadelphus texensis S.Y. Hu COAH, DGO

\section{Family Hydrocharitaceae}

Halophila decipiens Ostenf. VER

Halophila engelmannii Asch. TAMS, VER

Limnobium laevigatum (Humb. \& Bonpl. ex Willd.) Heine CDMX, HGO, MEX, MICH, SLP, TAB, VER

Najas guadalupensis (Spreng.) Magnus AGS, BCN, BCS, CAM, CHIS, CHIH, COAH, COL, CDMX, DGO, GTO, GRO, HGO, JAL, MEX, MICH, MOR, NAY, NLE, OAX, PUE, QRO, SLP, SIN, SON, TAB, TAMS, VER, YUC, ZAC

Najas marina L. AGS, BCN, BCS, CAM, COAH, DGO, GRO, JAL, MEX, MICH, MOR, NAY, NLE, QROO, SIN, SON, TAMS, VER

Najas wrightiana A. Br. CAM, CHIS, QROO, TAB, TAMS, VER, YUC

Thalassia testudinum Banks. \& Sol. ex K.D. Koenig CAM, QROO, TAMS, VER, YUC

Vallisneria americana Michx. CAM, MEX, MOR, QROO, TAB, TAMS, VER

\section{Family Hydroleaceae}

Hydrolea ovata Nutt. ex Choisy GRO, OAX, VER

Hydrolea spinosa L. CAM, CHIS, COL, DGO, GRO, HGO, JAL, MEX, MICH, MOR, NAY, OAX, PUE, QRO, QROO, SLP, SIN, SON, TAB, TAMS, VER, YUC, ZAC 


\section{Family Hydrophyllaceae}

Nemophila menziesii Hook. \& Arn. BCN

Nemophila pedunculata Dougl. BCN

Nemophila spathulata Coville BCN

Phacelia affinis A. Gray BCN, BCS, SON

Phacelia alba Rydb. CHIH

*Phacelia altotonga B.L. Turner VER

Phacelia bombycina Wooton \& Standl. BCS, SON

Phacelia brachyloba (Benth.) A. Gray BCN

* Phacelia carmenensis B.L. Turner COAH

* Phacelia cedrosensis Rose BCN, BCS

Phacelia cicutaria Greene BCN

Phacelia ciliata Benth. BCN

Phacelia coerulea Greene CHIH, COAH, SON

Phacelia congesta Hook. AGS, CHIH, COAH, DGO, NLE, SON, TAMS, ZAC

Phacelia corrugata A. Nelson $\mathrm{CHIH}$

*Phacelia coulteri Greenm. CDMX, HGO, MEX, PUE, TLAX

Phacelia crenulata Torr. ex S. Watson BCN, BCS, SON

Phacelia cryptantha Greene BCN, SON

Phacelia distans Benth. BCN, BCS, CHIH, SON

Phacelia floribunda Greene BCN

Phacelia fremontii Torr. BCN

* Phacelia gentryi Constance CHIH, SON

Phacelia grandiflora (Benth.) A. Gray BCN

*Phacelia gypsogenia I.M. Johnst. CHIH, COAH, DGO, NLE, ZAC

Phacelia heterophylla Pursh BCN, CHIH, COAH, CDMX, DGO, GRO, HGO, MEX, MICH, NLE, PUE, SON, TLAX, VER

*Phacelia hintoniorum B.L. Turner NLE

* Phacelia hirtuosa A. Gray BCN, BCS

Phacelia imbricata Greene BCN

Phacelia infundibuliformis Torr. CHIH, COAH, DGO

Phacelia integrifolia S. Watson CHIH, COAH, TAMS

Phacelia ixodes Kellogg BCN, BCS

* Phacelia marshall-johnstonii N.D. Atwood \& Pinkava COAH

Phacelia minor (Harv.) Thell. BCN

Phacelia minutiflora Voss ex Munz BCN, BCS, SON

*Phacelia neffii B.L. Turner NLE

Phacelia neglecta M.E. Jones SON

Phacelia pachyphylla A. Gray BCN, SON

Phacelia pallida I.M. Johnst. CHIH, COAH

Phacelia parryi Torr. BCN

* Phacelia parviflora Pursh TAMS

Phacelia patuliflora (Engelm. \& A. Gray) A. Gray CHIH, COAH, DGO, NLE, SIN, TAMS

*Phacelia pauciflora S. Watson BCN, BCS

Phacelia pedicellata A. Gray BCN, SON

* Phacelia phyllomanica A. Gray BCN

Phacelia platycarpa (Cav.) Spreng. CHIS, CHIH, COAH, COL, CDMX, DGO, GTO, GRO, HGO, JAL, MEX, MICH, MOR, NAY, NLE, OAX, PUE, QRO, SIN, SON, TLAX, VER

Phacelia popei Torr. \& A. Gray CHIH, COAH, NLE, SON

* Phacelia potosina B.L. Turner NLE

Phacelia ramosissima Dougl. ex Lehm. BCN, SON
Phacelia robusta (J.F. Macbr.) I.M. Johnst. CHIH, COAH, DGO, NLE

Phacelia rupestris Greene CHIH, COAH, NLE

* Phacelia scariosa Brandegee BCN, BCS, SON

Phacelia secunda J.F. Gmel. COAH, NLE, SLP, SON

*Phacelia stellaris Brand BCN

Phacelia suffrutescens Parry BCN

Phacelia tanacetifolia Benth. BCN

Phacelia umbrosa Greene BCN

Phacelia viscida (Benth.) Torr. BCN

*Phacelia vossii N.D. Atwood NLE, SLP, TAMS, ZAC

* Phacelia zaragozana B.L. Turner NLE, SLP, TAMS

Pholistoma auritum (Lindl.) Lilja BCN, SON

Pholistoma membranaceum (Benth.) Constance BCN

Pholistoma racemosum (Nutt. ex A. Gray) Constance BCN

\section{Family Hypericaceae}

Hypericum anagalloides Cham. \& Schltdl. BCN, BCS

*Hypericum collinum Schltdl. \& Cham. HGO, OAX, PUE, TAMS, VER

*Hypericum eastwoodianum I.M. Johnst. COL, JAL, ZAC

Hypericum epigeium Keller CHIS

Hypericum formosum Kunth CHIS, CHIH, COAH, CDMX, DGO, GTO, HGO, JAL, MEX, MICH, MOR, NAY, NLE, OAX, PUE, QRO, SLP, SIN, SON, TAMS, VER, ZAC

*Hypericum galinum S.F. Blake GTO, MICH, OAX, QRO, SLP Hypericum gnidioides Seem. GRO, MEX

Hypericum gymnanthum Engelm. \& A. Gray CHIS, PUE, VER Hypericum hypericoides (L.) Crantz CHIS, HGO, NLE, OAX, PUE, QRO, SLP, TAMS, VER

Hypericum macvaughii N. Robson CHIH, DGO, NLE, SIN, TAMS

Hypericum mutilum L. CHIH, DGO, MICH, OAX, PUE, VER Hypericum oaxacanum R. Keller CHIS, GRO, MEX, MICH, OAX, PUE, VER

*Hypericum paniculatum Lam. JAL, MICH, OAX

*Hypericum parvulum Greene BCS, DGO, JAL, ZAC

Hypericum pauciflorum Kunth AGS, CHIS, CHIH, COL, CDMX, DGO, GTO, GRO, HGO, JAL, MEX, MICH, MOR, NAY, NLE, OAX, QRO, SLP, SIN, SON, TAMS, VER, ZAC

*Hypericum peninsulare Eastw. BCS

Hypericum philonotis Schltdl. \& Cham. COL, CDMX, GTO, HGO, JAL, MEX, MICH, MOR, OAX, PUE, QRO, TLAX, VER

*Hypericum pratense Schltdl. \& Cham. CHIS, CHIH, GRO, OAX, PUE, SON, VER, ZAC

Hypericum punctatum Lam. AGS, CHIH, COAH, NLE, SON, TAMS, ZAC

*Hypericum seleri Keller VER

Hypericum silenoides Juss. AGS, BCS, CHIS, CHIH, COL, CDMX, DGO, GTO, GRO, HGO, JAL, MEX, MICH, MOR, NAY, OAX, PUE, QRO, SLP, SIN, SON, TAMS, TLAX, VER, ZAC

Hypericum sphaerocarpum Michx. AGS, JAL, MOR

Hypericum steyermarkii Standl. CHIS

* Hypericum umbraculoides N. Robson OAX

Thornea calcicola (Standl. \& Steyerm.) Breedlove \& E.M. 
McClint. CHIS

Thornea matudae (Lundell) Breedlove \& E.M. McClint. CHIS Vismia baccifera (L.) Triana \& Planch. CHIS, GRO, OAX, PUE, TAB, VER

Vismia camparaguey Sprague \& L. Riley CHIS, OAX, PUE, TAB, VER

\section{Family Hypoxidaceae}

Curculigo scorzonerifolia (Lam.) Baker CAM, CHIS, JAL, OAX, TAB, VER, YUC

*Hypoxis colliculata Sánchez-Ken CHIS, OAX

Hypoxis decumbens L. AGS, CHIS, CHIH, COL, CDMX, DGO, GTO, GRO, HGO, JAL, MEX, MICH, MOR, NAY, NLE, OAX, PUE, QRO, SLP, SON, TAB, TAMS, VER, ZAC

Hypoxis fibrata Brackett AGS, CHIH, COL, CDMX, DGO, GTO, GRO, JAL, MEX, MICH, MOR, OAX, PUE, QRO, SLP, VER, ZAC

Hypoxis hirsuta (L.) Coville CHIH, DGO, SON

Hypoxis humilis Kunth CHIS, GRO, HGO, JAL, MEX, OAX, VER

*Hypoxis lucens McVaugh COL, GTO, JAL, MICH, MOR, ZAC

Hypoxis mexicana Schult. \& Schult. f. AGS, CHIS, CHIH, COAH, COL, CDMX, DGO, GTO, GRO, HGO, JAL, MEX, MICH, MOR, NAY, NLE, OAX, PUE, QRO, SLP, SIN, SON, TAMS, TLAX, VER, ZAC

Hypoxis micrantha Pollard OAX

*Hypoxis pulchella G.L. Nesom COAH, NLE, TAMS

*Hypoxis tepicensis Brackett COL, GTO, GRO, MEX, MOR, NAY, OAX, SIN

\section{Family Icacinaceae}

Calatola costaricensis Standl. CHIS, OAX, VER

Calatola laevigata Standl. CHIS, COL, JAL, OAX, PUE, VER

* Calatola mollis Standl. CHIS, OAX, PUE, VER

Calatola uxpanapensis Vera-Caletti \& T. Wendt VER

*Mappia longipes Lundell CHIS, VER

* Mappia mexicana B.L. Rob. \& Greenm. SLP, TAMS, VER

Mappia racemosa Jacq. CHIS, OAX, PUE, TAB, VER

Oecopetalum greenmanii Standl. \& Steyerm. CHIS, TAB

Oecopetalum mexicanum Greenm. \& C.H. Thomps. CHIS, OAX, TAB, VER

Ottoschulzia pallida Lundell CAM, CHIS, QROO

\section{Family Iridaceae}

*Ainea conzattii (R.C. Foster) Ravenna OAX

Alophia drummondii (Graham) R.C. Foster CAM, CHIS, COAH, JAL, NLE, OAX, QRO, QROO, SLP, TAB, TAMS, VER, YUC

*Alophia intermedia (Ravenna) Goldblatt NAY, SIN

Alophia silvestris (Loes.) Goldblatt CAM, CHIS, QROO, TAB, VER, YUC

*Alophia veracruzana Goldblatt \& T.M. Howard OAX, VER

*Cardiostigma hintonii (R.C. Foster) Ravenna COL, JAL, $\mathrm{MICH}$

*Cardiostigma longispatha (Herb.) Baker DGO, GRO, JAL, MEX, MICH, NAY, OAX, ZAC

*Cardiostigma mexicana (Goldblatt \& Henrich) Ravenna GRO,
MEX, MICH

Cipura campanulata Ravenna CAM, CHIS, GRO, JAL, MEX, MICH, MOR, NAY, OAX, QROO, SLP, SIN, TAB, TAMS, VER, YUC

Cipura paludosa Aubl. CAM, CHIS, COL, GRO, JAL, MEX, MICH, MOR, NAY, OAX, QROO, SIN, SON, TAB, TAMS, VER, YUC, ZAC

* Colima convoluta (Ravenna) Aarón Rodr. \& Ortiz-Catedral COL, GRO, JAL

* Colima tuitensis Aarón Rodr. \& Ortiz-Catedral JAL

*Cypella mexicana C.V. Morton \& R.C. Foster COL, GRO, JAL, MICH

* Cypella rosei R.C. Foster JAL, NAY, SIN

Eleutherine bulbosa (Mill.) Urb. CHIS, QRO, SLP, VER

Eleutherine latifolia (Standl. \& L.O. Williams) Ravenna CHIS, GTO, MEX, NAY, OAX, QRO, SLP, VER

*Fosteria oaxacana Molseed OAX

Iris versicolor L. COAH, NLE, SON

Nemastylis tenuis (Herb.) S. Watson AGS, CHIH, COAH, CDMX, GTO, DGO, GRO, HGO, JAL, MEX, MICH, MOR, NAY, NLE, OAX, PUE, QRO, SLP, SIN, SON, TAMS, TLAX, VER, ZAC

* Nemastylis versicolor $\mathrm{S}$. Watson JAL, MICH, TAB

Neomarica variegata (M. Martens \& Galeotti) Henrich \& Goldblatt CHIS, HGO, OAX, PUE, TAB, VER

Orthrosanthus exsertus (R.C. Foster) Ravenna CHIS, CDMX, DGO, GRO, HGO, JAL, MEX, MICH, MOR, NLE, OAX, PUE, QRO, SLP, TAMS, VER

Orthrosanthus monadelphus Ravenna CHIS, HGO, OAX, VER

* Sessilanthera citrina Cruden GRO, MOR, PUE

* Sessilanthera heliantha (Ravenna) Cruden CHIS, GRO

Sessilanthera latifolia (Weath.) Molseed \& Cruden GRO, OAX

* Sisyrinchium abietum McVaugh COL, GRO, JAL

*Sisyrinchium alatum Hook. CHIS, HGO, OAX, VER

Sisyrinchium angustifolium Mill. COAH, CDMX, DGO, HGO, JAL, MEX, MICH, MOR, NAY, NLE, OAX, QRO, SIN, TAMS, VER, ZAC

*Sisyrinchium angustissimum (B.L. Rob. \& Greenm.) Greenm. \& C.H. Thomps. CHIS, CDMX, GTO, GRO, HGO, MEX, MICH, MOR, OAX, PUE, QRO, SLP, TAMS, TLAX, VER

*Sisyrinchium arguellesiae Ceja, Espejo \& López-Ferrari NLE, QRO, SLP, TAMS

Sisyrinchium arizonicum Rothr. CHIS, CHIH, COAH, CDMX, DGO, GRO, HGO, MEX, MICH, NLE, OAX, QRO, SLP, SIN, SON, VER

Sisyrinchium bellum S. Watson BCN, BCS

Sisyrinchium biforme E.P. Bicknell CHIS, COAH, GTO, NLE, OAX, SLP, TAMS, VER

*Sisyrinchium bracteatum Greenm. AGS, CDMX, MEX, NLE, OAX, SLP, TLAX

Sisyrinchium cernuum (E.P. Bicknell) Kearney AGS, CHIH, COAH, COL, CDMX, DGO, GTO, HGO, JAL, MEX, MICH, MOR, NAY, PUE, QRO, SLP, SIN, SON, TLAX, VER, ZAC Sisyrinchium convolutum Nocca AGS, CHIS, CHIH, COAH, COL, CDMX, DGO, GTO, GRO, HGO, JAL, MEX, MICH, MOR, NAY, NLE, OAX, PUE, QRO, SLP, SIN, SON, TLAX, VER, ZAC 
*Sisyrinchium conzattii Calderón \& Rzed. CDMX, MEX, $\mathrm{MICH}$

*Sisyrinchium coulterianum Klatt ex Baker GRO, HGO, MEX, $\mathrm{MICH}$

Sisyrinchium chiricanum Woodson CHIS

*Sisyrinchium cholewae Espejo, López-Ferrari \& Ceja DGO

Sisyrinchium demissum Greene BCS, CHIH, COAH, NLE, SON

Sisyrinchium elmeri Greene BCS, CHIH, DGO, SON

Sisyrinchium exalatum B.L. Rob. \& Greenm. CDMX, GRO, MEX, OAX

*Sisyrinchium guanajuatense Ceja, Espejo \& López-Ferrari GTO, JAL, SLP

*Sisyrinchium hintoniorum G.L. Nesom NLE, SLP, TAMS

Sisyrinchium johnstonii Standl. CHIS, GRO, OAX

*Sisyrinchium longispathum Conz. CHIS, GRO, OAX, PUE

*Sisyrinchium macrophyllum Greenm. CDMX, DGO, GTO, GRO, JAL, MEX, MICH, MOR, OAX, QRO, SLP

Sisyrinchium micranthum Cav. CHIS, CHIH, GRO, NLE, OAX, PUE, VER

* Sisyrinchium microbracteatum G.L. Nesom NLE, TAMS

*Sisyrinchium novoleonense G.L. Nesom \& L. Hern. COAH, NLE, SLP, TAMS

*Sisyrinchium parvum (E.P. Bicknell) Espejo \& López Ferrari COAH

*Sisyrinchium planicola Ceja \& Cholewa OAX

*Sisyrinchium platyphyllum S. Watson COL, JAL, ZAC

*Sisyrinchium polycladon B.L. Rob. \& Greenm. GRO, OAX

*Sisyrinchium pringlei B.L. Rob. \& Greenm. AGS, CHIH, COL, CDMX, DGO, GTO, JAL, MEX, MICH, NAY, SIN, SON, ZAC *Sisyrinchium quadrangulatum Klatt COL, CDMX, JAL, MEX, MICH, SLP, TAMS, VER

Sisyrinchium rosulatum E.P. Bicknell AGS, CHIH, HGO, TAMS, VER, ZAC

Sisyrinchium scabrum Cham. \& Schltdl. AGS, BCS, CHIS, CHIH, COAH, CDMX, DGO, GTO, GRO, HGO, JAL, MEX, MICH, MOR, NAY, NLE, OAX, PUE, QRO, SLP, SIN, SON, TAMS, TLAX, VER, ZAC

*Sisyrinchium schaffneri S. Watson AGS, BCS, CHIH, COAH, COL, CDMX, GTO, GRO, HGO, JAL, MEX, MICH, MOR, NAY, NLE, OAX, PUE, QRO, SLP, TAMS, VER

*Sisyrinchium serrulatum (E.P. Bicknell) Espejo \& López Ferrari GTO, MEX, MICH, OAX, QRO, VER

Sisyrinchium subalpinum Henrich \& Goldblatt CHIS

Sisyrinchium subcernuum (E.P. Bicknell) Henrich \& Goldblatt BCS, COAH, DGO, OAX

Sisyrinchium tenuifolium Humb. \& Bonpl. ex Willd. AGS, CHIS, CHIH, COAH, CDMX, DGO, GTO, GRO, HGO, JAL, MEX, MICH, MOR, NAY, NLE, OAX, PUE, QRO, SLP, TLAX, VER, ZAC

Sisyrinchium tinctorium Kunth CHIS, CHIH, GRO, HGO, MOR, OAX, SON, VER

*Sisyrinchium tolucense Peyr. AGS, CHIH, COL, CDMX, DGO, GTO, GRO, HGO, JAL, MEX, MICH, MOR, NAY, OAX, PUE, SIN, SON, VER, ZAC

*Sisyrinchium translucens (E.P. Bicknell) Espejo \& López Ferrari BCS

*Tigridia alpestris Molseed GTO, GRO, HGO, JAL, MEX,
MICH, QRO, VER

*Tigridia amatlanensis Aarón Rodr. \& García-Mend. OAX

*Tigridia augusta Drapiez AGS, COL, GTO, JAL, MEX, MICH, NAY, PUE, ZAC

*Tigridia bicolor Molseed OAX

*Tigridia catarinensis Cruden QRO, SLP

*Tigridia chiapensis Molseed ex Cruden CHIS

*Tigridia chrysantha Cruden JAL

*Tigridia dugesii S. Watson AGS, DGO, GTO, JAL, MICH, ZAC

*Tigridia durangense Molseed ex Cruden DGO, MEX, MICH, SIN

*Tigridia ehrenbergii (Schltdl.) Molseed COL, GTO, GRO, HGO, JAL, MEX, MICH, MOR, OAX, PUE, QRO, SLP, TLAX, VER

*Tigridia estelae López-Ferrari \& Espejo DGO, SIN

*Tigridia flammea (Lindl.) Ravenna GRO, MEX, MICH

*Tigridia galanthoides Molseed GRO, OAX, PUE, VER

*Tigridia gracielae Aarón Rodr. \& Ortiz-Catedral GRO, MEX

*Tigridia hallbergii Molseed CHIS, GRO, JAL, MEX, OAX, ZAC

*Tigridia hintonii Molseed GRO

*Tigridia huajuapanensis Molseed ex Cruden OAX, PUE

*Tigridia illecebrosa Cruden GRO, OAX

Tigridia immaculata (Herb.) Ravenna CHIS, OAX

*Tigridia inusitata (Cruden) Ravenna GRO, OAX

*Tigridia mariaetrinitatis Espejo \& López-Ferrari OAX

*Tigridia martinezii Calderón HGO, VER

*Tigridia matudae Molseed MEX, MOR

Tigridia meleagris (Lindl.) G. Nicholson AGS, COL, DGO, GRO, HGO, JAL, MEX, MICH, MOR, NAY, OAX, SIN, TLAX, VER, ZAC

*Tigridia mexicana Molseed COL, JAL, MEX, MICH, NAY, SIN, ZAC

Tigridia molseediana Ravenna OAX

*Tigridia mortonii Molseed GRO, MEX, MOR

*Tigridia multiflora (Herb.) Ravenna AGS, CHIH, COL, CDMX, DGO, GTO, GRO, HGO, JAL, MEX, MICH, MOR, NLE, PUE, QRO, SLP, TAMS, VER, ZAC

Tigridia orthantha (Lem.) Ravenna CHIS, GRO, OAX

Tigridia pavonia (L. f.) DC. CHIS, CHIH, COAH, COL, CDMX, DGO, GTO, GRO, HGO, JAL, MEX, MICH, MOR, NAY, NLE, OAX, PUE, QRO, SLP, SIN, SON, TAMS, TLAX, VER, ZAC

*Tigridia potosina López-Ferrari \& Espejo SLP

*Tigridia pugana Aarón Rodr. \& L. Ortiz-Catedral COL, JAL

*Tigridia pulchella B.L. Rob. COL, JAL, MICH

*Tigridia purpusii Molseed PUE

*Tigridia rzedowskiana Aarón Rodr. \& L. Ortiz-Catedral QRO, SLP

*Tigridia seleriana (Loes.) Ravenna OAX

*Tigridia suarezii Aarón Rodr. \& Ortiz-Catedral COL, JAL

*Tigridia tepoxtlana Ravenna MEX, MOR

*Tigridia vanhouttei Roezl ex Van Houtte CDMX, GTO, HGO, MEX, MICH, MOR, PUE, QRO, SLP, TAMS, TLAX

*Tigridia venusta Cruden MEX, MICH

Trimezia martinicensis (Jacq.) Herb. BCN, CHIS 
*Trimezia sobolifera Ravenna OAX, VER

Trimezia steyermarkii R.C. Foster CHIS, OAX, PUE, VER

Family Iteaceae

*Pterostemon bravoanus J. Jiménez Ram. \& Mart. Gord. GRO, OAX

*Pterostemon mexicanus S. Schauer GTO, HGO, QRO, SLP, VER

*Pterostemon rotundifolius Ramírez OAX, PUE

\section{Family Juglandaceae}

Alfaroa costaricensis Standl. CHIS, VER

Alfaroa guatemalensis (Standl.) L.O. Williams \& Ant. Molina CHIS

Alfaroa mexicana D.E. Stone CHIS, GRO, HGO, OAX, VER Carya illinoinensis (Wangenh.) K. Koch CHIH, COAH, COL, DGO, GTO, HGO, JAL, NLE, OAX, PUE, QRO, SLP, TAMS, VER

Carya myristiciformis (Michx. f.) Nutt. COAH, NLE, SLP, TAMS

Carya oliviformis (Marshall) Nutt. CHIH, HGO, NLE, SLP, TAMS

Carya ovata (Mill.) K. Koch COAH, GTO, HGO, MICH, NLE, PUE, QRO, SLP, TAMS, VER

* Carya palmeri W.E. Manning COAH, HGO, NLE, OAX, QRO, SLP, TAMS, VER

*Juglans hirsuta W.E. Manning COAH, HGO, JAL, NLE, PUE, QRO, SLP, VER, ZAC

Juglans major (Torr.) A. Heller CHIH, COAH, COL, DGO, GTO, GRO, HGO, JAL, MEX, MICH, MOR, NAY, NLE, OAX, QRO, SIN, SON, TAMS, VER

Juglans microcarpa Berl. CHIH, COAH, NLE

*Juglans mollis Engelm. COAH, GTO, GRO, HGO, JAL, MEX, MOR, NLE, OAX, PUE, QRO, SLP, TAMS, VER

Juglans olanchana Standl. \& L.O. Williams CHIS, COL, JAL, PUE, VER

*Juglans pyriformis Liebm. CHIS, CHIH, DGO, GRO, HGO, JAL, MEX, MICH, OAX, PUE, SON, TAMS, VER

Oreomunnea mexicana (Standl.) J.-F. Leroy CHIS, OAX, VER

\section{Family Juncaceae}

Juncus acuminatus Michx. AGS, CHIS, CHIH, COAH, CDMX, DGO, GTO, GRO, HGO, JAL, MEX, MICH, NAY, OAX, QRO, SLP, SON, VER, ZAC

Juncus acutus L. BCN, BCS, SON

Juncus aemulans Liebm. CHIS, CDMX, HGO, MEX, OAX, VER

Juncus articulatus L. BCS, CHIH

Juncus balticus Willd. BCN, BCS, CHIS, CHIH, COAH, CDMX, DGO, GTO, HGO, JAL, MEX, MICH, MOR, NLE, OAX, PUE, QRO, QROO, SLP, SIN, SON, TAMS, TLAX, VER, ZAC

Juncus bufonius L. BCN, CHIS, CHIH, COAH, CDMX, GTO, HGO, MEX, MICH, OAX, PUE, QRO, SLP, SON, TLAX, VER Juncus confusus Coville CHIH, SON

Juncus cooperi Engelm. BCN, SON

* Juncus chiapasensis Balslev CHIS

Juncus debilis A. Gray CHIS, OAX
Juncus dichotomus Elliott AGS, CHIS, CHIH, COAH, CDMX, DGO, GTO, HGO, JAL, MEX, MICH, OAX, PUE, QRO, SLP, SIN, SON, TAMS, TLAX, VER, ZAC

Juncus dubius Engelm. BCN

*Juncus dudleyi Wiegand COAH

Juncus ebracteatus E. Mey. AGS, CHIS, CHIH, CDMX, DGO, GTO, GRO, HGO, JAL, MEX, MICH, MOR, OAX, PUE, QRO, SLP, SON, TAMS, TLAX, VER, ZAC

Juncus effusus L. AGS, BCN, CHIS, CHIH, COL, CDMX, DGO, GTO, GRO, HGO, JAL, MEX, MICH, MOR, OAX, PUE, QRO, SLP, SIN, TLAX, VER, ZAC

Juncus ensifolius Wikstr. CHIH, COAH, SLP, SON, VER

Juncus imbricatus Laharpe DGO, HGO, MEX, OAX, TLAX, VER

Juncus interior Wiegand CHIH, COAH, SON

Juncus kelloggii Engelm. BCN

Juncus liebmannii J.F. Macbr. CHIS, CHIH, COL, CDMX, DGO, GTO, HGO, JAL, MEX, MOR, NAY, OAX, PUE, SLP, SIN, SON.VER, ZAC

Juncus longistylis Torr. CHIH, SON

Juncus macrophyllus Coville BCN

Juncus marginatus Rostk. BCS, CHIS, CHIH, DGO, GTO, GRO, JAL, MEX, MICH, NAY, OAX, SLP, SON, TAB, TAMS, VER, ZAC

Juncus microcephalus Kunth AGS, CHIS, CHIH, COL, CDMX, DGO, GTO, GRO, HGO, JAL, MEX, MICH, MOR, NAY, OAX, PUE, QRO, SLP, TLAX, VER, ZAC

*Juncus nevadensis $\mathrm{S}$. Watson CHIH, SON

Juncus nodosus L. CHIH, COAH, DGO, NLE, SLP, SON

Juncus oxymeris Engelm. BCN

Juncus patens $\mathrm{E}$. Mey. BCN

Juncus repens Michx. TAB

Juncus rugulosus Engelm. BCN

Juncus saximontanus A. Nelson CHIH, COAH, DGO, SON

Juncus tenuis Willd. AGS, CHIS, CHIH, COAH, CDMX, DGO, GTO, GRO, HGO, JAL, MEX, MICH, NAY, NLE, OAX, PUE, QRO, SLP, SIN, SON, TLAX, VER, ZAC

Juncus texanus (Engelm.) Cov. COAH, NLE

Juncus textilis Buchenau BCN

Juncus tiehmii Ertter BCN

Juncus torreyi Coville BCN, CHIH, COAH, SON

Juncus xiphioides E. Mey. BCN, CHIH, HGO, JAL, MEX

Luzula caricina E. Mey. CHIS, CHIH, COL, CDMX, DGO, GRO, HGO, JAL, MEX, MICH, MOR, OAX, PUE, SIN, VER Luzula comosa E. Mey. BCN, CHIH, DGO

Luzula denticulata Liebm. CHIS, CHIH, CDMX, DGO, GRO, HGO, JAL, MEX, MICH, MOR, OAX, PUE, SIN, SON, TLAX, VER, ZAC

Luzula racemosa Desv. CHIS, CHIH, CDMX, DGO, HGO, JAL, MEX, MICH, MOR, PUE, SIN, TLAX, VER

Luzula vulcanica Liebm. PUE, VER

Family Juncaginaceae

Triglochin maritima L. BCN, BCS, CHIH, MEX

Triglochin mexicana Kunth BCN, BCS, CDMX, GTO, MEX, MICH, PUE, TLAX

Triglochin scilloides (Poir.) Mering \& Kadereit AGS, BCN, 
CHIH, DGO, GTO, GRO, HGO, JAL, MEX, MICH, MOR, PUE, QRO, SLP, SON, TLAX, VER, ZAC

\section{Family Koeberliniaceae}

Koeberlinia spinosa Zucc. AGS, BCN, BCS, CHIH, COAH, DGO, GTO, HGO, NLE, PUE, QRO, SLP, SON, TAMS, VER, ZAC

\section{Family Krameriaceae}

Krameria bicolor S. Watson AGS, BCN, BCS, CHIH, COAH, DGO, HGO, JAL, MICH, NAY, NLE, QRO, SLP, SIN, SON, TAMS, ZAC

* Krameria cytisoides Cav. COAH, GTO, HGO, NLE, OAX, PUE, QRO, SLP, TAMS, VER, ZAC

Krameria erecta Willd. ex Schult. AGS, BCN, BCS, CHIH, COAH, DGO, JAL, NLE, SLP, SIN, SON, ZAC

Krameria ixine L. BCS, COL, DGO, GRO, JAL, MICH, NAY, OAX, SIN, SON

Krameria lanceolata Torr. AGS, CHIH, COAH, NLE, SLP, TAMS, ZAC

Krameria paucifolia (Rose) Rose BCS, CHIH, COAH, DGO, GTO, HGO, JAL, MICH, NLE, OAX, PUE, QRO, SLP, SON, ZAC

Krameria ramosissima (A. Gray) S. Watson AGS, BCN, COAH, JAL, NLE, SON, TAMS

Krameria revoluta O. Berg CHIS, GRO, MEX, OAX, PUE, VER, ZAC

Krameria secundiflora Moc. \& Sessé ex DC. AGS, CHIS, CHIH, COAH, CDMX, DGO, GTO, GRO, HGO, JAL, MEX, MICH, MOR, NAY, OAX, PUE, QRO, SLP, SIN, SON, TLAX, ZAC

\section{Family Lacistemataceae}

Lacistema aggregatum (P.J. Bergius) Rusby CHIS, GRO, OAX, PUE, SIN, TAB, TAMS, VER

\section{Family Lamiaceae}

Acanthomintha ilicifolia (A. Gray) A. Gray BCN

Aegiphila costaricensis Moldenke CHIS, OAX, TAB, VER

Aegiphila deppeana Steud. CHIS, COL, HGO, JAL, NAY, OAX,

PUE, TAB, TAMS, VER

Aegiphila elata Sw. CHIS, OAX, TAB, VER

Aegiphila falcata Donn. Sm. CHIS, VER

Aegiphila martinicensis Jacq. CHIS

Aegiphila monstrosa Moldenke CAM, CHIS, OAX, QROO,

TAB, VER

Aegiphila panamensis Moldenke CHIS

Aegiphila pauciflora Standl. CAM, QROO, YUC

*Aegiphila skutchii Moldenke CHIS, COL, JAL

Aegiphila valerioi Standl. CHIS

*Aegiphila wigandioides Lundell CHIS

*Agastache aurantiaca Linton \& Epling DGO

Agastache cana (Hook.) Wooton \& Standl. CHIH, SON

* Agastache coccinea (Greene) Lint \& Epling DGO, SIN

*Agastache eplingiana R.W. Sanders DGO

Agastache mearnsii Wooton \& Standl. CHIH, DGO

Agastache mexicana (Kunth) Link \& Epling CDMX, GTO, HGO, MEX, MICH, MOR, PUE, QRO, SLP, TAMS, TLAX, VER, ZAC

Agastache micrantha (A. Gray) Wooton \& Standl. CHIH,

\section{COAH, DGO, SON}

*Agastache palmeri (B.L. Rob.) Standl. CHIH, COAH, GTO, HGO, JAL, MEX, MICH, NLE, PUE, QRO, SLP, SON, VER Agastache pallida (Lindl.) Cory CHIH, DGO, MICH, SON Agastache pallidiflora (Heller) Rydb. CHIH, COAH, NLE, SON *Agastache pringlei (Briq.) Lint \& Epling CHIH Agastache wrightii (Greenm.) Wooton \& Standl. CHIH, SON Asterohyptis mociniana (Benth.) Epling CHIS, COL, DGO, GRO, JAL, MICH, MEX, MOR, NAY, OAX, QRO, SLP, SIN, VER, ZAC

*Asterohyptis nayarana B.L. Turner DGO, NAY

*Asterohyptis seemannii (A. Gray) Epling CHIH, DGO, NAY, SIN, SON

*Asterohyptis stellulata (Benth.) Epling CHIS, CHIH, COL, DGO, GTO, GRO, JAL, MEX, MICH, MOR, NAY, OAX, PUE, SIN, SON, VER, ZAC

Callicarpa acuminata Kunth CAM, CHIS, COAH, HGO, JAL, MICH, NLE, OAX, PUE, QRO, QROO, SLP, TAB, TAMS, VER, YUC

*Callicarpa parvifolia Hook. \& Arn. NAY

*Callicarpa subpubescens Hook. \& Arn. NAY

Cantinoa americana (Aubl.) Harley \& J.F.B. Pastore CAM, CHIS, GRO, JAL, MEX, MOR, OAX, QROO, SIN, VER, YUC Cantinoa mutabilis (Rich.) Harley \& J.F.B. Pastore CAM, CHIS, CHIH, COAH, COL, DGO, GTO, GRO, HGO, JAL, MEX, MICH, MOR, NAY, NLE, OAX, PUE, QRO, QROO, SLP, SIN, SON, TAB, TAMS, VER, ZAC

* Cantinoa pinetorum (Epling) Harley \& J.F.B. Pastore JAL

Catoferia capitata Benth. ex Hemsl. CHIS, VER

Catoferia chiapensis A. Gray ex Benth. CHIS

Catoferia martinezii Ramamoorthy CHIS, GRO

*Clerodendrum moldenkeanum Standl. CHIS

*Clinopodium amissum (Jiménez) Harley SIN

Clinopodium brownei (Sw.) Kuntze BCS, CHIS, COAH, GRO, HGO, JAL, NLE, PUE, QRO, SLP, SON, TAMS, VER, YUC Clinopodium chandleri (Brandegee) P.D. Cantino \& Wagstaff BCN

*Clinopodium ganderi (Epling) Govaerts BCN

*Clinopodium hintoniorum (B.L. Turner) Govaerts NLE, TAMS

*Clinopodium jaliscanum (McVaugh \& Schmid) Govaerts COL, JAL

Clinopodium ludens (Shinners) A. Pool CAM, QROO, YUC

*Clinopodium macrostemum (Moc. \& Sessé ex Benth.) Kuntze COL, CDMX, GTO, GRO, HGO, JAL, MEX, MICH, MOR, OAX, PUE, QRO, SIN, TLAX, VER

*Clinopodium maderensis (Henr.) Govaerts COAH

*Clinopodium mexicanum (Benth.) Govaerts CHIS, GTO, GRO, HGO, JAL, OAX, PUE, QRO, SLP, VER

*Clinopodium micromerioides (Hemsl.) Govaerts SLP

*Clinopodium palmeri (A. Gray) Kuntze BCN

*Clinopodium procumbens (Greenm.) Harley \& Jativa CHIS, HGO, OAX, PUE, QRO, VER

Clinopodium selerianum (Loes.) Govaerts CHIS, GRO

*Condea albida (Kunth) Harley \& J.F.B. Pastore AGS, BCS, CHIS, CHIH, COL, DGO, GTO, GRO, HGO, JAL, MEX, MICH, NAY, OAX, PUE, QRO, SLP, SIN, SON, TAMS, VER, $\mathrm{ZAC}$ 
*Condea anitae (Epling \& Játiva) Harley \& J.F.B. Pastore BCN, $\mathrm{BCS}$

*Condea decipiens (M.E. Jones) Harley \& J.F.B. Pastore BCN, BCS

Condea emoryi (Torr.) Harley \& J.F.B. Pastore BCN, BCS, CHIH, JAL, MICH, NAY, SIN, SON

*Condea iodantha (Epling) Harley \& J.F.B. Pastore GRO, NAY, $\mathrm{SON}$

* Condea laniflora (Benth.) Harley \& J.F.B. Pastore BCN, BCS

* Condea mixta (Epling) Harley \& J.F.B. Pastore OAX

Condea scandens (Epling) Harley \& J.F.B. Pastore CAM, CHIS, QROO

Condea subtilis (Epling) Harley \& J.F.B. Pastore JAL, MEX, MICH, OAX

*Condea tephrodes (A. Gray) Harley \& J.F.B. Pastore BCS, NAY, SIN

*Condea tomentosa (Poit.) Harley \& J.F.B. Pastore BCS, CHIS, GRO, HGO, JAL, OAX, PUE, QRO, VER, ZAC

Condea verticillata (Jacq.) Harley \& J.F.B. Pastore CAM, CHIS, COL, HGO, JAL, MICH, NAY, OAX, PUE, QRO, QROO, SLP, TAB, TAMS, VER, YUC

Cornutia pyramidata L. CAM, CHIS, OAX, PUE, QROO, TAB, VER, YUC

* Cunila crenata García-Peña \& Tenorio DGO, JAL, ZAC

* Cunila jaliscana García-Peña \& J.G. González JAL

Cunila leucantha Kunth ex Schltdl. \& Cham. CHIS, GRO, OAX, VER

*Cunila lythrifolia Benth. AGS, COL, CDMX, DGO, GRO, HGO, JAL, MEX, MICH, MOR, PUE, SIN, TLAX, VER

Cunila polyantha Benth. CHIS, COL, GTO, GRO, JAL, OAX, ZAC

*Cunila pycnantha B.L. Rob. \& Greenm. AGS, COL, GRO, JAL, MEX, MICH, MOR, NAY, OAX, ZAC

* Cunila ramamoorthiana M.R. García-Peña GRO

Hedeoma acinoides Scheele CHIH, COAH, NLE, TAMS

*Hedeoma bella (Epling) R.S. Irving JAL

*Hedeoma ciliolata (Epling) R.S. Irving COAH, NLE, SLP, TAMS

Hedeoma costata A. Gray AGS, CHIS, CHIH, COAH, DGO, HGO, JAL, MEX, MOR, NAY, NLE, PUE, QRO, SLP, TAMS, VER, ZAC

*Hedeoma chihuahuensis (Henr.) B.L. Turner CHIH, COAH

Hedeoma dentata Torr. SON

Hedeoma drummondii Benth. CHIH, COAH, DGO, HGO, NLE, QRO, SLP, SON, TAMS, VER

*Hedeoma floribunda Standl. CHIH, SON

Hedeoma hyssopifolia A. Gray SON

*Hedeoma irvingii B.L. Turner COAH, NLE

*Hedeoma johnstonii R.S. Irving COAH

*Hedeoma jucunda Greene DGO, SIN

*Hedeoma martirensis Moran BCN

*Hedeoma microphylla R.S. Irving COAH, PUE, SLP

Hedeoma mollis Torr. CHIH

*Hedeoma montana Brandegee COAH, DGO, NLE

Hedeoma nana (Torr.) Briq. AGS, BCN, CHIH, COAH, DGO, GTO, NLE, QRO, SLP, SON, ZAC

*Hedeoma oblatifolia Villarreal COAH
Hedeoma oblongifolia (A. Gray) Heller AGS, CHIH, SON, $\mathrm{ZAC}$

*Hedeoma palmeri Hemsl. COAH, GTO, HGO, NLE, QRO, SLP, TAMS

*Hedeoma patens M.E. Jones CHIH, DGO, NAY, SIN, SON, ZAC

*Hedeoma patrina Stewart AGS, CHIH, COAH, DGO, NLE, SLP, ZAC

Hedeoma piperita Benth. AGS, CHIS, CDMX, DGO, HGO, JAL, MEX, MICH, MOR, PUE, TLAX, VER, ZAC

Hedeoma plicata Torr. CHIH, COAH, DGO, NLE, SLP, ZAC

*Hedeoma pusilla (R.S. Irving) R.S. Irving COAH, NLE

*Hedeoma quercetora Epling NLE

*Hedeoma rzedowskii B.L. Turner AGS, NLE, SLP, ZAC

*Hedeoma tenuiflora Brandegee BCS

*Hedeoma tenuipes Epling TAMS

*Hypenia violacea Mart. Gord. \& Valencia-Avalos GRO, OAX Hyptis atrorubens Poit. CHIS, GRO, OAX, PUE, TAB, VER

Hyptis brevipes Poit. CHIS, GRO, PUE, TAB, VER

Hyptis capitata Jacq. CAM, CHIS, COL, DGO, GRO, HGO, JAL, MEX, MICH, MOR, NAY, OAX, PUE, QRO, QROO, SLP, SIN, TAB, TAMS, VER

Hyptis conferta Pohl ex Benth. CHIS, DGO, HGO, JAL, NAY, OAX, TAB, VER

*Hyptis cualensis J.G. González \& A. Castro JAL

Hyptis intermedia Epling CHIS

*Hyptis langlassei Fernald GRO, MICH

Hyptis lantanifolia Poit. CHIS, JAL, OAX, VER

*Hyptis macrocephala M. Martens \& Galeotti GRO, JAL, MEX,

MICH, MOR, NAY

*Hyptis macvaughii J.G. González \& A. Castro NAY

*Hyptis pseudolantana Epling GRO

Hyptis recurvata Poit. CHIS, VER

*Hyptis rhytidea Benth. AGS, COL, DGO, JAL, MICH, NAY, SIN

Hyptis sinuata Pohl ex Benth. CAM, CHIS, OAX, TAB

Lepechinia cardiophylla Epling BCN

Lepechinia caulescens (Ortega) Epling CHIS, CHIH, COL, CDMX, DGO, GTO, GRO, HGO, JAL, MEX, MICH, MOR, NAY, OAX, PUE, QRO, SLP, SIN, TLAX, VER, ZAC

*Lepechinia flammea Mart. Gord. \& Lozada-Pérez GRO, JAL, $\mathrm{OAX}$

*Lepechinia glomerata Epling GRO, JAL

Lepechinia hastata (A. Gray) Epling BCN, BCS, COL

*Lepechinia leucophylloides (Ramamoorthy, Hiriart \& Medrano) B.T. Drew, Cacho \& Sytsma HGO

Lepechinia mecistandra (Donn. Sm.) H.K. Moon CHIS

*Lepechinia mexicana (S. Schauer) Epling HGO, OAX, PUE, QRO, SLP, VER

*Lepechinia nelsonii (Fernald) Epling COL, GRO, JAL, MEX, $\mathrm{MICH}$

Lepechinia schiedeana (Schltdl.) Vatke CHIS, CHIH, GRO, HGO, JAL, MEX, MICH, NLE, OAX, PUE, QRO, SLP, TAMS, VER

*Lepechinia yecorana Henr., Fishbein \& Van Devender CHIH, SON

Marsypianthes chamaedrys (Vahl) Kuntze CHIS, JAL, OAX, 
VER

Mentha canadensis L. BCN, CHIH, COAH, GTO, GRO, HGO, MEX, MICH, MOR, OAX, PUE, QRO, SLP, SON, VER

*Mesosphaerum collinum (Brandegee) Harley \& J.F.B. Pastore $\mathrm{BCS}$

Mesosphaerum oblongifolium (Benth.) Kuntze CHIS, COL, GRO, JAL, MICH, NAY, OAX, TAB

Mesosphaerum pectinatum (L.) Kuntze CAM, CHIS, COL, GRO, JAL, MEX, MICH, MOR, NAY, OAX, PUE, QRO, QROO, SLP, TAB, VER, YUC, ZAC

Mesosphaerum pulegioides (Pohl ex Benth.) Kuntze CHIS

* Mesosphaerum septentrionale (Epling) Harley \& J.F.B. Pastore CHIH, DGO, GRO, MEX, MICH, OAX, SIN, SON

Mesosphaerum suaveolens (L.) Kuntze BCS, CAM, CHIS, CHIH, COL, DGO, GRO, JAL, MEX, MICH, NAY, MOR, OAX, PUE, QROO, SLP, SIN, SON, TAB, TAMS, VER, YUC Mesosphaerum urticoides (Kunth) Kuntze AGS, BCS, CHIS, COL, DGO, GRO, JAL, MEX, MICH, MOR, NAY, OAX, VER, $\mathrm{ZAC}$

*Monarda bartlettii Standl. TAMS, VER

Monarda citriodora Cerv. ex Lag. CHIH, COAH, DGO, MICH, NLE, SLP, SIN, SON, TAMS

Monarda fistulosa L. COAH, HGO, NLE, SON, TAMS, VER

* Monarda pringlei Fernald COAH, NLE

Monarda punctata L. TAMS

Monardella breweri A. Gray BCN

* Monardella lagunensis M.E. Jones BCS

Monardella linoides A. Gray BCN

Monardella macrantha A. Gray BCN

Monardella nana A. Gray BCN

*Monardella thymifolia Greene BCN, BCS

Ocimum basilicum L. AGS, CAM, CHIS, COL, DGO, GRO, HGO, JAL, MEX, MICH, MOR, NAY, NLE, OAX, PUE, QRO, QROO, SLP, SIN, TAB, TAMS, VER, YUC

Ocimum campechianum Mill. CAM, CHIS, COL, GRO, HGO, JAL, MOR, OAX, PUE, QRO, QROO, SLP, SIN, TAB, TAMS, VER, YUC

Ocimum carnosum (Spreng.) Link \& Otto ex Benth. CHIS, HGO, OAX, PUE, QRO, QROO, SLP, TAMS, VER

Physostegia correllii (Lundell) Shinners COAH, NLE

Physostegia pulchella Lundell COAH

Pogogyne nudiuscula A. Gray BCN

Pogogyne serpylloides (Torr.) A. Gray BCN

* Pogogyne tenuiflora A. Gray BCN

*Poliomintha bustamanta B.L. Turner NLE

*Poliomintha conjunctrix Epling \& Wiggins BCN

*Poliomintha dendritica B.L. Turner COAH, NLE

Poliomintha glabrescens A. Gray ex Hemsl. CHIH, COAH

Poliomintha incana (Torr.) A. Gray CHIH, NLE, SON

*Poliomintha longiflora A. Gray COAH, NLE, SLP, TAMS, ZAC

*Poliomintha maderensis Henr. COAH

*Poliomintha marifolia (Schauer) A. Gray GTO, HGO, QRO, SLP, TAMS, VER

*Salvia acerifolia B.L. Turner JAL, MICH

* Salvia adenophora Fernald GRO, OAX

* Salvia adglutinans Lag. PUE
* Salvia aequidistans Fernald DGO, JAL, NAY, SIN

* Salvia agnes Epling AGS, CHIS, DGO, JAL, MICH

*Salvia alamosana Rose CHIH, DGO, JAL, NAY, SIN, SON, ZAC

*Salvia albicalyx J.G. González DGO

* Salvia albiterrarum J.G. González \& A. Castro JAL

*Salvia albocaerulea Linden COL, GRO, JAL, MEX, MICH, MOR

*Salvia aliena Greene NAY

* Salvia altimitrata Epling CHIS

* Salvia anastomosans Ramamoorthy OAX, PUE

* Salvia angustiarum Epling JAL, NAY

Salvia apiana Jepson BCN

* Salvia arbuscula Fernald DGO, GRO, JAL, MICH, NAY, OAX

Salvia arizonica A. Gray CHIH, COAH

* Salvia arthrocoma Fernald COL, HGO, JAL, PUE, VER

* Salvia aspera M. Martens \& Galeotti OAX, PUE

* Salvia assurgens Kunth GTO, MICH, QRO, SLP

* Salvia atropaenulata Epling GRO, MEX, MICH, OAX

*Salvia axillaris Moc. \& Sessé ex Benth. AGS, DGO, GTO, HGO, JAL, MEX, OAX, PUE, QRO, SLP, VER, ZAC

Salvia azurea Lam. CHIH, NLE

Salvia ballotiflora Benth. AGS, CHIH, COAH, DGO, GTO, HGO, JAL, NLE, QRO, SLP, SON, TAMS, VER, ZAC

* Salvia betulifolia Epling CHIH, DGO, SON

* Salvia biserrata M. Martens \& Galeotti VER

* Salvia boegei Ramamoorthy OAX, PUE

* Salvia booleana B.L. Turner NLE, SLP, ZAC

* Salvia brachyodonta Briq. COL, JAL

* Salvia brandegei Munz BCN

* Salvia breviflora Moc. \& Sessé COL, GRO, JAL, MEX, MICH, MOR, OAX, PUE

* Salvia buchananii Hedge QRO, SLP

Salvia cacaliifolia Benth. CHIS, GRO, OAX, PUE, VER

*Salvia cacomensis J.G. González, J. Morales \& J. Rodr. COL, JAL

* Salvia calderoniae Bedolla \& Zamudio GTO

* Salvia californica Brandegee BCN, BCS

*Salvia candicans M. Martens \& Galeotti HGO, OAX, PUE, QRO, VER

Salvia capillosa Epling MEX, MICH

Salvia capitata Schltdl. SON

Salvia carduacea Benth. BCN

Salvia carnea Kunth CHIS, COAH, COL, GRO, HGO, JAL, MEX, MICH, MOR, NAY, NLE, OAX, PUE, SIN, TLAX, VER * Salvia carranzae Zamudio \& Bedolla QRO, SLP

* Salvia carreyesii J.G. González JAL

* Salvia caudata Epling COAH, NLE, TAMS

* Salvia cedrosensis Greene BCN, BCS

Salvia cinnabarina M. Martens \& Galeotti CHIS, COL, DGO, GRO, HGO, JAL, MEX, MICH, MOR, NAY, OAX, PUE, SIN, VER, ZAC

* Salvia clarkcowanii B.L. Turner GRO, OAX

Salvia clevelandii (A. Gray) Greene BCN

* Salvia clinopodioides Kunth DGO, GRO, JAL, MEX, MICH

* Salvia coahuilensis Fernald COAH, NLE, SLP

Salvia coccinea Buc'hoz ex Etl. AGS, CAM, CHIS, CHIH, 
COAH, DGO, GTO, GRO, HGO, JAL, MEX, MICH, MOR, NAY, NLE, OAX, PUE, QRO, QROO, SLP, SIN, TAB, TAMS, VER, YUC, ZAC

Salvia columbariae Benth. BCN, BCS, SON

* Salvia collinsii Donn. Sm. CHIS

* Salvia compsostachys Epling COAH, NLE, TAMS

* Salvia concolor Lamb. ex Benth. CHIS, COL, CDMX, DGO, GRO, HGO, JAL, MEX, MICH, MOR, OAX, PUE, QRO

*Salvia confertispicata I. Fragoso \& Mart. Gord. GRO, OAX

* Salvia connivens Epling GTO, HGO, QRO, SLP

* Salvia coulteri Epling COAH, DGO, HGO, NLE, QRO, SLP, TAMS, VER, ZAC

*Salvia crucis Epling DGO, JAL, NAY, SIN, ZAC

* Salvia cryptodonta Fernald DGO, JAL, ZAC

* Salvia cualensis J.G. González JAL

Salvia curtiflora Epling CHIS, GRO

* Salvia curviflora Benth. GTO, HGO, MEX, MICH, OAX, PUE, QRO

* Salvia cyanantha Epling JAL, MICH

* Salvia cyclophylla Fernald GRO, OAX

* Salvia chalarothyrsa Fernald JAL, MICH

* Salvia chamaedryoides Cav. CHIH, CDMX, HGO, MEX, MICH, MOR, NLE, OAX, QRO, SLP, VER, ZAC

*Salvia chapalensis Briq. JAL

* Salvia chia Fernald COAH, NLE, TAMS

* Salvia chiapensis Fernald CHIS

* Salvia chionopeplica Epling BCN

* Salvia chionophylla Fernald BCN, COAH

* Salvia dasycalyx Fernald GRO, MICH

* Salvia decora Epling COL, GRO, JAL, MICH

* Salvia dichlamys Epling GRO, JAL, MEX, MICH, OAX

* Salvia diegoae Mart. Gord. \& Lozada-Pérez GRO

Salvia disjuncta Fernald CHIS, OAX

*Salvia divinorum Epling \& Játiva OAX, PUE

* Salvia dryophila Epling GRO, MEX, MICH

*Salvia durangensis J.G. González DGO

* Salvia duripes Epling \& Mathias CHIS

*Salvia eizi-matudae Ramamoorthy CHIS

* Salvia elegans Vahl AGS, CHIS, CHIH, COL, CDMX, DGO, GTO, GRO, HGO, JAL, MEX, MICH, MOR, NAY, OAX, PUE, QRO, SLP, SIN, SON, TAMS, TLAX, VER, ZAC

* Salvia emaciata Epling CHIH, DGO, SON

Salvia eremostachya Jepson BCN

* Salvia eriocalyx Bertero ex Roem. \& Schult. MOR

* Salvia evadens J.G. González \& Art. Castro JAL

Salvia excelsa Benth. CHIS, GRO, JAL, MEX, MICH, NLE, OAX

* Salvia fallax Fernald NAY, SIN

Salvia farinacea Benth. COAH, NLE

* Salvia fasciculata Fernald OAX

*Salvia fernaldii Standl. CAM, QROO, YUC

* Salvia festiva Epling CHIS

* Salvia filifolia Ramamoorthy GTO, MEX, MICH, QRO, SLP, TLAX

* Salvia filipes Benth. AGS, GRO, HGO, JAL, PUE

* Salvia firma Fernald COL, JAL, NAY

Salvia flaccida Fernald CHIS
*Salvia flaccidifolia Fernald HGO, PUE, VER

* Salvia fluviatilis Fernald CHIS, COL, GRO, HGO, MEX, MICH, MOR, OAX, PUE, QRO, SLP, VER

* Salvia forreri Greene COAH, DGO, JAL, NAY, NLE, SLP, SIN, SON, TAMS, ZAC

* Salvia fruticulosa Benth. GRO, OAX

*Salvia fulgens Cav. AGS, COL, CDMX, DGO, GTO, GRO, HGO, JAL, MEX, MICH, MOR, OAX, PUE, QRO, TAMS, TLAX, VER, ZAC

*Salvia fusca Epling CHIH

*Salvia galinsogifolia Fernald CHIH, COL, DGO, GRO, SON, VER

* Salvia gesneriflora Lindl. \& Paxton AGS, COL, CDMX, DGO, GTO, GRO, HGO, JAL, MEX, MICH, MOR, NAY, NLE, PUE, QRO, SLP, SIN, TAMS, TLAX, ZAC

*Salvia glabra M. Martens \& Galeotti OAX, PUE

*Salvia glechomifolia Kunth AGS, COAH, DGO, GTO, GRO, HGO, JAL, MEX, MICH, MOR, NLE, OAX, PUE, SLP, TAMS, ZAC

*Salvia goldmanii Fernald CHIH, SIN, SON

* Salvia gonzalezii Fernald OAX

* Salvia graciliflora M. Martens \& Galeotti VER

*Salvia gravida Epling COL, JAL, MICH

Salvia greggii A. Gray AGS, CHIH, COAH, DGO, GTO, JAL, NLE, SLP, TAMS, ZAC

*Salvia guadalajarensis Briq. JAL, MEX, MICH, ZAC

*Salvia gypsophila B.L. Turner NLE, TAMS

* Salvia helianthemifolia Benth. GTO, GRO, HGO, JAL, MEX, MICH, NAY, OAX, PUE, QRO, SLP, TAMS, TLAX, VER

Salvia henryi A. Gray CHIH, SON

* Salvia herbacea Benth. CHIS, OAX

*Salvia heterotricha Fernald DGO, JAL, NAY, TAMS, ZAC

* Salvia hidalgensis Miranda HGO, QRO, VER

* Salvia hintonii Epling GRO, MICH

* Salvia hirsuta Jacq. AGS, CDMX, DGO, GTO, HGO, JAL, MEX, MICH, OAX, QRO, SLP, TLAX, VER, ZAC

Salvia hispanica L. CHIS, CHIH, COAH, COL, CDMX, DGO, GTO, GRO, HGO, JAL, MEX, MICH, MOR, NAY, NLE, OAX, QRO, SIN, SON, TAMS, VER

Salvia holwayi S.F. Blake CHIS

*Salvia ibugana J.G. González JAL

Salvia indigocephala Ramamoorthy CHIH, MICH

* Salvia infuscata Epling CHIS, OAX

* Salvia inornata Epling PUE

Salvia involucrata Cav. COAH, GTO, HGO, MEX, NLE, PUE, SLP, TAMS, VER

* Salvia iodantha Fernald CHIS, COL, DGO, GTO, GRO, JAL, MEX, MICH, MOR, NAY, OAX, SLP, SIN, VER

*Salvia iodophylla Epling PUE

* Salvia ionocalyx Epling CHIS

* Salvia isochroma (Fernald) B.L. Turner NLE, SLP, ZAC

* Salvia jacalana B.L. Turner HGO, QRO, SLP

*Salvia jacobii Epling GRO

* Salvia jaimehintoniana Ramamoorthy ex B.L. Turner COAH, DGO, NLE, TAMS

* Salvia jaliscana Briq. JAL

* Salvia jessicae B.L. Turner COAH 
Salvia karwinskii Benth. CHIS, COL, GRO, HGO, JAL, OAX, PUE, QRO, VER

*Salvia keerlii Benth. AGS, DGO, GTO, GRO, HGO, MEX, MICH, NLE, OAX, PUE, QRO, SLP, TAMS, VER, ZAC

* Salvia laevis Benth. AGS, COL, CDMX, DGO, GTO, GRO, HGO, JAL, MEX, MICH, MOR, NAY, PUE, QRO, SLP, TAMS, TLAX, VER, ZAC

*Salvia lanceolata Willd. GTO, HGO, JAL

* Salvia langlassei Fernald GRO, HGO, OAX, QRO, SLP

*Salvia languidula Epling COL, JAL, MICH

*Salvia lanicalyx Epling COAH

*Salvia lapazana B.L. Turner BCS

Salvia lasiantha Benth. CHIS, COL, DGO, GRO, JAL, MEX,

MOR, OAX, PUE, SLP, VER, ZAC

Salvia lasiocephala Hook. \& Arn. CHIS, CHIH, COL, DGO, GRO, JAL, MEX, MICH, NAY, OAX, PUE, QROO, SIN, SON, VER, ZAC

Salvia lavanduloides Kunth AGS, CHIS, COL, CDMX, DGO, GTO, GRO, HGO, JAL, MEX, MICH, MOR, NAY, OAX, PUE, QRO, SLP, SIN, TLAX, VER, ZAC

Salvia lemmonii A. Gray CHIH, SON

*Salvia lentiginosa Brandegee SLP

*Salvia leptostachys Benth. GTO, GRO, JAL, MEX, MICH, MOR, OAX, PUE, ZAC

Salvia leucantha Cav. CHIS, COAH, COL, GTO, GRO, HGO, JAL, MEX, MICH, MOR, OAX, PUE, SLP, TLAX, VER, ZAC

Salvia leucochlamys Epling CHIS

* Salvia lineata Benth. OAX, PUE

* Salvia littae Vis. MEX, MICH, OAX

*Salvia longispicata M. Martens \& Galeotti AGS, CHIS, CHIH, COL, GTO, GRO, JAL, MEX, MICH, MOR, NLE, OAX, PUE, SLP, VER

* Salvia longistyla Benth. COL, DGO, GTO, GRO, JAL, MEX, MICH, NLE, OAX, PUE, VER

*Salvia lophanthoides Fernald GRO, OAX

Salvia lycioides A. Gray AGS, CHIH, COAH, DGO, NLE, SLP, TAMS, ZAC

*Salvia macellaria Epling AGS, CHIH, COAH, NLE, SLP, ZAC

*Salvia madrensis Seem. DGO, SIN

* Salvia manantlanensis Ramamoorthy COL, JAL

* Salvia marci Epling BCS

* Salvia mazatlanensis Fernald DGO, NAY, SIN

* Salvia mcvaughii Bedolla, Lara \& Zamudio GRO, MOR

* Salvia meera Ramamoorthy ex J.G. González \& Santana Mich. COL, JAL

* Salvia melissodora Lag. AGS, CHIH, COL, DGO, GTO, GRO, HGO, JAL, MEX, MICH, NAY, OAX, PUE, QRO, SLP, SON, TAMS, VER, ZAC

Salvia mellifera Greene BCN

* Salvia mexiae Epling JAL

*Salvia mexicana L. AGS, CHIH, COL, CDMX, DGO, GTO, GRO, HGO, JAL, MEX, MICH, MOR, NAY, NLE, OAX, PUE, QRO, SLP, SIN, SON, TAMS, VER, ZAC

Salvia micrantha Vahl AGS, GRO, JAL, MEX, MICH, OAX, QROO, TAB, YUC, ZAC

Salvia microphylla Kunth AGS, CHIS, CHIH, COAH, CDMX,
DGO, GTO, GRO, HGO, JAL, MEX, MICH, MOR, NAY, NLE, OAX, PUE, QRO, SLP, SON, TAB, TAMS, TLAX, VER, ZAC Salvia miniata Fernald CHIS

Salvia misella Kunth AGS, BCS, CAM, CHIS, CHIH, COL, CDMX, DGO, GTO, GRO, HGO, JAL, MEX, MICH, MOR, NAY, NLE, OAX, PUE, QRO, QROO, SLP, SIN, SON, TAMS, VER, YUC, ZAC

Salvia mocinoi Benth. CHIS, COL, CDMX, GRO, HGO, JAL, MEX, MICH, MOR, NAY, OAX, PUE, QRO, TLAX, VER

*Salvia modesta Boiss. DGO, JAL, NAY, OAX, SON

*Salvia modica Epling DGO, NLE, TAMS

Salvia mohavensis Greene BCN, SON

*Salvia monclovensis Fernald COAH, MEX, MICH, NLE

*Salvia moniliformis Fernald CDMX, GRO, HGO, MEX, MICH, MOR, PUE

* Salvia moranii B.L. Turner BCS

*Salvia mucidiflora Fernald DGO

*Salvia muelleri Epling COAH, NLE, SLP

Salvia munzii Epling BCN

*Salvia muscarioides Kunth CHIH, NAY, SON

Salvia nana Kunth CHIS, DGO, GTO, JAL, OAX, PUE, QRO, SLP, VER, ZAC

* Salvia nelsonii Fernald GRO, HGO, MOR, PUE

*Salvia nepetoides Kunth COL, GTO, GRO, JAL, MEX, MICH, MOR, OAX, VER

Salvia nigriflora Epling JAL, MICH

*Salvia nitida Benth. GRO, OAX

* Salvia oaxacana Fernald OAX, PUE, VER

*Salvia oblongifolia M. Martens \& Galeotti CHIS, OAX

Salvia occidentalis Sw. BCS, CAM, CHIS, COL, GRO, HGO, JAL, MEX, MICH, MOR, NAY, OAX, PUE, QRO, QROO, SLP, SIN, TAMS, VER, YUC, ZAC

* Salvia occidua Epling OAX

* Salvia odam J.G. González DGO

* Salvia omissa J.G. González JAL

*Salvia oreopola Fernald CDMX, HGO, MEX, MOR, OAX, QRO

* Salvia oresbia Fernald NLE, SLP

*Salvia orizabensis Fernald OAX, VER

Salvia pachyphylla Epling BCN

* Salvia pachystachya Trautv. BCN

* Salvia palmeri A. Gray CHIH, SON

* Salvia pannosa Fernald OAX, PUE

* Salvia parrasana Brandegee COAH

Salvia parryi A. Gray CHIH, GRO, SON

*Salvia patens Cav. AGS, CDMX, GTO, GRO, HGO, JAL, MEX, MICH, MOR, OAX, PUE, QRO, SLP, TAMS, VER

* Salvia paupercula Epling PUE

*Salvia peninsularis Brandegee BCN, BCS

*Salvia pennellii Epling NLE, SLP

*Salvia pericona B.L. Turner OAX

*Salvia perlonga Fernald GRO

*Salvia pexa Epling OAX

Salvia pinguifolia (Fernald) Wooton \& Standl. AGS, CHIH, COAH, NLE, ZAC

*Salvia platycheila A. Gray BCN, BCS

* Salvia platyphylla Briq. COL, JAL 
*Salvia plurispicata Epling HGO, JAL, MEX, MICH, VER, ZAC

* Salvia podadena Briq. COAH, DGO, GRO, MOR, OAX, PUE, QRO, SLP

Salvia polystachia Cav. BCN, CHIS, COAH, COL, CDMX, DGO, GTO, GRO, HGO, JAL, MEX, MICH, MOR, NAY, NLE, OAX, PUE, QRO, SLP, SIN, TAMS, TLAX, VER, ZAC

*Salvia populifolia Fernald JAL, ZAC

* Salvia potus Epling COAH, NLE

* Salvia pringlei B.L. Rob. \& Greenm. DGO, JAL, NAY

*Salvia protracta Benth. CHIS, GRO, MEX, MICH, MOR, OAX, SIN, VER

*Salvia pruinosa Fernald JAL, ZAC

* Salvia prunelloides Kunth AGS, CHIS, CHIH, COAH, CDMX, DGO, GTO, GRO, HGO, JAL, MEX, MICH, MOR, NAY, NLE, OAX, QRO, SLP, SIN, SON, TAMS, VER, ZAC

*Salvia prunifolia Fernald DGO, NAY

* Salvia pseudoincisa Epling TAMS

*Salvia pseudomisella Moran \& G.A. Levin COL

*Salvia pseudopallida Epling COAH

* Salvia puberula Fernald GTO, HGO, NLE, QRO, SLP, TAMS

* Salvia pubescens Benth. GRO, OAX, PUE

*Salvia pugana J.G. González JAL

Salvia pulchella DC. CHIS, GTO, GRO, JAL, MEX, MICH

* Salvia purepecha Bedolla, Lara \& Zamudio JAL, MICH

Salvia purpurea Cav. AGS, CHIS, COL, DGO, GTO, GRO, JAL, MEX, MICH, MOR, OAX, PUE, QRO, TLAX, VER, ZAC

* Salvia purpusii Brandegee CHIH, COAH, DGO

* Salvia pusilla Fernald OAX, PUE

* Salvia querceto-pinorum Epling \& Játiva CHIS

* Salvia quercetorum Epling COL, JAL

* Salvia ramamoorthyana Espejo emend. J.G. González COL, CDMX, JAL, MEX, MICH, MOR

*Salvia ramirezii J.G. González JAL

* Salvia ramosa Brandegee OAX, PUE

* Salvia raveniana Ramamoorthy GRO, OAX

Salvia recurva Benth. CHIS, GRO, HGO, OAX, PUE, QRO, VER

Salvia reflexa Hornem. AGS, CHIH, COAH, CDMX, DGO, GTO, HGO, JAL, MEX, MICH, NLE, OAX, PUE, QRO, SLP, SON, TAMS, VER, ZAC

Salvia regla Cav. AGS, CHIH, COAH, DGO, GTO, HGO, JAL, MICH, NLE, OAX, PUE, QRO, SLP, SON, TAMS, VER, ZAC Salvia remissa Epling COL, JAL, MICH, SIN

* Salvia remota Benth. GRO, MEX, MICH, MOR

Salvia reptans Jacq. AGS, CHIS, CHIH, COAH, CDMX, GTO, GRO, HGO, JAL, MEX, MICH, NAY, OAX, PUE, QRO, SLP, ZAC

*Salvia rhyacophila (Fernald) Epling CHIS, GRO, JAL, MEX, MICH, MOR, OAX, SLP, ZAC

*Salvia richardsonii B.L. Turner TAMS

Salvia roemeriana Scheele CHIH, COAH, NLE, TAMS

* Salvia rogersiana Ramamoorthy ex J.G. González \& Cuevas COL, JAL

*Salvia roscida Fernald COL, DGO, GRO, JAL, NAY, SIN, SON, VER
*Salvia rosei Fernald JAL, ZAC

* Salvia rostellata Epling COL, JAL

Salvia rubiginosa Benth. CHIS, GRO, NAY, OAX, PUE, VER

* Salvia rubropunctata Fernald CHIH, NLE, SIN, SON

*Salvia rupicola Fernald AGS, HGO, QRO, SLP, ZAC

*Salvia rzedowskii Ramamoorthy MEX

*Salvia sacculus Epling CHIS, GRO, NLE, OAX, QRO, SLP, TAMS

* Salvia sanctae-luciae Seem. DGO, JAL, MICH, NAY, SIN, VER

* Salvia santanae Ramamoorthy ex J.G. González \& GuzmánHernández COL, JAL

*Salvia sapinea Epling COL, GRO, JAL, OAX, SIN

*Salvia scaposa Epling GRO, MEX

*Salvia schaffneri Fernald SLP, ZAC

* Salvia seemannii Fernald BCS, CHIH, DGO, GRO, MICH, NAY, SIN, SON

*Salvia semiatrata Zucc. GRO, OAX

*Salvia semiscaposa Epling ex Fragoso \& Mart. Gord. GRO, MEX

*Salvia serboana B.L. Turner OAX

Salvia serotina L. CAM, QROO, YUC

* Salvia serpyllifolia Fernald SLP

*Salvia sessei Benth. COL, CDMX, GRO, JAL, MEX, MICH, MOR, OAX, PUE, TAMS

Salvia setosa Fernald BCS, CHIH, DGO, JAL, MICH, NAY, OAX, SIN, SON

* Salvia setulosa Fernald COAH, HGO, MEX, MICH, MOR, NLE, OAX, PUE, QRO, SLP, TAMS, VER

Salvia shannonii Donn. Sm. CHIS, GRO, OAX

* Salvia sharpii Epling \& Mathias COAH, NLE, SLP

* Salvia similis Brandegee BCS, SON

* Salvia sinaloensis Fernald DGO, SIN

Salvia stachyoides Kunth BCN, MEX, MICH, PUE

*Salvia stolonifera Benth. GRO, OAX

* Salvia stricta Sessé \& Moc. AGS, CDMX, GTO, GRO, HGO, JAL, MEX, MICH, MOR, OAX, QRO, TLAX, VER, ZAC

*Salvia subhastata Epling GRO, MICH

Salvia subincisa Benth. AGS, CHIH, DGO, SLP, SON, ZAC

*Salvia subobscura Epling MICH

*Salvia subpatens Epling COL, GRO, JAL, MEX

Salvia summa A. Nelson $\mathrm{CHIH}$

* Salvia synodonta Epling MICH

* Salvia tehuacana Fernald GRO, OAX, PUE

*Salvia tenorioi Ramamoorthy ex B.L. Turner PUE

*Salvia tepicensis Fernald CHIH, NAY, SIN, SON

* Salvia teresae Fernald DGO, NAY

*Salvia tetramerioides Mart.Gord., Fragoso \& García-Peña OAX

Salvia texana (Scheele) Torr. COAH, NLE, SLP, TAMS, VER

* Salvia textitlana B.L. Turner OAX

* Salvia thymoides Benth. OAX, PUE, VER

* Salvia thyrsiflora Benth. COL, DGO, GRO, JAL, MICH, NAY, OAX, VER

*Salvia tilantongensis J.G. González \& R. Aguilar-Santelises OAX

Salvia tiliifolia Vahl AGS, CHIS, CHIH, COAH, COL, CDMX, 
DGO, GTO, GRO, HGO, JAL, MEX, MICH, MOR, NAY, NLE, OAX, PUE, QRO, SLP, SIN, SON, TAMS, TLAX, VER, ZAC

*Salvia tonalensis Brandegee CHIS

*Salvia tonaticensis Ramamoorthy ex Lara, Bedolla \& Zamudio GRO, MEX

*Salvia topiensis J.G. González DGO

*Salvia townsendii Fernald CHIH, JAL, NAY, SIN, SON, ZAC

*Salvia tricuspidata M. Martens \& Galeotti GRO, MICH, OAX Salvia tubifera Cav. CHIS, GRO, HGO, JAL, OAX, PUE, VER

* Salvia turneri Ramamoorthy GRO

* Salvia umbraticola Epling PUE

* Salvia unicostata Fernald NLE, SLP, TAMS

* Salvia univerticillata Ramamoorthy ex Klitgaard CHIS

Salvia urica Epling CHIS, GRO, OAX

*Salvia urolepis Fernald COAH, NLE, OAX, PUE

* Salvia uruapana Fernald COL, GRO, JAL, MICH

Salvia vaseyi (Porter) Parish BCN

* Salvia vazquezii H.H. Iltis \& Ramamoorthy JAL, MICH

*Salvia venturana B.L. Turner PUE, VER

* Salvia verecunda M.E. Jones CHIH, SON

* Salvia veronicifolia A. Gray ex S. Watson DGO, JAL, ZAC

* Salvia vialis Brandegee CHIS, OAX

*Salvia villosa Fernald AGS, JAL, NLE, OAX, SLP, TAMS, ZAC

* Salvia viscidifolia Epling COL, GRO, JAL, MICH

*Salvia vitifolia Benth. CHIS, GRO, OAX, PUE, VER

Salvia wagneriana Pol. CHIS

Salvia whitefoordiae Klitgaard CHIS

*Salvia whitehousei (Cory) Alziar COAH

*Salvia xalapensis Benth. CHIS, GRO, HGO, JAL, MEX,

MICH, MOR, OAX, PUE, SLP, VER

*Salvia xolocotzii Bedolla \& Zamudio QRO

* Salvia zacualpanensis Briq. CHIS, VER

* Salvia zacuapanensis Brandegee VER

* Salvia zamoranensis Zamudio \& Bedolla GTO, QRO

*Salvia zaragozana B.L. Turner NLE, TAMS

*Satureja sauderi Epling BCS

* Scutellaria aramberrana B.L. Turner NLE, TAMS

* Scutellaria atrocyanea Epling \& Játiva JAL

* Scutellaria aurea B.L. Rob. \& Greenm. OAX

*Scutellaria bartletti B.L. Turner NLE, SLP, TAMS

Scutellaria blepharophylla Epling MICH

* Scutellaria carmenensis Henr. COAH

*Scutellaria cuevasiana J.G. González \& A. Vázquez JAL

Scutellaria drummondii Benth. COAH, HGO, NLE, QRO, SLP, TAMS, VER

Scutellaria dumetorum Schltdl. CAM, CHIS, COL, CDMX, DGO, GTO, GRO, HGO, JAL, MEX, MICH, MOR, NAY, NLE, OAX, PUE, QRO, SLP, TAMS, TLAX, VER

* Scutellaria formosa Leonard GRO, OAX, VER

* Scutellaria fruticetorum Epling COAH, NLE, SLP, TAMS

*Scutellaria gaumeri Epling CAM, QROO, YUC

Scutellaria guatemalensis Leonard CHIS, QROO

Scutellaria havanensis Jacq. NLE, TAMS, VER

*Scutellaria hintoniana Epling JAL, MEX, MICH, OAX

* Scutellaria hintoniorum Henr. NLE

*Scutellaria hispidula B.L. Rob. COAH, NLE, SLP
*Scutellaria horridula Epling CHIH, SON

Scutellaria inflata Epling CHIS

*Scutellaria jaliscana Epling JAL

Scutellaria longifolia Benth. CHIS, GRO, OAX, VER

Scutellaria lundellii Epling CHIS

*Scutellaria lutilabia T.M. Lane \& G.L. Nesom NLE, TAMS

*Scutellaria macra Epling GTO, PUE, SLP, VER

Scutellaria mexicana (Torr.) A.J. Paton BCN, BCS, CHIH, COAH, DGO, NLE, SON

Scutellaria microphylla Moc. \& Sessé ex Benth. CHIH, COAH, DGO, NLE, OAX, PUE, TAMS

*Scutellaria molanguitensis Hiriart HGO, VER

* Scutellaria monterreyana B.L. Turner COAH, NLE

* Scutellaria multiflora Benth. GRO, MEX

*Scutellaria muzquiziana B.L. Turner COAH, NLE

*Scutellaria oaxacana Greenm. OAX, PUE

Scutellaria orichalcea Donn. Sm. CHIS, HGO, JAL, OAX, VER

*Scutellaria orizabensis Epling CHIS, PUE, VER

Scutellaria ovata Hill COAH, NLE

* Scutellaria pallidiflora Epling JAL, ZAC

* Scutellaria petersoniae B.L. Turner \& Reveal GRO

Scutellaria platyphylla (Epling) B.L. Turner \& Delprete SON

Scutellaria potosina Brandegee CHIH, COAH, DGO, GTO, HGO, NLE, OAX, PUE, QRO, SLP, SON, TAMS, VER, ZAC *Scutellaria pseudocearulea Briq. CHIS, GRO, HGO, MICH, PUE, QRO, SLP, TAMS, VER

Scutellaria purpurascens Sw. CHIS, COL, GRO, JAL, VER

Scutellaria racemosa Pers. CHIS, OAX, VER

Scutellaria resinosa Torr. CHIH, COAH, SON, TAMS

Scutellaria seleriana Loes. CHIS, COAH, GRO, HGO, NLE, OAX, PUE, QRO, QROO, SLP, TAB, TAMS, VER

*Scutellaria serboana B.L. Turner OAX

*Scutellaria sipilensis Cuevas COL, JAL

*Scutellaria splendens Link, Klotzsch \& Otto CHIS, OAX, TAB, VER

*Scutellaria stewartii B.L. Turner COAH

*Scutellaria sublitoralis J.G. González JAL, NAY

*Scutellaria suffrutescens S. Watson COAH, HGO, NLE, QRO, SLP, TAMS, VER

Scutellaria tuberosa Benth. BCN

*Scutellaria vitifolia Brandegee CHIS, OAX

Stachys agraria Schltdl. \& Cham AGS, CHIS, CHIH, COAH, COL, CDMX, DGO, GTO, GRO, HGO, JAL, MEX, MICH, MOR, NAY, NLE, OAX, PUE, QRO, SLP, SIN, SON, TAMS, TLAX, VER, ZAC

*Stachys albotomentosa Ramamoorthy GTO, HGO, QRO, SLP, TAMS

*Stachys aristata Greenm. JAL, SIN

Stachys bigelovii A. Gray BCS, CHIH, COAH, COL, DGO, JAL, MICH, NLE, SLP, SIN, SON, ZAC

*Stachys boraginoides Cham. \& Schltdl. CHIS, GRO, HGO, JAL, MEX, MICH, NAY, OAX, PUE, QRO, SLP, VER

Stachys coccinea Ortega AGS, BCS, CHIS, CHIH, COAH, COL, CDMX, DGO, GTO, GRO, HGO, JAL, MEX, MICH, MOR, NAY, OAX, PUE, QRO, SLP, SIN, SON, TAMS, TLAX, VER, ZAC 
* Stachys collina Brandegee GRO, OAX, PUE

Stachys drummondii Benth. MOR, NLE, PUE, SLP, TAMS

Stachys eriantha Benth. CDMX, GTO, HGO, JAL, MEX, MICH, MOR, NLE, OAX, PUE, QRO, SLP, TLAX, VER

*Stachys globosa Epling CDMX, HGO, MEX, NAY, SLP, VER

* Stachys grahamii Benth. CHIH, COAH, JAL, MICH, NAY, NLE, SLP, VER

*Stachys harkerae J.G. González JAL, ZAC

* Stachys harleyana A. Pool CHIS

* Stachys herrerana Rzed. \& Calderón HGO, VER

*Stachys hintoniorum B.L. Turner COAH, NLE

* Stachys inclusa Epling OAX, PUE

*Stachys keerlii Benth. GTO, HGO, JAL, MEX, MICH, MOR, NLE, OAX, PUE, TAMS, VER

* Stachys langmaniae Rzed. \& Calderón NLE, TAMS

Stachys lindenii Benth. CHIS, GRO, JAL, MEX, MICH, OAX, PUE, QRO, VER

*Stachys manantlanensis B.L. Turner COL, JAL

*Stachys moorei B.L. Turner HGO, VER

*Stachys nepetifolia Desf. CDMX, GTO, HGO, JAL, MEX, MICH, MOR, OAX, QRO, SLP, TAMS, TLAX, VER, ZAC

*Stachys pacifica B.L. Turner CHIH, COL, JAL, SIN, SON

*Stachys parvifolia M. Martens \& Galeotti CHIS, CHIH, CDMX, DGO, GTO, HGO, MEX, MICH, QRO, SLP, SON, TAMS, TLAX, VER

Stachys pilosissima M. Martens \& Galeotti CHIS, GRO, JAL, MEX, MICH, OAX, PUE, TAMS, VER

*Stachys pringlei Greenm. HGO, JAL, MEX, MICH, QRO, SLP, VER

Stachys radicans Epling CHIS, CDMX, HGO, JAL, MEX, MICH, TLAX, VER

Stachys rigida Nutt. BCN

*Stachys rotundifolia Sessé \& Moc. CHIS, COL, HGO, JAL, MEX, MOR, OAX, PUE, QRO, VER

*Stachys sanchezii Rzed. \& A. García CDMX, HGO, JAL, MEX, MICH, PUE, QRO, VER

*Stachys sandersii B.L. Turner SLP

* Stachys tamaulipana B.L. Turner NLE, TAMS

* Stachys tenerrima Epling BCS

* Stachys tlaxiacana B.L. Turner OAX

* Stachys torresii B.L. Turner OAX

* Stachys turneri Rzed. \& Calderón GTO

* Stachys vulnerabilis Rzed. \& Calderón COAH, NLE

Tetraclea coulteri A. Gray AGS, CHIH, COAH, DGO, GTO, HGO, JAL, NLE, PUE, SLP, SON, TAMS, ZAC

Teucrium canadense L. BCS, COAH, NLE, PUE, QRO, SLP, SON, TAMS, VER

Teucrium cubense Jacq. BCN, CAM, CHIH, COAH, DGO, GTO, HGO, JAL, MOR, NLE, PUE, QRO, QROO, SLP, SIN, SON, TAB, TAMS, VER, YUC, ZAC

Teucrium glandulosum Kellogg BCN, BCS, SIN, SON

Teucrium laciniatum Torr. AGS, CHIH, COAH, SLP, TAMS, ZAC

*Teucrium townsendii Vasey \& Rose COL

Teucrium vesicarium Mill. CAM, CHIS, GRO, MOR, NLE, OAX, PUE, QRO, QROO, SLP, TAB, TAMS, VER, YUC

Trichostema arizonicum A. Gray CHIH, COAH, DGO, SLP,

\section{SON}

Trichostema lanatum Benth. BCN

Trichostema lanceolatum Benth. BCN

*Trichostema mexicanum Epling COAH, GTO, JAL, NLE, SLP, ZAC

Trichostema micranthum A. Gray BCN

Trichostema parishii Vasey BCN

*Trichostema purpusii Brandegee OAX, PUE

Vitex gaumeri Greenm. CAM, CHIS, GRO, MEX, MICH, MOR, QROO, SIN, TAB, VER, YUC

Vitex hemsleyi Briq. CHIS, COL, GRO, JAL, MEX, MICH, MOR, NAY, OAX

*Vitex mollis Kunth BCN, BCS, CHIH, COL, DGO, GRO, JAL, MEX, MICH, MOR, NAY, OAX, PUE, SIN, SON, VER, ZAC *Vitex pyramidata B.L. Rob. CHIS, CHIH, COL, DGO, GRO, JAL, MEX, MICH, MOR, NAY, OAX, PUE, SIN, SON, YUC, ZAC

Volkameria aculeata L. QROO, VER

Volkameria ligustrina Jacq. CAM, CHIS, HGO, OAX, PUE, QRO, QROO, SLP, TAB, TAMS, VER, YUC

Warnockia scutellarioides (Engelm. \& A. Gray) M.W. Turner $\mathrm{COAH}$

\section{Family Lauraceae}

*Aiouea inconspicua van der Werff CHIS, OAX, TAB, VER

Beilschmiedia anay (S.F. Blake) Kosterm. CHIS, OAX, PUE, VER

*Beilschmiedia angustielliptica Lorea-Hern. GRO

Beilschmiedia hondurensis Kosterm. CHIS, OAX, VER

*Beilschmiedia manantlanensis Cuevas \& Cochrane COL, JAL Beilschmiedia mexicana (Mez) Kosterm. CHIS, GRO, HGO, OAX, PUE, QRO, SLP, VER

Beilschmiedia ovalioides Sa. Nishida CHIS, OAX

Beilschmiedia ovalis (S.F. Blake) C.K. Allen CHIS, OAX

Beilschmiedia riparia Matuda CHIS, GRO, JAL, MICH, SLP, OAX, VER

Beilschmiedia steyermarkii C.K. Allen CHIS

*Beilschmiedia zapoteoides (Lundell) Kosterm. CHIS

Cassytha filiformis L. CAM, CHIS, QROO, VER, YUC

*Cinnamomum amplexicaule (Cham. \& Schltdl.) Kosterm. GRO, OAX, VER

Cinnamomum areolatum (Lundell) Kosterm. CHIS, OAX

*Cinnamomum bractefoliaceum Lorea-Hern. QRO, SLP, TAMS

*Cinnamomum breedlovei (Lundell) Kosterm. CHIS, OAX

* Cinnamomum concinnum Lorea-Hern. CHIS, GRO, OAX

* Cinnamomum chiapense (Lundell) Kosterm. CHIS

*Cinnamomum effusum (Meisn.) Kosterm. HGO, OAX, PUE, QRO, SLP, TAMS, VER

*Cinnamomum glossophyllum Lorea-Hern. DGO, NAY

*Cinnamomum grisebachii Lorea-Hern. CHIS, OAX, TAB, VER

*Cinnamomum hartmannii (I.M. Johnst.) Kosterm. CHIH, COL, DGO, JAL, MICH, NAY, SON

* Cinnamomum kruseanum O. Téllez \& Villaseñor GRO

* Cinnamomum leptophyllum Lorea-Hern. VER

*Cinnamomum longipes (I.M. Jonst.) Kosterm. HGO, PUE, VER 
Cinnamomum molle (Mez) Kosterm. CHIS, GRO

Cinnamomum neurophyllum (Mez \& Pittier) Kosterm. OAX, $\mathrm{TAB}$

*Cinnamomum pachypodum (Nees) Kosterm. COAH, COL, GTO, GRO, HGO, JAL, MEX, MICH, NAY, NLE, OAX, PUE, QRO, SLP, TAMS, VER

Cinnamomum padiforme (Standl. \& Steyerm.) Kosterm. GRO, JAL

*Cinnamomum salicifolium (Nees) Kosterm. GTO, HGO, QRO, SLP, VER

*Cinnamomum subtriplinervium (Meisn.) Kosterm. VER

Cinnamomum trinerve (Lundell) Kosterm. CHIS

Cinnamomum triplinerve (Ruiz \& Pav.) Kosterm. CHIS, GRO, HGO, OAX, TAB, VER

*Cinnamomum velveti Lorea-Hern. GRO

Cinnamomum zapatae Lorea-Hern. CHIS, GRO, OAX

Licaria brittoniana C.K. Allen \& L.E. Greg. CHIS, OAX

Licaria campechiana (Standl.) Kosterm. CAM, CHIS, QRO, QROO, SLP, VER, YUC

Licaria capitata (Schltdl. \& Cham.) Kosterm. CHIS, GRO, HGO, OAX, PUE, QRO, SLP, TAB, VER

Licaria caudata (Lundell) Kosterm. CAM, CHIS, QROO

Licaria cervantesii (Kunth) Kosterm. CHIS, COL, GRO, JAL, NAY, OAX, TAB, VER

*Licaria chinanteca Lorea-Hern. OAX

Licaria excelsa Kosterm. CHIS, GRO, OAX, PUE, VER

*Licaria glaberrima (Lundell) C.K. Allen CHIS

* Licaria mexicana (Brandegee) Kosterm. HGO, VER

Licaria misantlae (Brandegee) Kosterm. CAM, CHIS, OAX, QROO, VER

Licaria multinervis H.W. Kurz CHIS

*Licaria nayaritensis (Lundell) Lundell COL, JAL, NAY

Licaria peckii (I.M. Johnst.) Kosterm. CAM, CHIS, OAX, QROO, TAB, VER

* Licaria phymatosa Lorea-Hern. GRO

* Licaria quercina Lorea-Hern. GRO

*Licaria siphonantha Lorea-Hern. GRO

Licaria urceolata Lundell GRO, OAX, QRO

*Licaria velutina van der Werff CHIS, OAX, VER

Litsea glaucescens Kunth AGS, CHIS, CHIH, COAH, COL, CDMX, DGO, GTO, GRO, HGO, JAL, MEX, MICH, MOR, NAY, NLE, OAX, PUE, QRO, SLP, SIN, SON, TAB, TAMS, VER, ZAC

*Litsea muelleri Rehder COAH, HGO, NLE, SLP, TAMS, VER

*Litsea novoleontis Bartlett COAH, NLE, SLP

*Litsea parvifolia (Hemsl.) Mez COAH, NLE, SLP

*Litsea pedicellata Bartlett COAH, NLE

*Litsea pringlei Bartlett COAH, HGO, NLE, SLP, TAMS, VER

* Mocinnodaphne cinnamomoidea Lorea-Hern. GRO, OAX

Nectandra ambigens (S.F. Blake) C.K. Allen CHIS, CHIH, GRO, MICH, NAY, OAX, TAB, VER

Nectandra belizensis (Lundell) C.K. Allen CHIS

Nectandra cissiflora Nees CHIS, VER

Nectandra colorata Lundell CHIS, OAX, VER

Nectandra coriacea (Sw.) Griseb. CAM, CHIS, OAX, QROO, SLP, TAB, TAMS, VER, YUC

Nectandra cuspidata Nees \& Mart. CHIS, OAX, PUE, TAB,

\section{VER}

Nectandra hihua (Ruiz \& Pav.) Rohwer CAM, CHIS, CHIH, COL, DGO, GRO, HGO, JAL, MEX, MICH, MOR, NAY, NLE, OAX, PUE, SIN, SON, TAB, TAMS, VER

*Nectandra leucocome Rohwer CHIS

Nectandra longicaudata (Lundell) C.K. Allen CAM, CHIS, OAX, VER

Nectandra lundellii C.K. Allen CHIS, OAX, PUE, TAB, VER

Nectandra martinicensis Mez CHIS, COL, GRO, JAL, TAB

* Nectandra matudae Lundell CHIS, OAX, VER

Nectandra membranacea (Sw.) Griseb. CHIS, OAX, PUE, TAB, VER

Nectandra nitida Mez CHIS, GRO, OAX, QROO, TAB, VER

Nectandra purpurea (Ruiz \& Pav.) Mez CHIS

Nectandra reticulata (Ruiz \& Pav.) Mez CHIS, HGO, OAX, PUE, SLP, TAB, VER

Nectandra rudis C.K. Allen CHIS

Nectandra salicifolia (Kunth) Nees CAM, CHIS, COL, GRO, HGO, JAL, MEX, MICH, MOR, NAY, NLE, OAX, PUE, QRO, QROO, SLP, SIN, TAB, TAMS, VER, YUC

Nectandra salicina C.K. Allen CHIS

Nectandra turbacensis (Kunth) Nees CHIS, OAX, VER

Ocotea acuminatissima (Lundell) Rohwer CHIS, GRO, JAL, NAY, OAX, VER

* Ocotea atacta Lorea-Hern. GRO, OAX

Ocotea bernoulliana Mez CAM, CHIS, GRO, OAX, TAB, VER

* Ocotea betazensis (Mez) van der Werff CHIS, OAX

Ocotea botrantha Rohwer CHIS, OAX

Ocotea bourgeauviana (Mez) van der Werff CHIS, HGO, OAX, TAB, VER

*Ocotea candidovillosa Lorea-Hern. GRO, OAX

Ocotea cernua (Nees) Mez CAM, CHIS, OAX, SIN, TAB, VER

* Ocotea congregata van der Werff CHIS

* Ocotea corrugata van der Werff OAX, VER

Ocotea chiapensis (Lundell) Standl. \& Steyerm. CHIS, GRO, OAX, VER

*Ocotea chrysobalanoides (Lundell) Lundell CHIS

Ocotea dendrodaphne Mez CHIS, OAX, TAB, VER

*Ocotea disjuncta Lorea-Hern. GRO, OAX, VER

Ocotea effusa (Meisn.) Hemsl. CHIS, HGO, OAX, PUE, QRO, SLP, VER

Ocotea helicterifolia (Meisn.) Hemsl. CHIS, GRO, HGO, OAX, PUE, TAB, VER

* Ocotea heribertoi $\mathrm{T}$. Wendt CHIS, OAX, VER

Ocotea heydeana (Mez \& Donn. Sm.) Bernardi CHIS, OAX

*Ocotea iridescens Lorea-Hern. \& van der Werff OAX

* Ocotea klotzschiana (Nees) Hemsl. HGO, OAX, PUE, QRO, SLP, VER

Ocotea laetevirens Standl. \& Steyerm. CHIS, OAX, VER Ocotea leucoxylon (Sw.) Laness. CHIS, OAX, PUE, VER Ocotea magnifolia (Lundell) Lundell CHIS, OAX, TAB, VER * Ocotea matudae Lundell CHIS

Ocotea oblonga (Meisn.) Mez CHIS, OAX

* Ocotea parvula (Lundell) van der Werff CHIS, OAX, VER

Ocotea platyphylla (Lundell) Rohwer CHIS, OAX, VER

*Ocotea psychotrioides Kunth GRO, HGO, JAL, OAX, PUE, QRO, SLP, VER 
Ocotea puberula (Rich.) Nees CHIS, HGO, OAX, PUE, VER Ocotea purpurea (Mez) van der Werff CHIS, OAX

* Ocotea rovirosae Lorea-Hern. \& van der Werff OAX, TAB, VER

Ocotea rubriflora Mez CHIS, OAX, PUE, TAB, VER

Ocotea salvinii Mez CAM, CHIS, HGO, QRO, SLP, TAB, TAMS, VER, YUC

* Ocotea sarcodes Lorea-Hern. CHIS, OAX

* Ocotea sauroderma Lorea-Hern. OAX

Ocotea sinuata (Mez) Rohwer CHIS, OAX

Ocotea standleyi C.K. Allen CHIS, OAX, VER

Ocotea subalata Lundell CHIS

*Ocotea tampicensis (Meisn.) Hemsl. HGO, NLE, OAX, QRO, SLP, TAMS, VER

* Ocotea tonii (Lundell) van der Werff CHIS, GRO, OAX

Ocotea truncata Lundell CHIS

* Ocotea uxpanapana T. Wendt \& van der Werff CHIS, OAX, VER

Ocotea veraguensis (Meisn.) Mez CHIS, DGO, GRO, JAL, MICH, NAY, OAX, PUE, QROO, SLP, SIN, VER

* Ocotea verticillata Rohwer OAX, VER

* Ocotea zoque Lorea-Hern. OAX

Persea albida Kosterm. CHIS, OAX

Persea americana Mill. CAM, CHIS, CHIH, COAH, COL, DGO, GRO, HGO, JAL, MEX, MICH, MOR, NAY, NLE, OAX, PUE, QRO, QROO, SLP, SIN, SON, TAB, TAMS, VER, YUC, ZAC

*Persea brevipetiolata van der Werff OAX, VER

* Persea cinerascens S.F. Blake OAX, PUE, VER

* Persea chamissonis Mez CHIS, GTO, HGO, OAX, PUE, QRO, SLP, VER

*Persea chrysantha Lorea-Hern. GRO, MICH

Persea donnell-smithii Mez CHIS, GRO, MICH, OAX, TAMS, VER

*Persea hintonii C.K. Allen COL, GRO, HGO, JAL, MEX, MICH, NAY, PUE, SIN, TAMS, VER

Persea liebmannii Mez CHIS, CHIH, COAH, DGO, GTO, GRO, HGO, JAL, MICH, NAY, NLE, OAX, PUE, QRO, SLP, SIN, SON, TAMS, VER, ZAC

* Persea obscura Lorea-Hern. OAX

Persea pallescens (Mez) Lorea-Hern. HGO, OAX, PUE, QRO, SLP, VER

* Persea purpusii L.E. Kopp HGO, QRO, SLP

* Persea rufescens Lundell CHIS, OAX

Persea schiedeana Nees CAM, CHIS, OAX, PUE, SLP, QROO, TAB, TAMS, VER

Persea vesticula Standl. \& Steyerm. CHIS, OAX

Umbellularia californica (Hook. \& Arn.) Nutt. BCN

Family Lecythidaceae

*Eschweilera mexicana T. Wendt, S.A. Mori \& Prance OAX, VER

Family Lentibulariaceae

Genlisea filiformis A. St.-Hil. CHIS

*Pinguicula acuminata Benth. CDMX, GRO, HGO, MEX, MICH, QRO, SLP, TLAX, VER

*Pinguicula agnata Casper GTO, HGO, QRO, SLP
* Pinguicula calderoniae Zamudio QRO, SLP

* Pinguicula caudata Schltdl. AGS, CHIH, HGO, JAL, MEX, MICH, MOR, QRO, SON, TAMS, VER, ZAC

Pinguicula clivorum Standl. \& Steyerm. CHIS

Pinguicula colimensis McVaugh \& Michel COL, MICH

* Pinguicula conzattii Zamudio \& van Marm OAX

* Pinguicula crassifolia Zamudio HGO, VER

Pinguicula crenatiloba A. DC. CHIS, COL, GRO, JAL, MEX,

MICH, NAY, OAX, SIN, SON

* Pinguicula cyclosecta Casper COAH, NLE, SLP

* Pinguicula debbertiana Speta \& Fuchs SLP

* Pinguicula ehlersiae Speta \& F. Fuchs NLE, QRO, SLP, TAMS

* Pinguicula elizabethiae Zamudio HGO, QRO

* Pinguicula emarginata Zamudio \& Rzed. PUE, VER

* Pinguicula esseriana B. Kirchn. HGO, NLE, QRO, SLP, TAMS

*Pinguicula gigantea Luhrs OAX

*Pinguicula gracilis Zamudio COAH, NLE

*Pinguicula greenwoodii Cheek OAX

* Pinguicula gypsicola Brandegee SLP

*Pinguicula hemiepiphytica Zamudio \& Rzed. OAX

* Pinguicula heterophylla Benth. GRO, MOR, OAX

* Pinguicula ibarrae Zamudio HGO, VER

* Pinguicula immaculata Zamudio \& Lux COAH, NLE, TAMS

*Pinguicula kondoi Casper NLE, SLP, TAMS

*Pinguicula laueana Speta \& F. Fuchs OAX

* Pinguicula laxifolia Luhrs TAMS

*Pinguicula lilacina Schltdl. \& Cham. CHIS, GRO, HGO, JAL, OAX, QRO, SLP, TAMS, VER, ZAC

*Pinguicula macrophylla Kunth GTO, JAL, MEX, MICH, MOR, OAX, QRO, SLP, VER

* Pinguicula martinezii Zamudio QRO, SLP

* Pinguicula medusina Zamudio \& Studnicka GRO, OAX

* Pinguicula mirandae Zamudio \& A. Salinas OAX

* Pinguicula moctezumae Zamudio \& R.Z. Ortega HGO, QRO

Pinguicula moranensis Kunth CHIS, CHIH, DGO, CDMX, DGO, GTO, GRO, HGO, JAL, MEX, MICH, MOR, OAX, PUE, QRO, SLP, SIN, SON, TAMS, TLAX, VER, ZAC

*Pinguicula oblongiloba DC. AGS, CHIH, COL, DGO, GTO, JAL, MEX, MICH, NAY, OAX, SIN, ZAC

Pinguicula orchidioides DC. GRO, OAX

*Pinguicula parvifolia B.L. Rob. COL, DGO, GRO, JAL, MEX, MICH, NAY, QRO, SLP, ZAC

* Pinguicula rotundiflora Studnicka COAH, NLE, SLP, TAMS

* Pinguicula sharpii Casper \& Kondo CHIS

*Pinguicula takakii Zamudio \& Rzed. SLP

* Pinguicula utricularioides Zamudio \& Rzed. OAX

* Pinguicula zecheri Speta \& Fuchs GRO

Utricularia amethystina A. St.-Hil. \& Girard CAM, CHIS, GRO, MEX, QROO, VER

Utricularia erectiflora A. St.-Hil. \& Girard CHIS

Utricularia foliosa L. CAM, CHIS, GTO, GRO, JAL, OAX, QRO, QROO, SLP, TAB, TAMS, VER

Utricularia gibba L. CAM, CHIS, CHIH, COAH, CDMX, DGO, GTO, GRO, HGO, JAL, MEX, MICH, MOR, OAX, PUE, QRO, QROO, SLP, SON, TAB, TAMS, VER, YUC, ZAC *Utricularia hintonii P. Taylor GRO, MEX 
Utricularia hispida Lam. CHIS, TAB, VER

Utricularia hydrocarpa Vahl TAB

Utricularia jamesoniana Oliver CHIS

Utricularia juncea Vahl CHIS, TAB, VER

Utricularia livida E. Mey. CHIS, CHIH, CDMX, DGO, GRO, HGO, JAL, MEX, MICH, MOR, NAY, OAX, PUE, SLP, SIN, SON, TLAX, VER, ZAC

Utricularia macrorhiza Leconte BCS, COAH, CDMX, MEX, MICH, PUE

*Utricularia perversa $\mathrm{P}$. Taylor CHIH, GTO, HGO, JAL, MEX, OAX, QRO

*Utricularia petersoniae P. Taylor GRO

Utricularia purpurea Walter QROO, TAB

Utricularia pusilla Vahl CAM, CHIS, GRO, JAL, NAY, QROO,

VER

Utricularia radiata Small TAB

*Utricularia regia Zamudio \& Olvera GRO, OAX

Utricularia resupinata B.D. Greene ex Bigelow TAB

Utricularia simulans Pilger CHIS

Utricularia subulata L. CAM, CHIS, HGO, JAL, MEX, NAY, OAX, QROO, TAB, VER, YUC

\section{Family Liliaceae}

Calochortus ambiguus (M.E. Jones) Ownbey SON

* Calochortus balsensis García-Mend. GRO, OAX

*Calochortus barbatus (Kunth) J.H. Painter AGS, CHIH, COAH, COL, CDMX, DGO, GTO, GRO, HGO, JAL, MEX, MICH, MOR, NLE, OAX, PUE, QRO, SLP, SIN, TAMS, TLAX, VER, ZAC

*Calochortus cernuus J.H. Painter GRO, MEX, MICH, MOR Calochortus concolor (Baker) Purdy BCN, BCS

Calochortus dunnii Purdy BCN

Calochortus flexuosus S. Watson BCN, SON

*Calochortus foliosus Ownbey MICH

Calochortus fuscus Schult. f. AGS, CHIH, COL, DGO, GTO, GRO, JAL, MEX, MICH, MOR, NAY, OAX, SON, VER, ZAC Calochortus ghiesbreghtii S. Watson CHIS, GTO, HGO, MEX, QRO

*Calochortus hartwegii Benth. AGS, DGO, JAL, NAY, ZAC

Calochortus kennedyi Porter SON

*Calochortus marcellae G.L. Nesom COAH, NLE, SLP, TAMS

*Calochortus mendozae Espejo, López-Ferrari \& Ceja GRO, MEX, SLP

*Calochortus nigrescens Ownbey OAX, PUE

*Calochortus pringlei B.L. Rob. MEX, MOR, OAX, PUE

*Calochortus purpureus (Kunth) Baker AGS, COL, DGO, GTO, GRO, JAL, MEX, MICH, MOR, NAY, OAX, ZAC

Calochortus splendens Douglas ex Benth. BCN

*Calochortus venustulus Greene CHIH, DGO, GRO, HGO,

JAL, MEX, MICH, NAY, QRO, SLP, SON, VER, ZAC

Calochortus weedii Alph. Wood BCN

Chlorogalum parviflorum S. Watson BCN, BCS

Lilium humboldtii Roezl \& Leichtl. BCN

Lilium pardalinum Kellog BCN

Lilium parryi S. Watson BCN, SON

\section{Family Linaceae}

Linum aristatum Engelm. CHIH, COAH, SON
Linum australe Heller CHIH, COAH, CDMX, DGO, GTO, GRO, HGO, MEX, OAX, PUE, SLP, SON, TAMS, VER, ZAC Linum berlandieri Hook. CHIH, COAH, DGO, NLE, TAMS

*Linum cruciatum Planch. DGO, JAL, NAY, SON

*Linum flagellare (Small) H.K.A. Winkl. COAH, DGO, SLP, ZAC

Linum guatemalense Benth. CHIS, CHIH

*Linum gypsogenium G.L. Nesom NLE, TAMS

*Linum lasiocarpum Rose COAH, HGO, NLE, SLP, VER

Linum lewisii Pursh BCN, CHIH, COAH, DGO, NLE, SLP, SON, ZAC

*Linum longipes Rose GRO, PUE

Linum lundellii C.M. Rogers NLE, TAMS

*Linum mexicanum Kunth AGS, CHIS, CDMX, GTO, GRO, HGO, JAL, MEX, MICH, MOR, OAX, PUE, QRO, SLP, TLAX, VER, ZAC

*Linum modestum C.M. Rogers COAH, NLE, SLP, TAMS

Linum nelsonii Rose CHIS, COAH, GTO, HGO, JAL, NAY, NLE, PUE, QRO, SLP, TAMS, VER

Linum neomexicanum Greene CHIH, SON

*Linum orizabae Planch. CHIH, CDMX, GTO, GRO, HGO, JAL, MEX, MICH, MOR, OAX, PUE, QRO, SLP, SON, TLAX, VER

Linum pratense (Norton) Small AGS

*Linum pringlei $\mathrm{S}$. Watson CHIH, DGO, NAY, SON

Linum puberulum (Engelm.) Heller CHIH, COAH, SON

Linum rupestre (A. Gray) Engelm. ex A. Gray AGS, CHIS, CHIH, COAH, DGO, GTO, GRO, HGO, NLE, OAX, PUE, QRO, SLP, TAMS, VER, ZAC

*Linum rzedowskii Arreguín MEX

*Linum scabrellum Planch. AGS, COAH, GTO, HGO, NLE, OAX, PUE, QRO, SLP, TAMS, VER, ZAC

Linum schiedeanum Schltdl. \& Cham. CHIS, CHIH, COAH, CDMX, DGO, GTO, GRO, HGO, JAL, MEX, MICH, NAY, NLE, OAX, PUE, QRO, SLP, SON, TAMS, VER

*Linum tenellum Schltdl. \& Cham. HGO, PUE, QRO, SLP, TAMS, VER

Linum vernale Wooton CHIH, COAH, NLE, SLP

\section{Family Linderniaceae}

Lindernia anagallidea (Michx.) Pennell AGS, CHIS, COL, DGO, GRO, JAL, TAB, VER, ZAC

Lindernia antipoda (L.) Alston TAB, VER

*Lindernia blancoi (Merr.) Philcox COL, JAL

Lindernia dubia (L.) Pennell CHIH, COL, GTO, JAL, MICH, MOR, OAX, PUE, SLP, SON, TAMS, VER

*Lindernia scandens Sessé \& Moc. ex Don HGO, QRO, SLP

*Lindernia tridentata (Small) D.Q. Lewis COL, HGO, JAL, NAY, QRO, SLP

*Micranthemum procerorum L.O. Williams VER

Micranthemum umbrosum S.F. Blake GTO, HGO, PUE, QRO, TAMS, VER

\section{Family Loasaceae}

Cevallia sinuata Lag. AGS, CHIH, COAH, DGO, GTO, HGO, NLE, QRO, SLP, SON, TAMS, ZAC

*Eucnide aurea (A. Gray) H.J. Thomps. \& W.R. Ernst BCN, BCS 
Eucnide bartonioides Zucc. CHIH, COAH, DGO, NLE, QRO, SLP, TAMS, VER

*Eucnide cordata (Kellogg) Kellogg ex Curran BCN, BCS, SIN, SON

*Eucnide chiapasana B.L. Turner CHIS

*Eucnide durangensis H.J. Thomps. \& Powell COAH, DGO

* Eucnide floribunda S. Watson COAH, DGO

*Eucnide grandiflora (Groenl.) Rose CHIS, OAX, PUE

Eucnide hirta (G. Don) H.J. Thomps. \& W.R. Ernst CHIS, GRO, HGO, JAL, MEX, MICH, MOR, OAX, QRO, SLP, VER, ZAC *Eucnide hypomalaca Standl. CHIH, SON

*Eucnide lobata (Hook.) A. Gray COAH, DGO, GTO, GRO, HGO, MICH, MOR, NLE, PUE, QRO, SLP, TAMS, VER

Eucnide rupestris (Baill.) H.J. Thomps. \& W.R. Ernst BCN, $\mathrm{BCS}, \mathrm{SON}$

*Eucnide tenella (I.M. Johnst.) H.J. Thomps. \& W.R. Ernst $\mathrm{BCN}, \mathrm{BCS}$

Eucnide urens (A. Gray) Parry BCN

*Eucnide xylinea C.H. Müll. COAH, NLE

*Gronovia longiflora Rose GRO, MEX, MICH, MOR, VER

Gronovia scandens L. CAM, CHIS, CHIH, COL, DGO, GTO, GRO, HGO, JAL, MEX, MICH, MOR, NAY, OAX, PUE, QRO, QROO, SLP, SIN, SON, TAB, TAMS, VER, YUC, ZAC

Klaprothia fasciculata (C. Presl) Poston CHIS, COL, DGO, GRO, JAL, MEX, MICH, NAY, SIN, VER, ZAC

* Mentzelia adhaerens Benth. BCN, BCS, SON

Mentzelia affinis Greene BCN, BCS, SON

Mentzelia albicaulis Dougl. ex Hook. BCN, BCS, CHIH, COAH, DGO, SON

* Mentzelia arborescens Urb. \& Gilg GRO, MICH, OAX

Mentzelia aspera L. AGS, BCN, BCS, CAM, CHIS, CHIH, COL, DGO, GTO, GRO, HGO, JAL, MEX, MICH, MOR, NAY, OAX, PUE, QRO, QROO, SLP, SIN, SON, TAMS, VER, YUC, ZAC

Mentzelia asperula Wooton \& Standl. AGS, CHIH, COAH, DGO, JAL, NLE, SON, ZAC

*Mentzelia conzattii Greenm. OAX

*Mentzelia desertorum (A. Davidson) H.J. Thomps. \& J. Roberts BCN, SON

Mentzelia dispersa S. Watson BCN

*Mentzelia gypsophila B.L. Turner COAH, NLE

* Mentzelia hintoniorum B.L. Turner \& Hempel NLE

Mentzelia hirsutissima S. Watson BCN, SON

Mentzelia hispida Willd. AGS, BCS, CHIS, CHIH, COAH,

COL, CDMX, DGO, GTO, GRO, HGO, JAL, MEX, MICH, MOR, NLE, OAX, PUE, QRO, SLP, SON, TAMS, TLAX, VER, ZAC

Mentzelia incisa Urb. \& Gilg CHIH, COAH, NLE, PUE, TAMS, VER

Mentzelia involucrata S. Watson BCN, SON

*Mentzelia isolata Gentry SON

Mentzelia lindheimeri Urb. \& Gilg CHIH, COAH, DGO, HGO, NLE, SON

Mentzelia mexicana H.J. Thomps. \& Zavort. CHIH, COAH, DGO, NLE

Mentzelia micrantha (Hook. \& Arn.) Torr. \& A. Gray BCN, $\mathrm{BCS}$
Mentzelia multiflora (Nutt.) A. Gray BCN, CHIH, COAH, NLE, SON

Mentzelia nitens Greene BCN

Mentzelia obscura H.J. Thomps. \& J. Roberts BCN, SON

Mentzelia oreophila J. Darl. BCN, SON

Mentzelia pachyrhiza I.M. Johnst. CHIH, COAH, DGO, NLE

Mentzelia pumila (Nutt.) Torr. \& A. Gray CHIH, COAH, DGO,

NLE, SON

Mentzelia reverchonii (Urb. \& Gilg) H.J. Thomps. \& Zavort. $\mathrm{COAH}, \mathrm{NLE}$

Mentzelia saxicola H.J. Thomps. \& Zavort. CHIH, COAH, NLE, ZAC

*Mentzelia sinuata $\mathrm{S}$. Watson GRO

Mentzelia veatchiana Kellogg BCN, SON

Nasa triphylla (Juss.) Weigend CHIS, OAX, VER

* Petalonyx crenatus A. Gray CHIH, COAH, DGO

Petalonyx linearis Greene BCN, BCS, SON

Petalonyx thurberi A. Gray BCN, SON

* Schismocarpus matudae Steyerm. CHIS

*Schismocarpus pachypus S.F. Blake CHIS, OAX

\section{Family Loganiaceae}

Cynoctonum mitreola (L.) Britton CHIS, COAH, GRO, NLE, SIN, TAMS, VER

Mitreola petiolata (J.F. Gmel.) Torr. \& A. Gray CHIS, GRO, MEX, OAX, QROO, TAB, TAMS, VER, YUC

Spigelia anthelmia L. CAM, CHIS, OAX, QROO, SLP, TAB, VER, YUC

Spigelia carnosa Standl. \& Steyerm. CHIS

Spigelia coelostyloides K.R. Gould CHIS, OAX, SLP, SON, TAMS, VER

*Spigelia chiapensis K.R. Gould CHIS

* Spigelia dolichostachya Fern. Casas CHIS

*Spigelia guerrerensis L.O. Alvarado \& J. Jiménez Ram. GRO Spigelia hedyotidea A. DC. CHIS, CHIH, COAH, SON, TAMS, VER

Spigelia humboldtiana Cham. \& Schltdl. CAM, CHIS, GTO, GRO, OAX, PUE, QRO, QROO, SLP, TAB, TAMS, VER, YUC * Spigelia longiflora M. Martens \& Galeotti GTO, GRO, HGO, PUE, QRO, SLP, VER

* Spigelia mexicana A. DC. GRO

Spigelia nicotianiflora Chodat \& Hassler CHIS, GRO, MEX

* Spigelia palmeri Rose CHIS, OAX, VER

Spigelia polystachya Klotzsch. ex Prog. CAM, CHIS, TAB

Spigelia pygmaea D. Gibson CAM, CHIS, QROO, YUC

* Spigelia scabrella Benth. COL, GTO, GRO, JAL, MICH, OAX, SLP, ZAC

*Spigelia speciosa Kunth GRO, HGO, MOR, OAX, PUE, VER

Spigelia splendens H. Wendl. ex Hook. CHIS, GRO, OAX

*Spigelia trispicata H. Hurley ex K.R. Gould GRO, MICH

Strychnos brachistantha Standl. CAM, CHIS, COL, GRO, JAL, NAY, PUE, QROO, TAB, VER

Strychnos panamensis Seem. CAM, CHIS, GRO, JAL, NAY, OAX, QROO, SIN, TAB, VER

Strychnos panurensis Sprague \& Sandwith VER

Strychnos peckii B.L. Rob. CHIS

\section{Family Loranthaceae}


*Cladocolea andrieuxii Tiegh. GRO, MEX, OAX, TLAX

*Cladocolea coyucae Kuijt GRO, MICH, PUE

*Cladocolea cupulata Kuijt DGO, JAL

*Cladocolea dimorpha Kuijt PUE

*Cladocolea diversifolia (Benth.) Kuijt COL, JAL, MEX, $\mathrm{MICH}$

*Cladocolea glauca Kuijt OAX, PUE, SIN

*Cladocolea gracilis Kuijt COL, GRO, JAL, MICH, OAX

*Cladocolea grahamii (Benth.) Tiegh. COL, DGO, GRO, JAL, MEX, MICH, MOR, NAY, OAX, SIN, SON, ZAC

*Cladocolea inconspicua (Benth.) Kuijt COL, JAL, MICH, NAY, SIN, ZAC

*Cladocolea inorna (B.L. Rob. \& Greenm.) Kuijt COL, GRO, JAL, MICH, OAX, ZAC

*Cladocolea kuijtii Martínez-Ambr. \& Cruz-Durán GRO

*Cladocolea loniceroides (Tiegh.) Kuijt COL, CDMX, GRO,

JAL, MEX, MICH, MOR, OAX

*Cladocolea mcvaughii Kuijt NAY, SIN, SON

*Cladocolea microphylla (Kunth) Kuijt COL, GRO, JAL, MEX, MICH, MOR, OAX

Cladocolea oligantha (Standl. \& Steyerm.) Kuijt CHIS, COL, GRO, JAL, MEX, MICH, MOR, OAX, VER

*Cladocolea pedicellata Kuijt GRO, JAL, MEX, MICH, PUE, QRO

*Cladocolea pringle $i$ Kuijt GRO, JAL, MICH, OAX, SIN, SON, TLAX

*Cladocolea stricta Kuijt GRO

Cladocolea tehuacanensis (Oliver) Tiegh. OAX, PUE, TAB, TLAX

Oryctanthus cordifolius (C. Presl) Urb. CAM, CHIS, OAX, QROO, TAB

Phthirusa pyrifolia (Kunth) Eichl. CHIS

Psittacanthus americanus (Jacq.) Mart. CAM, CHIS, GRO, MICH, NAY, OAX, QROO, VER, YUC

* Psittacanthus auriculatus (Oliv.) Eichler OAX, PUE

* Psittacanthus breedlovei Kuijt CHIS

Psittacanthus calyculatus (DC.) G. Don AGS, CAM, CHIS, COL, CDMX, DGO, GTO, GRO, HGO, JAL, MEX, MICH, MOR, NAY, OAX, PUE, QRO, QROO, SLP, SIN, SON, TAB, TAMS, TLAX, VER, YUC, ZAC

*Psittacanthus karwinskyanus (Schult.) Eichl. GRO, MEX, MOR

*Psittacanthus macrantherus Eichler DGO, JAL, MICH, OAX, SIN, ZAC

Psittacanthus mayanus Standl. \& Steyerm. CAM, CHIS, QROO, TAB, YUC

* Psittacanthus palmeri (S. Watson) Barlow \& Wiens AGS, CHIH, COL, DGO, GTO, GRO, JAL, MEX, MICH, MOR, NAY, OAX, PUE, QRO, SIN, SON, ZAC

Psittacanthus ramiflorus (DC.) G. Don CHIS, COL, GRO, JAL, NAY, OAX, PUE, TAB, VER

Psittacanthus rhynchanthus (Benth.) Kuijt CAM, CHIS, COL, GRO, JAL, NAY, OAX, QROO, SIN, VER

Psittacanthus schiedeanus (Schltdl. \& Cham.) Blume CAM, CHIS, COL, GTO, GRO, JAL, MEX, MICH, MOR, OAX, PUE, QRO, QROO, SLP, TAB, TAMS, TLAX, VER, YUC

*Psittacanthus sonorae (S. Watson) Kuijt BCN, BCS, SIN,
SON

*Struthanthus alnii Bartlett OAX

Struthanthus brachybotrys Standl. \& Steyerm. CHIS, MEX, SIN

*Struthanthus capitatus Lundell CHIS, OAX

Struthanthus cassythoides Millsp. ex Standl. CAM, CHIS, GRO, OAX, QROO, TAB, VER, YUC

*Struthanthus condensatus Kuijt COL, JAL, MICH, NAY, OAX, SIN

Struthanthus densiflorus (Benth.) Standl. CHIS, COL, GRO, JAL, MICH, OAX, VER

*Struthanthus deppeanus (Schltdl. \& Cham.) D. Don CHIS, GRO, HGO, OAX, PUE, VER

*Struthanthus grahamii (Benth.) Standl. GRO, MEX, MOR, OAX, SIN, SON, VER

Struthanthus haenkeanus (Presl) Standl. CHIS, CHIH, GRO, OAX, SON, VER

Struthanthus hartwegii (Benth.) Standl. CHIS, OAX

*Struthanthus inconspicuus (Benth.) Standl. GRO, NAY, SIN

*Struthanthus inornus (B.L. Rob. \& Greenm.) Standl. OAX

Struthanthus interruptus (Kunth) G. Don CHIS, COL, DGO, GRO, HGO, JAL, MEX, MICH, MOR, NAY, OAX, SIN, TAB, VER

Struthanthus johnstonii Standl. \& Steyerm. CHIS, OAX, VER

*Struthanthus macrostachyus Lundell CHIS, OAX

Struthanthus marginatus (Desr.) Blume CHIS, HGO, OAX, SLP, VER

Struthanthus matudae Lundell CHIS

Struthanthus orbicularis (Kunth) Blume CAM, CHIS, COL, GRO, JAL, OAX, QROO, TAB

*Struthanthus palmeri Kuijt CHIH, JAL, SIN, SON

Struthanthus phaneroneurus Standl. CAM, CHIS, OAX, VER

Struthanthus quercicola (Schltdl. \& Cham.) Blume CHIS, COL, GRO, HGO, JAL, MEX, OAX, PUE, QRO, SLP, TAB, TAMS, VER

Struthanthus tacanensis Lundell CHIS

\section{Family Lythraceae}

Adenaria floribunda Kunth CHIS, GRO, MICH, OAX, VER

Ammannia auriculata Willd. AGS, CAM, CHIS, COL, GRO, JAL, MEX, MICH, MOR, NAY, OAX, QROO, SIN, SON, TAMS, VER, YUC

Ammannia coccinea Rottb. BCN, BCS, CAM, CHIS, CHIH, COAH, COL, DGO, GRO, JAL, MEX, MOR, OAX, PUE, QROO, SIN, SON, TAB, TAMS, VER, YUC

Ammannia robusta Heer \& Regel BCN, BCS, CAM, CHIS, CHIH, JAL, NAY, OAX, QROO, SIN, SON, TAB, TAMS, VER, YUC

Cuphea aequipetala Cav. AGS, CHIS, COAH, CDMX, DGO, GTO, GRO, HGO, JAL, MEX, MICH, MOR, NLE, OAX, PUE, QRO, SLP, SIN, TAMS, TLAX, VER, ZAC

*Cuphea alaniana S.A. Graham GRO, MEX

Cuphea angustifolia Jacq. ex Koehne AGS, CDMX, GRO, HGO, JAL, MEX, MICH, MOR, OAX, PUE, QRO, SLP, TLAX, VER, ZAC

Cuphea appendiculata Benth. CHIS, COL, GRO, JAL, MEX, MICH, MOR, NAY, OAX, PUE, SIN, VER, ZAC 
*Cuphea aquilana S.A. Graham \& T.B. Cavalcanti MICH

* Cuphea avigera B.L. Rob. \& Seaton CHIS, COL, GRO, JAL, MEX, MICH, OAX, PUE, SIN

Cuphea axilliflora (Koehne) Koehne CHIS, GRO

* Cuphea baillonis Koehne JAL, OAX

*Cuphea bracteolosa Koehne GRO, MICH, OAX

*Cuphea bustamanta Lex. CHIS, GRO, JAL, MEX, MICH, MOR, OAX

*Cuphea caeciliae Koehne CHIS

* Cuphea caesariata S.A. Graham JAL, NAY

*Cuphea calaminthifolia Schltdl. COL, GRO, HGO, JAL, MICH, NAY, OAX, PUE, QRO, SLP, TAMS, VER, ZAC

*Cuphea calcarata Benth. AGS, DGO, GTO, GRO, JAL, MICH,

NAY, ZAC

Cuphea calophylla Cham. \& Schltdl. CHIS, GRO, MEX, MICH, MOR, OAX, QROO

* Cuphea crassiflora S.A. Graham COL, JAL, SIN

* Cuphea cristata Rose CHIS, GRO, MICH, NAY

Cuphea cyanea DC. CHIS, COAH, COL, GRO, HGO, JAL, MEX, MICH, MOR, NLE, OAX, PUE, QRO, SLP, TAMS, VER, ZAC

Cuphea decandra Aiton CHIS, JAL, NAY, QRO, SLP, TAB, TAMS, VER

* Cuphea delicatula Brandegee SIN

*Cuphea dibrachiata S.A. Graham GRO, OAX

Cuphea elliptica Koehne CHIS, OAX, VER

*Cuphea empetrifolia Rose CHIS, GRO, JAL, MICH, OAX

Cuphea epilobifolia Koehne CHIS, OAX, TAB

* Cuphea ferrisiae Bacig. COL, JAL, NAY, SIN

Cuphea flavisetula Bacig. CHIS, JAL, NAY, SIN

*Cuphea flavovirens S.A. Graham GRO, MICH, OAX

*Cuphea gaumeri Koehne CAM, CHIS, QROO, YUC

*Cuphea glossostoma Koehne CHIS, GRO, MOR

*Cuphea graciliflora Koehne CHIS, COL, JAL, OAX, VER

*Cuphea heteropetala Koehne OAX

*Cuphea heterophylla Benth. DGO, GRO, JAL, MEX, MICH, MOR, NAY, OAX, SIN

Cuphea hookeriana Walp. BCS, CHIS, COL, DGO, GTO, GRO, HGO, JAL, MEX, MICH, MOR, NAY, OAX, PUE, QRO, SLP, SIN, SON, VER, ZAC

*Cuphea humifusa S.A. Graham COL, HGO, JAL, MICH, NAY, QRO

Cuphea hyssopifolia Kunth BCS, CHIS, GRO, HGO, JAL, MEX, MOR, NAY, OAX, PUE, QRO, SLP, SIN, TAB, TAMS, VER

Cuphea ignea A. DC. CHIS, OAX, VER

*Cuphea inflata S.A. Graham COL, JAL, NAY, SIN, ZAC

Cuphea infundibulum Koehne CHIS, OAX

*Cuphea intermedia Hemsl. CHIS, OAX

* Cuphea itzocanensis Koehne GRO, MOR, OAX

*Cuphea jorullensis Kunth AGS, COL, CDMX, DGO, GTO, GRO, HGO, JAL, MEX, MICH, MOR, NAY, OAX, PUE, SIN, TLAX, VER, ZAC

*Cuphea karwinskii Koehne OAX

*Cuphea koehneana Rose GRO, MEX, MOR, OAX

* Cuphea laminuligera Koehne CHIH, DGO, GRO, JAL, MEX,

MICH, MOR, NAY, OAX, PUE, SIN, SON, ZAC
*Cuphea lanceolata W.T. Aiton AGS, CHIS, COAH, COL, CDMX, GTO, GRO, HGO, JAL, MEX, MICH, MOR, NAY, NLE, OAX, QRO, SLP, SON, TAMS, VER, ZAC

Cuphea leptopoda Hemsl. CHIS, CHIH, COL, DGO, GRO, JAL, MEX, MICH, MOR, NAY, OAX, PUE, SIN, SON, TLAX, ZAC

*Cuphea lobophora Koehne CHIS, COL, DGO, GRO, JAL, MEX, MICH, MOR, NAY, OAX, PUE, SIN, SON, ZAC

Cuphea lophostoma Koehne GRO, MEX, MOR

Cuphea lutea Rose CHIS, GRO, OAX, PUE, VER

Cuphea llavea Lex. AGS, BCS, CHIH, COL, DGO, GTO, GRO,

JAL, MEX, MICH, NAY, OAX, QRO, SLP, SIN, SON, ZAC

*Cuphea megalophylla S.F. Blake OAX

* Cuphea mexiae Bacig. JAL

Cuphea micrantha Kunth CHIS, JAL

* Cuphea micropetala Kunth BCS, CHIS, COL, GTO, GRO,

JAL, MEX, MICH, MOR, OAX, PUE, VER

*Cuphea michoacana R.C. Foster COL, JAL, MICH

Cuphea mimuloides Cham. \& Schltdl. CHIS

*Cuphea nitidula Kunth CHIS, GRO, OAX, PUE, VER

Cuphea nudicostata Hemsl. CHIS, OAX

*Cuphea ornithoides R.C. Foster GRO, OAX

*Cuphea ownbeyi S.A. Graham DGO, SIN

*Cuphea painteri Rose JAL, ZAC

*Cuphea palmeri S. Watson CHIS, CHIH, JAL, MICH

*Cuphea palustris Koehne OAX

*Cuphea paucipetala S.A. Graham GRO, JAL, MEX, MOR, OAX, ZAC

* Cuphea pertenuis R.C. Foster GRO, MEX, MICH

Cuphea pinetorum Benth. BCS, CHIS, GRO, MOR, OAX, VER

*Cuphea procumbens Ortega AGS, CDMX, DGO, GTO, GRO,

HGO, JAL, MEX, MICH, MOR, OAX, PUE, SLP, SIN, TLAX, VER, ZAC

*Cuphea pulcherrima R.C. Foster GRO, MEX

* Cuphea purpurascens Bacig. PUE

*Cuphea quaternata Bacig. DGO, JAL, NAY, ZAC

Cuphea racemosa (L. f.) Spreng. HGO, MICH, OAX, TAMS, VER

*Cuphea rasilis S.A. Graham JAL, NAY

* Cuphea retroscabra S. Watson JAL, NAY

* Cuphea roseana Koehne DGO, JAL, SIN

*Cuphea salicifolia Cham. \& Schltdl. CHIS, HGO, JAL, MEX,

MICH, OAX, PUE, QRO, SLP, VER

Cuphea salvadorensis (Standl.) Standl. CHIS, GRO, OAX

Cuphea sanguinea Koehne CHIS

*Cuphea schumannii Koehne GRO, JAL, MEX, MICH, OAX,

PUE, VER, ZAC

Cuphea secundiflora Sessé \& Moc. ex DC. CHIS, GRO, MEX, SIN

Cuphea setosa Koehne OAX, VER

*Cuphea spectabilis S.A. Graham GRO, OAX

* Cuphea subuligera Koehne CHIS, OAX

Cuphea tetrapetala Koehne OAX, TAB, VER

Cuphea tolucana Peyr. CHIS, COL, CDMX, DGO, GRO, HGO, JAL, MEX, MICH, MOR, OAX, QRO, VER, ZAC

*Cuphea trichochila R.C. Foster GRO, MICH

*Cuphea trochilus S.A. Graham CHIS, MICH 
Cuphea utriculosa Koehne CAM, CHIS, COL, GRO, JAL, MICH, NAY, OAX, QROO, SLP, TAB, VER, YUC

*Cuphea vesiculigera R.C. Foster CHIS, COL, GRO, JAL, MEX, MICH, SIN

*Cuphea viscosa Rose CHIS, GTO, GRO, MICH

Cuphea viscosissima Jacq. AGS, JAL, NLE, SIN, TAMS

*Cuphea watsoniana Koehne COL, DGO, GTO, JAL, MICH, OAX, QRO, SIN

Cuphea wrightii A. Gray AGS, CHIS, CHIH, COL, CDMX, DGO, GTO, GRO, HGO, JAL, MEX, MICH, MOR, NAY, NLE, OAX, PUE, QRO, SLP, SIN, SON, TLAX, VER, ZAC

*Ginoria nudiflora (Hemsl.) Koehne CHIS, MICH, OAX, VER Heimia salicifolia Link AGS, BCN, BCS, CHIS, COAH, COL, GTO, GRO, HGO, JAL, MEX, MICH, MOR, NAY, NLE, OAX, PUE, QRO, SLP, SIN, SON, TAB, TAMS, VER, ZAC

Lafoensia punicifolia DC. CHIS, GRO, OAX

Lythrum alatum Pursh CHIS, CHIH, COAH, MEX, MICH, MOR, QRO, SLP, TAMS, VER

*Lythrum album Kunth CDMX, GTO, HGO, JAL, MEX, MICH, QRO

*Lythrum bryantii Brandegee BCS

Lythrum californicum Torr. \& A. Gray AGS, BCN, CHIH, COAH, DGO, JAL, NLE, SON, TAMS, ZAC

Lythrum gracile Benth. AGS, BCN, BCS, CHIS, CDMX, DGO, GTO, GRO, HGO, JAL, MEX, MICH, NLE, OAX, PUE, QRO, QROO, SLP, TAB, TAMS, VER, ZAC

Lythrum ovalifolium (A. Gray) Koehne COAH

Lythrum vulneraria Aiton ex Schrank CHIS, COL, CDMX, GRO, HGO, JAL, MEX, MICH, OAX, PUE, ZAC

Nesaea longipes A. Gray CHIH, COAH, NLE, SON, TAMS

*Nesaea palmeri S.A. Graham SLP, TAMS

*Nesaea pringlei Rose PUE

Rotala mexicana Cham. \& Schltdl. CAM, CHIS, CHIH, GRO, QRO, VER

Rotala ramosior (L.) Koehne BCN, BCS, CHIS, CHIH, COL, GRO, JAL, MEX, MICH, NAY, OAX, QROO, SIN, SON, TAB, TAMS, VER

\section{Family Magnoliaceae}

*Magnolia dealbata Zucc. COAH, HGO, NLE, OAX, PUE, QRO, SLP, TAMS, VER

*Magnolia guerrerensis J. Jiménez Ram., K. Vega \& CruzDurán GRO

*Magnolia iltisiana A. Vázquez COL, GRO, JAL, MICH

* Magnolia jaliscana A. Vázquez \& R. Guzmán COL, JAL

* Magnolia krusei J. Jiménez Ram. \& Cruz-Durán GRO, OAX

Magnolia lacandonica A. Vázquez, Pérez-Farrera \& MartínezCamilo CHIS

*Magnolia lopezobradorii A. Vázquez VER

*Magnolia mayae A. Vázquez \& Pérez-Farrera CHIS

Magnolia mexicana DC. CHIS, COL, CDMX, GRO, HGO, JAL, MEX, MICH, MOR, OAX, PUE, VER

*Magnolia oaxacensis A. Vázquez OAX

*Magnolia pacifica A. Vázquez CHIH, DGO, JAL, NAY, SIN, SON, ZAC

*Magnolia perezfarrerae A. Vázquez \& H. Gómez CHIS

Magnolia poasana (Pittier) Dandy CHIS
*Magnolia pugana (H.H. Iltis \& A. Vázquez) A. Vázquez \& Carvajal JAL, ZAC

* Magnolia rzedowskiana A. Vázquez, R. Domíngue \& R. Pedraza HGO, QRO, SLP, VER

*Magnolia schiedeana Schltdl. CHIS, CHIH, COL, DGO, GRO, HGO, JAL, MICH, NAY, NLE, OAX, PUE, QRO, SLP, SIN, SON, TAMS, VER, ZAC

*Magnolia sharpii Miranda CHIS

*Magnolia sinacacolinii A. Vázquez VER

*Magnolia tamaulipana A. Vázquez NLE, TAMS

*Magnolia vazquezii $\mathrm{R}$. Cruz D. \& K. Vega GRO, OAX

Magnolia yoroconte Dandy CHIS, VER

*Magnolia zoquepopolucae A. Vázquez VER

\section{Family Malpighiaceae}

Adelphia hiraea (Gaertn.) W.R. Anderson CHIS, OAX, QROO, $\mathrm{TAB}$

*Aspicarpa brevipes (DC.) W.R. Anderson DGO, JAL, NAY, ZAC

*Aspicarpa humilis (Benth.) A. Juss. AGS, CHIH, DGO, SLP Aspicarpa hyssopifolia A. Gray CHIH, COAH, NLE

*Aspicarpa lanata Rose CHIH, DGO, JAL, TAB, ZAC

*Aspicarpa steinmannii W.R. Anderson JAL, ZAC

*Banisteria pallida (Brandegee) Standl. GRO, OAX

*Banisteria portillana (S. Watson) C.B. Rob. JAL

Banisteriopsis elegans (Triana \& Planch.) Sandwith CHIS

Banisteriopsis muricata (Cav.) Cuatrec. CHIS, OAX, QRO, SLP

Bronwenia acapulcensis (Rose) W.R. Anderson \& C. Davis CHIS, GRO, JAL, MICH, OAX

Bronwenia cornifolia (Kunth) W.R. Anderson \& C. Davis CHIS, GRO, MEX, OAX, QROO, TAB, VER

Bunchosia biocellata Schltdl. CHIS, GRO, HGO, JAL, MEX, MICH, MOR, OAX, PUE, QRO, SLP, SIN, TAMS, VER

*Bunchosia breedlovei W.R. Anderson CHIS

*Bunchosia canescens (W.T. Aiton) DC. CAM, CHIS, DGO, GRO, MEX, MICH, MOR, OAX, PUE, QROO, SIN

*Bunchosia caroli W.R. Anderson GRO, OAX

Bunchosia cornifolia Kunth CHIS, COL, GRO, NAY, OAX, $\mathrm{TAB}$

*Bunchosia cruciana W.R. Anderson VER

Bunchosia glandulosa (Cav.) DC. CAM, QROO, YUC

Bunchosia gracilis Nied. CHIS, OAX

Bunchosia guatemalensis Nied. CAM, CHIS, OAX, QROO, TAB, VER

*Bunchosia hedraiophylla W.R. Anderson OAX

Bunchosia lindeniana A. Juss. CAM, CHIS, COL, DGO, GRO, HGO, JAL, MEX, MICH, MOR, OAX, PUE, QRO, QROO, SLP, SIN, TAB, TAMS, VER, YUC

*Bunchosia luzmariae W.R. Anderson JAL, NAY

Bunchosia macrophylla Rose ex Donn. Sm. CHIS, OAX

*Bunchosia matudae Lundell CHIS, OAX

*Bunchosia mcvaughii W.R. Anderson COL, GRO, JAL, NAY

Bunchosia montana A. Juss. CHIS, GRO, JAL, MEX, MICH, MOR, OAX, PUE, VER

*Bunchosia monticola Brandegee CHIS

Bunchosia nitida (Jacq.) DC. GRO, OAX 
*Bunchosia palmeri S. Watson COL, DGO, GRO, JAL, MEX, MICH, MOR, NAY, OAX, PUE, SLP, SIN, TAMS, VER, ZAC *Bunchosia praecox W.R. Anderson JAL, SIN

*Bunchosia sonorensis Rose CHIH, DGO, NAY, SIN, SON

*Bunchosia strigosa Schltdl. COL, JAL, NAY, OAX

Bunchosia swartziana Griseb. CAM, CHIS, QROO, TAB, VER, YUC

Byrsonima bucidifolia Standl. CAM, CHIS, GRO, MEX, OAX, QROO, TAB, YUC

Byrsonima crassifolia (L.) Kunth CAM, CHIS, COL, DGO, GRO, HGO, JAL, MEX, MICH, MOR, NAY, OAX, PUE, QROO, SLP, SIN, TAB, TAMS, VER, YUC, ZAC

* Byrsonima oaxacana A. Juss. OAX

*Calcicola parvifolia (A. Juss.) W.R. Anderson \& C. Davis GRO, MEX, OAX, PUE, VER

*Calcicola sericea (Nied.) W.R. Anderson \& C. Davis CHIH, COAH, DGO, NLE, SLP, SON, ZAC

*Callaeum clavipetalum D.M. Johnson OAX

*Callaeum coactum D.M. Johnson GRO, MEX, MICH, OAX, PUE

*Callaeum chiapense (Lundell) D.M. Johnson CHIS

*Callaeum johnsonii W.R. Anderson PUE

Callaeum macropterum (Sessé \& Moc. ex DC.) D.M. Johnson

AGS, BCN, BCS, CHIH, COAH, COL, DGO, GRO, HGO, JAL, MEX, MICH, NAY, NLE, OAX, PUE, QRO, SLP, SIN, SON, TAMS, VER, ZAC

Callaeum malpighioides (Turcz.) D.M. Johnson CHIS, COL, GRO, JAL, OAX, VER

*Callaeum septentrionale (A. Juss.) D.M. Johnson COAH, GTO, HGO, NLE, OAX, QRO, SLP, SIN, TAMS, VER

Carolus sinemariensis (Aubl.) W.R. Anderson CAM, CHIS, JAL, NAY, QROO, SIN, YUC

*Cottsia californica (Benth.) W.R. Anderson \& C. Davis BCN, BCS, CHIH, SIN, SON

Cottsia gracilis (A. Gray) W.R. Anderson \& C. Davis BCN, BCS, CHIH, COAH, DGO, NLE, SON, ZAC

*Cottsia linearis (Wiggins) W.R. Anderson \& C. Davis SON

Christianella mesoamericana (W.R. Anderson) W.R. Anderson CHIS

*Diplopterys mexicana Gates VER

*Echinopterys eglandulosa (A. Juss.) Small CHIH, COL, DGO, GRO, JAL, MEX, MOR, NAY, OAX, PUE, SIN, SON, VER, ZAC

*Echinopterys setosa Brandegee COAH, DGO, NLE, ZAC

Galphimia angustifolia Benth. AGS, BCN, BCS, CHIH, COAH,

DGO, NLE, SIN, SON, TAMS

* Galphimia arenicola C.E. Anderson OAX

*Galphimia calliantha C.E. Anderson GRO, MICH, OAX

*Galphimia elegans Baill. OAX

*Galphimia floribunda C.E. Anderson CHIH, DGO, JAL, NAY, SIN, SON

*Galphimia glandulosa Cav. GRO, JAL, MICH, MOR, SIN, VER, ZAC

Galphimia glauca Cav. AGS, CAM, CHIS, COL, DGO, GTO, GRO, HGO, JAL, MEX, MICH, MOR, NAY, NLE, OAX, PUE, QRO, SLP, SIN, SON, TAMS, VER, ZAC

*Galphimia gracilis Bartl. CHIS, COL, DGO, GRO, JAL,
MICH, NAY, OAX, SLP, SIN, VER, ZAC

* Galphimia hirsuta Cav. COL, GRO, JAL, OAX

*Galphimia langlassei (S.F. Blake) C.E. Anderson COL, GRO,

JAL, MICH

* Galphimia mirandae C.E. Anderson GRO, OAX, PUE

*Galphimia multicaulis A. Juss. GRO, MICH, OAX

* Galphimia oaxacana C.E. Anderson OAX

*Galphimia paniculata Bartl. COL, GRO, JAL, MEX, MICH,

MOR, NAY, OAX, PUE, SIN, ZAC

*Galphimia radialis C.E. Anderson GRO, OAX

*Galphimia sessilifolia Rose OAX

Galphimia speciosa C.E. Anderson CHIS, GRO, OAX, PUE, VER

*Galphimia tuberculata (Rose) Nied. COL, DGO, GRO, JAL, MICH, NAY, SIN

*Galphimia vestita $\mathrm{S}$. Watson CHIH, NAY, SIN, SON

Gaudichaudia albida Schltdl. \& Cham. AGS, CAM, CHIS, CHIH, COL, DGO, GRO, HGO, JAL, MEX, MICH, MOR, NAY, OAX, PUE, QRO, SLP, SIN, SON, VER, YUC, ZAC

*Gaudichaudia andersonii S.L. Jessup MEX

*Gaudichaudia arnottiana A. Juss. JAL, MOR, SIN

*Gaudichaudia confertiflora Juss. AGS, CHIS, COL, JAL, OAX

*Gaudichaudia congestiflora A. Juss. OAX

*Gaudichaudia cycloptera (DC.) W.R. Anderson COL, GRO, JAL, MEX, MICH, MOR, NAY, OAX

*Gaudichaudia cynanchoides Kunth AGS, CHIS, COAH, COL, CDMX, DGO, GTO, HGO, JAL, MEX, MICH, MOR, NAY, OAX, PUE, QRO, SLP, TAMS, VER, ZAC

*Gaudichaudia chasei W.R. Anderson JAL, MOR

*Gaudichaudia diandra Chodat GTO, GRO, JAL, MEX, MICH, MOR, OAX

*Gaudichaudia galeottiana Chodat GRO, OAX, PUE, VER

Gaudichaudia hexandra (Nied.) Chodat CHIS, GRO

Gaudichaudia hirtella (Rich.) S.L. Jessup AGS, CHIH, COAH,

CDMX, DGO, GTO, GRO, HGO, JAL, MEX, MICH, NAY, NLE, OAX, PUE, QRO, SLP, SON, TAMS, ZAC

*Gaudichaudia implexa S.L. Jessup GTO, JAL, MICH, NLE, OAX, PUE, SLP, SON, TAMS, VER, ZAC

*Gaudichaudia intermixteca S.L. Jessup OAX, PUE

Gaudichaudia karwinskiana A. Juss. JAL, MEX, MOR, SLP, TAMS, ZAC

* Gaudichaudia krusei W.R. Anderson GRO

*Gaudichaudia mcvaughii W.R. Anderson COL, GRO, JAL, OAX, VER

*Gaudichaudia mollis Chodat AGS, JAL

Gaudichaudia mucronata (Moc. \& Sessé ex DC.) A. Juss. AGS, CHIH, COL, CDMX, DGO, GTO, GRO, HGO, JAL, MEX, MICH, MOR, NAY, OAX, PUE, QRO, QROO, SLP, SON, TLAX, VER, YUC, ZAC

*Gaudichaudia subverticillata Rose COL, DGO, GRO, JAL, NAY, ZAC

*Gaudichaudia symplecta S.L. Jessup MEX, MICH

*Gaudichaudia synoptera S.L. Jessup GRO, MEX, MOR

* Gaudichaudia webbiana A. Juss. ND

*Gaudichaudia zygoptera S.L. Jessup GRO, OAX, PUE

Heteropterys brachiata (L.) DC. CAM, CHIS, COL, GTO, GRO, HGO, JAL, MEX, MICH, MOR, NAY, OAX, PUE, 
QRO, QROO, SLP, SIN, TAB, TAMS, VER, YUC

*Heteropterys cotinifolia A. Juss. CHIS, COL, DGO, GRO, JAL, MEX, MICH, MOR, NAY, OAX, PUE, SIN, SON, VER, ZAC

Heteropterys laurifolia (L.) A. Juss. CAM, CHIS, COL, GRO, JAL, MEX, MICH, MOR, NAY, OAX, QROO, SIN, TAB, VER, YUC

Heteropterys lindeniana A. Juss. CAM, CHIS, QROO, TAB, YUC

Heteropterys macrostachya A. Juss. CHIS, OAX

*Heteropterys palmeri Rose CHIH, COL, GRO, JAL, MICH, MOR, NAY, OAX, SIN, SON

Heteropterys panamensis Cuatrec. \& Croat CHIS, VER

Hiraea barclayana Benth. CHIS, GRO, JAL, OAX, SIN, VER Hiraea fagifolia (DC.) A. Juss. CHIS, OAX, PUE, TAB, VER

*Hiraea purpusii Brandegee VER

Hiraea reclinata Jacq. CAM, CHIS, COL, GRO, JAL, NAY, OAX, QROO, TAB, VER, YUC

Hiraea smilacina Standl. OAX, TAB, VER

Hiraea ternifolia (Kunth) A. Juss NAY, OAX

*Lasiocarpus ferrugineus Gentry COL, DGO, JAL, MOR, NAY, OAX, PUE, SIN

*Lasiocarpus salicifolius Liebm. CHIS, GRO, MOR, OAX, PUE, SIN

* Malpighia davilae W.R. Anderson OAX, PUE

* Malpighia diversifolia Brandegee BCS

Malpighia emarginata Sessé \& Moc. ex DC. CAM, CHIS, CHIH, GRO, JAL, OAX, QROO, SIN, SON, VER, YUC

* Malpighia emiliae W.R. Anderson COL, JAL

*Malpighia galeottiana A. Juss. GRO, JAL, MEX, MICH, OAX, PUE, SLP

Malpighia glabra L. CAM, CHIS, COAH, GRO, HGO, JAL, MEX, NAY, NLE, OAX, PUE, QRO, QROO, SLP, SIN, SON, TAB, TAMS, VER, YUC

*Malpighia granitica F.K. Mey. GRO, MICH

*Malpighia hintonii Bullock MEX, MICH

Malpighia incana Mill. CAM, YUC

*Malpighia latifolia F.K. Mey. VER

*Malpighia leticiana (W.R. Anderson) W.R. Anderson \& C.

Davis OAX

Malpighia lundellii C.V. Morton CAM, QROO, TAB, YUC

* Malpighia macrocarpa F.K. Mey. CHIS, VER

* Malpighia mexicana A. Juss. CHIS, COL, DGO, GTO, GRO,

JAL, MEX, MICH, MOR, NAY, OAX, PUE, VER, ZAC

*Malpighia nayaritensis (Vivaldi) F.K. Mey. COL, JAL, NAY

*Malpighia novogaliciana W.R. Anderson COL, JAL, NAY, OAX

*Malpighia ovata Rose COL, GRO, JAL, MEX, MICH, OAX, PUE, SON, TAMS

Malpighia romeroana Cuatrec. CHIS, DGO, JAL, NAY, OAX, TAB, VER

* Malpighia rzedowskii W.R. Anderson COL, JAL, MICH, OAX

* Malpighia sessilifolia W.R. Anderson OAX, VER

*Malpighia souzae Miranda CAM, CHIS, OAX, VER, YUC

Malpighia spathulifolia F.K. Mey. CAM, QROO, YUC

*Malpighia tomentosa Pav. ex Moric. DGO, JAL, MOR, OAX

*Malpighia watsonii Rose BCS, SIN, SON
Malpighia wendtii W.R. Anderson CHIS, VER

* Malpighia wilburiorum W.R. Anderson COL, JAL

*Malpighia yucatanaea F.K. Mey. CAM, YUC

* Mascagnia gouania Small GRO, MOR

*Mascagnia lilacina (S. Watson) Nied. COAH, DGO, NLE, QRO, SLP, TAMS

*Mascagnia polybotrya (A. Juss.) Nied. GRO, MEX, MICH, MOR, OAX, PUE

Mascagnia polycarpa Brandegee CAM, CHIS, OAX, QROO, VER, YUC

Mascagnia sepium (A. Juss.) Griseb. CHIS, OAX, VER

Mascagnia sinemariensis (Aubl.) Griseb. CHIS, COL, JAL, NAY, OAX, TAB, VER

Mascagnia tomentosa C.E. Anderson CHIS, OAX, VER

Mascagnia vacciniifolia Nied. CHIS, GRO, MOR, OAX, PUE, TAB, VER

*Psychopterys dipholiphylla (Small) W.R. Anderson \& S. Corso CHIS, COL, GRO, JAL, MEX, MICH, MOR, OAX, PUE, SLP, TAMS, VER

*Psychopterys mcvaughii W.R. Anderson \& S. Corso COL, JAL

Psychopterys multiflora (Nied.) W.R. Anderson \& S. Corso CHIS, OAX

* Psychopterys ornata W.R. Anderson \& S. Corso CHIS

Psychopterys rivularis (C.V. Morton \& Standl.) W.R. Anderson \& S. Corso CHIS, QROO, VER

Stigmaphyllon bannisterioides (L.) C.E. Anderson CHIS, TAB, VER

Stigmaphyllon ellipticum (Kunth) A. Juss. CAM, CHIS, OAX, QROO, TAB, TAMS, VER, YUC

Stigmaphyllon lindenianum A. Juss. CAM, CHIS, OAX, PUE, QRO, QROO, SLP, TAB, VER, YUC

Stigmaphyllon pseudopuberum Nied. CHIS, OAX, TAB

Stigmaphyllon retusum Griseb. CHIS, HGO, OAX, PUE, QRO, SLP, TAB, TAMS, VER

*Stigmaphyllon selerianum Nied. CHIS, OAX

Tetrapterys arcana C.V. Morton CAM, CHIS, OAX, QROO, YUC

*Tetrapterys cotoneaster A. Juss. OAX

Tetrapterys discolor (G. Mey.) DC. CHIS, QROO, VER

*Tetrapterys donnell-smithii Small CHIS, OAX, VER

*Tetrapterys glabrifolia (Griseb.) Small CHIS, VER

Tetrapterys heterophylla (Griseb.) W.R. Anderson CHIS, GRO, JAL, MICH, OAX, VER

Tetrapterys macrocarpa I.M. Johnst. CHIS, VER

*Tetrapterys mexicana Hook. \& Arn. CHIS, COL, GRO, JAL, MEX, MICH, MOR, NAY, NLE, OAX, PUE, SIN, VER

Tetrapterys schiedeana Schltdl. \& Cham. CAM, CHIS, GRO, HGO, JAL, MICH, NAY, OAX, PUE, QRO, QROO, SLP, VER, YUC

Tetrapterys seleriana Nied. CAM, OAX, QROO, TAB, YUC

\section{Family Malvaceae}

Abutilon abutiloides (Jacq.) Garcke ex Hochr. AGS, CAM, CHIH, COAH, COL, DGO, GTO, GRO, JAL, MEX, MICH, NAY, NLE, OAX, PUE, QROO, SLP, SIN, SON, TAMS, YUC, ZAC 
Abutilon andrieuxii Hemsl. CHIS, GRO, MOR, NAY, OAX *Abutilon bastardioides Baker f. ex Rose COL, JAL Abutilon berlandieri A. Gray ex S. Watson COAH, NLE, TAMS *Abutilon bracteosum Fryxell CHIS, COL, NAY, OAX Abutilon californicum Benth. BCN, BCS, CHIH, COL, DGO, SIN, SON

*Abutilon coahuilae Kearney COAH, DGO

Abutilon divaricatum Turcz. CHIS, GRO, MEX, OAX, VER

*Abutilon dugesii S. Watson BCS, COL, GTO, HGO, JAL, MEX, MICH, NAY, NLE, OAX, PUE, QRO, SLP, ZAC

Abutilon fruticosum Guill. \& Perr. CHIH, COAH, COL, JAL, NLE, PUE, SLP, TAMS, VER, ZAC

Abutilon glabriflorum Hochr. CAM, COAH, HGO, NLE, OAX, PUE, QRO, SLP, TAMS, VER, YUC

*Abutilon grandidentatum Fryxell AGS, OAX, SIN, ZAC

*Abutilon haenkeanum C. Presl CHIS, COL, GRO, JAL, MEX, MICH, MOR, NAY, OAX, SIN, ZAC

Abutilon hulseanum (Torr. \& A. Gray) Torr. ex A. Gray PUE, TAB, TAMS, VER

Abutilon hypoleucum A. Gray COAH, DGO, HGO, MICH, NLE, OAX, PUE, QRO, SLP, TAMS, VER

Abutilon incanum (Link) Sweet AGS, BCN, BCS, CHIH, COAH, DGO, GTO, GRO, JAL, MICH, NLE, QRO, SLP, SIN, SON, TAMS

*Abutilon jaliscanum Standl. JAL

*Abutilon macvaughii Fryxell COL, JAL

Abutilon malacum S. Watson CHIH, COAH, DGO, GTO, HGO, NLE, QRO, SLP, SON, ZAC

Abutilon mollicomum (Willd.) Sweet CHIH, COAH, DGO, GRO, NLE, OAX, SON, VER

*Abutilon mucronatum J.E. Fryxell BCS, CHIH, COL, GTO, GRO, JAL, MICH, OAX, PUE, SLP, SIN, SON, ZAC

Abutilon pachecoanum Standl. \& Steyerm. CHIS

Abutilon palmeri A. Gray BCN, BCS, CHIH, OAX, SIN, SON, TAMS

Abutilon parvulum A. Gray BCN, CHIH, COAH, SON, TAMS Abutilon percaudatum Hochr. CHIS, GRO, HGO, OAX, QRO, SLP, VER

Abutilon permolle (Willd.) Sweet CAM, CHIS, QROO, YUC

*Abutilon pinkavae Fryxell CHIH, COAH, NLE

*Abutilon procerum Fryxell TAMS

Abutilon purpusii Standl. BCN, BCS, CHIS, HGO, MEX, OAX, PUE, QRO, SLP, TAMS, VER

Abutilon reventum $\mathrm{S}$. Watson CHIS, CHIH, COAH, COL, DGO, GTO, GRO, JAL, MEX, MICH, NAY, OAX, PUE, QRO, QROO, SLP, SIN, SON, ZAC

*Abutilon simulans Rose CHIH, COL, DGO, GTO, GRO, JAL, MEX, MICH, MOR, OAX, QRO, SLP, TAMS

*Abutilon sphaerostaminum Hochr. PUE, TAMS, VER

*Abutilon straminicarpum Fryxell PUE

*Abutilon tehuantepecense Fryxell OAX

Abutilon trisulcatum (Jacq.) Urb. CAM, CHIS, CHIH, COL, DGO, GRO, HGO, JAL, MICH, MOR, NAY, NLE, OAX, QRO, QROO, SLP, SIN, SON, TAB, TAMS, VER, YUC, ZAC Abutilon wrightii A. Gray CHIH, COAH, NLE, SLP, SON, TAMS, VER

*Abutilon xanti A. Gray BCS, JAL, MICH
Allosidastrum hilarianum (C. Presl) Krapov., Fryxell \& D. Bates CHIS, COL, DGO, GRO, JAL, MICH, NAY, PUE, SLP, SIN, SON, VER, ZAC

Allosidastrum interruptum (Balbis ex DC.) Krapov., Fryxell \& D.M. Bates COL, JAL, MICH, OAX

Allosidastrum pyramidatum (Cav.) Krapov., Fryxell \& D.M. Bates CAM, CHIS, COL, GRO, HGO, JAL, MICH, NAY, OAX, PUE, QRO, QROO, SLP, SIN, TAMS, VER

*Allowissadula chiangii M.C. Johnst. CHIH, DGO, GTO, JAL, SLP, ZAC

*Allowissadula floribunda (Schltdl.) Fryxell GTO, HGO, MICH, NLE, PUE, QRO, SLP, TAMS, ZAC

*Allowissadula glandulosa (Rose) D.M. Bates HGO, QRO

Allowissadula holosericea (Scheele) D.M. Bates BCS, CHIH, COAH, DGO, GTO, NLE, QRO, QROO, SLP, TAMS, YUC

Allowissadula lozanii (Rose) D.M. Bates NLE, TAMS

*Allowissadula pringlei (Rose) D.M. Bates GTO, HGO, JAL, OAX, PUE, QRO, SIN

*Allowissadula racemosa (Schltdl.) Fryxell GTO, HGO, MICH, QRO, SLP, VER

*Allowissadula rosei (R.E. Fr.) Bates GTO, NLE, OAX, PUE, QRO

*Allowissadula sessei (Lag.) D.M. Bates AGS, COL, CDMX, DGO, GTO, HGO, JAL, MEX, MICH, PUE, QRO, SLP, VER, ZAC

Anoda abutiloides A. Gray CHIH, DGO, JAL, NAY, SIN, SON, ZAC

Anoda acerifolia Cav. BCN, BCS, CAM, CHIS, CHIH, COL, DGO, GTO, GRO, HGO, JAL, MEX, MICH, MOR, NAY, OAX, PUE, QRO, QROO, SLP, SIN, SON, TAMS, VER, YUC

*Anoda albiflora Fryxell CHIH, GTO, JAL, MICH, NLE Anoda crenatiflora Ortega BCS, CHIS, CHIH, COAH, COL, CDMX, GTO, GRO, HGO, JAL, MEX, MICH, MOR, NLE, OAX, PUE, QRO, SLP, SIN, SON, TAMS, VER, ZAC

Anoda cristata (L.) Schltdl. AGS, BCN, BCS, CAM, CHIS, CHIH, COAH, COL, CDMX, DGO, GTO, GRO, HGO, JAL, MEX, MICH, MOR, NAY, NLE, OAX, PUE, QRO, QROO, SLP, SIN, SON, TAB, TAMS, TLAX, VER, YUC, ZAC

Anoda guatemalensis Fryxell CHIS, OAX, PUE

*Anoda henricksonii M.C. Johnst. GTO, HGO, QRO, SLP, ZAC

*Anoda hintoniorum Fryxell JAL, MEX, MICH, MOR

*Anoda hirta Fryxell BCS, TAMS

Anoda lanceolata Hook. \& Arn. AGS, BCN, BCS, CHIH, COAH, COL, GRO, HGO, JAL, MEX, MICH, NAY, NLE, OAX, QRO, SLP, SIN, SON, VER, TAMS, ZAC

*Anoda leonensis Fryxell COAH, NLE, TAMS

*Anoda maculata Fryxell BCS, COL, DGO, GTO, GRO, HGO, JAL, MICH, OAX, PUE, QRO, ZAC

*Anoda palmata Fryxell BCS, COL, DGO, GRO, JAL, MICH, NAY, OAX, SON, ZAC

*Anoda paniculata Hochr. GTO, GRO, MEX, MICH, SLP, SIN

*Anoda pedunculosa Hochr. GTO, HGO, OAX, QRO, SLP, TAMS, VER

Anoda pentaschista A. Gray BCN, BCS, CAM, CHIH, COAH, COL, DGO, GTO, GRO, HGO, JAL, MEX, MICH, MOR, OAX, QRO, QROO, SLP, SIN, SON, TAMS, VER, YUC, ZAC *Anoda polygyna Fryxell DGO, SIN, SON 
*Anoda pristina Fryxell CHIS

*Anoda pubescens Schltdl. COAH, GTO, GRO, HGO, JAL, MEX, MICH, MOR, OAX, PUE, QRO, SLP, VER, ZAC

Anoda reticulata $\mathrm{S}$. Watson DGO, NAY, SLP, SIN, SON

*Anoda speciosa Fryxell MEX

*Anoda succulenta Fryxell CHIH, NAY, SIN

Anoda thurberi A. Gray AGS, BCS, CHIH, COAH, COL, DGO, GTO, GRO, HGO, JAL, MEX, MICH, MOR, NLE, OAX, PUE, QRO, SLP, SON, TAMS, VER, ZAC

*Anotea flavida (DC.) Ulbr. GRO

Apeiba tibourbou Aubl. CHIS, GRO, HGO, JAL, NAY, OAX, PUE, VER

*Ayenia abutilifolia (Turcz.) Turcz. COL, GRO, JAL, MEX, MICH, QROO, SIN, SON, VER, YUC, ZAC

*Ayenia berlandieri S. Watson GRO, HGO, JAL, MEX, MICH, MOR, OAX, QRO, SLP, TAMS

*Ayenia fasciculata Millsp. CAM, QROO, YUC

* Ayenia fruticosa Rose OAX, PUE, QRO

*Ayenia glabra S. Watson BCN, BCS, COL, DGO, GRO, JAL, MICH, NAY, OAX, SIN, SON, ZAC

*Ayenia jaliscana S. Watson BCS, CHIS, CHIH, COL, DGO, GTO, GRO, JAL, MEX, MICH, QRO, SLP, SIN, SON, ZAC

Ayenia limitaris Cristóbal AGS, COAH, JAL, TAMS, ZAC

Ayenia magna L. CAM, CHIS, GRO, NLE, QROO, SLP, VER, YUC

*Ayenia mexicana Turcz. AGS, CAM, CHIS, COL, GRO, JAL, MEX, MICH, OAX, PUE, QRO, QROO, SON, YUC

Ayenia micrantha Standl. CHIS, COL, DGO, GRO, JAL, MEX, MICH, NAY, OAX, QRO, SIN, VER, ZAC

Ayenia microphylla A. Gray CHIH, COAH, DGO, NLE, SON, TAMS

*Ayenia mirandae Cristóbal GRO

* Ayenia neglecta Cristóbal CHIS, GRO, MOR, OAX

*Ayenia ovata Hemsl. GRO, HGO, MOR, OAX, PUE, SLP, VER

Ayenia palmeri $\mathrm{S}$. Watson $\mathrm{CHIH}, \mathrm{GRO}, \mathrm{MICH}, \mathrm{OAX}, \mathrm{SON}$

* Ayenia paniculata Rose SON

Ayenia pilosa Cristóbal AGS, CHIH, COAH, JAL, NLE, SLP, TAMS

Ayenia purpusii Brandegee DGO, HGO, JAL, MEX, MICH, MOR, TAMS, VER

Ayenia pusilla L. BCS, CHIS, CHIH, COAH, COL, DGO, GRO, JAL, MEX, MICH, NAY, NLE, OAX, PUE, QROO, SLP, SIN, SON, TAMS, VER, YUC, ZAC

*Ayenia rotundifolia Hemsl. GTO, HGO, QRO, SLP, VER

*Ayenia standleyi Cristóbal CHIS, DGO, GTO, HGO, JAL, NAY, OAX, PUE, QRO, SLP, SIN, VER

Ayenia wrightii B.L. Rob. BCN, BCS, COL, DGO, GRO, JAL, MICH, MOR, OAX, NAY, SIN, SON

*Bakeridesia amoena Fryxell OAX

* Bakeridesia bakeriana (Rose) D.M. Bates COL, GRO, JAL, OAX

*Bakeridesia ferruginea (Martyn) Krapov. MICH, OAX, QRO, SLP, TAMS, VER

Bakeridesia gaumeri (Standl.) D.M. Bates CAM, QROO, YUC

*Bakeridesia gloriosa D.M. Bates CHIS, OAX, VER

*Bakeridesia guerrerensis Donnell GRO
*Bakeridesia huastecana Donnell SLP, TAMS, VER

Bakeridesia integerrima (Hook. f.) D.M. Bates CHIS, GRO, HGO, OAX, QRO, SLP, SON, TAMS, VER, YUC

*Bakeridesia jaliscana Donnell COL, JAL

Bakeridesia nelsonii (Rose) D.M. Bates CHIS

*Bakeridesia notolophium (A. Gray) Hochr. CHIS, NLE, PUE, QRO, SLP, VER, ZAC

*Bakeridesia parvifolia Donnell COL, JAL

Bakeridesia pittieri (Donn. Sm.) D.M. Bates CHIS, OAX

*Bakeridesia yucatana (Standl.) D.M. Bates COL, JAL, QROO, YUC

*Bakeridesia zapoteca Donnell OAX

Bastardia bivalvis (Cav.) Kunth ex Griseb. BCS, COL, GTO, HGO, JAL, MICH, NAY, OAX, PUE, QRO, SLP, SIN, SON, TAMS, VER, ZAC

Bastardia viscosa (L.) Kunth CAM, CHIS, GRO, NLE, OAX, PUE, QRO, QROO, SLP, SON, TAMS, VER, YUC

*Bastardiastrum batesii Fryxell \& S.D. Koch COL, GRO, JAL

*Bastardiastrum cinctum (Brandegee) Bates CHIH, NAY, SIN, SON

*Bastardiastrum gracile (Hochr.) D.M. Bates CHIS, GRO, OAX, SIN

*Bastardiastrum hirsutiflorum (K. Presl) D.M. Bates COL, GRO, JAL, MICH, NAY, OAX, SIN

*Bastardiastrum incanum (Brandegee) D.M. Bates CHIS, COL, GRO, JAL, MICH, NAY, SIN

*Bastardiastrum tarasoides Fryxell SIN

* Bastardiastrum tricarpellatum (B.L. Rob. \& Greenm. ex Rose) Bates COL, GRO, JAL, MEX, MICH

*Bastardiastrum wissaduloides (Baker f.) D.M. Bates SIN

* Batesimalva lobata Villarreal \& Fryxell COAH, DGO

*Batesimalva pulchella Fryxell NLE, TAMS

Batesimalva violacea (Rose) Fryxell CHIH, COAH, NLE, TAMS

Bernoullia flammea Oliv. CAM, CHIS, COL, GRO, JAL, MICH, NAY, OAX, QROO, TAB, VER

*Bernoullia jaliscana Miranda \& McVaugh COL, JAL

Berrya cubensis (Griseb.) M. Gómez CHIS, COL, HGO, NAY, OAX, PUE, QRO, SLP, TAB, VER

Billieturnera helleri (Rose ex A.A. Heller) Fryxell COAH, NLE, TAMS

*Briquetia inermis Fryxell CHIH

* Briquetia sonorae Fryxell SON

Briquetia spicata (Kunth) Fryxell CHIS, COL, DGO, GRO, JAL, MICH, NAY, OAX, SIN, TAB, VER

Byttneria aculeata (Jacq.) Jacq. CAM, CHIS, CHIH, COL, DGO, GRO, HGO, JAL, MEX, MICH, MOR, NAY, OAX, PUE, QRO, QROO, SLP, SIN, SON, TAB, TAMS, VER, YUC, ZAC

*Byttneria atrata Bullock GRO, JAL, MEX, MICH

* Byttneria capillata Cristóbal OAX

Byttneria catalpifolia Jacq. CHIS, COL, DGO, GRO, JAL, MICH, NAY, OAX, SIN, VER, YUC

*Byttneria fluvialis Fryxell \& Guadarr. TAB

Callianthe tridens (Standl. \& Steyerm.) Donnell CHIS, PUE

Callirhoe involucrata (Nutt. ex Torr. \& A. Gray) A. Gray COAH, NLE, TAMS 
* Carpodiptera mariarum Standl. NAY

* Ceiba acuminata (S. Watson) Rose AGS, BCS, CHIS, CHIH, COL, DGO, GTO, GRO, JAL, MEX, MICH, OAX, QRO, SIN, SON, TAMS, VER, ZAC

Ceiba aesculifolia (Kunth) Britten \& Baker f. AGS, CAM, CHIS, CHIH, COL, CDMX, DGO, GTO, GRO, HGO, JAL, MEX, MICH, MOR, NAY, OAX, PUE, QRO, QROO, SIN, SON, TAB, VER, YUC, ZAC

Ceiba pentandra (L.) Gaertn. AGS, BCS, CAM, CHIS, CHIH, COL, GRO, HGO, JAL, MEX, MICH, MOR, NAY, OAX, PUE, QRO, QROO, SLP, SIN, SON, TAB, TAMS, VER, YUC, ZAC * Ceiba schottii Britten \& Baker f. CHIS, CAM, QROO, YUC

*Cienfuegosia intermedia Fryxell GTO, SLP

*Cienfuegosia rosei Fryxell OAX, VER

Cienfuegosia yucatanensis Millspaugh CAM, QROO, YUC

Corchorus aestuans L. CAM, CHIS, COL, GRO, JAL, MICH, NAY, OAX, SIN, VER, YUC

Corchorus hirtus L. CAM, CHIS, COL, GTO, GRO, JAL, MICH, MOR, NAY, OAX, PUE, QROO, SLP, SIN, SON, TAB, TAMS, VER, YUC

Corchorus siliquosus L. CAM, CHIS, CHIH, COL, GRO, HGO, JAL, MEX, MICH, MOR, NAY, OAX, PUE, QRO, QROO, SLP, SIN, SON, TAB, TAMS, VER, YUC

Chiranthodendron pentadactylon Larreat. CHIS, GRO, HGO, MEX, MICH, MOR, OAX, PUE, VER

*Dendrosida batesii Fryxell CHIS

*Dendrosida breedlovei Fryxell CHIS, OAX

* Dendrosida parviflora Fryxell OAX

*Dendrosida sharpiana (Miranda) Fryxell CHIS, GRO, MICH, OAX

*Dirhamphis mexicana Fryxell COL, GRO, JAL

Eremalche exilis (A. Gray) Greene BCN, BCS, CHIH, SON

Eremalche rotundifolia (A. Gray) Greene BCN, SON

Fremontodendron californicum (Torr.) Coville BCN

Fremontodendron mexicanum Davidson BCN

Fryxellia pygmaea (Correll) Bates COAH, NLE

Fuertesimalva jacens (S. Watson) Fryxell AGS, CHIS, CHIH, CDMX, DGO, GTO, HGO, JAL, MEX, MICH, MOR, OAX, PUE, QRO, SLP, SIN, TAMS, TLAX, VER, ZAC

Fuertesimalva limensis (L.) Fryxell CDMX, DGO, GTO, HGO, JAL, MEX, MICH, MOR, PUE, QRO, SLP, TLAX, VER

Gaya hermannioides Kunth CAM, CHIS, GRO, MOR, OAX, PUE, QROO, SLP, VER, YUC

Gaya minutiflora Rose AGS, CHIS, COL, DGO, GRO, HGO, JAL, MEX, MICH, MOR, OAX, PUE, QRO, SIN, VER

Gaya occidentalis (L.) Sweet CAM, CHIS, COL, JAL, QRO, QROO, SLP, TAMS, VER, YUC

*Gossypium aridum (Rose \& Standl.) Skovst. COL, DGO, GRO, JAL, MICH, NAY, OAX, PUE, SIN, VER, ZAC

*Gossypium armourianum Kearney BCN, BCS

* Gossypium davidsonii Kellogg BCS, SON

* Gossypium gossypioides (Ulbr.) Standl. OAX, PUE

* Gossypium harknessii Brandegee BCS

*Gossypium lanceolatum Todaro COL, GRO, MICH, NAY, OAX, QRO, SLP, VER

*Gossypium laxum L.L1. Phillips GRO, MICH

* Gossypium lobatum Gentry GRO, JAL, MICH
*Gossypium schwendimanii Fryxell \& S.D. Koch GRO, MICH Gossypium thurberi Todaro CHIH, SON

*Gossypium trilobum (DC.) Skovsted HGO, JAL, MEX, MICH, MOR, QRO, SIN

* Gossypium turneri Fryxell SON

Guazuma ulmifolia Lam. CAM, CHIS, CHIH, COL, DGO, GTO, GRO, HGO, JAL, MEX, MICH, MOR, NAY, OAX, PUE, QRO, QROO, SLP, SIN, SON, TAB, TAMS, VER, YUC, $\mathrm{ZAC}$

*Hampea breedlovei Fryxell CHIS

Hampea integerrima Schltdl. OAX, TAB, VER

Hampea latifolia Standl. CHIS

Hampea longipes Miranda CHIS, OAX, VER

*Hampea mexicana Fryxell CHIS, COL, JAL, OAX

*Hampea montebellensis Fryxell CHIS

*Hampea nutricia Fryxell CHIS, HGO, PUE, TAB, VER

Hampea rovirosae Standl. CAM, CHIS, TAB, VER

Hampea stipitata S. Watson CAM, CHIS, JAL, OAX, VER

Hampea tomentosa (C. Presl) Standl. CHIS, COL, GRO, JAL, MICH, OAX, QROO, VER, YUC

Hampea trilobata Standl. CAM, CHIS, QROO, TAB, VER, YUC

Helicteres baruensis Jacq. CAM, CHIS, COL, GRO, JAL, MICH, NAY, OAX, PUE, QROO, SLP, SIN, SON, TAB, TAMS, VER, YUC

Helicteres guazumifolia Kunth AGS, CAM, CHIS, COL, DGO, GRO, JAL, MEX, MICH, NAY, OAX, PUE, QROO, SIN, TAB, VER, YUC

*Helicteres rekoi Standl. COL, GRO, JAL, OAX

*Helicteres vegae Cristóbal SIN

Heliocarpus americanus L AGS, CAM, CHIS, COL, GTO, GRO, HGO, JAL, MEX, MICH, MOR, OAX, PUE, QRO, QROO, SLP, TAB, TAMS, VER, YUC, ZAC

Heliocarpus appendiculatus Turcz. CHIS, COL, DGO, GRO, HGO, JAL, MICH, OAX, PUE, QRO, SLP, SIN, TAB, VER

*Heliocarpus attenuatus S. Watson CHIH, SIN, SON

Heliocarpus donnellsmithii Rose CAM, CHIS, COL, GRO, HGO, JAL, MEX, MICH, MOR, NAY, OAX, PUE, QRO, QROO, SLP, SIN, TAB, TAMS, VER, YUC, ZAC

Heliocarpus mexicanus (Turcz.) Sprague CAM, CHIS, GRO, OAX, PUE, QRO, QROO, SLP, VER, YUC

Heliocarpus occidentalis Rose CHIS, CHIH, COL, DGO, GRO, JAL, MEX, MICH, MOR, NAY, OAX, SIN, SON, ZAC

*Heliocarpus palmeri E. Watson CHIH, COL, JAL, SIN, SON, ZAC

*Heliocarpus pallidus Rose CHIS, COL, GRO, JAL, MEX, MICH, MOR, NAY, OAX, VER

*Heliocarpus parvimontis Gual GRO, OAX

Heliocarpus terebinthaceus (DC.) Hochr. AGS, CHIS, COL, DGO, GTO, GRO, JAL, MEX, MICH, MOR, NAY, OAX, PUE, QRO, SON, VER, ZAC

*Heliocarpus velutinus Rose GRO, JAL, MEX, MICH, MOR, OAX, PUE

Herissantia crispa (L.) Brizicky AGS, BCN, BCS, CAM, CHIS, CHIH, COAH, COL, CDMX, DGO, GTO, GRO, HGO, JAL, MEX, MICH, MOR, NAY, NLE, OAX, PUE, QRO, QROO, SLP, SIN, SON, TAB, TAMS, VER, YUC, ZAC 
*Herissantia dressleri Fryxell TAMS

Hermannia inflata Link \& Otto CHIS, GRO, MEX, MICH, OAX, PUE

*Hermannia palmeri Rose BCS

Hermannia pauciflora S. Watson SLP, SON

Hermannia texana A. Gray COAH, NLE, SLP, TAMS

*Hibiscus acapulcensis Fryxell GRO

*Hibiscus acicularis Standl. CHIH, COAH, NLE, QRO, SLP, SON, TAMS, VER

Hibiscus biseptus $\mathrm{S}$. Watson BCN, BCS, CHIH, DGO, JAL, NAY, SIN, SON, ZAC

*Hibiscus citrinus Fryxell COL, JAL, NAY, SIN, SON

Hibiscus clypeatus L. CAM, CHIS, HGO, QRO, QROO, SLP,

TAMS, VER, YUC

*Hibiscus colimensis Fryxell COL, JAL

Hibiscus costatus A. Rich. CAM, CHIS, OAX, PUE, SIN, TAB, VER

Hibiscus coulteri Harvey ex A. Gray BCN, CHIH, COAH, DGO, GTO, HGO, NLE, QRO, SLP, SON, VER, ZAC

Hibiscus denudatus Benth. BCN, BCS, CHIH, COAH, DGO, HGO, NLE, QRO, SON, VER

Hibiscus diversifolius Jacq. CHIS, COL, GRO, VER

*Hibiscus elegans Standl. HGO, MOR, NLE, OAX, PUE, QRO, SLP, TAMS, VER, ZAC

Hibiscus furcellatus Desr. CAM, CHIS, GRO, NAY, PUE, QRO,

TAB, VER

*Hibiscus jaliscensis Fryxell CHIH, JAL, MEX, MICH, MOR, ZAC

*Hibiscus kochii Fryxell GRO, MICH, OAX

Hibiscus lasiocarpos Cav. $\mathrm{CHIH}$

Hibiscus lavateroides Moric. ex Ser. CHIS, HGO, PUE, QRO,

SLP, TAMS, VER

*Hibiscus longifilus Fryxell OAX, PUE

Hibiscus martianus Zucc. CHIH, COAH, DGO, GTO, HGO,

NLE, PUE, QRO, SLP, TAMS, VER

*Hibiscus nelsonii Rose \& Standl. OAX

*Hibiscus peripteroides Fryxell OAX, SLP, TAMS

Hibiscus phoeniceus Jacq. AGS, CHIS, CHIH, COL, GRO, JAL, MEX, OAX, PUE, SLP, SIN, SON, TAMS, VER, ZAC

Hibiscus poeppigii (Spreng.) Garcke CAM, CHIS, QROO, SLP, TAMS, VER, YUC

*Hibiscus purpusii Brandegee OAX, VER

*Hibiscus ribifolius A. Gray BCS

Hibiscus sororius L. CHIS, TAB, VER

*Hibiscus spiralis Cav. CDMX, MEX, MICH, MOR, OAX, VER, YUC

Hibiscus striatus Cav. TAB

*Hibiscus tenorii Fryxell OAX

Hibiscus tiliaceus L. BCS, CAM, CHIS, GRO, MEX, MICH, MOR, NAY, NLE, PUE, QROO, SIN, TAB, TAMS, VER, YUC Hibiscus uncinellus DC. CHIS, COL, GRO, HGO, JAL, MICH, NAY, OAX, PUE, SLP, SIN, TAB, VER

*Hibiscus zygomorphus Fryxell \& S.D. Koch GRO, MICH

Hochreutinera amplexifolia (DC.) Fryxell CHIS, COL, DGO, GRO, HGO, JAL, MICH, NAY, OAX, QRO, SLP, SIN, SON, TAMS, VER, ZAC

Horsfordia alata (S. Watson) A. Gray BCN, BCS, SON
*Horsfordia exalata Fryxell BCN, BCS, SON

Horsfordia newberryi (S. Watson) A. Gray BCN, BCS, SON

*Horsfordia rotundifolia S. Watson BCN, BCS, SLP, SON, TAMS

Kearnemalvastrum lacteum (Aiton) D.M. Bates CHIS, CDMX, GTO, GRO, HGO, JAL, MEX, MICH, OAX, PUE, QRO, SLP, TLAX, VER

Kearnemalvastrum subtriflorum (Lag.) D.M. Bates AGS, BCN, CHIS, COAH, COL, CDMX, GTO, GRO, HGO, JAL, MEX, MICH, MOR, OAX, PUE, QRO, SLP, TAMS, TLAX, VER, ZAC

*Kosteletzkya blanchardii Fryxell COL, JAL, MEX, MICH

Kosteletzkya depressa (L.) O.J. Blanch., Fryxell \& D.M. Bates BCN, BCS, CAM, CHIS, COL, GRO, JAL, MEX, MICH, MOR, NAY, OAX, PUE, QRO, QROO, SLP, SIN, SON, TAB, TAMS, VER, YUC

* Kosteletzkya flavicentrum Fryxell \& S.D. Koch GRO, SIN

*Kosteletzkya hispidula (Spreng.) Garcke BCS, QRO, SLP, SIN, SON

*Kosteletzkya ramosa Fryxell JAL, MICH

*Kosteletzkya reclinata Fryxell JAL, MICH

*Kosteletzkya thurberi A. Gray CHIH, GTO, SON

*Kosteletzkya tubiflora (DC.) O.J. Blanch. \& McVaugh AGS, CAM, CHIS, COL, DGO, GRO, JAL, MEX, MICH, NAY, QROO, SIN, SON, YUC, ZAC

Krapovickasia physaloides (C. Presl) Fryxell NLE, SLP, TAMS

*Lavatera lindsayi Moran BCN

* Lavatera occidentalis S. Watson BCN

* Lavatera venosa $\mathrm{S}$. Watson BCN, BCS

Lopimia malacophylla (Link. \& Otto) Mart. CHIS, GRO, OAX, TAB, VER

Luehea candida (Moc. \& Sessé ex DC.) Mart. CAM, CHIS, COL, DGO, GRO, JAL, MICH, NAY, OAX, QROO, SIN, TAB, VER, YUC

Luehea seemannii Triana \& Planch. CHIS, OAX

Luehea speciosa Willd. CAM, CHIS, COL, NAY, OAX, QROO, TAB, VER, YUC

Malacothamnus densiflorus (S. Watson) Greene BCN

Malacothamnus fasciculatus (Nutt. ex Torr. \& A. Gray) Greene $\mathrm{BCN}$

*Malacothamnus foliosus (S. Watson) Kearney BCN

Malacothamnus marrubioides (Durand \& Hilg.) Greene BCN

Malachra alceifolia Jacq. CAM, CHIS, COL, GRO, JAL, MEX, MICH, MOR, NAY, OAX, QROO, SLP, SIN, TAB, TAMS, VER, YUC

Malachra capitata (L.) L. CAM, CHIS, COL, GRO, JAL, MEX, MICH, MOR, NAY, OAX, PUE, QRO, QROO, SLP, TAB, TAMS, VER, YUC

Malachra fasciata Jacq. CAM, CHIS, COL, GRO, JAL, MEX, MICH, OAX, QRO, QROO, SLP, TAB, TAMS, VER, YUC

Malachra radiata (L.) L. CAM, JAL, NAY, SIN, TAB

Malvastrum americanum (L.) Torr. CHIS, COAH, COL, GTO, GRO, HGO, JAL, MEX, MICH, MOR, NAY, NLE, OAX, PUE, QRO, SLP, SIN, TAB, TAMS, VER, ZAC

Malvastrum bicuspidatum (S. Watson) Rose BCN, BCS, CHIH, COAH, COL, DGO, GTO, HGO, JAL, MEX, MICH, MOR, NAY, NLE, OAX, PUE, QRO, SLP, SIN, SON, ZAC 
Malvastrum corchorifolium (Desr.) Britton ex Small CAM, CHIS, NLE, QROO, SLP, TAB, YUC

Malvastrum coromandelianum (L.) Garcke AGS, BCN, BCS, CAM, CHIS, CHIH, COAH, COL, CDMX, DGO, GRO, GTO, HGO, JAL, MEX, MICH, MOR, NAY, NLE, OAX, PUE, QRO, QROO, SLP, SIN, SON, TAB, TAMS, VER, YUC, ZAC

*Malvastrum hillii Fryxell, León de la Luz \& M. Domínguez BCS

Malvaviscus achanioides (Turcz.) Fryxell CHIS, OAX, TAB, VER

Malvaviscus arboreus Cav. AGS, CAM, CHIS, CHIH, COL, CDMX, DGO, GTO, GRO, HGO, JAL, MEX, MICH, MOR, NAY, NLE, OAX, PUE, QRO, QROO, SLP, SIN, TAB, TAMS, TLAX, VER, YUC, ZAC

Malvaviscus drummondii Torr. \& A. Gray CHIS, HGO, MOR, SLP, TAB, TAMS, VER, YUC

Malvaviscus lanceolatus Rose CHIS, COL, GRO, JAL, MOR, NAY, OAX, PUE, VER

*Malvaviscus oaxacanus Standl. CHIS, OAX, VER

Malvaviscus penduliflorus DC. CAM, CHIS, COL, GRO, JAL, MEX, MICH, MOR, NAY, NLE, PUE, QROO, SLP, TAMS, VER, YUC

*Malvaviscus urticifolius (C. Presl) Fryxell CHIS, GRO, MEX, $\mathrm{MICH}, \mathrm{VER}$

Malvella lepidota (A. Gray) Fryxell CHIH, COAH, DGO, GTO, NLE, QRO, SLP, SON, ZAC

Malvella leprosa (Ortega) Krapov. BCN, BCS, CHIH, COAH, COL, CDMX, DGO, GTO, HGO, JAL, MEX, MICH, NLE, OAX, QRO, SLP, SIN, SON

Malvella sagittifolia (A. Gray) Fryxell CHIH, COAH, DGO, NLE, SLP, SON, TAMS

Melochia bernoulliana Donn. Sm. CHIS, TAB, VER

*Melochia corymbosa (C. Presl) Meisn. ex Steud. COL, GRO, JAL, MEX, MICH, MOR, NAY, OAX, PUE, SIN

Melochia glandulifera Standl. CHIS, GRO, JAL, MICH, OAX, SIN, ZAC

Melochia kerriifolia Triana \& Planch. CHIS, OAX, VER

Melochia lupulina Sw. CHIS, GRO, OAX, TAB, VER

Melochia manducata C. Wright CHIS, GRO, NAY, OAX, TAB, VER

Melochia nodiflora Sw. CHIS, COL, DGO, GRO, JAL, MEX, MICH, MOR, NAY, OAX, QRO, SLP, SIN, TAB, TAMS, VER Melochia nudiflora Standl. \& L.O. Williams CHIS, GRO, OAX * Melochia oaxacana Dorr \& L.C. Barnett OAX

Melochia pilosa (Mill.) Fawc. \& Rendle OAX, VER

Melochia pyramidata L. BCS, CAM, CHIS, COAH, COL, DGO, GTO, GRO, HGO, JAL, MEX, MICH, MOR, NAY, NLE, OAX, PUE, QRO, QROO, SLP, SIN, SON, TAB, TAMS, VER, YUC, ZAC

Melochia spicata (L.) Fryxell CAM, CHIS, COL, GRO, JAL, MICH, NAY, NLE, OAX, SLP, TAB, TAMS, VER, YUC, ZAC *Melochia tomentella (C. Presl) Hemsl. GRO, NAY, OAX, SIN, SON, TAB

Melochia tomentosa $\mathrm{L}$. BCN, BCS, CAM, CHIS, CHIH, COL, DGO, GRO, HGO, JAL, MEX, MICH, MOR, NAY, NLE, OAX, PUE, QROO, SLP, SIN, SON, TAB, TAMS, VER, YUC, ZAC * Melochia tragiifolia Standl. GRO, MICH, SIN, SON
Melochia urticifolia (Turcz.) Standl. GRO, JAL, MEX, MICH, MOR, NAY, OAX, QROO, SIN, VER

Meximalva filipes (A. Gray) Fryxell AGS, CHIH, COAH, HGO, NLE, SLP, TAMS, VER

*Meximalva venusta (Schltdl.) Fryxell HGO, MICH, SLP, TAMS, VER, ZAC

Mortoniodendron guatemalense Standl. \& Steyerm. CHIS, OAX, TAB, VER

Mortoniodendron hirsutum Standl. OAX

*Mortoniodendron ocotense Ishiki \& T. Wendt CHIS

*Mortoniodendron palaciosii Miranda CHIS, OAX, VER

Mortoniodendron ruizii Miranda CHIS, TAB

Mortoniodendron sulcatum Lundell CHIS, VER

*Mortoniodendron uxpanapense Dorr \& T. Wendt OAX, VER

Mortoniodendron vestitum Lundell CHIS, TAB

Neobrittonia acerifolia (G. Don) Hochr. CHIS, COL, JAL, MEX, MICH, MOR

Ochroma pyramidale (Cav. ex Lam.) Urb. CHIS, OAX, TAB, VER

Pachira aquatica Aubl. CAM, CHIS, GRO, JAL, MICH, NAY, OAX, PUE, QROO, SIN, TAB, VER, YUC

Pachira macrocarpa (Schltdl. \& Cham.) Walp. OAX, TAB, VER

* Pavonia alia Fryxell CHIH

* Pavonia anisaster (Standl.) Fryxell DGO, JAL, NAY, ZAC

* Pavonia arachnoidea C. Presl COL, GRO, JAL, SIN

* Pavonia baumliana Fryxell JAL, ZAC

Pavonia cancellata (L.) Cav. VER

* Pavonia candida (DC.) Fryxell AGS, COAH, COL, GTO, HGO, JAL, MEX, MICH, QRO, SLP, VER

*Pavonia chlorantha (Kunth) Fryxell GRO, MEX

*Pavonia durangensis Fryxell DGO, JAL, ZAC

* Pavonia firmiflora Schery JAL, NAY

* Pavonia fryxellii Krapov. COL, GRO, JAL, MICH, OAX, PUE, VER

*Pavonia gentryi Fryxell SIN

Pavonia hastata Cav. COL, TAMS

* Pavonia hirtiflora Benth. AGS, JAL

Pavonia integrifolia Standl. CHIS, VER

* Pavonia langlassei Hochr. GRO

Pavonia lasiopetala Scheele COAH, NLE, SLP, TAMS

*Pavonia macdougallii Fryxell OAX

* Pavonia monticola Fryxell CHIS, GRO, OAX

* Pavonia nayarensis Fryxell DGO, JAL, NAY, ZAC

*Pavonia nepetifolia (Standl.) Standl. COAH, GTO, NLE, SLP, TAMS

*Pavonia oxyphylla (DC.) Fryxell COL, GTO, GRO, JAL, MEX, MICH, MOR, PUE

* Pavonia paneroi Fryxell PUE

Pavonia paniculata Cav. CHIS, GRO, HGO, NAY, OAX, PUE, QRO, SLP, VER

*Pavonia pleuranthera (DC.) Fryxell COL, GRO, JAL, MEX, MICH, NAY, OAX

* Pavonia pulidoae Fryxell MEX, SLP

Pavonia purpusii Brandegee CHIS, HGO, OAX, VER

Pavonia schiedeana Steud. CAM, CHIS, GRO, HGO, OAX, PUE, QRO, SLP, TAB, TAMS, VER 
Pavonia sidifolia Kunth CHIS, OAX

* Pavonia spuria Fryxell OAX

* Pavonia submutica Fryxell CHIS, OAX

*Pavonia uniflora (Sessé \& Moc.) Fryxell GTO, HGO, NLE, OAX, PUE, QRO, SLP, TAMS, VER

*Pavonia venusta Fryxell JAL, NAY

Peltaea trinervis (C. Presl) Krapov. \& Cristóbal OAX

*Periptera ctenotricha Fryxell COL, JAL

* Periptera lobelioides Fryxell \& S.D. Koch GRO, MICH

* Periptera macrostelis Rose COL, GRO, JAL, MICH, SLP

Periptera punicea (Lag.) DC. AGS, CHIS, CHIH, COL, CDMX, DGO, GTO, GRO, HGO, JAL, MEX, MICH, MOR, NAY, OAX, PUE, QRO, SLP, SIN, SON, VER, ZAC

*Periptera trichostemon Bullock SIN

* Phymosia anomala Fryxell HGO, MEX, MICH, OAX, VER

* Phymosia crenulata (Brandegee) Fryxell PUE

* Phymosia floribunda (Schltdl.) Fryxell OAX, PUE

*Phymosia pauciflora (Baker f.) Fryxell GTO, HGO, OAX, QRO, SLP, TAMS

Phymosia rosea (DC.) Kearney CHIS, CHIH, COL, GRO, JAL, MEX, MICH, MOR, OAX, PUE, VER

*Phymosia rzedowskii Fryxell GTO, GRO, MEX, PUE, QRO, VER

Phymosia umbellata (Cav.) Kearney CHIS, CDMX, GTO, HGO, MEX, PUE, QRO, SLP, TAMS, VER

*Physodium adenodes (Goldberg) Fryxell COL, DGO, GRO, JAL, MEX, MICH, NAY, OAX, SIN, ZAC

Pseudabutilon ellipticum (Schltdl.) Fryxell AGS, CAM, CHIS, COL, CDMX, DGO, GTO, GRO, HGO, JAL, MEX, MICH, MOR, NAY, NLE, OAX, PUE, QRO, SLP, SIN, TAMS, VER, ZAC

Pseudabutilon orientale (Standl. \& Steyerm.) Fryxell CHIS, COL, GRO, JAL, OAX, SIN

Pseudabutilon scabrum (C. Presl) R.E. Fr. CHIS, COL, DGO, GRO, JAL, NAY, OAX, PUE, SIN, SON, VER, ZAC

Pseudabutilon thurberi (A. Gray) Fryxell CHIH, SON

Pseudabutilon umbellatum (L.) Fryxell CAM, COL, GRO, HGO, JAL, NAY, NLE, OAX, QROO, SLP, SIN, TAMS, VER, YUC, ZAC

Pseudobombax ellipticum (Kunth) Dugand CAM, CHIS, COL, DGO, GTO, GRO, HGO, JAL, MEX, MICH, MOR, NAY, NLE, OAX, PUE, QRO, QROO, SLP, SIN, TAB, TAMS, VER, YUC, ZAC

*Pseudobombax palmeri (S. Watson) Dugand BCS, CHIH, COL, JAL, NAY, SLP, SIN, SON, VER, ZAC

Quararibea funebris (La Llave) Vischer CAM, CHIS, HGO, OAX, PUE, QROO, TAB, VER, YUC

Quararibea guatemalteca (Donn. Sm.) Standl. \& Steyerm. CHIS, QROO, TAB, YUC

*Quararibea lopezperaltae Gallardo-Hernández \& Lorea-Hern. OAX, VER

*Quararibea mayanum Lorea-Hern. \& Gallardo-Hernández OAX

Quararibea yunckeri Standl. CHIS, OAX, TAB, VER

Rhynchosida physocalyx (A. Gray) Fryxell CHIH, COAH, DGO, GTO, HGO, NLE, PUE, QRO, SLP, SON, TAMS

*Robinsonella brevituba Fryxell CHIS, OAX, VER
Robinsonella cordata Rose \& Baker f. CHIS, COL, DGO, GRO, JAL, MICH, MOR, OAX, PUE

*Robinsonella chiangii Fryxell OAX, PUE, VER

Robinsonella densiflora Fryxell VER

*Robinsonella discolor Rose \& Baker f. ex Rose GTO, HGO, NAY, OAX, QRO, SLP, TAMS, VER

Robinsonella glabrifolia Fryxell CHIS

*Robinsonella hintonii Fryxell GRO, JAL, MEX, MICH, NAY

Robinsonella lindeniana (Turcz.) Rose \& Baker f. CHIS, OAX, TAB, VER

*Robinsonella macvaughii Fryxell COL, JAL, MICH

*Robinsonella mirandae Gómez-Pompa CHIS, OAX, TAB, VER

Robinsonella pilosa Rose SLP, TAMS, VER

*Robinsonella pilosissima Fryxell CHIS

*Robinsonella samaricarpa Fryxell CHIS, OAX, VER

Robinsonella speciosa Fryxell CHIS, COL, JAL, OAX

Sida abutifolia Mill. AGS, BCS, CAM, CHIS, CHIH, COAH, COL, CDMX, DGO, GTO, GRO, HGO, JAL, MEX, MICH, MOR, NAY, NLE, OAX, PUE, QRO, QROO, SLP, SIN, SON, TAB, TAMS, TLAX, VER, YUC, ZAC

Sida acuta Burm. f. BCS, CAM, CHIS, CHIH, COL, DGO, GTO, GRO, HGO, JAL, MEX, MICH, MOR, NAY, NLE, OAX, PUE, QRO, QROO, SLP, SIN, SON, TAB, TAMS, VER, YUC, ZAC

Sida aggregata C. Presl CHIS, CHIH, COL, DGO, GRO, HGO, JAL, MEX, MICH, NAY, OAX, QRO, SLP, SIN, SON, VER, ZAC

*Sida anodifolia Fryxell GRO, OAX

Sida barclayi Baker f. AGS, CHIS, COL, CDMX, GRO, JAL, MEX, MICH, OAX, PUE, TLAX, VER

Sida brachystemon DC. OAX

Sida ciliaris L. BCS, CAM, CHIS, CHIH, COL, DGO, GTO, GRO, HGO, JAL, MEX, MICH, MOR, NAY, OAX, PUE, QRO, QROO, SLP, SIN, SON, TAMS, VER, YUC, ZAC

Sida cordifolia L. CAM, CHIS, CHIH, GRO, MOR, NAY, OAX, SIN, SON, TAB, TAMS, VER, YUC

Sida cuspidata (A. Robyns) Krapov. CHIS, DGO, GRO, NAY, OAX, QRO, QROO, SLP, SIN, TAMS, VER

Sida elliottii Torr. \& A. Gray BCS, CHIS, COAH, GTO, HGO, MEX, MOR, NLE, QRO, SLP, TAMS, VER, ZAC

*Sida fastuosa Fryxell \& S.D. Koch GRO, MICH

Sida glabra Mill. BCS, CAM, CHIS, CHIH, COL, CDMX, DGO, GTO, GRO, HGO, JAL, MEX, MICH, MOR, NAY, NLE, OAX, PUE, QRO, QROO, SLP, SIN, SON, TAB, TAMS, VER, YUC, ZAC

Sida haenkeana C. Presl CAM, CHIS, COL, CDMX, GTO, GRO, JAL, MEX, MICH, MOR, NAY, OAX, PUE, QROO, SLP, TAMS, VER, YUC, ZAC

*Sida hyalina Fryxell BCS, SIN, SON

Sida hyssopifolia C. Presl CHIS, COL, DGO, GRO, JAL, MICH, MOR, NAY, NLE, OAX, SIN, SON, TAMS, VER

Sida jamaicensis L. CHIS, COL, CDMX, GRO, JAL, MEX, MICH, OAX, QROO, VER, YUC

Sida jussieana DC. CAM, CHIS, GRO, OAX, QROO, VER, YUC

Sida lindheimeri Engelm. \& A. Gray CHIH, COAH, NLE, 
TAMS, VER

* Sida linearis Cav. AGS, CHIS, COL, CDMX, DGO, GTO, HGO, JAL, MEX, MICH, PUE, QRO, SLP, VER, ZAC

Sida linifolia Cav. CAM, CHIS, COL, DGO, GRO, JAL, MEX, MICH, NAY, OAX, QROO, SIN, SON, TAB, TAMS, VER, YUC, ZAC

Sida longipes A. Gray CHIH, COAH, NLE

* Sida michoacana Fryxell GRO, MEX, MICH

* Sida monticola Fryxell COL, DGO, GRO, HGO, JAL, MEX, $\mathrm{MICH}, \mathrm{NAY}, \mathrm{OAX}, \mathrm{QRO}, \mathrm{SLP}, \mathrm{ZAC}$

Sida neomexicana A. Gray CHIH, COAH, DGO, NLE, SLP, SON, ZAC

* Sida nesogena I.M. Johnst. COL, DGO, GRO, SIN

*Sida potosina Brandegee NLE, SLP, TAMS, ZAC

* Sida prolifica Fryxell \& S.D. Koch COL, GRO, MEX, MICH, OAX, QRO

*Sida pueblensis Fryxell OAX, PUE

Sida rhombifolia L. AGS, BCS, CAM, CHIS, CHIH, COAH, COL, CDMX, DGO, GTO, GRO, HGO, JAL, MEX, MICH, MOR, NAY, NLE, OAX, PUE, QRO, QROO, SLP, SIN, SON, TAB, TAMS, TLAX, VER, YUC, ZAC

Sida salviifolia C. Presl CHIS, COL, GRO, JAL, MEX, MICH, MOR, NAY, OAX, PUE, SIN, ZAC

Sida spinosa L. CAM, CHIS, CHIH, COAH, COL, DGO, GTO, GRO, HGO, JAL, MEX, MICH, MOR, NAY, NLE, OAX, PUE, QRO, QROO, SLP, SIN, SON, TAMS, VER, YUC, ZAC

Sida tragiifolia A. Gray CHIH, COAH, NLE, SON, TAMS

* Sida turneroides Standl. COAH, HGO, NLE, QRO, SLP, TAMS

Sida urens L. CAM, CHIS, GRO, JAL, NAY, OAX, PUE, TAB, VER, YUC

Sida viarum A. St.-Hil. CHIS

*Sida xanti A. Gray BCS, COL, JAL, SIN

Sidalcea malviflora (DC.) A. Gray ex Benth. BCN

Sidalcea neomexicana A. Gray BCN, CHIH, COAH, DGO, SIN, SON

* Sidastrum burrerense Fryxell, León de la Luz \& M. Domínguez BCS

*Sidastrum lodiegense (Baker f. ex Rose) Fryxell DGO, NAY, SIN, SON

Sidastrum paniculatum (L.) Fryxell CHIS, JAL, OAX, PUE, SLP, SIN, TAB, TAMS, VER

*Sidastrum strictum (Standl.) Fryxell NAY, SLP, SIN, TAMS, VER

* Sidastrum tehuacanum (Brandegee) Fryxell HGO, OAX, PUE, QRO

Sphaeralcea ambigua A. Gray BCN, BCS, SON

Sphaeralcea angustifolia (Cav.) G. Don AGS, CHIS, CHIH, COAH, COL, CDMX, DGO, GTO, HGO, JAL, MEX, MICH, NLE, OAX, PUE, QRO, SLP, SON, TAMS, TLAX, VER, ZAC *Sphaeralcea axillaris $\mathrm{S}$. Watson BCN, BCS

Sphaeralcea coccinea (Nutt.) Rydb. CHIH, NLE

Sphaeralcea coulteri (S. Watson) A. Gray BCN, BCS, SIN, SON

Sphaeralcea digitata (Greene) Rydb. CHIH

Sphaeralcea emoryi Torr. ex A. Gray BCN, BCS, COAH, DGO, NLE, SIN, SON
*Sphaeralcea endlichii Ulbrich CHIH, COAH, DGO, NLE, TAMS, ZAC

Sphaeralcea fendleri A. Gray CHIH, COAH, DGO, SON

* Sphaeralcea fulva Greene BCN, BCS, CHIH, NAY, SON

*Sphaeralcea hainesii Brandegee BCN, BCS, SON

Sphaeralcea hastulata A. Gray CHIH, COAH, DGO, GTO, NLE, PUE, QRO, SLP, SON, TAMS, VER, ZAC

Sphaeralcea incana Torr. ex A. Gray CHIH, COAH, SON

Sphaeralcea laxa Wooton \& Standl. CHIH, SON

Sphaeralcea leptophylla (A. Gray) Rydb. CHIH, SON

Sphaeralcea orcuttii Rose BCN, BCS, SON

* Sphaeralcea palmeri Rose BCN

Sphaeralcea pedatifida (A. Gray) A. Gray COAH, TAMS

Sphaeralcea polychroma La Duke CHIH, SON

*Sphaeralcea reflexa Fryxell, Valdés-Reyna \& Villarreal $\mathrm{COAH}, \mathrm{DGO}$

* Sphaeralcea sulphurea S. Watson BCN

Sphaeralcea wrightii A. Gray CHIH, SON

Sterculia apetala (Jacq.) H. Karst. CHIS, OAX, TAB, VER

Sterculia mexicana R. Br. CHIS, OAX, TAB, VER

*Sterculia xolocotzii T. Wendt \& E.L. Taylor OAX, VER

Talipariti tiliaceum (L.) Fryxell CAM, CHIS, COL, GRO, JAL,

MICH, NAY, OAX, PUE, QROO, SLP, SIN, TAB, TAMS, VER

Tarasa antofagastana (Phil.) Krapov. CDMX, HGO, MEX, QRO, VER

*Tarasa geranioides (Schltdl. \& Cham.) Krapov. CDMX, HGO, MEX, QRO, TLAX, VER

Theobroma angustifolium Sessé \& Moc. ex DC. CHIS, TAB

Theobroma bicolor Bonpl. CHIS, GRO, OAX, TAB, VER

Thespesia populnea (L.) Sol. ex Correa CHIS, CAM, QROO,

SLP, TAMS, VER, YUC

Tilia americana L. CHIS, CHIH, COAH, COL, DGO, GRO, HGO, JAL, MEX, MICH, MOR, NAY, NLE, OAX, PUE, QRO, SLP, SIN, SON, TAMS, VER

*Trichospermum galeottii (Turcz.) Kosterm. CHIS, COAH, COL, GRO, JAL, MEX, MICH, NAY, OAX, PUE, SLP, SIN, VER

*Trichospermum grandifolium (Spreng.) Kosterm. VER

Trichospermum lessertianum (Hochr.) Dorr CAM, CHIS, GRO, HGO, JAL, OAX, QROO, TAB, VER, YUC

*Triumfetta acracantha Hochr. BCS, CHIS, COL, GRO, HGO, JAL, MICH, NAY, SIN, VER

*Triumfetta attenuata Lay CHIS

*Triumfetta barbosa Lay COL, JAL

Triumfetta bogotensis DC. CAM, CHIS, COL, DGO, GRO, HGO, JAL, MEX, MICH, NAY, OAX, PUE, QROO, VER, YUC

*Triumfetta brachistacantha Standl. OAX

*Triumfetta brevipes S. Watson CHIS, CHIH, DGO, JAL, $\mathrm{MICH}, \mathrm{NAY}, \mathrm{SON}$

Triumfetta calderonii Standl. CHIS, OAX

*Triumfetta calzadae Fryxell GRO, OAX

*Triumfetta columnaris Hochr. CHIS, COL, GRO, JAL, MEX, MICH, MOR, NLE, OAX, ZAC

*Triumfetta coriacea Hochr. GRO, MEX, MICH, NAY, OAX

*Triumfetta cucullata Fernald DGO, NAY

*Triumfetta chihuahuensis Standl. CHIH, DGO, NAY, SIN, 
SON

*Triumfetta dioica Brandegee CHIS, OAX

*Triumfetta discolor Rose CHIH, DGO, GRO, JAL, NAY, OAX, SIN, SON, ZAC

*Triumfetta falcifera Rose CHIS, GRO, MICH, NAY, OAX

*Triumfetta ferruginea Lay CHIS

*Triumfetta galeottiana Turcz. CHIS, CHIH, COL, DGO, GTO, GRO, JAL, MEX, MICH, NAY, OAX, SLP, SIN, SON, VER, ZAC

*Triumfetta goldmanii Rose BCS, CHIH, COL, DGO, GRO, JAL, MEX, MICH, NAY, OAX, SIN, SON, ZAC

*Triumfetta gonophora W.W. Thomas \& McVaugh COL, JAL

Triumfetta grandiflora Vahl CHIS, CHIH, COL, HGO, JAL, OAX, PUE, QRO, SLP, SON, VER

*Triumfetta guerrerensis Gual, Peralta \& N. Diego GRO, OAX

*Triumfetta heliocarpoides Bullock GRO, JAL, MEX, MICH, NAY, OAX

*Triumfetta indurata W.W. Thomas \& McVaugh JAL

*Triumfetta kochii Fryxell COL, JAL

Triumfetta lappula L. CAM, CHIS, COL, GRO, HGO, MEX, MICH, MOR, NAY, OAX, QRO, QROO, SLP, TAB, VER

*Triumfetta martinezalfaroi Gual \& F. Chiang OAX

*Triumfetta medusae W.W. Thomas \& McVaugh DGO, JAL, NAY

*Triumfetta mexiae C.V. Morton \& Lay GRO, MEX, MICH

*Triumfetta mexicana Turcz. CHIS, OAX, VER

*Triumfetta paniculata Hook. \& Arn. CHIS, COL, DGO, GRO, JAL, MICH, NAY, OAX, SIN

Triumfetta polyandra Sessé \& Moc. ex DC. CHIS, COL, DGO, GRO, JAL, MEX, MICH, NAY, OAX, SIN, VER, ZAC

*Triumfetta purpusii Standl. CHIS, OAX, VER

Triumfetta semitriloba Jacq. BCS, CAM, CHIS, CHIH, COL, DGO, GTO, GRO, HGO, JAL, MEX, MICH, MOR, NAY, OAX, PUE, QRO, QROO, SLP, SIN, SON, TAB, TAMS, VER, YUC, ZAC

*Triumfetta simplicifolia (Sessé \& Moc.) Fryxell COL, GRO, JAL, MEX, MICH, MOR, OAX, SIN

*Triumfetta socorrensis Brandegee COL, OAX

Triumfetta speciosa Seem. CHIS, GRO, NAY, OAX, VER

*Triumfetta stellata Lay MEX, MICH, OAX

Urena lobata L. CHIS, MICH, OAX, PUE, VER

Veeresia clarkii Monach. \& Moldenke HGO, SLP, VER

*Waltheria acapulcensis Rose CHIS, GRO, OAX

*Waltheria acuminata Rose DGO, JAL, NAY, SIN, SON

* Waltheria alamosana Standl. SON

*Waltheria bicolor J.G. Saunders COL, JAL

Waltheria brevipes Turcz. CHIS, OAX, TAB, VER

*Waltheria conzattii Standl. GRO, NAY, OAX, SIN

*Waltheria fryxellii J.G. Saunders GRO, JAL, MEX, MICH

Waltheria glomerata C. Presl CHIS, OAX, SIN, TAB, VER

Waltheria indica L. AGS, BCS, CAM, CHIS, CHIH, COAH, COL, DGO, GTO, GRO, HGO, JAL, MEX, MICH, MOR, NAY, NLE, OAX, PUE, QRO, QROO, SLP, SIN, SON, TAB, TAMS, VER, YUC, ZAC

*Waltheria lundelliana J.G. Saunders NAY

Waltheria operculata Rose GRO, OAX

Waltheria preslii Walp. CAM, CHIS, GRO, OAX, SON, YUC
*Waltheria pringlei Rose \& Standl. GRO, MEX, MICH, MOR, PUE

*Waltheria rotundifolia Schranck CAM, CHIS, QROO, YUC

*Waltheria tridentata J.G. Saunders GRO, OAX, PUE

Waltheria viscosissima A. St.-Hil. JAL, NAY, OAX, SIN

Wissadula amplissima (L.) R.E. Fr. CAM, CHIS, CHIH, COAH, COL, GTO, GRO, JAL, MEX, MICH, MOR, NAY, NLE, OAX, PUE, QRO, QROO, SLP, SIN, SON, TAB, TAMS, VER, YUC, ZAC

Wissadula contracta (Link) R.E. Fr CHIS, OAX

Wissadula excelsior (Cav.) C. Presl CAM, CHIS, GRO, OAX, PUE, SLP, TAB, VER

Wissadula periplocifolia (L.) C. Presl ex Thwaites CAM, CHIS, COL, GRO, JAL, NAY, OAX, PUE, QRO, QROO, SLP, TAB, TAMS, VER, YUC

\section{Family Marantaceae}

Calathea altissima (Poepp. \& Endl.) Horan. CHIS

Calathea allouia (Aubl.) Lindl. CAM, CHIS, GRO, MEX, OAX, TAB, VER

*Calathea atropurpurea Matuda CHIS, COL, GRO, JAL, MEX, MICH, NAY, SIN

Calathea coccinea Standl. \& Steyerm. CHIS, GRO, MICH, OAX, VER

Calathea crotalifera S. Watson CAM, CHIS, OAX, PUE, TAB, VER

* Calathea cyclophora Baker NAY, SIN

Calathea inocephala (Kuntze) H.A. Kenn. \& Nicholson CHIS

*Calathea leonoriae Lascurain, H. Oliva \& S. Avendaño VER

Calathea lutea Schult. CAM, CHIS, OAX, QROO, TAB, VER

*Calathea matudae H. Kenn. \& Ganders CHIS

Calathea microcephala (Poepp. \& Endl.) Körn. CAM, CHIS, NAY, OAX, PUE, TAB, VER

*Calathea misantlensis Lascurain VER

*Calathea ovandensis Matuda CHIS, MEX, OAX, SLP, SIN, TAB, VER

*Calathea soconuscum Matuda CHIS, COL, GRO, JAL, MICH, OAX

*Calathea sosandradeana H. Kenn. \& Ganders OAX, VER

Goeppertia matudae (H. Kenn. \& Ganders) Borchs. \& S. Suárez CHIS, OAX

*Goeppertia sousandreana (H. Kenn. \& Ganders) Borchs. \& S. Suárez OAX, VER

Ischnosiphon pruinosus (Regel) Petersen CHIS

Maranta arundinacea L. AGS, CAM, CHIS, COL, DGO, GRO, HGO, JAL, MEX, MICH, MOR, NAY, OAX, PUE, QRO, QROO, SLP, SIN, TAB, TAMS, VER, YUC, ZAC

Maranta divaricata Roscoe CAM, CHIS, GRO, OAX, QRO, SLP, TAB, VER, YUC

Maranta gibba Sm. CAM, CHIS, GRO, JAL, OAX, PUE, QROO, SLP, TAB, VER, YUC

Maranta leuconeura E. Morren VER

Stromanthe macrochlamys (Woodson \& Standl.) H.A. Kenn. \& Nicolson CHIS, OAX, PUE, TAB, VER

Stromanthe popolucana Cast.-Campos, Vovides \& Vázq. Torres VER

Stromanthe tonckat (Aubl.) Eichler CHIS, OAX, VER 
Thalia geniculata L. CAM, CHIS, COL, DGO, GRO, JAL, MEX, MICH, MOR, NAY, OAX, PUE, QROO, SLP, SIN, TAB, TAMS, VER, YUC, ZAC

\section{Family Marcgraviaceae}

Marcgravia brownei (Triana \& Planch.) Krug \& Urb. CHIS

Marcgravia mexicana Gilg CHIS, OAX, PUE, TAB, VER

Marcgravia nepenthoides Seem. CHIS, OAX, VER

Marcgravia pittieri Gilg. CHIS, OAX

* Marcgravia stonei Utley CHIS, OAX, PUE, VER

Ruyschia enervia Lundell CHIS, VER

Souroubea exauriculata Delpino CHIS, GRO, OAX, PUE, TAB, VER

Souroubea loczyi (V.A. Richt.) de Roon CAM, CHIS, OAX, TAB, VER

\section{Family Martyniaceae}

Martynia annua L. CAM, CHIS, CHIH, COL, DGO, GRO, HGO, JAL, MEX, MICH, MOR, NAY, NLE, OAX, PUE, QRO, QROO, SLP, SIN, SON, TAMS, VER, YUC, ZAC

Proboscidea altheifolia (Benth.) Decne. BCN, BCS, CHIH, COAH, DGO, SLP, SIN, SON

*Proboscidea diversifolia Hevly JAL, MICH

Proboscidea louisiana (Mill.) Thell. AGS, BCN, CHIH, COAH, COL, CDMX, DGO, GTO, GRO, HGO, JAL, MEX, MICH, MOR, NAY, NLE, OAX, PUE, QRO, SLP, SON, TAMS, TLAX, VER, ZAC

Proboscidea parviflora (Wooton) Wooton \& Standl. BCN, BCS, CHIH, COAH, DGO, MOR, SIN, SLP, SON, TAMS

Proboscidea spicata Correll CHIH, COAH

Proboscidea triloba (Schltdl. \& Cham.) Decne. CHIS, COL, GRO, MEX, MICH, MOR, OAX, PUE, SLP, VER

\section{Family Mayacaceae}

Mayaca fluviatilis Aubl. BCS, CHIS, JAL, MEX, MICH, NAY, TAB, VER, ZAC

\section{Family Melanthiaceae}

Anticlea fremontii Torr. BCN

*Anticlea frigida (Schltdl. \& Cham.) Zomlefer \& Judd CDMX, HGO, JAL, MEX, MICH, MOR, OAX, PUE, TLAX, VER

*Anticlea hintoniorum (B.L. Turner) Zomlefer \& Judd COAH, NLE, TAMS

*Anticlea neglecta (Espejo, López-Ferrari \& Ceja) Zomlefer \& Judd GTO, QRO, SLP

Anticlea virescens (Kunth) Rydb. AGS, CHIH, COAH, COL, DGO, GTO, GRO, HGO, JAL, MEX, MICH, NAY, NLE, OAX, PUE, QRO, SLP, SIN, SON, TAMS

Anticlea volcanica (Benth.) Baker CHIS, OAX

* Schoenocaulon calcicola Greenm. OAX, PUE

*Schoenocaulon caricifolium (Schltdl.) A. Gray AGS, GTO, HGO, OAX, QRO, SLP, VER, ZAC

*Schoenocaulon comatum Brinker JAL, OAX, PUE, ZAC

*Schoenocaulon conzattii Brinker OAX

* Schoenocaulon coulteri Baker COAH, SIN

* Schoenocaulon framei Zomlefer \& Judd OAX, PUE

*Schoenocaulon ghiesbreghtii Greenm. CHIS, CHIS, VER, YUC

*Schoenocaulon ignigenum Frame NLE, TAMS
Schoenocaulon intermedium Baker SLP

*Schoenocaulon jaliscense Greenm. COL, GRO, JAL

*Schoenocaulon lauricola McVaugh ex D. Frame AGS, JAL

Schoenocaulon macrocarpum Brinker COAH, NLE

*Schoenocaulon madidorum Frame OAX, PUE, VER

*Schoenocaulon megarrhizum M.E. Jones CHIH, DGO, GTO,

JAL, NAY, SIN, SON, ZAC

*Schoenocaulon mortonii Brinker JAL, MICH, OAX

* Schoenocaulon oaxacense (Frame) Zomlefer \& Judd OAX

*Schoenocaulon obtusum Brinker HGO, VER

Schoenocaulon officinale (Schltdl. \& Cham.) A. Gray ex Benth.

CHIS, COL, GRO, HGO, MEX, MICH, MOR, NAY, NLE, OAX, QRO, SLP, VER, YUC

* Schoenocaulon pellucidum Frame JAL, MICH, NAY

* Schoenocaulon plumosum Frame COAH, NLE, TAMS

*Schoenocaulon pringlei Greenm. CDMX, HGO, MEX, MICH, MOR, NAY, PUE, VER

*Schoenocaulon rzedowskii Frame MEX, PUE

*Schoenocaulon tenorioi Frame OAX, PUE

* Schoenocaulon tenue Baker GTO, MEX, MOR, OAX, SLP

*Schoenocaulon tenuifolium (M. Martens \& Galeotti) B.L. Rob. \& Greenm. OAX, PUE

Schoenocaulon texanum Scheele CHIH, COAH, DGO, NLE, SLP, TAMS, ZAC

* Schoenocaulon tigrense Frame JAL

Veratrum californicum Durand CHIH, DGO, SON

Zigadenus paniculatus (Nutt.) S. Watson CHIH, SON

Zigadenus venenosus $\mathrm{S}$. Watson $\mathrm{BCN}$

\section{Family Melastomataceae}

Aciotis indecora (Bonpl.) Triana CHIS, TAB, VER

Acisanthera limnobios (DC.) Triana VER

Acisanthera quadrata Pers. CHIS, GRO, TAB, VER

Adelobotrys adscendens (Sw.) Triana CHIS, OAX, VER

Arthrostemma alatum Triana COL, DGO, GRO, JAL, MEX, MICH, NAY, QRO, SLP, VER

Arthrostemma ciliatum Pav. ex D. Don CHIS, GRO, HGO, OAX, PUE, QRO, SLP, TAB, TAMS, VER

Arthrostemma parvifolium Cogn. CHIS, TAB

*Arthrostemma primaevum Almeda CHIS, HGO, OAX, PUE, VER

Bellucia grossularioides (L.) Triana CHIS, OAX, TAB, VER

Bellucia pentamera Naudin CHIS, OAX, TAB, VER

Blakea guatemalensis Donn. Sm. CHIS, GRO

Blakea purpusii Brandegee CHIS

Centradenia floribunda Planch. CHIS, OAX, VER

Centradenia grandifolia (Schltdl.) Endl. CHIS, HGO, OAX, PUE, SLP, VER

Centradenia inaequilateralis (Schltdl. \& Cham.) G. Don CHIS, OAX, VER

Clidemia capitellata (Bonpl.) D. Don CAM, CHIS, GRO, MICH, OAX, QROO, TAB, VER

Clidemia cymifera Donn. Sm. CHIS

Clidemia dentata Pav. ex D. Don CHIS, JAL, NAY, OAX, PUE,

TAB, VER

Clidemia donnell-smithii Cogn. CHIS

Clidemia fulva Gleason CHIS, OAX, PUE, VER 
*Clidemia gracilipes Gleason OAX, PUE, VER

Clidemia hirta (L.) D. Don CHIS, GRO, OAX, PUE, TAB, VER Clidemia inopinata Almeda CHIS, OAX, TAB, VER

Clidemia laxiflora (Schltdl.) Walp. ex Naudin CHIS, NAY, OAX, VER

Clidemia matudae L.O. Williams CHIS, COL, GRO, JAL, MEX, NAY, OAX

Clidemia monantha L.O. Williams CHIS, OAX, VER

Clidemia octona (Bonpl.) L.O. Williams CAM, CHIS, OAX, PUE, QROO, TAB, VER

Clidemia petiolaris (Schltdl. \& Cham.) Schltdl. ex Triana CHIS, HGO, JAL, NAY, OAX, PUE, QRO, SLP, SIN, SON, TAB, VER Clidemia ruddae Wurdack DGO, NAY

*Clidemia scopulina (Brandegee) L.O. Williams CHIS, OAX

Clidemia septuplinervia Cogn. CHIS, OAX

Clidemia sericea D. Don CAM, CHIS, GRO, OAX, QROO,

TAB, VER

Clidemia setosa (Triana) Gleason CHIS, GRO, OAX, VER

Clidemia strigillosa (Sw.) DC. CHIS, TAB

*Clidemia submontana Rose ex Gleason CHIS, COL, GRO,

JAL, NAY, OAX, SIN

Clidemia urceolata DC. CHIS

*Conostegia arborea (Schltdl.) Steud. CHIS, GRO, HGO, OAX, PUE, VER

Conostegia caelestis Standl. CHIS, OAX, PUE, VER

Conostegia hirtella Cogn. CHIS

Conostegia icosandra (Sw. ex Wikstr.) Urb. CHIS, GRO, HGO,

MICH, OAX, PUE, SLP, TAB, VER

*Conostegia jaliscana Standl. COL, JAL, OAX

*Conostegia mexicana Cogn. OAX, VER

Conostegia montana (Sw.) D. Don ex DC. CHIS

Conostegia plumosa L.O. Williams CHIS

Conostegia superba D. Don ex Naudin CHIS, OAX, VER

Conostegia volcanalis Standl. \& Steyerm. CHIS, COL, GRO,

JAL, MICH, OAX, PUE, VER, ZAC

Conostegia xalapensis (Bonpl.) D. Don ex DC. CHIS, COL, DGO, GRO, HGO, JAL, MEX, MICH, MOR, NAY, OAX, PUE, QRO, SLP, SIN, TAB, TAMS, VER, YUC

Graffenrieda galeottii (Naudin) L.O. Williams CHIS, OAX

Henriettea seemannii (Nuadin) L.O. Williams CHIS

Henriettea succosa (Aubl.) DC. CHIS, QROO

Henriettella fascicularis (Sw.) C. Wright CHIS, OAX

*Heterocentron alatum Rose \& Standl. CHIS, OAX

*Heterocentron chimalapanum Todzia OAX, VER

Heterocentron elegans (Schltdl.) Kuntze CHIS, HGO, PUE, VER

Heterocentron hirtellum (Cogn.) L.O. Williams CHIS, JAL, NAY

*Heterocentron laxiflorum Standl. COL, GRO, JAL, MICH

*Heterocentron mexicanum Hook. \& Arn. COL, DGO, GRO, JAL, MEX, MICH, NAY, SIN

*Heterocentron muricatum Gleason CHIS, OAX

Heterocentron subtriplinervium (Link \& Otto) A. Braun \& C.D.

Bouché CHIS, COL, GRO, HGO, JAL, MEX, NAY, OAX, PUE, SIN, VER

Heterocentron suffruticosum Brandegee CHIS

Leandra cornoides (Schltdl. \& Cham.) Cogn. COL, HGO, JAL,
MEX, MOR, OAX, PUE, QRO, SLP, TAMS, VER

Leandra dichotoma (Pav. ex D. Don) Cogn. CHIS, OAX

Leandra longicoma Cogn. OAX, VER

Leandra melanodesma (Naudin) Cogn. CHIS, COL, GRO, HGO, JAL, MICH, OAX, VER

Leandra mexicana (Naudin) Cogn. CHIS, HGO, OAX, TAB, VER

Leandra multiplinervis (Naudin) Cogn. CHIS, GRO, OAX

Meriania macrophylla (Benth.) Triana CHIS

Miconia aeruginosa Naudin CHIS

Miconia affinis DC. CHIS, OAX, PUE, TAB, VER

Miconia albicans (Sw.) Steud. CHIS, COL, GRO, JAL, NAY, OAX, TAB, VER

Miconia alpestris Cogn. CHIS

Miconia ampla Triana CHIS, OAX, VER

*Miconia anisotricha (Schltdl.) Triana GRO, HGO, MICH, OAX, PUE, QRO, SLP, TAB, VER

Miconia argentea $(\mathrm{Sw}$.$) DC. CAM, CHIS, OAX, PUE, QROO,$ TAB, VER, YUC

Miconia bubalina (D. Don) Naudin OAX, VER

Miconia calvescens DC. CHIS, GRO, OAX, TAB, VER

Miconia caudata (Bonpl.) DC. CHIS, OAX

Miconia ciliata (Rich.) DC. CAM, CHIS, OAX, QROO, VER

Miconia costaricensis Cogn. CHIS, OAX

Miconia chamissois Naudin CHIS, GRO, JAL, MICH, OAX,

TAB, VER

Miconia chinantlana (Naudin) Almeda CHIS, OAX, VER

*Miconia chrysoneura Triana HGO, OAX, PUE, SLP, VER

Miconia chrysophylla (Rich.) Urb. CHIS, OAX

Miconia decurrens Cogn. CHIS

Miconia desmantha Benth. CHIS, MICH, OAX, VER

Miconia dodecandra Cogn. CHIS, GRO, MICH, OAX, PUE, VER

Miconia donaeana Naudin GRO

Miconia elata (Sw.) DC. CHIS, VER

*Miconia erythrantha Naudin OAX

Miconia fulvostellata L.O. Williams CHIS, OAX, VER

Miconia glaberrima (Schltdl.) Naudin CHIS, COL, GRO, HGO,

JAL, MEX, MICH, MOR, OAX, PUE, SIN, VER

* Miconia glabrata Cogn. CHIS, GRO, OAX

* Miconia globulifera Naudin CHIS, GRO, OAX, VER

Miconia gracilis Triana CHIS, GRO, OAX, VER

Miconia guatemalensis Cogn. CHIS, HGO, OAX, VER

Miconia hemenostigma Naudin CHIS, GRO, HGO, MEX, MICH, OAX, PUE, VER

* Miconia heterothrix Gleason \& Wurdack GRO, MICH

Miconia holosericea (L.) DC. CHIS, VER

Miconia hondurensis Donn. Sm. CHIS

Miconia hyperprasina Naudin CAM, CHIS, OAX, PUE, QROO, TAB, VER

Miconia ibaguensis (Bonpl.) Triana CHIS, GRO, MICH, OAX, PUE, TAB, VER

* Miconia ibarrae Almeda VER

Miconia impetiolaris (Sw.) D. Don ex DC. CAM, CHIS, GRO, NAY, OAX, PUE, QROO, TAB, VER

Miconia insularis Gleason OAX, VER

*Miconia jitotolana Wurdack CHIS 
Miconia lacera (Humb. \& Bonpl.) Naudin CHIS, OAX, TAB, VER

Miconia laevigata (L.) D. Don CAM, CHIS, GRO, HGO, MOR, OAX, PUE, QROO, SLP, TAB, VER

Miconia lateriflora Cogn. CHIS, PUE, VER

* Miconia liebmannii Cogn. OAX

Miconia livida Triana CHIS, GRO, OAX

Miconia lonchophylla Naudin CHIS, OAX, PUE, VER

Miconia longifolia (Aubl.) DC. CHIS, OAX

*Miconia madrensis Standl. DGO, JAL, NAY, SIN

Miconia matthaei Naudin CHIS, OAX, TAB, VER

* Miconia mazatecana de Santiago OAX

* Miconia mcvaughii Wurdack COL, JAL

Miconia mexicana (Bonpl.) Naudin CHIS, GRO, HGO, JAL,

OAX, PUE, VER

*Miconia militis Wurdack GRO, OAX

Miconia minutiflora (Bonpl.) DC. CHIS, GRO, OAX, PUE,

TAB, VER

Miconia mirabilis (Aubl.) L.O. Williams GRO

* Miconia moorei Wurdack HGO, PUE, QRO, SLP, VER

Miconia nervosa (Sm.) Triana CHIS

Miconia nutans Donn. Sm. CHIS

*Miconia obconica Gleason \& Wurdack GRO, OAX

Miconia oinochrophylla Donn. Sm. CHIS, OAX, TAB, VER

Miconia oligocephala Donn. Sm. CHIS

Miconia oligotricha (DC.) Naudin CHIS, GRO, HGO, OAX, PUE, QRO, SLP, VER

*Miconia phaeotricha Naudin OAX

Miconia prasina (Sw.) DC. CHIS, GRO, OAX, PUE, QROO,

TAB, VER

Miconia punctata (Desr.) D. Don ex DC. CHIS, OAX, VER

Miconia purulensis Donn. Sm. OAX

* Miconia ravenii Wurdack CHIS

Miconia reducens Triana CHIS

* Miconia rzedowskii de Santiago GRO, OAX

*Miconia saxicola Brandegee DGO, JAL, NAY, SIN

Miconia schippii Standl. CHIS

Miconia schlechtendalii Cogn. CAM, CHIS, GRO, OAX, TAB, VER

Miconia schlimii Triana CHIS

Miconia serrulata (DC.) Naudin CHIS, OAX, VER

Miconia silvestris Gleason OAX

Miconia smaragdina Naudin GRO, OAX, VER

Miconia splendens ( $\mathrm{Sw}$.) Griseb. CHIS

Miconia stenostachya DC. CHIS, OAX, TAB, VER

Miconia sylvatica (Schltdl.) Naudin CHIS, HGO, OAX, PUE,

QRO, SLP, VER

Miconia tacanensis Wurdack CHIS

*Miconia teotepecensis de Santiago GRO, OAX

* Miconia tepicana Standl. JAL, NAY

Miconia tomentosa (L. Rich.) D. Don CHIS, VER

Miconia trinervia (Sw.) D. Don ex Loudon CHIS, GRO, NAY,

OAX, PUE, TAB, VER

Miconia triplinervis Ruiz \& Pav. CHIS, TAB

Miconia tuerckheimii Cogn. CHIS, OAX, TAMS

Miconia zemurrayana Standl. \& L.O. Williams CHIS

Monochaetum alpestre Naudin CHIS, GRO, HGO, MICH,
OAX, VER

*Monochaetum calcaratum (DC.) Triana DGO, GRO, JAL, MEX, MICH, MOR, NAY, OAX, SIN, VER, ZAC

Monochaetum deppeanum (Schltdl. \& Cham.) Naudin CHIS, VER

Monochaetum floribundum (Schltdl.) Naudin CHIS, DGO, HGO, MEX, OAX, PUE, SIN, VER

*Monochaetum pulchrum Decne. CHIS, HGO, OAX, PUE, VER

*Monochaetum rubescens Gleason CHIS

Monochaetum tenellum Naudin CHIS

Mouriri exilis Gleason CHIS

Mouriri gleasoniana Standl. CHIS, OAX, TAB, VER

*Mouriri muelleri Cogn. CHIS, GRO, OAX

Mouriri myrtilloides (Sw.) Poir. CAM, CHIS, JAL, NAY, OAX, PUE, SLP, TAB, VER

Ossaea micrantha (Sw.) Macfad. ex Cogn. CHIS, OAX, VER

Ossaea trichocalyx Pittier CHIS

Pterolepis stenophylla Gleason CHIS, VER

Pterolepis trichotoma (Rottb.) Cogn. CHIS, COL, GRO, JAL,

MICH, MOR, NAY, OAX, TAB, VER

Rhynchanthera grandiflora (Aubl.) DC. OAX, VER

Rhynchanthera medialis Standl. \& Steyerm. CHIS

Rhynchanthera mexicana (L.) DC. TAB, VER

Schwackaea cupheoides (Benth.) Cogn. CHIS, OAX

Stanmarkia medialis (Standl. \& Steyerm.) Almeda CHIS

*Tibouchina almedae Todzia DGO, NAY, SIN

*Tibouchina araneicalyx de Santiago GRO

*Tibouchina breedlovei Wurdack CHIS

*Tibouchina congestiflora Todzia GRO, OAX

*Tibouchina connata Gleason ex Todzia GRO, MICH

*Tibouchina chiapensis Wurdack CHIS, OAX

*Tibouchina durangensis Standl. DGO, GRO

*Tibouchina ferrariana Cogn. PUE

*Tibouchina galeottiana (Naudin) Cogn. GRO, HGO, MEX, MOR, OAX, PUE, QRO, SLP, TAMS, VER

*Tibouchina hintonii Gleason ex Todzia GRO, JAL, OAX

Tibouchina longifolia (Vahl) Baill. CHIS, COL, GRO, HGO, JAL, MEX, MICH, NAY, OAX, PUE, QRO, TAB, VER, YUC Tibouchina longisepala Cogn. CHIS, HGO, OAX, VER

*Tibouchina macvaughii Todzia COL, JAL, NAY

Tibouchina monticola (Naudin) Cogn. COL, GRO, OAX

*Tibouchina nanifolia Todzia GRO, MEX

*Tibouchina naudiniana (Decne.) Cogn. CHIS, GRO, HGO,

OAX, PUE, QRO, SLP, VER

*Tibouchina patens Todzia GRO

*Tibouchina roseotincta Todzia GRO

*Tibouchina rufipilis (Schltdl.) Cogn. HGO, PUE, QRO, SLP, VER

*Tibouchina scabriuscula (Schltdl.) Cogn. CHIS, COL, GRO, JAL, MEX, MICH, OAX, PUE

Tibouchina schiedeana (Schltdl. \& Cham.) Cogn. CHIS, GRO, HGO, OAX, SLP, VER

*Tibouchina sericea de Santiago CHIS, GRO, OAX

*Tibouchina sinaloensis Todzia DGO, OAX, SIN

*Tibouchina thulia Todzia SIN

*Tibouchina tortuosa (Bonpl.) Almeda CHIS, GRO, HGO, 
MEX, MICH, MOR, OAX, PUE, QRO, SLP, VER

*Tibouchina xochiatencana de Santiago GRO

Tococa guianensis Aubl. CHIS, TAB, VER

Topobea calycularis Naudin CHIS, OAX

Topobea laevigata (D. Don) Naudin CHIS, OAX, PUE, VER

Topobea maurofernandeziana Cogn. GRO, OAX, VER

Topobea watsonii Cogn. CHIS

*Triolena scorpioides Naudin CHIS, OAX, PUE, TAB, VER

\section{Family Meliaceae}

Cedrela angustifolia Moc. \& Sessé ex DC. MICH, VER

*Cedrela discolor S.F. Blake DGO, GRO

*Cedrela oaxacensis C. DC. \& Rose CHIS, GRO, MEX, MOR, OAX, PUE

Cedrela odorata L. AGS, CAM, CHIS, CHIH, COL, DGO, GTO, GRO, HGO, JAL, MEX, MICH, MOR, NAY, NLE, OAX, PUE, QRO, QROO, SLP, SIN, SON, TAB, TAMS, VER, YUC, ZAC

Cedrela salvadorensis Standl. CHIS, COL, DGO, GRO, JAL, MEX, MICH, MOR, NAY, OAX, PUE, SIN

Cedrela tonduzii C. DC. CHIS, VER

Guarea glabra Vahl CAM, CHIS, COL, CDMX, DGO, GRO, HGO, JAL, MEX, MICH, MOR, NAY, OAX, PUE, QROO, SIN, TAB, VER

Guarea grandifolia DC. CHIS, OAX, PUE, TAB, VER

Guarea mexicana Coronado CHIS

Guarea petenensis Coronado CAM, CHIS, COL, JAL, NAY, OAX, PUE, QROO, TAB, VER

Swietenia humilis L. CAM, CHIS, COL, DGO, GRO, JAL, MEX, MICH, MOR, NAY, OAX, PUE, QROO, SLP, SIN, VER Swietenia macrophylla G. King CAM, CHIS, COL, GTO, GRO, HGO, JAL, MEX, MICH, MOR, NAY, OAX, PUE, QRO, QROO, SIN, TAB, VER, YUC

Trichilia americana (Sessé \& Moc.) T.D. Penn. AGS, CAM, CHIS, CHIH, COL, DGO, GRO, JAL, MEX, MICH, MOR, NAY, OAX, PUE, QROO, SLP, SIN, SON, TAB, VER, YUC, ZAC

Trichilia breviflora S.F. Blake \& Standl. CHIS, MICH, OAX, VER

Trichilia chirriactensis (Standl. \& Steyerm.) T.D. Penn. CHIS Trichilia erythrocarpa Lundell CAM, CHIS, OAX, QROO

Trichilia glabra L. CAM, CHIS, OAX, QROO, VER, YUC Trichilia havanensis Jacq. CAM, CHIS, COL, GTO, GRO, HGO, JAL, MEX, MICH, MOR, NAY, NLE, OAX, PUE, QRO, QROO, SLP, SIN, TAB, TAMS, VER, YUC

Trichilia hirta L. CAM, CHIS, CHIH, COL, DGO, GRO, HGO, JAL, MEX, MICH, MOR, NAY, OAX, PUE, QRO, QROO, SLP, SIN, SON, TAB, TAMS, VER, YUC, ZAC

*Trichilia japurensis C. DC. CHIS, VER

Trichilia martiana C. DC. CAM, CHIS, GRO, JAL, MICH, NAY, OAX, TAB, VER

Trichilia minutiflora Standl. CAM, CHIS, JAL, NAY, OAX, PUE, QROO, TAB, VER, YUC

Trichilia moschata Sw. CAM, CHIS, COL, JAL, OAX, QROO, TAB, VER

*Trichilia oligantha C. DC. VER

Trichilia pallida Sw. CAM, CHIS, OAX, QROO, TAB, VER
Trichilia trifolia L. CAM, CHIS, COL, GRO, JAL, MEX, MICH, NAY, OAX, PUE, QROO, SLP, SIN, TAMS, VER, YUC

\section{Family Menispermaceae}

Abuta chiapasensis Krukoff \& Barneby CHIS

Abuta panamensis (Standl.) Krukoff \& Barneby CHIS, OAX, TAB, VER

Abuta steyermarkii (Standl.) Standl. CHIS, OAX

Cissampelos fasciculata Benth. OAX, SLP, TAMS, VER

Cissampelos grandifolia Triana \& Planch. CAM, CHIS, GRO, MICH, PUE, QROO, SLP, TAB, TAMS, VER

Cissampelos pareira L. BCS, CAM, CHIS, COL, GTO, GRO, HGO, JAL, MEX, MICH, MOR, NAY, OAX, PUE, QRO, QROO, SLP, SIN, SON, TAB, TAMS, VER, YUC

Cissampelos tropaeolifolia DC. CHIS, GRO, HGO, NAY, OAX, QROO, TAB, VER

Cocculus carolinus (L.) DC. CHIH, COAH, NLE, TAMS

Cocculus diversifolius DC. BCS, CHIH, COAH, COL, GTO, GRO, HGO, JAL, MEX, MICH, MOR, NAY, NLE, OAX, PUE, QRO, SLP, SIN, SON, TAMS, VER

Disciphania calocarpa Standl. CHIS, OAX, TAB, VER

Disciphania cardiophylla Standl. VER

*Disciphania mexicana Bullock COL, JAL, MEX, MICH, NAY, SIN

*Disciphania nesiotes Standl. NAY

Disciphania spadicea Barneby JAL, OAX

*Hyperbaena ilicifolia Standl. COL, GRO, JAL, MICH

*Hyperbaena jalcomulcensis Pérez-Cueto \& Cast.-Campos VER

Hyperbaena mexicana Miers CAM, CHIS, GRO, MICH, OAX, QROO, TAB, VER, YUC

Hyperbaena standleyi Mathias \& W.L. Theob. CHIS, OAX

Hyperbaena winzerlingii Standl. CAM, QROO, YUC

Menispermum canadense L. COAH, NLE, QRO, SLP

Odontocarya mexicana Barneby CHIS, GRO, OAX, PUE, VER

\section{Family Menyanthaceae}

Nymphoides fallax Ornduff AGS, CHIS, CHIH, CDMX, DGO, GTO, HGO, JAL, MEX, MICH, NAY, OAX, PUE, QRO, TLAX, VER, ZAC

Nymphoides humboldtiana (Kunth) Kuntze CHIS, VER

Nymphoides indica (L.) Kuntze CAM, CHIS, COL, GRO, HGO, JAL, MICH, NAY, PUE, QRO, QROO, TAB, TAMS, VER, YUC

Family Mitrastemonaceae

Mitrastemon matudae Yamamoto CHIS, GRO, OAX

\section{Family Molluginaceae}

Glinus radiatus (Ruiz \& Pav.) Rohrb. BCN, BCS, CAM, CHIS, CHIH, COAH, COL, DGO, GRO, JAL, MOR, SLP, SIN, SON, TAMS, VER

Mollugo verticillata $\mathrm{L}$. BCN, BCS, CHIS, CHIH, COL, DGO, GTO, GRO, HGO, JAL, MEX, MICH, MOR, OAX, PUE, QRO, SLP, SIN, SON, TAB, TAMS, VER

\section{Family Monimiaceae}

* Mollinedia butleriana Standl. OAX, VER

*Mollinedia jerzyi Lorence OAX 
*Mollinedia mexicana Perkins CHIS, TAB, VER

* Mollinedia oaxacana Lorence OAX

* Mollinedia orizabae Perkins VER

Mollinedia pallida Lundell CHIS

* Mollinedia torresiorum Lorence OAX

Mollinedia viridiflora Tul. CHIS, GRO, OAX, PUE, TAB, VER

Family Montiaceae

Calandrinia acaulis Kunth CHIS, CDMX, MEX, PUE, VER

Calandrinia ciliata (Ruiz \& Pav.) DC. BCN, BCS, CHIS, CDMX, GTO, HGO, JAL, MEX, MICH, PUE, QRO, SLP, SON, TLAX, VER, ZAC

Claytonia parviflora Hook. BCN

Claytonia perfoliata Donn ex Willd. BCN, CHIS, COL, CDMX, DGO, GTO, HGO, JAL, MEX, MICH, MOR, OAX, PUE, QRO, SIN, TLAX, VER

Lewisia brachycalyx Engelm. ex A. Gray BCN

Montia calcicola Standl. \& Steyerm. CHIS, GRO, OAX

Montia chamissoi (Ledeb. ex Spreng.) Greene CDMX, GTO, HGO, MEX, MICH, QRO, VER

Montia fontana L. MEX

Phemeranthus aurantiacus (Engelm.) Kiger CHIH, COAH, DGO, GTO, HGO, NLE, QRO, SLP, SON

Phemeranthus brevicaulis (S. Watson) Kiger CHIH, COAH

Phemeranthus calycinus (Engelm.) Kiger $\mathrm{COAH}$

Phemeranthus humilis (Greene) Kiger AGS, CDMX, DGO, JAL, MEX, ZAC

Phemeranthus marginatus (Greene) Kiger CHIH, JAL, NAY, SON

*Phemeranthus mexicanus (Hemsl.) G. Ocampo SLP, ZAC

*Phemeranthus multiflorus (Rose \& Standl.) G. Ocampo CHIH, DGO, GTO, HGO, OAX, QRO, VER

*Phemeranthus napiformis (DC.) G. Ocampo AGS, CDMX, DGO, GTO, HGO, JAL, MEX, QRO, SLP, VER, ZAC

*Phemeranthus oligospermus (Brandegee) G. Ocampo OAX, PUE

Phemeranthus parviflorus (Nutt.) Kiger AGS, CHIH, COAH, DGO, JAL, NLE, SON, ZAC

Phemeranthus parvulus (Rose \& Standl.) D.J. Ferguson \& T.M. Price CHIH, DGO, NAY, SON

\section{Family Moraceae}

Brosimum alicastrum Sw. CAM, CHIS, COL, DGO, GRO, HGO, JAL, MEX, MICH, MOR, NAY, OAX, PUE, QRO, QROO, SLP, SIN, SON, TAB, TAMS, VER, YUC, ZAC

Brosimum costaricanum Liebm. CHIS, TAB, VER

Brosimum guianense (Aubl.) Huber CHIS, OAX, TAB, VER

Brosimum lactescens (S. Moore) C.C. Berg CHIS, OAX, TAB, VER

Castilla elastica Sessé ex Cerv. CAM, CHIS, COL, GRO, HGO, JAL, MEX, MICH, NAY, OAX, PUE, QRO, QROO, SLP, SIN, TAB, TAMS, VER, YUC

Clarisia biflora Ruiz \& Pav. CHIS, OAX, VER

Clarisia recemosa Ruiz \& Pav. OAX

Dorstenia contrajerva L. CAM, CHIS, GRO, HGO, JAL, MEX, MICH, MOR, NAY, OAX, PUE, QRO, QROO, SLP, TAB, VER, YUC

Dorstenia choconiana S. Watson CHIS
Dorstenia drakena L. CHIS, CHIH, COL, DGO, GRO, JAL, MEX, MICH, MOR, NAY, OAX, PUE, QRO, SLP, SIN, SON, TAMS, VER, ZAC

*Dorstenia excentrica Moric. HGO, QRO, SLP, VER

Dorstenia lindeniana Bureau CHIS, TAB

*Dorstenia uxpanapana C.C. Berg \& T. Wendt VER

Ficus americana Aubl. CAM, CHIS, HGO, PUE, QRO, QROO, SLP, TAB, VER

Ficus apollinaris Dugand CAM, CHIS, GRO, OAX, PUE, VER Ficus aurea Nutt. CAM, CHIS, COL, GRO, HGO, JAL, MEX, MICH, MOR, NAY, OAX, PUE, QRO, QROO, SLP, TAB, TAMS, VER, YUC, ZAC

Ficus citrifolia Mill. CAM, CHIS, COL, DGO, GRO, JAL, MEX, MICH, NAY, OAX, QROO, TAB, VER, YUC

Ficus colubrinae Standl. CHIS, OAX, TAB, VER

Ficus cotinifolia Kunth CAM, CHIS, CHIH, COL, DGO, GTO, GRO, HGO, JAL, MEX, MICH, MOR, NAY, OAX, PUE, QRO, QROO, SLP, SIN, SON, TAB, TAMS, VER, YUC, ZAC

Ficus crassinervia Desf. ex Willd. CAM, CHIS, OAX, QROO, TAB, VER, YUC

Ficus crocata (Miq.) Miq. CAM, CHIS, COL, DGO, GRO, JAL, MEX, MICH, MOR, NAY, OAX, PUE, QRO, QROO, SLP, SIN, SON, TAB, TAMS, VER, YUC, ZAC

*Ficus fuscescens (Liebm.) Miq. VER

Ficus guajavoides Lundell CHIS, TAB

Ficus insipida Willd. CAM, CHIS, CHIH, COL, DGO, GRO, HGO, JAL, MEX, MICH, MOR, NAY, OAX, PUE, QRO, SLP, SIN, SON, TAB, TAMS, VER, ZAC

*Ficus lapathifolia (Liebm.) Miq. CHIS, GRO, JAL, MEX, NAY, OAX, PUE, TAB, VER

Ficus maxima Mill. CAM, CHIS, CHIH, COL, DGO, GRO, HGO, JAL, MEX, MICH, MOR, NAY, OAX, PUE, QRO, QROO, SLP, SIN, SON, TAB, VER, YUC

Ficus membranacea $\mathrm{C}$. Wright COL, DGO, GRO, HGO, JAL, MEX, MICH, MOR, NAY, OAX, QRO, SLP, SIN, ZAC

Ficus obtusifolia Kunth CAM, CHIS, COL, GRO, HGO, JAL, MEX, MICH, MOR, NAY, OAX, PUE, QRO, QROO, SLP, SIN, TAB, TAMS, VER, YUC

Ficus paraensis (Miq.) Miq. CHIS, OAX, TAB, VER

Ficus pertusa L. f. CAM, CHIS, CHIH, COL, DGO, GTO, GRO, HGO, JAL, MEX, MICH, MOR, NAY, OAX, PUE, QRO, QROO, SLP, SIN, SON, TAB, TAMS, VER, YUC, ZAC * Ficus petiolaris Kunth AGS, BCN, BCS, CHIS, CHIH, COL, DGO, GTO, GRO, JAL, MEX, MICH, MOR, NAY, OAX, PUE, SIN, SON, VER, ZAC

Ficus popenoei Standl. CHIS

*Ficus pringlei $\mathrm{S}$. Watson COL, GRO, JAL, MICH, NAY, OAX, ZAC

*Ficus rzedowskiana Carvajal \& Cuevas-Figueroa CHIS, GRO, HGO, OAX, PUE, QRO, SLP, VER

Ficus trigonata L. CAM, CHIS, CHIH, COL, DGO, GRO, HGO, JAL, MEX, MICH, MOR, NAY, OAX, PUE, QRO, QROO, SLP, SIN, SON, TAB, TAMS, VER, YUC, ZAC

Ficus turrialbana W.C. Burger CAM, CHIS, GRO, JAL, OAX, PUE, QROO, TAB, VER

Ficus velutina Humb. \& Bonpl. ex Willd. CAM, CHIS, COL, DGO, GRO, HGO, JAL, MEX, MICH, MOR, NAY, OAX, PUE, 
SLP, SIN, VER, ZAC

Ficus yoponensis Desv. CAM, CHIS, GRO, OAX, PUE, QROO, TAB, VER

Maclura tinctoria (L.) D. Don ex Steud. CAM, CHIS, CHIH, COL, DGO, GRO, HGO, JAL, MICH, NAY, OAX, PUE, QRO, QROO, SLP, SIN, SON, TAB, TAMS, VER, YUC

Morus celtidifolia Kunth CHIS, CHIH, COAH, COL, CDMX, DGO, GTO, HGO, JAL, MEX, MICH, MOR, NLE, OAX, PUE, QRO, SLP, SIN, SON, TAMS, VER, YUC, ZAC

Morus insignis Bureau CHIS

Morus microphylla Buckley CHIH, COAH, DGO, NLE, SON

Poulsenia armata (Miq.) Standl. CHIS, OAX, TAB, VER

Pseudolmedia glabrata (Liebm.) C.C. Berg CAM, CHIS, GRO, HGO, MICH, OAX, PUE, QROO, TAB, VER, YUC

Pseudolmedia simiarum Standl. \& Steyerm. CHIS

Pseudolmedia spuria (Sw.) Griseb. CAM, CHIS, OAX, QROO

Sorocea affinis Hemsl. CHIS, OAX, TAB, VER

Sorocea trophoides W.C. Burger TAB, VER

Trophis cuspidata Lundell CHIS, HGO, OAX, PUE, VER

Trophis chiapensis Brandegee CHIS, OAX, PUE, VER

Trophis mexicana (Liebm.) Bureau CHIS, COL, GRO, HGO,

JAL, MICH, NAY, OAX, PUE, TAB, VER

*Trophis noraminervae Cuevas \& Carvajal COL, JAL

Trophis racemosa (L.) Urb. CAM, CHIS, CHIH, COL, DGO, GRO, HGO, JAL, MEX, MICH, MOR, NAY, OAX, PUE, QRO, QROO, SLP, SIN, SON, TAB, TAMS, VER, YUC

\section{Family Muntingiaceae}

Dicraspidia donnell-smithii Standl. OAX

Muntingia calabura L. CAM, CHIS, COL, GRO, HGO, JAL, MEX, MICH, MOR, NAY, OAX, PUE, QRO, QROO, SLP, TAB, TAMS, VER, YUC

\section{Family Musaceae}

Musa mexicana Matuda TAB, VER

\section{Family Myricaceae}

Morella cerifera (L.) Small CAM, CHIS, COL, DGO, GRO, HGO, JAL, MEX, MICH, NAY, OAX, PUE, QRO, QROO, SLP, TAB, TAMS, VER, YUC

Morella lindeniana (C. DC.) S. Knapp CHIS, GRO, MICH, OAX, VER

Morella pringlei (Greenm.) Wilbur HGO, OAX, PUE, QRO, VER

\section{Family Myristicaceae}

Compsoneura mexicana (Hemsl.) Janovec CHIS, OAX, TAB, VER

Compsoneura sprucei (DC.) Warb. CHIS, TAB, VER

Virola guatemalensis (Hemsl.) Warb. CHIS, OAX, TAB, VER

Virola koschnyi Warb. CHIS

\section{Family Myrtaceae}

*Calycorectes mexicanus O. Berg HGO, OAX, QRO, SLP, VER Calyptranthes calderonii Standl. CHIS

Calyptranthes contrerasii Lundell CHIS

Calyptranthes chiapensis Lundell CHIS, VER

Calyptranthes chytraculia (L.) Sw. CHIS, OAX, QROO, VER, YUC
*Calyptranthes hernandezii McVaugh SLP, VER

Calyptranthes hylobates Standl. ex Amshoff CHIS, OAX, PUE Calyptranthes karlingii Standl. CAM, CHIS, QROO, TAB, VER

*Calyptranthes karwinskyana O. Berg CHIS, PUE, TAB, VER Calyptranthes lindeniana O. Berg CHIS, OAX, QROO, TAB, VER

Calyptranthes macrantha Standl. \& Steyerm. CHIS

Calyptranthes mayana Lundell CHIS

Calyptranthes megistophylla Standl. CHIS, OAX, VER

Calyptranthes millspaughii Urb. CHIS, OAX, QROO, TAB, VER, YUC

Calyptranthes pallens Griseb. CAM, CHIS, COL, JAL, NAY, OAX, QROO, SIN, VER, YUC

Calyptranthes paxillata McVaugh CHIS, VER

Calyptranthes pendula O. Berg CHIS, GRO, JAL, NAY, OAX, SIN

Calyptranthes perlaevigata Lundell CHIS

Calyptranthes schiedeana O. Berg CAM, CHIS, COL, DGO, GRO, HGO, NAY, OAX, PUE, SIN, VER

*Calyptranthes tenuipes McVaugh OAX, PUE, VER

Calyptranthes tonii Lundell CHIS

*Calyptranthes venulosa Lundell CHIS

Calyptranthes zuzygium (L.) Sw. MICH, NAY

Chamguava gentlei (Lundell) Landrum CHIS

Chamguava schippii (Standl.) Landrum CHIS, GRO

Eugenia acapulcensis Steud. CAM, CHIS, COL, DGO, GRO, HGO, JAL, MEX, MICH, MOR, NAY, OAX, PUE, QROO, SLP, SIN, SON, TAB, TAMS, VER, YUC

Eugenia aeruginea DC. CAM, CHIS, GRO, OAX, QROO, TAB, VER

*Eugenia alnifolia McVaugh JAL, MEX, MICH

Eugenia amatenangensis Lundell CHIS

*Eugenia avicenniae Standl. GRO

Eugenia axillaris (Sw.) Willd. CAM, CHIS, GRO, NAY, OAX, QRO, QROO, SLP, VER, YUC

*Eugenia balancanensis Lundell CHIS, TAB

Eugenia biflora (L.) DC. CHIS, QROO

*Eugenia breedlovei Barrie CHIS

Eugenia bumelioides Standl. CHIS, TAB

Eugenia capuli (Schltdl. \& Cham.) Hook. \& Arn. CAM, CHIS, COL, GRO, HGO, JAL, MEX, MICH, MOR, NAY, OAX, PUE, QRO, QROO, SLP, SIN, TAB, TAMS, VER, YUC

Eugenia capulioides Lundell CHIS

Eugenia cerrocacaoensis Barrie OAX

Eugenia citroides Lundell CHIS

*Eugenia colipensis O. Berg VER

*Eugenia crenularis Lundell COL, DGO, GRO, HGO, JAL, MEX, MICH, MOR, NAY, QRO, SLP, SIN, VER

*Eugenia culminicola McVaugh COL, GRO, JAL, MEX

Eugenia chiapensis Lundell CHIS

Eugenia chinajensis Standl. \& Steyerm. CHIS

Eugenia choapamensis Standl. CHIS, OAX, PUE, TAB, VER

Eugenia farameoides A. Rich. CHIS, OAX, QROO, TAB, VER

Eugenia flavoviridis Lundell CHIS

Eugenia foetida Pers. CAM, CHIS, OAX, QROO, YUC

Eugenia galalonensis (C. Wright ex Griseb.) Krug \& Urb. CAM, 
CHIS, OAX, QROO, TAB, VER

Eugenia gaumeri Standl. CAM, QROO, YUC

Eugenia guatemalensis Donn. Sm. CHIS, DGO, GRO, MEX, OAX, SIN, VER

*Eugenia hintonii Lundell GRO

Eugenia hypargyraea Standl. CHIS, VER

*Eugenia inconspicua Standl. DGO, JAL, SIN

*Eugenia inirebensis P.E. Sánchez VER

*Eugenia karwinskyana O. Berg CHIS, HGO, PUE, QRO, QROO, SLP, TAB, VER, YUC

Eugenia laevis O. Berg CAM, CHIS, QROO, YUC

*Eugenia ledophylla (Standl.) McVaugh HGO, VER

Eugenia letreroana Lundell CHIS

*Eugenia liebmannii Standl. CHIS, OAX, QRO, SLP, TAMS, VER

* Eugenia matudae Lundell CHIS, GRO

*Eugenia mexicana Steud. CHIS, GRO, HGO, NAY, OAX, SLP, TAMS, VER

*Eugenia michoacanensis Lundell COL, GRO, JAL, MICH, MOR

*Eugenia mirandae Ramírez-Cantú MOR

*Eugenia mozomboensis P.E. Sánchez VER

* Eugenia nigrita Lundell CHIS

Eugenia octopleura Krug \& Urb. CHIS

Eugenia oerstediana O. Berg CAM, CHIS, COL, GRO, HGO, JAL, MEX, MOR, NAY, OAX, PUE, QRO, QROO, SLP, TAB, TAMS, VER

*Eugenia ovandensis Lundell CHIS

Eugenia percivalii Lundell CHIS, VER

*Eugenia pleurocarpa Standl. COL, GRO, JAL, NAY

*Eugenia praeterita McVaugh CHIS, VER

Eugenia principium McVaugh COL, JAL

*Eugenia pueblana Lundell HGO, PUE, QRO, SLP, VER

*Eugenia purpusii Standl. CHIS, GRO, MOR, OAX

*Eugenia ravenii Lundell CHIS

* Eugenia rekoi Standl. COL, GRO, JAL, MICH, OAX, SIN

Eugenia rhombea (O. Berg) Krug. \& Urb. ex Urb. CHIS, GRO, OAX, QROO, VER, YUC

Eugenia riograndis Lundell CAM, CHIS, TAB

Eugenia rubella Lundell CHIS

Eugenia salamensis Donn. Sm. COL, DGO, GRO, JAL, MICH, NAY, OAX, SIN

Eugenia savannarum Standl. \& Steyerm. CHIS

Eugenia siltepecana Lundell CHIS

*Eugenia sinaloae Standl. SIN

*Eugenia sotoesparzae P.E. Sánchez OAX, PUE, VER

*Eugenia standleyi McVaugh CHIS, OAX

*Eugenia symphoricarpos McVaugh TAMS, VER

*Eugenia teapensis McVaugh CHIS, TAB, VER

*Eugenia tenejapensis Lundell CHIS

Eugenia tikalana Lundell CAM, CHIS, QROO

Eugenia toledinensis Lundell CHIS, QROO

*Eugenia tonii Lundell CHIS

Eugenia trikii Lundell CAM, CHIS, QROO

Eugenia trunciflora (Schltdl. \& Cham.) O. Berg CHIS, HGO, OAX, PUE, SLP, TAB, VER

*Eugenia turneri McVaugh MICH
* Eugenia uliginosa Lundell CHIS, OAX

*Eugenia uxpanapensis P.E. Sánchez \& L.M. Ortega VER

Eugenia venezuelensis O. Berg CHIS, GRO, JAL, MICH, OAX, SLP, TAB, TAMS, VER

Eugenia verapazensis Lundell CHIS

Eugenia vesca Lundell CHIS

Eugenia winzerlingii Standl. CAM, CHIS, QROO, TAB, YUC

Eugenia xalapensis (Kunth) DC. CHIS, GRO, HGO, OAX,

QRO, SLP, TAMS, VER

*Eugenia xilitlensis McVaugh QRO, SLP, VER

*Eugenia yautepecana Lundell MEX, MOR, TAB, VER

Eugenia yucatanensis Standl. CAM, QROO, YUC

Eugenia yunckeri Standl. CHIS

Mosiera contrerasii (Lundell) Landrum CAM, QROO

* Mosiera ehrenbergii (O. Berg) Landrum HGO, QRO, SLP, TAMS, VER

Myrcia fallax (Rich.) DC. CHIS

Myrcia leptoclada DC. CHIS

Myrcia splendens (Sw.) DC. CHIS, MICH, OAX, TAB, VER

Myrcianthes fragrans (Sw.) McVaugh CAM, CHIS, COL, DGO, GRO, HGO, JAL, MEX, MICH, MOR, NAY, OAX, QRO, QROO, SLP, SIN, TAB, TAMS, VER, YUC

Myrciaria floribunda (H. West ex Willd.) O. Berg CAM, CHIS, GRO, OAX, QROO, TAB, VER, YUC

Myrciaria ibarrae Lundell CAM, QROO

*Psidium chiapasense Lundell CHIS

Psidium friedrichsthalianum (O. Berg) Nied. CHIS, OAX, VER Psidium guajava L. BCS, CAM, CHIS, CHIH, COAH, COL, DGO, GTO, GRO, HGO, JAL, MEX, MICH, MOR, NAY, NLE, OAX, PUE, QRO, QROO, SLP, SIN, SON, TAB, TAMS, VER, YUC, ZAC

Psidium guineense Sw. CAM, CHIS, COL, DGO, GRO, JAL, MICH, NAY, OAX, QRO, QROO, SLP, SIN, TAB, VER, YUC Psidium salutare (Kunth) O. Berg CHIS, GRO, NAY, OAX, QROO, TAB, VER, YUC

Psidium sartorianum (O. Berg) Nied. AGS, BCS, CAM, CHIS, CHIH, COL, DGO, GRO, HGO, JAL, MEX, MICH, MOR, NAY, OAX, PUE, QRO, QROO, SLP, SIN, SON, TAB, TAMS, TLAX, VER, YUC, ZAC

*Psidium socorrense I.M. Johnst. COL

Ugni myricoides (Kunth) O. Berg CHIS, HGO, OAX, PUE, VER

\section{Family Nelumbonaceae}

Nelumbo lutea (Willd.) Pers. CAM, CHIS, COL, JAL, NAY, QROO, SLP, TAB, TAMS, VER

\section{Family Nitrariaceae}

Peganum mexicanum A. Gray AGS, CHIH, COAH, DGO, NLE, SLP, TAMS, ZAC

Family Nyctaginaceae

Abronia angustifolia Greene CHIH, COAH

*Abronia carterae R.S. Ferris BCS

Abronia fragrans Nutt. ex Hook. CHIH, COAH

Abronia gracilis Benth. BCN, BCS

Abronia maritima Nutt. ex S. Watson BCN, BCS, COL, JAL, NAY, SIN, SON 
Abronia umbellata Lam. BCN, BCS

Abronia villosa $\mathrm{S}$. Watson $\mathrm{BCN}, \mathrm{BCS}, \mathrm{SON}$

Acleisanthes acutifolia Standl. CHIH, COAH, NLE, SLP, ZAC Acleisanthes angustifolia (Torr.) R.A. Levin CHIH, COAH, DGO

Acleisanthes anisophylla A. Gray COAH, NLE

Acleisanthes crassifolia A. Gray COAH, NLE, QRO, TAMS

Acleisanthes chenopodioides (A. Gray) R.A. Levin CHIH, $\mathrm{COAH}, \mathrm{SON}$

Acleisanthes lanceolatus (Wooton) R.A. Levin CHIH

Acleisanthes longiflora A. Gray CHIH, COAH, DGO, NLE, SLP, SON

*Acleisanthes nana I.M. Johnst. COAH, GTO, JAL, NLE, SLP, ZAC

Acleisanthes obtusa (Choisy) Standl. AGS, COAH, GTO, HGO, JAL, NLE, QRO, SLP, TAMS

*Acleisanthes palmeri (Hemsl.) R.A. Levin COAH, NLE

*Acleisanthes purpusiana (Heimerl) R.A. Levin CHIH, COAH, DGO, NLE

*Acleisanthes undulata (B.A. Fowler \& B.L. Turner) R.A. Levin COAH, NLE

Allionia choisyi Standl. AGS, CHIH, COAH, CDMX, DGO, GTO, GRO, HGO, JAL, MEX, NLE, OAX, PUE, QRO, SLP, SON, TAMS, TLAX, VER, ZAC

Allionia incarnata L. AGS, BCN, BCS, CHIH, COAH, COL, CDMX, DGO, GTO, GRO, HGO, JAL, MEX, MICH, MOR, NLE, OAX, PUE, QRO, SLP, SIN, SON, TAMS, TLAX, VER, ZAC

Anulocaulis eriosolenus (A. Gray) Standl. CHIH, COAH, DGO, NLE

*Anulocaulis hintoniorum B.L. Turner COAH

Anulocaulis leiosolenus (Torr.) Standl. CHIH, COAH

Anulocaulis reflexus I.M. Johnst. CHIH

*Boerhavia alata S. Watson SON

Boerhavia anisophylla Torr. AGS, CHIH, COAH, DGO, JAL, MICH, NLE, OAX, SLP, SIN, ZAC

* Boerhavia ciliata Brandegee SLP, TAMS

Boerhavia coccinea Mill. AGS, BCN, BCS, CAM, CHIS, CHIH, COAH, COL, CDMX, DGO, GTO, GRO, HGO, JAL, MEX, MICH, MOR, NAY, NLE, OAX, PUE, QRO, QROO, SLP, SIN, SON, TAB, TAMS, TLAX, VER, YUC, ZAC

Boerhavia coulteri (Hook. f.) S. Watson BCN, BCS, CHIH, NAY, SIN, SON

Boerhavia erecta L. AGS, BCN, BCS, CAM, CHIS, CHIH, COAH, COL, CDMX, DGO, GTO, GRO, HGO, JAL, MEX, MICH, MOR, NAY, NLE, OAX, PUE, QRO, QROO, SLP, SIN, SON, TAB, TAMS, VER, YUC, ZAC

Boerhavia gracillima Heimerl AGS, BCN, BCS, CHIH, COAH, DGO, GTO, GRO, HGO, MEX, MOR, NLE, OAX, PUE, QRO, SLP, SIN, SON, TAMS

Boerhavia intermedia M.E. Jones BCN, BCS, CHIH, COAH, DGO, JAL, MOR, SLP, SIN, SON, TAMS, ZAC

Boerhavia linearifolia A. Gray CHIH, COAH, NLE, VER

* Boerhavia maculata Standl. BCS, SON

Boerhavia pterocarpa S. Watson SON

Boerhavia purpurascens A. Gray CHIH, SON

*Boerhavia repens L. SIN
Boerhavia spicata Choisy BCN, BCS, CHIH, COAH, DGO, NLE, SIN, SON

Boerhavia torreyana (S. Watson) Standl. CHIH, COAH, SON

*Boerhavia traubae Spellenb. SON

Boerhavia triquetra S. Watson BCN, BCS, CHIH, SON

Boerhavia wrightii A. Gray BCN, CHIH, COAH, DGO, SON

*Boerhavia xanti S. Watson BCN, BCS, SIN, SON

*Commicarpus brandegeei Standl. BCN, BCS

*Commicarpus coctoris N.A. Harriman GRO, OAX

*Commicarpus praetermissus N.A. Harriman MICH

Commicarpus scandens (L.) Standl. BCN, BCS, CAM, CHIS, CHIH, COAH, COL, DGO, GTO, GRO, HGO, JAL, MEX, MICH, MOR, NAY, OAX, PUE, QRO, QROO, SLP, SIN, SON, TAMS, VER, YUC, ZAC

Cryptocarpus pyriformis Kunth OAX

Cyphomeris crassifolia (Standl.) Standl. COAH, HGO, NLE, TAMS, OAX, PUE, QRO, SLP, VER

Cyphomeris gypsophiloides (M. Martens \& Galeotti) Standl. AGS, CHIH, COAH, DGO, HGO, NLE, OAX, PUE, QRO, SLP, SIN, TAMS, VER, ZAC

Grajalesia fasciculata (Standl.) Miranda CHIS, OAX

Guapira costaricana (Standl.) Woodson CAM, CHIS, QROO, YUC

Guapira linearibracteata (Heimerl) Lundell CAM, CHIS, GRO, OAX, SLP, QROO, SIN, TAB, YUC

Guapira petenensis (Lundell) Lundell CHIS, COL, GRO, JAL, MEX, MICH, MOR, OAX, PUE, QROO, VER

Mirabilis aggregata (Ortega) Cav. AGS, CHIH, COAH, CDMX, DGO, GTO, HGO, MEX, OAX, QRO, VER, ZAC

Mirabilis albida (Walter) Heimerl AGS, BCS, CHIS, CHIH, COAH, CDMX, DGO, GTO, HGO, JAL, MEX, MOR, NLE, OAX, PUE, QRO, SLP, SON, TAMS, VER, ZAC

Mirabilis californica A. Gray BCN, BCS

Mirabilis coccinea (Torr.) Benth. \& Hook. f. CHIH, SON

* Mirabilis donahooiana Le Duc MICH

* Mirabilis exserta Brandegee BCS

Mirabilis glabra (S. Watson) Standl. CHIH, COAH

Mirabilis glabrifolia (Ortega) I.M. Johnst. AGS, CHIH, COAH, DGO, GTO, HGO, JAL, MEX, MICH, NLE, OAX, PUE, QRO, SLP, SON, TAMS, VER, ZAC

*Mirabilis gracilis (Standl.) Le Duc CHIH, DGO, MEX, MICH, NAY

*Mirabilis grandiflora (Standl.) Standl. COL, JAL, OAX

* Mirabilis hintoniorum Le Duc MICH

Mirabilis jalapa L. AGS, BCN, BCS, CAM, CHIS, CHIH, COAH, COL, CDMX, DGO, GTO, GRO, HGO, JAL, MEX, MICH, MOR, NAY, NLE, OAX, PUE, QRO, QROO, SLP, SIN, SON, TAB, TAMS, TLAX, VER, YUC, ZAC

Mirabilis laevis (Benth.) Curran BCN, BCS, OAX, SIN, SON

Mirabilis linearis (Pursh) Heimerl AGS, CHIH, COAH, DGO, NLE, SLP, SON, ZAC

Mirabilis longiflora L. AGS, CHIH, COAH, COL, CDMX, DGO, GTO, GRO, HGO, JAL, MEX, MICH, MOR, NAY, NLE, OAX, PUE, QRO, SLP, SIN, SON, TAMS, TLAX, VER, ZAC

Mirabilis melanotricha (Standl.) Spellenb. CHIH, COAH, DGO, GTO, HGO, MEX, PUE, QRO, SLP, SON, TLAX, VER 
Mirabilis multiflora (Torr.) A. Gray BCN, BCS, CHIH, COAH, GTO, NLE, SLP, SON, ZAC

*Mirabilis nesomii B.L. Turner COAH, NLE, TAMS

Mirabilis nyctaginea (Michx.) Weath. AGS, COL, GTO, HGO, JAL, OAX, PUE, QRO, SON, VER, ZAC

* Mirabilis oaxacae Heimerl CHIS, OAX

*Mirabilis oligantha (Standl.) J.F. Macbr. BCN

Mirabilis oxybaphoides (A. Gray) A. Gray CHIH, COAH, DGO, NLE, SLP, SON

*Mirabilis polonii Le Duc CHIH, COAH, COL, JAL, NAY, NLE, OAX, SLP, SIN, TAMS

*Mirabilis pringlei Weath. GRO, GTO, JAL, MEX, MICH, NAY, PUE

Mirabilis pulchella Standl. \& Steyerm. CHIS, OAX

*Mirabilis sanguinea Heimerl. CHIS, COL, GRO, JAL, MEX,

MICH, MOR, NLE, OAX, PUE, TAMS

*Mirabilis suffruticosa (Standl.) Standl. OAX

Mirabilis tenuiloba S. Watson BCN, BCS, SON

Mirabilis texensis (J.M. Coult.) B.L. Turner CHIH, COAH, DGO, SLP, VER

*Mirabilis triflora Benth. BCS, JAL, NLE, ZAC

* Mirabilis urbani Heimerl GRO, MICH

Mirabilis violacea (L.) Heimerl CHIS, COAH, CDMX, GTO, GRO, HGO, JAL, MEX, MICH, NLE, OAX, PUE, QRO, SLP, VER, YUC

Mirabilis viscosa Cav. AGS, CHIS, CHIH, COAH, COL, DGO, GTO, GRO, HGO, JAL, MEX, MICH, MOR, NLE, OAX, PUE, QRO, SLP, SON, TAMS, TLAX, VER, ZAC

Neea amplifolia Donn. Sm. CAM, CHIS, QROO, TAB

*Neea chiapensis Standl. CHIS

Neea choriophylla Standl. CAM, CHIS, QROO, YUC

*Neea fagifolia Heimerl CAM, CHIS, QROO, YUC

Neea psychotrioides Donn. Sm. CAM, CHIS, COL, GRO, HGO,

JAL, MICH, OAX, PUE, QRO, QROO, SLP, SIN, TAB, TAMS,

VER, YUC

Neea stenophylla Standl. CAM, CHIS, QROO

Neea tenuis Standl. CAM, CHIS, OAX, VER, YUC

*Neea turbinata Lundell CHIS

Nyctaginia capitata Choisy CHIH, COAH, DGO, NLE, SLP, TAMS, ZAC

Okenia hypogaea Schltdl. \& Cham. CAM, CHIS, COL, GRO, HGO, JAL, MEX, MICH, MOR, NAY, OAX, QRO, QROO, SLP, SIN, SON, TAB, TAMS, VER, YUC, ZAC

Pisonia aculeata L. CAM, CHIS, CHIH, COL, DGO, GRO, HGO, JAL, MEX, MICH, MOR, NAY, OAX, PUE, QRO, QROO, SLP, SIN, SON, TAB, TAMS, VER, YUC, ZAC

*Pisonia calafia León de la Luz \& Levin BCS

* Pisonia capitata (S. Watson) Standl. BCS, CHIH, COL, DGO, GRO, JAL, MICH, NAY, SIN, SON, VER

Pisonia donnell-smithii Heimerl ex Standl. CHIS, MICH, OAX

* Pisonia flavescens Standl. BCS, SIN

Pisoniella arborescens (Lag. \& Rodr.) Standl. COL, CDMX, GTO, HGO, JAL, MEX, MICH, OAX, PUE, QRO, SLP, VER

* Salpianthus aequalis Standl. GRO, MICH

* Salpianthus arenarius Bonpl. CHIS, COL, GRO, JAL, MEX, MICH, MOR, OAX, SON

*Salpianthus macrodontus Standl. BCS, COL, GRO, JAL,
MOR, OAX, SIN, SON

Salpianthus purpurascens (Cav. ex Lag.) Hook. \& Arn. CHIS, CHIH, COL, DGO, GRO, HGO, JAL, MEX, MICH, MOR, NAY, OAX, PUE, QRO, SIN, SON, VER

Tripterocalyx carneus (Greene) L.A. Galloway CHIH

\section{Family Nymphaeaceae}

Nuphar advena (Aiton) W.T. Aiton COAH, SLP, TAMS, VER

Nymphaea amazonum Mart. \& Zucc. CAM, CHIS, GRO, OAX, QROO, TAB, TAMS, VER, YUC

Nymphaea ampla (Salisb.) DC. CAM, CHIS, COAH, COL, GRO, HGO, JAL, MICH, NAY, NLE, OAX, PUE, QRO, QROO, SLP, SON, TAB, TAMS, VER, YUC, ZAC

Nymphaea conardii Wiersema CAM, CHIS, OAX, QROO, TAB, VER

Nymphaea elegans Hook. BCS, COL, GRO, HGO, JAL, MOR, NAY, OAX, PUE, QROO, SIN, SON, TAMS, VER

*Nymphaea gracilis Zucc. AGS, CDMX, DGO, GTO, JAL, MEX, MICH, OAX

Nymphaea jamesoniana Planch. CAM, CHIS, COL, GRO, JAL, NAY, OAX, QROO, SLP, TAB, TAMS, VER, YUC

Nymphaea mexicana Zucc. CDMX, HGO, MEX, MICH, VER Nymphaea novogranatensis Wiersema OAX

Nymphaea odorata Aiton CDMX, DGO, HGO, MEX, MICH, VER

Nymphaea prolifera Wiersema TAB, VER

Nymphaea pulchella DC. CAM, GRO, JAL, MICH, MOR, NAY, OAX, PUE, TAB, YUC

\section{Family Ochnaceae}

Ouratea acuminata (DC.) Engl. GRO, OAX

Ouratea curvata (A. St.-Hil.) Engl. ex Dwyer CHIS, VER

*Ouratea jaliscensis McVaugh JAL

* Ouratea jurgensenii (Planch.) Engl. CHIS, OAX

Ouratea lucens (Kunth) Engl. CAM, CHIS, GRO, NAY, OAX, QROO, TAB, VER, YUC

* Ouratea madrensis L. Riley COL, GRO, JAL, MICH, NAY

Ouratea mexicana (Bonpl.) Engl. CHIS, COL, GRO, JAL, MICH, NAY, OAX, SIN

Ouratea nitida (Sw.) Engl. CAM, CHIS, GRO, OAX, QROO, TAB, TAMS, VER

*Ouratea pyramidalis L. Riley CHIS, TAB, VER

Ouratea theophrasta (Planch.) Baill. CHIS, TAB, VER

Sauvagesia erecta L. CHIS, OAX, PUE, TAB, VER

Sauvagesia pulchella Planch. ex Seem. CHIS

Sauvagesia tenella Lam. GRO

\section{Family Olacaceae}

Heisteria acuminata (Bonpl.) Engl. CHIS

Heisteria macrophylla Oerst. CHIS

Heisteria media S.F. Blake CHIS, VER

\section{Family Oleaceae}

Cartrema americana (L.) Raf. CHIS, CHIH, COAH, COL, DGO, GTO, GRO, HGO, JAL, MEX, NLE, OAX, PUE, QRO, SLP, SON, TAMS, VER

Chionanthus ligustrinus (Sw.) Pers. CHIS, VER

Chionanthus oblanceolatus (B.L. Rob.) P.S. Green CHIS, MICH, OAX, TAB, VER 
Chionanthus panamensis (Standl.) Stearn CHIS, VER

Forestiera angustifolia Torr. CHIH, COAH, DGO, GTO, HGO, NLE, OAX, PUE, QRO, SLP, SON, TAMS, VER, ZAC

Forestiera cartaginensis Donn. Sm. CHIS

*Forestiera durangensis Standl. DGO, GTO, HGO, OAX, PUE, QRO, SIN, TAMS, ZAC

*Forestiera macrocarpa Brandegee BCS

Forestiera neomexicana A. Gray AGS, BCN, BCS, CHIH, COAH, DGO, ZAC

Forestiera phillyreoides (Benth.) Torr. AGS, BCS, CHIH, COAH, COL, DGO, GTO, GRO, HGO, JAL, MEX, MICH, MOR, NLE, OAX, PUE, QRO, SLP, SON, TLAX, VER, ZAC Forestiera pubescens Nutt. BCS, CHIH, COAH, SON

Forestiera reticulata Torr. CHIS, COAH, COL, GTO, GRO, HGO, JAL, MEX, NLE, OAX, PUE, QRO, SLP, TAMS, VER Forestiera rhamnifolia Griseb. CHIS, COL, JAL, NAY, OAX, QROO, SIN, SON, TAB, VER

* Forestiera rotundifolia (Brandegee) Standl. OAX, PUE, VER

* Forestiera tomentosa S. Watson AGS, COL, GTO, GRO, HGO,

JAL, MEX, MICH, OAX, PUE, QRO, SLP, ZAC

Fraxinus americana $\mathrm{L}$. AGS, CHIH, COAH, DGO, NLE

Fraxinus anomala Torr. ex S. Watson NLE, SLP

Fraxinus cuspidata Torr. CHIH, COAH, DGO, NLE, SLP, TAMS

Fraxinus dipetala Hook. \& Arn. BCN, BCS

*Fraxinus dubia (Willd. ex Schult. \& Schult. f.) P.S. Green \& M. Nee CHIS, OAX, QRO, SLP, TAMS, VER

Fraxinus gooddingii Little $\mathrm{CHIH}, \mathrm{SON}$

Fraxinus greggii A. Gray CHIH, COAH, DGO, HGO, JAL, NLE, QRO, SLP, SON, TAMS, VER, ZAC

Fraxinus papillosa Lingelsh. AGS, CHIH, HGO, JAL, SON, ZAC

Fraxinus pennsylvanica Marsh CHIH, COAH, DGO, NLE, SLP, SON, TAMS, VER

*Fraxinus pringlei Lingelsh. CHIH, GRO, HGO, MICH, OAX, SON, VER

Fraxinus purpusii Brandegee CHIS, GRO, JAL, MEX, MICH, OAX, PUE, SLP, TAMS

*Fraxinus rufescens Lingelsh. DGO, GTO, HGO, QRO, SLP, VER

Fraxinus uhdei (Wenz.) Lingelsh. AGS, CHIS, COAH, COL, CDMX, DGO, GTO, GRO, HGO, JAL, MEX, MICH, MOR, NAY, NLE, OAX, PUE, QRO, SLP, SIN, TLAX, VER, ZAC

Fraxinus velutina Torr. AGS, BCN, BCS, CHIS, CHIH, COAH, DGO, NLE, PUE, SLP, SON, TAMS, VER, ZAC

*Hesperelaea palmeri A. Gray BCN

* Menodora coulteri A. Gray AGS, COAH, DGO, GTO, HGO, NLE, PUE, QRO, SLP, TAMS, VER, ZAC

*Menodora chumleyi B.L. Turner COAH

*Menodora geohintonii B.L. Turner COAH

*Menodora gypsophila B.L. Turner COAH, NLE

*Menodora helianthemoides Bonpl. AGS, COAH, CDMX, GTO, HGO, MEX, MICH, NLE, OAX, PUE, QRO, SLP, VER, ZAC

Menodora heterophylla Moric. ex A. DC COAH, NLE, TAMS

*Menodora hintoniorum B.L. Turner COAH, NLE, TAMS

*Menodora intricata Brandegee PUE, SLP, TAMS
*Menodora jaliscana B.L. Turner COL, JAL, MICH

Menodora longiflora Engelm. ex A. Gray CHIH, COAH, DGO, NLE

*Menodora magniflora (Steyerm.) B.L. Turner COAH, NLE, SLP

*Menodora mexicana (A. DC.) A. Gray OAX

*Menodora muellerae Rehder COAH, NLE, TAMS

*Menodora potosiensis Henr. ex B.L. Turner GTO, SLP

Menodora scabra A. Gray AGS, BCN, CHIH, COAH, DGO, GTO, NLE, SLP, SON

Menodora spinescens A. Gray BCN

* Menodora tehuacana B.L. Turner PUE

* Menodora yecorana T. Van Devender \& B.L. Turner CHIH, SON

\section{Family Onagraceae}

Calylophus drummondianus Spach COAH, NLE, TAMS

Calylophus hartwegii (Benth.) P.H. Raven AGS, CHIH, COAH, DGO, GTO, HGO, JAL, NLE, QRO, SLP, SON, TAMS, ZAC

Calylophus lavandulifolius (Torr. \& A. Gray) P.H. Raven CHIH, COAH, NLE, SLP

*Calylophus toumeyi (Small) Towner CHIH, SON

Calylophus tubicula (A. Gray) P.H. Raven CHIH, COAH, DGO, NLE, ZAC

Camissonia arenaria (A. Nelson) P.H. Raven SON

Camissonia boothii (Douglas) P.H. Raven BCN, SON

Camissonia cardiophylla (Torr.) P.H. Raven BCN, BCS, SON

Camissonia claviformis (Torr. \& Frem.) P.H. Raven BCN, SON Camissonia chamaenerioides (A. Gray) P.H. Raven BCN, BCS, CHIH, COAH, SON

Camissonia graciliflora (Hook. \& Arn.) P.H. Raven BCN

Camissonia strigulosa (Fisch. \& C.A. Mey.) P.H. Raven BCN

Camissoniopsis bistorta (Nutt. ex Torr. \& A. Gray) W.L. Wagner \& Hoch BCN

Camissoniopsis cheiranthifolia (Hornem. ex Spreng.) W.L. Wagner \& Hoch BCN

Camissoniopsis guadalupensis (S. Watson) W.L. Wagner \& Hoch BCN

Camissoniopsis hirtella (Greene) W.L. Wagner \& Hoch BCN

Camissoniopsis ignota (Jeps.) W.L. Wagner \& Hoch BCN

Camissoniopsis intermedia (P.H. Raven) W.L. Wagner \& Hoch $\mathrm{BCN}$

Camissoniopsis lewisii (P.H. Raven) W.L. Wagner \& Hoch BCN Camissoniopsis micrantha (Hornem. ex Spreng.) W.L. Wagner \& Hoch BCN

Camissoniopsis pallida (Abrams) W.L. Wagner \& Hoch BCN

*Camissoniopsis proavita (P.H. Raven) W.L. Wagner \& Hoch $\mathrm{BCN}$

Camissoniopsis robusta (P.H. Raven) W.L. Wagner \& Hoch $\mathrm{BCN}$

Clarkia delicata (Abrams) A. Nelson \& J. Macbr. BCN

Clarkia epilobioides (Nutt.) A. Nelson \& J. Macbr. BCN

Clarkia purpurea (Curtis) A. Nelson \& J. Macbr. BCN, CHIH, COAH, DGO

Clarkia rhomboidea Dougl. ex Hook. BCN

Clarkia unguiculata Lindl. BCN

*Diplandra lopezioides Hook. \& Arn. NAY 
Epilobium canum (Greene) P.H. Raven BCN, SON

Epilobium ciliatum Raf. BCN, BCS, CHIS, CHIH, COAH, CDMX, DGO, GTO, HGO, JAL, MEX, MICH, MOR, NLE, OAX, PUE, QRO, SIN, SON, TLAX, VER

*Epilobium denticulatum Ruiz \& Pav. COL, CDMX, HGO, JAL, MEX, QRO, VER

Epilobium densiflorum (Lindl.) P. Hoch \& P.H. Raven BCN

Epilobium foliosum (Torr. \& A. Gray) Suksd. BCN

*Epilobium glaberrimum Barbey BCN, BCS

Epilobium halleanum Haussk. CHIH, SON

*Epilobium maysillesii Munz CHIH, DGO, NAY, SIN

Epilobium pygmaeum (Speg.) P. Hoch \& P.H. Raven BCN

*Eulobus angelorum (S. Watson) W.L. Wagner \& Hoch BCN, BCS

Eulobus californicus Nutt. ex Torr. \& A. Gray BCN, BCS, SON *Eulobus crassifolius (Greene) W.L. Wagner \& Hoch BCN, BCS

*Eulobus sceptrostigma (Brandegee) W.L. Wagner \& Hoch BCN, BCS

Fuchsia arborescens Sims CHIS, COL, DGO, GTO, GRO, HGO, JAL, MEX, MICH, MOR, OAX, PUE, SIN, VER

Fuchsia bacillaris Lindl. CHIS, JAL, MICH, OAX

*Fuchsia cylindracea Lindl. COL, GRO, JAL, MEX, MICH, MOR, OAX

*Fuchsia decidua Standl. COL, GRO, JAL, MEX, MOR, OAX Fuchsia encliandra Steud. CHIS, COL, CDMX, GTO, GRO, HGO, JAL, MEX, MICH, MOR, NAY, OAX, PUE, QRO, SLP, VER

* Fuchsia exoniensis J. Paxton VER

*Fuchsia fulgens DC. COL, GRO, JAL, MEX, MICH, MOR

Fuchsia lycioides Andrews CHIS, COAH, DGO, GRO, HGO, JAL, MEX, MICH, MOR, NAY, OAX, PUE, QRO, SLP

Fuchsia magellanica Lam. OAX, VER

Fuchsia microphylla Kunth CHIS, COL, CDMX, GTO, GRO, HGO, JAL, MEX, MICH, MOR, OAX, PUE, QRO, SLP, TLAX, VER

Fuchsia minutiflora Hemsl. CHIS, MEX, MICH, VER

*Fuchsia obconica Breedlove COL, GTO, JAL, MEX, MICH

Fuchsia paniculata Lindl. CHIS, OAX, PUE, VER

* Fuchsia ravenii Breedlove OAX

Fuchsia skutchiana Munz CHIS

Fuchsia splendens Zucc. CHIS, GRO, OAX

Fuchsia tetradactyla Lindl. CHIS, GRO, OAX

Fuchsia thymifolia Kunth CHIS, COL, CDMX, DGO, GTO, GRO, HGO, JAL, MEX, MICH, MOR, NAY, OAX, PUE, QRO, SIN, TLAX, VER, ZAC

Gaura boquillensis P.H. Raven \& Gregory CHIH, COAH, NLE Gaura calcicola P.H. Raven \& Gregory COAH, DGO, NLE, QRO

Gaura drummondii (Spach.) Torr. \& A. Gray BCN, BCS, COAH, DGO, GTO, HGO, MEX, MICH, NLE, OAX, QRO, SLP, TAMS, ZAC

* Gaura grandiflora Rose CHIH, DGO

Gaura mckelveyae (Munz) P.H. Raven \& D.P. Greg. COAH, NLE

* Gaura mollis Kunth PUE

*Gaura mutabilis Cav. CHIH, CDMX, DGO, GTO, HGO, JAL,
MEX, MICH, MOR, OAX, PUE, QRO, SON, TLAX, VER, ZAC

Gaura sinuata Nutt. ex Ser. BCN, TAMS

Gaura suffulta A. Gray CHIH, COAH

Gayophytum diffusum Torr. \& A. Gray BCN

*Gongylocarpus fruticulosus (Benth.) Brandegee BCS

Gongylocarpus rubricaulis Schltdl. \& Cham. CHIS, CHIH,

COL, DGO, GTO, JAL, MEX, MICH, MOR, NAY, OAX, SIN, SON, VER, ZAC

Hauya elegans DC. CHIS, COL, GTO, GRO, HGO, JAL, MEX, MICH, MOR, OAX, PUE, QRO, SLP, VER

Hauya heydeana Donn. Sm. CHIS

*Hauya rusbyi Donn. Sm. \& Rose GRO, MICH, OAX

*Lopezia ciliatula Plitmann, P.H. Raven \& Breedlove DGO, SIN

* Lopezia clavata Brandegee BCS

*Lopezia concinna P.H. Raven DGO, SIN

*Lopezia conjugens Brandegee SIN

*Lopezia cornuta S. Watson CHIH, DGO, SON

*Lopezia galeottii Planch. OAX

*Lopezia gentryi (Munz) Plitmann, P.H. Raven \& Breedlove DGO, SIN

*Lopezia gracilis S. Watson CHIH, DGO, SON

Lopezia grandiflora Zucc. BCN, CHIS, JAL, OAX

*Lopezia hintonii R.C. Foster GRO

*Lopezia integrifolia DC. CHIS

*Lopezia laciniata (Rose) M.E. Jones DGO, JAL, NAY, SIN, ZAC

*Lopezia langmaniae Miranda CHIS

*Lopezia lopezioides (Hook. \& Arn.) Plitmann, P.H. Raven \& Breedlove JAL, NAY

Lopezia miniata Lag. ex DC. AGS, COL, CDMX, DGO, GTO, GRO, HGO, JAL, MEX, MICH, MOR, NAY, OAX, QRO, SLP, SIN, TLAX, VER, ZAC

*Lopezia nuevo-leonis Plitman \& Breedlove COAH, HGO, NLE, QRO, SLP

*Lopezia oaxacana Rose OAX

*Lopezia ovata (Plitmann, P.H. Raven \& Breedlove) Plitmann, P.H. Raven \& Breedlove DGO, SIN

Lopezia racemosa Cav. CHIS, COAH, COL, CDMX, DGO, GTO, GRO, HGO, JAL, MEX, MICH, MOR, NAY, NLE, OAX, PUE, QRO, SLP, SIN, TAMS, TLAX, VER, ZAC

* Lopezia riesenbachia Plitmann, P.H. Raven \& Breedlove COL, DGO, GRO, JAL, NAY, OAX

*Lopezia semeiandra Plitmann, P.H. Raven \& Breedlove COL, DGO, JAL, MICH, NAY, SIN

*Lopezia sinaloensis Munz SIN

*Lopezia smithii Rose OAX

*Lopezia suffrutescens Munz DGO, SIN

*Lopezia trichota Schltdl. AGS, CHIH, COAH, CDMX, DGO, GTO, HGO, JAL, MEX, MICH, OAX, PUE, QRO, SLP, VER

*Lopezia violacea Rose MEX, MICH, MOR

*Ludwigia adscendens (L.) H. Hara HGO, JAL, MICH, VER

*Ludwigia bonariensis (Micheli) H. Hara CHIH, DGO, MEX, MICH, PUE, QRO, TAB, TAMS, VER

Ludwigia clavellina M. Gómez CHIS, CHIH, COAH, GRO, JAL, MEX, MICH, MOR, PUE, QRO, SLP, TAMS, VER, ZAC 
Ludwigia decurrens Walter CAM, CHIS, COL, JAL, MICH, NAY, TAB

Ludwigia erecta (L.) H. Hara CAM, CHIS, COL, GRO, JAL, MEX, MICH, NAY, OAX, QROO, SIN, TAB, VER, YUC

Ludwigia foliobracteolata (Munz) H. Hara CHIS, TAB

Ludwigia helminthorrhiza (C. Mart.) H. Hara CHIS, GRO, JAL, NAY, OAX, TAB, VER

Ludwigia hyssopifolia (G. Don) Exell CHIS, GRO, OAX, TAB, VER

Ludwigia inclinata (L. f.) M. Gómez CHIS, GRO, OAX, TAB, VER

Ludwigia leptocarpa (Nutt.) H. Hara CAM, CHIS, COL, GRO, JAL, MOR, NAY, OAX, PUE, QROO, SLP, SIN, TAB, TAMS,

VER, YUC

Ludwigia linifolia Poir. TAB

Ludwigia nervosa (Poir.) H. Hara CHIS, TAB

Ludwigia octovalvis (Jacq.) P.H. Raven AGS, BCN, BCS, CAM, CHIS, CHIH, COAH, COL, DGO, GTO, GRO, HGO, JAL, MEX, MICH, MOR, NAY, NLE, OAX, PUE, QRO, QROO, SLP, SIN, SON, TAB, TAMS, VER, YUC, ZAC

Ludwigia palustris (L.) Elliot CHIS, CHIH, CDMX, DGO, GRO, HGO, JAL, MEX, MICH, MOR, NLE, QRO, QROO, SLP, SIN, SON, TAB, TAMS, VER

Ludwigia peploides (Kunth) P.H. Raven AGS, BCN, BCS, CAM, CHIS, CHIH, COAH, COL, CDMX, DGO, GTO, GRO, HGO, JAL, MEX, MICH, MOR, NAY, OAX, PUE, QRO, SLP, SIN, SON, TAB, TAMS, TLAX, VER, ZAC

Ludwigia peruviana (L.) H. Hara CHIS, MICH, OAX, QROO, TAB, VER

Ludwigia rigida (Miq.) Sandwith CHIS, TAB

Ludwigia sedoides (Humb. \& Bonpl.) H. Hara CAM, CHIS,

TAB, VER

Ludwigia suffruticosa Walter CHIS, OAX, VER

Ludwigia torulosa (Arn.) H. Hara OAX, TAB, VER

* Megacorax gracielanus S. González \& W.L. Wagner DGO

Oenothera albicaulis Nutt. CHIH, SON

Oenothera arizonica (Munz) W.L. Wagner BCN, SON

* Oenothera biennis L. CHIH, PUE

Oenothera brachycarpa A. Gray CHIH, COAH, DGO, SON

* Oenothera brandegeei P.H. Raven BCN

* Oenothera breedlovei W. Dietrich \& W.L. Wagner BCS

Oenothera caespitosa Nutt. CHIH, SON

Oenothera cardiophylla Torr. BCN, BCS

Oenothera cespitosa Nutt. BCN, BCS, SON

Oenothera curtiflora W.L. Wagner \& Hoch BCN, BCS, CHIH, COAH, DGO, NLE, SIN, SON, TAMS, ZAC

Oenothera decorticans (Hook. \& Arn.) Greene BCN, CHIH, SON

Oenothera deltoides Torr. \& A. Gray BCN, SON

*Oenothera deserticola (Loes.) Munz COAH, COL, CDMX, DGO, GTO, HGO, JAL, MEX, MICH, MOR, OAX, PUE, QRO, SLP, SIN, TLAX, VER, ZAC

*Oenothera dissecta A. Gray ex S. Watson COAH, DGO, NLE, SLP, ZAC

Oenothera drummondii Hook. BCS, HGO, QRO, SLP, SON, TAB, TAMS, VER

Oenothera elata Kunth AGS, BCN, CHIH, CDMX, DGO, GRO,
JAL, MEX, MICH, PUE, SIN, SON, TLAX, VER, ZAC

Oenothera epilobiifolia Kunth CHIS, CDMX, GTO, HGO, MEX, MICH, OAX, PUE, VER

Oenotheraflava (A. Nelson) Garrett BCS, CHIH, CDMX, DGO, GTO, HGO, JAL, MEX, SLP, SON, VER

Oenothera grandis (Britton) Smyth DGO, MICH, TAMS

*Oenothera greggii A. Gray CHIH, SON

Oenothera havardii S. Watson CHIH, DGO, SON

Oenothera hexandra (Ortega) W.L. Wagner \& Hoch AGS, CHIS, CHIH, COL, CDMX, DGO, GTO, GRO, HGO, JAL, MEX, MICH, OAX, PUE, QRO, SLP, SON, TLAX, VER, ZAC Oenothera hookeri Torr. \& A. Gray BCN, CHIH, SON

Oenothera jamesii Torr. \& A. Gray COAH, NLE

Oenothera kunthiana (Spach) Munz AGS, CHIS, CHIH, COAH, COL, CDMX, DGO, GTO, GRO, HGO, JAL, MEX, MICH, MOR, NLE, OAX, QRO, SLP, SIN, SON, TAMS, VER, ZAC Oenothera laciniata Hill AGS, BCS, CHIH, COAH, GRO, HGO, JAL, MEX, MICH, MOR, NLE, OAX, PUE, QRO, SLP, SIN, SON, TAMS, VER, ZAC

* Oenothera luciae-julianae W.L. Wagner CHIH, DGO, GTO, JAL, MICH, NAY, QRO, SIN

Oenothera macrocarpa Nutt. COAH

*Oenothera macrosceles A. Gray CHIH, COAH, DGO, NLE, SLP, ZAC

*Oenothera maysillesii Munz DGO, SIN

Oenothera micrantha Hornem. ex Spreng. BCN

*Oenothera muelleri Munz NLE, TAMS

Oenothera multicaulis Ruiz \& Pav. CHIS

Oenothera pallida Lindl. CHIH, SON

*Oenothera pennellii Munz NLE, TAMS

Oenothera primiveris A. Gray BCN, BCS, CHIH, COAH, NLE, SON

Oenothera pubescens Willd. ex Spreng. AGS, BCS, CHIS, CHIH, COAH, COL, CDMX, DGO, GTO, HGO, JAL, MEX, MICH, MOR, NAY, NLE, OAX, PUE, QRO, SLP, SIN, TLAX, VER, ZAC

Oenothera refracta S. Watson BCN

Oenothera rosea L'Hér. ex Aiton AGS, BCS, CHIS, CHIH, COAH, COL, CDMX, DGO, GTO, GRO, HGO, JAL, MEX, MICH, MOR, NAY, NLE, OAX, PUE, QRO, SLP, SIN, SON, TAMS, TLAX, VER, ZAC

Oenothera speciosa Nutt. BCS, CHIH, COAH, DGO, MOR, NLE, SLP, SON, TAMS, ZAC

*Oenothera stubbei W. Dietrich, P.H. Raven \& W.L. Wagner NLE, TAMS

Oenothera suffrutescens (Ser.) W.L. Wagner \& Hoch AGS, CHIS, CHIH, COAH, CDMX, DGO, GTO, GRO, HGO, JAL, MEX, MICH, NLE, OAX, PUE, QRO, SLP, SIN, SON, TAMS, TLAX, VER, ZAC

* Oenothera tamrae W. Dietr. \& W.L. Wagner DGO, NAY Oenothera tetraptera Cav. BCS, CHIH, COAH, CDMX, DGO, GTO, HGO, MEX, MICH, MOR, NLE, OAX, PUE, QRO, SLP, SON, TAMS, TLAX, VER, ZAC

Oenothera texensis P.H. Raven \& D.R. Parnell CHIH, COAH, DGO, NLE, TAMS

Oenothera triloba Nutt. BCN, CHIH, COAH, NLE, TAMS

* Oenothera tubifera Seringe CDMX, HGO, GTO, MEX, 
MICH, MOR

*Oenothera wigginsii W. Klein BCN

*Xylonagra arborea (Kellogg) Donn. Sm. \& Rose BCN, BCS

\section{Family Opiliaceae}

*Agonandra obtusifolia Standl. CHIS, GTO, OAX, PUE, QRO, QROO, SLP, TAMS, VER, YUC

*Agonandra ovatifolia Miranda CAM, CHIS

Agonandra racemosa (DC.) Standl. CHIS, CHIH, COL, DGO, GTO, GRO, JAL, MEX, MICH, MOR, NAY, OAX, PUE, QRO, QROO, SLP, SIN, SON, TAMS, VER, YUC, ZAC

\section{Family Orchidaceae}

Acianthera angustifolia (Lindl.) Luer CHIS, OAX, QRO, SLP, TAMS, VER

Acianthera angustisepala (Ames \& Correll) Pridgeon \& M.W. Chase CHIS

Acianthera breedlovei Soto Arenas \& Salazar CHIS

Acianthera circumplexa (Lindl.) Pridgeon \& M.W. Chase CHIS, GRO, OAX, PUE, VER

*Acianthera chrysantha (Lindl.) Pridgeon \& M.W. Chase COL, GRO, JAL, MEX, MICH, MOR, NAY, OAX

*Acianthera eximia (L.O. Williams) R. Solano OAX

*Acianthera greenwoodii Soto Arenas OAX

*Acianthera hartwegiifolia (H. Wendl. \& Kraenzl.) R. Solano \& Soto Arenas GRO, OAX

Acianthera herrerae (Luer) Solano \& Soto Arenas CHIS

Acianthera hondurensis (Ames) Pridgeon \& M.W. Chase CHIS, VER

Acianthera johnsonii (Ames) Pridgeon \& M.W. Chase CHIS, OAX

*Acianthera majakoluckae Soto Arenas \& R. Solano CHIS, OAX

*Acianthera obscura (A. Rich. \& Galeotti) Pridgeon \& M.W. Chase CHIS, QRO, SLP, VER

*Acianthera pollardiana Solano OAX

Acianthera pubescens (Lindl.) Pridgeon \& M.W. Chase CHIS, TAB, VER

*Acianthera sotoana $\mathrm{R}$. Solano OAX, VER

Acianthera testifolia (Sw.) R. Solano VER

Acianthera tikalensis (Correll \& C. Schweinf.) Pridgeon \& M.W. Chase CAM, CHIS, QROO, VER

*Acianthera unguicallosa (Ames \& C. Schweinf.) R. Solano COL

Acianthera violacea (A. Rich. \& Galeotti) Pridgeon \& M.W. Chase CHIS, HGO, OAX, VER

*Acineta barkeri (Bateman) Lindl. CHIS, GRO, OAX, PUE, VER

*Acineta hagsateri Salazar \& Soto Arenas GRO, OAX

Acineta salazarii Soto Arenas CHIS, OAX

*Alamania punicea Lex. AGS, HGO, JAL, MEX, MICH, OAX, PUE, QRO, SLP, TLAX, VER, ZAC

*Amoana kienastii (Rchb. f.) Leopardi \& Carnevali OAX

*Amoana latipetala Leopardi \& Hágsater OAX

Anathallis abbreviata (Schltr.) Pridgeon \& M.W. Chase CHIS Anathallis barbulata (Lindl.) Pridgeon \& M.W. Chase CHIS, JAL, NAY, OAX, ZAC

Anathallis comayaguensis (Ames) Pridgeon \& M.W. Chase

\section{CHIS}

Anathallis dolichopus (Schltr.) Pridgeon \& M.W. Chase CHIS *Anathallis greenwoodii Soto Arenas \& Salazar OAX

*Anathallis involuta (L.O. Williams) R. Solano \& Soto Arenas GRO, JAL, MICH, NAY, OAX

Anathallis lewisiae (Ames) R. Solano \& Soto Arenas CHIS, OAX, VER

Anathallis minutalis (Lindl.) Pridgeon \& M.W. Chase CHIS, COL, GRO, HGO, JAL, MICH, OAX

*Anathallis oblanceolata (L.O. Williams) R. Solano \& Soto Arenas CHIS, OAX

Anathallis scariosa (Lex.) Pridgeon \& M.W. Chase CHIS, GRO, MEX, MICH, MOR, OAX

Anathallis sertularioides (Sw.) Pridgeon \& M.W. Chase CHIS, OAX, TAB, VER

Anathallis yucatanensis (Ames \& C. Schweinf.) R. Solano \& Soto Arenas CAM, CHIS, OAX, QROO

Arpophyllum alpinum Lindl. CHIS, VER

*Arpophyllum giganteum Hartw. ex Lindl. CHIS, OAX, VER

*Arpophyllum laxiflorum Pfitzer HGO, OAX, PUE, QRO, SLP, TAMS, VER

*Arpophyllum medium Rchb. f. CHIS, OAX, PUE, VER

*Arpophyllum spicatum Lex. CHIS, GRO, MEX, MICH, OAX, TAMS, VER

*Artorima erubescens (Lindl.) Dressler \& G.E. Pollard GRO, OAX

Aspidogyne stictophylla (Schltr.) Garay CHIS, OAX, VER Aulosepalum hemichreum (Lindl.) Garay CHIS, COL, GRO, JAL, MICH, OAX

*Aulosepalum nelsonii (Greenm.) Garay COL, GRO, JAL, $\mathrm{MICH}, \mathrm{OAX}$

*Aulosepalum oestlundii (Burns-Bal.) Catling GRO, OAX

Aulosepalum pyramidale (Lindl.) M.A. Dix \& M.W. Dix CHIS, COL, CDMX, DGO, GRO, HGO, JAL, MEX, MICH, MOR, OAX, QRO, SLP, TLAX, VER, ZAC

*Aulosepalum ramentaceum (Lindl.) Garay JAL, MICH, QRO, SLP, TAMS

*Aulosepalum riodelayense (Burns-Bal.) Salazar OAX

*Aulosepalum tenuiflorum (Greenm.) Garay GRO, JAL, MEX, $\mathrm{MICH}, \mathrm{MOR}, \mathrm{OAX}$

Barbosella prorepens (Rchb. f.) Schltr. GRO, OAX, VER

*Barkeria barkeriola Rchb. f. COL, JAL, NAY, SIN

* Barkeria dorotheae Halb. JAL

* Barkeria fritz-halbingeriana Soto Arenas OAX

*Barkeria melanocaulon A. Rich. \& Galeotti JAL, NAY, OAX

*Barkeria naevosa (Lindl.) Schltr. GRO, MICH, OAX

Barkeria obovata (Presl) Christenson CHIS, COL, GRO, JAL, MEX, MICH, MOR, NAY, OAX

* Barkeria palmeri (Rolfe) Schltr. COL, JAL, MICH, NAY, SIN

*Barkeria scandens (Lex.) Dressler \& Halb. GRO, MEX, MICH, OAX

*Barkeria shoemakeri Halb. GRO, MICH, OAX, SIN

*Barkeria skinneri (Bateman ex Lindl.) A. Rich. CHIS

*Barkeria spectabilis Bateman ex Lindl. CHIS

Barkeria strophinx (Rchb. f.) Halb. MEX, MICH

Barkeria uniflora (Lex.) Dressler \& Halb. GRO, JAL, MEX, MICH, MOR, OAX, PUE 
*Barkeria vanneriana Rchb. f. GRO, OAX, PUE

*Barkeria whartoniana (C. Schweinf.) Soto Arenas OAX Beloglottis costaricensis (Rchb. f.) Schltr. CHIS, COL, HGO, JAL, NAY, OAX, QRO, SLP, TAB, TAMS, VER

Beloglottis hameri Garay \& Hamer OAX

*Beloglottis mexicana Garay \& Hamer CHIS, JAL, OAX, TAMS, VER

*Bletia adenocarpa Rchb. f. COL, DGO, GRO, JAL, MEX, MICH, MOR, NAY, NLE, OAX, SIN, TAB, ZAC

*Bletia amabilis C. Schweinf. CHIH, COL, DGO, GRO, JAL, MICH, NAY, SON, ZAC

Bletia campanulata Lex. AGS, CHIS, COL, CDMX, DGO, GTO, GRO, HGO, JAL, MEX, MICH, MOR, NAY, OAX, PUE, SLP, SIN, SON, TLAX, VER, ZAC

*Bletia coccinea Lex. CDMX, GRO, JAL, MEX, MICH, MOR, OAX, PUE, QRO

* Bletia concolor Dressler GRO, JAL, MEX, MICH

*Bletia ensifolia L.O. Williams DGO, JAL, NAY, ZAC

Bletia gracilis Lodd. CHIH, COL, DGO, GRO, HGO, JAL, MEX, MICH, MOR, NAY, OAX, PUE, SIN, SON, VER, ZAC *Bletia greenmaniana L.O. Williams CDMX, DGO, JAL, MEX, MICH, MOR, OAX, SIN, VER

*Bletia greenwoodiana Sosa DGO, JAL

*Bletia lilacina A. Rich. \& Galeotti GRO, MEX, MOR, OAX

*Bletia macristhmochila Greenm. COL, CDMX, DGO, GRO, JAL, MEX, MICH, MOR, NAY, OAX, PUE, SIN, ZAC

*Bletia neglecta Sosa COL, CDMX, GTO, HGO, JAL, MEX, MOR, OAX, PUE, VER

* Bletia nelsonii Ames CHIS, OAX

*Bletia parkinsonii Hook. CHIS, COL, GRO, JAL, MEX, MOR, NLE, OAX, PUE, SLP, VER

*Bletia punctata Lex. CDMX, GRO, JAL, MEX, MICH, MOR, OAX, PUE, ZAC

Bletia purpurata A. Rich. \& Galeotti BCN, BCS, CHIS, COL, CDMX, DGO, GRO, JAL, MEX, MICH, MOR, NAY, OAX, QROO, SIN, TAMS, VER, ZAC

Bletia purpurea (Lam.) DC. CAM, CHIS, COL, GTO, GRO, HGO, JAL, MEX, MICH, MOR, NAY, OAX, PUE, QRO, QROO, SLP, SON, TAB, TAMS, VER, YUC, ZAC

Bletia reflexa Lindl. AGS, CHIS, CHIH, COL, CDMX, DGO, GTO, GRO, HGO, JAL, MEX, MICH, MOR, NAY, OAX, PUE, QRO, SLP, SIN, SON, VER, ZAC

* Bletia riparia Sosa \& Palestina VER

* Bletia similis Dressler JAL, MEX, MICH

Bletia tenuifolia Ames \& C. Schweinf. CHIS

* Bletia urbana Dressler CDMX, MEX, OAX

* Bletia villae Soto Arenas DGO

* Bletia warfordiana Sosa JAL

* Bletia $\times$ tamayoana S. Rosillo ex R. Soltero JAL

Brachystele guayanensis (Lindl.) Schltr. CHIS, VER

*Brachystele luzmariana Szlach. \& R. González JAL

*Brachystele tamayoana Szlach., Rutk. \& Mytnik BCS, GTO

Brassavola cucullata (L.) R. Br. CAM, CHIS, COL, JAL, NAY, OAX, QRO, QROO, SLP, SIN, SON, TAB, TAMS, VER, YUC Brassavola grandiflora Lindl. CAM, QROO, VER

Brassavola nodosa (L.) Lindl. CAM, CHIS, MOR, QROO, SLP, TAB, TAMS, VER, YUC
* Brassavola venosa Lindl. CAM, CHIS, OAX, QROO, YUC Brassia caudata (L.) Lindl. CAM, CHIS, GRO, OAX, QROO, TAB, VER

*Brassia maculata R. Br. CAM, CHIS, OAX, QROO, TAB, VER, YUC

*Brassia signata $\mathrm{Rchb}$. f. GRO, OAX

Brassia verrucosa Bateman ex Lindl. CHIS, HGO, OAX, PUE, VER

*Bulbophyllum cirrhosum L.O. Williams COL, GRO, JAL, OAX

Bulbophyllum jamaicense Cogn. COL, JAL, OAX

*Bulbophyllum nagelii L.O. Williams COL, GRO, JAL, MEX, MICH, MOR, OAX

Bulbophyllum oerstedii (Rchb. f.) Hemsl. CHIS, GRO, QROO, VER

Bulbophyllum sordidum Lindl. CHIS, OAX, VER

Calanthe calanthoides (A. Rich. \& Galeotti) Hamer \& Garay CHIS, GTO, GRO, HGO, OAX, PUE, QRO, SLP, SIN, TAMS, VER

Campylocentrum fasciola (Lindl.) Cogn. CHIS

Campylocentrum hondurense Ames CHIS, OAX, VER

Campylocentrum micranthum (Lindl.) Rolfe CAM, CHIS, COL, GRO, JAL, NAY, OAX, PUE, QROO, TAB, VER

Campylocentrum microphyllum Ames \& Correll CHIS

Campylocentrum pachyrrhizum (Rchb. f.) Rolfe CAM, CHIS, QROO

Campylocentrum poeppigii (Rchb. f.) Rolfe CAM, CHIS, QROO

*Campylocentrum schiedei (Rchb. f.) Benth. ex Hemsl. CHIS, OAX, VER

Catasetum integerrimum Hook. CAM, CHIS, HGO, OAX, PUE, QRO, QROO, SLP, TAB, TAMS, VER, YUC

* Catasetum laminatum Lindl. GRO, MEX, MICH, OAX

*Catasetum pendulum Dodson CHIH, COL, JAL, MICH, NAY, SIN

Caularthron bilamellatum (Rchb. f.) R.E. Schult. CHIS

*Clowesia dodsoniana E. Aguirre CHIS, COL, GRO, JAL, MICH, NAY, OAX, SIN

*Clowesia glaucoglossa (Rchb. f.) Dodson JAL, MICH, NAY

Clowesia rosea Lindl. GRO, MICH, NAY, OAX

Clowesia russelliana (Hook.) Dodson CHIS, COL, JAL

*Clowesia thylaciochila (Lem.) Dodson CHIS, GRO, JAL, MEX, MICH, MOR, OAX, PUE

* Cochleanthes flabelliformis (Sw.) R.E. Schult. \& Garay CHIS

* Coelia bella (Lem.) Rchb. f. CHIS

Coelia densiflora Rolfe CHIS

Coelia guatemalensis Rchb. f. CHIS

Coelia macrostachya Lindl. CHIS, GRO, OAX, PUE, VER

*Coelia triptera (Sm.) G. Don ex Steud. CHIS, OAX, PUE, TAB, VER

Comparettia falcata Poepp. \& Endl. CHIS, OAX, PUE, VER Comparettia tuerckheimii (Schltr.) M.W. Chase \& N.H. Williams CHIS, OAX

*Corallorrhiza bulbosa A. Rich. \& Galeotti COAH, GTO, HGO, JAL, MEX, MOR, NLE, OAX, QRO, VER

Corallorrhiza macrantha Schltr. COAH, CDMX, MEX, OAX, PUE, TLAX, VER 
Corallorrhiza maculata (Raf.) Raf. CHIS, COAH, COL, CDMX, DGO, GTO, GRO, HGO, JAL, MEX, MICH, MOR, NAY, NLE, OAX, PUE, QRO, SON, TLAX, VER, ZAC

Corallorrhiza odontorrhiza (Willd.) Poir. CHIS, CHIH, CDMX, GRO, HGO, JAL, MEX, MICH, MOR, OAX, PUE, SLP, TAMS, TLAX, VER

Corallorrhiza striata Lindl. CHIH, COAH, CDMX, HGO, JAL, MEX, MICH, MOR, NLE, OAX, PUE, SLP, SON, TLAX, VER Corallorrhiza williamsii Correll MEX, MOR

Corallorrhiza wisteriana Conrad BCS, CHIS, CHIH, COAH, COL, CDMX, HGO, JAL, MEX, MICH, MOR, NLE, OAX, SON, TLAX

Coryanthes picturata Rchb. f. CAM, CHIS, OAX, QROO, VER

*Corymborkis forcipigera (Rchb. f. \& Warsz.) L.O. Williams CHIS, GRO, OAX, VER

Cranichis apiculata Lindl. CHIS, COL, JAL, NAY, OAX

Cranichis ciliata (Kunth) Kunth CHIS, JAL, OAX

*Cranichis ciliilabia C. Schweinf. CHIS, HGO, JAL, OAX, VER

*Cranichis cochleata Dressler CHIS, OAX

Cranichis diphylla Sw. CHIS, MICH

Cranichis gracilis L.O. Williams DGO, JAL, NAY, SIN

*Cranichis hieroglyphica Ames \& Correll CHIS

Cranichis muscosa Sw. CHIS

Cranichis revoluta Hamer \& Garay CHIS

*Cranichis subumbellata A. Rich. \& Galeotti GRO, JAL, MEX,

MICH, MOR, OAX, QRO, SLP, TAMS

Cranichis sylvatica A. Rich. \& Galeotti CHIS, COL, JAL, MEX, NAY, OAX, QRO, SLP, TAMS, VER

Cranichis wageneri Rchb. f. CHIS

Crossoliparis wendlandii (Rchb. f.) Marg. CHIS, COL, GRO,

JAL, MEX, OAX

Cryptarrhena guatemalensis Schltr. CHIS

Cryptarrhena lunata R. Br. CHIS, OAX, VER

Cuitlauzina candida (Lindl.) Dressler \& N.H. Williams CHIS

Cuitlauzina convallarioides (Schltr.) Dressler \& N.H. Williams CHIS

*Cuitlauzina dubia (S. Rosillo) E. Yáñez \& Soto Arenas ex R.

Solano COL, GRO, JAL, MEX, MICH

Cuitlauzina egertonii (Lindl.) Dressler \& N.H. Williams CHIS, GRO, OAX

*Cuitlauzina pendula Lex. CHIS, COL, GRO, JAL, MEX, MICH, NAY, OAX, SIN

Cuitlauzina pulchella (Bateman ex Lindl.) Dressler \& N.H. Williams CHIS, JAL, MICH

*Cuitlauzina pygmaea (Lindl.) M.W. Chase \& N.H. Williams HGO, VER

Cyclopogon comosus (Rch. f.) Burns-Bal. \& E.W. Greenw. CHIS, GRO, OAX, SLP, VER

Cyclopogon cranichoides (Griseb.) Schltr. CHIS, OAX, QROO, $\mathrm{TAB}$

Cyclopogon elatus (Sw.) Schltr. CHIS, COL, GRO, HGO, JAL, MEX, MOR, NAY, OAX, QRO, SLP, VER

*Cyclopogon luteo-albus (A. Rich. \& Galeotti) Schltr. CHIS, HGO, OAX, QRO, SLP, VER

Cyclopogon obliquus (J.J. Sm.) Szlach. CHIS

Cyclopogon papilio Szlach. CHIS
Cyclopogon prasophyllum (Rchb. f.) Schltr. CAM, CHIS, OAX, QROO

*Cyclopogon pringlei (S. Watson) Soto Arenas JAL

*Cyclopogon saccatus (A. Rich. \& Galeotti) Schltr. CHIS, JAL, MEX, MICH, MOR, OAX, PUE, VER, ZAC

Cyclopogon violaceus (A. Rich. \& Galeotti) Schltr. CHIS, HGO, MOR, OAX, QRO, SLP, TAMS, VER

Cycnoches egertonianum Bateman CHIS, OAX, TAB, VER

Cycnoches ventricosum Bateman CHIS, OAX, TAB, VER

*Cypripedium dickinsonianum Hágsater CHIS

Cypripedium irapeanum Lex. CHIS, COL, DGO, GTO, GRO,

JAL, MEX, MICH, MOR, NAY, OAX, PUE, SIN, TAB, VER, ZAC

Cyrtochiloides ochmatochila (Rchb. f.) N.H. Williams \& M.W. Chase CHIS

Cyrtopodium macrobulbon (Lex.) G.A. Romero-Gonzalez \& Carnevali CAM, CHIS, COL, CDMX, DGO, GRO, HGO, JAL, MEX, MICH, MOR, NAY, OAX, PUE, QRO, QROO, SLP, SIN, SON, TAMS, VER, YUC, ZAC

* Chysis addita Dressler CHIS

*Chysis bractescens Lindl. CHIS, OAX, QRO, SLP, TAB, VER

*Chysis laevis Lindl. CHIS, OAX, PUE, VER

*Chysis limminghei Linden \& Rchb. f. OAX, TAB

*Deiregyne albovaginata (C. Schweinf.) Garay COL, CDMX, JAL, MEX, MICH, MOR

*Deiregyne callifera Salazar \& Hernández-Cardona HGO, VER

* Deiregyne chartacea (L.O. Williams) Garay DGO, JAL

*Deiregyne densiflora (C. Schweinf.) Salazar \& Soto Arenas COL, DGO, GTO, HGO, JAL, MEX, MICH, MOR, NAY, NLE, OAX, QRO, SLP, TLAX, VER

*Deiregyne diaphana (Lindl.) Garay OAX, PUE

Deiregyne eriophora (B.L. Rob. \& Greenm.) Garay CHIS, CHIH, CDMX, DGO, GRO, HGO, JAL, MEX, MICH, MOR, NLE, OAX, PUE, SON, TAMS, TLAX, VER

*Deiregyne falcata (L.O. Williams) Garay CHIH, DGO, GRO, JAL, OAX

*Deiregyne nonantzin (R. González ex McVaugh) Catling COL, JAL

*Deiregyne pandurata Garay DGO

*Deiregyne pseudopyramidalis (L.O. Williams) Garay CHIS, GRO, MEX, OAX

*Deiregyne rhombilabia Garay COL, GTO, GRO, JAL, MEX, MICH, MOR, OAX, PUE, VER

*Deiregyne tenorioi Soto Arenas \& Salazar GRO, HGO, MEX, OAX, PUE, TLAX

*Deiregyne velata (B.L. Rob. \& Fernald) Garay CHIH

Dendrophylax porrectus (Rchb. f.) Carlsward \& Whitten CAM, CHIS, COL, JAL, NAY, OAX, QROO, TAMS, VER, YUC

*Dichaea glauca (Sw.) Lindl. CHIS, GRO, HGO, OAX, PUE, QRO, SLP, VER

Dichaea graminoides (Sw.) Lindl. CHIS, OAX, VER

*Dichaea intermedia Ames \& Correll CHIS, OAX, VER

Dichaea muricatoides Hamer \& Garay CHIS, OAX, PUE, QRO, SLP, VER

*Dichaea neglecta Schltr. CHIS, HGO, OAX, PUE, QRO, SLP, VER

Dichaea panamensis Lindl. CHIS, OAX, TAB, VER 
Dichaea squarrosa Lindl. CHIS, GRO, JAL, MEX, MICH, MOR, OAX, PUE, VER

Dichaea suaveolens Kraenzl. CHIS, OAX

*Dichaea trichocarpa (Sw.) Lindl. CHIS, MEX, MICH

Dichromanthus aurantiacus (Lex.) Salazar \& Soto Arenas AGS, CHIS, CHIH, COL, CDMX, DGO, GTO, GRO, HGO, JAL, MEX, MICH, MOR, NAY, NLE, OAX, PUE, QRO, SLP, SIN, SON, TAMS, VER, ZAC

Dichromanthus cinnabarinus (Lex.) Garay AGS, BCS, CHIS, CHIH, COAH, CDMX, DGO, GTO, GRO, HGO, JAL, MEX, MICH, MOR, NAY, NLE, OAX, PUE, QRO, QROO, SLP, TAMS, VER, ZAC

Dichromanthus michuacanus (Lex.) Salazar \& Soto Arenas AGS, CHIS, CHIH, COAH, COL, CDMX, DGO, GTO, GRO, HGO, JAL, MEX, MICH, MOR, NAY, NLE, OAX, PUE, QRO, SON, TLAX, VER, ZAC

*Dichromanthus yucundaa Salazar \& García-Mend. OAX

Dimerandra emarginata (G. Mey.) Hoehne CAM, CHIS, OAX, QROO, TAB, VER

Dinema polybulbon (Sw.) Lindl. CHIS, HGO, OAX, PUE, VER Domingoa gemma (Rchb. f.) Van den Berg \& Soto Arenas PUE *Domingoa kienastii (Rchb. f.) Dressler CDMX, GRO, HGO, MEX, OAX, PUE, VER

Domingoa purpurea (Lindl.) van de Berg \& Soto Arenas CHIS, OAX, PUE, VER

Dracula pusilla (Rolfe) Luer CHIS, OAX

*Dryadella greenwoodiana Soto Arenas, Salazar \& R. Solano CHIS, OAX

Dryadella guatemalensis (Schltr.) Luer CHIS, OAX, VER

Dryadella linearifolia (Ames) Luer CHIS, OAX, VER

Elleanthus caricoides Nash CHIS, OAX

Elleanthus cynarocephalus (Rchb. f.) Rchb. f. CHIS, HGO, OAX, PUE, VER

Elleanthus graminifolius (Barb. Rodr.) Lojtnant CHIS

Elleanthus hymenophorus (Rchb. f.) Rchb. f. CHIS

*Elleanthus teotepecensis Soto Arenas GRO

Encyclia adenocarpa (Lex.) Schltr. CHIS, CHIH, COL, DGO, GRO, JAL, MEX, MICH, MOR, NAY, OAX, PUE, SIN, SON

*Encyclia adenocaula (Lex.) Schltr. CHIH, COL, DGO, GRO, JAL, MEX, MICH, NAY, OAX, SIN, ZAC

Encyclia aenicta Dressler \& G.E. Pollard CHIS, COL, DGO, GRO, JAL, MICH, MOR, NAY, OAX, SIN, ZAC

Encyclia alata (Bateman) Schltr. CAM, CHIS, OAX, QROO, TAB, TAMS, VER, YUC

Encyclia amanda (Ames) Dressler CHIS, OAX, QROO

Encyclia ambigua (Lindl.) Schltr. CHIS

*Encyclia asperula Dressler \& G.E. Pollard CHIS

*Encyclia atrorubens (Rolfe) Schltr. GRO, OAX

Encyclia bractescens (Lindl.) Hoehne CAM, CHIS, OAX, QROO, TAB, VER

*Encyclia calderoniae Soto Arenas OAX

*Encyclia candollei (Lindl.) Schltr. HGO, MICH, OAX, PUE, QRO, SLP, TAMS, VER

Encyclia ceratistes (Lindl.) Schltr. CHIS, OAX

*Encyclia contrerasii R. González COL

Encyclia cordigera (Kunth) Dressler CHIS, OAX, VER

*Encyclia chiapasensis Withner \& D.G. Hunt CHIS
*Encyclia diota (Lindl.) Schltr. CHIS, OAX

*Encyclia dressleri Beutelsp. \& Moreno-Molina CHIS

*Encyclia flabellata (Lindl.) B.F. Thurst. \& W.R. Thurst. VER Encyclia gravida (Lindl.) Schltr. CHIS, OAX, PUE, VER

Encyclia guatemalensis (Klotzsch) Dressler \& G.E. Pollard CAM, CHIS, HGO, OAX, QROO, PUE, QRO, SLP, TAB, TAMS, VER, YUC

*Encyclia halbingeriana Hágsater \& Soto Arenas MICH

*Encyclia hanburyi (Lindl.) Schltr. CHIS, GRO, OAX, PUE

*Encyclia huertae Soto Arenas \& R. Jiménez DGO, GRO, JAL, MEX, MICH, MOR, NAY, OAX

Encyclia incumbens (Lindl.) Mabb. AGS, CHIS, ZAC

*Encyclia lorata Dressler \& G.E. Pollard GRO

*Encyclia meliosma (Rchb. f.) Schltr. COL, GRO, JAL, MEX, MICH, MOR, NAY, OAX, SIN

*Encyclia microbulbon (Hook.) Schltr. CHIH, COL, DGO, GRO, JAL, MEX, MICH, MOR, NAY, OAX, SIN, SON

Encyclia nematocaulon (A. Rich.) Acuña CAM, QROO, TAB, VER, YUC

*Encyclia nizandensis Pérez-García \& Hágsater OAX

*Encyclia oestlundii (Ames, F.T. Hubbard \& C. Schweinf.) Hágsater \& Stermitz GRO, MICH

*Encyclia papillosa (Bateman) Ag.-Olav. CHIS, OAX, YUC

*Encyclia parviflora (Regel) Withner MICH, NAY, OAX, SLP, VER

*Encyclia pollardiana (Withner) Dressler \& G.E. Pollard JAL, $\mathrm{MICH}$

*Encyclia rzedowskiana Soto Arenas OAX

*Encyclia selligera (Lindl.) Schltr. CHIS

*Encyclia spatella (Rchb. f.) Schltr. COL, GRO, JAL, MEX, MICH, MOR, NAY

*Encyclia suaveolens Dressler JAL, NAY, SIN

*Encyclia trachycarpa (Lindl.) Schltr. COL, GRO, JAL, NAY, SIN

*Encyclia tuerckheimii Schltr. CHIS, OAX

*Encyclia uxpanapensis Salazar OAX, VER

Epidendrum aberrans Schltr. CHIS, OAX

Epidendrum acunnae Dressler CHIS, OAX

*Epidendrum alabastrialatum G.E. Pollard ex Hágsater CHIS, OAX

Epidendrum alticola Ames \& Correll CHIS

Epidendrum alvarezdeltoroi Hágsater CHIS, OAX

*Epidendrum anisatum Lex. COL, CDMX, DGO, GRO, JAL, MEX, MICH, MOR, NAY, OAX, SIN, ZAC

Epidendrum arbuscula Lindl. CHIS, OAX

*Epidendrum atroscriptum Hágsater CHIS, OAX, TAB, VER

Epidendrum beharorum Hágsater CHIS

*Epidendrum camposii Hágsater GRO, OAX

*Epidendrum cardiochilum L.O. Williams CHIS, OAX, VER

Epidendrum cardiophorum Schltr. CAM, CHIS, COL, GRO, HGO, OAX, PUE, QRO, QROO, SLP, TAB, TAMS, VER, YUC

Epidendrum carolii Schltr. CHIS, COL, GRO, JAL, MICH, OAX

Epidendrum centropetalum Rchb. f. CHIS, OAX

Epidendrum cerinum Schltr. CHIS

Epidendrum ciliare L. CAM, CHIS, CHIH, COL, DGO, GRO, 
JAL, MEX, MICH, NAY, OAX, PUE, QROO, SIN, TAB, VER *Epidendrum cilioccidentale Hágsater \& L. Sánchez CHIS, GRO, JAL, MEX, MICH, NAY, SIN

*Epidendrum citrosmum Hágsater COL, GRO, JAL, MICH, OAX

Epidendrum clowesii Bateman ex Lindl. CAM, CHIS, COL, GRO, JAL, MEX, MICH, NAY, OAX, SIN, VER

Epidendrum cnemidophorum Lindl. CHIS, OAX

Epidendrum coronatum Ruiz \& Pav. OAX, VER

*Epidendrum costatum A. Rich. \& Galeotti OAX

Epidendrum cristatum Ruiz \& Pav. CAM, CHIS, OAX, QRO, QROO, SLP, TAMS, VER

*Epidendrum cusii Hágsater GRO, JAL, MEX, MICH, MOR

*Epidendrum cystosum Ames CHIS

Epidendrum chloe Rchb. f. CHIS

Epidendrum chlorocorymbos Schltr. CAM, CHIS, NAY, OAX, QROO, SLP, TAB, VER, YUC

*Epidendrum diffusum Sw. CHIS, OAX, SLP, VER

Epidendrum dixiorum Hágsater CHIS

*Epidendrum dorsocarinatum Hágsater MEX, MICH

*Epidendrum dressleri Hágsater VER

*Epidendrum erectifolium Hágsater \& L. Sánchez CHIS

Epidendrum eustirum Ames, F.T. Hubb. \& C. Schweinf. CHIS,

OAX, PUE, VER

*Epidendrum examinis S. Rosillo COL, JAL

Epidendrum eximium L.O. Williams CHIS, GRO, MEX, MICH,

MOR, OAX

*Epidendrum falcatum Lindl. DGO, GRO, OAX, PUE, SIN

Epidendrum flexuosum G. Mey. CAM, CHIS, OAX, QROO,

TAB, VER, YUC

Epidendrum fruticosum Pav. ex Lindl. CHIS, OAX

*Epidendrum galeottianum A. Rich. \& Galeotti CAM, CHIS,

OAX, QROO, TAB, VER

*Epidendrum gasteriferum Scheeren OAX

*Epidendrum gomezii Schltr. COL, GRO, JAL, MICH, NAY, OAX

*Epidendrum gonzalez-tamayoi Hágsater CHIS, COL, GRO, JAL, NAY, OAX

Epidendrum greenwoodii Hágsater OAX

* Epidendrum guerrerense Hágsater \& García-Cruz GRO, OAX

*Epidendrum hagsateri Christenson GRO, OAX

*Epidendrum hueycantenangense Hágsater \& García-Cruz GRO, OAX

*Epidendrum ibarrae R. González JAL

Epidendrum incomptoides Ames, F.T. Hubb \& C. Schweinf. CHIS

Epidendrum incomptum Rchb. f. CHIS

Epidendrum isomerum Schltr. CHIS, OAX, TAB, VER

Epidendrum isthmii Schltr. CHIS, OAX, QROO, TAB, VER

*Epidendrum juergensenii Rchb. f. GRO, MEX, OAX

Epidendrum lacertinum Lindl. CHIS

Epidendrum laucheanum Rolfe CHIS, OAX, VER

*Epidendrum lignosum Lex. COL, GRO, HGO, JAL, MEX,

MICH, MOR, NAY, OAX, PUE, QRO, SLP, VER

*Epidendrum longicaule (L.O. Williams) L.O. Williams COL, JAL, SIN

*Epidendrum longipetalum A. Rich. \& Galeotti GRO, HGO,
JAL, MICH, MOR, OAX, PUE, QRO, SLP, VER

Epidendrum lowilliamsii García-Cruz MICH

*Epidendrum macdougallii (Hágsater) Hágsater CHIS, OAX

*Epidendrum macroclinium Hágsater CHIS, OAX, TAB, VER

*Epidendrum magnificum Schltr. GRO, MICH

Epidendrum magnoliae Muhl. MOR, NLE, QRO, SLP, TAMS

*Epidendrum marmoratum A. Rich. \& Galeotti GRO, JAL,

$\mathrm{MICH}, \mathrm{OAX}$

*Epidendrum martinezii L. Sánchez \& Carnevali CAM, CHIS, OAX, QROO

*Epidendrum matudae L.O. Williams GRO, MEX, MICH, MOR

*Epidendrum melistagoides Hágsater \& L. Sánchez CHIS, OAX Epidendrum melistagum Hágsater CAM, CHIS, OAX, QROO, TAB, VER

Epidendrum mesocarpum Hágsater CHIS, OAX

Epidendrum microcharis Rchb. f. CHIS

*Epidendrum miserum Lindl. COL, GRO, JAL, MEX, MICH, MOR, OAX

*Epidendrum mixtecanum Hágsater \& García-Cruz OAX

*Epidendrum mixtum Schltr. CHIS, GRO, OAX, VER

*Epidendrum mocinnoi Hágsater GRO, OAX

*Epidendrum motozintlensis Hágsater \& L. Sánchez CHIS

Epidendrum myrianthum Lindl. CHIS, OAX, TAB

Epidendrum nelsonii Hágsater CHIS

*Epidendrum neogaliciense Hágsater \& R. González COL, JAL

Epidendrum nitens Rchb. f. CHIS, COL, GRO, OAX, VER

Epidendrum nocturnum Jacq. CAM, CHIS, MICH, OAX,

QROO, SLP, TAB, VER, YUC

*Epidendrum oaxacanum Rolfe OAX

Epidendrum pachyrhachis Ames OAX

Epidendrum pansamalae Schltr. CHIS

Epidendrum paranthicum Rchb. f. CHIS

Epidendrum parkinsonianum Hook. CHIS, COL, GRO, JAL,

MEX, MICH, MOR, OAX, PUE, VER

*Epidendrum pastranae Hágsater GRO, MICH, OAX

Epidendrum pentadactylum Rchb. f. CHIS

Epidendrum peperomia Rchb. f. CHIS

Epidendrum polyanthum Lindl. CHIS, COL, GRO, HGO, JAL,

MICH, OAX, PUE, SLP, TAB, VER

Epidendrum polychromum Hágsater CHIS

*Epidendrum pollardii Hágsater GRO, OAX

*Epidendrum propinquum A. Rich. \& Galeotti CHIS, HGO,

OAX, PUE, QRO, SLP, TAMS, VER

*Epidendrum pseudoramosum Schltr. CHIS, GRO, OAX, VER Epidendrum radicans Pav. ex Lindl. CHIS, OAX, PUE, TAB,

TAMS, VER

Epidendrum radioferens (Ames, F.T. Hubb. \& C. Schweinf.)

Hágsater CHIS, OAX, PUE, VER

Epidendrum ramosum Jacq. CHIS, GRO, OAX, PUE, VER

Epidendrum repens Cogn. CHIS, OAX, VER

Epidendrum roseoscriptum Hágsater CHIS

*Epidendrum rosilloi Hágsater JAL, NAY

*Epidendrum rowleyi Withner \& G.E. Pollard GRO, OAX

Epidendrum santaclarense Ames CHIS

Epidendrum sculptum Rchb. f. CHIS

*Epidendrum singuliflorum Schltr. CHIS 
Epidendrum skutchii Ames, F.T. Hubb. \& C. Schweinf. CHIS Epidendrum sobralioides Ames \& Correll CHIS

Epidendrum stamfordianum Bateman CAM, CHIS, OAX, QROO, TAB, VER, YUC

Epidendrum strobiliferum Rchb. f. CHIS, OAX, QROO, TAB, VER

*Epidendrum succulentum Hágsater GRO, OAX

*Epidendrum tacanaense Hágsater, Soto Arenas \& E. Santiago

A. CHIS

*Epidendrum tortipetalum Scheeren GRO, JAL, MICH, OAX

Epidendrum trachythece Schltr. CHIS, OAX

Epidendrum trianthum Schltr. GRO, OAX

*Epidendrum tuxtlense Hágsater, García-Cruz \& L. Sánchez OAX, VER

Epidendrum tziscaoense Hágsater CHIS

*Epidendrum vandifolium Lindl. DGO, JAL, NAY, SIN, ZAC

Epidendrum veroscriptum Hágsater CHIS, GRO, OAX, PUE,

VER

Epidendrum verrucosum Sw. CHIS, OAX, TAB, VER

Epidendrum wendtii Hágsater \& Salazar CHIS, OAX

Epipactis gigantea Douglas ex Hook. BCN, BCS, CHIH, COAH, GTO, HGO, JAL, NLE, SLP, SON, ZAC

Erycina crista-galli (Rchb. f.) N.H. Williams \& M.W. Chase CHIS, OAX, VER

*Erycina echinata (Kunth) Lindl. COL, GRO, JAL, MICH, NAY, OAX, SIN

*Erycina hyalinobulbon (Lex.) N.H. Williams \& M.W. Chase COL, GRO, JAL, MEX, MICH, MOR, NAY, OAX

Erycina pumilio (Rchb. f.) N.H. Williams \& M.W. Chase CHIS Erycina pusilla (L.) N.H. Williams \& M.W. Chase CAM, CHIS, JAL, OAX, QROO, TAB, VER, YUC

Eulophia alta (L.) Fawc. \& Rendle CAM, CHIS, GRO, JAL, NAY, OAX, QROO, TAB, VER

Eurystyles borealis A.H. Heller CHIS

*Fernandezia mexicana (Dressler \& Hágsater) M.W. Chase GRO

Funkiella hyemalis (A. Rich. \& Galeotti) Schltr. CHIS, CHIH, COL, CDMX, GRO, HGO, JAL, MEX, MICH, MOR, OAX, PUE, QRO, QROO, TLAX, VER

*Funkiella laxispica (Catling) Salazar \& Soto Arenas OAX

*Funkiella markowskiana (Szlach.) Salazar \& Soto Arenas OAX

Funkiella minutiflora (A. Rich. \& Galeotti) Salazar \& Soto Arenas CHIS, CDMX, HGO, MEX, MICH, MOR, NLE, OAX, SLP, TLAX, VER

Funkiella parasitica (A. Rich. \& Galeotti) Salazar \& Sto Arenas CHIS, CHIH, COAH, CDMX, DGO, HGO, JAL, MEX, MICH, MOR, NLE, OAX, PUE, QRO, SLP, SON, TAMS, VER

Funkiella rubrocallosa (B.L. Rob. \& Greenm.) Salazar \& Soto Arenas CHIH, COAH, CDMX, HGO, JAL, MEX, MICH, MOR, NLE, OAX, PUE, SON, TLAX, VER

Funkiella stolonifera (Ames \& C. Schweinf.) Garay CHIS

Galeandra batemanii Rolfe CHIS, GRO, NAY, OAX

Galeandra baueri Lindl. CHIS, JAL, NAY, OAX

* Galeandra greenwoodiana Warford COL, NAY, OAX

* Galeoglossum cactorum Salazar \& Chávez-Rendón OAX

*Galeoglossum thysanochilum (Rob. \& Greenm.) Salazar OAX
Galeoglossum tubulosum (Lindl.) Salazar CHIS, COAH, CDMX, DGO, GRO, HGO, JAL, MEX, MICH, MOR, NLE, OAX, PUE, QRO, SLP, SIN, TAMS, TLAX

Galeottia grandiflora A. Rich. \& Galeotti OAX

Galeottiella sarcoglossa (A. Rich. \& Galeotti) Schltr. CHIS, CDMX, DGO, GTO, GRO, HGO, JAL, MEX, MICH, MOR, OAX, QRO, VER, ZAC

*Gongora galeata (Lindl.) Rchb. f. CHIS, HGO, OAX, PUE, SLP, VER

*Gongora galeottiana A. Rich. \& Galeotti COL, GRO, JAL, MICH, NAY, OAX

Gongora leucochila Lem. CHIS, OAX, TAB, VER

Gongora tridentata Whitten CHIS

Gongora truncata Lindl. CHIS, OAX, VER

*Gongora unicolor Schltr. CHIS, OAX, TAB, VER

Goodyera brachyceras (A. Rich. \& Galeotti) Garay \& G.A. Romero OAX

*Goodyera dolabripetala (Ames) Schltr. CHIS, OAX, VER

*Goodyera fimbrilabia Ormerod CHIS

Goodyera oblongifolia Raf. COAH, NLE, SON

*Goodyera purpusii Ormerod VER

Goodyera striata Rchb. f. CHIS, CHIH, COL, CDMX, DGO, GRO, HGO, JAL, MEX, MICH, MOR, NAY, NLE, OAX, PUE, QRO, SLP, SIN, SON, TAMS, VER

*Goodyera zacuapanensis Ormerod HGO, VER

*Govenia alba A. Rich. \& Galeotti CHIS, SLP

*Govenia bella E.W. Greenw. CHIS, OAX

*Govenia capitata Lindl. CDMX, GTO, GRO, HGO, MEX, MICH, MOR, OAX

*Govenia dressleriana E.W. Greenw. CHIS, GRO, JAL, MEX, MICH, OAX, SIN

Govenia greenwoodii Dressler \& Soto Arenas CHIS, GRO, OAX

*Govenia jouyana R. González JAL

Govenia lagenophora Lindl. COAH, CDMX, DGO, GRO, HGO, JAL, MEX, MICH, MOR, NLE, OAX, TAMS, TLAX, VER

Govenia liliacea (Lex.) Lindl. AGS, CHIS, CHIH, COAH, COL, CDMX, DGO, GTO, HGO, JAL, MEX, MICH, MOR, NLE, OAX, SLP, SON, TAMS, TLAX, VER, ZAC

Govenia matudae E.W. Greenw. \& Soto Arenas CHIS, OAX Govenia mutica Rchb. f. CHIS, OAX, HGO, JAL, QRO, SLP, TAMS, VER

*Govenia pauciflora Lindl. COAH, NLE, SLP

*Govenia praecox Salazar \& E.W. Greenw. HGO, VER

Govenia purpusii Schltr. CHIS, COL, DGO, GRO, HGO, JAL, MEX, MICH, OAX, PUE, QRO, VER, ZAC

*Govenia rubellilabia García-Cruz CHIS

Govenia superba (Lex.) Lindl. ex Lodd. CHIS, COL, CDMX, DGO, GRO, HGO, JAL, MEX, MICH, MOR, OAX, PUE, TAMS, TLAX, VER

*Govenia tequilana Dressler \& Hágsater JAL, MICH, OAX, PUE

Guarianthe aurantiaca (Bateman ex Lindl.) Dressler \& W.E. Higgins CHIS, COL, DGO, GRO, JAL, MEX, MICH, MOR, NAY, OAX, PUE, SIN, SON, VER

Guarianthe bowringiana (J.H. Veitch. ex O’Brien) Dressler \& 
W.E. Higgins CHIS

Guarianthe skinneri (Bateman) Dreesler \& W.E. Higgins CHIS, OAX

Guarianthe $\times$ deckeri (Klotzsch) Soto Arenas CHIS

*Habenaria acalcarata Espejo \& López-Ferrari CHIS

*Habenaria agapitae R. González \& Reynoso JAL, OAX

*Habenaria agrestis R. González \& Cuevas-Figueroa JAL

Habenaria alata Hook. CHIS, COL, GRO, JAL, MEX, MOR,

NAY, OAX, SLP, SIN, VER

*Habenaria atrata R. González \& Cuevas-Figueroa JAL

*Habenaria brevilabiata A. Rich. \& Galeotti COL, GRO, JAL, MICH, MOR, NAY, OAX

Habenaria brownelliana Catling CHIS, TAB, VER

*Habenaria calicis R. González GRO, MEX, MICH, MOR

*Habenaria carvajaliana R. González \& Cuevas-Figueroa JAL, ZAC

*Habenaria castroi R. González \& Cuevas-Figueroa ZAC

Habenaria clypeata Lindl. AGS, BCS, CHIS, CHIH, COL, CDMX, DGO, GRO, HGO, JAL, MEX, MICH, MOR, NAY, OAX, PUE, SIN, SON, VER, ZAC

*Habenaria cortesii R. González \& Cuevas-Figueroa JAL

Habenaria crassicornis Lindl. BCN, BCS, CHIS, CHIH, COL, CDMX, DGO, GRO, JAL, MEX, MICH, MOR, NAY, OAX, PUE, SIN

*Habenaria cuevasiana R. González \& Cuevas-Figueroa JAL Habenaria diffusa A. Rich. \& Galeotti CHIH, COL, GRO, JAL, MEX, MICH, MOR, NAY, OAX, PUE, SLP, VER

Habenaria distans Griseb. CAM, CHIS, COL, GRO, JAL, MICH, NAY, OAX, PUE, SLP, QROO, SIN, TAMS, VER, ZAC Habenaria eustachya Rchb. f. CHIS, OAX, TAB, VER

*Habenaria felipensis Ames OAX

*Habenaria filifera S. Watson BCS, COL, CDMX, JAL, MEX, MICH, MOR, OAX, ZAC

*Habenaria flexuosa Lindl. CHIS, COL, JAL, MEX, MICH, MOR, OAX

Habenaria floribunda Lindl. CAM, CHIS, HGO, MICH, OAX, PUE, QROO, TAB, TAMS, VER

*Habenaria gonzaleztamayoi García-Cruz, R. Jiménez \& L. Sánchez GRO, MEX, MOR

Habenaria guadalajarana S. Watson AGS, BCS, CHIS, CHIH, COL, CDMX, DGO, GRO, HGO, JAL, MEX, MICH, MOR, NAY, NLE, OAX, PUE, SIN, SON, TLAX, VER, ZAC

*Habenaria horaliae R. González MICH

* Habenaria ibarrae R. González JAL

*Habenaria ixtlanensis E.W. Greenw. OAX

*Habenaria jaliscana S. Watson AGS, CHIH, COL, DGO, GRO, HGO, JAL, MEX, MICH, NAY, OAX, SLP, SIN, TAB, VER, ZAC

*Habenaria jardeliana R. González \& Cuevas-Figueroa COL, JAL

*Habenaria karinae R. González \& Cuevas-Figueroa DGO, JAL

*Habenaria leon-ibarrae R. Jiménez \& Carnevali QROO

*Habenaria lizbethae R. González \& Cuevas-Figueroa JAL, NAY, SIN

*Habenaria luzmariana R. González JAL, MEX, NAY

*Habenaria macvaughiana R. González MEX, MICH
*Habenaria mariae R. González \& Cuevas-Figueroa JAL

* Habenaria matudae Salazar CHIS, OAX

*Habenaria micheliana R. González \& Cuevas-Figueroa JAL, NAY

*Habenaria mitodes Garay \& W. Kittr. COL, JAL, NAY

Habenaria monorrhiza (Sw.) Rchb. f. CHIS, OAX, TAB

*Habenaria nogeriana R. González \& Cuevas-Figueroa JAL, ZAC

Habenaria novemfida Lindl. BCS, CHIS, CHIH, COL, CDMX, GRO, HGO, JAL, MEX, MICH, MOR, NAY, OAX, PUE, SLP, SIN, SON, TLAX, VER, ZAC

*Habenaria oreophila Greenm. CHIH, GRO, JAL, MEX, MOR, VER

*Habenaria ortiziana R. González MEX

*Habenaria pinzonii R. González \& Cuevas-Figueroa GRO, JAL, NAY, OAX

Habenaria pringlei B.L. Rob. CAM, CHIS, MEX, OAX, QRO, QROO, SLP, TAB, TAMS, VER

*Habenaria pseudofilifera R. González \& Cuevas-Figueroa JAL

Habenaria quinqueseta (Michx.) Eaton BCS, CHIS, CHIH, COL, GRO, JAL, MEX, MICH, MOR, NAY, OAX, PUE, QROO, SON, TAB, TAMS, VER, YUC, ZAC

Habenaria repens Nutt. CAM, CHIS, GTO, GRO, HGO, JAL, MICH, QRO, QROO, SLP, TAB, TAMS, VER, YUC

*Habenaria rosilloana $\mathrm{R}$. González JAL, MICH

*Habenaria rosulifolia Espejo \& López-Ferrari MEX, MOR

*Habenaria ruizii R. González JAL

*Habenaria rzedowskiana R. González COL, GRO, JAL, MEX, MICH, NAY, OAX

*Habenaria rzedowskii R. González MICH

*Habenaria schaffneri S. Watson CHIH, DGO, JAL, NAY, SLP, SIN, VER

*Habenaria socorroae R. González \& Cuevas-Figueroa JAL Habenaria strictissima Rchb. f. CHIS, CDMX, GTO, GRO, HGO, JAL, MEX, MICH, MOR, NAY, OAX, PUE, QRO, SLP, SIN, SON, VER, ZAC

Habenaria subauriculata B.L. Rob. \& Greenm. CHIS, JAL, OAX, PUE

*Habenaria szlachetkoana R. González \& Cuevas-Figueroa JAL

*Habenaria talaensis R. González \& Cuevas-Figueroa JAL

*Habenaria tequilana R. González \& Cuevas-Figueroa JAL

Habenaria tetranema Schltr. CHIS

Habenaria trifida Kunth CHIS, DGO, GRO, JAL, MEX, NAY, OAX, SLP, TAB, VER, ZAC

*Habenaria uncata R. Jiménez, L. Sánchez \& García-Cruz MEX, MOR, OAX

*Habenaria virens A. Rich. \& Galeotti CHIS, COL, GRO, JAL, MEX, MOR, NAY, OAX, SON, VER

*Habenaria xochitlae R. González JAL

*Habenaria zamudioana R. González JAL, MEX, MICH

*Hagsatera brachycolumna (L.O. Williams) R. González GRO, MEX, MICH, OAX

*Hagsatera rosilloi $\mathrm{R}$. González COL, JAL

Hapalorchis lineatus (Lindl.) Schltr. CHIS

*Helleriella guerrerensis Dressler \& Hágsater GRO, OAX 
Helleriella nicaraguensis A.D. Hawkes CHIS

*Hexalectris brevicaulis L.O. Williams COAH, COL, GRO,

JAL, MEX, MICH, MOR, NAY, OAX

*Hexalectris fallax M.I. Rodr. \& R. González JAL, ZAC

Hexalectris grandiflora (A. Rich. \& Galeotti) L.O. Williams CHIH, COAH, GRO, JAL, MEX, MICH, MOR, NLE, OAX, PUE, SLP, SON, TAMS, VER, ZAC

Hexalectris nitida L.O. Williams COAH, OAX, TAMS

*Hexalectris parviflora L.O. Williams JAL, SON, ZAC

Hexalectris revoluta Correll CHIH, COAH, JAL, NLE, ZAC

Hexalectris spicata (Walter) Barnhart CHIH, NLE, SON

Hexalectris warnockii Ames \& Correll BCS, CHIH, COAH, SON

*Hintonella mexicana Ames COL, GRO, JAL, MEX, MICH, MOR, NAY, OAX

*Homalopetalum pachyphyllum (L.O. Williams) Dressler GRO, JAL, MEX, MICH, OAX

Homalopetalum pumilio (Rchb. f.) Schltr. CHIS, GRO, JAL, MEX, MICH, OAX, VER, ZAC

*Homalopetalum pumilum (Ames) Dressler CHIS, HGO, OAX, QRO, SLP, TAMS, VER

Ionopsis satyrioides (Sw.) Rchb. f. CHIS

Ionopsis utricularioides (Sw.) Lindl. CAM, CHIS, GRO, JAL, MICH, NAY, OAX, QROO, TAB, VER, YUC

Isochilus alatus Schltr. CHIS, OAX

*Isochilus aurantiacus Hamer \& Garay CHIS

*Isochilus bracteatus (Lex.) Salazar \& Soto Arenas ex Espejo \& López-Ferrari COL, JAL, MEX, MICH, MOR, OAX

Isochilus carnosiflorus Lindl. CAM, CHIS, OAX, QROO, TAB, VER

Isochilus chiriquensis Schltr. CHIS, COL, GRO, HGO, JAL, MEX, MICH, MOR, OAX, PUE, QRO, SLP, VER

*Isochilus langlassei Schltr. GRO, OAX

*Isochilus latibracteatus A. Rich. \& Galeotti CHIS, OAX, PUE, TAB, VER

Isochilus linearis (Jacq.) R. Br. CHIS, COL, HGO, JAL, MEX, MICH, MOR, OAX, QRO, SLP, SIN, TAMS, VER

Isochilus major Schltdl. \& Cham. CAM, CHIS, HGO, OAX, PUE, TAB, VER

*Isochilus oaxacanus Salazar \& Soto Arenas OAX

*Isochilus unilateralis B.L. Rob. HGO, PUE, QRO, SLP, TAMS, VER

*Jacquiniella cernua (Lindl.) Dressler GRO, MEX, MICH, OAX

Jacquiniella cobanensis (Ames \& Schltr.) Dressler CHIS, OAX Jacquiniella equitantifolia (Ames) Dressler CHIS, GRO, MICH, OAX, TAB, VER

Jacquiniella gigantea Dressler, Salazar \& García-Cruz CHIS Jacquiniella globosa (Jacq.) Schltr. CHIS, OAX, TAB, VER

Jacquiniella leucomelana (Rchb. f.) Schltr. CHIS, COL, GRO, JAL, MEX, MICH, NAY, OAX, PUE, VER, ZAC

Jacquiniella teretifolia (Sw.) Britton \& P. Wilson CHIS, GRO, OAX, PUE, VER

Kefersteinia tinschertiana Pupulin CHIS, GRO, OAX

Kegeliella atropilosa L.O. Williams \& A. H. Heller OAX, VER

* Kionophyton sawyeri (Standl. \& L.O. Williams) Garay DGO,

GTO, GRO, JAL, MEX, MICH, MOR, OAX, QRO, SLP, SIN
Kionophyton seminudum (Schltr.) Garay CHIS, CHIH, DGO, GRO, HGO, JAL, MEX, MOR, OAX, PUE, QRO, SLP, VER Kraenzlinella erinacea (Rchb. f.) R. Solano CHIS, OAX

* Kraenzlinella hintonii (L.O. Williams) R. Solano GRO

Kreodanthus casillasii R. González COL, JAL

Kreodanthus ovatilabius (Ames \& Correll) Garay CHIS, OAX

*Kreodanthus secundus (Ames) Garay CHIS, GRO, OAX

*Lacaena bicolor Lindl. CHIS, GRO, OAX, PUE

*Laelia albida Bateman ex Lindl. COL, DGO, GRO, JAL, MICH, NAY, OAX, PUE, SIN, ZAC

Laelia anceps Lindl. CHIS, GTO, GRO, HGO, JAL, MEX, MOR, NLE, OAX, PUE, QRO, SLP, TAMS, VER

* Laelia aurea A.V. Navarro DGO, JAL, NAY, SIN

*Laelia autumnalis (Lex.) Lindl. CHIH, COL, CDMX, DGO, GTO, GRO, HGO, JAL, MEX, MICH, MOR, NAY, OAX, PUE, QRO, SIN, SON, TLAX, ZAC

*Laelia crawshayana Rchb. f. JAL

*Laelia eyermaniana Rchb. f. CHIH, DGO, GTO, JAL, MICH, QRO, SIN, SON, ZAC

*Laelia furfuracea Lindl. OAX, PUE

*Laelia gouldiana Rchb. f. HGO, VER

*Laelia halbingeriana Salazar \& Soto Arenas OAX

Laelia rubescens Lindl. CAM, CHIS, COL, DGO, GRO, JAL, MEX, MICH, NAY, OAX, PUE, QROO, VER, YUC

*Laelia speciosa (Kunth) Schltr. AGS, DGO, GTO, GRO, HGO, JAL, MEX, MICH, OAX, QRO, SLP, TAMS, VER, ZAC

Laelia superbiens Lindl. CHIS, OAX

* Laelia $\times$ oaxacana Salazar \& R. Jiménez OAX

Leochilus carinatus (Knowles \& Westc.) Lindl. CHIS, MEX, MICH, MOR, OAX, VER

*Leochilus crocodiliceps (Rchb. f.) Kraenzl. COL, GRO, JAL, OAX

*Leochilus johnstonii Ames \& Correll GRO, OAX

Leochilus labiatus (Sw.) Kuntze CHIS, JAL, NAY, OAX, VER

*Leochilus leiboldii Rchb. f. OAX, VER

*Leochilus oncidioides Knowles \& Westc. CHIS, COL, GRO, HGO, JAL, MICH, OAX, QRO, SLP, TAMS, VER

Leochilus scriptus (Scheidw.) Rchb. f. CAM, CHIS, OAX, QROO, YUC

Lepanthes acuminata Schltr. CHIS, OAX, VER

Lepanthes almolongae Luer \& Behar CHIS

*Lepanthes ancylopetala Dressler CHIS

Lepanthes appendiculata Ames CHIS, OAX

*Lepanthes aprica Catling \& V.R. Catling OAX

Lepanthes arachnion Luer \& Dressler CHIS

*Lepanthes attenuata Salazar, Soto Arenas \& O. Suárez OAX, VER

*Lepanthes avis Rchb. f. OAX, VER

*Lepanthes brachystele Salazar \& Soto Arenas GRO, OAX

*Lepanthes breedlovei Salazar \& Soto Arenas CHIS, OAX

*Lepanthes calopetala Salazar \& Soto Arenas OAX, VER

* Lepanthes camposii Salazar \& Soto Arenas GRO, OAX

*Lepanthes catlingii Salazar, Soto Arenas \& O. Suárez OAX

*Lepanthes cryptostele Salazar \& Soto Arenas GRO

*Lepanthes chiangii Salazar, Soto Arenas \& O. Suárez OAX

Lepanthes denticulata Luer \& Behar CHIS

*Lepanthes erythroxantha Salazar \& Soto Arenas OAX 
*Lepanthes gabriellae Salazar \& Soto Arenas OAX

* Lepanthes galeottiana Salazar \& Soto Arenas OAX

* Lepanthes greenwoodii Salazar \& Soto Arenas OAX

Lepanthes guatemalensis Schltr. CHIS

* Lepanthes guerrerensis Salazar \& Soto Arenas GRO, OAX

*Lepanthes hagsateri Salazar \& Soto Arenas GRO

Lepanthes johnsonii Ames CHIS

Lepanthes lenticularis Luer \& Behar CHIS

* Lepanthes machorroi Salazar \& Soto Arenas OAX

*Lepanthes maldonadoae Soto Arenas CHIS

* Lepanthes mariae Salazar \& Soto Arenas OAX

*Lepanthes martinezii Salazar \& Soto Arenas CHIS, OAX

* Lepanthes matudiana Salazar \& Soto Arenas CHIS

*Lepanthes maxima Salazar \& Soto Arenas CHIS

*Lepanthes mazatlanensis R. Solano \& Reynaud OAX

*Lepanthes minima Salazar \& Soto Arenas CHIS, OAX

* Lepanthes mixe Salazar \& Soto Arenas OAX

*Lepanthes moorei C. Schweinf. HGO, OAX, VER

* Lepanthes motozintlensis Salazar \& Soto Arenas CHIS

*Lepanthes nagelii Salazar \& Soto Arenas GRO, MEX, MICH, MOR, OAX

*Lepanthes oaxacana Salazar, Soto Arenas \& O. Suárez OAX

Lepanthes oreocharis Schltr. CHIS, OAX

*Lepanthes oreophila Catling \& V.R. Catling OAX

*Lepanthes papilionacea Salazar, Soto Arenas \& O. Suárez

OAX, QRO, SLP

Lepanthes papillipetala Dressler CHIS

Lepanthes parvula Dressler CHIS, OAX

*Lepanthes pollardii Hespenh. GRO, OAX

Lepanthes pristidis Rchb. f. CHIS, OAX, VER

*Lepanthes rekoi R.E. Schult. HGO, OAX, PUE, VER

Lepanthes samacensis Ames CHIS, OAX

Lepanthes scopula Schltr. CHIS, OAX

*Lepanthes schiedei Rchb. f. OAX, VER

*Lepanthes schultesii Salazar \& Soto Arenas OAX

* Lepanthes sousae Salazar \& Soto Arenas OAX

Lepanthes stenophylla Schltr. CHIS

*Lepanthes suarezii Salazar \& Soto Arenas GTO, OAX

Lepanthes tecpanica Luer \& Behar CHIS

Lepanthes tenuiloba R.E. Schult. \& G.W. Dillon CHIS, OAX

*Lepanthes thurstoniorum Salazar, Soto Arenas \& O. Suárez CHIS, OAX

*Lepanthes totontepecensis Salazar \& Soto Arenas OAX

* Lepanthes vivipara Salazar \& Soto Arenas CHIS

* Lepanthes wendtii Salazar \& Soto Arenas OAX

*Lepanthes williamsii Salazar \& Soto Arenas CHIS

Lepanthes yunckeri Ames CHIS

*Lepanthes yuvilensis Catling GRO, OAX

Lepanthopsis floripecten (Rchb. f.) Ames CHIS

Ligeophila clavigera (Rchb. f.) Garay CHIS, OAX

*Liparis arnoglossophylla (Rchb. f.) Rchb. f. ex Hemsl. CHIS, GRO, OAX, VER

Liparis cordiformis C. Schweinf. JAL, MEX, MICH, MOR, OAX

*Liparis draculoides E.W. Greenw. GRO, HGO, JAL, MEX, MICH, MOR, OAX, VER

Liparis elata Lindl. CHIS, GRO, OAX, TAB, VER
Liparis fantastica Ames \& C. Schweinf. CHIS, GRO, JAL, MICH, OAX

*Liparis greenwoodiana Espejo CDMX, MEX, MICH, MOR, OAX

*Liparis lindeniana (A. Rich. \& Galeotti) Hemsl. OAX, VER

*Liparis madrensis Soto Arenas, Salazar \& R. Jiménez AGS, CHIH, DGO, ZAC

Liparis vexillifera (Lex.) Cogn. AGS, CHIS, CHIH, COL, CDMX, DGO, GRO, HGO, JAL, MEX, MICH, MOR, NAY, OAX, QRO, SLP, SIN, VER, ZAC

*Liparis volcanica R. González \& Zamudio JAL, MICH, NAY Lockhartia galeottiana A. Rich. ex Soto Arenas GRO, JAL, NAY, OAX

Lockhartia oerstedii Rchb. f. CHIS, COL, GRO, JAL, NAY, OAX, VER

Lockhartia verrucosa Lindl. ex Rchb. f. CHIS, OAX

*Lycaste aromatica (Graham ex Hook.) Lindl. CHIS, HGO, OAX, PUE, QRO, SLP, TAB, TAMS, VER

* Lycaste cochleata Lindl. \& Paxton CHIS, VER

*Lycaste consobrina Rchb. f. CHIS, HGO, OAX, PUE, QRO, SLP, TAMS, VER

*Lycaste crinita Lindl. COL, GRO, HGO, JAL, MICH, NAY, OAX, QRO, SLP

* Lycaste cruenta (Lindl.) Lindl. CHIS

* Lycaste deppei (Lodd.) Lindl. CHIS, OAX, PUE, TAMS, VER

Lycaste dowiana Endrés ex Rchb. f. CHIS

* Lycaste lassioglossa Rchb. f. CHIS

* Lycaste skinneri (Bateman ex Lindl.) Lindl. CHIS

Lyroglossa pubicaulis (L.O. Williams) Garay VER

Macradenia brassavolae Rchb. f. CHIS, VER

Macroclinium bicolor (Lindl.) Dodson CHIS, OAX

*Macroclinium lexarzanum (Hágsater \& R. González) Dodson GRO, JAL, MEX, MICH, NAY, OAX, SIN

*Macroclinium pachybulbon (Hágsater \& R. González) Dodson OAX, VER

Malaxis abieticola Salazar \& Soto Arenas BCS, CHIH, CDMX, DGO, HGO, JAL, MEX, MICH, MOR, NLE, OAX, SON, VER Malaxis acianthoides (Schltr.) Ames CHIS, COL, JAL, OAX

* Malaxis adenotropa R. González, L. Hern. \& E. Ramírez JAL

*Malaxis alvaroi García-Cruz, R. Jiménez \& L. Sánchez GTO, MEX, MOR, OAX

*Malaxis amplexicolumna E.W. Greenw. \& R. González CHIS, MEX, MICH

*Malaxis aurea Ames CHIS, DGO, GRO, JAL, NAY, OAX

*Malaxis brachystachys (Rchb. f.) Kuntze COAH, GTO, HGO, JAL, NLE, OAX, PUE, SLP, TAMS, VER

Malaxis carnosa (Kunth) C. Schweinf. CHIS, COL, CDMX, HGO, JAL, MEX, MICH, MOR, OAX, QRO, SLP, VER

*Malaxis casillasii R. González JAL

* Malaxis contrerasii R. González JAL

Malaxis corymbosa (S. Watson) Kuntze AGS, BCS, CHIS, CHIH, COAH, COL, CDMX, DGO, GRO, HGO, JAL, MEX, MICH, MOR, NLE, OAX, PUE, QRO, SLP, SON, TAMS, TLAX, VER, ZAC

*Malaxis crispata (Lindl.) R. González DGO, JAL, NAY, ZAC

* Malaxis chiarae R. González, L. Hernández \& E. Ramírez COL, JAL 
*Malaxis chica Todzia NLE, TAMS

*Malaxis elviae R. González DGO, JAL, NAY

Malaxis elliptica A. Rich. \& Galeotti CHIS, CHIH, DGO, GRO, HGO, JAL, MEX, MICH, MOR, NAY, OAX, PUE, SLP, SIN, SON, VER

*Malaxis espejoi R. González, L. Hernández \& E. Ramírez COL, HGO, JAL, MEX, MOR

Malaxis excavata (Lindl.) Kuntze CHIS, HGO, OAX, PUE, QRO, SLP, VER

Malaxis fastigiata (Rchb.f.) Kuntze AGS, CHIS, CHIH, COAH, COL, CDMX, DGO, GTO, GRO, HGO, JAL, MEX, MICH, MOR, NAY, NLE, OAX, PUE, QRO, SLP, SIN, SON, TAMS, TLAX, VER, ZAC

* Malaxis greenwoodiana Salazar \& Soto Arenas CHIS, OAX

* Malaxis hagsateri Salazar COL, GRO, JAL

*Malaxis hintonii Todzia COAH, NLE, TAMS

Malaxis histionantha (Link, Klotsch \& Otto) Garay \& Dunst. CAM, CHIS, COL, GTO, GRO, HGO, JAL, MEX, MICH, NAY, OAX, QRO, QROO, SLP, VER

*Malaxis javesiae (Rchb. f.) Ames CHIS, MICH, OAX, PUE Malaxis lepanthiflora (Schltr.) Ames CHIS, OAX, VER

Malaxis lepidota (Finet) Ames CHIS, COL, GRO, JAL, MEX, MICH, MOR, NAY, OAX, VER

*Malaxis lizbethiae R. González \& E. Ramírez JAL

* Malaxis luceroana R. González JAL, ZAC

*Malaxis lyonnetii Salazar MEX, MOR

Malaxis macrostachya (Lex.) O. Kuntze CHIH, COAH, DGO, HGO, SON, VER

*Malaxis macvaughiana R. González, L. Hernández \& E. Ramírez COL, JAL

Malaxis maianthemifolia Schltdl. \& Cham. CHIS, GRO, HGO, MEX, MICH, MOR, OAX, PUE, QRO, SLP, TAMS, VER

*Malaxis marthaleidae R. González, L. Hernández \& E. Ramírez JAL, ZAC

*Malaxis martinezii R. González JAL

* Malaxis molotensis Salazar \& de Santiago GRO

*Malaxis myurus (Lindl.) Kuntze AGS, CHIH, CDMX, DGO, HGO, JAL, MEX, MICH, MOR, NAY, OAX, SIN, SON, ZAC

*Malaxis nelsonii Ames DGO

*Malaxis novogaliciana R. González ex McVaugh AGS, CHIH, DGO, GTO, JAL, NAY, SLP, SIN, ZAC

*Malaxis ochreata (S. Watson) Ames JAL, MOR, NAY, OAX, PUE

Malaxis pandurata (Schltr.) Ames CHIS, GRO, OAX

Malaxis perezii R. González JAL

*Malaxis pringlei (S. Watson) Ames CHIH, COL, DGO, JAL, NAY, ZAC

*Malaxis ramirezii $\mathrm{R}$. González COL, JAL

*Malaxis reichei (Schltr.) Ames \& C. Schweinf. GRO, MEX

* Malaxis ribana Espejo \& López-Ferrari MEX, MOR

* Malaxis roblesgilana $\mathrm{R}$. González JAL, NAY, ZAC

*Malaxis rodrigueziana R. González COAH, JAL, NAY, SLP, TAMS

*Malaxis rosei Ames AGS, CHIH, COL, DGO, GRO, HGO, JAL, MEX, MICH, MOR, OAX, SON, VER, ZAC

*Malaxis rosilloi R. González \& E.W. Greenw. COL, DGO, GRO, JAL, MEX, MICH, MOR, NAY, OAX, SIN
*Malaxis rositae R. González, L. Hernández \& E. Ramírez JAL

* Malaxis ruizii R. González JAL, ZAC

*Malaxis rzedowskiana $\mathrm{R}$. González MEX

Malaxis soulei L.O. Williams AGS, BCS, CHIS, CHIH, COAH, COL, CDMX, DGO, GTO, GRO, HGO, JAL, MEX, MICH, MOR, NAY, NLE, OAX, PUE, QRO, QROO, SLP, SON, TAMS, TLAX, VER, ZAC

Malaxis steyermarkii Correll OAX

*Malaxis streptopetala (B.L. Rob. \& Greenm.) Ames CDMX, DGO, GRO, HGO, JAL, MEX, MOR, NAY, OAX, VER

*Malaxis stricta L.O. Williams COL, JAL, MEX, MOR

* Malaxis tamayoana Garay \& W. Kittr. COL, JAL, ZAC

* Malaxis tepicana Ames JAL, NAY, ZAC

*Malaxis tequilensis R. González, L. Hernández \& E. Ramírez JAL

Malaxis triangularis Dressler CHIS, VER

Malaxis unifolia Michx. BCN, BCS, CHIS, CHIH, COAH, COL, DGO, GTO, GRO, HGO, JAL, MEX, MICH, MOR, NAY, NLE, OAX, PUE, SLP, SIN, SON, TAMS, TLAX, VER, ZAC

*Malaxis urbana E.W. Greenw. OAX

*Malaxis zempoalensis López-Ferrari \& Espejo MEX, MOR Masdevallia adamsii Luer CHIS

* Masdevallia floribunda Lindl. CHIS, MICH, OAX, PUE, VER

*Masdevallia tuerckheimii Ames CHIS, OAX

Maxillaria aciantha Rchb. f. CAM, CHIS, OAX, TAB, QROO

Maxillaria alba (Hook.) Lindl. CHIS

Maxillaria anceps Ames \& C. Schweinf. CHIS, OAX, VER

* Maxillaria atrata Rchb. f. CHIS, COL, JAL

Maxillaria cobanensis Schltr. CHIS

Maxillaria crassifolia (Lindl.) Rchb. f. CAM, CHIS, OAX, QROO, TAB, VER

Maxillaria cucullata Lindl. CHIS, COL, GRO, JAL, MEX, MICH, MOR, OAX, PUE, VER

* Maxillaria chimalapana Soto Arenas \& Salazar OAX

Maxillaria chiriquensis Schltr. ND

*Maxillaria densa Lindl. CHIS, HGO, JAL, OAX, PUE, QRO, SLP, VER

*Maxillaria elatior Rchb. f. CHIS, MICH, OAX, TAB, VER

Maxillaria friedrichsthallii Rchb. f. CAM, CHIS, OAX, QROO, TAB

* Maxillaria hagsateriana Soto Arenas CHIS, OAX Maxillaria hedwigae Hamer \& Dodson CHIS, TAB

* Maxillaria houtteana Rchb. f. CHIS, GRO, JAL, MEX, OAX Maxillaria hystrionica (Rchb. f.) L.O. Williams CHIS, GRO, OAX

*Maxillaria lexarzana Soto Arenas \& F. Chiang GRO, JAL, MEX, MICH, MOR

Maxillaria macleei Bateman ex Lindl. OAX, TAB

Maxillaria maleolens Schltr. CHIS

*Maxillaria meleagris Lindl. CHIS, GRO, HGO, OAX, PUE, VER

*Maxillaria mexicana J.T. Atwood GRO, MEX, MICH, OAX, SIN

Maxillaria moralesii Carnevali \& J.T. Atwood CHIS

Maxillaria nasuta Rchb. f. CHIS

*Maxillaria oestlundiana L.O. Williams GRO 
Maxillaria parviflora (Poepp. \& Endl.) Garay CHIS, OAX, VER

* Maxillaria praestans Rchb. f. CHIS, OAX, VER

*Maxillaria pulchra (Schltr.) L.O. Williams ex Correll CHIS, OAX, TAB, VER

Maxillaria punctostriata Rchb. f. ND

* Maxillaria rhombea Lindl. GRO, OAX

Maxillaria ringens $\mathrm{Rchb}$. f. CHIS, GRO, OAX, TAB, VER

Maxillaria scorpioidea Kraenzl. CHIS

Maxillaria soconuscana Breedlove \& D. Mally CHIS

*Maxillaria sotoana Carnevali \& Gómez-Juárez CHIS

Maxillaria tenuifolia Lindl. CAM, CHIS, OAX, PUE, QROO, TAB, VER

Maxillaria tonsoniae Soto Arenas CHIS, GRO, OAX

Maxillaria tuerckheimii Schltr. CHIS

Maxillaria uncata Lindl. CHIS, TAB

Maxillaria variabilis Bateman ex Lindl. CHIS, COL, DGO, GRO, HGO, JAL, MEX, MICH, MOR, NAY, OAX, PUE, QRO, SLP, SIN, TAB, VER

*Maxillaria $\times$ yucatanensis Carnevali \& R. Jiménez CAM, QROO

Meiracyllium gemma Rchb. f. COL, DGO, GRO, JAL, MICH, NAY, OAX, SIN

Meiracyllium trinasutum Rchb. f. CHIS, COL, DGO, GRO, JAL, MICH, NAY, OAX, PUE, SIN, VER

Mesadenella petenensis (L.O. Williams) Garay CAM, CHIS, OAX, QROO, TAB, VER

*Mesadenus chiangii (M.C. Johnst.) Garay COAH, NLE

Mesadenus lucayanus (Britton) Schltr. CHIS, OAX, QRO, SLP, VER

Mesadenus polyanthus (Rchb. f.) Schltr. CHIS, CHIH, COAH, CDMX, GTO, GRO, HGO, JAL, MEX, MICH, MOR, NLE, OAX, PUE, QRO, SLP, SIN, SON, TAMS, TLAX, VER, YUC *Mesadenus tenuissimus (L.O. Williams) Garay GRO, MEX, MOR, OAX

*Mexipedium xerophyticum (Soto Arenas, Salazar \& Hágsater) V.A. Albert \& M.W. Chase OAX

Microchilus luniferus (Schltr.) Ormerod CHIS, OAX, PUE, VER

*Microchilus mexicanus (Ames) Ormerod CHIS

Microchilus vesicifer (Rchb. f.) Ormerod CHIS

*Microepidendrum subulatifolium (A. Rich. \& Galeotti) W.E.

Higgins COL, GRO, JAL, MEX, MICH, OAX

*Mormodes aromatica Lindl. CHIS, GRO, MEX, OAX

*Mormodes badia Rolfe ex W. Watson COL, JAL, NAY, SIN

* Mormodes cozticxochitl Salazar GRO, OAX

Mormodes lineata Bateman ex Lindl. CHIS, OAX

*Mormodes luxata Lindl. COL, DGO, JAL, MICH, NAY, SIN

*Mormodes maculata (Klotzsch) L.O. Williams CHIS, GRO, HGO, JAL, OAX, PUE, QRO, SLP, VER

*Mormodes nagelii L.O. Williams CHIS

*Mormodes oestlundiana Salazar \& Hágsater GRO, MICH

*Mormodes pardalinata S. Rosillo JAL, NAY

* Mormodes porphyrophlebia Salazar OAX

*Mormodes ramirezii S. Rosillo COL, JAL

* Mormodes sanguineoclaustra Fowlie GRO

*Mormodes sotoana Salazar CHIS
*Mormodes tezontle S. Rosillo GRO, JAL, MICH

*Mormodes tuxtlensis Salazar OAX, VER

*Mormodes uncia Rchb. f. OAX

*Mormodes williamsii hort. ex G. Nicholson COL, JAL

*Mormolyca ringens (Lindl.) Schltr. CAM, CHIS, OAX, QROO, TAB, VER

Myoxanthus octomeriae (Schltr.) Luer CHIS

Myoxanthus octomerioides (Lindl.) Luer CHIS, GRO, OAX, VER

Myrmecophila brysiana (Lem.) G.C. Kenn. CAM, QROO

*Myrmecophila christinae Carnevali \& Gómez-Juárez QROO, YUC

Myrmecophila exaltata (Kraenzl.) G.C. Kenn. CHIS

* Myrmecophila galeottiana (A. Rich.) Rolfe COL, GRO, JAL, MICH, MOR, NAY, OAX

* Myrmecophila grandiflora (Lindl.) Carnevali, Tapia-Muñoz \& I. Ramírez OAX, SLP, VER

Myrmecophila laguna-guerrerae Carnevali, Ibarra-González \& Tapia-Muñoz QROO

Myrmecophila tibicinis (Bateman) Rolfe CAM, CHIS, HGO, MOR, OAX, QROO, SLP, TAB, TAMS, VER, YUC

*Nemaconia dressleriana (Soto Arenas) van den Berg, Salazar $\&$ Soto Arenas MEX, MOR

Nemaconia glomerata (Correll) van den Berg, Salazar \& Soto Arenas CHIS, OAX, VER

Nemaconia graminifolia Knowles \& Westc. CHIS, OAX

*Nemaconia longipetala (Correll) van den Berg, Salazar \& Soto Arenas GRO, OAX

Nemaconia pellita (Rchb. f.) Van den Berg, Salazar \& Soto Arenas CHIS

Nemaconia striata (Lindl.) van den Berg, Salazar \& Soto Arenas CAM, CHIS, MOR, OAX, QROO, TAB, VER

Nidema boothii (Lindl.) Schltr. CAM, CHIS, COL, GRO, HGO, JAL, MEX, MICH, OAX, PUE, QRO, QROO, SLP, TAB, TAMS, VER

Notylia barkeri Lindl. CAM, CHIS, COL, GRO, JAL, MICH, NAY, OAX, PUE, QRO, QROO, SLP, TAB, TAMS, VER, YUC

*Notylia leucantha Salazar OAX

Notylia orbicularis A. Rich. \& Galeotti CAM, CHIS, OAX, QROO, VER, YUC

* Notylia trisepala Lindl. \& Paxton CAM, CHIS, VER

*Oestlundia cyanocolumna (Ames, F.T. Hubb. \& C. Schweinf.) W.E. Higgins HGO, NLE, OAX, PUE, QRO, SLP, TAMS, VER Oestlundia distantiflora (A. Rich. \& Galeotti) W.E. Higgins CHIS, VER

*Oestlundia ligulata (Lex.) Soto Arenas COL, GRO, JAL, MEX, MICH, MOR, NAY, OAX

Oestlundia luteorosea (A. Rich. \& Galeotti) W.E. Higgins CHIS, GRO, MICH, OAX, PUE, VER

Oncidium brachyandrum Lindl. CHIS, COL, DGO, GRO, JAL, MEX, MICH, MOR, NAY, OAX, SIN, ZAC

* Oncidium durangense Hágsater DGO, SIN

Oncidium endocharis Rchb. f. CHIS

* Oncidium ensatum Lindl. CAM, CHIS, COL, GRO, JAL, MICH, NAY, OAX, QROO

* Oncidium exauriculatum (Hamer \& Garay) R. Jiménez CHIS Oncidium geertianum C. Morren COL, DGO, GRO, JAL, MEX, 
MICH, MOR, NAY, OAX, SIN, SON

* Oncidium ghiesbreghtianum A. Rich. \& Galeotti COL, GRO, JAL, MEX, MICH, OAX

Oncidium guatemalenoides M.W. Chase \& N.H. Williams CHIS Oncidium hagsaterianum R. Jiménez \& Soto Arenas CHIS, OAX, VER

* Oncidium hastatum (Bateman) Lindl. COL, GRO, JAL, MEX, MICH, MOR, NAY, OAX

* Oncidium hintonii L.O. Williams CHIS, COL, DGO, GRO, JAL, MICH, NAY, OAX, SIN

* Oncidium incurvum Barker ex Lindl. CHIS, OAX, PUE, VER

* Oncidium iricolor Rchb. f. OAX

* Oncidium karwinskii (Lindl.) Lindl. COL, DGO, GRO, JAL, MEX, MICH, NAY, OAX, SIN

* Oncidium laeve (Lindl.) Beer CHIS

* Oncidium leleui $\mathrm{R}$. Jiménez \& Soto Arenas COL, JAL, MICH, NAY, OAX

* Oncidium leucochilum Bateman ex Lindl. CHIS

Oncidium lindleyi (Lindl.) R. Jiménez \& Soto Arenas CHIS, OAX

Oncidium maculatum (Lindl.) Lindl. CHIS, OAX, SLP, VER

*Oncidium mexicanum (L.O. Williams) M.W. Chase \& N.H. Williams GRO

* Oncidium microstigma Rchb. f. MEX, MOR

*Oncidium oblongatum Lindl. CHIS, GRO, OAX

Oncidium oliganthum (Rchb. f.) L.O. Williams ex Correll CHIS, OAX, VER

Oncidium pergamineum Lindl. CHIS, OAX, VER

Oncidium poikilostalix (Kränzl.) M.W. Chase \& N.H. Williams CHIS

Oncidium reflexum Lindl. COL, GRO, JAL, MEX, MICH, MOR, NAY, OAX, PUE

Oncidium reichenheimii (Linden \& Rchb. f.) Garay \& Stacy COL, GRO, JAL, MEX, MICH, MOR, OAX

Oncidium sotoanum R. Jiménez \& Hágsater CHIS, OAX, TAB, VER

*Oncidium sphacelatum Lindl. CAM, CHIS, COL, GRO, HGO, OAX, PUE, QRO, QROO, SLP, TAB, TAMS, VER, YUC

*Oncidium stelligerum Rchb. f. COL, GRO, JAL, MICH, OAX Oncidium stenoglossum (Schltr.) Dressler \& N.H. Wms. CHIS, VER

*Oncidium suave Lindl. COL, GRO, JAL, MEX, MICH, MOR, NAY, OAX, SIN

Oncidium subcruciforme (A.H. Heller) Garay \& Stacy OAX

*Oncidium suttonii Bateman ex Lindl. CHIS

Oncidium tenuipes Kraenzl. CHIS, OAX

Oncidium tigrinum Lex. AGS, COL, JAL, MEX, MICH

*Oncidium unguiculatum Lindl. GRO, MEX, MICH, MOR, OAX

Oncidium wentworhtianum Bateman ex Lindl. CHIS

*Oncidium $\times$ oviedomotae Hágsater JAL, MICH

Ornithocephalus bicornis Lindl. ex Benth. CHIS, OAX, QROO, VER

*Ornithocephalus biloborostratus Salazar \& R. González JAL, MICH, OAX

Ornithocephalus inflexus Lindl. CAM, CHIS, JAL, OAX, QRO, QROO, SLP, TAB, VER, YUC
Ornithocephalus iridifolius Rchb. f. CHIS, JAL, NAY, OAX, TAB, VER

*Ornithocephalus obergiae Soto Arenas CHIS, VER

* Ornithocephalus torresii Salazar \& Soto Arenas CHIS, OAX

*Ornithocephalus tripterus Schltr. CHIS, OAX, VER

*Pachyphyllum mexicanum Dressler \& Hágsater GRO, OAX

Pelexia adnata (Sw.) Poit. ex Spreng. HGO, OAX, QRO, SLP, TAMS, VER

Pelexia congesta Ames \& C. Schweinf. CHIS

Pelexia funckiana (A. Rich. \& Galeotti) Schltr. CHIS, OAX, PUE, VER

*Pelexia gutturosa (Rchb. f.) Garay CHIS, QRO, SLP, VER

Pelexia longipetiolata (Rchb. f.) Schltr. CHIS, OAX, PUE, SLP, VER

Phloeophila peperomioides (Ames) Garay CHIS

Phragmipedium exstaminodium Castaño, Hágsater \& E. Aguirre CHIS

Phragmipedium warscewiczii (Rchb. f.) Christenson CHIS

* Physogyne garayana R. González \& Szlach. COL, JAL

*Physogyne gonzalezii (L.O. Williams) Garay COL, JAL, NAY, OAX

*Physogyne sparsiflora (C. Schweinf.) Garay COL, JAL, MEX, MOR

Platanthera brevifolia (Greene) Kraenzl. BCN, CHIH, COAH, COL, CDMX, DGO, GRO, HGO, JAL, MEX, MICH, MOR, NLE, OAX, SON, TLAX, VER, ZAC

* Platanthera calderoniae López-Ferrari \& Espejo MICH

Platanthera cooperi (S. Watson) R.M. Bateman BCN

Platanthera limosa Lindl. BCN, BCS, CHIS, CHIH, COAH, CDMX, DGO, GRO, HGO, JAL, MEX, MICH, MOR, NAY, NLE, OAX, QRO, SLP, SIN, SON, TAB, TAMS, TLAX, VER, ZAC

* Platanthera nubigena A. Rich. \& Galeotti VER

Platanthera sparsiflora (S. Watson) Schltr. BCN

Platystele caudatisepala (C. Schweinf.) Garay CHIS

Platystele compacta (Ames) Ames CHIS

Platystele jungermannioides (Schltr.) Garay CHIS

Platystele minimiflora (Schltr.) Garay CHIS, OAX, VER

Platystele ovatilabia (Ames \& C. Schweinf.) Garay CHIS, VER

Platystele oxyglossa (Schltr.) Garay CHIS, OAX, VER

Platystele pedicellaris (Schltr.) Garay CHIS, OAX

Platystele repens (Ames) Garay VER

Platystele stenostachya (Rchb. f.) Garay CHIS, OAX, PUE, TAB, VER

Platythelys maculata (Hook.) Garay CHIS, OAX, TAB, VER

Platythelys vaginata (Hook.) Garay CAM, CHIS, COL, GRO, HGO, JAL, NAY, OAX, PUE, QROO, TAB, VER

Platythelys venustula (Ames) Garay CHIS

Plectrophora alata (Rolfe) Garay CHIS

Pleurothallis antonensis L.O. Williams CHIS, OAX, VER

Pleurothallis cardiothallis Rchb. f. CHIS, MICH, OAX, PUE, VER

Pleurothallis correllii Luer CHIS, GRO

Pleurothallis crocodiliceps Rchb. f. CHIS, GRO

Pleurothallis homolantha Schltr. ND

Pleurothallis leucantha Schltr. TAB

Pleurothallis matudana C. Schweinf. CHIS, OAX 
Pleurothallis pansamalae Schltr. CHIS, GRO

Pleurothallis saccatilabia C. Schweinf. CHIS

Pleurothallis sanchoi Ames CHIS

Pleurothallopsis ujarrensis Pridgeon \& M.W. Chase CHIS

Polystachya foliosa (Hook.) Rchb. f. CAM, CHIS, GRO, JAL, MICH, NAY, OAX, PUE, QROO, TAB, TAMS, VER, YUC

*Polystachya lineata Rchb. f. CHIS, SLP, VER

Polystachya masayensis Rchb. f. CHIS

* Polystachya mcvaughiana Soto Arenas GRO, JAL, NAY, OAX

* Ponera exilis Dressler COL, GRO, JAL, MEX, MICH

Ponera juncifolia Lindl. CHIS, OAX, PUE, VER

*Ponthieva brittoniae Ames CAM, QROO

Ponthieva ephippium Rchb. f. CHIS, COL, CDMX, GRO, HGO, JAL, MEX, MOR, NLE, OAX, PUE, QRO, SLP, TAMS, VER Ponthieva formosa Schltr. CHIS, COL, GRO, JAL, MEX, MOR, OAX, PUE, TAB, VER

*Ponthieva hildae R. González \& R. Soltero JAL, MEX, MOR Ponthieva mexicana (A. Rich. \& Galeotti) Salazar CHIS, GTO, GRO, HGO, JAL, MEX, MICH, MOR, NLE, OAX, QRO, SLP, TAMS

*Ponthieva parvula Schltr. CHIS

Ponthieva racemosa (Walter) C. Mohr CHIS, CDMX, GRO, HGO, JAL, MEX, MICH, MOR, NAY, NLE, OAX, PUE, QRO, SLP, SIN, TAMS, VER

*Ponthieva rinconii Salazar VER

Ponthieva schaffneri (Rchb. f.) E.W. Greenw. CHIS, CHIH, COL, CDMX, DGO, GTO, GRO, HGO, JAL, MEX, MICH, MOR, NAY, NLE, OAX, PUE, QRO, SLP, SIN, SON, TAMS, VER, ZAC

Ponthieva triloba Schltr. CHIS, GRO

*Ponthieva trilobata (L.O. Williams) L.O. Williams CHIS, OAX, VER

*Ponthieva tuerckheimii Schltr. CHIS, HGO, OAX, VER

Prescottia oligantha (Sw.) Lindl. CHIS, VER

Prescottia stachyodes (Sw.) Lindl. CAM, CHIS, HGO, MEX, OAX, PUE, QROO, SLP, TAB, VER

Prosthechea abbreviata (Schltr.) W.E. Higgins CHIS

Prosthechea baculus (Rchb. f.) W.E. Higgins CHIS, OAX, VER

*Prosthechea bicamerata (Rchb. f.) W.E. Higgins DGO, GRO, OAX

Prosthechea boothiana (Lindl.) W.E. Higgins CAM, COL, JAL, NAY, QROO, TAB, YUC

*Prosthechea brachiata (A. Rich. \& Galeotti) W.E. Higgins GRO, OAX

Prosthechea brassavolae (Rchb. f.) W.E. Higgins CHIS, GRO, JAL, MEX, MICH, OAX, VER, ZAC

*Prosthechea citrina (Lex.) W.E. Higgins COL, DGO, GRO, JAL, MEX, MICH, NAY, OAX, PUE, SIN, TLAX, VER

Prosthechea cochleata (L.) W.E. Higgins CAM, CHIS, COL, GTO, GRO, HGO, JAL, MICH, NAY, OAX, PUE, QRO, QROO, SLP, TAB, TAMS, VER

*Prosthechea concolor (Lex.) W.E. Higgins GRO, JAL, MEX, MICH, MOR, OAX, PUE

*Prosthechea cretacea (Dressler \& G.E. Pollard) W.E. Higgins COL, JAL, MEX, MICH, OAX

Prosthechea chacaoensis (Rchb. f.) W.E. Higgins CHIS, COL, GRO, JAL, MICH, NAY, OAX, TAB, VER
Prosthechea chondylobulbon (A. Rich. \& Galeotti) W.E. Higgins CHIS, COL, GRO, JAL, MEX, MICH, NAY, OAX, VER, $\mathrm{ZAC}$

*Prosthechea favoris (Rchb. f.) Salazar \& Soto Arenas COL, JAL, NAY

Prosthechea fragrans (Sw.) W.E. Higgins CHIS, GRO, OAX

*Prosthechea ghiesbreghtiana (A. Rich. \& Galeotti) W.E. Higgins GRO, OAX

Prosthechea glauca Knowles \& Westc. CHIS, GRO, OAX, PUE, VER

* Prosthechea greenwoodiana (Ag.-Olav.) W.E. Higgins OAX

*Prosthechea hastata (Lindl.) W.E. Higgins CHIS, GRO, MOR, OAX

* Prosthechea karwinskii (Mart.) J.M.H. Shaw OAX

Prosthechea livida (Lindl.) W.E. Higgins CAM, CHIS, HGO, NAY, OAX, QRO, QROO, SLP, TAB, TAMS, VER

Prosthechea maculosa (Ames, F.T. Hubb. \& C. Schweinf.) W.E. Higgins CHIS, OAX

Prosthechea madrensis (Schltr.) Karremans GRO, MICH, OAX *Prosthechea magnispatha (Ames, F.T. Hubb. \& C. Schweinf.) W.E. Higgins GRO, OAX

*Prosthechea mariae (Ames) W.E. Higgins GTO, HGO, PUE, QRO, SLP, TAMS, VER

*Prosthechea micropus (Rchb. f.) W.E. Higgins GRO, JAL, MEX, MICH, MOR, NAY, OAX, SIN

Prosthechea michuacana (Lex.) W.E. Higgins CHIS, CDMX, GRO, HGO, JAL, MEX, MICH, MOR, OAX, PUE, VER

* Prosthechea mulasii Soto Arenas \& L. Cerv. GRO, JAL, MEX

Prosthechea neurosa (Ames) W.E. Higgins CHIS

*Prosthechea obpiribulbon (Hágsater) W.E. Higgins GRO, NAY, OAX

Prosthechea ochracea (Lindl.) W.E. Higgins CHIS, GRO, HGO, OAX, PUE, VER

* Prosthechea panthera (Rchb. f.) W.E. Higgins CHIS, OAX

*Prosthechea pastoris (Lex.) Espejo \& López-Ferrari COL, DGO, JAL, MEX, MICH, NAY, SIN, ZAC

*Prosthechea pringlei (Rolfe) W.E. Higgins GRO, MEX, MICH, MOR, OAX

Prosthechea pseudopygmaea (Finet) W.E. Higgins CHIS, OAX, PUE, VER

* Prosthechea pterocarpa (Lindl.) W.E. Higgins CHIS, COL, GRO, JAL, MEX, MICH, NAY, OAX, SIN

* Prosthechea punctulata (Rchb. f.) Soto Arenas \& Salazar COL, GRO, JAL, MEX, MICH, MOR, OAX, SIN

Prosthechea pygmaea (Hook.) W.E. Higgins CHIS, OAX, PUE, TAB, VER

Prosthechea radiata (Lindl.) W.E. Higgins CAM, CHIS, HGO, OAX, PUE, QRO, SLP, TAB, VER

Prosthechea rhynchophora (A. Rich. \& Galeotti) W.E. Higgins CHIS, OAX, PUE, VER

*Prosthechea semiaperta (Hágsater) W.E. Higgins GRO, OAX, PUE

*Prosthechea squalida (Lex.) Soto Arenas \& Salazar COL, DGO, GRO, JAL, MEX, MICH, MOR, NAY, OAX, SIN, VER * Prosthechea trulla (Rchb. f.) W.E. Higgins COL, JAL, NAY, OAX

Prosthechea vagans (Ames) W.E. Higgins CHIS, COL, OAX 
Prosthechea varicosa (Bateman ex Lindl.) W.E. Higgins CHIS, COL, GRO, HGO, JAL, MEX, MICH, MOR, OAX, PUE, SLP, TAMS, VER

Prosthechea vitellina (Lindl.) W.E. Higgins CHIS, HGO, OAX, PUE, VER

*Pseudogoodyera pseudogoodyerioides (L.O. Williams) R. González \& Szlach. CHIS, OAX, PUE, QRO, SLP, VER

Psilochilus macrophyllus (Lindl.) Ames CHIS, OAX, PUE, VER

Pteroglossa roseoalba (Rchb. f.) Salazar \& M.W. Chase CHIS, OAX

Restrepia muscifera (Lindl.) Rchb. f. ex Lindl. CHIS, GRO, OAX

Restrepia trichoglossa F. Lehm. ex Sander CHIS

*Restrepiella ophiocephala (Lindl.) Garay \& Dunst. CHIS, GRO, OAX, SLP, TAB, VER

Rhyncholaelia digbyana (Benth.) Schltr. CAM, QROO, YUC

*Rhyncholaelia glauca (Lindl.) Schltr. CAM, CHIS, OAX, VER

* Rhynchostele aptera (Lex.) Soto Arenas \& Salazar COL, DGO, GRO, JAL, MEX, MICH, MOR, OAX, SIN, TAB

Rhynchostele beloglossa (Rchb. f.) Dressler \& N.H. Wiliams. CHIS, GRO, OAX, VER

* Rhynchostele bictoniensis (Bateman) Soto Arenas \& Salazar CHIS, HGO, OAX, PUE, VER

*Rhynchostele candidula (Rchb. f.) Soto Arenas \& Salazar GRO, OAX

*Rhynchostele cervantesii (Lex.) Soto Arenas \& Salazar COL, DGO, GRO, JAL, MEX, MICH, MOR, NAY, OAX, SIN

*Rhynchostele cordata (Lindl.) Soto Arenas \& Salazar CHIS, MICH, OAX, PUE, VER

*Rhynchostele ehrenbergii (Link, Klotzsch \& Otto) Soto Arenas \& Salazar HGO, OAX, PUE, VER

*Rhynchostele galeottiana (A. Rich.) Soto Arenas \& Salazar GRO, OAX

*Rhynchostele londesboroughiana (Rchb. f.) Soto Arenas \& Salazar GRO

Rhynchostele maculata (Lex.) Soto Arenas \& Salazar CHIS, COL, GRO, JAL, MEX, MICH, MOR, OAX, PUE, VER

*Rhynchostele madrensis (Rchb. f.) Soto Arenas \& Salazar GRO, OAX

Rhynchostele majalis (Rchb. f.) Soto Arenas \& Salazar CHIS, OAX

Rhynchostele pygmaea (Lindl.) Rchb. f. CHIS

*Rhynchostele rossii (Lindl.) Soto Arenas \& Salazar CHIS, HGO, OAX, PUE, QRO, SLP, VER

Rhynchostele stellata (Lindl.) Soto Arenas \& Salazar CHIS, VER

Rhynchostele uroskinneri (Lindl.) Soto Arenas \& Salazar CHIS

*Rodriguezia dressleriana R. González COL, JAL, NAY

Rossioglossum grande (Lindl.) Garay \& G.C. Kenn. CHIS

*Rossioglossum hagsaterianum Soto Arenas JAL, NAY

*Rossioglossum insleayi (Baker ex Lindl.) Garay \& G.C. Kenn. GRO, JAL, MEX, MICH, NAY, OAX

*Rossioglossum splendens (Rchb. f.) Garay \& G.C. Kenn. COL, GRO, JAL, NAY, OAX

*Rossioglossum williamsianum (Rchb. f.) Garay \& G.C. Kenn. CHIS, OAX
Sacoila lanceolata (Aubl.) Garay BCS, CAM, CHIS, CHIH, COL, GRO, JAL, MEX, MICH, MOR, NAY, OAX, PUE, QRO, QROO, SLP, SIN, SON, TAB, TAMS, VER, YUC, ZAC

*Sarcoglottis assurgens (Rchb. f.) Schltr. CAM, CHIS, OAX, SLP, TAMS, YUC

Sarcoglottis cerina (Lindl.) P.N. Don CHIS, DGO, HGO, OAX, VER

Sarcoglottis corymbosa Garay CHIS, CHIH, GRO, JAL, MEX, NAY, OAX, SON

* Sarcoglottis lobata (Lindl.) P.N. Don HGO

Sarcoglottis richardiana (Schltr.) Salazar \& Soto Arenas CHIS, QRO, VER

Sarcoglottis rosulata (Lindl.) P.N. Don CHIS, COL, GRO, JAL, MOR, OAX, QRO, SLP, VER

Sarcoglottis sceptrodes (Rchb. f.) Schltr. CAM, CHIS, JAL, MOR, NAY, OAX, QRO, QROO, SLP, SIN, TAB, TAMS, VER, YUC

*Sarcoglottis scintillans (E.W. Greenw.) Salazar \& Soto Arenas OAX

Sarcoglottis schaffneri (Rchb. f.) Ames CHIS, COAH, COL, CDMX, DGO, GTO, GRO, HGO, JAL, MEX, MICH, MOR, NAY, NLE, OAX, PUE, QRO, SLP, SIN, TAMS, TLAX, VER, ZAC

Scaphosepalum microdactylum Rolfe CHIS

Scaphosepalum standleyi Ames CHIS

Scaphyglottis behri (Rchb. f.) Benth. \& Hook. ex Hemsl. CAM, CHIS, OAX, QROO

*Scaphyglottis crurigera (Bateman ex Lindl.) Ames \& Correll CHIS, OAX

Scaphyglottis fasciculata Hook. CHIS, OAX, TAB, VER

Scaphyglottis graminifolia (Ruiz \& Pav.) Poepp. \& Endl. CAM, CHIS, OAX, PUE, VER

Scaphyglottis hondurensis (Ames) L.O. Williams CHIS, GRO, OAX

Scaphyglottis imbricata (Lindl.) Dressler CHIS, COL, GRO, JAL, MICH, NAY, OAX, VER

Scaphyglottis leucantha Rchb. f. CAM, CHIS, QROO

Scaphyglottis lindeniana (A. Rich. \& Galeotti) L.O. Williams CHIS, GRO, MICH, OAX, VER

Scaphyglottis minutiflora Ames \& Correll CHIS, OAX, VER

Scaphyglottis prolifera (Sw.) Cogn. CHIS

Scaphyglottis sessilis (Rchb. f.) Foldats CHIS, COL, GRO, JAL, MEX, MICH, NAY, OAX, SIN, VER

*Schiedeella affinis (C. Schweinf.) Salazar BCS, GTO, JAL, MEX, MICH, MOR

*Schiedeella crenulata (L.O. Williams) Espejo \& López-Ferrari COL, CDMX, HGO, JAL, MEX, MOR, PUE, VER

Schiedeella durangensis (Ames \& C. Schweinf.) Burns-Bal. COAH, CDMX, DGO, GRO, HGO, JAL, MEX, MICH, NAY, NLE, OAX, QRO, SLP, SIN, VER, ZAC

* Schiedeella garayana R. González JAL, MEX, MOR

Schiedeella llaveana (Lindl.) Schltr. AGS, CHIS, CHIH, COL, CDMX, DGO, GTO, GRO, HGO, JAL, MEX, MICH, MOR, NAY, OAX, PUE, QRO, SLP, SON, TLAX, VER

*Schiedeella nagelii (L.O. Williams) Garay NLE, QRO, SLP

* Schiedeella romeroana Szlach. OAX, PUE

* Schiedeella tenella (L.O. Williams) Burns-Bal. CHIH, DGO, 
JAL, MEX

Schiedeella trilineata (Lindl.) Burns-Bal. CHIS

Schiedeella wercklei (Schltr.) Garay OAX

*Schiedeella williamsiana Szlach., Rutk. \& Mytnik HGO, JAL

Sobralia crispissima Dressler CHIS

Sobralia decora Bateman CHIS, GRO, JAL, NAY, OAX, TAB, VER

Sobralia fragrans Lindl. CHIS, OAX, VER

*Sobralia galeottiana A. Rich. CHIS, GRO, JAL, MEX, MICH, NAY, OAX, SIN

Sobralia lindleyana $\mathrm{Rchb}$. f. CHIS

*Sobralia macdougallii Soto Arenas, Pérez-García \& Salazar CHIS, OAX

*Sobralia macrantha Lindl. CHIS, GRO, MICH, OAX, PUE, VER

Sobralia mucronata Ames \& C. Schweinf. CHIS, OAX

Sobralia warscewiczii Rchb. f. CHIS

Sobralia xantholeuca hort. ex B.S. Williams CHIS, OAX

* Sotoa confusa (Garay) Salazar COAH, CDMX, GTO, HGO, MEX, OAX, QRO, VER, ZAC

Specklinia alata (A. Rich. \& Galeotti) R. Solano \& Soto Arenas CHIS, VER

Specklinia blancoi (Pupulin) Soto Arenas \& R. Solano CHIS, VER

Specklinia brighamii (S. Watson) Pridgeon \& M.W. Chase CHIS, OAX, TAB, VER

*Specklinia digitale (Luer) Pridgeon \& M.W. Chase CHIS, OAX, TAB, VER

Specklinia emarginata Lindl. CHIS

Specklinia fimbriata (Ames \& C. Schweinf.) R. Solano CHIS, OAX, VER

Specklinia fuegii (Rchb. f.) R. Solano \& Soto Arenas CHIS, GRO, OAX

Specklinia glandulosa (Ames) Pridgeon \& M.W. Chase CHIS, OAX, VER

Specklinia grobyi (Bateman ex Lindl.) F. Barros CHIS, QROO, VER

Specklinia lateritia (Endres ex Rchb. f.) Pridgeon \& M.W. Chase CHIS, OAX, VER

Specklinia marginata (Lindl.) Pridgeon \& M.W. Chase CAM, CHIS, OAX, QROO, TAB, VER

Specklinia pisinna (Luer) R. Solano \& Soto Arenas CHIS, OAX, TAB, VER

Specklinia samacensis (Ames) Pridgeon \& M.W. Chase ND Specklinia segregatifolia (Ames \& C. Schweinf.) R. Solano CHIS, GRO, HGO, VER

Specklinia spectabilis (Ames \& C. Schweinf.) Pupulin \& Karremans CHIS, OAX

Specklinia tribuloides (Sw.) Pridgeon \& M.W. Chase CHIS, OAX, QRO, SLP, TAB, VER

Spiranthes graminea Lindl. CHIH, COL, CDMX, DGO, GRO, HGO, JAL, MEX, MICH, MOR, OAX, PUE, QRO, SLP, VER Spiranthes nebulorum Catling \& V.R. Catling BCN, CHIS, GRO, HGO, MEX, MICH, NLE, OAX, PUE, TAMS, VER Spiranthes torta (Thunb.) Garay \& H.R. Sweet QROO, VER * Stanhopea dodsoniana Salazar \& Soto Arenas OAX, VER

* Stanhopea ecornuta Lem. CHIS
Stanhopea graveolens Lindl. CHIS, OAX, TAB, VER

*Stanhopea hernandezii (Kunth) Schltr. CHIS, MEX, MICH, MOR

*Stanhopea intermedia Klotzsch COL, GRO, JAL, MICH, NAY, OAX

*Stanhopea maculosa Knowles \& Westc. COL, DGO, GRO, JAL, MICH, NAY, SIN, SON

*Stanhopea martiana Bateman ex Lindl. COL, GRO, JAL, NAY, OAX

Stanhopea oculata (G. Lodd.) Lindl. CHIS, OAX, PUE, VER

*Stanhopea pseudoradiosa Jenny \& R. González OAX

* Stanhopea radiosa Lem. COL, DGO, GRO, JAL, MICH, NAY, OAX, SIN, SON

Stanhopea ruckeri Lindl. CHIS, OAX, VER

Stanhopea saccata Bateman CHIS, COL, JAL, NAY, OAX

Stanhopea tigrina Bateman ex Lindl. CHIS, HGO, OAX, PUE, QRO, SLP, TAMS, VER

Stanhopea whittenii Soto Arenas, Salazar \& G. Gerlach CHIS

Stelis aemula Schltr. CHIS

Stelis aeolica $\mathrm{R}$. Solano \& Soto Arenas CHIS

* Stelis annedamoniae R. Solano CHIS

Stelis argentata Lindl. GRO, OAX, VER

* Stelis aristocratica (L.O. Williams) R. Solano \& Soto Arenas GRO

*Stelis bidentata Schltr. CHIS, OAX, VER

* Stelis ciliaris Lindl. CAM, CHIS, OAX, QROO, TAB, VER

* Stelis cleistogama Schltr. CHIS, OAX

Stelis cobanensis (Schltr.) Pridgeon \& M.W. Chase CHIS, OAX, VER

* Stelis chiapensis Solano CHIS, OAX

Stelis deregularis Barb. Rodr. CHIS

* Stelis desantiagoi R. Solano \& Salazar GRO

Stelis despectans Schltr. CHIS

Stelis emarginata (Lindl.) Soto Arenas \& R. Solano CHIS, COL, DGO, GRO, JAL, MEX, MICH, MOR, NAY, OAX, PUE, SIN, VER

* Stelis fulva Schltr. CHIS

Stelis gracilis Ames CAM, CHIS, OAX, TAB, VER

Stelis greenwoodii Soto Arenas \& R. Solano CHIS, COL, DGO, GRO, JAL, MEX, MICH, NAY, OAX, SIN

Stelis guatemalensis Schltr. CHIS, OAX

* Stelis guerrerensis Soto Arenas \& R. Solano GRO

* Stelis hagsateri R. Solano CHIS

Stelis hymenantha Schltr. CHIS, GRO, OAX

Stelis immersa (Linden \& Rchb. f.) Pridgeon \& M.W. Chase CHIS, GRO, OAX, VER

Stelis jalapensis (Kraenzl.) Pridgeon \& M.W. Chase CHIS, GRO

Stelis johnsonii Ames CHIS

* Stelis kaiae R. Solano \& C. Dietz CHIS

* Stelis lapinerae Soto Arenas \& R. Solano MICH

* Stelis martinezii R. Solano CHIS, OAX

Stelis megachlamys (Schltr.) Pupulin CHIS, GRO, OAX, VER

Stelis microchila Schltr. CHIS, OAX

* Stelis nagelii R. Solano OAX, PUE, VER

Stelis nicaraguensis (Liebm.) R. Solano \& Soto Arenas CHIS, OAX, QRO, SLP, VER 
*Stelis nigriflora (L.O. Williams) Pridgeon \& M.W. Chase MEX, MOR

*Stelis nonresupinata $\mathrm{R}$. Solano \& Soto Arenas GRO, OAX

* Stelis oaxacana R. Solano OAX

*Stelis oestlundiana (L.O. Williams) Pridgeon \& M.W. Chase GRO, MEX, MICH, MOR

* Stelis ornata (Rchb. f.) Pridgeon \& M.W. Chase CHIS, HGO, OAX, PUE, VER

*Stelis ovatilabia Schltr. CHIS

*Stelis oxypetala Schltr. CHIS, TAB

Stelis pachyglossa (Lindl.) Pridgeon \& M.W. Chase CHIS, GRO, OAX, PUE, VER

*Stelis perplexa Ames CHIS, OAX, VER

Stelis platystylis (Schltr.) Solano \& Soto Arenas CHIS, HGO,

OAX, PUE, VER

Stelis purpurascens A. Rich. \& Galeotti CAM, CHIS, OAX, PUE, VER

Stelis quadrifida (Lex.) R. Solano \& Soto Arenas CHIS, COL, DGO, GRO, MEX, JAL, MICH, NAY, OAX, PUE, SIN, TAMS, VER

*Stelis resupinata (Ames) Pridgeon \& M.W. Chase COL, GRO, JAL, MICH

*Stelis retusa (Lex.) Pridgeon \& M.W. Chase COL, GRO, JAL, MEX, MICH, MOR, OAX

* Stelis rubens Schltr. CHIS, OAX, PUE, VER

*Stelis rufobrunnea (Lindl.) L.O. Williams DGO, GRO, JAL, MICH, OAX, SIN

*Stelis salazarii $\mathrm{R}$. Solano CHIS, OAX

*Stelis sanguinolenta (Garay \& W. Kittr.) R. Solano \& Soto Arenas COL, JAL, MICH, ZAC

Stelis segoviensis (Rchb. f.) Pridgeon \& M.W. Chase CHIS

*Stelis soconuscana R. Solano CHIS

* Stelis sotoana $\mathrm{R}$. Solano OAX

*Stelis sotoarenasii R. Solano OAX

Stelis tacanensis R. Solano \& Soto Arenas CHIS

* Stelis tenuissima Schltr. CHIS

* Stelis veracrucensis $\mathrm{R}$. Solano OAX, PUE, VER

Stelis vespertina $\mathrm{R}$. Solano \& Soto Arenas CHIS, OAX

Stelis villosa (Knowles \& Westc.) Pridgeon \& M.W. Chase

CHIS, COL, DGO, GRO, JAL, MEX, MICH, NAY, MOR,

OAX, PUE, SIN, VER

*Stelis wendtii $\mathrm{R}$. Solano OAX

*Stelis xerophila (Schltr.) Soto Arenas CHIH, COL, DGO, JAL, MEX, MICH, MOR, NAY, SIN

*Stelis zootrophionoides Castañeda-Zárate \& Ramos-Castro CHIS

Stenorrhynchos speciosum (Jacq.) Rich. ex Spreng. CHIS, GRO, OAX, PUE, VER

Stenotyla lendyana (Rchb. f.) Dressler CHIS, OAX

*Svenkoeltzia congestiflora (L.O. Williams) Burns-Bal. COL, JAL, MEX, MICH, MOR, OAX

Tamayorkis ehrenbergii (Rchb. f.) R. González \& Szlach. CHIS, CHIH, COAH, COL, CDMX, DGO, GRO, HGO, JAL, MEX, MICH, MOR, NLE, OAX, PUE, QRO, SON, TAMS, TLAX, VER

*Tamayorkis hintonii (Todzia) R. González \& Szlach. COAH, NLE
Tamayorkis wendtii (Salazar) R. González \& Szlach. COAH, NLE, QRO

*Telipogon amoanus Bogarín GRO

Telipogon helleri (L.O. Williams) N.H. Williams \& Dressler CHIS

Teuscheria pickiana (Schltr.) Garay CHIS, OAX, VER

*Trichocentrum andreanum (Cogn.) R. Jiménez \& Carnevali CHIS, COL, GRO, JAL, MICH, NAY, OAX

*Trichocentrum andrewsiae (R. Jiménez \& Carnevali) R. Jiménez \& Carnevali CAM, YUC

Trichocentrum ascendens (Lindl.) M.W. Chase \& N.H. Williams CAM, CHIS, OAX, QROO, SON, TAB, TAMS, VER, YUC

Trichocentrum bicallosum (Lindl.) M.W. Chase \& N.H. Williams CHIS, OAX

*Trichocentrum biorbiculare (Balam \& Cetzal) R. Jiménez \& R. Solano PUE, QRO, SLP, TAMS, VER, ZAC

Trichocentrum brachyphyllum (Lindl.) R. Jiménez CHIS, CHIH, COL, DGO, GRO, JAL, MEX, MICH, MOR, OAX, SIN, SON *Trichocentrum candidum Lindl. CHIS, OAX, PUE, QRO, SLP, VER

Trichocentrum cebolleta (Jacq.) M.W. Chase \& N.H. Williams CAM, CHIS, CHIH, COL, DGO, GRO, HGO, JAL, MEX, MICH, MOR, NAY, OAX, QRO, QROO, SLP, SIN, SON, TAMS, VER, YUC

*Trichocentrum cosymbephorum (C. Morren) R. Jiménez \& Carnevali CHIS, OAX, QRO, SLP, TAB, TAMS, VER

*Trichocentrum chrysops (Rchb. f.) Soto Arenas \& R. Jiménez GRO, OAX

*Trichocentrum flavovirens (L.O. Williams) W.W. Chase \& N.H. Williams COL, GRO, JAL, MICH, NAY

*Trichocentrum hoegei Rchb. f. GRO, OAX

*Trichocentrum leptotifolium (Cetzal \& Carnevali) R. Jiménez \& R. Solano CHIH, DGO, SIN, SON

*Trichocentrum lindenii (Brogn.) M.W. Chase \& N.H. Williams CAM, CHIS, OAX, QROO, TAB, VER, YUC

Trichocentrum luridum (Lindl.) M.W. Chase \& N.H. Williams CAM, CHIS, GRO, HGO, JAL, MEX, MICH, NAY, OAX, PUE, SLP, SIN, TAB, VER

Trichocentrum microchilum (Bateman ex Lindl.) M.W. Chase \& N.H. Williams CHIS, OAX

*Trichocentrum natalieae (Balam \& Carnevali) R. Jiménez \& R. Solano JAL

Trichocentrum oerstedii (Rchb. f.) R. Jiménez \& Carnevali CAM, CHIS, OAX, QROO, TAB, VER, YUC

*Trichocentrum oestlundianum (L.O. Williams) M.W. Chase \& N.H. Williams COL, JAL, MICH, MOR, NAY, SIN

*Trichocentrum pachyphyllum (Hook.) R. Jiménez \& Carnevali CHIS, COL, GRO, JAL, MEX, MICH, MOR, NAY, OAX, VER

*Trichocentrum pendulum (Carnevali \& Cetzal) R. Jiménez \& R. Solano COL, DGO, JAL, NAY

*Trichocentrum stramineum (Bateman ex Lindl.) M.W. Chase \& N.H. Williams HGO, PUE, VER

*Trichocentrum tapiae (Balam \& Carnevali) J.M.H.Shaw CAM

*Trichocentrum $\times$ teaboanum (R. Jiménez, Carnevali \& J.L. Tapia) R. Jiménez \& Carnevali CAM, CHIS, QROO, YUC

*Trichocentrum yucatanense (Cetzal-Ix \& Carnevali) R. Jiménez \& R. Solano YUC 
*Trichopilia galeottiana A. Rich. \& Galeotti CHIS, OAX

Trichopilia subulata (Sw.) Rchb. f. CHIS

*Trichopilia tortilis Lindl. CHIS, OAX, VER

Trichosalpinx blaisdellii (S. Watson) Luer CHIS, GRO, OAX, PUE, VER

Trichosalpinx cedralensis (Ames) Luer CHIS

Trichosalpinx ciliaris (Lindl.) Luer CAM, CHIS, COL, GRO, JAL, NAY, OAX, PUE, QROO, SLP, TAB, VER

Trichosalpinx dura (Lindl.) Luer CHIS, OAX, QROO, VER

Trichosalpinx memor (Rchb. f.) Luer CHIS

*Trichosalpinx nageliana Soto Arenas GRO, OAX

*Trichosalpinx pringlei (Schltr.) Luer CHIS, GRO, OAX

*Trichosalpinx tamayoana Soto Arenas COL, DGO, GRO, JAL,

NAY, OAX, SIN, ZAC

Trichosalpinx trachystoma (Schltr.) Luer CHIS

Trigonidium egertonianum Bateman ex Lindl. CAM, CHIS, GRO, OAX, QROO, TAB, VER

Triphora debilis (Schltr.) Schltr. CHIS

Triphora trianthophora (Sw.) Rydb. BCS, CHIS, CHIH, COL, CDMX, GRO, HGO, JAL, MEX, MICH, MOR, NAY, NLE, OAX, PUE, QRO, SLP, SIN, VER

Triphora yucatanensis Ames CAM, CHIS, OAX, QROO, TAB, VER, YUC

*Tropidia polystachya (Sw.) Ames CAM, CHIS, OAX, QROO, SLP, TAMS, VER

Vanilla cribbiana Soto Arenas CHIS, OAX

Vanilla hartii Rolfe CHIS

Vanilla helleri A.D. Hawkes OAX

Vanilla inodora Schiede CHIS, COL, GRO, JAL, OAX, PUE, TAB, VER

Vanilla insignis Ames CAM, CHIS, OAX, PUE, QROO, TAB, VER, YUC

Vanilla odorata C. Presl. CAM, CHIS, OAX, QROO, TAB, VER

Vanilla phaeantha Rchb. f. QROO, YUC

Vanilla planifolia G. Jacks. CAM, CHIS, OAX, PUE, QRO, QROO, SLP, TAB, VER, YUC

Vanilla pompona Schiede CHIS, COL, GRO, JAL, MICH, NAY, OAX, VER

Warrea costaricensis Schltr. CHIS

Wullschlaegelia aphylla (Sw.) Rchb. f. CHIS, OAX, VER

Xylobium elongatum (Lindl. \& Paxton) Hemsl. CHIS, OAX, VER

Xylobium foveatum (Lindl.) G. Nicholson CHIS, OAX, VER

Xylobium sulfurinum (Lem.) Schltr. CHIS, OAX, VER

\section{Family Orobanchaceae}

Agalinis calycina Pennell COAH, TAMS

*Agalinis gypsophila B.L. Turner NLE

Agalinis harperi Pennell QROO, VER

Agalinis heterophylla (Nutt.) Small TAMS

Agalinis hispidula (Mart.) D'Arcy CHIS, TAB

Agalinis peduncularis (Benth.) Pennell AGS, CHIS, CHIH, CDMX, DGO, GTO, HGO, JAL, MEX, MICH, MOR, NAY, OAX, PUE, QRO, SLP, SIN, TAMS, TLAX, VER, ZAC Agalinis spiciflora (Engelm.) Pennell CHIS, TAB, VER *Agalinis wrightii (A. Gray) Tidestr. CHIH, SON
*Aureolaria greggii (S. Watson) Pennell COAH, DGO, NLE, SLP, TAMS, ZAC

Buchnera americana L. CAM, CHIS, QROO, SLP, TAB, TAMS, VER, YUC

*Buchnera lithospermifolia Kunth CHIS, TAB, VER

*Buchnera mexicana Hemsl. CHIS, GTO, GRO, JAL, MEX, MICH, NAY, OAX, VER

Buchnera obliqua Benth. CHIH, COAH, COL, CDMX, DGO, GTO, GRO, HGO, JAL, MEX, MICH, NAY, NLE, OAX, PUE, QRO, SON, TLAX, VER, ZAC

Buchnera palustris (Aubl.) Spreng. TAB

Buchnera pusilla Kunth AGS, BCS, CAM, CHIS, CHIH, COAH, COL, CDMX, DGO, GTO, GRO, HGO, JAL, MEX, MICH, MOR, NAY, OAX, PUE, QRO, QROO, SLP, SIN, SON, TAB, VER, YUC, ZAC

*Buchnera retrorsa Philcox GTO, MICH, VER

Castilleja affinis Hook. \& Arn. BCN

*Castilleja albobarbata Iltis \& G.L. Nesom COL, JAL

Castilleja angustata (B.L. Rob. \& Seaton) Eastw. MICH

*Castilleja angustifolia (Nutt.) G. Don CHIH, DGO, JAL, MOR, SIN, SON, VER

Castilleja arvensis Schltdl. \& Cham. AGS, BCN, CHIS, COL, CDMX, DGO, GTO, GRO, HGO, JAL, MEX, MICH, MOR, NAY, NLE, OAX, PUE, QRO, QROO, SLP, SIN, TAMS, TLAX, VER, YUC, ZAC

*Castilleja aspera Eastw. CHIH, DGO, SON

Castilleja attenuata (A. Gray) T.I. Chuang \& Heckard BCN

*Castilleja aurea B.L. Rob. \& Greenm. GRO, MEX, MOR, OAX

*Castilleja auriculata Eastw. COL, GRO, JAL, MEX, OAX, PUE

*Castilleja bella Standl. COAH, NLE

*Castilleja bryantii Brandegee BCN, BCS

Castilleja californica Abrams BCN

* Castilleja conzattii Fernald OAX

* Castilleja cryptandra Eastw. COL, DGO, JAL, NAY

Castilleja ctenodonta Eastw. CHIS, GRO, OAX

* Castilleja chiapensis Brandegee CHIS

*Castilleja chlorosceptron G.L. Nesom CHIH, DGO, SIN

*Castilleja dendridion G.L. Nesom OAX

Castilleja densiflora (Benth.) T.I. Chuang \& Heckard BCN

*Castilleja durangensis G.L. Nesom DGO, SIN

Castilleja exilis A. Nelson BCN, SON

Castilleja exserta (A. Heller) T.I. Chuang \& Heckard BCN, SON

*Castilleja falcata Eastw. PUE, VER

Castilleja foliolosa Hook. \& Arn. BCN

*Castilleja fruticosa Moran BCN

*Castilleja galehintoniae G.L. Nesom NLE

* Castilleja genevievana G.L. Nesom COAH

*Castilleja gonzalezii G.L. Nesom DGO

*Castilleja gracilis Benth. COL, CDMX, GTO, GRO, HGO,

JAL, MEX, MICH, MOR, NAY, OAX, QRO

*Castilleja guadalupensis Brandegee BCN

*Castilleja hidalgensis J.M. Egger HGO, VER

*Castilleja hirsuta M. Martens \& Galeotti HGO, PUE, VER

*Castilleja holmgrenii J.M. Egger DGO, SIN 
Castilleja integra A. Gray BCN, CHIH, COAH, DGO, HGO, NLE, QRO, SLP, SON, VER, ZAC

Castilleja integrifolia L. f. CHIS, COAH, COL, GRO, HGO, JAL, MEX, MICH, NLE, OAX, QRO, SLP, TAMS, VER, ZAC Castilleja jepsonii Bacig. \& Heckard BCN

*Castilleja jiquilpana G.L. Nesom JAL, MICH

Castilleja lanata A. Gray BCN, CHIH, COAH, DGO, GTO,

NLE, SLP, SON, ZAC

*Castilleja lebgueana G.L. Nesom CHIH, SON

*Castilleja linifolia N.H. Holmgren DGO, SIN, ZAC

*Castilleja lithospermoides Kunth AGS, CHIS, CHIH, COAH, COL, CDMX, DGO, GTO, HGO, JAL, MEX, MICH, MOR, NAY, NLE, OAX, PUE, QRO, SLP, SON, TAMS, TLAX, VER, ZAC

*Castilleja longiflora Kunze MICH, OAX, PUE

* Castilleja macrostigma B.L. Rob. MEX, MICH

Castilleja martinii Abrams BCN

*Castilleja mcvaughii N.H. Holmgren COL, JAL

Castilleja mexicana (Hemsl.) A. Gray AGS, CHIH, COAH,

DGO, NLE, SLP, TAMS, ZAC

*Castilleja moranensis Kunth CDMX, HGO, JAL, MEX, MICH, MOR, PUE, SLP, VER

Castilleja nervata Eastw. CHIH, COAH, COL, DGO, JAL, MICH, NAY, NLE, OAX, SLP, SIN, SON, TAMS

*Castilleja nitricola Eastw. SLP

*Castilleja nivibractea G.L. Nesom OAX

* Castilleja obovata Benth. VER

* Castilleja ornata Eastw. CHIH, DGO, SIN, SON

*Castilleja ortegae Standl. CHIH, DGO, SIN, SON

*Castilleja palmeri B.L. Rob. DGO

*Castilleja papilionacea G.L. Nesom TAMS

Castilleja patriotica Fernald CHIH, DGO, SON

Castilleja pectinata M. Martens \& Galeotti JAL, MEX, PUE,

VER

*Castilleja perelegans G.L. Nesom DGO, SIN

* Castilleja porphyrosceptron G.L. Nesom TAMS

*Castilleja pterocaulon N.H. Holmgren COL, JAL

*Castilleja quiexobrensis G.L. Nesom OAX

*Castilleja racemosa (Breedlove \& Heckard) T.I. Chuang \& Heckard SIN

*Castilleja rhizomata N.H. Holmgren CHIH, DGO

*Castilleja rigida Eastw. CHIH, COAH, DGO, NLE, QRO,

SLP, VER, ZAC

*Castilleja roei Crosswh. DGO, NAY, SIN

*Castilleja saltensis Eastw. DGO, SIN

*Castilleja scorzonerifolia Kunth AGS, COAH, CDMX, DGO, GTO, GRO, HGO, JAL, MEX, MICH, MOR, NLE, OAX, PUE, QRO, SLP, SIN, TAMS, TLAX, VER, ZAC

Castilleja sessiliflora Pursh AGS, COAH, JAL, NLE, SON,

ZAC

*Castilleja socorrensis Moran COL

*Castilleja speciosa M. Martens \& Galeotti VER

*Castilleja sphaerostigma B.L. Rob. DGO, SIN, ZAC

*Castilleja spiranthoides Standl. DGO, SIN

Castilleja stenantha A. Gray BCN

*Castilleja stenophylla M.E. Jones CHIH, DGO, JAL, SON

*Castilleja stipifolia G.L. Nesom JAL, MEX, MICH
*Castilleja subalpina Eastw. OAX

Castilleja tapeinoclada Loes. CHIS, OAX

Castilleja tenuiflora Benth. AGS, BCN, BCS, CHIH, COAH, COL, CDMX, DGO, GTO, GRO, HGO, JAL, MEX, MICH, MOR, NAY, NLE, OAX, PUE, QRO, SLP, SIN, SON, TAMS, TLAX, VER, ZAC

*Castilleja tenuifolia M. Martens \& Galeotti COL, DGO, GTO, GRO, JAL, MEX, MICH, MOR, OAX, SIN

*Castilleja toluccensis Kunth MEX, MICH, PUE, TLAX, VER

*Castilleja venusta Rzed. GRO

*Castilleja zempoaltepetlensis G.L. Nesom OAX

*Clevelandia beldingii (Greene) Greene BCS

Conopholis alpina Liebm. AGS, BCS, CHIS, CHIH, COAH, CDMX, DGO, GTO, GRO, HGO, JAL, MEX, MICH, MOR, NLE, OAX, PUE, QRO, SLP, SIN, SON, TAMS, TLAX, VER, ZAC

Cordylanthus filifolius Nutt. ex Benth. BCN

Cordylanthus maritimus Nutt. BCN

Cordylanthus nevinii A. Gray BCN

Cordylanthus orcuttianus A. Gray BCN

Cordylanthus rigidus (Benth.) Jeps. BCN

Cordylanthus wrightii A. Gray CHIH, SON

Epifagus virginiana (L.) W.P.C. Barton TAMS

*Escobedia crassipes Pennell CHIH, DGO, JAL, MICH, NAY, PUE, SIN, VER, ZAC

Escobedia grandiflora (L. f.) Kuntze CHIH, JAL, MOR, OAX

Escobedia guatemalensis Loes. CHIS

Escobedia laevis Schltdl. \& Cham. CHIS, CHIH, COL, CDMX, DGO, JAL, MEX, MICH, NAY, NLE, OAX, PUE, QRO, SLP, TAB, VER, ZAC

Escobedia longiflora Pennell CHIS, CHIH, GRO, JAL, MOR, OAX, SON, VER, ZAC

*Escobedia michoacana E. Carranza \& C. Medina JAL, MICH

* Escobedia peduncularis Pennell CHIH, DGO, MICH, NAY

*Lamourouxia brachyantha Greenm. CDMX, HGO, JAL, MEX, QRO

*Lamourouxia colimae W.R. Ernst \& Baad COL, JAL

*Lamourouxia dasyantha (Cham. \& Schltdl.) W.R. Ernst COAH, CDMX, DGO, GTO, GRO, HGO, JAL, MEX, MICH, NLE, OAX, PUE, QRO, SLP, TAMS, TLAX, VER, ZAC

Lamourouxia dependens Benth. CHIS, GRO

*Lamourouxia gracilis B.L. Rob. \& Greenm. GRO, MOR

*Lamourouxia jaliscana W.R. Ernst \& Baad JAL

Lamourouxia longiflora Benth. CHIS, DGO, GRO, JAL, MOR, OAX, ZAC

*Lamourouxia macrantha M. Martens \& Galeotti CHIS, DGO, GTO, JAL, OAX

*Lamourouxia microphylla M. Martens \& Galeotti OAX

Lamourouxia multifida Kunth AGS, CHIS, COL, CDMX, DGO, GTO, GRO, HGO, JAL, MEX, MICH, MOR, NAY, OAX, PUE, QRO, SLP, SIN, TAMS, TLAX, VER, ZAC

*Lamourouxia nelsonii B.L. Rob. \& Greenm. CDMX, GRO, HGO, MEX, MOR, OAX, PUE, QRO, SLP

*Lamourouxia ovata M. Martens \& Galeotti DGO, OAX, PUE

*Lamourouxia paneroi B.L. Turner OAX

* Lamourouxia parayana W.R. Ernst MEX

*Lamourouxia pringlei B.L. Rob. \& Greenm. ex Pringle HGO, 
OAX, PUE, QRO, SLP, VER

*Lamourouxia rhinanthifolia Kunth AGS, CDMX, DGO, GTO, GRO, HGO, JAL, MEX, MICH, MOR, NAY, NLE, OAX, PUE, QRO, SLP, SIN, TAMS, TLAX, VER, ZAC

*Lamourouxia smithii B.L. Rob. \& Greenm. OAX, PUE

*Lamourouxia tenuifolia M. Martens \& Galeotti AGS, CDMX, MEX, OAX, QRO, SLP, ZAC

Lamourouxia viscosa Kunth AGS, CHIS, CHIH, COL, DGO, GTO, GRO, HGO, JAL, MEX, MICH, MOR, NAY, NLE, OAX, PUE, QRO, SLP, SIN, SON, TAMS, VER, ZAC

Lamourouxia xalapensis Kunth CHIS, COL, CDMX, GRO, HGO, JAL, MEX, MICH, OAX, PUE, TLAX, VER

Melasma physalodes (D. Don) Melch. CHIS, GRO, HGO, MEX, MICH, MOR, OAX, QRO, SLP, TAMS, VER

*Ophiocephalus angustifolius Wiggins BCN

Orobanche bulbosa (A. Gray) G. Beck BCN

Orobanche cooperi (A. Gray) Heller BCN, BCS, CHIH, COAH, DGO, SIN, SON

*Orobanche dugesii (S. Watson) Munz COL, GTO, HGO, JAL, MEX, MICH, OAX, PUE, QRO

Orobanche fasciculata Nutt. BCN

Orobanche grayana Beck BCN, BCS

Orobanche ludoviciana Nutt. AGS, BCN, BCS, CHIH, COAH, DGO, HGO, JAL, MEX, MICH, NAY, NLE, PUE, OAX, QRO, SLP, SIN, SON, TAMS, VER, ZAC

Orobanche multicaulis Brandegee BCN, BCS, COAH, NLE, OAX, SLP, SON

Orobanche parishii (Jepson) Heckard BCN

Pedicularis angustifolia Benth. CHIH, DGO, JAL, MICH, OAX, SIN, ZAC

Pedicularis canadensis L. COAH, HGO, JAL, MEX, NLE, QRO, SLP, TAMS, TLAX, VER, ZAC

*Pedicularis chihuahuensis G.L. Nesom CHIH

Pedicularis densiflora Benth. BCN

*Pedicularis glabra McVaugh \& Mellich. COL, DGO, JAL, $\mathrm{MICH}$

*Pedicularis gordonii McVaugh \& Koptur JAL, ZAC

*Pedicularis hintonii McVaugh \& Mellich. GRO

*Pedicularis mexicana Zucc. ex Benth. CDMX, DGO, HGO, JAL, MEX, MICH, MOR, NAY, OAX, PUE, TLAX, ZAC

Pedicularis orizabae Schltdl. \& Cham. CDMX, DGO, HGO, JAL, MEX, MICH, OAX, PUE, TLAX, VER, ZAC

*Pedicularis tripinnata M. Martens \& Galeotti COL, DGO, JAL, MICH

Seymeria bipinnatisecta Seem. CHIH, COAH, DGO, JAL, NAY, NLE, SIN, SON

* Seymeria coahuilana (Pennell) Standl. COAH, DGO, NLE

* Seymeria cualana B.L. Turner JAL

* Seymeria decurva Benth. CHIH, COAH, CDMX, DGO, GTO, HGO, JAL, MEX, MICH, NLE, OAX, PUE, QRO, SLP, TLAX, VER, ZAC

* Seymeria deflexa Eastw. COAH, NLE

* Seymeria falcata B.L. Turner CHIH, COAH, NLE, SLP

* Seymeria gypsophila B.L. Turner NLE, TAMS

* Seymeria integrifolia Greenm. DGO, JAL, NAY

*Seymeria laciniata (M. Martens \& Galeotti) Standl. GRO, OAX, PUE
*Seymeria pailana B.L. Turner COAH

* Seymeria pennellii B.L. Turner DGO, JAL, TAMS

Seymeria scabra A. Gray CHIH, COAH, NLE, SLP, ZAC

* Seymeria sinaloana (Pennell) Standl. CHIH, SIN, SON

*Seymeria tamaulipana B.L. Turner NLE, TAMS

* Seymeria virgata (Kunth) Benth. ex DC. AGS, COAH, DGO, GTO, HGO, JAL, NLE, OAX, QRO, SLP, SIN, VER, ZAC

* Silviella prostrata (Kunth) Pennell CDMX, HGO, MEX, OAX, PUE, QRO, TLAX, VER

*Silviella serpyllifolia (Kunth) Pennell CDMX, HGO, MEX, PUE, SLP, TLAX, VER

\section{Family Oxalidaceae}

*Biophytum cowanii $\mathrm{T}$. Wendt TAB, VER

Biophytum dendroides (Kunth) DC. CHIS, OAX, TAB, VER

*Biophytum latifolium Durán-Esp. \& Lorea-Hern. VER

Oxalis alpina (Rose) Knuth BCS, CHIS, CHIH, COL, COAH, CDMX, DGO, GTO, GRO, HGO, JAL, MEX, MICH, MOR, OAX, PUE, QRO, SLP, SIN, SON, TLAX, VER, ZAC

* Oxalis angustifolia L. GRO, OAX

* Oxalis bipartita Rose CDMX, MEX, MOR, OAX

Oxalis caerulea (Small) Knuth CHIH, DGO

Oxalis clematodes Donn. Sm. CHIS

Oxalis corniculata L. AGS, BCN, BCS, CAM, CHIS, CHIH, COAH, COL, CDMX, DGO, GTO, GRO, HGO, JAL, MEX, MICH, MOR, NAY, NLE, OAX, PUE, QRO, QROO, SLP, SIN, SON, TAB, TAMS, TLAX, VER, YUC, ZAC

Oxalis decaphylla Kunth AGS, CHIH, COAH, CDMX, DGO, GTO, GRO, HGO, JAL, MEX, MICH, MOR, NLE, OAX, PUE, QRO, SLP, SON, TAMS, VER, ZAC

Oxalis dichondrifolia A. Gray COAH, NLE, SLP, TAMS

Oxalis dillenii Jacq. COAH, NLE, QRO, SLP, VER

Oxalis dimidiata Donn. Sm. CHIS, GRO, MEX, OAX

* Oxalis discolor Klotzsch CHIS, GRO, MEX, MOR, PUE

Oxalis divergens Benth. ex Lindl. CHIS, CHIH, CDMX, DGO, GTO, GRO, HGO, JAL, MEX, MICH, OAX, PUE, QRO, SLP, SON, TLAX, VER

Oxalis drummondii A. Gray BCN, BCS, CHIH, COAH, NLE, SLP, SON, TAMS

Oxalis frutescens L. CAM, CHIS, COAH, COL, DGO, GRO, JAL, MEX, MICH, NAY, NLE, OAX, PUE, QROO, SIN, TAB, TAMS, VER, YUC

*Oxalis gregaria (Rose) Knuth MEX, MICH, MOR, SIN

*Oxalis hernandezii DC. AGS, CHIH, COL, CDMX, DGO, GTO, GRO, HGO, JAL, MEX, MICH, MOR, NAY, OAX, PUE, QRO, SLP, SIN, SON, TLAX, ZAC

Oxalis jacquiniana Kunth CHIS, CHIH, COL, CDMX, GTO, GRO, HGO, JAL, MEX, MICH, MOR, OAX, PUE, SLP, TAMS, VER, ZAC

* Oxalis lasiandra Zucc. OAX

Oxalis latifolia Kunth CAM, CHIS, CHIH, COAH, COL, CDMX, DGO, GTO, GRO, HGO, JAL, MEX, MICH, MOR, NLE, OAX, PUE, QRO, QROO, SLP, SIN, SON, TAMS, TLAX, VER, YUC, ZAC

Oxalis lunulata Zucc. AGS, CHIS, CDMX, GTO, GRO, HGO, JAL, MEX, MICH, MOR, OAX, QRO, SLP, TLAX, VER, ZAC

*Oxalis macrocarpa (Small) Kunth COL, JAL 
* Oxalis madrensis $\mathrm{S}$. Watson COAH, NLE, SLP, TAMS

* Oxalis magnifica (Rose) R. Kunth MICH, OAX

Oxalis microcarpa Benth. CHIS, COL, JAL, MEX, MICH, NAY, SIN

* Oxalis morelosi Pérez-Calix MICH

Oxalis nelsonii (Small) R. Knuth CHIS, CDMX, GRO, HGO, MEX, OAX, TLAX

* Oxalis nudiflora Moc. \& Sessé BCS, SIN

* Oxalis primavera (Rose) Kunth JAL, NAY, SIN

Oxalis rhombifolia Jacq. CHIS, GRO, HGO, OAX, QRO, SLP,

VER

* Oxalis robusta (Rose ex Small) R. Knuth VER

Oxalis stricta L. CHIH, COAH, NLE, SON, TAMS

Oxalis tetraphylla Cav. AGS, CHIS, CDMX, GRO, HGO, JAL,

MEX, MICH, MOR, NAY, OAX, PUE, VER, ZAC

Oxalis violacea $\mathrm{L}$. CHIH, COAH, NLE, SON, TAMS

Oxalis yucatanensis (Rose) L. Riley CAM, CHIS, GRO, OAX,

QROO, SIN, TAB, YUC

\section{Family Papaveraceae}

Argemone aenea G.B. Ownbey COAH, NLE, TAMS

*Argemone alba $\mathrm{F}$. Lestib. CHIH, SON

Argemone albiflora Hornem. CHIH, COAH, NLE

Argemone chisosensis G.B. Ownbey CHIH, COAH

*Argemone echinata G.B. Ownbey COAH, NLE, SLP

*Argemone fruticosa Thurb. ex A. Gray COAH, DGO, NLE,

SLP

Argemone gracilenta Greene BCN, BCS, SON

*Argemone grandiflora Sweet COAH, GTO, HGO, NLE, QRO,

SLP, SON, TAMS, VER

Argemone intermedia Sweet BCN, BCS, CHIH, DGO, GTO, NLE, QRO, SLP, SON, ZAC

Argemone mexicana L. AGS, BCN, BCS, CAM, CHIS, CHIH, COAH, COL, DGO, GTO, GRO, HGO, JAL, MEX, MICH, MOR, NAY, NLE, OAX, PUE, QRO, QROO, SLP, SIN, SON, TAB, TAMS, TLAX, VER, YUC, ZAC

Argemone munita Durand \& Hilg. BCN

Argemone ochroleuca Sweet AGS, BCN, BCS, CHIS, CHIH, COAH, COL, CDMX, DGO, GTO, GRO, HGO, JAL, MEX, MICH, MOR, NAY, NLE, OAX, PUE, QRO, QROO, SLP, SIN, SON, TAMS, TLAX, VER, ZAC

Argemone platyceras Link \& Otto BCN, BCS, CHIH, COAH, CDMX, DGO, GRO, HGO, JAL, MEX, MICH, MOR, NLE, OAX, PUE, QRO, SON, TAMS, TLAX, VER, ZAC

Argemone pleiacantha Greene CHIH, SON

*Argemone subalpina J.A. McDonald NLE, TAMS

*Argemone subintegrifolia G.B. Ownbey BCN

*Argemone superba G.B. Ownbey GTO, HGO, QRO, SLP

*Argemone turnerae A.M. Powell CHIH, COAH

Bocconia arborea $\mathrm{S}$. Watson BCN, CHIS, COL, DGO, GTO, GRO, HGO, JAL, MEX, MICH, MOR, NAY, NLE, OAX, PUE, SLP, SIN, SON, TAMS, VER, ZAC

Bocconia frutescens L. CHIS, COAH, COL, GTO, GRO, HGO, JAL, MEX, MICH, MOR, NAY, NLE, OAX, PUE, QRO, SLP, SIN, TAMS, VER

Bocconia glaucifolia Hutch. CHIS

Bocconia gracilis Hutch. CHIS
*Bocconia hintoniorum B.L. Turner OAX

Bocconia integrifolia Bonpl. HGO, OAX, VER

*Bocconia latisepala S. Watson COAH, NLE

Bocconia vulcanica Donn. Sm. CHIS

Corydalis aurea Willd. BCN, CHIH, DGO, NLE, SIN, SON

Corydalis curvisiliqua (A. Gray) Engelm. ex A. Gray CHIH

Corydalis micrantha (Engelm.) A. Gray TAMS, VER

Corydalis pseudomicrantha Fedde COAH, GTO, NLE, PUE, QRO, SLP, TAMS, VER

Dendromecon rigidum Benth. $\mathrm{BCN}$

Eschscholzia californica Cham. AGS, BCN, CHIS, MEX, OAX, SIN

Eschscholzia elegans Greene BCN

Eschscholzia mexicana Greene BCN, BCS, CHIH, SON

Eschscholzia minutiflora S. Watson BCN, BCS, SON

* Eschscholzia palmeri Rose BCN

Eschscholzia parishii Greene BCN

Eschscholzia ramosa (Greene) Greene BCN

*Hunnemannia fumariifolia Sweet COAH, GTO, HGO, NLE, OAX, PUE, SLP, TAMS, VER, ZAC

*Hunnemannia hintoniorum G.L. Nesom NLE, TAMS

Meconella denticulata Greene BCN

Platystemon californicus Benth. BCN, SON

Romneya coulteri Harvey BCN

Romneya trichocalyx Eastw. BCN

Stylomecon heterophylla (Benth.) G. Taylor BCN

Family Passifloraceae

Erblichia odorata Seem. CHIS, NAY, OAX, PUE, QROO, SIN, TAB, TAMS, VER

Passiflora adenopoda DC. CHIS, HGO, MEX, OAX, PUE, QRO, SLP, TAMS, VER

Passiflora affinis Engelm. COAH, NLE, TAMS

Passiflora alata Curtis CHIS, OAX

Passiflora ambigua Hemsl. CHIS, OAX, TAB, VER

Passiflora apetala Killip CHIS

* Passiflora arida (Mast. \& Rose) Killip BCN, BCS, SIN, SON

Passiflora bicornis Mill. CAM, CHIS, OAX, QROO, VER, YUC

Passiflora biflora Lam. CAM, CHIS, COL, GRO, HGO, JAL, MEX, MICH, MOR, NAY, OAX, PUE, QRO, QROO, SLP, SIN, TAB, TAMS, VER, YUC

Passiflora brevipes Killip CAM, CHIS, QROO

Passiflora bryonoides Kunth AGS, CHIS, CHIH, DGO, GTO, HGO, JAL, MEX, MICH, MOR, NAY, OAX, PUE, QRO, SLP, SIN, SON, TAMS, ZAC

Passiflora capsularis L. HGO, JAL, OAX, PUE, QRO, QROO, SLP, VER

Passiflora clypeophylla Mast. CHIS

Passiflora cobanensis Killip CAM, CHIS, QROO

*Passiflora colimensis Mast. \& Rose COL, GRO, JAL, MEX, MICH, NAY

*Passiflora complanata J.M. MacDougal OAX

* Passiflora conzattiana Killip CHIS, OAX, PUE, QRO, QROO, SLP, TAMS, VER

Passiflora coriacea Juss. CAM, CHIS, COL, GRO, HGO, JAL, MEX, MICH, MOR, NAY, OAX, PUE, QRO, QROO, SLP, SIN, 
TAB, TAMS, VER, YUC

Passiflora costaricensis Killip OAX, VER

Passiflora dolichocarpa Killip CHIS

*Passiflora exsudans Zucc. AGS, CAM, COAH, COL, CDMX, DGO, GTO, GRO, HGO, JAL, MEX, MICH, MOR, NLE, OAX, PUE, QRO, SLP, TAMS, TLAX, VER, ZAC

Passiflora filipes Benth. CHIS, COL, GRO, JAL, MEX, MICH, NAY, OAX, PUE, TAMS, VER

Passiflora foetida L. BCS, CAM, CHIS, CHIH, COL, COAH, GTO, GRO, HGO, JAL, MEX, MICH, MOR, NAY, NLE, OAX, PUE, QRO, QROO, SLP, SIN, SON, TAB, TAMS, VER, YUC, ZAC

* Passiflora fruticosa Killip BCS

*Passiflora goniosperma Killip. COL, GRO, JAL, MICH, OAX Passiflora hahnii (E. Fourn.) Mast. CHIS, GRO, OAX, PUE, TAB, VER

Passiflora helleri Peyr. CHIS, COL, JAL, MICH, OAX, PUE, QROO, TAB, VER, YUC

Passiflora holosericea L. CHIS, COL, GRO, JAL, MICH, NAY, OAX, SIN, VER

Passiflora jorullensis Kunth CHIS, COL, GRO, JAL, MEX, MICH, NAY, OAX

*Passiflora juliana J.M. MacDougal COL, GRO, JAL, MICH

* Passiflora karwinskii Mast. JAL, MICH, OAX, PUE, ZAC

Passiflora lancearia Mast. CHIS

* Passiflora lauana J.M. MacDougal PUE

* Passiflora liebmannii Mast. OAX, PUE

Passiflora ligularis Juss. CHIS, MEX, MICH, OAX, PUE, VER

* Passiflora manantlanensis J.M. MacDougal COL, GRO, JAL

Passiflora mayarum J.M. MacDougal CAM, CHIS, QROO

* Passiflora mcvaughiana J.M. MacDougal COL, JAL, OAX

Passiflora membranacea Benth. CHIS, GRO, JAL, NAY, OAX, VER

Passiflora mexicana Juss. CHIS, CHIH, COL, GRO, JAL, MEX, MICH, MOR, NAY, OAX, PUE, SIN, SON, VER, ZAC

*Passiflora microstipula J.L. Gilbert \& J.M. MacDougal OAX, VER

Passiflora morifolia Mast. CHIS, GRO

Passiflora nelsonii Mast. \& Rose CHIS, VER

* Passiflora oaxacensis J.M. MacDougal OAX

Passiflora obovata Killip CAM, CHIS, OAX, QROO, YUC

Passiflora oerstedii Mast. CHIS, VER

Passiflora ornithoura Mast. CAM, CHIS, GRO, NAY, QROO

Passiflora palmeri Rose BCN, BCS, CAM, QROO, SON, YUC

Passiflora pavonis Mast. CHIS, COL, GRO, JAL, MEX, MICH, MOR, OAX

Passiflora pedata L. CAM, QROO, YUC

Passiflora pilosa Ruiz \& Pav. ex DC. CHIS, GRO, HGO, OAX, QRO, VER

Passiflora platyloba Killip CHIS, QROO

* Passiflora podadenia Killip COL, JAL, MICH, NAY

Passiflora porphyretica Mast. CHIS, COL, GRO, JAL, MEX, MICH, NAY, OAX, SIN, SON

Passiflora prolata Mast. CAM, CHIS, QROO

Passiflora pusilla J.M. MacDougal OAX

* Passiflora quercetorum Killip CHIH, SON

Passiflora quetzal J.M. MacDougal CHIS
Passiflora quinquangularis S. Calderón ex J.M. MacDougal OAX

Passiflora rovirosae Killip CAM, CHIS, OAX, PUE, QROO, TAB, VER

Passiflora sanctae-mariae J.M. MacDougal CHIS

Passiflora seemannii Griseb. CHIS

Passiflora serratifolia L. CAM, CHIS, HGO, OAX, PUE, QRO, QROO, SLP, TAB, TAMS, VER, YUC

Passiflora sexflora Juss. CHIS, GRO, HGO, MOR, OAX, PUE, QRO, SLP, VER

Passiflora sicyoides Schltdl. \& Cham. CHIS, GRO, HGO, JAL, MEX, MICH, NAY, NLE, OAX, PUE, QRO, SLP, VER

Passiflora standleyi Killip CHIS

Passiflora suberosa L. AGS, CAM, CHIS, CHIH, COAH, COL, DGO, GTO, GRO, HGO, JAL, MEX, MICH, MOR, NAY, NLE, OAX, PUE, QRO, QROO, SLP, SIN, SON, TAMS, VER, YUC, ZAC

Passiflora subpeltata Ortega CAM, CHIS, CDMX, GRO, HGO, JAL, MEX, MICH, MOR, NAY, OAX, PUE, QRO, SLP, VER, ZAC

Passiflora tarminiana Coppens \& V.E. Barney CHIS, CDMX, MICH, OAX

Passiflora tenuiloba Engelm. COAH, NLE, SLP, TAMS

* Passiflora trisetosa DC. VER

* Passiflora uncinata J.M. MacDougal GRO

* Passiflora viridiflora Cav. GRO, JAL, MICH, OAX

Passiflora xiikzodz J. MacDougal CAM, CHIS, QROO, YUC

* Passiflora yucatanensis Killip CAM, QROO, YUC

Piriqueta cistoides (L.) Griseb. CAM, CHIS, GRO, OAX, QROO, TAB, VER, YUC

*Piriqueta mexicana Fryxell \& S.D. Koch GRO, MICH

*Piriqueta mortonii S.D. Koch \& Fryxell OAX

*Turnera callosa Urb. GTO, JAL, MEX, MICH, MOR, ZAC

Turnera diffusa Willd. ex Schult. BCN, BCS, CAM, CHIS, CHIH, COAH, COL, GTO, GRO, HGO, JAL, MICH, MOR, NAY, NLE, OAX, PUE, QRO, QROO, SLP, SIN, SON, TAMS, VER, YUC, ZAC

Turnera odorata L. Rich. CAM, QROO

Turnera pumilea L. BCS, CHIS, CHIH, COAH, JAL, MICH, NAY, NLE, OAX, PUE, QRO, SLP, SIN, SON, TAB, TAMS, VER

Turnera ulmifolia L. CAM, CHIS, CHIH, COL, DGO, GRO, JAL, MEX, MICH, MOR, NAY, OAX, QROO, SIN, SON, TAB, TAMS, VER, YUC, ZAC

*Turnera violacea Brandegee CHIS

\section{Family Pentaphylacaceae}

* Cleyera cernua (Tul.) Kobuski OAX, VER

* Cleyera integrifolia (Benth.) Choisy CHIS, COL, DGO, GRO, HGO, JAL, MEX, MICH, MOR, NAY, OAX, SIN, VER, ZAC * Cleyera serrulata Choisy HGO, VER

Cleyera theoides (Sw.) Choisy CHIS, HGO, OAX, PUE, QRO, SLP, VER

*Cleyera velutina B.M. Barthol. GRO, MICH, OAX

Freziera candicans Tul. CHIS, GRO, OAX

Freziera grisebachii Krug \& Urb. CHIS, OAX, VER

Freziera guatemalensis (Donn. Sm.) Kobuski CHIS, OAX, VER 
* Symplococarpon flavifolium Lundell CHIS

Symplococarpon purpusii (Brandegee) Kobuski CHIS, COL, GRO, JAL, MEX, MICH, NAY, OAX, VER

*Ternstroemia dentisepala B.M. Barthol. COL, DGO, JAL, NAY, OAX, SIN

*Ternstroemia hemsleyi Hochr. OAX, PUE

*Ternstroemia huasteca B.M. Barthol. HGO, PUE, QRO, SLP, VER

Ternstroemia lineata DC. AGS, CHIS, COL, DGO, GRO, HGO, JAL, MEX, MICH, MOR, NAY, OAX, SIN, TAMS, VER

*Ternstroemia maltbyi Rose JAL, NAY, SIN

Ternstroemia sphaerocarpa (Rose) Melchior CHIS, GRO, OAX Ternstroemia sylvatica Schltdl. \& Cham. CDMX, GTO, GRO, HGO, JAL, MEX, MICH, MOR, OAX, PUE, QRO, SLP, TAMS, VER

Ternstroemia tepezapote Schltdl. \& Cham. CAM, CHIS, GRO, HGO, MEX, MICH, OAX, PUE, QRO, QROO, SLP, TAB, VER

\section{Family Peraceae}

Pera barbellata Standl. CHIS, OAX, TAB, VER

\section{Family Petenaeaceae}

Petenaea cordata Lundell CHIS, TAB

\section{Family Phrymaceae}

Diplacus stellatus Kellogg BCN, BCS

Erythranthe arvensis (Greene) G.L. Nesom BCN

*Erythranthe brevinasuta G.L. Nesom BCN, BCS

Erythranthe calciphila (Gentry) G.L. Nesom CHIH, DGO, SIN, SON

Erythranthe cordata (Greene) G.L. Nesom CHIH, COAH, SON Erythranthe dentiloba (B.L. Rob. \& Fernald) G.L. Nesom AGS, BCS, CHIH, DGO, JAL, SIN, SON

Erythranthe geyeri (Torr.) G.L. Nesom BCN, BCS, CHIH, SON Erythranthe glabrata (Kunth) G.L. Nesom AGS, BCN, BCS, CHIS, CHIH, COAH, COL, CDMX, DGO, GTO, GRO, HGO, JAL, MEX, MICH, MOR, NAY, NLE, OAX, QRO, PUE, QRO, SLP, SIN, SON, TAMS, TLAX, VER, ZAC

Erythranthe guttata (Fisch. ex DC.) G.L. Nesom BCN, BCS, CHIH, COAH, SON

Erythranthe inamoena (Greene) G.L. Nesom CHIH, COAH

*Erythranthe lagunensis G.L. Nesom BCS

*Erythranthe madrensis (Seem.) G.L. Nesom CHIH, DGO, SIN

Erythranthe nasuta (Greene) G.L. Nesom BCN, SON

*Erythranthe nelsonii (A.L. Grant) G.L. Nesom \& N.S. Fraga DGO

Erythranthe orizabae (Benth.) G.L. Nesom CHIS, HGO, OAX, PUE, VER

*Erythranthe pallens (Greene) G.L. Nesom CHIH, DGO, MEX, MICH, SIN, SON

Erythranthe parvula (Wooton \& Standl.) G.L. Nesom CHIH, SON

*Erythranthe pennellii (Gentry) G.L. Nesom CHIH, SIN, SON *Erythranthe rupestris (Greene) G.L. Nesom \& N.S. Fraga MEX, MOR

Erythranthe unimaculata (Pennell) G.L. Nesom CHIH, SON

* Erythranthe visibilis G.L. Nesom MICH

*Hemichaena coulteri (A. Gray) Thieret GTO, HGO, QRO,

\section{SLP}

Hemichaena fruticosa Benth. CHIS, GRO, HGO, OAX, TAMS *Hemichaena levigata (B.L. Rob. \& Greenm.) Thieret OAX, PUE, VER

Hemichaena rugosa (Benth.) Thieret CHIS

*Hemichaena spinulosa (S. Watson) Thieret COAH, DGO, NLE

Leucocarpus perfoliatus (Kunth) Benth. CHIS, COL, GRO, HGO, JAL, OAX, PUE, QRO, SLP, VER

Mimulus aridus (Abrams) A.L. Grant BCN

Mimulus aurantiacus Curtis BCN, MOR

Mimulus bigelovii (A. Gray) A. Gray BCN

Mimulus brevipes Benth. BCN

Mimulus cardinalis Douglas ex Benth. BCN, CHIH, DGO, HGO, JAL, MEX, MOR, NAY, QRO, SIN, SON, VER, ZAC

Mimulus clevelandii Brandegee BCN

Mimulus diffusus A.L. Grant BCN

* Mimulus exiguus A. Gray BCN

Mimulus floribundus Douglas ex Lindl. BCN, BCS, CHIH, COL, JAL, SIN, SON, ZAC

Mimulus fremontii (Benth.) A. Gray BCN

Mimulus guttatus Fisch. ex DC. BCN, BCS, CHIH, SON

Mimulus latidens (A. Gray) Greene BCN

Mimulus latifolius A. Gray BCN

Mimulus longiflorus (Nutt.) A.L. Grant BCN

*Mimulus minimus E. Pérez-Calix \& Zamudio MEX, MICH

Mimulus pilosus (Benth.) S. Watson BCN

Mimulus puniceus (Nutt.) Steud. BCN

*Mimulus purpureus A.L. Grant BCN

Mimulus rubellus A. Gray BCN, SON

Phryma leptostachya L. COAH

\section{Family Phyllanthaceae}

Andrachne microphylla (Lam.) Baill. BCN, BCS, SON

*Astrocasia diegoae J. Jiménez Ram. \& Mart. Gord. GRO

*Astrocasia neurocarpa (Müll. Arg.) I.M. Johnst. ex Standl. GRO, HGO, NAY, OAX, PUE, QRO, SLP, TAMS

*Astrocasia peltata Standl. COL, GRO, JAL, NAY, SIN

Astrocasia tremula (Griseb.) G.L. Webster CAM, CHIS, COL, JAL, NAY, QROO, YUC

Hieronyma alchorneoides Allemao CHIS

Hieronyma oblonga (Tul.) Müll. Arg. CHIS, OAX, PUE, VER Margaritaria nobilis L. f. CAM, CHIS, COL, GRO, JAL, MEX, MICH, NAY, OAX, QRO, QROO, SLP, SIN, TAB, TAMS, VER, YUC

Meineckia bartlettii (Standl.) G.L. Webster CHIS, COL, JAL Meineckia neogranatensis (Müll. Arg.) G.L. Webster CHIS

Phyllanthopsis arida (Warnock \& M.C. Johnst.) Voronts. \& Petra Hoffm. CHIH, COAH

Phyllanthus acuminatus Vahl BCS, CAM, CHIS, COL, GRO, HGO, JAL, MICH, NAY, OAX, QROO, SLP, SIN, SON, TAB, TAMS, VER, YUC

*Phyllanthus adenodiscus Müll. Arg. CHIS, HGO, JAL, NAY, PUE, QRO, SLP, TAMS, VER, ZAC

Phyllanthus amarus Schumach. \& Thonn. CAM, CHIS, COL, JAL, MICH, OAX, QROO, TAB, VER, YUC

*Phyllanthus barbarae M.C. Johnst. QRO, SLP, TAMS 
Phyllanthus botryanthus Müll. Arg. COL, JAL

*Phyllanthus brandegeei Millsp. BCS

Phyllanthus carolinensis Walter CHIS, GRO, JAL, MEX, MICH, MOR, NAY, NLE, SON, TAB, TAMS, VER, YUC

*Phyllanthus coalcomanensis Croizat COL, GRO, MICH, NAY, SIN

Phyllanthus compressus Kunth CHIS, HGO, JAL, MOR, OAX, PUE, QRO, SLP, TAB, VER

*Phyllanthus chiapensis Sprague CHIS

Phyllanthus elsiae Urb. CAM, CHIS, CHIH, COL, GRO, JAL,

MICH, NAY, OAX, QROO, SIN, TAMS

Phyllanthus ericoides Torr. CHIH

Phyllanthus evanescens Brandegee BCS, SIN, SON

Phyllanthus fluitans Benth. ex Müll. Arg. TAB

*Phyllanthus fraguensis M.C. Johnst. COAH

*Phyllanthus galeottianus Baill. BCS, CHIS, GRO, JAL, MEX, MICH, OAX, VER

Phyllanthus grandifolius L. CAM, CHIS, COL, CDMX, GRO, HGO, JAL, MEX, MICH, NAY, OAX, QRO, QROO, SLP, TAMS, VER, YUC, ZAC

Phyllanthus graveolens Kunth CAM, CHIS, CHIH, COL, GTO, GRO, JAL, MEX, MICH, MOR, NAY, OAX, QRO, QROO, SLP, SIN, SON, TAMS, VER, YUC

*Phyllanthus gypsicola McVaugh COL, JAL

*Phyllanthus harrimanii G.L. Webster TAMS

*Phyllanthus hexadactylus McVaugh COL, GRO, JAL, MICH, SON

Phyllanthus liebmannianus Müll. Arg. CAM, CHIS, GRO, HGO, NLE, OAX, QROO, SLP, TAMS, VER, YUC

Phyllanthus mcvaughii G.L. Webster CHIS

*Phyllanthus mickelii McVaugh COL, JAL

Phyllanthus mocinianus Baill. CAM, CHIS, CHIH, COL, DGO, GTO, GRO, JAL, MEX, MICH, MOR, NAY, OAX, PUE, QRO, QROO, SLP, SIN, SON, TAMS, VER, YUC

*Phyllanthus neoleonensis Croizat COAH, NLE

Phyllanthus niruri L. CAM, CHIS, COL, GRO, HGO, JAL, MICH, MOR, NLE, OAX, PUE, QRO, QROO, SLP, SIN, TAB, TAMS, VER, YUC

*Phyllanthus oaxacanus Brandegee OAX, PUE

*Phyllanthus peninsularis Brandegee BCS, COAH, JAL, NAY

*Phyllanthus petaloideus Paul G. Wilson MEX

Phyllanthus polygonoides Nutt. ex Spreng. AGS, CHIH, COAH,

DGO, GTO, NLE, QRO, SLP, SON, TAMS, ZAC

Phyllanthus purpusii Brandegee CHIS, OAX

*Phyllanthus standleyi McVaugh COL, JAL, MICH, SIN

Phyllanthus stipulatus (Raf.) G.L. Webster CHIS, COL, JAL, NAY, TAB, VER

*Phyllanthus subcuneatus Greenm. COL, JAL, OAX, PUE, QRO, SLP, VER

*Phyllanthus tequilensis B.L. Rob. \& Greenm. CHIS, COL, DGO, GRO, HGO, JAL, MICH, NAY, OAX, PUE, SIN, TAB, TAMS, VER, ZAC

Phyllanthus tuerckheimii G.L. Webster CHIS, OAX

Savia sessiliflora (Sw.) Willd. CAM, COL, HGO, JAL, NAY, QRO, QROO, SLP, TAMS, VER, YUC

Family Phyllonomaceae
Phyllonoma laticuspis (Turcz.) Engl. CHIS, DGO, GRO, HGO, JAL, MEX, MICH, MOR, OAX, PUE, QRO, SLP, SIN, VER

\section{Family Phytolaccaceae}

Agdestis clematidea Moc. \& Sessé ex DC. CHIS, COL, GRO, HGO, JAL, MICH, NAY, NLE, OAX, PUE, QRO, SLP, SIN, TAB, TAMS, VER, YUC

Ledenbergia macrantha Standl. CHIS, COL, JAL, MICH, MOR, VER

*Nowickea glabra J. Martínez \& J.A. McDonald MOR

*Nowickea xolocotzii J. Martínez \& J.A. McDonald GTO, JAL

Petiveria alliacea L. BCS, CAM, CHIS, CHIH, COL, DGO, GTO, GRO, HGO, JAL, MEX, MICH, MOR, NAY, OAX, PUE, QRO, QROO, SLP, SIN, SON, TAB, TAMS, VER, YUC

Phytolacca americana L. CHIH, COAH, HGO, JAL, NLE, OAX, PUE, QRO, SLP, SON, TAMS, VER

Phytolacca heterotepala $\mathrm{H}$. Walter TAMS

Phytolacca icosandra L. AGS, BCN, BCS, CAM, CHIS, CHIH, COAH, COL, CDMX, DGO, GTO, GRO, HGO, JAL, MEX, MICH, MOR, NAY, NLE, OAX, PUE, QRO, QROO, SLP, SIN, SON, TAB, TAMS, TLAX, VER, YUC, ZAC

Phytolacca rivinoides Kunth \& C.D. Bouché CAM, CHIS, GRO, HGO, MEX, NLE, OAX, PUE, QRO, QROO, SLP, TAB, TAMS, VER

Phytolacca rugosa A. Braun \& C.D. Bouché CHIS, COL, CDMX, GTO, GRO, HGO, JAL, MEX, MICH, MOR, NAY, OAX, PUE, TAMS, TLAX, VER

Rivina humilis L. BCS, CAM, CHIS, CHIH, COAH, COL, DGO, GTO, GRO, HGO, JAL, MEX, MICH, MOR, NAY, NLE, OAX, PUE, QRO, QROO, SLP, SIN, SON, TAB, TAMS, VER, YUC, ZAC

Trichostigma octandrum (L.) H. Walter CHIS, COL, GRO, JAL, MEX, MICH, MOR, NAY, OAX, PUE, QRO, SLP, SIN, TAB, TAMS, VER

\section{Family Picramniaceae}

Alvaradoa amorphoides Liebm. CAM, CHIS, CHIH, COL, DGO, GRO, JAL, MEX, MICH, MOR, NAY, OAX, PUE, QROO, SIN, SON, VER, YUC, ZAC

Picramnia antidesma Sw. CAM, CHIS, COL, GRO, HGO, JAL, MEX, MICH, MOR, NAY, OAX, PUE, QRO, QROO, SLP, SIN, TAB, VER, YUC

Picramnia brachybotryosa Donn. Sm. CHIS

* Picramnia deflexa W.W. Thomas CHIS

*Picramnia guerrerensis W.W. Thomas CHIS, COL, GRO, JAL, MEX, MICH, SIN

Picramnia hirsuta W.W. Thomas CHIS, OAX, VER

Picramnia polyantha (Benth.) Planch. CHIS, COAH, GRO, NLE, OAX, PUE, QRO, SLP, VER

Picramnia quaternaria Donn. Sm. CHIS, OAX

Picramnia teapensis Tul. CHIS, OAX, PUE, QROO, TAB, VER, YUC

Picramnia tetramera Turcz. CHIS, GRO, MICH, TAB, VER

*Picramnia thomasii González-Martínez \& J. Jiménez Ram. GRO

*Picramnia xalapensis Planch. HGO, OAX, PUE, QRO, SLP, VER 


\section{Family Picrodendraceae}

* Piranhea mexicana (Standl.) Radcl. Sm. COL, JAL, NAY, SIN

*Tetracoccus capensis (I.M. Johnst.) Croizat BCS

Tetracoccus dioicus Parry BCN

Tetracoccus fasciculatus (S. Watson) Croizat BCN, BCS, CHIH, COAH, DGO, NLE

\section{Family Piperaceae}

Peperomia acuminata Ruiz \& Pav. CHIS, OAX, VER

Peperomia alata Ruiz \& Pav. CHIS, HGO, PUE, VER

Peperomia alpina (Sw.) A. Dietr. CHIS, OAX

Peperomia angularis C. DC. CHIS, COL, GRO, JAL, OAX, PUE, VER

Peperomia angustata Kunth CAM, CHIS, OAX, QROO, SLP, TAMS, VER, YUC

Peperomia arboricola C. DC. CHIS, GRO, OAX, VER

Peperomia asarifolia Schltdl. \& Cham. CHIS, COL, DGO, GRO, JAL, MEX, NAY, OAX, VER

Peperomia berlandieri Miq. CHIS, COAH, NLE, OAX, SLP, TAMS, VER

Peperomia blanda (Jacq.) Kunth CHIS, COAH, COL, HGO, JAL, NLE, OAX, QRO, SLP, TAMS, VER

*Peperomia botteri C. DC. VER

Peperomia bracteata A.W. Hill AGS, BCS, CHIS, CHIH, COAH, COL, CDMX, DGO, GTO, GRO, HGO, JAL, MEX, MICH, MOR, NAY, NLE, OAX, PUE, QRO, SLP, SIN, SON, TAMS, TLAX, VER, ZAC

* Peperomia calderoniae Barrios, Cota \& Medina-Cota HGO, VER

*Peperomia camptotricha Miq. VER

* Peperomia cavispicata G. Mathieu GRO, MEX

*Peperomia chazaroi G. Mathieu \& T. Krömer VER

Peperomia claytonioides Kunth CHIS, COL, JAL, MICH, MOR, NLE, OAX, TAMS

Peperomia cobana C. DC. CAM, CHIS, OAX, VER

Peperomia condormiens Trel. CHIS, OAX

*Peperomia consoquitlana C. DC. CHIS, OAX, VER

* Peperomia cordovana C. DC. COL, GRO, JAL, VER

Peperomia crassiuscula Millsp. CAM, CHIS, GRO, OAX, QROO, SIN, TAB, TAMS, VER, YUC

Peperomia cyclophylla Miq. COL, JAL, PUE, VER

Peperomia dendrophila Schltdl. \& Cham. CHIS, GRO, OAX, VER

Peperomia deppeana Schltdl. \& Cham. CAM, CHIS, COL, GRO, HGO, JAL, OAX, PUE, QROO, VER

Peperomia distachya (L.) A. Dietr. CHIS, COL, JAL, VER

*Peperomia donaguiana C. DC. HGO, OAX, VER

Peperomia dorstenioides Standl. \& Steyerm. CHIS, TAB

Peperomia emiliana C. DC. CHIS, VER

*Peperomia epidendron C. DC. CHIS, GRO, VER

Peperomia flagitans Trel. CHIS

Peperomia floribunda (Miq.) Dahlstedt CHIS, TAB, VER

* Peperomia fugax C. DC. VER

*Peperomia galeottii Miq. VER

Peperomia galioides Kunth CAM, CHIS, COL, CDMX, DGO, GTO, GRO, HGO, JAL, MEX, MICH, MOR, NAY, OAX, PUE, QRO, QROO, SLP, SIN, VER
Peperomia glabella (Sw.) A. Dietr. CAM, CHIS, COL, JAL, OAX, PUE, QRO, SLP, TAB, TAMS, VER

Peperomia glutinosa Millsp. CAM, CHIS, GRO, NAY, OAX, QROO, TAB, TAMS

*Peperomia gracillima S. Watson CDMX, GRO, JAL, MEX, $\mathrm{MICH}, \mathrm{OAX}$

Peperomia granulosa Trel. CAM, CHIS, HGO, OAX, PUE, QRO, QROO, SLP, TAB, TAMS, VER

Peperomia guatemalensis C. DC. CHIS, OAX

Peperomia hernandiifolia (Vahl) A. Dietr. CHIS, OAX, VER

Peperomia heterodoxa Standl. \& Steyerm. CHIS, OAX

Peperomia heterophylla Miq. CHIS, OAX, TAB

*Peperomia hintonii Yunck. CHIS, CDMX, HGO, JAL, MEX,

MICH, VER

Peperomia hirta C. DC. CHIS, NAY, VER

Peperomia hispidula (Sw.) A. Dietr. CHIS, COL, CDMX, DGO, GRO, HGO, JAL, MEX, MICH, MOR, OAX, QRO, TAB, VER Peperomia hispiduliformis Trel. DGO, GRO, HGO, MEX, MICH, MOR, OAX, PUE, SIN, VER

* Peperomia hobbitoides $\mathrm{T}$. Wendt VER

Peperomia hoffmannii C. DC. CHIS, COL, HGO, JAL, OAX, SLP, VER

Peperomia hondoana Trel. \& Standl. CHIS, HGO, OAX, QRO, SLP

*Peperomia huatuscoana C. DC. VER

Peperomia humilis A. Dietr. CHIS, GRO, MEX, MICH, OAX, PUE, QROO, VER

*Peperomia jaliscana $\mathrm{S}$. Watson JAL, SON

Peperomia lanceolatopeltata C. DC. CHIS, GRO, JAL, MEX, MICH, NAY, OAX

Peperomia lancifolia Hook. CHIS, OAX, VER

Peperomia lenticularis Dahlst. CHIS

* Peperomia leptophylla Miq. OAX

Peperomia liebmannii C. DC. CHIS, OAX, PUE, QRO, SLP, VER

Peperomia lignescens C. DC. CHIS, GRO, OAX

Peperomia lindeniana Miq. CHIS, COL, GRO, HGO, JAL, MEX, MICH, NAY, NLE, OAX, SLP, TAMS, VER

Peperomia macrandra C. DC. CHIS, GRO, HGO, MEX, OAX, PUE

Peperomia macrostachya (Vahl) A. Dietr. CHIS, COL, GRO, JAL, OAX, QRO, SLP, TAB, VER

Peperomia maculosa (L.) Hook. CHIS, MEX, MICH, OAX, PUE, QRO, SLP, VER

Peperomia major (Miq.) C. DC. CHIS, VER, YUC

Peperomia martiana Miq. COL, JAL, OAX, PUE

* Peperomia metapalcoensis C. DC. VER

Peperomia mexicana (Miq.) Miq. CHIS, COL, GRO, JAL, OAX, VER

Peperomia microphylla Kunth QRO, SLP, VER

Peperomia molithrix Trel. \& Standl. CHIS, COL, GRO, JAL, MEX, NAY, OAX

*Peperomia monticola Miq. CDMX, GRO, HGO, MEX, MICH, OAX, PUE, QRO, VER

*Peperomia muelleri C. DC. VER

Peperomia nigropunctata Miq. CHIS, COL, JAL, OAX, PUE, TAB, VER 
*Peperomia novaea-hispani Trel. CHIS, VER

Peperomia obtusifolia (L.) A. Dietr. CAM, CHIS, DGO, GRO, HGO, JAL, NAY, OAX, PUE, QRO, QROO, SLP, SIN, TAB, VER

Peperomia olivacea C. DC. CHIS, COL, JAL, OAX

*Peperomia papantlacensis C. DC. PUE, VER

*Peperomia parastriata G. Mathieu CHIS, OAX, VER

Peperomia pecuniifolia Trel. \& Standl. CHIS, OAX

Peperomia peltata C. DC. CHIS, COL, GRO, JAL, MEX, OAX, VER

Peperomia peltilimba C. DC. ex Trel. CHIS, GRO, HGO, OAX, PUE, SLP, VER

Peperomia pellucida (L.) Kunth CAM, CHIS, NAY, OAX, PUE, QROO, SIN, TAB, VER, YUC

Peperomia pereskiifolia (Jacq.) Kunth CAM, CHIS, OAX, QROO, SLP, VER, YUC

Peperomia petrophila C. DC. GRO, OAX, PUE, VER

Peperomia portobellensis Beurl. CHIS, COL, JAL, NAY, OAX, TAB, VER

Peperomia praeteruentifolia Trel. CHIS, OAX, VER

* Peperomia pseudoalpina Trel. CHIS, PUE, VER

Peperomia pseudopereskiifolia C. DC. CHIS, OAX, VER

Peperomia puberulilimba C. DC. CHIS, OAX

* Peperomia purpurinervis DC. HGO, VER

Peperomia quadrifolia (L.) Kunth CHIS, COAH, COL, CDMX, DGO, GTO, GRO, HGO, JAL, MEX, MICH, NAY, NLE, OAX, PUE, QRO, SLP, SIN, TAB, TAMS, VER

Peperomia rotundifolia (L.) Kunth CHIS, COL, GRO, JAL, MICH, NAY, OAX, PUE, QRO, SLP, TAB, VER

Peperomia santa-helenae Trel. CHIS

*Peperomia schizandra Trel. COL, JAL, MICH, OAX

Peperomia serpens (Sw.) Loudon CHIS, OAX, VER

*Peperomia socorronis Trel. COL

Peperomia subblanda C. DC. CHIS, OAX, SLP, TAMS, VER

Peperomia succulenta Trel. CHIS, COL, GRO, HGO, JAL, MICH, NAY, OAX, PUE, QRO, SLP, TAMS, VER

Peperomia suchitanensis Trel. \& Standl. CHIS

Peperomia tacanana Trel. \& Standl. CHIS, VER

Peperomia tenella (Sw.) A. Dietr. CHIS

Peperomia tenerrima Schltdl. \& Cham. HGO, OAX, PUE, VER

Peperomia tetraphylla (G. Forst.) Hook \& Arn. CHIS, COL, GRO, HGO, JAL, MEX, MICH, MOR, OAX, PUE, QRO, SLP, SON, VER

*Peperomia tlapacoyoensis C. DC. CHIS, VER

Peperomia tuerckheimii C. DC. ex Donn. Sm. CHIS, VER

Peperomia tuisana C. DC. CHIS, VER

Peperomia umbilicata Ruiz \& Pav. BCS, CHIS, COAH, CDMX, GRO, HGO, JAL, MEX, MICH, MOR, OAX, QRO, SLP, VER Peperomia urocarpa Fisch. \& C.A. Mey. CHIS, VER

Peperomia urocarpoides C. DC. CHIS, OAX, PUE, VER

*Peperomia vazquezii G. Mathieu \& D. Vergara-Rodríguez VER

Peperomia vegana Trel. \& Standl. CHIS

* Piper abalienatum Trel. COL, JAL, MICH

* Piper acutiusculum C. DC. VER

Piper achoteanum Trel. CHIS

Piper adamatum Trel. \& Standl. CHIS
Piper aduncum L. CHIS, COL, DGO, GRO, HGO, JAL, MEX, MICH, MOR, NAY, OAX, PUE, QRO, QROO, SLP, SIN, TAB, TAMS, VER, YUC

Piper aequale Vahl CAM, CHIS, OAX, PUE, SLP, TAB, VER Piper aeruginosibaccum Trel. CAM, CHIS, QROO, TAB, VER Piper amalago L. CAM, CHIS, COL, DGO, GTO, GRO, HGO, JAL, MEX, MICH, MOR, NAY, NLE, OAX, PUE, QRO, QROO, SLP, TAB, TAMS, VER, YUC

Piper arboreum Aubl. CHIS, COL, GRO, JAL, NAY, OAX, PUE, SLP, TAB, VER

Piper augustum Rudge HGO, OAX, VER

Piper auritum Kunth CAM, CHIS, COAH, GRO, HGO, MEX, MICH, MOR, NLE, OAX, PUE, QRO, QROO, SLP, SON, TAB, TAMS, VER, YUC

* Piper austro-mexicanum Trel. CHIS

Piper berteroanum C. DC. GRO, SLP, VER

Piper bisasperatum Trel. CHIS, COL, JAL, OAX, TAB, VER

* Piper bourgeaui C. DC. MOR

* Piper brachypus Trel. COL, JAL

Piper bredemeyeri Jacq. CHIS, OAX

*Piper brevipedicellatum Bornstein COL, GRO, JAL, SIN

Piper caladiifolium (Miq.) C. DC. ND

* Piper cihuatlanense Bornstein COL, JAL

* Piper colipanum C. DC. VER

* Piper colotlipanense Bornstein GRO, MICH

* Piper commutatum Steud. CHIS, VER

Piper cordillerianum C. DC. TAMS, VER

* Piper cordovanum C. DC. VER

Piper curtispicum C. DC. ex Pittier PUE, VER

Piper curvatipes Trel. CHIS

Piper chamissonis (Miq.) Steud. ex C. DC. HGO, OAX, PUE, SLP, VER

* Piper chinantlense M. Martens \& Galeotti CHIS, OAX, VER

* Piper decipiens (Miq.) C. DC. VER

Piper decurrens C. DC. CHIS

* Piper descourtilsianum C. DC. VER

Piper dilatatum Rich. CHIS, OAX, PUE, QRO, SLP, TAB, VER

* Piper disjunctum C. DC. OAX, VER

Piper divaricatum G. Mey. TAB, VER

Piper donnell-smithii C. DC. VER

Piper fallens Trel. DGO, GRO, MICH, NAY, OAX

* Piper fenianum Trel. CHIS

* Piper fischerianum C. DC. ND

Piper flavidum C. DC. ex Donn. Sm. CHIS, TAB

*Piper fortunyoanum Trel. VER

* Piper galeottianum M. Martens \& Galeotti VER

*Piper gibbosum C. DC. OAX, PUE, VER

Piper glabrescens (Miq.) C. DC. CHIS, OAX, VER

* Piper glaucescens Jacq. VER

Piper grandilimbum C. DC. CHIS

Piper guazacapanense Trel. \& Standl. CHIS, OAX

Piper heydei C. DC. CHIS, COL, GRO, JAL, OAX

Piper hispidum Sw. CAM, CHIS, CHIH, COL, DGO, GRO, HGO, JAL, MEX, MICH, NAY, OAX, PUE, QRO, QROO, SLP, SON, TAB, VER

* Piper instabilipes Trel. GRO

Piper jacquemontianum Kunth CAM, CHIS, OAX, PUE, 
QROO, TAB, VER

Piper jalapensis M. Martens \& Galeotti CHIS, OAX, VER

* Piper jaliscanum $\mathrm{S}$. Watson COL, JAL, NAY, SIN, SON, ZAC

*Piper karwinskianum Kunth SLP, TAMS, VER

Piper kerberi C. DC. CHIS, OAX, PUE, TAB, VER

* Piper kunthii (Miq.) C. DC. CHIS, OAX, VER

Piper lacunosum Kunth CHIS, GRO

Piper lapathifolium (Kunth) Steud. CHIS, COL, HGO, JAL, OAX, PUE, TAB, VER

*Piper laurifolium Mill. VER

Piper lepturum Kunth OAX, VER

*Piper leucophyllum (Miq.) C. DC. COL, GRO, JAL, MEX, MICH, MOR, PUE, SIN

* Piper liebmannii C. DC. CHIS, PUE, VER

*Piper lindenii (Miq.) C. DC. TAB, VER

Piper littorale C. DC. OAX, VER

Piper luxii C. DC. GRO, OAX

* Piper malpasoensis Tebbs CHIS, TAB

Piper marginatum Jacq. CAM, CHIS, GRO, JAL, MICH, NAY,

OAX, PUE, QROO, TAB, VER, YUC

Piper martensianum C. DC. CAM, CHIS, OAX, QROO, SLP,

TAB, VER

*Piper matudae Lundell CHIS

Piper maxonii C. DC. CHIS, VER

* Piper mcvaughii Bornstein COL, JAL

*Piper melanostictum (Miq.) C. DC. TAB

*Piper melastomoides Schldtl. \& Cham. HGO, VER

* Piper mexicanum (Miq.) DC. JAL, OAX, VER

*Piper michelianum C. DC. COL, JAL, MICH

Piper misantlense C. DC. CHIS, HGO, OAX, PUE, SLP, TAB, VER

Piper muelleri C. DC. JAL, VER

Piper neesianum C. DC. CAM, CHIS, OAX, PUE, QROO, VER, YUC

*Piper nigrum L. VER

*Piper novogalicianum Bornstein COL, JAL, MEX

*Piper nudum C. DC. VER

* Piper oaxacanum C. DC. OAX, VER

Piper obliquum Ruiz \& Pav. CHIS, OAX, VER

Piper oblongum Kunth VER

Piper oophorum C. DC. OAX, VER

Piper oradendron Trel. \& Standl. OAX, VER

* Piper orizabanum C. DC. VER

* Piper pachoanum C. DC. VER

* Piper palmeri C. DC. COL, JAL, OAX, SON

Piper patzulinum Trel. \& Standl. OAX

Piper peltatum L. CHIS, COL, JAL, OAX, TAB, TAMS, VER

Piper peracuminatum C. DC. OAX, VER

Piper pergamentifolum Trel. \& Standl. CHIS, TAB, VER

Piper phytolaccifolium Opiz OAX, TAB

Piper pogonioneuron Trel. \& Standl. OAX

* Piper pothomorphe (Miq.) C. DC. OAX, VER

Piper pseudoasperifolium C. DC. CHIS, GRO, JAL, NAY, OAX

Piper pseudofuligineum C. DC. CHIS, COL, JAL, MICH, OAX, TAB, VER

Piper pseudolindenii C. DC. CHIS, COL, JAL, MEX, MICH, MOR, OAX, QROO, SLP, TAB, VER
Piper psilorhachis C. DC. CAM, CHIS, DGO, GRO, MICH, MOR, NAY, OAX, PUE, QROO, TAB, VER, YUC

Piper punctulatum Standl. \& Steyerm. CHIS, OAX

* Piper purpusianum Trel. CHIS, OAX

Piper rohrii C. DC. OAX

* Piper rosei C. DC. COL, JAL, SIN

Piper sancti-felicis Trel. CHIS, OAX, TAB, VER

Piper sanctum (Miq.) Schltdl. ex C. DC. CAM, CHIS, COL, GRO, HGO, JAL, MEX, MICH, MOR, NAY, OAX, PUE, QRO, QROO, SLP, SIN, TAB, VER, ZAC

Piper schiedeanum Steud. CHIS, HGO, OAX, PUE, QRO, SLP, TAB, VER

Piper schippianum Trel. \& Standl. CAM, CHIS, HGO, OAX, PUE, QROO, VER

Piper silvivagum C. DC. MOR

* Piper stipulaceum Opiz COL, GRO, JAL, MICH, SIN

Piper subeburneum Trel. \& Standl. CHIS

Piper tacticanum Trel. \& Standl. OAX

* Piper teapense C. DC. CHIS, TAB

Piper tecutlanum Trel. \& Standl. OAX, PUE, VER

Piper telanum Trel. CHIS, OAX, VER

Piper trigonum C. DC. CHIS, OAX, VER

Piper tuberculatum Jacq. CHIS, COL, DGO, GRO, JAL, MICH, NAY, OAX, TAB, VER

Piper tuerckheimii C. DC. CHIS, COL, VER

*Piper tuxpenyana (Trel.) Lundell CAM, QROO

*Piper uhdei C. DC. GRO, OAX, VER

Piper umbellatum L. CHIS, COL, GRO, HGO, JAL, MEX, MICH, MOR, NAY, OAX, PUE, QRO, SLP, TAB, TAMS, VER Piper umbricola C. DC. CHIS, NAY, OAX, VER

Piper unguiculatum Ruiz \& Pav. GRO, NAY, OAX, VER

Piper uspantanense C. DC. CHIS, GRO, OAX

* Piper usumacintense Lundell TAB

* Piper variifolium (Miq.) C. DC. OAX, VER

*Piper velutinovarium $\mathrm{C}$. DC. VER

Piper veraguense C. DC. CHIS, VER

Piper verbascifolium (Miq.) C. DC. HGO, MOR, OAX

Piper vergelense Trel. \& Standl. CHIS

Piper villiramulum C. DC. CHIS, CHIH, COL, JAL, SON, VER

Piper virginicum Trel. \& Standl. CHIS

Piper wydlerianum (Miq.) C. DC. VER, YUC

Piper xanthostachyum C. DC. CHIS, OAX, TAB, VER

Piper yucatanense C. DC. CAM, CHIS, QROO, TAB, YUC

Piper yzabalanum C. DC. CHIS, NAY, OAX, PUE, TAB, VER

\section{Family Plantaginaceae}

Angelonia angustifolia Benth. CAM, CHIS, GRO, OAX, PUE, QROO, SLP, TAB, VER, YUC

Angelonia ciliaris B.L. Rob. CAM, CHIS, QROO, TAB, VER, YUC

*Antirrhinum costatum Wiggins BCS, SIN, SON

Antirrhinum coulterianum Benth. BCN

Antirrhinum cyathiferum Benth. BCN, BCS, CHIH, SON

Antirrhinum filipes A. Gray SON

Antirrhinum kelloggii Greene BCN

Antirrhinum nuttallianum Benth. BCN, BCS, SON

Antirrhinum watsonii Vasey \& Rose BCN, BCS, SON 
Asarina filipes (A. Gray) Pennell BCN, SON

Asarina stricta (Hook. \& Arn.) Pennell BCN, BCS

Bacopa lacertosa Pennell ex Standl. CAM, CHIS, OAX, QROO, TAB, VER, YUC

Bacopa monnieri (L.) Wettst. AGS, BCN, BCS, CAM, CHIS, COAH, COL, CDMX, GTO, GRO, HGO, JAL, MEX, MICH, MOR, NAY, NLE, OAX, PUE, QRO, QROO, SLP, SIN, SON, TAB, TAMS, VER, YUC, ZAC

Bacopa repens (Sw.) Wettst. CHIS, CHIH, COL, DGO, GRO, HGO, JAL, NAY, OAX, QRO, SIN, SON, TAB, VER

Bacopa rotundifolia (Michx.) Wettst. AGS, GTO, JAL, QRO, SLP, TAMS, ZAC

Bacopa salzmannii (Benth.) Wetts. ex Edwall CHIS, GTO, JAL, MICH, TAB, VER

Bacopa sessiliflora (Benth.) Edwall CHIS

Bacopa valerioi Standl. \& L.O. Williams CHIS, OAX

Benjaminia reflexa (Benth.) D'Arcy QROO, TAB, VER

Callitriche deflexa A. Braun ex Hegelm. CDMX, GTO, HGO, MEX, MICH, PUE, QRO, SLP, SON, TLAX, VER

Callitriche heterophylla Pursh AGS, BCN, CHIS, CHIH, COL, DGO, GTO, HGO, JAL, MEX, MICH, OAX, PUE, QRO, SLP, SIN, SON, VER, ZAC

* Callitriche marginata Torr. BCN

Callitriche palustris L. AGS, BCN, JAL, VER

Callitriche peploides Nutt. JAL, NAY, PUE, SLP, VER

Collinsia concolor Greene BCN

Collinsia childii Parry ex A. Gray BCN

Collinsia heterophylla Buist ex R. Grah. BCN

Conobea scoparioides (Cham. \& Schltdl.) Benth. CHIS, TAB, VER

*Galvezia juncea (Benth.) Ball BCN, BCS, SON

* Galvezia rupicola Brandegee BCS

Galvezia speciosa (Nutt.) A. Gray BCN, BCS

Hippuris vulgaris L. BCN

Keckiella antirrhinoides (Benth.) Straw BCN, SON

Keckiella cordifolia (Benth.) Straw BCN

Keckiella ternata (Torr. ex A. Gray) Straw BCN

Linaria canadensis (L.) Dum. Cours. GTO, JAL, MEX, SLP, SON, VER

* Linaria rupestris (L.) Mill. TAMS

*Lophospermum breedlovei Elisens CHIS

*Lophospermum chiapense Elisens CHIS

*Lophospermum purpurascens Elisens OAX, VER

*Lophospermum purpusii (Brandegee) Rothm. OAX, PUE

Lophospermum turneri Elisens CHIS, OAX

* Mabrya coccinea (I.M. Johnst.) Elisens COAH

* Mabrya erecta (Hemsl.) Elisens COAH, DGO, JAL, NLE

* Mabrya geniculata (B.L. Rob. \& Fernald) Elisens BCN, SON

* Mabrya rosei (Munz) Elisens JAL, ZAC

Maurandya antirrhiniflora Humb. \& Bonpl. ex Willd. AGS, CHIH, COAH, CDMX, DGO, GTO, GRO, HGO, JAL, MEX, MICH, NAY, NLE, OAX, PUE, QRO, SLP, SON, TAMS, VER, $\mathrm{ZAC}$

Maurandya barclayana Lindl. COAH, GTO, HGO, MEX, NLE, OAX, PUE, QRO, SLP, TAMS, VER

* Maurandya erecta Hemsl. CHIH, COAH, DGO, JAL, ZAC

Maurandya erubescens (D. Don) A. Gray GRO, HGO, MEX,
MOR, OAX, PUE, QRO, SLP, TAMS, VER

Maurandya scandens (Cav.) Pers. AGS, CHIS, DGO, GRO, HGO, JAL, MEX, MOR, OAX, PUE, SLP, ZAC

Maurandya wislizenii Engelm. ex A. Gray CHIH, DGO

* Mecardonia exilis (Brandegee) Pennell BCS

Mecardonia procumbens (Mill.) Small AGS, BCS, CAM, CHIS, CHIH, COAH, COL, CDMX, DGO, GTO, GRO, HGO, JAL, MEX, MICH, MOR, NAY, NLE, OAX, PUE, QRO, QROO, SLP, SIN, SON, TAB, TAMS, TLAX, VER, YUC, ZAC

Mecardonia vandellioides (Kunth) Pennell BCS, CHIH, COAH, COL, DGO, GRO, JAL, NLE, SLP, SON, TAMS, ZAC

Mohavea confertiflora (Benth.) A. Heller BCN, BCS, SON

Nuttallanthus canadensis (L.) D.A. Sutton AGS, BCN, BCS, CHIH, COAH, COL, CDMX, GTO, HGO, JAL, MEX, MICH, PUE, SLP, SON, TAMS, TLAX, VER, ZAC

Penstemon ambiguus Torr. CHIH

*Penstemon amphorellae Crosswh. COAH, GTO, HGO, NLE, QRO, SLP, ZAC

Penstemon baccharifolius Hook. CHIH, COAH, GTO, JAL, SLP

Penstemon barbatus (Cav.) Roth AGS, CHIS, CHIH, COAH, CDMX, DGO, GTO, HGO, JAL, MEX, MICH, MOR, NLE, OAX, PUE, QRO, SLP, SIN, SON, TAMS, TLAX, VER, ZAC *Penstemon bolanus Straw JAL, ZAC

Penstemon californicus (Munz \& I.M. Johnst.) D.D. Keck BCN Penstemon campanulatus (Cav.) Willd. AGS, CHIH, COAH, CDMX, DGO, GTO, GRO, HGO, JAL, MEX, MICH, MOR, NAY, NLE, OAX, PUE, QRO, SLP, SIN, SON, TAMS, TLAX, VER, ZAC

*Penstemon cedrosensis Krautter BCN

Penstemon centranthifolius Benth. BCN

Penstemon clevelandii A. Gray BCN

Penstemon dasyphyllus A. Gray CHIH, COAH, NLE, SLP, SON

*Penstemon eximius Keck BCN, BCS

*Penstemon fasciculatus A. Gray CHIH, SON

Penstemon fendleri Torr. \& A. Gray CHIH, COAH

*Penstemon filisepalis Straw CHIH

*Penstemon galloensis G.L. Nesom COAH, NLE

Penstemon gentianoides (Kunth) Poir. AGS, CHIS, COAH, CDMX, DGO, GTO, HGO, JAL, MEX, MICH, MOR, NLE, OAX, PUE, QRO, TAMS, TLAX, VER, ZAC

*Penstemon gentryi Standl. CHIH

Penstemon glaber Pursh CHIH

*Penstemon hartwegii Benth. HGO, MEX, OAX, QRO, SLP, VER

Penstemon havardii A. Gray CHIH, COAH, NLE

*Penstemon henricksonii Straw COAH

*Penstemon hidalgensis Straw AGS, HGO, JAL, OAX, QRO, SLP, ZAC

*Penstemon imberbis (Kunth) Trautv. AGS, CHIH, COL, GTO, HGO, JAL, MEX, MICH, MOR, QRO, SLP, ZAC

*Penstemon isophyllus B.L. Rob. JAL, NAY, OAX, PUE, VER Penstemon jamesii Benth. COAH, NLE

Penstemon labrosus (A. Gray) Mast. ex Hook. f. BCN

Penstemon lanceolatus Benth. AGS, CHIH, COAH, DGO, JAL, NAY, NLE, SLP, SON, TAMS, VER, ZAC

*Penstemon leonensis Straw AGS, COAH, JAL, NLE, TAMS, 
ZAC

Penstemon linarioides A. Gray BCN, SON

*Penstemon luteus G.L. Nesom COAH, NLE

Penstemon miniatus Lindl. AGS, CHIH, COL, CDMX, DGO, GTO, GRO, HGO, JAL, MEX, MICH, MOR, NAY, NLE, OAX, PUE, QRO, SLP, SIN, SON, TAMS, TLAX, VER, ZAC

*Penstemon minutifolius Straw DGO, JAL, OAX, PUE, SIN, ZAC

*Penstemon mohinoranus Straw CHIH

*Penstemon occiduus Straw CHIH, DGO

*Penstemon pallidus Small VER

Penstemon parryi (A. Gray) A. Gray CHIH, SON

Penstemon perfoliatus Brongn. OAX

Penstemon pinifolius Greene CHIH, HGO, QRO, SON, TAMS

*Penstemon plagapineus Straw DGO, NAY, SIN

* Penstemon potosinus Straw SLP, ZAC

Penstemon pseudospectabilis M.E. Jones SON

Penstemon pulchellus Lindl. CHIH, COAH, SON

*Penstemon punctatus Brandegee $\mathrm{COAH}$

*Penstemon roseus (Sweet) G. Don AGS, CHIH, COL, CDMX, DGO, GTO, GRO, HGO, JAL, MEX, MICH, MOR, NAY, NLE, OAX, PUE, QRO, SLP, SIN, SON, TLAX, VER, ZAC

Penstemon rostriflorus Kellogg BCN

*Penstemon rotundifolius A. Gray CHIH

*Penstemon schaffneri (Hemsl.) Straw AGS, GTO, JAL, SLP, ZAC

Penstemon secundiflorus Benth. CHIH, SON

Penstemon spectabilis Thurb. ex A. Gray BCN

Penstemon stenophyllus A. Gray CHIH, DGO, JAL, SON, ZAC

*Penstemon tenuifolius Benth. AGS, COAH, DGO, JAL, QRO,

SLP, ZAC

*Penstemon tepicensis Straw DGO, NAY

Penstemon thurberi Torr. BCN, COAH

Penstemon triflorus Heller COAH

Penstemon virgatus A. Gray COL, JAL, SLP

*Penstemon wendtiorum B.L. Turner COAH

*Penstemon wislizenii (A. Gray) Straw CHIH, DGO, NLE, SLP, SON

*Plantago alismatifolia Pilger CHIS, CHIH, CDMX, DGO, GTO, GRO, HGO, JAL, MEX, MICH, MOR, PUE, QRO, SLP, SON, VER

Plantago australis Lam. AGS, BCS, CHIS, CHIH, COAH, COL, CDMX, DGO, GTO, GRO, HGO, JAL, MEX, MICH, MOR, NLE, OAX, PUE, QRO, SLP, SIN, SON, TAB, TAMS, TLAX, VER, ZAC

* Plantago floccosa Decne. CHIS, DGO, GTO, HGO, MEX, NLE, QRO, SLP, TAMS, VER

Plantago helleri Small CHIH, COAH, NLE

Plantago heterophylla Nutt. BCN, SON

Plantago hookeriana Fisch. \& C.A. Mey. BCN, BCS, CHIH, COAH, NLE, TAMS

Plantago linearis Kunth AGS, BCN, BCS, CHIS, CHIH, CDMX, DGO, GTO, GRO, HGO, JAL, MEX, MICH, MOR, NAY, NLE, OAX, QRO, SLP, SIN, SON, TLAX, VER, ZAC

Plantago nivea Kunth AGS, BCS, CDMX, DGO, GTO, GRO, HGO, JAL, MEX, MICH, NLE, OAX, PUE, QRO, SLP, SIN, TAMS, TLAX, VER, ZAC
Plantago ovata Forssk. AGS, BCN, BCS, CHIH, COAH, NLE, SLP, SON, ZAC

Plantago patagonica Jacq. BCN, CHIH, COAH, NLE, SON

Plantago rhodosperma Decne. BCN, CHIH, COAH, NLE, SLP, SON, TAMS, VER

* Plantago tolucensis Pilg. MEX, PUE, TLAX, VER

Plantago tubulosa Decne. MEX, VER

Plantago virginica L. CHIH, COAH, HGO, NLE, OAX, QRO, SON, TAMS, VER

Plantago wrightiana Decne. CHIH, COAH, DGO, TAMS

Rhodochiton atrosanguineum (Zucc.) Rothm. OAX

*Rhodochiton hintonii (Elisens) D.A. Sutton GRO, OAX

*Rhodochiton nubicola (Elisens) D.A. Sutton CHIS

Russelia acuminata Carlson CHIS

Russelia campechiana Standl. CAM, CHIS, HGO, QROO, TAB, VER, YUC

Russelia coccinea (L.) Wettst. CAM, CHIS, COL, GRO, HGO, JAL, MEX, MICH, MOR, NLE, OAX, PUE, QRO, SLP, TAMS, VER

Russelia contrerasii B.L. Turner CHIS, VER

* Russelia conzattii Carlson GRO, OAX

*Russelia cora Méndez-Larios \& O. Téllez NAY

*Russelia cuneata B.L. Rob. COL, DGO, GRO, HGO, JAL, MEX, MICH, MOR, NAY, OAX, QRO, SLP, ZAC

Russelia chiapensis Lundell CHIS, OAX, QROO, TAB

*Russelia elongata Carlson DGO, NAY, SIN, SON

Russelia equisetiformis Schltdl. \& Cham. CAM, CHIS, HGO, JAL, MEX, MICH, MOR, NAY, OAX, PUE, QRO, QROO, SLP, SIN, TAB, TAMS, VER, YUC

*Russelia floribunda Kunth CHIS, COL, GRO, JAL, MEX, MICH, OAX, QROO, SIN, VER, YUC

*Russelia furfuracea Brandegee GRO, NAY, SIN, SON

*Russelia grandidentata Carlson BCS

*Russelia hintonii Lundell GRO

*Russelia iltisneeana B.L. Turner COL, JAL

*Russelia jaliscensis B.L. Rob. GRO, JAL, MEX, MOR, NAY, PUE, ZAC

*Russelia lanceifolia Lundell GRO

*Russelia leptopoda Lundell MICH

Russelia longifolia Carlson CHIS, TAB

Russelia longisepala Carlson CHIS

*Russelia maculosa Lundell GRO, HGO, QRO, SLP

* Russelia manantlana B.L. Turner COL, JAL

*Russelia obtusata S.F. Blake COL, GRO, JAL, OAX, PUE, SLP, VER

Russelia parvifolia Carlson GRO, JAL, MEX, MICH, OAX

*Russelia polyedra Zucc. AGS, CHIH, GTO, GRO, HGO, JAL, MEX, MICH, MOR, NLE, OAX, QRO, SLP, TAMS, VER, ZAC

*Russelia pringlei B.L. Rob. COL, GRO, JAL, OAX

*Russelia pubescens Lundell DGO, GRO, JAL, NAY

*Russelia purpusii Brandegee CHIS, OAX, VER

*Russelia retrorsa Greene AGS, BCS, CHIS, COL, CDMX, DGO, GTO, GRO, JAL, MEX, MICH, MOR, NAY, OAX, PUE, QRO, SIN, TAMS, VER

* Russelia rotundifolia Cav. DGO, GRO, JAL, OAX

Russelia sarmentosa Jacq. CAM, CHIS, CHIH, COL, GTO, 
GRO, JAL, MEX, MICH, MOR, NAY, OAX, PUE, QRO, QROO, SLP, SIN, SON, TAB, TAMS, VER, YUC

*Russelia sonorensis Carlson CHIH, NAY, SON

Russelia staleyae Carlson OAX

Russelia steyermarkii Carlson CHIS

*Russelia syringifolia Schldtl. \& Cham. HGO, OAX, PUE, QRO, SLP, TAMS, VER

*Russelia tenuis Lundell COL, DGO, GRO, JAL, MEX, MICH, MOR, NAY, SLP, SIN

*Russelia tepicensis B.L. Rob. COL, DGO, JAL, NAY, SIN

*Russelia teres Lundell MICH

*Russelia ternifolia Kunth CHIS, JAL, MEX, MICH, NAY, OAX, SLP

Russelia tetraptera S.F. Blake COL, GRO, JAL, NAY, OAX, SIN, ZAC

Russelia verticillata Kunth CHIS, HGO, MEX, MICH, OAX, QRO, SLP, VER

*Russelia villosa Lundell GRO, MEX, MICH, MOR

*Russelia worthingtonii B.L. Turner DGO, NAY, SIN

Scoparia аппиа Schltdl. \& Cham. GRO, OAX, PUE

Scoparia dulcis L. BCS, CAM, CHIS, COL, DGO, GRO, HGO, JAL, MEX, MICH, MOR, NAY, NLE, OAX, PUE, QRO, QROO, SLP, SIN, TAB, TAMS, VER, YUC

*Scoparia mexicana R.E. Fr. CHIS, OAX

Schistophragma intermedium (A. Gray) Pennell BCN, BCS, CHIH, COAH, DGO, NLE, SON, ZAC

Schistophragma mexicanum Benth. ex D. Dietr. CAM, CHIS, COL, GRO, JAL, MEX, MICH, MOR, OAX, PUE, QROO, VER, YUC

*Schistophragma polystachyum (Brandegee) B.L. Turner BCN, BCS

Schistophragma pusillum Benth. CHIS, COL, GRO, JAL, MEX, MICH, MOR, NAY, OAX, PUE, SIN, SON, TAB, TAMS, VER, YUC, ZAC

Sibthorpia rotundifolia (Ruiz \& Pav.) Edwin CHIS, COL, CDMX, DGO, GTO, GRO, HGO, JAL, MEX, MICH, MOR, NLE, OAX, PUE, QRO, SLP, TLAX, VER

Stemodia angulata Oerst. CHIS

*Stemodia bartsioides Benth. DGO, GTO, HGO, JAL, OAX, QRO, SIN, ZAC

Stemodia durantifolia (L.) Sw. AGS, BCN, BCS, CAM, CHIS, CHIH, COL, DGO, GTO, GRO, HGO, JAL, MEX, MICH, MOR, NAY, NLE, OAX, PUE, QRO, QROO, SLP, SIN, SON, TAB, TAMS, VER, YUC, ZAC

Stemodia jorullensis Kunth CHIS, GRO, MICH

Stemodia lanata Sessé \& Moc. TAMS, VER

Stemodia macrantha B.L. Rob. COL, JAL, MEX, MICH, NAY, OAX, QRO, SLP, VER

Stemodia maritima L. CAM, QROO

* Stemodia micrantha Brandegee CHIS

*Stemodia palmeri A. Gray CHIS, CHIH, DGO, MOR, SIN, SON

Stemodia peduncularis Benth. CHIS, GRO, MICH, PUE, VER

*Stemodia purpusii Brandegee COAH

Stemodia pusilla Benth. BCN, BCS, JAL, NAY, SIN, SON

Stemodia schottii Holz. CHIH, COAH, DGO, NLE, SLP, TAMS, VER
*Stemodia tenuifolia Minod COL, JAL

Stemodia tomentosa (Mill.) Greenm. \& C.H. Thomps. TAMS, VER

Stemodia verticillata (Mill.) Hassl. CHIS, GRO, OAX, TAB, VER

*Tetranema megaphyllum (Brandegee) L.O. Williams CHIS Tetranema roseum (M. Martens \& Galeotti) Standl. \& Steyerm. CHIS, HGO, OAX, PUE, VER

*Uroskinnera almedae T.F. Daniel \& Breedlove OAX

*Uroskinnera flavida Lundell CHIS, OAX, TAB, VER

*Uroskinnera hirtiflora Hemsl. OAX, PUE, VER

Veronica americana (Raf.) Schwein. ex Benth. BCN, COAH, CDMX, DGO, HGO, JAL, MEX, MICH, MOR, NLE, SON, VER

*Veronica mexicana S. Watson CHIH, SON

Veronica peregrina L. AGS, BCN, BCS, CHIS, CHIH, COAH, COL, CDMX, DGO, GTO, GRO, HGO, JAL, MEX, MICH, MOR, NAY, NLE, OAX, PUE, QRO, QROO, SLP, SIN, SON, TAB, TAMS, TLAX, VER, ZAC

Veronica tenella All. CHIS, VER

\section{Family Platanaceae}

* Platanus gentryi Nixon \& J.M. Poole CHIH, SIN, SON

Platanus mexicana Moric. CHIS, COAH, CDMX, GTO, HGO, MEX, NLE, OAX, PUE, QRO, SLP, TAMS, VER

Platanus occidentalis L. COAH, NLE, SLP

Platanus racemosa Nutt. BCN, CHIH, SIN, SON

* Platanus rzedowskii Nixon \& J.M. Poole COAH, NLE, QRO,

TAMS, SLP, VER

\section{Family Plocospermataceae}

Plocosperma buxifolium Benth. GRO, JAL, MICH, OAX, PUE, VER

Family Plumbaginaceae

Limonium californicum (Boiss.) Heller BCN, BCS

Limonium limbatum Small COAH

* Plumbago pulchella Boiss. AGS, COL, CDMX, DGO, GTO, GRO, HGO, JAL, MEX, MICH, MOR, NAY, NLE, OAX, PUE, QRO, SLP, TAMS, TLAX, VER, ZAC

\section{Family Poaceae}

Aakia tuerckheimii (Hack.) J.R. Grande CHIS, OAX, PUE, VER Acroceras zizanioides (Kunth) Dandy CAM, CHIS, HGO, MOR, OAX, PUE, SLP, TAB, VER

*Achnatherum acutum (Swallen) Valdés-Reyna \& Barkworth COAH, NLE

Achnatherum altum (Swallen) Hoge \& Barkworth $\mathrm{CHIH}$, COAH, NLE

*Achnatherum aridum (M.E. Jones) Barkworth COAH

*Achnatherum bracteatum (Swallen) Valdés-Reyna \& Barkworth $\mathrm{BCN}$

*Achnatherum constrictum (Hitchc.) Valdés-Reyna \& Barkworth COAH, CDMX, GTO, HGO, MEX, MOR, NLE, OAX, PUE, QRO, SLP, TAMS, TLAX, VER, ZAC

Achnatherum curvifolium (Swallen) Barkworth CHIH

Achnatherum diegoense (Swallen) Barkworth BCN

*Achnatherum editorum (E. Fourn.) Valdés-Reyna \& Barkworth AGS, COAH, HGO, MEX, NLE, PUE, SLP, TAMS, TLAX, 
VER, ZAC

Achnatherum eminens (Cav.) Barkworth AGS, BCN, CHIS, CHIH, COAH, CDMX, DGO, GTO, HGO, JAL, MEX, MICH, NAY, NLE, OAX, PUE, QRO, SLP, SIN, SON, TAMS, TLAX, VER, ZAC

*Achnatherum hirticulme (S.L. Hatch, Valdés-Reyna \& Morden) Valdés-Reyna \& Barkworth COAH, GTO, JAL, NLE, SLP, ZAC

Achnatherum hymenoides (Roem. \& Schult.) Barkworth BCN, $\mathrm{CHIH}$

*Achnatherum multinode (Scribn. ex Beal) Valdés-Reyna \& Barkworth CHIH, COAH, DGO, NLE, PUE, QRO, SLP, ZAC Achnatherum nelsonii (Scribn.) Barkworth NLE

Achnatherum robustum (Vasey) Barkworth CHIH, COAH, NLE, SLP, TAMS, ZAC

Aegopogon cenchroides Humb. \& Bonpl. ex Willd. AGS, BCN, BCS, CHIS, CHIH, COAH, COL, CDMX, DGO, GTO, GRO, HGO, JAL, MEX, MICH, MOR, NAY, OAX, PUE, QRO, SLP, SIN, SON, TAMS, TLAX, VER, ZAC

*Aegopogon solisii G.A. Levin COL

Aegopogon tenellus (DC.) Trin. AGS, BCN, BCS, CHIS, CHIH, COL, CDMX, DGO, GTO, GRO, HGO, JAL, MEX, MICH, MOR, NAY, NLE, OAX, PUE, QRO, SLP, SIN, SON, TLAX, VER, ZAC

*Agrostis bourgeaei E. Fourn. CDMX, GTO, HGO, MEX, MICH, MOR, OAX, PUE, QRO, TLAX, VER

*Agrostis calderoniae Acosta MEX

Agrostis exarata Trin. BCN, BCS, CHIH, COAH, CDMX, DGO, GTO, HGO, SON

Agrostis ghiesbreghtii E. Fourn. CHIS, GRO, HGO, MEX, MICH, MOR, OAX, PUE, VER

Agrostis hyemalis (Walter) Britton, Stearn \& Pogg. AGS, BCN, CHIS, CHIH, COAH, COL, CDMX, DGO, GTO, HGO, JAL, MEX, MICH, MOR, NLE, OAX, PUE, QRO, SLP, SIN, SON, TLAX, VER, ZAC

Agrostis laxissima Swallen CHIS

*Agrostis liebmannii (E. Fourn.) Hitchc. CDMX, DGO, MEX, OAX, PUE, QRO, VER

Agrostis mertensii Trin. MEX, PUE

Agrostis microphylla Steud. BCN

*Agrostis novogaliciana McVaugh COL, JAL

Agrostis pallens Trin. BCN

Agrostis perennans (Walter) Tuck. CHIS, CHIH, CDMX, DGO, GTO, GRO, HGO, JAL, MEX, MICH, MOR, OAX, PUE, QRO, SLP, TLAX, VER

*Agrostis rosei Scribn. \& Merr. DGO, HGO, JAL, MEX, MICH, QRO, SLP, ZAC

*Agrostis schaffneri E. Fourn. CHIS, CDMX, HGO, JAL, MEX, MICH, MOR, OAX, PUE, QRO, TLAX, VER

Agrostis subpatens Hitchc. CHIS, CDMX, HGO, JAL, MEX, MICH, OAX, PUE, TLAX, VER

Agrostis subrepens (Hitchc.) Hitchc. CHIH, SON

Agrostis tolucensis Kunth CHIS, COL, CDMX, DGO, GTO, GRO, HGO, JAL, MEX, MICH, MOR, OAX, PUE, TLAX, VER

Agrostis turrialbae Mez CHIS

Alopecurus geniculatus L. BCN, CHIH
Alopecurus magellanicus Lam. BCN

Alopecurus saccatus Vasey BCN

Allolepis texana (Vasey) Soderstr. \& H.F. Decker CHIH, COAH, DGO, NLE, TAMS

Amelichloa clandestina (Hack.) Arriaga \& Barkworth AGS, COAH, HGO, MEX, MICH, NLE, PUE, SLP, TLAX, VER, ZAC

Andropogon angustatus (J. Presl) Steud. CHIS, COL, GRO, JAL, MEX, MICH, MOR, NAY, OAX, SIN, ZAC

Andropogon bicornis L. CAM, CHIS, COL, GRO, HGO, JAL, MEX, MICH, NAY, OAX, PUE, QROO, TAB, TAMS, VER, YUC

Andropogon bourgaei Hack. CHIS, OAX, TAB, VER, YUC

Andropogon fastigiatus Sw. CAM, CHIS, CHIH, COL, DGO, GRO, JAL, MEX, MICH, MOR, NAY, OAX, PUE, SIN, SON, TAMS, VER, ZAC

Andropogon gerardii Vitman AGS, CHIS, CHIH, COAH, CDMX, DGO, GTO, HGO, JAL, MEX, MICH, MOR, NLE, OAX, PUE, QRO, SLP, SIN, TLAX, VER, ZAC

Andropogon glomeratus (Walter) Britton, Stearn \& Pogg. AGS, BCN, BCS, CAM, CHIS, CHIH, COAH, COL, DGO, GRO, HGO, JAL, MEX, MICH, MOR, NAY, NLE, OAX, PUE, QRO, QROO, SLP, SIN, SON, TAB, TAMS, VER, YUC, ZAC

Andropogon gyrans Ashe CHIS, PUE, SLP, VER

Andropogon leucostachyus Kunth CAM, CHIS, GRO, OAX, PUE, TAB, VER

Andropogon liebmannii Hack. DGO, HGO, JAL, MEX, MICH, NAY, OAX, PUE, VER, ZAC

*Andropogon pringlei Scribn. \& Merr. CHIS, COL, CDMX, DGO, JAL, MEX, MICH, OAX, PUE, SLP, TLAX, VER, ZAC Andropogon selloanus (Hack.) Hack. CAM, CHIS, OAX, TAB, VER

Andropogon ternarius Michx. COAH, MEX, MICH

Andropogon virgatus Desv. ex Ham. CAM, CHIS, OAX

Andropogon virginicus L. CAM, CHIS, COAH, JAL, NLE, OAX, PUE, QROO, TAB, TAMS, VER, YUC

Anthaenantia lanata (Kunth) Benth. CAM, CHIS, COL, GTO, GRO, JAL, MEX, MICH, NAY, OAX, PUE, QROO, SLP, TAB, TAMS, VER, YUC

*Anthaenantia villaregalis (McVaugh \& R. Guzmán) Espejo \& López-Ferrari COL, GRO, JAL

Anthephora hermaphrodita (L.) Kuntze BCN, BCS, CAM, CHIS, COL, CDMX, DGO, GRO, JAL, MEX, MICH, MOR, NAY, OAX, PUE, QROO, SIN, SON, VER, YUC, ZAC

*Anthoxanthum mexicanum (Rupr. ex E. Fourn.) Mez CHIS, MEX, MICH, MOR, OAX

Aristida adscensionis L. AGS, BCN, BCS, CAM, CHIS, CHIH, COAH, COL, CDMX, DGO, GTO, GRO, HGO, JAL, MEX, MICH, MOR, NAY, NLE, OAX, PUE, QRO, QROO, SLP, SIN, SON, TAMS, TLAX, VER, YUC, ZAC

Aristida appressa Vasey AGS, CAM, CHIS, CHIH, COL, CDMX, DGO, GTO, GRO, JAL, MEX, MICH, MOR, NAY, OAX, PUE, QRO, SLP, SIN, TLAX, YUC, ZAC

Aristida arizonica Vasey BCN, CHIH, COAH, CDMX, DGO, GTO, GRO, HGO, JAL, MEX, MICH, MOR, NLE, OAX, PUE, QRO, SLP, SIN, SON, TAMS, VER, ZAC

Aristida californica Thurb. ex S. Watson BCN, BCS, SON 
Aristida capillacea Lam. CHIS, COL, CDMX, JAL, MEX, NAY, OAX, SIN, VER

Aristida divaricata Humb. \& Bonpl. ex Willd. AGS, BCN, CHIH, COAH, CDMX, DGO, GTO, HGO, JAL, MEX, MICH, MOR, NLE, OAX, PUE, QRO, SLP, SON, TAMS, TLAX, VER, ZAC

*Aristida eludens Allred \& Valdés-Reyna CHIH, COAH, DGO, GTO, JAL, NLE, OAX, QRO, SLP

Aristida floridana (Chapman) Vasey CAM, QROO, YUC

Aristida gibbosa (Nees) Kunth AGS, CAM, CHIS, CHIH, COAH, COL, CDMX, DGO, GTO, GRO, JAL, MEX, MICH, NAY, OAX, PUE, SIN, SON, TLAX, VER, ZAC

Aristida gypsophila Beetle CHIH, COAH, NLE, SLP

Aristida havardii Vasey AGS, BCN, BCS, CHIH, COAH, CDMX, DGO, GTO, HGO, JAL, MEX, NLE, OAX, PUE, QRO, SLP, SON, TLAX, VER, ZAC

*Aristida hintonii Hitchc. COL, CDMX, GTO, GRO, JAL, MEX, MICH, NAY, OAX, PUE, TAMS, ZAC

*Aristida hitchcockiana Henrard GTO, GRO, JAL, NAY, OAX, PUE, QRO, ZAC

*Aristida jaliscana R. Guzmán \& V. Jaram. GTO, JAL, ZAC Aristida jorullensis Kunth CAM, CHIS, COL, CDMX, DGO, GRO, JAL, MEX, MICH, NAY, OAX, PUE, QROO, SIN, SON, VER, ZAC

Aristida laxa Cav. AGS, CHIS, CHIH, COL, CDMX, DGO, GTO, GRO, HGO, JAL, MEX, MICH, MOR, NAY, OAX, PUE, QRO, SLP, SIN, SON, TAB, TLAX, VER, ZAC

Aristida liebmannii E. Fourn. CHIS, OAX, TLAX, VER

Aristida longespica Poir. HGO, QRO, VER

Aristida pansa Wooton \& Standl. AGS, BCN, CHIH, COAH, COL, CDMX, DGO, GTO, HGO, JAL, MEX, MOR, NAY, NLE, OAX, PUE, QRO, SLP, SON, TAMS, VER, ZAC

*Aristida petersonii Allred \& Valdés-Reyna OAX

Aristida purpurascens Poir. CHIS, CHIH, COAH, SON

Aristida purpurea Nutt. AGS, BCN, BCS, CHIS, CHIH, COAH, CDMX, DGO, GTO, HGO, JAL, MEX, MICH, NLE, OAX, PUE, QRO, SLP, SIN, SON, TAMS, VER, YUC, ZAC

*Aristida purpusiana Hitchc. BCN, BCS

Aristida recurvata Kunth CAM, OAX, TLAX, YUC

*Aristida scribneriana Hitchc. AGS, DGO, GTO, GRO, HGO, JAL, MICH, QRO, SLP, ZAC

Aristida schiedeana Trin. \& Rupr. AGS, BCN, BCS, CHIS, CHIH, COAH, COL, CDMX, DGO, GTO, GRO, HGO, JAL, MEX, MICH, MOR, NAY, NLE, OAX, PUE, QRO, SLP, SIN, SON, TAMS, TLAX, VER, ZAC

*Aristida spanospicula Allred, Valdés-Reyna \& Sánchez-Ken CHIH, DGO, GTO, JAL, SON

*Aristida tenuifolia Hitchc. COL

Aristida ternipes Cav. AGS, BCN, BCS, CAM, CHIS, CHIH, COAH, COL, CDMX, DGO, GTO, GRO, HGO, JAL, MEX, MICH, MOR, NAY, NLE, OAX, PUE, QRO, QROO, SLP, SIN, SON, TAB, TAMS, TLAX, VER, YUC, ZAC

*Aristida tuitensis Sánchez-Ken \& Dávila COL, JAL

*Aristida vaginata Hitchc. COL, JAL

Arthropogon villosus Nees OAX

Arthrostylidium excelsum Griseb. CHIS

Arundinella berteroniana (Schult.) Hitchc. \& Chase CHIS,
COAH, COL, CDMX, GRO, HGO, JAL, MEX, MICH, MOR, NAY, NLE, OAX, PUE, QRO, SLP, SIN, SON, TAB, TAMS, VER

Arundinella deppeana Nees ex Steud. CAM, CHIS, COL, CDMX, DGO, GRO, HGO, JAL, MEX, MICH, MOR, NAY, NLE, OAX, PUE, QRO, QROO, SLP, SIN, TAB, VER, YUC Arundinella hispida (Humb. \& Bonpl. ex Willd.) Kuntze CHIS, CHIH, COL, CDMX, DGO, GRO, JAL, MEX, MICH, MOR, NAY, OAX, SON, TAB, VER

*Aulonemia laxa (F. Maek.) McClure CHIS, GRO, MEX, $\mathrm{MICH}, \mathrm{VER}$

Avenella flexuosa (L.) Drejer CHIH, DGO, GRO, HGO, MEX, NLE, QRO, TLAX

Axonopus arsenei Swallen CHIS, COL, CDMX, GRO, JAL, MEX, MICH, OAX, TLAX

Axonopus aureus $\mathrm{P}$. Beauv. CHIS, OAX, TAB

Axonopus centralis Chase CHIS, COL, GRO, JAL, MICH, NAY, SIN

Axonopus compressus (Sw.) P. Beauv. CAM, CHIS, COL, CDMX, DGO, GTO, GRO, HGO, JAL, MEX, MICH, MOR, NAY, NLE, OAX, PUE, QROO, SLP, SIN, SON, TAB, TAMS, VER, YUC

*Axonopus deludens Chase COL, DGO, JAL, NAY, SIN

Axonopus fissifolius (Raddi) Kuhlm. CAM, CHIS, HGO, JAL, OAX, PUE, QRO, TAB, TAMS, VER, YUC

Axonopus poiophyllus Chase CHIS, GRO, OAX, PUE, TAMS, VER

Axonopus purpusii (Mez) Chase CAM, CHIS, COL, GRO, OAX, PUE, QROO, TAB, VER

*Axonopus rosei (Scribn. \& Merr.) Chase COL, JAL, NAY, SIN, ZAC

*Bealia mexicana Scribn. CHIH, DGO, SON

Blepharidachne bigelovii (S. Watson) Hack. CHIH, COAH, NLE

*Blepharoneuron shepherdii (Vasey) P.M. Peterson \& Annable CHIH, DGO, SON

Bothriochloa alta (Hitchc.) Henrard CHIS, CHIH, COAH, DGO, GRO, GTO, HGO, JAL, MEX, NAY, OAX, PUE, QRO, SLP, TAMS, VER, ZAC

Bothriochloa barbinodis (Lag.) Herter AGS, BCN, BCS, CHIS, CHIH, COAH, COL, CDMX, DGO, GTO, GRO, HGO, JAL, MEX, MICH, MOR, NAY, NLE, OAX, PUE, QRO, SLP, SIN, SON, TAMS, TLAX, VER, ZAC

Bothriochloa edwardsiana (Gould) Parodi COAH, NLE, OAX, PUE

Bothriochloa hirtifolia (J. Presl) Henrard AGS, CHIS, CHIH, COAH, COL, CDMX, DGO, GTO, GRO, HGO, JAL, MEX, MICH, MOR, NAY, OAX, PUE, QRO, SLP, TLAX, VER, ZAC Bothriochloa hybrida (Gould) Gould AGS, COAH, CDMX, DGO, GTO, GRO, HGO, JAL, MEX, MICH, NLE, OAX, QRO, SLP, TAMS, VER, ZAC

Bothriochloa laguroides (DC.) Herter AGS, BCN, CHIS, CHIH, COAH, COL, CDMX, DGO, GTO, GRO, HGO, JAL, MEX, MICH, MOR, NAY, NLE, OAX, PUE, QRO, SLP, SIN, SON, TAB, TAMS, TLAX, VER, ZAC

Bothriochloa longipaniculata (Gould) Allred \& Gould CHIH, COAH, DGO, JAL, MICH, NLE, SLP, TAMS 
Bothriochloa springfieldii (Gould) Parodi AGS, CHIH, COAH, DGO, GTO, GRO, HGO, JAL, MICH, NAY, OAX, PUE, SLP, VER, ZAC

Bothriochloa wrightii (Hack.) Henrard AGS, CHIH, DGO, GRO, MEX, MOR, NLE, OAX, SLP, VER, ZAC

Bouteloua alamosana Vasey CHIS, GRO, JAL, MEX, OAX, PUE, SIN, SON, ZAC

Bouteloua americana (L.) Scribn. CAM, CHIS, YUC

*Bouteloua annua Swallen BCN, BCS, SON

Bouteloua aristidoides (Kunth) Griseb. AGS, BCN, BCS, CHIH, COAH, COL, CDMX, DGO, GTO, JAL, MEX, MICH, NAY, OAX, PUE, QRO, SLP, SIN, SON, ZAC

Bouteloua barbata Lag. AGS, BCN, BCS, CHIH, COAH, COL, CDMX, DGO, GTO, GRO, HGO, JAL, MEX, MICH, NAY, NLE, OAX, PUE, QRO, SLP, SIN, SON, TAMS, TLAX, ZAC *Bouteloua bracteata (McVaugh) J.T. Columbus MICH

Bouteloua breviseta Vasey CHIH, COAH, DGO, NLE, SLP, TAMS, VER, ZAC

Bouteloua curtipendula (Michx.) Torr. AGS, BCN, BCS, CHIS, CHIH, COAH, COL, CDMX, DGO, GTO, GRO, HGO, JAL, MEX, MICH, MOR, NAY, NLE, OAX, PUE, QRO, SLP, SIN, SON, TAMS, TLAX, VER, ZAC

*Bouteloua chasei Swallen COAH, HGO, NLE, QRO, SLP, VER, ZAC

*Bouteloua chihuahuana (M.C. Johnst.) J.T. Columbus CHIH, ZAC

Bouteloua chondrosioides (Kunth) Benth. ex S. Watson AGS, BCN, CHIS, CHIH, COAH, CDMX, DGO, GTO, GRO, JAL, MEX, MICH, MOR, NAY, NLE, OAX, PUE, SLP, SON, TAMS, TLAX, ZAC

Bouteloua dactyloides (Nutt.) J.T. Columbus AGS, CHIH, COAH, CDMX, DGO, GTO, HGO, JAL, MEX, MOR, NLE, PUE, QRO, SLP, SON, TAMS, TLAX, VER, ZAC

Bouteloua dimorpha J.T. Columbus CAM, CHIS, COL, GRO, HGO, JAL, MEX, MICH, MOR, NAY, OAX, PUE, QRO, QROO, SLP, VER, YUC

*Bouteloua distans Swallen NLE, OAX, PUE

Bouteloua disticha (Kunth) Benth. CAM, GTO, GRO, JAL, MEX, MICH, MOR, NAY, OAX, TAMS, YUC

Bouteloua diversispicula J.T. Columbus AGS, CHIS, CHIH, COL, CDMX, GTO, GRO, JAL, MEX, MICH, MOR, NAY, OAX, PUE, SLP, SIN, SON, ZAC

*Bouteloua elata Reeder \& C. Reeder CHIS, COL, GRO, JAL, NAY, OAX

Bouteloua eludens Griffiths CHIH, COAH, SIN, SON

Bouteloua erecta (Vasey \& Hack.) J.T. Columbus BCN, CHIH, COAH, COL, GTO, JAL, MEX, MICH, MOR, NLE, OAX, SIN, SON, TAMS, ZAC

Bouteloua eriopoda (Torr.) Torr. BCN, CHIH, COAH, DGO, NLE, SLP, SON, TAMS, ZAC

*Bouteloua eriostachya (Swallen) Reeder COAH

Bouteloua gracilis (Kunth) Lag. ex Griffiths AGS, BCN, CHIS, CHIH, COAH, CDMX, DGO, GTO, GRO, HGO, JAL, MEX, MICH, NAY, NLE, OAX, PUE, QRO, SLP, SIN, SON, TAMS, TLAX, VER, ZAC

*Bouteloua griffithsii J.T. Columbus GRO, JAL, MEX, MICH, MOR, OAX, PUE, QRO
*Bouteloua johnstonii Swallen COAH, DGO, NLE, ZAC Bouteloua juncea (Desv. ex P. Beauv.) Hitchc. HGO, MOR, QRO, VER, YUC

*Bouteloua karwinskii (E. Fourn.) Griffiths AGS, COAH, NLE, SLP, TAMS, ZAC

Bouteloua media (E. Fourn.) Gould \& Kapadia CHIS, COAH, COL, CDMX, DGO, GRO, JAL, MEX, MICH, MOR, NAY, NLE, OAX, PUE, SLP, SON, VER, ZAC

Bouteloua mexicana (Scribn.) J.T. Columbus GRO, MEX

*Bouteloua multifida (Griffiths) J.T. Columbus CHIH, CDMX, GRO, JAL, MEX, MICH, MOR, OAX, PUE, SON, TAMS

*Bouteloua nervata Swallen HGO, MEX, VER

Bouteloua parryi (E. Fourn.) Griffiths CHIH, COAH, COL, CDMX, DGO, GTO, GRO, JAL, MEX, MOR, NLE, PUE, SLP, SIN, SON, ZAC

*Bouteloua pedicellata Swallen GTO, HGO, PUE, SLP, TLAX, VER

*Bouteloua polymorpha (E. Fourn.) J.T. Columbus AGS, COL, CDMX, DGO, GTO, GRO, HGO, JAL, MEX, MICH, MOR, NAY, OAX, PUE, QRO, SLP, SIN, SON, TLAX, ZAC

*Bouteloua purpurea Gould \& Kapadia CDMX, GTO, HGO, JAL, MEX, MICH, QRO, SLP

*Bouteloua quiriegoensis Beetle SIN, SON

*Bouteloua reederorum J.T. Columbus AGS, COAH, DGO, GRO, HGO, MEX, MICH, OAX, PUE, SLP, ZAC

*Bouteloua reflexa Swallen BCN, BCS, NAY, SIN, SON

Bouteloua repens (Kunth) Scribn. \& Merr. AGS, BCN, BCS, CAM, CHIS, CHIH, COAH, COL, CDMX, DGO, GTO, GRO, HGO, JAL, MEX, MICH, MOR, NAY, NLE, OAX, PUE, QRO, QROO, SLP, SIN, SON, TAB, TAMS, TLAX, VER, YUC, ZAC

Bouteloua rigidiseta (Steud.) Hitchc. CHIH, COAH, NLE, SON, TAMS

Bouteloua scabra (Kunth) J.T. Columbus CHIS, COL, GRO, HGO, NAY, OAX, QRO, TAB

*Bouteloua scorpioides Lag. AGS, CHIH, COAH, CDMX, DGO, GTO, HGO, JAL, MEX, MICH, MOR, NAY, NLE, OAX, PUE, QRO, SLP, TAMS, TLAX, VER, ZAC

Bouteloua simplex Lag. AGS, BCN, BCS, CHIH, COAH, CDMX, DGO, GTO, GRO, HGO, JAL, MEX, MICH, MOR, NLE, OAX, PUE, QRO, SLP, SON, TAMS, TLAX, VER, ZAC *Bouteloua tamaulipensis G.J. Pierce ex D. Pacheco \& J.T. Columbus TAMS

Bouteloua triaena (Trin. ex Spreng.) Scribn. CAM, CHIS, CHIH, COAH, COL, CDMX, GTO, GRO, HGO, JAL, MEX, MICH, MOR, NAY, NLE, OAX, PUE, QRO, SLP, SIN, TLAX, YUC

Bouteloua trifida Thurb. BCN, BCS, CHIH, COAH, DGO, GTO, NLE, SLP, SON, TAMS, VER, ZAC

Bouteloua uniflora Vasey AGS, CHIH, COAH, DGO, GTO, HGO, JAL, MEX, NLE, OAX, PUE, QRO, SLP, TAMS, TLAX, VER, ZAC

*Bouteloua varia (Swallen) J.T. Columbus GRO, JAL, MICH, OAX, PUE

Bouteloua warnockii Gould \& Kapadia CHIH, COAH, NLE, SON, TAMS

Bouteloua williamsii Swallen AGS, CHIS, DGO, GRO, JAL, 
MEX, MICH, NAY, OAX, PUE, SLP, ZAC

Brachypodium mexicanum (Roem. \& Schult.) Link AGS, BCS, CHIS, COAH, COL, CDMX, DGO, GTO, GRO, HGO, JAL, MEX, MICH, MOR, NLE, OAX, PUE, QRO, SLP, TAMS, TLAX, VER, ZAC

*Brachypodium pringlei Scribn. ex Beal COAH, DGO, NLE, TAMS

Bromidium tandilense (Kuntze) Rúgolo BCN

Bromus anomalus Rupr. ex E. Fourn. AGS, BCN, BCS, CAM, CHIS, CHIH, COAH, CDMX, DGO, GTO, GRO, HGO, JAL, MEX, MICH, MOR, NLE, OAX, PUE, QRO, SLP, SON, TAMS, TLAX, VER, ZAC

Bromus arizonicus (Shear) Stebbins BCN, CHIH, COAH, NLE, SON

*Bromus attenuatus Swallen HGO, MICH, NLE, TAMS

Bromus carinatus Hook. \& Arn. AGS, BCN, BCS, CAM, CHIS, CHIH, COAH, COL, CDMX, DGO, GTO, GRO, HGO, JAL, MEX, MICH, MOR, NAY, NLE, OAX, PUE, QRO, SLP, SIN, SON, TAMS, TLAX, VER, ZAC

Bromus ciliatus L. BCN, CHIH, COAH, DGO, JAL, MICH, NAY, NLE, SIN, SON, VER, ZAC

*Bromus densus Swallen COAH, NLE, TAMS

Bromus dolichocarpus Wagnon CHIS, COL, CDMX, HGO, JAL, MEX, MICH, MOR, OAX, PUE, TLAX, VER

Bromus exaltatus Bernh. CHIS, CHIH, COL, CDMX, DGO, GTO, GRO, HGO, JAL, MEX, MICH, MOR, OAX, PUE, SLP, TLAX, VER, ZAC

Bromus frondosus (Shear) Wooton \& Standl. CHIH, COAH, SON

Bromus lanatipes (Shear) Rydb. COAH, SLP

Bromus madritensis L. BCN

Bromus marginatus Nees ex Steud. BCN, CHIH, COAH, NLE, SON

Bromus mucroglumis Wagnon BCS, CHIH, COAH, DGO, NLE, SON

Bromus polyanthus Scribn. ex Shear CHIH, SON, TAMS

Bromus porteri (J.M. Coult.) Nash BCS, CAM, CHIS, CHIH, COAH, CDMX, DGO, HGO, JAL, MEX, NLE, OAX, PUE, QRO, SLP, SON, TAMS

Bromus pseudolaevipes Wagnon $\mathrm{BCN}$

Bromus texensis (Shear) Hitchc. COAH, HGO, SLP

*Bromus thysanoglottis Soderst. \& Beaman CHIH, DGO, SIN

*Calamagrostis coahuilensis P.M. Peterson, Soreng \& Valdés-

Reyna COAH, NLE

Calamagrostis densa Vasey BCN

*Calamagrostis divaricata P.M. Peterson, Soreng \& Valdés-

Reyna DGO, SIN

*Calamagrostis eriantha (Kunth) Steud. CDMX, MEX, OAX, PUE, VER

*Calamagrostis mcvaughii Sohns COL, DGO, JAL, MICH, SIN

*Calamagrostis orizabae (Rupr. ex E. Fourn.) Beal COL, CDMX, HGO, JAL, MEX, MICH, MOR, OAX, PUE, TLAX, VER

Calamagrostis pringlei Scribn. ex Beal AGS, CHIS, CHIH, COAH, DGO, HGO, JAL, MICH, NLE, SIN, ZAC

Calamagrostis purpurascens $\mathrm{R}$. Br. COAH, NLE, TAMS

Calamagrostis recta (Kunth) Trin. ex Steud. GRO, OAX, VER
Calamagrostis tolucensis (Kunth) Trin. ex Steud. CHIS, CDMX, GRO, JAL, MEX, MICH, MOR, OAX, PUE, QRO, TLAX, VER Calamagrostis vulcanica Swallen CHIS

Cenchrus complanatus (Nees) Morrone CAM, CHIS, MOR, SIN, TAB, VER

Cenchrus echinatus L. AGS, BCN, BCS, CAM, CHIS, CHIH, COAH, COL, DGO, GTO, GRO, HGO, JAL, MEX, MICH, MOR, NAY, NLE, OAX, PUE, QRO, QROO, SLP, SIN, SON, TAB, TAMS, TLAX, VER, YUC, ZAC

Cenchrus incertus M.A. Curtis AGS, BCN, BCS, CAM, CHIS, CHIH, COAH, COL, CDMX, DGO, GTO, GRO, HGO, JAL, MEX, MICH, MOR, NAY, NLE, OAX, PUE, QRO, QROO, SLP, SIN, SON, TAB, TAMS, TLAX, VER, YUC, ZAC

Cenchrus longispinus (Hack.) Fernald CAM, CHIS, CHIH, COL, DGO, GTO, GRO, JAL, MEX, MICH, NAY, OAX, QROO, SLP, SON, TAMS

Cenchrus multiflorus J. Presl AGS, CAM, CHIS, CHIH, COAH, COL, DGO, GRO, JAL, MEX, MICH, MOR, NAY, OAX, SON, $\mathrm{ZAC}$

Cenchrus myosuroides Kunth AGS, BCN, BCS, CHIS, CHIH, COAH, COL, DGO, GTO, GRO, HGO, JAL, MEX, MICH, MOR, NAY, NLE, OAX, PUE, QRO, SLP, SON, TAMS, VER, $\mathrm{ZAC}$

Cenchrus palmeri Vasey BCN, BCS, SIN, SON

Cenchrus pilosus Kunth CAM, CHIS, COL, CDMX, GRO, JAL, MEX, MICH, MOR, NAY, OAX, PUE, QRO, QROO, SIN, VER, YUC, ZAC

*Cenchrus prolificus (Chase) Morrone GRO, MEX, MICH, OAX, SLP, VER

Cinna poiformis (Kunth) Scribn. \& Merr. CHIS, CDMX, GRO, HGO, MEX, MICH, MOR, OAX, PUE, TLAX, VER

Coleataenia caricoides (Nees ex Trin.) Soreng CHIS

Coleataenia stenodes (Griseb.) Soreng CHIS

Cottea pappophoroides Kunth CHIH, COAH, DGO, GTO, JAL, NLE, OAX, PUE, SLP, SON, TAMS, ZAC

Cryptochloa strictiflora (E. Fourn.) Swallen CHIS, OAX, PUE, TAB, VER

*Ctenium planifolium (J. Presl) Kunth CHIS, GRO, OAX, PUE *Ctenium plumosum (Hitchc.) Swallen COL, JAL, NAY, SIN, ZAC

* Chaboissaea decumbens (Swallen) Reeder \& C. Reeder CHIH *Chaboissaea ligulata E. Fourn. AGS, CHIH, CDMX, DGO, GTO, JAL, MEX, SLP, ZAC

*Chaboissaea subbiflora (Hitchc.) Reeder \& C. Reeder CHIH, DGO, SON

Chaetium bromoides (J. Presl) Benth. ex Hemsl. CHIS, CHIH, COL, CDMX, GRO, HGO, JAL, MEX, MICH, MOR, NAY, OAX, PUE, TLAX, VER, ZAC

Chascolytrum subaristatum (Lam.) Desv. AGS, CHIS, COAH, CDMX, DGO, GTO, HGO, JAL, MEX, MICH, NLE, OAX, PUE, QRO, SLP, TAMS, TLAX, VER, ZAC

*Chasmanthium curvifolium (Valdés-Reyna, Morden \& S.L. Hatch) Wipff \& S.D. Jones GTO, JAL, SLP, TAMS, ZAC

Chasmanthium latifolium (Michx.) H.O. Yates COAH, NLE, VER

Chloris andropogonoides E. Fourn. CHIH, COAH, NLE, SLP, TAMS 
Chloris ciliata Sw. CAM, CHIS, COAH, GRO, HGO, NLE, OAX, PUE, QRO, QROO, SLP, TAB, TAMS, VER, YUC Chloris cucullata Bisch. CAM, COAH, NLE, TAMS, YUC Chloris radiata (L.) Sw. CHIS, CHIH, COL, GTO, GRO, JAL, MICH, NLE, OAX, PUE, SLP, TAB, VER

Chloris rufescens Lag. AGS, CHIS, CHIH, COAH, COL, CDMX, DGO, GTO, GRO, HGO, JAL, MEX, MICH, MOR, NLE, OAX, PUE, QRO, SLP, TLAX, VER, ZAC

Chloris submutica Kunth AGS, CHIS, CHIH, COAH, COL, CDMX, DGO, GTO, HGO, JAL, MEX, MICH, MOR, NAY, NLE, OAX, PUE, QRO, SLP, SON, TAMS, TLAX, VER, ZAC Chloris verticillata Nutt. BCS, COAH, NLE

Chloris $\times$ subdolichostachya Müll. Hal CHIH, COAH, NLE, TAMS

Chondrosum hirsutum (Lag.) Sweet AGS, BCN, BCS, CHIS, CHIH, COAH, COL, CDMX, DGO, GTO, GRO, HGO, JAL, MEX, MICH, MOR, NAY, NLE, OAX, PUE, QRO, SLP, SIN, SON, TAB, TAMS, TLAX, VER, YUC, ZAC

*Chusquea aperta L.G. Clark OAX

*Chusquea bilimekii E. Fourn. MEX, MICH, OAX, PUE, SLP, VER

*Chusquea circinata Soderstr. \& C.E. Calderón CHIS, COL, DGO, JAL, MICH, NAY, OAX, SIN

Chusquea coronalis Soderstr. \& C.E. Calderón CHIS, COL, OAX, SIN

Chusquea cortesii L.G. Clark \& Ruiz-Sanchez CHIS, GRO, JAL, MEX, MICH, NAY

Chusquea foliosa L.G. Clark CHIS

*Chusquea galeottiana Rupr. ex Munro CHIS, GRO, OAX

*Chusquea gibcooperi Ruiz-Sánchez, Mejía-Saulés, Cortés \&

L.G. Clark HGO, PUE, VER

*Chusquea glauca L.G. Clark HGO, PUE, VER

Chusquea lanceolata Hitchc. CHIS, VER

Chusquea liebmannii E. Fourn. CHIS, COL, DGO, GRO, HGO,

JAL, MICH, NAY, OAX, PUE, SIN, TAB, VER, ZAC

Chusquea longifolia Swallen CHIS, SIN

*Chusquea matlatzinca L.G. Clark \& Ruiz-Sanchez MICH

*Chusquea mulleri Munro VER

*Chusquea nedjaquithii Ruiz-Sánchez, Mejía-Saulés \& L.G. Clark OAX

Chusquea nelsonii Scribn. \& J.G. Sm. CHIS, COL, GRO, JAL, MEX, MICH, NAY, OAX

*Chusquea perotensis L.G. Clark, G. Cortés \& Cházaro OAX, VER

Chusquea pittieri Hack. CHIS, GRO, MICH, OAX

*Chusquea repens L.G. Clark \& Londoño CHIS, OAX

Chusquea simpliciflora Munro CHIS, COL, OAX

Chusquea sulcata Swallen CHIS, OAX

*Danthonia chiapasensis Davidse CHIS

Danthonia secundiflora J. Presl CHIS, HGO, PUE

Danthonia spicata (L.) P. Beauv. ex Roem. \& Schult. COAH, HGO, PUE, TAMS, VER

Dasyochloa pulchella (Kunth) Willd. ex Rydb. AGS, BCN, CHIH, COAH, CDMX, DGO, GTO, HGO, JAL, MEX, MICH, MOR, NLE, QRO, SLP, SON, TAMS, VER, ZAC

Deschampsia caespitosa (L.) P. Beauv. COAH, NLE, SON

Deschampsia danthonioides (Trin.) Munro BCN, CHIH, SON
Deschampsia elongata (Hook.) Munro BCN, CHIH, COL, CDMX, DGO, HGO, JAL, MEX, MICH, MOR, NAY, SON, TLAX, VER, ZAC

*Deschampsia liebmanniana (E. Fourn.) Hitchc. CDMX, HGO, JAL, MEX, MICH, PUE, VER

Dichanthelium aciculare (Desv. ex Poir.) Gould \& C.A. Clark CAM, CHIS, NLE, OAX, TAB, TAMS, VER

Dichanthelium acuminatum (Sw.) Gould \& C.A. Clark BCN, CAM, CHIS, CHIH, COAH, GRO, NLE, OAX, PUE, SLP, SON, TAB, TAMS, VER

Dichanthelium commutatum (Schult.) Gould CHIS, COL, GRO, HGO, JAL, MEX, MICH, MOR, NAY, NLE, OAX, PUE, QRO, TAMS, VER

Dichanthelium dichotomum (L.) Gould CHIS, HGO, MEX, OAX, PUE, QROO, SLP, TAMS, VER

Dichanthelium ensifolium (Baldwin ex Elliott) Gould HGO, SLP, VER

Dichanthelium lanuginosum (Elliott) Gould BCN

Dichanthelium laxiflorum (Lam.) Gould CHIS, CHIH, COL, HGO, JAL, MEX, MICH, OAX, PUE, QRO, SLP, SON, TAMS, VER

*Dichanthelium macrospermum Gould QRO, SLP, TAMS

Dichanthelium nodatum (Hitchc. \& Chase) Gould TAMS

Dichanthelium oligosanthes (Schult.) Gould $\mathrm{CHIH}, \mathrm{COAH}$, NLE, SON, TAMS, VER

Dichanthelium ovale (Elliott) Gould \& C.A. Clark CHIS, MEX, PUE, SLP, TAMS, VER

Dichanthelium pedicellatum (Vasey) Gould COAH, NLE, OAX, TAMS

Dichanthelium portoricense (Desv. ex Harm.) B.F. Hansen \& Wunderlin TAMS, VER

Dichanthelium sphaerocarpon (Elliott) Gould CHIS, CHIH, COAH, COL, CDMX, DGO, GTO, GRO, HGO, JAL, MEX, MICH, NAY, NLE, OAX, PUE, QROO, SLP, SON, TAMS, VER, YUC

Dichanthelium strigosum (Muhl. ex Elliott) Freckmann CAM, CHIS, OAX, QROO

Dichanthelium umbonulatum (Swallen) Davidse OAX

Dichanthelium viscidellum (Scribn.) Gould CAM, CHIS, HGO, OAX, PUE, QROO, VER

Digitaria arenicola (Swallen) Beetle TAMS

Digitaria argillacea (Hitchc. \& Chase) Fernald AGS, CHIS, CHIH, COL, DGO, GTO, GRO, HGO, JAL, MEX, MICH, MOR, NAY, OAX, PUE, QRO, SIN, SON, ZAC

*Digitaria badia (Scribn. \& Merr.) Fernald CHIS, COL, CDMX, GTO, HGO, JAL, MEX, MICH, OAX, QRO, TLAX, VER, ZAC

Digitaria bakeri (Nash) Fernald CHIS, VER

Digitaria bicornis (Lam.) Roem. \& Schult. AGS, BCN, BCS, CAM, CHIS, CHIH, COAH, COL, CDMX, GTO, GRO, HGO, JAL, MEX, MICH, MOR, NAY, NLE, OAX, PUE, QRO, QROO, SLP, SIN, SON, TAB, TAMS, VER, YUC, ZAC

*Digitaria breedlovei R.W. Pohl \& Davidse CHIS, DGO

Digitaria californica (Benth.) Henrard AGS, BCN, BCS, CHIH, COAH, DGO, GTO, HGO, JAL, NLE, PUE, QRO, SLP, SIN, SON, TAMS, ZAC

Digitaria cayoensis Swallen CHIS, OAX, QROO, YUC 
Digitaria curtigluma Hitchc. CHIS, COL, CDMX, GTO, JAL, MEX, MICH, OAX, PUE

Digitaria filiformis (L.) Koeler AGS, CHIS, CHIH, COAH, COL, CDMX, DGO, GTO, GRO, JAL, MEX, MICH, MOR, NAY, NLE, OAX, SON, TAMS, VER, YUC, ZAC

Digitaria hitchcockii (Chase) Stuck. COAH, GTO, JAL, NLE, OAX, QRO, SLP, TAMS, ZAC

Digitaria horizontalis Willd. BCS, CAM, CHIS, COAH, COL, CDMX, GRO, HGO, JAL, MEX, MICH, MOR, NAY, NLE, OAX, PUE, QRO, QROO, SLP, SIN, SON, TAB, TAMS, VER, YUC, ZAC

Digitaria insularis (L.) Mex ex Ekman AGS, BCN, CAM, CHIS, CHIH, COAH, COL, DGO, GTO, GRO, HGO, JAL, MEX, MICH, MOR, NAY, NLE, OAX, PUE, QRO, QROO, SLP, SIN, SON, TAB, TAMS, VER, YUC, ZAC

Digitaria leucites (Trin.) Henrard BCS, CHIS, COL, CHIH, CDMX, DGO, GRO, HGO, JAL, MEX, MICH, MOR, NLE, OAX, PUE, SLP, TAB, TLAX, VER, ZAC

Digitaria leucocoma (Nash) Urb. CHIS

*Digitaria michoacanensis Sánchez-Ken MEX, MICH

Digitaria obtusa Swallen CHIS, VER

Digitaria panicea (Sw.) Urb. CHIS, CHIH, COAH, DGO, GRO, JAL, OAX, SON, ZAC

*Digitaria paniculata Soderstr. ex McVaugh COL, JAL, ZAC

Digitaria patens (Swallen) Henrard CHIH, COAH, DGO, GTO,

JAL, NLE, QRO, SLP, SON, TAMS, VER

Digitaria pubiflora (Vasey) Wipff CHIH, COAH, DGO, GTO, JAL, MOR, NLE, PUE, QRO, SLP, SON, TAMS, VER, ZAC

Digitaria sellowii (Müll. Hal.) Henrard CHIS, COAH, HGO, NLE, OAX, PUE, QRO, QROO, SLP, TAB, TAMS, VER, YUC Digitaria texana Hitchc. VER

Digitaria violascens Link CHIS, VER

Disakisperma dubium (Kunth) P.M. Peterson \& N. Snow AGS, BCN, BCS, CAM, CHIS, CHIH, COAH, COL, CDMX, DGO, GTO, HGO, JAL, MEX, MICH, MOR, NAY, NLE, OAX, PUE, QRO, SLP, SIN, SON, TAMS, TLAX, VER, YUC, ZAC

Dissanthelium californicum (Nutt.) Benth. BCN

Dissanthelium calycinum (J. Presl) Hitchc. MEX, PUE, SLP, TLAX

*Distichlis bajaensis H.L. Bell BCN

Distichlis eludens (Soderstr. \& H.F. Decker) H.L. Bell \& J.T. Columbus DGO, SLP

Distichlis littoralis (Engelm.) H.L. Bell \& J.T. Columbus BCN, BCS, CAM, CHIS, CHIH, COAH, MICH, NAY, NLE, OAX, QROO, SLP, SIN, SON, TAMS, VER, YUC

Distichlis palmeri (Vasey) Fasset ex I.M. Johnst. BCN, BCS, DGO, SON

Distichlis spicata (L.) Greene AGS, BCN, BCS, CAM, CHIS, CHIH, COAH, COL, CDMX, DGO, GTO, GRO, HGO, JAL, MEX, MICH, NAY, NLE, OAX, PUE, QRO, QROO, SLP, SIN, SON, TAB, TAMS, TLAX, VER, YUC, ZAC

Echinochloa crus-pavonis (Kunth) Schult. AGS, BCN, BCS, CAM, CHIS, CHIH, COAH, COL, CDMX, DGO, GTO, GRO, HGO, JAL, MEX, MICH, MOR, NAY, OAX, PUE, QRO, SLP, SIN, SON, TAB, TAMS, TLAX, VER, ZAC

Echinochloa holciformis (Kunth) Chase AGS, CAM, CHIS, CHIH, COAH, CDMX, DGO, GTO, HGO, JAL, MEX, MICH,
MOR, NAY, PUE, QRO, SLP, TLAX, VER, ZAC

*Echinochloa jaliscana McVaugh AGS, GTO, JAL, MEX, $\mathrm{MICH}, \mathrm{ZAC}$

Echinochloa muricata (P. Beauv.) Fernald CHIH, COAH, DGO, SON, VER

Echinochloa oplismenoides (E. Fourn.) Hitchc. AGS, CHIH, CDMX, DGO, GTO, GRO, HGO, JAL, MEX, MICH, OAX, PUE, QRO, SON, TAB, TLAX, VER, ZAC

Echinochloa polystachya (Kunth) Hitchc. AGS, CAM, CHIS, CHIH, CDMX, DGO, GTO, GRO, JAL, MEX, MICH, OAX, PUE, SLP, SIN, SON, TAB, TAMS, TLAX, VER, YUC, ZAC Echinochloa walteri (Pursh) A. Heller CAM, CHIS, CHIH, COAH, MICH, QROO, SLP, TAB, VER, YUC

Echinolaena gracilis Swallen CHIS, PUE, VER

Echinolaena standleyi (Hitchc.) Stieber CHIS

Elionurus barbiculmis Hack. AGS, BCN, BCS, CHIS, CHIH, COAH, CDMX, DGO, GTO, GRO, JAL, MEX, MICH, NAY, PUE, QRO, SLP, SIN, SON, TAMS, TLAX, ZAC

Elionurus ciliaris Kunth AGS, CHIS, CHIH, COL, DGO, GTO, GRO, JAL, MEX, MICH, MOR, NAY, NLE, OAX, PUE, QRO, SLP, TAB, TLAX, VER, ZAC

Elymus canadensis L. CHIH, COAH, NLE, QRO, SLP, SON, VER

Elymus elymoides (Raf.) Swezey BCN, CHIH, COAH, CDMX, DGO, GTO, HGO, JAL, MEX, NLE, OAX, PUE, SLP, SON, VER, ZAC

Elymus glaucus Buckley BCN

*Elymus pringlei Scribn. \& Merr. AGS, COAH, GTO, HGO, MEX, NLE, PUE, QRO, SLP, TAMS, VER, ZAC

Elymus riparius Wiegand DGO

Elymus trachycaulus (Link) Gould ex Shinners BCN, CHIH, COAH, DGO, HGO, NLE, PUE, SON, TAMS, ZAC

Elymus $\times$ pseudorepens (Scribn. \& J.G. Sm.) Barkworht \& D.R. Dewey COAH, DGO, GRO, NLE, OAX

Eragrostis acutiflora (Kunth) Nees CHIS, COL, GRO, JAL, MICH, NAY, OAX, TAB, VER

Eragrostis contrerasii R.W. Pohl CAM, QROO, TAB, VER

Eragrostis curtipedicellata Buckley COAH, NLE

Eragrostis elliottii S. Watson CAM, CHIS, JAL, MICH, NLE, OAX, QROO, TAB, TAMS, TLAX, VER, YUC

Eragrostis erosa Scribn. ex Beal CHIH, COAH, DGO, NLE, SLP, SON, TAMS, ZAC

Eragrostis excelsa Griseb. CAM, QROO, VER, YUC

*Eragrostis glandulosa L.H. Harv. CHIS, CDMX, DGO, GRO, JAL, MEX, MICH, MOR, NAY, OAX, TAMS, ZAC

Eragrostis guatemalensis Witherspoon CHIS, MEX, PUE

Eragrostis hirsuta (Michx.) Nees CHIS, GTO, JAL, NLE, OAX, SLP, TAB, TAMS, VER

Eragrostis hirta E. Fourn. CHIS, CDMX, JAL, MEX, MICH, NLE, OAX, SLP, TAMS, VER, ZAC

Eragrostis hondurensis R.W. Pohl CHIS, OAX

Eragrostis hypnoides (Lam.) Britton, Sterns \& Poggenb. BCN, BCS, CAM, CHIS, COAH, GTO, GRO, JAL, NAY, NLE, OAX, SLP, SON, TAB, TAMS, VER

Eragrostis intermedia Hitchc. AGS, BCN, BCS, CHIS, CHIH, COAH, CDMX, DGO, GTO, GRO, HGO, JAL, MEX, MICH, MOR, NAY, NLE, OAX, PUE, QRO, SLP, SON, TAMS, TLAX, 


\section{VER, ZAC}

Eragrostis japonica (Thunb.) Trin. CHIS, COL, GRO, MEX, MICH, MOR, NAY, OAX, TLAX, VER

Eragrostis lugens Nees AGS, BCN, CHIS, CHIH, COAH, DGO, GTO, HGO, JAL, MEX, MICH, MOR, NLE, OAX, PUE, QRO, SLP, SIN, SON, TAMS, TLAX, VER, ZAC

Eragrostis lutescens Scribn. COAH, NLE, SLP

Eragrostis maypurensis (Kunth) Steud. CHIS, CHIH, COL, CDMX, DGO, GRO, JAL, MEX, MICH, MOR, NAY, OAX, PUE, QROO, SIN, SON, TAB, VER, YUC, ZAC

Eragrostis mexicana (Hornem.) Link AGS, BCN, BCS, CHIS, CHIH, COAH, COL, CDMX, DGO, GTO, GRO, HGO, JAL, MEX, MICH, MOR, NAY, NLE, OAX, PUE, QRO, SLP, SIN, SON, TAMS, TLAX, VER, ZAC

Eragrostis palmeri $\mathrm{S}$. Watson BCN, CHIH, COAH, DGO, NLE, OAX, SON, TAMS, VER, ZAC

Eragrostis pectinacea (Michx.) Nees AGS, BCN, BCS, CAM, CHIS, CHIH, COAH, COL, CDMX, DGO, GTO, GRO, HGO, JAL, MEX, MICH, MOR, NAY, NLE, OAX, PUE, QRO, QROO, SLP, SIN, SON, TAB, TAMS, TLAX, VER, YUC, ZAC

*Eragrostis plumbea Scribn. ex Beal AGS, COL, GTO, JAL, MEX, MICH, PUE, SLP, VER, ZAC

*Eragrostis pringlei Mattei CHIH, DGO, JAL, MICH, SON, ZAC

Eragrostis prolifera (Sw.) Steud. CAM, CHIS, COL, GRO, JAL, NAY, OAX, PUE, QROO, SIN, TAB, VER, YUC

Eragrostis reptans (Michx.) Nees BCS, CHIS, COAH, GRO, NLE, OAX, TAB, TAMS, VER

Eragrostis secundiflora J. Presl BCN, CAM, CHIS, CHIH, COAH, OAX, QROO, TAB, TAMS, VER, YUC

Eragrostis sessilispica Buckley CHIH, TAMS, VER

Eragrostis silveana Swallen COAH, NLE, OAX, QRO, SLP, TAMS

*Eragrostis simplex Scribn. CHIH, COAH, DGO

Eragrostis simpliciflora (J. Presl) Steud. CHIS

Eragrostis spectabilis (Pursh) Steud. CHIH, COAH, NLE, PUE, SLP, TAMS

Eragrostis spicata Vasey BCN, BCS, SON, TAMS

Eragrostis swallenii Hitchc. CHIH, COAH, DGO, GTO, GRO, JAL, MEX, MICH, OAX, PUE, QRO, SLP, TAMS, VER

Eragrostis trichodes (Nutt.) Wood CHIH, GTO, JAL, TAMS

Eriochloa acuminata (J. Presl) Kunth AGS, BCN, BCS, CHIH, COAH, COL, DGO, GTO, GRO, HGO, JAL, MEX, MICH, MOR, NAY, NLE, OAX, PUE, QRO, SLP, SIN, SON, TAMS, TLAX, VER, ZAC

Eriochloa aristata Vasey BCN, BCS, CAM, CHIS, CHIH, COL, DGO, GTO, GRO, JAL, MEX, MOR, NAY, OAX, PUE, SIN, SON, VER, YUC, ZAC

Eriochloa contracta Hitchc. BCN, CHIH, COAH, DGO, JAL, NAY, NLE, OAX, SIN, SON, TAMS

Eriochloa lemmonii Vasey \& Scribn. BCS, CHIH, COAH, COL, DGO, JAL, MEX, MICH, MOR, NAY, SIN, SON

Eriochloa nelsonii Scribn. \& J.G. Sm. CAM, CHIS, CHIH, COL, DGO, GRO, JAL, MEX, MICH, MOR, NAY, OAX, PUE, SIN, SON, YUC, ZAC

Eriochloa polystachya Kunth CHIS, CHIH
Eriochloa punctata (L.) Desv. ex Ham. CHIS, CHIH, COAH, COL, CDMX, DGO, GTO, GRO, HGO, JAL, MEX, MICH, MOR, NAY, NLE, OAX, PUE, SLP, TAB, TAMS, VER, YUC, ZAC

Eriochloa sericea (Scheele) Munro ex Vasey COAH, TAMS Eriochrysis cayennensis P. Beauv. CHIS, OAX, TAB, VER Erioneuron avenaceum (Kunth) Tateoka AGS, CHIH, COAH, CDMX, DGO, GTO, HGO, JAL, MEX, MICH, NLE, OAX, PUE, QRO, SLP, SON, TAMS, TLAX, VER, ZAC

Erioneuron nealleyi (Vasey) Tateoka AGS, CHIH, COAH, DGO, GTO, HGO, NLE, QRO, SLP, TAMS, ZAC

Erioneuron pilosum (Buckley) Nash AGS, CHIH, COAH, DGO, HGO, NLE, OAX, PUE, QRO, SLP, TAMS, VER, ZAC

*Eustachys floridana Chapm. TAMS

Festuca aguana E.B. Alexeev CHIS

Festuca amplissima Rupr. BCN, CHIS, CHIH, COAH, COL, CDMX, DGO, GTO, GRO, HGO, JAL, MEX, MICH, MOR, NAY, NLE, OAX, PUE, QRO, SIN, SON, TAMS, TLAX, VER Festuca arizonica Vasey COAH, DGO, MEX, NLE, SON, ZAC *Festuca bajacaliforniana M. González-Ledezma \& S.D. Koch $\mathrm{BCN}$

* Festuca bidenticulata E.B. Alexeev OAX, VER

Festuca breviglumis Swallen AGS, CHIS, CHIH, COL, DGO, GTO, GRO, JAL, MEX, MICH, MOR, OAX, TLAX, ZAC

*Festuca callosa (Piper) St.-Yves OAX, PUE

* Festuca coahuilana M. González-Ledema \& S.D. Koch COAH

* Festuca diclina Darbysh. CHIH

Festuca hephaestophila Nees ex Steud. CHIS, CDMX, MEX, MOR, NLE, PUE, TAMS, TLAX, VER

* Festuca hintoniana E.B. Alexeev COAH, NLE

* Festuca jaliscana E.B. Alexeev COL, JAL

Festuca ligulata Swallen CHIH, COAH, NLE

Festuca livida (Kunth) Willd. ex Spreng. CHIS, CDMX, MEX, PUE, TLAX, VER

* Festuca longiligula Darbysh. COAH, NLE

Festuca lugens (E. Fourn.) Hitchc. ex Hern.-Xol. CHIS, CHIH, CDMX, GTO, HGO, JAL, MEX, MICH, MOR, OAX, PUE, QRO, SLP, TAMS, TLAX, VER

*Festuca orizabensis E.B. Alexeev CHIS, CHIH, CDMX, DGO, GTO, HGO, JAL, MEX, MICH, MOR, NLE, PUE, VER

* Festuca pinetorum Swallen COAH, NLE, TAMS

* Festuca pringlei St.-Yves AGS, CHIH, COL, DGO, JAL, MICH, NLE, ZAC

*Festuca roblensis M. González-Ledezma GTO, JAL, ZAC Festuca rosei Piper AGS, CHIS, COL, CDMX, DGO, GRO, HGO, JAL, MEX, MICH, MOR, PUE, QRO, SLP, TAMS, TLAX, VER, ZAC

*Festuca rzedowskiana E.B. Alexeev HGO, JAL, MEX, MICH Festuca swallenii E.B. Alexeev GRO

*Festuca tancitaroensis M. González-Ledezma \& S.D. Koch JAL, MICH

Festuca thurberi Vasey COAH, NLE, TAMS Festuca tolucensis Kunth CHIS, CHIH, CDMX, DGO, HGO, JAL, MEX, MICH, MOR, PUE, SLP, TLAX, VER

*Festuca valdesii M. González-Ledezma \& S.D. Koch COAH, NLE, TAMS

Festuca viridula Vasey COAH, DGO 
Festuca willdenowiana Schult. \& Schult. f. CHIS, CDMX, GTO, GRO, HGO, JAL, MEX, MICH, OAX, PUE, QRO, TLAX, VER *Festuca $\times$ gonzalez-ledesmae Darbysh. MEX, TLAX, VER

*Festuca $\times$ miscella Darbysh. MEX

Glyceria borealis (Nash) Batsch CHIH, SON

Glyceria elata (Nash) Hitchc. GRO, SON

Glyceria septentrionalis Hitchc. MEX, QRO, SLP

Glyceria striata (Lam.) Hitchc. CHIS, CHIH, COAH, CDMX, HGO, MEX, MOR, NLE, OAX, PUE, SON

*Gouinia isabelensis J.J. Ortiz NAY

Gouinia latifolia (Griseb.) Vasey CAM, OAX, QROO, YUC

*Gouinia mexicana (Scribn.) Vasey QRO, SLP

*Gouinia papillosa Swallen CAM, QROO, TAMS, YUC

Gouinia virgata (J. Presl) Scribn. BCS, CAM, CHIS, CHIH, COL, DGO, GRO, JAL, MICH, MOR, NAY, NLE, OAX, QRO, QROO, SLP, SIN, SON, TAMS, VER, YUC, ZAC

Guadua amplexifolia J. Presl CHIS, MOR, OAX, SLP, SIN, TAB, TAMS, VER, YUC

Guadua angustifolia Kunth CAM, CHIS, GRO, JAL, OAX, PUE, QRO, SLP, TAB, TAMS, VER, ZAC

Guadua longifolia (E. Fourn.) R.W. Pohl CAM, CHIS, CHIH, DGO, JAL, MOR, NAY, OAX, PUE, QRO, QROO, SLP, SIN, SON, TAB, VER

Guadua paniculata Munro CHIS, COL, GRO, JAL, NAY, OAX, PUE, SLP, VER

*Guadua tuxtlensis Londoño \& Ruiz-Sánchez VER

Guadua velutina Londoño \& L.G. Clark OAX, SLP, TAB, TAMS, VER

Gymnopogon spicatus (Spreng.) Kuntze CHIS, VER

Gynerium sagittatum (Aubl.) P. Beauv. CAM, CHIS, HGO, OAX, PUE, QROO, SLP, TAB, VER

Hesperostipa neomexicana (Thurb.) Barkworth $\mathrm{BCN}, \mathrm{CHIH}$, COAH, DGO, NLE, SLP, SON, TAMS, ZAC

*Hesperostipa saxicola (Hitchc.) Valdés-Reyna \& Barkworth PUE

Heteropogon contortus (L.) P. Beauv. ex Roem. \& Schult. AGS, BCN, BCS, CAM, CHIS, CHIH, COAH, COL, CDMX, DGO, GTO, GRO, HGO, JAL, MEX, MICH, MOR, NAY, NLE, OAX, PUE, QRO, QROO, SLP, SIN, SON, TAB, TAMS, TLAX, VER, YUC, ZAC

Heteropogon melanocarpus (Elliott) Benth. AGS, BCN, BCS, CHIS, CHIH, COAH, COL, CDMX, DGO, GTO, GRO, JAL, MEX, MICH, MOR, NAY, NLE, OAX, PUE, QRO, SLP, SIN, SON, TAMS, VER, ZAC

*Hilaria annua Reeder \& C. Reeder COL, JAL

Hilaria belangeri (Steud.) Nash AGS, BCN, BCS, CHIS, CHIH, COAH, DGO, GTO, GRO, HGO, JAL, MEX, MICH, MOR, NAY, NLE, OAX, PUE, QRO, SLP, SIN, SON, TAMS, VER, ZAC

Hilaria cenchroides Kunth AGS, BCN, BCS, CHIS, CHIH, CDMX, DGO, GTO, GRO, HGO, JAL, MEX, MICH, MOR, NAY, NLE, OAX, PUE, QRO, SLP, SIN, SON, TAMS, TLAX, VER, ZAC

*Hilaria ciliata (Scribn.) Nash BCN, BCS, CHIH, COL, GTO, GRO, JAL, MICH, NAY, OAX, QRO, SLP, SIN, SON, TAMS, VER, ZAC

*Hilaria hintonii Sohns GRO, MEX, MICH, MOR, OAX, QRO
*Hilaria semplei Sohns JAL, MICH

Hilaria swallenii Cory AGS, CHIH, COAH, DGO, GRO, JAL, MEX, MICH, NLE, SLP, ZAC

Homolepis aturensis (Kunth) Chase CHIS, OAX, PUE, TAB, VER

Homolepis glutinosa (Sw.) Zuloaga \& Soderstr. CHIS, HGO, OAX, PUE, TAB, VER

Hopia obtusa (Kunth) Zuloaga \& Morrone AGS, CHIH, COAH, DGO, GTO, HGO, JAL, MEX, MICH, MOR, NLE, OAX, PUE, QRO, SLP, SON, TLAX, VER, ZAC

Hordeum arizonocum Covas BCN, DGO, SON

Hordeum brachyantherum Nevski BCN, SON

Hordeum depressum (Scribn. \& J.G. Sm.) Rydb. BCN

Hymenachne amplexicaulis (Rudge) Nees CAM, CHIS, COL, GRO, JAL, MICH, NAY, NLE, OAX, QROO, SIN, TAB, TAMS, VER, YUC

Hymenachne donacifolia (Raddi) Chase OAX

Ichnanthus inconstans (Trin. ex Nees) Doell OAX

Ichnanthus lanceolatus Scribn. \& J.G. Sm. CAM, CHIS, QROO,

TAB, YUC

Ichnanthus nemoralis (Schrad.) Hitchc. \& Chase QROO, YUC Ichnanthus nemorosus (Sw.) Döll CAM, CHIS, COL, GRO, HGO, JAL, OAX, PUE, QRO, QROO, SLP, SIN, VER

Ichnanthus pallens (Sw.) Munro ex Benth. CAM, CHIS, HGO, OAX, PUE, QROO, SLP, TAB, VER, YUC

Ichnanthus tenuis (J. Presl \& C. Presl) Hitchc. \& Chase CAM, CHIS, HGO, OAX, PUE, QROO, TAB, VER

Imperata brasiliensis Trin. BCN, BCS, CAM, CHIS, OAX, QRO, QROO, SLP, TAB, VER

Imperata brevifolia Vasey BCN, CHIH, JAL, NAY, SON, ZAC Imperata contracta (Kunth) Hitchc. CAM, CHIS, JAL, NAY, TAB, VER

Isachne arundinacea (Sw.) Griseb. CHIS, GRO, HGO, OAX, PUE, TAMS, VER

Isachne polygonoides (Lam.) Döll CHIS, GRO

Isachne pubescens Swallen CHIS, GRO, VER

Ischaemum latifolium (Spreng.) Kunth CHIS, OAX, PUE, QROO, VER

Ixophorus unisetus (J. Presl) Schltdl. AGS, CHIS, COL, DGO, GTO, GRO, JAL, MEX, MICH, MOR, NAY, OAX, PUE, QROO, SLP, SIN, TAMS, VER, ZAC

Jarava ichu Ruiz \& Pav. CHIS, COAH, CDMX, GTO, HGO, MEX, MICH, MOR, NLE, OAX, PUE, QRO, SLP, TLAX, VER Jouvea pilosa (J. Presl) Scribn. BCN, BCS, CHIS, COL, GRO, JAL, MICH, NAY, OAX, SIN, SON

Jouvea straminea E. Fourn. BCS, CHIS, COL, GRO, JAL, MICH, NAY, OAX, SIN, SON

Kalinia obtusiflora (E. Fourn.) H.L. Bell \& J.T. Columbus CHIH, COAH, COL, CDMX, GTO, GRO, HGO, JAL, MEX, MICH, NLE, OAX, SON, VER, ZAC

Koeleria macrantha (Ledeb.) Schult. COAH, NLE, SON

Koeleria pyramidata (Lam.) P. Beauv. BCN, BCS, CHIH, COAH, CDMX, DGO, GTO, HGO, JAL, MEX, NLE, OAX, SLP, SON, TAMS, TLAX, VER, ZAC

Lasiacis divaricata (L.) Hitchc. BCN, BCS, CAM, CHIS, COAH, COL, DGO, GRO, HGO, JAL, MEX, MICH, MOR, NAY, NLE, OAX, PUE, QRO, QROO, SLP, SIN, TAB, TAMS, 
VER, YUC, ZAC

Lasiacis grisebachii (Nash) Hitchc. CAM, CHIS, GRO, HGO, JAL, OAX, PUE, QRO, QROO, SLP, TAB, TAMS, VER, YUC Lasiacis linearis Swallen CHIS

Lasiacis nigra Davidse AGS, CAM, CHIS, COL, CDMX, DGO, GTO, GRO, HGO, JAL, MEX, MICH, MOR, NAY, NLE, OAX, PUE, QRO, QROO, SLP, SIN, TAB, TAMS, VER, ZAC

Lasiacis oaxacensis (Steud.) Hitchc. CHIS, COL, GRO, JAL, MEX, MICH, NAY, OAX, TAB, VER

Lasiacis procerrima (Hack.) Hitchc. CHIS, COL, DGO, GRO, HGO, JAL, MEX, MICH, MOR, NAY, OAX, PUE, SLP, SIN, SON, TAB, TAMS, VER, ZAC

Lasiacis rhizophora (E. Fourn.) Hitchc. CHIS, GRO, MICH, OAX, PUE, VER

Lasiacis rugelii (Griseb.) Hitchc. CAM, CHIS, GRO, HGO, OAX, QROO, SLP, TAB, TAMS, VER, YUC

Lasiacis ruscifolia (Kunth) Hitchc. BCN, BCS, CAM, CHIS, CHIH, COL, DGO, GRO, HGO, JAL, MEX, MICH, MOR, NAY, NLE, OAX, PUE, QRO, QROO, SLP, SIN, SON, TAB, TAMS, VER, YUC, ZAC

Lasiacis scabrior Hitchc. CHIS, GRO, OAX, VER

Lasiacis sloanei (Griseb.) Hitchc. CAM, CHIS, OAX, QROO, SLP, TAB, TAMS, VER, YUC

Lasiacis sorghoidea (Desv. ex Ham.) Hitchc. \& Chase BCN, CHIS, GRO, JAL, MEX, MICH, MOR, OAX, PUE, SLP, SON, TAB, TAMS, VER

Lasiacis standleyi Hitchc. CHIS, VER

Leersia hexandra Sw. CAM, CHIS, CHIH, COAH, COL, CDMX, DGO, GTO, GRO, HGO, JAL, MEX, MICH, MOR, NAY, OAX, PUE, QROO, SLP, TAB, TAMS, VER, YUC, ZAC Leersia ligularis Trin. CAM, CHIS, GRO, HGO, NLE, OAX, PUE, QRO, QROO, SLP, TAMS, VER, YUC

Leersia monandra Sw. CAM, CHIS, COAH, NLE, OAX, PUE, QRO, QROO, SLP, TAB, TAMS, VER, YUC

Leersia oryzoides (L.) Sw. BCN, BCS, COAH, NLE, SLP, SON, TAMS

*Leptochloa aquatica Scribn. \& Merr. COL, GTO, GRO, JAL, MEX, MICH, MOR, NAY, QRO, SIN, ZAC

Leptochloa crinita (Lag.) P. M. Peterson \& N. Snow BCS, CHIH, COAH, DGO, NLE, SLP, SIN, SON, TAMS, YUC

Leptochloa fusca (L.) Kunth AGS, BCN, BCS, CAM, CHIS, CHIH, COAH, COL, CDMX, DGO, GTO, GRO, HGO, JAL, MEX, MICH, MOR, NAY, NLE, OAX, PUE, QRO, QROO, SLP, SIN, SON, TAMS, TLAX, VER, YUC, ZAC

Leptochloa nealleyi Vasey CAM, QROO, TAB, TAMS, VER, YUC

Leptochloa panicea (Retz.) Ohwl BCN, BCS, CAM, CHIS, CHIH, COAH, COL, DGO, GTO, GRO, JAL, MEX, MICH, MOR, NAY, NLE, OAX, PUE, QRO, QROO, SLP, SIN, SON, TAB, TAMS, TLAX, VER, YUC, ZAC

Leptochloa panicoides (J. Presl) Hitchc. BCN, BCS, CHIH, COL, DGO, GRO, JAL, MICH, MOR, NAY, OAX, SIN, SON, $\mathrm{TAB}, \mathrm{ZAC}$

Leptochloa pluriflora (E. Fourn.) P. M. Peterson \& N. Snow CAM, CHIH, COAH, DGO, GTO, JAL, MICH, NLE, OAX, PUE, QROO, SLP, TAMS, YUC

Leptochloa scabra Nees CHIS, CHIH, COL, JAL, MICH, MOR,
OAX, QRO, SLP, SIN, SON, TAB, TAMS, VER, YUC Leptochloa virgata (L.) P. Beauv. CAM, CHIS, COAH, COL, DGO, GTO, GRO, JAL, MICH, NAY, NLE, OAX, PUE, QROO, SLP, SON, TAB, TAMS, VER, YUC, ZAC

Leptochloa viscida (Scribn.) Beal BCN, BCS, CHIH, SIN, SON Leymus condensatus (J. Presl) A. Löve BCN, COAH, DGO Leymus triticoides (Buckley) Pilg. BCN, SON, TAMS Limnodea arkansana (Nutt.) L.H. Dewey COAH, TAMS Lithachne pauciflora (Sw.) P. Beauv. CHIS, COL, JAL, NLE, OAX, PUE, QRO, QROO, SLP, TAB, TAMS, VER, YUC Luziola fluitans (Michx.) Terrell \& H. Rob. AGS, CHIH, GTO, HGO, JAL, MEX, MICH, NAY, QRO, SLP, ZAC

Luziola gracillima Prodoehl GTO, JAL, SIN, SON

Luziola peruviana Juss. ex J.F. Gmel. CAM, CHIS, CDMX, HGO, JAL, MEX, MICH, OAX, QRO, TAB, VER

Luziola spruceana Benth. CAM, TAB

Luziola subintegra Swallen CHIS, TAB, VER

Melica frutescens Scribn. BCN, BCS

Melica imperfecta Trin. BCN, BCS

Melica montezumae Piper CHIH, COAH, DGO, NLE

Melica mutica $\mathrm{T}$. Walter TAMS

Melica nitens (Nutt.) Nutt. ex Piper COAH, NLE, TAMS

Melica porteri Scribn. CHIH, COAH, OAX, QRO, SON

Mesosetum blakei Swallen CHIS, TAB, VER

Mesosetum chaseae Luces CHIS, TAB

Mesosetum filifolium F.T. Hubb. OAX

Mesosetum pittieri Hitchc. CHIS, OAX, VER

* Metcalfia mexicana (Scribn.) Conert CHIS, COAH, DGO, HGO, MEX, NLE, OAX, PUE, QRO, SLP, TAMS, VER, ZAC Microchloa kunthii Desv. AGS, BCN, BCS, CHIS, CHIH, COAH, COL, CDMX, DGO, GTO, GRO, HGO, JAL, MEX, MICH, MOR, NAY, NLE, OAX, PUE, QRO, SLP, SIN, SON, TAMS, TLAX, VER, ZAC

Mnesithea aurita (Steud.) de Koning \& Sosef CHIS

Mnesithea granularis (L.) de Koning \& Sosef BCN, BCS, CAM, CHIS, CHIH, COL, DGO, GTO, GRO, JAL, MEX, MICH, MOR, NAY, OAX, PUE, SLP, SIN, SON, TAB, TAMS, VER, YUC, ZAC

Mnesithea ramosa (E. Fourn.) de Koning \& Sosef CAM, CHIS, GTO, JAL, MEX, MICH, NAY, PUE, TAB, VER

Mnesithea subgibbosa (Winkl. ex Hack.) de Koning \& Sosef CHIS

*Muhlenbergia aguascalientensis Y. Herrera \& de la Cerda AGS, ZAC

*Muhlenbergia alamosae Vasey AGS, BCS, CHIH, CDMX, DGO, GTO, GRO, JAL, MEX, MOR, OAX, PUE, SLP, SIN, SON, ZAC

Muhlenbergia alopecuroides (Griseb.) P.M. Peterson \& J.T. Columbus AGS, CHIH, COAH, DGO, GTO, JAL, QRO, SLP, SIN, SON, ZAC

*Muhlenbergia annua (Vasey) Swallen CHIH, DGO, SON Muhlenbergia appressa C.O. Goodd. BCN, BCS

Muhlenbergia arenacea (Buckley) Hitchc. CHIH, COAH, NLE, SLP, SON, ZAC

Muhlenbergia arenicola Buckley CHIH, COAH, DGO, GTO, JAL, NLE, SLP, SON, TAMS, ZAC

*Muhlenbergia argentea Vasey CHIH, SON 
Muhlenbergia arizonica Scribn. BCN, BCS, CHIH, DGO, SIN, SON

Muhlenbergia arsenei Hitchc. BCN, CHIH

Muhlenbergia articulata Scribn. CHIH, GTO, GRO, HGO, JAL, PUE, QRO, SLP, SON, TAMS

Muhlenbergia asperifolia (Nees \& Meyen ex Trin.) Parodi BCN, CHIH, COAH, DGO, JAL, SLP, SON

*Muhlenbergia brandegeei $\mathrm{C}$. Reeder BCS

*Muhlenbergia brevifolia Scribn. ex Beal COL, DGO, GTO, JAL, NAY, ZAC

Muhlenbergia breviligula Hitchc. CHIS, GRO

Muhlenbergia brevis C.O. Goodd. CHIH, CDMX, DGO, JAL, MEX, SLP, ZAC

*Muhlenbergia breviseta Griseb. ex E. Fourn. COAH, GRO, JAL, MEX, MICH, NAY, SLP, VER, ZAC

*Muhlenbergia brevivaginata Swallen AGS, DGO, JAL, NAY, ZAC

Muhlenbergia calcicola Swallen CHIS, MEX, TLAX

Muhlenbergia californica Vasey BCN

Muhlenbergia capillaris (Lam.) Trin. CHIS, CHIH, CDMX, DGO, GTO, GRO, HGO, JAL, MEX, MICH, MOR, NAY, OAX, PUE, QRO, QROO, SLP, TAMS, TLAX, VER, YUC

*Muhlenbergia capillipes (M.E. Jones) P.M. Peterson \& Annable CHIH

Muhlenbergia ciliata (Kunth) Trin. AGS, BCN, BCS, CHIS, CHIH, COL, CDMX, DGO, GTO, GRO, HGO, JAL, MEX, MICH, MOR, NAY, OAX, PUE, QRO, SLP, SIN, SON, VER, ZAC

Muhlenbergia crispiseta Hitchc. AGS, CHIH, DGO, GTO, JAL, NAY, QRO, SLP, SIN, SON, ZAC

*Muhlenbergia cualensis Y. Herrera \& P.M. Peterson JAL

Muhlenbergia depauperata Scribn. AGS, CHIH, COAH, CDMX, DGO, GTO, HGO, JAL, MEX, MICH, NLE, OAX, PUE, QRO, SLP, VER, ZAC

Muhlenbergia distans Swallen AGS, CDMX, DGO, GTO, GRO, HGO, JAL, MEX, MICH, NLE, OAX, PUE, QRO, SLP, TAMS, TLAX, VER, ZAC

Muhlenbergia distichophylla (J. Presl) Kunth CHIS, CHIH, COL, GRO, JAL, MEX, MICH, OAX, PUE, SIN

Muhlenbergia diversiglumis Trin. CHIS, CHIH, COL, DGO, GRO, HGO, JAL, MEX, MICH, MOR, NAY, OAX, SIN, SON, VER, ZAC

Muhlenbergia dubia E. Fourn. AGS, BCS, CHIH, COAH, CDMX, DGO, GTO, HGO, JAL, MEX, MICH, NLE, OAX, PUE, QRO, SLP, SON, TAMS, TLAX, VER, ZAC

* Muhlenbergia durangensis Y. Herrera CHIH, DGO, JAL, SLP Muhlenbergia elongata Scribn. ex Beal CHIH, HGO, SIN, SON Muhlenbergia eludens $\mathrm{C}$. Reeder CHIH, DGO, GRO, SON

Muhlenbergia emersleyi Vasey AGS, BCS, CHIS, CHIH, COAH, CDMX, DGO, GTO, GRO, HGO, JAL, MEX, MICH, MOR, NAY, NLE, OAX, PUE, QRO, SLP, SIN, SON, TAMS, TLAX, VER, ZAC

*Muhlenbergia eriophylla Swallen AGS, CDMX, DGO, GTO, JAL, MEX, MICH, SLP, ZAC

Muhlenbergia expansa (Poir.) Trin. DGO, VER

Muhlenbergia filiculmis Vasey DGO, SIN

Muhlenbergia filiformis (Thurb. ex S. Watson) Rydb. BCN,
BCS, DGO, SON

*Muhlenbergia flavida Vasey CHIH, DGO, HGO, JAL, MICH, NAY, SIN, SON, ZAC

*Muhlenbergia flaviseta Scribn. CHIH, DGO, SIN, SON, ZAC Muhlenbergia fragilis Swallen AGS, BCN, CHIS, CHIH, COAH, DGO, GTO, GRO, HGO, JAL, MEX, MICH, MOR, NAY, OAX, PUE, QRO, SLP, SIN, SON, VER, ZAC

*Muhlenbergia gigantea (E. Fourn.) Hitchc. CHIS, COL, DGO, GTO, GRO, HGO, JAL, MEX, MICH, OAX, PUE, SLP, SIN, VER, ZAC

Muhlenbergia glauca (Nees) B.D. Jacks. AGS, BCN, CHIH, COAH, CDMX, DGO, GTO, HGO, JAL, MEX, NLE, PUE, SLP, SON, VER, ZAC

*Muhlenbergia grandis Vasey CHIH, COL, GTO, JAL, NAY, SIN, SON, ZAC

*Muhlenbergia gypsophila Reeder \& C. Reeder COAH, NLE, SLP

*Muhlenbergia hintonii Swallen MEX, MICH, VER

Muhlenbergia implicata (Kunth) Trin. AGS, CAM, CHIS, CHIH, COL, CDMX, DGO, GTO, GRO, HGO, JAL, MEX, MICH, MOR, NAY, OAX, PUE, QRO, SLP, TLAX, VER, ZAC

*Muhlenbergia iridifolia Soderstr. JAL

*Muhlenbergia jaime-hintonii P.M. Peterson \& Valdés-Reyna NLE, TAMS

* Muhlenbergia jaliscana Swallen AGS, COL, JAL

* Muhlenbergia laxa Hitchc. VER

Muhlenbergia lindheimeri Hitchc. CHIH, COAH, NLE

*Muhlenbergia longiglumis Vasey AGS, COL, DGO, GTO, JAL, MICH, NAY, SON, ZAC

Muhlenbergia longiligula Hitchc. CHIH, DGO, JAL, PUE, SLP, SON

*Muhlenbergia lucida Swallen CHIH, GTO, MEX

Muhlenbergia macroura (Kunth) Hitchc. AGS, CHIS, CHIH, COAH, COL, CDMX, DGO, GTO, HGO, JAL, MEX, MICH, MOR, NLE, OAX, PUE, QRO, SLP, SON, TAMS, TLAX, VER, ZAC

*Muhlenbergia majalcensis P.M. Peterson CHIH

Muhlenbergia mexicana (L.) Trin. CHIH, DGO, NLE

Muhlenbergia microsperma (DC.) Kunth AGS, BCN, BCS, CHIS, CHIH, COAH, CDMX, DGO, GTO, HGO, JAL, MEX, MICH, MOR, NAY, NLE, OAX, PUE, QRO, SLP, SIN, SON, TAMS, TLAX, VER, ZAC

*Muhlenbergia michisensis Y. Herrera \& P.M. Peterson DGO, GRO

Muhlenbergia minutissima (Steud.) Swallen AGS, BCN, CHIH, CDMX, DGO, GTO, HGO, JAL, MEX, MICH, MOR, NAY, NLE, OAX, PUE, QRO, SLP, SIN, SON, TLAX, VER, ZAC Muhlenbergia montana (Nutt.) Hitchc. AGS, BCN, CHIS, CHIH, COAH, CDMX, DGO, GTO, GRO, HGO, JAL, MEX, MICH, OAX, PUE, QRO, SLP, SON, TLAX, VER, ZAC

*Muhlenbergia mutica (Rupr. ex E. Fourn.) Hitchc. CHIS, PUE, VER

Muhlenbergia nigra Hitchc. CHIS, CDMX, GTO, HGO, JAL, MEX, MICH, MOR, OAX, PUE, SLP, TLAX, VER

Muhlenbergia orophila Swallen CHIS, CDMX, HGO, MEX, MOR, PUE, TLAX

Muhlenbergia palmeri Vasey CHIH, COAH, MICH, SLP, SON 
Muhlenbergia pauciflora Buckley BCN, CHIH, COAH, DGO, GTO, JAL, MEX, NLE, SON, TAMS

Muhlenbergia pectinata C.O. Goodd. CHIH, DGO, GRO, JAL, MEX, MICH, MOR, NAY, OAX, SIN, SON, ZAC

Muhlenbergia pereilema P.M. Peterson AGS, BCN, BCS, CHIS, CHIH, COL, DGO, GRO, HGO, JAL, MEX, MICH, MOR, NAY, OAX, PUE, QRO, SLP, SIN, SON, VER, ZAC

Muhlenbergia peruviana (P. Beauv.) Steud. AGS, CHIS, CHIH, COL, CDMX, DGO, GTO, HGO, JAL, MEX, MICH, MOR, NAY, OAX, PUE, QRO, SLP, SON, TLAX, VER, ZAC

Muhlenbergia phalaroides (Kunth) P.M. Peterson AGS, BCS, CHIS, CHIH, COAH, COL, CDMX, DGO, GTO, HGO, JAL, MEX, MICH, MOR, NAY, NLE, OAX, PUE, QRO, SLP, TAMS, TLAX, VER, ZAC

Muhlenbergia phleoides (Kunth) J.T. Columbus AGS, BCN, BCS, CHIH, COAH, CDMX, DGO, GTO, HGO, JAL, MEX, MICH, MOR, NAY, NLE, OAX, PUE, QRO, SLP, SON, TAMS, TLAX, VER, ZAC

*Muhlenbergia pilosa P.M. Peterson, Wipff \& S.D. Jones GRO, MEX, MICH

Muhlenbergia plumbea (Trin.) Hitchc. CDMX, DGO, HGO, MEX, MICH, PUE, TLAX, ZAC

Muhlenbergia polycaulis Scribn. AGS, BCN, BCS, CHIH, COAH, DGO, GTO, HGO, JAL, MEX, NAY, OAX, QRO, SLP, SIN, SON, ZAC

Muhlenbergia porteri Scribn. ex Beal BCN, BCS, CHIH, COAH, DGO, NLE, SLP, SIN, SON, TAMS, ZAC

*Muhlenbergia pubescens (Kunth) Hitchc. AGS, CHIH, COL, CDMX, DGO, GTO, HGO, JAL, MEX, MICH, NAY, NLE, OAX, PUE, QRO, SLP, SON, TAMS, TLAX, VER, ZAC

* Muhlenbergia pubigluma Swallen CHIH, COAH, NLE, TAMS *Muhlenbergia purpusii Mez CHIH, COAH, NLE, SLP, TAMS Muhlenbergia quadridentata (Kunth) Trin. AGS, CHIS, CHIH, COAH, COL, CDMX, DGO, GTO, GRO, HGO, JAL, MEX, MICH, MOR, NLE, OAX, PUE, QRO, SLP, SON, TLAX, VER, ZAC

Muhlenbergia racemosa (Michx.) Britton, Stern \& Poggenb. COAH, NLE, TAMS

Muhlenbergia ramulosa (Kunth) Swallen BCN, BCS, CHIS, CHIH, COAH, COL, CDMX, DGO, GTO, GRO, HGO, JAL, MEX, MICH, MOR, OAX, PUE, QRO, SLP, SON, TAMS, TLAX, VER, ZAC

* Muhlenbergia reederorum Soderstr. CHIH, DGO, JAL, MICH, SIN

Muhlenbergia repens (J. Presl) Hitchc. AGS, BCN, BCS, CHIS, CHIH, COAH, CDMX, DGO, GTO, GRO, HGO, JAL, MEX, MICH, MOR, NLE, OAX, PUE, QRO, SLP, SON, TAMS, TLAX, VER, ZAC

Muhlenbergia richardsonis (Trin.) Rydb. BCN, CHIH, CDMX, DGO, HGO, MEX, PUE, SLP, SON, TLAX

Muhlenbergia rigens (Benth.) Hitchc. AGS, BCN, BCS, CHIH, COAH, CDMX, DGO, GTO, JAL, MEX, MICH, MOR, NLE, PUE, SIN, SON, TAMS, ZAC

Muhlenbergia rigida (Kunth) Kunth AGS, BCN, BCS, CHIS, CHIH, COAH, CDMX, DGO, GTO, GRO, HGO, JAL, MEX, MICH, MOR, NAY, NLE, OAX, PUE, QRO, SLP, SIN, SON, TAMS, TLAX, VER, ZAC
Muhlenbergia robusta (E. Fourn.) Hitchc. AGS, CHIS, CHIH, COAH, COL, CDMX, DGO, GTO, GRO, HGO, JAL, MEX, MICH, MOR, NAY, NLE, OAX, PUE, QRO, SLP, SIN, SON, TLAX, VER, ZAC

*Muhlenbergia scoparia Vasey CHIH, COL, DGO, JAL, MICH, NAY, SIN, SON, ZAC

*Muhlenbergia schmitzii Hack. CHIH, HGO, MEX, MICH Muhlenbergia schreberi J.F. Gmel. COAH, HGO, NLE, OAX, PUE, QRO, SLP, TAMS, TLAX, VER

*Muhlenbergia seatonii Scribn. CDMX, HGO, MEX, PUE, TLAX

Muhlenbergia setarioides E. Fourn. CHIS, PUE, TLAX, VER Muhlenbergia setifolia Vasey CHIH, COAH, DGO, GTO, NLE, SLP, TAMS

*Muhlenbergia speciosa Vasey AGS, CHIH, COL, DGO, GTO, JAL, MICH, NAY, SIN, ZAC

Muhlenbergia spiciformis Trin. CHIS, CHIH, COAH, DGO, GTO, HGO, JAL, MEX, MICH, NLE, OAX, PUE, QRO, SLP, TAMS, TLAX, VER

Muhlenbergia straminea Hitchc. CHIH, SON

*Muhlenbergia stricta (J. Presl) Kunth COL, DGO, GTO, JAL, MEX, MICH, MOR, NAY, OAX, VER, ZAC

*Muhlenbergia strictior Scribn. ex Beal CHIH, CDMX, DGO, MEX, SLP, SON

*Muhlenbergia subaristata Swallen DGO, JAL, MEX, OAX, SIN, VER

*Muhlenbergia sylvatica (Torr.) Torr. ex A. Gray VER

* Muhlenbergia tarahumara P.M. Peterson \& J.T. Columbus $\mathrm{CHIH}$

Muhlenbergia tenella (Kunth) Trin. CHIS, CHIH, COL, DGO, GTO, GRO, HGO, JAL, MEX, MICH, MOR, NAY, OAX, PUE, QRO, SLP, SIN, SON, TLAX, VER, ZAC

Muhlenbergia tenuifolia (Kunth) Kunth AGS, CHIS, CHIH, COAH, COL, CDMX, DGO, GTO, GRO, HGO, JAL, MEX, MICH, MOR, NAY, NLE, OAX, PUE, QRO, SLP, SON, TAB, TAMS, TLAX, VER, ZAC

Muhlenbergia tenuissima (J. Presl) Kunth AGS, CHIS, COAH, COL, JAL, NAY, NLE, SLP, SIN, ZAC

Muhlenbergia texana Buckley BCN, BCS, CHIH, DGO, NAY, SIN, SON

Muhlenbergia torreyi (Kunth) Hitchc. ex Bush CHIH, COAH, DGO, NLE, SLP, SON

Muhlenbergia tricholepis (Torr.) J.T. Columbus AGS, BCN, CHIH, COAH, COL, CDMX, DGO, GTO, HGO, JAL, MEX, MICH, MOR, NLE, PUE, SLP, SIN, SON, TAMS, TLAX, VER, ZAC

*Muhlenbergia uniseta (Lag.) J.T. Columbus DGO, SLP Muhlenbergia utilis (Torr.) Hitchc. AGS, BCN, CHIS, CHIH, COAH, CDMX, DGO, GTO, HGO, JAL, MEX, MICH, NLE, OAX, PUE, QRO, SLP, SON, TAB, TLAX, VER, ZAC

Muhlenbergia vaginata Swallen CHIS, CHIH, COL, CDMX, DGO, GTO, HGO, JAL, MEX, MICH, MOR, OAX, PUE, QRO, SIN, TLAX, VER

Muhlenbergia versicolor Swallen CHIS, COL, CDMX, GTO, GRO, HGO, JAL, MEX, MICH, MOR, NAY, OAX, PUE, VER *Muhlenbergia villiflora Hitchc. CHIH, COAH, DGO, HGO, NLE, SLP, TAMS, ZAC 
Muhlenbergia virescens (Kunth) Trin. AGS, CHIH, COAH, COL, DGO, GTO, GRO, JAL, MEX, MICH, NAY, NLE, QRO, SLP, SIN, SON, ZAC

*Muhlenbergia virletti (E. Fourn.) Soderstr. CHIS, CDMX, DGO, HGO, JAL, MEX, MICH, MOR, PUE, SLP, TLAX, $\mathrm{ZAC}$

*Muhlenbergia watsoniana Hitchc. AGS, DGO, JAL, SLP, ZAC Muhlenbergia wrightii Vasey ex J.M. Coult. BCN, CHIH, COAH, DGO, SON, TAMS

*Muhlenbergia xanthodas Soderstr. CHIS

Munroa squarrosa (Nutt.) Torr. CHIH, COAH, DGO

Nassella cernua (Stebbins \& Löve) Barkworth BCN

Nassella lepida (Hitchc.) Barkworth BCN, BCS, GTO, JAL, OAX, PUE

Nassella leucotricha (Trin. \& Rupr.) R.W. Pohl AGS, BCN, CHIS, COAH, CDMX, DGO, GTO, HGO, MEX, NLE, OAX, PUE, QRO, SLP, TAMS, ZAC

Nassella linearifolia (E. Fourn.) R.W. Pohl CHIS, CDMX, HGO, MEX, OAX, PUE, TLAX, VER

Nassella mexicana (Hitchc.) R.W. Pohl CHIS, CDMX, HGO, MEX, NLE, OAX, PUE, QRO, SLP, TLAX, VER

Nassella mucronata (Kunth) R.W. Pohl AGS, CHIS, CHIH, COAH, COL, CDMX, DGO, GTO, HGO, JAL, MEX, MICH, MOR, NLE, OAX, PUE, QRO, SLP, TAMS, TLAX, VER, ZAC Nassella pulchra (Hitchc.) Barkworth BCN, BCS

Nassella tenuissima (Trin.) Barkworth AGS, CHIH, COAH, DGO, HGO, MEX, MICH, NLE, PUE, SLP, TAMS, TLAX, VER, ZAC

Ocellochloa biglandulare (Scribn. \& J.G. Sm.) Zuloaga \& Morrone CHIS, GRO, OAX

*Ocellochloa craterifera (Sohns) Zuloaga \& Morrone GRO, OAX

Ocellochloa pulchella (Raddi) Zuloaga \& Morrone CHIS, OAX, TAB, VER

Ocellochloa stolonifera (Poir.) Zuloaga \& Morrone CHIS, GRO, OAX, PUE, QROO, TAB, VER

*Olmeca clarkiae (Davidse \& R.W. Pohl) Ruiz-Sanchez, E., Sosa \& Mejía Saulés CHIS

* Olmeca fulgor (Soderstr.) Ruiz-Sanchez, Sosa \& Mejía Saulés OAX, VER

* Olmeca recta Soderstr. VER

* Olmeca reflexa Soderstr. CHIS, OAX, VER

*Olmeca zapotecorum Ruiz-Sánchez, Sosa \& Mejía Saulés OAX

Olyra glaberrima Raddi CAM, CHIS, OAX, QROO, SLP, TAB, VER, YUC

Olyra latifolia L. CAM, CHIS, COL, GRO, JAL, MEX, MICH, MOR, NAY, OAX, PUE, QROO, SLP, SIN, TAB, TAMS, VER, YUC

Oplismenus burmannii (Retz.) P. Beauv. AGS, BCS, CAM, CHIS, CHIH, COL, CDMX, DGO, GTO, GRO, JAL, MEX, MICH, MOR, NAY, OAX, PUE, QRO, SLP, SIN, SON, TAMS, VER, YUC, ZAC

Oplismenus compositus (L.) P. Beauv. CHIS, COL, DGO, GTO, GRO, HGO, JAL, MEX, MICH, MOR, NAY, OAX, PUE, QRO, SLP, SIN, SON, TAMS, VER, ZAC

Oplismenus hirtellus (L.) P. Beauv. AGS, BCN, BCS, CAM,
CHIS, CHIH, COAH, COL, GRO, HGO, JAL, MEX, MICH, MOR, NAY, NLE, OAX, PUE, QRO, QROO, SLP, SIN, SON, TAB, TAMS, VER, YUC

Orcuttia californica Vasey BCN

Orthoclada laxa (Rich.) P. Beauv. CHIS, OAX, TAB, VER

Oryza latifolia Desv. CAM, CHIS, COL, GRO, JAL, NAY, OAX, QROO, SIN, TAB, VER, YUC

*Otatea acuminata (Munro) C.E. Calderón \& Soderstr. AGS, CHIS, CHIH, COL, DGO, GTO, GRO, HGO, JAL, MEX, MICH, MOR, NAY, OAX, PUE, QRO, SLP, SIN, SON, TAMS, VER, ZAC

* Otatea carrilloi Ruiz-Sánchez, Sosa \& Mejía-Saulés CHIS

Otatea fimbriata Soderst. CHIS, JAL, MICH, NAY, OAX, PUE

* Otatea glauca L.G. Clark \& G. Cortés CHIS

* Otatea ramirezii Ruiz-Sánchez HGO, QRO

* Otatea reynosoana Ruiz-Sánchez \& L.G. Clark DGO, GRO, JAL, NAY

* Otatea transvolcanica Ruiz-Sánchez \& L.G. Clark COL, JAL, MEX, MICH

* Otatea ximenae Ruiz-Sánchez \& L.G. Clark OAX

Panicum alatum Zuloaga \& Morrone BCN, BCS, CAM, CHIS, CHIH, COAH, COL, DGO, GRO, JAL, MICH, MOR, NAY, OAX, SLP, SIN, SON, TAMS, VER

Panicum altum Hitchc. \& Chase QROO

Panicum amarum Elliott CAM, QROO, TAB, TAMS, VER, YUC

Panicum aquaticum Poir. CAM, COL, JAL, QROO, VER

Panicum arundinariae Trin. ex E. Fourn. CHIS, COL, GTO, JAL, MEX, OAX, SLP, VER

*Panicum aztecanum Zuloaga \& Morrone CDMX, JAL, MEX, $\mathrm{MICH}$, SIN

Panicum bartlettii Swallen CAM, CHIS, HGO, OAX, QRO, QROO, SLP, TAB, VER, YUC

Panicum brevifolium L. NAY, VER

Panicum capillare L. BCN, BCS, COAH, MEX, MICH, NLE, SLP, SON, TAMS, VER

Panicum capillarioides Vasey COAH, NLE, SLP, TAMS

Panicum cayennense Lam. CAM, CHIS, NAY, OAX, QROO, TAB

Panicum cayoense Swallen CAM, CHIS, QROO, YUC

Panicum cyanescens Nees ex Trin. CHIS, TAB

* Panicum decolorans Kunth BCS, CHIS, CHIH, COAH, DGO, GTO, GRO, HGO, JAL, MEX, MICH, OAX, PUE, QRO, SLP, SIN, SON, ZAC

Panicum dichotomiflorum Michx. BCN, CAM, CHIS, CHIH, COL, DGO, GRO, JAL, MEX, NAY, OAX, PUE, SIN, SON

Panicum elephantipes Nees ex Trin. COL, CDMX, GTO, HGO, JAL, MEX, MICH, QROO, SLP, SIN, TAB, YUC, ZAC

Panicum flexile (Gatt.) Scribn. CHIH, COAH, DGO, NLE Panicum ghiesbreghtii E. Fourn. AGS, CAM, CHIS, CHIH, COAH, DGO, HGO, JAL, MEX, MICH, MOR, NAY, OAX, PUE, QROO, SLP, SIN, SON, TAB, TAMS, VER, YUC

Panicum grande Hitchc. \& Chase CHIS, TAB, VER

Panicum guatemalense Swallen CHIS, VER

Panicum haenkeanum J. Presl CHIS, VER

Panicum hallii Vasey AGS, CHIS, CHIH, COAH, CDMX, DGO, GTO, GRO, HGO, JAL, MEX, MICH, MOR, NLE, 
OAX, PUE, QRO, SLP, SON, TAMS, VER, ZAC

Panicum hillmanii Chase CHIS, CHIH, COAH, DGO

Panicum hirsutum Sw. CAM, CHIS, COL, JAL, OAX, QROO, SLP, TAB, TAMS, VER, YUC

Panicum hirticaule J. Presl AGS, BCN, BCS, CAM, CHIS, CHIH, COAH, COL, DGO, GTO, GRO, JAL, MEX, MICH, MOR, NAY, OAX, PUE, QROO, SLP, SIN, SON, TAB, TAMS, VER, YUC, ZAC

Panicum hirtum Lam. CHIS, OAX

Panicum hispidifolium Swallen CHIS, OAX

Panicum hylaeicum Mez CAM, CHIS, OAX, PUE, QROO, SLP, TAB, VER, YUC

Panicum incumbens Swallen CHIS

*Panicum langei (Fourn.) Nash COAH, NLE, VER

Panicum lepidulum Hitchc. \& Chase AGS, CHIH, COAH, COL, CDMX, DGO, GTO, HGO, JAL, MEX, MICH, MOR, NAY, OAX, PUE, QRO, SLP, SON, ZAC

*Panicum longum Hitchc. \& Chase VER

Panicum mertensii Roth JAL, TAB, VER

Panicum millegrana Poir. CHIS, VER

Panicum pampinosum Hitchc. \& Chase BCS, CHIH, DGO, GTO, OAX, SIN, SON

Panicum parcum Hitchc. \& Chase CHIS, CHIH, COAH, COL, DGO, GTO, GRO, HGO, JAL, MEX, MICH, MOR, NAY, OAX, PUE, SIN, SON, TLAX, VER, ZAC

Panicum parvifolium Lam. CHIS, TAB, VER

Panicum parviglume Hack. CHIS, COL, JAL, MEX, MICH, OAX, VER

Panicum pilosum Sw. CAM, CHIS, COL, GRO, JAL, MICH, MOR, NAY, OAX, PUE, QRO, QROO, SLP, TAB, VER

Panicum plenum Hitchc. \& Chase CHIH, COAH, CDMX, DGO, GTO, GRO, JAL, MEX, MOR, NLE, SON, VER

Panicum polygonatum Schrad. CAM, CHIS, NAY, OAX, PUE, QROO, TAB, VER

Panicum rigidulum Nees CAM, CHIS, COAH, QROO, YUC

Panicum rudgei Roem. \& Schult. TAB, VER

Panicum sellowii Nees CHIS, HGO, OAX, PUE, VER

Panicum stagnatile Hitchc. \& Chase CHIS, PUE, TAB, VER

Panicum stramineum Hitchc. \& Chase CHIS, CHIH, COAH, DGO, GRO, JAL, MICH, NAY, NLE, OAX, SIN, SON

Panicum sublaeve Swallen CHIS, COL, JAL

* Panicum tamaulipense F.R. Waller \& Morden PUE, SLP, TAMS

Panicum trichanthum Nees CAM, CHIS, COL, GRO, OAX, PUE, QROO, SLP, TAB, TAMS, VER

*Panicum trichidiachne Döll CHIS, OAX, PUE, SLP, VER

Panicum trichoides Sw. BCN, BCS, CAM, CHIS, CHIH, COL, DGO, GRO, JAL, MEX, MICH, MOR, NAY, OAX, PUE, QRO, QROO, SLP, SIN, SON, TAB, TAMS, VER, YUC, ZAC

Panicum urvilleanum Kunth BCN, BCS, SON

*Panicum vaseyanum Scribn. ex Beal AGS, CHIS, CHIH, CDMX, DGO, GTO, GRO, JAL, MEX, MICH, PUE, QRO, SIN, SON, ZAC

Pappophorum bicolor E. Fourn. CHIH, COAH, DGO, GTO, GRO, MEX, NLE, SLP, TAMS, VER, ZAC

Pappophorum mucronulatum Nees BCS, CHIH, COAH, DGO, NLE, PUE, SLP, ZAC
Pappophorum pappiferum (Lam.) Kuntze COAH, NAY, OAX, SLP, VER

Pappophorum philippianum Parodi SLP, SON, TAMS, VER

Pappophorum vaginatum Buckley CHIH, COAH, DGO, GTO, NLE, PUE, SLP, SON, TAMS, ZAC

Parodiophyllochloa cordovensis (E. Fourn.) Zuloaga \& Morrone CHIS, HGO, PUE, VER

Pascopyrum smithii (Rydb.) Barkworth \& D.R. Dewey CHIH, COAH, SLP, ZAC

Paspalidium chapmanii (Vasey) R.W. Pohl CAM, QROO, YUC Paspalidium geminatum (Forssk.) Stapf AGS, BCN, BCS, CAM, CHIS, COAH, COL, DGO, GTO, GRO, HGO, JAL, MEX, MICH, MOR, NAY, NLE, OAX, PUE, QRO, QROO, SLP, SIN, SON, TAB, TAMS, TLAX, VER, YUC, ZAC

Paspalum acuminatum Raddi GTO, GRO, MICH, MOR, PUE, TAB, TAMS, VER

Paspalum acutum Chase CHIS, TAB, VER

Paspalum adoperiens (E. Fourn.) Chase CHIS, DGO, HGO, OAX, QRO, SIN, VER

Paspalum affine Steud. CHIS, HGO, MEX, OAX, PUE, VER

* Paspalum arsenei Chase AGS, GTO, GRO, HGO, JAL, MEX, MICH, MOR, NAY, PUE, QRO, SLP, TAMS, VER, ZAC

Paspalum arundinaceum Poir. CAM, QROO, TAMS, VER, YUC

Paspalum blodgetii Chapm. CAM, CHIS, OAX, QROO, TAMS, VER, YUC

Paspalum botterii (E. Fourn.) Chase CAM, CHIS, CHIH, COAH, COL, DGO, GTO, GRO, HGO, JAL, MEX, MICH, MOR, NAY, NLE, OAX, PUE, QRO, QROO, SLP, SIN, SON, TAB, TAMS, VER, YUC, ZAC

Paspalum caespitosum Flüggé CAM, CHIS, OAX, PUE, QRO, QROO, TAB, TAMS, VER, YUC

Paspalum campylostachyum (Hack.) S. Denham CHIS, OAX, PUE, TAB, VER

Paspalum candidum (Humb. \& Bonpl. ex Flüggé) Kunth CHIS, COL, GRO, HGO, JAL, MEX, MICH, MOR, PUE, TAB, VER Paspalum centrale Chase CHIS, COL, GRO, JAL, MICH, OAX, VER

Paspalum clavuliferum C. Wright CAM, CHIS, COL, GRO, JAL, MICH, NAY, OAX, QROO, SIN, SON, TAMS, VER, YUC

Paspalum conjugatum P.J. Bergius AGS, CAM, CHIS, COAH, COL, DGO, GRO, HGO, JAL, MEX, MICH, MOR, NAY, NLE, OAX, PUE, QRO, QROO, SLP, SIN, TAB, TAMS, VER, YUC Paspalum conspersum Schrad. CAM, CHIS, COL, JAL, MEX, MICH, MOR, NAY, OAX, PUE, QROO, SLP, TAMS, VER

Paspalum convexum Humb. \& Bonpl. ex Flüggé AGS, CAM, CHIS, CHIH, COL, CDMX, DGO, GTO, GRO, HGO, JAL, MEX, MICH, MOR, NAY, OAX, PUE, QRO, QROO, SIN, SON, TAB, TLAX, VER, ZAC

Paspalum corcovadense Raddi CAM, CHIS, OAX, QROO, TAMS, VER, YUC

Paspalum coryphaeum Trin. CAM, QROO, YUC

Paspalum costaricense Mez CHIS, OAX

* Paspalum crinitum Chase AGS, CHIS, COAH, COL, DGO, GTO, GRO, JAL, MICH, NLE, OAX, PUE, SLP, ZAC

Paspalum culiacanum Vasey CHIS, SIN 
Paspalum cymbiforme E. Fourn. CHIS, GRO, MEX, OAX, VER * Paspalum chiapense Sánchez-Ken CHIS

Paspalum decumbens Sw. CHIS, DGO, HGO, QRO, VER

Paspalum denticulatum Trin. CAM, CHIS, CHIH, COAH, COL, CDMX, DGO, GTO, GRO, HGO, JAL, MEX, MICH, MOR, NAY, NLE, OAX, PUE, QRO, QROO, SLP, SIN, TAB, TAMS, VER, YUC, ZAC

Paspalum distichum L. AGS, BCN, BCS, CAM, CHIS, CHIH, COAH, COL, CDMX, DGO, GTO, GRO, HGO, JAL, MEX, MICH, MOR, NAY, NLE, OAX, PUE, QRO, QROO, SLP, SIN, SON, TAB, TAMS, TLAX, VER, YUC, ZAC

* Paspalum erectum Chase COL, GTO, JAL, MICH

Paspalum erianthum Nees ex Trin. CHIS, GRO, OAX

Paspalum fasciculatum Willd. ex Flüggé CAM, CHIS, GTO, JAL, NAY, OAX, QROO, TAB, VER

Paspalum fimbriatum Kunth CAM, CHIS, MEX, QROO, YUC Paspalum foliiforme S. Denham CHIS

* Paspalum guyanerum Beetle JAL, NAY, SIN, SON

Paspalum hartwegianum E. Fourn. AGS, CAM, CHIS, COAH, GTO, GRO, JAL, MICH, MOR, NLE, OAX, PUE, QRO, QROO, SLP, SON, TAMS, VER, YUC, ZAC

Paspalum heterotrichon Trin. CHIS

* Paspalum hintonii Chase COL, GRO, JAL, MEX, MICH, NAY, OAX

Paspalum humboldtianum Flüggé AGS, CHIS, CHIH, COAH, COL, CDMX, DGO, GTO, GRO, HGO, JAL, MEX, MICH, MOR, NAY, NLE, OAX, PUE, QRO, SLP, SIN, SON, TAMS, VER, ZAC

Paspalum intermedium Munro ex Morong \& Britton CHIS, GRO, JAL, MEX, VER

Paspalum jaliscanum Chase CHIS, COL, CDMX, HGO, JAL, MEX, MICH, NAY, OAX, PUE, VER, ZAC

Paspalum langei (E. Fourn.) Nash CAM, CHIS, CHIH, COAH, COL, GRO, HGO, JAL, MEX, MICH, MOR, NAY, NLE, OAX, PUE, QRO, QROO, SLP, SIN, SON, TAB, TAMS, VER, YUC Paspalum lentiginosum J. Presl AGS, BCN, CHIS, COL, DGO, JAL, MEX, MICH, MOR, NAY, OAX, PUE, SIN, SON, VER

*Paspalum leptachne Chase NAY, SIN, VER

Paspalum ligulare Nees COL, JAL, OAX, SIN, VER

Paspalum lineare Trin. CHIS, PUE, TAB

*Paspalum longicuspe Nash CHIS, COL, GRO, JAL, MICH, MOR, NAY, OAX, SIN, VER

*Paspalum longum Chase COL

*Paspalum luxurians R. Guzmán \& L. Rico COL, GTO, JAL, MEX, MICH

Paspalum malacophyllum Trin. CAM, CHIS, GTO, QROO, SLP, TAB, TAMS, VER, YUC

*Paspalum mayanum Chase CAM, TAB, YUC

Paspalum melanospermum Desv. ex Poir. JAL, MOR, NAY, OAX, TAB

Paspalum microstachyum J. Presl GRO, NAY, TAB, VER

Paspalum millegrana Schrad. CAM, CHIS, PUE, QROO, TAB, TAMS, VER, YUC

Paspalum minus E. Fourn. CHIS, GTO, MEX, MICH, OAX, PUE, SLP, TAB, TAMS, VER

Paspalum monostachyum Vasey TAMS, VER

Paspalum multicaule Poir. CHIS, GRO, JAL, MEX, NAY, OAX,
TAB, VER

*Paspalum mutabile Chase COAH, GTO, HGO, NLE, OAX, PUE, QRO, SLP, TAMS, VER

*Paspalum nelsonii Chase CHIS, JAL, NAY

Paspalum notatum Alain ex Flüggé AGS, CAM, CHIS, COAH, COL, CDMX, DGO, GTO, GRO, HGO, JAL, MEX, MICH, MOR, NAY, NLE, OAX, PUE, QRO, QROO, SLP, SIN, SON, TAB, TAMS, VER, YUC, ZAC

Paspalum nutans Lam. CHIS, QROO

Paspalum orbiculatum Poir. CHIS, GRO, OAX, SLP, SIN, TAB, VER

* Paspalum palmeri Chase JAL, MOR, SON

Paspalum paniculatum L. AGS, BCN, BCS, CAM, CHIS, COL, DGO, GRO, HGO, JAL, MEX, MICH, MOR, NAY, OAX, PUE, QRO, QROO, SLP, SIN, SON, TAB, TAMS, VER, YUC, ZAC Paspalum pectinatum Nees ex Trin. CHIS, OAX, TAB, VER Paspalum pilosum Lam. CHIS, GRO, MEX, MICH, OAX, TAB, VER

Paspalum plenum Chase CHIS, COL, JAL, MEX, MICH, MOR, NAY, TAB, VER

Paspalum plicatulum Michx. AGS, CAM, CHIS, CHIH, COL, DGO, GTO, GRO, HGO, JAL, MEX, MICH, MOR, NAY, NLE, OAX, PUE, QROO, SLP, SIN, TAB, TAMS, VER, YUC, ZAC Paspalum prostratum Scribn. \& Merr. CHIS, COL, CDMX, DGO, GTO, GRO, HGO, JAL, MEX, MICH, MOR, OAX, PUE, VER

Paspalum pubiflorum Rupr. ex Fourn. AGS, BCN, BCS, CAM, CHIH, COAH, COL, DGO, GTO, GRO, HGO, JAL, MEX, MICH, MOR, NAY, NLE, OAX, PUE, QRO, SLP, SON, TAMS, VER, ZAC

Paspalum pulchellum Kunth CHIS, OAX, TAB, VER

Paspalum pygmaeum Hack. CDMX, DGO, GRO, JAL, MEX, MICH, MOR, SIN, TLAX, VER

Paspalum repens P.J. Bergius CAM, CHIS, TAB, VER

* Paspalum rudimentosum Steud. OAX

Paspalum serpentinum Hochst. ex Steud. TAB

Paspalum setaceum Michx. CHIS, CHIH, COAH, DGO, GTO, JAL, MEX, MICH, NAY, NLE, OAX, PUE, SLP, SON, TAB, TAMS, VER

* Paspalum sparsum Chase CAM, YUC

Paspalum squamulatum E. Fourn. BCS, CHIS, CHIH, COL, DGO, GRO, JAL, MEX, MICH, MOR, NAY, OAX, PUE, QRO, SLP, SIN, SON, TAMS, VER

Paspalum stellatum Humb. \& Bonpl. ex Flüggé CHIS, OAX Paspalum tenellum Willd. AGS, CHIS, CDMX, DGO, GTO, GRO, JAL, MEX, MICH, MOR, PUE, QRO, SLP, SIN, SON, TLAX, VER, ZAC

Paspalum tinctum Chase BCN, CHIS, CDMX, DGO, GTO, GRO, JAL, MEX, MICH, MOR, NAY, NLE, PUE

*Paspalum tolucense R. Guzmán MEX

Paspalum trachycoleon Steud. CHIS, OAX

Paspalum tumidum Kuhlm. CHIS, COL, GRO, JAL, MEX, $\mathrm{MICH}$

Paspalum turriforme R.W. Pohl CHIS

Paspalum unispicatum (Scribn. \& Merr.) Nash COAH, COL, GRO, JAL, MOR, NLE, OAX, PUE, QRO, SLP, TAMS, VER Paspalum variabile (E. Fourn.) Nash CHIS, COL, HGO, JAL, 
MEX, MICH, MOR, OAX, PUE, QRO, TAMS, VER

Paspalum virgatum L. CAM, CHIS, COL, GRO, JAL, MEX, MICH, MOR, NAY, OAX, PUE, QROO, SLP, TAB, TAMS, VER, YUC

Paspalum virletii E. Fourn. CHIS, GTO, HGO, NAY, NLE, PUE, QRO, SLP, SIN, SON, TAMS, VER

Paspalum wrightii Hitchc. \& Chase CAM, QROO, TAB, YUC

* Paspalum yecorae Sánchez-Ken SON

Pennisetum bambusiforme (E. Fourn.) Hemsl. ex B.D. Jacks. CHIS, COL, HGO, JAL, MICH, OAX, PUE, QRO, SLP, VER

*Pennisetum crinitum (Kunth) Spreng. AGS, CHIS, COL, CDMX, DGO, GTO, GRO, JAL, MEX, MICH, MOR, NAY, OAX, PUE, VER, ZAC

Pennisetum distachyum (E. Fourn.) Rupr. ex Chase CHIS, MEX, MICH, OAX, PUE, VER

* Pennisetum durum Beal CHIH, MEX, OAX, SON, VER

Pennisetum nervosum (Nees) Trin. CHIS, GRO, OAX, PUE, SLP, TAMS, VER

Pereilema beyrichianum (Kunth) Hitchc. CHIS

Pereilema ciliatum E. Fourn. CHIS, COL, GRO, JAL, MEX, MICH, MOR, NAY, OAX, PUE, VER, ZAC

Peyritschia deyeuxioides (Kunth) Finot AGS, CHIS, CHIH, COAH, COL, CDMX, DGO, GTO, GRO, HGO, JAL, MEX, MICH, MOR, NAY, NLE, OAX, PUE, QRO, SLP, SON, TAMS, TLAX, VER, ZAC

*Peyritschia koelerioides (Peyr.) E. Fourn. CDMX, GTO, HGO, JAL, MEX, MICH, MOR, OAX, PUE, TLAX, VER

Peyritschia pinetorum (Swallen) Finot \& P.M. Peterson CHIS Peyritschia pringlei (Scribn.) S.D. Koch AGS, BCN, BCS, CHIS, CHIH, CDMX, DGO, GTO, HGO, JAL, MEX, MICH, OAX, PUE, QRO, SLP, SON, TLAX, VER, ZAC

Phalaris caroliniana Walter BCN, CHIH, COAH, NLE, SON

Phalaris lemmonii Vasey BCN

Pharus lappulaceus Aubl. CAM, CHIS, SLP, TAB, VER

Pharus latifolius L. CAM, CHIS, GRO, MEX, MICH, OAX, QROO, TAB, VER

Pharus mezii Prodoehl CHIS, COL, GRO, JAL, MICH, ZAC

Pharus parvifolius Nash CHIS, OAX, TAB, VER

Phragmites australis (Cav.) Trin. ex Steud. BCN, BCS, CAM, CHIS, CHIH, COAH, COL, DGO, GTO, GRO, JAL, MEX, MICH, MOR, NAY, NLE, OAX, PUE, QRO, QROO, SLP, SIN, SON, TAB, TAMS, VER, YUC

*Piptochaetium angustifolium (Hitchc.) Valencia \& Costas COAH, NLE, OAX, PUE, TAMS

Piptochaetium avenaceum (L.) Parodi NLE, PUE, TAMS

*Piptochaetium brevicalyx (Fourn.) Ricker AGS, CDMX, DGO, GTO, HGO, JAL, MEX, NLE, PUE, QRO, SLP, TLAX, VER, ZAC

Piptochaetium fimbriatum (Kunth) Hitchc. AGS, BCN, BCS, CAM, CHIS, CHIH, COAH, COL, CDMX, DGO, GTO, GRO, HGO, JAL, MEX, MICH, MOR, NAY, NLE, OAX, PUE, QRO, QROO, SLP, SIN, SON, TAMS, TLAX, VER, ZAC

Piptochaetium pringlei (Beal.) Parodi CHIH, COAH, DGO, GTO, JAL, NLE, OAX, PUE, SLP, SON

Piptochaetium seleri (Pilg.) Henrard CHIS, COAH, CDMX, HGO, MEX, MICH, MOR, NLE, PUE, TLAX, VER

Piptochaetium stipoides (Trin. \& Rupr.) Hack. ex Arechav.
GTO, JAL, NLE, PUE, SLP, TAMS

Piptochaetium virescens (Kunth) Parodi AGS, CHIS, COAH, COL, CDMX, GTO, GRO, HGO, JAL, MEX, MICH, MOR, NLE, OAX, PUE, QRO, SLP, TAMS, TLAX, VER, ZAC

Pleuraphis jamesii Torr. CHIH, COAH, DGO

Pleuraphis mutica Buckley BCS, CHIH, COAH, DGO, NAY, NLE, SON, TAMS, TLAX, ZAC

Pleuraphis rigida Thurb. BCN, CHIH, OAX, SON

*Poa bajaensis Soreng BCN

Poa bigelovii Vasey \& Scribn. BCN, BCS, CHIH, COAH, NLE, OAX, SON

Poa fendleriana (Steud.) Vasey BCN, BCS, CHIH, COAH, NLE, SON

Poa howellii Vasey \& Scribn. BCN

* Poa matri-occidentalis P.M. Peterson \& Soreng CHIH, DGO

* Poa mulleri Swallen NLE

Poa orizabensis Hitchc. CHIS, MEX, NLE, PUE, TLAX, VER

* Poa ruprechtii Peyr. COAH, CDMX, MEX, NLE, OAX

Poa scaberula Hook. f. CHIS, CDMX, HGO, MEX, PUE, TLAX, VER

Poa secunda J. Presl BCN

Poa seleri Pilg. CHIS, HGO, MEX, MOR, OAX, PUE, VER

*Poa sharpii Swallen CHIS, CDMX, MEX, SLP, VER

Poa strictiramea Hitchc. CHIH, COAH, DGO, JAL, NLE, ZAC

Poa tacanae Swallen CHIS

Poa villaroeli Philippi MEX, PUE

Polypogon australis Brongn. BCN

Polypogon elongatus Kunth AGS, CHIS, CHIH, COAH, COL, CDMX, DGO, GTO, GRO, HGO, JAL, MEX, MICH, MOR, NAY, NLE, OAX, PUE, QRO, SLP, SIN, TAMS, TLAX, VER, ZAC

Pseudechinolaena polystachya (Kunth) Stapf CHIS, HGO, OAX, PUE, TAB, VER

Pseudoroegneria spicata (Pursh) A. Löve CHIH, COAH, DGO, GTO, JAL, MOR, NLE, SLP, SON, ZAC

*Reimarochloa acuta (Flüggé) Hitchc. OAX

Rhipidocladum bartlettii (McClure) McClure CAM, CHIS, QROO, TAMS, YUC

*Rhipidocladum martinezii Davidse \& R.W. Pohl CHIS

Rhipidocladum pittieri (Hack.) McClure CAM, CHIS, MICH, VER

Rhipidocladum racemiflorum (Steud.) McClure CHIS, COL, DGO, HGO, JAL, MICH, NAY, OAX, SIN, TAMS, VER, ZAC Rhytachne guianensis (Hitchc.) Clayton CHIS, OAX, TAB

*Rhytachne rottboellioides Desv. CHIS

Sacciolepis myuros (Lam.) Chase CHIS, JAL, MEX, NAY, OAX, PUE, VER

Sacciolepis striata (L.) Nash TAB, VER

Saccharum villosum Steud. CHIS, NLE, PUE, SLP

Sarga trichoclada (Rupr. ex Hack.) Spangler CHIS, COL, DGO, GRO, JAL, MEX, MICH, NAY, OAX, SIN, ZAC

Scleropogon brevifolius Phil. AGS, CHIH, COAH, DGO, GTO, HGO, JAL, MEX, NLE, PUE, QRO, SLP, SON, TAMS, VER, ZAC

*Schaffnerella gracilis (Benth.) Nash SLP, ZAC

Schizachyrium brevifolium (Sw.) Nees ex Büse AGS, CHIS, CHIH, COL, DGO, GTO, GRO, JAL, MEX, MICH, MOR, 
NAY, OAX, SIN, SON, TAB, VER, ZAC

Schizachyrium cirratum (Hack.) Wooton \& Standl. AGS, BCN, BCS, CHIS, CHIH, COAH, COL, CDMX, DGO, GTO, GRO, HGO, JAL, MEX, MICH, MOR, NAY, NLE, OAX, PUE, QRO, SLP, SIN, SON, TAMS, TLAX, VER, ZAC

Schizachyrium condensatum (Kunth) Nees CAM, CHIS, GRO, HGO, JAL, MEX, MICH, MOR, NAY, OAX, PUE, QRO, QROO, SLP, SIN, SON, TAB, TAMS, TLAX, VER, YUC

*Schizachyrium gaumeri Nash CAM, CHIS, QROO, YUC

* Schizachyrium maderensis Swallen COAH

Schizachyrium malacostachyum (J. Presl) Nash BCS, CAM, CHIS, GRO, JAL, MOR, NAY, OAX, PUE, QROO, VER, YUC * Schizachyrium mexicanum (Hitchc.) A. Camus CHIS, CHIH, COL, GTO, JAL, MEX, MICH, MOR, NAY, NLE, SLP, SON, VER, ZAC

*Schizachyrium muelleri Nash VER

Schizachyrium salzmannii (Trin. ex Steud.) Nash CHIS, OAX, TAB, TAMS, VER

Schizachyrium sanguineum (Retz.) Alston AGS, BCN, BCS, CAM, CHIS, CHIH, COAH, COL, CDMX, DGO, GTO, GRO, HGO, JAL, MEX, MICH, MOR, NAY, NLE, OAX, PUE, QRO, QROO, SLP, SIN, SON, TAB, TAMS, TLAX, VER, YUC, ZAC Schizachyrium scoparium (Michx.) Nash CAM, CHIH, COAH, DGO, HGO, MICH, NAY, NLE, OAX, PUE, QRO, SLP, SON, TAB, TAMS, VER, YUC, ZAC

Schizachyrium semitectum (Swallen) Reeder BCN, BCS, CHIS, CHIH, GRO, JAL, MEX, MOR, OAX, SON, YUC

Schizachyrium tenerum Nees AGS, CHIS, CHIH, COL, CDMX, DGO, GTO, GRO, HGO, JAL, MEX, MICH, MOR, NAY, OAX, PUE, QRO, SLP, SIN, SON, TAMS, TLAX, VER, ZAC

Setaria arizonica Rominger SON

Setaria corrugata (Elliott) Schult. VER

Setaria grisebachii E. Fourn. AGS, BCS, CAM, CHIS, CHIH, COAH, COL, CDMX, DGO, GTO, GRO, HGO, JAL, MEX, MICH, MOR, NAY, NLE, OAX, PUE, QRO, QROO, SLP, SON, TAMS, TLAX, VER, YUC, ZAC

* Setaria latifolia (Scribn.) R.A.W. Herm. AGS, DGO, GTO, JAL, MICH, OAX, QRO, ZAC

Setaria leucopila (Scribn. \& Merr.) K. Schum. AGS, BCS, CHIH, COAH, DGO, GTO, NLE, OAX, PUE, QRO, SLP, SON, TAMS, ZAC

Setaria liebmannii E. Fourn. BCN, BCS, CHIS, CHIH, COL, DGO, GTO, GRO, JAL, MEX, MICH, MOR, NAY, OAX, PUE, QRO, SLP, SIN, SON, VER, YUC, ZAC

Setaria longipila E. Fourn. CHIS, COL, JAL, MICH, NAY, OAX, SIN, SON

Setaria macrostachya Kunth AGS, BCN, BCS, CHIS, CHIH, COAH, DGO, GTO, GRO, HGO, JAL, MEX, MICH, MOR, NAY, NLE, OAX, PUE, QRO, SLP, SIN, SON, TAMS, VER, ZAC

Setaria magna Griseb. CAM, COAH, QROO, TAMS, YUC

*Setaria palmeri Henrard BCS

Setaria palmifolia (J. König) Stapf BCS, CHIS, COL, HGO, JAL, MICH, MOR, NAY, OAX, PUE, QRO, QROO, SLP, TAB, VER, YUC

Setaria parviflora (Poir.) Kerguélen AGS, BCN, BCS, CAM, CHIS, CHIH, COAH, COL, CDMX, DGO, GTO, GRO, HGO,
JAL, MEX, MICH, MOR, NAY, NLE, OAX, PUE, QRO, QROO, SLP, SIN, SON, TAB, TAMS, TLAX, VER, YUC, ZAC

Setaria reverchonii (Vasey) Pilger COAH, NLE, TAMS

Setaria scandens Schrad. CAM, CHIS, COL, HGO, JAL, MEX, OAX, QROO, VER, YUC

Setaria scheelei (Steud.) Hitchc. CHIS, CHIH, COAH, DGO, HGO, JAL, MICH, NLE, PUE, SLP, TAMS, VER

Setaria sphacelata (K. Schum.) Stapf \& C.E. Hubb. PUE, TAB, YUC

Setaria sulcata Raddi CHIS, NAY, OAX, PUE, SLP, SIN, TAMS, VER

Setaria tenax (Rich.) Desv. CAM, CHIS, GRO, JAL, NLE, OAX, PUE, QROO, SLP, TAB, VER, YUC

Setaria texana Emery NLE, TAMS

Setaria variifolia (Swallen) Davidse CAM, QROO, YUC

Setaria villosissima (Scribn. \& Merr.) K. Schum. CHIH, COAH, NLE, SIN, SON, TAMS

Setaria vulpiseta (Lam.) Roem. \& Schult. CAM, CHIS, COAH, DGO, MEX, NLE, OAX, PUE, QROO, SLP, TAB, VER, YUC Setariopsis auriculata (E. Fourn.) Scribn. BCN, BCS, CAM, CHIS, CHIH, COAH, COL, GTO, GRO, JAL, MEX, MICH, MOR, NAY, OAX, PUE, QRO, QROO, SLP, SIN, SON, TAMS, VER, YUC, ZAC

*Setariopsis latiglumis (Vasey) Scribn. CAM, CHIS, CHIH, COL, DGO, GTO, GRO, HGO, JAL, MEX, MICH, MOR, NAY, OAX, PUE, QRO, SON, ZAC

Sitanion jubatum J.G. Sm. BCN

*Sohnsia filifolia (E. Fourn.) Airy Shaw QRO, SLP

Sorghastrum brunneum Swallen CHIS, COAH, GTO, GRO, HGO, JAL, MICH, NAY, NLE, OAX, PUE, QRO, SLP, TAMS, VER

Sorghastrum incompletum (J. Presl) Nash CAM, CHIS, COAH, COL, DGO, GTO, GRO, HGO, JAL, MEX, MICH, MOR, NAY, OAX, PUE, SON, VER, YUC, ZAC

*Sorghastrum nudipes Nash CHIH, COL, DGO, GRO, JAL, OAX, SON

Sorghastrum nutans (L.) Nash AGS, BCN, CHIS, CHIH, COAH, COL, CDMX, DGO, GTO, GRO, HGO, JAL, MEX, MICH, NAY, NLE, OAX, PUE, QRO, SLP, SON, TAMS, VER, ZAC

*Sorghastrum pohlianum Dávila, L.I. Cabrera \& Lira COL

Sorghastrum setosum (Griseb.) Hitchc. CHIS, TAB, TAMS, VER

Sorghastrum stipoides (Kunth) Nash CHIS, COL, GRO, JAL, MICH, MOR, NAY, OAX, VER

Spartina alterniflora Loisel. PUE, TAMS, VER

Spartina cynosuroides (L.) Roth CHIH, TAMS

* Spartina densiflora Brongn. SLP, SIN, TAMS, VER

Spartina foliosa Trin. BCN, BCS

Spartina patens (Aiton) Muhl. QROO, TAB, TAMS

Spartina spartinae (Trin.) Merr. ex Hitchc. CAM, COAH, GRO, NLE, OAX, QROO, SLP, TAB, TAMS, VER, YUC, ZAC Sphenopholis interrupta (Buckley) Scribn. BCN, NLE, SON Sphenopholis obtusata (Michx.) Scribn. AGS, BCN, CHIH, COAH, CDMX, DGO, GTO, JAL, MEX, NLE, OAX, PUE, SIN, SON, ZAC 
Sporobolus airoides (Torr.) Torr. AGS, BCN, CHIH, COAH, DGO, GTO, JAL, NLE, OAX, PUE, SLP, SIN, SON, TAMS, VER, ZAC

*Sporobolus atrovirens (Kunth) Kunth AGS, BCN, BCS, COAH, COL, CDMX, DGO, GTO, GRO, HGO, JAL, MEX, MICH, MOR, NLE, OAX, PUE, QRO, QROO, SLP, TAMS, TLAX, VER, YUC, ZAC

Sporobolus buckleyi Vasey CAM, COAH, NLE, OAX, PUE, QROO, SLP, TAMS, VER, YUC

Sporobolus coahuilensis Valdés-Reyna CHIH, COAH, DGO

Sporobolus contractus Hitchc. BCN, BCS, CHIH, COAH, DGO, JAL, NLE, SLP, SON, ZAC

Sporobolus cryptandrus (Torr.) A. Gray BCN, BCS, CHIH, COAH, DGO, NLE, OAX, SON, TAMS, YUC, ZAC

Sporobolus cubensis Hitchc. CHIS, OAX, TAB, VER Sporobolus domingensis (Trin.) Kunth CAM, QROO, YUC Sporobolus erectus Hitchc. CHIS, VER

Sporobolus flexuosus (Thurb. ex Vasey) Rydb. BCN, CHIH, COAH, DGO, SIN, SON

Sporobolus giganteus Nash $\mathrm{CHIH}, \mathrm{COAH}$

*Sporobolus hintonii T.G. Hartley GRO, MEX, MICH

Sporobolus junceus (P. Beauv.) Kunth CHIS, CHIH, MICH, VER

Sporobolus macrospermus Scribn. ex Beal AGS, CHIS, COL, GTO, GRO, JAL, MEX, MICH, NAY, OAX, PUE, SIN, ZAC Sporobolus nealleyi Vasey COAH, DGO, NLE, SLP

*Sporobolus palmeri Scribn. COAH, DGO, NLE, SLP, ZAC

*Sporobolus potosiensis Wipff \& S.D. Jones SLP, TAMS

Sporobolus purpurascens (Sw.) Ham. CHIS, COL, JAL, OAX, PUE, TAMS, VER

Sporobolus pyramidatus (Lam.) Hitchc. AGS, BCN, BCS, CAM, CHIS, CHIH, COAH, COL, CDMX, DGO, GTO, GRO, HGO, JAL, MEX, MICH, NAY, NLE, OAX, PUE, QRO, QROO, SLP, SIN, SON, TAMS, TLAX, VER, YUC, ZAC

*Sporobolus spiciformis Swallen CHIH, COAH, DGO, NLE

*Sporobolus splendens Swallen CHIS, COL, GRO, JAL, NAY, OAX

*Sporobolus teretifolius Harper CHIH, COAH, DGO

Sporobolus texanus Buckley CHIH, SON

*Sporobolus trichodes Hitchc. AGS, CHIH, DGO, GTO, GRO, JAL, MEX, MICH, MOR, OAX, PUE, QRO, VER, ZAC

Sporobolus virginicus (L.) Kunth BCN, BCS, CAM, CHIS, COL, JAL, NLE, OAX, QROO, SLP, SIN, SON, TAB, TAMS, TLAX, VER, YUC

*Sporobolus viscidus Sohns MEX, MICH

Sporobolus wrightii Munro ex Scribn. AGS, BCN, BCS, CHIH, COAH, CDMX, DGO, GTO, GRO, HGO, MEX, MICH, NLE, PUE, QRO, SLP, SIN, SON, TAMS, VER, ZAC

*Steinchisma cupreum (Hitchc. \& Chase) W.V. Br. AGS, DGO, GTO, JAL, MEX, MICH, PUE, VER, ZAC

Steinchisma hians (Elliott) Nash AGS, CHIS, DGO, GTO, HGO, JAL, MEX, MICH, QRO, SLP, SON, VER, ZAC

Steinchisma laxum (Sw.) Zuloaga CAM, CHIS, COL, DGO, GRO, JAL, MEX, MICH, NAY, NLE, OAX, PUE, QROO, SLP, SIN, TAB, TAMS, VER, YUC, ZAC

Stipa coronata Thurb. BCN

Stipa speciosa Trin. \& Rupr. BCN, SON
*Stipa virlettii E. Fourn. SLP

Streptochaeta sodiroana Hack. CHIS, TAB

Streptochaeta spicata Schrad. ex Nees CHIS, OAX, TAB, VER Streptogyna americana C.E. Hubb. CHIS, VER

*Tetrapogon brandegeei (Vasey) P.M. Peterson BCN, BCS, SIN Tetrapogon chlorideus (J. Presl) P.M. Peterson AGS, BCN, BCS, CHIS, COL, CDMX, GTO, JAL, MEX, MICH, MOR, NAY, NLE, OAX, SLP, SIN, SON, TAMS, VER, ZAC

Thrasya campylostachya (Hack.) Chase CHIS, OAX, PUE, TAB, VER

Thrasya petrosa (Trin.) Chase CHIS

Trachypogon spicatus (L. f.) Kuntze AGS, BCN, BCS, CHIS, CHIH, COL, CDMX, DGO, GTO, GRO, HGO, JAL, MEX, MICH, MOR, NAY, NLE, OAX, PUE, QRO, SLP, SIN, SON, TAB, TAMS, VER, YUC, ZAC

*Trachypogon vestitus Audas OAX

Tridens albescens (Vasey) Wooton \& Standl. CHIH, COAH, DGO, NLE, TAMS

Tridens ambiguus (Elliott) Schult. TAMS

Tridens eragrostoides (Vasey \& Scribn.) Nash CHIH, COAH, GRO, NAY, NLE, OAX, QROO, SLP, SON, TAMS, TLAX, YUC

Tridens muticus (Torr.) Nash BCN, CHIH, COAH, DGO, HGO, NLE, QRO, SLP, SON, TAMS, ZAC

Tridens texanus (S. Watson) Nash CHIH, COAH, NLE, SLP, TAMS

*Triniochloa gracilis Gómez-Sánchez \& Gonz.-Led. GRO, OAX

*Triniochloa laxa Hitchc. CHIH, SON

*Triniochloa micrantha (Scribn.) Hitchc. GRO, MEX, MICH, MOR

Triniochloa stipoides (Kunth) Hitchc. CHIS, COL, CDMX, GTO, GRO, HGO, JAL, MEX, MICH, MOR, OAX, PUE, QRO, SLP, VER

*Triniochloa talpensis M. González-Ledezma \& M. GómezSánchez JAL

Triplasis purpurea (Walter) Chapm. TAB, VER

Tripogon spicatus (Nees) Ekman AGS, CAM, CHIH, CDMX, DGO, GTO, HGO, JAL, MEX, SLP, VER, YUC, ZAC

Tripsacum andersonii J.R. Gray CAM, CHIS, VER

*Tripsacum bravum J.R. Gray GRO, JAL, MEX, MICH, NAY

Tripsacum dactyloides (L.) L. AGS, BCS, CAM, CHIS, CHIH, COAH, COL, CDMX, DGO, GTO, GRO, HGO, JAL, MEX, MICH, MOR, NAY, NLE, OAX, PUE, QRO, QROO, SLP, SIN, SON, TAMS, TLAX, VER, ZAC

Tripsacum intermedium de Wet \& J.R. Harlan CHIS, GRO

Tripsacum jalapense de Wet \& Brink AGS, CHIS, GRO, JAL, OAX

Tripsacum lanceolatum Rupr. ex E. Fourn. AGS, BCN, BCS, CAM, CHIH, COAH, COL, CDMX, DGO, GTO, GRO, HGO, JAL, MEX, MICH, MOR, NAY, NLE, OAX, PUE, QRO, SLP, SIN, SON, TAMS, VER, YUC, ZAC

Tripsacum latifolium Hitchc. CAM, CHIS, CHIH, DGO, MICH, NAY, OAX, PUE, QROO, VER

Tripsacum laxum Nash CHIS, COL, DGO, GRO, JAL, MICH, NAY, OAX, VER

Tripsacum maizar Hern.-Xol. \& Randolph CHIS, COL, GRO, 
JAL, NAY, OAX

*Tripsacum manisuroides de Wet \& J.R. Harlan CHIS, OAX

Tripsacum pilosum Scribn. \& Merr. CHIS, CHIH, COL, DGO, GTO, GRO, JAL, MEX, MICH, NAY, OAX, SLP, ZAC

Tripsacum zopilotense Hern.-Xol. \& Randolph CHIS, CHIH, COL, DGO, GTO, GRO, JAL, MEX, MICH, OAX, PUE, SLP, SIN, TAMS, TLAX, ZAC

Trisetum angustum Swallen CHIS

*Trisetum curvisetum Morden \& Valdés-Reyna NLE, TAMS

*Trisetum durangense Finot \& P.M. Peterson DGO, SIN

*Trisetum filifolium Scribn. ex Beal CHIH, COAH, DGO, HGO, NLE

Trisetum irazuense (Kuntze) Hitchc. CHIS, CDMX, DGO, HGO, MEX, MICH, MOR, OAX, PUE, SLP, TLAX, VER

*Trisetum ligulatum Finot \& Zuloaga VER

*Trisetum martha-gonzaleziae P.M. Peterson \& Finot CHIH, DGO, NAY

*Trisetum mexicanum (Swallen) S.D. Koch JAL, MEX, ZAC Trisetum pringlei (Scribn. ex Beal) Hitchc. CHIS, OAX, PUE

*Trisetum spellenbergii Soreng, Finot \& P.M. Peterson CHIH, SON

Trisetum spicatum (L.) K. Richt. CHIS, CHIH, COAH, CDMX, GTO, HGO, JAL, MEX, MICH, NLE, PUE, QRO, SLP, TLAX, VER

Trisetum viride (Kunth) Kunth CHIH, COAH, CDMX, DGO, GTO, GRO, HGO, JAL, MEX, MICH, NAY, NLE, OAX, QRO, SIN, SON, TAMS, VER, ZAC

*Trisetum virletii E. Fourn. CHIS, CDMX, DGO, GTO, GRO, HGO, JAL, MEX, MICH, MOR, PUE, QRO, SLP, SIN, TLAX, VER

*Tristachya angustifolia Hitchc. JAL, NAY, ZAC

Tristachya avenacea (J. Presl) Scribn. \& Merr. CHIS, COL, GRO, JAL, MEX, MICH, NAY, OAX, SIN, ZAC

*Tristachya contrerasii R. Guzmán JAL, ZAC

*Tristachya laxa Scribn. \& Merr. DGO, JAL, NAY, SIN, ZAC

*Tristachya papillosa R. Guzmán JAL, NAY, ZAC

*Tuctoria fragilis (Swallen) Reeder BCS

Uniola pittieri Hack. BCN, CHIS, COL, JAL, NAY, OAX, SIN

Urochloa adspersa (Trin.) R.D. Webster YUC

Urochloa arizonica (Scribn. \& Merr.) Morrone \& Zuloaga BCN, BCS, CHIH, COAH, COL, DGO, GRO, JAL, MICH, NAY, NLE, OAX, SIN, SON, TAMS, ZAC

Urochloa ciliatissima (Buckley) R.D. Webster COAH, NLE, TAMS, VER

*Urochloa discifera (E. Fourn.) Morrone \& Zuloaga AGS, DGO, GTO, JAL, MEX, MICH, NAY, OAX, QRO, SLP, ZAC Urochloa fusca (Sw.) B.F. Hansen \& Wunderlin BCN, BCS, CAM, CHIS, CHIH, COAH, COL, DGO, GTO, GRO, JAL, MEX, MICH, MOR, NAY, NLE, OAX, PUE, QRO, QROO, SLP, SIN, SON, TAB, TAMS, TLAX, VER, YUC, ZAC

Urochloa jaliscana (Santana Mich.) Espejo \& López-Ferrari JAL

*Urochloa meziana (Hitchc.) Morrone \& Zuloaga AGS, CHIH, COAH, COL, CDMX, DGO, GTO, HGO, JAL, MEX, MICH, NLE, OAX, PUE, QRO, SLP, TAMS, TLAX, VER, YUC, ZAC Urochloa mollis (Sw.) Morrone \& Zuloaga CAM, CHIS, COAH, COL, DGO, GRO, JAL, MEX, NAY, OAX, PUE, SIN, SON,
VER, YUC

*Urochloa olivacea Sánchez-Ken COL, JAL, NAY, SIN

*Urochloa ophryodes (Chase) Morrone \& Zuloaga COAH, NLE, TAMS

*Urochloa pauciflora Sánchez-Ken JAL, MICH

Urochloa platyphylla (Munro ex C. Wright) R.D. Webster COL, GTO, JAL, MICH, OAX, QRO

Urochloa texana (Buckley) R.D. Webster COAH, MICH, NAY, NLE, SLP, TAMS, ZAC

*Urochloa venosa (Swallen) Morrone \& Zuloaga JAL, MICH Vulpia microstachys (Nutt.) Munro BCN, BCS

Vulpia octoflora (Walter) Rydb. BCN, BCS, CHIH, SON

*Zea diploperennis H.H. Iltis, Doebley \& R. Guzmán COL, JAL

Zea luxurians (Durieu \& Asch.) R.M. Bird CHIS, OAX

Zea mays L. AGS, BCN, BCS, CAM, CHIS, CHIH, COAH, COL, CDMX, DGO, GTO, GRO, HGO, JAL, MEX, MICH, MOR, NAY, NLE, OAX, PUE, QRO, QROO, SLP, SIN, SON, TAB, TAMS, TLAX, VER, YUC, ZAC

*Zea perennis (Hitchc.) Reeves \& Mangelsd. COL, JAL, MEX, $\mathrm{MICH}, \mathrm{VER}$

Zeugites americanus Willd. CAM, CHIS, COL, GRO, HGO, JAL, MEX, MICH, MOR, NAY, OAX, PUE, QRO, SLP, TAMS, VER, YUC

*Zeugites capillaris (Hitchc.) Swallen COL, GRO, JAL, MEX, MICH, OAX, VER

*Zeugites hackelii Swallen JAL, MEX, MICH, SIN, ZAC

*Zeugites hintonii T.G. Hartley GRO, MEX

*Zeugites latifolius (E. Fourn.) Hemsl. CHIS, GRO, JAL, OAX, SIN, ZAC

Zeugites munroanus Hemsl. CHIS, VER

Zeugites pittieri Hack. CHIS, GRO, JAL, OAX, ZAC

*Zeugites sagittatus T.G. Hartley MEX, MICH

*Zeugites smilacifolius Scribn. COL, GRO, HGO, JAL, MEX, MICH, MOR, OAX

Zizaniopsis miliacea (Michx.) Döll \& Asch. CHIS, JAL, MICH, VER

Zuloagaea bulbosa (Kunth) Bess AGS, BCN, BCS, CAM, CHIS, CHIH, COAH, COL, CDMX, DGO, GTO, GRO, HGO, JAL, MEX, MICH, MOR, NAY, NLE, OAX, PUE, QRO, QROO, SLP, SIN, SON, TAMS, TLAX, VER, ZAC

\section{Family Podostemaceae}

Marathrum minutiflorum Engl. CHIS, OAX, TAB, VER

* Marathrum plumosum (Novelo \& C.T. Philbrick) C.T. Philbrick \& C.P. Bove JAL, OAX

*Marathrum rubrum Novelo \& C.T. Philbrick GRO, JAL

Marathrum schiedeanum (Cham.) Tul. CHIS, COL, GRO, JAL, MICH, MOR, NAY, OAX, VER

Marathrum tenue Liebm. CHIS, MOR, OAX, PUE, VER

*Noveloa coulteriana (Tul.) C.T. Philbrick BCS, CHIH, COL, GRO, JAL, MICH, MOR, NAY, SIN, SON, TAMS, ZAC

*Noveloa longifolia (Novelo \& C.T. Philbrick) C.T. Philbrick COL, JAL

Podostemum rutifolium Warm. OAX, PUE, TAB, VER

Tristicha trifaria (Bory ex Willd.) Spreng. CHIS, COL, GRO, HGO, JAL, MEX, MOR, NAY, OAX, PUE, SLP, TAB, TAMS, 
VER, ZAC

\section{Family Polemoniaceae}

*Acanthogilia gloriosa (Brandegee) A.G. Day \& Moran BCN Allophyllum gilioides (Benth.) A.D. Grant \& V.E. Grant BCN, SON

Allophyllum glutinosum (Benth.) A.D. Grant \& V.E. Grant BCN *Allophyllum nemophilophyllum J.M. Porter \& L.A. Johnson $\mathrm{BCN}$

Bonplandia geminiflora Cav. CHIS, CHIH, COL, DGO, GTO, GRO, HGO, JAL, MEX, MICH, MOR, NAY, OAX, QRO, SLP, SIN, SON, TAMS, ZAC

*Bryantiella palmeri (S. Watson) J.M. Porter BCN, BCS

* Cobaea biaurita Standl. CHIS, OAX, VER

Cobaea lutea D. Don CHIS, COL, GRO, JAL, MICH

Cobaea minor M. Martens \& Galeotti VER

Cobaea pachysepala Standl. CHIS, PUE, VER

*Cobaea paneroi L.A. Prather HGO, MOR, PUE, QRO, VER

*Cobaea pringlei (House) Standl. CHIS, COAH, GTO, NLE, QRO, SLP, TAMS

* Cobaea rotundiflora L.A. Prather CHIS

Cobaea scandens Cav. CHIS, COAH, CDMX, GTO, GRO, HGO, MEX, MICH, MOR, NLE, OAX, PUE, QRO, SLP, TAMS, VER

Cobaea skutchii I.M. Johnst. CHIS

*Cobaea stipularis Benth. CHIS, GTO, HGO, OAX, PUE, QRO, SLP, VER

*Dayia grantii J.M. Porter BCS

Dayia havardii (A. Gray) J.M. Porter CHIH, COAH

*Dayia scabra (Brandegee) J.M. Porter BCS

* Dayia sonorae (Rose) J.M. Porter SIN, SON

Eriastrum densifolium (Benth.) Mason BCN

Eriastrum diffusum (A. Gray) H.L. Mason BCN, CHIH, SON

Eriastrum eremicum (Jepson) H.L. Mason BCN

Eriastrum filifolium (Nutt.) Wooton \& Standl. BCN

Eriastrum sapphirinum (Eastw.) H.L. Mason BCN

Gilia angelensis V.E. Grant BCN

Gilia australis (H. Mason \& A.D. Grant) V.E. Grant \& A.D.

Grant BCN, SON

Gilia caruifolia Abrams BCN

Gilia diegensis (Munz) A.D. Grant \& V.E. Grant BCN

Gilia flavocincta A. Nelson BCN, SON

Gilia latifolia S. Watson BCN, SON

Gilia mexicana A.D. Grant \& V.E. Grant BCN, BCS, CHIH, SON

Gilia minor A.D. Grant \& V.E. Grant SON

Gilia nevinii A. Gray BCN

Gilia sinuata Douglas BCN, SON

Gilia stellata A. Heller BCN, BCS, SON

* Giliastrum gypsophilum (B.L. Turner) J.M. Porter NLE

Giliastrum incisum (Benth.) J.M. Porter CHIH, COAH, GTO, HGO, NLE, QRO, SLP, TAMS, VER

Giliastrum insigne (Brand) J.M. Porter CHIH, COAH, DGO, NLE

Giliastrum ludens (Shinners) J.M. Porter CHIH, COAH, DGO Giliastrum purpusii (Brandegee) J.M. Porter AGS, CHIH, COAH, DGO, GTO, HGO, NLE, QRO, SLP, TAMS, ZAC
Giliastrum rigidulum (Benth.) Rydb. AGS, BCN, BCS, CHIH, COAH, DGO, NLE, SLP, SON, TAMS, ZAC

*Giliastrum stewartii (I.M. Johnst.) J.M. Porter CHIH, COAH, DGO, SLP

Ipomopsis aggregata (Pursh) V.E. Grant BCN, CHIH, COAH, DGO, NLE, SLP, SON

Ipomopsis effusa (A. Gray) Moran BCN

*Ipomopsis guttata (A. Gray) Moran BCN

Ipomopsis laxiflora (Coult.) V.E. Grant CHIH, COAH, DGO

Ipomopsis longiflora (Torr.) V.E. Grant CHIH, COAH, DGO, SON

Ipomopsis macombii (Torr. ex A. Gray) V.E. Grant CHIH, COAH, NLE, SON

*Ipomopsis monticola J.M. Porter \& L.A. Johnson SIN

*Ipomopsis pinnata (Cav.) V.E. Grant AGS, CHIH, COAH, DGO, GTO, HGO, JAL, MEX, NLE, SLP, SON, TLAX, VER, ZAC

Ipomopsis polycladon (Torr.) V.E. Grant CHIH, COAH, DGO, SON

Ipomopsis pringlei (A. Gray) W.C. Martin \& C.R. Hutchins CHIH, SON

Ipomopsis tenuifolia (A. Gray) V.E. Grant BCN

Ipomopsis thurberi (A. Gray) V.E. Grant CHIH, SON

*Ipomopsis wendtii Henr. COAH

Langloisia matthewsii (A. Gray) Greene BCN, SON

Langloisia schottii (Torr.) Greene BCN, SON

Langloisia setosissima (Torr.) Greene BCN, SON

Leptodactylon pungens (Torr.) Rydb. BCN, BCS

*Leptodactylon veatchii (Parry ex Greene) Wherry BCN, BCS

Linanthus aureus (Nutt.) Greene BCN, SON

Linanthus bellus (A. Gray) Greene BCN

Linanthus bigelovii (A. Gray) Greene BCN, SON

Linanthus dianthiflorus (Benth.) Greene BCN

Linanthus floribundus (A. Gray) Greene BCN, BCS, CHIH, SIN, SON

*Linanthus jamauensis Moran BCN

Linanthus jonesii (A. Gray) Greene BCN, SON

*Linanthus laxus (Vasey \& Rose) Wherry BCN

Linanthus lemmonii (A. Gray) Greene BCN

*Linanthus melingii (Wiggins) V.E. Grant BCN

Linanthus orcuttii (Parry \& A. Gray) Jepson BCN

Linanthus parviflorus (Benth.) Greene BCN

Linanthus pygmaeus (Brand) J.T. Howell BCN

*Linanthus uncialis (Brandegee) Greene BCN, BCS

*Linanthus vizcainensis Moran BCS

*Loeselia amplectens (Hook. \& Arn.) Benth. COL, GRO, JAL, MICH, NAY, SON, ZAC

Loeselia caerulea (Cav.) G. Don AGS, CHIH, COAH, COL, CDMX, DGO, GTO, GRO, HGO, JAL, MEX, MICH, MOR, NLE, OAX, PUE, QRO, SLP, TAMS, TLAX, VER, ZAC

*Loeselia campechiana Gutiérrez-Báez \& R. Duno CAM Loeselia ciliata L. BCN, BCS, CHIS, CHIH, COL, DGO, GRO, JAL, NAY, OAX, SLP, SIN, SON, VER, ZAC

Loeselia glandulosa (Cav.) G. Don AGS, CHIS, CHIH, COL, CDMX, DGO, GTO, GRO, HGO, JAL, MEX, MICH, MOR, NAY, OAX, PUE, QRO, SLP, SIN, SON, TLAX, VER, ZAC Loeselia greggii $\mathrm{S}$. Watson AGS, CHIH, COAH, DGO, GTO, 
HGO, JAL, NLE, QRO, SLP, TAMS, ZAC

Loeselia mexicana (Lam.) Brand AGS, CHIS, CHIH, COL, CDMX, DGO, GTO, GRO, HGO, JAL, MEX, MICH, MOR, NAY, OAX, PUE, QRO, SLP, SIN, SON, TAMS, TLAX, VER, ZAC

Loeselia pumila (M. Martens \& Galeotti) Walp. CHIS, CHIH, COL, DGO, GTO, GRO, HGO, JAL, MEX, MICH, MOR, NAY, OAX, PUE, QRO, SLP, SON, TAMS, VER, ZAC

* Loeselia purpusii Brandegee OAX, PUE

Loeselia ramosissima (M. Martens \& Galeotti) Walp. CHIS

*Loeselia rupestris DC. OAX

*Loeselia rzedowskii McVaugh JAL, ZAC

*Loeselia spectabilis J.M. Porter \& V.M. Steinm. MICH

*Loeselia tancitaroensis J.M. Porter \& V.M. Steinm. JAL, $\mathrm{MICH}$

Navarretia atractyloides (Benth.) Hook. \& Arn. BCN

Navarretia fossalis Moran BCN

Navarretia hamata Greene BCN

Navarretia intertexta (Benth.) Hook. BCN

Navarretia peninsularis Greene BCN

Phlox austromontana Coville BCN, SON

Phlox gracilis (Dougl. ex Hook.) Greene BCN, SON

* Phlox mexicana Wherry DGO

Phlox nana Nutt. CHIH, COAH, SON

Phlox pilosa L. COAH

Phlox triovulata Thurb. ex Torr. CHIH, COAH, SON

*Polemonium grandiflorum Benth. CHIH, CDMX, DGO, GTO, HGO, MEX, OAX, PUE, QRO, SLP, TLAX, VER

*Polemonium melindae Rzed., Calderón \& Villarreal CHIH, DGO, GTO, QRO, SON

*Polemonium mexicanum Cerv. ex Lag. CDMX, HGO, MEX, MICH, MOR, PUE, TLAX

Polemonium pauciflorum S. Watson CHIH, COAH, DGO, JAL, NLE, QRO, SON, ZAC

Family Polygalaceae

Bredemeyera lucida (Benth.) Klotzsch ex Hassk. CAM, CHIS, OAX, QROO

*Hebecarpa americana (Mill.) J.R.I. Wood \& S. Beck HGO, MOR, OAX, QRO, VER

Hebecarpa barbeyana (Chodat) J.R. Abbot AGS, BCS, CHIH, COAH, DGO, GTO, GRO, HGO, JAL, MICH, NAY, NLE, OAX, PUE, QRO, SLP, SON, TAMS, VER, ZAC

*Hebecarpa buxifolia (Kunth) J.R. Abbott \& J.F.B. Pastore AGS, GTO, HGO, NLE, QRO, SLP, TAMS, VER

Hebecarpa costaricensis (Chodat) J.R. Abbott \& J.F.B. Pastore CHIS, GRO, OAX, VER

Hebecarpa greggii (S. Watson) J.R. Abbott COAH, NLE, SLP, TAMS

Hebecarpa macradenia (A. Gray) J.R. Abbott BCN, CHIH, COAH, GTO, HGO, NLE, QRO, SLP, SON, TAMS, VER, ZAC

*Polygala myrtilloides (Willd.) J.R. Abbott \& J.F.B. Pastore COL, CDMX, DGO, GTO, HGO, JAL, MEX, MICH, NLE, QRO, SLP, VER

Hebecarpa obscura (Benth.) J.R. Abbott AGS, CHIS, CHIH, COAH, DGO, GTO, GRO, JAL, NAY, OAX, PUE, QRO, SLP,
SIN, SON, ZAC

Hebecarpa ovatifolia (A. Gray) J.R. Abbott CHIH, COAH, NLE, TAMS

Hebecarpa palmeri (S. Watson) J.R. Abbott COAH

Hebecarpa platycarpa (Benth.) J.R. Abbott \& J.F.B. Pastore CHIS, GRO, OAX, TAB

Hebecarpa rectipilis (S.F. Blake) J.R. Abbott CHIH, COAH, DGO, ZAC

*Hebecarpa rivinifolia (Kunth) J.R. Abbott \& J.F.B. Pastore AGS, CHIS, COL, DGO, GRO, HGO, JAL, MEX, MICH, MOR, NAY, OAX, QRO, SIN, VER, ZAC

*Hebecarpa robinsonii (S.F. Blake) J.R. Abbott \& J.F.B. Pastore JAL

*Hebecarpa tehuacana (Brandegee) J.R. Abbott \& J.F.B. Pastore OAX, PUE

*Monnina ciliolata DC. CHIH, COL, CDMX, GTO, GRO, HGO, JAL, MEX, MICH, MOR, NAY, OAX, QRO, SLP, SIN, SON, TLAX, VER, ZAC

Monnina guatemalensis Chodat CHIS, VER

*Monnina subserrata Chodat ND

Monnina sylvatica Schltdl. \& Cham. CHIS, COL, GRO, HGO, JAL, MICH, OAX, QRO, SLP, VER

Monnina wrightii A. Gray AGS, CHIH, COL, DGO, GTO, JAL, MICH, SON, ZAC

Monnina xalapensis Kunth AGS, CHIS, COL, CDMX, DGO, GTO, GRO, HGO, JAL, MEX, MICH, MOR, NAY, OAX, PUE, QRO, SLP, SIN, TAMS, TLAX, VER, ZAC

Polygala adenophora DC. CHIS, OAX, TAB, VER

Polygala alba Nutt. CHIS, CHIH, COAH, CDMX, DGO, GTO, GRO, HGO, JAL, MEX, MICH, NAY, NLE, OAX, PUE, QRO,

SLP, SIN, SON, TAMS, VER, ZAC

*Polygala amphothrix S.F. Blake CHIH, SON

* Polygala annectans S.F. Blake PUE

Polygala aparinoides Hook. \& Arn. CHIS, DGO, GTO, JAL, MICH, NAY, NLE, QRO, SLP, TAMS

* Polygala apopetala Brandegee BCS, CHIH

*Polygala appressipilis S.F. Blake CDMX, DGO, JAL, MEX, MICH, MOR, NAY, OAX, PUE, SIN, ZAC

Polygala asperuloides Kunth CHIS, OAX, TAB

Polygala berlandieri S. Watson AGS, BCS, CAM, CHIS, CHIH, GTO, GRO, JAL, MEX, MICH, MOR, NAY, OAX, SLP, SIN, SON, TAMS, VER, ZAC

*Polygala biformipilis S.F. Blake MICH, VER

* Polygala brachyanthema S.F. Blake SLP

*Polygala brachysepala S.F. Blake COL, GRO, JAL, MEX, MICH, MOR, SLP

*Polygala brachytropis S.F. Blake HGO, MEX, MICH, MOR

* Polygala brandegeeana Chodat HGO, PUE, QRO

*Polygala caerulescens S.F. Blake GTO, PUE

*Polygala compacta Rose AGS, BCS, COAH, CDMX, DGO, GTO, GRO, HGO, JAL, MEX, MICH, MOR, OAX, PUE, QRO, SLP, TAMS, VER, ZAC

Polygala conferta A.W. Benn. ex Hemsl. CHIS, JAL, MICH, VER, ZAC

* Polygala conzattii Rose OAX

* Polygala crinita Chodat COL, JAL, OAX, SIN

* Polygala cuspidulata S.F. Blake OAX, PUE 
* Polygala chiapensis S.F. Blake CHIS

* Polygala decidua S.F. Blake DGO, GTO, MICH

*Polygala dolichocarpa S.F. Blake AGS, COAH, GTO, HGO,

JAL, NLE, OAX, PUE, QRO, SLP, TAMS, ZAC

Polygala floribunda Benth. CHIS, CHIH, GRO, OAX

*Polygala galeottii Chodat CHIS, VER

Polygala glochidiata Kunth AGS, BCS, CHIS, CHIH, COL, DGO, GTO, GRO, JAL, MEX, MICH, MOR, NAY, OAX, QRO, SIN, SON, TAB, VER, ZAC

Polygala gracillima S. Watson CHIS, COL, DGO, GTO, GRO, JAL, MEX, MICH, MOR, NAY, VER

Polygala hemipterocarpa A. Gray CHIH, HGO, NLE, SON, TAMS

Polygala hygrophila Kunth CHIS, TAB, VER

Polygala incarnata L. CHIS, HGO, TAB, VER

Polygala jamaicensis Chodat CAM, CHIS, QROO, YUC

*Polygala leptosperma Chodat OAX, VER

Polygala longicaulis Kunth CHIS, GRO, JAL, NAY, OAX, TAB, VER

*Polygala longipes S.F. Blake COL, GRO, JAL, MICH, OAX

*Polygala lozanii Rose PUE

* Polygala magdalenae Brandegee BCS

* Polygala microtricha S.F. Blake CHIS, HGO, MEX, MICH, OAX, QRO

* Polygala neurocarpa Brandegee HGO, QRO

* Polygala oaxacana Chodat OAX, PUE

* Polygala oedophylla S.F. Blake NLE, TAMS

* Polygala oophylla S.F. Blake OAX, PUE

Polygala paniculata L. CAM, CHIS, COL, GRO, HGO, JAL,

MOR, OAX, PUE, QRO, QROO, SLP, TAB, VER, YUC

* Polygala parrasana Brandegee COAH, DGO, NLE

* Polygala pavoni Chodat ND

* Polygala pedicellata S.F. Blake CHIS, HGO, OAX, VER

* Polygala perennis S.F. Blake OAX, VER

*Polygala phoenicistes S.F. Blake SLP

* Polygala polyedra Brandegee SIN

* Polygala pterocarya Chodat GRO, OAX

* Polygala retifolia S.F. Blake JAL

Polygala rhysocarpa S.F. Blake CHIS, VER

* Polygala russelliana S.F. Blake BCS, MOR, SON

Polygala salviniana A.W. Benn. CHIS, GRO, OAX

*Polygala scoparia Kunth AGS, CDMX, GTO, GRO, HGO,

JAL, MEX, MICH, OAX, PUE, QRO, SLP, TLAX, VER, ZAC

Polygala scoparioides Chodat CHIH, COAH, DGO, GTO, NLE,

SLP, SON, TAMS, ZAC

*Polygala semialata S. Watson CHIH, COAH, DGO, NLE, SLP, ZAC

*Polygala serpens S.F. Blake COL, GRO, JAL, OAX

*Polygala shinnersii W.H. Lewis COAH, NLE, SLP, ZAC

* Polygala sinaloae S.F. Blake CHIH, SIN, SON

*Polygala subalata S. Watson COAH, CDMX, DGO, GTO, GRO, HGO, JAL, MEX, MICH, NLE, OAX, PUE, QRO, SLP, VER

Polygala tenella Willd. CHIS, GRO, JAL, MEX, MOR, OAX, TAB, VER

*Polygala turgida Rose COAH, NLE, SLP

Polygala variabilis Kunth CHIS, OAX, TAB, VER
* Polygala velata S.F. Blake CHIS, GRO, OAX

*Polygala vergrandis W.H. Lewis COAH, GTO, HGO, NLE, OAX, PUE, QRO, SLP, TAMS

Polygala violacea Aubl. CAM, CHIS, COL, GRO, JAL, MICH, MOR, NAY, OAX, SIN, TAB, VER, YUC, ZAC

*Polygala viridis $\mathrm{S}$. Watson COAH, NLE

Polygala watsonii Chodat CHIH, COAH, DGO, NLE, SLP, ZAC

*Polygala xanti A. Gray BCS

Rhinotropis cornuta (Kellogg) J.R. Abbott BCN

*Rhinotropis desertorum (Brandegee) J.R. Abbott BCN, BCS

Rhinotropis lindheimeri (A. Gray) J.R. Abbott AGS, CHIH, COAH, DGO, GTO, HGO, NLE, QRO, SLP, SON, TAMS, VER, ZAC

*Rhinotropis madrensis (T. Wendt) J.R. Abbott NLE, TAMS

Rhinotropis maravillasensis (Correll) J.R. Abbott CHIH, COAH *Rhinotropis minutifolia (Rose) J.R. Abbott COAH, NLE

Rhinotropis nitida (Brandegee) J.R. Abbott HGO, JAL, NLE, QRO, SLP, TAMS, VER, ZAC

Rhinotropis nudata (Brandegee) J.R. Abbott CHIH, COAH, NLE, TAMS

*Rhinotropis parryi (A.W. Benn.) J.R. Abbott DGO, SLP

Rhinotropis purpusii (Brandegee) J.R. Abbott CHIS, HGO, OAX, PUE, VER

Securidaca diversifolia (L.) S.F. Blake CAM, CHIS, COL, GRO, HGO, JAL, MEX, MICH, MOR, OAX, PUE, QRO, QROO, SLP, TAB, TAMS, VER, YUC, ZAC

Securidaca sylvestris Schltdl. CHIS, GRO, JAL, MICH, NAY, OAX, PUE, VER

\section{Family Polygonaceae}

Antigonon cinerascens M. Martens \& Galeotti CHIS, COL, GRO, OAX, PUE, SLP, TAMS, VER

Antigonon flavescens S. Watson CHIS, COL, GRO, JAL, MEX, MICH, NAY, OAX, QRO, SLP, SIN, VER

Antigonon guatimalense Meisn. CHIS, GRO, OAX, VER

Antigonon leptopus Hook. \& Arn. BCN, BCS, CAM, CHIS, CHIH, COAH, COL, DGO, GTO, GRO, HGO, JAL, MEX, MICH, MOR, NAY, NLE, OAX, QRO, QROO, SLP, SIN, SON, TAB, TAMS, VER, YUC, ZAC

Centrostegia thurberi A. Gray ex Benth. BCN

Coccoloba acapulcensis Standl. CAM, CHIS, GRO, JAL, MICH, OAX, PUE, QROO, TAB, YUC

Coccoloba acuminata Kunth CHIS, QROO

Coccoloba barbadensis Jacq. CAM, CHIS, COL, GRO, HGO, JAL, MEX, MICH, MOR, NAY, OAX, PUE, QRO, QROO, SLP, SIN, TAB, TAMS, VER, YUC

Coccoloba belizensis Standl. CAM, CHIS, QROO, VER

Coccoloba caracasana Meisn. CHIS

Coccoloba cozumelensis Hemsl. CAM, CHIS, GRO, QROO, TAB, YUC

* Coccoloba chiapensis Standl. CHIS, OAX, VER

Coccoloba diversifolia Jacq. CHIS, OAX, QROO, SLP, VER, YUC

Coccoloba escuintlensis Lundell CHIS, VER

* Coccoloba floresii Ortiz-Díaz \& Arnelas CHIS

Coccoloba floribunda (Benth.) Lindau CHIS, COL, GRO, JAL, 
OAX, QROO, VER

* Coccoloba goldmannii Standl. CHIH, SIN, SON

Coccoloba grandifolia Jacq. OAX, VER

Coccoloba hirtella Lundell OAX, VER

Coccoloba hondurensis Lundell CHIS, OAX, TAB, VER

*Coccoloba humboldtii Meisn. CAM, CHIS, OAX, QROO, SLP, TAB, TAMS, VER, YUC

*Coccoloba jurgenseni Lindau COL, JAL, NAY, OAX

* Coccoloba lapathifolia Standl. GRO

Coccoloba lehmannii Lindau CHIS, TAB

Coccoloba liebmannii Lindau CHIS, COL, GRO, JAL, MICH, OAX, VER

Coccoloba lindaviana R.A. Howard CHIS, VER

Coccoloba lindeniana (Benth.) Lindau CHIS, TAB, VER

Coccoloba montana Standl. CHIS, GRO, MICH, OAX, QROO, TAB, VER

*Coccoloba ortizii R.A. Howard QROO, YUC

Coccoloba reflexiflora Standl. CAM, QROO, YUC

Coccoloba spicata Lundell CAM, CHIS, PUE, QROO, TAB, VER, YUC

Coccoloba swartzii Meisn. QROO

Coccoloba tuerckheimii Donn. Sm. CHIS, TAB, VER

Coccoloba uvifera L. CAM, OAX, QROO, SIN, TAB, TAMS, VER, YUC

Coccoloba venosa L. CHIS, COL, GRO, JAL, OAX

Chorizanthe brevicornu Torr. BCN, SON

Chorizanthe corrugata (Torr.) Torr. \& A. Gray BCN, SON

Chorizanthe fimbriata Nutt. BCN

* Chorizanthe flava Brandegee BCN, BCS

* Chorizanthe inequalis Stokes BCN

*Chorizanthe interposita Goodman BCN, BCS

* Chorizanthe jonesiana Goodman BCN

Chorizanthe leptotheca Goodman BCN

* Chorizanthe mutabilis Brandegee BCN, BCS

Chorizanthe parryi $\mathrm{S}$. Watson $\mathrm{BCN}$, BCS

Chorizanthe polygonoides Torr. \& A. Gray BCN

Chorizanthe procumbens Nutt. BCN

* Chorizanthe pulchella Brandegee BCN, BCS

Chorizanthe rigida (Torr.) Torr. \& A. Gray BCN, BCS, SON

* Chorizanthe rosulenta Reveal BCN

* Chorizanthe turbinata Wiggins BCN, BCS

Eriogonum abertianum Torr. BCN, CHIH, COAH, SLP, SON

*Eriogonum angelense Moran $\mathrm{BCN}$

Eriogonum angulosum Benth. BCN

Eriogonum annuum Nutt. CHIH

Eriogonum atrorubens Engelm. CHIH, COAH, DGO, NLE, SIN, SON, ZAC

Eriogonum austrinum (S. Stokes) Reveal BCN, SON

Eriogonum brachyanthum Coville BCN

Eriogonum brachypodum Torr. \& A. Gray BCN

*Eriogonum ciliatum Torr. COAH, NLE, SLP, TAMS

*Eriogonum daduchum Hess \& Reveal COAH

Eriogonum davidsonii Greene BCN

Eriogonum deflexum Torr. BCN, SON

Eriogonum deserticola $\mathrm{S}$. Watson $\mathrm{BCN}$, SON

Eriogonum elongatum Benth. BCN, BCS

*Eriogonum encelioides Reveal \& Hanson BCN, BCS
Eriogonum fasciculatum Benth. BCN, BCS, SON

*Eriogonum fastigiatum Parry BCN

*Eriogonum fimbriatum W.J. Hess \& Reveal COAH, NLE, ZAC

Eriogonum foliosum S. Watson BCN

*Eriogonum galioides I.M. Johnst. BCN

Eriogonum gracile Benth. BCN

Eriogonum grande Greene BCN

Eriogonum greggii Torr. \& A. Gray CHIH, COAH, NLE

Eriogonum hemipterum (Torr. \& A. Gray) S. Stokes CHIH, COAH, DGO, NLE

*Eriogonum henricksonii Reveal COAH

Eriogonum hieracifolium Benth. CHIH, COAH

Eriogonum inflatum Torr. \& Frem. BCN, BCS, CHIH, SON

*Eriogonum intricatum Benth. BCN, BCS

Eriogonum jamesii Benth. AGS, CHIH, COAH, CDMX, DGO, GTO, HGO, MEX, NLE, SLP, SON, TAMS, ZAC

Eriogonum lonchophyllum Torr. \& A. Gray CHIH, SON

*Eriogonum molle Brandegee BCN, BCS

*Eriogonum moranii Reveal BCN, BCS

Eriogonum nudum Dougl. ex Benth. BCN

*Eriogonum orcuttianum S. Watson BCN, BCS

Eriogonum parishii S. Watson $\mathrm{BCN}$

Eriogonum polycladon Benth. CHIH, COAH, DGO, SON

*Eriogonum pondii Greene BCN, BCS

*Eriogonum preclarum Reveal BCS

Eriogonum reniforme Torr. \& Frém. BCN

*Eriogonum repens (S. Stokes) Reveal BCS

Eriogonum rotundifolium Benth. CHIH, COAH, NLE

*Eriogonum scalare $\mathrm{S}$. Watson $\mathrm{BCN}, \mathrm{BCS}$

Eriogonum tenellum Torr. CHIH, COAH, NLE

Eriogonum thomasii Torr. BCN, BCS, SON

Eriogonum thurberi Torr. BCN, BCS, SON

Eriogonum trichopes Torr. BCN, BCS, SON

*Eriogonum turneri Reveal NLE

*Eriogonum viscanum W.J. Hess \& Reveal NLE, SLP, ZAC

Eriogonum wrightii Torr. ex Benth. AGS, BCN, BCS, CHIH, COAH, DGO, GTO, JAL, SLP, SON, ZAC

*Eriogonum zapatoense Moran BCN

Gymnopodium floribundum Rolfe CAM, CHIS, OAX, QROO, TAB, VER, YUC

*Harfordia macroptera (Benth.) Greene \& Parry BCN, BCS

* Lastarriaea coriacea (Goodman) Hoover BCN

Lastarriaea chilensis Remy BCN, BCS

* Lastarriaea ptilota Reveal BCN, BCS

Muehlenbeckia tamnifolia (Kunth) Meisn. CHIS, GRO, MEX, MOR, OAX, PUE, VER

Muehlenbeckia volcanica (Benth.) Endl. CHIS

Nemacaulis denudata Nutt. BCN, BCS, SON

Neomillspaughia emarginata (H. Gross) S.F. Blake CAM, QROO, YUC

Oxytheca trilobata A. Gray BCN

Periscaria segeta (Kunth) Small AGS, BCN, CAM, CHIS, CHIH, DGO, GTO, GRO, HGO, JAL, MEX, MICH, MOR, NAY, OAX, PUE, QRO, QROO, SLP, SON, TAB, TAMS, TLAX, VER, ZAC

Persicaria amphibia (L.) Gray CHIS, BCN, BCS, CHIH, CDMX, GTO, HGO, JAL, MEX, MICH, MOR, OAX, PUE, 
SON, TLAX, ZAC

Persicaria bistortoides (Pursh) H.R. Hinds CHIH

Persicaria ferruginea (Wedd.) Soják CHIS, TAMS, VER

Persicaria glabra (Willd.) M. Gómez BCS, CHIS, CHIH, COAH, COL, JAL, MOR, NLE, QRO, SLP, TAB, TAMS, VER

Persicaria hispida (Kunth) M. Gómez CHIS, GRO, OAX, SIN Persicaria hydropiperoides (Michx.) Small AGS, BCN, BCS, CAM, CHIS, CHIH, COAH, COL, CDMX, DGO, GTO, GRO, HGO, JAL, MEX, MICH, MOR, NAY, NLE, OAX, PUE, QRO, QROO, SLP, SIN, SON, TAB, TAMS, TLAX, VER, YUC, ZAC Persicaria longistyla (Small) Small MICH

Persicaria meisneriana (Cham. \& Schltdl.) M. Gómez CHIS, VER

Persicaria pennsylvanica (L.) M. Gómez AGS, CHIH, COAH, DGO, MEX, MICH, NLE, QRO, SLP, SON, TAB, TAMS, ZAC Persicaria punctata (Elliott) Small AGS, BCN, BCS, CAM, CHIS, CHIH, COAH, COL, CDMX, DGO, GTO, GRO, HGO, JAL, MEX, MICH, MOR, NAY, NLE, OAX, PUE, QRO, QROO, SLP, SIN, SON, TAB, TAMS, TLAX, VER, ZAC

Persicaria setacea (Baldwin) Small MICH, SLP, TAMS

Persicaria virginiana (L.) Gaertn. PUE, VER

*Podopterus cordifolius Rose \& Standl. COL, GRO, JAL, $\mathrm{MICH}, \mathrm{OAX}$

Podopterus guatemalensis S.F. Blake OAX, VER

Podopterus mexicanus Bonpl. CAM, CHIS, COL, GRO, HGO, JAL, MICH, NAY, OAX, PUE, QRO, QROO, SLP, TAMS, VER, YUC

Polygonum acuminatum Kunth CAM, CHIS, GRO, JAL, MEX, MICH, MOR, QROO, TAB, TAMS, VER

*Polygonum aubertii L. SLP, TAMS, VER

Polygonum douglasii Greene BCN, SON

*Polygonum longiocreatum Bartlett CHIS, TAB, VER

*Polygonum paniculatum L. VER

*Polygonum pringlei Small QRO, SLP

Polygonum scabrum Moench MICH

Pterostegia drymarioides Fisch. \& C.A. Mey. BCN, BCS, CHIH

Rumex altissimus Wood CHIH, SLP, SON

Rumex berlandieri Meisn. OAX, TAMS, VER

Rumex californicus Rech. f. BCN

Rumex chrysocarpus Moris TAMS

*Rumex flexicaulis Rech. f. CDMX, HGO, JAL, MEX, MICH, TLAX

Rumex hymenosepalus Torr. BCN, BCS, CHIH, COAH, SON

Rumex inconspicuus Rech. f. BCS, SIN, SON

Rumex mexicanus Meisn. AGS, BCS, CHIS, CHIH, COAH, CDMX, DGO, GTO, GRO, HGO, JAL, MEX, MICH, MOR, NLE, OAX, PUE, QRO, SLP, SON, TLAX, VER, ZAC

Rumex nematopodus Rech. f. CHIH

Rumex salicifolius Weinm. BCN, CHIH, SON

Rumex triangulivalvis (Danser) Rech. f. CHIH, SLP

Rumex verticillatus $\mathrm{L}$. CHIH, COAH, DGO

Rumex violascens Rech. f. BCN, BCS, CHIH, COAH, SON

Ruprechtia chiapensis Lundell CAM, CHIS, DGO, OAX, QRO, QROO, SLP, TAB, TAMS, VER, YUC

Ruprechtia costata Meisn. GRO, OAX, VER

*Ruprechtia fusca Fernald COL, GRO, JAL, MEX, MICH,
MOR, OAX, PUE, SIN, TAB, VER

*Ruprechtia laevigata Pendry JAL, OAX, VER, YUC

Ruprechtia pallida Standl. COL, GRO, JAL, MICH

*Ruprechtia standleyana Cocucci GRO, MEX, MICH, MOR, OAX, PUE

Triplaris americana L. CHIS

Triplaris melaenodendron (Bertol.) Standl. \& Steyerm. CHIS

Triplaris tomentosa Wedd. OAX

\section{Family Pontederiaceae}

Eichhornia azurea (Sw.) Kunth COL, GRO, JAL, MICH, OAX, SON, TAB, VER

Eichhornia heterosperma Alexander CHIS, TAB

Heteranthera dubia (Jacq.) MacMill. CAM, CHIH, COAH, DGO, HGO, JAL, NAY, OAX, QROO, SLP, SON, TAB, TAMS, VER, YUC

Heteranthera limosa (Sw.) Willd. AGS, CHIS, CHIH, COAH, COL, CDMX, DGO, GTO, GRO, HGO, JAL, MEX, MICH, MOR, NAY, OAX, PUE, QRO, QROO, SLP, SIN, SON, TAMS, VER, YUC, ZAC

Heteranthera mexicana S. Watson COAH, QRO, QROO, SLP, TAMS, YUC

Heteranthera oblongifolia C. Mart. ex Roem. \& Schult. OAX

Heteranthera peduncularis Benth. AGS, CHIH, COL, CDMX, DGO, GTO, HGO, JAL, MEX, MICH, MOR, NAY, OAX, PUE, QRO, SLP, SIN, VER, ZAC

Heteranthera reniformis Ruiz \& Pav. CHIS, COAH, COL, GTO, HGO, JAL, MEX, MICH, MOR, NAY, OAX, PUE, QRO, SLP, TAB, TAMS, VER

Heteranthera rotundifolia (Kunth) Griseb. AGS, CHIS, CHIH, COAH, DGO, GTO, GRO, HGO, JAL, MEX, MICH, MOR, NAY, OAX, PUE, QRO, SLP, SIN, SON, TAMS, VER, YUC, ZAC

Heteranthera seubertiana Solms CHIS, QROO, YUC

Heteranthera spicata C. Presl JAL

Pontederia rotundifolia $\mathrm{L}$. f. GRO, MOR, OAX, TAB, VER Pontederia sagittata C. Presl CAM, CHIS, GRO, JAL, MICH, OAX, PUE, QROO, TAB, VER

\section{Family Portulacaceae}

Cistanthe ambigua (S. Watson) Carolin ex Hershkovitz BCN, SON

*Cistanthe guadalupensis (Dudley) Carolin BCN

Cistanthe maritima (Nutt.) Carolin BCN, BCS

Cistanthe monandra (Nutt.) Hershkovitz BCN, SON

Cistanthe parryi (A. Gray) Hershkovitz BCN, BCS, SON

Cistanthe umbellata (Torr.) Hershkovitz BCN

* Portulaca californica D. Legrand BCS

Portulaca conzattii P. Wilson CHIS, HGO, OAX

Portulaca grandiflora Hook. CAM, MOR, NAY, TAB, TAMS, VER

* Portulaca guanajuatensis G. Ocampo GTO, QRO, SLP

Portulaca halimoides L. AGS, BCN, BCS, CAM, CHIH, COAH, DGO, GTO, OAX, QROO, SLP, SON, YUC

* Portulaca johnstonii Henr. COAH, SON

*Portulaca matthewsii G. Ocampo QRO, SLP

Portulaca mexicana P. Wilson CDMX, GTO, HGO, JAL, MEX, MICH, MOR, OAX, PUE, QRO, TLAX, VER 
Portulaca oleracea L. AGS, BCN, BCS, CAM, CHIS, CHIH, COAH, COL, CDMX, DGO, GTO, GRO, HGO, JAL, MEX, MICH, MOR, NAY, NLE, OAX, PUE, QRO, QROO, SLP, SIN, SON, TAB, TAMS, TLAX, VER, YUC, ZAC

Portulaca pilosa L. AGS, BCN, BCS, CAM, CHIS, CHIH, COAH, COL, CDMX, DGO, GTO, GRO, HGO, JAL, MEX, MICH, MOR, NAY, NLE, OAX, PUE, QRO, QROO, SLP, SIN, SON, TAB, TAMS, VER, YUC, ZAC

Portulaca rubricaulis Kunth CAM, CHIS, QROO, YUC

* Portulaca rzedowskiana G. Ocampo MICH

Portulaca suffrutescens Engelm. BCN, BCS, CHIH, COAH, DGO, SON

Portulaca umbraticola Kunth BCN, BCS, CAM, CHIH, DGO, JAL, OAX, PUE, SLP, SIN, SON, TAMS, VER, ZAC

\section{Family Potamogetonaceae}

Potamogeton amplifolius Tuck. MICH

Potamogeton diversifolius Raf. AGS, CHIH, COAH, GTO, JAL, MICH, NAY, QRO, SLP, SON, ZAC

Potamogeton foliosus Raf. AGS, BCN, BCS, CHIH, COL, CDMX, DGO, GTO, GRO, HGO, JAL, MEX, MICH, MOR, OAX, PUE, QRO, SLP, SON, TAB, TAMS, VER, ZAC

Potamogeton illinoensis Morong BCN, BCS, CAM, CHIS, CHIH, COAH, CDMX, DGO, HGO, JAL, MEX, MICH, MOR, NAY, NLE, QRO, QROO, SLP, TAB, TAMS, VER, YUC

Potamogeton natans L. BCN, CDMX, MEX, MICH

Potamogeton nodosus Poir. AGS, BCN, CAM, CHIS, CHIH, COAH, COL, CDMX, DGO, GTO, HGO, JAL, MEX, MICH, NAY, NLE, OAX, PUE, QRO, QROO, SLP, SIN, SON, TAB, TAMS, TLAX, VER, ZAC

Potamogeton pusillus L. AGS, BCN, CHIS, CHIH, COL, CDMX, DGO, GTO, HGO, JAL, MEX, MICH, MOR, PUE, QRO, SLP, SON, TAMS, VER, ZAC

Stuckenia pectinata (L.) Börner BCN, BCS, CAM, COAH, COL, CDMX, GTO, GRO, HGO, JAL, MEX, MICH, MOR, NAY, NLE, OAX, PUE, QRO, SLP, SIN, SON, TAMS, TLAX, VER, ZAC

Stuckenia striata (Ruiz \& Pav.) Holub COAH, GTO, GRO, JAL, MICH, MOR, NAY, QRO, SON, TAMS, VER

Zannichellia palustris L. AGS, BCN, BCS, CHIH, COAH, CDMX, DGO, GTO, HGO, JAL, MEX, MICH, MOR, NLE, OAX, PUE, QRO, SLP, SIN, SON, TAMS, VER, ZAC

\section{Family Primulaceae}

Anagallis pumila Sw. CHIS, GRO, JAL, MEX, MOR, NAY, OAX, VER, ZAC

Androsace elongata $\mathrm{L} . \mathrm{BCN}$

Androsace septentrionalis L. COAH, NLE, SON

*Ardisia bastonalensis Ricketson \& Pipoly VER

Ardisia bracteosa A. DC. CAM, CHIS, COL, GRO, JAL, NAY, OAX, PUE, QROO, VER

*Ardisia breedlovei Lundell CHIS

Ardisia compressa Kunth CAM, CHIS, COL, DGO, GRO, HGO, JAL, MEX, MICH, MOR, NAY, OAX, PUE, QRO, QROO, SLP, SIN, TAB, TAMS, VER

Ardisia copeyana Standl. CHIS, OAX

Ardisia escallonioides Schltdl. \& Cham. CAM, CHIS, COL, GRO, HGO, JAL, MEX, MICH, OAX, PUE, QRO, QROO,
SLP, TAMS, TAB, TAMS, VER, YUC

*Ardisia fendleri Lundell JAL, NAY, OAX

Ardisia hintonii Lundell CHIS, DGO, JAL, MICH, OAX, SLP, VER

*Ardisia hyalina Lundell SLP, VER

*Ardisia liebmannii Oerst. CHIS, HGO, OAX, PUE, VER

*Ardisia mexicana Lundell CHIS, COL, JAL, NAY, OAX, VER Ardisia nigrescens Oerst. CHIS, GRO, HGO, JAL, MICH, OAX, PUE, QRO, SLP, TAB, TAMS, VER

Ardisia nigropunctata Oerst. CHIS, OAX, TAB

Ardisia paschalis Donn. Sm. CHIS, HGO, OAX, TAB, VER

Ardisia pellucida Oerst. CHIS, OAX, PUE, SLP, TAB, VER

Ardisia rarescens Standl. CHIS

Ardisia revoluta Kunth CAM, CHIS, CHIH, COL, DGO, GRO,

HGO, JAL, MEX, MICH, MOR, NAY, OAX, PUE, QRO, QROO, SLP, SIN, SON, TAB, VER, YUC

Ardisia schippii Standl. CHIS

Ardisia tuerckheimii Donn. Sm. CHIS, OAX, TAB, VER

Ardisia verapazensis Donn. Sm. CHIS, OAX, TAB, VER

*Ardisia verdisepala Ricketson \& Pipoly CHIS

Bonellia albiflora (Lundell) B. Ståhl \& Källersjö CAM, QROO, YUC

Bonellia flammea (Millsp. ex Mez) B. Ståhl \& Källersjö CAM, QROO, YUC

Bonellia longifolia (Standl.) B. Ståhl \& Källersjö CAM, QROO Bonellia macrocarpa (Cav.) B. Ståhl \& Källersjö BCN, BCS, CAM, CHIS, CHIH, COL, DGO, GRO, JAL, MEX, MICH, MOR, NAY, OAX, PUE, QRO, QROO, SLP, SIN, SON, TAB, TAMS, VER, YUC

Bonellia nervosa (C. Presl) B. Ståhl \& Källersjö CAM, CHIS, COL, GRO, JAL, MICH, OAX, QROO, VER, YUC

Bonellia paludicola (Standl.) B. Ståhl \& Källersjö CAM, QROO *Bonellia pringlei (Bartlett) B. Ståhl \& Källersjö GRO, MICH, OAX, PUE

*Bonellia seleriana (Urb. \& Loes. ex Mez) B. Ståhl \& Källersjö OAX, VER

*Ctenardisia purpusii (Brandegee) Lundell CHIS

Deherainia matudae Lundell CHIS, OAX

Deherainia smaragdina (Planch. ex Linden) Decne. CHIS, OAX, TAB, VER

Dodecatheon clevelandii Greene BCN

Dodecatheon pulchellum (Raf.) Merr. $\mathrm{CHIH}$

Gentlea austin-smithii (Lundell) Lundell CHIS, OAX

*Gentlea cuneifolia Lundell VER

Gentlea micranthera (Donn. Sm.) Lundell CHIS, COL, JAL, OAX, VER

*Gentlea penduliflora (A. DC.) Pipoly \& Ricketson CHIS, GTO, GRO, HGO, OAX, PUE, QRO, SLP, VER

Gentlea tacanensis (Lundell) Lundell CHIS

Gentlea vatterii (Standl. \& Steyerm.) Lundell CHIS

Gentlea venosissima (Ruiz \& Pav.) Lundell CHIS, OAX

Jacquinia arborea Vahl QROO

*Jacquinia morenoana Cast.-Campos \& Medina Abreo PUE, VER

* Lysimachia mexicana $\mathrm{R}$. Knuth HGO, OAX, QRO, VER

Lysimachia steyermarkii Standl. CHIS, GRO

Myrsine coriacea (Sw.) R. Br. ex Roem. \& Schult. CAM, CHIS, 
CHIH, COL, DGO, GRO, HGO, JAL, MEX, MICH, MOR, NAY, NLE, OAX, PUE, QRO, QROO, SLP, SIN, SON, TAMS, VER

Myrsine cubana A. DC. CAM, CHIS, QROO, TAB, VER

Myrsine guianensis (Aubl.) Kuntze CAM, CHIS, OAX, QROO, VER

Myrsine juergensenii (Mez) Ricketson \& Pipoly CAM, CHIS, COL, GRO, JAL, MEX, MICH, MOR, NAY, OAX, PUE, QROO, TAB, VER

Myrsine pellucidopunctata Oerst. CHIS

Parathesis aeruginosa Standl. CHIS, TAB

Parathesis belizensis Lundell CHIS

* Parathesis bracteolata Lundell CHIS, TAB

*Parathesis breedlovei Lundell CHIS, VER

Parathesis calophylla Donn. Sm. CHIS

*Parathesis calzadae Lundell OAX, VER

*Parathesis cintalapana Lundell CHIS

Parathesis columnaris Lundell CHIS, OAX, TAB

* Parathesis conzattii (S.F. Blake) Lundell OAX, VER

Parathesis cubana (A. DC.) Molinet \& M. Gómez CAM, CHIS, GRO, MICH, MOR, OAX, QROO, TAB, VER, YUC

*Parathesis cuspidata Lundell OAX

Parathesis chiapensis Fernald CHIS, OAX, VER

Parathesis donnell-smithii Mez CHIS, GRO, HGO, JAL, OAX, PUE, QRO, SLP, TAMS, VER

* Parathesis ferruginea Lundell COL, JAL, NAY

* Parathesis gracilis Lundell OAX

*Parathesis kochii Lundell OAX, VER

Parathesis lanceolata Brandegee CHIS

Parathesis latifolia Lundell CHIS

*Parathesis lenticellata Lundell VER

Parathesis leptopa Lundell CHIS, GRO, HGO, OAX, QRO, SLP, VER

*Parathesis macronema Bullock CHIS, GRO, JAL, MICH, OAX, PUE, VER

Parathesis melanosticta (Schltdl.) Hemsl. CHIS, DGO, GRO, HGO, JAL, MEX, MICH, OAX, PUE, SIN, VER

Parathesis microcalyx Donn. Sm. CHIS

*Parathesis minutiflora Lundell OAX

*Parathesis multiflora Lundell CHIS

* Parathesis navarretei Lundell VER

*Parathesis neei Lundell PUE, VER

* Parathesis oerstediana Mez OAX

* Parathesis pajapanensis Lundell VER

Parathesis papillosa Lundell CHIS

*Parathesis parvissima Lundell CHIS

*Parathesis perpunctata Lundell TAB, VER

Parathesis pleurobotryosa Donn. Sm. VER

* Parathesis prionophylla Standl. NAY

*Parathesis pseudocalophylla Ricketson \& Pipoly CHIS

* Parathesis psychotrioides Lundell CHIS, HGO, OAX, PUE, TAB, VER

Parathesis reflexa Brandegee CHIS

* Parathesis rekoi Standl. OAX

*Parathesis rubriflora Lundell CHIS

Parathesis rufa Lundell CHIS

*Parathesis schultesii Lundell OAX
Parathesis serrulata (Sw.) Mez CHIS, GRO, JAL, MICH, NAY, OAX, QRO, SLP, TAB, TAMS, VER

Parathesis sessilifolia Donn. Sm. CHIS, TAB, VER

* Parathesis subcoriacea Lundell CHIS, QRO, SLP

Parathesis subulata Lundell CHIS

Parathesis tartarea Lundell CHIS

*Parathesis tenuis Standl. OAX, VER

Parathesis trichogyne Hemsl. CHIS

*Parathesis tuxtlensis Lundell VER

* Parathesis villalobosii Lundell GRO, OAX, VER

*Parathesis villosa Lundell CHIS, COL, DGO, GRO, JAL, MEX, MICH, NAY, OAX, SIN, VER

Parathesis vulgata Lundell CHIS, GRO, OAX

* Parathesis wendtii Lundell OAX, VER

Primula rusbyi Greene CHIH, COAH, DGO, SON

*Samolus cinerascens (B.L. Rob.) Pax \& R. Knuth NLE, SLP, TAMS

*Samolus dichondrifolius Channell COAH

Samolus ebracteatus Kunth BCS, CAM, CHIS, CHIH, COAH, DGO, GTO, GRO, HGO, MOR, NLE, OAX, PUE, QRO, QROO, SLP, SON, TAB, TAMS, VER, YUC

Samolus floribundus Kunth BCN, CHIS, CHIH, COAH, DGO, GTO, GRO, HGO, JAL, NLE, OAX, PUE, QRO, SLP, SON, TAMS, VER, ZAC

Samolus vagans Greene BCS, CHIH, SON

Stylogyne guatemalensis S.F. Blake CHIS, OAX

Stylogyne turbacensis (Kunth) Mez CHIS, OAX, TAB, VER

Synardisia venosa (Mast.) Lundell AGS, CHIS, COL, GRO, HGO, JAL, MEX, MICH, OAX, VER

\section{Family Proteaceae}

*Roupala mexicana K.S. Edwards \& Prance OAX, TAB, VER Roupala montana Aubl. CHIS, GRO, HGO, OAX, QRO, SLP, TAB, VER

\section{Family Putranjivaceae}

Drypetes brownii Standl. CAM, CHIS, QROO, TAB, VER

*Drypetes gentryi Monach. CHIH, COL, GRO, JAL, MICH, NAY, SIN, SON

Drypetes lateriflora (Sw.) Krug \& Urb. CAM, CHIS, CHIH, COL, GTO, GRO, HGO, JAL, MEX, OAX, PUE, QRO, QROO, SLP, SON, TAB, TAMS, VER, YUC

\section{Family Ranunculaceae}

Aconitum columbianum Nutt. CHIH, SON

Anemone heterophylla Nutt. SLP, TAMS

Anemone mexicana Kunth CHIS, GTO, GRO, JAL, MEX, MICH, OAX, PUE, QRO, SLP, VER

Anemone tuberosa Rydb. BCN, COAH, NLE, SON

Aquilegia canadensis L. COAH, DGO, NLE

Aquilegia chrysantha A. Gray CHIH, COAH, SON

Aquilegia elegantula Greene COAH, NLE

Aquilegia formosa Fisch. ex DC. BCN, CHIH, SON

Aquilegia longissima A. Gray CHIH, COAH, NLE, SON

*Aquilegia skinneri Hook. F. CHIS, CHIH, COL, DGO, GRO, HGO, JAL, MEX, OAX, SIN, SON, VER, ZAC

Clematis caleoides Standl. \& Steyerm. CHIS

*Clematis coahuilensis D.J. Keil COAH, DGO, NLE, SLP 
Clematis dioica L. AGS, CAM, CHIS, COAH, COL, CDMX, DGO, GTO, GRO, HGO, JAL, MEX, MICH, MOR, NAY, NLE, OAX, PUE, QRO, QROO, SLP, SIN, TAB, TAMS, TLAX, VER, YUC, ZAC

Clematis drummondii Torr. \& A. Gray AGS, BCS, CHIH, COAH, DGO, JAL, NLE, OAX, PUE, QRO, SLP, SIN, SON, TAMS, ZAC

*Clematis grahamii Benth. HGO, MICH, NLE, PUE, QRO, SLP, TAMS, VER

Clematis grossa Benth. CAM, CHIS, COL, CDMX, GTO, GRO, HGO, JAL, MEX, MICH, MOR, NAY, OAX, PUE, QRO, QROO, SLP, TAMS, VER

Clematis haenkeana C. Presl CHIS, VER

Clematis lasiantha Nutt. BCN

Clematis ligusticifolia $\mathrm{Nutt}$. BCN, CHIH, SON

*Clematis malacocoma W.T. Wang OAX, PUE

Clematis pauciflora Nutt. BCN, BCS, CHIH, SON

Clematis pitcheri Torr. \& A. Gray CHIH, COAH, GTO, HGO, NLE, QRO, SLP, SIN, TAMS

Clematis polygama Jacq. CAM, CHIS, HGO, MOR, OAX, PUE, QROO, SLP, TAB, TAMS, VER, YUC

*Clematis rhodocarpa Rose CHIS, CHIH, COL, CDMX, GTO, GRO, HGO, JAL, MEX, MICH, MOR, OAX, PUE, QRO, QROO, SIN, TAMS, VER, ZAC

Delphinium andesicola Ewan CHIH, SON

*Delphinium bicornutum Hemsl. GRO, MEX, MOR, OAX, PUE

*Delphinium calcar-equitis Standl. CHIH

Delphinium cardinale Hook. BCN

Delphinium carolinianum Walt. CHIH, COAH, TAMS

*Delphinium divaricatum Ledeb. VER

*Delphinium leptophyllum Hemsl. CHIH, SLP

Delphinium madrense S. Watson COAH, NLE, SLP, TAMS

Delphinium parishii A. Gray BCN

Delphinium parryi A. Gray BCN

Delphinium patens Benth. BCN

*Delphinium pedatisectum Hemsl. AGS, DGO, GTO, HGO,

JAL, MEX, MICH, MOR, OAX, QRO, SLP, VER, ZAC

Delphinium scaposum Greene SON

Delphinium scopulorum A. Gray CHIH, SON

*Delphinium subscandens Ewan CHIH, COL, GTO, JAL, $\mathrm{MICH}$

Delphinium tenuisectum Greene CHIH, DGO

*Delphinium valens Standl. COAH, NLE

*Delphinium viride $\mathrm{S}$. Watson CHIH, DGO

*Delphinium wislizenii Engelm. CHIH, DGO

Delphinium wootonii Rydb. COAH, SON

Myosurus cupulatus S. Watson BCN, SON

Myosurus minimus L. BCN, SON

Paeonia californica Nutt. ex Torr. \& A. Gray BCN

Ranunculus alismifolius Benth. BCN

Ranunculus aquatilis L. BCN, CHIH, COAH, CDMX, DGO,

GRO, HGO, MEX, MOR, PUE, SON, TLAX, VER

Ranunculus arizonicus Lemmon CHIH, SON

Ranunculus californicus Benth. BCN

Ranunculus circinatus Sibth. CHIH

Ranunculus cymbalaria Pursh AGS, BCN, CHIH, COAH,
CDMX, DGO, GTO, GRO, HGO, MEX, MICH, MOR, PUE, QRO, SLP, TLAX, VER, ZAC

Ranunculus dichotomus Moc. \& Sessé ex DC. CDMX, DGO, GTO, GRO, HGO, JAL, MEX, MICH, MOR, OAX, PUE, TLAX, VER

Ranunculus donianus Pritzel CHIS, CDMX, HGO, JAL, MEX, MICH, MOR, PUE, TLAX, VER

Ranunculus fasciculatus Sessé \& Moc. CHIH, COAH, CDMX, DGO, HGO, JAL, MEX, MICH, MOR, NAY, NLE, OAX, PUE, QRO, SLP, SON, TAMS, TLAX, VER

Ranunculus flagelliformis Sm. CHIS, GTO, HGO, OAX, PUE, VER

*Ranunculus forreri Greene CHIH, DGO, SON

Ranunculus harveyi (A. Gray) Britton BCS

Ranunculus hebecarpus Hook. \& Arn. BCN

Ranunculus hydrocharoides A. Gray BCN, BCS, CHIS, CHIH, CDMX, DGO, GTO, HGO, JAL, MEX, MICH, MOR, QRO, SLP, SON, TLAX, VER, ZAC

Ranunculus macranthus Scheele AGS, CHIH, HGO, JAL, MEX, MICH, QRO, SLP, SON, TLAX, VER

*Ranunculus mexiae (L. Benson) T. Duncan GRO, JAL, NAY

Ranunculus multicaulis D. Don CDMX, MEX, PUE, VER

Ranunculus peruvianus Pers. CHIS, COAH, CDMX, HGO, MEX, MICH, NLE, OAX, TAMS, TLAX, VER

Ranunculus petiolaris Kunth ex DC. AGS, CHIS, CHIH, COAH, COL, CDMX, DGO, GTO, GRO, HGO, JAL, MEX, MICH, MOR, NLE, OAX, PUE, QRO, SLP, SIN, SON, TAMS, TLAX, VER, ZAC

Ranunculus praemorsus Kunth ex DC. AGS, CHIS, CDMX, GRO, HGO, JAL, MEX, MICH, MOR, NLE, OAX, PUE, VER *Ranunculus pueblensis W.B. Drew PUE

Ranunculus sierrae-orientalis (L. Benson) G.L. Nesom CHIS, COAH, CDMX, DGO, GTO, GRO, HGO, JAL, MEX, MICH, MOR, NLE, OAX, QRO, SLP, TAMS, VER, ZAC

*Thalictrum cuernavacanum Rose GRO, MEX, MOR

Thalictrum fendleri Engelm. ex A. Gray BCN, BCS, CHIH, COAH, DGO, JAL, NAY, SIN, SON, ZAC

*Thalictrum galeottii Lecoyer CHIS, COL, VER

*Thalictrum gibbosum Lecoy. AGS, CDMX, DGO, GTO, GRO, HGO, JAL, MEX, MICH, MOR, OAX, PUE, QRO, SLP, TAMS, TLAX, VER

*Thalictrum grandidentatum $\mathrm{S}$. Watson COAH, NLE

*Thalictrum grandifolium S. Watson CHIH, COAH, DGO, HGO, JAL, NLE, QRO, SLP, TAMS, VER, ZAC

Thalictrum guatemalense C. DC. \& Rose CHIS, GRO, JAL, MICH, TAMS

*Thalictrum hernandezii Tausch GRO, HGO, JAL, MEX, MICH, QRO, VER, ZAC

Thalictrum hintonii Boivin MEX, MICH

Thalictrum johnstonii Standl. \& Steyerm. CHIS

*Thalictrum lanatum Lecoy. OAX, PUE, VER

*Thalictrum madrense Rose DGO, NAY

*Thalictrum nelsonii B. Boivin OAX

*Thalictrum occidentalis A. Gray CHIH, SON

*Thalictrum pachucense Rose AGS, GRO, HGO, JAL, MEX, VER, ZAC

*Thalictrum parvifructum Boivin CHIH, DGO, SON 
*Thalictrum peltatum DC. COL, JAL, MEX, MICH, MOR, ZAC

*Thalictrum peninsulare Rose BCS

*Thalictrum pennellii Boivin DGO, SIN

*Thalictrum pinnatum $\mathrm{S}$. Watson CHIH, COAH, DGO, SON

Thalictrum polycarpum (Torr.) S. Watson $\mathrm{BCN}$

*Thalictrum pringlei S. Watson COL, JAL, MICH, NAY, ZAC

*Thalictrum pubigerum Benth. CDMX, DGO, GTO, GRO, HGO, JAL, MEX, MICH, OAX, PUE, QRO, TLAX, VER, ZAC

*Thalictrum pudicum Standl. \& B. Boivin JAL, MICH

Thalictrum standleyi Steyerm. CHIS

Thalictrum steyermarkii Standl. CHIS, VER

*Thalictrum strigillosum Hemsl. CHIS, COL, CDMX, DGO, GTO, HGO, JAL, MEX, MICH, MOR, NLE, OAX, PUE, QRO, SLP, TAMS, TLAX, VER

*Thalictrum treleasii B. Boivin GRO, OAX

\section{Family Resedaceae}

Oligomeris linifolia (Vahl) J.F. Macbr. BCN, BCS, CHIH, COAH, COL, DGO, JAL, NLE, SLP, SON, ZAC

\section{Family Rhamnaceae}

Adolphia californica S. Watson BCN

Adolphia infesta (Kunth) Meisn. AGS, BCS, CHIH, COAH, CDMX, DGO, GTO, HGO, JAL, MEX, OAX, PUE, QRO, SLP, SON, TLAX, VER, ZAC

*Ceanothus buxifolius Willd. ex Schult. f. CHIH, COAH, DGO, GTO, HGO, JAL, MEX, MICH, NLE, PUE, QRO, SLP, SON, TAMS, TLAX, VER, ZAC

Ceanothus caeruleus Lag. AGS, CHIS, CHIH, COAH, COL, CDMX, DGO, GTO, GRO, HGO, JAL, MEX, MICH, MOR, NAY, NLE, OAX, PUE, QRO, QROO, SLP, SIN, SON, TAMS, TLAX, VER, YUC, ZAC

Ceanothus cordulatus Kellogg BCN

Ceanothus crassifolius Torr. BCN

Ceanothus cuneatus (Hook.) Nutt. BCN

Ceanothus cyaneus Eastw. BCN

*Ceanothus depressus Benth. CHIH, COAH, DGO, GTO, JAL, MICH, NAY, PUE, SLP, SIN, SON, TAMS, VER, ZAC

Ceanothus divaricatus Nutt. BCN

Ceanothus fendleri A. Gray AGS, CHIH, COAH, DGO, NLE, SLP, SON, TAMS, ZAC

Ceanothus greggii A. Gray AGS, BCN, BCS, CHIH, COAH, DGO, GTO, HGO, MOR, NLE, OAX, PUE, QRO, SLP, SON, TAMS, VER, ZAC

Ceanothus herbaceus Raf. COAH

*Ceanothus lanuginosus (M.E. Jones) Rose AGS, CHIH, COAH, DGO, NLE, SLP, ZAC

Ceanothus leucodermis Greene BCN

*Ceanothus ochraceus Suess. CHIH, DGO, NAY, SIN, SON

Ceanothus oliganthus Nutt. BCN, BCS

*Ceanothus pauciflorus DC. AGS, COAH, DGO, NLE, OAX,

SLP, TAMS, VER, ZAC

Ceanothus spinosus Nutt. BCN

Ceanothus thyrsiflorus Eschsch. BCN

Ceanothus tomentosus Parry BCN

Ceanothus verrucosus Nutt. BCN
Ceanothus vestitus Greene BCN, GTO, JAL, PUE, SLP

*Colubrina angustior (M.C. Johnst.) G.L. Nesom SLP, TAMS, VER

Colubrina arborescens (Mill.) Sarg. CAM, CHIS, GRO, QROO, TAB, VER, YUC

Colubrina californica I.M. Johnst. BCN, BCS, SON

Colubrina celtidifolia (Schltdl. \& Cham.) Schltdl. CAM, GRO, HGO, JAL, MEX, MICH, MOR, OAX, QRO, VER

*Colubrina ehrenbergii Schltdl. COAH, GTO, HGO, JAL, NLE, OAX, PUE, QRO, SLP, TAMS, VER

Colubrina elliptica (Sw.) Brizicky \& W.L. Stern CAM, CHIS, COL, COAH, DGO, GTO, GRO, HGO, JAL, MEX, MICH, NAY, OAX, PUE, QRO, QROO, SLP, TAMS, VER, YUC, ZAC Colubrina greggii S. Watson BCN, CAM, CHIS, COAH, DGO, GTO, GRO, HGO, JAL, MEX, MOR, NLE, OAX, PUE, QRO, QROO, SLP, TAMS, VER, YUC, ZAC

Colubrina guatemalensis Standl. CHIS

Colubrina heteroneura (Griseb.) Standl. CAM, CHIS, COL, DGO, GTO, GRO, HGO, JAL, MICH, NAY, OAX, QRO, SLP, SIN, TAMS, VER, YUC

*Colubrina johnstonii T. Wendt OAX, VER

*Colubrina macrocarpa (Cav.) G. Don COAH, GRO, JAL, MEX, MICH, MOR, OAX, PUE, QRO, TAMS

*Colubrina sordida M.C. Johnst. GRO, MICH

Colubrina spinosa Donn. Sm. GRO, NAY

Colubrina stricta Engelm. CHIH, COAH, DGO, NLE, TAMS

Colubrina triflora Brongn. ex Sweet AGS, BCS, CHIS, CHIH, COL, DGO, GTO, GRO, JAL, MEX, MICH, MOR, NAY, OAX, PUE, QRO, SLP, SIN, SON, TAMS, VER, ZAC

*Colubrina viridis (M.E. Jones) M.C. Johnst. BCN, BCS, COAH, DGO, SIN, SON

Colubrina yucatanensis (M.C. Johnst.) G.L. Nesom CAM, QROO, YUC

*Condalia brandegeei I.M. Johnst. BCN, BCS

Condalia correllii M.C. Johnst. CHIH, COAH, SON

Condalia ericoides (A. Gray) M.C. Johnst. AGS, CHIH, COAH, DGO, HGO, NLE, QRO, SLP, ZAC

*Condalia fasciculata I.M. Johnst. CHIH, COAH, GTO, HGO, NLE, OAX, PUE, QRO, SLP, TAMS, VER, ZAC

Condalia globosa I.M. Johnst. BCN, BCS, SIN, SON

Condalia hookeri M.C. Johnst. COAH, NLE, SLP, TAMS, VER Condalia lycioides (A. Gray) Weberb. BCN, BCS, CHIH, COAH, DGO, NLE, SLP, SON, TAMS, ZAC

Condalia mexicana Schltdl. CHIH, COAH, CDMX, DGO, GTO, GRO, HGO, JAL, MEX, MICH, NLE, OAX, PUE, QRO, SLP, SIN, SON, TAMS, TLAX, VER, ZAC

*Condalia mirandana M.C. Johnst. SLP, TAMS, VER

Condalia parryi (Torr.) Weberb. BCN, BCS

*Condalia rigida Wiggins BCS

*Condalia sonorae Henr. SON

Condalia spathulata A. Gray BCN, CHIH, COAH, DGO, NLE, SLP, SIN, SON, TAMS, ZAC

*Condalia velutina I.M. Johnst. GTO, HGO, JAL, MEX, MICH, QRO, SLP, SON, VER, ZAC

Condalia viridis I.M. Johnst. AGS, CHIH, COAH, DGO, NLE, SLP

Condalia warnockii M.C. Johnst. AGS, CHIH, COAH, DGO, 
JAL, NLE, SLP, SON, TAMS, ZAC

Frangula betulifolia (Greene) Grubov CHIH, COAH, DGO, HGO, JAL, NAY, NLE, QRO, SLP, SIN, SON, TAMS, VER

Frangula breedlovei (L.A. Johnst. \& M.C. Johnst.) A. Pool CHIS

Frangula californica (Eschsch.) A. Gray BCN, CHIH, SON

Frangula capreifolia (Schltdl.) Grubov CHIS, COL, GRO, HGO, JAL, MICH, NAY, OAX, PUE, QRO, SLP, TAMS, VER

*Frangula chimalapensis (R. Fernández) A. Pool OAX

* Frangula dianthes (L. Riley) Grubov DGO

Frangula discolor (Donn. Sm.) Grubov CHIS, COL, JAL, NAY, OAX

Frangula grandiflora A. Pool CHIS

*Frangula hintonii (M.C. Johnst. \& L.A. Johnst.) A. Pool COL, GRO, JAL, MEX, MICH, MOR, OAX, ZAC

* Frangula inconspicua A. Pool OAX

* Frangula longistyla (C.B. Wolf) A. Pool HGO, MEX, OAX, PUE, QRO, SLP, VER

*Frangula mcvaughii (L.A. Johnst. \& M.C. Johnst.) A. Pool CHIS, GRO, HGO, OAX, PUE, VER

* Frangula microphylla (Humb. \& Bonpl. ex Schult.) Grubov AGS, CHIH, COAH, DGO, GTO, HGO, JAL, MEX, MICH, NAY, NLE, OAX, PUE, QRO, SLP, SIN, TAMS, TLAX, VER, ZAC

Frangula mucronata (Schltdl.) Grubov CHIS, CHIH, COAH, COL, CDMX, DGO, GRO, HGO, JAL, MEX, MICH, MOR, NAY, OAX, PUE, QRO, SLP, SIN, SON, TLAX, VER, ZAC

*Frangula palmeri (S. Watson) Grubov JAL, MEX, MICH, NAY

* Frangula pinetorum (Standl.) Grubov CHIH, SON

Frangula pringlei (Rose) Grubov CHIS, GRO, OAX, VER

*Frangula scopulorum (M.E. Jones) A. Pool CHIH, DGO, JAL, SIN, SON

*Frangula surotatensis (Gentry) A. Pool SIN

*Frangula wendtii (Ishiki) A. Pool OAX

*Gouania conzattii Greenm. COL, GRO, JAL, MEX, MICH, NAY, OAX, PUE

Gouania eurycarpa Standl. CAM, CHIS, QROO, YUC

*Gouania guiengolensis A. Pool OAX

Gouania lupuloides (L.) Urb. CAM, CHIS, CHIH, COL, DGO,

GRO, HGO, JAL, MEX, MICH, MOR, NAY, OAX, PUE, QRO,

QROO, SLP, SIN, TAB, TAMS, VER, YUC, ZAC

Gouania obamana A. Pool OAX, PUE, TAB, VER

Gouania polygama (Jacq.) Urb. CAM, CHIS, COL, GRO, HGO, JAL, OAX, PUE, QRO, QROO, SLP, TAB, VER, YUC

Gouania pubidisca A. Pool CHIS, GRO, JAL, NAY, OAX

*Gouania rosei Wiggins BCS, CHIH, COL, DGO, GRO, JAL, MICH, NAY, OAX, SIN, SON

*Gouania stipularis DC. CHIS, COL, GRO, MEX, JAL, MICH, NAY, OAX

Gouania velutina Reissek OAX

Karwinskia calderonii Standl. CAM, CHIS, GRO, OAX, QROO, SIN, YUC

Karwinskia humboldtiana (Schult.) Zucc. AGS, BCN, BCS, CAM, CHIS, CHIH, COAH, COL, DGO, GTO, GRO, HGO, JAL, MEX, MICH, MOR, NAY, NLE, OAX, PUE, QRO, QROO, SLP, SIN, SON, TAMS, VER, YUC, ZAC
*Karwinskia johnstonii R. Fernández GRO, JAL, MEX, MICH, MOR

*Karwinskia latifolia Standl. AGS, COL, DGO, JAL, MICH, NAY, SIN, ZAC

*Karwinskia mollis Schltdl. GTO, GRO, HGO, JAL, MEX, MICH, NLE, OAX, PUE, QRO, SLP, TAMS, VER, ZAC

*Karwinskia rzedowskii R. Fernández DGO, JAL, NAY, ZAC

*Karwinskia subcordata Schltdl. HGO, QRO

*Karwinskia tehuacana R. Fernández \& N. Waksman MICH, PUE

* Karwinskia umbellata (Cav.) Schldtl. GRO, MOR, OAX, PUE

*Karwinskia venturae R. Fernández GRO, MEX, PUE

Krugiodendron ferreum (Vahl) Urb. CAM, CHIS, GTO, GRO, HGO, MEX, OAX, PUE, QRO, QROO, SLP, TAMS, VER, YUC

*Rhamnus biglandulosa Sessé \& Moc. GRO

* Rhamnus calderoniae R. Fernández OAX

Rhamnus caroliniana Walter AGS, COAH, NLE, TAMS

Rhamnus crocea Nutt. BCN, BCS, CHIH, SON

Rhamnus ilicifolia Kellogg BCN, CHIH, SON

*Rhamnus macrocarpa Standl. JAL, MICH, SLP

Rhamnus pirifolia Greene BCN

* Rhamnus rosei M.C. Johnst. \& L.A. Johnst. CHIH, DGO, ZAC

Rhamnus serrata Humb. \& Bonpl. ex Willd. CHIS, CHIH, COAH, CDMX, DGO, GTO, HGO, MEX, MOR, NLE, OAX, PUE, QRO, SLP, SON, TAMS, TLAX, VER

Rhamnus sharpii M.C. Johnston \& L.A. Johnston CHIS, COL, JAL, NAY, OAX

*Rhamnus standleyana C.B. Wolf COAH, NLE

Sageretia elegans (Kunth) Brongn. CAM, CHIS, GRO, JAL, MEX, MICH, NAY, OAX, PUE, QRO, QROO, SIN, TAB, VER, ZAC

*Sageretia mexicana G.L. Nesom JAL, MEX, MICH

Sageretia wrightii S. Watson BCS, CHIH, COAH, DGO, JAL, SON

Ziziphus acuminata Benth. GRO, MOR, OAX

*Ziziphus amole (Sessé \& Moc.) M.C. Johnst. BCS, CHIS, CHIH, COAH, COL, DGO, GTO, GRO, HGO, JAL, MEX, MICH, MOR, NAY, NLE, OAX, PUE, QRO, SLP, SIN, SON, TAMS, VER, ZAC

Ziziphus guatemalensis Hemsl. CHIS, OAX

*Ziziphus lloydii (Standl.) M.C. Johnst. COAH, NLE, SLP, ZAC

*Ziziphus mexicana Rose CHIS, COL, GRO, JAL, MICH, OAX, PUE, QROO, SIN, VER, ZAC

Ziziphus obtusifolia (Torr. \& A. Gray) A. Gray BCN, BCS, CHIH, COAH, DGO, NLE, SLP, SIN, SON, TAMS, VER, ZAC

*Ziziphus pedunculata (Brandegee) Standl. GRO, OAX, PUE

*Ziziphus yucatanensis Standl. CAM, QROO, YUC

\section{Family Rhizophoraceae}

Cassipourea guianensis Aubl. CAM, CHIS, OAX, QROO, TAB, VER

Rhizophora harrisonii Leechm. CHIS, TAMS, VER

Rhizophora mangle L. BCN, BCS, CAM, CHIS, COL, GRO, JAL, MICH, NAY, OAX, PUE, QROO, SIN, SON, TAB, TAMS, 
VER, YUC

\section{Family Rosaceae}

Acaena elongata L. CHIS, COL, CDMX, GRO, HGO, JAL, MEX, MICH, MOR, OAX, PUE, TLAX, TAMS, VER

Adenostoma fasciculatum Hook. \& Arn. BCN, BCS

Adenostoma sparsifolium Torr. BCN

Agrimonia gryposepala Wallr. CHIS, CHIH, COAH, HGO, NLE, TAMS, VER

*Agrimonia pringlei Rydb. HGO, PUE, QRO, SLP, TAMS, VER

Amelanchier utahensis Koehne BCN, CHIH

Aphanes occidentalis (Nutt.) Rydb. BCN

Cercocarpus betuloides Nutt. BCN, CHIH, COAH, SON

Cercocarpus breviflorus A. Gray CHIH, COAH, SON

Cercocarpus eximius (C. Schneid.) Rydb. CHIH, COAH, SON

*Cercocarpus fothergilloides Kunth COAH, GRO, HGO, MEX,

NLE, OAX, PUE, QRO, SLP, TAMS, VER, ZAC

Cercocarpus ledifolius Nutt. BCN

*Cercocarpus macrophyllus C.K. Schneid. AGS, COAH, COL, CDMX, DGO, GTO, GRO, HGO, JAL, MEX, MICH, NAY, NLE, OAX, PUE, QRO, SLP, SIN, TAMS, VER, ZAC

*Cercocarpus mexicanus Henr. NLE, TAMS

*Cercocarpus mojadensis C.K. Schneid. CHIH, COAH, DGO, GRO, NLE, OAX, QRO, SLP, TAMS, ZAC

Cercocarpus montanus Raf. AGS, BCN, CHIH, COAH, HGO, NLE, QRO, SLP, SON, TAMS, ZAC

*Cercocarpus pringlei (C.K. Schneid.) Rydb. GRO, HGO, OAX, PUE, QRO, VER

Cercocarpus rotundifolius $\mathrm{Rydb}$. BCN

*Cercocarpus rzedowskii Henr. NLE, SLP, TAMS

*Crataegus aurescens J.B. Phipps COAH, NLE

*Crataegus baroussana Eggl. COAH, NLE, TAMS

Crataegus crus-galli L. COAH, NLE

*Crataegus cuprina J.B. Phipps COAH, NLE

*Crataegus gracilior J.B. Phipps CHIS, COL, CDMX, GTO, GRO, HGO, JAL, MEX, MICH, NLE, OAX, PUE, QRO, SLP, TAMS, VER

*Crataegus grandifolia J.B. Phipps COAH, NLE

Crataegus greggiana Eggl. COAH, NLE, TAMS

*Crataegus johnstonii J.B. Phipps COAH

Crataegus mexicana DC. AGS, CHIS, COAH, COL, CDMX, DGO, GTO, GRO, HGO, JAL, MEX, MICH, MOR, NLE, OAX, PUE, QRO, SLP, SIN, TAMS, TLAX, VER, ZAC

*Crataegus nelsoni Eggl. CHIS

*Crataegus rosei Eggl. CHIH, COAH, DGO, GTO, HGO, JAL, NLE, QRO, SLP, SIN, TAMS, VER

*Crataegus sulfurea J.B. Phipps COAH, NLE

Crataegus tracyi Ashe ex Eggl. COAH, NLE, SLP

Crataegus uniflora Moench COAH, TAMS

Chamaebatia australis (Brandegee) Abrams BCN

Fallugia paradoxa (D. Don) Endl. ex Torr. CHIH, COAH, DGO, SON, ZAC

Fragaria californica Cham. \& Schltdl. BCN, BCS, COAH, NLE, TAMS

* Fragaria mexicana Schltdl. AGS, BCS, CHIH, COL, CDMX, DGO, HGO, JAL, MEX, MICH, MOR, NAY, NLE, OAX, PUE,
QRO, SLP, SIN, TAMS, TLAX, VER, ZAC

Fragaria virginiana Duchesne COAH, NLE, SLP, SON

Geum aleppicum Jacq. CHIH, COAH, CDMX, HGO, MEX, NLE, PUE

Geum canadense Jacq. CHIS, CDMX, MEX

*Geum mexicanum Rydb. MEX, MOR

Heteromeles arbutifolia (Aiton) M. Roem. BCN, BCS

Holodiscus argenteus (L. f.) Maxim. CHIS, OAX

Holodiscus discolor (Pursh) Maxim. CHIH, COAH, DGO, JAL, MEX, MOR, NLE, SLP, SON

Holodiscus fissus (Lindl.) C.K. Schneid. CHIS, COAH, COL, DGO, GTO, GRO, JAL, MEX, MICH, MOR, NLE, OAX, QRO, SLP, SIN, TAMS, VER

Holodiscus microphyllus Rydb. BCN

*Holodiscus orizabae F.A. Ley VER

*Holodiscus pachydiscus (Rydb.) Standl. CDMX, GTO, GRO,

HGO, JAL, MEX, MICH, PUE, QRO

Horkelia bolanderi A. Gray BCN

Horkelia cuneata Lindl. BCN

Horkelia truncata Rydb. BCN

*Ivesia argyrocoma $\mathrm{Rydb}$. BCN

Lachemilla aphanoides (Mutis ex L. f.) Rothm. BCS, CHIS, CHIH, COL, CDMX, DGO, GTO, GRO, HGO, JAL, MEX, MICH, NLE, OAX, QRO, SLP, SIN, SON, TAMS, TLAX, VER, ZAC

Lachemilla orbiculata (Ruiz \& Pav.) Rydb. CHIS, CDMX, GRO, HGO, JAL, MEX, MICH, MOR, OAX, PUE, QRO, SLP, TLAX, VER

*Lachemilla orizabensis Rydb. VER

Lachemilla pinnata (Ruiz \& Pav.) Rothm. CHIS, GRO, MEX, MICH, PUE, VER

Lachemilla procumbens (Rose) Rydb. CHIS, CHIH, COAH, COL, CDMX, DGO, GTO, GRO, HGO, JAL, MEX, MICH, MOR, NLE, OAX, PUE, QRO, SLP, SIN, TAMS, TLAX, VER, ZAC

Lachemilla sibbaldiifolia (Kunth) Rydb. CHIS, CHIH, COL, CDMX, DGO, GTO, GRO, HGO, JAL, MEX, MICH, MOR, NLE, OAX, PUE, SLP, TLAX, VER, ZAC

*Lachemilla velutina (S. Watson) Rydb. AGS, CHIH, CDMX, GTO, GRO, HGO, MEX, PUE, QRO, SLP, TLAX, VER, ZAC Lachemilla venusta (Schltdl. \& Cham.) Rydb. CHIS, GRO, OAX, PUE, VER

Lachemilla vulcanica (Schltdl. \& Cham.) Rydb. CHIS, COAH, CDMX, HGO, JAL, MEX, MICH, NLE, OAX, PUE, TLAX, VER

*Lindleya mespiloides Kunth AGS, CHIH, COAH, DGO, GTO, HGO, NLE, OAX, PUE, QRO, SLP, TAMS, VER, ZAC

Malacomeles denticulata (Kunth) G.N. Jones AGS, CHIS, CHIH, COAH, CDMX, DGO, GTO, HGO, JAL, MEX, NLE, OAX, PUE, QRO, SLP, TAMS, TLAX, VER, ZAC

Malacomeles nervosa (Decne.) G.N. Jones CHIS, MEX, NLE, OAX, SLP, TAMS, VER

*Malacomeles paniculata (Rehder) J.B. Phipps COAH, NLE, SLP, TAMS, VER

*Malacomeles pringlei (Koehne) B.L. Turner CHIH, COAH, NLE

*Malacomeles psilantha (C.K. Schneid.) B.L. Turner COAH, 
DGO, GTO, NLE, OAX, PUE, QRO, SLP, VER

Petrophytum caespitosum (Nutt.) Rydb. CHIH, COAH, NLE

*Photinia guerreris J.B. Phipps GRO

Photinia matudae Lundell CHIS, VER

* Photinia mexicana (Baill.) Hemsl. CHIS, JAL, MICH, OAX, QRO, SLP, VER

Photinia microcarpa Standl. CHIS, GRO, JAL, MEX, MICH, OAX, QRO, SLP, VER

*Photinia oblongifolia Standl. JAL, MICH, NAY

* Photinia parviflora L.O. Williams COL, JAL

Physocarpus opulifolius (L.) Maxim. COAH, NLE, SON

Potentilla anserina L. CHIH, DGO, SON

Potentilla biennis Greene BCN

*Potentilla candicans Humb. \& Bonpl. ex Nestl. CDMX, HGO, MEX, MICH, MOR, PUE, TLAX, VER

* Potentilla durangensis Rydb. DGO, SIN

*Potentilla ehrenbergiana Schltdl. HGO, OAX, VER

Potentilla glandulosa Lindl. BCN

Potentilla goldmanii Painter ex Rydb. CHIS, OAX, VER

Potentilla haematochrous Lehm. CDMX, GTO, HGO, JAL, MEX, MICH, MOR, TLAX, VER

Potentilla heterosepala Fritsch CHIS, GRO, PUE, VER

Potentilla hippiana Lehm. COAH, NLE, SON

* Potentilla horrida Rydb. CHIH, SON

* Potentilla leonina Standl. COAH, NLE

*Potentilla leptopetala Lehm. CHIH, DGO

* Potentilla macdonaldii B.L. Turner OAX

* Potentilla mexiae Standl. CHIH, DGO, NLE, SIN

* Potentilla oaxacana Rydb. OAX

Potentilla oblanceolata Rydb. CHIH

Potentilla paradoxa Nutt. DGO, ZAC

*Potentilla queretarensis Rzed. \& Calderón QRO

* Potentilla ranunculoides Kunth. AGS, COAH, CDMX, GRO, HGO, MEX, MICH, MOR, NLE, OAX, PUE, TLAX, VER, ZAC

*Potentilla richardii Lehm. JAL, MEX, PUE, TLAX, VER

Potentilla rivalis Nutt. CDMX, HGO, MEX, MICH, SON

*Potentilla rubra Willd. CDMX, DGO, HGO, MEX, MICH, MOR, VER

Potentilla saxosa Lemmon ex Greene BCN

Potentilla staminea Rydb. CHIS, CDMX, DGO, HGO, JAL, MEX, MOR, NAY, OAX, PUE, TLAX, VER

Potentilla subviscosa Greene SON

Potentilla thurberi A. Gray CHIH, COAH, DGO, SON

*Potentilla townsendii Rydb. CHIH, SON

Potentilla wheeleri S. Watson BCN, SON

Prunus annularis Koehne CHIS, HGO, VER

Prunus barbata Koehne CHIS, GRO, MICH

Prunus brachybotrya Zucc. CHIS, COL, CDMX, GRO, HGO, JAL, MEX, MICH, MOR, NLE, OAX, PUE, QRO, SLP, SIN, TAMS, VER, ZAC

*Prunus cercocarpifolia Villarreal COAH

Prunus cortapico Kerber ex Koehne CHIS, COL, GRO, JAL, MEX, MICH, MOR, OAX, PUE, SIN, VER, ZAC

Prunus chiapensis Standl. \& L.O. Williams ex Ant. Molina CHIS

*Prunus erythroxylon Koehne COL, DGO, GRO, MICH, NAY,
VER

Prunus fasciculata (Torr.) A. Gray BCN

*Prunus ferruginea (Ser.) Steud. DGO, GRO, JAL, MEX,

MICH, MOR, NAY, SIN, ZAC

Prunus fremontii S. Watson BCN, BCS

* Prunus gentryi Standl. CHIH, HGO, SIN, SON, VER

Prunus guatemalensis I.M. Johnst. CHIS, OAX

Prunus ilicifolia (Nutt.) Walp. BCN, BCS

Prunus lundelliana Standl. CHIS, GRO, OAX, VER

Prunus lyonii (Eastw.) Sarg. BCN, BCS

*Prunus matudae Lundell CHIS, HGO, OAX, PUE, VER

Prunus mexicana S. Watson COAH, NLE, SLP, TAMS

* Prunus microphylla (Kunth) Hemsl. CDMX, DGO, GTO,

HGO, JAL, MEX, NLE, OAX, PUE, QRO, SLP, TLAX, VER,

$\mathrm{ZAC}$

Prunus minutiflora Engelm. AGS, CHIH, COAH

Prunus myrtifolia (L.) Urb. CAM, QROO

Prunus occidentalis Sw. CHIS

*Prunus ochoterenae Ramírez-Cantú MOR

Prunus rhamnoides Koehne CHIS, CHIH, DGO, GTO, GRO, HGO, JAL, MEX, MICH, OAX, SLP, SON, TAMS, TLAX,

VER

Prunus salasii Standl. CHIS

*Prunus samydoides Schltdl. HGO, PUE, QRO, SLP, VER

Prunus serotina Ehrh. AGS, BCS, CHIS, CHIH, COAH, COL, CDMX, DGO, GTO, GRO, HGO, JAL, MEX, MICH, MOR, NAY, NLE, OAX, PUE, QRO, SLP, SIN, SON, TAMS, TLAX, VER, ZAC

Prunus skutchii I.M. Johnst. CHIS, VER

* Prunus tartarea Lundell CHIS, OAX

*Prunus tetradenia Koehne CHIS, COL, HGO, JAL, MEX, MICH, NAY, OAX, PUE, QRO, SLP, TAMS, VER

* Prunus tuberculata Koehne CHIS, OAX

*Prunus zinggii Standl. CHIH, COL, GRO, JAL, MICH, MOR, OAX, SON

Purshia ericifolia (Torr. ex A. Gray) Henr. CHIH, COAH, NLE, TAMS

Purshia mexicana (D. Don) Henr. AGS, CHIH, COAH, DGO, GTO, JAL, NLE, QRO, SLP, SON, ZAC

*Purshia plicata (D. Don) Henr. AGS, COAH, DGO, GTO, JAL, NLE, SLP, TAMS, ZAC

Purshia stansburiana (Torr.) Henr. CHIH, SON

Rosa californica Cham. \& Schltdl. BCN

Rosa carolina L. COAH, NLE, TAMS

Rosa minutifolia Parry BCN

* Rosa moschata Benth. MICH, VER

*Rosa noisettiana Rose OAX, VER

Rosa serrulata Raf. COAH, NLE, TAMS

Rosa woodsii Lindl. CHIH, COAH, SON

Rubus aboriginum Rydb. NLE, SLP, TAMS

Rubus adenotrichos Schltdl. CHIS, COL, CDMX, GRO, HGO, JAL, MEX, MICH, MOR, OAX, PUE, QRO, SLP, TAMS, VER

*Rubus alnifolius Rydb. VER

Rubus arizonensis Focke CHIH, SON

*Rubus caudatisepalus Calderón CDMX, JAL, MEX, MICH, MOR, NLE, TLAX

Rubus coriifolius Liebm. CHIS, COL, DGO, GRO, HGO, JAL, 
MEX, MICH, MOR, OAX, PUE, SIN, TAMS, VER

*Rubus cymosus Rydb. CDMX, GRO, JAL, MEX, MICH

Rubus eriocarpus Liebm. CHIS, COL, HGO, JAL, MOR, OAX, PUE, SLP, VER

Rubus fagifolius Schltdl. \& Cham. CHIS, HGO, OAX, VER

Rubus flagellaris Willd. COAH, NLE, TAMS

Rubus glaucus Benth. CHIS, COL, JAL, MOR, OAX

Rubus hadrocarpus Standl. \& Steyerm. CHIS

*Rubus humistratus Steud. CHIS, CHIH, COAH, COL, GTO, GRO, HGO, JAL, NLE, OAX, PUE, QRO, SLP, VER

Rubus idaeus L. COAH, MEX, MICH, NLE, TAMS

Rubus irasuensis Liebm. CHIS, MEX, MICH, MOR, OAX, VER

Rubus leucodermis Douglas ex Torr. \& A. Gray CHIH

*Rubus liebmannii Focke COL, CDMX, GRO, HGO, JAL,

MEX, MICH, MOR, OAX, QRO, SLP, TLAX, VER

*Rubus macvaughianus Rzed. \& Calderón GTO, NLE, QRO

Rubus miser Liebm. CHIS, OAX

*Rubus nelsonii Rydb. CHIS, GRO, OAX

Rubus neomexicanus A. Gray CHIH, SON

Rubus oligospermus Thornber BCS, CDMX, DGO, JAL, MEX,

SLP, SON

*Rubus palmeri Rydb. CHIH, COL, DGO, JAL, NAY, SIN, SON, VER

Rubus parviflorus Nutt. CHIH, SON

*Rubus philyrophyllus Rydb. HGO, OAX, PUE, QRO, VER

Rubus pringlei Rydb. CHIS, COL, CDMX, DGO, GTO, GRO,

HGO, JAL, MEX, MICH, MOR, NAY, OAX, PUE, QRO, SLP,

TAMS, TLAX, VER

*Rubus pseudofagifolius Huan C. Wang OAX, VER

* Rubus pumilus Focke CHIH, COL, CDMX, DGO, HGO, JAL,

MEX, MICH, MOR, SIN, TLAX

Rubus sapidus Schltdl. CHIS, HGO, MICH, OAX, PUE, QRO,

TAMS, VER

*Rubus schiedeanus Steud. GRO, HGO, JAL, MEX, MICH, OAX, PUE, TLAX, VER

*Rubus sierrae Laferr. CHIH

Rubus strigosus Michx. CHIH, COAH, NLE, SON, VER

Rubus trilobus Moc. \& Sessé ex Ser. CHIS, OAX, PUE, VER

Rubus trivialis Michx. COAH, NLE, SLP, TAMS, VER

* Rubus uhdeanus Focke MEX, MOR

Rubus ursinus Cham. \& Schltdl. BCN

Rubus urticifolius Poir. CHIS, GRO, MOR, OAX, PUE, VER

*Rubus verae-crucis Rydb. HGO, OAX, PUE, VER

Rubus vulcanicola (Donn. Sm.) Rydb. HGO, VER

Sericotheca dumosa (Nutt.) Rydb. BCN, CHIH, DGO, NLE, QRO, SLP, SON, ZAC

Sericotheca fissa (Lindl.) Rydb. MICH, OAX, VER

Sericotheca schaffneri Rydb. SLP, ZAC

Sericotheca velutina Rydb. OAX

Sibbaldia procumbens L. MEX, PUE, VER

*Vauquelinia australis Standl. OAX, PUE

Vauquelinia californica (Torr.) Sarg. BCN, BCS, CHIH, COAH, DGO, SON

Vauquelinia corymbosa Bonpl. CHIH, COAH, DGO, GTO, HGO, NLE, QRO, SLP, TAMS, VER, ZAC

Vauquelinia pauciflora Standl. DGO, SON
*Xerospiraea hartwegiana (Rydb.) Henr. COAH, DGO, HGO, NLE, OAX, PUE, QRO, SLP, VER

\section{Family Rubiaceae}

Alibertia edulis (Rich.) A. Rich. ex DC. CAM, CHIS, OAX, PUE, QROO, SIN, TAB, VER, YUC

Alseis hondurensis Standl. CHIS, OAX, VER

Alseis yucatanensis Standl. CAM, CHIS, QROO, TAB, YUC

Amaioua corymbosa Kunth CHIS, OAX, TAB, VER

Appunia guatemalensis Donn. Sm. CHIS, QROO, TAB, VER

*Arachnothryx angustispicata Borhidi, E. Martínez \& Ramos VER

*Arachnothryx atravesadensis (Lorence) Bohr. OAX

Arachnothryx bertieroides (Standl.) Bohr. CHIS

*Arachnothryx bourgaei (Standl.) Bohr. OAX, VER

*Arachnothryx brachythyrsa Bohr. OAX

*Arachnothryx breviflora Bohr. CHIS

Arachnothryx buddleioides (Benth.) Planch. CHIS, COL, GRO, JAL, MEX, MICH, NAY, OAX, TAB, VER

Arachnothryx capitellata (Hemsl.) Bohr. CHIS, GRO, HGO, JAL, OAX, PUE, VER

Arachnothryx chiapensis (Brandegee) Bohr. CHIS

*Arachnothryx chimalaparum Lorence ex Bohr. OAX

Arachnothryx chinajensis (Standl. \& Steyerm.) Bohr. CHIS

Arachnothryx flocculosa Bohr. CHIS

*Arachnothryx ginetteae (Lorence) Bohr. OAX

*Arachnothryx gonzaleoides (Standl.) Bohr. CHIS, VER

Arachnothryx gracilis (Hemsl.) Bohr. VER

*Arachnothryx gracilispica (Standl.) Bohr. HGO, OAX, PUE, VER

*Arachnothryx guerrerensis (Lorence) Bohr. GRO

*Arachnothryx heteranthera (Brandegee) Bohr. HGO, OAX, PUE, QRO, SLP, VER

*Arachnothryx hirtinervis Bohr. OAX

*Arachnothryx jaliscensis Bohr. \& E. Martínez JAL

Arachnothryx jurgensenii (Hemsl.) Bohr. CHIS, COL, GTO, GRO, JAL, MEX, MICH, NAY, OAX, SIN

Arachnothryx laniflora (Benth.) Planch. CHIS, GRO, OAX, VER

*Arachnothryx latiloba Bohr. GRO

*Arachnothryx leucophylla (Kunth) Planch. CAM, CHIS, COL, DGO, GRO, JAL, MEX, MICH, MOR, NAY, OAX, PUE, QRO, SLP, SIN, VER, YUC, ZAC

*Arachnothryx lineolata Bohr. CHIS

Arachnothryx linguiformis (Hemsl.) Bohr. CHIS

Arachnothryx macrocalyx (Standl. \& Steyerm.) Bohr. CHIS, OAX

*Arachnothryx manantlanensis (Lorence) Bohr. COL, JAL

*Arachnothryx mexicana (Turcz.) Bohr. OAX

*Arachnothryx michoacana Bohr. MICH

*Arachnothryx monticola Bohr. GRO

Arachnothryx myriantha (Standl. \& Steyerm.) Bohr. CHIS

*Arachnothryx nitida (Hemsl.) Bohr. CHIS, OAX

Arachnothryx ovandensis (Lundell) Bohr. CHIS, OAX

*Arachnothryx pauciflora Bohr. CHIS

*Arachnothryx pumae Torres-Montúfar \& H. Ochoterena VER

*Arachnothryx purpurea (Lorence) Bohr. CHIS, OAX 
*Arachnothryx pyramidalis (Lundell) Bohr. CHIS, GRO, OAX

*Arachnothryx rekoi (Standl.) Bohr. OAX

*Arachnothryx ricoi (Lorence) Bohr. OAX

Arachnothryx rufescens (B.L. Rob.) Bohr. CHIS, OAX

*Arachnothryx rzedowskii (Lorence) Bohr. GRO

*Arachnothryx sanchezii Bohr. \& Salas-Morales OAX

*Arachnothryx scabra (Hemsl.) Bohr. CHIS, GRO, OAX

*Arachnothryx scoti (Lorence) Bohr. OAX

Arachnothryx secundiflora (B.L. Rob.) Bohr. CHIS, OAX, TAB, VER

Arachnothryx septicidalis (B.L. Rob.) Bohr. CHIS, OAX, VER

*Arachnothryx sessilis Bohr. \& G. Ortiz TAB

*Arachnothryx sinaloae Bohr. SIN

Arachnothryx skutchii (Standl. \& Steyerm.) Bohr. CHIS

*Arachnothryx sousae Bohr. CHIS

Arachnothryx stachyoidea (Donn. Sm.) Bohr. CHIS, OAX, TAB, VER

*Arachnothryx subglabra Bohr. \& N.C. Jiménez TAB

Arachnothryx tacanensis (Lundell) Bohr. CHIS

*Arachnothryx tenorioi (Lorence) Bohr. GRO, OAX

*Arachnothryx tenuisepala Bohr. VER

*Arachnothryx tuxtlensis (Lorence \& Cast. Campos) Bohr. VER

*Arachnothryx uxpanapensis (Lorence \& Cast.-Campos) Bohr. OAX, VER

*Arachnothryx villosa (Hemsl.) Bohr. CHIS, OAX, TAB, VER

*Arachnothryx wendtii (Lorence \& Cast.-Campos) Bohr. OAX, VER

Arcytophyllum fasciculatum (A. Gray) Terrell \& H. Rob. CHIH, $\mathrm{COAH}$

Arcytophyllum serpyllaceum (Schltdl.) Terrell CHIS, OAX, VER

Asemnantha pubescens Hook. f. CAM, QROO, YUC

Augusta rivalis (Benth.) J.H. Kirkbr. CHIS, COL, GRO, JAL, MEX, MICH, NAY, OAX, PUE, TAB, TAMS, VER

Balmea stormiae Martínez CHIS, COL, GRO, JAL, MEX, MICH, NAY, OAX, PUE, TAB, VER

*Bellizinca scoti (J.H. Kirkbr.) Bohr. OAX, PUE

Bertiera guianensis Aubl. CHIS, OAX, TAB, VER

Blepharidium guatemalense Standl. CAM, CHIS, TAB

Borreria brownii (Rusbyi) Standl. CHIS, GRO, OAX

Borreria densiflora DC. CAM, CHIS, COL, GRO, JAL, NAY, OAX, QROO, SIN, TAB, VER, YUC

Borreria exilis L.O. Williams COL, JAL, VER

*Borreria myrtilloides Bohr. \& Salas-Morales OAX

Borreria ocymoides (Burm. f.) DC. CAM, CHIS, COL, CDMX, GRO, HGO, JAL, MEX, MICH, MOR, NAY, OAX, PUE, QRO, SLP, SIN, TAB, VER, YUC

Borreria remota (Lam.) Bacigalupo \& E.L. Cabral CAM, CHIS, COAH, COL, GTO, GRO, HGO, JAL, MEX, MICH, NAY, NLE, OAX, PUE, QRO, QROO, SLP, SIN, SON, TAB, TAMS, VER, YUC

Borreria suaveolens G. Mey AGS, CAM, CHIS, CHIH, COL, DGO, GRO, JAL, MEX, MICH, MOR, NAY, OAX, QROO, SIN, SON, TAB, VER, YUC, ZAC

Borreria verticillata (L.) G. Mey. AGS, CAM, CHIS, COL, CDMX, DGO, GTO, GRO, HGO, JAL, MEX, MICH, MOR, NAY, OAX, PUE, QRO, QROO, SLP, SIN, TAB, TAMS, VER,
YUC, ZAC

*Bouvardia amplexicaulis Bohr. \& E. Martínez MEX

* Bouvardia Bohr.ana Lozada-Pérez GRO, OAX

Bouvardia bouvardioides (Seem.) Standl. CHIS, DGO, GRO, JAL, NAY, OAX, QRO, SIN, TAMS, VER

* Bouvardia candidissima Bohr. \& E. Martínez OAX

* Bouvardia capitata Bullock COL, GRO, JAL, MEX, MOR

* Bouvardia castilloi Bohr. \& García Gonz. VER

*Bouvardia conzattii Greenm. MICH, OAX

*Bouvardia cordifolia DC. AGS, CHIS, COL, GRO, JAL, MEX, MICH, MOR, NAY, OAX, PUE, ZAC

*Bouvardia chrysantha Mart. COL, GRO, HGO, JAL, MEX, MICH, MOR, NAY, OAX, PUE, QRO, SLP, ZAC

Bouvardia dictyoneura Standl. CHIS, GRO, OAX

* Bouvardia elegans Bohr. OAX

*Bouvardia erecta (DC.) Standl. HGO, MEX, OAX, PUE, QRO, TLAX, VER

*Bouvardia ferruginea Bohr. OAX

* Bouvardia fragilis Bohr. \& Salas-Morales OAX

Bouvardia glabra Pol. CHIS, GRO, OAX, PUE, TAB

*Bouvardia gracilipes B.L. Rob. COL, JAL, NAY

*Bouvardia hernan-maganae Bohr. \& Serrano-Cárdenas HGO, QRO

*Bouvardia hintoniorum B.L. Turner GRO, OAX

* Bouvardia juarezana Bohr. \& Salas-Morales OAX

* Bouvardia karwinskyi Standl. NLE, TAMS

* Bouvardia keniae Bohr. \& Saynes OAX

Bouvardia laevis M. Martens \& Galeotti CHIS, COL, GTO, GRO, HGO, JAL, MEX, MICH, MOR, NAY, OAX, PUE, QRO, SLP, TAMS, VER

*Bouvardia lancifolia Bohr. \& Salas-Morales OAX

*Bouvardia langlassei Standl. GRO, MICH

Bouvardia leiantha Benth. CHIS, GRO, NAY, OAX

*Bouvardia loeseneriana Standl. COL, GRO, JAL, MEX, MICH, NAY, OAX

*Bouvardia longiflora (Cav.) Kunth AGS, CHIS, COL, CDMX, GTO, GRO, HGO, JAL, MEX, MICH, MOR, OAX, PUE, QRO,

SLP, SIN, TLAX, VER, ZAC

*Bouvardia lottae Bohr. COL, JAL

*Bouvardia mitlensis Bohr. \& Salas-Morales OAX

Bouvardia multiflora (Cav.) Schult. \& Schult. f. AGS, CHIS, CHIH, COL, CDMX, DGO, GTO, GRO, HGO, JAL, MEX, MICH, MOR, NAY, OAX, PUE, QRO, SLP, SIN, SON, TAB, TLAX, VER, ZAC

* Bouvardia nivea Bohr. OAX

* Bouvardia nodiflora Bohr. \& I. García OAX

*Bouvardia oaxacana Standl. OAX

*Bouvardia obovata Kunth CDMX, HGO, JAL, MEX, MICH, MOR, NAY, OAX, TLAX

*Bouvardia pascualii Bohr. OAX

*Bouvardia pedicellaris Bohr. GRO, MICH

* Bouvardia pulverulenta Bohr. \& Salas-Morales OAX

*Bouvardia pungens Bohr. SLP, TAMS

* Bouvardia quinquenervata Standl. CHIS, OAX

* Bouvardia rekoi Standl. OAX

*Bouvardia rosea Schltdl. GTO, HGO, JAL, QRO, SLP

*Bouvardia rosei Standl. DGO, SIN 
*Bouvardia rzedowskii Terrell \& S.D. Koch GRO, OAX

* Bouvardia sancaroli Bohr. \& M. Martínez TAMS

*Bouvardia scabra Hook. \& Arn. GRO, JAL, NAY, OAX, ZAC *Bouvardia scabrida M. Martens \& Galeotti AGS, COAH, DGO, GTO, HGO, JAL, NLE, OAX, QRO, SLP, TAMS, VER, ZAC

*Bouvardia sinaloae Bohr. \& E. Martínez SIN

*Bouvardia standleyana W.H. Blackw. COL, GRO, JAL, MEX, $\mathrm{MICH}, \mathrm{OAX}$

*Bouvardia stenosiphon Bohr. \& Salas-Morales OAX

*Bouvardia subcordata Standl. CHIH, SIN

*Bouvardia tenuifolia Standl. DGO, JAL, NAY, SIN

*Bouvardia tenuis Bohr. \& Salas-Morales OAX

Bouvardia ternifolia (Cav.) Schltdl. AGS, CHIS, CHIH, COAH, CDMX, DGO, GTO, GRO, HGO, JAL, MEX, MICH, MOR, NAY, NLE, OAX, PUE, QRO, SLP, SIN, SON, TAMS, TLAX, VER, ZAC

*Bouvardia tubicalyx Bohr. \& Salas-Morales OAX

*Bouvardia viminalis Schltdl. CHIS, GRO, OAX, PUE

*Bouvardia xestosperma (B.L. Rob. \& Greenm.) Terrell \& S.D. Koch OAX

*Bouvardia xylosteoides Hook. \& Arn. HGO, OAX, QRO, SLP, VER

Calycophyllum candidissimum (Vahl) DC. CAM, CHIS, GRO, OAX, PUE, QROO, VER

*Carterella alexanderae (Carter) Terrell BCS

Cephalanthus occidentalis L. CAM, CHIS, CHIH, COAH, COL, DGO, GRO, HGO, JAL, MEX, MICH, MOR, NAY, NLE, OAX, PUE, SLP, SIN, SON, TAB, TAMS, VER, ZAC

Cephalanthus salicifolius Bonpl. CHIH, COAH, COL, DGO, GRO, HGO, JAL, MEX, MICH, MOR, NAY, NLE, OAX, PUE, SLP, SIN, SON, TAMS, VER, ZAC

*Cigarrilla mexicana (Zucc. \& Mart. ex DC.) Aiello GTO, HGO, NLE, QRO, SLP, VER

Coccocypselum cordifolium Nees \& Mart. CHIS, HGO, OAX, PUE, TAB, VER

Coccocypselum guianense (Aubl.) Schum. CHIS, OAX, PUE, QRO, SLP, TAB, TAMS, VER

Coccocypselum herbaceum Lam. CHIS, VER

Coccocypselum hirsutum Bartl. ex DC. CHIS, GRO, HGO, JAL, NAY, OAX, PUE, SLP, TAB, VER

Coccocypselum lanceolatum (Ruiz \& Pav.) Pers. CHIS

Cosmibuena matudae (Standl.) L.O. Williams CHIS

*Cosmocalyx spectabilis Standl. CAM, GRO, MICH, QROO,

YUC

*Coussarea chiapensis Bohr. CHIS

Coussarea imitans L.O. Williams CHIS, OAX, VER

*Coussarea impetiolaris Donn. Sm. CHIS, VER

*Coussarea mexicana Standl. CHIS, OAX, TAB, VER

* Coussarea rafa-torresii Bohr. OAX

*Coutaportla ghiesbreghtiana (Baill.) Urb. HGO, OAX, PUE, QRO, SLP, VER

*Coutaportla pailensis Villarreal COAH

Coutarea hexandra (Jacq.) Schum. CAM, CHIS, GTO, GRO,

OAX, QROO, TAB, VER

*Crusea andersoniorum Lorence OAX

*Crusea calcicola Greenm. OAX, PUE
Crusea calocephala DC. CHIS, COL, GRO, HGO, JAL, MEX, MICH, MOR, NAY, OAX, PUE, QRO, SLP, TAB, VER

Crusea coccinea DC. CHIS, COL, CDMX, DGO, GRO, HGO, JAL, MEX, MICH, MOR, NAY, OAX, PUE, SIN, VER

*Crusea coronata B.L. Rob. \& Greenm. CHIH, COL, JAL, MEX, MOR, NAY, SLP, SON

Crusea diversifolia (Kunth) W.R. Anderson AGS, BCS, CAM, CHIS, CHIH, COAH, COL, CDMX, DGO, GTO, GRO, HGO, JAL, MEX, MICH, MOR, NAY, NLE, OAX, PUE, QRO, SLP, SIN, SON, TAMS, TLAX, VER, YUC, ZAC

Crusea hispida (Mill.) B.L. Rob. CHIS, CHIH, COL, GRO, JAL, MEX, MICH, MOR, NAY, OAX, PUE, SIN, SON, TAB, VER, YUC, ZAC

Crusea longiflora (Willd. ex Roem. \& Schult.) W.R. Anderson AGS, CHIS, CHIH, COL, CDMX, DGO, GTO, GRO, HGO, JAL, MEX, MICH, MOR, NAY, OAX, PUE, QRO, SLP, SON, VER, ZAC

*Crusea lucida Benth. OAX, SIN, SON

*Crusea megalocarpa (A. Gray) S. Watson CHIS, COL, DGO, GTO, GRO, JAL, MEX, MICH, MOR, NAY, OAX

Crusea parviflora Hook. \& Arn. BCS, CHIS, COL, GRO, JAL, MEX, MICH, NAY, OAX, SIN, SON

*Crusea psyllioides (Kunth) W.R. Anderson CHIS, CHIH, COL, GRO, JAL, MEX, MICH, MOR, NAY, OAX, SIN, SON

*Crusea pulcherrima Bohr. \& Salas-Morales OAX

Crusea setosa (M. Martens \& Galeotti) Standl. \& Steyerm. BCS, CAM, CHIS, CHIH, DGO, GRO, JAL, MEX, MICH, MOR, NAY, OAX, SON, VER

Crusea wrightii A. Gray CHIS, CHIH, COL, GRO, JAL, MEX, MICH, MOR, NAY, OAX, PUE, SIN, SON

*Csapodya challengeri Bohr. \& Reyes-García CHIS, OAX

*Csapodya sousae Bohr. \& Reyes-García CHIS, OAX

*Csapodya splendens (Breedlove \& Lorence) Bohr. CHIS

Chiococca alba (L.) Hitchc. BCS, CAM, CHIS, COAH, COL, DGO, GTO, GRO, HGO, JAL, MEX, MICH, MOR, NAY, NLE, OAX, PUE, QRO, QROO, SLP, SIN, SON, TAB, TAMS, VER, YUC, ZAC

Chiococca belizensis Lundell CAM, CHIS, OAX, QROO, TAB, VER

*Chiococca coriacea M. Martens \& Galeotti CAM, QROO, TAMS, VER

*Chiococca filipes Lundell CHIS, GRO, JAL, OAX

*Chiococca henricksonii M.C. Johnst. COAH

*Chiococca oaxacana Standl. CHIS, JAL, MOR, OAX, PUE, TAMS

Chiococca pachyphylla Wernham CHIS, CAM, COAH, COL, DGO, GRO, JAL, MEX, MICH, MOR, NAY, NLE, OAX, PUE, QRO, SLP, TAB, TAMS, VER, ZAC

Chiococca parviflora Wullschl. ex Griseb. CAM, CHIS, QROO, YUC

*Chiococca petrina Wiggins CHIH, SIN, SON

Chiococca phaenostemon Schltdl. CHIS, GRO, OAX, PUE, SLP, VER, YUC

*Chiococca pubescens Humb. \& Bonpl. ex Roem. \& Schult. BCS, GRO, MOR, OAX, PUE, SIN, TAMS

Chiococca rubriflora Lundell CHIS

Chiococca semipilosa Standl. \& Steyerm. CHIS, GRO, MOR, 
OAX, PUE, TAMS, VER, ZAC

*Chiococca sessilifolia Miranda CHIS, OAX, VER

Chiococca steyermarkii Standl. CHIS

Chione venosa (Sw.) Urb. CHIS, HGO, MICH, NLE, OAX, PUE, QRO, SLP, TAB, TAMS, VER

* Chomelia anisophylla Ramos \& Borhidi VER

* Chomelia barbata Standl. COL, JAL, NAY, OAX, SIN, VER

Chomelia brachypoda Donn. Sm. CHIS, OAX

* Chomelia breedlovei Bohr. CHIS, OAX

* Chomelia crassifolia Bohr. OAX, TAB

Chomelia longituba (Bohr.) Bohr. CHIS, VER

*Chomelia pringlei S. Watson QRO, SLP, VER

Chomelia protracta (Bartl. ex DC.) Standl. CHIS, GRO, JAL, MICH, NAY, OAX, TAB, VER

Chomelia spinosa Jacq. CHIS, GRO, OAX, TAB, VER

Chomelia tenuiflora Benth. VER

Declieuxia fruticosa (Willd. ex Roem. \& Schult.) Kuntze CHIS

Deppea amaranthina Standl. \& Steyerm. CHIS, OAX

Deppea anisophylla L.O. Williams CHIS, OAX

* Deppea arachnipoda (Bohr. \& Salas-Morales) Bohr. OAX

*Deppea chimalaparum Bohr. \& E. Martínez OAX

*Deppea cornifolia (Benth.) Benth. CHIS, CHIH, COL, CDMX, DGO, GRO, HGO, JAL, MEX, MICH, NAY, NLE, OAX, PUE, QRO, SLP, SON, TAMS, VER

*Deppea ehrenbergii Standl. OAX

*Deppea erythrorhiza Schltdl. \& Cham. CHIS, OAX, TAB, VER

*Deppea foliosa Bohr., E. Martínez \& Salas-Morales OAX

Deppea grandiflora Schltdl. CHIS, GRO, JAL, OAX, SLP, TAB,

VER

* Deppea hernandezii Lorence HGO, OAX, VER

* Deppea hintonii Bullock CHIS, GRO, MEX, OAX

Deppea inaequalis Standl. \& Steyerm. CHIS, VER

*Deppea keniae Bohr., E. Martínez \& A. Saynes OAX

*Deppea longifolia Bohr. OAX

*Deppea martinez-calderonii Lorence OAX

* Deppea microphylla Greenm. HGO, PUE, VER

* Deppea nitida Bohr. \& Salas-Morales OAX

*Deppea obtusiflora (Benth.) Benth. HGO, OAX, VER

*Deppea pauciflora Bohr. \& E. Martínez NLE, TAMS

Deppea pubescens Hemsl. CHIS, OAX

*Deppea purpurascens Lorence OAX, VER

*Deppea purpusii Standl. HGO, OAX, PUE, QRO, SLP, TAMS, VER

*Deppea rubrinervis Bohr. VER

* Deppea tenuiflora Benth. CHIS

*Deppea tubaeana Bohr. OAX

* Deppea umbellata Hemsl. HGO, OAX, VER

Didymaea alsinoides (Schltdl. \& Cham.) Standl. CHIS, COL, CDMX, HGO, GTO, GRO, JAL, MEX, MICH, MOR, OAX, PUE, QRO, SLP, TLAX, VER

*Didymaea crassifolia Bohr. OAX

*Didymaea floribunda Rzed. CHIS, COL, CDMX, GRO, JAL, MEX, MICH, MOR, OAX, VER

*Didymaea hispidula L.O. Williams GRO, OAX

*Didymaea ixtepejiensis Bohr. \& E. Martínez OAX

* Didymaea linearis Standl. JAL, OAX
Didymaea mexicana Hook. f. MICH, OAX, PUE, VER

Didymaea microphylla L.O. Williams CHIS

* Didymaea naniflora Bohr. \& E. Martínez OAX

*Diodella apiculata (Willd. ex Roem. \& Schult.) Delprete OAX, TAB, TAMS, VER

* Diodella crassifolia (Benth.) Bohr. NAY, SIN

Diodella sarmentosa (Sw.) Bacigalupo \& E.L. Cabral CHIS, COL, JAL, OAX, PUE, QRO, SLP, SON, TAB, VER

*Diodia aspera Brandegee CHIS

*Diodia crassifolia Benth. NAY, SIN

Diodia rigida (Willd. ex Roem. \& Schult.) Schltdl. \& Cham. CHIS, GRO, JAL, MICH, OAX, TAB

Diodia saponariifolia (Cham. \& Schltdl.) K. Schum. PUE, VER

Diodia serrulata (P. Beauv.) G. Taylor VER

Diodia teres Walter AGS, BCN, BCS, CAM, CHIS, CHIH, COL, DGO, GTO, GRO, JAL, MEX, MICH, MOR, NAY, NLE, OAX, PUE, QRO, QROO, SLP, SIN, SON, TAMS, VER, YUC, ZAC

Diodia virginiana L. YUC

*Diphragmus hexasepalus Bohr. \& Lozada-Pérez OAX

*Diphragmus scaber C. Presl BCS, CHIS, COL, GRO, JAL, MEX, MICH, MOR, NAY, OAX, SIN, SON

Donnellyanthus deamii (Donn. Sm.) Bohr. CHIS, OAX

* Edithea floribunda Standl. GRO, OAX

* Edithea guerrerensis (Dwyer \& Lorence) Bohr. \& Stranczinger GRO, JAL, MEX, MICH, OAX

*Edithea miahuatlanica (Lorence) Bohr. OAX

* Edithea oaxacana (Lorence) Bohr. \& Stranczinger GRO, OAX

*Edithea rupicola (Bohr. \& Velasco-Gutiérrez) Bohr. OAX

*Edithea schiblii Bohr., Saynes \& Velasco OAX

*Edithea serboi (Bohr. \& Velasco-Gutiérrez) Bohr. OAX

*Edithea sousae (Bohr., Mart. Gord., M. \& Cruz-Durán) Bohr. GRO

Eizia mexicana Standl. CHIS

Erithalis fruticosa L. CAM, QROO, VER, YUC

Ernodea littoralis Sw. CAM, QROO, VER, YUC

Exostema caribaeum (Jacq.) Roem. \& Schult. CAM, CHIS, COL, GTO, GRO, HGO, JAL, MEX, MICH, MOR, NLE, OAX, PUE, QRO, QROO, SLP, TAB, TAMS, VER, YUC, ZAC Faramea brachysiphon Standl. CHIS, OAX, VER

Faramea cobana Donn. Sm. CHIS, OAX

* Faramea chiapensis Bohr. CHIS

Faramea glandulosa Poepp. \& Endl. CHIS, OAX, TAB, VER

* Faramea liebmannii Standl. OAX

* Faramea oaxacensis Bohr. OAX

Faramea occidentalis (L.) A. Rich. CHIS, COL, GRO, JAL, NAY, NLE, OAX, PUE, QRO, SLP, TAB, VER

*Faramea schultesii Standl. CHIS, OAX, VER

Galianthe angulata (Benth.) Bohr. CHIS, HGO, OAX, PUE, TAB, VER, YUC

Galium andrewsii A. Gray BCN

* Galium angulosum A. Gray BCN

Galium angustifolium Nutt. BCN

Galium aparine L. BCN, CHIH, COAH, JAL, NLE, OAX, SON, TAMS

Galium aschenbornii S. Schauer AGS, CHIS, COL, CDMX, GTO, GRO, HGO, JAL, MEX, MICH, MOR, OAX, PUE, 
QRO, SLP, TLAX, VER, ZAC

* Galium carmenicola Dempster COAH

* Galium carterae Dempster BCS

* Galium coronadoense Dempster BCN

Galium correllii Dempster COAH

*Galium dempsterae B.L. Turner NLE

*Galium denticulatum Bartl. ex DC. HGO, VER

*Galium diabolense Dempster BCN

Galium fendleri A. Gray CHIH, SON

*Galium fuscum M. Martens \& Galeotti DGO, GTO, GRO, JAL, OAX, PUE, VER

*Galium glaberrimum Hemsl. OAX

*Galium hintoniorum B.L. Turner TAMS

*Galium hypadenium Schauer QRO, VER

*Galium hystricocarpum Greenm. CHIH

* Galium iltisii Dempster GRO

*Galium juniperinum Standl. NLE

*Galium lacrimiforme Dempster NLE, TAMS

*Galium martirense Dempster BCN

*Galium mechudoense Dempster BCS

Galium mexicanum Kunth AGS, CHIS, CHIH, COAH, COL, CDMX, DGO, GTO, GRO, HGO, JAL, MEX, MICH, MOR, NAY, NLE, OAX, PUE, QRO, SLP, SIN, SON, TAMS, VER, ZAC

*Galium moranii Dempster BCS

Galium nelsonii Greenm. CHIS, COAH

Galium nuttallii A. Gray BCN

* Galium oresbium Greenm. COAH, NLE

Galium orizabense Hemsl. CHIS, GRO, HGO, JAL, OAX, PUE, QRO, SLP, TAMS, VER

*Galium pendulum Greenm. HGO, PUE, VER

*Galium pennellii Dempster HGO, SLP, TAMS

Galium porrigens Dempster BCN

*Galium praetermissum Greenm. CDMX, GTO, JAL, MEX, MICH, PUE

*Galium pringlei Greenm. COAH, NLE

Galium proliferum A. Gray CHIH, COAH, NLE, SLP, SON, TAMS

*Galium rzedowskii Dempster COAH, NLE, SLP

*Galium seatonii Greenm. CDMX, HGO, JAL, MEX, MICH, PUE, TLAX, VER

Galium sphagnophilum (Greenm.) Dempster CDMX, GTO, HGO, JAL, MEX, MICH, OAX, PUE, TLAX

Galium stellatum Kellogg BCN, BCS, SON

Galium trifidum L. BCS, CDMX, GRO, HGO, MEX, MICH, MOR, PUE

Galium triflorum Michx. TAMS, VER

Galium uncinulatum DC. AGS, BCS, CHIS, CHIH, COAH, CDMX, DGO, GTO, GRO, HGO, JAL, MEX, MICH, MOR, NLE, OAX, PUE, QRO, SLP, SIN, SON, TAMS, TLAX, VER, ZAC

*Galium volcanense Dempster BCN, BCS

*Galium wigginsii Dempster BCN

Galium wrightii A. Gray BCN, CHIH, COAH, SON

Genipa americana L. AGS, CAM, CHIS, COL, GRO, JAL, MICH, NAY, OAX, SIN, TAB, VER, ZAC

Geophila macropoda (Ruiz \& Pav.) DC. CHIS, OAX, PUE,
VER

Geophila repens (L.) I.M. Johnst. CAM, CHIS, OAX, QROO, TAB, VER

*Glossostipula blepharophylla (Standl.) Lorence DGO, JAL, NAY, SIN

Glossostipula concinna (Standl.) Lorence CHIS, COL, GTO, GRO, JAL, OAX, QRO, VER

Gonzalagunia chiapasensis (Standl.) Standl. \& Steyerm. CHIS, GRO, TAB, VER

Gonzalagunia panamensis (Cav.) K. Schum. CHIS, COL, GRO, HGO, JAL, MICH, OAX, PUE, TAB, VER

Gonzalagunia rudis Standl. CHIS

Gonzalagunia thyrsoidea (Donn. Sm.) B.L. Rob. CHIS, PUE, $\mathrm{TAB}$

*Guettarda coatzacoalcensis Ramos \& Bohr. VER

* Guettarda colubrinoides Standl. TAB

Guettarda combsii Urb. CAM, CHIS, QROO, TAB, VER, YUC Guettarda crispiflora Vahl CHIS

* Guettarda dealbata M. Martens \& Galeotti OAX

Guettarda deamii Standl. CAM, CHIS, QROO, YUC

Guettarda elliptica Sw. CAM, CHIS, COL, GRO, JAL, MEX, MICH, NAY, OAX, QROO, SIN, TAB, VER, YUC

*Guettarda elongata Borh., K. Velasco-Gutiérrez \& VázquezMartínez OAX

*Guettarda filipes Standl. CAM, DGO, GRO, JAL, MICH, NAY, OAX, QROO, SIN, YUC

Guettarda foliacea Standl. CHIS, TAB

*Guettarda galeottii Standl. CHIS, NAY, OAX, SIN

Guettarda gaumeri Standl. CAM, CHIS, OAX, QROO, YUC

* Guettarda guerrerensis Bohr. GRO, JAL

Guettarda macrosperma Donn. Sm. CAM, CHIS, COL, GRO, JAL, MICH, NAY, OAX, PUE, QROO, SIN, TAB, VER

* Guettarda quadrifida Bohr. \& Reyes-García CHIS

* Guettarda sotonunezii Bohr., E. Martínez \& Ramos GRO

Guettarda subcapitata C.M. Taylor CAM, CHIS, OAX, QROO

Guettarda tikalana Lundell CHIS, TAB, VER

*Habroneuron radicans (Wernham) S.P. Darwin OAX

Hamelia axillaris $\mathrm{Sw}$. CHIS, JAL, OAX, SLP, TAB, VER

Hamelia barbata Standl. CHIS, OAX, TAB, VER

Hamelia calycosa Donn. Sm. CHIS, COL, GRO, JAL, MICH, NAY, OAX, TAB, VER

Hamelia longipes Standl. CHIS, GRO, OAX, TAB, VER

Hamelia magnifolia Weinh. TAB

Hamelia patens Jacq. CAM, CHIS, COL, CDMX, DGO, GRO, HGO, JAL, MEX, MICH, MOR, NAY, NLE, OAX, PUE, QRO, QROO, SLP, SIN, TAB, TAMS, VER, YUC

Hamelia rovirosae Wernham CAM, CHIS, TAB, VER

*Hamelia versicolor A. Gray CHIS, COL, DGO, GRO, JAL, MEX, MICH, MOR, NAY, OAX, SIN, VER

*Hamelia xorullensis Kunth CHIS, CHIH, COL, CDMX, DGO, GRO, JAL, MEX, MICH, MOR, NAY, OAX, SIN, SON, ZAC Hedyotis acerosa A. Gray CHIH, COAH, NLE, SLP, TAMS, ZAC

*Hedyotis drymarioides (Standl.) W.H. Lewis SLP, TAMS

*Hedyotis exigula W.H. Lewis OAX, VER

Hedyotis greenei (A. Gray) W.H. Lewis SON

Hedyotis humifusa A. Gray CHIH, DGO 
Hedyotis intricata Fosberg CHIH, COAH, NLE

Hedyotis mullerae Fosberg CHIH, COAH, NLE

Hedyotis nigricans (Lam.) Fosberg AGS, CHIH, COAH, NLE, OAX, SLP, TAMS, ZAC

Hedyotis palmeri (A. Gray) W.H. Lewis COAH, NLE, SLP, ZAC

Hedyotis rubra (Cav.) A. Gray AGS, CHIH, COAH, DGO, GTO, HGO, JAL, NLE, PUE, QRO, SLP, SON, VER, ZAC

*Hedyotis sharpii (Terrell) G.L. Nesom HGO, VER

*Hedyotis sinaloae W.H. Lewis SIN, SON

*Hedyotis spellenbergii G.L. Nesom \& Vorobik CHIH, SON

*Hedyotis teretifolia (Terrell) G.L. Nesom COAH

*Hedyotis umbratilis (B.L. Rob.) W.H. Lewis NLE

*Hedyotis vergrandis W.H. Lewis BCS, SON

Hedyotis wrightii (A. Gray) Fosberg AGS, CHIH, CDMX, DGO, GTO, GRO, HGO, JAL, MEX, MICH, MOR, NLE, OAX, PUE, QRO, SLP, SON, TAMS, TLAX, VER, ZAC

Hemidiodia ocymifolia (Willd. ex Roem. \& Schult.) K. Schum. CHIS, GRO, OAX, QROO, VER

Hillia chiapensis Standl. CHIS

Hillia loranthoides Standl. CHIS, OAX

*Hillia maxonii Standl. CHIS

*Hillia oaxacana C.M. Taylor OAX

Hillia panamensis Standl. CHIS

Hillia tetrandra Sw. CHIS, OAX, PUE, TAB, VER

Hillia triflora (Oerst.) C.M. Taylor CHIS

Hintonia latiflora (Sessé \& Moc. ex DC.) Bullock AGS, CAM, CHIS, CHIH, COL, DGO, GRO, JAL, MEX, MICH, MOR, NAY, OAX, PUE, SIN, SON, VER, ZAC

Hintonia lumaeana (Baill.) Bullock CHIS, VER

*Hintonia octomera (Hemsl.) Bullock CAM, QROO, YUC

*Hoffmannia altipetens Dwyer ex A. Torres-Montúfar \& H. Ochoterena VER

Hoffmannia amplexifolia Standl. OAX

Hoffmannia angustifolia Standl. CHIS, OAX, VER

Hoffmannia cauliflora Hemsl. CHIS

Hoffmannia conzattii B.L. Rob. CHIS, GRO, HGO, JAL, MOR, OAX, PUE, QRO, SLP, TAB, TAMS, VER

Hoffmannia cryptoneura Standl. CHIS, GRO, OAX, SLP, TAMS, VER

Hoffmannia culminicola Standl. \& L.O. Williams CHIS, GRO, JAL, OAX, TAB, VER

*Hoffmannia cuneatissima B.L. Rob. CHIS, COL, GRO, JAL, MEX, MICH, MOR, NAY, OAX, SON

Hoffmannia discolor (Lem.) Hemsl. CHIS, OAX, TAB, TAMS, VER

Hoffmannia excelsa (Kunth) K. Schum. CHIS, HGO, OAX, QRO, SLP, TAMS, VER

Hoffmannia gesnerioides (Oerst.) Kuntze CHIS, OAX, VER

Hoffmannia ghiesbreghtii (Lem.) Hemsl. CHIS, TAB, VER

*Hoffmannia guerrerensis Bohr. \& J. Rojas GRO

*Hoffmannia hidalgensis Bohr. HGO, VER

Hoffmannia huehueteca Standl. \& Steyerm. CHIS, OAX

*Hoffmannia ixtlanensis Lorence OAX, TAMS

Hoffmannia macrosiphon Standl. CHIS

*Hoffmannia minuticarpa Dwyer \& Lorence OAX, VER

Hoffmannia montana L.O. Williams CHIS, HGO, OAX, QRO,
SLP, TAMS, VER

Hoffmannia nicotianifolia (M. Martens \& Galeotti) L.O. Williams CHIS, GRO, HGO, MICH, NAY, OAX, PUE, QRO, SLP, TAMS, VER

*Hoffmannia oaxacensis Lorence \& Dwyer ex Bohr. OAX, VER

*Hoffmannia orizabensis Standl. HGO, OAX, VER

Hoffmannia phoenicopoda K. Schum. CHIS, OAX, VER

Hoffmannia psychotriifolia (Benth.) Griseb. CHIS, HGO, OAX, PUE, SLP, VER

Hoffmannia quadrifolia Standl. \& Steyerm. CHIS, OAX

Hoffmannia racemifera Standl. \& Steyerm. CHIS, VER

Hoffmannia refulgens (Hook. f.) Hemsl. CHIS, VER

Hoffmannia regalis (Hook. f.) Hemsl. CHIS, OAX, TAB, VER

Hoffmannia rhizantha Standl. VER

Hoffmannia riparia Standl. CHIS, TAB, VER

Hoffmannia rotata Donn. Sm. CHIS, HGO, VER

Hoffmannia rzedowskiana Cast. Campos, Bautista-Bello \& Lorence CHIS, NLE, OAX, VER

*Hoffmannia silviarum Bohr. OAX

Hoffmannia steyermarkii Standl. CHIS

*Hoffmannia strigillosa Hemsl. SLP, TAMS

*Hoffmannia tonduzii Standl. OAX

Hoffmannia wilsonii Standl. CHIS, PUE, VER

*Houstonia angustifolia Michx. CHIH, HGO, QRO, SON, VER

*Houstonia gracilis Brandegee OAX, VER

*Houstonia macvaughii (Terrell) Govaerts ex Bohr. JAL

Isertia haenkeana DC. CHIS

* Ixora acuminata Roxb. CHIS, TAB, VER

Lorencea guatemalensis (Standl.) Bohr. CHIS, VER

Machaonia acuminata Kunth CAM, CHIS, COL, JAL, MICH, OAX, PUE, QROO, SLP, SIN, TAB, TAMS, VER, YUC

*Machaonia coulteri (Hook. f.) Standl. GTO, HGO, MICH, OAX, QRO, SLP, TAMS, VER

Machaonia erythrocarpa (Standl.) Bohr. CHIS, COL, HGO, JAL, MICH, NAY, OAX, PUE, SIN, VER

* Machaonia hahniana Baill. OAX, PUE

Machaonia lindeniana Baill. CAM, CHIS, QROO, TAB, YUC

Machaonia martinezorum Lorence CHIS, OAX

* Machaonia pringlei A. Gray COAH, DGO

Manettia flexilis Brandegee CHIS, VER

*Manettia hirtella M. Martens \& Galeotti OAX

Manettia reclinata Mutis ex L. CHIS, OAX, TAB, VER

Margaritopsis microdon (DC.) C.M. Taylor CAM, CHIS, COL, GRO, JAL, MEX, MICH, OAX, PUE, QRO, QROO, SLP, SIN, TAB, TAMS, VER, YUC

* Martensianthus albiflorus (Bohr. \& E. Martínez) Bohr. CHIS

*Martensianthus breviflorus (Bohr. \& Salas-Morales) Bohr. OAX

* Martensianthus macdougallii Lorence OAX

* Martensianthus micranthus (Bohr.) Bohr. OAX

*Martensianthus viticellus (Lorea-Hern. \& Lozada-Pérez) Bohr. GRO

*Mexotis galeottii (M. Martens \& Galeotti) Terrell \& H. Rob. CHIS, OAX, VER

* Mexotis kingii (Terrell) Terrell \& H. Rob. OAX

*Mexotis latifolia (M. Martens \& Galeotti) Terrell \& H. Rob. 
CHIS, OAX, PUE, VER

*Mexotis lorencei Terrell \& H. Rob. GRO, OAX

*Mexotis terrellii (Lorence) Terrell \& H. Rob. OAX

Mitchella repens L. CAM, CHIS, GRO, HGO, NLE, OAX, PUE, QRO, SLP, TAMS, VER

* Mitracarpus aristatus Bohr. \& Lozada-Pérez SIN

Mitracarpus breviflorus A. Gray BCN, BCS, CHIH, COL, DGO, GRO, HGO, JAL, MICH, NAY, NLE, PUE, SLP, SIN, SON, TAMS, VER

* Mitracarpus capitatus Lozada-Pérez \& Bohr. GRO, OAX

* Mitracarpus carnosus Bohr. \& Lozada-Pérez OAX

* Mitracarpus falcatus Lozada-Pérez \& Bohr. GRO, MICH

* Mitracarpus floribundus Bohr. \& Lozada-Pérez BCS

Mitracarpus glabrescens (Griseb.) Urb. BCN, CAM, CHIS, GRO, OAX, QROO, VER, YUC

* Mitracarpus gypsophilus Bohr. \& E. Martínez CAM

Mitracarpus hirtus (L.) DC. AGS, BCS, CAM, CHIS, CHIH, COAH, COL, DGO, GTO, GRO, JAL, MEX, MICH, MOR, NAY, NLE, OAX, PUE, QROO, SLP, SIN, SON, TAMS, TLAX, VER, YUC, ZAC

Mitracarpus linearifolius A. Rich. CHIS, COL, GRO, JAL, MICH, OAX, TAB, VER

*Mitracarpus linearis Benth. BCS, CAM, QROO

* Mitracarpus micranthus Borh. \& E. Martínez OAX, TAB

*Mitracarpus schizangius DC. BCS, COL, GRO, JAL, MICH, NAY, OAX, SIN, VER

*Mitracarpus trichanthus Bohr. \& Lozada-Pérez SIN

Morinda panamensis Seem. CHIS, OAX, QROO, TAB, VER

Morinda royoc L. CAM, CHIS, QROO, TAB, YUC

Morinda yucatanensis Greenm. CAM, CHIS, QROO, TAB, YUC

*Neomartensia breviflora (Bohr. \& Salas-Morales) Bohr. \& Lozada-Pérez OAX

*Neomartensia macdougallii (Lorence) Bohr. \& Lozada-Pérez CHIS, OAX

*Neomartensia micrantha (Bohr.) Bohr. \& Lozada-Pérez OAX

*Nernstia mexicana (Zucc. \& Mart. ex DC.) Urb. HGO, QRO, SLP, VER

Nertera granadensis (Mutis. ex L. f.) Druce CHIS, GRO, HGO, MICH, OAX, PUE, QRO, SLP, VER

Notopleura anomothyrsa (K. Schum. \& Donn. Sm.) C.M. Taylor CHIS, OAX, TAB, VER

Notopleura epiphytica (K. Krause) C.M. Taylor OAX

Notopleura guadalupensis (DC.) C.M. Taylor CHIS, OAX, VER

Notopleura hondurensis C.M. Taylor CHIS, OAX

Notopleura macrophylla (Ruiz \& Pav.) C.M. Taylor CHIS, OAX, TAB, VER

Notopleura siggersiana (Standl.) C.M. Taylor OAX

Notopleura tolimensis (Wernham) C.M. Taylor CHIS, TAB, VER

Notopleura uliginosa (Sw.) Bremek. CHIS, OAX, PUE, VER

Oldenlandia corymbosa L. CHIS, NAY, QROO, TAB, YUC

Oldenlandia herbacea (L.) DC. CHIS, TAB, VER

Oldenlandia lancifolia (Schumach.) DC. CAM, CHIS, OAX, TAB, VER

Oldenlandia microtheca (Schltdl. \& Cham.) DC. CHIS, OAX,
PUE, VER

* Oldenlandia ovata S. Watson COAH, NLE, PUE, SLP, TAMS, VER

*Oldenlandia pringlei B.L. Rob. QRO, SLP

Oldenlandiopsis callitrichoides (Griseb.) Terrell \& W.H. Lewis YUC

*Omiltemia longipes Standl. GRO, OAX

* Omiltemia parvifolia Bohr. \& Velasco-Gutiérrez OAX

* Paederia ciliata (Bartl. ex DC.) Standl. COL, CDMX, GRO, JAL, MEX, MICH, MOR, OAX, PUE, VER

Palicourea acuminata (Benth.) Bohr. CHIS, HGO, OAX, TAB, VER

Palicourea berteriana (DC.) Bohr. CHIS, OAX, PUE, TAMS, VER

Palicourea brachiata (Sw.) Bohr. CHIS, OAX, PUE, VER

*Palicourea breedlovei (Lorence) Lorence CHIS

Palicourea capitata (Ruiz \& Pav.) Bohr. CHIS, OAX, TAB, VER

Palicourea crocea (Sw.) Roem. \& Schult. CAM, CHIS, GRO, OAX, TAB, VER

Palicourea chiapensis (Standl.) Borh. CHIS, GRO, JAL, OAX

* Palicourea chlorobotrya (Standl.) Borh. CHIS, OAX, VER

Palicourea deflexa (DC.) Borh. CHIS, GRO, OAX, PUE, VER

Palicourea domingensis (Jacq.) DC. CHIS, OAX, PUE, TAB, VER

Palicourea elata (Sw.) Borh. CHIS, OAX, PUE, TAB, VER

*Palicourea faxlucens (Lorence \& Dwyer) Lorence OAX, TAB, VER

Palicourea furcata (DC.) Borh. CHIS, OAX, TAB, VER

Palicourea galeottiana M. Martens CAM, CHIS, GRO, HGO, OAX, PUE, QRO, QROO, SLP, TAB, TAMS, VER, YUC

*Palicourea gardenioides (Scheidw.) Hemsl. TAB, VER Palicourea glomerulata (Donn. Sm.) Borh. CHIS

Palicourea guianensis Aubl. CAM, CHIS, OAX, TAB, VER

Palicourea hebeclada (DC.) Borh. OAX, QROO, VER

Palicourea heydei (Standl.) Lorence CHIS

Palicourea hoffmannseggiana (Willd. ex Roem. \& Schult.) Borh. CHIS, OAX, TAB, VER

Palicourea izabalensis (L.O. Williams) Borh. CHIS, OAX

*Palicourea juarezana (C.M. Taylor \& Lorence) Borh. CHIS, OAX

Palicourea leucantha Loes. OAX

Palicourea lozadae (Borh. \& Lorea-Hern.) Borh. GRO

Palicourea macrantha Loes. CHIS, OAX, VER

* Palicourea megalantha (Lorence) Lorence CHIS, OAX, VER Palicourea minarum (Standl. \& Steyerm.) Borh. CHIS, OAX

Palicourea neopurpusii C.M. Taylor CHIS

Palicourea oreodoxa (L.O. Williams) Borh. GRO

Palicourea padifolia (Willd. ex Roem. \& Schult.) C.M. Taylor $\&$ Lorence CHIS, GRO, HGO, OAX, PUE, SLP, TAB, VER

* Palicourea perotensis (Cast.-Campos) Borh. VER

Palicourea phanerandra Standl. \& Steyerm. CHIS, GRO, HGO, OAX, PUE, TAB, VER

Palicourea pubescens (Sw.) Borh. CAM, CHIS, COL, GRO, JAL, MICH, NAY, OAX, PUE, QRO, QROO, SLP, TAB, VER, YUC

Palicourea racemosa (Aubl.) Borh. CHIS, OAX, TAB, VER 
Palicourea simiarum (Standl.) Borh. CHIS, GRO, HGO, OAX, PUE, TAB, VER

*Palicourea sousae (Lorence \& Dwyer) Lorence OAX, VER Palicourea steyermarkiana Borh. CHIS

Palicourea tetragona (Donn. Sm.) C.M. Taylor \& Lorence CHIS, GRO, OAX, PUE, SLP, TAB, VER

*Palicourea thornei (Lorence) Lorence CHIS

Palicourea triphylla DC. CHIS, TAB, VER

*Palicourea veracruzensis (Lorence \& Dwyer) Borh. CHIS, OAX, PUE, TAB, VER

Pinarophyllon bullatum Standl. CHIS, VER

Pinarophyllon flavum Brandegee CHIS

Pittoniotis trichantha Griseb. CHIS

* Placocarpa mexicana Hook. f. OAX, VER

Plocaniophyllum flavum Brandegee CHIS

Pogonopus exsertus (Oerst.) Oerst. CHIS, VER

Posoqueria coriacea M. Martens \& Galeotti CHIS, GRO, OAX, TAB, VER

Posoqueria latifolia (Rudge.) Roem. \& Schult. CHIS, GRO, OAX, TAB, VER

*Pseudomiltemia davidsonii Martínez-Camilo \& Lorence CHIS

* Pseudomiltemia filisepala (Standl.) Bohr. CHIS, GRO, OAX

Psychotria aguilarii Standl. \& Steyerm. CAM, CHIS, VER

Psychotria aubletiana Sw. CHIS, OAX

* Psychotria balancanensis C.W. Ham. TAB

Psychotria buchtienii (H.J.P. Winkl.) Standl. CHIS

Psychotria calophylla Standl. OAX, QROO, VER

Psychotria calopogon L.O. Williams CHIS, OAX

Psychotria carthagenensis Jacq. CAM, CHIS, OAX, QROO, SLP, TAB, TAMS, VER

Psychotria clivorum Standl. \& Steyerm. CHIS, OAX, TAB, VER

Psychotria costivenia Griseb. CAM, CHIS, GRO, HGO, OAX, PUE, QRO, QROO, SLP, TAB, VER, YUC

Psychotria cyanococca Dombrain CHIS

Psychotria chagrensis Standl. CHIS, QROO, VER

*Psychotria chamelaensis C.M. Taylor \& E. Domínguez-Licona JAL

*Psychotria chamissoana Standl. VER

* Psychotria diegoae Bohr. QRO, SLP

Psychotria dispersa Standl. CHIS

* Psychotria dwyeri C.W. Ham. OAX, VER

Psychotria erecta (Aubl.) Standl. \& Steyerm. CHIS, VER

Psychotria erythrocarpa Schltdl. CAM, CHIS, COL, GTO, GRO, HGO, JAL, MEX, MOR, OAX, PUE, QRO, SLP, TAB, TAMS, VER

Psychotria flava Oerst. ex Standl. CHIS, GRO, OAX, PUE, QRO, SLP, TAB, VER

Psychotria gracilenta Müll. Arg. OAX, VER

Psychotria graciliflora Benth. CAM, CHIS, GRO, HGO, JAL, OAX, QRO, QROO, SLP, TAB, TAMS, VER, YUC

Psychotria grandis Sw. CHIS, GRO, OAX, TAB, VER, YUC

* Psychotria hidalgensis Bohr. HGO, PUE, QRO, SLP, TAMS

Psychotria horizontalis Sw. CHIS, COL, GRO, JAL, MEX, MICH, NAY, OAX, QROO, SIN, TAB, VER

* Psychotria inegi I. García-González \& Borh. JAL

Psychotria limonensis K. Krause CAM, CHIS, JAL, MICH,
NAY, OAX, PUE, QRO, SLP, TAB, VER

Psychotria lorenciana C.M. Taylor CHIS

Psychotria lundellii Standl. CHIS, VER

Psychotria marginata Sw. CHIS, OAX, TAB, VER

Psychotria mexiae Standl. CHIS, GRO, JAL, NAY, OAX, PUE, SON, VER

Psychotria micrantha Kunth CAM, CHIS, VER

*Psychotria miradorensis (Oerst.) Hemsl. CAM, CHIS, OAX, QROO, TAB

*Psychotria mirandae C.W. Ham. CAM, CHIS, OAX, QROO, VER

Psychotria mombachensis Standl. CHIS, TAB, VER

Psychotria nervosa Sw. CAM, CHIS, GTO, OAX, QRO, QROO, SLP, TAB, VER, YUC

* Psychotria oaxacensis Bohr. \& Salas-Morales OAX

Psychotria officinalis (Aubl.) Sandwith CHIS, TAB, VER

Psychotria panamensis Standl. CHIS, GRO, OAX, PUE, TAB, VER

*Psychotria papantlensis (Oerst.) Hemsl. CHIS, MICH, QRO, SLP, TAB, TAMS, VER, YUC

Psychotria pittieri Standl. CHIS

Psychotria pleuropoda Donn. Sm. CHIS, TAB

Psychotria poeppigiana Müll. Arg. CHIS, OAX, TAB, VER

Psychotria pulverulenta Urb. SLP

Psychotria quinqueradiata Pol. CHIS, OAX, PUE, TAB, VER

* Psychotria sarapiquensis Standl. CHIS, OAX, VER

Psychotria tenuifolia Sw. CAM, CHIS, COL, JAL, NAY, OAX, QRO, QROO, SLP, TAB, TAMS, VER, YUC

Psychotria trichotoma M. Martens \& Galeotti CHIS, GRO, HGO, JAL, OAX, PUE, TAB, VER

Psychotria viridis Ruiz \& Pav. CHIS, TAB

Rachicallis americana (Jacq.) Hitchc. QROO, YUC

* Randia aciculiflora Bohr. \& Saynes OAX

Randia aculeata L. CAM, CHIS, COL, DGO, GRO, HGO, JAL, MEX, MICH, MOR, NAY, NLE, OAX, PUE, QRO, QROO, SLP, SIN, TAB, TAMS, VER, YUC

* Randia alvarocamposii Bohr. \& E. Martínez OAX

Randia armata (Sw.) DC. BCN, BCS, CAM, CHIS, COL, DGO, GRO, JAL, MICH, NAY, OAX, PUE, QROO, SIN, TAB, TAMS, VER, YUC

* Randia brachysiphon Bohr. \& Salas-Morales OAX

*Randia canescens Greenm. COL, GTO, GRO, MICH, MOR, OAX, QRO

Randia capitata DC. BCS, CAM, CHIS, CHIH, COL, DGO, GTO, GRO, HGO, JAL, MEX, MICH, MOR, NAY, NLE, OAX, PUE, QRO, SLP, SIN, SON, TAB, TAMS, VER, ZAC

* Randia cinerea (Fernald) Standl. CHIS, COL, GRO, HGO, JAL, MEX, MICH, NAY, OAX, QRO, VER

* Randia colimensis Bohr. \& E. Martínez COL

* Randia confusa Bohr. \& Diego GRO

Randia cookii Standl. CHIS, OAX, VER

* Randia coronata Bohr. GRO

* Randia chiapensis Standl. CAM, CHIS, GRO, QROO, TAB

*Randia crucis Bohr. \& Salas-Morales OAX

* Randia denticulata Bohr. GRO, MICH

* Randia dionisi Bohr. \& Salas-Morales OAX

Randia echinocarpa Sessé \& Moc. ex DC. CAM, CHIH, COL, 
CDMX, DGO, GRO, JAL, MEX, MICH, MOR, NAY, OAX, PUE, SIN, SON, TAMS, VER

Randia genipifolia (Standl. \& Steyerm.) Lorence CHIS

Randia grandifolia (Donn. Sm.) Standl. CHIS, VER

Randia guatemalensis Standl. CHIS

*Randia guerrerensis Lorence \& Rodr. Acosta GRO

Randia habrophlebia Standl. \& Steyerm. CHIS

*Randia hidalgensis Lorence GTO, HGO, QRO, SLP

* Randia hypoleuca Bohr. \& E. Martínez MICH, OAX

* Randia induta Standl. GRO, JAL, NAY, SIN

Randia laetevirens Standl. CAM, CHIS, COAH, COL, GRO, HGO, JAL, MICH, NAY, NLE, OAX, PUE, QRO, QROO, SLP, SIN, TAMS, VER, YUC

*Randia laevigata Standl. CAM, CHIH, COL, DGO, GRO, JAL, MEX, MICH, NAY, OAX, QROO, SIN, SON, VER, ZAC

*Randia laevigatoides Bohr. GRO, MICH, OAX

*Randia lanuginosa Bohr. \& García-González COL, JAL, OAX

Randia longiloba Hemsl. CAM, CHIS, QROO, YUC

Randia loniceroides Dwyer \& Lorence CHIS, TAB, VER

*Randia lorenceana J. Jiménez Ram. \& Cruz-Durán GRO, OAX

Randia malacocarpa Standl. COL, GRO, JAL, MICH, NAY, OAX, SIN, TAB

*Randia manglaris Bohr. \& E. Martínez VER

Randia matudae Lorence \& Dwyer CHIS, OAX, VER

* Randia mendozae Bohr. GRO, MICH

*Randia mixe Bohr. \& E. Martínez OAX

*Randia mollifolia Standl. CHIH, COL, GRO, JAL, NAY, SIN, SON

Randia monantha Benth. CAM, CHIS, DGO, GRO, JAL, MEX, OAX, QROO, SLP, TAMS, VER, YUC

Randia nodiflora Bohr. \& I. García OAX

*Randia oaxacana Standl. CHIS, OAX

*Randia obcordata S. Watson BCN, BCS, CAM, COL, DGO, GRO, JAL, MEX, MICH, MOR, NLE, OAX, PUE, QRO, QROO, SLP, SIN, SON, TAMS, VER, YUC

* Randia ovalifolia Bohr. GRO, OAX

*Randia pascualii Bohr. \& Salas-Morales OAX

Randia petenensis Lundell CHIS, OAX, VER

*Randia pringlei (S. Watson) A. Gray CHIH, COAH, DGO, HGO, NLE, QRO, SON, TAMS, ZAC

*Randia pterocarpa Lorence \& Dwyer OAX, VER

* Randia pueblensis Bohr. \& E. Martínez PUE

*Randia purpusii Greenm. \& C.H. Thomps. HGO, QRO, SLP, VER

*Randia retroflexa Lorence \& M. Nee CHIS, OAX, TAB, VER Randia rhagocarpa Standl. NLE, QRO, SLP, TAMS, VER

*Randia sepium Bohr. \& E. Martínez CHIS

*Randia serboi Bohr. \& Saynes OAX

*Randia similis Bohr. \& Salas-Morales OAX

*Randia sonorensis Wiggins CHIH, SON

*Randia tetracantha (Cav.) DC. CAM, CHIS, COL, DGO, GRO, JAL, MEX, MICH, MOR, NAY, OAX, PUE, SIN, TAB, VER

*Randia thurberi S. Watson BCN, CAM, CHIS, CHIH, COL, GTO, GRO, JAL, MEX, MICH, MOR, NAY, NLE, OAX, PUE, QRO, QROO, SIN, SON, TAMS, VER
Randia tomatillo Loes. CAM, TAB, TAMS, VER

*Randia truncata Greenm. \& C.H. Thomps. CAM, QROO, TAB, YUC

*Randia tubericollis Bohr., E. Martínez \& A. Nava JAL, OAX

* Randia vazquezii Lorence \& Dwyer CHIS, OAX, VER

*Randia veracruzana Bohr. \& E. Martínez VER

*Randia xalapensis M. Martens \& Galeotti CAM, CHIS, HGO, MICH, NLE, OAX, PUE, QRO, QROO, SLP, TAB, TAMS, VER, YUC

Relbunium hypocarpium (L.) Hemsl. CHIS, HGO, MEX, OAX, PUE, QRO, SLP, TAMS, TLAX, VER

Relbunium laevigatum (DC.) Hemsl. BCS, CHIS, CHIH, COAH, COL, CDMX.DGO, GTO, GRO, HGO, JAL, MEX, MICH, MOR, NAY, NLE, OAX, PUE, QRO, SLP, SIN, SON, TAMS, TLAX, VER, ZAC

*Renistipula galeottii (Standl.) Bohr. CAM, CHIS, OAX, TAB, VER

Renistipula izabalensis (Standl. \& Steyerm.) Bohr. OAX, VER *Resinanthus aromaticus (Cast.-Campos \& Lorence) Bohr. OAX, VER

Richardia brasiliensis Gomes OAX, TAMS, VER

*Richardia gandarae Rzed. GTO, JAL, SLP

Richardia scabra L. BCS, CHIS, COAH, COL, CDMX, DGO, GTO, GRO, JAL, MEX, MICH, MOR, NAY, OAX, PUE, SLP, SIN, SON, TAB, TAMS, TLAX, VER, YUC

Richardia tricocca (Torr. \& A. Gray) Standl. AGS, CHIH, COAH, CDMX, DGO, GTO, HGO, JAL, MEX, MICH, MOR, NLE, OAX, PUE, QRO, SLP, TAMS, TLAX, VER, ZAC

Rogiera amoena Planch. CHIS, COL, GRO, JAL, OAX, SIN, VER

*Rogiera breedlovei (Lorence) Bohr. CHIS

Rogiera cordata (Benth.) Planch. CHIS, GRO, JAL, OAX, VER Rogiera edwardsii (Standl.) Bohr. CHIS, OAX

Rogiera gratissima Planch. \& Linden CHIS, GRO, JAL, OAX, TAB, VER

*Rogiera langlassei (Standl.) Bohr. CHIS, COL, GTO, GRO, JAL, MICH, OAX, SIN, VER

*Rogiera ligustroides (Hemsl.) Bohr. CHIS, OAX, VER

*Rogiera macdougallii (Lorence) Bohr. OAX

*Rogiera oaxacensis Bohr. \& Velasco-Gutiérrez OAX

Rogiera stenosiphon (Hemsl.) Bohr. CHIS, GRO, OAX, TAB, TAMS, VER, YUC

Rogiera subscandens (Lundell) Bohr. CHIS, TAB

*Rogiera tabascensis Bohr. TAB

*Rogiera variiflora Bohr. CHIS

Rondeletia belizensis Standl. CHIS

Rovaeanthus strigosus (Benth.) Bohr. CHIS, COL, JAL, OAX, VER

Rovaeanthus suffrutescens (Brandegee) Bohr. CHIS

*Rudgea alvarezii Bohr. \& Lozada-Pérez CAM, GRO, OAX, QROO, YUC

Rudgea cornifolia (Kunth) Standl. CHIS, GRO, NAY, OAX, TAB, VER, YUC

* Sabicea mexicana Wernham OAX, PUE, VER

Sabicea villosa Willd. ex Roem. \& Schult. CHIS, OAX, TAB, VER

Sickingia vestita Lundell CAM, CHIS, QROO, TAB, VER 
Simira lancifolia (Lundell) E. Martínez \& Bohr. CHIS, TAB, VER

* Simira mexicana (Bullock) Steyerm. CHIS, CDMX, GRO, MEX, JAL, MICH, MOR

Simira rhodoclada (Standl.) Steyerm. CHIS, MOR, OAX, PUE, TAB, TAMS, VER

Simira salvadorensis (Standl.) Steyerm. CAM, CHIS, GRO, JAL, MICH, OAX, QROO, TAB, VER, YUC

Solenandra mexicana (A. Gray) Bohr. CAM, CHIS, COL, GRO, HGO, JAL, MICH, OAX, QRO, QROO, SLP, TAB, TAMS, VER, YUC

*Sommera arborescens Schltdl. CHIS, COL, OAX, PUE, VER Sommera chiapensis Brandegee CHIS, PUE, VER

* Sommera fusca Oerst. ex Standl. OAX

Sommera grandis (Bartl. ex DC.) Standl. CHIS, COL, DGO, GRO, JAL, MICH, NAY, OAX, SIN, VER

Sommera guatemalensis Standl. CHIS, OAX, VER

* Sommera parva Lorence CHIS

Spermacoce alata Aubl. CAM, CHIS, GRO, OAX, SLP, TAB, VER

Spermacoce confusa Rendle CAM, CHIS, COL, GTO, GRO, JAL, MEX, MICH, NAY, NLE, OAX, QRO, QROO, SLP, SIN, SON, TAMS, VER, YUC

*Spermacoce haenkeana Hemsl. MEX, MOR, QRO, VER

*Spermacoce nesiotica (B.L. Rob.) G.A. Levin COL

Spermacoce ovalifolia (M. Martens \& Galeotti) Hemsl. CAM, CHIS, COL, GRO, JAL, MEX, MICH, MOR, OAX, QROO, TAB, VER, YUC

Spermacoce tenuior L. CAM, CHIS, COAH, COL, CDMX, DGO, GTO, GRO, HGO, JAL, MEX, MICH, MOR, NAY, NLE, OAX, PUE, QRO, QROO, SLP, SIN, SON, TAB, TAMS, VER, YUC

Spermacoce tetraquetra A. Rich. CAM, CHIS, OAX, QROO, VER, YUC

Stenostomum lucidum (Sw.) C.F. Gaertn. CAM, CHIS, QROO, TAB, VER, YUC

*Stenotis arenaria (Rose) Terrell BCS

*Stenotis asperuloides (Benth.) Terrell BCS

*Stenotis australis (I.M. Johnst.) Terrell BCS

*Stenotis brevipes (Rose) Terrell BCN, BCS

*Stenotis gracilenta (I.M. Johnst.) Terrell BCS

* Stenotis mucronata (Benth.) Terrell BCS

* Stenotis peninsularis (Brandegee) Terrell BCS

Steyermarkia guatemalensis Standl. CHIS

Strumpfia maritima Jacq. QROO, YUC

* Stylosiphonia glabra Brandegee CHIS

* Syringantha coulteri (Hook. f.) T. McDowell GTO, HGO, QRO, SLP, TAMS, VER

*Warszewiczia uxpanapensis (Lorence) C.M. Taylor CHIS, OAX, TAB, VER

\section{Family Ruppiaceae}

Ruppia didyma Sw. ex Wikstr. COL, JAL, NAY, OAX, SIN, YUC

Ruppia maritima L. BCN, BCS, CAM, CHIS, COAH, COL, CDMX, GRO, JAL, MEX, MOR, NAY, OAX, PUE, QROO, SLP, SIN, SON, TAB, TAMS, VER, YUC
*Ruppia mexicana Den Hartog \& Van Tussenbroek CAM, CDMX, PUE, QROO, YUC

\section{Family Rutaceae}

Amyris attenuata Standl. CHIS, QROO, VER

Amyris balsamifera L. CAM, CHIS, GRO, MOR, NAY, OAX, QROO, SIN, SON

*Amyris carterae J. Rebman \& F. Chiang BCS, COAH, NLE

*Amyris cordata I.M. Johnst. TAMS

*Amyris chiapensis Lundell CHIS

Amyris elemifera L. CAM, CHIS, QROO, VER, YUC

Amyris madrensis S. Watson COAH, COL, GTO, JAL, NLE,

SLP, TAMS, VER, ZAC

*Amyris marshii Standl. COAH, NLE

*Amyris mexicana Lundell COL, JAL, MICH

*Amyris monophylla Brandegee NLE, OAX, PUE, TAMS

*Amyris purpusii $\mathrm{P}$. Wilson VER

*Amyris rekoi S.F. Blake CHIS, COL, GRO, HGO, JAL, MEX, MOR, NAY, OAX, PUE, QRO, SLP, VER

Amyris sylvatica Jacq. CAM, CHIS, COL, GRO, JAL, MICH, OAX, PUE, QROO, SLP, TAB, TAMS, VER, YUC

Amyris texana (Buckley) P.G. Wilson NLE, SLP, TAMS, VER

*Amyris thyrsiflora Turcz. HGO, VER

Angostura granulosa (Kallunki) Kallunki NAY

* Casimiroa calderoniae F. Chiang \& Medrano OAX, PUE

Casimiroa edulis La Llave \& Lex. AGS, BCS, CHIS, CHIH,

COL, CDMX, DGO, GTO, GRO, HGO, JAL, MEX, MICH,

MOR, NAY, OAX, PUE, QRO, SLP, SIN, SON, TAB, TAMS,

TLAX, VER, ZAC

*Casimiroa greggii (S. Watson) F. Chiang COAH, HGO, NLE, QRO, SLP, TAMS, VER

Casimiroa microcarpa Lundell CHIS

*Casimiroa pringlei (S. Watson) Engler COAH, DGO, NLE, SLP, TAMS

*Casimiroa pubescens Ramírez GTO, HGO, NLE, OAX, QRO, SLP, TAMS, VER

Casimiroa sapota Oerst. CHIS, COL, DGO, JAL, NAY, OAX, QRO, QROO, SLP, TAB, TAMS, VER, ZAC

Casimiroa tetrameria Millsp. CAM, CHIS, JAL, MICH, OAX, QROO, VER, YUC

*Casimiroa watsonii Engl. COL, JAL

Cneoridium dumosum (Nutt.) Hook. f. BCN

Choisya dumosa (Torr.) A. Gray CHIH, COAH, NLE

*Choisya katherinae C.H. Müll. CHIH, COAH, DGO

Choisya mollis Standl. MEX, SON, TAMS

* Choisya neglecta C.H. Müll. PUE

*Choisya palmeri A. Gray COAH, NLE, SLP, ZAC

*Choisya ternata (La Llave) Kunth GRO, HGO, MEX, OAX, PUE, QRO, SLP, TAMS, VER

*Decatropis bicolor (Zucc.) Radlk. CHIS, COAH, GTO, HGO, NLE, QRO, SLP, TAMS, VER

Decatropis paucijuga (Donn. Sm.) Loes. CHIS

*Decazyx esparzae F. Chiang CHIS, OAX, TAB, VER

Ertela trifolia (L.) Kuntze COL, JAL, MICH, OAX

Erythrochiton lindenii Planch. CHIS, OAX, TAB, VER

Esenbeckia berlandieri Baill. ex Hemsl. CAM, CHIS, CHIH,

COAH, COL, GTO, GRO, HGO, JAL, MICH, NAY, NLE, 
OAX, QRO, QROO, SLP, SIN, TAMS, VER, YUC, ZAC

*Esenbeckia bicolor Ramos CHIS

*Esenbeckia collina Brandegee JAL, MICH, OAX

*Esenbeckia conspecta (Kaastra) Ramos COL, JAL, MICH

*Esenbeckia dorantesii Ramos \& E. Martínez VER

Esenbeckia feddemae Kaastra COL, JAL, MICH

*Esenbeckia flava Brandegee BCS

* Esenbeckia hartmanii B.L. Rob. \& Fernald CHIH, SIN, SON

* Esenbeckia macrantha Rose OAX, PUE, SLP, VER

*Esenbeckia nesiotica Standl. COL, JAL, MICH, NAY

*Esenbeckia ovata Brandegee VER

Esenbeckia runyonii C.V. Morton COAH, HGO, NLE, QRO, SLP, TAMS

*Esenbeckia stephani Ramos OAX

*Esenbeckia vazquezii Ramos \& E. Martínez MOR

* Esenbeckia velutina Ramos GRO

*Helietta lottiae F. Chiang COL, GRO, JAL, OAX

*Helietta lucida Brandegee OAX, PUE

Helietta parvifolia (A. Gray ex Hemsl.) Benth. COAH, GTO, HGO, NLE, PUE, QRO, SLP, TAMS, VER

*Megastigma balsense F. Chiang \& J. Jiménez Ram. GRO, OAX

*Megastigma chiangii J. Jiménez Ram. \& Cruz-Durán GRO

* Megastigma galeottii Baill. OAX, PUE

*Megastigma morenoi Cuevas \& Guzm.-Hern. COL

Megastigma skinneri Hook. f. CHIS

Peltostigma guatemalense (Standl. \& Steyerm.) Gereau CHIS,

TAB, VER

Peltostigma pteleoides (Hook.) Walp. CHIS, DGO, HGO, JAL,

MICH, SLP, SIN, VER

Pilocarpus racemosus Vahl CAM, GRO, MICH, NAY, OAX, QROO, YUC

*Polyaster boronioides Hook. f. ex Benth. \& Hook. GTO, HGO, OAX, PUE, QRO, TAMS, VER

*Ptelea aptera Parry BCN

Ptelea trifoliata L. AGS, CHIH, COAH, COL, DGO, GTO, GRO, HGO, JAL, MEX, MICH, MOR, NLE, OAX, PUE, QRO, SLP, SON, TAMS, VER, ZAC

*Stauranthus conzattii Rose \& Standl. OAX

Stauranthus perforatus Liebm. CHIS, OAX, VER

Thamnosma montanum Torr. \& Frém. BCN, SON

*Thamnosma pailense M.C. Johnst. COAH

*Thamnosma stanfordii I.M. Johnst. COAH, DGO

Thamnosma texana (A. Gray) Torr. CHIH, COAH, DGO, HGO,

NLE, QRO, SLP, SON, TAMS, ZAC

*Thamnosma trifoliatum I.M. Johnst. BCS

Zanthoxylum acuminatum (Sw.) Sw. CAM, CHIS, OAX, QROO, QRO, SLP, TAB, TAMS, VER

*Zanthoxylum arborescens Rose BCS, CHIS, COL, DGO, HGO, JAL, MICH, MOR, NAY, OAX, PUE, SIN, SON, VER

Zanthoxylum caribaeum Lam. CAM, CHIS, COL, DGO, GRO, JAL, MOR, NAY, OAX, QRO, QROO, SLP, SIN, TAB, TAMS, VER, YUC

Zanthoxylum ciliatum Engl. CHIS, OAX, SIN, SON

Zanthoxylum clava-herculis L. COAH, HGO, QRO, SLP, TAMS, VER

Zanthoxylum ekmanii (Urb.) Alain CHIS, OAX, TAB, VER
*Zanthoxylum elegantissimum (Engl.) P.G. Wilson CHIS, GTO, QRO, SLP, VER

Zanthoxylum fagara (L.) Sarg. AGS, BCS, CAM, CHIS, CHIH, COAH, COL, DGO, GTO, GRO, HGO, JAL, MEX, MICH, MOR, NAY, NLE, OAX, PUE, QRO, QROO, SLP, SIN, SON, TAB, TAMS, VER, YUC, ZAC

*Zanthoxylum ferrisiae Standl. NAY

Zanthoxylum flavum Vahl CAM, QROO, VER, YUC

Zanthoxylum foliolosum Donn. Sm. CHIS, GRO, HGO, OAX, PUE, SLP, VER

Zanthoxylum limoncello Planch. \& Oerst. CHIS, GRO, JAL, MEX, MICH, MOR, OAX, PUE, VER

Zanthoxylum melanostictum Schltdl. \& Cham. CHIS, COL, GRO, JAL, OAX, PUE, TAB, VER

Zanthoxylum mollissimum (Engl.) P. Wilson CHIS, COL, GRO, JAL, MOR, OAX

Zanthoxylum monophyllum (Lam.) P. Wilson CHIS, SIN

*Zanthoxylum purpusii Brandegee OAX, PUE

Zanthoxylum quassiifolium (Donn. Sm.) Standl. \& Steyerm. CHIS, VER

Zanthoxylum rhoifolium Lam. CHIS, OAX, TAB

Zanthoxylum riedelianum Engl. CHIS, OAX, PUE, QROO, TAB, VER, YUC

*Zanthoxylum xicense Miranda HGO, VER

\section{Family Sabiaceae}

Meliosma alba (Schltdl.) Walp. COAH, HGO, NLE, PUE, QRO, SLP, TAMS, VER

Meliosma dentata (Liebm.) Urb. CHIS, CHIH, COL, CDMX, DGO, GRO, HGO, JAL, MEX, MICH, MOR, NAY, OAX, QRO, SLP, SIN, TAMS, VER

Meliosma dives Standl. \& Steyerm. CHIS

Meliosma glabrata (Liebm.) Urb. OAX, VER

Meliosma grandifolia (Liebm.) Urb. CHIS, OAX, VER

Meliosma idiopoda S.F. Blake CHIS, COL, JAL, OAX, VER

*Meliosma matudae Lundell CHIS

*Meliosma mexicana V.W. Steinm. QRO, SLP, TAMS

*Meliosma nesites I.M. Johnst. COL, JAL

* Meliosma oaxacana Standl. CHIS, OAX, TAMS

Meliosma occidentalis Cuatrec. OAX, VER

Meliosma seleriana Urb. CHIS

\section{Family Salicaceae}

*Abatia mexicana Standl. GRO, HGO, OAX, PUE, SLP, VER Banara dioica Benth. VER

Bartholomaea sessiliflora (Standl.) Standl. \& Steyerm. CHIS, OAX

Casearia aculeata Jacq. CAM, CHIS, COL, GRO, HGO, JAL, MEX, MICH, NAY, OAX, PUE, QRO, QROO, SLP, SIN, TAB, TAMS, VER, YUC

Casearia arborea (Rich.) Urb. CHIS, OAX, VER

* Casearia argentea Kunth CHIS

Casearia arguta Kunth CHIS, COL, DGO, GRO, HGO, JAL, MICH, MOR, NAY, OAX, PUE, SIN, TAB, VER, ZAC

Casearia bartlettii Lundell CHIS, MICH, TAB

Casearia commersoniana Cambess. CHIS, GRO, OAX, TAB, VER

Casearia emarginata Wright ex Griseb. CAM, QROO, YUC 
* Casearia guevarana Cast.-Campos \& E. Medina VER Casearia guianensis (Aubl.) Urb. CAM, CHIS, TAB, VER Casearia javitensis Kunth CHIS, OAX, TAB, VER

Casearia nitida (L.) Jacq. CAM, CHIS, COL, DGO, GRO, HGO, JAL, MEX, MICH, NAY, OAX, PUE, QRO, QROO, SLP, SIN, TAB, TAMS, VER, YUC, ZAC

* Casearia orizabana Briq. VER

Casearia sanchezii J. Linares \& D. Angulo CHIS

Casearia spiralis J.R. Johnston CHIS, OAX

Casearia sylvestris Sw. CAM, CHIS, COL, DGO, GRO, JAL, MICH, NAY, OAX, PUE, QRO, QROO, SLP, SIN, TAB, VER, YUC

Casearia tacanensis Lundell CHIS, OAX, VER

Casearia tremula (Griseb.) Griseb. ex C. Wright CAM, CHIS, COL, GRO, JAL, MEX, MICH, NAY, OAX, PUE, QROO, SIN Dendrostylis suaveolens Triana \& H. Karst. VER

Hasseltia guatemalensis Warb. CHIS

Hasseltiopsis dioica (Benth.) Sleumer CHIS, COL, JAL, OAX, TAB, VER

Homalium racemosum Jacq. CHIS, GRO, JAL, MICH, OAX, VER

Laetia thamnia L. CAM, CHIS, QROO, TAB, VER, YUC

Lindackeria laurina C. Presl CHIS

Lunania mexicana Brandegee CHIS, OAX, VER

*Neopringlea integrifolia (Hemsl.) S. Watson COAH, GTO, HGO, MEX, NLE, QRO, SLP, TAMS, VER

Neopringlea viscosa (Liebm.) Rose CHIS, COL, GRO, JAL, MEX, MOR, OAX, PUE, QRO, SLP

Olmediella betschleriana (Göpp.) Loes. CHIS, VER

Pleuranthodendron lindenii (Turcz.) Sleumer CHIS, HGO, OAX, PUE, QRO, SLP, TAB, TAMS, VER

Populus acuminata Rydb. CHIH, SON

Populus angustifolia James CHIH, COAH, SON

* Populus brandegeei Schneider BCS, CHIH, SON

Populus deltoides Marshall AGS, CHIH, COAH, DGO, JAL, NLE, SON, VER, ZAC

Populus fremontii S. Watson AGS, BCN, BCS, CHIS, CHIH, COAH, CDMX, DGO, GTO, HGO, JAL, MEX, NLE, PUE, QRO, SLP, SON, TAMS, ZAC

*Populus guzmanantlensis A. Vázquez \& Cuevas COL, JAL

*Populus mexicana Wesm. ex DC. CHIS, COAH, DGO, HGO, JAL, NLE, OAX, PUE, QRO, SLP, SIN, SON, TAMS, VER, ZAC

Populus palmeri Sarg. CHIH, COAH, SON

* Populus simaroa Rzed. GRO, JAL, MEX, MICH, OAX

Populus tremuloides Michx. AGS, BCN, CHIH, COAH, DGO, GTO, HGO, JAL, MEX, MICH, NLE, QRO, SLP, SIN, SON, TAMS, VER, ZAC

Populus trichocarpa Torr. \& A. Gray BCN

Prockia crucis P. Browne ex L. CAM, CHIS, COL, GRO, JAL, MEX, MICH, MOR, NAY, OAX, PUE, QROO, SLP, SIN, TAMS, VER, YUC

Prockia krusei J. Jiménez Ram. \& Cruz-Durán GRO

* Prockia oaxacana J. Jiménez Ram. \& Cruz-Durán OAX

* Salix aeruginosa E. Carranza MEX, MICH

Salix bonplandiana Kunth AGS, BCN, BCS, CAM, CHIS, CHIH, COAH, COL, CDMX, DGO, GTO, GRO, HGO, JAL,
MEX, MICH, MOR, NAY, NLE, OAX, PUE, QRO, SLP, SIN, SON, TAB, TLAX, VER, ZAC

*Salix cana M. Martens \& Galeotti COL, CDMX, HGO, JAL, MEX, MICH, MOR, OAX, PUE, TLAX, VER

Salix exigua Nutt. BCN, CHIH, COAH, DGO, SON, TAMS, VER

Salix exilifolia Dorn CHIH, COAH

Salix gooddingii C.R. Ball BCN, BCS, CHIH, COAH, COL, GRO, JAL, NLE, OAX, SIN, SON

* Salix hartwegii Benth. DGO, GRO, HGO, JAL, MEX, MICH, MOR, NAY

Salix hindsiana Benth. BCN

Salix humboldtiana Willd. AGS, CAM, CHIS, COAH, COL, CDMX, DGO, GTO, GRO, HGO, JAL, MEX, MICH, MOR, NAY, NLE, OAX, PUE, QRO, SLP, SON, TAB, TAMS, TLAX, VER, ZAC

*Salix jaliscana M.E. Jones CHIH, COL, DGO, JAL, MEX, MICH, NAY, NLE, OAX, SIN, SON, ZAC

Salix laevigata Bebb BCN, SON

Salix lasiolepis Benth. AGS, BCN, BCS, CHIH, COAH, DGO, GTO, HGO, JAL, MEX, MOR, NLE, QRO, SLP, SIN, SON, TAMS, VER, ZAC

* Salix mexicana Seemen GTO, HGO, MEX, PUE, TLAX, VER Salix nigra Marshall CHIH, COAH, DGO, NAY, NLE, OAX, PUE, SLP, SIN, TAMS, ZAC

* Salix paradoxa Kunth COAH, COL, CDMX, DGO, GTO, GRO, HGO, JAL, MEX, MICH, MOR, NLE, OAX, PUE, QRO, SLP, TAMS, TLAX, VER, ZAC

* Salix riskindii M.C. Johnst. COAH

Salix scouleriana Barratt COAH, SON

Salix taxifolia Kunth AGS, BCN, BCS, CHIS, CHIH, COAH, COL, DGO, GTO, GRO, HGO, JAL, MEX, MICH, MOR, NAY, NLE, OAX, PUE, QRO, SLP, SIN, SON, TAMS, VER, ZAC

Salix thurberi Rowlee CHIH, COAH, HGO, NLE, TAMS, VER Salix wrightii Andersson CHIH, SON

* Samyda mexicana Rose COL, GRO, JAL, MICH, OAX

* Samyda yucatanensis Standl. CAM, QROO, YUC

*Xylosma chiapensis Lundell CHIS, SIN

Xylosma chlorantha Donn. Sm. CHIS, OAX, TAB, VER

Xylosma flexuosa (Kunth) Hemsl. CAM, CHIS, COAH, COL, GTO, GRO, HGO, JAL, MEX, MICH, MOR, NAY, NLE, OAX, PUE, QRO, QROO, SLP, SIN, SON, TAB, TAMS, VER, YUC *Xylosma horrida Rose CHIS, COL, GRO, JAL, MEX, MICH, OAX

Xylosma intermedia (Seem.) Triana \& Planch. CHIS, COL, GTO, GRO, JAL, MEX, MICH, OAX, SIN, VER

Xylosma oligandra Donn. Sm. CHIS, OAX, VER

Xylosma panamensis Turcz. CHIS, COL, GRO, MICH, NAY, OAX, PUE, QROO, TAB, VER

Xylosma quichensis Donn. Sm. CHIS, OAX, PUE, VER

Xylosma sessilis Standl. \& Steyerm. CHIS

Xylosma velutina (Tul.) Triana \& Planch CHIS, COL, GRO, HGO, JAL, MEX, MICH, NAY, OAX, PUE, SLP, SIN, TAMS, VER, ZAC

Zuelania guidonia (Sw.) Britton \& Millsp. CAM, CHIS, HGO, OAX, PUE, QRO, QROO, SLP, TAB, TAMS, VER, YUC 


\section{Family Santalaceae}

Antidaphne viscoidea Poepp. \& Endl. CHIS

Arceuthobium abietinum (Engelm.) Hawksw. \& Wiens BCN, $\mathrm{CHIH}$

*Arceuthobium abietis-religiosae Heil. COAH, CDMX, GTO, HGO, JAL, MEX, MICH, NLE, PUE, QRO, TLAX

Arceuthobium apachecum Hawksw. \& Wiens COAH, SON

Arceuthobium blumeri A. Nelson DGO, SON

Arceuthobium campylopodum Engelm. BCN, NLE

Arceuthobium cyanocarpum J.M. Coult. \& A. Nelson CHIH

Arceuthobium divaricatum Engelm. BCN

Arceuthobium douglasii Engelm. COAH, DGO, NLE, SON

*Arceuthobium durangense (Hawskw. \& Wiens) Hawskw. \&

Wiens DGO, JAL, SIN

Arceuthobium gillii Hawksw. \& Wiens CHIH, GTO, JAL, SON, ZAC

Arceuthobium globosum Hawksw. \& Wiens CHIS, CHIH, COL, CDMX, DGO, GRO, HGO, JAL, MEX, MICH, MOR, OAX, PUE, SIN, SON, TLAX, VER, ZAC

Arceuthobium nigrum (Hawskw. \& Wiens) Hawskw. \& Wiens CHIS, CDMX, DGO, GTO, GRO, HGO, MEX, MICH, OAX, PUE, QRO, SIN, TLAX, VER, ZAC

*Arceuthobium oaxacanum Hawskw. \& Wiens OAX

*Arceuthobium pendens Hawksw. \& Wiens PUE, SLP, VER

*Arceuthobium rubrum Hawskw. \& Wiens DGO, SIN

*Arceuthobium strictum Hawskw. \& Wiens DGO

Arceuthobium vaginatum (Humb. \& Bonpl. ex Willd.) J. Presl CHIS, CHIH, COAH, CDMX, DGO, GRO, HGO, JAL, MEX, MICH, MOR, NAY, NLE, OAX, PUE, QRO, SIN, SON, TAB, TAMS, TLAX, VER, ZAC

*Arceuthobium verticilliflorum Engelm. DGO

*Arceuthobium yecorense Hawskw. \& Wiens CHIH, SON

Comandra pallida A. DC. CHIH, COAH, SON

Dendrophthora costaricensis Urb. VER

*Dendrophthora mexicana Kuijt CHIS

Dendrophthora obliqua (C. Presl) Wiens CHIS

Phoradendron aguilarii Standl. \& Steyerm. CHIS, OAX

Phoradendron angustifolium (Kunth) Nutt. OAX

Phoradendron annulatum Oliv. CAM, CHIS, OAX, PUE, QROO, VER, YUC

Phoradendron aurantiacum Trel. CHIS

Phoradendron bolleanum (Seem.) Eichler AGS, BCN, CHIH, COAH, DGO, GTO, HGO, JAL, NAY, NLE, PUE, QRO, SLP, SIN, SON, ZAC

Phoradendron brachystachyum (DC.) Nutt. AGS, BCN, BCS, CHIS, CHIH, COAH, COL, CDMX, DGO, GTO, GRO, HGO, JAL, MEX, MICH, MOR, NAY, NLE, OAX, PUE, QRO, SLP, SIN, SON, TAMS, TLAX, VER, ZAC

*Phoradendron brevifolium Oliv. OAX, PUE

Phoradendron californicum Nutt. BCN, BCS, SIN, SON

Phoradendron capitellatum Torr. ex Trel. CHIH, SON

*Phoradendron carneum Urb. AGS, CHIS, COL, CDMX, DGO, GTO, GRO, JAL, MEX, MICH, MOR, NAY, OAX, PUE, QRO, SIN, VER, ZAC

Phoradendron cockerellii Trel. CHIH

Phoradendron coryae Trel. BCN, CHIH, COAH, DGO, JAL,
SON, ZAC

Phoradendron crassifolium (Pohl ex DC.) Eichler CHIS, GRO, TAB

Phoradendron cheirocarpum Trel. CHIS

Phoradendron chrysocladon A. Gray CHIS

Phoradendron densum Torr. ex Trel. BCN, CHIH, COAH, NLE, SLP, SON

*Phoradendron diguetii Tiegh. BCN, BCS, SON

Phoradendron dipterum Eichler JAL, OAX, PUE, VER

* Phoradendron dolichocarpum Kuijt GRO, JAL, MICH

Phoradendron engelmannii Trel. CHIH, COAH

Phoradendron falcatum Eichler CHIS, COL, GTO, GRO, JAL, MICH, MEX, MOR, NAY, OAX, PUE, QRO, SON, VER, ZAC *Phoradendron falcifer Kuijt CHIS, COL, HGO, JAL, MICH, OAX, PUE, SLP, VER

Phoradendron flavescens Millsp. CHIH, COAH, DGO, MICH, QROO, SLP, SIN, TAMS, VER

*Phoradendron flavum I.M. Johnst. COAH, DGO, NLE, SLP, ZAC

*Phoradendron forestierae B.L. Rob. \& Greenm. AGS, GTO, HGO, JAL, MICH, OAX, PUE, QRO, SLP, TLAX, ZAC

*Phoradendron galeottii Trel. DGO, GTO, GRO, HGO, JAL, MEX, OAX, PUE, QRO, SLP, TLAX, VER

*Phoradendron greggii Trel. COAH, NLE

*Phoradendron hawksworthii Wiens COAH, NLE

Phoradendron herbert-smithii Trel. CHIS, OAX, VER

Phoradendron hexastichum (DC.) Griseb. GRO, PUE, VER

Phoradendron heydeanum Trel. CHIS

*Phoradendron iltisiorum Kuijt OAX

Phoradendron juniperinum Engelm. BCN, CHIH, COAH, DGO, SIN, SON, TLAX

*Phoradendron kingii Kuijt CHIS

*Phoradendron lanatum Trel. CHIH, HGO, OAX, PUE, QRO, VER

*Phoradendron lanceolatum Engelm. ex A. Gray AGS, CHIH, COAH, DGO, GTO, GRO, HGO, JAL, MEX, MICH, MOR, NLE, OAX, PUE, QRO, SLP, TAMS, TLAX, VER, ZAC

Phoradendron leucarpum (Raf.) Reveal \& M.C. Johnst. BCN, BCS, CHIS, CHIH, COAH, DGO, HGO, MICH, NAY, NLE, OAX, PUE, QRO, SLP, SIN, SON, TAMS, TLAX, ZAC

Phoradendron libertadanum Trel. CHIS

*Phoradendron longifolium Eichler ex Trel. CHIS, CHIH, COL, DGO, GTO, GRO, HGO, JAL, MEX, MICH, NAY, OAX, PUE, QRO, SLP, SIN, SON, TAMS, ZAC

*Phoradendron minutifolium Urb. COAH, COL, DGO, JAL, PUE, SIN, TLAX, VER

Phoradendron mucronatum (DC.) Krug. \& Urb. CAM, CHIS, QROO, TAB, VER, YUC

Phoradendron naviculare Kuijt CHIS

Phoradendron nervosum Oliv. CAM, CHIS, GRO, HGO, OAX, PUE, QRO, QROO, SLP, VER

*Phoradendron nudum Kuijt JAL, MICH, MOR

*Phoradendron olae Kuijt GRO, OAX, PUE

* Phoradendron olivae Wiens COL, JAL

* Phoradendron oliverianum Trel. PUE, VER

* Phoradendron palmeri Greenm. DGO, QRO, SLP, SIN, SON

Phoradendron pedicellatum (Tiegh.) Kuijt CHIS, GRO, OAX, 
PUE, QROO, TAB, VER

*Phoradendron perredactum Rzed. \& Calderón GRO, MEX, MOR, OAX

Phoradendron piperoides (Kunth) Trel. CHIS, GRO, MEX, OAX, PUE, TAB, VER

*Phoradendron puberulum Trel. MICH, VER

*Phoradendron purpusii Trel. OAX, VER

Phoradendron quadrangulare (Kunth) Griseb. CAM, CHIS, CHIH, COL, CDMX, DGO, GTO, GRO, HGO, JAL, MEX, MICH, MOR, NAY, OAX, PUE, QRO, QROO, SLP, SIN, SON, TAB, TAMS, VER, YUC

*Phoradendron ramosissimum Kuijt CHIS

Phoradendron reichenbachianum (Seem.) Oliv. AGS, CHIS, COL, CDMX, DGO, GTO, GRO, HGO, JAL, MEX, MICH, MOR, NAY, OAX, PUE, QRO, SLP, SIN, TLAX, VER, ZAC *Phoradendron rhipsalinum Rzed. GTO, JAL, MEX, MICH, QRO

Phoradendron robinsonii Urb. CHIS, COL, GTO, GRO, HGO, JAL, MEX, MICH, MOR, NAY, OAX, PUE, QRO, SLP, TAMS, VER, YUC

Phoradendron robustissimum Eichler CAM, CHIS, OAX, QROO, VER

Phoradendron rondeletiae Trel. CHIS

*Phoradendron saltillense Trel. COAH, NLE

Phoradendron spathulatum Kuijt CHIS, GRO, MEX, OAX, PUE, TLAX

*Phoradendron teretifolium Kuijt VER

*Phoradendron tetrapterum Krug \& Urb. COL, JAL, VER

* Phoradendron thyrsoideum Trel. SLP, TAMS

Phoradendron tonduzii Trel. CHIS, COL, GRO, JAL, MICH, VER

Phoradendron treleaseanum Standl. \& Steyerm. CHIS, OAX Phoradendron undulatum (Pohl ex DC.) Eichler CHIS

Phoradendron velutinum (DC.) Oliv. CHIS, CHIH, COL, CDMX, GTO, GRO, HGO, JAL, MEX, MICH, MOR, OAX, PUE, QRO, SLP, SON, TAMS, TLAX, VER

Phoradendron villosum (Nutt.) Nutt. ex Engelm. AGS, BCN, BCS, CHIH, COAH, DGO, GTO, JAL, NLE, SLP, SON, ZAC Phoradendron wattii Krug \& Urb. CAM, CHIS, COL, JAL, MEX, MICH, MOR, NAY, OAX, PUE, QROO, VER, YUC * Phoradendron wawrae Trel. CHIS, VER

\section{Family Sapindaceae}

Acer brachypterum Wooton \& Standl. CHIH, SON

Acer grandidentatum Nutt. CHIH, COAH, NLE, SON, TAMS

*Acer mexicanum A. Gray NLE, TAMS

Acer negundo L. AGS, CHIS, CHIH, COAH, COL, CDMX, DGO, GRO, HGO, JAL, MEX, MICH, NLE, OAX, PUE, QRO, SLP, SON, TAMS, TLAX, VER

Acer skutchii Rehder CHIS, CHIH, COAH, COL, GRO, JAL, MICH, SLP, TAMS, VER

*Aesculus parryi A. Gray BCN

Allophylus camptostachys Radlk. CAM, CHIS, JAL, NAY, OAX, QROO, TAB, VER, YUC, ZAC

Allophylus cominia (L.) Sw. CAM, CHIS, GRO, MEX, MICH, OAX, QROO, TAB, VER, YUC

Allophylus psilospermus Radlk. CAM, CHIS, OAX, QROO,
VER, YUC

Allophylus racemosus Sw. CHIS, TAB, VER

*Averrhoidium spondioides (Standl.) Acev.-Rodr. \& M.S. Ferrucci COL, GRO, JAL, NAY

*Balsas guerrerensis Cruz-Durán \& K. Vega GRO, OAX Billia hippocastanum Peyr. CHIS, GRO, OAX, PUE, VER Blomia prisca (Standl.) Lundell CAM, CHIS, QROO, YUC

*Cardiospermum cuchujaquense M.S. Ferrucci \& Acev.-Rodr. SON

*Cardiospermum dissectum (S. Watson) Radlk. CHIH, NLE, TAMS

Cardiospermum grandiflorum Sw. CAM, CHIS, GRO, HGO, MEX, MICH, OAX, PUE, TAB, VER, ZAC

Cardiospermum halicacabum L. AGS, BCN, BCS, CAM, CHIS, CHIH, COAH, COL, CDMX, DGO, GTO, GRO, HGO, JAL, MEX, MICH, MOR, NAY, NLE, OAX, PUE, QRO, QROO, SLP, SIN, SON, TAB, TAMS, TLAX, VER, YUC, ZAC

* Cardiospermum spinosum Radlk. BCS

* Cardiospermum tortuosum Benth. BCS

Cupania belizensis Standl. CAM, CHIS, GRO, OAX, PUE, QROO, VER, YUC

Cupania dentata Moc. \& Sessé ex DC. CAM, CHIS, COL, GRO, HGO, JAL, MICH, NAY, OAX, PUE, QRO, QROO, SLP, SIN, TAB, TAMS, VER, YUC

Cupania glabra Sw. CAM, CHIS, COL, GRO, HGO, JAL, MEX, MICH, NAY, OAX, PUE, QROO, SLP, SIN, TAB, TAMS, VER, YUC

Cupania guatemalensis (Turcz.) Radlk. CHIS, JAL, MICH, OAX, TAMS, VER

Cupania juglandifolia A. Rich. CHIS, VER

Cupania macrophylla A. Rich. CHIS, COL, GRO, JAL, MICH, OAX, TAB, TAMS, VER

Cupania mayana Lundell CHIS, TAB, VER

Cupania mollis Standl. CHIS, OAX, VER

Cupania rufescens Triana \& Planch. CHIS, PUE, VER

Cupania scrobiculata Rich. CHIS, OAX, VER

Cupania spectabilis Radlk. CHIS, TAB, VER

Cupania sylvatica Seem. CHIS

Dodonaea viscosa (L.) Jacq. AGS, BCN, BCS, CHIS, CHIH, COAH, COL, CDMX, DGO, GTO, GRO, HGO, JAL, MEX, MICH, MOR, NAY, NLE, OAX, PUE, QRO, QROO, SLP, SIN, SON, TAMS, TLAX, VER, YUC, ZAC

*Exothea copalillo (Schltdl.) Radlk. CHIS, GTO, HGO, QRO, SLP, TAMS, VER

Exothea diphylla (Standl.) Lundell CAM, QROO, YUC

Exothea paniculata (Juss.) Radlk. CAM, CHIS, COL, GTO, HGO, JAL, MICH, OAX, QRO, SLP, QROO, TAMS, VER

*Houssayanthus biternatus (Weath.) Rzed. \& Calderón GRO

*Houssayanthus serjanioides Rzed. \& Calderón HGO, QRO, SLP

Matayba clavelligera Radlk. CHIS, OAX, VER

* Matayba floribunda Radlk. OAX

Matayba glaberrima Radlk. CHIS, VER

Matayba mexicana Radlk. VER

Matayba oppositifolia (A. Rich.) Britton CAM, CHIS, COL, OAX, QROO, SLP, TAB, VER, YUC

Matayba scrobiculata (Kunth) Radlk. CHIS, COL, GRO, JAL, 
OAX, VER

Paullinia clavigera Schltdl. CAM, CHIS, COL, GRO, HGO, JAL, MICH, NAY, OAX, QROO, TAB, VER

Paullinia costaricensis Radlk. CAM, CHIS, COL, JAL, OAX, PUE, QROO, TAB, VER, YUC

Paullinia costata Schltdl. \& Cham. CHIS, COL, JAL, OAX, TAB, TAMS, VER

Paullinia cururu L. CAM, CHIS, COL, GRO, JAL, MICH, NAY, OAX, QROO, YUC

Paullinia fuscescens Kunth CAM, CHIS, COL, GRO, JAL, MOR, NAY, OAX, QROO, SLP, SIN, SON, TAMS, VER, YUC Paullinia jamaicensis Macfad. COL, JAL

Paullinia pinnata L. CAM, CHIS, COL, GRO, HGO, JAL, MICH, NAY, OAX, PUE, QROO, TAB, TAMS, VER, YUC

Paullinia scarlatina Radlk. CHIS

Paullinia sessiliflora Radlk. COL, GRO, JAL, MICH, NAY, OAX, QROO

*Paullinia sonorensis $\mathrm{S}$. Watson BCN, BCS, JAL, SIN, SON

Paullinia tomentosa Jacq. CAM, CHIS, COL, GRO, HGO, JAL, MEX, MICH, NLE, OAX, PUE, QRO, QROO, SLP, SIN, TAB, TAMS, VER, YUC, ZAC

Paullinia turbacensis Kunth CHIS

*Paullinia venosa Radlk. VER

Sapindus saponaria L. BCS, CAM, CHIS, CHIH, COAH, COL, DGO, GTO, GRO, HGO, JAL, MEX, MICH, MOR, NAY, NLE, OAX, PUE, QRO, QROO, SLP, SIN, SON, TAB, TAMS, VER, YUC

Serjania acuta Triana \& Planch. CHIS, HGO, VER

Serjania adiantoides Radlk. CAM, CHIS, QROO, VER, YUC

*Serjania albida Radlk. BCS, SIN

Serjania atrolineata C. Wright CHIS, GRO, NAY, OAX, QROO, SIN, TAB, VER, YUC

Serjania brachycarpa A. Gray ex Raldk. AGS, COAH, COL, JAL, MICH, NLE, SLP, TAMS, VER

*Serjania brachylopha Radlk. JAL

*Serjania brachystachya Radlk. CHIS, COL, GRO, MICH, OAX, VER

*Serjania californica Radlk. BCS

*Serjania cambessedeana Schltdl. \& Cham. OAX, SLP, TAMS, VER

Serjania caracasana (Jacq.) Willd. CHIS, GRO, MEX, MICH, OAX, QROO, VER

Serjania cardiospermoides Schltdl. \& Cham. CHIS, GRO, HGO, JAL, MEX, MICH, MOR, NAY, OAX, PUE, QRO, SLP, SIN, TAMS, VER, ZAC

*Serjania cystocarpa Radlk. COAH, NLE, SLP, TAMS

Serjania depauperata Radlk. CHIS

*Serjania emarginata Kunth GRO, MICH

* Serjania flaviflora Radlk. COL, JAL, MICH, OAX, PUE, VER

*Serjania fuscopunctata Radlk. COL, JAL

Serjania goniocarpa Radlk. CAM, CHIS, COL, GRO, HGO, JAL, MICH, OAX, QROO, TAB, VER, YUC

Serjania grosii Schltdl. CAM, CHIS, COL, DGO, GRO, JAL, MICH, NAY, OAX, QROO, TAB, VER, YUC

*Serjania heterocarpa Standl. OAX, PUE

Serjania hispida Standl. \& Steyerm. CHIS

*Serjania impressa Radlk. VER
Serjania incisa Torr. AGS, COAH, JAL, NLE

Serjania insignis Radlk. CHIS

Serjania lobulata Standl. \& Steyerm. CHIS, DGO, MICH, NAY, OAX, PUE, VER

Serjania lundellii Croat CAM, CHIS, GRO, JAL

Serjania macrocarpa Standl. \& Steyerm. CHIS, GRO, MICH, OAX, TAB, VER

*Serjania macrococca Radlk. OAX

Serjania mexicana (L.) Willd. CAM, CHIS, CHIH, COL, GRO, JAL, MEX, MICH, MOR, NAY, OAX, QROO, SIN, SON, TAB, VER, ZAC

*Serjania oaxacana Standl. OAX

* Serjania pacifica Standl. JAL, NAY, SIN

* Serjania palmeri S. Watson SIN, SON

Serjania paniculata Kunth CAM, CHIS, GRO, OAX, QROO, VER, YUC

Serjania paucidentata DC. CHIS, OAX, TAB, VER

*Serjania plicata Radlk. COL, JAL, TAB, VER, YUC

Serjania polystachya (Turcz.) Radlk. JAL, OAX

Serjania psilophylla Radlk. CHIS, COL, JAL, OAX

Serjania pterantha Standl. CAM, CHIS, QROO, TAB, VER, YUC

Serjania punctata Radlk. CHIS, MEX, NAY, OAX, VER

*Serjania pygmaea (Radlk.) Ferrucci \& Medina GRO, OAX, PUE

Serjania racemosa Schumach. CAM, CHIS, COL, GTO, GRO, JAL, MEX, MICH, MOR, NAY, NLE, OAX, PUE, QRO, QROO, SLP, SIN, TAMS, VER, YUC, ZAC

Serjania rachiptera Radlk. CAM, CHIS, QRO, SLP, VER

* Serjania rekoi Standl. OAX, VER

* Serjania rhytidococca Acev.-Rodr. GRO, QRO

* Serjania rutifolia Radlk. SIN, SON

*Serjania schiedeana Schltdl. AGS, COL, GRO, JAL, MEX, MICH, MOR, NAY, OAX, PUE, TAMS, ZAC

Serjania sordida Radlk. OAX, TAB, VER

*Serjania sphenocarpa Radlk. SON

*Serjania subtriplinervis Radlk. COL, GRO, JAL, MICH, OAX Serjania trachygona Radlk. CAM

*Serjania trifoliolata Radlk. COL, GRO, JAL, MEX, MICH, MOR, SIN

Serjania triquetra Radlk. CAM, CHIS, COL, GRO, JAL, MEX, MICH, MOR, NAY, OAX, PUE, QROO, SLP, SIN, TAB, VER, YUC, ZAC

* Serjania vesicosa Radlk. QRO

Serjania yucatanensis Standl. CAM, CHIS, QROO, YUC

Talisia floresii Standl. CAM, CHIS, QROO, TAB, VER, YUC

Talisia oliviformis (Kunth) Radlk. CAM, CHIS, OAX, QROO, TAB, VER, YUC

Thinouia myriantha Triana \& Planch. VER

Thinouia tomocarpa Standl. CAM, CHIS, QROO, VER

Thouinia acuminata S. Watson CHIS, CHIH, COL, GRO, JAL, MICH, NAY, OAX, PUE, QRO, SLP, SON, VER, ZAC

Thouinia paucidentata Radlk. CAM, CHIS, COL, GRO, JAL, MEX, MICH, NAY, OAX, QROO, YUC

*Thouinia serrata Radlk. COL, JAL, NAY, OAX

*Thouinia villosa DC. CAM, CHIH, COL, DGO, GRO, HGO, JAL, MEX, MICH, MOR, NLE, OAX, PUE, QRO, QROO, 
SLP, SIN, SON, TAMS, ZAC

Thouinidium decandrum (Bonpl.) Radlk. CAM, CHIS, COL, DGO, GRO, JAL, MEX, MICH, MOR, NAY, OAX, SIN, TAB, VER, ZAC

*Thouinidium insigne (Brandegee) Radlk. OAX, PUE

*Thouinidium oblongum Radlk. HGO, PUE, VER

Ungnadia speciosa Endl. CHIH, COAH, NLE, SLP, SON, TAMS, VER

Urvillea ulmacea Kunth CAM, CHIS, COAH, COL, DGO, GTO, GRO, HGO, JAL, MEX, MICH, MOR, NAY, NLE, OAX, PUE, QRO, QROO, SLP, SIN, TAB, TAMS, VER, YUC

\section{Family Sapotaceae}

Chrysophyllum mexicanum Brandegee CAM, CHIS, GRO, HGO, OAX, PUE, QRO, QROO, SLP, TAB, TAMS, VER, YUC

Chrysophyllum oliviforme L. CHIS, SLP

Chrysophyllum venezuelanense (Pierre) T.D. Penn. CAM, CHIS, OAX, PUE, QROO, TAB, VER

Manilkara chicle (Pittier) Gilly CAM, CHIS, OAX, PUE, QROO, TAB, VER

Manilkara zapota (L.) P. Royen CAM, CHIS, CHIH, COL, DGO, GRO, HGO, JAL, MEX, MICH, MOR, NAY, OAX, PUE, QRO, QROO, SLP, SIN, SON, TAB, TAMS, VER, YUC, ZAC

Micropholis melinoniana Pierre CHIS, GRO, OAX, TAB, VER Pouteria amygdalina (Standl.) Baehni CAM, CHIS, QROO

Pouteria belizensis (Standl.) Cronquist CHIS, TAB, VER

Pouteria briocheoides Lundell CHIS

Pouteria campechiana (Kunth) Baehni CAM, CHIS, COL, GRO, HGO, JAL, MEX, MICH, MOR, NAY, OAX, PUE, QRO, QROO, SLP, SIN, TAB, VER, YUC

Pouteria durlandii (Standl.) Baehni CAM, CHIS, OAX, PUE, QROO, SLP, TAB, VER

Pouteria glomerata (Miq.) Radlk. CAM, CHIS, HGO, PUE, QRO, SLP, TAB, VER, YUC

Pouteria izabalensis (Standl.) Baehni CHIS

Pouteria reticulata (Engl.) Eyma CAM, CHIS, OAX, QROO, TAB, VER, YUC

* Pouteria rhynchocarpa T.D. Penn. OAX, VER

Pouteria sapota (Jacq.) H.E. Moore \& Stearn CAM, CHIS, COL, GTO, GRO, JAL, MEX, MICH, MOR, OAX, PUE, QRO, QROO, SLP, SIN, TAB, VER, YUC

Pouteria squamosa Cronquist OAX, VER

Pouteria torta (Mart.) Radlk. CHIS, OAX, TAB, VER

Pouteria viridis (Pittier) Cronquist CHIS, VER

* Sideroxylon altamiranoi (Rose \& Standl.) T.D. Penn. GTO, HGO, QRO, SLP, VER

Sideroxylon americanum (Mill.) T.D. Penn. CAM, GRO, MOR, OAX, QROO, VER, YUC

Sideroxylon capiri (A. DC.) Pittier CHIS, CHIH, COL, DGO, GRO, JAL, MEX, MICH, MOR, NAY, OAX, PUE, QRO, QROO, SLP, SIN, SON, TAB, VER, ZAC

*Sideroxylon cartilagineum (Cronquist) T.D. Penn. BCS, COL, GRO, JAL, MEX, MICH, MOR, NAY, OAX, SIN, ZAC

Sideroxylon celastrinum (Kunth) T.D. Penn. CAM, CHIS, CHIH, COAH, COL, DGO, GRO, HGO, JAL, MICH, NLE,
OAX, QRO, QROO, SLP, SIN, TAB, TAMS, VER, YUC

Sideroxylon contrerasii (Lundell) T.D. Penn. CHIS, HGO, OAX, PUE, QRO, VER

Sideroxylon durifolium (Standl.) T.D. Penn. CHIS

*Sideroxylon eriocarpum (Greenm. \& Conz.) T.D. Penn. GRO, MOR, OAX

Sideroxylon eucoriaceum (Lundell) T.D. Penn. CHIS, VER

*Sideroxylon eucuneifolium (Lundell) T.D. Penn. CHIS

*Sideroxylon excavatum T.D. Penn. GRO, OAX

Sideroxylon foetidissimum Jacq. CAM, CHIS, QROO, YUC

Sideroxylon lanuginosum Michx. AGS, CHIH, COAH, JAL, NLE, SLP, SIN, SON, TAMS, VER

Sideroxylon leucophyllum S. Watson BCN, BCS, SON

Sideroxylon obtusifolium (Roem. \& Schult.) T.D. Penn. CAM, CHIS, COL, GRO, JAL, MICH, OAX, PUE, QROO, TAB, VER, YUC

*Sideroxylon occidentale (Hemsl.) T.D. Penn. BCN, BCS, CHIH, JAL, MICH, SIN, SON, VER

*Sideroxylon palmeri (Rose) T.D. Penn. CHIS, DGO, GTO, HGO, JAL, MEX, MICH, NAY, OAX, PUE, QRO, QROO, SLP, SIN, TAB, TAMS, VER

*Sideroxylon peninsulare (Brandegee) T.D. Penn. BCS, JAL, MICH, SIN

Sideroxylon persimile (Hemsl.) T.D. Penn. CAM, CHIS, CHIH, COL, DGO, GRO, HGO, JAL, MICH, MOR, NAY, OAX, PUE, QROO, SIN, SON, TAB, VER, ZAC

Sideroxylon portoricense Urb. CHIS, COL, GRO, HGO, JAL, MEX, MOR, OAX, SLP, TAB, VER

Sideroxylon salicifolium (L.) Lam. CAM, CHIS, COL, GRO, JAL, OAX, PUE, QROO, TAMS, VER, YUC

* Sideroxylon socorrense (Brandegee) T.D. Penn. COL, NAY, SIN

Sideroxylon stenospermum (Standl.) T.D. Penn. CHIS, COL, GRO, JAL, MICH, NAY, OAX, SIN, VER

Sideroxylon stevensonii (Standl.) T.D. Penn. CHIS

Sideroxylon tepicense (Standl.) T.D. Penn. CHIS, CHIH, COL, GRO, MEX, NAY, OAX, SLP, SIN, SON

*Sideroxylon verruculosum (Cronquist) T.D. Penn. CHIS, HGO, OAX, QRO, SLP, VER

\section{Family Saururaceae}

Anemopsis californica (Nutt.) Hook. \& Arn. AGS, BCN, BCS, CHIH, COAH, CDMX, DGO, JAL, MEX, NLE, QRO, SLP, SIN, SON, ZAC

Saururus cernuus L. COAH, GRO, MICH

\section{Family Saxifragaceae}

*Heuchera acutifolia Rose HGO, OAX, PUE, VER

*Heuchera amoena Rosend., Butters \& Lakela COAH, NLE

Heuchera brevistaminea Wiggins BCN

Heuchera hemsleyana Rosend., Butters \& Lakela DGO, HGO, JAL, MEX, MICH, MOR, OAX, QRO

*Heuchera lakelae Folk COAH

*Heuchera longipetala Moc. ex Ser. COL, JAL, MOR, OAX, ZAC

*Heuchera mexicana W. Schaffn. ex Small \& Rydb. COAH, CDMX, DGO, GTO, JAL, MEX, MICH, MOR, NLE, OAX, PUE, QRO, SLP, TAMS, VER, ZAC 
*Heuchera orizabensis Hemsl. AGS, CDMX, DGO, GTO, GRO, HGO, JAL, MEX, MICH, MOR, OAX, PUE, QRO, SLP, SIN, TAMS, TLAX, VER, ZAC

Heuchera parvifolia Nutt. ex Torr. \& A. Gray CHIH, SON

Heuchera rubescens Torr. AGS, BCN, CHIH, COAH, DGO, NLE, OAX, SLP, SIN, SON, ZAC

Heuchera sanguinea Engelm. CHIH, NLE, SON

Jepsonia parryi (Torr.) Small BCN

Lithophragma affine A. Gray BCN

Lithophragma cymbalaria Torr. \& A. Gray BCN

Lithophragma heterophyllum (Hook. \& Arn.) Hook. \& Arn. $\mathrm{BCN}$

Lithophragma tripartitum (Greene) Greene BCN

* Micranthes mexicana (Engelm. \& Irmsch.) Brouillet \& Gornall CHIH, COAH, DGO, SON

* Saxifraga eriophora $\mathrm{S}$. Watson $\mathrm{CHIH}$

\section{Family Scrophulariaceae}

Alectra aspera (Cham. \& Schltdl.) L.O. Williams CHIS

Alonsoa meridionalis (L. f.) Kuntze CHIS, GRO, OAX, PUE, VER

Brachystigma wrightii (A. Gray) Pennell CHIH, SON

Buddleja americana L. CAM, CHIS, CDMX, GTO, GRO, HGO, JAL, MEX, MICH, MOR, NAY, OAX, PUE, QRO, QROO, SLP, TAMS, VER

Buddleja cordata Kunth AGS, CHIS, CHIH, COAH, COL, CDMX, DGO, GTO, GRO, HGO, JAL, MEX, MICH, MOR, NAY, NLE, OAX, PUE, QRO, SLP, SIN, SON, TAMS, TLAX, VER, ZAC

*Buddleja corrugata M.E. Jones BCS, SON

Buddleja crotonoides A. Gray BCS, CHIS, CHIH, GRO, JAL, MICH, MOR, OAX, PUE, VER

*Buddleja chapalana B.L. Rob. JAL

*Buddleja elliptica M. Martens \& Galeotti MICH

Buddleja marrubiifolia Benth. AGS, CHIH, COAH, DGO,

GTO, JAL, NLE, SLP, TAMS, ZAC

Buddleja megalocephala Donn. Sm. CHIS, OAX, VER

Buddleja nitida Benth. CHIS, VER

*Buddleja ovandensis Lundell ex E.M. Norman CHIS

*Buddleja parviflora Kunth AGS, CHIS, CHIH, COAH, COL, CDMX, DGO, GTO, GRO, HGO, JAL, MEX, MICH, MOR, NAY, NLE, OAX, PUE, QRO, SLP, SIN, SON, TAMS, TLAX, VER, ZAC

*Buddleja perfoliata Kunth AGS, CDMX, GTO, GRO, HGO, JAL, MEX, MOR, OAX, PUE, QRO, SLP, TAMS, TLAX, VER, ZAC

Buddleja scordioides Kunth AGS, CHIH, COAH, CDMX, DGO, GTO, HGO, JAL, MEX, NLE, OAX, QRO, SLP, TAMS, VER, ZAC

Buddleja sessiliflora Kunth AGS, BCN, CHIH, COAH, COL, CDMX, DGO, GTO, GRO, HGO, JAL, MEX, MICH, MOR, NAY, NLE, OAX, PUE, QRO, SLP, SIN, SON, TAMS, TLAX, VER, ZAC

Buddleja skutchii C.V. Morton CHIS, GRO, HGO, OAX, VER Capraria biflora L. CAM, CHIS, COL, GTO, GRO, HGO, JAL, MEX, MICH, MOR, NAY, OAX, PUE, QRO, QROO, SLP, SIN, SON, TAB, TAMS, VER, YUC
Capraria frutescens (Mill.) Britton CAM, CHIS, COL, DGO, GRO, HGO, JAL, MEX, MICH, MOR, OAX, QRO, QROO, SLP, SIN, TAMS, VER, YUC

Capraria mexicana Moric. ex Benth. CAM, CHIS, GTO, GRO, JAL, MICH, OAX, QRO, QROO, SLP, TAB, TAMS, VER, YUC

*Emorya rinconensis Mayfield COAH

Emorya suaveolens Torr. CHIH, COAH, DGO, NLE

Eremogeton grandiflorus (A. Gray) Standl. \& L.O. Williams CHIS

*Leucophyllum alejandrae G.L. Nesom NLE

*Leucophyllum ambiguum Bonpl. HGO, JAL, QRO, SLP, VER, ZAC

Leucophyllum candidum I.M. Johnst. CHIH, COAH, DGO, HGO, NLE, SLP, ZAC

*Leucophyllum coahuilensis Henr. COAH

*Leucophyllum flyrii B.L. Turner SLP

Leucophyllum frutescens (Berland.) I.M. Johnst. CHIH, COAH, DGO, HGO, JAL, NLE, QRO, SLP, SON, TAMS, VER, ZAC

*Leucophyllum hintoniorum G.L. Nesom NLE, TAMS

*Leucophyllum laevigatum Standl. AGS, CHIH, COAH, DGO, GTO, NLE, SLP, ZAC

*Leucophyllum langmaniae Flyr COAH, NLE, TAMS

Leucophyllum minus A. Gray CHIH, COAH, DGO, NLE, SLP, TAMS, ZAC

*Leucophyllum pringlei (Greenm.) Standl. OAX, PUE

*Leucophyllum pruinosum I.M. Johnst. HGO, NLE, QRO, SLP, TAMS, VER

*Leucophyllum revolutum Rzed. NLE, SLP, TAMS, ZAC

*Leucophyllum ultramonticola Flyr DGO, NAY, ZAC

*Leucophyllum virescens I.M. Johnst. DGO, SLP, ZAC

*Leucophyllum zygophyllum I.M. Johnst. COAH, NLE, SLP, TAMS, ZAC

Limosella aquatica L. AGS, BCN, CHIS, CHIH, COAH, COL, CDMX, GTO, GRO, HGO, JAL, MEX, MICH, MOR, OAX, PUE, QRO, SLP, SON, TLAX, VER, ZAC

Pseudorontium cyathiferum (Benth.) Rothm. BCS, SON

Scrophularia californica Cham. \& Schltdl. BCN

*Scrophularia mexicana Mayfield \& G.L. Nesom NLE, TAMS Scrophularia villosa Pennell BCN

\section{Family Schisandraceae}

Illicium floridanum J. Ellis HGO, PUE, SLP, TAMS, VER Schisandra glabra (Brickell) Rehder BCS, HGO, VER

\section{Family Schlegeliaceae}

Gibsoniothamnus cornutus (Donn. Sm.) A.H. Gentry CHIS, OAX, VER

Schlegelia nicaraguensis Standl. CHIS, VER

Schlegelia parviflora (Oerst.) Monach. CHIS, OAX, VER

\section{Family Schoepfiaceae}

*Schoepfia californica Brandegee BCN, BCS, OAX Schoepfia flexuosa (Ruiz \& Pav.) Schult. f. CAM, CHIS, CHIH, COL, GTO, GRO, HGO, JAL, MEX, MICH, NAY, NLE, OAX, PUE, QRO, QROO, SLP, SIN, SON, TAB, TAMS, VER, YUC, ZAC

*Schoepfia pringlei B.L. Rob. GRO, MEX, MICH 
*Schoepfia shreveana Wiggins SON

Family Setchellanthaceae

* Setchellanthus caeruleus Brandegee COAH, DGO, OAX, PUE

Family Simaroubaceae

Castela emoryi (A. Gray) Moran \& Felger BCN, COAH, QRO, SLP, SON, ZAC

Castela erecta Turpin CHIH, COAH, COL, DGO, GRO, HGO, NLE, OAX, PUE, QRO, SLP, TAMS, ZAC

*Castela peninsularis Rose BCS

* Castela polyandra Moran \& Felger BCN, BCS, SON

* Castela retusa Liebm. OAX

Castela stewartii (C.H. Müll.) Moran \& Felger CHIH, COAH, NLE, SLP

* Picrasma mexicana Brandegee CHIS, COL, JAL, NAY, VER, ZAC

Quassia amara L. CHIS, COL, GRO, JAL, MICH, MOR, OAX Simarouba amara Aubl. CAM, CHIS, COL, GRO, JAL, MICH, MOR, OAX, PUE, QROO, TAB, VER, YUC

\section{Family Simmondsiaceae}

Simmondsia chinensis (Link) C.K. Schneid. BCN, BCS, CHIH, SON

\section{Family Siparunaceae}

Siparuna gesnerioides (Kunth) A. DC. CHIS, GRO, OAX, VER Siparuna grandiflora (Kunth) Perkins CHIS, OAX, VER Siparuna thecaphora (Poepp. \& Endl.) A. DC. CHIS, COL, GRO, HGO, JAL, MICH, NAY, OAX, PUE, SLP, TAB, VER

\section{Family Smilacaceae}

Smilax aristolochiifolia Mill. CAM, CHIS, COAH, COL, GRO, HGO, JAL, MEX, MICH, MOR, NAY, NLE, OAX, PUE, QRO, QROO, SLP, TAB, TAMS, VER, YUC

Smilax bona-nox L. CHIS, COAH, GRO, HGO, NLE, OAX, PUE, QRO, SLP, TAMS, VER

* Smilax cordifolia Humb. \& Bonpl. ex Willd. COL, GRO, HGO, JAL, MEX, MICH, MOR, OAX, TAB, TAMS, VER

* Smilax chiapensis Lundell CHIS

Smilax glauca Walter CHIS, COAH, COL, HGO, NLE, OAX, PUE, QRO, SLP, TAMS, VER

Smilax laurifolia L. CAM, CHIS, COAH, COL, GRO, HGO, JAL, MEX, MICH, NAY, NLE, OAX, PUE, QRO, QROO, SLP, TAB, TAMS, VER

Smilax luculenta Killip \& C.V. Morton CAM, CHIS, QROO, YUC

Smilax lundellii Killip \& C.V. Morton CAM, CHIS, QROO, VER, YUC

Smilax mollis Humb. \& Bonpl. ex Willd. CAM, CHIS, GRO, HGO, JAL, MEX, MICH, MOR, OAX, PUE, QRO, QROO, SLP, SIN, TAB, TAMS, VER, YUC

Smilax moranensis M. Martens \& Galeotti CHIS, CHIH, COAH, COL, CDMX, DGO, GTO, GRO, HGO, JAL, MEX, MICH, MOR, NAY, NLE, OAX, PUE, QRO, SLP, SIN, SON, TAMS, TLAX, VER, ZAC

Smilax munda Killip \& C.V. Morton CHIS, OAX

Smilax officinalis Kunth CHIS, HGO, OAX, VER

*Smilax pringlei Greenm. CHIS, COAH, COL, GRO, HGO,
JAL, MEX, MICH, MOR, NLE, OAX, QRO, SLP, SIN, TAMS, VER

*Smilax purpusii Brandegee CHIS, OAX, PUE, QROO, VER Smilax regelii Killip \& C.V. Morton CHIS, COL, GRO, OAX, TAB, TAMS, VER

Smilax spinosa Mill. CAM, CHIS, COL, GRO, HGO, JAL, MICH, MOR, NAY, OAX, PUE, QRO, QROO, SLP, SIN, TAB, TAMS, VER, YUC

Smilax subpubescens A. DC. CAM, CHIS, GRO, HGO, MEX, MICH, OAX, PUE, QROO, TAB, TAMS, VER

Smilax tamnoides L. NLE, SLP, TAMS

Smilax tomentosa Kunth HGO, OAX, TAB, VER

Smilax velutina Killip \& C.V. Morton CHIS, GRO, OAX, PUE, TAB, VER

\section{Family Solanaceae}

Acnistus arborescens (L.) Schltdl. CHIS, GTO, GRO, JAL, MEX, MICH, QRO

*Athenaea purpusii Brandegee VER

* Bouchetia arniatera B.L. Rob. AGS, COAH, DGO, GTO, JAL, MICH, NLE, OAX, SLP

Bouchetia erecta DC. ex Dunal BCN, CHIS, COAH, CDMX, DGO, GTO, GRO, HGO, JAL, MEX, MICH, NLE, OAX, PUE, QRO, SLP, SON, TLAX, VER, ZAC

*Bouchetia procumbens DC. ex Dunal SLP

Brachistus affinis (C.V. Morton) D'Arcy, J.L. Gentry \& Averett CHIS

Brachistus nelsonii (Fernald) D'Arcy, J.L. Gentry \& Averett CAM, CHIS, OAX, QROO, TAB, VER

Brachistus stramoniifolius (Kunth) Miers CHIS, CHIH, COL, GRO, HGO, JAL, MEX, MICH, MOR, NAY, OAX, PUE, QRO, SON, TAMS, VER

Browallia americana L. CHIS, JAL, MICH, MOR, OAX, TAB, VER

Browallia eludens R.K. Vandevender \& P.D. Jenkins CHIH, SON

Brugmansia sanguinea (Ruiz \& Pav.) D. Don CHIS, MOR, PUE

Calibrachoa parviflora (A. Juss.) D'Arcy AGS, BCN, BCS, CHIH, COAH, COL, CDMX, DGO, GTO, GRO, HGO, JAL, MEX, MICH, MOR, NLE, OAX, PUE, QRO, SLP, SIN, SON, TAMS, TLAX, VER, ZAC

Capsicophysalis potosina (B.L. Rob. \& Greenm.) Averett \& M. Martínez CHIS, GRO, OAX, QRO, SLP, TAMS

Capsicum annuum L. AGS, BCN, BCS, CAM, CHIS, CHIH, COAH, COL, DGO, GTO, GRO, HGO, JAL, MEX, MICH, MOR, NAY, NLE, OAX, PUE, QRO, QROO, SLP, SIN, SON, TAB, TAMS, VER, YUC, ZAC

Capsicum frutescens L. BCS, CHIS, CHIH, COAH, GRO, HGO, JAL, MICH, NLE, SLP, SIN, SON, TAMS, VER

Capsicum lanceolatum (Greenm.) C.V. Morton \& Standl. CHIS, OAX, VER

Capsicum rhomboideum (Dunal) Kuntze CAM, CHIS, COAH, COL, DGO, GTO, GRO, HGO, JAL, MEX, MICH, MOR, NLE, OAX, PUE, QRO, QROO, SLP, TAMS, VER

Cestrum alternifolium (Jacq.) O.E. Schulz CAM, GRO, QROO Cestrum aurantiacum Lindl. CHIS, COL, GRO, HGO, JAL, 
MEX, MICH, MOR, OAX, SLP, TAMS, VER

Cestrum dasyanthum Donn. Sm. CHIS, MOR

Cestrum diurnum L. CAM, CHIS, QROO, SIN, TAMS, YUC

Cestrum dumetorum Schltdl. CHIS, COL, DGO, GRO, HGO,

JAL, MOR, NLE, OAX, PUE, QRO, SLP, SIN, TAMS, VER,

ZAC

*Cestrum elegans (Brongn.) Schltdl. HGO, OAX, PUE, QRO,

TAMS, VER

Cestrum elegantissimum C.V. Morton CHIS

*Cestrum endlicheri Miers CAM, CHIS, HGO, TAMS, VER

Cestrum fasciculatum (Schltdl.) Miers HGO, OAX, PUE, QRO,

SLP, TAMS, VER

*Cestrum flavescens Greenm. CHIS, GRO, HGO, MEX, MICH,

MOR, NLE, OAX, QRO, SLP, TAMS, VER

*Cestrum flavinervium Francey GRO, MICH

Cestrum formosum C.V. Morton CHIS

Cestrum fraternum C.V. Morton CHIS

*Cestrum fulvescens Fernald CDMX, GTO, GRO, HGO, MEX,

MICH, NLE, OAX, PUE, QRO, TAMS

*Cestrum galeottianum Francey OAX

Cestrum glanduliferum Kerber ex Francey CHIS, COL, GRO,

JAL, MICH, OAX, PUE, VER

Cestrum guatemalense Francey CHIS

Cestrum laurifolium L'Hér. GRO, JAL, MICH, VER

Cestrum laxum Benth. CHIS, COAH, CDMX, GTO, GRO, HGO, JAL, MEX, MICH, MOR, NLE, OAX, PUE, QRO, SLP, TAMS, TLAX, VER

Cestrum luteo-virescens Francey CHIS, OAX, VER

*Cestrum miradorense Francey OAX, VER

Cestrum mortonianum J.L. Gentry CHIS, COL, GRO, JAL, MICH, OAX

*Cestrum nitidum M. Martens \& Galeotti CHIS, COL, CDMX, GTO, GRO, HGO, JAL, MEX, MICH, MOR, OAX, PUE, QRO, TAB, TAMS, TLAX

Cestrum nocturnum L. CAM, CHIS, COAH, COL, GRO, HGO, JAL, MEX, MICH, MOR, NAY, OAX, PUE, QRO, QROO, SLP, SIN, TAB, TAMS, VER, YUC, ZAC

*Cestrum oblongifolium Schltdl. COAH, CDMX, GTO, GRO, HGO, JAL, MEX, MICH, NLE, OAX, PUE, QRO, SLP, TAMS, TLAX, VER

Cestrum pacayense Francey CHIS

* Cestrum pacificum Brandegee COL

Cestrum racemosum Ruiz \& Pav. CHIS, OAX, PUE, SIN, QROO, VER

Cestrum regelii Planch. CHIS, MEX, MICH

Cestrum roseum Kunth CHIS, CDMX, GTO, HGO, MEX,

MICH, OAX, PUE, QRO, SLP, TAMS, TLAX, VER

Cestrum scandens Vahl CHIS, OAX, VER

Cestrum schlechtendalii G. Don CHIS, OAX, TAB, VER

*Cestrum sotonunezii Mont.-Castro GRO, MEX, MICH

Cestrum subcordatum Francey GRO, MICH

Cestrum thyrsoideum Kunth CHIS, COL, CDMX, DGO, GTO,

GRO, HGO, JAL, MEX, MICH, MOR, NAY, OAX, PUE, QRO,

SIN, TLAX, ZAC

Cestrum tomentosum L. f. AGS, CHIS, CHIH, COL, DGO, GTO, GRO, HGO, JAL, MEX, MICH, MOR, NAY, OAX,

PUE, SIN, SON, VER, ZAC
Chamaesaracha cernua (Donn. Sm.) Hunz. CHIS, COL, GRO, JAL, NAY, OAX, SIN, TAMS, VER

Chamaesaracha conioides (Moric. ex Dunal) Britton AGS, CHIH, COAH, DGO, NLE, SLP, SON, TAMS, ZAC

Chamaesaracha coronopus (Dunal) A. Gray AGS, CHIH, COAH, DGO, GTO, NLE, SLP, SON, TAMS, ZAC

*Chamaesaracha crenata Rydb. COAH

*Chamaesaracha geohintonii Averett \& B.L. Turner NLE Chamaesaracha luteiflorum (Dunal) Henr. COAH, NLE, SLP Chamaesaracha pallida Averett COAH, DGO, NLE, ZAC

*Chamaesaracha rzedowskiana Hunz. QRO, SLP, VER

Chamaesaracha texensis Henr. CHIH, COAH, NLE

Chamaesaracha villosa Rydb. CHIH, COAH, DGO, NLE

*Datura ceratocaula Ortega AGS, CHIH, COAH, CDMX, DGO, GTO, HGO, JAL, MEX, MICH, MOR, OAX, PUE, QRO, SLP, TAMS, TLAX, VER, ZAC

Datura discolor Bernh. AGS, BCN, BCS, CAM, CHIS, CHIH, COAH, COL, DGO, GTO, GRO, JAL, MEX, MICH, MOR, NAY, NLE, OAX, QRO, SLP, SIN, SON, TAMS, VER, YUC, ZAC

Datura inoxia Mill. AGS, BCN, BCS, CAM, CHIS, CHIH, COAH, COL, DGO, GTO, GRO, HGO, JAL, MEX, MICH, MOR, NAY, NLE, OAX, PUE, QRO, QROO, SLP, SIN, SON, TAMS, VER, YUC, ZAC

*Datura kymatocarpa A.S. Barclay COL, GRO, JAL, MEX, $\mathrm{MICH}$

*Datura lanosa A.S. Barclay ex R. Bye CHIH, JAL, NAY, SIN, SON, ZAC

Datura metel L. CAM, CHIS, OAX, QROO, TAB

Datura pruinosa Greenm. GRO, OAX, PUE, VER

Datura quercifolia Kunth AGS, CHIH, COAH, COL, CDMX, DGO, GTO, HGO, JAL, MEX, MICH, NLE, QRO, SLP, SIN, SON, TAMS, VER, ZAC

* Datura reburra A.S. Barclay SIN, SON

Datura stramonium L. AGS, BCS, CHIS, CHIH, COAH, COL, CDMX, DGO, GTO, GRO, HGO, JAL, MEX, MICH, MOR, NAY, NLE, OAX, PUE, QRO, QROO, SLP, SIN, SON, TAB, TAMS, TLAX, VER, ZAC

Datura wrightii Regel BCS, CHIH, COAH, DGO, NLE, SON, TAMS

Grabowskia geniculata (Fernald) C.L. Hitchc. MICH, OAX, PUE

*Hunzikeria coulteri (A. Gray) D'Arcy HGO, QRO

Hunzikeria texana (Torr.) D'Arcy COAH, HGO, NLE, QRO, SLP, TAMS

* Jaltomata bohsiana Mione \& D.M. Spooner MEX, MICH

*Jaltomata chihuahuensis (Bitter) Mione \& Bay CHIH, DGO, SIN, SON

*Jaltomata grandiflora (B.L. Rob. \& Greenm.) D'Arcy, Mione \& T. Davis MICH

Jaltomata procumbens (Cav.) J.L. Gentry AGS, BCS, CHIS, CHIH, COAH, COL, CDMX, DGO, GTO, GRO, HGO, JAL, MEX, MICH, MOR, NAY, NLE, OAX, PUE, QRO, SLP, SIN, SON, TAB, TAMS, TLAX, VER, ZAC

*Jaltomata repandidentata (Dunal) Hunz. COL, JAL, OAX, PUE

Juanulloa mexicana (Schltdl.) Miers CHIS, JAL, OAX, TAB, 
VER

Lycianthes acapulcensis (Baill.) D'Arcy COL, GRO, JAL, MEX, MOR, OAX, PUE

Lycianthes amatitlanensis (J.M. Coult. \& Donn. Sm.) Bitter CHIS, OAX, TAB, VER

* Lycianthes anomala Bitter OAX, VER

Lycianthes armentalis J.L. Gentry CAM, CHIS, GRO, OAX, QROO, VER, YUC

Lycianthes arrazolensis (J.M. Coult. \& Donn. Sm.) Bitter CHIS, GRO, MEX, MICH, MOR, OAX

Lycianthes barbatula Standl. \& Steyerm. CHIS, OAX

Lycianthes ceratocalycia (Donn. Sm.) Bitter CHIS, OAX

Lycianthes ciliolata (M. Martens \& Galeotti) Bitter CHIS, COL,

DGO, GTO, GRO, HGO, JAL, MEX, MICH, MOR, OAX, PUE, QRO, ZAC

Lycianthes connata J.L. Gentry CHIS, JAL

Lycianthes cuchumatanensis J.L. Gentry CHIS

Lycianthes chiapensis (Brandegee) Standl. CHIS, GRO, OAX, VER

*Lycianthes dejecta (Fernald) Bitter CDMX, DGO, GTO, HGO, JAL, MEX, MICH, NLE, PUE, QRO, SIN, TAMS, VER

Lycianthes geminiflora (M. Martens \& Galeotti) Bitter CHIS, OAX, PUE, TAB, VER

Lycianthes gorgonea Bitter CHIS, JAL, OAX

Lycianthes heteroclita (Sendtn.) Bitter CHIS, COL, GRO, JAL, MICH, OAX, TAB, VER

*Lycianthes hintonii E. Dean NLE, TAMS

Lycianthes hypoleuca Standl. CAM, CHIS, QROO

*Lycianthes jaliscensis E. Dean COL, JAL, MICH

Lycianthes lenta (Cav.) Bitter CAM, CHIS, COL, GRO, HGO, JAL, MICH, NAY, OAX, PUE, QROO, SLP, SIN, TAB, TAMS, VER, YUC

Lycianthes limitanea (Standl.) J.L. Gentry CAM, CHIS, QROO, YUC

* Lycianthes luisana Standl. SLP

*Lycianthes manantlanensis Aarón Rodr. \& O. Vargas CHIS, COL, JAL, MICH, OAX

*Lycianthes michaelneei E. Dean VER

Lycianthes moziniana (Dunal) Bitter AGS, CHIS, COAH, COL, CDMX, DGO, GTO, GRO, HGO, JAL, MEX, MICH, NLE, OAX, PUE, QRO, SLP, TAMS, TLAX, VER, ZAC

Lycianthes nitida Bitter CHIS, OAX, TAB, VER

Lycianthes ocellata (Donn. Sm.) Morton \& Standl. CHIS, OAX Lycianthes orogenes Standl. \& Steyerm. CHIS, OAX

*Lycianthes peduncularis (Schltdl.) Bitter CDMX, GTO, HGO, MEX, MICH, MOR, OAX, PUE, QRO, SLP

*Lycianthes pilifera (Benth.) Bitter GRO, MEX, MICH, OAX

Lycianthes pilosissimum (M. Martens \& Galeotti) Bitter CHIS, OAX, VER

*Lycianthes pringlei (B.L. Rob. \& Greenm.) Bitter COL, GRO, JAL, MEX, MICH

Lycianthes purpusii (Brandegee) Bitter CHIS, OAX, PUE, TAB, VER

Lycianthes quichensis (J.M. Coult. \& Donn. Sm.) Bitter CHIS, GRO, OAX, VER

*Lycianthes rzedowskii E. Dean MEX, MICH, MOR, OAX

Lycianthes sideroxyloides (Schltdl.) Bitter CAM, CHIS, OAX,
QROO, VER, YUC

*Lycianthes starbuckii E. Dean GRO, MEX

Lycianthes stephanocalyx (Brandegee) Bitter CHIS, COL, GRO, JAL, MICH, NAY, OAX, QRO, SLP, VER

*Lycianthes surotatensis J.L. Gentry COL, GRO, JAL, MEX, NAY, OAX, SIN, SON

Lycianthes synanthera (Sendt.) Bitter CHIS, TAB, VER

Lycianthes tricolor (Sessé \& Moc. ex Dunal) Bitter CHIS, GRO, MEX, MOR, OAX, PUE, VER

*Lycianthes venturana E. Dean PUE

Lycium andersonii A. Gray BCN, BCS, CHIH, SIN, SON

* Lycium arochae F. Chiang, T. Wendt \& E.J. Lott COAH

*Lycium barbinodum Miers CHIH, COAH, SLP, SIN, SON, ZAC

Lycium berlandieri Dunal BCN, BCS, CHIH, COAH, DGO, GTO, HGO, JAL, NLE, QRO, SLP, SIN, SON, TAMS, VER, ZAC

Lycium brevipes Benth. BCN, BCS, SIN, SON

Lycium californicum Nutt. ex A. Gray BCN, BCS, COAH, NLE, SLP, SIN, SON, ZAC

Lycium carolinianum Walter BCS, CAM, COL, GTO, GRO, HGO, JAL, MEX, MICH, NLE, OAX, QRO, QROO, SLP, SIN, TAMS, VER, YUC, ZAC

Lycium cooperi A. Gray BCN

*Lycium densiflorum Wiggins BCN, BCS

Lycium exsertum A. Gray BCN, BCS, SIN, SON

Lycium fremontii A. Gray BCN, BCS, SIN, SON

Lycium isthmense F. Chiang OAX, PUE

* Lycium leiospermum I.M. Johnst. COAH, NLE, SLP, ZAC

Lycium macrodon A. Gray SON

*Lycium megacarpum Wiggins BCN, BCS

Lycium pallidum Miers BCS, CHIH, COAH, NLE, SLP, SON, ZAC

Lycium parishii A. Gray BCN, COAH, NLE, SLP, SON, TAMS

Lycium puberulum A. Gray CHIH, COAH, DGO

*Lycium schaffneri Hemsl. GTO, JAL, NLE, SLP, ZAC

Lycium torreyi A. Gray BCN, BCS, CHIH, HGO, SON

Markea megalandra (Dunal) D'Arcy CHIS

Melananthus guatemalensis (Benth.) Solereder CHIS, OAX

Nectouxia formosa Kunth CHIH, COL, COAH, CDMX, GTO, HGO, JAL, MEX, MICH, MOR, NLE, OAX, SLP, PUE, TLAX, VER, ZAC

Nicotiana acuminata (Graham) Hook. SIN

Nicotiana attenuata Torr. ex S. Watson BCN, SIN, SON

Nicotiana clevelandii A. Gray BCN, BCS, SON

*Nicotiana nudicaulis S. Watson COAH, NLE, TAMS

Nicotiana obtusifolia M. Martens \& Galeotti AGS, BCN, BCS, CHIH, COAH, DGO, GTO, HGO, JAL, MEX, NAY, NLE, OAX, PUE, QRO, SLP, SIN, SON, TAMS, VER, ZAC

Nicotiana palmeri A. Gray BCN, BCS, CHIH, COAH, GTO, HGO, JAL, QRO, SLP, SON, TAMS, ZAC

Nicotiana plumbaginifolia Viv. CHIS, COAH, COL, GTO, GRO, HGO, JAL, MEX, MICH, MOR, NAY, NLE, OAX, PUE, QRO, SLP, SIN, SON, TAB, TAMS, VER

Nicotiana repanda Willd. ex Lehm. CHIH, COAH, GTO, QRO, NLE, SLP, TAMS, VER

*Nicotiana stocktonii Brandegee COL 
* Nicotiana $\times$ sanderae W. Watson CHIS

*Nierembergia angustifolia Kunth AGS, COAH, CDMX, GTO, HGO, JAL, MEX, MICH, NLE, QRO, SLP, TLAX, ZAC

*Petunia axillaris (Lam.) Britton, Stearn \& Poggenb. CDMX, MEX, MOR, SLP

*Petunia nyctaginiflora Juss. CDMX, MEX, VER

Petunia violacea Lindl. CHIH, CDMX, MEX, MOR, SON, VER

Physalis acutifolia (Miers) Sandwith AGS, BCN, BCS, CHIH, COL, DGO, JAL, MICH, SIN, SON, ZAC

* Physalis aggregata Waterf. GRO, JAL, OAX, QRO

*Physalis ampla Waterf. CHIH, GTO, GRO, JAL, NAY, OAX, QRO, SIN, SON, ZAC

Physalis angulata L. AGS, BCN, BCS, CAM, CHIS, CHIH, COL, DGO, GTO, GRO, JAL, MEX, MICH, MOR, NAY, OAX, PUE, QROO, SIN, SON, TAB, VER, ZAC

*Physalis angustior Waterf. MOR

Physalis angustiphysa Waterf. CHIS, GTO, JAL, MICH, NAY, TAB

*Physalis arborescens L. CAM, CHIS, OAX, QROO, VER, YUC

* Physalis breviloba O. Vargas, M. Martínez \& Dávila JAL Physalis campanula Standl. \& Steyerm. HGO, OAX, QRO, SLP, VER

Physalis campechiana L. CAM, OAX, QROO, YUC

Physalis caudella Standl. CHIH, OAX, SLP, SON, ZAC

Physalis cinerascens (Dunal) Hitchc. AGS, CAM, CHIS, CHIH,

COAH, CDMX, DGO, GTO, GRO, HGO, JAL, MEX, MICH,

NLE, OAX, PUE, QRO, SLP, TAMS, VER, YUC, ZAC

* Physalis cinerea Waterf. SLP

Physalis cordata Mill. AGS, CAM, CHIS, COAH, COL, DGO, GRO, HGO, JAL, NAY, OAX, QROO, SLP, SIN, VER, YUC *Physalis coztomatl Moc. \& Sessé ex Dunal AGS, CHIS, COL, CDMX, GTO, GRO, HGO, JAL, MEX, MICH, NAY, MOR, OAX, PUE, QRO, SLP, TAMS, TLAX, VER, ZAC

Physalis crassifolia Benth. BCN, BCS, CHIH, GTO, MICH, SON

Physalis fendleri A. Gray QRO, SON

*Physalis filipendula Brandegee BCS

*Physalis flava Wiggins BCN

*Physalis glabra Benth. BCS

*Physalis glutinosa Schltdl. AGS, CHIH, CDMX, DGO, GTO, GRO, HGO, JAL, MEX, NLE, PUE, QRO, SLP, ZAC

Physalis gracilis Miers CAM, CHIS, COL, GTO, GRO, HGO, JAL, MEX, MICH, MOR, NAY, NLE, OAX, PUE, QRO, QROO, SLP, TAB, TAMS, VER

Physalis greenei Vasey \& Rose BCN, BCS, DGO, MICH, SON * Physalis greenmanii Waterf. CHIS, HGO, OAX, PUE, VER

* Physalis hastatula Waterf. AGS, GTO, JAL, SLP, ZAC

Physalis hederifolia A. Gray AGS, BCN, CHIH, COAH, DGO, GRO, HGO, JAL, NLE, OAX, SLP, SIN, SON, TAMS, ZAC

*Physalis hintonii Waterf. MEX, NLE, VER

Physalis hirsuta M. Martens \& Galeotti CHIS, VER

* Physalis hunzikeriana M. Martínez NLE, TAMS

Physalis ignota Britton CHIS, OAX

Physalis lagascae Roem. \& Schult. AGS, CAM, CHIS, CHIH, COL, DGO, GTO, GRO, JAL, MEX, MICH, MOR, NAY, OAX,
PUE, QRO, QROO, SIN, SON, VER, YUC, ZAC

Physalis lanceolata Michx. CHIH, QRO, SON, TAMS

Physalis lassa Standl. \& Steyerm. CHIS, COL, GRO, JAL,

OAX

*Physalis latecorollata Waterf. OAX

Physalis latiphysa Waterf. GTO, MICH, OAX, SON

Physalis leptophylla B.L. Rob. \& Greenm. BCS, CHIH, COL, DGO, GRO, JAL, MICH, NAY, OAX, SIN, SON, ZAC

* Physalis lignescens Waterf. COL, JAL

Physalis lobata Torr. CHIH, COAH, DGO, NLE, SON, TAMS

*Physalis longicaulis Waterf. COAH, NLE

Physalis longifolia Nutt. GTO, NLE, SLP, SON

*Physalis longiloba O. Vargas, M. Martínez \& Dávila COL, JAL, OAX

*Physalis longipedicellata Waterf. COL, JAL

Physalis macrophysa Rydb. NLE

*Physalis mcvaughii Waterf. JAL

Physalis melanocystis (B.L. Rob.) Bitter CAM, CHIS, COL, GRO, HGO, OAX, QRO, QROO, SLP, TAMS, VER, YUC

Physalis microcarpa Urb. \& Ekman CHIS, CHIH, JAL, MEX, MICH, OAX

*Physalis microphysa A. Gray CHIH, COAH, DGO, GTO, HGO, JAL, NLE, QRO, SLP, TAMS, ZAC

*Physalis michoacanensis Waterf. MICH

* Physalis minimaculata Waterf. GRO, MICH

Physalis minuta Griggs CAM, CHIS, CHIH, COL, GRO, JAL, MICH, NAY, OAX, QROO, TAB

Physalis mollis Nutt. CHIS, CHIH, COAH, GRO, MEX, NLE, QRO, TAMS, VER, ZAC

*Physalis muelleri Waterf. COAH, NLE

*Physalis muriculata Greene BCN, BCS

Physalis nicandroides Schltdl. BCS, CHIS, CHIH, COL, CDMX, DGO, GTO, GRO, HGO, JAL, MEX, MICH, MOR, NAY, OAX, PUE, QRO, SLP, SIN, SON, TAMS, VER, YUC, ZAC

*Physalis orizabae Dunal AGS, COAH, COL, CDMX, DGO, GTO, GRO, HGO, JAL, MEX, MICH, MOR, NAY, NLE, OAX, PUE, QRO, TAMS, TLAX, VER, ZAC

*Physalis parvianthera Waterf. MOR, NAY

*Physalis patula Mill. AGS, CHIH, COL, CDMX, DGO, GTO, GRO, HGO, JAL, MEX, MICH, MOR, OAX, PUE, QRO, SLP, SIN, SON, TAB, TAMS, TLAX, VER, ZAC

* Physalis pennellii Waterf. GTO, NLE, SLP

Physalis peruviana L. AGS, CAM, CHIS, CHIH, COAH, COL, CDMX, DGO, GTO, GRO, HGO, JAL, MEX, MICH, MOR, NLE, OAX, PUE, QRO, QROO, SLP, SIN, TAMS, TLAX, VER, ZAC

Physalis philadelphica Lam. AGS, BCN, BCS, CHIS, CHIH, COAH, COL, CDMX, DGO, GTO, GRO, HGO, JAL, MEX, MICH, MOR, NAY, NLE, OAX, PUE, QRO, QROO, SLP, SIN, SON, TAB, TAMS, TLAX, VER, ZAC

*Physalis philippensis Fernald OAX

Physalis porrecta Waterf. CHIS, GRO, OAX

*Physalis pringlei Greenm. CHIS, CHIH, CDMX, DGO, GRO, HGO, JAL, MEX, MICH, MOR, NLE, OAX, SON, TAMS

Physalis pruinosa L. AGS, BCS, CAM, CHIS, CHIH, COL, DGO, GRO, HGO, JAL, MEX, MICH, NAY, NLE, OAX, PUE, 
QRO, QROO, SIN, SON, TAMS, VER, YUC, ZAC

Physalis pubescens L. BCN, BCS, CAM, CHIS, CHIH, COAH, COL, DGO, GRO, HGO, JAL, MEX, MICH, MOR, NAY, NLE, OAX, PUE, QRO, QROO, SLP, SIN, SON, TAB, TAMS, VER, YUC, ZAC

* Physalis purpurea Wiggins SON

*Physalis queretaroensis M. Martínez \& L. Hern. QRO, SLP

*Physalis rydbergii Fernald SIN

*Physalis sancti-josephi Dunal GTO, JAL, NAY, OAX, QRO, SLP

Physalis solanacea (Schltdl.) Axelius AGS, CHIS, CHIH, COAH, DGO, GTO, HGO, JAL, MEX, MICH, MOR, NLE, OAX, PUE, QRO, SLP, SIN, SON, TAMS, VER, ZAC

*Physalis sordida Fernald COAH, CDMX, DGO, GTO, HGO, JAL, MEX, MICH, NLE, OAX, PUE, QRO, SLP, SIN, TAMS, TLAX, ZAC

* Physalis subrepens Waterf. COL, HGO, JAL, MEX, VER

*Physalis sulphurea (Fernald) Waterf. AGS, CHIH, CDMX, DGO, GTO, GRO, HGO, JAL, MEX, MICH, OAX, PUE, QRO, SON, TLAX, ZAC

* Physalis tamayoi O. Vargas, M. Martínez \& Dávila COL, JAL

*Physalis tehuacanensis Waterf. OAX, PUE

*Physalis turbinatoides Waterf. MOR

Physalis versicolor Rydb. CHIH, SIN, SON

*Physalis vestita Waterf. SIN

Physalis virginiana Mill. CHIH, COAH, DGO, GTO, HGO, JAL, NLE, QRO, SLP, SIN, SON, TAMS, VER, ZAC

Physalis viscosa L. CAM, CHIS, CHIH, COAH, CDMX, GTO, HGO, MEX, MICH, NLE, OAX, PUE, QRO, SLP, TAMS, VER, YUC, ZAC

*Physalis volubilis Waterf. GTO, JAL, MEX, MICH, QRO, SLP, TAMS

Physalis walteri Nutt. COAH, NLE

*Physalis waterfallii O. Vargas, M. Martínez \& Dávila GTO, JAL

Plowmania nyctaginoides (Standl.) Hunz. \& Subils CHIS

*Salpiglossis arniatera (B.L. Rob.) D'Arcy JAL, MICH, ZAC

Schraderanthus viscosus (Schrad.) Averett CHIS, GRO, HGO, OAX, VER

Schultesianthus leucanthus (Donn. Sm.) Hunz. CHIS, OAX

Schultesianthus uniflorus (Lundell) S. Knapp CHIS

Schwenckia americana D. Royen ex L. CAM, CHIS, OAX, QROO, YUC

Solandra brevicalyx Standl. TAMS

Solandra grandiflora Sw. CHIS, COL, GRO, JAL, MOR, OAX, TAB, VER

* Solandra guerrerensis Martínez COL, CDMX, GRO, HGO, JAL, MEX, MICH, MOR, OAX, VER

* Solandra guttata D. Don CAM, CDMX, DGO, GRO, HGO, JAL, MEX, MICH, MOR, NLE, OAX, QRO, SIN, QROO, SLP, TAMS, VER, ZAC

Solandra nitida Zuccagni CAM, CHIS, COL, GRO, HGO, JAL, MEX, MICH, MOR, NAY, NLE, OAX, PUE, QRO, SLP, SIN, TAB, TAMS, VER

Solandra nizandensis Matuda CHIS, JAL, MICH, OAX

Solanum acerifolium Dunal CHIS, GRO, HGO, OAX, QRO, SLP, TAMS, VER
Solanum aculeolatum M. Martens \& Galeotti CHIS, GRO, OAX Solanum adhaerens Roem. \& Schult. CAM, CHIS, OAX, PUE, QROO, TAB, VER

Solanum adscendens Sendtn. AGS, BCN, BCS, CHIS, CHIH, COL, DGO, GTO, GRO, JAL, MEX, MICH, MOR, NAY, OAX, QRO, SLP, SIN, SON, VER

Solanum agrimonifolium Rydb. CHIS, GRO, OAX

Solanum aligerum Schltdl. CHIS, COL, GRO, HGO, JAL, MEX, MICH, MOR, OAX, PUE, QRO, SLP, VER

Solanum americanum Mill. BCN, BCS, CAM, CHIS, CHIH, COAH, COL, CDMX, DGO, GTO, GRO, HGO, JAL, MEX, MICH, MOR, NAY, NLE, OAX, PUE, QRO, QROO, SLP, SIN, SON, TAB, TAMS, TLAX, VER, YUC, ZAC

*Solanum amictum Moric. ex Dunal PUE, TAMS, VER

Solanum angustifolium Mill. CHIS, COL, GTO, GRO, JAL, MEX, MICH, MOR, OAX, PUE, QRO, SIN, TAMS, VER

Solanum aphyodendron S. Knapp CAM, CHIS, COL, GRO, HGO, JAL, MEX, MICH, NAY, OAX, PUE, QRO, QROO, SLP, VER

Solanum appendiculatum Humb. \& Bonpl. ex Dunal AGS, CHIS, COL, CDMX, GRO, HGO, JAL, MEX, MICH, MOR, OAX, PUE, QRO, SLP, SIN, TAMS, VER

Solanum asperum Rich. CAM, CHIS, COL, GRO, HGO, JAL, MICH, OAX, PUE, QRO, QROO, SLP, TAB, TAMS, VER

Solanum atitlanum Roe QROO

Solanum aturense Dunal CAM, CHIS, OAX, QROO, SLP, TAMS, VER

*Solanum axillifolium K.E. Roe GRO, OAX, PUE, SIN

*Solanum bicorne Dunal COL, GRO, JAL, MICH, NAY

Solanum blodgettii Chapm. CHIS, QROO

*Solanum brachistotrichum (Bitter) Rydb. AGS, CHIH, DGO, JAL, MICH, SON, ZAC

Solanum brevipedicellatum K.E. Roe CHIS, COL, JAL

Solanum bulbocastanum Dunal CHIS, COL, CDMX, DGO, GTO, GRO, HGO, JAL, MEX, MICH, MOR, NAY, OAX, PUE, QRO, SIN, TLAX, VER, ZAC

Solanum campechiense L. CAM, CHIS, COL, GRO, JAL, NAY, OAX, QROO, SLP, TAB, TAMS, VER, YUC

Solanum candidum Lindl. CAM, CHIS, CHIH, COL, DGO, GRO, JAL, MEX, MICH, NAY, OAX, PUE, QROO, SIN, SON, TAB, VER, YUC, ZAC

*Solanum cardiophyllum Lindl. AGS, CHIH, COAH, COL, CDMX, DGO, GTO, GRO, HGO, JAL, MEX, MICH, MOR, NAY, NLE, OAX, PUE, QRO, SLP, SIN, SON, TLAX, VER, ZAC

Solanum carolinense L. NLE, SON, TAMS

Solanum circinatum Bohs CHIS, OAX, TAB, VER

*Solanum citrinum M. Nee GTO, NLE, QRO, SLP, TAMS, VER

Solanum citrullifolium A. Br. CHIH, COAH, DGO, SON, ZAC Solanum clarum Correll CHIS

Solanum cobanense J.L. Gentry CHIS

Solanum cordovense Sessé \& Moc. CHIS, OAX, QROO, VER Solanum corymbosum Jacq. AGS, CDMX, GTO, GRO, HGO, JAL, MEX, MICH, MOR, OAX, PUE, QRO, SLP, TLAX, VER, ZAC

Solanum chiapasense K.E. Roe CHIS, OAX, QROO 
Solanum chrysotrichum Schltdl. CHIS, COL, GTO, GRO, HGO, JAL, MEX, MICH, OAX, PUE, QRO, SLP, VER

*Solanum dasyadenium Bitter CDMX, GTO, HGO, JAL, MEX, OAX, QRO, SLP, VER, ZAC

Solanum dasyanthum Brandegee CDMX, MEX, OAX, PUE, YUC

Solanum dasyneuron S. Knapp CHIS

* Solanum davisense Whalen $\mathrm{CHIH}, \mathrm{COAH}$

Solanum demissum Lindl. AGS, CHIS, CHIH, CDMX, DGO, GTO, GRO, HGO, JAL, MEX, MICH, MOR, OAX, PUE, QRO, SIN, SON, TLAX, VER, ZAC

Solanum dimidiatum Raf. COAH

Solanum diphyllum L. CAM, CHIS, CHIH, COL, GRO, HGO, JAL, MEX, MICH, MOR, NAY, OAX, PUE, QRO, QROO, SLP, SON, TAB, TAMS, VER

Solanum donianum Walp. AGS, CAM, CHIS, CHIH, COAH, DGO, HGO, JAL, MEX, MICH, MOR, NAY, NLE, OAX, PUE, QRO, QROO, SLP, SIN, SON, TAB, TAMS, VER, YUC, ZAC Solanum douglasii Dunal AGS, BCN, BCS, CHIS, CHIH, COAH, COL, CDMX, DGO, GTO, GRO, HGO, JAL, MEX, MICH, MOR, NAY, NLE, OAX, PUE, QRO, SLP, SON, TAMS, VER, ZAC

Solanum dulcamaroides Dunal CHIS, COL, GTO, GRO, HGO, JAL, MEX, MICH, MOR, OAX, PUE, QRO, QROO, SLP, VER *Solanum edmundoi Cuevas \& N.M. Núñez COL, JAL

*Solanum ehrenbergii (Bitter) Rydb. AGS, COAH, CDMX, GTO, HGO, JAL, MEX, MICH, NAY, PUE, QRO, SLP, ZAC

Solanum elaeagnifolium Cav. AGS, BCN, BCS, CHIS, CHIH, COAH, COL, CDMX, DGO, GTO, GRO, HGO, JAL, MEX, MICH, MOR, NLE, OAX, PUE, QRO, SLP, SIN, SON, TAMS, VER, ZAC

Solanum erianthum D. Don BCS, CAM, CHIS, CHIH, COAH, COL, DGO, GTO, GRO, HGO, JAL, MEX, MICH, MOR, NAY, NLE, OAX, PUE, QRO, QROO, SLP, SIN, SON, TAB, TAMS, VER, YUC, ZAC

*Solanum erythrotrichum Fernald CHIS, GRO, OAX

Solanum fendleri A. Gray CHIH, COAH, DGO

Solanum fontium Standl. \& Steyerm. CHIS

*Solanum fructu-tecto Cav. CHIH, COL, CDMX, DGO, GTO, GRO, HGO, JAL, MEX, MICH, PUE, SON, TLAX

Solanum gardneri Sendtn. CHIS, OAX, PUE, YUC

*Solanum glaucescens Zucc. CHIS, GRO, OAX, PUE, TAB

*Solanum grayi Rose BCS, CHIH, COL, DGO, GRO, JAL, MICH, NAY, OAX, PUE, SIN, SON

*Solanum guamuchilenese Cast.-Campos JAL, NAY

*Solanum guerreroense Correll GRO, JAL

Solanum hazenii Britton CHIS, COL, DGO, GRO, JAL, MICH, NAY, OAX, QRO, SLP, SIN, TAMS

Solanum hernandesii Dunal CHIS, TAB

Solanum heterodoxum Dunal AGS, CHIH, CDMX, DGO, GTO, HGO, JAL, MEX, MOR, PUE, QRO, SLP, SIN, SON, VER, ZAC

Solanum hindsianum Benth. BCN, BCS, CHIH, SON

*Solanum hintonii Correll COL, GTO, HGO, JAL, MEX, $\mathrm{MICH}, \mathrm{QRO}, \mathrm{SLP}$

Solanum hirtum Vahl CAM, CHIS, GRO, HGO, JAL, MICH, NAY, OAX, QRO, QROO, SLP, SIN, TAB, TAMS, VER, YUC
Solanum hispidum Pers. CHIS, COL, DGO, GRO, HGO, JAL, MEX, MICH, MOR, OAX, PUE, QRO, SLP, TAB, VER

*Solanum hjertingii Hawkes COAH, HGO, NLE, QRO, SLP, TAMS

*Solanum hougasii Correll COL, GRO, JAL, MEX, MICH, OAX

*Solanum ionidium Bitter CDMX, GRO, HGO, MEX, OAX, PUE, VER

*Solanum iopetalum (Bitter) Hawkes COL, CDMX, GTO, GRO, HGO, JAL, MEX, MICH, MOR, OAX, PUE, QRO, SLP, SIN, TLAX, VER

Solanum jamaicense Mill. CHIS, GRO, HGO, OAX, PUE, SLP, TAB, TAMS, VER

Solanum jamesii Torr. CHIH, DGO, HGO, JAL, QRO, SLP, SON, ZAC

*Solanum johnstonii Whalen COAH, DGO

Solanum lanceifolium Jacq. CAM, CHIS, COL, GTO, GRO, HGO, JAL, NAY, OAX, PUE, QRO, QROO, SLP, TAB, TAMS, VER

Solanum laurifolium Mill. AGS, CAM, CHIS, COL, CDMX, GTO, GRO, HGO, JAL, MEX, MICH, MOR, NAY, OAX, PUE, QRO, QROO, SLP, SIN, TAB, TLAX, VER, YUC, ZAC

Solanum lepidotum Humb. \& Bonpl. ex Dunal CHIS, OAX, VER

*Solanum lesteri Hawkes \& Hjert. OAX

* Solanum leucandrum Whalen GRO, OAX, PUE

*Solanum leucocarpum A. Rich. MICH

*Solanum lignescens Fernald CHIS, COL, GRO, JAL, OAX, QROO

*Solanum lumholtzianum Bartlett CHIH, SON

*Solanum malacothrix S. Knapp GRO, JAL, MICH

*Solanum michoacanum (Bitter) Rydb. GTO, MICH

*Solanum minensis C.V. Morton GRO, MOR

Solanum mitlense Dunal CHIS, GRO, MEX, MICH, MOR, OAX, PUE, QRO, SLP, VER

Solanum morelliforme Bitter \& Münch CHIS, COL, GRO, HGO, JAL, MEX, MICH, OAX, PUE, QRO, SLP, VER

Solanum mиenscheri Standl. \& Steyerm. CHIS

Solanum myriacanthum Dunal CHIS, CDMX, GRO, HGO, JAL, MICH, NLE, OAX, PUE, QRO, SLP, TAMS, VER

*Solanum nannodes Correll CHIH, DGO, SON

Solanum nigrescens $\mathrm{M}$. Martens \& Galeotti AGS, BCN, BCS, CAM, CHIS, CHIH, COAH, COL, CDMX, DGO, GTO, GRO, HGO, JAL, MEX, MICH, MOR, NAY, NLE, OAX, PUE, QRO, QROO, SLP, SIN, SON, TAB, TAMS, TLAX, VER, YUC, ZAC Solanum nigricans M. Martens \& Galeotti CAM, CHIS, COL, GTO, GRO, HGO, JAL, MEX, MICH, MOR, OAX, PUE, QROO, SLP, SIN, TAB, TAMS, VER

Solanum nudum Dunal CAM, CHIS, COL, GRO, HGO, JAL, MEX, MICH, MOR, NAY, OAX, PUE, QRO, QROO, SLP, TAB, TAMS, VER, YUC

*Solanum nyctaginoides Dunal HGO, JAL, MOR, OAX

*Solanum oxycarpum Schiede CHIS, HGO, MEX, MICH, OAX, PUE, VER

* Solanum palmeri Vasey \& Rose BCN, BCS

* Solanum palmillae Standl. CHIS, VER

Solanum parishii A. Heller BCN 
Solanum pectinatum Dunal CHIS, GRO, OAX, TAB, VER

*Solanum pedunculare Schltdl. CDMX, GTO, GRO, HGO, MEX, MICH, OAX, QRO

Solanum phaseoloides Polak. CHIS

*Solanum pinnatisectum Dunal COL, GTO, JAL, MEX, MICH, QRO

*Solanum plumense Fernald CHIS, GRO, MICH, OAX

*Solanum polyadenium Greenm. COL, CDMX, GTO, HGO, JAL, MEX, MICH, OAX, PUE, QRO, SLP, VER

*Solanum pringlei B.L. Rob. \& Greenm. JAL, MICH

Solanum pubigerum Dunal AGS, CHIS, CHIH, COL, CDMX, GTO, GRO, HGO, JAL, MEX, MICH, MOR, OAX, PUE, QRO, SLP, TAMS, TLAX, VER, ZAC

*Solanum pulverulentifolium K.E. Roe CHIS

* Solanum refractum Hook. \& Arn. CHIS, COL, DGO, GTO, GRO, JAL, MEX, MICH, MOR, NAY, OAX, QRO, SLP, SIN, SON, ZAC

*Solanum roei Ugent \& H.M. Iltis CHIS

Solanum rojasianum (Standl. \& Steyerm.) Bohs CHIS

Solanum rostratum Dunal AGS, CAM, CHIS, CHIH, COAH, COL, CDMX, DGO, GTO, GRO, HGO, JAL, MEX, MICH, MOR, NAY, NLE, OAX, PUE, QRO, SLP, SIN, SON, TAB, TAMS, TLAX, VER, ZAC

Solanum rovirosanum Donn. Sm. CAM, CHIS, COL, JAL, OAX, TAB, VER

Solanum rudepannum Dunal BCS, CAM, CHIS, COL, GRO, HGO, JAL, MEX, MICH, NAY, OAX, PUE, QROO, SLP, SIN, TAB, TAMS, VER, YUC

*Solanum sambucinum Rydb. GTO, HGO, JAL, MICH, QRO, SLP

*Solanum schenkii Bitter GTO, JAL, OAX, PUE, QRO, SLP, VER

*Solanum sideroxyloides Schltdl. CAM, MICH, QROO, VER, YUC

Solanum skutchii Correll HGO, MICH, OAX, PUE, QRO, SLP, VER

*Solanum sousae S. Knapp OAX, PUE

*Solanum stenophyllidium Bitter AGS, CHIH, COAH, COL, DGO, GTO, JAL, MEX, MICH, NAY, NLE, QRO, SLP, SON, $\mathrm{ZAC}$

*Solanum stephanocalyx Brandegee VER

Solanum stoloniferum Schltdl. \& C.D. Bouché AGS, BCS, CHIH, COAH, COL, CDMX, DGO, GTO, GRO, HGO, JAL, MEX, MICH, NAY, NLE, OAX, PUE, QRO, SLP, SIN, SON, TLAX, VER, ZAC

Solanum suaveolens Kunth \& C.D. Bouché CHIS, OAX, PUE, QRO, SLP, VER

*Solanum subvelutinum Rydb. DGO, SIN

*Solanum sylvicola Brandegee CHIS

Solanum tacanense Lundell CHIS

Solanum tampicense Dunal CAM, CHIS, GRO, JAL, NAY, OAX, PUE, SLP, TAB, TAMS, VER, YUC

*Solanum tarnii Hawkes \& Hjert. HGO, QRO, VER

Solanum tenuilobatum Parish BCN

Solanum tenuipes Bartlett CHIH, COAH, DGO, NLE

Solanum torvum Sw. CAM, CHIS, CHIH, COL, DGO, GTO, GRO, HGO, JAL, MEX, MICH, MOR, NAY, NLE, OAX, PUE,
QRO, QROO, SLP, SIN, SON, TAB, TAMS, VER, YUC, ZAC * Solanum tribulosum Schauer GTO, HGO, OAX, PUE, QRO, SLP, VER

* Solanum tridynamum Dunal CAM, CHIH, COL, DGO, GRO, HGO, JAL, MICH, NAY, OAX, PUE, QRO, QROO, SLP, SIN, SON, TAMS, VER, YUC, ZAC

*Solanum trifidum Correll COL, JAL, MICH

Solanum triquetrum Cav. CHIH, COAH, DGO, GTO, NLE, QRO, SLP, TAMS, ZAC

Solanum trizygum Bitter CHIS, GRO, OAX, PUE, VER

Solanum tuerckheimii Greenm. CHIS, QROO, VER, YUC

Solanum umbellatum Mill. BCN, CAM, CHIS, CHIH, COL, DGO, GTO, GRO, HGO, JAL, MEX, MICH, MOR, NAY, NLE, OAX, PUE, QRO, QROO, SLP, SIN, SON, TAB, TAMS, VER, YUC, ZAC

Solanum umbelliferum Eschsch. BCN

* Solanum verrucosum Schltdl. COAH, COL, CDMX, GTO, GRO, HGO, JAL, MEX, MICH, MOR, NLE, OAX, PUE, QRO, SLP, TAMS, TLAX, VER, ZAC

Solanum volubile $\mathrm{Sw}$. CHIS, OAX, PUE, TAB, VER

Solanum wallacei (A. Gray) Parish BCN

Solanum wendlandii Hook. f. CAM, CHIS, GRO, JAL, OAX, PUE, QRO, SLP, TAB, VER

*Solanum $\times$ edinenese P. Berhault CDMX, GTO, HGO, MEX, MICH, PUE, SLP, TLAX, VER

*Solanum $\times$ michoacanum (Bitter) Rydb. MICH

*Solanum $\times$ vallis-mexici Juz. CDMX, HGO, MEX, VER

Solanum xanti A. Gray BCN, BCS

*Solanum yucatanum Standl. CAM, QROO, YUC

Tzeltalia amphitricha (Bitter) E. Estrada \& M. Martínez CHIS, HGO, QRO, SLP, VER

Tzeltalia calidaria (Standl. \& Steyerm.) E. Estrada \& M. Martínez CHIS

*Tzeltalia esenbeckii M. Martínez \& O. Vargas CHIS

Witheringia cuneata (Standl.) Hunz. CHIS, GRO, OAX, TAMS, VER

Witheringia meiantha (Donn. Sm.) Hunz. CHIS, OAX, TAB, VER

*Witheringia mexicana (B.L. Rob.) Hunz. CHIS, COAH, GRO, HGO, NLE, OAX, QRO, SLP, TAMS, VER

Witheringia solanacea L'Hér. CAM, CHIS, GRO, HGO, MICH, NLE, OAX, PUE, QRO, QROO, SLP, TAB, TAMS, VER, YUC *Witheringia stellata (Greenm.) Hunz. HGO, OAX, PUE, QRO, SLP, VER

\section{Family Staphyleaceae}

*Staphylea pringlei $\mathrm{S}$. Watson COAH, HGO, NLE, SLP, TAMS, VER

Turpinia insignis (Kunth) Tul. CHIS, GRO, HGO, OAX, PUE, VER

Turpinia occidentalis (Sw.) G. Don CHIS, COL, DGO, GRO, HGO, JAL, NAY, OAX, PUE, QRO, SLP, TAB, TAMS, VER

Turpinia parvifoliola L.O. Williams CHIS

Turpinia tricornuta Lundell CHIS, GRO

Family Stegnospermataceae

Stegnosperma cubense A. Rich. BCN, CHIS, COL, GRO, JAL, MEX, MICH, NAY, OAX, SIN, VER 
* Stegnosperma sanchezii Medrano \& Medina OAX, PUE Stegnosperma scandens (Lunan) Standl. BCN, BCS, CHIS, COL, GRO, JAL, MEX, MICH, NAY, OAX, SIN, SON, VER

\section{Family Styracaceae}

Styrax argenteus $\mathrm{C}$. Presl BCS, CHIS, COL, DGO, GRO, JAL, MEX, MICH, NAY, OAX, QRO, SLP, SIN, TAMS, VER

* Styrax austromexicanus P.W. Fritsch GRO, OAX

Styrax conterminus Donn. Sm. CHIS, GRO, OAX

* Styrax gentryi P.W. Fritsch BCS, SIN

Styrax glabrescens Benth. CHIS, GTO, GRO, HGO, MEX,

MICH, OAX, PUE, QRO, SLP, TAMS, VER

* Styrax jaliscana $\mathrm{S}$. Watson JAL, NAY

* Styrax lanceolatus P.W. Fritsch HGO, QRO, SLP, TAMS

Styrax magnus Lundell CHIS

Styrax platanifolius Engelm. ex Torr. COAH, NLE, TAMS

* Styrax radians P.W. Fritsch CHIS, COL, DGO, GRO, JAL, MEX, MICH, NAY, OAX, SIN, VER

* Styrax ramirezii Greenm. AGS, CHIS, COL, DGO, GRO, JAL, MEX, MICH, MOR, NAY, OAX, SLP, SIN, TAMS, VER

* Styrax tuxtlensis P.W. Fritsch VER

*Styrax uxpanapensis P.W. Fritsch OAX, VER

Styrax warscewiczii Perkins CHIS, GRO, OAX, SLP

\section{Family Surianaceae}

* Recchia connaroides (Loes. \& Soler.) Standl. OAX

* Recchia mexicana Moc. \& Sessé ex DC. CHIS, COL, GRO, JAL, MICH, NAY, OAX, TAB

*Recchia sessiliflora González-Murillo \& Cruz-Durán GRO

*Recchia simplicifolia T. Wendt \& E.J. Lott CHIS, OAX, TAB, VER

Suriana maritima L. CAM, QROO, VER, YUC

\section{Family Symplocaceae}

*Symplocos austromexicana Almeda OAX

Symplocos bicolor L.O. Williams CHIS, OAX, VER

Symplocos breedlovei Lundell CHIS, OAX

*Symplocos citrea Lex. ex La Llave \& Lex. AGS, COL, CDMX, GTO, GRO, HGO, JAL, MEX, MICH, MOR, NAY, OAX, QRO, SLP, VER, ZAC

* Symplocos coccinea Bonpl. CHIS, HGO, OAX, PUE, VER

*Symplocos excelsa L.O. Williams CHIS, OAX, VER

Symplocos hartwegii DC. CHIS, PUE, VER

*Symplocos hintonii Lundell GRO

Symplocos johnstonii Standl. CHIS, OAX, VER

*Symplocos jurgensenii Hemsl. OAX

Symplocos limoncillo Bonpl. CHIS, HGO, OAX, VER

Symplocos longipes Lundell CHIS, GRO, OAX, VER

Symplocos matudae Lundell CHIS, OAX, VER

*Symplocos novogaliciana L.M. González COL, JAL, NAY

*Symplocos pachycarpa L.M. Kelly \& Almeda GRO, OAX

Symplocos pycnantha Hemsl. CHIS, GRO, HGO, OAX, VER

*Symplocos sousae Almeda COL, GRO, JAL, OAX

Symplocos speciosa Hemsl. HGO, MICH, OAX, VER

Symplocos tacanensis Lundell CHIS

\section{Family Talinaceae}

*Grahamia coahuilensis (S. Watson) G.D. Rowley COAH, NLE
Talinum angustissimum (A. Gray) Wooton \& Standl. CHIH, COAH, DGO, GTO, JAL, SON, TAMS, ZAC

*Talinum attenuatum Rose \& Standl. GRO, SIN

Talinum fruticosum (L.) Juss. BCS, CHIS, COL, GRO, JAL, MEX, MICH, MOR, NAY, OAX, PUE, QRO, QROO, SLP, SIN, SON, TAB, TAMS, VER, YUC

Talinum lineare Kunth AGS, CHIH, COAH, CDMX, DGO, GTO, HGO, JAL, MEX, NLE, OAX, PUE, QRO, SLP, SON, TAMS, VER, ZAC

Talinum paniculatum (Jacq.) Gaertn. AGS, BCS, CAM, CHIS, CHIH, COAH, COL, CDMX, DGO, GTO, GRO, HGO, JAL, MEX, MICH, MOR, NAY, NLE, OAX, PUE, QRO, QROO, SLP, SIN, SON, TAB, TAMS, VER, YUC, ZAC

Talinum pulchellum Wooton \& Standl. CHIH, COAH, ZAC

Talinum tuberosum (Benth.) P.G. Wilson MICH

\section{Family Tapisciaceae}

Huertea cubensis Griseb. CHIS, PUE, VER

Family Tetrachondraceae

Polypremum procumbens L. CHIS, GTO, GRO, OAX, SLP, SIN, SON, TAB, TAMS

\section{Family Theaceae}

Gordonia brenesii (Standl.) Q. Jiménez CHIS, GRO, OAX, VER

\section{Family Thymelaeaceae}

Daphnopsis americana (Mill.) J.R. Johnst. CHIS, COL, GRO, HGO, JAL, MEX, MICH, MOR, OAX, PUE, QROO, VER, YUC

*Daphnopsis brevifolia Nevling OAX, TAMS, VER

Daphnopsis ficina Standl. \& Steyerm. CHIS, GRO, NAY, OAX * Daphnopsis flavida Lundell CHIS

*Daphnopsis lagunae Breedlove \& León de la Luz BCS

*Daphnopsis liebmannii Nevling CHIS, OAX

Daphnopsis malacophylla Standl. \& Steyerm. CHIS

*Daphnopsis megacarpa Nevling \& Barringer VER

*Daphnopsis mexiae Nevling JAL, NAY

*Daphnopsis mollis (Cham. \& Schltdl.) Standl. CHIS, HGO, JAL, OAX, QRO, QROO, SLP, TAMS, VER

Daphnopsis monocephala Donn. Sm. CHIS

*Daphnopsis nevlingii J. Jiménez Ram. \& J.L. Contr. GRO, OAX

*Daphnopsis purpusii Brandegee OAX, PUE

Daphnopsis radiata Donn. Sm. CHIS, OAX, VER

Daphnopsis tuerckheimiana Donn. Sm. CHIS, VER

*Dirca mexicana G.L. Nesom \& Mayfield NLE, TAMS

Family Ticodendraceae

Ticodendron incognitum Gómez-Laur. \& L.D. Gómez CHIS, OAX

Family Tovariaceae

Tovaria pendula Ruiz \& Pav. CHIS, GRO, HGO, OAX, PUE, VER

Family Trigoniaceae

Trigonia eriosperma (Lam.) Fromm \& E. Santos CAM, CHIS, OAX, QROO, YUC

Trigonia rugosa Benth. CHIS 
Family Triuridaceae

*Lacandonia schismatica E. Martínez \& Ramos CHIS

Triuris hyalina Miers CHIS

Family Tropaeolaceae

Tropaeolum moritzianum Klotzsch CHIS

\section{Family Typhaceae}

Sparganium americanum Nutt. DGO, SIN

Sparganium eurycarpum Engelm. BCN, MEX

Typha domingensis Pers. AGS, BCN, BCS, CAM, CHIS, CHIH, COAH, COL, CDMX, DGO, GTO, GRO, HGO, JAL, MEX, MICH, MOR, NAY, NLE, OAX, PUE, QRO, QROO, SLP, SIN, SON, TAB, TAMS, TLAX, VER, YUC, ZAC

Typha latifolia L. AGS, BCN, BCS, CAM, CHIS, CHIH, COAH, COL, CDMX, DGO, GTO, HGO, JAL, MEX, MICH, MOR, NAY, NLE, OAX, PUE, QRO, SLP, SIN, SON, TAB, TAMS, TLAX, VER

\section{Family Ulmaceae}

Ampelocera hottlei (Standl.) Standl. CAM, CHIS, OAX, PUE, QROO, TAB, VER

Phyllostylon brasiliense Capan. ex Benth. \& Hook. f. CAM, COL, GTO, HGO, JAL, NLE, OAX, PUE, QRO, SLP, TAMS, VER, YUC

Ulmus americana L. CHIH, COAH, DGO, NLE, QRO, SON

Ulmus crassifolia Nutt. COAH, NLE, TAMS

Ulmus ismaelis Todzia \& Panero OAX

Ulmus mexicana (Liebm.) Planch. CHIS, GRO, HGO, JAL, OAX, PUE, QRO, SLP, TAB, VER, ZAC

Ulmus serotina Sarg. COAH, NLE

\section{Family Urticaceae}

Boehmeria aspera Wedd. HGO, VER

Boehmeria caudata Sw. CHIS, GRO, JAL, OAX, VER

Boehmeria cylindrica (L.) Sw. COAH, GRO, HGO, MEX, MOR, NLE, PUE, QRO, QROO, SLP, TAB, TAMS, VER

Boehmeria pavonii Wedd. CHIS

Boehmeria radiata W.C. Burger CHIS, COL, GRO, JAL, MEX, MICH, NAY, OAX, PUE, SIN

Boehmeria ramiflora Jacq. CHIS, OAX, PUE, TAB, VER

Boehmeria ulmifolia Wedd. CHIS, COL, GRO, HGO, JAL, MEX, MICH, OAX, PUE, QRO, SLP, TAB, TAMS, VER

Cecropia angustifolia Trécul OAX, PUE

Cecropia obtusifolia Bertol. CAM, CHIS, CHIH, COL, DGO, GRO, HGO, JAL, MEX, MICH, MOR, NAY, OAX, PUE, QRO, QROO, SLP, SIN, TAB, TAMS, VER, YUC

Cecropia peltata L. CAM, CHIS, HGO, GRO, MICH, NAY, OAX, PUE, QRO, QROO, SLP, TAB, VER, YUC

Coussapoa oligocephala Donn. Sm. CAM, CHIS, PUE, QRO, QROO, SLP, TAB

Coussapoa purpusii Standl. CAM, CHIS, COL, GRO, JAL, NAY, OAX, TAB, VER

Discocnide mexicana (Liebm.) Chew CHIS, COL, GRO, HGO, JAL, MEX, MICH, MOR, OAX, PUE, QRO, SLP, VER, YUC Gyrotaenia microcarpa (Wedd.) Fawc. \& Rendle CHIS, OAX, TAB, YUC

Hemistylus odontophylla Wedd. CHIS

Hesperocnide tenella Torr. $\mathrm{BCN}$
Laportea canadensis (L.) Wedd. NLE, PUE, QRO, SLP, TAMS, VER

Myriocarpa bifurca Liebm. CHIS, OAX, VER

* Myriocarpa brachystachys S. Watson CHIS, GTO, GRO, JAL, MEX, MICH, MOR, NAY, OAX, VER, ZAC

*Myriocarpa cordifolia Liebm. CHIS, HGO, OAX, PUE, TAMS, VER

Myriocarpa cubilgueitzensis A.K. Monro CHIS, OAX, TAB, VER

Myriocarpa heterospicata Donn. Sm. CHIS, GRO, OAX, TAB, VER

Myriocarpa longipes Liebm. CHIS, COL, GRO, HGO, JAL, MEX, MICH, MOR, NAY, OAX, PUE, QRO, SLP, TAB, TAMS, VER

Myriocarpa obovata Donn. Sm. CHIS, OAX, VER

* Myriocarpa trifurca A.K. Monro CHIS, VER

Parietaria debilis G. Forst. BCN, BCS, CHIS, COAH, COL, JAL, MEX, NLE, OAX, PUE, SIN, SON, VER

*Parietaria decoris N.G. Mill. COAH, NLE, TAMS

* Parietaria macrophylla B.L. Rob. \& Greenm. COL, JAL, MEX, MICH, MOR, OAX, QRO, SLP

Parietaria pensylvanica Muhl. ex Willd. BCS, CHIH, COAH, CDMX, GTO, GRO, HGO, JAL, MEX, MICH, MOR, NLE, OAX, PUE, QRO, SLP, SON, TAMS, TLAX, VER, ZAC

Phenax hirtus (Sw.) Wedd. CHIS, COL, GRO, HGO, JAL, MEX, MICH, MOR, NAY, OAX, PUE, QRO, SLP, SIN, TAB, VER

Phenax mexicanus Wedd. CHIS, COL, GRO, HGO, JAL, MICH, OAX, PUE, QRO, VER

Phenax rugosus (Poir.) Wedd. CAM, CHIS, GRO, OAX, QROO, SLP, TAMS, VER

Phenax sonneratii (Poir.) Wedd. CHIS, OAX, VER

Pilea acuminata Liebm. OAX, QRO, SLP, VER

Pilea auriculata Liebm. CHIS, SLP

Pilea cadierei Gagnep. \& Guillaumin CHIS, VER

Pilea chiapensis Killip CHIS, TAB

Pilea daguensis Killip CHIS, OAX

Pilea dauciodora Wedd. CHIS, OAX

Pilea ecboliophylla Donn. Sm. CHIS, QRO, SLP, TAB

* Pilea falcata Liebm. CHIS, OAX, VER

Pilea glabra S. Watson CHIS, OAX, QRO, SLP, TAMS, VER

Pilea gracilipes Killip CHIS, GRO, OAX

Pilea herniarioides (Sw.) Wedd. CAM, GRO, QROO, VER, YUC

Pilea hyalina Fenzl CHIS, COL, GRO, JAL, MOR, NAY, OAX, VER

Pilea imparifolia Wedd. OAX, QRO, SLP, VER

Pilea irrorata Donn. Sm. CHIS, OAX, PUE, VER

Pilea killipiana Standl. \& Steyerm. CHIS

*Pilea mexicana Wedd. CHIS, GRO, OAX, QRO, SLP, VER

Pilea microphylla (L.) Liebm. CAM, CHIS, CHIH, COAH, COL, GTO, GRO, HGO, JAL, MEX, MICH, MOR, NAY, NLE, OAX, PUE, QRO, QROO, SLP, SIN, TAB, TAMS, VER, YUC Pilea pansamalana Donn. Sm. CHIS, TAB, VER

Pilea parietaria (L.) Blume CHIS, HGO, OAX, QRO, VER, YUC

* Pilea pteridophylla A.K. Monro CHIS, OAX, TAB, VER 
Pilea pubescens Liebm. CAM, CHIS, HGO, MICH, OAX, PUE, QRO, QROO, SLP, TAB, TAMS, VER

Pilea purulensis Donn. Sm. CHIS, GRO, MICH, OAX

Pilea quercifolia Killip CHIS, COL, GRO, JAL, MEX, MICH, OAX, VER

Pilea quichensis Donn. Sm. CHIS

Pilea riparia Donn. Sm. CHIS, OAX, VER

* Pilea serpyllifolia (Poir.) Wedd. OAX, TAMS

Pilea skutchii Killip CHIS

Pilea tridentata Killip CHIS, OAX

Pilea tuerckheimii Donn. Sm. CHIS, OAX

* Pilea vulcanica Liebm. OAX, PUE, VER

Pourouma aspera Trecul. CHIS

Pouzolzia guatemalana (Blume) Wedd. CHIS, GRO, JAL, MEX,

MICH, OAX, SIN, SON, VER, ZAC

Pouzolzia obliqua (Poepp.) Wedd. CHIS, TAB

Pouzolzia occidentalis (Liebm.) Wedd. CHIS, COL, GTO, GRO, HGO, JAL, MEX, MICH, MOR, NAY, OAX, PUE, QRO, SLP, SIN, SON, VER

*Pouzolzia pringlei Greenm. OAX, PUE

*Pouzolzia purpusii Brandegee CHIS, OAX, PUE

Rousselia humilis (Sw.) Urb. CAM, QROO, YUC

Urera alceifolia Gaudich. CHIS, GRO, HGO, OAX, QRO, SLP,

TAB, VER

Urera baccifera (L.) Gaudich. ex Wedd. CAM, CHIS, CHIH, COL, DGO, GTO, GRO, HGO, JAL, MEX, MICH, MOR, NAY,

OAX, PUE, QROO, SIN, SON, TAB, TAMS, VER, YUC

Urera elata (Sw.) Griseb. CHIS, GRO, HGO, OAX, QRO, TAB, VER

Urera glabriuscula V.W. Steinm. CHIS, OAX, TAB, VER

Urera killipiana Standl. \& Steyerm. CHIS, HGO, OAX, QRO,

TAB, VER

Urera lianoides A.K. Monro \& Al. Rodr. CHIS, VER

*Urera martiniana V.W. Steinm. HGO, MEX, MICH, QRO, SLP, TAMS

*Urera pacifica V.W. Steinm. COL, GRO, JAL, MEX, MICH, NAY, OAX, SIN

Urera rzedowskii V.W. Steinm. VER

Urera simplex Wedd. CHIS, HGO, OAX, PUE, QRO, SLP, TAB, VER

Urera verrucosa V.W. Steinm. CHIS, COL, GRO, HGO, JAL, MEX, MICH, MOR, OAX, PUE, QRO, VER

Urtica chamaedryoides Pursh CHIS, CHIH, COAH, CDMX, GRO, HGO, JAL, MEX, MICH, MOR, NLE, OAX, PUE, QRO, SLP, SON, TAMS, TLAX, VER

Urtica dioica L. CHIS, CHIH, CDMX, HGO, JAL, MEX, MICH, MOR, NLE, OAX, PUE, QRO, SON, TAB, TAMS, TLAX, VER

Urtica gracilenta Greene CHIH, COAH, DGO, NLE, TAMS, ZAC

Urtica gracilis Aiton CDMX, MEX, PUE, VER

Urtica holosericea Nutt. BCN

Urtica mexicana Liebm. CHIS, CDMX, GRO, HGO, JAL, MEX, MICH, MOR, OAX, PUE, QRO, SLP, VER

*Urtica orizabae Liebm. VER

*Urtica praetermissa V.W. Steinm. CDMX, GRO, MEX, MICH, OAX
*Urtica spiralis Blume COAH, GTO, NLE, OAX, SLP, VER

*Urtica subincisa Benth. CDMX, GTO, HGO, JAL, MEX, MICH, PUE, QRO, TLAX, VER

\section{Family Verbenaceae}

*Aloysia barbata (Brandegee) Moldenke BCS

*Aloysia chiapensis Moldenke CHIS, MOR, OAX

Aloysia citriodora Paláu AGS, HGO, JAL, MEX, MICH, MOR, NAY, NLE, PUE, QRO, SLP, TLAX, ZAC

Aloysia gratissima (Gillies \& Hook.) Tronc. AGS, CHIH, COAH, DGO, GTO, HGO, JAL, NLE, OAX, PUE, QRO, SLP, SON, TAMS, VER, ZAC

Aloysia macrostachya (Torr.) Moldenke COAH, GTO, HGO, MOR, NLE, QRO, SLP, TAMS, ZAC

*Aloysia nahuire A.H. Gentry \& Moldenke SIN

*Aloysia sonorensis Moldenke SIN, SON

Aloysia wrightii (A. Gray) Heller CHIH, COAH, DGO, NLE, SLP, SON, ZAC

*Bouchea dissecta S. Watson COL, GRO, JAL, MICH, SIN, SON

*Bouchea flabelliformis M.E. Jones BCS, COL, JAL

Bouchea linifolia A. Gray CHIH, COAH

Bouchea nelsonii Grenzeb. CHIS, GRO, OAX, VER

Bouchea prismatica (L.) Kuntze AGS, CAM, CHIS, CHIH, COAH, COL, CDMX, DGO, GTO, GRO, HGO, JAL, MEX, MICH, MOR, NAY, NLE, OAX, PUE, QRO, QROO, SLP, SIN, SON, TAMS, VER, YUC, ZAC

Bouchea spathulata Torr. CHIH, COAH, DGO, NLE, ZAC

* Burroughsia fastigiata (Brandegee) Moldenke BCN, BCS

Citharexylum affine D. Don AGS, CHIS, COL, CDMX, GTO, GRO, HGO, JAL, MEX, MICH, NAY, OAX, PUE, QRO, SIN, TAB, VER, ZAC

*Citharexylum altamiranum Greenm. DGO, GTO, HGO, QRO, SLP, ZAC

Citharexylum berlandieri B.L. Rob. COL, DGO, GTO, GRO, HGO, JAL, MEX, NLE, OAX, QRO, SLP, SIN, TAMS, VER

*Citharexylum bourgeauianum Greenm. GRO, OAX, PUE, VER

Citharexylum brachyanthum (A. Gray) A. Gray CHIH, COAH, DGO, GTO, HGO, NLE, QRO, SLP, TAMS, ZAC

Citharexylum caudatum L. CHIS, MICH, OAX, QRO, QROO, SLP, TAB, VER, YUC

Citharexylum crassifolium Greenm. CHIS, QROO

*Citharexylum danirae León de la Luz \& F. Chiang COL

Citharexylum donnell-smithii Greenm. CHIS, COL, GRO, JAL, NAY, OAX

*Citharexylum ellipticum D. Don CAM, PUE, QROO, TAB, VER

*Citharexylum flabellifolium S. Watson BCS, SON

*Citharexylum fulgidum Moldenke CHIS, HGO, SLP, VER

*Citharexylum glabrum (S. Watson) Greenm. COL, GRO, JAL, MEX, NAY, OAX, QRO, SLP

Citharexylum hexangulare Greenm. CAM, CHIS, COL, DGO, GRO, JAL, MICH, NAY, OAX, QROO, SLP, TAB, VER, YUC *Citharexylum hidalgense Moldenke HGO, JAL, MEX, MICH, NLE, PUE, QRO, SLP, TAMS, VER, ZAC

*Citharexylum hintonii Moldenke CHIS, GRO, MEX, MOR 
Citharexylum hirtellum Standl. CAM, CHIS, COL, JAL, OAX, QROO, TAB, VER

*Citharexylum incanum D. Don BCS, NAY, SIN, SON

*Citharexylum kerberi Greenm. VER

*Citharexylum ligustrinum Van Houtte CDMX, HGO, PUE, QRO, SIN, VER

*Citharexylum lycioides D. Don COAH, DGO, GTO, HGO, MICH, QRO, SLP, VER, ZAC

*Citharexylum mexicanum Moldenke COL, GRO, JAL, OAX, PUE, VER

Citharexylum mocinnoi D. Don CHIS, COL, DGO, GRO, JAL, MEX, NAY, OAX, VER

*Citharexylum oleinum (Benth.) Moldenke GTO, HGO, NLE, OAX, PUE, QRO, SLP, TAMS, VER

*Citharexylum ovatifolium Greenm. DGO, GRO, HGO, MEX, MOR, NLE, OAX, TAMS

*Citharexylum racemosum Sessé \& Moc. GTO, MICH, OAX, PUE

*Citharexylum rosei Greenm. DGO, GTO, HGO, QRO, SLP, ZAC

*Citharexylum roxanae Moldenke BCS

*Citharexylum scabrum Sessé \& Moc. ex D. Don NAY, SIN, SON

Citharexylum schottii Greenm. CAM, CHIS, QROO, YUC

*Citharexylum shrevei Moldenke SON

Citharexylum spathulatum Moldenke \& Lundell CHIH, NLE, QRO, SLP, TAMS, ZAC

Citharexylum standleyi Moldenke COL, JAL, MICH

Citharexylum steyermarkii Moldenke CHIS, VER

*Citharexylum tetramerum Brandegee HGO, OAX, PUE, QRO, SLP

Duranta erecta L. BCS, CAM, CHIS, COL, CDMX, GRO, HGO, JAL, MEX, MICH, MOR, OAX, PUE, QRO, QROO, SLP, SIN, TAB, TAMS, VER, YUC

Duranta guatemalensis Moldenke CHIS

* Glandularia alejandrana B.L. Turner NLE, SLP, ZAC

*Glandularia amoena (Paxton) Umber MEX, MICH, QRO, SLP

*Glandularia bajacalifornica (Moldenke) Umber BCS

Glandularia bipinnatifida (Nutt.) Nutt. AGS, CHIS, CHIH, COAH, COL, CDMX, DGO, GTO, GRO, HGO, JAL, MEX, MICH, MOR, NAY, NLE, OAX, PUE, QRO, SLP, SIN, SON, TAB, TAMS, TLAX, VER, ZAC

* Glandularia brachyrhynchos G.L. Nesom \& Vorobik SLP, TAMS

Glandularia delticola (Small) Umber AGS, CHIS, COAH, HGO, JAL, MICH, NLE, OAX, PUE, QRO, SLP, SON, TAMS, VER

Glandularia elegans (Kunth) Umber CHIS, CHIH, COAH, CDMX, DGO, GTO, GRO, HGO, MEX, MICH, MOR, NAY, NLE, OAX, QRO, SLP, SIN, SON, TAMS, VER, ZAC Glandularia gooddingii (Briq.) Solbrig BCN, BCS, SON

* Glandularia lilacina (Greene) Umber BCN, BCS

* Glandularia malpaisana Van Devender \& Nesom SON

Glandularia polyantha Umber COAH, NLE, TAMS

Glandularia pumila (Rydb.) Umber COAH, NLE, SON, TAMS,

ZAC
Glandularia quadrangulata (A. Heller) Umber CHIH, COAH, NLE, TAMS

Glandularia teucriifolia (M. Martens \& Galeotti) Umber AGS, CDMX, DGO, GTO, HGO, JAL, MEX, MICH, MOR, NLE, OAX, PUE, SLP, TLAX, VER, ZAC

Glandularia tumidula (L.M. Perry) Umber COAH, TAMS

*Glandularia turneri G.L. Nesom COAH, NLE

Lantana achyranthifolia Desf. AGS, CHIS, CHIH, COAH, COL, DGO, GTO, GRO, HGO, JAL, MEX, MICH, MOR, NAY, NLE, OAX, PUE, QRO, SLP, SIN, SON, TAMS, TLAX, VER, ZAC

Lantana camara L. AGS, BCN, BCS, CAM, CHIS, CHIH, COAH, COL, CDMX, DGO, GTO, GRO, HGO, JAL, MEX, MICH, MOR, NAY, NLE, OAX, PUE, QRO, QROO, SLP, SIN, SON, TAB, TAMS, TLAX, VER, YUC, ZAC

Lantana canescens Kunth CAM, COAH, COL, DGO, GTO, GRO, HGO, JAL, MEX, MICH, MOR, NLE, OAX, PUE, QRO, QROO, SLP, TAMS, VER, YUC, ZAC

*Lantana chiapasensis Moldenke CHIS

* Lantana dwyeriana Moldenke CAM, QROO

Lantana glandulosissima Hayek AGS, CAM, CHIS, CHIH, COL, DGO, GTO, GRO, HGO, JAL, MEX, MICH, MOR, NAY, OAX, PUE, QRO, QROO, SLP, SIN, SON, VER, YUC Lantana involucrata L. AGS, CAM, CHIH, COL, GTO, GRO, HGO, JAL, MEX, MICH, MOR, NLE, OAX, PUE, QRO, QROO, SLP, SIN, SON, TAB, TAMS, VER, YUC, ZAC

* Lantana jaliscana Moldenke COL, JAL

* Lantana kingii Moldenke CHIS, OAX, PUE

*Lantana langlassei Moldenke COL, GRO, JAL, MEX, MICH, MOR, NAY, SIN

Lantana macropoda Torr. CHIH, COAH, COL, DGO, GTO, HGO, JAL, NLE, QRO, SLP, SON, TAMS, VER, ZAC

Lantana montevidensis Briq. SIN

*Lantana notha Moldenke COAH, HGO, NLE, OAX, QRO, SLP, SIN, SON, VER

Lantana trifolia L. CAM, CHIS, GRO, HGO, MEX, MOR, OAX, TAB, TAMS, VER

Lantana urticoides Hayek BCN, BCS, CHIS, CHIH, COAH, COL, CDMX, GTO, GRO, HGO, JAL, MEX, MICH, MOR, NAY, NLE, OAX, PUE, QRO, SLP, SIN, SON, TAB, TAMS, VER

Lantana $\times$ strigocamara R.W. Sanders CAM, CHIS, QROO, YUC

Lippia alba (Mill.) N.E. Br. ex Britton \& P. Wilson BCS, CAM, CHIS, COL, GTO, GRO, HGO, JAL, MEX, MICH, MOR, NAY, OAX, PUE, QRO, QROO, SLP, SIN, TAB, TAMS, VER, YUC, ZAC

Lippia americana $\mathrm{L}$. VER

*Lippia appendiculata B.L. Rob. \& Greenm. CHIH, COAH, DGO

Lippia bicolor Kunth \& Bouché GRO, MEX, MICH, MOR

Lippia bracteosa (M. Martens \& Galeotti) Moldenke CHIS, COL, GTO, JAL, NAY, OAX, PUE, ZAC

Lippia cardiostegia Benth. CAM, CHIS, QROO

Lippia chiapasensis Loes. CHIS, COAH, DGO, NAY

*Lippia chrysantha Greenm. CHIS, MOR, OAX

*Lippia durangensis Moldenke CHIH, COAH, DGO, SIN, ZAC 
* Lippia formosa Brandegee BCS

* Lippia gentryi Standl. DGO, SIN, SON

Lippia graveolens Kunth AGS, CAM, CHIS, CHIH, COAH, COL, CDMX, DGO, GTO, GRO, HGO, JAL, MEX, MICH, MOR, NAY, NLE, OAX, PUE, QRO, QROO, SLP, SIN, SON, TAMS, VER, YUC, ZAC

Lippia incisa (Small) E.D. Schulz BCN, BCS, CHIH, COAH, DGO, HGO, NLE, QRO, QROO, SLP, VER

*Lippia inopinata Moldenke AGS, CHIS, JAL, MICH, NAY, OAX, PUE, ZAC

Lippia macrostachya (Torr.) S. Watson COAH, DGO, NLE, QRO, SLP, TAMS, ZAC

*Lippia mcvaughii Moldenke CHIS, COL, JAL, MICH, OAX

*Lippia mexicana G.L. Nesom CHIS, COL, CDMX, GTO, HGO, JAL, MEX, MICH, MOR, PUE, QRO, SON

Lippia myriocephala Schltdl. \& Cham. AGS, CAM, CHIS, DGO, GTO, GRO, HGO, JAL, MEX, MICH, MOR, NAY, OAX, PUE, QRO, QROO, SLP, SIN, TAB, TAMS, VER, YUC *Lippia nutans B.L. Rob. \& Greenm. CHIS, OAX, PUE, VER *Lippia oaxacana B.L. Rob. \& Greenm. GRO, JAL, MICH, MOR, OAX, PUE

*Lippia palmeri S. Watson BCN, BCS, JAL, MICH, SIN, SON *Lippia queretarensis Kunth COL, GTO, JAL, MICH, PUE, QRO, SLP, TAMS, VER

*Lippia tepicana Moldenke JAL, MICH, NAY

Lippia umbellata Cav. AGS, CAM, CHIS, CHIH, COAH, COL, CDMX, DGO, GTO, GRO, HGO, JAL, MEX, MICH, MOR, NAY, OAX, PUE, QRO, QROO, SLP, SIN, SON, TAB, TAMS, VER, YUC, ZAC

Lippia wrightii A. Gray CHIH, COAH, DGO, SON, ZAC

Lippia yucatana Loes. QROO, YUC

Petrea volubilis L. CAM, CHIS, COAH, GRO, HGO, JAL, MEX, MICH, MOR, OAX, PUE, QRO, QROO, SLP, TAB, TAMS, VER, YUC

Phyla cuneifolia (Torr.) Greene CHIH, SLP, SON, TAMS

Phyla dulcis (Trevir.) Moldenke CAM, CHIS, COL, GRO, HGO, JAL, MEX, MICH, MOR, OAX, PUE, QRO, QROO, SLP, TAB, TAMS, VER, YUC, ZAC

Phyla fruticosa (Mill.) K. Kenn. ex Wunderlin \& B.F. Hansen CAM, CHIS, COAH, GTO, HGO, JAL, MICH, NLE, OAX, PUE, QRO, QROO, SLP, SIN, TAB, TAMS, VER, YUC

Phyla lanceolata (Michx.) Greene BCN, NLE, SIN, TAMS

Phyla nodiflora (L.) Greene AGS, BCN, BCS, CAM, CHIS, CHIH, COAH, COL, CDMX, DGO, GTO, GRO, HGO, JAL, MEX, MICH, MOR, NAY, NLE, OAX, PUE, QRO, QROO, SLP, SIN, SON, TAB, TAMS, TLAX, VER, YUC, ZAC Phyla stoechadifolia (L.) Small CAM, CHIS, OAX, QROO, SLP, TAB, TAMS, VER, YUC

Phyla strigulosa (M. Martens \& Galeotti) Moldenke CAM, CHIS, CHIH, COAH, DGO, GTO, GRO, HGO, JAL, MEX, MICH, MOR, NLE, OAX, PUE, QRO, QROO, SLP, SIN, SON, TAB, TAMS, VER, YUC

* Priva armata $\mathrm{S}$. Watson NLE

Priva aspera Kunth CAM, CHIS, CHIH, COL, CDMX, DGO, GRO, HGO, JAL, MEX, MICH, MOR, NAY, NLE, OAX, PUE, QRO, QROO, SLP, SIN, SON, TAMS, VER

* Priva grandiflora (Ortega) Moldenke AGS, CHIH, CDMX,
DGO, GTO, HGO, JAL, MEX, MICH, MOR, OAX, QRO, SLP, VER, ZAC

* Priva ibugana Rzed. \& Calderón AGS, GTO, ZAC

Priva lappulacea (L.) Pers. AGS, BCS, CAM, CHIS, CHIH, COL, DGO, GTO, GRO, HGO, JAL, MEX, MICH, MOR, NAY, NLE, OAX, PUE, QRO, QROO, SLP, SIN, SON, TAB, TAMS, VER, YUC, ZAC

Priva mexicana (L.) Pers. AGS, CHIH, COAH, COL, CDMX, DGO, GTO, GRO, HGO, JAL, MEX, MICH, MOR, NLE, OAX, PUE, QRO, QROO, SLP, SON, TAB, TAMS, TLAX, VER, ZAC

Rehdera penninervia Standl. \& Moldenke CHIS

Rehdera trinervis (S.F. Blake) Moldenke CAM, QROO, YUC

* Stachytarpheta acuminata DC. GRO, HGO, JAL, MEX, MOR, OAX, PUE, QRO, VER

*Stachytarpheta albiflora DC. HGO, OAX

Stachytarpheta angustifolia (Mill.) Vahl CAM, CHIS, GRO, MICH, OAX, QROO, TAB, TAMS, VER, YUC

Stachytarpheta calderonii Moldenke QROO

Stachytarpheta cayennensis (L. Rich.) Vahl CAM, CHIS, GRO, OAX, QROO, TAB, VER, YUC

Stachytarpheta frantzii Pol. CAM, CHIS, COL, GRO, JAL, MICH, NAY, OAX, PUE, QRO, QROO, SLP, SIN, SON, TAB, VER, YUC

*Stachytarpheta hintoni Moldenke GRO, MEX, NAY, SON

Stachytarpheta jamaicensis (L.) Vahl CAM, CHIS, GRO, JAL, MICH, MOR, OAX, PUE, QROO, SLP, TAB, TAMS, VER, YUC

*Stachytarpheta luisana (Standl.) Standl. PUE

*Stachytarpheta mexicana Moldenke GRO, MEX

Stachytarpheta miniacea Moldenke CAM, CHIS, OAX, QROO, YUC

Stachytarpheta mutabilis (Jacq.) Vahl AGS, CAM, CHIS, GRO, HGO, JAL, MEX, MICH, NAY, OAX, PUE, QRO, QROO, SLP, SIN, VER, YUC

*Stachytarpheta nelsonii B.L. Rob. \& Greenm. OAX

*Stachytarpheta velutina Moldenke GRO, HGO, MEX, MICH, MOR, OAX, QRO, VER

Tamonea curassavica (L.) Pers. CAM, CHIS, HGO, NLE, OAX, PUE, QRO, QROO, SLP, TAMS, VER, YUC

*Tamonea euphrasiifolia B.L. Rob. SLP, TAMS, VER

Tamonea spicata Aubl. CAM, HGO, QRO, QROO, SLP

Verbena bracteata Lag. \& Rodr. BCN, CHIH, COAH, DGO, HGO, NLE, QRO, SON, VER, ZAC

Verbena canescens Kunth AGS, CHIH, COAH, CDMX, DGO, GTO, GRO, HGO, JAL, MEX, MICH, NLE, OAX, PUE, QRO, SLP, TAMS, VER, ZAC

Verbena carolina L. AGS, BCS, CHIS, CHIH, COAH, COL, CDMX, DGO, GTO, GRO, HGO, JAL, MEX, MICH, MOR, NAY, NLE, OAX, PUE, QRO, SLP, SIN, SON, TAMS, TLAX, VER, ZAC

Verbena ehrenbergiana Schauer CHIH, COAH, GTO, HGO, JAL, MICH, NLE, PUE, QRO, SLP, SON, VER

*Verbena erecta Cav. HGO, MOR, QRO

*Verbena gentryi Moldenke DGO, OAX, SIN

Verbena gracilis Desf. AGS, CHIH, COAH, CDMX, DGO, GTO, HGO, JAL, MEX, MICH, NAY, OAX, PUE, QRO, SLP, 
SON, TLAX, VER, ZAC

Verbena halei Small CHIH, COAH, HGO, JAL, MICH, NLE, QRO, SIN, SON, TAMS, VER

*Verbena integrifolia M. Martens \& Galeotti MICH

*Verbena johnstonii (Moldenke) G.L. Nesom COAH, NLE, ZAC

Verbena lasiostachys Link BCN

Verbena litoralis Kunth AGS, CHIS, COAH, COL, CDMX, DGO, GTO, GRO, HGO, JAL, MEX, MICH, MOR, NAY, NLE, OAX, PUE, QRO, SLP, SIN, TAMS, TAB, VER, ZAC

*Verbena macrodonta Perry BCS

*Verbena madrensis Nesom NLE, TAMS

Verbena menthifolia Benth. AGS, BCN, BCS, CHIS, CHIH, COAH, CDMX, DGO, GTO, HGO, JAL, MEX, MICH, NLE, OAX, PUE, QRO, SLP, SIN, SON, TAMS, TLAX, VER, ZAC *Verbena moctezumae Nesom \& Van Devender SON

Verbena neomexicana (A. Gray) Small BCN, CHIH, COAH, DGO, JAL, NLE, SLP, SON, TAMS, ZAC

*Verbena orcuttiana Perry BCN

Verbena perennis Wooton CHIH, COAH, NLE, SLP, SON

Verbena pinetorum Moldenke CHIH, HGO, MEX, QRO, VER Verbena plicata Greene BCN, COAH, NLE, SON, TAMS

*Verbena recta Kunth COL, CDMX, GRO, GTO, HGO, JAL, MEX, MICH, MOR, NLE, OAX, PUE, QRO, TLAX, VER, ZAC

Verbena scabra Vahl BCN, BCS, CHIH, COAH, NLE, OAX

*Verbena sphaerocarpa Perry COL

Verbena xutha Lehm. CHIH, COAH, SON, TAMS

Xolocotzia asperifolia Miranda CHIS

\section{Family Violaceae}

Corynostylis arborea (L.) S.F. Blake CAM, CHIS, GRO, OAX, QROO, TAB, VER, YUC

Hybanthus attenuatus (Humb. \& Bonpl. ex Roem. \& Schult.) Schulze-Menz AGS, BCS, CHIS, CHIH, COL, DGO, GTO, GRO, HGO, JAL, MEX, MICH, MOR, NAY, OAX, PUE, QRO, QROO, SLP, SIN, SON, TAB, TAMS, VER, YUC, ZAC Hybanthus calceolaria (L.) Schulze-Menz CHIS, TAB, VER *Hybanthus chiapensis Lundell CHIS

Hybanthus elatus (Turcz.) C.V. Morton CHIS, COL, JAL, OAX, VER

*Hybanthus fruticulosus (Benth.) I.M. Johnst. BCN, BCS, SIN, SON

Hybanthus galeotti (Turcz.) C.V. Morton ex L.O. Williams CHIS, OAX, VER

*Hybanthus longipes (Dowell) Standl. CHIS, QRO

Hybanthus oppositifolius (L.) Taub. CAM, CHIS, GRO, HGO, MEX, MICH, MOR, OAX, PUE, QRO, QROO, SLP, TAB, TAMS, VER, YUC

*Hybanthus pennellii (C.V. Morton) B.L. Turner NLE, TAMS

*Hybanthus potosinus C.V. Morton SLP, TAMS

*Hybanthus serrulatus Standl. COL, JAL, SIN, SON

Hybanthus sylvicola Standl. \& Steyerm. CAM, CHIS, QROO

Hybanthus thiemei (Donn. Sm.) C.V. Morton CAM, CHIS, OAX, QROO, YUC

Hybanthus verbenaceus (Kunth) Loes. CHIS, COAH, COL, CDMX, GTO, GRO, HGO, JAL, MEX, MICH, MOR, NLE,
OAX, PUE, QRO, SLP, TAMS, TLAX, VER

Hybanthus verticillatus (Ortega) Baill. AGS, BCN, BCS, CHIH, COAH, CDMX, DGO, GTO, HGO, JAL, MEX, MICH, NLE, OAX, PUE, QRO, SLP, SON, TAMS, ZAC

Hybanthus yucatanensis Millsp. CAM, CHIS, COL, JAL, QROO, YUC

Ixchelia mexicana (Ging.) H.E. Ballard \& Wahlert BCS, CHIH, COL, GTO, GRO, HGO, JAL, MEX, MICH, OAX, QRO, SLP, SIN, SON, TAMS, VER, YUC

Ixchelia uxpanapana (T. Wendt) Wahlert \& H.E. Ballard OAX, VER

Orthion malpighiifolium (Standl.) Standl. \& Steyerm. CHIS, OAX, VER

*Orthion montanum Lundell CHIS

Orthion oblanceolatum Lundell CHIS, OAX, VER

Orthion subsessile (Standl.) Steyerm. \& Standl. CAM, CHIS,

TAB, VER

*Orthion veracrucense Lundell OAX, VER

Rinorea deflexiflora Bartlett OAX, VER

Rinorea guatemalensis (S. Watson) Bartlett CAM, CHIS, OAX, PUE, QROO, TAB, VER

Rinorea hummelii Sprague CAM, CHIS, OAX, QROO, TAB, VER, YUC

*Rinorea mexicana Lundell CHIS, VER

Rinorea sylvatica (Seem.) Kuntze OAX

Viola adunca J.E. Sm. CHIS, CHIH, SON

*Viola barroetana Hemsl. AGS, DGO, GTO, JAL, SLP, SIN,

TAMS, ZAC

*Viola beamanii Calderón MEX, VER

*Viola cochranei Ballard GTO, JAL, QRO, SLP

Viola douglasii Steudel BCN

*Viola flagelliformis Hemsl. CHIH, NLE, QRO, SLP, TAMS

*Viola galeanaensis M.S. Baker COAH, NLE

Viola grahamii Benth. CHIS, CHIH, COL, CDMX, DGO, GTO, GRO, HGO, JAL, MEX, MICH, MOR, NAY, NLE, OAX, PUE, QRO, SLP, SIN, SON, TAMS, TLAX, VER

Viola guatemalensis W. Becker CHIS, CDMX, GTO, GRO, HGO, JAL, MEX, MICH, MOR, OAX, SLP, VER

*Viola hemsleyana Calderón CDMX, HGO, JAL, MEX, MICH, OAX, VER

*Viola hookeriana Kunth CHIS, CHIH, CDMX, DGO, GTO, HGO, JAL, MEX, MICH, MOR, NAY, NLE, OAX, PUE, QRO, SLP, TAMS, VER

Viola humilis Kunth CHIS, CDMX, HGO, JAL, MEX, MICH, MOR, OAX, PUE, QRO, VER

*Viola jalapaensis W. Becker TAMS, VER

Viola lobata Benth. BCN

Viola lovelliana Brainerd COAH, NLE

Viola nannei Pol. CHIS, DGO, GTO, GRO, JAL, MEX, MICH, OAX, SLP, VER

*Viola nelsonii W. Becker CHIS, VER

Viola nephrophylla Greene COAH, NLE, SON

*Viola oxyodontis H.E. Ballard COL, GRO, JAL, MEX, MICH, SIN

*Viola painteri Rose \& House CDMX, HGO, MEX, MICH, MOR, OAX, PUE, TLAX, VER

Viola palustris L. CHIS 
Viola pedunculata Torr. \& A. Gray BCN

Viola purpurea Kellogg BCN

Viola scandens Humb. \& Bonpl. ex Roem. \& Schult. CHIS, GRO, OAX, VER

Viola seleriana Becker CHIS

Viola sororia Willd. COAH, NLE, VER

Viola umbraticola Kunth AGS, CHIH, CDMX, DGO, HGO, JAL, MEX, SLP, SON, TLAX, VER, ZAC

\section{Family Vitaceae}

Ampelocissus acapulcensis (Kunth) Planch. CHIS, COL, GRO, JAL, MEX, MICH, MOR, NAY, OAX, PUE, SLP, SIN, SON, VER, YUC

Ampelocissus erdvendbergiana Planch. CHIS, OAX, PUE, QRO, QROO, SLP, TAB, TAMS, VER, YUC

Ampelocissus mesoamericana Lombardi CHIS, JAL, VER

Ampelopsis cordata Michx. CHIH, GRO, MOR, SON, VER

Ampelopsis denudata Planch. BCS, CHIS, COL, GRO, JAL, MEX, MICH, MOR, NAY, OAX, SIN, SON, VER

Cissus alata Jacq. CAM, CHIS, COL, GRO, HGO, JAL, MEX, MICH, MOR, NAY, OAX, PUE, QRO, QROO, SLP, SIN, TAB, TAMS, VER, YUC

Cissus biformifolia Standl. CAM, CHIS, COL, GRO, MICH, MOR, OAX, PUE, QROO, TAB, VER

Cissus cacuminis Standl. CAM, CHIS, GRO, OAX, PUE, QROO, SLP, TAB, VER

*Cissus cucurbitina Standl. COL, GRO, JAL, MEX, MOR, NAY, OAX, SIN

Cissus erosa Rich. CAM, CHIS, GRO, MICH, MOR, NAY, OAX, QROO, SIN, TAB, VER, YUC

Cissus gossypifolia Standl. CAM, CHIS, GRO, OAX, QROO, TAB, VER, YUC

Cissus martiniana Woodson \& Seibert CHIS, OAX

*Cissus mexicana DC. BCS, NAY, OAX, SIN, SON

Cissus microcarpa Vahl AGS, CAM, CHIS, COL, GRO, JAL, MEX, MICH, MOR, NAY, OAX, PUE, QRO, QROO, SLP, SIN, TAB, TAMS, VER, YUC

*Cissus subtruncata Rose GRO, MEX, MOR, OAX, PUE, TAB Cissus tiliacea Kunth CAM, CHIS, CDMX, DGO, GTO, GRO, HGO, JAL, MEX, MICH, MOR, NAY, OAX, PUE, QRO, QROO, SIN, SON, TAMS, VER

Cissus trianae Planch. CHIS, GRO, OAX

Cissus trifoliata (L.) L. BCN, BCS, CAM, CHIH, COAH, COL, DGO, GTO, GRO, HGO, JAL, MEX, MICH, MOR, NLE, OAX, PUE, QRO, QROO, SLP, SIN, SON, TAMS, VER, YUC, ZAC *Cissus tuberosa DC. DGO, GRO, JAL, MEX, NAY, OAX, PUE

Cissus ulmifolia (Baker) Planch. CHIS, TAB, VER

Cissus verticillata (L.) Nicolson \& C.E. Jarvis AGS, CAM, CHIS, CHIH, COL, CDMX, DGO, GTO, GRO, HGO, JAL, MEX, MICH, MOR, NAY, OAX, PUE, QRO, QROO, SLP, SIN, SON, TAB, TAMS, TLAX, VER, YUC, ZAC

Parthenocissus quinquefolia (L.) Planch. BCS, CHIS, CHIH, COAH, COL, DGO, GTO, GRO, HGO, JAL, MEX, MICH, NAY, NLE, OAX, PUE, QRO, QROO, SLP, SIN, SON, TAMS, VER, YUC

Parthenocissus vitacea (Knerr) Hitchc. COAH
Vitis arizonica Engelm. BCN, BCS, CHIH, COAH, DGO, NLE, SIN, SON, TAMS

Vitis berlandieri Planch. COAH, COL, DGO, HGO, JAL, MICH, NAY, NLE, PUE, QRO, SLP, TAMS, VER, ZAC

*Vitis blancoi Munson emend. Comeaux COL, GRO, JAL, MEX, MICH, MOR, OAX, PUE, SLP

*Vitis bloodworthiana Comeaux DGO, JAL, NAY, ZAC

Vitis bourgaeana Planch. CAM, CHIS, GRO, HGO, JAL, MEX, MICH, MOR, OAX, PUE, QRO, QROO, SLP, TAB, TAMS, VER, ZAC

Vitis cinerea (Engelm.) Engelm. ex Millardet CHIH, COAH, DGO, GRO, HGO, JAL, MEX, MICH, NAY, NLE, OAX, PUE, SLP, SIN, SON, TAMS, VER, ZAC

Vitis girdiana Munson BCN, BCS

*Vitis jaegeriana Comeaux QRO, SLP, VER

Vitis mustangensis Buckley TAMS

*Vitis nesbittiana Comeaux VER

*Vitis peninsularis M.E. Jones BCS

*Vitis popenoei J.L. Fennell CHIS, HGO, OAX, PUE, QRO, SLP, TAB, VER

Vitis riparia Michx. COAH

Vitis rotundifolia Michx. OAX, SIN, TAMS, VER

Vitis tiliifolia Humb. \& Bonpl. ex Roem. \& Schult. BCS, CAM, CHIS, COL, DGO, GTO, GRO, HGO, JAL, MEX, MICH, MOR, NAY, NLE, OAX, PUE, QRO, QROO, SLP, SIN, TAB, TAMS, VER, YUC

\section{Family Vochysiaceae}

Vochysia guatemalensis Donn. Sm. CHIS, OAX, TAB, VER *Vochysia tabascana Sprague TAB, VER

\section{Family Winteraceae}

Drimys granadensis L. f. CHIS, GRO, HGO, OAX, PUE, QRO, SLP, VER

\section{Family Ximeniaceae}

Ximenia americana L. CAM, CHIS, COL, GRO, HGO, JAL, MICH, NAY, OAX, PUE, QROO, TAB, VER, YUC, ZAC

*Ximenia parviflora Benth. AGS, BCS, CHIS, COL, DGO, GTO, GRO, HGO, JAL, MEX, MICH, NAY, OAX, PUE, QRO, SLP, SIN, TAMS, ZAC

*Ximenia pubescens Standl. COL, JAL, OAX, SIN

\section{Family Xyridaceae}

Xyris ambigua Beyr. ex Kunth CAM, CHIS, QROO, TAB, TAMS, VER

Xyris jupicai L. Rich. CAM, CHIS, QROO, TAB, TAMS, VER *Xyris mexicana $\mathrm{S}$. Watson DGO, GTO, HGO, JAL, MICH, NAY, SLP, VER, ZAC

\section{Family Zingiberaceae}

Renealmia alpinia (Rottb.) Maas CHIS, OAX, PUE, TAB, VER Renealmia aromatica (Aubl.) Griseb. CAM, CHIS, OAX, SLP, TAB, VER

Renealmia mexicana Klotzsch ex Petersen CAM, CHIS, HGO, OAX, PUE, SLP, TAB, VER

Renealmia occidentalis (Sw.) Sw. CAM, CHIS, COL, JAL, OAX, VER

Renealmia pacifica (Maas) Maas \& H. Maas CHIS, GRO, NAY, 
OAX

\section{Family Zosteraceae}

Phyllospadix scouleri Hook. BCN, BCS

Phyllospadix torreyi S. Watson BCN, BCS

Zostera marina L. BCN, BCS, SIN, SON

\section{Family Zygophyllaceae}

* Fagonia barclayana (Benth.) Rydb. BCN, BCS, SON

Fagonia californica Benth. BCN, BCS, SON

*Fagonia densa I.M. Johnst. BCN, BCS, SON

Fagonia laevis Standl. BCN, BCS, SON

Fagonia longipes Standl. SON

Fagonia pachyacantha Rydb. BCN, BCS, SON

* Fagonia palmeri Vasey \& Rose BCN, BCS, SON

* Fagonia scoparia Brandegee CHIH, COAH, DGO

*Fagonia villosa D.M. Porter BCN, BCS

Guaiacum angustifolium Engelm. CHIH, COAH

Guaiacum coulteri A. Gray BCN, CHIS, CHIH, COAH, COL, GRO, JAL, MICH, NAY, OAX, PUE, SIN, SON, VER

Guaiacum sanctum L. CAM, CHIS, MICH, NAY, OAX, QROO,

TAB, VER, YUC

* Guaiacum unijugum Brandegee BCS

Kallstroemia californica (S. Watson) Vail AGS, BCN, BCS, CHIH, COAH, DGO, GRO, JAL, MICH, NAY, NLE, OAX, QRO, SLP, SIN, SON, TAMS, VER, ZAC

Kallstroemia caribaea Rydb. GRO, OAX, YUC

Kallstroemia grandiflora Torr. BCN, BCS, CHIS, CHIH, COAH, COL, DGO, GRO, JAL, NAY, SIN, SON, VER, ZAC

* Kallstroemia hintonii D. Porter JAL, MICH

Kallstroemia hirsutissima Vail CHIH, COAH, DGO, GTO,
HGO, NLE, PUE, QRO, SLP, SIN, SON, TAMS, VER, ZAC

*Kallstroemia longipes Rydb. CHIH, SON

Kallstroemia maxima (L.) Hook. \& Arn. BCS, CAM, CHIS, COL, DGO, GRO, HGO, JAL, MEX, MICH, MOR, NAY, NLE, OAX, PUE, QRO, QROO, SLP, SIN, SON, TAB, TAMS, VER, YUC, ZAC

Kallstroemia parviflora Norton AGS, BCS, CHIH, COAH, CDMX, DGO, GTO, GRO, HGO, JAL, MEX, MICH, MOR, NAY, NLE, PUE, QRO, SLP, SIN, SON, ZAC

* Kallstroemia peninsularis D.M. Porter BCS

Kallstroemia perennans B.L. Turner CHIH, COAH, DGO

Kallstroemia pubescens (G. Don) Dandy CAM, COL, GRO, JAL, MICH, OAX, QROO, SIN, VER

*Kallstroemia rosei Rydb. AGS, CHIS, CHIH, COL, CDMX, DGO, GTO, GRO, HGO, JAL, MEX, MICH, MOR, NLE, OAX, PUE, QRO, SLP, SIN, TAMS, TLAX, VER, ZAC

* Kallstroemia standleyi D.M. Porter OAX

Larrea tridentata (DC.) Coville AGS, BCN, BCS, CHIH, COAH, DGO, GTO, HGO, JAL, NLE, QRO, SLP, SIN, SON, TAMS, VER, ZAC

*Morkillia acuminata Rose \& Painter HGO, QRO, TAMS, VER

*Morkillia mexicana (Moc. \& Sessé) Rose \& Painter GTO, HGO, OAX, PUE, QRO, SLP, VER

Porlieria angustifolia (Engelm.) A. Gray CHIH, COAH, NLE, SLP, TAMS, VER

*Sericodes greggii A. Gray CHIH, COAH, DGO, NLE, ZAC

*Viscainoa geniculata (Kellogg) Greene BCN, BCS, SON

\section{References}

APG III. (2009). An update of the Angiosperm Phylogeny Group classification for the orders and families of flowering plants: APG III. Botanical Journal of the Linnean Society, 161, 105-121.

BFG (The Brazil Flora Group). (2015). Growing knowledge: An overview of seed plant diversity in Brazil. Rodriguesia, 66, 1-29.

Bisby, F. A. (2000). The quiet revolution: Biodiversity informatics and the Internet. Science, 289, 2309-2312.

Chase, M. W., \& Reveal, J. L. (2009). A phylogenetic classification of the land plants to accompany APG III. Botanical Journal of the Linnean Society, 161, 122-127.

Christenhusz, M. J. M., Chun, Z. X., \& Scheider, H. (2011). A linear sequence of extant families and genera of lycophytes and ferns. Phytotaxa, 19, 7-54.

Christenhusz, M. J. M., Reveal, J. L., Farjon, A., Garder, M. F., Mill, R. R., \& Chase, M. W. (2011). A new classification and linear sequence of extant gymnosperms. Phytotaxa, 19, 55-70.

Conabio (Comisión Nacional para el Conocimiento y Uso de la Biodiversidad) (2008). Capital natural de México. Vol. I: conocimiento actual de la biodiversidad. México, D.F.: Comisión Nacional para el Conocimiento y Uso de la Biodiversidad.

Conabio (Comisión Nacional para el Conocimiento y Uso de la Biodiversidad). (2012). Estrategia mexicana para la conservación vegetal, 2012-2030. México, D.F.: Comisión Nacional para el Conocimiento y Uso de la Biodiversidad.

Dávila, P., Mejía-Saulés, M. T., Gómez-Sánchez, M., Valdés-Reyna, J., Ortíz, J. J., Morín, C., et al. (2006). Catálogo de las gramíneas de México. México, D.F.: Universidad Nacional Autónoma de México, Comisión Nacional para el Conocimiento y Uso de la Biodiversidad.

Corlett, R. T. (2011). Trouble with the gray literature. Biotropica, 43, 3-5.
Ertter, B. (2000). Floristic surprises in North America North of México. Annals of the Missouri Botanical Garden, 87, 81-109.

García-Mendoza, A., \& Meave, J. A. (Eds.). (2011). Diversidad florística de Oaxaca: de musgos a angiospermas (colecciones y lista de especies). México, D.F.: Instituto de Biología, Universidad Nacional Autónoma de México, Comisión Nacional para el Conocimiento y Uso de la Biodiversidad.

Gaston, K. L., \& Williams, P. H. (1996). Spatial patterns in taxonomic diversity. In K. J. Gaston (Ed.), Biodiversity. A biology of numbers and difference (pp. 202-229). Oxford: Blackwell Science.

Germishuizen, G., Meyer, N. L., Steenkamp, Y., \& Keith, M. (2006). A checklist of South African plants. Southern African Botanical Diversity Network Report No. 41. Pretoria: SABONET.

Graham, C. H., Ferrier, S., Huettman, F., Moritz, C., \& Peterson, A. T. (2004). New developments in museum-based informatics and applications in biodiversity analysis. Trends in Ecology and Evolution, 19, 497-503.

Guzmán, U., Arias, S., \& Dávila, P. (2003). Catálogo de cactáceas mexicanas. México, D.F.: Universidad Nacional Autónoma de México, Comisión Nacional para el Conocimiento y Uso de la Biodiversidad.

Haston, E., Richardson, J. E., Stevens, P. F., Chase, M. W., \& Harris, D. J. (2009). The linear Angiosperm Phylogeny Group (LAPG) III: A linear sequence in APG III. Botanical Journal of the Linnaean Society, 161, $128-131$.

Ibarra-Manríquez, G., Villaseñor, J. L., \& Durán, G. R. (1995). Riqueza de especies y endemismo del componente arbóreo de la península de Yucatán, México. Boletín de la Sociedad Botánica de México, 57, 49-77.

Llorente-Bousquets, J., \& Ocegueda, S. (2008). Estado del conocimiento de la biota. In J. Soberón, G. Halffter, \& J. Llorente-Bousquets (Comps.), Capital natural de México. Conocimiento actual de la biodiversidad. Vol. I, (pp. 283-322). México, D.F.: Comisión Nacional para el Conocimiento y Uso de la Biodiversidad. 
Luna-Vega, I., Espinosa, D., Rivas, G., \& Contreras-Medina, R. (2013). Geographical patterns and determinants of species richness in Mexico across selected families of vascular plants: Implications for conservation. Systematics and Biodiversity, 11, 237-256.

May, R. M. (1990). Taxonomy as destiny. Nature, 347, 129-130.

Nimis, P. L. (1996). Towards a checklist of Mediterranean lichens. Bocconea, $6,5-17$.

Qian, H., \& Ricklefs, R. E. (1999). A comparison of the taxonomic richness of vascular plants in China and the United States. American Naturalist, 154, $160-181$.

Ramírez-Delgadillo, R., Vargas-Ponce, O., Arreola-Nava, H. J., CedanoMaldonado, M., González-Tamayo, R., González-Villarreal, L. M., et al. (2010). Catálogo de plantas vasculares de Jalisco. Guadalajara, Jalisco: Universidad de Guadalajara, Sociedad Botánica de México y Universidad Autónoma Metropolitana.

Rangel, C. J. O. (2015). La riqueza de las plantas con flores de Colombia. Caldasia, 37, 279-307.

Rivera-Hernández, J. E., \& Flores-Hernández, N. (2013). Flora y vegetación del Distrito Federal. Conservación y problemática. México, D.F.: Universidad Autónoma Metropolitana, Unidad Iztapalapa, Centro de Estudios Geográficos, Biológicos y Comunitarios, S.C.

Rodríguez, A. M., Villaseñor, J. L., Coombes, A. J., \& Cerón, C. A. B. (2014). Flora del estado de Puebla, México. Puebla, México: Benemérita Universidad Autónoma de Puebla.

Rzedowski, J. (1991). Diversidad y orígenes de la flora fanerogámica de México. Acta Botanica Mexicana, 14, 3-21.

Semarnat. (2010). Norma Oficial Mexicana NOM-059-SEMARNAT-2010. Protección ambiental-especies nativas de México de flora y fauna
silvestres-Categorías de riesgo y especificaciones para su inclusión, exclusión o cambio-Lista de especies en riesgo. Diario Oficial. Thursday December 30, 2010.

Soberón, J., \& Peterson, A. T. (2004). Biodiversity informatics: Managing and applying primary biodiversity data. Philosophical Transactions of the Royal Society B, 359, 689-698.

Villarreal, Q. J. A., \& Estrada, C. E. (2008). Flora de Nuevo León. Listados florísticos de México, XXIV. México, D.F.: Instituto de Biología, Universidad Nacional Autónoma de México.

Villaseñor, J. L. (2003). Diversidad y distribución de las Magnoliophyta de México. Interciencia, 28, 160-167.

Villaseñor, J. L. (2004). Los géneros de plantas vasculares de la flora de México Boletín de la Sociedad Botánica de México, 75, 105-135.

Villaseñor, J. L. (2015). ¿La crisis de la biodiversidad es la crisis de la taxonomía? Botanical Sciences, 93, 3-14.

Villaseñor, J. L., \& Espinosa-García, F. (2004). The alien flowering plants of Mexico. Diversity and Distributions, 10, 113-123.

Villaseñor, J. L., \& Ortiz, E. (2014). Biodiversidad de las plantas con flores (División Magnoliophyta) en México. Revista Mexicana de Biodiversidad, 85 (Suppl.), S134-S142.

Villaseñor, J. L., Ortiz, E., Beutelspacher, C. R., \& Gómez-López, J. A. (2013). La familia Asteraceae en el municipio de San Cristóbal de Las Casas, Chiapas, México. Lacandonia, 7, 31-55.

Walter, K. S., \& Gillett, H. J. (1998). 1997 IUCN red list of threatened plants. Cambridge: IUCN, The World Conservation Union.

Wearn, J. A., Chase, M. W., Mabberley, D. J., \& Couch, C. (2013). Utilizing a phylogenetic plant classification for systematic arrangements in botanic gardens and herbaria. Botanical Journal of the Linnaean Society, 172, 127-141. 